\title{
ESICM LIVES 2021: Part 2
}

\author{
Virtual. 03-06 October 2021 \\ Published: 11 October 2021
}

\section{0}

Relationship between the Clinical Frailty Scale and short-term mortality in patients $\geq \mathbf{8 0}$ years old acutely admitted to the ICU-a prospective cohort study

J. Fronczek ; K. Polok'; D. De Lange ${ }^{2}$; C. Jung ${ }^{3}$; M. Beil'; ; A. Rhodes ; J.

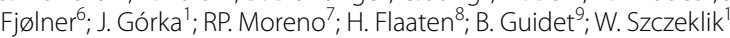
${ }^{1}$ Centre for Intensive Care and Perioperative Medicine, Jagiellonian University Medical College, Kraków, Poland; ${ }^{2}$ Department of Intensive Care Medicine, Utrecht University, Utrecht, Netherlands: ${ }^{3}$ Division of Cardiology, Pulmonology and Vascular Medicine, University Hospital of Düsseldorf, Düsseldorf, Germany; ${ }^{4}$ Medical Intensive Care Unit, Hadassah Medical Center, Jerusalem, Israel; ${ }^{5}$ Anaesthesia and Intensive Care Medicine, St George's University Hospitals NHS Foundation Trust, London, United Kingdom; ${ }^{6}$ Department of Intensive Care, Aarhus University Hospital, Aarhus, Denmark; ${ }^{7}$ Unidade de cuidados intensivos neurocríticos, Hospital de São José, Centro Hospitalar de Lisboa Central, Nova Médical School, Lisbon, Portugal; ${ }^{8}$ Department of Anaesthesia and Intensive Care, University of Bergen, Bergen, Norway; ${ }^{9}$ Réanimation Médicale, Hôpital Saint-Antoine, Paris, France

Correspondence: J. Fronczek

Intensive Care Medicine Experimental 2021, 9(1): 000440

Introduction. The Clinical Frailty Scale (CFS) is frequently used to measure frailty in critically ill adults. There is wide variation in the approach to analysing the relationship between the CFS score and mortality after admission to the ICU.

Methods. We used data from two multicentre prospective cohort studies which enrolled very old intensive care unit patients in 26 countries to evaluate the influence of modelling approach on the association between the CFS score and mortality. The primary outcome was mortality within 30-days from admission to the ICU. Logistic regression models for both ICU and 30-day mortality included the CFS score as either a categorical, continuous or dichotomous variable and were adjusted for patient's age, sex, reason for admission to the ICU, and admission Sequential Organ Failure Assessment score.

Results. The median age in the sample of 7487 consecutive patients was 84 years (IQR 81-87). The highest fraction of new prognostic information from frailty in the context of 30-day mortality was observed when the CFS score was treated as either a categorical variable using all original levels of frailty or a nonlinear continuous variable and was equal to $9 \%$ using these modelling approaches $(p<0.001)$. The relationship between the CFS score and mortality was nonlinear $(p<0.01)$. Conclusion. Knowledge about a patient's frailty status adds a substantial amount of new prognostic information at the moment of admission to the ICU. Arbitrary simplification of the CFS score into fewer groups than originally intended leads to a loss of information and should be avoided.
000444

Respiratory muscle strength is associated with functional exercise capacity in survivors of critical illness: a prospective cohort study with 6-month follow-up

M. Major ; M. Van Egmond'; S. Ramaekers, ${ }^{2}$; R. Engelbert, ${ }^{2}$; M. Van Der

Schaaf ${ }^{3}$

${ }^{1}$ Faculty of health, center of expertise urban vitality, Hogeschool van Amsterdam (HvA), Amsterdam University of Applied Sciences, Amsterdam, Netherlands; ${ }^{2}$ Faculty of health, center of expertise urban vitality, Amsterdam University of Applied Sciences, Amsterdam, Netherlands; ${ }^{3}$ Department of rehabilitation medicine, Amsterdam UMC, University of Amsterdam, Amsterdam Movement Sciences, Amsterdam, Netherlands

Correspondence: M. Major

Intensive Care Medicine Experimental 2021, 9(1): 000444

Introduction. The detrimental physiological effects of critical illness on skeletal muscle have been well described, leaving survivors with severely decreased muscle strength, contractile function and muscle endurance, also known as ICU-acquired weakness [1-9].To date, little information exists about the long-term effect of critical illness and mechanical ventilation on respiratory muscles, and if respiratory muscle strength is associated with (recovery of) other physical outcomes.

Objectives. The aim of this study was to describe the 6-month course of (recovery) of respiratory muscle strength and its' association with functional exercise capacity in survivors of critical illness.

Methods. A prospective cohort study was conducted with a 6-month follow up on survivors of critical illness who had been mechanically ventilated for $\geq 48 \mathrm{~h}$. Measurements were conducted at 1-2 weeks, 3- and 6 months after hospital discharge. Primary outcomes were maximum inspiratory and expiratory pressure (PImax and PEmax), converted to predicted values adjusted for age and gender. Secondary outcome was functional exercise capacity, tested with the two-minute step test (TMST) [10]. Ethical approval was granted (Amsterdam UMC, location AMC, ref: NL68475.018.19). Descriptive analyses were conducted for sample characteristics, primary and secondary outcome variables. Bivariate correlations were investigated with the Pearson's or Spearman's rank test as appropriate for (non)parametric data. Mixed model analysis was performed to determine the course of recovery and establish potential longitudinal relationships between (prognostic) variables.

Results. We included 59 participants (age mean/SD $59 \pm 12.6,64.4 \%$ male) with a median ICU and hospital length of stay (LOS) of 11 and 35 days respectively. PImax improved significantly between all timepoints but did not reach predicted values at 6 months. PEmax recovered to predicted values at 3 months. Functional exercise capacity improved significantly between timepoints. Hospital LOS and age were independently, significantly associated with decreased PImax 
and functional exercise capacity. Significant positive correlations at each of the timepoints as well as a significant positive longitudinal relationship were found between PImax/PEmax and functional exercise capacity. Table: change in outcomes over time.

\begin{tabular}{|c|c|c|c|c|c|c|c|}
\hline Variable & Baseline & $\begin{array}{l}3 \text { months } \\
\text { F.U. }\end{array}$ & $\begin{array}{l}\Delta \text { T1-T0 } \\
(95 \% \\
\text { C.I) }\end{array}$ & $\begin{array}{l}\mathrm{p} \\
\text { value } \\
\text { T0-T1 }\end{array}$ & $\begin{array}{l}6 \text { months } \\
\text { F.U. }\end{array}$ & $\begin{array}{l}P \\
\text { value } \\
\text { T1-T2 }\end{array}$ & $\begin{array}{l}\Delta \mathrm{T} 2-\mathrm{T} 1 \\
95 \% \mathrm{Cl}\end{array}$ \\
\hline & $n=45$ & $n=47$ & & & $n=44$ & & \\
\hline \multirow{2}{*}{$\begin{array}{l}\text { PImax \% } \\
\text { predicted } \\
\mathrm{cmH}_{2} \mathrm{O} \\
(\text { mean, } \\
95 \% \text { C.I) }\end{array}$} & $\begin{array}{l}68.1 \\
\quad(62.0- \\
74.2)\end{array}$ & $\begin{array}{l}91.2 \\
\quad(83.6-98.9)\end{array}$ & $\begin{array}{l}30.4 \\
\quad(24.5- \\
36.3)\end{array}$ & 0.000 & $\begin{array}{l}98.5 \\
\quad(90.7-1.06)\end{array}$ & 0.002 & $\begin{array}{l}7.2 \\
(2.9- \\
11.6)\end{array}$ \\
\hline & $n=44$ & $n=47$ & & & $n=44$ & & \\
\hline \multirow{2}{*}{$\begin{array}{l}\text { PEmax \% } \\
\text { predicted } \\
\mathrm{CmH}_{2} \mathrm{O} \\
\text { (mean, } \\
95 \% \text { C.I) }\end{array}$} & $\begin{array}{c}76.6 \\
(69.4- \\
83.8)\end{array}$ & $\begin{array}{c}100.6(93.0- \\
108.3)\end{array}$ & $\begin{array}{l}28.5 \\
\quad(21.6- \\
35.3)\end{array}$ & 0.000 & $\begin{array}{c}1.05(97.9- \\
112.4)\end{array}$ & 0.100 & $\begin{array}{l}4.3 \\
\quad(-0.9- \\
9.5)\end{array}$ \\
\hline & $n=41$ & $n=46$ & & & $n=40$ & & \\
\hline $\begin{array}{c}\text { Functional } \\
\text { exercise } \\
\text { capacity } \\
\text { (mean } \\
\text { steps, } \\
\text { (95\% C.I) }\end{array}$ & $\begin{array}{l}54.3 \\
\quad(47.3- \\
61.3)\end{array}$ & $\begin{array}{l}79.7 \\
\quad(72.4-87.1)\end{array}$ & $\begin{array}{l}31.9 \\
\quad(22.7- \\
41.0)\end{array}$ & 0.000 & $\begin{array}{l}86.2 \\
\quad(78.1-94.2)\end{array}$ & 0.042 & $\begin{array}{l}6.6 \\
(0.3- \\
12.9)\end{array}$ \\
\hline
\end{tabular}

Conclusion. Significant-but not full-recovery was observed for inspiratory muscle strength and exercise capacity up to 6 months after critical illness, while a shorter hospital LOS and younger age were prognostic for recovery. As respiratory muscle strength was significantly associated with functional exercise capacity, it seems justified to further explore the effectiveness of respiratory muscle training beyond ICU and hospital discharge.

\section{Reference(s)}

1. [10] Rikli RE, Jones CJ. Development and validation of a functional fitness test for community-residing older adults. Journal of aging and physical activity. 1999 Apr 1;7(2):129-61.

2. [9] Mayer KP, Welle MM, Evans CG, Greenhill BG, Montgomery-Yates AA, Dupont-Versteegden EE, Morris PE, Parry SM. Muscle power is related to physical function in patients surviving acute respiratory failure: a prospective observational study. The American Journal of the Medical Sciences. 2021 Mar 1:361(3):310-8.

3. [8] Schefold JC, Wollersheim T, Grunow JJ, Luedi MM, Z'Graggen WJ, Weber-Carstens S. Muscular weakness and muscle wasting in the critically ill. Journal of cachexia, sarcopenia and muscle. 2020 Dec;11(6):1399-412.

4. [7] Saccheri C, Morawiec E, Delemazure J, Mayaux J, Dubé BP, Similowski T, Demoule A, Dres M. ICU-acquired weakness, diaphragm dysfunction and long-term outcomes of critically ill patients. Annals of intensive care. 2020 Dec;10(1):1-9.

5. [6] van Gassel RJ, Baggerman MR, van de Poll MC. Metabolic aspects of muscle wasting during critical illness. Current opinion in clinical nutrition and metabolic care. 2020 Mar;23(2):96.

6. [5] Wandrag L, Brett SJ, Frost GS, Bountziouka V, Hickson M. Exploration of muscle loss and metabolic state during prolonged critical illness: Implications for intervention?. PloS one. 2019 Nov 14;14(11):e0224565.

7. [4] Piva S, Fagoni N, Latronico N. Intensive care unit-acquired weakness: unanswered questions and targets for future research. F1000Research. 2019;8.

8. [3] Parry SM, Puthucheary ZA. The impact of extended bed rest on the musculoskeletal system in the critical care environment. Extreme physiology \& medicine. 2015 Dec;4(1):16.

9. [2] McClave SA, Wischmeyer PE, Miller KR, van Zanten AR. Mitochondrial dysfunction in critical illness: implications for nutritional therapy. Current nutrition reports. 2019 Dec;8(4):363-73.

10. [1] Latronico N, Herridge M, Hopkins RO, Angus D, Hart N, Hermans $G$ Iwashyna T, Arabi Y, Citerio G, Ely EW, Hall J. The ICM research agenda on intensive care unit-acquired weakness. Intensive care medicine. 2017 Sep;43(9):1270-81.

11. Mel Major's PhD research is funded by the Dutch Research Council (NWO).

\section{5}

Quality Improvement Project on Social History Taking and Frailty Assessments for ICU Admissions at Buckinghamshire Healthcare

\section{NHS Trust}

S. De Soysa'; A. Nadeem ${ }^{1}$; K. Panikkarr';. Ali ${ }^{1}$

${ }^{1}$ Intensive care medicine, Stoke Mandeville Hospital, Aylesbury, United Kingdom

Correspondence: S. De Soysa

Intensive Care Medicine Experimental 2021, 9(1): 000455

Introduction. Undertaking a thorough social history and frailty assessment appropriately informs treatment escalation plans for patients admitted to the intensive care unit (ICU) [1]. Studies have found that pre-ICU admission frailty correlates with increased risk of post-ICU disability, new discharge to nursing homes and mortality $[2,3,4,5,6]$.

Despite this clear importance and implication on patient care, there is great variation in how thoroughly such assessments are undertaken. This was therefore, evaluated at the ICU at Buckinghamshire Healthcare NHS Trust (BHT).

Objectives. To review and improve the quality of documentation for social history and frailty assessment.

Methods. Due to a lack of existing guidelines on social history taking and frailty assessments within the critical care department a standard for comparison had to first be determined and set. This was discussed with the clinical team to establish the questions that were felt necessary for a high-quality social history and frailty assessment. Studies have also found that the Clinical Frailty Scale can help identify patients at increased risk of adverse outcomes [7].

Standardised questions were agreed by a group of ICU consultants. A retrospective unselected assessment of 20 consecutive patients admitted to ICU was undertaken using 'Evolve', an electronic database of medical records. The admission notes were analysed to assess whether the social history and frailty assessment documentation were in line with our set standard.

A social history and frailty assessment proforma was subsequently designed to ensure standardised questions were asked and documented. It was then trialled in 17 new admissions in February 2021. The quality of documentation pre and post proforma was compared to assess for improvement.

Results. The results are summarised as follows:

\begin{tabular}{lccc}
\hline & $\begin{array}{l}\text { Pre-proforma } \\
\text { (\% documented) }\end{array}$ & $\begin{array}{l}\text { Post-proforma } \\
\text { (\% documented) }\end{array}$ & $\begin{array}{l}\text { Fold of } \\
\text { improvement }\end{array}$ \\
\hline Smoking & 15 & 100 & 6.7 \\
Alcohol & 10 & 88 & 8.8 \\
Illicit drugs & 5 & 94 & 18.8 \\
Occupation & 20 & 100 & 5.0 \\
Type of accommodation & 5 & 94 & 18.8 \\
Living alone & 80 & 100 & 1.3 \\
Current care package & 15 & 100 & 6.7 \\
Previous DNA CPR & 20 & 94 & 4.7 \\
Walking ability & 55 & 100 & 1.8 \\
Walking aids & 47 & 100 & 2.1 \\
Walking distance & 11 & 100 & 9.0 \\
$\quad$ exercise tolerance & & & 56 \\
What limits exercise & 0 & 56 & 6.7 \\
$\quad$ tolerance & & 100 & 71 \\
Stairs ability & 15 & 71 & \\
Stair aids & 0 & &
\end{tabular}




\begin{tabular}{lccc}
\hline & $\begin{array}{l}\text { Pre-proforma } \\
\text { (\% documented) }\end{array}$ & $\begin{array}{l}\text { Post-proforma } \\
\text { (\% documented) }\end{array}$ & $\begin{array}{l}\text { Fold of } \\
\text { improvement }\end{array}$ \\
\hline $\begin{array}{l}\text { Number of stairs man- } \\
\text { aged }\end{array}$ & 6 & 78 & 14.0 \\
Exercise frequency & 5 & 65 & 12.9 \\
$\begin{array}{l}\text { Activities of daily living } \\
\text { Clinical Frailty Scale }\end{array}$ & 25 & 94 & 3.8 \\
\hline
\end{tabular}

Conclusion. Prior to the introduction of the proforma the documentation of social history and frailty for ICU admissions in BHT was poor and did not meet the standard. The standardised proforma demonstrated significant improvement in documentation across all domains. We recommend implementation of the proforma for critical care admissions through inclusion within the admission pack in order to better inform treatment escalation plans. We also aim for periodic review of its use through repeat audits and review of our set standards. Furthermore, we would recommend and encourage its use in other clinical departments.

\section{Reference(s)}

1. [1] Falvey, J.R. and Ferrante, L.E., 2019. Frailty assessment in the ICU: translation to'real-world'clinical practice.

2. [2] Ferrante, L.E., Pisani, M.A., Murphy, T.E., Gahbauer, E.A., Leo-Summers, L.S. and Gill, T.M., 2018. The association of frailty with post-ICU disability, nursing home admission, and mortality: a longitudinal study. Chest, 153(6), pp.1378-1386.

3. [3] Flaatten, H., De Lange, D.W., Morandi, A., Andersen, F.H., Artigas, A. Bertolini, G., Boumendil, A.. Cecconi, M., Christensen, S., Faraldi, L. and Fjølner, J., 2017. The impact of frailty on ICU and 30-day mortality and the level of care in very elderly patients ( $\geq 80$ years). Intensive care medicine, 43(12), pp.1820-1828

4. [4] Bagshaw, S.M., Stelfox, H.T., McDermid, R.C., Rolfson, D.B., Tsuyuki, R.T. Baig, N., Artiuch, B., Ibrahim, Q., Stollery, D.E., Rokosh, E. and Majumdar, S.R., 2014. Association between frailty and short-and long-term outcomes among critically ill patients: a multicentre prospective cohort study. Cmaj, 186(2), pp.E95-E102

5. [5] Darvall, J.N., Boonstra, T., Norman, J., Murphy, D., Bailey, M., Iwashyna, T.J., Bagshaw, S.M. and Bellomo, R., 2019. Retrospective frailty determination in critical illness from a review of the intensive care unit clinical record. Anaesthesia and intensive care, 47(4), pp.343-348.

6. [6] Muscedere, J., Waters, B., Varambally, A., Bagshaw, S.M., Boyd, J.G., Maslove, D., Sibley, S. and Rockwood, K., 2017. The impact of frailty on intensive care unit outcomes: a systematic review and meta-analysis. Intensive care medicine, 43(8), pp.1105-1122.

7. [7] Flaatten, H., Guidet, B., Andersen, F.H., Artigas, A., Cecconi, M. Boumendil, A., Elhadi, M., Fjølner, J., Joannidis, M., Jung, C. and Leaver, S., 2021. Reliability of the Clinical Frailty Scale in very elderly ICU patients: a prospective European study. Annals of intensive care, 11(1), pp.1-7.

\section{7}

Incidence, risk factors and impact on outcome of iatrogenic events: a one-year study in intensive care unit

S. AYED ${ }^{1}$; R. Jemmali ${ }^{1}$; A. Jamoussi' ${ }^{1}$; E. Rachdi ${ }^{1}$; F. Jarraya ${ }^{1}$; J. Benkhelil

${ }^{1}$ Intensive care, Hôpital Abderrahmen Mami de pneumo-phtisiologie, Ariana, Tunisia

Correspondence: S. AYED

Intensive Care Medicine Experimental 2021, 9(1): 000467

Introduction. Intensive care units (ICU) are structures with a high risk of iatrogenic events (IE) due to the often invasive and urgent nature of the procedures performed and the precarious state of patients hospitalized. In Tunisia, few data are available concerning the incidence andcharacteristics of these incidents.

Objectives. The aim of this study was to find out the incidence of IE in ICU, describe their different types and analyze their risk factors.

Methods. This was a prospective observational study performed at the ICU of the Abderrahmen Mami Hospital over a period of one year. It included all patients hospitalized in the ICU for more than $24 \mathrm{~h}$. Patients who had IE in another ward or in end of life stage were excluded. We collected demographic characteristics, severity scores, reason for admission, IE types and time of occurrence, ICU workload expressed by the Omega score, duration of mechanical ventilation and icu length of stay.

Results. During the study period, 384 patients were enrolled. Mean age was $59 \pm 17$ years. IEs were observed in 125 patients with 316 episodes, representing an incidence of $36 \%$ and an incidence density of 3 per 100 days-patients. Out of $316 \mathrm{IE}, 81,9 \%$ were classified as preventable and $46,8 \%$ were severe. The average time to occurrence of IE was $8 \pm 8$ days. IE were care-related in $67,1 \%$ of cases, procedure-related in $17,7 \%$ of cases, and drug-related in $15,2 \%$ of cases. The occurrence of IE was associated with an increase of duration of mechanical ventilation and length of stay. Mortality in IE group was significantly higher with $10.3 \%$ mortality directly related to an IE. In univariate analysis, identified risk factors for occurrence of IE were age, history of asthma, severity on admission, ICU workload expressed by the Omega score, duration of mechanical ventilation and length of stay. Multivariate analysis showed that ICU workload was the only independent predictor of the occurrence of an IE with a cut-off of 98 (AUC $=0.796$, $p<10-3$, sensitivity $=72 \%$, specificity $=73 \%$ ).

Dead patients were significantly older and severe on admission. They experienced more IEs especially the care-related ones. Life-threatening IEs, OMEGA score, ICU length of stay and MV duration were significantly higher in dead patient. Multivariate analysis showed that severity on admission, IE occurrence and OMEGA score were independent factors associated to mortality.

Conclusion. IE are frequent and preventable. They are associated with a high morbidity and mortality. ICU workload was an independent predictor of their occurrence. Reporting and classifying IE may allow a better targeting of preventive measures and implementing of safety practices.

\section{6}

Using long-term predicted Quality of Life in ICU clinical practice: a feasibility study

N. Wubben ${ }^{1}$; M. Zegers, ${ }^{1}$; J. Ramjith, ${ }^{2}$; LL. Bisschops, ${ }^{1} ;$ T. Frenzel, $^{1}$; JGVD.

Hoeven, ; MVD. Boogaard,

'Department of intensive care medicine, Radboud University Medical Center, Nijmegen, Netherlands; ${ }^{2}$ Health evidence, Radboud University

Medical Center, Nijmegen, Netherlands

Correspondence: $\mathrm{N}$. Wubben

Intensive Care Medicine Experimental 2021, 9(1): 000516

Introduction. Decision-making in the ICU is largely based on doctors experience and intuition, due to a lack of patient-reported outcome information regarding long-term physical and mental functioning. To increase the incorporation of long-term patient-reported outcome data in ICU clinical practice, we have developed the PREdicting PAtients' long-term outcome for REcovery (PREPARE) prediction model for change in QoL one year after ICU admission.

Objectives. To examine the feasibility of using the PREPARE prediction model for long-term QoL in ICU clinical practice as a means of discussing and preparing patients for life post-ICU in preparation for a larger trial.

Methods. This feasibility study was carried out between June 2020 and February 2021 in a tertiary center. The model, and intervention, are focused on expected ICU survivors. The intervention consists of three parts: 1. the PREPARE prediction for change in QoL 1 year after ICU admission, visualized in a bar chart; 2 . a conversation about life post-ICU and quality of life; and 3. an extensive information leaflet about recovery after an ICU stay. The intervention was incorporated in 25 conversations between physicians and patients and family members. The involved physicians, nurses and patients and/or family members were interviewed to evaluate their experiences with the PREPARE model in ICU practice. Transcripts of the conversations, as well as field notes and the evaluation interviews, were analysed qualitatively using thematic coding with the five distinctive features of feasibility studies 1, such as the acceptability of the intervention and study procedures and a preliminary evaluation of the intervention, as a guiding principle. 
Results. Five patients (20.0\%) were female. Median age was 68.0. Seven patients $(28.0 \%)$ died within the ICU admission after having been previously selected as an expected survivor. Using the intervention in conversations regarding expected survivors posed a challenge, as these patients were often discharged before conversations could take place. The intervention was generally deemed acceptable by clinicians and patients and family members. Participants' feelings about the prediction, the conversation and the leaflet ranged from indifferent, often when participants expressed a general scepticism about using predictions, to positive, often when family members felt reassured and heartened by the information. Thoughts on the best time point during admission for intervention execution varied. Some participants felt the model needed more data or wanted more information on different recovery trajectories, while other participants were glad the intervention provided direct motivation to talk about treatment wishes and recovery expectations.

Conclusion. The results indicate that future use of the intervention may be of use for ICU clinicians and patients and family members, though intervention improvements may be necessary before further testing is carried out.

\section{Reference(s)}

1. 1. Orsmond Gl, Cohn ES. The Distinctive Features of a Feasibility Study: Objectives and Guiding Questions. OTJR (Thorofare N J). 2015 Jul;35(3):169-77. https://doi.org/10.1177/1539449215578649. PMID: 26594739.

\section{3}

\section{Latrogenic blood loss in Critical Care}

J. Taylor ; N. Manning, ${ }^{2}$; D. Parekh, ${ }^{2}$

${ }^{1}$ Intensive care department, Queen Elizabeth Hospital Birmingham, Birmingham, France; ${ }^{2}$ Intensive care department, Queen Elizabeth Hospital Birmingham, Birmingham, United Kingdom

Correspondence: J. Taylor

Intensive Care Medicine Experimental 2021, 9(1): 000523

Introduction. Critical Care patients generally require more monitoring than other hospital patients. Arterial blood gases (ABGs) and blood sampling form an essential part of this. It is common to draw full tubes of blood from adults despite this often being many times the volume necessary for analysis (1). A 'discard' further increases the volume taken from each patient (1). Anaemia is common in intensive care unit (ICU) patients with haemodilution, blood loss and blood sampling contributing $(2,3)$. Reduced phlebotomy volumes show association with reduced blood transfusions in prolonged stay ICU patients (4). Volumes may be reduced, and anaemia potentially prevented, by using blood conserving arterial line systems $(5,6)$ and by using paediatric size blood bottles (7) for collection.

Objectives. To investigate potential causes of iatrogenic blood loss in critical care patients and potential solutions for this

Methods. Data collection was done in a tertiary centre ICU in the West Midlands, UK. The ICU has over 100 beds in four units with specialised cardiac, neurology, liver, trauma, and burns patients. Two auditors collected the data on all patients in ICU without major haemorrhage over two sequential $24 \mathrm{~h}$ periods. The following were collected: number of blood gases done; number and type of sample tube used; documented indications for clotting tests. Discard volumes varied between 5-10 $\mathrm{ml}$ each time a vascular access line was used for a blood sample. A $5 \mathrm{ml}$ discard was assumed to avoid overestimation of volume. Sample tube volumes were: serum tube $3.5 \mathrm{ml}$; EDTA tube $4 \mathrm{ml}$; sodium citrate tube $3.5 \mathrm{ml}$. ABG syringe volume was $2 \mathrm{ml}$.

Results. The average number of $A B G s / 24 h$ was 7.6 and blood loss was $53.2 \mathrm{ml} /$ patient $/ 24 \mathrm{~h}$. The average number of blood taken for routine sampling over $24 \mathrm{~h}$ was $20 \mathrm{ml} /$ patient ( 1.26 serum tubes; 1.12 EDTA tubes; 1.18 sodium citrate tubes). Total blood loss was $73.2 \mathrm{ml} /$ patient/24 h. Over $48 \mathrm{~h}, 92$ sodium citrate samples were taken for clotting profiles of which 12/91 were deranged and 23/91 had a documented indication.

Conclusion. This study demonstrated the average ICU patient loses $73.2 \mathrm{ml}$ blood$/ 24 \mathrm{~h}$ to blood sampling. There are cost implications with the sampling in this study costing approximately $£ 67.80 /$ patient $/ 24 \mathrm{~h}$ (8). The average ICU stay is four days (9) which translates to $292.8 \mathrm{ml}$ of blood venesected. Considering many patients require longer stays the burden can be much higher. In 13.5 days $1 \mathrm{~L}$ of blood may be taken from the average ICU patient. Anecdotally, a contributor to this burden may be regular ABGs not specifically requested by medical staff and repeated requests for clotting profiles which are unlikely to change day-to-day. Moving forward we suggest: implementing paediatric bottles for routine sampling where possible; introducing blood conservation devices for arterial and venous access lines; review the necessity of discards and if required implementing standardised volumes; documenting indications for daily clotting profiles.

\section{Reference(s)}

1. 1) Dale JC, Pruett SK. Phlebotomy - a minimalist approach. Mayo Clin Proc. 1993; Mar;68(3):249-55. https://doi.org/10.1016/s0025-6196(12) 60044-5.

2. 2) Retter A, Wyncoll D, Pearse R, Carson D, McKechnie S, Stanworth S, Allard S, Thomas D, Walsh T; British Committee for Standards in Haematology. Guidelines on the management of anaemia and red cell transfusion in adult critically ill patients. Br J Haematol. 2013; Feb;160(4):445-64. https://doi.org/10.1111/bjh.12143.

3. 3) Thavendiranathan P, Bagai A, Ebidia A, Detsky AS, Choudhry NK. Do blood tests cause anemia in hospitalized patients? The effect of diagnostic phlebotomy on hemoglobin and hematocrit levels. J Gen Intern Med. 2005;20(6):520-524.

4. 4) Harber CR, Sosnowski KJ, Hegde RM. Highly conservative phlebotomy in adult intensive care-a prospective randomized controlled trial. Anaesth Intensive Care. 2006; Aug;34(4):434-7. https://doi.org/10.1177/ $0310057 X 0603400414$

5. 5) Mukhopadhyay A, Yip HS, Prabhuswamy D, Chan YH, Phua J, Lim TK, Leong P. The use of a blood conservation device to reduce red blood cell transfusion requirements: a before and after study. Crit Care. 2010;14(1):R7. https://doi.org/10.1186/cc8859

6. 6) Maclsaac CM, Presneill J Boyce CA, Byron KL, Cade JF The influence of a blood conserving device on anaemia in intensive care patients. Anaesth Intensive Care. 2003 Dec;31(6):653-7. https://doi.org/10.1177/ $0310057 X 0303100607$

7. 7) Wu Y, Spaulding AC, Borkar S, Shoaei M, Mendoza M, Grant R, Barber B, Johns G, Franco PM. Reducing blood loss by changing to small volume tubes for laboratory testing. Mayo Clin Proc. 2021; 5(1): 72-83. https://doi. org/10.1016/j.mayocpiqo.2020.08.007.

8. 8) National Clinical Guideline Centre, Preoperative tests: Routine preoperative tests for elective surgery [NG45, Appendix M: Economic considerations for Delphi]. 2016. Available from: https://www.nice.org.uk/ guidance/ng45/documents/guideline-appendices-13. [Accessed 11th April 2021]

9. 9) NHS Digital. Hospital Admitted Patient Care and Adult Critical Care Activity. Available from: https://files.digital.nhs.uk/F2/E70669/hosp-episstat-admi-summ-rep-2018-19-rep.pdf [Accessed 11th April 2021].

10. No grants

\section{7}

Does ethnicity play a role in incidence of Intensive Care Unit (ICU) admissions for patients infected with COVID-19?

MMA. Zaki ${ }^{1}$; TW. Hla ${ }^{2}$; H. James ${ }^{2}$; N. sabir ${ }^{3}$; J. Gross, ${ }^{2}$; J. Borkowski ${ }^{4} ;$ K.

Dombrowsky,

${ }^{1}$ Anaesthesia and Intensive Care, Northwick Park Hospital, London, United Kingdom; ${ }^{2}$ Department of anaesthesia and critical care, London North West University Healthcare NHS Trust, London, United Kingdom; ${ }^{3}$ Critical care, Northwick Park Hospital, London, United Kingdom; ${ }^{4}$ Intensive care, London North West University Healthcare NHS Trust, London, United Kingdom

Correspondence: M.M.A. Zaki

Intensive Care Medicine Experimental 2021, 9(1): 000547

Introduction. There is evidence that incidence of COVID-19 (SARSCoV-2) is higher in black, Asian and minority ethnic groups (BAME) and is associated with disproportionately higher incidence of critical illness and mortality $[\mathbf{1}, \mathbf{2}, \mathbf{3}]$. London North West University 
Healthcare NHS Trust serves one of the most ethnically diverse population in UK [4] and was one of the first hospitals in the UK to see a rapid surge of critically ill COVID-19 admissions [5,6].

Objectives. Our aim was to evaluate whether the incidence of COVID-19 Intensive Care (ICU) admissions by ethnicity is comparable to the proportion of ethnicity in strongly diverse local ethnic population of North West London.

Methods. We conducted a retrospective analysis of all COVID-19 ICU admissions in our Trust for 12 months, between 1st March 2020 to 28th February 2021, by ethnicity. This data was compared to the data from the Office of National Statistics for the populations of ethnic groups in Harrow, Ealing and Brent, a total population of 920,000. Results. It was possible to assign ethnicity to $704(96 \%)$ of the 734 individuals who had tested positive for SARS-CoV-2 between $01 / 03 / 2020$ to $28 / 02 / 2021$. These individuals were categorized into four ethnic groups: White $(n=126)$, Black $(n=85)$, Asian $(n=380)$ and Mix/Other $(n=113)$. When compared to local population data, ICU admission rates were disproportionately higher in those of Asian ethnic origin $(\mathbf{5 4 \%}$ versus $\mathbf{3 3} \%$ ) and disproportionately lower in those of White ethnic origin ( $18 \%$ versus $\mathbf{4 2} \%$ ). The incidence of COVIDrelated-ICU admissions was proportional in Black and Mixed ethnic groups (see figure).

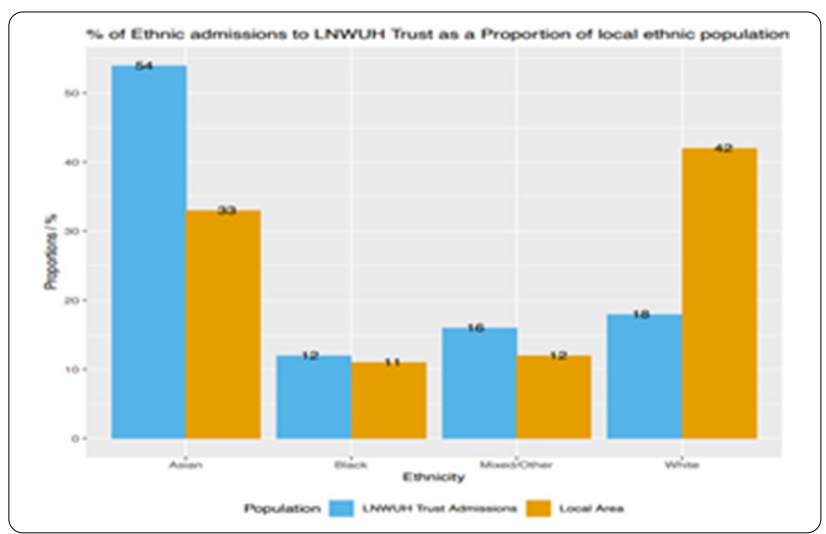

Conclusion. Our results are suggestive that people of Asian ethnic origin are more likely and those of White origin are less likely to develop critical illness in the context of SARS-CoV-2 infection. Further research is required to confirm or refute these findings and to suggest plausible explanations for differences relating to the development of critical illness across different ethnic groups.

\section{Reference(s)}

1. 5. Rao GG, Allen A, Papineni P, Wang L, Anderson C, McGregor A, Whittington A, John L, Harris M, Hiles S, Nicholas T. Cross-sectional observational study of epidemiology of COVID-19 and clinical outcomes of hospitalised patients in North West London during March and April 2020 BMJ open. 2021 Feb 1;11(2):e044384. https://www.bbc.co.uk/news/ukengland-london-52812457

2. 4. Garnett C, Foldes D, Bailey C, Nesr G, Hui T, Hinton R, Gurung K, Phillips J, Saleem Z, Koshy R, Aduwa E. Outcome of hospitalized patients with hematological malignancies and COVID-19 infection in a large urban healthcare trust in the United Kingdom. Leukemia \& Lymphoma. 2021 Jan 28;62(2):469-72.

3. 3. Kar P. Partha Kar: Covid-19 and ethnicity — why are all our angels white? BMJ. 2020 May 5:369.

4. 2. Razieh C, Zaccardi F, Davies MJ, Khunti K, Yates T. Body mass index and the risk of COVID-19 across ethnic groups: analysis of UK Biobank. Diabetes, Obesity and Metabolism. 2020 Oct;22(10):1953-4.

5. 1. Pan D, Sze S, Minhas JS, Bangash MN, Pareek N, Divall P, Williams CM, Oggioni MR, Squire IB, Nellums LB, Hanif W. The impact of ethnicity on clinical outcomes in COVID-19: a systematic review. EClinicalMedicine. 2020 Jun 1:23:100,404.

6. No conflict of interest to declare

\section{8}

Impact of Ethnicity on Mortality in Critically III Covid-19 Patients

MMA. Zaki' ; TW. Hla ${ }^{2}$; H. James ${ }^{2}$; N. sabiri ${ }^{3}$, J. Gross, ${ }^{2}$; J. Borkowski, ${ }^{4}$; K.

Dombrowsky, ${ }^{4}$

${ }^{1}$ Anaesthesia and Intensive Care, Northwick Park Hospital, London, United Kingdom; ${ }^{2}$ Department of anaesthesia and critical care, London North West University Healthcare NHS Trust, London, United Kingdom; ${ }^{3}$ Critical care, Northwick Park Hospital, London, United Kingdom; ${ }^{4}$ Intensive care, London North West University Healthcare NHS Trust, London, United Kingdom

Correspondence: M.M.A. Zaki

Intensive Care Medicine Experimental 2021, 9(1): 000548

Introduction. Relationship between ethnicity and COVID-19 remains unclear [1] but there is mounting evidence of a higher incidence and poorer outcomes among Black and minority ethnic (BAME) groups $[2,3]$. Our institution, London North West University Healthcare (LNWUH) NHS Trust serves one of the most widely ethnically diverse populations in the United Kingdom.

Objectives. To compare survival outcome amongst different ethnic groups for patients admitted to the ICU at our institution with COVID-19 infection.

Methods. This retrospective analysis included all patients with COVID19 infection who were admitted to the ICU at either Northwick Park or Ealing Hospitals between $\mathbf{1 / 3 / 2 0}$ to $\mathbf{2 8 / 2 / 2 1}$. Patients were categorised into one of 4 ethnic groups: Asian, Black, White and Mixed/ Other. Relevant baseline characteristics including acute illness severity at the point of ICU admission (defined by Intensive Care National Audit and Research Centre - ICNARC) was recorded for each patient. Primary outcome was mortality at the point of ICU discharge.

Results. During the study period, $\mathbf{7 1 3}$ patients with COVID-19 infection were admitted to one of our ICUs. The proportion of ICU admissions across Asian, Black, White and mixed/other ethnic groups were $\mathbf{5 1 . 3 \%}, \mathbf{1 1 . 8} \%$, $\mathbf{1 6 . 5 \%}$ and $\mathbf{2 0 . 3 \%}$ respectively. Mortality at the point of ICU discharge was $31 \%$ overall and comparable across all ethnic groups (32\%, 35\%, 30\%, $\mathbf{2 8 \%}$ for Asian, Black, White and Mixed/Other ethnic groups respectively).

\begin{tabular}{|c|c|c|c|c|c|}
\hline & ASIAN & BLACK & WHITE & MIX/OTHER & TOTAL \\
\hline $\begin{array}{l}\text { No. of } \\
\text { PATIENTS(\%)* }\end{array}$ & $366(51.3 \%)^{*}$ & $84(\mathbf{1 1 . 8 \%})^{*}$ & $118(\mathbf{1 6 . 5} \%)^{*}$ & $145(\mathbf{2 0 . 3} \%)^{*}$ & 713 \\
\hline MEAN AGE (SD) & $\mathbf{5 9 . 5 7 ( 1 2 . 0 6 )}$ & $\mathbf{5 7 . 5 6}(12.32)$ & $\mathbf{6 0 . 1 3}(12.40)$ & $\mathbf{5 8 . 0 5 ( 1 1 . 6 6 )}$ & $\mathbf{5 9 . 1 2 ( 1 2 . 0 8 )}$ \\
\hline MALE:FEMALE & $283: 83$ & $59: 25$ & $86: 32$ & $107: 38$ & $535: 178$ \\
\hline MEAN BMI (SD) & $26.76(4.98)$ & $29.35(6.75)$ & $28.72(6.41)$ & 27.76(5.78) & $\mathbf{2 7 . 5 9}(5.70)$ \\
\hline $\begin{array}{l}\text { MEDIAN ICNARC } \\
\text { sCore [INTER- } \\
\text { QUARTILE } \\
\text { RANGE] }\end{array}$ & $\begin{array}{l}24.0 \\
\quad[19.0-27.8]\end{array}$ & $\begin{array}{l}23.0 \\
{[20.0-28.0]}\end{array}$ & $\begin{array}{l}24.0[19.0- \\
28.0]\end{array}$ & $\begin{array}{l}23.0[19.0- \\
27.0]\end{array}$ & $\begin{array}{l}23.0[19.0- \\
28.0]\end{array}$ \\
\hline ICU MORTALITY \% & 31.6 & 35.4 & 29.7 & 27.5 & 30.9 \\
\hline
\end{tabular}

Conclusion. Our findings show comparable mortality outcomes across all ethnic groups for patients admitted to ICU with COVID-19 infection. This is in contrast to findings elsewhere that are suggestive of disproportionately worse outcomes for ethnic minority groups [4]. Further research is required to assess the role ethnicity plays in relation to critical illness and survival outcomes for those admitted to ICU with COVID-19 infection.

\section{Reference(s)}

1. Apea VJ, Wan YI, Dhairyawan R, Puthucheary ZA, Pearse RM, Orkin CM Prowle JR. Ethnicity and outcomes in patients hospitalised with COVID-19 infection in East London: an observational cohort study. BMJ open. 2021 Jan 1;11(1):e042140

2. 3. Kar P. Partha Kar: Covid-19 and ethnicity—why are all our angels white?. BMJ. 2020 May 5;369.

3. 2. Razieh C, Zaccardi F, Davies MJ, Khunti K, Yates T. Body mass index and the risk of COVID-19 across ethnic groups: analysis of UK Biobank. Diabetes, Obesity and Metabolism. 2020 Oct;22(10):1953-4.

4. 1. Pan D, Sze S, Minhas JS, Bangash MN, Pareek N, Divall P, Williams CM, Oggioni MR, Squire IB, Nellums LB, Hanif W. The impact of ethnicity on clinical outcomes in COVID-19: a systematic review. EClinicalMedicine. 2020 Jun 1;23:100,404.

5. No conflict of interest to declare 
000553

Admission criteria in critically ill COVID-19 patients: a physiology-based approach

A. Glotta ${ }^{1}$; M. Biggiogero'2; P. Maida, ${ }^{3}$; C. Garzoni, ${ }^{3}$ 'S. Ceruti ${ }^{4}$

${ }^{1}$ Department of critical care, Clinica Luganese Moncucco, Lugano,

Switzerland; ${ }^{2}$ Clinical research unit, Clinica Luganese Moncucco, Lugano,

Switzerland; Internal medicine department, Clinica Luganese Moncucco,

Lugano, Switzerland; ${ }^{4}$ Department of critical care, Clinica Luganese

Moncucco, Via Moncucco, Lugano, Switzerland, Lugano, Switzerland

Correspondence: $\mathrm{S}$. Ceruti

Intensive Care Medicine Experimental 2021, 9(1): 000553

Introduction. During the COVID-19 pandemic, principles of triage have been developed in Switzerland to better evaluate ICUs admission criteria, reserving ICU admission for patients who will benefit of an intensive medical intervention. A standardized procedure was implemented in our COVID-19 Center, based on standardized criteria for ICU admission.

Objectives. Aim of this study was to analyze the mortality rate and the clinical characteristics in patients assessed for the ICU admission, both in admitted and in rejected, established on standard pathophysiological criteria.

Methods. A retrospective analysis was conducted on all consecutive patients with acute respiratory distress syndrome due to COVID19 pneumonia triaged for ICU admission. Main exclusion criteria was a non-resuscitation order (DNR). For a standardized evaluation of patients admitted to the ward and eligible for ICU admission, the Early Warning Score (EWS) was applied based on the clinical condition. The EWS score was based on the patient's symptom assessment, peripheral oxygen saturation $\left(\mathrm{SpO}_{2}\right)$, blood gas values, and clinical status. For an EWS equal to or greater than 7, an intensive consultation was required. Patients with a partial respiratory failure with an $\mathrm{SpO}_{2}$ lower than $85 \%$ and dyspnea and patients with dyspnea alone were eligible for ICU admission.Patients who did not meet ICU inclusion criteria were followed in their clinical course.

Results. From March 16, 2020 to January 15, 2021, the Intensivist consultant evaluated 100 (7\%) patients of 1415 admitted to COVID-19 center. Sixty-six (66\%) of consulted patients were subsequently admitted to the ICU, while 34 (34\%) patients were not admitted and followed in the internal medicine ward. At 4 weeks, the death rate in the rejected group was $0 \%$, all rejected patients progressively improved their conditions and some of them had already been discharged from the hospital (Figure 1.).

Using the $\mathrm{SpO}_{2}<92 \%$ criteria, the hypoxia rate distribution in both groups did not appear to be correlated with ICU admission (Fig. 2): in the rejected group, 24 (70.6\%) patients had an $\mathrm{SpO} 2$ of less than $92 \%$, while in the admitted group there were $54(81.8 \%)$ patients who had an $\mathrm{SpO}_{2}<92 \%$, with an identical distribution (Chi-square $\mathrm{p}=0.19$ ). Contrariwise, using the $\mathrm{SpO}_{2}<85 \%$ criteria, a correlation was found regarding SpO2 distribution and ICU admission (Chi-square $\mathrm{p}=0.009$ ); similarly, dyspnea and ICU admission were correlated $(p=0.0004)$. The mortality distribution in the ICU-admitted group and in the ICUrejected group resulted also significant $(p<0.001)$.

Conclusion. A conservative approach based on strict surveillance until the onset of dyspnea or $\mathrm{SpO}_{2}<85 \%$ seems to be a valid method to safely reduce ICU admission, avoiding any ICU overload and healthcare workload. An approach based on physiology in terms of symptoms and/or signs of lung damage due to hypoxia appears to be more appropriate for approaching this type of patient.

\section{Reference(s)}

1. 4. Alhazzani W, Møller MH, Arabi YM, et al. (2020) Surviving Sepsis Campaign: guidelines on the management of critically ill adults with Coronavirus Disease 2019 (COVID-19). Intensive Care Med 46:854-887

2. 3. Poston JT, Patel BK, Davis AM (2020) Management of Critically III Adults with COVID-19. JAMA—J Am Med Assoc 323:1839-1841

3. 2. Bouadma L, Lescure FX, Lucet JC, Yazdanpanah Y, Timsit JF (2020) Severe SARS-CoV-2 infections: practical considerations and management strategy for intensivists. Intensive Care Med 46:579-582
4. 1. Swiss Academy Of Medical Sciences (2020) COVID-19 pandemic: triage for intensive-care treatment under resource scarcity. Swiss Med Wkly 150:w20229

\section{0}

Implementation of "ABC-team" at Regional Hospital West Jutland S. Birkebaek'; RP. Nielsen, ; MN. Kristensen, ${ }^{2}$; N. Dey, ${ }^{1}$

${ }^{1}$ Anesthesia and Intensive, Regionshospitalet Herning, Herning, Denmark; ${ }^{2}$ Anesthesia and intensive, Regionshospitalet i Viborg, Viborg, Denmark Correspondence: S. Birkebaek

Intensive Care Medicine Experimental 2021, 9(1): 000560

Introduction. Rapid response system (RRS) teams have been widely adopted by many health systems. It is known that RRS teams are associated with a reduction in hospital mortality and cardiopulmonary arrest (1-2). Therefore, we implemented the $A B C$ team at Regional Hospital West Jutland. An ABC team consists of a nurse anesthetist, an anesthesiologist and a porter. The team functions as a RRS team with equipment and skills to offer ABCDE guided treatment and can be activated for patients with an immediate life-threatening condition involving airway, breathing, circulation, or disability when there is indication for treatment (the $A B C$ code).

Objectives. The purpose of this study was to investigate whether the implementation of the $A B C$ team in Regional Hospital West Jutland has reduced activation of the resuscitation team. Furthermore, we wanted to investigate if there was relevant indication for activating the $A B C$ team, the number of subsequent admissions to the intensive care unit (ICU), operating theater and other hospitals, the time-consumption of the $A B C D E$ assessment made by the $A B C$ team and the department activating the $A B C$ team.

Methods. A retrospective evaluation was made of all $A B C$ codes registered during a six-month period from December 1st, 2019 until May 31st, 2020 at Regional Hospital West Jutland. Information was provided through questionnaires completed by the anesthesiologist who participated in the $A B C$ code.

Results. A total of $68 \mathrm{ABC}$ codes occurred at Regional Hospital West Jutland during the study period. $55(81 \%)$ of the questionnaires were completed. During the study period, there were 63 codes for the resuscitation team compared to 67 codes during a six-month period the year before (Figure 1). When activating the $A B C$ code $75 \%$ of the patients $(41 / 55)$ were in an acute or potentially life-threatening condition, and $9 \%(5 / 55)$ had problems with the airways, $22 \%(12 / 55)$ had problems with breathing, $13 \%(7 / 55)$ had problems with the circulation, $24 \%(13 / 55)$ had problems with disability, and $25 \%$ of the patients had more than one of the mentioned problems. After ABCDE rescue $35 \%$ of the patients $(19 / 55)$ were transferred to ICU, the operating theater or another hospital. An average $A B C D E$ assessment made by the $A B C$ team was done in $32 \mathrm{~min}$. However, a time interval from 5 to $140 \mathrm{~min}$ was recorded. The most frequent departments activating the ABC code were the Department of Internal Medicine (19/55), Department of Radiology (10/55), Department of Neurology (9/55) and Department of Cardiology (6/55).

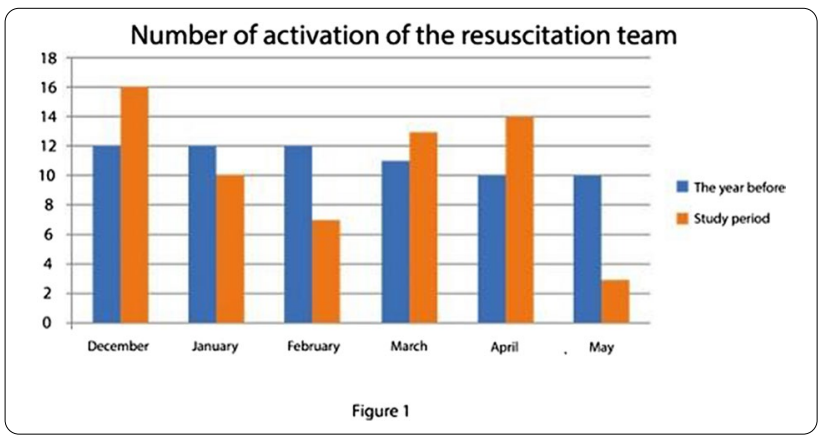

Conclusion. The implementation of $A B C$ codes has not reduced activation of the resuscitation team. However, the $A B C$ team was mainly 
used for patients in acute or potentially life-threatening conditions. The high proportion of patients transferred to the ICU, operating theater and other hospitals demonstrates the need for a RRS team. The implemented $A B C$ code is recommended to continue based on the mentioned results.

\section{Reference(s) \\ 1. Maharaj R., et al., Rapid response system: a systematic review and meta- analysis. Critical Care (2015) 19:254 \\ 2. Semeraro F., et al., European Resuscitation Council Guidelines 2021: Systems saving lives. Resusciation 8901. 2021. \\ 3. No grants \\ 000569 \\ Muscle function and physical function outcomes in critically ill survivors \\ S. Chhiba'; S. Hanekom ${ }^{1}$; A. Lupton-Smith \\ 'Physiotherapy, Stellenbosch University, Stellenbosch, South Africa Correspondence: S. Chhiba \\ Intensive Care Medicine Experimental 2021, 9(1): 000569}

Introduction. Despite improvements in intensive care unit (ICU) management, many critically ill survivors experience respiratory and peripheral muscle weakness and profound long-term physical function impairments, which may persist years after hospital discharge. $(1,2)$ However, it is unknown what the relationship between respiratory and peripheral muscle function is with physical function in critically ill survivors.

Objectives. To describe respiratory and peripheral muscle function and physical function outcomes in critically ill survivors of a South African population at hospital discharge. Secondly, to determine the correletion between respiratory and peripheral muscle function with physical function at hospital discharge.

Methods. A prospective observational cohort study was conducted at a Tertiary Academic Hospital. The respiratory and peripheral muscle function and physical function assessments were conducted $24 \mathrm{~h}$ prior to hospital discharge. Assessments of respiratory and peripheral muscle function included maximal inspiratory pressure (MIP), respiratory muscle endurance and the Medical Research Council-Sum Score (MRC-SS). The physical function assessment included the Six-Minute Walk Test (6-MWT).

Results. The mean (SD) APACHE II score was 14.6 (6.4) points, and the mean (SD) SOFA score was 8 (2.6) points. The median (IQR) ICU length of stay (LOS) was 4 (2.9-6.3) days and the total hospital LOS was 10 (5.8-16.7) days. At hospital discharge, the mean (SD) MIP score was $32.8(12.5) \mathrm{cmH}_{2} \mathrm{O}$, and the median (IQR) time for the completion of the respiratory muscle endurance test was $2(0.9-3.6)$ minutes. The mean (SD) MRC-SS was 53.3 (6) with 3 participants (23.1\%) scoring less than 48 on the MRC-SS thus presenting with intensive care unitacquired weakness (ICU-AW). Thirteen participants (8 Male; 5 Female) with a mean (SD) age of $31.6(12.5)$ years completed the 6-MWT. The mean (SD) six-minute walk distance (6-MWD) for the first 6-MWT was $338.5 \pm 111.5 \mathrm{~m}$ and for second 6-MWT, the mean (SD) 6-MWD was $349.3 \pm 87.2 \mathrm{~m}$. At hospital discharge, the correlation between MIP and the 6-MWT was a strong positive significant correlation $(r h o=0.75$, $p=0.01$ ) whereas, the correlation between respiratory muscle endurance and the 6-MWT was a moderate positive non-significant correlation $(r h o=0.49 ; p=0.13$ ). The correlation between the MRC-SS and the 6-MWT was a moderate strong positive significant correlation $(r h o=0.57 ; p=0.04)$

Conclusion. Despite a relative short ICU and hospital LOS, decreased respiratory muscle strength and endurance as well as decreased functional exercise capacity was observed at hospital discharge. Furthermore, almost a quarter of a small sample of participants also presented with ICU-AW. The clinical implications of the medium to strong correlation established between peripheral and respiratory muscle strength and functional exercise capacity in critically ill survivors at hospital discharge should be explored.

\section{Reference(s)}

1. 2. Wieske L, Dettling-Ihnenfeldt DS, Verhamme C, Nollet F, van Schaik IN Schultz MJ, et al. Impact of ICU-acquired weakness on post-ICU physical functioning: A follow-up study. Crit Care. 2015;19(1):1-8.

2. 1. Hermans $G$, Van Aerde N, Meersseman P, Van Mechelen H, Debaveye Y, Wilmer A, et al. Five-year mortality and morbidity impact of prolonged versus brief ICU stay: a propensity score matched cohort study. Thorax. 2019;74(11):1037-45

\section{0}

Implementation of Indirect calorimetry measurement in ICUs, Regional Hospital West Jutland, Denmark

N. dey ${ }^{1}$; R. Winding ; M. Biilmann ${ }^{1}$; B. Husted ${ }^{1}$; DG. Pedersen ${ }^{1}$; L. Rokkjær'; B. Muldbjerg ${ }^{1}$; A. Erichsen ${ }^{2}$

${ }^{1}$ Anesthesia and Intensive, Regionshospitalet Herning, Herning, Denmark; ${ }^{2}$ Department of nutrition, Regionshospitalet Herning, Herning, Denmark

Correspondence: $\mathrm{N}$. dey

Intensive Care Medicine Experimental 2021, 9(1): 000580

Introduction. Malnutrition in critically ill patient is the known cause of increased morbidity and mortality in ICU 1. Physicians working in the ICU needs to supplement adequate protein and calorie intake, without underfeeding or overfeeding the patient. In critically ill patients, indirect calorimetry (IC) measurements is the gold standard to determine the energy needs, as the use of predictive equations can result in underfeeding or overfeeding 1,2. Little data on the ease and success of implementation of indirect calorimetry systems in the ICU exist 2. The aim of this quality improvement (QI) initiative was to describe the rationale for implementation of a process and evaluate whether the implementation was easy and successful.

Objectives. In 2020 an internal audit was performed in our ICU. During that time, Indirect calorimetry was measured using Ecovx, GE, Helsinki, Finland. The audit showed indirect calorimetry was performed on $25 \%$ of our mechanically ventilated patients. The objective of this study was to examine whether a new indirect calorimetric measurement system (Beacon Caresysten) could be easily and successfully implemented in the ICU, during a three-month implementation period, therein one month of focused theory and hands-on training. For implementation to be successful, $80 \%$ of patients admitted to the ICU, requiring mechanical ventilation should have indirect calorimetry measured and a dietist or ICU nurse accordingly must assess the IC measurement to create a patient oriented plan (POP) in the patient journal.

Methods. As a part of implementation, a dedicated nutrition team comprising of $4 \mathrm{ICU}$ nurses and $2 \mathrm{ICU}$ physician coordinators along with a dietist, was established. Team was supposed to work on a quality improvement project guided by PDSA model. Team meet regularly and planned the project strategy. The first part was the implementation of IC measurements and creating POP and second part to focus on clinical application. Indirect calorimetry measurement system (Beacon Care system), Mermaid care A/S, Noerresundby, Denmark is installed in our ICU. An internal guideline document explaining the system and measurement procedures was published. Indirect calorimetry was to be performed on mechanically ventilated patients admitted in ICU every Monday and Thursday night during a 15 min to 2-h period and POP to be created by dietist and ICU nurses every Tuesday and Friday. All ICU staff (nurses, physicians) were given theory teaching followed by hands-on training on the device along with individual teaching on POP given to ICU nurses. As a part of teaching, quizes was organized, with feedback questionnaires.

Results. Teaching and hands on training was given to $81 \%(35 / 40)$ and $74 \%(60 / 81)$ of doctors and nurses respectively during the month of January,2021. Almost all who participated in teaching and training participated in the quiz and feedback questionnaires. We used the feedback answers to improve on our internal IC measurement and POP guidelines. We observed that a total of 46 patients were admitted to ICU requiring mechanical ventilation during the 2 months starting February 2021. A total of 16 patients requiring MV for less than 2 days was excluded. IC was measured in $53 \%(16 / 30)$ of patients as compared to $25 \%$ in 2020 . POP was created in $56 \%$ (17/30) of patients, there was no previous POP guidelines. 
Conclusion. Implementation of the Beacon indirect calorimetric measurement system in the ICU is easy but to be successful requires a dedicated team and planning beside focused feedback oriented teaching and training of the ICU staff.

\section{Reference(s)}

1. 2. Preventing Underfeeding and Overfeeding: A Clinician's Guide to the Acquisition and Implementation of Indirect Calorimetry. Ladd AK, Skillman HE, Haemer MA, Mourani PM.Nutr Clin Pract. 2018 Apr;33(2):198-205.

2. 1. ESPEN guideline on clinical nutrition in the intensive care unit. Singe P, Blaser AR, Berger MM, Alhazzani W, Calder PC, Casaer MP, Hiesmayr M, Mayer K, Montejo JC, Pichard C, Preiser JC, van Zanten ARH, Oczkowski S, Szczeklik W, Bischoff SC. Clin Nutr. 2019 Feb;38(1):48-79.

3. No grants

\section{4}

Physical, social and psychological functioning in COVID-19 ICU survivors and their family members 12 months after ICU discharge

I. van der Meulen ${ }^{1}$; M. Onrust ${ }^{1}$; N. Van Veenendaal ${ }^{2}$; ML. Luttik ${ }^{3}$; W.

Dieperink'; P. Van Der Voort

'Department of Critical Care, University Medical Center Groningen, Groningen, Netherlands; ${ }^{2}$ Department of Critical Care / Department of Anesthesiology, University Medical Center Groningen, Groningen, Netherlands; ${ }^{3}$ Professorship Family Nursing, School of nursing, Hanze University of Applied Sciences Groningen, Groningen, Netherlands Correspondence: I. van der Meulen

Intensive Care Medicine Experimental 2021, 9(1): 000584

Introduction. ICU survivors of COVID-19 are prone to developing Post Intensive Care Syndromes (PICS)1, mainly as a result of prolonged and deep sedation. In addition, family members are also susceptible to the acute and chronic psychological effects, also known as the PICS-F2. Up to $30 \%$ of family or the caregivers experience stress, anxiety, depression, and complicated grief3. It is possible that this rate is even higher in family members of COVID-19 ICU survivors because the consequences of the disease are unknown and family members are in most cases prohibited to visit the patient during hospital admission. Since the SARS-CoV-2 infection is relatively new, the long term attributable burden for COVID-19 ICU survivors and their family members are largely unknown.

Objectives. This study provides insight into the physical-, social- and psychological functioning of COVID-19 ICU-survivors and their family members 12-months after ICU discharge.

Methods. A single-center, prospective cohort study was conducted in COVID-19 ICU-survivors and their family members. Enrolled participants received a questionnaire which included the Clinical frailty scale, physical symptoms list, MOS Short-Form General Health Survey, McMaster Family Assessment Device (FAD-GF6+), return to work, the Hospital Anxiety and Depression Scale and the Spiritual Needs Questionnaire (SpNQ-15).

Results. Results reflects the physical-, social- and psychological functioning of COVID-19 ICU-survivors and their family members 12 months after ICU discharge.

Conclusion. This study provides insight into whether and what burden is experienced by COVID-19 ICU-survivors and their family members. These results can be used to guide rehabilitation in COVID-19 ICU-survivors and their family members.

\section{Reference(s)}

1. 1 Needham DM, Davidson J, Cohen $\mathrm{H}$, et al. Improving long-term outcomes after discharge from intensive care unit: report from a stakeholders' conference. Crit Care Med 2012; 40: 502-9

2. 2 Davidson JE, Jones C, Bienvenu OJ. Family response to critical illness: postintensive care syndrome-family. Crit Care Med 2012; 40: 618-24

3. 3 Rawal G, Yadav S, Kumar R. Post-intensive Care Syndrome: an Overview. J Transl Int Med 2017: 5: 90-92.

\section{1}

Assessment of two telemedical services in the setting of intensive care

Y. Hage ${ }^{1}$; S. Hollerbach, ; M. Tübben, ${ }^{2}$; M. Höckel, ${ }^{3}$; A. Hettel, ${ }^{4}$; MR

Muellenbach ${ }^{5}$; C. Reyher, ; N. Kessemeier, ${ }^{3}$

${ }^{1}$ Kassel School of Medicine, Campus Kassel of the University of Southampton, Kassel, Germany; ${ }^{2}$ Anaesthesiology and critical care, Stadtkrankenhaus Korbach, Korbach, Germany; ${ }^{3}$ Pharmacy, Gesundheit Nordhessen Holding, Kassel, Germany; ${ }^{4}$ Anaesthesiology and Critical

Care, Asklepios Klinikum Schwalmstadt, Schwalmstadt, Germany;

${ }^{5}$ Anaesthesiology and Critical Care, Campus Kassel of the University

of Southampton, Kassel, Germany

Correspondence: $Y$. Hage

Intensive Care Medicine Experimental 2021, 9(1): 000591

Introduction. Telemedicine, the delivery of healthcare using telecommunication, allows for the distribution of specialised and highly skilled medical knowledge to institutions which would otherwise not have the local infrastructure. In the setting of intensive care, which requires a vast number of multidisciplinary specialists, the advantages of these services might be beneficial. Nonetheless, while there has not been an assessment of the quality of telemedicine yet, within the setting of intensive care units, newly delivered services like telepharmaceutical ward rounds need assessment of practicability as well.

Methods. We conducted two pilot studies. The first study was a service evaluation of a newly implemented telepharmaceutical service within the setting of intensive care. The second study performed a clinical audit remotely to assess the quality of care in an intensive care unit. Both studies used a newly developed telemedical app which allowed communication between a hospital of maximum care (service provider) and a hospital of basic care (service customer).The analysis for the telepharmaceutical pilot study was descriptive using ATC drug classification, ADKA DokuPik Categories and High-Alert Medications list of ISMP. The clinical audit used quality indicators set by the German Interdisciplinary Society for Intensive Care Medicine (DIVI) and reassessed them one week after suggestions for improvement were presented to the intensive care team. The service provider was the same hospital whereas the service customer consisted of two remote hospitals of basic and customary care.

Results. Within the service evaluation acceptance rate of suggested recommendations pronounced by the telepharmacist was $90 \%$. Greatest category of suggestions according to ADKA classification was the group of interactions with 16 out of 51 suggestions. $58 \%$ of the prescribed ISMP high alert medications generated an intervention by the clinical pharmacist. For the clinical audit data of 19 patients was analysed and showed that it is possible to assess the adherence to DIVI QIs remotely.

Conclusion. The pilot studies gave a first insight into the implementation of initialising a new telemedical application within intensive care, both evaluating a service tailored to pharmaceutical consultations and assessing the quality of a telemedical service. Yet both projects show that it can be practical to provide specialists knowledge via distance and that a service for remote auditing of critical care is feasible. This shows that patient safety can be increased by continuously offering good quality assessment whilst redistributing rare specialist knowledge.

Reference(s)

1. Kahn JM, Hill NS, Lilly CM, et al. The research agenda in ICU telemedicine: a statement from the Critical Care Societies Collaborative. Chest 2011;140(1):230-38

2. Practices IfSM. High-Alert Medications in Acute Care Settings. Home Page: Institute for Safe Medication Practices, 2018:

3. Authority HPR. ATC Codes http://www.hpra.ie/homepage/medicines/ medicinesinformation/atc-codes\# (accessed 23.09.2020).

4. Methodology WHOCCfDS. ATC structure and principles https://www. whocc.no/atc/structure and_principles/ (accessed 23.09.2020).

5. Kantelhardt DCLP. DokuPIK —Ein Dokumentationssystem für Medikationsfehler und Interventionen, 2009. Translation: A documentation system for medication errors and interventions

6. Kumpf O, Braun J-P, Brinkmann A, Bause H, Bellgardt M, Bloos F, et al. Quality indicators in intensive care medicine for Germany-third edition 2017. GMS Ger Med Sci. 2017:15. 


\section{4}

The oxygen supply situation in COVID-19 hospitals: Ukraine's national survey

MM. Pylypenko'; O. Khomenko 2 ; D. Osin ${ }^{3}$

${ }^{1}$ Anaesthesia and Intensive Care Unit, Romodanov Neurosurgery Institute, National Academy of Medical Sciences of Ukraine, Kyiv, Ukraine;

${ }^{2}$ Anaesthesiology and Intensive Care, A. A. Shalimov National Institute of Surgery and Transplantology, Kyiv, Ukraine; ${ }^{3}$ Biomedical engineer, Consultant, Kyiv, Ukraine

Correspondence: $\mathrm{O}$. Khomenko

Intensive Care Medicine Experimental 2021, 9(1): 000594

Introduction. During the epidemic of COVID-19, the typical situation for almost all principal COVID-19 hospitals in Ukraine was lack of oxygen. However, there was no systematic analysis of the situation in Ukrainian clinics performed to investigate the possibility to provide oxygenation to critically ill COVID-19 patients. Nowadays, there is still a lack of data about components of the hospital's oxygen supply systems in Ukraine.

Objectives. To evaluate the oxygen performance of the principal COVID-19 hospitals in Ukraine totally and its different regions.

Methods. We have surveyed more than 450 principal COVID-19 hospitals in Ukraine and near $99 \%$ of them have responded and filled in the questionnaire. The hospitals' oxygen supply performance was determined by the consumption of oxygen from reliable sources through the hospital oxygen distribution system (liquefied gas, oxygen cylinders, and powerful PSA concentrators) and via the available mobile oxygen concentrators and the value of their performance. We calculated the value of maximum potential oxygen delivery for all hospitals in $\mathrm{l} / \mathrm{min}$ from all available sources, incl. mobile oxygen concentrators. The parameter of maximum potential oxygen delivery was calculated based on the data of the oxygen consumption by the hospital from all reliable sources with adding the cumulative performance of the mobile oxygen concentrators, available at the hospital at the date of the survey. This parameter in $1 / \mathrm{min}$ we calculated for each principal hospital and as a mean potential performance to deliver oxygen parameter for the different regions of Ukraine.

Results. The potential performance of oxygen delivery depends on the region. Typically, the maximum potential performance to deliver oxygen for analysed Ukrainian hospitals is $375 \mathrm{l} / \mathrm{min}$ from different sources. It allows supporting simultaneously near 70 patients with severe COVID-19 (5 I/min per patient) or near 35 patients with critical Covid-19 (10 l/min oxygen flow for each), despite that principal hospitals declare the possibility to treat over 100 patients with COVID-19 and hypoxic respiratory failure who required oxygen therapy. At the time of finalizing the survey data collection, hospitals typically had 25 mobile oxygen concentrators that could produce a total of $240 \mathrm{l} / \mathrm{min}$ of oxygen. It is alarming that about one third of hospitals surveyed, mobile oxygen concentrators provide $90 \%$ to $100 \%$ of the maximum potential oxygen production capacity in $\mathrm{I} / \mathrm{min}$. Even more, about 60 hospitals receiving oxygen only from mobile oxygen concentrators.

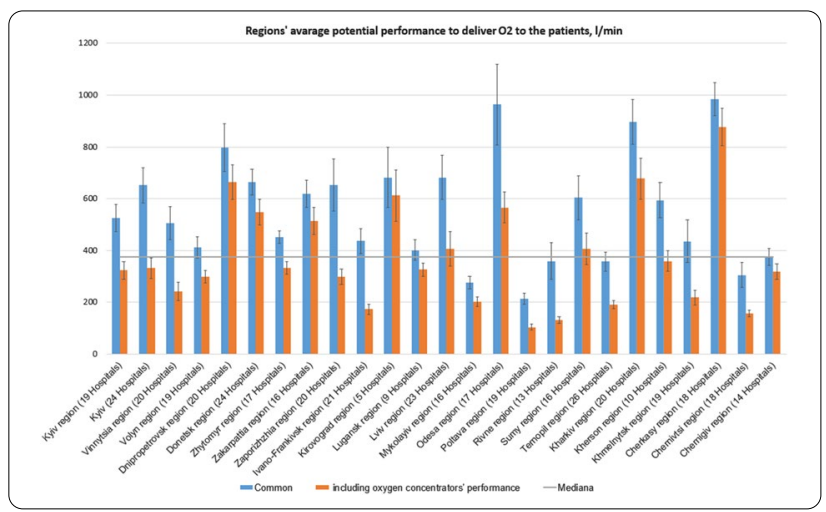

Conclusion. This survey, based on the actual state of hospital equipment for storage and supply of oxygen, as well as on the value of oxygen consumption, reviles information on the potential capacity of hospital oxygen supply systems. It allows predicting the number of patients that could be provided with an adequate amount of oxygen. Preliminary, the findings can serve as a road map for management decisions to improve the situation in preparation for future waves of COVID-19 or epidemics of similar severe acute respiratory infections. Future deep analysis of the data requires for having recommendations that are more precise.

\section{7}

Behavior of the ICU of the Hospital Virgen de la Concha de Zamora during the Sars-Cov-2 pandemic. Impact of the Contingency Plan during the first and second waves

N. Rodrigo Castroviejo'; LL. Beltran Martínez'1; G. Ferrigno²; R. Beltrán Bernáldez ; C. Sanmartino Gonzalez'; M. Berenguer Rodriguez ; D. Monge Donaire ; A. Marcos Gutiérrez ; ;. Tarancón Maján ${ }^{1}$; S. Cortés Díaz

${ }^{1}$ Intensive Care, Hospital Virgen De La Concha, Zamora, Spain; ${ }^{2}$ Intensive Care Unit, Hospital Virgen De La Concha, Zamora, Spain

Correspondence: $\mathrm{G}$. Ferrigno

Intensive Care Medicine Experimental 2021, 9(1): 000597

Introduction. The SARS-CoV-2 pandemic (COVID-19) has had an unprecedented impact in the recent history of medicine. Despite advances, the rapid expansion of the disease and the number of admissions to intensive care units has meant that we have had to develop contingency plans and protocols that are constantly changing to improve the care of our patients.

Objectives. To analyze ICU performance from an organizational point of view and the impact on morbimortality and critical patient care.

Methods. Retrospective descriptive study analyzing the human and material resources available during the pandemic and the characteristics of the population attended. Management and quality indicators and morbimortality variables are evaluated. The data are collected from March 10 to May 31, 2020 being this the first wave and from $15 / 08 / 2020$ to $28 / 11 / 2020$ the second wave.

Results. Our ICU usually has 11 beds, 14 ventilators and 3 portable monitors. It has 7 intensivists for a care ratio of 1.5 beds per physician and the pre-pandemic occupancy rate ranges between $60-80 \%$. For care during the first wave, 11 extra beds were opened for a total of 22 functioning beds and 20 functioning beds were left for the second wave.

In the first wave the staffing was adjusted to 9 intensivists versus 8 in the second wave and in both peaks medical support was received from residents, anesthesiologists and pulmonologists, as well as a change in the on-call schedule. The bed/physician ratio for intensivists became 2.5:1. The nursing staff increased their staffing levels, maintaining a bed-patient/nurse ratio of 2:1 during the two waves.

During the first wave $89 \%$ of patients were admitted for Sars-Cov-2 pneumonia with a peak admission on April 1 (20 patients) for an occupancy rate of $181 \%$ of usual capacity. In the second wave, 59 patients were admitted for Sars-Cov-2 Pneumonia with a peak admission in November (17 patients) for an occupancy rate of $154.54 \%$ of its usual capacity.

\begin{tabular}{lll}
\hline VARIABLE & FIRST WAVE & SECOND WAVE \\
\hline Sexo & Men 81\% & Men 69\% \\
& Women 19\% & Women 31\% \\
Mean age & $64,5($ IC $95 \%$ 61,71-67,3) & $64,6($ IC $95 \%$ 61,6-67,6) \\
Mean SOFA scale & $6($ IC 95\% 5,2-6,7), & 5 (IC 95\% 4,3-5,6) \\
Mean APACHE scale & 14,24 (IC 95\% 12,1-16,2) & $14,13($ IC $95 \% 11,7-16,5)$ \\
Mean stay in ICU & 14,55 days (IC95\% & 16,75 days (IC 95\% 11,7-21,21) \\
Mean number of days IMV & $14,07-15,05)$ & \\
Use of IMV & 13,71 days (IC 10,3-17.07) & 14,36 days (IC 10,7-17,8) \\
Non-invasive oxygen therapy & $100 \%$ of admissions & $74 \%$ of admissions \\
Percentage of prone therapy & $73 \%$ & $26 \%$ of admissions \\
\hline
\end{tabular}




\begin{tabular}{lll}
\hline VARIABLE & FIRST WAVE & SECOND WAVE \\
\hline Complications & FMO 38.1\% & Sepsis 33\% \\
& VAP 35.7\% & VAP 32\% \\
Reintubation rate & $18,3 \%$ & $17 \%$ \\
Percutaneous tracheostomy & $13 \%$ & $28,6 \%$ \\
Readmission rate & $10 \%$ & $0,016 \%$ \\
Intra-ICU Mortality & $52,3 \%$ & $25 \%$ \\
\hline
\end{tabular}

Conclusion. The increase in material resources was necessary for the care of critical patients. The unit doubled its capacity during the two waves and presented a maximum occupancy almost double its usual capacity considering its acceptable performance.

During the two waves, the admission and stay characteristics of the patients were similar, but the significant decrease in intra-ICU mortality in the second wave and the number of patients who resolved their critical illness with non-invasive oxygen therapy stand out.

\section{3}

Use of a Local safety standard for invasive procedures (LocSSIP) for arterial line insertion in Stoke Mandeville Hospital Intensive Care Unit

E. Wright ${ }^{1}$; D. Jittasaiyapan ${ }^{1}$; M. Dada ${ }^{1}$; T. Ali ${ }^{1}$

${ }^{1}$ Intensive Care Department, Stoke Mandeville Hospital, Aylesbury, United Kingdom

Correspondence: $\mathrm{E}$. Wright

Intensive Care Medicine Experimental 2021, 9(1): 000623

Introduction. NHS England have set out national safety standards for invasive procedures (NatSSIPs) which are designed to work as a framework for local safety standards for invasive procedures (LocSSIPs). These aim to standardise patient care by establishing important steps within invasive procedures (1). Safety standard checklists have been shown to improve compliance when used for other invasive procedures (2), and insertion and maintenance bundles have been shown to reduce incidence of central-line-associated bloodstream infections (3). Arterial lines are frequently used in the Intensive Care Unit for accurate monitoring of blood pressure, frequent blood sampling for blood gas analysis and biochemical and haematological analysis. Although serious complications are rare, there is the potential for vascular or infectious complications (4). With the vygon insertion system, there is the potential for a retained guidewire, which is a 'never event' (5).

Objectives. To determine whether implementation of a LocSSIP for arterial line insertion improves documentation of key steps undertaken within this procedure.

Methods. We established a set of essential steps requiring completion and documentation for the insertion of all arterial lines. We collected data from patient notes to review what documentation existed from the time of insertion. We implemented a LocSSIP designed to ensure safe insertion and appropriate documentation. The LocSSIPs were then reviewed in order to assess adherence to this proforma.

Results. We collected data from 50 arterial line insertions, which occurred in the Stoke Mandeville Hospital Intensive Care Unit between February and March 2021. Of these, 20 had used the LocSSIP proforma for arterial line insertion. Alternative sources for line insertion documentation included clinical notes and arterial line daily monitoring sheets.

We established 13 criteria which should be documented following arterial line insertion. These are documented in Table 1.

\begin{tabular}{lll}
\hline Criteria & $\begin{array}{l}\text { Number documented } \\
\text { before implementation } \\
\text { of LocSSIP checklist (\%) }\end{array}$ & $\begin{array}{l}\text { Number documented } \\
\text { after implementation of } \\
\text { LocSSIP checklist (\%) }\end{array}$ \\
\hline $\begin{array}{l}\text { Indication for arterial line } \\
\begin{array}{l}\text { Confirmation of patient } \\
\text { identity }\end{array}\end{array}$ & $0(0)$ & $20(100)$ \\
$\begin{array}{l}\text { Presence of coagu- } \\
\text { lopathy }\end{array}$ & $1(3.3)$ & $19(95)$ \\
\hline
\end{tabular}

\begin{tabular}{lll}
\hline Criteria & $\begin{array}{l}\text { Number documented } \\
\text { before implementation } \\
\text { of LocSSIP checklist (\%) }\end{array}$ & $\begin{array}{l}\text { Number documented } \\
\text { after implementation of } \\
\text { LocSSIP checklist (\%) }\end{array}$ \\
\hline $\begin{array}{l}\text { Type of arterial cannula } \\
\begin{array}{l}\text { Site of arterial line (artery/ } \\
\text { side) }\end{array}\end{array}$ & $8(26.7)$ & $20(100)$ \\
$\begin{array}{l}\text { Use of local anaesthetic } \\
\text { Use of ultrasound }\end{array}$ & $1(3.3)$ & $20(100)$ \\
$\begin{array}{l}\text { Number of attempts } \\
\text { Aseptic Non-Touch Tech- } \\
\text { nique (ANTT) used }\end{array}$ & $16(10)$ & $20(100)$ \\
$\begin{array}{l}\text { Guidewire removed } \\
\text { (For vygon/seldinger } \\
\text { technique only) }\end{array}$ & $0(0)$ & $20(100)$ \\
Line transduced & $15(50)$ & $10(100)$ \\
$\begin{array}{l}\text { Line secured } \\
\text { Sharps disposed of } \\
\text { appropriately }\end{array}$ & $11(36.7)$ & $10(100)$ \\
\hline
\end{tabular}

Table 1: Documentation of essential criteria for arterial line insertion before and after LocSSIP checklist implementation

Conclusion. Introduction of a Local Safety Standard for Invasive Procedures (LocSSIP) for arterial line insertion led to improved documentation of all criteria. We aim to repeat the audit yearly in order to ensure compliance.

\section{Reference(s)}

1. 1. NHS England patient Safety Domain. National Safety Standards for Invasive Procedures (NatSSIPS). NHS Improv. 2015;1-49.

2. 2. Mason MC, Griggs RK, Withecombe R, Xing EY, Sandberg C, Molyneux MK. Improvement in staff compliance with a safety standard checklist in endoscopy in a tertiary centre. BMJ Open Qual. 2018;7(3).

3. 3. Ista E, van der Hoven B, Kornelisse RF, van der Starre C, Vos MC, Boersma E, et al. Effectiveness of insertion and maintenance bundles to prevent central-line-associated bloodstream infections in critically ill patients of all ages: a systematic review and meta-analysis. Lancet Infect Dis. 2016;16(6):724-34.

4. 4. Blackburn J, Walton B. Risks associated with arterial lines; Time for a National Safety Standard? J Anaesth Pract [Internet]. 2016; Available from: http://japractice.co.uk/2016/11/risks-associated-arterial/12248

5. 5. Bamford P, Smith G. LocSSIPS - The quest to improve patient safety. J Intensive Care Soc. 2017;18(3):180-3.

6. No grants received

000628

Long-term mental health impairment after critical illness

J. Tejero Aranguren ${ }^{1}$; ME. Poyatos Aguilera²; O. Moreno Romero ${ }^{3}$; R. Garcia Del Moral ${ }^{2}$

${ }^{1}$ Intensive Care Unit, Hospital Universitario San Cecilio, Granada, Spain; ${ }^{2}$ Intensive Care Unit, Hospital Universitario Clínico San Cecilio, Granada, Spain; ${ }^{3}$ Intensive Care Unit, Regional Hospital Santa Ana de Motril, Motril, Spain

Correspondence: J. Tejero Aranguren

Intensive Care Medicine Experimental 2021, 9(1): 000628

Introduction. After critical illness, new or worsening impairments in mental health function are common among patients who have survived. Methods. A follow-up hospital visit was performed to patients admitted to an Intensive Care Unit (ICU) between 1 and 3 months after discharge, from January 2018 to August 2020.

The follow-up criteria were: Length of stay in the ICU of at least one week and one of the following: sepsis, need for mechanical ventilation for at least 3 days, shock or delirium in the ICU. We considered impairment in mental health if they presented traumatic memories of their admission to ICU or a score $>11$ points in the HADS test.

Results. During the study period 92 patients were included. Average age was 58.1 (sd 13.8), 34.9\% of the patients $(n=32)$ were admitted for COVID-19 pneumonia. Considering an impairment in mental 
health as the appearance of any of the previous items, the incidence was $35.2 \%(\mathrm{~N}=30)$. The variables associated with the development of impairment mental health in our cohort are length of stay in the ICU $(1.02, p=0.03)$ and treatment with non invasive mechanical ventilation $(2.96, p=0.03)$. In the subgroup of patients undergoing invasive mechanical ventilation, age acts as a protective factor for the appearance of delirium (OR $0.96, p=0.04$ ).

Conclusion. The incidence of patients discharged from ICU with mental health impairment was $35.2 \%$. The variables associated with this event were non-invasive mechanical ventilation and length of stay in the ICU.

\section{Reference(s)}

1. Mark E Mikkelsen et al. :Society of Critical Care Medicine's International Consensus Conference on Prediction and Identification of Long-Term Impairments After Critical IIIness.Crit Care Med. 2020 Nov:48(11):1670-1679.

2. The author have disclosed that they do not have any potential conflicts of interest.

\section{4}

\section{Comparison between first and second wave among critically} ill COVID-19 survivors admitted to a Portuguese ICU: what are the long-term outcomes?

T. Vieira'; J. Fernandes, ${ }^{1} ;$ S. Martins, ${ }^{2} ;$ N. Reis, ${ }^{1}$; AR. Ferreira, ${ }_{1}^{3}$; L. Fontes, ${ }^{1} ;$, Coimbra, ${ }_{1}$; L. Fernandes, ${ }^{4}$; JA. Paiva,

${ }^{1}$ Intensive care, São João Universitary Hospital Center, Porto, Portugal;

${ }^{2}$ Clinical neurosciences and mental health, Faculdade de Medicina

da Universidade do Porto-FMUP, Porto, Portugal; ${ }^{3}$ Center for health technology and services research/cintesis, Faculdade de Medicina da Universidade do Porto—FMUP, Porto, Portugal; ${ }^{4}$ Psychiatry service, São João Universitary Hospital Center, Porto, Portugal

Correspondence: T. Vieira

Intensive Care Medicine Experimental 2021, 9(1): 000654

Introduction. Survivors of critical illness frequently have long-term physical and neuropsychiatric impairments, with consequent poor long-term health-related quality of life (HRQoL). There are limited data describing long-term outcomes of critically ill coronavirus disease 2019 (COVID-19) patients.

Objectives. We aimed to compare 1 st and 2 nd waves critically ill COVID-19 survivors in terms of long-term health consequences and HRQoL.

Methods. This prospective study involved critically ill COVID-19 survivors admitted to Intensive Care Medicine Service of a University Hospital in Portugal, during the 1st and 2nd waves, (March to May 2020, October to December 2020, respectively). Patients with ICU length of stay (LoS) $\leq 24 \mathrm{~h}$, terminal illness, major sensory loss or inability to communicate at assessment time were excluded. All participants were assessed by a telephone follow-up evaluation performed 1 to 3 months after hospital discharge. HRQoL was explored by EuroQol five-dimension five-level questionnaire (EQ-5D-5L) and visual analogue scale (EQ-VAS). This study reports preliminary results of the ongoing multidisciplinary longitudinal project.

Results. A total of 90 patients were included ( $n=30-1 \mathrm{st}$ wave $/ \mathrm{n}=60-2 \mathrm{nd}$ wave), with a median age of 64,5 years, most being male $(60 \%)$, with a median SAPSII score of 34 , without differences between groups. The most frequent comorbidity was arterial hypertension $(58.9 \%)$, being more frequent in the 1 st wave $(73.3 \%$ vs. $51.7 \%, p=0.049)$. Invasive Mechanical Ventilation (IMV) $(86.7 \%$ vs $31.7 \%, p=0.001)$, deep sedation $(86.7 \%$ vs $31.7 \%, p=0.001)$ and neuromuscular blockade $(79.3 \%$ vs $28.3 \%, p=0.001)$ were more frequently used in the 1st wave. ICU and hospital LoS (median 35.5 vs 7 days; $p=0.001$; median $=79.5$ vs 22 days, $p=0.001$; respectively) were longer in the 1st wave. First wave's patients reported more problems in self-care $(43,3 \%$ vs $36,7 \%)$, daily life activities $(83,3 \%$ vs $76,7 \%$ ), pain/discomfort(73,3\% vs $63,3 \%$ ) and anxiety/depression ( 73,3 vs $70 \%)$ of the EQ-5D-5L domains, but not reaching statistical significance. However, the 2nd wave patients had a worse self-perception of HRQoL (median 70 vs $35, p=0.001$ ) on EQ-VAS. Fatigue, sleep disorders and dyspnea were the most frequent self-reported symptoms, without differences between the two groups.

Conclusion. Firstwave critically ill CoVID-19 survivors reported more problems in most of the domains evaluated in EQ-5D-5L compared to the 2 nd wave patients, but 2 nd wave patients had a worst perception of their HRQoL, which could be explained by an early discharge in this wave. Moreover, larger studies are needed to understand risk factors and, namely in ventilation support, sedation and use of NMBAs, that may impact health-related outcomes for long-term QoL impairment and an organized post-intensive care follow-up structure is fundamental to improve COVID-19 survivors long-term outcome.

\section{9}

Factors influencing ICU bed utilisation post liver transplantation

D. Patel'; K. Kohler

'John Farman Intensive Care Unit, Addenbrooke's Hospital, Cambridge,

United Kingdom; ${ }^{2}$ Division of Anaesthesia, Department of Medicine,

University of Cambridge, Cambridge, United Kingdom

Correspondence: D. Patel

Intensive Care Medicine Experimental 2021, 9(1): 000659

Introduction. Liver transplantation (LT) is the only effective treatment for end-stage liver disease. Advances in peri-operative care have led to an expansion in the annual number of liver transplants undertaken. Clinical activity is set to increase with the introduction of the national opt-out scheme. In tandem, recipients will have a greater risk profile given an ageing population and the rise of non-alcoholic fatty liver disease. This will place a greater strain on intensive care (ICU) resources, so investigating factors that increased bed utilisation are important to determine.

Objectives. To investigate factors contributing to ICU bed utilisation in patients admitted to a single ICU post LT over an 8-year period.

Methods. Retrospective analysis using the ICNARC dataset of all consecutive adult liver-only transplants from 1st January 2012 to 31st December 2019 at Addenbrooke's Hospital, Cambridge.

Results. 690 patients were included (males 437, females 253) with a median age 56.4 years (range 17.4-73.9 years) and admitting APACHE score 15 (range $0-31$ ). The number of LT at this centre increased annually (Fig. 1a). 427 patients (61\%) were admitted to ICU out of hours (2000-0800), with an additional 142 patients $(21 \%)$ admitted between 1600-2000 (Fig. 1b). Median duration of level 0 and 1 care was 0 days. Median duration of level 2 care was 1 day (range 1-13), and level 3 care was 2 days (range $0-53$ ). In keeping with this, median duration of advanced respiratory support was 2 days (range $0-53$ ). Most patients did not require advanced cardiovascular or renal support. The median ICU length of stay (LOS) was 2 days (range $0-58.9$ ). LOS was strongly correlated with advanced respiratory, basic cardiac, and gastrointestinal support, and weakly correlated with age, sex, and APACHE II score (Fig. 1c). 8 patients died during the ICU admission. The remainder were discharged to high dependency level care (598 patients) or the ward (84 patients). Patients discharged to HDU left ICU faster (Fig. 1d).

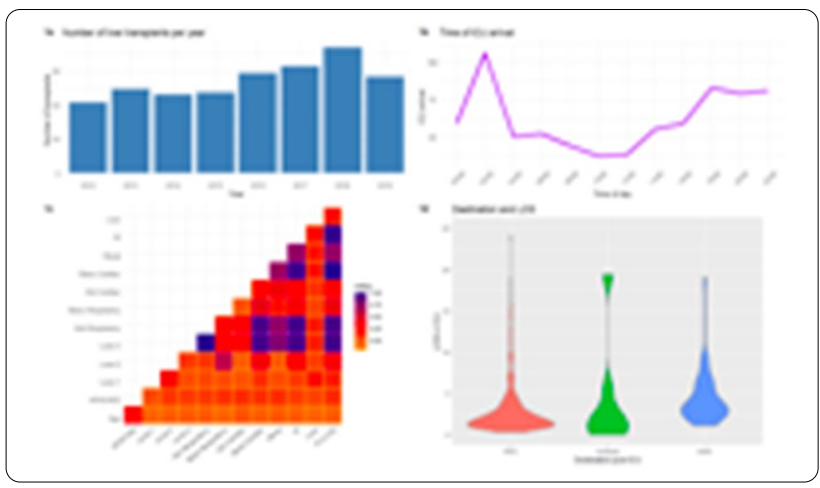

Conclusion. Increasing LT activity increases demands on ICU resources. Advanced respiratory support heavily influences ICU LOS. 
Several LT centres are now advocating a fast-track extubation strategy and have demonstrated physiological and resource benefit. Adoption of this approach in our centre could significantly reduce LOS given the projected rise in LT. However, the proportion of LT that arrive on ICU between 2000-0800 may reduce the number of eligible patients. Age and APACHE score did not influence LOS suggesting that intra-operative factors such as graft function and blood loss have a greater impact on post-operative trajectory.

\section{4}

Traumatic memories in severe COVID-19 survivors

J. Fernandes ${ }^{1}$; T. Vieira'; S. Martins, ${ }^{2}$; I. Coimbra ${ }^{1}$; L. Fontes, ${ }^{1}$; AR. Ferreira, ${ }_{1}^{3}$; L. Fernandes, ${ }^{4}$; JA. Paiva

${ }^{1}$ Intensive Care Medicine Department, São João Universitary Hospital

Center, Porto, Portugal; ${ }^{2}$ Department of Clinical Neurosciences and Mental Health, Faculty of Medicine, University of Porto, Porto, Portugal; ${ }^{3}$ Center for Health Technology and Services Research/Cintesis, Faculty of Medicine, University of Porto, Porto, Portugal; ${ }^{4}$ Psychiatry service, São João Universitary Hospital Center, Porto, Portugal

Correspondence: J. Fernandes

Intensive Care Medicine Experimental 2021, 9(1): 000664

Introduction. Traumatic memories have been described in survivors of critical illness, but little is known about long-term psychological impairments and its impact on health-related quality of life (HRQoL) following discharge from Intensive Care Medicine (ICM) in COVID-19 patients.

Objectives. To assess memories in survivors of severe COVID-19 and to identify stressor factors in critically ill patients, as well as their impact on $\mathrm{HRQ}$ oL after discharge.

Methods. We retrospectively analyzed clinical hospital data of severe COVID-19 survivors admitted to the Intensive Care Department of an University Hospital between 21 March and 13 May 2020. Follow-up evaluation was performed between the 2 nd and the 6th month after hospital discharge. Patients were asked to recall items from a checklist of possible memories from the ICU Memory Tool (ICU-MT) and HRQoL was explored by EuroQol five-dimension five-level questionnaire (EQ$5 \mathrm{D}-5 \mathrm{~L})$. This study reports preliminary results of a multidisciplinary longitudinal project.

Results. Fifty-nine patients were included, $66 \%$ men with a median age of 65 (IQR 55-74) years. Invasive mechanical ventilation (IMV) was performed in $75 \%$ (median 20 days, IQR 16-45) and ICM length of stay was 21 (IQR 9-49) days. Follow-up evaluation occurred a median of 105 days after hospital discharge (IQR 81-128). Any type of memories was described in $81 \%$. Factual memories were reported in $73 \%$ and emotional memories in $64 \%$. Delusional memories were described in $56 \%$ of patients (39\% reporting nightmares, $24 \%$ guessing that people were trying to hurt them and $19 \%$ presenting visual or auditive hallucinations). Patients with ICM stay memories were younger (63 vs 76 years, $p=0.008$ ). Patients with delusional memories had higher SAPS II score (42 vs $28, p=0.022$ ) and nightmares during ICM stay occurred more frequently in difficult to wean patients $(65 \%$ vs $36 \%$, $p=0.036$ ). No difference was observed between delusional memories and occurrence of nosocomial infections, intensive care length of stay, duration of deep sedation or support with extracorporeal membrane oxygenation. Panic sensation experience was associated to sleep disorders $(29 \%$ vs $5 \%, p=0.017)$ and nightmares $(36 \%$ vs $4 \%, p=0,006)$ after discharge. In EQ-5D-5L, the group of patients with delusional memories reported higher incidence of anxiety/depression $(67 \%$ vs $50 \%)$, usual activities ( $76 \%$ vs $54 \%$ ) and self-care (30\% vs $27 \%$ ) problems after hospital discharge in comparison to those without those type of memories, despite no statistically significant association.

Conclusion. Most critically ill COVID-19 patients described memories of their ICM stay, often of a delusional nature, and traumatic memories during ICM stay may be correlated with lower HRQoL 2 to 6 months after discharge. More studies are needed to understand delusional associated factors in critically ill patients and the association between those memories and psychological sequelae, affecting HRQoL after discharge.

\section{Reference(s)}

1. Granja C, Lopes A, Moreira S, et al. Patients' recollections of experiences in the intensive care unit may affect their quality of life. Crit Care. 2005;9:R96-R109. https://doi.org/10.1186/cc3026

2. Train S, Kydonaki K, Rattray J, Stephen J, Weir CJ, Walsh TS. Frightening and Traumatic Memories Early after Intensive Care Discharge. Am J Respir Crit Care Med. 2019;199:120-123. https://doi.org/10.1164/rccm. 201804-0699LE.

3. Vlake JH, van Genderen ME, Schut A, et al. Patients suffering from psychological impairments following critical illness are in need of information. J Intensive Care 8, 6 (2020). https://doi.org/10.1186/s40560-019-0422-0

\section{2}

Early Predictors of Mortality in Cardiogenic Shock-ten year experience of a tertiary cardiac centre

L. Stewart'; S. Gillon,

${ }^{1}$ Medical School, The University of Edinburgh, Edinburgh, United Kingdom; ${ }^{2}$ Critical Care Unit, Royal Infirmary of Edinburgh, NHS Lothian, Edinburgh, United Kingdom

Correspondence: $L$. Stewart

Intensive Care Medicine Experimental 2021, 9(1): 000672

Introduction. Cardiogenic shock (CGS) is a common indication for admission to the Intensive Care Unit (ICU) and is associated with a high-mortality rate [1]. For patients with refractory CGS, mechanical circulatory support (MCS) devices may offer an advantage over conventional management with vasopressors and inotropes alone [2]. There is however, limited guidance to aid decision making with regards to the use of MCS devices in this patient group [3]. Given the resource implications and the risk of complications associated with MCS, there is a pressing need to identify CGS patients at high risk of death towards whom MCS resources may be better directed.

Objectives. To describe the population of patients presenting to a large, regional ICU, at a tertiary care centre with cardiogenic shock; to identify factors associated with hospital mortality.

Methods. This was a retrospective evaluation of service involving patients admitted to the Royal Infirmary of Edinburgh's general intensive care unit between 1st January 2010 and 29th January 2020. Approval was granted by the local Quality Improvement Team committee.

Patients with cardiogenic shock, of primary cardiac aetiology were identified from the WardWatcher ICU database. Continuous variables were summarised by mean and standard deviation or median and interquartile range as appropriate. Categorical variables were summarised by proportion. Patients were stratified into those who died directly due to CGS and those who did not. Univariate and subsequent, stepwise multivariate analysis were carried out to identify factors independently associated with CGS-related in-hospital mortality. Statistics were performed in the computing environment $R$.

Results. 476 eligible patients were admitted during the study period median age 63 years (IQR 53.75-72), 67.2\% of the cohort was male. Acute myocardial infarction was the most common aetiology of CGS accounting for $67 \%$ of cases. Overall mortality was $57.1 \%$, with risk of in-hospital mortality remaining stable across the 10 years. Several factors were associated with cardiogenic shock related mortality (Table 1).

Table 1. Factors associated with cardiogenic shock mortality

\begin{tabular}{lllr}
\hline Variable & $\begin{array}{l}\text { Non-CGS related } \\
\text { mortality } \\
(\mathbf{n}=\mathbf{2 7 3})\end{array}$ & $\begin{array}{l}\text { CGS-related } \\
\text { mortality } \\
(\mathbf{n}=\mathbf{2 0 3})\end{array}$ & P value \\
\hline Age & $60(52-69)$ & $68(58-75)$ & $<0.001$ \\
APACHE score & $25.0(19.0-30.0)$ & $28.0(22.8-35.0)$ & $<0.001$ \\
$\begin{array}{l}\text { Severe cardiovascular } \\
\text { disease }\end{array}$ & $6.2 \%$ & $13.9 \%$ & 0.005 \\
Lowest SBP in first 24h h & $90(79-95)$ & $80(62-95)$ & $<0.001$
\end{tabular}

(mmHg) 


\begin{tabular}{|c|c|c|c|}
\hline Variable & $\begin{array}{l}\text { Non-CGS related } \\
\text { mortality } \\
(n=273)\end{array}$ & $\begin{array}{l}\text { CGS-related } \\
\text { mortality } \\
(n=203)\end{array}$ & $P$ value \\
\hline $\begin{array}{l}\text { Average hourly urine } \\
\text { ouput in first } 24 \mathrm{~h} \\
\text { (ml) }\end{array}$ & $54(32-80)$ & $17(3-46)$ & $<0.001$ \\
\hline $\begin{array}{l}\text { Highest } \mathrm{H}+\text { in first } \\
24 \mathrm{~h}(\mathrm{mmol} / \mathrm{l})\end{array}$ & $57(46-68)$ & 72 (56-96) & $<0.001$ \\
\hline $\begin{array}{l}\text { Highest creatinine in } \\
\text { first } 24 \mathrm{~h}(\mu \mathrm{mol} / \mathrm{l})\end{array}$ & $122(84-179)$ & 166 (119-228) & $<0.001$ \\
\hline $\begin{array}{l}\text { Admission lactate } \\
(\mathrm{mmol} / \mathrm{l})\end{array}$ & $3.1(2.0-6.3)$ & $5.8(3.3-9.2)$ & $<0.001$ \\
\hline $\begin{array}{l}\text { Highest noradrenaline } \\
\text { dose in first } 24 \mathrm{~h} \\
\text { (mcg/hr) }\end{array}$ & $0.19(0.03-0.37)$ & $0.37(0.21-0.62)$ & 0.018 \\
\hline $\begin{array}{l}\text { Lowest } \mathrm{PaO}_{2} / \mathrm{FiO}_{2} \text { ratio } \\
\text { in first } 24 \mathrm{~h}\end{array}$ & $14.0(10.0-21.2)$ & $11.1(8.3-15.9)$ & 0.001 \\
\hline
\end{tabular}

Multivariate analysis demonstrated those factors independently associated with in-hospital mortality due to CGS to be age OR 1.04 (95\% $\mathrm{Cl} 1.01-1.07)$, hourly urine-output OR 0.98 (0.97-0.99) and admission lactate OR 1.12 (1.03-1.22).

Conclusion. In our population, CGS is associated with a high mortality rate, which has not improved with time. Age, admission lactate and urine output were all independently associated with CGS-related mortality in this cohort. In the future these findings may assist decision making with regards to the use of MCS devices, by identifying patients at a higher risk of death due to CGS who would benefit most from additional circulatory support.

\section{Reference(s)}

1. 1. Vahdatpour C, Collins D, Goldberg S. Cardiogenic Shock. J Am Heart Assoc. 04 2019;8(8):e011991. https://doi.org/10.1161/JAHA.119.011991

2. 2. Parissis $\mathrm{H}$, Soo A, Al-Alao B. Intra aortic balloon pump: literature review of risk factors related to complications of the intraaortic balloon pump. J Cardiothorac Surg. Nov 2011;6:147. https://doi.org/10.1186/ 1749-8090-6-147

3. 3. Helgestad OKL, Josiassen J, Hassager C, et al. Contemporary trends in use of mechanical circulatory support in patients with acute $\mathrm{Ml}$ and cardiogenic shock. Open Heart. 2020;7(1):e001214. https://doi.org/10. 1136/openhrt-2019-001214

\section{5}

Availability and use of supports during the COVID-19 health crisis A. Fournier ' ;. Deltour ${ }^{2}$; F. Lheureux ${ }^{3}$; AL. Poujol ${ }^{4}$; C. Binquet ${ }^{5}$; JP. Quenot ${ }^{6}$; A. Laurent ${ }^{2}$

${ }^{1}$ Psychology, University of Burgundy, Dijon, France; ${ }^{2}$ Psy-drepi, University of Burgundy, Dijon, France; ${ }^{3}$ Psychologie, University of Franche-Comté, Besançon, France; ${ }^{4}$ Psychopathologies, Ecole de Psychologues Praticiens, Paris, France; Inserm, cic 1432, Clinical Epidemiology, Hospital Center University François Mitterand, Dijon, France; ${ }^{6}$ Medical intensive care unit, Chu Dijon, Dijon, France

Correspondence: A. Fournier

Intensive Care Medicine Experimental 2021, 9(1): 000685

Introduction. To support intensive care unit (ICU) professionals in coping with difficulties associated with the COVID-19 health crisis, various supports have been proposed at national level. Professionals were offered support by psychologists (internal or external to the service) and telephone hotlines provided by psychologists or psychiatrists. Healthcare professionals could also turn to their colleagues and superiors, and outside of work, to those around them, to discuss the difficulties they encountered. In addition, volunteers, directly or indirectly, supported the healthcare workers by offering free services, gifts, equipment or meals, and the population applauded the health workers for the work they did every evening during the first wave of the crisis.
Objectives. To develop optimal care, the objective of the PsyCOVIDICU research team was to estimate whether professionals have taken advantage of the supports available to them and to identify which supports are preferred by carers according to their professional category.

Methods. From 3 June to 21 December 2020, three online questionnaires (June, October and December) were offered in the intensive care units of 77 French hospitals. These questionnaires assessed perceived stress [adapted versions of the PS-ICU (1) and Khalid (2) scales], and closed questions (availability and use) on the use of six supports.

Results. In June, 1941 ICU professionals agreed to participate in the study; in October 1595 professionals; in December 1348 professionals. Across the different times of the crisis, support from the hierarchy and volunteers were the least available supports, while support from the entourage was the most available. The availability of support remained stable throughout the crisis, except for the telephone hotline, whose availability increased between June and October $(p=.004)$. When they were available, the most used supports were those of relatives and colleagues, while the least used were the telephone hotline and psychologists. The use of colleague, hierarchy, volunteer and relative supports decreased between June and December and between October and December; while the use of psychologists decreased between June and October and between June and December (all $p<.05$ ). However, there was no variation over time in the use of the telephone hotline. Despite a gradual decrease in the use of supports, the analyses reveal a significant increase in the level of perceived stress between the months of June and October $(p<.001)$, and a significant decrease between the months of October and December, $\mathrm{p}<.001$.

Conclusion. Despite the nnational establishment of supports and their availability, professionals made little use of the supports and seemed to prefer support from the known social environment. The hierarchy was one of the least available supports. Further studies are needed to identify the most effective supports for reducing the perceived stress associated with the health crisis.

\section{Reference(s)}

1. 1. Laurent A, Fournier A, Lheureux F, Martin Delgado MC, Bocci MG, Prestifilippo A, et al. An international tool to measure perceived stressors in intensive care units: the PS-ICU scale. Annals of intensive care. Accepted

2. 2. Khalid I, Khalid TJ, Qabajah MR, Barnard AG, Qushmaq IA. Healthcare Workers Emotions, Perceived Stressors and Coping Strategies During a MERS-CoV Outbreak. Clin Med Res [Internet]. 2016 Mar 1:14(1):7-14.

3. This study was funded by a grant (PHRC-COVID 2020) from the Programme Hospitalier de Recherche Clinique National, funded by the French Ministry of Health.

000686

Predicting ICU outcome in patients with very long stay in the ICU ( $>\mathbf{3 0}$ days) after cardiac surgery

B. Zapletal, ${ }^{1} ;$ T. Stamm ${ }^{2}$; T. Neugebauer ${ }^{3}$; E. Tschernko ${ }^{4}$

'Division of Cardiac Thoracic Vascular Anaesthesia and Intensive Care, Medical University of Vienna, Wien, Austria; ${ }^{2}$ Center for Medical Statistics, Informatics, and Intelligent Systems, Section for Outcomes Resear, Medical University of Vienna, Wien, Austria; ${ }^{3}$ Division of Cardiac Thoracic Vascular Anaesthesia and Intensive Care Medicine, Medical University of Vienna, Wien, Austria: ${ }^{4}$ Cardiothoracic and vascular anaesthesia,

Medical University of Vienna, Wien, Austria

Correspondence: B. Zapletal

Intensive Care Medicine Experimental 2021, 9(1): 000686

Introduction. Patients with very long stay in the ICU (>30 d) have been shown to have a high mortality and morbidity during their ICU stay (Friedrich JO, Crit Care 2006). The need of ICU resources for such patients has been shown to be high and their exposure to stress, pain and dyspnoea during invasive respiratory weaning, invasive procedures, complications may be significant.

Scores such as SAPS or APACHE are of limited value in these patients (Suistomaa M, ICM 2002). Alternative outcome prediction models showed advanced age, immunosuppression and mechanical ventilation for $>90$ days to be risk factors for death in the ICU\{Friedrich:2006 cd\}. 
However, they may be of limited help for decision making when ICU clinicians are faced with questions of futility of their treatment for example in an old, transplanted patient before day 90 . The aim of our retrospective study was to investigate the impact of 10 clinically significant parameters of organ dysfunction in order to predict ICU mortality of these patients at day 31 in the ICU.

Methods. A total of 138 cardiac surgical patients with ICU length of stay $>30$ day were treated in our 16 bed ICU between 2016 and 2020 in our tertiary care centre. We compared utilisation of resources between survivors and non-survivors, a model including parameters of hemodynamic, renal and respiratory support was created in order to enable clinicians to prognose ICU outcome at day 31.

Results. ICU mortality in patients with very long stay stay in the ICU was $26 \%$, length of stay did not differ clinically significantly between ICU survivors ( 55 days) and ICU non-survivors ( 52 days). Utilisation of resources differed significantly in regard to renal support $14.2 \pm 17.4$ for ICU survivors vs. $23 \pm 8.6$ days of CVVHD for non-survivors. Of all the investigated parameters, only duration of norepinephrine support $>0,1 \mathrm{mcg} / \mathrm{kg} / \mathrm{min}$ correlated significantly and clinically relevantly with ICU survival, meaning that patients with NA support $<23$ days had a predicted ICU survival of $82 \%$.

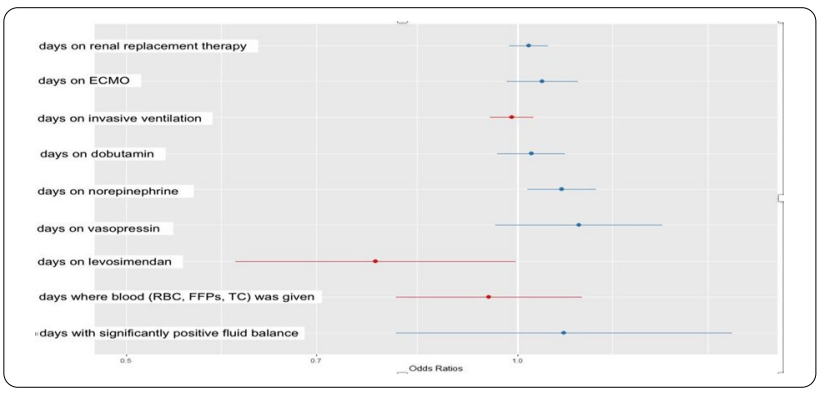

Conclusion. In patients with very long stay in the ICU after cardiac surgery, length of stay in the ICU alone does not predict ICU outcome. However, utilisation of ICU resources is significantly increased in ICU non-survivors compared to ICU survivors. On day 31, clinically significant doses of Norepinephrine support on less than 23 days in the ICU are highly predictable of ICU survival with $82 \%$ certainty in this population. Among other factors, this may serve as an additional guide for clinicians in order to facilitate communication with patients and their families and avoid premature withdrawal of care.

\section{Reference(s)}

1. Suistomaa M, Niskanen M, Kari A, Hynynen M, Takala J. Customized prediction models based on APACHE II and SAPS II scores in patients with prolonged length of stay in the ICU. Intensive Care Med. Springer-Verlag; 2002 Apr:28(4):479-85.

2. Friedrich JO, Wilson G, Chant C. Long-term outcomes and clinical predictors of hospital mortality in very long stay intensive care unit patients: a cohort study. Crit Care. BioMed Central; 2006;10(2):R59-9.

\section{8}

Psychological Safety in an Adult ICU Following COVID-19

ME. Stewart ${ }^{1}$; JS. Needham ${ }^{1}$; AJ. Chadwick

${ }^{1}$ Adult Intensive Care Unit, John Radcliffe Hospital, Oxford, United

Kingdom

Correspondence: M.E. Stewart

Intensive Care Medicine Experimental 2021, 9(1): 000698

Introduction. 'Psychological safety' is the belief that one will be not humiliated or punished when asking questions, taking risks or voicing concerns within a team. Organisations need psychological safety to learn via error reporting 1 and quality improvement2. It has been recognised as a driver of high-quality patient care in nursing literature3, but is understudied in intensive care. Following the burden placed on ICU staff by COVID-19, the promotion of psychologically safe workplaces will be key in ensuring patient safety and staff retention following the pandemic4. Here we present an assessment of psychological safety in the multi-disciplinary team of an adult ICU after the first COVID wave. This study is one of the first assessments of psychological safety in a UK ICU, and the first presented globally since the start of the pandemic.

Objectives. 1. To quantify overall psychological safety in our adult ICU's MDT. 2. To investigate whether psychological safety varied based on a) profession b) time spent working in the unit

Methods. We investigated psychological safety of MDT members in the adult ICU at a university teaching hospital. Psychological safety was assessed using a validated 7 item survey5. Each item was quantified with a 7-point Likert scale ('strongly disagree' to 'strongly agree'). Items assessed features of psychological safety including cultures of blame, willingness to help and perceptions of interpersonal risk. Staff were recruited anonymously without incentive October-November 2020.

Results. 110 staff completed the survey, with 107 included after excluding those with poor understanding of survey items. These included 38 doctors, (36\%, 8 consultants and 30 juniors), 57 nurses (53\%, 28 staff grade and 29 senior banded nurses) and 12 other allied health professionals (11\%).

Overall mean ( \pm standard deviation) scores were $5.0( \pm 1.5)$ for positive statements (higher scores = more psychological safety) and $3.0( \pm 1.7)$ for negative statements (lower scores $=$ more psychological safety). There was no significant difference between mean scores in positive/ negative statements for doctors, nurses or AHPs. Finally, we compared mean scores of all staff who had been part of the team for $<3$ months against all others, allowing us to capture juniors temporarily rotating through the unit. There was no significant difference in mean scores compared to staff that had been on the team for $>3$ months. Staff felt least psychologically safe when responding to "People on this team sometimes reject others who think in a different way", with $40 \%$ agreeing.

Conclusion. After the first COVID wave, our ICU demonstrated a medium level of psychological safety6. There was no significant association between psychological safety and profession, or duration of working in the unit. However, our results suggest a rigidity of thought in at least some aspects of the MDT. These data highlight deficits in our working culture and provide a baseline from which to judge the efficacy of future initiativess

We plan to use this data to inform further psychological safety interventions to address these deficits and ultimately correlate with clinical outcome measures.

\section{Reference(s)}

1. 2. Tucker, A. L., Nembhard, I. M. \& Edmondson, A. C. Implementing new practices: An empirical study of organizational learning in hospital intensive care units. Manage. Sci. 53, 894-907 (2007).

2. 3. Pfeifer, L. E. \& Vessey, J. A. Psychological safety on the healthcare team. Nurs. Manage. 50, 32-38 (2019).

3. 4. Rangachari, P. \& Woods, J. L. Preserving organizational resilience, patient safety, and staff retention during covid-19 requires a holistic consideration of the psychological safety of healthcare workers. International Journal of Environmental Research and Public Health vol. 17 1-12 (2020).

4. 5. Edmondson, A. Psychological Safety and Learning Behavior in Work Teams. Adm. Sci. Q. 44, 350 (1999).

5. 6. O'Donovan, R. \& McAuliffe, E. Exploring psychological safety in healthcare teams to inform the development of interventions: Combining observational, survey and interview data. BMC Health Services Research vol. $20810(2020)$

6. $\mathrm{Nil}$

7. 1. Edmondson, A. C. Learning from Mistakes is Easier Said Than Done: Group and Organizational Influences on the Detection and Correction of Human Error. J. Appl. Behav. Sci. 32, 5-28 (1996). 


\section{8}

Changes in the ICU patient profile after COVID-19 pandemic in a low-middle income country

R. Santos Oliveira $\mathrm{Jr}^{1}$; M. Pina ${ }^{1}$; M. Pina'; R. Menezes, ${ }^{2}$; R. Menezes, ${ }^{2}$; I. Ferreira $_{1}{ }^{3} ;$ I. Ferreira, ${ }^{3}$; L. Pamplona Neto ${ }^{4}$; L. Pamplona Neto ${ }^{4}$; S. Agareno ${ }^{5}$; S. Agareno ${ }^{5}$; NM. Filgueiras Filho ${ }^{6}$

${ }^{1}$ Research, Teaching and Communication Center, Hospital da Cidade, Salvador, Brazil; ${ }^{2}$ Inflammation and Biomarkers Laboratory, Oswaldo Cruz Foundation, Salvador, Brazil; ${ }^{3}$ Life Sciences Department, University of State of Bahia, Salvador, Brazil; ${ }^{4}$ Critical Care, Hospital da Cidade, Salvador, State of Bahia, Brazil, Brazil; ${ }^{5}$ Board of directors, Hospital da Cidade, Salvador, Brazil; ${ }^{6}$ Medicine, Salvador University, Campus Teacher Barros, Salvador, Brazil

Correspondence: N.M. Filgueiras Filho

Intensive Care Medicine Experimental 2021, 9(1): 000708

Introduction. Due to the coronavirus (COVID) pandemic, the need for medical assistance rose, since this virus has spread with ease due to its high transmissibility, (1). As a result, many hospitals began to receive a different patient profile in their intensive care units (ICU), serving mainly the most serious patients due to COVID. Thus, knowing this new patient profile is necessary to properly manage the ICUs $(2,3)$

Objectives. To compare the epidemiological profile of patients one year before and one year after COVID, in a referral hospital in Salvador. Methods. Prospective cohort that evaluated secondary data of patients admitted in the ICU in a tertiary hospital between February 2019 and February 2021. Patients were divided into 2 groups based on differences between one year before (group A) and one year after (group B) the start of the pandemic. Results were described using mean (standard deviation) or frequency (percentage). Differences between continuous variables were analyzed using T-test, while categorical variables were assessed using $\mathrm{Chi}^{2}$ test or Fisher's exact test. This study was approved by the ethical and research committee.

Results. Of the 1,402 patients included in the study, 787 patients were in group A (56.1\%) and 615 patients were in group B (43.8\%). Group A had a mean age of 67 years $( \pm 19), 54.6 \%$ were woman and the mean $\mathrm{BMI}$ was $25.6 \mathrm{~kg} / \mathrm{m} 2( \pm 5.9)$. In addition, the group B had a mean age of $63 \pm 18$ years, $51.1 \%$ were woman, the mean BMI was $26.6 \pm 6 \mathrm{~kg} / \mathrm{m}^{2}$ and 110 patients $(17.9 \%)$ had confirmed COVID. Regarding the ventilatory profile in the first hour of admission, respiratory failure increased from $1.4 \%$ to $38 \%(p<0,001)$ and the need of mechanical ventilation increased from $4.4 \%$ to $11.9 \%(p<0,001)$ in group B. The use of vasopressors in the first hour of admission had an increase from $7.1 \%$ to $10.7 \%(p<0,022)$ in group B. The mean SAPS3 score increased from $46 \pm 12$ points to $47 \pm 12$ points $(p<0,024)$ in group $B$. The mean duration of mechanical ventilation use increased from $5 \pm 6$ days to $10 \pm 12$ $(p<0,001)$ in group B. As well, there was an increase in ventilator-associated pneumonia (VAP) from $0.6 \%$ to $2.1 \%(p<0,006)$.

Conclusion. In the population admitted after February 2020, a higher mean age and BMI were found. In addition, they presented a higher frequency of respiratory failure, the need for mechanical ventilation, the duration of use of this ventilation, and a greater use of vasopressor. Furthermore, these patients had a higher score on SAPS3, and had more VAP. However, despite the change in the profile, mortality has not changed significantly.

\section{Reference(s)}

1. Petrilli CM, Jones SA, Yang J, Rajagopalan H, O'Donnell L, Chernyak Y, et al. Factors associated with hospital admission and critical illness among 5279 people with coronavirus disease 2019 in New York City: Prospective cohort study. BMJ. 2020;369.
2. Ferreira JC, Ho YL, Besen BAMP, Malbuisson LMS, Taniguchi LU, Mendes P $V$. et al. Characteristics and outcomes of patients with covid-19 admitted to the icu in a university hospital in são paulo, brazil-study protocol. Clinics. 2020;75(9):1-7.

3. Goh KJ, Wong J, Tien JCC, Ng SY, Duu Wen S, Phua GC, et al. Preparing your intensive care unit for the COVID-19 pandemic: Practical considerations and strategies. Crit Care. 2020;24(1):1-12.

4. No funding has been required.

000712

Development of a novel severity scoring system for COVID-19 patients at hospital admission

R. Menezes, ; M. Pina ${ }^{2}$; R. Santos Oliveira Jr ${ }^{2}$; I. Ferreira, ${ }^{3}$; B. Darzé, ${ }^{4}$; C. Cunha, ${ }^{5}$; L. Amorim, ; D. Vicente, ${ }^{6}$; R. Vieira, ${ }^{6}$; J. Ramos, ; E. Darzé, ; L. Ritt, NM. Filgueiras Filho ${ }^{9}$

${ }^{1}$ Inflammation and Biomarkers Laboratory, Oswaldo Cruz Foundation, Salvador, Brazil; ${ }^{2}$ Research, Teaching and Communication Center, Hospital da Cidade, Salvador, Brazil; ${ }^{3}$ Life sciences department, University of State of Bahia, Salvador, Brazil; ${ }^{4}$ Research Center, Hospital Cárdio Pulmonar, Salvador, Brazil; ${ }^{5}$ School of Medicine, BAHIANA — School of Medicine and Public Health, Salvador, Brazil; ${ }^{6}$ Critical care, Hospital e Clínica São Roque, Ipiaú, Brazil; ; Board of directors, Hospital Cárdio Pulmonar, Salvador, Brazil; ${ }^{8}$ Training, Teaching and Research Manager, Hospital Cárdio Pulmonar, Salvador, Brazil; ${ }^{9}$ Medicine, Salvador University, Campus Teacher Barros, Salvador, Brazil

Correspondence: N.M. Filgueiras Filho

Intensive Care Medicine Experimental 2021, 9(1): 000712

Introduction. Due to the COVID-19 pandemic, the world has seen an increase in demand for intensive care and material resources, as well as a reduction in health teams, as professionals are also affected by the pandemic. In this context, the possibility of classifying patients more accurately is extremely important, allowing resources to be allocated more efficiently. The prognostic scores, especially those that demand less technology, provide a valuable aid to medical decisions in lowmiddle income countries.

Objectives. To develop a score that predicts the mortality of patients with COVID-19 upon hospital admission.

Methods. Multi-center, retrospective, observational cohort, which evaluated medical records of COVID-19 patients admitted in 6 tertiary care hospitals in Brazil, from March 2020 to October 2020. Demographic, clinical, laboratorial, and outcomes data. Results were described using mean \pm standard deviation or frequency (percentage). A binary logistic regression, backwards stepwise method, was used to assess the characteristics independently associated with hospital mortality. The optimal cut-off points for the continuous variables were determined with the Youden's criteria. To assess the model calibration, the Hosmer-Lemeshow test was used. This study was approved by the ethical and research committee.

Results. There were 430 patients included in the analysis. Mean age was $63 \pm 18$ years; 257 (59.8\%) were men, and mean BMI was $29 \pm 6 \mathrm{~kg} / \mathrm{m}^{2}$. The prognostic predictive model included the following variables: age $>70$ years, asthenia, hypertension, lactate $>1.08 \mathrm{mg} / \mathrm{dl}$ and respiratory rate $>20$ incursions per minute. One point was attributed to the presence of each of these characteristics. Then, the model presented good accuracy for the prediction of mortality, with an AUC of 0.83 (Cl $0.79-0.86)$, sensitivity of $77 \%$, and specificity $76 \%$ at a cutoff of more than two points, and good calibration, shown by the Hosmer-Lemeshow test, $\mathrm{p}$-value 0.99 . 


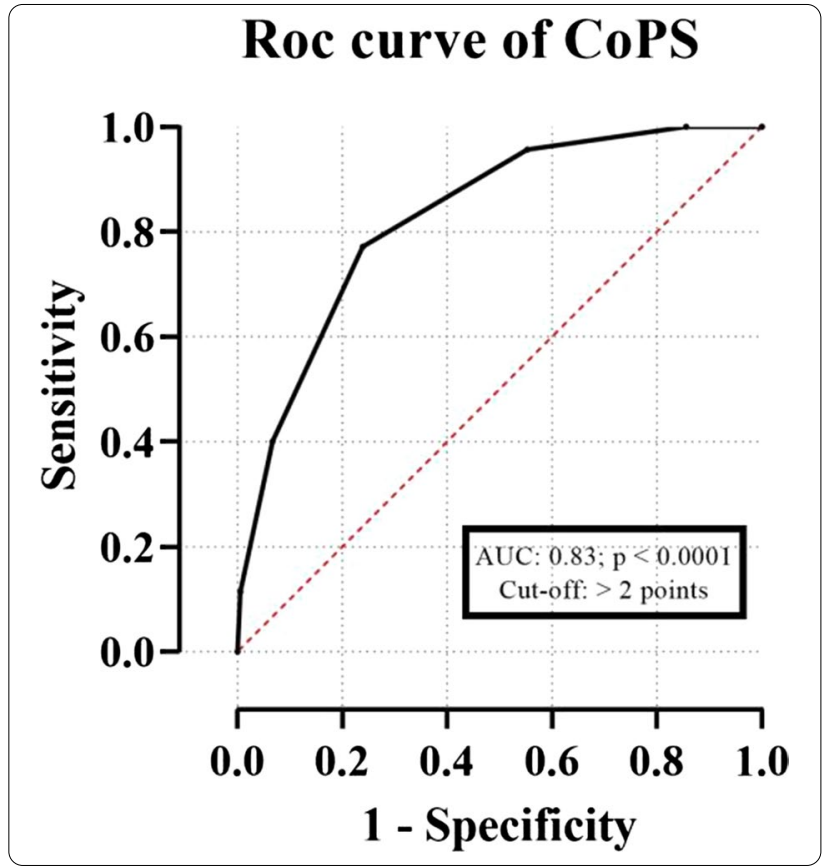

Conclusion. The COPS score is an accurate prognostic tool based on simple variables, readily available at the time of admission, compatible with the reality of limited resources, in low-middle income countries. We hope that it can assist the management of risks and the use of resources of COVID-19 patients.

\section{Reference(s)}

1. Silva Karla Rona da, Souza Fernanda Gonçalves de, Roquete Fátima Ferreira, Faria Shirlei Moreira da Costa, Peixoto Bruno César Ferreira, Vieira Adriane. Allocation of resources for health care in COVID-19 pandemic times: integrative review. Rev. Bras. Enferm. [Internet]. 2020 [cited 2021 Apr 17]; 73( Suppl 2): e20200244. Available from: http://www.scielo.br/ scielo.php?script=sci_arttext\&pid=S0034-71672020001400303\&lng=en. Epub Sep 18, 2020. https://doi.org/10.1590/0034-7167-2020-0244.

2. Menezes RC, Ferreira IBB, Carmo TA, Telles GP, Pugas PLD, Otero ML, Arriaga MB, Fukutani KF, Neto LP, Agareno S, Filqueiras Filho NM, Akrami KM, Andrade BB. Are prognostic tools losing accuracy? Development and performance of a novel age-calibrated severity scoring system for critically ill patients. PLoS One. 2020 Nov 4;15(11):e0240793. https://doi.org/ 10.1371/journal.pone.0240793. PMID: 33147243; PMCID: PMC7641388.

3. Carmo TA, Ferreira IB, Menezes RC, Telles GP, Otero ML, Arriaga MB, Fukutani KF, Neto LP, Agareno S, Filgueiras Filho NM, Andrade BB, Akrami KM. Derivation and Validation of a Novel Severity Scoring System for Pneumonia at Intensive Care Unit Admission. Clin Infect Dis. $2021 \mathrm{Mar}$ 15;72(6):942-949. https://doi.org/10.1093/cid/ciaa183. PMID: 32,146,482. PMCID: PMC7958772.

4. Bradley P, Frost F, Tharmaratnam K, Wootton DG; NW Collaborative Organisation for Respiratory Research. Utility of established prognostic scores in COVID-19 hospital admissions: multicentre prospective evaluation of CURB-65, NEWS2 and qSOFA. BMJ Open Respir Res. 2020;7(1):e000729. https://doi.org/10.1136/bmjresp-2020-000729

5. No funding has been required.
000713

Assessment of the correlation between burnout and spirituality in health professionals during the covid-19 pandemic B. Fialho, ${ }^{1}$; R. Silva ${ }^{1}$; K. Aguiar, ${ }^{2}$; R. Menezes, ${ }^{3}$; I. Ferreira, ${ }^{4}$; NM. Filgueiras Filho ${ }^{5}$

${ }^{1}$ Research, Teaching and Communication Center, Hospital da Cidade, Salvador, Brazil; ${ }^{2}$ Critical care, Hospital Emec, Salvador, Brazil; ${ }^{3}$ Inflammation and Biomarkers Laboratory, Oswaldo Cruz Foundation, Salvador, Brazil; ${ }^{4}$ Life Sciences Department, University of State of Bahia, Salvador, Brazil; ${ }^{5}$ Medicine, Salvador University, Campus Teacher Barros, Salvador, Brazil

Correspondence: N.M. Filgueiras Filho

Intensive Care Medicine Experimental 2021, 9(1): 000713

Introduction. The negative effects of the COVID-19 pandemic have left health professionals that work on the front line even more vulnerable to Burnout Syndrome. Understanding the determinants of this syndrome is essential to assist in its confrontation. It is known that spirituality participates in the phenomenon of coping, but its correlation with burnout has not yet been determined.

Objectives. To investigate the correlation between the Burnout Syndrome and the support of spirituality as a coping mechanism.

Methods. Observational cross-sectional study conducted between March and April 2020. Self-administered questionnaires were applied to health professionals in a general hospital in the state of Bahia, Brazil, containing the Oldenburg Burnout Inventory (OLBI) and the Beliefs scale. The scores were compared using Spearman's correlation.

Results. There were included 102 professionals in our study, being 25 nurses (24.5\%), 24 doctors (23.5\%), 13 physical therapists (12.7\%) and 38 nursing technicians ( $37.2 \%)$ who met the inclusion criteria during the study period, with mean age $22 \pm 58$ and prevalence of women (68.6\%). The average of Beliefs was $1 \pm 4$ with 51 (50\%) professionals obtaining the highest score. The OLBI average was 1,125 $\pm 3,375$, with 90 (88.2\%) of professionals at medium/high risk of developing burnout. There was no significant correlation between the scores $(r-13.5 ; 95 \% \mathrm{Cl}-0.32-0.06$; $\mathrm{p}=0.174)$.

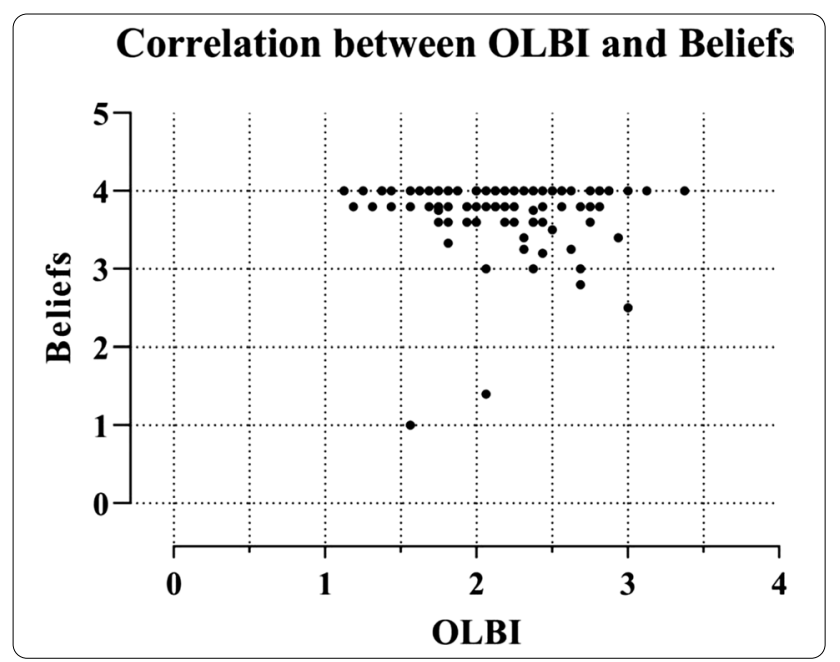

Conclusion. There is a high prevalence of religiosity and a high risk of burnout in our population. However, there was no significant correlation between the two phenomena. Beliefs' high marks may have impacted the analysis. 
Reference(s)

1. 5. Demerouti, E. (2008). Measurement of Burnout and Engagement. Handbook of Stress and Burnout in Health Care, 1-25.

2. 4. Esperandio, M. R., Michel, R. B., Trebien, H. A. C., \& Menegatti, C. L.(2017). Spiritual/Religious Coping in an ICU: Reflections on the Integration of Spirituality Into Health Care. p. 308

3. 3. Group, A. W. (n.d.). Multidimensional Measurement of Religiousness/ Spirituality for Use in Health Research: A Report of the Fetzer Institute/ National Institute on Aging Working Group.

4. 2. Embriaco, N., Azoulay, E., Barrau, K., Kentish, N., Pochard, F., Loundou, A., \& Papazian, L. (2007). High level of burnout in intensivists: Prevalence and associated factors. American Journal of Respiratory and Critical Care Medicine, 175(7), 686-692. https://doi.org/10.1164/rccm.200608-11840C

5. 1. Oliveira, W. A. de. (2020). COVID-19: Desafios e oportunidades da enfermagem brasileira COVID-19: Challenges and opportunities for brazilian nursing Wender Antonio de Oliveira 1*. Revista de Saúde-RSF, 7(2), 22-39.

6. No funding has been needed.

\section{4}

Risk assessment for burnout in health professionals during covid-19 pandemic shows a prevalence of exhaustion R. Silva ${ }^{1}$; B. Fialho, ${ }^{1}$; K. Aguiar, ${ }^{2}$; R. Menezes, ${ }^{3} ;$ I. Ferreira, ${ }^{4}$; NM. Filgueiras Filho

${ }^{1}$ Research, Teaching and Communication Center, Hospital da Cidade, Salvador, Brazil; ${ }^{2}$ Critical care, Hospital Emec, Salvador, Brazil; ${ }^{3}$ Inflammation and biomarkers laboratory, Oswaldo Cruz Foundation, Salvador, Brazil; ${ }^{4}$ Life Sciences Department, University of State of Bahia, Salvador, Brazil; ${ }^{5}$ Medicine, Salvador University, Campus Teacher Barros, Salvador, Brazil

Correspondence: N.M. Filgueiras Filho

Intensive Care Medicine Experimental 2021, 9(1): 000714

Introduction. The negative effects of the COVID-19 pandemic have left health professionals that work on the front line even more vulnerable. Fear, stress and anxiety intensify physical and mental exhaustion, favoring the development of Burnout Syndrome. It is known that this is already a more vulnerable population, but the impacts of the current events are yet to be defined.

Objectives. To investigate the profile of the Burnout syndrome of health professionals who work on the front lines during the covid pandemic, and its determinants.

Methods. Investigate the profile of the Burnout syndrome of health professionals who work on the front lines during the covid pandemic and its determinants.

Results. There were included 102 professionals in our study, being 25 nurses (24.5\%), 24 doctors (23.5\%), 13 physical therapists $(12.7 \%)$ and 38 nursing technicians (37.2\%) who met the inclusion criteria during the study period, with mean age $22 \pm 58$ and prevalence of women (68.6\%). None of the demographic and comorbidity data was significant in relation to differences in burnout rates. In this population, 80 (78.4\%) worked in the ICU. The data also point out that $18(17.6 \%)$ of these have chronic disease while only $17(16.7 \%)$ makes use of medication, 59 (57.8\%) do not perform physical activities and 53 (52\%) does not do nutritional monitoring. Most, 71 (69.60\%), agree that during work they feel emotionally drained, in contrast, 96 (94.11\%) participants consider the work a positive challenge. According to OLBI, 90 (88.2\%) professionals were at medium/high risk for burnout syndrome, and regarding the domains of Burnout, 75 (73.5\%) were exhausted and $22(21.5 \%)$ depersonalized.

Conclusion. The results show a high prevalence of the exhaustion component of Burnout in most of these professionals, despite having a low rate of comorbidities and low use of medications in general. Also these data suggest that these professionals, despite being exhausted are able to see a positive challenge at work.

\section{Reference(s)}

1. 3. Demerouti, E. (2008). Measurement of Burnout and Engagement. Handbook of Stress and Burnout in Health Care, 1-25.

2. 2. Embriaco, N., Azoulay, E., Barrau, K., Kentish, N., Pochard, F., Loundou, A., \& Papazian, L. (2007). High level of burnout in intensivists: Prevalence and associated factors. American Journal of Respiratory and Critical Care Medicine, 175(7), 686-692. https://doi.org/10.1164/rccm.200608-1184OC.

3. 1. Oliveira, W. A. de. (2020). COVID-19: Desafios e oportunidades da enfermagem brasileira COVID-19: Challenges and opportunities for brazilian nursing Wender Antonio de Oliveira $1^{*}$. Revista de Saúde-RSF, 7(2), 22-39.

\section{0}

COVID-19 as a springboard for developing Intensive Care Medicine in Mauritania

F. Sigaud ; L. Valencia ${ }^{2}$; A. Salem³ ; AHEH. Saleck ${ }^{3}$; M. Roux ${ }^{1}$; S. Calvino Günther $^{1}$; B. Camara ${ }^{4}$; S. Dia ${ }^{4} ;$ AS. Gueye $^{4}$; D. Pestaña ${ }^{5}$; N. Terzi ${ }^{1}$

${ }^{1}$ Médecine Intensive Réanimation, Chu Grenoble Alpes, La Tronche, France; ${ }^{2}$ Anesthesiology and surgical intensive care, University Hospital of Gran Canaria Dr. Negrin, Las Palmas de Gran Canaria, Spain; ${ }^{3}$ Intensive

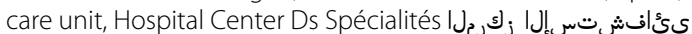
, Nouakchott, Mauritania; ${ }^{4}$ World heath organization, WHO MRT (World Health Organization Mauritania), Nouakchott, Mauritania; ${ }^{5}$ Anesthesiology and Surgical Intensive Care Unit, Hospital Ramón y Cajal, Madrid, Spain

Correspondence: F. SIGAUD

Intensive Care Medicine Experimental 2021, 9(1): 000730

Introduction. COVID-19 pandemic spreads worldwide for more than a year. Beside the strongest health systems, many countries, like Mauritania, don't have material and human resources to take care of the most severe patients. Starting in October 2020 Mauritanian health ministry and World Health Organization (WHO) sent an international group of Spanish and French nurses and intensivists to evaluate the needs, organize and implement an adapted unit in order to face the crisis and share their experience in intensive care. A new 6 beds unit was created in Nouakchott, where this international group trains, develops and emulates intensive care culture among health care local providers.

Objectives. To assess the impact of this new ICU on mortality of severe Covid-19 patients requiring intensive care support in Mauritania.

Methods. We performed a prospective study to determine the mortality rate among inpatients adults in the new COVID ICU in Nouakchott between 2020/12/31 and 2021/03/28. Mortality rate, demographic, respiratory support, adjunctive interventions, ICU length-of-stay data were collected.

Results. From 2020/12/31 to 2021/03/28, 27 patients were admitted and treated according to best practices and international guidelines. Overall mortality and among intubated patients was 67\%. Data are reported in Table 1

\begin{tabular}{|c|c|c|c|}
\hline & \multirow[t]{2}{*}{$\mathbf{N}$} & \multicolumn{2}{|l|}{ By status } \\
\hline & & $\begin{array}{l}\text { Non-survivors } \\
\text { n (\%) }\end{array}$ & $\begin{array}{l}\text { Survivors } \\
\text { n (\%) }\end{array}$ \\
\hline Total population & 27 & $18(66.67)$ & $9(33.33)$ \\
\hline $\begin{array}{l}\text { Age } \\
\text { Years } \pm D E\end{array}$ & $57 \pm 14.5$ & $63.5 \pm 17.5$ & $51 \pm 23$ \\
\hline $\begin{array}{l}\text { Male gender } \\
\mathrm{n}(\%)\end{array}$ & $14(51.85)$ & $10(55.55)$ & $4(44.44)$ \\
\hline $\begin{array}{l}\text { Median ICU length of stay } \\
\text { Days } \pm \text { DE }\end{array}$ & $7 \pm 14$ & $5 \pm 9.75$ & $17 \pm 13$ \\
\hline $\begin{array}{l}\text { Intermitttent haemodyalisis } \\
\mathrm{n}(\%)\end{array}$ & $4(14.81)$ & $2(7.41)$ & $2(7.41)$ \\
\hline $\begin{array}{l}\text { Mechanically ventilated } \\
\text { patients }\end{array}$ & 21 & $14(66.67)$ & $7(33.33)$ \\
\hline $\begin{array}{l}\text { Median length of Mechanical } \\
\text { ventilation } \\
\text { Days } \pm \text { DE }\end{array}$ & $6 \pm 8$ & $5 \pm 5.5$ & $10 \pm 10.5$ \\
\hline $\begin{array}{l}\text { Tracheostomy } \\
\mathrm{n}\end{array}$ & 4 & 2 & 2 \\
\hline
\end{tabular}


Table 1: Demographic, mortality, respiratory support, adjunctive interventions, ICU length-of-stay data

Mechanically ventilated patients' mortality was ranging up to $100 \%$ before the intervention of the task force and decreased to $67 \%$. Nevertheless, mortality remains higher than reported in European ICUs, (actually slightly over $25 \% 1$ ). Among several factors, management of infectious events could be a decisive element with an evident lack of hygiene's procedures, microbiological diagnosis techniques and a short panel of available antimicrobials treatments with a high risk of resistance emergence. There are still many challenges to address to sustain a culture of modern intensive care in Mauritania after COVID crisis. Pursued political commitment seems to be necessary in order to warrant logistical supply and the training of dedicated staff. European teams of Grenoble Alpes, Annecy (France), Gran Canaria and Madrid (Spain) are uniting their efforts to pursue the support in the area of Mauritanian staff mentoring.

Conclusion. COVID-19 pandemic has highlighted resources and competencies discrepancies triggering new areas of cooperation network.

\section{1}

High propensity to Burnout syndrome contrast with low rates of psychological monitoring among health professionals in the face of the Covid-19 pandemic

B. Fialho, ${ }^{1}$; R. Silva ${ }^{1}$; K. Aguiar, ${ }^{2}$; R. Menezes, ${ }^{3}$; I. Ferreira, ${ }^{4}$; NM. Filgueiras Filho ${ }^{5}$

${ }^{1}$ Research, Teaching and Communication Center, Hospital da

Cidade, Salvador, Brazil; ${ }^{2}$ Critical care, Hospital Emec, Salvador, Brazil;

${ }^{3}$ Inflammation and biomarkers laboratory, Oswaldo Cruz Foundation,

Salvador, Brazil; ${ }^{4}$ Life sciences department, University of State of Bahia,

Salvador, Brazil; ${ }^{5}$ Medicine, Salvador University, Campus Teacher Barros,

Salvador, Brazil

Correspondence: N.M. Filqueiras Filho

Intensive Care Medicine Experimental 2021, 9(1): 000731

Introduction. Faced with the Covid-19 pandemic, with a high workload and under intense concern for the lives of patients, their own lives and that of their families, health professionals are in a condition of professional exhaustion that has led them to a mental and physical breakdown. Portrait of exhaustion, stress and fear, these professionals experience a condition of physical and psychological vulnerability, reflecting the triggering of the burnout syndrome.

Objectives. To evaluate the prevalence of burnout and psychological support in health professionals working on the front lines of the pandemic.

Methods. Cross-sectional observational study carried out between March and April 2020. Self-administered questionnaires were applied to health professionals at a general hospital in the state of Bahia, Brazil, seeking demographic and health information and the Oldenburg Burnout Inventory (OLBI), a scale validated for Burnout investigation.

Results. 102 professionals were included in our study, being 25 nurses (25.5\%), 24 doctors (24.5\%), 13 physiotherapists (12.7\%) and 38 nursing technicians (37.3\%) who attended the inclusion criteria during the study period, with a mean age of $22 \pm 58$ and a prevalence of women $(68.6 \%)$. Of this population, it is noted that $80(78.4 \%)$ work in the ICU. According to OLBI, 90 (88.2\%) professionals had medium/ high risk for Burnout syndrome and, as for the Burnout domains, 75 (67\%) were exhausted and $22(19 \%)$ depersonalized. Another fact to be highlighted is that 98 (95.5\%) of the evaluated professionals do not undergo psychological counseling.

Conclusion. The results reflect a high propensity for the development of Burnout Syndrome among health professionals, but in contrast there is a low rate of professionals in psychological counseling. Therefore, it is suggested that there is a possible impact on the development of strategies aimed at disseminating the importance of psychotherapy, emphasizing the importance of this self-care. Having hospital psychologists focused on the care of these professionals is a plausible alternative.

\section{Reference(s)}

1. Demerouti, E. (2008). Measurement of Burnout and Engagement. Handbook of Stress and Burnout in Health Care, 1-25.

2. Embriaco, N., Azoulay, E., Barrau, K., Kentish, N., Pochard, F., Loundou, A., \& Papazian, L. (2007). High level of burnout in intensivists: Prevalence and associated factors. American Journal of Respiratory and Critical Care Medicine, 175 (7), 686-692. https://doi.org/10.1164/rccm.200608-11840C

3. Oliveira, W. A. de. (2020). COVID-19: Challenges and opportunities for Brazilian nursing COVID-19: Challenges and opportunities for brazilian nursing Wender Antonio de Oliveira 1 * . Revista de Saúde-RSF, 7 (2), 22-39.

\section{2}

Logistic and Transport of two Severely Hypoxic CoViD19 Patients by a single ambulance

A. Andreotti ${ }^{1}$; S. Busani ; M. Girardis ${ }^{2}$

${ }^{1}$ Anesthesia \& Intensive Care, Policlinico di Modena Azienda

Ospedaliero-Universitaria di Modena, Modena, Italy; ${ }^{2}$ Intensive Care Unit, Policlinico of Modena University Hospital of Modena, Modena, Italy

Correspondence: $A$. Andreotti

Intensive Care Medicine Experimental 2021, 9(1): 000732

Introduction. One of the biggest problems during the covid19 pandemic was the overcrowding of hospitals and the need to transport numerous critically ill patients elsewhere.

Objectives. We report the first case of a long inter-hospital double transport of COVID-19 severely hypoxic patients on a single vehicle.

Methods. The choice of the ambulance was dictated by the ability to support a second "field" stretcher. In addition to the standard set-up, were implemented 2 advanced multi-parameter monitors, 2 syringe pumps for continuous drug administration, 2 advanced pneumatic transport ventilators and an additional medical gas compartment with 3 supplementary cylinders of $7 \mathrm{~L}$ of $\mathrm{O}_{2}$. The transportation team only consisted of an ambulance driver, an ICU nurse and an ICU doctor. To minimize SARS-CoV-2 aerosolization, the ambulance was set up with negative pressure by means of air expulsion systems from sanitary cells. Operators were fully protected with a full Tyvek suit, two pairs of gloves, boots and Turbo Fans Powered Air-Purifying Respirators with Helmets and Pro2000 PF10 filters.

Results. The patients we had to take over for the transfer were a 59-year-old woman and a 65-year-old man, both hospitalized in a hospital $125 \mathrm{~km}$ away from our CoViD19-ICU HUB Center. The estimated time was $2 \mathrm{~h}$ totally including the time for loading and unloading the patient. In an attempt to save oxygen during transport, we decided to set up non-invasive mechanical ventilation on both patients and sedated both with dexmedetomidine at dosages between 0.6 and $0.8 \mathrm{mcg} / \mathrm{kg} / \mathrm{h}$. During the transport, the female patient showed a progressive decreasing of oxygen down to $60 \% \mathrm{FiO}_{2}$ and minute volume ventilation of $16 \mathrm{~L} / \mathrm{min}$ (less than $10 \mathrm{l} / \mathrm{min}$ of $\mathrm{O}_{2}$ ); male patient showed a progressive decreasing of oxygen down to $40 \% \mathrm{FiO}_{2}$ and minute volume ventilation of $14 \mathrm{~L} / \mathrm{min}$ (less than $7 \mathrm{l} / \mathrm{min}$ of $\mathrm{O} 2$ ) for $\mathrm{SpO}_{2}>90 \%$.

Conclusion. The oxygen requirement for the two patients was around $40 \mathrm{~L} / \mathrm{min}$ in spontaneous breathing with reservoir mask (at least $4800 \mathrm{~L}$, consider unforeseen events such as breakdowns or accidents). Optimizing the positive pressure ventilation and sedation, we reduced $\mathrm{FiO}_{2}$ on the basis of progressive lung recruitment and compliance optimization. Thanks to this strategy the overall consumption during the ambulance transport was of $2200 \mathrm{~L}$ of oxygen (less than half the expected need). Our case has shown that during an epidemic emergency, multiple transport of critically ill patients with SARS-CoV-2 infection on a single ground vehicle is feasible. This also allows savings in economic resources, time and a decrease in the number of operators exposed to a biological risk.

\section{Reference(s)}

1. 7. Semeraro F, Gamberini L, Tartaglione M, et al. An integrated response to the impact of coronavirus outbreak on the Emergency Medical Services of Emilia Romagna. Resuscitation. 2020;151:1-2. 
2. 6. Grasselli G, Pesenti A, Cecconi M. Critical Care Utilization for the COVID19 Outbreak in Lombardy, Italy: Early Experience and Forecast During an Emergency Response [published online ahead of print, 2020 Mar 13]. JAMA. 2020;10.1001/jama.2020.4031

3. 5. https://www.cdc.gov/infectioncontrol/basics/transmission-basedprecautions.html

4. 4. Schilling S, Maltezou HC, Fusco FM, et al. Transportation capacity for patients with highly infectious diseases in Europe: a survey in 16 nations. Clin Microbiol Infect. 2019;21S:e1-e5.

5. 3. http://www.aricar.it/wp-content/uploads/2018/05/Safety-II-WEB.pdf.

6. 2. Holmes JL, Brake S, Docherty M, Lilford R, Watson S. Emergency ambulance services for heart attack and stroke during UK's COVID-19 lockdown. Lancet. 2020 May 23;395(10,237):e93-e94.

7. 1. Phua J, Weng L, Ling $L$, et al. Intensive care management of coronavirus disease 2019 (COVID-19): challenges and recommendations. Lancet Respir Med. 2020 May;8(5):506-517

\section{0}

Predictive value of the APACHE II and IV score for ICU mortality in critically ill patients with COVID-19: a retrospective cohort study

L. Piccart ${ }^{1}$; J. Vandenbrande, ${ }^{2}$; J. Dubois, ${ }^{2}$; I. Callebout, ${ }^{2}$; B. Stessel, ${ }^{2}$

${ }^{1}$ Virga Jessa, Hasselt, Belgium; ${ }^{2}$ Anesthesie en intensieve zorgen, Virga

Jessa, Hasselt, Belgium

Correspondence: L. Piccart

Intensive Care Medicine Experimental 2021, 9(1): 000740

Introduction. Severity scores play a crucial role in comparing predicted versus observed outcomes, allowing evaluation of treatment and benchmarking of ICU performance. Acute physiology and chronic health evaluation (APACHE) scoring systems are designed for ICU mortality prediction. APACHE scoring systems are not validated in critically ill COVID19 patients. This study aims to assess and compare the predictive value of APACHE II and IV for ICU mortality in critically ill COVID-19 patients.

Methods. All adult patients with a laboratory-confirmed diagnosis of COVID-19 pneumonia admitted to the ICU of Jessa Hospital, Hasselt, Belgium between March 13, 2020, and October 17, 2020, were included in this retrospective, cohort study. APACHE II and APACHE IV scores were calculated within $24 \mathrm{~h}$ after admission. The performances of the APACHE scoring systems were evaluated by means of logistic regression models with ICU mortality as the dependent variable and one of the scoring systems as an independent variable. Discrimination for mortality was assessed by receiver operating characteristic curves. Tenfold cross-validation was used to obtain more conservative estimates of the discriminative abilities of the prediction tools. The HosmerLemeshow goodness-of-fit (HL) test was used to assess calibration.

Results. In total, 116 COVID-19 patients were admitted to the ICU between March 13, 2020, and October 17, 2020. 13 COVID-19 patients were excluded for various reasons, leaving 103 patients in the statistical analysis of the overall population. Baseline characteristics are presented in Table 1. Table 2 shows the results of the cross-validated discriminative abilities and the HL test.

Conclusion. APACHE IV on admission provided the best discrimination and calibration for ICU mortality in critically ill COVID-19 patients compared to APACHE II. Nevertheless, discriminative abilities of APACHE IV scores were only moderate (AUC 0.67) after correction for optimism.

\section{1}

Three-Months Outcome in COVID-19 Critical Care Patients: Results from a Follow-Up Study

S. Gatti ${ }^{1}$; P. Matteo, ${ }^{2}$; F. Mauri²; A. Lucchini ${ }^{1}$;V. Bellin'²; F. Bettini²; V. Cogliandro $^{3}$; N. Squillace ${ }^{3}$; P. Faverio ${ }^{4}$; R. Rona ${ }^{1}$; A. Pesci ${ }^{4} ;$ P. Bonfanti ${ }^{5} ; G$. Foti

${ }^{1}$ Department of Emergency and Intensive Care, ASST Monza, University of Milano Bicocca, Monza, Italy; ${ }^{2}$ Department of emergency and intensive care, ASST Monza, Monza, Italy; ${ }^{3}$ Clinic of infectious diseases, ASST Monza, Monza, Italy; ${ }^{4}$ Respiratory unit, ASST Monza, University of Milano Bicocca, Monza, Italy; ${ }^{5}$ Clinic of infectious diseases, ASST Monza, University of Milano Bicocca, Monza, Italy

Correspondence: S. Gatti

Intensive Care Medicine Experimental 2021, 9(1): 000741
Introduction. Long term outcome of COVID-19 patients, in particular for those admitted to ICU is not completely known.

Objectives. To describe functional, respiratory and neuropsychological long term outcome in a cohort of COVID-19 ICU patients.

Methods. In this prospective observational study, we enrolled hospitalized patients who were admitted to ICU with a diagnosis of COVID19 pneumonia, received invasive mechanical ventilation for $>72 \mathrm{~h}$ and were successfully discharged from ICU. Pre-admission Clinical Frailty scale (CFS) and Charlson Comorbidity Index was recorded. A multidisciplinary assessment was performed at 3 months after ICU discharge, including: CFS, Short Physical Performance Battery (SPPB), 6 min walking test (6MWT), Medical Research Council (MRC) Scale, Handgrip Strength measurement. Respiratory function was evaluated with Pulmonary Function Test, chest X-Ray and modified MRC (mMRC) dyspnea score. Hospital Anxiety and Depression Scale (HADS), Post-Traumatic Symptom Scale (PTSS-10) and Montreal Cognitive Assessment test (MoCA) were collected in order to investigate neuropsychological outcome. Euroquality Five Levels Five Dimensions (HQ5D) was used in assessing self-reported quality of life.

Results. 111 patients were enrolled after ICU discharge from March to December 2020, 4 patients died after discharge and 17 (15\%) were lost to follow-up. 90 patients were so evaluated at 3 months (see Table 1 for characteristics). Most of patients ( $60 \%$ of previously actively employed) resumed the previous job, and experienced no or minimal change in CFS (see table). In a multivariate analysis only pre-admission Charlson Index, but not age, sex or pre-admission CFS, is associated with relative increase of CFS $(p<0.001)$. Impaired muscular strength (Handgrip $<20 \mathrm{~kg}$ for females and $<30 \mathrm{~kg}$ for males or MRC $<56$ ) was present in $39 \%$ of patients. $64 \%$ of patients presented a reduced functional capacity $(6 \mathrm{MWT}<400 \mathrm{mt}$, Dispnoea or desaturation during $6 \mathrm{MWT}$, SPPB $<10$ ). $39 \%$ of patients had at least one positive neuropsychological test suffering for anxiety, depression or post-traumatic stress disease symptoms or a neurocognitive impairment. $41 \%$ of patients suffered for persistent respiratory alterations (FEV1 $<80 \%$, mMRC $>1$ or a positive chest X-ray). Median HQ5D-VAS was $80+16 \%$. In a multivariate analysis no variable is associated to self-reported quality of life except mMRC scale ( $p=0.0019)$.

Table 1. Pre-admission patients characteristic and 3 Months Outcome. BMI: Body Mass Index; CFS: Clinical Frailty Scale. If not otherwise specified, data are represented ad $\mathrm{n}(\%)$.

\begin{tabular}{ll}
\hline Age $(\mathrm{yrs})$ & $60+11$ \\
Sex M/F (\%) & $76 / 24 \%$ \\
BMI $\left(\mathrm{kg} / \mathrm{m}^{2}\right)$ (median + IQR) & $28,8+5,2$ \\
Active workers (\%) & $(60) 67 \%$ \\
Pre-admission CFS & 1-Very Fit: $15(17)$ \\
& 2-Well: 61 (67) \\
& 3-Managing Well: 14 (16)
\end{tabular}

Conclusion. Among our ICU COVID-19 cohort a most patients didn't experienced a major worsening in clinical frailty. Nevertheless, a significative part of them suffers for physical or neuropsychological sequalae at 3 months from discharge. No measured variable is independently associated with self-reported quality of life except selfreported dyspnea.

Functional and physical recovery seems to be encouraging; however, further evaluations and longer follow-up programs are necessary in order to monitor patients' progression.

000763

Methylprednisolone vs. Tocilizumab as Treatment for Severe

COVID 19-Observational Study

M. Lana, ; S. Cavinato'; J. Blake1; S. Eswarappa”; R. Kumar

${ }^{1}$ Anaesthetics and intensive care, Kingston Hospital, London, United

Kingdom

Correspondence: M. Lana

Intensive Care Medicine Experimental 2021, 9(1): 000763 
Introduction. Severe acute respiratory syndrome coronavirus 2 (SARSCoV-2) infection can be associated with hyper immune response that may lead to acute respiratory distress syndrome (ARDS).[1] Various drugs have been used in the management of ARDS in SARS-CoV-2 including antiviral and immunomodulatory agents.[2] Small number of studies show reduced mortality after intravenous methylprednisolone administration. $[3,4]$ The efficacy of IL- 6 antagonists is unclear although there are published studies that support its positive effect on mortality.[5]

Objectives. The aim of this retrospective study was to compare different treatment strategies involving intravenous glucocorticoid Methylprednisolone and IL- 6 antagonist Tocilizumab. The effect of these drugs on mortality and possible synergic role of tocilizumab and methylprednisolone in limiting the immune response leading to ARDS.

Methods. We conducted a single centre retrospective, observational study on patients with confirmed SARS-COV-2 infection who were admitted to a district general hospital intensive care unit (ICU) in London between 20.8.2020 and 31.01.2021. Inclusion criteria were clinical deterioration or lack of response to initial treatment $(\mathrm{LDH} \geq 350$ or $\mathrm{CRP} \geq 75$ and $\mathrm{FiO}_{2} \geq 0.4$ ). We divided patients into three groups: 43 of them received a minimum of one dose of Methylprednisolone $(1 \mathrm{~g} /$ day or $1 / \mathrm{mg} / \mathrm{kg} /$ day), 24 patients received a minimum of one dose of Tocilizumab (380-800 mg depending on weight) and 13 patients received at least one dose of Tocilizumab and Methylprednisolone. The 28-day mortality, median $\mathrm{FiO}_{2}$ and CRP levels on days 1 and 7 of treatment were compared. Demographics amongst the groups were also observed.

Results. Of the 157 patients admitted to the ICU 80 met our inclusion criteria. 28-day mortality in the Methylprednisolone group, Tocilizumab group and combined treatment group were $47 \%, 13 \%$ and $23 \%$ respectively $(p=0.03)$. Mortality was highest in Methylprednisolone group and lowest in patients only receiving Tocilizumab. There was no significant difference in ages amongst the groups. In all three groups patients were predominantly male between 60-79 years of age. Across the 3 groups $42 \%-50 \%$ patients were of white ethnicity, followed by ethnicities classified as other (23\%-29\%), Asian (13\%$23 \%$ ) and Black ethnicity (7\%-8\%). The highest reduction in median $\mathrm{FiO}_{2}$ on day 7 was observed in the Tocilizumab group $(55 \%, \mathrm{p}<0.01)$ followed by the Methylprednisolone group $(50 \%, p<0.01)$ and dual treatment group ( $33 \%, p=0.02$ ). Maximum reduction of median CRP levels was seen with Tocilizumab treatment $(98 \%, p<0.01)$.

Conclusion. From this observational study we conclude that Tocilizumab significantly reduces 28 -day mortality and $\mathrm{FiO}_{2}$ requirements on day 7. It is also effective in suppressing inflammatory response measured by CRP reduction. Tocilizumab in combination with Methylprednisolone is effective in reducing mortality and $\mathrm{FiO}_{2}$ requirements on day 7.

\section{Reference(s)}

1. 1. Huang $C$, Wang Y, Li X, et al. Clinical features of patients infected with 2019 novel coronavirus in Wuhan, China. Lancet. 2020; 395: 497-506

2. 2. G Guaraldi, M Meschiari, A Cozzi-Lepri, J Milic, et al. Tocilizumab in patients with severe COVID-19: a retrospective cohort. Lancet. June 24, 2020; https://doi.org/10.1016/S2665-9913(20)30173-9study

3. 3. M Edalatifard, M Akhtari, M Salehi, Z Naderi, A Jamshidi, S Mostafaei, S R Najafizadeh, et al. Intravenous methylprednisolone pulse as a treatment for hospitalised severe COVID-19 patients: results from a randomised controlled clinical trial. European Respiratory Journal. 2020; https://doi. org/10.1183/13993003.02808-2020

4. 4. The WHO Rapid Evidence Appraisal for COVID-19 Therapies (REACT) Working Group. Association between administration of systemic corticosteroids and mortality among critically ill patients with COVID-19: a meta-analysis. JAMA 2020;324:1330- 1341

5. 5. Anthony C. Gordon, M.B., B.S., M.D., Paul R. Mouncey, M.Sc., et al. Interleukin-6 Receptor Antagonists in Critically III Patients with Covid-19. NEJM. February 25, 2021; https://nejm.org/ https://doi.org/10.1056/ NEJMoa2100433
000764

Do Covid-19 patients needing ICU admission have worse 6 months follow up outcomes when compared with hospitalized non-ICU patients? A prospective cohort study

S. Yıldırım ; Ö. Ediboğlü² S. Susam³ ; P. Cimen ${ }^{4}$; S. Yapicioglu ${ }^{4}$; O. Sunecli ${ }^{5}$; C. Kirakli ${ }^{2}$

${ }^{1}$ Intensive Care, Dr. Suat Seren Göğüs Hastalıkları Ve Cerrahisi Eğitim Ve Araştırma Hastanesi Acil Servisi, Izmir, Turkey; ${ }^{2}$ Intensive care unit, Dr. Suat Seren Chest Diseases Hospital, Izmir, Turkey; ${ }^{3}$ Department of radiology,

Dr. Suat Seren Chest Diseases Hospital, Izmir, Turkey; ${ }^{4}$ Department

of chest disease, Dr. Suat Seren Chest Diseases Hospital, Izmir, Turkey;

${ }^{5}$ Department of Physiotherapy, Dr. Suat Seren Chest Diseases Hospital, Izmir, Turkey

Correspondence: S. Yıldırım

Intensive Care Medicine Experimental 2021, 9(1): 000764

Introduction. The long-term outcomes of COVID-19 patients who are discharged from the ICU are unclear. We investigated the effect of COVID-19 on lung structure, pulmonary function, exercise capacity and quality of life in patients discharged from ICU and medical wards. Methods. A prospective single-centre study conducted on PCR confirmed COVID-19 patients who have been discharged from University of Health Sciences, Dr. Suat Seren Chest Disease and Thoracic Surgery Teaching and Research Hospital between the March 19 and September 1, 2020. Patients who were followed up on for more than $48 \mathrm{~h}$ in ICU and more than $72 \mathrm{~h}$ in medical wards were included in the study. Computed tomography scores, pulmonary function tests (PFT), 6-min walking distance and health related quality of life (assessed by SF-36), were compared between ICU and medical ward patients at 6 months after discharge.

Results. Seventy patients were included in the final analyses and 31 of them were discharged from ICU. ICU patients had higher CT scores than non-ICU patients at admission (17 vs 11) and follow-up visits (6 vs 0 ). Two-thirds of ICU patients had at least one abnormal finding on a follow-up CT. Advanced age (OR 1.08, 95\% Cl 1.02-1.15) and higher CT score at admission (OR 1.13,95\% Cl 1.01-1.27) were risk factors for having radiological abnormalities on the follow-up CT. $90 \%$ of the patients discharged from the ICU had at least one persistent symptom. Impairment of PFT and 6-min walking distance were similar in both ICU and medical ward patients, and higher CT scores on the follow-up CT was associated with impairment of PFT.

Conclusion. A number of COVID-19 survivors could not fully recover 6 months after discharge from hospital. COVID-19 survivors need support therapies during the recovery phase of the disease.

\section{3}

Interpretation of the patient's medical condition. Is there concordance between family members of ICU-patients and healthcare staff?

N. Liere, ${ }_{1}^{\text {; P. Berg, }}{ }_{1}^{\text {; }}$ A. Bouwman, ${ }^{2}$; M. Buise

${ }^{1}$ Intensive care, Catharina Ziekenhuis, Eindhoven, Netherlands;

${ }^{2}$ Anesthesiology, Catharina Ziekenhuis, Eindhoven, Netherlands

Correspondence: M. Buise

Intensive Care Medicine Experimental 2021, 9(1): 000773

Introduction. A digital intensive care unit (ICU) diary provides unique opportunities to gain insight into the perspective of family members on the medical condition of the ICU-patient throughout the ICU admission. We aimed to explore if there is a concordance between family members of ICU-patients and healthcare staff in the interpretation of the patient's medical condition. Furthermore, we looked into the satisfaction of family members with ICU care.

Methods. The retrospective multiple-case study was executed in an tertiary teaching hospital in the Netherlands.

Data was retrieved from digital ICU-diaries, medical records and the European quality questionnaire (euroQ2). The first two sources provided both qualitative and quantitative data, the latter solely quantitative data. Diary entries and medical records were scored as negative, 
stable or positive. The Linguistic Inquiry and Word Count (LIWC) program was used to quantify the emotional content of the diary notes. Substantial changes in the patient's medical condition were objectified with the Sequential Organ Failure Assessment (SOFA) score. Two to four weeks after ICU discharge or death, family members received the euroQ2 questionnaire.

Results. We included ten family members, representing ten ICUpatients. A total of 109 diary notes were used in the analysis. Healthcare staff and family members agreed on the ICU-patient's medical condition in $67,9 \%$ of the cases. Family members seem to be more positive compared to healthcare staff. The interrater reliability for the two groups was Kappa 0,426, indicating a moderate agreement. Based on the euroQ2 questionnaire, families were satisfied with the overall quality of ICU care and with the given information.

Statistically significant more negative (positive) emotions were used in negative (positive) diary notes. A substantial increase (decrease) in the SOFA score correlated with a negative (positive) score given by healthcare staff for that day.

Conclusion. There is a moderate agreement between family members of ICU-patients and healthcare staff in the interpretation of the patient's medical condition. Satisfaction with ICU care does not seem to be influenced by disagreements in the patient's medical condition. Further research should be executed to confirm these results

\section{6}

Telemedicine in the Brazilian Unique Health System (SUS) during the Covid-19 pandemic - an experience report

D. Cunha Birriel ${ }^{1}$; HM. Constant ${ }^{1}$; MC. Cotta Matte ${ }^{1}$; VC. Jacovas, ${ }^{1}$; S. Stiehl Alves' ${ }^{1}$; FC. Cabral,

${ }^{1}$ Proadi-sus, Moinhos de Vento Hospital, Porto Alegre, Brazil

Correspondence: D. Cunha Birrie

Intensive Care Medicine Experimental 2021, 9(1): 000786

Introduction. The Covid-19 pandemic has impacted global health systems. Admissions in intensive care units increased significantly, leading to an emergency health crisis in Brazil. It exposed the scarcity of resources, mainly of health care providers trained in critical care. In this context, telemedicine emerges as an important alternative in the health care sector to support critical global health situations.

However, studies on covid-19 pandemic and telemedicine in the ICU setting are still scarce. Thus, we highlight the importance of studies that discuss the applicability of this resource for critically ill patients.

Objectives. Describe the experience of a hospital of excellence, Hospital Moinhos de Vento (HMV), using telemedicine to qualify COVID ICUs in regions with shortage of critical care specialists.

Methods. Retrospective, descriptive study with data collection from a secondary source, nested in the program qualification of assistance in ICUs.

Covid ICUs from all over Brazil were selected by the Ministry of Health through the SUS Institutional Support Program, and divided between 5 centers of excellence. The HMV was responsible for 8 ICUs, defined as remote centers. Daily videoconferences were held between centers and the HMV intensive care physician. Both centers used the same platform to access and record patient data and recommendations. Remote centers were responsible for entering patient data on admission as well as removing it after discharge or death. HMV was responsible for recording the discussion of cases and the recommendations given. The physician directly responsible for the patient was free to decide whether or not to accept the recommendations, but it was mandatory for the remote centers to acknowledge them through the platform.

Results. Each ICU had an average of 18 beds for Covid-19 patients The program carried out a total of 3075 consultations for more than 700 patients. Altogether, more than 4000 recommendations were made, of which approximately $70 \%$ were accepted by the remote centers. Regarding the profile of the patients, $58 \%$ were male and the median age was 62 years (IQ 25-75:50-72). The median ICU stay was 9 days (IQ 25-75: 6-16) and ICU mortality was $49,1 \%$. The physicians at the remote centers reported a positive and beneficial perception regarding participating in tele-rounds.

Conclusion. Pandemics pose unique challenges for health care delivery. Our experience illustrates that while telemedicine in the ICU environment does not solve all challenges, it can provide rapid access to specialists who would not be available in person.

\section{Reference(s)}

1. Lilly CM, Zubrow MT, Kempner KM, Reynolds HN, Subramanian S, Eriksson EA, Jenkins CL, Rincon TA, Kohl BA, Groves RH Jr, Cowboy ER, Mbekeani KE, McDonald MJ, Rascona DA, Ries MH, Rogove HJ, Badr AE, Kopec IC; Society of Critical Care Medicine Tele-ICU Committee. Critical care telemedicine: evolution and state of the art. Crit Care Med. 2014 Nov:42(11):2429-36. https://doi.org/10.1097/CCM.00000000000000539. Erratum in: Crit Care Med. 2015 Feb;43(2):e64. PMID: 25080052

2. Kahn JM, Cicero BD, Wallace DJ, Iwashyna TJ. Adoption of ICU telemedicine in the United States. Crit Care Med. 2014 Feb;42(2):362-8. https:// doi.org/10.1097/CCM.0b013e3182a6419f. PMID: 24145839; PMCID: PMC3947050.

3. Rosenfeld BA, Dorman T, Breslow MJ, Pronovost P, Jenckes M, Zhang $\mathrm{N}$, Anderson G, Rubin H. Intensive care unit telemedicine: alternate paradigm for providing continuous intensivist care. Crit Care Med. 2000 Dec;28(12):3925-31. https://doi.org/10.1097/00003246-20001200000034. PMID: 11153637

4. Grundy BL, Crawford P, Jones PK, Kiley ML, Reisman A, Pao YH, Wilkerson EL, Gravenstein JS. Telemedicine in critical care: an experiment in health care delivery. JACEP. 1977 Oct;6(10):439-44. https://doi.org/10.1016/ s0361-1124(77)80239-6. PMID: 71365

5. Hong Z, Li N, Li D, et al. Telemedicine During the COVID-19 Pandemic: Experiences From Western China. J Med Internet Res. 2020;22(5):e19577. Published 2020 May 8. https://doi.org/10.2196/19577

6. Lurie N, Carr BG. The Role of Telehealth in the Medical Response to Disasters. JAMA Intern Med. 2018 Jun 01;178(6):745-746. https://doi.org/ 10.1001/jamainternmed.2018.1314.

7. Hollander JE, Carr BG. Virtually Perfect? Telemedicine for Covid-19. N Engl J Med. 2020 Apr 30;382(18):1679-1681. https://doi.org/10.1056/NEJMp 2003539

8. The data presented was obatained in partnership with the Ministry of Health of Brazil, through the Institutional Development Support Program of the Unified Health System (PROADI-SUS).

\section{4}

Predictive value of combined do-not-resuscitate (DNR)/ do-not-intubate (DNI) code for ICU mortality in critically ill patients with COVID-19: a retrospective cohort study

G. Vercruysse ${ }^{1}$; J. Dubois ${ }^{2}$; B. Michiel' ; I. Callebaut ${ }^{3}$; J. Vandenbrande, ; B. Stessel $\left.\right|^{2}$

${ }^{1}$ Intensive Care, Virga Jessa, Hasselt, Belgium; ${ }^{2}$ Anesthesiology and intensive care, Virga Jessa, Hasselt, Belgium. ${ }^{3}$ Department

of anesthesiology and intensive care, Jessa Ziekenhuis, Hasselt, Belgium;

${ }^{4}$ Anesthesie en intensieve zorgen, Virga Jessa, Hasselt, Belgium

Correspondence: G. Vercruysse

Intensive Care Medicine Experimental 2021, 9(1): 000794

Introduction. The current Coronavirus Disease 2019 (COVID-19) pandemic has placed enormous strain on health systems worldwide. The availability of ICU beds in Belgium is high with 15.9 beds per 100.000 inhabitants 1 . Nevertheless, we were forced to implement a critical care triage system to allocate the scarce mechanical ventilators towards patients most likely to benefit from them 2. Therefore, some patients received a do-not-intubate code at admission to Intensive Care Unit (ICU).

Objectives. Our goal was to compare patient characteristics, applied therapies and outcomes between ICU patients with full code and ICU patients with a (DNI)-code.

Methods. All adult patients admitted to ICU of JESSA hospital, Hasselt, Belgium with a laboratory-confirmed COVID-19 pneumonia between March 13th 2020 and October 17th 2020 were included in his retrospective, cohort study. Data covering patient demographics 
and medical history, disease severity, applied therapies, laboratory results and clinical outcomes were prospectively collected and retrospectively reviewed. Categorical variables are presented as frequency (\%) and groups were compared using Fisher's exact test. Continuous variables are presented as median with interquartile range and groups were compared using Wilcoxon rank sum test.

Results. From March 13th until October 17th 2020, 116 COVID-19 patients were admitted to the ICU. Thirteen COVID-19 patients were admitted for other reasons than pneumonia, resulting in 103 patients for final analysis (Figure 1). Baseline demographics, medical history and clinical characteristics at admission to ICU, stratified for code, are presented in Table 1. Older age, female gender, history of malignancy, higher clinical frailty index and apache IV score were significantly more present in the DNI-group (Table 1). Outcomes stratified for code, are presented in Table 2 . Mortality and sepsis were respectively positively and negatively associated with a DNI-code (table2). Mortality in patients admitted to ICU with a DNI-code was $68.7 \%$.

Conclusion. Mortality in patients admitted to ICU with a DNI-code is very high. Policy makers and Intensive care doctors should discuss the admission policy to ICU of this patient group in terms of cost-benefit ratio.

\section{Reference(s)}

1. 1 Rhodes A, Ferdinande P, Flaatten H, Guidet B, Metnitz PG, Moreno RP. The variability of critical care bed numbers in Europe. Intensive Care Med. 2012;38(10):1647-53.

2. 2 Maves RC, Downar J, Dichter JR, Hick JL, Devereaux A, Geiling JA, Kissoon N, Hupert N, Niven AS, King MA, Rubinson LL, Hanfling D, Hodge JG Jr, Marshall MF, Fischkoff K, Evans LE, Tonelli MR, Wax RS, Seda G, Parrish JS, Truog RD, Sprung CL, Christian MD; ACCP Task Force for Mass Critical Care. Triage of Scarce Critical Care Resources in COVID-19 An Implementation Guide for Regional Allocation: An Expert Panel Report of the Task Force for Mass Critical Care and the American College of Chest Physicians. Chest. 2020 Jul;158(1):212-225.

\section{3}

Evaluation of the individual perceptions of the experience in a novel Intensive Care Unit for COVID-19 patients in a Portuguese Center

M. Joana ${ }^{1}$; S. Duarte, ; M. Carvalho, ${ }^{1}$; Z. Moreira, ${ }^{1}$; E. Neutel,

${ }^{1}$ Department of Anesthesia Intensive Care and Emergency, University

Hospital Centre of Porto (CHUPorto), Porto, Portugal

Correspondence: M. Joana

Intensive Care Medicine Experimental 2021, 9(1): 000813

Introduction. The importance of the human factors are essential for the success of a team, but they are not readily measurable or appreciated. The goal of this study was to evaluate the individual perceptions of the experience associated with the work in a novel Intensive Care Unit: Neoclassic-ICU (NICU), in Porto, Portugal, in a context of the SARS CoV-2 pandemic, in March 2020.

Methods. A questionnaire with 110 items was made to the health professionals involved in the ICU (doctors, nurses and operational assistants $(\mathrm{OA})$ ). The Safety Attitudes Questionnaire (SAQ) - Short Version 2006 PT was integrated in this evaluation, as well as an adaptation of the TeamSTEPPS Team Work Attitudes Questionnaire (T-TAQ). The principles of the Declaration of Helsinki have been observed. Each participant consented to the use of the data, on a voluntary basis, guaranteeing its confidentiality and anonymity. The results were examined by means of descriptive analysis (Microsoft Excel software).

Results. Among the 76 participants, $43(56.6 \%)$ responded to the survey: $47 \%$ doctors, $28 \%$ nurses and $26 \% \mathrm{AO}$. The medical team consisted of Hospital Assistants, Trainees in Anesthesiology and a Graduate Hospital Assistant with a specialty in Anesthesiology and Intensive Care Medicine. The nursing team came entirely from the operating room, being mostly anesthesiology nurses. A substantial part of the AO team were inexperienced in Intensive Care.
The predominant feeling at the time of joining the team was one of trust, linked to the acquaintance of the team members and the ease of communication.

During the months of March and April, responses to SAQshort PT were positive (score > 75) in 4 of the 6 domains evaluated: teamwork (89), safety climate (80), job satisfaction (91), perception of management (85).

Non-technical skills were assessed by adapting the T-TAQ with positive responses in all 5 domains assessed: communication, team work, work coordination, leadership and decision making. It should be noted that communication between team members was facilitated by sharing the same mental model (score of 81.5).

The feelings perceived during the trajectory at the NICU were: companionship (93\%), responsibility (88\%), sense of mission $(81 \%)$ and togetherness $(79 \%)$. The predominant form of emotional compensation was the mutual support of professionals $(95 \%)$, as well as the sense of mission (72\%).

After the closure of the NICU, there was a perception of a change in individual and team behaviors, with a notion of professional growth (94\%).

Conclusion. The fragile structural conditions of this novel ICU, the insecurity and fear in the face of the clinical challenge of the unknown, as well as the inexperience of part of the team in intensive medicine, were supplemented by a team spirit, in which leadership, confidence, resilience, sharing and communication generated a positive integrative experience. The participation in the implementation and organization of the NICU motivated a behavioral change in the professionals involved. As a future investigation, the same survey will be applied to the team of professionals involved in the same unit, in a later period.

\section{Reference(s)}

1. Saraiva, Dora Maria Ricardo Fonseca, \& Almeida, Anabela Antunes de (2017). Tradução e adaptação cultural do Safety Attitudes Questionnaire: Short Form 2006 para Portugal. Portuguese Journal of Public Health, 35 (3)

\section{8}

Prognostic Value of Muscle and Fat Tissue Volume Measurements in Chest CT images of COVID-19 Patients

SB. Akınc1; E. Akpınar ${ }^{2}$; C. Balci Altin ${ }^{1}$; ND. Bulut Yüksel ${ }^{1}$; N. Abdurrahimli

${ }^{1}$ Anesthesiology and Reanimation, Intensive Care Unit, Hacettepe

University Hospitals, Ankara, Turkey; ${ }^{2}$ Radiology, Hacettepe University

Hospitals, Ankara, Turkey

Correspondence: $C$. Balci Altin

Intensive Care Medicine Experimental 2021, 9(1): 000828

Introduction. Presence of sarcopenia causes prolonged mechanical ventilation and intensive care unit (ICU) stay, in critically ill patients. Sarcopenia is common in patients with COVID-19 who are admitted to the ICU. Cross-sectional muscle area measurement calculated from thoracic tomography imaging is a method to evaluate presence of sarcopenia.

Objectives. In our study, we aimed to investigate the prognostic value of the muscle and Fat Tissue Volume Measurements which are calculated from the chest CT images taken at ICU admission in COVID-19 pneumonia patients, in terms of duration of mechanical ventilation, ICU stay and the rate of mortality.

Methods. After ethical committee approval, adult patients who were admitted to the Hacettepe Hospital Anesthesia Intensive Care Unit between August 2020 and February 2021, with positive COVID PCR and respiratory distress were included into the study. Patients who did not have chest CT imaging before ICU admission or who did not have sufficient image quality were excluded from the study. The demographic data of the patients, laboratory parameters, admission APACHEII, SOFA, NUTRIC scores, durations of mechanical ventilation, ICU and hospital stay, rates of mortality at 28 and 90 days were recorded from the hospital data system. Chi-Square 
Tests, Mann-Whitney U tests, Spearman correlations, multivariate binary logistic regression tests were used for statistical analyses.

Results. A total of 139 patients ( 55 female, 84 male) were included into the analysis. The median duration of mechanical ventilation was 7 (0-121) days, ICU stay was 17 (1-132) days, and the 90-day mortality rate was $43 \%$ (Table 1 ). There were no statistically significant correlations between the measurement of the muscle/fat tissue volume and the NUTRIC score, the durations of the mechanical ventilation, ICU or hospital stay. We found significant correlations between NUTRIC score and the duration of invasive mechanical ventilation $(p<0.01, r=0.364)$, ICU stay $(p=0.011, r=0.216)$, the prealbumin value on the 14 th day $(p<0.01, r=0.496)$. The NUTRIC score $(B=0.558, p .026)$ and SOFA score $(B=0.760, p .001)$ at admission day were independent predictors of mortality.

Conclusion. The measurements of muscle and fat tissue volume from chest CT images don't have prognostic value for predicting duration of mechanical ventilation, ICU stay and mortality whereas NUTRIC score is a predictor for mortality in critically ill COVID-19 patients.

\section{Reference(s)}

1. Medrinal, C., et al., Muscle weakness, functional capacities and recovery for COVID-19 ICU survivors. BMC Anesthesiol, 2021. 21(1): p. 64.

2. Nemec, U., et al., Diagnosing Sarcopenia on Thoracic Computed Tomography: Quantitative Assessment of Skeletal Muscle Mass in Patients Undergoing Transcatheter Aortic Valve Replacement. Academic Radiology, 2017. 24(9): p. 1154-1161.

3. Fintelmann, F.J, et al., Thoracic Skeletal Muscle Is Associated With Adverse Outcomes After Lobectomy for Lung Cancer. Ann Thorac Surg, 2018 105(5): p. 1507-1515

4. Hermans, G. and G. Van den Berghe, Clinical review: intensive care unit acquired weakness. Crit Care, 2015. 19: p. 274

\section{5}

\section{A prognostic value for the Lactate trend in the ICU? First $48 \mathrm{~h}$ Lactate whole variation was strongly predictive of in-Hospital Mortality}

A. Oliveira" ; F. Marino ; J. Silva'; M. Vera-Cruz ; J. Gonçalves-Pereira 'Intensive Care Unit, Hospital de Vila Franca de Xira, Estrada Carlos Lima Costa, Vila Franca de Xira, Portugal, Vila Franca de Xira, Portugal

Correspondence: A. Oliveira

Intensive Care Medicine Experimental 2021, 9(1): 000845

Introduction. In critical illness lactate is a marker of stress and strongly correlates with severity. Accordingly, lactate is part of the initial evaluation of emergent patients and lactate clearance has been used to evaluate initial therapy.

The evaluation of lactate clearance as a prognostic marker usually rely on only a two point difference, and this approach may miss important variations (either an increase or a decrease in lactate) occurring during the initial approach to the patient.

Objectives. Evaluation of lactate variation during the whole first $48 \mathrm{~h}$ of treatment as a prognostic marker of all cause Intensive Care Unit (ICU) and Hospital mortality.

Methods. Retrospective, cohort study. All patients admitted for $>48 \mathrm{~h}$ to a mixed ICU between 01/2017-03/2019 were screened. Those with $\geq 2$ lactate samplings, of which at least one $>2 \mathrm{mmol} / \mathrm{L}$ measured during the first $24 \mathrm{~h}$ of the ICU admission, were segregated for further analyses. The trend of all lactate determinations during the first $48 \mathrm{~h}$ of ICU stay, according to the time of sampling, was computed for all included patients.

A logistic regression model (LogR) with a forward stepwise selection of factors and a Neural Network Analysis with Multilayer Perceptron (MLP) were performed including the variables: maximum lactate in either the first $24 \mathrm{~h}$ or $48 \mathrm{~h}$ and the lactate trend. Area under the Curve (AUC) were plotted for each model.

Results. We screened 2205 ICU admissions of which 358 were selected. The mean age was $68.3 \pm 14.5$ and $55 \%$ were male, mean SAPSII score $50 \pm 17.9$, and 115 (32.1\%) died in the hospital, of which $71(19.8 \%)$ in the ICU.
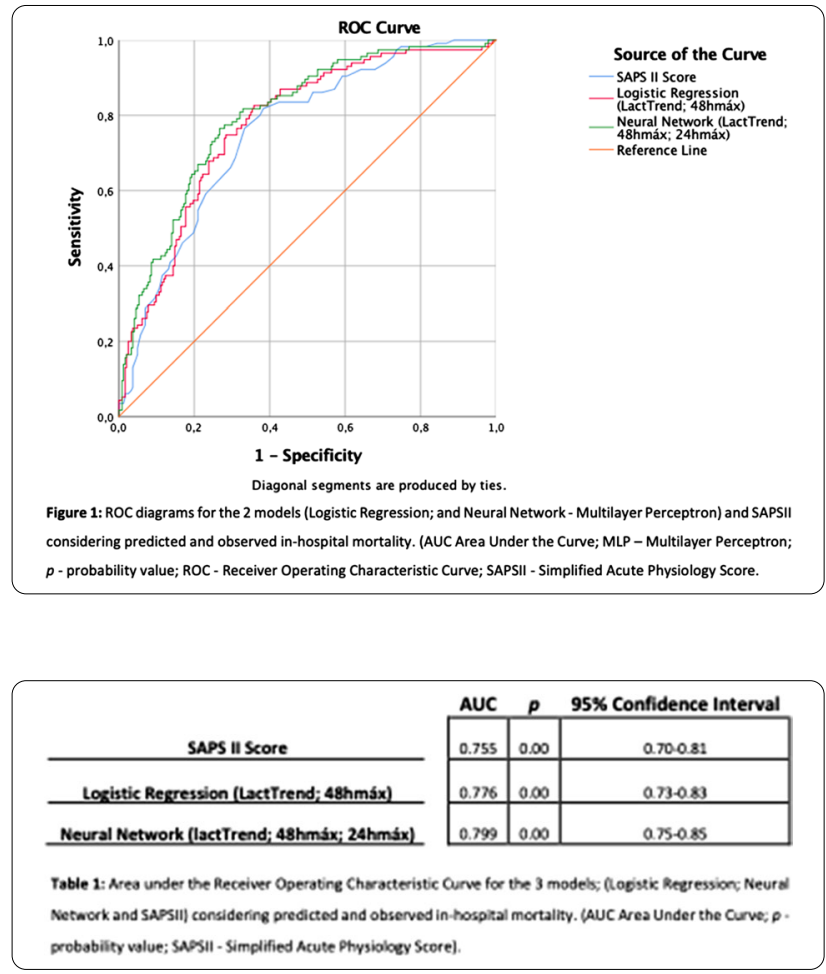

Both the LogR and MLP models performed well for predicting the in-hospital mortality, including only these two indicators, with AUC slightly higher than SAPS II, as shown in Fig. 1 and Table 1.

\begin{tabular}{|c|c|c|}
\hline & \multicolumn{2}{|c|}{ Relative importance in the neural network } \\
\hline & Importance & Normalized importance \\
\hline Lact. Trend & 0.461 & $100 \%$ \\
\hline 48hmáx & 0.340 & $73.3 \%$ \\
\hline 24hmáx & 0.199 & $43.3 \%$ \\
\hline
\end{tabular}

In the neural network the activation function was Sofmax, which used $70 \%$ of cases for training and $30 \%$ for testing. In this model, the lactate trend proved to be the most informative variable. (Table 2)

Conclusion. In critically ill patients with lactate $\geq 2 \mathrm{mmol} / \mathrm{L}$ on admission, the lactate trend in the whole first $48 \mathrm{~h}$ of admission along with its maximum value was strongly predictive of in-hospital mortality (similar to SAPS II). This easy to calculate value may help to identify patients with a high risk of death and to guide the therapeutic approach.

\section{Reference(s)}

1. Mahmoodpoor A, Shadvar K, Saghaleini SH, et al. Which one is a better predictor of ICU mortality in septic patients? Comparison between serial serum lactate concentrations and its removal rate. J Crit Care. 2018;44:5156. https://doi.org/10.1016/j.jcrc.2017.10.019

2. Masyuk $M$, Wernly $B$, Lichtenauer $M$, et al. Prognostic relevance of serum lactate kinetics in critically ill patients. Intensive Care Med. 2019;45(1):5561. https://doi.org/10.1007/s00134-018-5475-3 
3. Vincent JL, e Silva AQ, Couto L, Taccone FS. The value of blood lactate kinetics in critically ill patients: A systematic review. Crit Care. 2016;20(1):1-14. https://doi.org/10.1186/S13054-016-1403-5

4. Zhou X, Liu D, Su L, et al. Use of stepwise lactate kinetics-oriented hemodynamic therapy could improve the clinical outcomes of patients with sepsis-associated hyperlactatemia. Crit Care. 2017;21(1):1-10. https://doi. org/10.1186/s13054-017-1617-1

\section{0}

Treating critically ill patients experiencing SARS-CoV-2 severe infection with Ig-M enriched Ig-G infusion

A. CORONA ${ }^{1}$; G. Richini ${ }^{1}$; I. Gatti ${ }^{1}$; M. Pasqua ${ }^{1}$; M. Zangrandi ${ }^{1}$; S. Simoncini ${ }^{1}$. C. Santorsola ${ }^{1}$; M. Biasini ${ }^{1}$; E. Zendra ${ }^{1}$; A. Capone

'DEPARTMENT OF EMERGENCY AND CASUALTY AND ICU, ASST

Vallecamonica, Esine, Province of Brescia, Italy, Esine, Italy

Correspondence: A. CORONA

Intensive Care Medicine Experimental 2021, 9(1): 000870

Introduction. In SARS-CoV-2 patients who need Intensive Care (ICU), the condition is associated with a mortality ranging from 10 to $40-45 \%$, with a catastrophic increase in morbidity and mortality in case of sepsis. We hypothesized that $\lg M$ and $\lg A$ enriched immunoglobulin may support the sepsis-related phase improving patient outcome.

Methods. We have conducted a retrospective case-control study on 47 consecutive patients admittedto our ICU; at the admission, patients received therapy with anticoagulants (heparin sodium) besides the standard supportive treatment. We decided to add $\lg M$ and $\lg A$ enriched immunoglobulin to the standard therapy. Patients receiving $\lg \mathrm{M}$ and $\lg \mathrm{A}$ enriched immunoglobulin were compared with patients with similar baseline characteristics and treatment, receiving only standard therapy.

Results. The mortality resulted significantly higher in patients with standard therapy $(56.5$ vs. $37.5 \%, p<0.01)$. At day 7 , the death probability was 3 times higher in this group. Variable life adjustment display (VLAD) was 2.4 and - 2.2 (in terms of lives saved in relation with those expected and derived from Simplified Acute Physiology Score II), respectively in the treated and not treated group.

Conclusion. The treatment based on $\lg M$ and $\lg A$ enriched immunoglobulin infusion seems to give an advantage on survival to SARSCoV-2 severe infection

\section{Reference(s)}

1. Carannante, N. et al. Administration of immunoglobulins in SARS-CoV-

2-positive patient is associated with fast clinical and radiological healing: Case report. Front Med (Lausanne). 7, 388 (2020).

2. None.

\section{4}

\section{Quality Improvement: The role of the intensive care recovery} co-ordinator

C. Rainey ${ }^{1}$; O. Peter ${ }^{1}$; M. Pauline ${ }^{1}$;W. Aimee

${ }^{1}$ Intensive Care Medicine, NHS Ayrshire and Arran, Kilmarnock, United Kingdom

Correspondence: $C$. Rainey

Intensive Care Medicine Experimental 2021, 9(1): 000884

Introduction. Patients who survive an Intensive Care (ICU) stay are left with significant psychological, social and physical morbidity following discharge. Poor preparation for discharge and poor psychological support contribute to this, with a lack of specific support for ICU survivors between ICU and hospital discharge being identified by patients and their families. The InS:PIRE (Intensive care Syndrome: Promoting Independence and REcovery) programme seeks to improve long term outcomes for ICU patients following hospital discharge. The Realistic Medicine's Value Improvement Fund has successfully funded 2 years of a new role, the Recovery co-ordinator, developed to support ICU survivors and families in this period between ICU and hospital discharge.

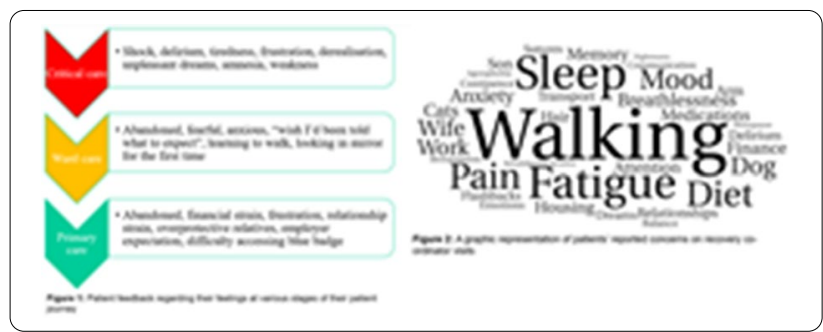

Methods. All ICU patients are visited on the wards as many times as required by the recovery co-ordinator on site. The interventions provided are tailored to each patient, and take multiple forms: from leaflets to explain to the patient why they are feeling how they feel, or struggling with for example sleep, to links to a national peer support group of other ICU survivors, including onwards referrals for therapies, and orientation tools for patients to help remind them what has happened to them so far in their patient journey. Patients' hospital anxiety and depression scores are assessed, as well as a patient-centred concerns checklist and EQ5D heath scoring questionnaire reflecting functional impairment.

73 patients' data, reflecting all ICU survivors who engaged with the recovery coordinator between November 2019 and February 202, was accessed, including paper and electronic notes taken by the recovery coordinator on their visit, as well as their paper and electronic medical records.

Results. Patients median self-assessed health score (out of 100) was 50 , their median hospital anxiety and depression scores (out of 21) were 9 and 7 respectively-with a score of 8 being suggestive of a mood disorder. Patients on the EQ5D questionnaire report a median value of "moderate" impairment in the fields of self-care, mobility and ability to undertake their usual activities, and "slight" levels of pain and anxiety. The median number of interventions for each patient was 3.

Conclusion. A low median health score amongst ICU survivors, alongside median depression and anxiety scores around the 8/21 cut off score suggestive of mood disorder demonstrate quantitatively the need for the role of the recovery co-ordinator, adding to qualitative feedback from previous ICU survivors' experiences. Reassessment of returning survivors to the InS:PIRE programme will further demonstrate the value added by this role in addressing the significant issue of post ICU morbidity commonly referred to as Post Intensive Care Syndrome (PICS). Further feedback from IN:SPIRE programmes will provide an opportunity for tests of change within the Recovery Coordinator role, with an aim to improve the outcome measures associated with the psychological, physical and social consequences.

Reference(s)

1. We wish to thank all previous patients who have engaged with the InS:PIRE programme in a bid to improve care for future patients, and to all the staff involved with programmes.

\section{6}

Chronic Critical Illness Among COVID-19 Patients Admitted to Intensive Care Unit

M. Yildirim¹; B. Halacli ; E. Ulusoydan ${ }^{1}$; Y. Gunegul ${ }^{1}$; G. Guven ${ }^{1}$; E. Ortac Ersoy ${ }^{1}$; A. Topeli ${ }^{1}$

${ }^{1}$ Division of Intensive Care, Department of Internal Medicine, Hacettepe University Faculty of Medicine, Ankara, Turkey

Correspondence: M. Yildirim

Intensive Care Medicine Experimental 2021, 9(1): 000886

Introduction. Chronic critical illness $(\mathrm{CCl})$, a serious and a growing healthcare problem all over the world, has been defined as prolonged ICU stay with persistent organ dysfunctions leading to higher morbidity and mortality rates (1). CCl patients have additional problems 
such as myopathy, neuropathy, malnutrition, immunosuppression, persistent infections, brain dysfunction, delirium, neuroendocrine and hematologic disorders, anxiety and depression. Of patients on mechanical ventilation, $10 \%$ develop $\mathrm{CCl}$ (2).

Objectives. We aimed to investigate the presence of $\mathrm{CCl}$ in critically-ill COVID-19 patients and to evaluate and compare clinical findings and outcomes of critically-ill COVID 19 patients with and without CCI.

Methods. Patients with laboratory confirmed COVID-19 diagnosis who were admitted our ICU between 21st March 2020 and 21st March 2021 were retrospectively evaluated. CCI was defined as a length-of-ICU stay (LOS) of $\geq 14$ days with persistent organ dysfunction on the 14th day of ICU admission, defined as a cardiovascular SOFA score $\geq 1$ and score in any other organ system $\geq 2$ on day 14 of ICU admission (1).

Results. 328 patients were admitted during the study period. 109 (33.2\%) of them met with the CCl criteria. There was no difference in terms of sex and comorbidities between patients with $\mathrm{CCl}$ and others. Patients with $\mathrm{CCl}$ were older $(p=0.04)$, were more frail on admission $(p<0.01)$, had higher APACHE II and SOFA scores $(p<0.001)$ and had lower $\mathrm{PaO}_{2} / \mathrm{FiO}_{2}$ ratio $(p<0.01)$. The rate of IMV requirement, presence of septic shock on admission and steroid use during follow-up were higher in patients with $\mathrm{CCI}(\mathrm{p}<0.01)$. Patients with $\mathrm{CCl}$ had longer ICU and hospital LOS than patients without $\mathrm{CCl}(\mathrm{p}<0.01)$. ICU and hospital mortality rates were higher in patients with $\mathrm{CCl}$ than others $(p<0.01)$. Binary logistic regression analysis revealed that each point increase in APACHE II score (OR 1.04, 95\% Cl [1.00-1.08]) and septic shock on admission (OR 2.07 [1.06-4.09]) were the independent predictors for $\mathrm{CCl}$ when adjusted for age, ECOG, $\mathrm{CFS}, \mathrm{PaO}_{2} / \mathrm{FiO}_{2}, \mathrm{IMV}$ and septic shock on admission.

Conclusion. Current study showed that chronic critical illness was present in about one third of the critically-ill COVID-19 patients, which was more than previously reported (2). Mortality is significantly higher in chronic critically ill patients.

Table: General characteristics of patients with chronic critical illness and others.

*median [IQR], Ton admission, text in bold in $\mathrm{p}$ value indicated statistical significance.

\begin{tabular}{|c|c|c|c|c|}
\hline & Total, $\mathrm{n}=328$ & $\mathrm{CCl}, \mathrm{n}=109$ & Others, $n=219$ & $\mathrm{p}$ value \\
\hline Age $^{*}$ & $66[55-76]$ & $67[60-78]$ & $64[54-74]$ & 0.04 \\
\hline Male sex, n (\%) & $214(65.2)$ & $70(64.2)$ & $144(65.8)$ & 0.78 \\
\hline \multicolumn{5}{|l|}{ Comorbidities, n (\%) } \\
\hline Hypertension & $173(52.7)$ & $61(56.5)$ & $112(51.4)$ & 0.38 \\
\hline Diabetes & $117(35.7)$ & $40(37.0)$ & $77(35.3)$ & 0.75 \\
\hline Cardiac disease & $110(33.6)$ & $41(38.0)$ & $69(31.8)$ & 0.26 \\
\hline Chronic lung disease & $56(17.1)$ & $23(21.3)$ & $33(15.1)$ & 0.16 \\
\hline $\mathrm{ECOG}^{*}$ & $1[0-2]$ & $1[1-3]$ & $1[0-2]$ & 0.02 \\
\hline $\mathrm{CFS}^{*}$ & $3[2-6]$ & $4[3-7]$ & $3[2-6]$ & $<0.01$ \\
\hline APACHE II* & 15 [12-19] & 17 [14-22] & $14[11-18]$ & $<0.01$ \\
\hline SOFA* & $4[3-6]$ & $5[3-7]$ & $3[2-5]$ & $<0.01$ \\
\hline $\mathrm{PaO}_{2} / \mathrm{FiO}_{2} \mathrm{~T}^{*}$ & 144 [111-214] & $129[98-172]$ & $160[120-232]$ & $<0.01$ \\
\hline IMVT, n (\%) & $133(40.5)$ & $81(74.3)$ & $52(23.7)$ & $<0.01$ \\
\hline IMV, days* & $11[3-20]$ & $17[10-25]$ & $4[2-9]$ & $<0.01$ \\
\hline AKIT, n (\%) & $70(21.3)$ & $30(27.5)$ & $40(18.3)$ & 0.14 \\
\hline Septic shockT, n (\%) & $49(14.9)$ & $26(23.9)$ & $23(10.5)$ & $<0.01$ \\
\hline ICU LOS*, days & $11[5-18]$ & 24 [18-33] & $7[4-11]$ & $<0.01$ \\
\hline Hospital LOS*, days & 19 [13-32] & $25[32-44]$ & 14 [10-23] & $<0.01$ \\
\hline ICU mortality, n (\%) & $106(32.3)$ & $59(54.1)$ & $47(21.5)$ & $<0.01$ \\
\hline Hospital mortality, n (\%) & 111 (33.8) & $59(54.1)$ & $52(23.7)$ & $<0.01$ \\
\hline
\end{tabular}

\section{Reference(s)}

1. 1. Gardner AK et al. The Development of Chronic Critical IIIness Determines Physical Function, Quality of Life, and Long-Term Survival Among Early Survivors of Sepsis in Surgical ICUs. Crit Care Med. (2019) 47(4):566-73.
2. 2. Nelson, JE et al. Chronic critical illness. Am J Respir Crit Care Med. (2010) 182(4): 446-54

\section{8}

Ten Year Outcome of ICU Survivors: A Single Center Experience

M. Yildirim ${ }^{1}$; B. Halacli'; S. Bozkurt ${ }^{1}$; Y. Gunegul ${ }^{1}$; TK. Sahin ${ }^{1}$; B. Celikten ${ }^{1}$. P. Ercan'; S. Maden ${ }^{1}$; C. Okur ${ }^{1}$; DA. Ozbek'; G. Guven ${ }^{1}$; E. Ortac Ersoy ${ }^{1}$; A. Topeli ${ }^{1}$

'Division of Intensive Care, Department of Internal Medicine, Hacettepe University Faculty of Medicine, Ankara, Turkey

Correspondence: $M$. Yildirim

Intensive Care Medicine Experimental 2021, 9(1): 000888

Introduction. A subset of critically-ill patients who are discharged from ICU suffer from post intensive care syndrome and they are at higher risk for mortality and for poor quality of life compared with general population. One fifth of ICU survivors pass away in the first year of discharge (1). Therefore, to improve post ICU outcomes, more information is needed on risk factors for mortality in ICU survivors.

Objectives. We aimed to define short and long-term mortality in different times in ICU survivors and to evaluate whether admission reason is related with mortality.

Methods. Patients admitted to our ICU between 1st January 2007 and 1st July 2015 were included in the study. We retrospectively obtained patients' admission and discharge dates. ICU admission reasons of patients were noted from patients' electronic medical records. Admission reasons were grouped as acute respiratory failure (ARF), sepsis, metabolic disorders, intoxications, gastrointestinal system disorders, cardiac disorders, postoperative care and neurological disorders. We also searched mortality date of patients who were discharged from ICU for up to ten years.

Results. 1612 patients were admitted to our ICU in the study period and 122 of whom medical records could not have been reached were excluded. Total number of 1490 patients were included the study. ARF (44.6\%) and sepsis (29.7\%) were the most common admission reasons. Overall ICU mortality was $30.9 \%(n=461)$. 3 months, 6 months, 1 year, 5 years and 10 years mortality rates of ICU survivors were $19.1 \%, 23.4 \%, 28.7 \%, 42.8 \%, 51.6 \%$, respectively. Although 1-year mortality rates of ICU survivors of patients with ARF and sepsis were $22.9 \%$ and $17.2 \%$, respectively, majority of these patients passed away in the following 5 years after ICU discharge $(60.2 \%$ and $75.6 \%$, respectively) (Table).

Table: Short- and Long-Term Mortality of ICU Survivors

\begin{tabular}{|c|c|c|c|c|c|c|}
\hline \multirow[b]{2}{*}{$\begin{array}{l}\text { Admission } \\
\text { reasons, } \mathrm{n}(\%)\end{array}$} & \multirow[b]{2}{*}{$\begin{array}{l}\text { ICU } \\
\text { Mortality } \\
(n=1490)\end{array}$} & \multirow[b]{2}{*}{$\begin{array}{l}3 \text { months } \\
\text { mortality }\end{array}$} & \multicolumn{4}{|c|}{ ICU survivors $(n=1029)$} \\
\hline & & & $\begin{array}{l}6 \text { months } \\
\text { mortality }\end{array}$ & $\begin{array}{l}1 \text { year } \\
\text { mortality }\end{array}$ & $\begin{array}{l}5 \text { years } \\
\text { mortality }\end{array}$ & $\begin{array}{l}10 \text { years } \\
\text { mortality }\end{array}$ \\
\hline All patients & $461(30.9)$ & $197(19.1)$ & $241(23.4)$ & $296(28.7)$ & $441(42.8)$ & $531(51.6)$ \\
\hline $\begin{array}{l}\text { ARF } \\
664(44.6)\end{array}$ & $151(22.7)$ & $98(14.8)$ & $122(18.4)$ & $152(22.9)$ & $400(60.2)$ & $454(68.4)$ \\
\hline $\begin{array}{l}\text { Sepsis } \\
443(29.7)\end{array}$ & $244(55.1)$ & $54(12.2)$ & $64(14.4)$ & $76(17.2)$ & $335(75.6)$ & $348(78.6)$ \\
\hline $\begin{array}{l}\text { Metabolic } \\
90(6.1)\end{array}$ & $15(16.7)$ & $9(10.0)$ & $11(12.2)$ & $12(13.3)$ & $31(34.4)$ & $37(41.1)$ \\
\hline $\begin{array}{l}\text { Intoxications } \\
74(5.0)\end{array}$ & $2(2.7)$ & $0(0)$ & $0(0)$ & $0(0)$ & $6(8.1)$ & $8(10.8)$ \\
\hline $\begin{array}{l}\text { GIS } \\
71(4.8)\end{array}$ & $14(19.7)$ & $17(23.9)$ & $21(29.6)$ & $23(32.4)$ & $37(52.1)$ & $42(59.2)$ \\
\hline $\begin{array}{l}\text { Cardiac } \\
57(3.8)\end{array}$ & $18(31.6)$ & $9(15.8)$ & $9(15.8)$ & $16(28.1)$ & $33(57.9)$ & $36(63.2)$ \\
\hline $\begin{array}{l}\text { Postoperative } \\
49(3.3)\end{array}$ & $6(12.2)$ & $3(6.1)$ & $5(10.2)$ & $6(12.2)$ & $16(32.7)$ & $18(36.7)$ \\
\hline $\begin{array}{l}\text { Neurological } \\
40(2.7)\end{array}$ & $11(27.5)$ & $6(15.0)$ & $8(20.0)$ & $10(25.0)$ & $22(55.0)$ & $24(60.0)$ \\
\hline
\end{tabular}

ICU: intensive care unit, ARF: acute respiratory failure, GIS: gastrointestinal system. 
Conclusion. Our findings revealed that as main admission reasons, patients with ARF and sepsis have poor prognosis especially between 1st and 5th years after ICU discharge. Further studies are needed to identify predictors of long-term mortality in ICU survivors.

\section{Reference(s)}

1. 1. Gayat, E et al. Determinants of long-term outcome in ICU survivors: results from the FROG-ICU study. Critical care (2018) 22(1):8.

\section{9}

Comparison of Critically-III Patients with Short and Prolonged ICU Length of Stay

M. Yildirim ${ }^{1}$; B. Halacli, ; C. Okur ' ;. Maden'; P. Ercan ${ }^{1}$; S. Bozkurt ${ }^{1}$; B.

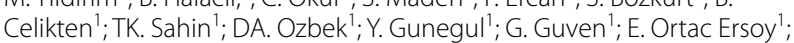
A. Topeli ${ }^{1}$

'Division of Intensive Care, Department of Internal Medicine, Hacettepe University Faculty of Medicine, Ankara, Turkey

Correspondence: $M$. Yildirim

Intensive Care Medicine Experimental 2021, 9(1): 000889Intensive Care Medicine Experimental 2021, 9(1): 000889

Introduction. Some critically-ill patients have prolonged ICU length of stay (LOS). Many studies showed that prolonged ICU stay is associated with poor outcomes and consume remarkable amount of ICU resources (1)

Objectives. We aimed to investigate predictors of prolonged ICU stay and compare clinical features and outcomes of patients with short and prolonged ICU-LOS.

Methods. Patients admitted to our ICU between 1st January 2007 and 1st July 2015 were included in the study. We compared clinical characteristics and outcomes of patients with ICU LOS $\leq 7$ days and those with ICU-LOS $>7$ days.

Results. 1490 patients were evaluated. $827(55.5 \%)$ patients stayed in ICU for $\leq 7$ days and 663 (44.5\%) patients stayed for $>7$ days. Comparison of critically-ill patients with short and prolonged ICU length of stay were shown in table.Binary logistic regression analysis revealed that the history of chronic lung disease (OR $1.75,95 \% \mathrm{Cl}[1.27-2.41]$; $p<0.01)$, neurological diseases (1.60 [1.09-2.37]; $p=0.02)$, admission due to respiratory failure $(1.59[1.03-2.48] ; p=0.04)$, sepsis $(1.71[1.04-$ 2.81]; $p=0.03)$, requirement of IMV (2.99 [2.18-4.10]; $p<0.01)$ and RRT $(2.20[1.59-3.06] ; p<0.01)$ were independent predictors of prolonged ICU stay.

Table: Comparison of Short and Prolonged ICU stay groups

\begin{tabular}{|c|c|c|c|c|}
\hline & All $(n=1490)$ & $\begin{array}{l}\leq 7 \text { days } \\
(n=827)\end{array}$ & $\begin{array}{l}>7 \text { days } \\
(n=663)\end{array}$ & $p$ value \\
\hline Age $^{*}$ & $63[45-74]$ & 59 [39-72] & $66[52-76]$ & $<0.01$ \\
\hline Male sex, n (\%) & $788(52.9)$ & $448(54.1)$ & $340(51.2)$ & 0.26 \\
\hline \multicolumn{5}{|l|}{ Comorbidities, n (\%) } \\
\hline Cardiac disease & $553(37.1)$ & $268(32.4)$ & $285(43.0)$ & $<0.01$ \\
\hline Hypertension & $496(33.3)$ & $254(30.7)$ & $242(36.5)$ & 0.01 \\
\hline Chronic lung disease & $418(28.1)$ & $179(21.6)$ & $239(36.0)$ & $<0.01$ \\
\hline Neurological disease & $196(13.1)$ & $87(10.5)$ & $109(16.4)$ & $<0.01$ \\
\hline $\mathrm{CFS}^{*}$ & $4[3-6]$ & $4[3-6]$ & $5[4-6]$ & $<0.01$ \\
\hline $\begin{array}{l}\text { Charlson Comorbidity } \\
\text { Index* }\end{array}$ & $3[2-5]$ & $3[1-5]$ & $4[2-6]$ & $<0.01$ \\
\hline APACHE $\|^{*}$ & 19 [13-25] & $17[11-24]$ & $21[16-27]$ & $<0.01$ \\
\hline SOFA $^{*}$ & $6[4-10]$ & $5[3-9]$ & $7[5-10]$ & $<0.01$ \\
\hline \multicolumn{5}{|c|}{ Admission reason, $\mathrm{n}(\%)$} \\
\hline Acute respiratory failure & $664(44.6)$ & $320(38.7)$ & $344(51.9)$ & $<0.01$ \\
\hline Sepsis & $443(29.7)$ & $228(27.6)$ & $215(32.4)$ & 0.04 \\
\hline Gastrointestinal disorders & $71(4.7)$ & $53(6.4)$ & $18(2.7)$ & $<0.01$ \\
\hline Metabolic disorders & $90(6.0)$ & $69(8.3)$ & $21(3.2)$ & $<0.01$ \\
\hline $\mathrm{PaO}_{2} / \mathrm{FiO}_{2} \mathrm{~T}$ & $186[127-257]$ & $\begin{array}{l}203[140- \\
300]\end{array}$ & $\begin{array}{l}\text { 167[120- } \\
217]\end{array}$ & $<0.01$ \\
\hline
\end{tabular}

\begin{tabular}{lllll}
\hline & All $(\mathbf{n}=\mathbf{1 4 9 0})$ & $\begin{array}{c}\leq \mathbf{7} \text { days } \\
(\mathbf{n}=\mathbf{8 2 7})\end{array}$ & $\begin{array}{l}>\mathbf{7} \text { days } \\
(\mathbf{n}=\mathbf{6 6 3})\end{array}$ & p value \\
\hline RRT $\rightarrow, n(\%)$ & $361(24.2)$ & $155(18.9)$ & $206(31.3)$ & $<\mathbf{0 . 0 1}$ \\
IMVT, n (\%) & $755(50.7)$ & $319(38.6)$ & $436(65.7)$ & $<\mathbf{0 . 0 1}$ \\
Tracheostomy, n (\%) & $134(9.0)$ & $10(12.1)$ & $124(18.7)$ & $<\mathbf{0 . 0 1}$ \\
ICU LOS*, days & $6[3-13]$ & $4[2-5]$ & $15[10-26]$ & $<\mathbf{0 . 0 1}$ \\
Hospital LOS*, days & $17[9-34]$ & $11[5-22]$ & $27[16-47]$ & $<0.01$ \\
ICU mortality, n (\%) & $461(30.9)$ & $228(27.5)$ & $233(35.1)$ & $<\mathbf{0 . 0 1}$ \\
Hospital mortality, n (\%) & $608(40.8)$ & $296(35.7)$ & $312(47.0)$ & $<\mathbf{0 . 0 1}$ \\
\hline
\end{tabular}

*median $[\mathrm{IQR}]$, Ton admission, text in bold in $\mathrm{p}$ value indicated statistical significance. CFS: clinical frailty scale, APACHE: acute physiology and chronic health evaluation, SOFA: sequential organ failure assessment, $\mathrm{PaO}_{2} / \mathrm{FiO}_{2}$ : Partial pressure of oxygen/Fraction of inspired oxygen, RRT: renal replacement therapy, IMV: invasive mechanical ventilation, ICU: intensive care unit, LOS: length of stay.

Conclusion. Current study showed that a significant number of patients stayed more than 7 days in the ICU. Comorbidities, admission reasons and utilisation of IMV and RRT were main determinants of prolonged ICU stay which was associated with poor ICU and hospital outcomes.

\section{Reference(s)}

1. 1. Arabi $Y$ et al. A prospective study of prolonged stay in the intensive care unit: predictors and impact on resource utilization. Int J Qual Health Care. (2002) 14(5):403-10

\section{0}

Short- and Long-Term Mortality of Patients Admitted to the Intensive Care Unit

M. Yildirim ' ; B. Halacli'; Y. Gunegul'; TK. Sahin '; S. Bozkurt'; S. Maden '; C. Okur'; P. Ercan'; B. Celikten'; DA. Ozbek'; G. Guven '; E. Ortac Ersoy'; A. Topeli ${ }^{1}$

'Division of Intensive Care, Department of Internal Medicine, Hacettepe University Faculty of Medicine, Ankara, Turkey

Correspondence: $M$. Yildirim

Intensive Care Medicine Experimental 2021, 9(1): 000890

Introduction. Although intensive care outcomes have improved almost $40 \%$ of the critically-ill patients admitted to the ICU die within 3 years after admission (1). There has been no definition for short-term mortality among critically-ill ICU patients yet. Generally, first 6-months is accepted as a cut-off for short and long-term outcome for ICU patients (2).

Objectives. We aimed to investigate short-term and long-term mortality of critically-ill patients.

Methods. Patients admitted to our ICU between 1st January 2007 and 1st July 2015 were included. Clinical parameters, outcomes of patients were retrospectively noted. We have also searched time of mortality in patients who were discharged from ICU. Patients who died in the 6 months period after ICU admission were included in short-term mortality group and long-term mortality group consisted of patients who died after 6 months from ICU admission.

Results. 1612 patients were admitted to our ICU in the study period. Medical records of 1490 patients could be reached and were included in the study. $993(66.6 \%)$ of the patients passed away as of 1st January 2021. $705(71.0 \%)$ of the 993 patients died in the first 6 months period after ICU admission while 288 (29\%) patients died after 6 months from ICU admission. Comparison of short- and longterm mortality groups were shown in table. Binary logistic regression analysis revealed that only history of chronic lung disease was an independent predictor of long-term mortality (OR $1.83,95 \% \mathrm{Cl}$ $[1.31-2.35] ; p<0.001)$ whereas chronic liver disease $(0.28,[0.11-$ $0.70] ; p<0.01)$, requirement of dialysis (0.64 [0.44-0.94]; $p=0.02)$, IMV (0.34, [0.25-0.48]; $p<0.01)$, vasopressor use on admission (0.38, [0.25-0.56]; $p<0.01)$, sepsis on admission (0.61, [0.40-0.93]; $\mathrm{p}=0.02$ ) were independent predictors of short-term mortality.

Table: Short- and Long-Term Mortality of Patients Admitted to ICU 


\begin{tabular}{|c|c|c|c|c|}
\hline & All, $n=993$ & $\begin{array}{l}\text { Short-term } \\
\text { mortality, } \\
n=705\end{array}$ & $\begin{array}{l}\text { Long-term } \\
\text { mortality, } \\
n=288\end{array}$ & $\mathrm{p}$ value \\
\hline Age $^{*}$ & $67[54-76]$ & $65[52-76]$ & $68[58-78]$ & 0.52 \\
\hline Male sex, n(\%) & $546(55)$ & $402(57)$ & $144(50)$ & $<0.01$ \\
\hline \multicolumn{5}{|c|}{ Comorbidities, n (\%) } \\
\hline $\begin{array}{l}\text { Cardiac } \\
\text { disease }\end{array}$ & $420(42.3)$ & $284(40.3)$ & $11(3.8)$ & 0.04 \\
\hline Hypertension & $350(35.2)$ & $236(33.5)$ & $114(39.6)$ & 0.06 \\
\hline CLD & $314(31.6)$ & $177(25.1)$ & $137(47.6)$ & $<0.01$ \\
\hline Diabetes & $269(27.1)$ & $185(26.2)$ & $84(29.2)$ & 0.34 \\
\hline $\begin{array}{l}\text { Hematologic } \\
\text { malignancy }\end{array}$ & $123(12.4)$ & $105(14.9)$ & $18(6.3)$ & $<0.01$ \\
\hline $\begin{array}{l}\text { Chronic liver } \\
\text { disease }\end{array}$ & $55(5.5)$ & $49(7)$ & $6(2.1)$ & $<0.01$ \\
\hline $\begin{array}{l}\text { Clinical Fraility } \\
\text { Scale* }\end{array}$ & $5[4-7]$ & $5[4-7]$ & $5[4-6]$ & 0.11 \\
\hline APACHE $\|^{*}$ & 21 [16-28] & 23 [18-30] & 17 [13-23] & $<0.01$ \\
\hline SOFA* & $7[5-11]$ & 8 [6-12] & $5[3-7]$ & $<0.01$ \\
\hline ARFT, n(\%) & $461(46.4)$ & $276(39.1)$ & $185(64.2)$ & $<0.01$ \\
\hline SepsisT, n(\%) & $354(35.6)$ & $307(43.5)$ & $47(16.3)$ & $<0.01$ \\
\hline $\mathrm{PaO}_{2} / \mathrm{FiO}_{2} \mathrm{~T}^{*}$ & $168[120-229]$ & $\begin{array}{l}165 \\
{[113-224]}\end{array}$ & $\begin{array}{l}187 \\
{[137-241]}\end{array}$ & 0.45 \\
\hline IMV, n (\%) & 575 (57.9) & $483(68.5)$ & $92(32.0)$ & $<0.01$ \\
\hline $\begin{array}{l}\text { Vasopressor } \mathrm{T}, \\
\mathrm{n}(\%)\end{array}$ & $472(47.5)$ & $416(59.0)$ & $56(19.4)$ & $<0.01$ \\
\hline RRT, n (\%) & $284(28.6)$ & $235(33.3)$ & $49(17.0)$ & $<0.01$ \\
\hline $\begin{array}{l}\text { ICU LOS*, } \\
\text { days }\end{array}$ & 7 [4-15] & $8[3-17]$ & $7[4-11]$ & 0.31 \\
\hline $\begin{array}{l}\text { Hospital LOS*, } \\
\text { days }\end{array}$ & 19 [10-36] & 20 [10-39] & 17 [11-27] & 0.07 \\
\hline
\end{tabular}

*median [IQR], Ton admission, text in bold in p value indicated statistical significance. CLD: chronic lung disease, ARF:acute respiratory failure, $\mathrm{PaO}_{2} / \mathrm{FiO}_{2}$ : Partial pressure of oxygen/Fraction of inspired oxygen, RRT: renal replacement therapy, IMV: invasive mechanical ventilation, ICU: intensive care unit, LOS: length of stay.

Conclusion. Current study revealed that majority of the mortality was seen in the first 6 month period in critically-ill patients. Chronic iver disease, requirement of dialysis, IMV, vasopressor use and sepsis on admission were independently associated with 6 months mortality.

\section{Reference(s)}

1. 1. Atramont, A et al. Association of Age With Short-term and Long-term Mortality Among Patients Discharged From Intensive Care Units in France. JAMA Network Open (2019) vol. 2,5 e193215.

2. 2. Hadique, $\mathrm{S}$ et al. Derivation and Validation of a Prognostic Model to Predict 6-Month Mortality in an Intensive Care Unit Population. Ann Am Thorac Soc. (2017) 14(10):1556-61

\section{2}

\section{A National Multi-disciplinary Approach to Critical Care System} Response to Surge during COVID-19 Pandemic

V. Hamilton"; M. Power, ; R. Dwyer ${ }^{3}$; D. Cribbon, 2, G. Flynn, ${ }^{4}$; R. Mcdermott, ${ }^{4}$; D. Murphy, ; R. Morgan, ; D. Doherty, ; D. Menzies, ; B Mcnicholas, ${ }^{2}$, D. Clarke, ; U. Quill, ${ }^{2}$; F. King, ${ }^{10}$; B. Moran, ${ }^{11}$; F. Treanor, ${ }^{11}$; E. Whelan, 12; E. Benton, 13, E. Brown, 13 ; L. Woods, ${ }^{13}$

${ }^{1} \mathrm{Ncagl}$, Acute Operations, HSE, Dublin, Ireland; ${ }^{2}$ Critical care programme, College of Anaesthesiologists of Ireland, Dublin, Ireland; ${ }^{3} \mathrm{cu}$ Business
Information System, NOCA, Dublin, Ireland; ${ }^{4}$ Medical Equipment, HSE, Dublin, Ireland; ${ }^{5}$ Respiratory Medicine, Cork University Hospital, Cork, Ireland; ${ }^{6}$ Respiratory Medicine, Beaumont Hospital, Dublin, Ireland; ${ }^{7}$ Micas, HSE Ireland, Dublin, Ireland; ${ }^{8}$ Micas, HSE, Dublin, Ireland; ${ }^{9}$ Estates, HSE, Dublin, Ireland; ${ }^{10}$ Pharmacy, HSE, Dublin, Ireland; ${ }^{11} \mathrm{Noca}, \mathrm{RCSI}$, Dublin, Ireland; ${ }^{12}$ Dublin Midlands Hospital Group, HSE, Dublin, Ireland;

${ }^{13}$ Acute operations, HSE, Dublin, Ireland

Correspondence: $V$. Hamilton

Intensive Care Medicine Experimental 2021, 9(1): 000892

Introduction. COVID-19 pandemic has placed unprecedented challenges to health care delivery requiring innovations in healthcare provision and systems organisation. Ireland, population 4.9 million with a public hospital system delivered through 7 hospital groups and governance from the Acute Operations Division of the Health Service Executive, took a national multi-disciplinary approach to pandemic management. This paper provides a brief overview on how Wave 3 surge management was supported.

In March 2020, engagement with the critical care programme, estates, medical equipment and the acute operations drug management programme commenced a programme of work to quantify and augment critical care delivery, oxygen delivery, match medical equipment to surge management plans, secure critical drugs supply and maximise infrastructure in terms of isolation and environment control rooms. The critical care business information system dashboard was operationalised to provide real time data on critical care utilisation.

Objectives. To ensure timely and equitable access to critical care for all patients with COVID and non-COVID critical care needs, to ensure that each acute hospital managed its resources optimally and to provide immediate expert intervention to effect solutions to problems as they arose, driven by live data on occupancy, acuity and resource utilisation.

Methods. A daily multi-disciplinary critical care surge meeting, informed by data from all acute hospitals on case numbers, staffing, resilience, oxygen utilisation, interventions including invasive ventilation and renal replacement therapy in the ICU and CPAP and HFNO on the wards. The mobile intensive care unit provided daily update on transfers planned and their outcomes.

Results. During wave 3 in January/February 2021, all ICUs were in surge, requiring redeployed staff to support critical care. This was delivered through a structured nurse surge escalation plan designed by the National Critical Care Programme, and implemented through a protocol starting with a 'buddy' system with 1:1 critical care nurse to redeployed staff, then increasing ratios of $1: 2$ and 1:3, as required. Interventions arising included working with a unit to develop their nursing surge plan and offering, advice and, on occasion, direction on staff ratios and skill mixes. Such interventions were effected immediately and the outcome reported and reviewed at the following meeting. On occasions multiple interventions were required to effect the necessary support to prevent a unit from 'falling over'. Informal contact was encouraged between the sites and the national surge working group in the event concerns weren't being addressed locally. A 'concierge' service for critical care transfer was offered to support busy units, which identified and organised transfers. 158 critical care transfers occurred during the third wave, 2.6 times the typical workload. This was enabled by the successful deployment of critical care paramedics for the first time.

Conclusion. No ICU 'fell over' despite all being in surge and there being a large number of staff on COVID related leave. Oxygen and equipment utilisation remained well within capacity. ICU mortality for COVID-19 patients admitted to Critical Care during the third wave was $39.9 \%, 34.7 \%$ in the second and $21 \%$ in the first wave. For every one patient admitted to Critical Care there were approximately 2 patients managed with CPAP or High Flow on the ward. 


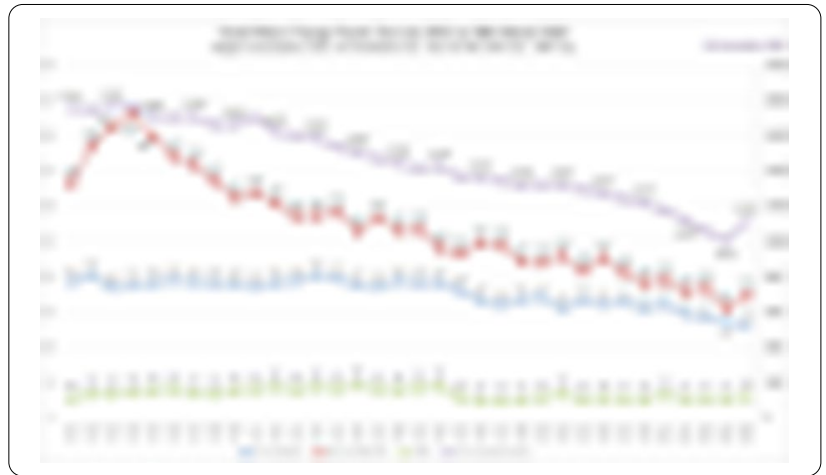

Reference(s)

1. Cucinotta D, Vanelli M. WHO Declares COVID-19 a Pandemic. Acta Biomed. 2020 Mar 19;91(1):157-160. https://doi.org/10.23750/abm.v91i1. 9397. PMID: 32191675; PMCID: PMC7569573.

\section{0}

Risk factors for prolonged intensive care unit stay in patients with hypercapnic respiratory failure

N. Naurzvai ; A. Mammadova'; G. Gursel ${ }^{2}$

${ }^{1}$ Department of Pulmonary Critical Care Medicine, Gazi University School of Medicine, Ankara, Turkey; ${ }^{2}$ Department of Pulmonary Critical Care Medicine, Gazi University School of Medicine, Professor of Respiratory Medicine, Ankara, Turkey

Correspondence: N. Naurzvai

Intensive Care Medicine Experimental 2021, 9(1): 000900

Introduction. The long intensive care unit (ICU) duration is the main reason for increased mortality rates and high hospitalization costs. Hypercapnic respiratory failure (HRF) is one of the most common causes requiring intensive care admission. HRF may prolong the intensive care treatment duration if it is not corrected immediately. The indication and duration of non-invasive mechanical ventilation (NIV) and the risk factors for NIV failure in patients with HRF have been studied well in previous studies. However, other factors have not been investigated in detail in patients with HRF. The hospital duration might be affected by the severity of the underlying disease, sepsis, comorbidities, and complications. This study aimed to investigate the factors that prolong the ICU duration in patients with HRF.

Methods. Patients with a partial pressure of carbon dioxide $\left(\mathrm{PaCO}_{2}\right)>45$ were included in this retrospective cohort study. Patients having incomplete file records or staying in the ICU less than $24 \mathrm{~h}$ were not enrolled in the study.

Impact of demographics, vitals at the admission, disease severity, respiratory parameters, admission diagnoses, comorbidities, NIV application time and settings, arterial blood gases, blood biochemistry results were analyzed in patients with HRF in order to investigate risk factors for longer ICU stay.

Determination of threshold values for hospitalization period: The number of days that each patient stayed in the ICU was recorded. The ICU duration period was divided into time frames and analyzed whether the investigated parameters were significant for prolonging ICU duration for each time frame. Since the length of ICU stay of patients with HRF ranges between 8 and 13 days in the literature, threshold values were chosen within this range. Risk factors for prolonging ICU hospitalizations were investigated following threshold values, which were 7 days, 10 days, and 15 days.

Statistical analysis: All patients were analyzed in two groups according to the chosen threshold values for ICU stay. Those who stayed in ICU less than 7, 10, and 15 days, and those who stayed longer. SPSS for Windows 25.0 (SPSS for Windows; Chicago, IL, USA) software package was used for the statistical analysis. In order to determine the risk factors that prolong the hospital stay, parameters that were found to be significantly different with univariate analysis were further evaluated with multivariate analysis in terms of being independent risk factors. Receiver operating curve analysis was used to determine the optimal cut-point value of potential risk factors and to calculate the sensitivity and specificity for this value.

Results. A total of 210 patients who were admitted to ICU with HRF were included in this study. The mean age of the patients was $69 \pm 12$ years, and the mean ICU duration was $9 \pm 7$ days. Forty-five percent of the patients stayed in the ICU for more than one week and $\% 10$ of them stayed more than 15 days. Risk factors for prolonged stay in ICU for more than 7 days were higher SOFA score, acute renal failure (ARF) at the admission, low $\mathrm{PaO}_{2} / \mathrm{FiO}_{2}$ on the 2 nd day of the admission, and high TSH level. Low FEV1 and FEV1/FVC ratio, ARF at the admission, and low $\mathrm{PaO}_{2} / \mathrm{FiO}_{2}$ ratio on the 2 nd day of the admission were found to be risk factors for prolonged stay in ICU for longer than 10 days. Significant risk factors prolonging the ICU duration more than 15 days were high SOFA score, low FEV1, low FEV1/FVC ratio $(P=0.008)$, and hypothyroidism $(P=0.037)$. FEV1\% predicted less than $25.5 \%$ and FEV1/FVC\% less than 46.5 were significantly associated with ICU stay longer than 10 days.

Conclusion. Earlier diagnosis and treatment of patients with hypothyroidism and severe airflow obstruction could shorten the length of ICU stay of hypercapnic patients.

\section{Reference(s)}

1. 2-Türk M, Aydoğdu M, Gürsel G. Effects of modes, obesity, and body position on non-invasive positive pressure ventilation success in the intensive care unit: a randomized controlled study. Turkish thoracic journal. 2018;19(1):28.

2. 1-Wunsch H, Gershengorn H, Scales DC. Economics of ICU organization and management. Crit Care Clin. 2012;28(1):25

\section{1}

Change in characteristics and outcome of critically ill COVID-19 patients over two periods of a pandemic

T. Mayerhöfer ${ }^{1}$; S. Klein ${ }^{1}$; F. Perschinka, ${ }^{1}$; M. Joannidis

${ }^{1}$ Division of Intensive Care and Emergency Medicine, Medizinische

Universität Innsbruck, Innsbruck, Austria

Correspondence: T. Mayerhöfer

Intensive Care Medicine Experimental 2021, 9(1): 000901

Introduction. With the beginning of the pandemic in Tyrol, Austria, a register study was established, in order to observe characteristics and outcomes of critically ill COVID-19 patients at a regional level (1).

Objectives. The aim of this study was to identify important differences in baseline characteristics, resource use and outcomes over two waves of the COVID-19 pandemic.

Methods. This multicenter prospective register study included all 508 patients with a SARS-CoV-2 infection confirmed by polymerase chain reaction, who were treated in one of the 12 dedicated ICUs (from 8 hospitals in Tyrol, Austria) during two defined periods (February 1st, 2020 until July 17th [first wave] and July 18th, 2020 until February 22nd, 2021 [second wave]) of the COVID-19 pandemic. According to the median age of the total cohort, patients were divided in an older and younger age group and analyzed with Kaplan-Meier survival curves.

Results. The majority of the 508 patients were enrolled during the second period. Patients were significantly older in the second wave. The male/female ratio was 69.9 to $30.1 \%$ and was quite similar over the two periods. This also applies to the most common comorbidities: hypertension and cardiovascular diseases. The median BMI was 27.55, the SAPS III score and SOFA score were 56 and 5, respectively and did not show any difference between the two periods. Invasive mechanical ventilation (IMV) was more frequently used during the first period, as were vasopressors. The use of renal replacement therapy decreased over time and was less frequent in the second wave. Hospital and ICU length of stay (LOS) were shorter, but hospital mortality was significantly higher in the second period. In an overall analysis older age 
(> 71 years) was significantly associated with impaired hospital survival (Figure 1).

Table 1: Comparison between critically ill COVID-19 patients of the first and second wave

\begin{tabular}{|c|c|c|c|c|}
\hline & $\begin{array}{l}\text { Overall } \\
(n=508)\end{array}$ & $\begin{array}{l}\text { First wave } \\
(n=107)\end{array}$ & $\begin{array}{l}\text { Second wave } \\
(n=401)\end{array}$ & $p$ value \\
\hline \multicolumn{5}{|c|}{ Patient characteristics and Scores } \\
\hline $\begin{array}{l}\text { Sex: male/female } \\
(\%)\end{array}$ & $\begin{array}{l}355 / 153 \\
(69.9 / 30.1)\end{array}$ & 77/30 (72.0/28.0) & $\begin{array}{l}278 / 123 \\
\quad(69.3 / 30.7)\end{array}$ & 0.682 \\
\hline $\begin{array}{l}\text { Age (median } \\
{[\text { IQR]) }}\end{array}$ & $\begin{array}{l}71.00[60.00 \\
78.00]\end{array}$ & $\begin{array}{l}64.00[54.00 \\
74.00]\end{array}$ & $\begin{array}{l}72.00[62.00, \\
78.00]\end{array}$ & $<0.001$ \\
\hline $\begin{array}{l}\text { BMI (median } \\
{[\text { IQR]) }}\end{array}$ & $\begin{array}{l}27.55[24.98 \\
30.86]\end{array}$ & $\begin{array}{l}26.83[25.08 \\
30.15]\end{array}$ & $\begin{array}{c}27.73[24.96 \\
31.05]\end{array}$ & 0.309 \\
\hline $\begin{array}{l}\text { SOFA score } \\
\text { (median [IQR]) }\end{array}$ & $5.00[4.00,8.00]$ & $6.00[4.00,10.00]$ & $5.00[4.00,8.00]$ & 0.109 \\
\hline $\begin{array}{l}\text { SAPS III score } \\
\text { (median [IQR]) }\end{array}$ & $\begin{array}{l}56.00[49.00 \\
64.00]\end{array}$ & $\begin{array}{l}56.00[49.00 \\
64.00]\end{array}$ & $\begin{array}{l}55.00[49.00 \\
64.00]\end{array}$ & 0.877 \\
\hline \multicolumn{5}{|l|}{ Comorbidities } \\
\hline Hypertension (\%) & $327(64.4)$ & $71(66.4)$ & $256(63.8)$ & 0.712 \\
\hline $\begin{array}{l}\text { Cardiovascular } \\
(\%)\end{array}$ & $217(42.7)$ & $45(42.1)$ & $172(42.9)$ & 0.964 \\
\hline \multicolumn{5}{|l|}{ Treatment } \\
\hline IMV (\%) & $272(53.8)$ & $72(67.3)$ & $200(50.1)$ & 0.002 \\
\hline RRT (\%) & $69(13.7)$ & $21(19.6)$ & $48(12.1)$ & 0.062 \\
\hline $\begin{array}{l}\text { Corticosteroids } \\
(\%)\end{array}$ & $355(88.5)$ & $0(\mathrm{Na})$ & $355(88.5)$ & $\mathrm{Na}$ \\
\hline \multicolumn{5}{|l|}{ Outcome } \\
\hline $\begin{array}{l}\text { ICU LOS (median } \\
\text { [IQR]) }\end{array}$ & $\begin{array}{c}11.00[5.00 \\
22.00]\end{array}$ & $\begin{array}{c}18.00[5.00 \\
31.50]\end{array}$ & $\begin{array}{c}10.00[5.00 \\
18.00]\end{array}$ & $<0.001$ \\
\hline $\begin{array}{l}\text { Hospital LOS } \\
\quad \text { (median [IQR]) }\end{array}$ & $\begin{array}{l}21.00[13.00 \\
35.00]\end{array}$ & $\begin{array}{c}27.00[14.50 \\
41.50]\end{array}$ & $\begin{array}{c}19.50[13.00 \\
33.00]\end{array}$ & 0.010 \\
\hline Death in ICU (\%) & $139(27.4)$ & $23(21.5)$ & $116(28.9)$ & 0.159 \\
\hline $\begin{array}{l}\text { Death in Hospital } \\
(\%)\end{array}$ & $158(31.1)$ & $24(22.4)$ & $134(33.4)$ & 0.039 \\
\hline
\end{tabular}

Figure 1: Age and hospital survival.

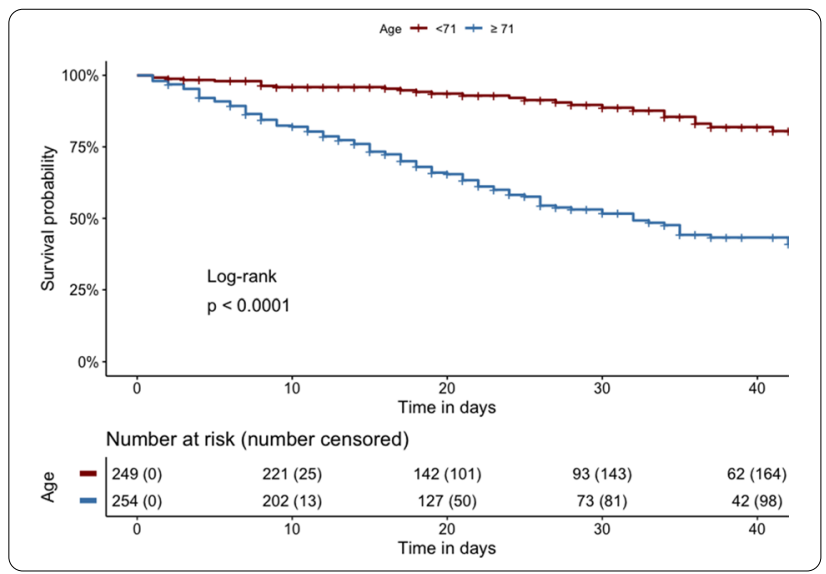

Conclusion. The significant reduction of IMV, vasopressor use and RRT and the shorter ICU as well as hospital LOS, might be attributed to the general use of dexamethasone in the second period, due to the results of the RECOVERY trial. However, mortality increased, which was probably due to a substantial increase of median age of patients during the second period. Independent of improvements in treatment, age appears to be a major determinant of hospital survival.
Reference(s)

1. 1. Klein SJ, Bellmann R, Dejaco H, Eschertzhuber S, Fries D, Furtwängler $W$, et al. Structured ICU resource management in a pandemic is associated with favorable outcome in critically ill COVID-19 patients. Wien Klin Wochenschr. 2020;132(21-22):653-63.

\section{5}

Describing Two Populations of Invasively Ventilated Covid-19 Positive to Explain Changes in Survival Outcomes

M. Crawford'; S. Stott ${ }^{2}$

${ }^{1}$ Department of Medicine, University of Aberdeen School of Medicine and Dentistry, Foresterhill, United Kingdom; ${ }^{2}$ CRITICAL CARE, ABEREDEEN ROYAL iNFIRMARY, Aberdeen, United Kingdom

Correspondence: $\mathrm{S}$. Stott

Intensive Care Medicine Experimental 2021, 9(1): 000905

Introduction. Survival between invasively ventilated Covid-19 positive patients was reduced from wave one to wave two of the pandemic in Aberdeen Royal Infirmary (ARI) Critical Care (CC) Department. Known risk factors which potentially lead to poorer outcomes in Covid-19 infections were investigated, such as age, sex, diabetes, hypertension and obesity1-3.

Objectives. To compare the two wave populations to identify any possible explanation for the change in survival outcomes.

Methods. Invasively ventilated Covid-19 patients who were admitted to ARI CC during wave one and wave two were identified. Two patient databases were used to retrieve 32 demographic and physiological patient variables which were then compared. Whole population (primary) and non-survivor (secondary) comparisons are reported.

Results. In total 32 patients in wave 1 and 35 patients in wave 2 were invasively ventilated. In wave 1 , there were 21 survivors and 11 nonsurvivors. In wave 2 , there were 10 survivors and 25 non-survivors. The survival outcome changed from wave 1,65.63\% surviving, decreasing to $28.57 \%$ in wave $2(p=0002)$. From the 32 variables identified and compared, 7 were found to be significantly different in both the primary and secondary outcome. BMI was found to be significantly higher in wave 2 populations (Primary 28.76 vs $33.1, p=0.007$. Secondary 28.09 vs $34.16, p=0.0116$ ). Administration of steroids prior to admission (Primary 0 vs 13 receiving, $p=0.001$. Secondary 0 vs $9, p=0.0006$ ) and during admission (Primary 14 vs $32, p=0.0000026$. Secondary 3 vs $24, p=0.0000012$ ) was found to higher in wave 2 . Additionally, tocilizumab administration was significantly increased within the wave 2 population (Primary 0 vs $12, p=0.003$. Secondary 0 vs $9, p=0.02$ ). The average $\mathrm{Pa} / \mathrm{FiO}_{2}$ ratio change in response to prone positioning was found to be lower in wave 2 (Primary 12.97 vs $7.5, p=0.006$. Secondary 10.42 vs $7.55, p=0.05)$. The platelet count on admission was significantly lower in wave 2 (Primary 284.64 vs $200.01, p=0.03$. Secondary 285.5 vs $201.45, p=0.018$ ). Finally, the CRP level on admission was lower in wave 2 (Primary 185.86 vs $81.95, p=0.0000035$. Secondary 194.25 vs $87, p=0.001)$. The rest of the variables compared did not show significant differences between the two populations.

Conclusion. There were statically significant differences between the two waves and non survivors. Non survivors in wave two had higher BMI, lower platelet counts and CRP and had received steroid before admission. They also showed less response to proning manoeuvres. These results suggest invasively ventilated wave 2 patients had identifiable risk factors that may explain the differences in outcomes observed.

\section{Reference(s)}

1. 1. Williamson EJ, Walker AJ, Bhaskaran K, Bacon S, Bates C, Morton CE, Curtis HJ, Mehrkar A, Evans D, Inglesby P, Cockburn J. OpenSAFELY: factors associated with COVID-19 death in 17 million patients. Nature. 2020 Jul 8:1-1.

2. 2. Rod J, Oviedo-Trespalacios O, Cortes-Ramirez J. A brief-review of the risk factors for covid-19 severity. Revista de Saúde Pública. 2020;54:60

3. 3. Zheng Z, Peng F, Xu B, Zhao J, Liu H, Peng J et al. Risk factors of critical \& mortal COVID-19 cases: A systematic literature review and meta-analysis. Journal of Infection. 2020;81 (2):e16-e25.

4. No grants received. 


\section{2}

The association between measures of ICU strain and adverse event reporting before and during the COVID surge: Mind the gap W. Roberts ${ }^{1}$; DJ. Kirkin ${ }^{2} ;$ T. Williams ${ }^{3} ;$ N. Singh, ${ }^{3} ;$ J. Bedford ${ }^{2}$

${ }^{1}$ Foundation year 2 doctor, Medway Maritime Hospital, Gillingham, United Kingdom; ${ }^{2}$ Anaesthetics, King's College Hospital, London, United Kingdom; ${ }^{3}$ Intensive Care Medicine, King's College Hospital, London, United Kingdom

Correspondence: D.J. Kirkin

Intensive Care Medicine Experimental 2021, 9(1): 000952

Introduction. ICU strain is defined as the inability to provide a recognised standard of care for all critically ill patients using the available resources. 1 Adverse events (AEs) are injury or harm related to (or from) the delivery of care.2 Both ICU strain and increased incidence of AEs have been associated with unfavourable outcomes for patients (increased length of hospital stay and/or mortality).3\&4 Identification of AEs often relies of the successful reporting by individuals through a detection system, it is unknown how ICU strain may affect the likelihood of AEs being reported by individuals.

Objectives. We aimed to establish the relationship between measures of strain and AE reporting in our ICU before and during the COVID-19 pandemic. We hypothesised AEs may have been under reported during the COVID period due to the strain placed on resources.

Methods. Data from January 2018 to May 2020 was included. Patient level data was obtained from the ICNARC registry. Staffing and $A E$ data was obtained from local databases. Measures of ICU strain were based on those identified by Rewa et al.5 We fitted a negative binomial regression model (NBM) for the reference period and applied this model to the COVID period.

Results. The reference period included data from the $01 / 01 / 2018$ to $31 / 01 / 2020$ and COVID period from the $01 / 02 / 2020$ to $31 / 05 / 2020$ There were 333 and 48 AEs reported during the reference and COVID period respectively. The NBM provided a good fit for our data. Mode coefficients for all included measures of ICU strain were non-significant. The model estimated there may have been $240 \mathrm{AEs}$ during the COVID period ( $95 \% \mathrm{Cl} 85-763)$.

Conclusion. The potential under reporting of AEs in our ICU during the first wave is a missed opportunity to understand how ICU care provision changes under extreme strain and enable better planning for future waves of COVID-19 and other pandemics.

\section{Reference(s)}

1. 1. Bagshaw, S. M. et al. Association between strained capacity and mortality among patients admitted to intensive care: A path-analysis modeling strategy. J. Crit. Care 43, 81-87 (2018).

2. 2. IHI Intensive Care Unit (ICU) Adverse Event Trigger Tool | IHI-Institute for Healthcare Improvement. Available at: http://www.ihi.org/resources/ Pages/Tools/ICUAdverseEventTriggerTool.aspx\#: :text=The ICU Adverse Event Trigger,and degree of harmful events. (Accessed: 7th December 2020)

3. 3. Wilcox ME, Harrison DA, Patel A, Rowan KM. Higher ICU Capacity Strain Is Associated With Increased Acute Mortality in Closed ICUs. Crit Care Med. 2020 May;48(5):709-716.

4. 4. Roque KE, Tonini T, Melo EC. Adverse events in the intensive care unit: impact on mortality and length of stay in a prospective study. Cad Saude Publica. 2016 Oct 20;32(10):e00081815. English, Portuguese.

5. 5. Rewa, O. G. et al. Indicators of intensive care unit capacity strain: A systematic review. Crit. Care 22, 1-13 (2018).

\section{9}

COVID-19 Pandemic Communication Survey: A true collateral damage

A. Simas ; P. Ramos ${ }^{1}$; V. Silveira,

${ }^{1}$ Intensive Care Unit, Hospital Vila Franca de Xira, Vila Franca de Xira, Portugal

Correspondence: A. Simas

Intensive Care Medicine Experimental 2021, 9(1): 000959

Introduction. COVID-19 pandemic led to a global health crisis with deep impact on communication. Social detachment contributed to ineffective communication and to isolation of patients. Poor and ineffective communication has a significant impact, leading to anxiety, depression, sadness, anguish and, when the outcome is death, to complicated grief and post-traumatic stress. To minimize this negative impact, many hospitals focused on the humanization of health care, whether it is through promoting phone or video calls, sending emails, or even permitting exceptional face-to-face visits. These strategies strengthen health care professionals-patients-relatives' relationships and minimize the family-patient gap, involving them in the disease process and making them part of the treatment.

Communication between health care staff and patients' families is essential, and communication skills are just as valuable as clinical skills, or even more.

Methods. Online survey shared from February 17th to April 13th, 2021, through personal contact network, between Portuguese physicians and nurses involved in direct care setting.

Results. 520 health care professionals responded the survey, with fair distribution between physicians and nurses, mostly women and between 20 and 40 years of age. Most of them treated patients with COVID-19; nearly $50 \%$ worked in intensive care units.

$91 \%$ considered that communication is an essential and crucial part of the healing process, with high impact on the outcome of the patient. $89 \%$ considered communication an important element for professional performance, but only $1 / 3$ had training in clinical communication. $88 \%$ of the professionals identified themselves verbally to the patient and only $27 \%$ identified themselves when wearing the individual protective equipment.

Families were often contacted by more than a half of responders $(>4 \times)$ week) and $98 \%$ established phone calls between the patients and their family, frequently allowing face-to-face visits in end-of-life situations. Almost $2 / 3$ recognized difficulties in communication with the family and $78 \%$ identify fatigue as a barrier to an efficient communication. $87 \%$ perceived family discomfort about visiting restrictions and many feel a sense of emptiness given the insufficient means available to establish a good connection.

Conclusion. The outbreak of COVID-19 created challenges for health care providers at different levels. The importance of effective communication between medical staff and families became amplified and even more relevant due to the imposed restrictions and isolation.

The inability to see, touch and talk to loved ones increases the burden of critical illness on the patients and their families. The lack of face-toface communication has also greatly changed the dynamic of interactions between healthcare providers and families.

COVID-19 pandemic stole us the ability to communicate, the core of the doctor-patient relationship. It is our job to develop different strategies to achieve our purpose: the patient's health.

\section{Reference(s)}

1. Walid Al-Zyoud et al., The Psychological Effects of Physicians' Communication Skills on COVID-19 Patients, Patient Preference and Adherence 2021:15 677-690.

2. Anthony Back, MD et al., Communication Skills in the Age of COVID-19, Ann Intern Med. 2020:172:759-760.

3. Marra et al., How COVID-19 pandemic changed our communication with families: losing nonverbal cues, Critical Care 2020 24:297.

\section{0}

ROSe (Rural Outreach Support e-ICU) platform for community

\section{critical care}

D. Burke ; O. Ahmad ${ }^{2}$; N. Mclean ${ }^{3} ;$ I. Ahmed ${ }^{3}$; M. Slabbert ${ }^{4}$; M.

Francispragasam ${ }^{3}$; A. Thomas ${ }^{2}$

${ }^{1}$ Intensive Care Medicine, Fraser Health Authority, Abbotsford, Canada; ${ }^{2}$ Intensive care medicine, BC Health Authorities-Island Health, Victoria,

Canada; ${ }^{3}$ Intensive care medicine, Fraser Health Authority, Vancouver,

Canada; ${ }^{4}$ Intensive Care Medicine, Northern Health Authority, Prince

George, Canada

Correspondence: D. Burke

Intensive Care Medicine Experimental 2021, 9(1): 000960 
Introduction. The current pandemic has highlighted the importance of virtual care and knowledge exchange in medicine. Most virtual medical networks in North America provide a connection between the patient and a primary provider, and always in a non-urgent setting. None are designed to manage acutely ill patients. Under-resuscitation or having the wrong diagnosis at presentation can lead to worse outcomes. This underscores the fact that the management of very ill patients needs to start at the primary facility, whether it be a rural or urban setting. Valuable time is often wasted in attempting to transport a patient to a higher level of care, when many of the resources are already available at the primary facility to initiate proper treatment. Additionally, a major reason for rural physician dissatisfaction is lack of support during urgent situations. This translates into a decrease in the recruitment of physicians into smaller communities, suboptimal patient outcomes, and wasted resources.

Objectives. What is needed is a robust system that links any rural emergency physician with an intensive care specialist, in the most direct and simplest manner possible (eg.. cellular phones), using a high level of encryption to allow real-time data and knowledge-sharing with high-fidelity video, to achieve the best possible outcomes.

The second major objective is to reduce the need for unnecessary patient transfers, by empowering physicians in smaller healthcare facilities to maximize their available resources. In this way, large savings in healthcare expenses can be realized.

The third objective is to support physicians at all levels of training who are at the frontlines in smaller urban and rural communities, by way of immediate knowledge-exchange and problem-solving, as well as video-assisted skills training, and simulation-based learning.

Methods. ROSe went through a one-year pilot phase in British Columbia, engaging a wide range of rural and smaller-community hospitals, during which several quality measures were evaluated, including patient outcomes, the frequency of patient transfers to larger facilities, healthcare cost savings, healthcare provider satisfaction with the support, recruitment and retention of rural providers and quality performance of the platform itself.

Results. On all measures, ROSe performed better than anticipated. Based upon historical controls in each sector, the ROSe platform provided better patient outcomes, less need for transfer to higher levels of care in urban centres, thereby significantly decreasing both healthcare costs and larger urban hospital congestion. Data on rural physician recruitment and retention was harder to capture within the one year pilot study, but based upon feedback surveys and using historical controls, but rural physician satisfaction was very significantly improved.The platform allows for the encrypted transmission of imaging and other clinical data, including bedside ultrasound, to assist in the acute management of a patient. The specialist is also able to screen-share with the rural provider, any relevant guidelines, videos or other relevant data. Immediately following the live interaction, a consultation note can be dictated by the specialist via the app, directly to the referring physician, for the patient's medical record.

The greatest value was the 'virtual bedside' support of rural physicians faced with critical care issues. The program focuses on crisis resource management, helping rural physicians utilize local resources and empowering them with the knowledge and confidence to manage such patients successfully. This feature of ROSe received the most accolades. The support and trust developed between physician and specialist were key strengths of the program.

Conclusion. The ROSe virtual telehealth program has become transformative in its management of the critically ill patient in a rural or smaller-community healthcare facility. It has been demonstrated to improve patient outcomes, substantially reduce healthcare costs, improve family satisfaction, and empower rural practitioners to make optimal use of their resources to improve patient outcomes. Knowing that they have an 'intensivist in their pocket' has improved the confidence and skill level of practitioners working in smaller facilities who are confronted with critically ill patients. ROSe is now involved in critical care simulation-learning with residents at all levels. The Royal College of Physicians and Surgeons of Canada and the British Columbia
Ministry of Health have endorsed the value of the platform for critical care outreach teaching and knowledge exchange.

\section{7}

Central venous access-a doctor's job? A comparison of over 300 lines and the case for advanced critical care practitioners

N. Gardner, ; A. Wong ${ }^{2}$

${ }^{1}$ King's Critical Care, King's College Hospital, London, United Kingdom;

${ }^{2}$ Critical care, Royal Surrey County Hospital, Guildford, United Kingdom

Correspondence: N. Gardner

Intensive Care Medicine Experimental 2021, 9(1): 000967

Introduction. The recent COVID19 pandemic has placed an unprecedented strain in healthcare systems across the world. In such challenging times, it is a timely reminder that the delivery of high-quality care is dependent upon the availability of trained staff. Central venous access is ubiquitous in modern intensive care practice and has traditionally been the remit of doctors rather than allied healthcare professionals. Given the challenges of staffing (made more acute by COVID), we aimed to see if this dogma can be challenged. In some healthcare systems (USA and UK), advanced critical care practitioners (ACCPs) are increasingly part of the intensive care workforce, taking on this role. This is less true in Europe.

Objectives. To compare the effectiveness and safety profile of ACCPs in the provision of central venous access in a busy, tertiary-level critical care department.

Methods. A prospective cohort study of all central lines (CVCs) inserted by the critical care team during a 6-week period were analysed. Insertion locations included all critical care units, and all outreach locations attended by the critical care team. For the purpose of this study, central lines included vascular catheters for renal replacement therapy but excluded peripherally inserted central lines and lines inserted by interventional radiology.

Results. A total of 335 CVCs were inserted. ACCPs inserted $26 \%$ of all lines while making up $6 \%$ of the daytime medical teams. Across the groups complication rates were $4.44 \%$ for ACCPs, $5.20 \%$ for ST 3 s and above, $7.14 \%$ for CT2/ST2 or below, and $0 \%$ for Consultant grade doctors. When grouped together the overall complication rate for doctors was $5.60 \%$. Full compliance with the use of checklists were $85.5 \%$ for ACCPs, $79.89 \%$ for ST3s and above, $79.31 \%$ for CT2/ST2 and below, and $33.3 \%$ for Consultants.

Conclusion. Despite the near universal use of ultrasound to guide CVC insertions, complications still occur. The rate of complications reported varies between 6.8 and $15 \%$. In our study, the overall complication rate was $5.27 \%$. The most common immediate complications encountered were multiple anatomical puncture sites before success, line malposition, and haematoma formation. Crucially, we found that there were lower complication rates amongst ACCPs compared to non-consultant doctors, although this rate was not statistically significant $(p>0.999)$. In conclusion, our data supports the fact that ACCPs can safely and effectively insert CVCs, challenging the dogma that it is a doctor's role. Throughout the pandemic, the ESICM has consistently championed the multidisciplinary approach of ICM, our study is further evidence of this.

\section{Reference(s)}

1. Wong AV, Arora N, Olusanya O, et al. Insertion rates and complications of central lines in the UK population: A pilot study. Journal of the Intensive Care Society. 2018:19(1):19-25.

2. Waghorn, DJ. Intravascular device-associated systemic infections: a 2 year analysis of cases in a district general hospital. J Hosp Infect 1994; 28: 91-101.

3. McGee, DC, Gould, MK. Preventing complications of central venous catheterization. N Engl J Med 2003; 348: 1123-1133. 
001001

Outcome for patients subject to EWS monitoring failure-a retrospective cohort study of the NEWS-protocol leading up to MET-calls

HES. Seitz-Rasmussen ${ }^{1}$; M. Føns-Sønderskov, ${ }^{2}$; AM. Kodal, ; M. Bestle, 'Department of Anaesthesiology and Intensive Care, Nordsjaellands Hospital, Hilleroed, Denmark; ${ }^{2}$ Department of Anaestesiology and Intensive Care, Nordsjaellands Hospital, Hilleroed, Denmark Correspondence: H.E.S. Seitz-Rasmussen

Intensive Care Medicine Experimental 2021, 9(1): 001001

Introduction. The afferent limb of the rapid response system is designed as a track and trigger system that is meant to recognize deteriorating of patients outside the ICU-setting, responding, and triggering clinical action (1). It is arguably the most important component of the RRS, and monitoring needs to be timely and correct to be effective, as sever adverse events such as in hospital cardiac arrest, mortality and ICU-admission are preceded by vital sign deviations. (2-4). The National Early Warning Score (NEWS) is the track and trigger system used in the capital region of Denmark to monitor and detect deteriorating patients in the general ward.

Objectives. The objective of this study was to retrospectively evaluate compliance toward the NEWS-protocol $72 \mathrm{~h}$ prior to Medical emergency team-event (MET-event) and illuminate whether poor compliance translates into a worse patient outcome.

Methods. In this study we investigated every patient subject to a MET-event from 31/12/19 to $31 / 20 / 20$ all admitted non-obstetric and non-psychiatric patients above 18 years where included. Included patients electronical medical journal were evaluated in detail. Age, gender, Length of stay, 30-day mortality, ICU-admission and surviving to discharge was registered. EWS recordings was reviewed in the timeframes 72-48-, 48-24-, 24-16, 16-8, 8-0-h before MET-event. EWS monitoring failure was defined as not monitoring vital signs within the time limits recommended by the NEWS-monitoring protocol used at North Zealand Hospital-Hillerød ( $\mathrm{NOH}$-Hillerød).

Results. 521 MET-events where registered in the quality assurance database of North Zealand Hospital-Hillerød from 31/12/19 to $31 / 12 / 20$. 92 where excluded resulting in 429 MET-events that were included in the final analysis. EWS-monitoring failure was observed in more than half the patients within all the timeframes.

Adjusting for LOS, age, and gender patients subject to EWS-monitoring failure had an odds ratio (OR) of 1.9, 2.0, 2.1, 2.3 for mortality in the time frames 72-48-, 24-16-, 16-8- and 8-0-h before MET-call respectively. The adjusted OR for ICU-admission was 1.6 and 1.7 for patients subject to EWS monitoring failure in the timeframes $16-8$ - and 8-0-h before MET-event respectively (table 4 below).

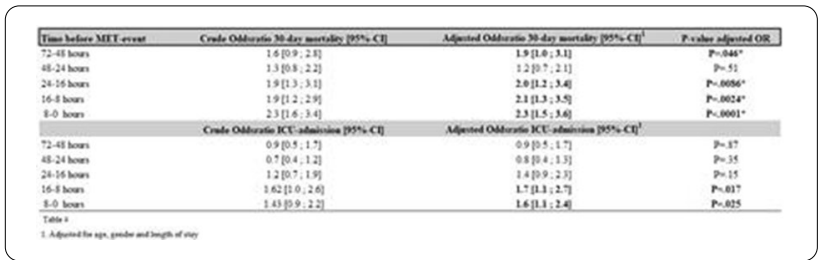

Conclusion. Compliance toward the NEWS-protocol $72 \mathrm{~h}$ prior to MET-event is poor, with more than half of patience being subject to monitoring failure. Monitoring failure is associated with a significant higher mortality and ICU-admission rate and therefore represents a serious liability for patient safety and quality of care.

\section{Reference(s)}

1. 4. Hillman KM, Bristow PJ, Chey T, Daffurn K, Jacques T, Norman SL, et al. Antecedents to hospital deaths. Intern Med J. 2001:31(6):343-8.

2. 3. Goldhill DR, McNarry AF. Physiological abnormalities in early warning scores are related to mortality in adult inpatients. Br J Anaesth. 2004;92(6):882-4.

3. 2. Buist M, Bernard S, Nguyen TV, Moore G, Anderson J. Association between clinically abnormal observations and subsequent in-hospital mortality: a prospective study. Resuscitation. 2004;62(2):137-41.
4. 1. Devita MA, Bellomo R, Hillman K, Kellum J, Rotondi A, Teres D, et al. Findings of the first consensus conference on medical emergency teams. Crit Care Med. 2006:34(9):2463-78

5. The author(s) received no financial support for the research, and/or authorship.

001002

Underweight is associated with mortality in sepsis-A retrospective analysis of the eICU Collaborative Research Database

R. Rezar $^{1}$;T. Danninger ${ }^{2}$; V. Osmani ${ }^{3}$; B. Mamandipoor ${ }^{3}$; B. Wernly ${ }^{2}$

${ }^{1}$ Cardiology and Intensive Care, Paracelsus Medical University Salzburg,

Salzburg, Austria; ${ }^{2}$ Anesthesiology and intensive care, Paracelsus

Medical University Salzburg, Salzburg, Austria; ${ }^{3}$ E-health Research Group,

Fondazione Bruno Kessler Research Institute, Trento, Italy

Correspondence: R. Rezar

Intensive Care Medicine Experimental 2021, 9(1): 001002

Introduction. Once dubbed a disease of affluence, overweight is now on the rise in low- and middle-income countries, and in 2016 , the World Health Organization (WHO) reported 1.9 billion overweight adults worldwide, whereas $8.9 \%$ of all adults worldwide were listed as underweight. Different studies have attributed better outcomes to overweight individuals in the context of various illnesses such as sepsis or heart failure. This phenomenon is also known as the "obesity paradox". In this study, we investigated outcomes of septic patients from different body mass index (BMI) categories in the large, multicenter elCU Collaborative Research Database.

Methods. A total of 16,612 septic patients were included in this analysis from the multicenter elCU Collaborative Research Database. They were divided into four BMI categories (according to the WHO classification): (1) underweight $\left(<18.5 \mathrm{~kg} / \mathrm{m}^{2}\right)$, (2) normal weight $\left(18.5\right.$ to $\left.<25 \mathrm{~kg} / \mathrm{m}^{2}\right)$, (3) overweight $\left(25\right.$ to $\left.<30 \mathrm{~kg} / \mathrm{m}^{2}\right)$ and (4) obesity $\left(\geq 30 \mathrm{~kg} / \mathrm{m}^{2}\right.$ ). ICU mortality was used as the primary endpoint and intensive care measures as secondary outcomes. A multilevel regression analysis was performed, three models (1: ICU as random effect; 2 : baseline characteristics; 3: management strategies) were fitted. Furthermore, a stratified sensitivity analysis was performed.

Results. In total, $964(6 \%)$ patients were classified as underweighted, 4,402 (26\%) as overweighted and 6,027 (36\%) as obese. Furthermore $5,219(31 \%)$ patients were of normal weight. Obese patients were younger $(64+19$ yrs. vs. $68+23$ yrs.; $p=0.001)$, but evidenced SOFA-scores $>10$ pts. more often $(14 \%$ vs $12 \%$; $p<0.001)$. Underweight patients showed serum lactate concentrations $>2.0 \mathrm{mmol} / \mathrm{I}$ ( $47 \%$ vs. $44 \%$ in normal weight patients) more often and received higher amounts of fluid per kg bodyweight $(>30 \mathrm{ml} / \mathrm{kg} / \mathrm{h}$ in the first $24 \mathrm{~h}$. after admission; $\mathrm{p}<0.001)$. Furthermore, underweight patients evidenced higher ICU mortality rates. This finding remained significant after adjustment for all three models (model 2: aOR $1.5495 \% \mathrm{Cl}$ 1.15-2.06; $p=0.004$; model 3: aOR $1.5795 \% \mathrm{Cl} 1.16-2.12 ; \mathrm{p}=0.003$ ). No difference was shown between normal weight and overweighted patients, whereas a lower risk for ICU mortality was observed in obese patients. This finding remained significant after adjustment for all three models (model 3: aOR 0.82 95\% Cl 0.68-0.98; $p=0.03$ ).

Conclusion. Our analysis demonstrates a better outcome in obese patients with sepsis, whereas underweight patients evidenced a higher mortality. This study supports the thesis of the "obesity paradox" and should be thesis generating for further analyses regarding underweight as a prognostic factor in critically ill patients.

\section{5}

Provision of critical care for the elderly with COVID-19 in Europe-a retrospective comparison of national healthcare frameworks in intensive care units

B. Wernly ; M. Beil ${ }^{2} ;$ RR. Bruno ${ }^{3}$; M. Kelm³; S. Leaver ${ }^{4}$; W. Szczeklik $;$ J. Fjølner ${ }^{6}$; S. Sigal ${ }^{7}$; P. Vernon Van Heerden ${ }^{8}$; D. De Lange ; B. Guidet ${ }^{10} ; H$. Flaaten $^{11}$; C. Jung ${ }^{12}$

${ }^{1}$ Department of Anaesthesiology, Perioperative Medicine and Intensive Care Medicine, Paracelsus Medical University, Salzburg, Austria;

${ }^{2}$ Medical intensive care unit, Hadassah Medical Center, London, 
United Kingdom; ${ }^{3}$ Cardiology, Pulmonary Diseases, Vascular Medicine, Universität Düsseldorf, Düsseldorf, Germany; ${ }^{4}$ Research Lead Critical Care Directorate, St George's Hospital, London, United Kingdom; ${ }^{5}$ ntensive Care and Perioperative Medicine, Jagiellonian University Medical College, Kraków, Poland; ${ }^{6}$ Department of Intensive Care, Aarhus University Hospital, Aarhus, Denmark; ${ }^{7}$ Medical Intensive Care unit, Hadassah Medical Center, Jerusalem, Israel, Israel; ${ }^{8}$ Medical Intensive Care Unit, Hadassah Medical Center, Jerusalem, Israel; ${ }^{9}$ Department of Intensive Care Medicine, Utrecht University, Utrecht, Netherlands; ${ }^{10}$ Réanimation Médicale, Hôpital Saint-Antoine, Paris, France; ${ }^{11}$ Department

of Anaestesia and Intensive Care, University of Bergen, Bergen, Norway; ${ }^{12}$ Dep. of Cardiology, Pulmonology and Angiology, University Hospital of Düsseldorf, Düsseldorf, Germany

Correspondence: B. Wernly

Intensive Care Medicine Experimental 2021, 9(1): 001005

Introduction. A rough distinction can be made between two health care systems in Europe, the tax-based healthcare system (THS) and the social health insurance system (SHI). This study sought to evaluate potential differences in baseline risk distribution, management, and outcome of older critically ill COVID-19 patients between the two systems. Methods. In total, 2338 critically ill older ( ${ }^{3} 70$ years) patients were included in this study, 878 in THS and 1460 in the SHI systems. Frailty was assessed using the Clinical Frailty Scale (CFS). We chose generalised estimation equations (GEE) with robust standard errors to produce population average adjusted odds ratios (aOR). We adjusted for patient-specific variables, health economic data and treatment limitations. The primary outcome was 30 -day-mortality.

Results. The patients were slightly older in the SHI ( $75 \pm 6$ vs. $74 \pm 6$ years; $p=0.03)$, evidenced higher SOFA scores ( $5 \pm 5$ vs. $4 \pm 4$; $p=0.008$ ) and were more often frail (CFS $>5 ; 15 \%$ vs. $13 \% ; P=0.04)$. We found higher rates of renal replacement therapy (18\% vs $11 \% ; p<0.001)$ in $\mathrm{SHI}$. However, neither we did not detect any differences in intubation rates $(70 \%$ vs $72 \% ; p=0.37)$, vasopressor use $(69 \%$ vs. $69 \% ; p=0.94)$ nor in 30 -day-mortality rates ( $47 \%$ vs. $50 \%$; aOR 1.26 ; $95 \% \mathrm{Cl} 0.82-1.93$; $\mathrm{p}=0.30$ ). Mortality remained the same between $\mathrm{SHI}$ and THS even after multivariable adjustment and in extensive sensitivity analyses.

Conclusion. Baseline risk distribution was similar, and we could not detect any indirect signals for differences in ICU admission criteria between SHI and THS. Also, the mortality rates were similar in both systems at all timepoints. During the COVID-19 pandemic, the macroscopic organization of the health care system does not appear to have played a role in the intensive care treatment of the elderly in Europe.

\section{Reference(s)}

1. Corona Virus disease (COVID19) in Very Elderly Intensive care Patients (VIPs). A VIP network study.

\section{0}

No ethnic disparities in critically ill septic patients in the multi-center eICU Collaborative Research Database

A. Koköfer'; B. Mamandipoor ${ }^{2}$; V. Osmani' ${ }^{2}$ B. Wernly ${ }^{1}$

${ }^{1}$ Department of Anaesthesiology, Perioperative Medicine and Intensive Care Medicine, Paracelsus Medical University, Salzburg, Austria; ${ }^{2} \mathrm{E}-\mathrm{Health}$ Research Group, Fondazione Bruno Kessler Research Institute, Trento, Italy Correspondence: B. Wernly

Intensive Care Medicine Experimental 2021, 9(1): 001010

Introduction. Previous studies suggested racial disparities in sepsis. This study sought to evaluate the impact of ethnic background on outcomes and management of critically ill septic patients.

Methods. This analysis included 17,146 patients with sepsis of the multi-center elCU Collaborative Research Database, Caucasian $(n=13,336)$, African American $(n=1807)$, Hispanic $(n=663)$ and others $(n=1340)$. We chose generalized estimated equation (GEE) population-averaged models to fit three sequential regression models for the binary primary outcome ICU mortality.

Results. Caucasian patients had the highest proportion of elderly (>65 years; 55\%) patients compared to African American (39\%; $p<0.001)$, Hispanic (51\%; $p=0.03)$ and others $(46 \% ; p<0.001)$.
The mortality rates were similar in Caucasian (10\%) compared to African American (10\%; aOR 0.98 95\% Cl 0.82-1.16), Hispanic (9\%; aOR 1.06 $95 \% \mathrm{Cl} 0.80-1.41)$ and others (11\%; aOR $1.1295 \% \mathrm{Cl} 0.92-1.36)$. This finding persisted after adjustment for patient and hospital level variables, as well as in sensitivity analyses for patients with septic shock, sex, age above 65 and treatment in teaching versus non-teaching hospitals.

Conclusion. We could not detect any racial disparities in the management and outcomes of critically ill septic patients in this cohort.

\section{7}

Safety and Efficacy of a Post Anaesthetic Care Unit as an alternative to Critical Care during the Covid-19 Pandemic R. Loveless' ; S. Brown ${ }^{1}$; E. Mathers ${ }^{1}$

${ }^{1}$ Anaesthetic Department, Royal Surrey County Hospital, Guildford, United Kingdom

Correspondence: R. Loveless

Intensive Care Medicine Experimental 2021, 9(1): 001027

Introduction. Royal Surrey County Hospital is a Regional Cancer Centre in Southern England. The Covid-19 pandemic required hospital trusts in England to reduce their elective surgical activity to re-allocate resources to Intensive Care. We sought to continue urgent cancer operating and this required provision of level 2 critical care capacity that the Intensive Care Unit was unable to provide. The existing Post Anaesthetic Care Unit (PACU) was repurposed, and existing PACU staff were given critical care training and protocols. Exclusion criteria for admission to PACU were ongoing mechanical ventilation, requirement for renal replacement therapy, or significant cardiovascular failure requiring a second agent (eg dobutamine). Procedures admitted included complex gynae-oncology laparotomy, Robotic cystectomy, liver resection, partial gastrectomy, Whipple's procedure and distal pancreatectomy. A total of 110 patients underwent a cancer procedure requiring post-operative critical care during the second wave of Covid-19 in January and February 2021. Here we compare length of stay, incidence of complications and pain scores to a similar cohort of patients who were admitted to the Intensive Care Unit prior to the pandemic.

Methods. We collected data using the electronic patient record system, ICCA(TM-Phillips) identifying all patients admitted to PACU level 2 during January to March 2021. The data collected was age, procedure, length of stay, maximum pain score, mode of analgesia, ASA score and incidence of complications. Length of stay was deemed to end when the patient was identified as fit for discharge. Pain score was graded 0-3. ASA score was calculated. This data was then compared with a second group of patients who had undergone matched procedures in October to December 2019 and were admitted to ICU post-operatively. To demonstrate equity between these two patient pathways we compared the length of critical care stay as a primary outcome, and then compared pain scores and rate of complications as secondary outcomes. We excluded the most complex cases from both groups (Robotic cystectomy, liver resection $>3$ segments and Whipples) as these patients would not be suitable for an overnight intensive recovery after the pandemic.

Results. 55 patients were included in the PACU group over three months. 25 matched cases were identified in the ICU group over a prepandemic three month period. Mean length of stay was lower in the PACU group (1.43 days) than the ICU group (2.13 days). Incidence of complications (prolonged hypotension, severe pain, requirement for blood transfusion, severe electrolyte abnormalities) was 20\% in PACU group compared with $43 \%$ in the ICU group. The groups were similar in mean age (PACU: 64 years, ICU: 60 years) and ASA grade (71\% ASA 2 in PACU group, $79 \%$ in ICU group). The PACU group had a lower proportion of epidurals (36\% vs $67 \%$ ) and pain scores were higher in the PACU group (51\% at least 1 episode of moderate or severe pain vs $42 \%$ in ICU group), but this was not statistically significant $(p=0.45$, Chi square test).

Conclusion. Our data shows that during the Covid-19 pandemic, our Post Anaesthetic Care Unit was able to deliver level 2 care safely and effectively, with lower mean length of critical care stay and a lower 
incidence of complications than a similar group of patients admitted to Critical Care prior to the pandemic. It should be noted that the use of epidurals for analgesia was higher in the ICU group, and could lead to both increased hypotension and prolonged length of stay. A similar model could be implemented in future to provide safe level 2 care following specific procedures to alleviate pressures on Critical Care capacity.

\section{Reference(s)}

1. Office for National Statistics website. https://www.ons.gov.uk/peopl epopulationandcommunity/healthandsocialcare/conditionsanddiseases/ articles/coronaviruscovid19/latestinsights\#hospitalisations

2. Royal Surrey NHS Foundation Trust website. https://www.royalsurrey.nhs. uk/about-us.

\section{9}

Use of Non Vented BAIN Circuit in Refractory Hypoxic COVID Patients With NRBM and HFNO in Resource Poor Set Up SC. dutta ${ }^{1}$

${ }^{1}$ hod Medicine and Crit Care, St. Ann Hospital, VISAKHAPATNAM, India Correspondence: S.C. dutta

Intensive Care Medicine Experimental 2021, 9(1): 001029

Introduction. In resource poor hospitalised severe covid patients due to critical scarcity of oxygen in this part of world we have been working on different oxygen preserving modalities without compromising patient's oxygenation status.We found that modified conventional Bain circuit with amodified BIPAP MASK eith APL Valve for release of patient's expired air and oxygenation through BAIN has saved many precious lives whose hypoxia was refractory with HFNO and NRBM.

Objectives. To find out effectiveness of Bain circuit with BIPAP Mask is a better option in hospitalised severe and critical covid patients who are on HFNO or NRBM and having refractory hypoxia despite very high oxygen flow and proning.

Methods. From 15 APR 2021 to 14 May 2021 in our state government cont covrolled covid hospital 15 severe and critical covid patients were studied. These patients were on NRBM initially and subsequently shifted to HFNO as satisfactory oxygen levels were not achieved despite maximum $\mathrm{FiO}_{2}$ /Proning and medical therapy including IV Methyl prednisolone as well as Remdesivir. Then they were subjected to BAIN CIRCUIT with a modified BIPAP Mask with APL Valve at patient end.Drastic improvement was seen from hour onwards in term of $\mathrm{SPO}_{2}$, Respiratory rate reduction, Decreased oxygen requirement to maintain satisfactory $\mathrm{SPO}_{2}$ Level and significant increased recovery and discharge.

Results. Out of our cohort of 15 severe and refractory hypoxic patient despite protocolized NRBM AND HFNO with very high Oxygen requirement and deteriorating clinically when changed over to Bain circuit with modified face niv mask, 11 patients recovered smoothly and we lost only 4 patients as they had critical covid and severe comorbid conditions. $\mathrm{CO}_{2}$ retention as also not raised in patients with Bain circuit ventilation with bipap mask.

Conclusion. Modified Bains circuit with modified Bipap mask with APL valve near patient end should be the firsr line of choice for severe and ctitical covid hospitalized patient rather than NRBM AND HFNO as the previous one saves huge amount of oxygen, easy to use, cheap (appx cost 10 Dollar total set cost) and suprior outcome than later two methods.

\section{1}

\section{Factors affecting health related quality of life in intensive care} survivors four months after discharge

I. Soultati ${ }^{1}$; G. Manomenidis ${ }^{2}$; T. Bellali ${ }^{3}$

${ }^{1}$ Anesthesiologist_-Intensivist of ICU, "Ahepa" University General

Hospital and Hellenic Open University, Thessaloniki, Greece; ${ }^{2}$ Chief nurse of internal medicine, "Bodosakeio" General Hospital, Ptolemaida, Greece;

${ }^{3}$ Professor of Nursing Department, International Hellenic University and Hellenic Open University, Thessaloniki, Greece

Correspondence: I. Soultati

Intensive Care Medicine Experimental 2021, 9(1): 001051
Introduction. Intensive care (IC) development has managed to reach the goal of reduced mortality. However, the real aim of IC is to help patients return to their previous state of health, which is not always accomplished due to complications related to patient's stay in the intensive care unit (ICU).

Objectives. To explore the factors that affect health related quality of life in intensive care survivors four months after discharge from ICU.

Methods. A cross-sectional study was conducted in a University Hospital in Greece. Quality of care was assessed four months after patients' discharge from the ICU. Data was collected by a completion of battery of questionnaires after telephonic interviews, which included patients demographic characteristics and mortality predictors of ICU, the Medical Outcomes Study Health Survey-Short Form (SF-12) Questionnaire, the Numeric Pain Rating Scale (NRPS) and the Short Form Social Support Questionnaire, (SSQ-6).

Results. 40 patients, 16 women and 12 men, with Glasgow Coma Scale (GCS) 10.67, SOFA score 2.87 and APACHE II score 12, were recruited. Participants reported good levels of physical and mental state (SF12 subscales), $42.18( \pm 12.1)$ and 40.13 ( \pm 12.82$)$, respectively Patients with better level of QOL were active employees $(b=-0.426,95 \%$ confidence interval $[\mathrm{CI}] 0.012,0.003)$, had higher $\mathrm{GOS}(b=0.361,95 \%$ confidence interval $[\mathrm{Cl}] 0.017,0.009)$ and lower levels of NRPS $(b=-0.378$ $95 \%$ confidence interval $[\mathrm{Cl}] 0.015,0.012$ ) during their stay in ICU. Participants' better quality of physical state was associated with marital status (married) $(p=0.042)$, professional status (active employee) $(p=0.037)$ and low levels of pain in ICU $(p=0.003)$. Participants' better quality of mental state was associated with GOS $(p=0.033)$, and social support $(p=0.002)$.

Conclusion. Findings provide information about the factors that affect ICU survivors' QoL, i.e. active employment after ICU discharge, high level of consciousness and low level of pain in the ICU. Early treatment of these factors might have a positive effect on outcomes related to QoL. To this direction, a future longitudinal study exploring the impact of these factors 12 months after patients' discharge from ICU, might be especially useful for health professionals in addressing their patients' needs.

\section{Reference(s)}

1. Zeggwagh, Z., Abidi, K., Kettani, M. N., Iraqi, A., Dendane, T., \& Zeggwagh, A. A. (2020). Health-related Quality of Life Evaluated by MOS SF-36 in the Elderly Patients 1 Month before ICU Admission and 3 Months after ICU Discharge. Indian journal of critical care medicine: peer-reviewed, official publication of Indian Society of Critical Care Medicine, 24(7), 531-538.

2. Kyösti, E., Ala-Kokko, T.l., Ohtonen, P. et al. (2018). Factors associated with health-related quality of life 6 years after ICU discharge in a Finnish paediatric population: a cohort study. Intensive Care Med 44, 1378-1387.

3. Alfheim H.B., Cvancarova M., Hofsø K., Tøien K., Rosseland L.A., Tone Rustøen T., (2018). Quality of life in family caregivers of patients in the intensive care unit: A longitudinal study. PLoS One. 2018; 13(6): e0199656.

\section{9}

Predictors of mortality in COVID-19 patients admitted to ECMO

ICU

H. Asogan ${ }^{1}$; A. Kalyanasundaram²; N. Lin Myint

${ }^{1}$ Intensive care unit, Royal Papworth Hospital, Cambridge, United Kingdom; ${ }^{2}$ Cardiology, Royal Papworth Hospital, Cambridge, United Kingdom

Correspondence: $\mathrm{H}$. Asogan

Intensive Care Medicine Experimental 2021, 9(1): 001059

Introduction. The coronavirus disease 2019 (COVID-19) pandemic has caused significant mortality and morbidity worldwide.

The predominant clinical manifestation is viral pneumonia, which can progress into ARDS, but it can also cause cardiovascular complications. In view of these presentations, extracorporeal membrane oxygenation (ECMO) has emerged as a therapeutic intervention for those with severe disease.

Previous studies have shown the risk factors for mortality include age, male sex, deranged cardiac and inflammatory biomarkers, and multiple co-morbidities. In this study, we investigated the predictors of 
mortality in COVID positive patients admitted to Intensive Care Unit in the Royal Papworth Hospital (RPH), one of the largest COVID ECMO centres in the United Kingdom.

Objectives. To assess the predictive value of biomarkers, ECMO use, and co-morbidities on mortality in COVID positive patients admitted to the RPHICU.

Methods. 197 patients were admitted to the ICU with a PCR-confirmed diagnosis of COVID-19 between 1st March 2020 and 3rd February 2021. Their health records were interrogated for demographics, co-morbidities, admission blood test results, and ECMO use. Binary logistical regression models were created using the $\mathrm{R}$ programming language to assess the prognostic value of individual variables and combinations of variables on the mortality.

Results. The mean age was 52.3 years with $70.6 \%$ being male and $61.7 \%$ white. $50.8 \%$ were admitted during the first wave (prior to $1 \mathrm{st}$ October 2020) and $45 \%$ were on ECMO for an average of 36.2 days. On univariate analysis, only a high NT-pro BNP $(p=0.0092)$ and older age $(p=0.0101)$ were significant predictors of mortality. The mean BNP level was $1572 \mathrm{pg} / \mathrm{mL}$ in those who survived vs. $5622 \mathrm{pg} / \mathrm{mL}$ in those who died, and the mean age of those who survived was 51.2 vs 56.6 in those who died. No co-morbidities, other admission blood results, or ECMO usage independently predicted mortality.

On multivariate analysis of blood markers alone, the best predictors of mortality (defined by the lowest Akaike Information Criterion) were high BNP and CRP together, though the BNP level was the only component that was statistically significant within this model $(p=0.00738)$. When ECMO data was included in the analysis, the best predictors were high BNP, high repeat high-sensitivity troponin-I (within $24 \mathrm{~h}$ of admission), high lymphocytes, and long ECMO duration, though no factors were individually significant within the model. Finally, if co-morbidities were also included, the best model used smoking, obesity, asthma, white race, high D-dimer, high repeat troponin, and high BNP to predict mortality, though no factors were individually significant within the model.

Conclusion. NT- proBNP is strongly and independently associated with mortality. Therefore, using it as a marker for severity may improve early prognostic stratification for COVID-19 ICU patients.

\section{Reference(s) \\ 1. None.}

\section{3}

Stress Levels among healthcare professionals during COVID 19 crisis response in a tertiary Cardiothoracic unit

H. Ahmed'; M. Osman 2. L. Mwaura, ${ }^{3}$ : B. Ripoll,2. S. George, ${ }^{4}$

${ }^{1}$ Anaesthesia and Critical Care, Faculty of Medicine, Kasr Alainy Cairo University, Cairo, Egypt; ${ }^{2}$ Surgery and transplant, Royal Papworth NHS Foundation Trust, Cambridge, United Kingdom; ${ }^{3}$ Anaesthesia, Oxford NHS Foundation Trust, Oxford, United Kingdom; ${ }^{4}$ Anaesthesia and Critical Care, Royal Papworth NHS Foundation Trust, Cambridge, United Kingdom

Correspondence: $\mathrm{H}$. Ahmed

Intensive Care Medicine Experimental 2021, 9(1): 001063

Introduction. The World Health Organization declared a pandemic of COVID 19 virus in March 2020. Health care professionals experienced a challenging time due to increased workload, fear of infection transmission and working outside their scope of practice.

High stress levels negatively affect healthcare professional's wellbeing but could affect patient safety and lowers the level of care. Hence, we wished to consider the psychological burden of COVID 19 pandemic on healthcare professionals.

Objectives. The aim of this survey was to assess the stress levels among healthcare professionals during this crisis using a previously validated tool "PERCEIVED STRESS SCALE".

Methods. The Royal Papworth hospital is a tertiary unit that responded to the crisis by providing a surge increase in capacity of Critical care beds (70\% increase in capacity from 46 to 65 ) and delivery of ECMO care (500\% increase from 4 to 21 beds).

Perceived stress scale (PSS) is a classic stress assessment instrument, originally developed in 1983, which has been previously validated.
Healthcare professionals in Royal Papworth Hospital, including doctors and nurses, were invited to participate via email and participation was voluntary. The surge practice of enforced changes in work patterns and roles was introduced on 6th April 2020 and the study was conducted between $27 / 04 / 2020$ and $26 / 05 / 2020$. They were asked to complete PSS, in addition to completion of basic demographic data age, sex, race and experience.

Results. A total 306 health professional (117 doctors and 189 of nurses) who were working regularly in either the critical care unit or theatres took part in the survey. Perceived Stress Scale (PSS) scores ranged between 0 and 40, mean was 19 and SD 7.13\% of participants reported high stress levels (27-40), 70\% reported moderate stress levels (13-26), while $17 \%$ reported low stress levels $(0-13)$.

Females reported higher PSS compared to males 19(3) and 17(7) respectively ( $p$ 0.042). Young participants (18-34) reported a higher PSS when compared to older participants (35-54) and +55 years old $20(6), 18(6)$ and $16(6), p<0.05$. Those of Asian race reported the highest PSS followed by black race then white race 22(6), 20(4) and 18(7), $\mathrm{p}<0.05$.

Interestingly, nurses reported a statistically significant higher perceived stress levels when compared to doctors $20(6)$ vs $17(6), p<0.05$ In addition, race showed a significant correlation with PSS ( $p 0.002)$.

Conclusion. COVID 19 pandemic resulted in high stress levels among healthcare professionals and might affect their well-being. Females, young age groups and certain races experienced higher stress levels in comparison with their colleagues.

\section{Reference(s)}

1. Roberti JW, Harrington LN, Storch EA: Further Psychometric Support for the 10-Item Version of the Perceived Stress Scale. [Internet]]. Coll. Couns. [Internet] John Wiley \& Sons, Ltd, 9:135-47, 2006 [cited 2020] Retrieved from: $h$ ttp://doi.wiley.com/10.1002/j.2161-1882.2006.tb00100.x

2. Andreou E, Alexopoulos EC, Lionis C, et al: Perceived Stress Scale: Reliability and Validity Study in Greece. [Internet]Int. J. Environ. Res. Public Health [Internet] Molecular Diversity Preservation International, 8:3287-98, 2011 [cited 2020]Retrieved from: http://www.mdpi.com/1660-4601/8/8/3287.

3. Sharp LK, Kimmel LG, Kee R, et al.: Assessing the Perceived Stress Scale for African American Adults with Asthma and Low Literacy. [Internet]J. Asthma [Internet] Taylor \& Francis, 44:311-6, 2007 [cited 2020] Retrieved from: http://www.tandfonline.com/doi/full/10.1080/027709007013441 65.

4. Wongpakaran N, Wongpakaran T: The Thai version of the PSS-10: An Investigation of its psychometric properties. 2010.

5. A Global Measure of Perceived Stress Author ( $\mathrm{s}$ ): Sheldon Cohen, Tom Kamarck and Robin Mermelstein Source: Journal of Health and Social Behavior, Vol. 24, No. 4 ( Dec., 1983), pp. 385-396 Published by: American Sociological Association Stable U. 24:385-96, 1983.

6. Hall LH, Johnson J, Watt I, et al.: Healthcare Staff Wellbeing, Burnout, and Patient Safety: A Systematic Review. [Internet]Harris F, ed. (ed): PLoS One [Internet] Public Library of Science, 11:e0159015, 2016 [cited 2020] Retrieved from: https://dx.plos.org/10.1371/journal.pone.0159015.

\section{0}

The introduction of an emergency algorithm folder to reduce cognitive overload and improve teamwork and non-technical skills in an Intensive Care Unit

L. Guscoth ${ }^{1}$; K. Goodchild, ${ }^{1}$; F. Kelly,

${ }^{1}$ Intensive Care Unit, Royal United Hospitals Bath, BATH, United kingdom, United Kingdom

Correspondence: L. Guscoth

Intensive Care Medicine Experimental 2021, 9(1): 001070

Introduction. The management of emergency scenarios requires prompt action, leadership and effective teamwork with clear and concise communication. Decision making in emergency scenarios can be hampered by environmental and organisational factors and cognitive overload [1-3]. Emergency scenario communication has been further challenged by the physical and mental strain of the COVID-19 pandemic [4]. Cognitive aids have been shown to improve clinician performance [3] and improve non-technical skills $[5,6]$. Reducing the mental workload of recalling an 
emergency algorithm sequence may ensure that all critical steps are followed, enabling more focus on team function $[5,6]$. We proposed that a folder of emergency algorithms, readily available in our Intensive Care Unit (ICU), may enhance the running of emergency scenarios.

Objectives. We aimed to introduce an ICU algorithm folder for use in emergency scenarios, design and deliver an education programme and measure the effectiveness of this teaching in improving staff awareness of decision-making aids and adherence to emergency algorithms.

Methods. We designed and developed a 12-tabbed emergency algorithm folder including national and local algorithms, divided into clear categories for ease of use and located in an easily accessible central ICU location. We conducted 'tea trolley teaching' [7] to educate staff with the location and folder content and collect feedback regarding content and method of use. We designed a questionnaire to assess the perceived impact of the algorithm folder for emergency scenarios and evaluate confidence in locating and using the algorithms following teaching, including close-ended questions (Yes/No), Likert 5-point Scale (Always-Never) questions and free text.

Results. Thirty-seven multidisciplinary (MDT) ICU team members received teaching (8 doctors, 17 nurses, 1 ACCP, 2 physiotherapists, 9 others). Questionnaire response rate $37 / 37$ (100\%). Results are described in Table 1. Confidence in locating the emergency algorithm folder on the ICU improved from 32 to $96 \%$ following teaching and $100 \%$ of staff would recommend the initiative to other units. Table 1:

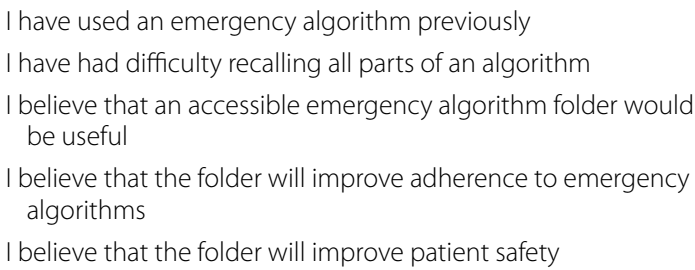

I have had difficulty recalling all parts of an algorithm

I believe that an accessible emergency algorithm folder would be useful

I believe that the folder will improve adherence to emergency algorithms

I believe that the folder will improve patient safety

I believe that the folder will improve performance in an emergency

$86 \%$

$98 \%$

$100 \%$

Conclusion. Our findings demonstrate that an ICU emergency algorithm folder could improve recall, performance and adherence to algorithms in emergency scenarios and could improve patient safety. Tea trolley teaching was shown to be an effective method of educating the wider multidisciplinary team and raised awareness of the use of emergency algorithms. We now plan to trial the emergency algorithm folder in MDT simulation scenario training and are investigating digital options for the folder.

\section{Reference(s)}

1. Not applicable.

2. 1. Edelman DA, Perkins EJ, Brewster DJ. Difficult airway management algorithms: a directed review. Anaesthesia. 2019 Sep;74(9):1175-1185.

3. 2. Cook TM, Woodall N, Frerk C; Fourth National Audit Project. Major complications of airway management in the UK: results of the Fourth National Audit Project of the Royal College of Anaesthetists and the Difficult Airway Society. Part 1: anaesthesia. Br J Anaesth. 2011 May;106(5):617-31

4. 3. Low D, Clark N, Soar J, Padkin A, Stoneham A, Perkins GD, Nolan J. A randomised control trial to determine if use of the iResus@ application on a smart phone improves the performance of an advanced life support provider in a simulated medical emergency. Anaesthesia. 2011 Apr;66(4):255-62.

5. 4. Coughlan C, Nafde C, Khodatars S, Jeanes AL, Habib S, Donaldson E, Besi C, Kooner GK. COVID-19: lessons for junior doctors redeployed to critical care. Postgrad Med J. 2021 Mar;97(1145):188-191.

6. 5. Marshall SD, Mehra R. The effects of a displayed cognitive aid on non-technical skills in a simulated 'can't intubate, can't oxygenate' crisis. Anaesthesia. 2014 Jul;69(7):669-77.

7. 6. Neal JM, Hsiung RL, Mulroy MF, Halpern BB, Dragnich AD, Slee AE. ASRA checklist improves trainee performance during a simulated episode of local anesthetic systemic toxicity. Reg Anesth Pain Med. 2012 Jan-Feb;37(1):8-15.

8. 7. O'Farrell G, McDonald M, Kelly FE. 'Tea trolley' difficult airway training. Anaesthesia. 2015 Jan:70(1):104
001072

The impact of age on covid 19 patients

A. Meftah, ; Y. Touil, ${ }^{2}$; E. Abid, ${ }^{3}$; S. Ben Nessira³ ; F. Daly, ${ }^{2}$; A. Trifi²; S.

Abdellatif,; LS. Ben²

${ }^{1}$ Hospital la rabta, Intensive Care Unit, Tunis, Tunisia; ${ }^{2}$ nntensive Care

Unit, Hospital la Rabta, Tunis, Tunisia; ${ }^{3}$ Hospital la rabta, intensive care medecine, Tunis, Tunisia

Correspondence: $Y$. Touil

Intensive Care Medicine Experimental 2021, 9(1): 001072

Introduction. As the number of covid-19 patients has risen rapidly throughout the world, it has become essential to identify the risk factors associated to the severe forms.

The ageing is associated with certain changes in pulmonary physiology, pathology and function.

Objectives. To identify the age-related impact on covid-19 patients.

Methods. A retrospective observational study was conducted in the ICU at a university hospital in Tunis (Tunisia).

Patients diagnosed with covid-19 (determined by a positive PCR result for SARS-cov-2) and admitted to the intensive care unit for respiratory distress were included.

They were divided in two groups taking age 60 years as the threshold. Data on demographics, biological tests, different therapies and outcomes were analyzed in this study.

Results. Forty-nine patients were included. Twenty patients (40.8\%) were aged $<60$ years and the other 29 cases $(59.18 \%)$ were aged $>=60$ years. There was no significant difference in BMI $(p<0.88)$.

Compared with the younger group, patients aged $>=60$ years had significantly lower $\mathrm{PaO}_{2} / \mathrm{FiO}_{2}(66$ versus $96, \mathrm{p}<0.05)$ and higher CRP levels ( 200 versus $123, \mathrm{p}<0,003$ ).

There was no significant difference in the lymphocyte counts $(p=1)$, nor in the lactate dehydrogenase levels $(p=0.94)$ between the two groups.

As for the therapies, the proportion of patients requiring endo-tracheal intubation was significantly higher in patients aged $>=60$ years than in patients aged $<60$ years ( 22 versus $10, p<0,022$ ).

Concerning the outcomes, the mortality rate was greater in the agedgroup than in the other group and the difference was statistically significant (22 versus $10, \mathrm{p}<0,022$ ).

Conclusion. The prognosis of this disease varies with age.

A thorough assessment of age may help clinicians to establish riskstratification for all covid-19 patients.

\section{6}

\section{Post-ICU COVID-19 Long Term Outcomes}

L. Alegria Vargas ${ }^{1}$; P. Garcia²; M. Henriquez ${ }^{3}$; P. Repetto ${ }^{3}$; R. Severino ${ }^{1}$

${ }^{1}$ Departamento de medicina intensiva, Pontificia Universidad Católica

de Chile, Santiago, Chile; ${ }^{2}$ Escuela de kinesiología, Pontificia Universidad

Católica de Chile, Santiago, Chile; ${ }^{3}$ Escuela de psicología, Pontificia

Universidad Católica de Chile, Santiago, Chile

Correspondence: L. Alegria Vargas

Intensive Care Medicine Experimental 2021, 9(1): 001076

Introduction. Patients critically ill with coronavirus disease 2019 (COVID-19) spend significant time on mechanical ventilation and have prolonged hospitalization duration (1)(2). Prior studies of patient survivorship after an intensive care unit (ICU) stay suggest that many critically ill patients with COVID-19 will face long-lasting physical, cognitive and/or mental health impairments (3)(4)(5).

Objectives. The main purpose of this study is to observe and describe physical, psychological and cognitive sequelae at 3 and 6 months post discharge in patients admitted with respiratory failure due to Covid-19 to a polyvalent ICU of a health center.

Methods. A prospective, observational study, carried out in the Intensive Care Unit at Hospital Clínico UC-CHRISTUS, between May and August 2020. Patients with severe COVID-19 and mechanical ventilation for over $48 \mathrm{~h}$. were admitted and underwent follow-up approximately at 3 and 6 months following discharge. Post COVID-19 functional status, anxiety and depression symptoms, and cognitive function were assessed with validated scales. 
Results. A total of 27 patients completed the follow-up. The median age was 58 years and the median number of days on mechanical ventilation was 20 days. The mean Sequential Organ Failure Score and the Acute Physiology were $5.2 \pm 2.5$ and $11 \pm 7.1$, respectively. Median ICU and hospital length of stay were 26 days and 36 days, respectively. At follow-up at 3 months, $37.5 \%$ of patients experience mild functional limitation, and $25 \%$ have no functional limitations, while at 6 months, $31 \%$ of patients experiment undetectable functional limitations and $68 \%$ have no functional limitations. One of the patients required supplemental oxygen. None of the patients had clinically diagnosed depression, or cognitive impairment prior to admission. At follow-up at 3 months, 9 patients had moderate to severe anxiety symptoms and $45 \%$ experimented moderate depression symptoms while at 6 months, $25 \%$ of patients had severe anxiety symptoms and $60 \%$ had no depression symptoms. Five patients had mild cognitive impairment at 3 months of follow-up, which was maintained at 6 months post-ICU discharge.

Conclusion. This was a single center case series. The long-term impact of this disease remains unclear, but our study suggests a high prevalence of physical dysfunction at ICU discharge, which recovers over time, and a low burden of neurocognitive morbidity at long-term follow-up. The high prevalence of symptoms of anxiety and depression that persists high in long-term follow-up is worrisome.

\section{Reference(s)}

1. Ramani Ch. Post-ICU COVID-19 Outcomes. Serie cases. 2019;215-8.

2. Biehl M, Sese D. Post-intensive care syndrome and COVID-19—Implications post pandemic. Cleve Clin J Med. 2020;19-21.

3. Guan WJ, Ni ZY, Hu Y, Liang WH, Ou CQ, He JX, et al. Clinical Characteristics of Coronavirus Disease 2019 in China. N Engl J Med. 2020;323(16):1545-6.

4. Grasselli G, Zangrillo A, Zanella A, Antonelli M, Cabrini L, Castelli A, et al. Baseline Characteristics and Outcomes of 1591 Patients Infected with SARS-CoV-2 Admitted to ICUs of the Lombardy Region, Italy. JAMAAm Med Assoc. 2020;1-8.

\section{8}

Clinical and laboratory factors predicting the prognosis of patients with COVID-19

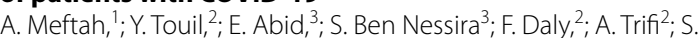
Abdellatif, ${ }^{2} ;$ LS. Ben ${ }^{2}$

${ }^{1}$ Hospital la rabta, Intensive Care Unit, Tunis, Tunisia; ${ }^{2}$ Intensive Care Unit, Hospital la Rabta, Tunis, Tunisia; ${ }^{3}$ Hospital la rabta, Intensive Care Medecine, Tunis, Tunisia

Correspondence: Y. Touil

Intensive Care Medicine Experimental 2021, 9(1): 001078

Introduction. Covid-19 infection is an emergent disease. Nowadays, patients' prognosis is a debate subject and predictive mortality factors still aren't clearly identified. Therefore, epidemiological, clinical and laboratory characteristics are important to analyse in intensive care unit.Covid-19 infection is an emergent disease. Nowadays, patients prognosis is a debate subject and predictive mortality factors still aren't clearly identified. Therefore, epidemiological, clinical and laboratory characteristics are important to analyse in intensive care unit.

Objectives. To evaluate the predictive factors of mortality in critically ill COVID-19 patients.

Methods. This retrospective study was conducted in ICU of Rabta Universitary Hospital of Tunis, Tunisia between September 2020 to Mars 2021.

One hundred patients diagnosed with covid-19 (determined by a positive PCR result for SARS-cov-2) were included.

They were divided into two groups: survivors and no-survivors.

Data of demographics parameters, clinical characteristics, laboratory tests and outcomes were collected.

Results. There were 60 patients $(60 \%)$ in the no-survivors group and 40 patients (40\%) in the survivors group.

No-survivors were older than the survivors (64 years [56-72] versus 57 years [49-66]) but the difference wasn't significant $(p=0,1)$. Death rate was higher within females (78\%) then males (58\%).

No-survivors had more comorbidities $46(76.7 \%)$ without significant difference $(p=0.042)$. Body mass index (BMI) wasn't associated with higher risk of mortality. In fact, there was no significant difference between the two groups $(p=0.9)$.

Respiratory symptoms were more frequent in the no-survivors groupe especially cough (42 (70\%) versus $21(52.5 \%)),(p=0.076)$.

No-survivors had a significantly higher SOFA score at admission (4 [4-5] versus $3[2-4], p<0,001)$, higher white blood cells count $(12,580$ versus 9350; $p<0,001)$, higher LDH level (445 versus $319 p<0,001)$ and lower $\mathrm{PaO}_{2} / \mathrm{FiO}_{2}$ (68 [60-91] versus 108 [90-189], $\left.\mathrm{p}<0,001\right)$. However there was no significant difference in the lymphocyte counts (p: 0,09).

The percentage of patients requiring mechanical ventilation was significantly higher in the no-survivors group $(81.5 \%$ versus $15.4 \%$, $\mathrm{p}<0,001$ ).

The area under the curve (AUC) of CRP used to assess covid-19 mortality was 0.78 (and the optimal cut off for predicting mortality was a CRP value of $138.4 \mathrm{mg} / \mathrm{l}$ (Specificity: $70 \%$ and sensitivity: $75 \%$ ).

In univariate analysis: A value of CRP greater than $138.4 \mathrm{mg} / \mathrm{l}$ was associated with mortality $(p<0,001)$.

In multivariate analysis: gender-ratio, respiratory symptoms: cough, mechanical ventilation, CRP level greater than 138.4, higher white blood cells count were the predictive factors of mortality with a significant difference: $\mathrm{p}, \mathrm{OR}, \mathrm{Cl} 95 \%$ were respectively $=0.009 / 12 /[1.8-81]$ $0.005 / 18 /[2-146] ;<0,001 / 65 /[8-1503] ; 0.035 / 5 /[1-22]$ and $0.005 / 1 /$ [1-1].

Conclusion. Gender-ratio, respiratory symptoms: cough, mechanical ventilation, CRP level greater than 138.4, higher white blood cells count were correlated with mortality. Thus, they can be used to help identify the severe forms of the coronavirus disease.

\section{2}

Measuring the Incidence of and Risk Factors for Severe Maternal Morbidity and its Impact on Critical Care

J. Masterson'; I. Adamestam²; M. Beatty ${ }^{1}$; J. Boardman ${ }^{3}$; P. Johnston ${ }^{4}$; J. Joss $^{4} ;$ H. Lawrence ${ }^{5}$; K. Litchfield ${ }^{6} ;$ T. Walsh $^{1} ;$ A. Wise ${ }^{1}$; C. Weir ${ }^{2}$; F. Denison ${ }^{3}$; N. Lone ${ }^{2}$

${ }^{1}$ Department of Anaesthesia, Critical Care, and Pain Medicine, Royal Infirmary of Edinburgh, University of Edinburgh, Edinburgh, United Kingdom; ${ }^{2}$ Usher institute, The University of Edinburgh, Edinburgh, United Kingdom: ${ }^{3}$ Mrc Centre for Reproductive Health, The Queen's Medical Research Institute, The University of Edinburgh, Edinburgh, United Kingdom; ${ }^{4}$ Department of Anaesthesia, Critical Care, and Pain Medicine, Ninewells Hospital, Dundee, United Kingdom; ${ }^{5}$ Patient

Representative, The University of Edinburgh, Edinburgh, United Kingdom; ${ }^{6}$ Department of Anaesthesia, Critical Care, and Pain Medicine, Glasgow

Royal Infirmary, Glasgow, United Kingdom

Correspondence: J. Masterson

Intensive Care Medicine Experimental 2021, 9(1): 001082

Introduction. Whilst the global rate of maternal mortality is declining and is low in high income countries, there has been an increase in the incidence of women experiencing Severe Maternal Morbidity (SMM). [1] Consequently, high-income countries have implemented SMM as an alternative quality marker for obstetric care services.[2,3] Current research has suggested that some cases of SMM are avoidable with early anticipation, appropriate treatment and optimising obstetric critical care services.[4] However, with a paucity of research into the burden of SMM and its sequelae in Scotland, there is a lack of information necessary for guiding improvements in maternal critical care.

Objectives. This study has three objectives: (1) To quantify the incidence of SMM in the Scottish population, (2) To identify factors that are associated with SMM and (3) To report outcomes of SMM and understand the impact of SMM on critical care services.

Methods. A national cohort study of first pregnancies in Scotland between 01/01/2005 and 31/12/2019. Cohort data were sourced using database linkage including obstetric, critical care and statutory birth and death records.

Objectives 1 and 2

SMM was measured as a binary outcome using a recently established 26-criterion indicator for deriving SMM in administrative databases by Nair et al. [5] The incidence was reported as the number of women experiencing any SMM event for every 1,000 pregnancies. 
Baseline characteristics were measured as exposure variables which included sociodemographic measures, health and obstetric history and current pregnancy information

Objective 3

For objective 3, SMM was the exposure variable. The primary outcome was an admission to Intensive Care Units (ICUs) following an SMM event. Secondary outcomes included admission to ICU or generalHigh Dependency Units (HDUs) and admission to ICU or general-HDU or obstetric-HDU.

Additional outcomes compared the length of stay, level of care required and mortality between those with and without SMM.

Statistical analysis

Multivariable logistic regression was used for objective 2 to identify characteristics independently associated with SMM, and for objective 3 to quantify the association between SMM and critical care admission while adjusting for confounders.

Results. Amongst 350,195 first-time pregnancies, 4,297 women experienced SMM $(12.3 / 1,000)$. Women with multiple gestations, African or Asian birthplaces or co-morbidities had a greater risk of SMM. There was a strong association measured between ICU admissions and SMM (78.87(65.14, 95.48)). Additionally, both secondary outcomes were significantly associated with SMM; however, the data showed a substantial proportion of admissions to o-HDU without a classified SMM (3.6\%). Mortality, stillbirths, longer hospital stays, and organ support were more common amongst women experiencing SMM.

Conclusion. The incidence of SMM is higher than previously measured in Scotland and multiple factors increase a woman's risk of these events. Women experiencing SMM require a high level of care and are at risk of life-changing outcomes.

\section{Reference(s)}

1. Grant funding: Obstetric Anaesthetists' Association

2. 1. Geller S, Koch A, Garland C, MacDonald E, Storey F, Lawton B. A global view of severe maternal morbidity: moving beyond maternal mortality. Reproductive Health [Internet]. 2018 [cited 7 March 2021];15(S1). Available from: https://reproductive-health-journal.biomedcentral.com/artic les/10.1186/s12978-018-0527-2

3. 2. Baskett T. Epidemiology of obstetric critical care. Best Practice \& Research Clinical Obstetrics \& Gynaecology. 2008;22(5):763-774.

4. 3. Van Roosmalen J, Zwart J. Severe acute maternal morbidity in highincome countries. Best Practice \& Research Clinical Obstetrics \& Gynaecology. 2009;23(3):297-304.

5. 4. Geller SE, Rosenberg D, Cox SM, Brown ML, Simonson L, Driscoll CA, et al. The continuum of maternal morbidity and mortality: Factors associated with severity. American Journal of Obstetrics and Gynecology. 2004:191(3):939-44.

6. 5. Nair M, Kurinczuk JJ, Knight M. Establishing a National Maternal Morbidity Outcome Indicator in England: A Population-Based Study Using Routine Hospital Data. PLoS One. 2016 Apr 7;11(4):e0153370. https://doi.org/10.1371/journal.pone.0153370. PMID: 27054761; PMCID: PMC4824476.

7. Statistician acknowledgments: We are grateful to Roz Pollock for deriving the cohort and linking the datasets.

\section{2}

Adrenaline Infusion Practices in Intensive Care Units-A National Scottish Survey

N. Dowie'; T. Keast, ${ }^{2}$

${ }^{1}$ School of Medicine, University of Glasgow, Glasgow, UK, Glasgow, United Kingdom; ${ }^{2}$ Dept. of Anaesthetics, Crosshouse Hospital, West of Scotland Deanery, Glasgow UK, United Kingdom

Correspondence: N. Dowie

Intensive Care Medicine Experimental 2021, 9(1): 001092

Introduction. The movement to a unified standardisation of intravenous drugs in intensive care has been grumbling for years with guidelines and recommendations being produced to mixed response. Whilst some commonplace drugs have achieved a level of standardisation, others have been left behind causing a schism in practice. The UK Intensive Care Society has introduced recommended concentrations for 16 infusion drugs commonly used in intensive care. Standardisation not only lends to easier preparation and administration; it also directly impacts patient safety and cost (1).

Objectives. Following similar UK wide studies carried out between 2007 and 2017, this survey was concerned with adrenaline prescribing in Scottish ICUs and wanted to ascertain the concentrations being used, how the drug was being administered, and by which dosing method. These data would help clarify the use of adrenaline in Scottish ICUs and elucidate any common practices or indeed highlight the lack of standardisation.

Methods. From April to May 2021 a series of telephone questionnaires were carried out in all 23 Scottish ICUs. During each phone call the most senior nurse or medic was requested and a series of questions were asked:

- What concentration of adrenaline is being used in the unit?

- Do you administer adrenaline through syringe drivers or volumetric pumps?

- Do you dose in micrograms $/ \mathrm{kg} / \mathrm{min}$ or $\mathrm{mls} / \mathrm{hr}$ ?

We compared our data to ICS guidelines and results of previous UK wide surveys.

Results. All 23 sites gave responses to key questions and any additional anecdotal data was collected. There was no consensus on concentration of adrenaline used in ICU with one of two preparations being preferred (either $4 \mathrm{mg} / 50 \mathrm{ml}$-nine units, $5 \mathrm{mg} / 50 \mathrm{ml}-11$ units, or other-three units). There was an overall agreement that $\mathrm{mls} / \mathrm{hr}$ is the preferred dosing method with all but four units using this method. Respondents were in favour of using a syringe pump (15 units) with the remaining units using volumetric pumps with greater infusion volumes (eight units). Results outlined in Figure 1.

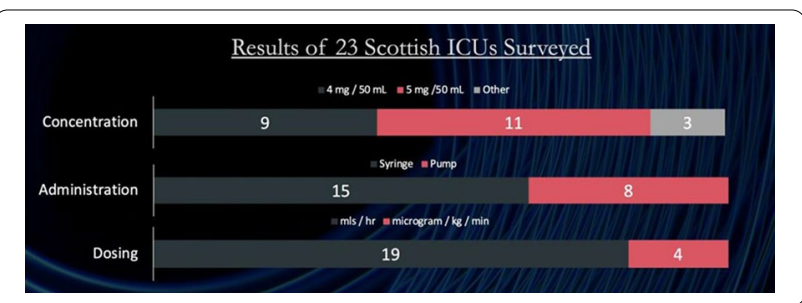

Figure 1

Conclusion. There is no clear consensus in Scotland with regards to the concentration of intravenous adrenaline infusion used in ICU. Units tend to prefer one of two common concentrations with only $39 \%$ using the ICS recommended concentration of $4 \mathrm{mg} / 50 \mathrm{ml}$. This is in keeping with the findings of the UK wide surveys conducted previously. Volumetric pumps were used primarily for convenience and not for clinical reasons. Medical staff seem to be most comfortable in dosing in $\mathrm{mls} / \mathrm{hr}$ with few units showing progression to a weight based, microgram/min regimen. Units surveyed cited infrequent use as the main reason for out-of-date practice; however, it could be argued that this is precisely why seldom used medications should be standardised.

\section{Reference(s)}

1. 1. Titiesari YD, Barton G, Borthwick M, Keeling S, Keeling P. Infusion medication concentrations in UK's critical care areas: Are the Intensive Care Society's recommendations being used? J Intensive Care Soc. 2017 Feb 1;18(1):30-5.

2. None. 


\section{5}

Geriatric Patients with COVID-19 Pneumonia in the Intensive Care

Unit-1 year retrospective study

P. Oliveira1; D. Adrião1; R. Veríssimo, 2; D. Miranda,

${ }^{1}$ Department of Intensive Care, Centro Hospitalar Vila Nova de Gaia/

Espinho, Vila Nova de Gaia, Portugal; ${ }^{2}$ Serviço de medicina interna, Centro hospitalar Vila Nova de Gaia /Espinho, Vila Nova de Gaia, Portugal

Correspondence: P. Oliveira

Intensive Care Medicine Experimental 2021, 9(1): 001095

Introduction. COVID-19 pandemics was a challenge to Health Care Systems and to Intensive Care Units (ICU). Geriatric patients (65 years old (yo) or older) are more common in ICU. Older patients were frequently admitted in the ICU with COVID-19 pneumonia and doubts existed about some admissions considering their frailty and comorbidities.

Objectives. We intended to understand differences in comorbidities, ventilation, Length of stay (LOS) and mortality between young and older patients.

Methods. Observational retrospective study of all patients admitted at the adult ICU from March 15th 2020 and March 14th 2021 with COVID-19 pneumonia, comparing adults under 65 yo and geriatric patients (65 yo or older). Data were analyzed using Qui-square and Mann-Whitney Test.

Results. We treated 237 COVID 19 pneumonia patients during 12 months, 168 (70.9\%) males, median age of 65 yo, ranging from 27 to 85 yo [interquartile ranges (IQR) 58; 71] (48.9\% under 65 yo and $51.1 \%$ 65 yo or older, median APACHE score 16.5, SAPSII score 34, ICU LOS 11 days [IQR 4.9; 19.5] and mechanical ventilation (MV) of 13 days [IQR 8; 22]. 62 patients $(26.2 \%)$ were submitted to non-invasive ventilation (NIV), 134 (56.5\%) to High Flow Nasal Oxygen (HFNO) and 155 (65.4\%) to MV. The ICU mortality rate was $24.9 \%$ and in-hospital mortality rate was $27.8 \%$ for patients admitted in the ICU. From the most common comorbidities, hypertension was the most frequent $(62.4 \%)$, followed by Diabetes mellitus (33.8\%), obesity (33.8\%), chronic respiratory diseases $(14.8 \%)$, cardiac disease $(13.5 \%)$ and chronic renal disease $(7.6 \%)$ When we divided our patients in adults (18-64 yo) and geriatric ( $\geq 65$ yo) we found no difference in oxygenation support as HFNO $(p=0.53)$, NIV $(p=0.27)$ and MV (0.16), days on MV $(p=0.14)$ and APACHE score $(p=0.146)$. SAPSII score was higher on geriatric patients (Median 32 vs 37$)(p=0.004)$. LOS was higher in older patients $(p=0.047)$. Hypertension and Diabetes mellitus were more frequent in geriatric patients $(p<0.001$ and $p=0.025)$. Other comorbidities showed no difference between groups. ICU mortality rate was $18.1 \%$ in patients $<65$ yo and $32.3 \%$ in geriatric patients $(p=0.012)$ and in Hospital mortality was $19.8 \%$ in the younger group and $35.5 \%$ in the older $(p=0.007)$.

Conclusion. Geriatric patients represent an important part of ICU COVID-19 patients. There was no difference on ventilation support and days on MV. As expected, they had more frequently hypertension and diabetes. ICU LOS was higher in geriatric patients, as well as ICU and hospital mortality. Older patients are a challenge in ICU during COVID 19 pandemics, needing more time of treatment, having more comorbidities to manage, with worse outcomes.

\section{Reference(s)}

1. Liu K, Chen Y, Lin R, Han K. Clinical features of COVID-19 in elderly patients: A comparison with young and middle-aged patients. J Infect. 2020 Jun;80(6):e14-e18

2. Görgülü Ö, Duyan M. Effects of Comorbid Factors on Prognosis of Three Different Geriatric Groups with COVID-19 Diagnosis. SN Compr Clin Med. 2020 Nov 18:1-12

3. None
001101

Frailty biomarkers

A. Simas ${ }^{1}$; P. Ramos '; A. Oliveira'; I. Correia ${ }^{2}$; J. Gonçalves-Pereira

${ }^{1}$ Intensive Care Unit, Hospital Vila Franca de Xira, Vila Franca de Xira,

Portugal; ${ }^{2}$ Palliative Care Unit, Fernando Fonseca Hospital—Portugal,

Lisboa, Portugal

Correspondence: A. Simas

Intensive Care Medicine Experimental 2021, 9(1): 001101

Introduction. Frailty is an increasingly common clinical condition characterized as a decreased physiological reserve and enhanced vulnerability, with an increased risk for poor health outcomes, including falls, incident disability, hospitalization, and mortality.

With demographic changes in society, such as population aging, physicians, namely intensivists, are facing a great challenge, when dealing with this population.

The screening of frailty in the Intensive Care Units (ICU) presents potential benefits for both patients and decision-makers, optimizing the real notion of prognosis, risk of complications, expectations of recovery and use of resources. This may create the stage for patient empowerment and realistic therapeutic goals.

Some biomarkers may be related to frailty and may help to identify patients at high risk of morbidity and mortality.

Human serum albumin is an important parameter for the routine assessment of the nutritional status as well as for monitoring inflammatory activity, and chronic anaemia, may be a good frailty and outcome predictor.

Objectives. To determine laboratory values suitable to discriminate frailty patients at risk for a poor outcome.

Methods. Prospective, multicentre, cohort, observational study in Portuguese ICU. All adult patients admitted for more than $48 \mathrm{~h}$ to one of the 23 participating ICU, during a 15-day consecutive period were included.

All patients were evaluated according to the clinical frailty score by the doctor and nurse in charge as well as by a family member. Frailty was considered to be present when the mean frailty score of the 3 assessments was $\geq 5$. We grouped patients according to the measured clinical frailty score: low frailty (score 1-3), mild frailty (score 4-6), high frailty (score 7-9).

The more deviated laboratory value, during the first $24 \mathrm{~h}$ of ICU admission, was collected for each patient, namely the lower haemoglobin and the lower albumin concentration. The ICU and hospital length of stay (LOS), the presence of organ disfunctions, SAPS II score and ICU and hospital mortality were recorded. Spearman correlation test was applied between the 2 biomarkers and the presence of frailty.

Results.

\begin{tabular}{|c|c|c|c|c|c|c|}
\hline \multirow{2}{*}{ Frailty Score } & \multirow{2}{*}{ Age } & \multirow{2}{*}{ Gender (Male) } & \multirow{2}{*}{ SAPS ॥ } & \multicolumn{2}{|c|}{ Mortality } & \multirow{2}{*}{ Hospital LoS } \\
\hline & & & & ICU & ICU 6-months & \\
\hline 1 (N 58) & $59.9 \pm 17.1$ & 43 (74.1\%) & $30.7 \pm 15.7$ & $5(8.6 \%)$ & $12(20.7 \%)$ & $27.8 \pm 34.1$ \\
\hline 2 (N 53) & $65.2 \pm 13.8$ & $40(75.5)$ & $38.2 \pm 16.8$ & $3(5.7 \%)$ & $8(15.4 \%)$ & $34.22 \pm 45.8$ \\
\hline 3 (N80) & 69.8土14.6 & 50 (62.5\%) & $42 \pm 15.1$ & $8(10 \%)$ & 20 (25\%) & $28.43 \pm 3.5$ \\
\hline 4 (N 74) & $69.1 \pm 12.2$ & 42 (56.8\%) & $44.7 \pm 16.5$ & $8(10.8 \%)$ & 23 (31.1\%) & $28.46 \pm 25.6$ \\
\hline 5 (N 34) & $72.1 \pm 13$ & $24(70.6 \%)$ & $49.1 \pm 16.9$ & $5(14.7 \% 6)$ & $17(50 \%)$ & $35.56 \pm 28$ \\
\hline 6 (N 27) & $79 \pm 13.7$ & 16 (59.3\%) & $49.4 \pm 17.5$ & 3 (11.1.1\%) & 12 (44.4\%) & $25.89 \pm 21$ \\
\hline 7 (N 3$)$ & $76.00 \pm 6.6$ & $2(66.786)$ & $57.7 \pm 10.5$ & 1(33.3\%) & 1(33.3\%) & $9.67 \pm 6.8$ \\
\hline $8(\mathrm{~N} 4)$ & $82.5 \pm 6.3$ & $4(100 \%)$ & $55.8 \pm 19.7$ & 125\%) & $3(75 \%)$ & $58.0 \pm 44.1$ \\
\hline 9 (N2) & $59.9 \pm 2.1$ & 0 (0\%) & $70.5 \pm 26.2$ & $1(50 \%)$ & 2 (100\%) & $15.5 \pm 10.6$ \\
\hline
\end{tabular}

The study sample included 335 patients, with a mean age of $63.2 \pm 16.8$, (66\% Male), with an average SAPSII score of $41.8 \pm 17.4$ LOS was $9.4 \pm 11.4$ days and six-month all-cause mortality was $29.3 \%$. The prevalence of frailty $($ score $\geq 5)$ in our cohort of patients was $20.9 \%$. These patients had higher mortality, $50 \%$. Six-month all-cause mortality on non-frailty patients was $23.9 \%$ (Table 1). 


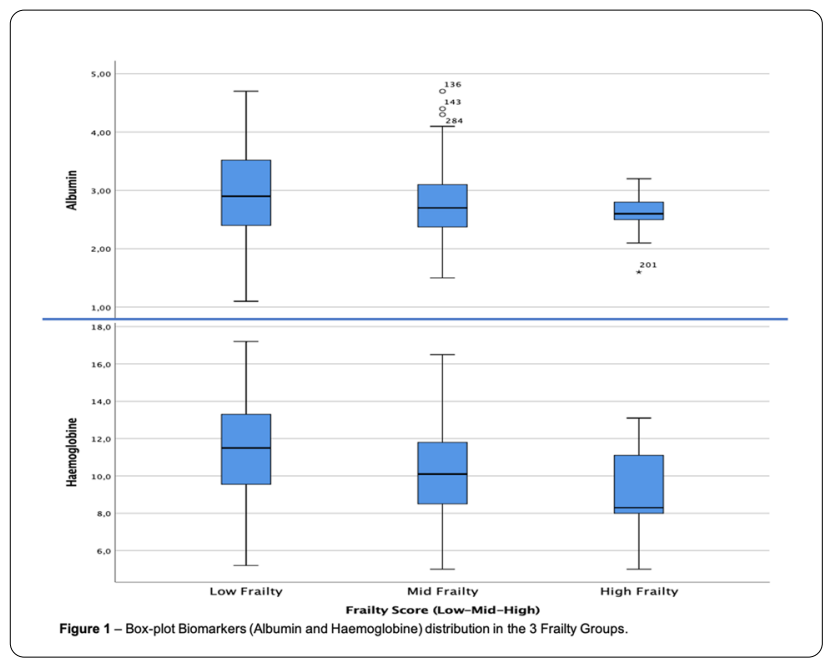

Spearman correlations unveiled a statistically significant negative relationship between these 2 biomarkers and the presence of frailty (Table 2), that is, as frailty increased, low mean concentration of haemoglobin and albumin were noted (Fig. 1).

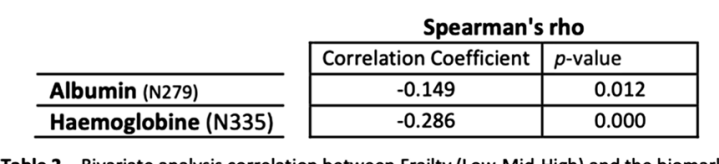

Table 2 - Bivariate analysis correlation between Frailty (Low-Mid-High) and the biomarkers

(Albumin and Haemoglobin). Statistical significancy with p-value $<0.05$.

Conclusion. Clinical frailty score is associated with poor prognosis, with increased mortality. Commonly used biomarkers, haemoglobin and albumin, may help to identify patients with frailty and can contribute to a decision-making tool, permitting a more realistic therapeutic approach.

\section{Reference(s)}

1. Ferrucci, L. et al.; Biomarkers of frailty in older persons. Journal of Endocrinological Investigation 2002, 25(10 Suppl), 10-15.

2. Aurélie Mailliez et al.; Circulating biomarkers characterizing physical frailty: CRP, hemoglobin, albumin, 25OHD and free testosterone as best biomarkers. Results of a meta-analysis; Experimental Gerontology, October 2020, 111014

3. Ahmed Al Saedi et al.; Current and emerging biomarkers of frailty in the elderly; Clinical Interventions in Aging 2019:14 389-398

\section{0}

Medical students supporting the critical care workforce during the COVID-19 pandemic

R. Gray'; J. Pateman, ${ }^{2}$; H. Baid ${ }^{3}$; L. White, ${ }_{1}^{3}$; O. Boyd, ${ }^{4}$

${ }^{1}$ Department of Intensive Care Medicine, University Hospitals Sussex, Brighton, United Kingdom; ${ }^{2}$ Department of Anaesthesia, Royal Sussex County Hospital, Brighton, United Kingdom; ${ }^{3}$ School of Health Sciences, University of Brighton, Brighton, United Kingdom; ${ }^{4}$ Department of Intensive Care Medicine, Royal Sussex County Hospital, Brighton, United Kingdom

\section{Correspondence: R. Gray}

Intensive Care Medicine Experimental 2021, 9(1): 001120

Introduction. Enrolling medical students to work in Intensive Care (ICU) could rapidly expand the workforce during a COVID-19 surge in ICU bed requirement (1-3). Hospitals have used medical students in ICU during previous viral pandemics (4). It has been shown that medical students on HCA placements benefit by developing an understanding of multidisciplinary teams $(5,6)$ However, the use of medical students as HCAs is controversial and has been considered 'too menial' (9).

December 2020 saw a COVID-19 surge in the UK and medical students from the Brighton and Sussex Medical School (BSMS), with Health Education England approval, were invited to work one 12-h shift per week. A fast track recruitment and virtual induction process was developed. A dedicated consultant coordinated the scheme. Pastoral care support sessions were provided weekly by a consultant and senior nurse using Microsoft Teams.

Objectives. A service evaluation of using medical students as HCAs on ICU during the COVID-19 pandemic explored if this model could be used again in the future to benefit medical students and the workforce.

Methods. Medical students, who joined the HCA scheme, were invited to complete an online questionnaire using Jisc online academic surveys. The questions included Likert scales and free text answers.

Results. 182 medical students worked as HCAs in ICU, 108 have continued to work as HCAs since the COVID-19 surge.

The survey had a $43 \%$ response rate. $46.1 \%$ found the enrolment process easy. $78 \%$ undertook the virtual induction, with just under $50 \%$ of those undertaking it finding it of practical use. $88 \%$ received the shifts they requested.

Only $42 \%$ received pay in the correct time scale, $52.6 \%$ found the practical aspects of the shifts straightforward (e.g. uniforms and parking).

$97 \%$ of medical students felt welcomed by the ICU staff, and $83 \%$ thought their roles and responsibilities were made clear. $100 \%$ received adequate breaks.

$98 \%$ reported an overall positive experience, with $76 \%$ describing the experience as having a positive impact on their medical student development, However $68 \%$ found their experience had an emotional, though often positive, impact on them; "seeing very ill and dying patients was tough, but I feel it has better prepared me for being a doctor". 94\% indicated they would work again as an HCA in another surge of COVID-19. $70 \%$ of medical students found the weekly debrief sessions helpful.

Conclusion. Medical students working as HCAs in the ICU was an excellent experience for the students and helped address the ICU workforce shortage during the COVID-19 surge. The medical students benefited from a personal and professional development perspective. The rapid set-up created some practical difficulties for working and pay. However, a model is now in place that the hospitals and BSMS can quickly reinstate if needed again with mutual benefits for both medical students and the workforce. There may be longer term benefit in showcasing HCA work as rewarding and useful for the students.

\section{Reference(s)}

1. 1. Bauchner H, Sharfstein J. A Bold Response to the COVID-19 Pandemic: Medical Students, National Service, and Public Health. JAMA. 2020;323(18):1790-1.

2. 2. Bazan D, Nowicki M, Rzymski P. Medical students as the volunteer workforce during the COVID-19 pandemic: Polish experience. Int J Disaster Risk Reduct. 2021:55:102109.

3. 3. Rasmussen S, Sperling P, Poulsen MS, Emmersen J, Andersen S. Medical students for health-care staff shortages during the COVID-19 pandemic. Lancet. 2020;395(10234):e79-e80.

4. 4. Lassen HC. A preliminary report on the 1952 epidemic of poliomyelitis in Copenhagen with special reference to the treatment of acute respiratory insufficiency. Lancet. 1953;1 (6749):37-41.

5. 5. Seaman K, Saunders R, Dugmore H, Tobin C, Singer R, Lake F. Shifts in nursing and medical students' attitudes, beliefs and behaviours about interprofessional work: An interprofessional placement in ambulatory care. J Clin Nurs. 2018;27(15-16):3123-30.

6. 6. Butterworth K, Rajupadhya R, Gongal R, Manca T, Ross S, Nichols D. A clinical nursing rotation transforms medical students' interprofessional attitudes. PLoS One. 2018;13(5):e0197161.

7. 7. Brand PLP. COVID-19: a unique learning opportunity if the well-being of learners and frontline workers is adequately supported. Perspect Med Educ. 2020;9(3):129-31. 
8. 8. Aase I, Hansen BS, Aase K. Norwegian nursing and medical students' perception of interprofessional teamwork: a qualitative study. BMC Med Educ. 2014;14:170.

9. 9. Kituno N. Students call for end of reassignment to 'menial' ICU shifts HSJ. 2021 20th January 2021. https://www.hsj.co.uk/workforce/stude nts-call-for-end-of-reassignment-to-menial-icu-shifts/7029340.article (accessed 11-5-21)

\section{5}

\section{Intensive Care Unit-specific Virtual Reality for COVID-19 ICU}

\section{survivors}

JH. Vlake'; J. Van Bommel ${ }^{2}$; EJ. Wils, ${ }^{3} ;$ OJ. Bienvenu ${ }^{4}$; M. Hellemons ${ }^{5}$.

T. Korevaar, ${ }^{6}$; A. Schut ${ }^{7}$; J. Labout ${ }^{8}$; M. Van Bavel ${ }^{2}$; L. Schreuder²; D.

Gommers $^{2}$; M. van Genderen ${ }^{2}$

${ }^{1}$ Department of Intensive Care, Erasmus MC, Franciscus Gasthuis, Rotterdam, Netherlands; ${ }^{2}$ Department of Intensive Care, Erasmus MC, Rotterdam, Netherlands; ${ }^{3}$ Department of Intensive Care, Franciscus Gasthuis, Rotterdam, Netherlands; ${ }^{4}$ Department of Psychiatry and Behavioral Sciences, John Hopkins University School of Medicine, Maryland, Baltimore, United States of America; ${ }^{5}$ Department of Pulmonology, Erasmus MC, Rotterdam, Netherlands; ${ }^{6}$ Department of Internal Medicine, Environmental Epidemiology, Erasmus MC, Rotterdam, Netherlands; ${ }^{7}$ Department of Intensive Care, Ikazia hospital, Rotterdam, Netherlands; ${ }^{8}$ Department of Intensive Care, Maasstad hospital, Rotterdam, Netherlands

\section{Correspondence: J.H. Vlake}

Intensive Care Medicine Experimental 2021, 9(1): 001125

Introduction. One-third of ICU survivors develop psychological impairments in the aftermath of their critical illness (1), that negatively impact one's health-related quality of life (2). No robustly effective treatment modality is currently available. Recently, virtual reality (VR) demonstrated to be an innovative technique that could improve psychological ICU-related sequelae, and could be safely used in a postICU COVID-19 patient, but clinical trials are unavailable $(3,4)$.

Objectives. To determine the effect of ICU-VR, incorporated in followup clinics three months after hospital discharge, on PICS-related psychological sequelae, mental quality of life, and patients' satisfaction with and rating of ICU care and aftercare, and their perspectives on ICU-VR.

Methods. A multicenter, randomized controlled trial in COVID-19 patients treated in one of the four participating mixed medical-surgical ICUs in Rotterdam, the Netherlands. Patients were randomly assigned to either the ICU-VR (intervention) or control group. Three months after hospital discharge, all patients were invited to a postCOVID-19 outpatient clinic during which patients in the intervention group received ICU-VR. Primary outcomes were psychological distress, i.e., symptoms of post-traumatic stress disorder, anxiety, and depression, and quality of life up to six months after discharge. Secondary outcomes were the effect on patients' satisfaction with and rating of ICU care and aftercare, and patients' perspectives on ICU-VR.

Results. 89 patients (median age: 58 years; 63 [70\%] male) were included. No differences in psychological distress or quality of life were observed between groups, except ICU-VR patients reporting less anxiety four months post-hospital discharge (ICU-VR, 6 [14\%], vs. control, 21 [50\%], $p=0.02$ ). ICU-VR did improve aftercare satisfaction (median [IQR] satisfaction score: ICU-VR, 9 [8-10], vs. control, 7 [7-9], p<0.001) and overall rating of aftercare (median rating [IQR]: 9 [8-10] vs. 8 [7-9], $\mathrm{p}<0.01$ ). VR added to the quality of aftercare according to $80 \%$ of ICU-VR patients and all ICU-VR patients would recommend ICU-VR to others.

Conclusion. We did not observe an improvement in PICS-related psychological symptomatology, and found a rather low incidence of psychological sequelae in our COVID-19 ICU survivors. Nevertheless, ICU-VR did increase satisfaction and rating, and added to the quality of ICU aftercare. ICU-VR could serve as a tool to improve quality of ICU aftercare and fulfill patients' information needs, but future research is warranted.

\section{Reference(s)}

1. 4. Vlake JH, van Bommel J, Hellemons ME, et al. Intensive Care UnitSpecific Virtual Reality for Psychological Recovery After ICU Treatment for COVID-19; A Brief Case Report. Frontiers in Medicine 2021;7(1143).

2. BeterKeten (foundation) kindly funds the PhD-trajectory of Johan $\mathrm{H}$. Vlake. Stichting Coolsingel (foundation), DSW, Stichting Thea (foundation), and Stichting SGS (foundation) funded the development of COVID19 ICU-VR. The funding sources had no role in writing this manuscript.

3. 1. Bienvenu OJ, Friedman LA, Colantuoni E, et al. Psychiatric symptoms after acute respiratory distress syndrome: a 5-year longitudinal study. Intensive Care Med 2018;44(1):38-47.

4. 2. Needham DM, Davidson J, Cohen $\mathrm{H}$, et al. Improving long-term outcomes after discharge from intensive care unit: report from a stakeholders' conference. Crit Care Med 2012;40(2):502-509.

5. 3. Vlake JH, Wils E-J, Van Bommel J, et al. Intensive Care specific Virtual Reality (ICU-VR) to improve Post-Intensive Care Syndrome-related psychological sequelae in survivors of critical illness. ICMx 2020;8(suppl.2).

\section{8}

Alcohol Related Admissions To Intensive Care During The COVID-19 Pandemic

O. Nichoileann, ; F. Fallon, 2; M. Pecak²; J. Mulvihill²; C. Angus ${ }^{3}$; N. Fitzgerald ${ }^{4}$

${ }^{1}$ Anaesthesiology \& Critical Care, Cork University Hospital, Cork, Ireland; ${ }^{2}$ Anaesthesiology \& Critical Care, Beaumont Hospital, Dublin, Ireland; ${ }^{3}$ Sheffield Alcohol Research Group, School of Health and Related Research, University of Sheffield, Sheffield, United Kingdom; ${ }^{4}$ Institute of Social Marketing and Health, University of Stirling, Dublin, Ireland

Correspondence: $\mathrm{O}$. Nichoileann

Intensive Care Medicine Experimental 2021, 9(1): 001128

Introduction. Across the world, critical care units are struggling to deal with the outbreak of coronavirus disease 2019 (COVID-19) caused by severe acute respiratory syndrome coronavirus 2 (SARSCoV-2) $(1,2)$. Intensive Care Units (ICUs) in both Britain and Ireland are particularly poorly resourced (3), with only 6.0 critical care beds per 100,000 population in Republic of Ireland, compared with the European average of 11.5 per 100,000 (4). As the healthcare crisis caused by the pandemic continues, other indirect effects of the pandemic are becoming apparent. Concerns regarding increased alcohol misuse, possibly related to stress and public health measures placed on individuals during COVID-19, have been voiced (5).

Objectives. To establish the impact of the COVID-19 pandemic on alcohol-related admissions to critical care in an Irish teaching hospital. Methods. Admissions to critical care from 1st January to 31st December for both 2019 and 2020 were screened for alcohol-involvement in the cause of admission. Patients whose admissions were directly related to excessive alcohol consumption were included in the study. The demographics, nature of admission and clinical outcomes of patients admitted to ICU between 1st January to 31 st December in both 2019 and 2020 were reviewed. ICU admissions during the Republic of Ireland's three waves of peak COVID-19 cases were compared to the same time interval in 2019.

Results. Alcohol related admissions were $11.34 \%$ of total admissions to critical care in 2019 , compared to $11.15 \%$ in 2020 . In terms of bed occupancy, alcohol-related ICU admissions accounted for $22.01 \%$ of non-COVID bed days used during the first COVID-19 surge period in 2020 , as opposed to $18.30 \%$ for the same time interval in 2019 . Bed occupancy for non-COVID admissions fell sharply during the study period in 2020.

Conclusion. Alcohol-related admissions placed a significant burden on critical care resources during the Covid-19 pandemic in Dublin. This burden was greater than the equivalent bed usage by alcohol-related admissions in 2019, despite the closure of licensed on-trade premises. This study highlights an urgent need to implement interventions to reduce harmful alcohol consumption in order to protect vital ICU beds. 
Reference(s)

1. Clay JM, Parker MO. Alcohol use and misuse during the COVID-19 pandemic: a potential public health crisis? Lancet Public Health. 2020;5(5):e259.

2. Health Protection Surveillance Centre H. Epidemiology of COVID-19 in Ireland. Report prepared by HSPC on 23/05/2020 for NPHET. 2020

3. National Office of Clinical Audit N. Irish National ICU Audit Annual Report 2018. 2020

4. Rhodes A, Ferdinande P, Flaatten H, Guidet B, Metnitz PG, Moreno RP. The variability of critical care bed numbers in Europe. Intensive Care Med. 2012;38(10):1647-53.

5. Wu Z, McGoogan JM. Characteristics of and Important Lessons From the Coronavirus Disease 2019 (COVID-19) Outbreak in China: Summary of a Report of 72314 Cases From the Chinese Center for Disease Control and Prevention. Jama. 2020.

6. Grasselli G, Pesenti A, Cecconi M. Critical Care Utilization for the COVID-19 Outbreak in Lombardy, Italy: Early Experience and Forecast During an Emergency Response. Jama. 2020.

\section{0}

Psychological distress and health-related quality after ICU treatment for COVID-19

JH. Vlake'; J. Van Bommel'; M. Hellemons ${ }^{3}$; EJ. Wils, ${ }^{4}$ OJ. Bienvenu ${ }^{5}$; A Schut $^{6}$; E. Klijn²; M. Van Bavel²; D. Gommers²; M. van Genderen²

${ }^{1}$ Intensive Care, Erasmus MC, Franciscus Gasthuis, Rotterdam,

Netherlands: ${ }^{2}$ Department of Intensive Care, Erasmus MC, Rotterdam,

Netherlands; ${ }^{3}$ Department of Pulmonology, Erasmus MC, Rotterdam,

Netherlands: ${ }^{4}$ Department of Intensive Care, Franciscus Gasthuis,

Rotterdam, Netherlands; ${ }^{5}$ Department of Psychiatry and Behavioral

Sciences, John Hopkins University School of Medicine, Maryland

Baltimore, United States of America; ${ }^{6}$ Department of Intensive Care, Ikazia hospital, Rotterdam, Netherlands

Correspondence: J.H. Vlake

Intensive Care Medicine Experimental 2021, 9(1): 001130

Introduction. ICU survivors are, in general, at risk of developing psychological distress, as part of the post-intensive care syndrome (PICS), negatively impacting quality of life $(1,2)$. Risk factors for PICS, such as acute respiratory distress syndrome, episodes of dyspnea, and prolonged mechanical ventilation and ICU stay, were highly prevalent during COVID-19 ICU treatment. Limited patient- and family-centered care due to changing in-ICU circumstances during the pandemic might have further increased this risk $(3,4)$. To date, limited data is available concerning the psychological recovery of COVID-19 ICU survivors.

Objectives. To quantify short- and long-term psychological distress, i.e., symptoms of post-traumatic stress disorder, anxiety, and depression, and health-related quality of life in COVID-19 ICU survivors.

Methods. This observational, prospective, multicenter study was conducted in three mixed medical-surgical ICUs in Rotterdam, the Netherlands. All consecutive adult ( $\geq 18$ years) patients admitted to the ICU with a PCR-proven SARS-CoV-2 infection between March 2 and October 17, 2020, who visited our post-COVID-19 follow-up clinic were eligible. The primary outcomes were psychological distress and overall and mental health-related quality of life, assessed using validated questionnaires, six weeks, three months, and six months post-hospital discharge. Secondly, we compared three-month psychological outcomes and mental health-related quality of life with a historical critical illness survivor cohort, and overall and mental health-related quality of life with the Dutch general population.

Results. 118 patients were included (median age 61 years [95\% range 36-77], 79 [68\%] male) with a median ICU length of stay of 13 days (95\% range 1-49), and $93(79 \%)$ were mechanically ventilated with a median duration of $284 \mathrm{~h}$ (10-629). At six weeks, 13 patients (23\%) reported psychological distress, co-presence of probable PTSD, anxiety, and depression was common, and no changes in psychological distress were observed throughout follow-up. COVID-19 ICU survivors tend to report probable PTSD more frequently than patients in the historical cohort (COVID-19, 12 (11\%), vs. historical cohort, 24 (20\%), OR 4.1 [95\% Cl 0.7-25.1], $p=0.13$ ), and reported more severe symptoms of anxiety (HADS anxiety score: $3(0-17)$ vs. $5(0-16)$, estimated mean difference 2.33 [95\% $\mathrm{Cl} 0.0-4.7], p=0.05$ ) and depression (HADS depression score: $3(0-15)$ vs. $5(0-16)$, estimated mean difference 2.4 [95\% Cl 0.1-2.4], $p=0.04)$. Overall and mental health-related quality of life both increased over time. COVID-19 ICU survivors reported a better mental health-related quality of life than historical controls, but overall and mental health-related quality of life was still poorer than in the Dutch general population.

Conclusion. Psychological distress prevalence was relatively low in COVID-19 ICU survivors and remained similar up to six months after hospital discharge. Health-related quality of life increased over time, was higher than in a historical controls, but was lower than in the Dutch general population. Our findings highlight that COVID-19 ICU survivors should be monitored after ICU treatment to detect possible psychological disorders.

\section{Reference(s)}

1. 4. Marin-Corral J, Garcia-Sanz A, Masclans JR. Humanizing ICU Coronavirus Disease 2019 Care. Chest 2021;159(2):489-491.

2. 3. Worsham CM, Banzett RB, Schwartzstein RM. Dyspnea, Acute Respiratory Failure, Psychological Trauma, and Post-ICU Mental Health: A Caution and a Call for Research. Chest 2021:159(2):749-756.

3. 2. Bienvenu OJ, Friedman LA, Colantuoni E, et al. Psychiatric symptoms after acute respiratory distress syndrome: a 5-year longitudinal study. Intensive Care Med 2018;44(1):38-47.

4. 1. Needham DM, Davidson J, Cohen H, et al. Improving long-term outcomes after discharge from intensive care unit: report from a stakeholders' conference. Crit Care Med 2012;40(2):502-509.

5. BeterKeten (foundation) funds the PhD-trajectory of Johan H. Vlake, and had no role in writing this abstract.

\section{9}

In hospital mortality due to respiratory failure in COVID 19 patients: A comparison between CPAP, early intubation and delayed intubation groups

S. Eswarappa'; M. Agarwal' ; J. Blake1; M. Lana, ; S. Cavinato ; R. Kumar ${ }^{1}$ Anaesthetics and Intensive Care, Kingston Hospital NHS Trust, London, United Kingdom

Correspondence: S. eswarappa

Intensive Care Medicine Experimental 2021, 9(1): 001139

Introduction. During the first wave of the covid-19 pandemic almost three quarters of patients who were admitted to critical care received invasive ventilation and one in two patients received it within $24 \mathrm{~h}$ of admission (1). As the pandemic unfolded, various non-invasive options such as CPAP (continuous positive air pressure) and HFNO (High Flow Nasal Oxygen) were tried for respiratory support. CPAP has been used successfully in patients with COVID 19 respiratory failure as a simple and cost-effective intervention (2). We present a study to evaluate and compare the effectiveness of continuous positive air pressure (CPAP) versus intubated patients in the management of respiratory failure associated with COVID-19 infection.

Objectives. The aim of the study was to compare the in-hospital mortality due to respiratory failure in COVID infected patients who were managed on CPAP with those who were intubated (early or delayed). Intubation was considered early when the patients were intubated within $48 \mathrm{~h}$ of initiation of CPAP and delayed when patients were intubated after $48 \mathrm{~h}$ of initiation of CPAP.

Methods. We retrospectively reviewed the records of the patients who were admitted to the Critical Care Unit at Kingston Hospital from March 2020 to February 2021 with COVID 19 infection and required either CPAP or intubation for the treatment.

Results. The total number of patients with COVID 19 pneumonia who needed CPAP only or invasive ventilation over the above-mentioned period was 295. 


\begin{tabular}{llll}
\hline Feature & $\begin{array}{l}\text { CPAP } \\
\text { Early } \\
\text { Intuba- } \\
\text { tion }\end{array}$ & $\begin{array}{l}\text { Delayed } \\
\text { Intuba- } \\
\text { tion }\end{array}$ \\
\hline Number of patients & 184 & 80 & 31 \\
Number of deaths & 54 & 38 & 20 \\
Number of survivors & 130 & 42 & 11 \\
Mortality (\%) & 29.34 & 47.5 & 64.5 \\
Deaths within 30 days & 50 & 35 & 17 \\
\hline
\end{tabular}

Conclusion. CPAP as a treatment modality for type 1 respiratory failure in COVID 19 pneumonia is a simple and effective option. Although our study is a retrospective analysis of a small number of patients, comparing 30-day mortality in patients given CPAP only versus intubated patients, the significantly decreased mortality in the CPAP group shows that CPAP is a feasible option which can avoid intubation altogether. This is consistent with other studies using CPAP in COVID 19 respiratory failure $(2,3)$. It is certainly a preferable treatment option for patients with isolated respiratory failure without respiratory distress and in patients who are not for resuscitation.

The increased mortality in the intubated group as compared to CPAP group indicates no advantage of early or delayed invasive ventilation over CPAP. High mortality in the delayed intubation group suggests that if patients need to be intubated after starting CPAP, the decision to intubate must be made early. As our study is a single centre, retrospective study with limited number of cases, the results indicate that it will be worthwhile doing this study on a larger number of patients as a multicentre prospective study.

\section{Reference(s)}

1. 1. Intensive Care National Audit and Research Centre. ICNARC report on covid-19 in critical care: England, Wales and Northern Ireland 31 December 2020.Available at https://www.icnarc.org/Our-Audit/Audits/Cmp/ Reports [Accessed 10 May 2021]

2. 2. Ashish A, Unsworth A, Martindale J, et alCPAP management of COVID19 respiratory failure: a first quantitative analysis from an inpatient service evaluation BMJ Open Respiratory Research 2020;7:e000692. https://doi. org/10.1136/bmjresp-2020-000692

3. 3. Oranger M, Gonzalez-Bermejo J, Dacosta-Noble P, Llontop C, Guerder A, Trosini-Desert $V$ et al. Continuous positive airway pressure to avoid intubation in SARS-CoV-2 pneumonia: a two-period retrospective casecontrol study. European Respiratory Journal Jan 2020, 2001692; https:// doi.org/10.1183/13993003.01692-2020

4. None.

\section{8}

The impact of a "one day basic intensive care training program" on knowledge of non-intensivists during the COVID-19 pandemic SS. Siddiqui'; S. Saran '; S. Saxena'; S. Agrawal ${ }^{2}$; SN. Muzaffar'; A. Ahmed'. A. Lohiya ${ }^{3}$; A. Agrawal ${ }^{1}$

'Department of Critical Care Medicine, King George's Medical University, Lucknow, India; ${ }^{2}$ Department of Obstetrics and Gynecology, King George's Medical University, Lucknow, India; ${ }^{3}$ Department of public health, Super Specialty Cancer Institute and Hospital, Lucknow, India Correspondence: S.S. Siddiqui

Intensive Care Medicine Experimental 2021, 9(1): 001148

Introduction. The Covid-19 pandemic posed a big challenge all over the world reflecting the scarcity of trained manpower. 1 The utility of the basic ICU training comprising of a "one day course", has been scientifically evaluated in very few studies.2

Objectives. The objective of the study was to assess the utility of such training and evaluate its effectiveness in improving the knowledge through a pre and post test model.

Methods. This single centre, prospective observational study done in a 4000 bedded medical university of India from April 2020 to June 2020. The study included all the participants who underwent this training and completed pre \& post-test. The participants were divided based on their prior ICU experience as stated in the information sheet viz. <1 month, 1-6 months \& $\geq 6$ months as Group I, II \& III respectively. The assessment was done using a pre and post-test model containing 25 multiple choice questions with single best answer. Data were summarised as Mean \pm SE (standard error of the mean). Pre and post groups were compared by paired t test. Pre to post change in outcome measures of 3 independent groups were compared by one-way ANOVA \& the significance of mean difference between the groups was done by Newman-Keuls post hoc test. Discrete (categorical) groups were summarised in number $(n)$ and percentage $(\%) \&$ compared by Chi-square $\left(x^{2}\right)$ test. A two-tailed $(a=2) p<0.05$ was considered statistically significant.

Results. 302 doctors were trained for "one day basic intensive care training". Their mean $( \pm \mathrm{SE})$ age was $31.66( \pm 0.30 \mathrm{yrs}), 198(65.6 \%)$ were males, 29 (9.6\%) were faculties, 98 (32.5\%) were Senior Residents and 175 (57.9\%) were Junior Residents. Finally, 252 doctors were analysed who fulfilled the inclusion criteria (Group I; $n=66$, Group II; $n=120$ \& Group III; $n=66$ ).

The mean total marks at pre-test were highest in Group II (II $>$ III $>$ I) [II: $14.77 \pm 0.31$; III: $14.64 \pm 0.41$; I: $11.94 \pm 0.36$ ]. The mean total marks at post-test were highest in Group III (III > II > I) [III: 19.97 \pm 0.46 ; II: $19.42 \pm 0.30$; I: $17.21 \pm 0.55]$. Within each group there was significant $(p<0.001)$ increase in mean total marks at post-test compared to pretest [l: $5.27 \pm 0.31 ;$ II: $4.65 \pm 0.16$; III: $5.33 \pm 0.24]$.

In comparing the difference in mean total marks between the groups, at pre-test, Group II (2.83 marks) and Group III (2.70 marks) had significantly $(p<0.001)$ higher marks as compared to Group I and at posttest, Group II (2.20 marks) and Group III (2.76 marks) had significantly $(p<0.001)$ higher marks as compared to Group I.

Conclusion. The study focused on training non-intensivists for one day through a structured program revealed that such courses can be useful in improving the knowledge. Knowledge gain was similar in all participants irrespective of their duration of prior ICU experience.

\section{Reference(s)}

1. Engberg, M. et al. Training non-intensivist doctors to work with COVID-19 patients in intensive care units. Acta Anaesthesiol. Scand. 65, (2021).

2. Ashcroft, J., Byrne, M. H. V., Brennan, P. A., Davies, R. J. \& Davies, R. J. Preparing medical students for a pandemic: A systematic review of student disaster training programmes. Postgrad. Med. J. (2020) https://doi.org/10. 1136/postgradmedj-2020-137906

3. $\mathrm{Ni}$

\section{1}

A summary review of clinical characteristics and outcomes of patients with Covid-19 admitted to Intensive care in Hull R. Martin'; P. Gunasekera

${ }^{1}$ Intensive and Critical Care Department, Hull Teaching Hospitals NHS Foundation Trust, Hull, United Kingdom

Correspondence: R. Martin

Intensive Care Medicine Experimental 2021, 9(1): 001151

Introduction. As many as $30 \%$ of those infected with Covid-19 will require intensive care treatment with an associated mortality of $40 \%$ (1). Admission PF ratio has been proposed to be independently associated with poor ICU outcome (2). The defining characteristics and admission parameters of patients who will have an eventual poor outcome remains obscure.

Objectives. To explore the relationship between patient characteristics and outcome in Covid-19 patients in ICU and identify trends which might predict care requirements and outcome.

Methods. We conducted an observational retrospective case series review of patients admitted to ICU at Hull Teaching Hospitals University Trust (HUTH) with Covid-19 between March 2020 and March 2021. All patient information was obtained from casenote review and electronic medical records. Only patients with known outcomes were included.

Results. 2206 patients attended HUTH for treatment of Covid-19, 250 received basic respiratory support outside of ICU. 191 were admitted to ICU. Of these $72 \%$ were male, predominantly White-British and had 
a median age of 63 years. $74 \%$ had a high BMI. $40 \%$ had pre-existing hypertension, $20 \%$ had chronic lung disease and $18 \%$ were diabetic. Disease limiting daily activity was recorded in $8 \%$. APACHE II score mean was 15 (range 6-32) with scores evenly distributed across all levels of respiratory support and outcome. The observed ICU mortality was $42.8 \%$ with an uneven distribution towards advanced respiratory support.

The median ratio of arterial partial pressure of oxygen $\left(\mathrm{PaO}_{2}\right)$ to inspired fraction of oxygen $\left(\mathrm{FiO}_{2}\right)(\mathrm{P} / \mathrm{F}$ ratio) ratio on admission was 13.7 (IQR 9-18). 91 patients received basic respiratory support. 45 patients were intubated at the point of admission. 55 received a median of 4 days basic support (IQR 2-7) before being escalated to advanced support.Median PF ratios of 13.3 (IQR 10-18) and 11.5 (IQR 8-16) were observed for the basic and advanced groups respectively. This worsened between wave 1 and Wave 2, falling from a median of 14 (IQR 11-21) to 11 (IQR 8-14) in the basic group and from 13 (IQR $10-20)$ to 10 (IQR 8-12) in the advanced group. The lowest recorded $\mathrm{PaO}_{2}$ had a median value of $6 \mathrm{kPa}$ (range 4.3-7.7 kPa) regardless of month of admission or level of support received.

Conclusion. The hypoxic state of patients being admitted to critical care worsened significantly during the pandemic. Hypoxaemia in isolation was not enough to trigger an escalation to advanced respiratory support. Although a low P/F ratio was associated high mortality in patients receiving advanced care, we found this was less applicable when patients only received basic level respiratory support. Our findings support evidence that traditional severity scoring systems are of limited value in predicting the care trajectory and outcome of patients with Covid-19(3).

\section{Reference(s)}

1. 3. Douville NJ, Douville CB, Mentz G, Pancaro C, Tremper KK, Engoren M (2021) Clinically applicable approach for predicting mechanical ventilation in patients with COVID-19. British Journal of Anaesthesia Vol 126(3) Pg 590

2. 2. Thompson RJ, Hunter J, Dutton J, Schenider J, Khosravi M, Casement A, Dhadwal K, Martin D (2021) Clinical characteristics and outcomes of critically ill patients with COVID-19 admitted to an intensive care unit in London: A prospective observational cohort study. PLoS ONE https://doi. org/10.1371/journal.pone.0243710

3. 1. Abate SM, Ahmed Ali S, Mantfardo B, Basu B (2020) Rate of Intensive Care Unit admission and outcomes among patients with coronavirus: A systematic review and Meta- analysis. PLoS ONE 15(7): e0235653. https:// doi.org/10.1371/journal.pone.0235653

\section{7}

Improving the quality of patient care in the ICU through a latent threats response system building on multidisciplinary in-situ simulation

S. Stallworthy' ${ }^{1}$ D. Worley' ${ }^{1}$ L. Ma

${ }^{1}$ Intensive Care Unit, The Whittington Hospital, London, United Kingdom

Correspondence: $\mathrm{S}$. Stallworthy

Intensive Care Medicine Experimental 2021, 9(1): 001157

Introduction. In-situ simulation training is a well-established educational tool. After the UK's first COVID-19 pandemic surge, our department wanted to ensure quality of patient care was maintained. Latent threats are potential risks to the delivery of effective, safe, and highquality care that are not necessarily revealed through everyday practice but identified through safety analysis. This allows a proactive response to future potential risks with the goal of preventing them from materialising. We present the development and implementation of a verbally-undertaken safety analysis protocol built into our in-situ simulation debriefing sessions on the intensive care unit (ICU) to identify and respond to latent threats to high quality patient care.

Objectives. To integrate a verbal safety analysis into in-situ simulation debriefing sessions to identify latent threats and reach a consensus agreement on a robust response plan to reduce the potential risk of patient harm.

Methods. Utilising existing regular multidisciplinary in-situ simulation sessions, we developed a verbal safety analysis process building on the existing literature on safety analysis in in-situ simulation. Trained faculty members observed in-situ simulations to identify latent threats that posed either a current or potential risk to patient safety. During debriefing sessions, participants were supported in raising latent threats they observed with a reduced risk of framing bias. Faculty meetings after each simulation session discussed the latent threats identified and appropriate risk reduction plans were agreed with the rest of the department. Following this, a repeat simulation on the same topic was undertaken to re-assess latent threats regardless of whether the risk reduction plan had been implemented, to reduce the risk of expectation bias masking additional latent threats.

Results. 9 in-situ simulation sessions were conducted over a 10-month period covering 4 separate themes of patient care. 7 latent threats to patient care were identified with risk reduction plans devised and partially implemented, ranging from direct changes to the ICU environment, adjustments to electronic patient care record proformas, and escalation within the hospital's quality management system. Repeat simulation was undertaken for 2 themes resulting in the identification of 4 new latent threats across 5 repeat sessions. The faculty agreed that additional verbal safety analysis did not burden the delivery of the sessions and enhanced the perceived quality of debriefing sessions.

Conclusion. The additional of verbal safety analysis into in-situ simulation sessions on a COVID ICU was easily undertaken and effective means of identifying latent threats to patient care that were amenable to risk reduction plans without hindering session delivery. Repeat latent threat analysis within the same patient care theme enhanced the utility of the latent threat response program and reduced the risk of expectation bias.

\section{Reference(s)}

1. Couto, T.B., Barreto, J.K.S., Marcon, F.C. et al. Detecting latent safety threats in an interprofessional training that combines in situ simulation with task training in an emergency department. Adv Simul 3, 23 (2018). https://doi. org/10.1186/s41077-018-0083-4

2. Knight $P$, MacGloin H, Lane M, Lofton L, Desai A, Haxby E, Macrae D, Korb C, Mortimer P, Burmester M. Mitigating latent threats identified through an embedded in situ simulation program and their comparison to patient safety incidents: a retrospective review. Frontiers in pediatrics. 2018 Feb 1:5:281.

3. Goldshtein D, Krensky C, Doshi S, et alln situ simulation and its effects on patient outcomes: a systematic reviewBMJ Simulation and Technology Enhanced Learning 2020;6:3-9

4. Patterson MD, Geis GL, Falcone RA, et alln situ simulation: detection of safety threats and teamwork training in a high risk emergency departmentBMJ Quality \& Safety 2013;22:468-477.

5. Rosen, M.A. Hunt, E.A., Pronovost, P.J.,Federowicz, M.A. and Weaver, S.J. (2012), In situ simulation in continuing education for the health care professions: A systematic review. J. Contin. Educ. Health Prof., 32: 243-254. https://doi.org/10.1002/chp.21152

6. No grants or funding have been received for this work

\section{0}

Post Intensive Care Syndrome in patients with cancer following critical illness

J. O'Neil ${ }^{1}$; P. Henderson ${ }^{1}$; M. Shaw, ${ }_{1}^{1}$; J. McPeake ${ }^{1}$; T. Quasim²; D. Morrison ${ }^{1}$; P. Kathryn ${ }^{2}$

'School of Medicine, University of Glasgow, Glasgow, United Kingdom; ${ }^{2}$ Intensive Care Unit, Glasgow Royal Infirmary, Glasgow, United Kingdom Correspondence: $J$. O’Neil

Intensive Care Medicine Experimental 2021, 9(1): 001160

Introduction. PICS is a relatively newly reported syndrome and describes physical, cognitive, and psychological symptoms resulting after a critical illness. Symptoms can include neuromuscular weakness, chronic pain, impairments of memory and attention and anxiety and depression [1]. It can also present as PICS-Family in patients' caregiver's who may suffer from similar psychological symptoms [1] One potential method of reducing severity and symptom burden is a post-ICU clinic in order to screen patients for symptoms of PICS and address any that arise by referring them to relevant care providers. An 
example is the Intensive Care Syndrome: Promoting Independence and Return to Employment (In:SPIRE) study where data for this study was collected.

Objectives. This study evaluates the impact of Post Intensive Care Syndrome (PICS) in patients both with and without cancer, whilst also considering the impact on their families, through the use of standardised questionnaires in the hopes of assessing any differences in symptom prevalence and severity.

Methods. 206 patients were part of the In:SPIRE study, at Glasgow Royal Infirmary, which evaluated the use of a Post-ICU clinic. Any patient receiving level 3 care or level 2 care for more than 7 days was eligible. Exclusion criteria included a terminal diagnosis, traumatic brain injury and psychiatric inpatients. Cancer patients required a diagnosis no more than 5 years before or within 1 month of admission with 29 meeting these criteria. Propensity score matching was used to account for differences in baseline characteristics between cancer and non-cancer patients and created a cohort of 29 non-cancer patients from 159.

Results. No statistically significant differences were found in the patient outcomes measures between the two cohorts. EQ5D scores which looked at Health-related quality of life were similar for the two cohorts. Cancer patients reported slightly more interference from pain in their daily lives as well as a higher worst pain score on the Brief Pain Inventory and higher rates of anxiety and depression in the Hospital Anxiety and Depression Scale (HADS). However, these differences were not statistically significant. This was the same for caregiver outcomes using HADS and the caregiver strain and insomnia severity indices. Conclusion. PICS is an area of critical care requiring further research including on how underlying diseases processes can alter symptom prevalence and severity. However, this study found no differences in patients with cancer or their caregivers, indicating that they should therefore be managed the same as any other post-ICU patients.

\section{Reference(s)}

1. 1. Needham DM, Davidson J, Cohen H, Hopkins RO, Weinert C, Wunsch H, et al. Improving long-term outcomes after discharge from intensive care unit: Report from a stakeholders' conference*. Critical Care Medicine. 2012 Feb:40(2):502-9.

\section{8}

\section{Assessing knowledge gap of emergency department nurses} and physicians regarding ischemic strokes

C. Rossis'; E. Giannelou'; M. Karanikola'; C. Andreou'; N. Middleton ${ }^{1}$; G.

Papageorgiou'; E. Papathanassoglou²; M. Mpouzika ${ }^{1}$

'Nursing Department, Cyprus University of Technology, Limassol, Cyprus;

${ }^{2}$ Faculty of Nursing, University of Alberta, Edmonton, Canada

Correspondence: C. Rossis

Intensive Care Medicine Experimental 2021, 9(1): 001168

Introduction. Data show that ischemic stroke is one of the leading causes of death and disability worldwide (1-3). Knowledge of ischemic stroke-related guidelines is vital for health care professionals working in the Emergency Departments (EDs) because it affects the early diagnosis and timely treatment to combating stroke (4).

Objectives. The investigation of knowledge of Cyprus health care personnel regarding the recognition and treatment of the ischemic stroke.

Methods. This was a descriptive cross-sectional correlation study. From November 2019 to April 2020 Greek-speaking nurses and physicians employed in 3 private and 7 public EDs in Cyprus were invited to complete anonymously a self-administered questionnaire developed by a multidisciplinary group of experts in stroke based on the latest guidelines (2018) of American Stroke Association.

Results. 243 nurses [Response Rate (RR): 74.34\%] and 26 physicians (RR; $46.42 \%$ ) completed the questionnaire. $94 \%$ of the participants responded that EDs play an important role in the rapid recognition and treatment of ischemic stroke. Previous education based on ischemic strokes is correlated in higher levels of knowledge with Mean difference $=1.4(t=8.423$ and $p=0.004)$ with $128(48.51 \%)$ participants stated that they had previous education. Regarding overall knowledge, nurses and physicians reported poor to moderate (Mean: $12.6 \pm 4.1$; Scale Range: 1-28) and moderate (Mean: 15.7 \pm 4 ) level, respectively. Awareness of IS was higher among physicians $(p<0.001)$. Conclusion. Cypriot health care professionals in EDs reported poor to moderate knowledge about ischemic stroke and further study of factors related to this maybe of interest. Also, the development and implementation of evidence-based protocols and enhanced education regarding ischemic stroke should be considered essential interventions for emergency health care professionals.

\section{Reference(s)}

1. - (1) Stevens, E. G. V. et al. (2017) The burden of stroke in Europe. Available at http://www.strokeeurope.eu/downloads/TheBurdenOfStrokelnE uropeReport.pdf. • (2) Virani, S. S. et al. (2020) 'Heart disease and stroke statistics-2020 update: A report from the American Heart Association', Circulation. Lippincott Williams and Wilkins, pp. E139-E596. https://doi. org/10.1161/CIR.0000000000000757. • (3) WHO | Stroke, Cerebrovascular accident' (2015b) WHO. World Health Organization. Available at: http://www.who.int/topics/cerebrovascular_accident/en/ • (4) Powers, W. J. et al. (2019) Guidelines for the Early Management of Patients with Acute Ischemic Stroke: 2019 Update to the 2018 Guidelines for the Early Management of Acute Ischemic Stroke: A Guideline for Healthcare Professionals from the American Heart Association/American Stroke, Stroke. https://doi.org/10.1161/STR.0000000000000211

001179

Survival After Intensive Care Unit Admission in Cancer Patients: A Matched Cohort Study

E. Cullen ${ }^{1}$; K. Puxty ${ }^{2}$; D. Morrison

${ }^{1}$ School of Medicine, University of Glasgow, Glasgow, United Kingdom;

${ }^{2}$ Academic Unit of Anaesthesia, critical care and Peri-operative Medicine, University of Glasgow, Glasgow, United Kingdom

Correspondence: E. Cullen

Intensive Care Medicine Experimental 2021, 9(1): 001179

Introduction. Admission to the Intensive Care Unit (ICU) is an important part of cancer treatment providing post-operative care and treatment for complications of cancer therapies or immunocompromised events. Despite its necessary use, ICU is traumatic so risk calculations are performed to ensure only patients with a reasonable expectation of survival are admitted. Cancer has been often cited as a reason for refusal of ICU admission due to perceived low survival and high personal cost. However, there is limited information regarding impact of ICU on long term survival of cancer patients who survive ICU treatment

Objectives. This study aims to compare outcomes among cancer patients discharged from ICU with a matched group who were not admitted to ICU.

Methods. A retrospective, observational cohort study using data from four linked Scottish datasets was designed. 3509 cancer patients who had survived ICU treatment were matched to 3444 non-ICU cancer patients. A descriptive analysis of both groups was performed. T-test of independent samples was used to test differences in means between cohorts and Pearson's Chi-square used to test differences in distributions of categorical variables. Kaplan-Meier curves and log-rank tests were used to compare survival up to 5 years between the ICU and the nonICU cancer patients. Curves were also constructed to compare survival depending on admission type; non-ICU, elective and emergency admission. A univariate and multivariable logistic regression was carried out to assess effects of different variables on 1- and 5-year survival.

Results. There was no statistically significant difference between survival of ICU and non-ICU patients $(p=0.099)$ and median survival times were 38.3 months (34.0-43.2) and 36.0 months (31.4-41.7) respectively. Survival of cancer patients stratified by admission type found elective ICU patients had the best survival outcomes followed by non-ICU patients and finally emergency ICU patients $(p<0.001)$. Median survival times were 22.3 months, 36.0 months and 56.0 months respectively. Multiple logistic regression analysis showed elective admission significantly increased survival odds at 30 days. Elective admission, female sex and higher SIMD score increased survival at 5 years. 
Conclusion. Cancer patients admitted to ICU after elective admission are likely to have long term outcomes that are as good as, or better than, cancer patients not admitted to ICU. However, ICU support following emergency admission has a poorer prognosis. Consequently, cancer patients should not be excluded from ICU based on underlying malignancy alone and instead factors associated with survival should be considered.

\section{Reference(s)}

1. Koutsoukou A. Admission of critically ill patients with cancer to the ICU: many uncertainties remain. ESMO Open. 2017;2(4):e000105. Published 2017 Oct 12. https://doi.org/10.1136/esmoopen-2016-000105

2. McDermid RC, Bagshaw SM. Prolonging life and delaying death: the role of physicians in the context of limited intensive care resources. Philos Ethics Humanit Med. 2009:4:3. Published 2009 Feb 12. https://doi.org/10. 1186/1747-5341-4-3

\section{6}

\section{Evaluation of clinical severity scores in predicting ICU mortality}

\section{for patients with COVID-19}

M. Sileli'; E. Lazoudi'; E. Kerezidou'; I. Karageorgiou'; E. Siomos'; A.

Kosmas ${ }^{1}$; D. Kampranis ${ }^{1}$; N. Kapravelos ${ }^{1}$; C. Iasonidou

'B ICU, GENERAL HOSPITAL "GEORGE PAPANIKOLAOU", Thessaloniki,

Greece, Greece

Correspondence: M. Sileli

Intensive Care Medicine Experimental 2021, 9(1): 001186

Introduction. The assessment of disease severity in COVID-19 patients on ICU admission is challenging. It could effectively help in clinical decision-making and in guiding treatment options.

Objectives. We aimed to assess the correlation of severity indexes APACHE II, SOFA score and the newly introduced COVID-Gram Critical illness Risk score and Veterans Health Administration COVID-19 (VACO index) with ICU mortality. The secondary endpoint was to evaluate and compare their predictive capacity.

Methods. Observational single-center cohort study on PCR confirmed COVID-19 severe pneumonia adult patients that were necessitated mechanical ventilation (December 2020-May 2021). Patients who succumbed within the first $24 \mathrm{~h}$ of admission were excluded. Demographics, Body mass index (BMI), ICU length of stay (ICU LOS), comorbidities expressed through Charlson's comorbidity index $(\mathrm{CCl})$ and ICU mortality were recorded. APACHE II, SOFA score, COVID-Gram Risk score and VACO index were calculated for each patient within the first $24 \mathrm{~h}$ of ICU admission. Categorical data were compared with the chi-square test, while continuous data with the Mann-Witney $\mathrm{U}$ test. Receiver operating characteristics (ROC) curves were constructed for the scores mentioned above. The area under the ROC curve (AUC) was calculated to evaluate and compare their prediction performance on ICU mortality.

Results. We analyzed 67 COVID-19 patients with a median age of 62 (IQR: 55 to 69.5 ); $67 \%$ were males. Patients had a median BMI of 31 (IQR: 27 to 36.5) and a median CCl of 2 (IQR: 1 to 3). The median APACHE II score was 16 (IQR: 13 to 18), and the median SOFA score 7 (IQR: 6 to 8). The overall ICU mortality was $37 \%$, with a median length of ICU stay of 17 (IQR: 8.5 to 22) days. Regarding mortality, no statistically significant difference was observed between survivors and nonsurvivors in terms of sex, age and BMI ( $p>0.05)$. In contrast, patients who didn't survive had increased comorbidities quantified by the $\mathrm{CCl}$ ( 3 vs $2, p=0.009$ ) and longer median ICU LOS ( 21 days vs 13 days, $p=0.016$ ). There was no evidence of differences in severity risk scores APACHE II, SOFA score and COVID-Gram risk score between survivors and non-survivors $(p>0.05)$, except for VACO index (5 vs 7 respectively, $p=0.035$ ). The tested severity scoring systems showed low predictive accuracy in the studied population. Based on the ROC curves, the Apache II score had the worst discriminative ability (AUC: 0,518, $95 \% \mathrm{Cl}: 0,393$ to 0,642 ). The AUC was 0,547 (95\% Cl: 0,421 to 0,669$)$ and 0,622 ( $95 \%$ Cl: 0,495 to 0,738 ) for the COVID-Gram Risk score and SOFA score respectively. Although the VACO index performed better, it still had poor accuracy (AUC: $0.655,95 \% \mathrm{Cl}: 0.529$ to 0.767 ).
Conclusion. According to our findings, only the VACO index showed a significant correlation with mortality. Nevertheless, none of the severity scores support any reliable predictive performance. There is a need for more extensive studies to trace the impact of these scores on ICU COVID-19 patients.

\section{Reference(s)}

1. Zou Xiaojing, Li Shusheng, Fang Minghao, Hu Ming, Bian Yi, Ling Jianmin, Yu Shanshan, Jing Liang, Li Donghui, Huang Jiao. Acute Physiology and Chronic Health Evaluation II Score as a Predictor of Hospital Mortality in Patients of Coronavirus Disease 2019. Crit Care Med. 2020 Aug;48(8):e657-e665

2. None.

\section{6}

Peripheral nerve injuries and Covid pneumonitis: Who is prone? E. Barton ${ }^{1}$; G. Crosbie'; J. Livesey ; S. Hobson²; J. Harvey²; A. Abu-Arafeh ${ }^{3}$; E. Wilson'

${ }^{1}$ Royal Infirmary of Edinburgh, Department of Critical Care, NHS Lothian, Edinburgh, United Kingdom; ${ }^{2}$ Royal Infirmary of Edinburgh, Department of Physiotherapy, NHS Lothian, Edinburgh, United Kingdom; ${ }^{3}$ Western General Hospital, Department of Critical Care, NHS Lothian, Edinburgh, United Kingdom

Correspondence: E. Barton

Intensive Care Medicine Experimental 2021, 9(1): 001196

Introduction. The importance of multi-disciplinary critical care followup in influencing the recovery profile for ICU survivors is widely recognised. These services holistically identify and manage unmet health needs associated with post intensive care syndrome (PICS) and currently play an essential added role in characterising the long term sequelae associated with COVID-19.

Prone ventilation reduces mortality in patients with acute respiratory distress (1), a common feature of COVID-19 pneumonitis. However this intervention is not without risk. Recent small studies have identified a concerning trend of nerve injury, potentially related to prone positioning (2). At our ICU recovery clinic we also identified patients with peripheral nerve injuries and questioned an association with our prone positioning technique.

Objectives. Quantify peripheral nerve injuries in recovery clinic patients, clarify any association with prone positioning, address changes in practice required with a quality improvement process.

Methods. A retrospective cohort study was undertaken which identified all mechanically ventilated patients with a diagnosis of COVID-19 pneumonitis admitted to the Royal Infirmary Edinburgh ICU between $15 / 03 / 2020$ and $25 / 12 / 2020$. Patients with persisting symptoms of PICS, including peripheral nerve injury were invited to the ICU recovery clinic, held 2-3 months post hospital discharge. Additional patients referred to the clinic from other hospital sites within our Health Board, with similar proning protocols, were also included. Peripheral nerve injuries were diagnosed by a consultant intensivist and/or a senior physiotherapist. Results. Thirty five patients recovering from COVID-19 pneumonitis were included in the study cohort. The results are shown in Table 1, with subgroup analysis of patient demographics and ventilation/proning requirements. A spectrum of nerve injuries were evident, affecting 13 (37\%) patients who attended the clinic. The nature of the nerve injuries implicated prone positioning as a factor in the aetiology. Only one patient with a nerve injury did not undergo prone positioning. This patient had meralgia paraesthetica, which can occur de novo in patients with obesity and diabetes mellitus, both of which were present in this case.

We subsequently made adaptations to our prone ventilation guidance document (Figure 1) to address these findings with a focus on shoulder, upper limb, pelvis and knee positioning. 


\begin{tabular}{|c|c|c|}
\hline TABLE 1: Study cohort $(\mathrm{N}=35)$ & & \\
\hline Nerve injury, $\mathbf{N}(\%)$ & \multicolumn{2}{|c|}{$13(37 \%)$} \\
\hline Injury Classification & \multicolumn{2}{|r|}{$\mathrm{N}$} \\
\hline Meralgia paresthetica & \multicolumn{2}{|r|}{7} \\
\hline Multiple upper limb neuropathies & \multicolumn{2}{|r|}{2} \\
\hline Brachial plexopathy & \multicolumn{2}{|r|}{1} \\
\hline Bilateral ulnar nerve injury & \multicolumn{2}{|r|}{1} \\
\hline Foot drop & \multicolumn{2}{|r|}{2} \\
\hline$\underline{\text { Subgroup Analysis }}$ & Nerve injury $(N=13)$ & No Nerve injury $\mathrm{N}=(22)$ \\
\hline Age years, median (IQR) & $47.1(43,57)$ & $56(50,63)$ \\
\hline BMI, median (IQR) & $38.2(3142)$ & $30.7(22,33)$ \\
\hline Diabetes Mellitus (\%) & $30 \%$ & $18 \%$ \\
\hline Requiring prone positioning (\%) & $91 \%$ & $67 \%$ \\
\hline Required $\geq 2$ episode of proning (\%) & $63 \%$ & $38 \%$ \\
\hline Number of days requiring ventilation, median (IQR) & $24.5(9,38)$ & $24(11,33)$ \\
\hline \multicolumn{3}{|c|}{$\begin{array}{l}\text { Table } 1 \text { key: Summary of total number of peripheral nerve injuries identified in the recovery clinic. Nerve injury } \\
\text { classification describes meralgia paresthetica, a syndrome resulting from compression of the lateral femoral cutaneous } \\
\text { nerve. Multiple upper limb nerve injuries and brachial plexopathy describe cases with } 2 \text { or more upper limb neuropathies } \\
\text { or brachial plexus injury identified by clinical examination or neurophysiology investigations. Foot drop describes patients } \\
\text { with clinical examination consistent with common peroneal nerve palsy, both loss of ankle dorsi-flexion and sensory } \\
\text { impairment over the dorsum and lateral aspect of foot. Other factors relevant to patient demographics and ICU } \\
\text { interventions were compared for patients with and without a peripheral nerve injury. }\end{array}$} \\
\hline
\end{tabular}

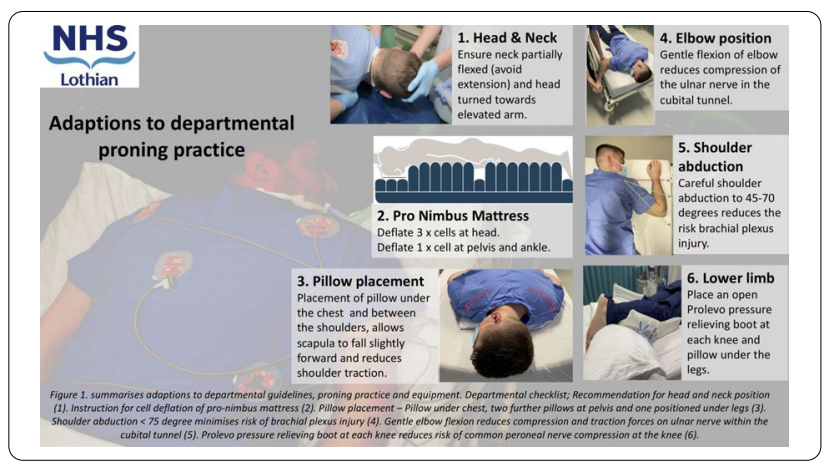

Conclusion. This study highlights the importance of a multi-disciplinary ICU recovery clinic in identifying problems, which can inform a quality improvement process to enhance current practice, improve patient outcomes and minimise complications.

\section{Reference(s)}

1. 2) Caroline Miller, Joel O'Sullivan, Jack Jeffrey, Dominic Power, Brachial Plexus Neuropathies During the COVID-19 Pandemic: A Retrospective Case Series of 15 Patients in Critical Care, Physical Therapy, Volume 101, Issue 1, January 2021, https://doi.org/10.1093/ptj/pzaa191.

2. 1) Guérin C, Reignier J, Richard JC, Beuret P, Gacouin A, Boulain T, Mercier E, Badet M, Mercat A, Baudin O, Clavel M, Chatellier D, Jaber S, Rosselli S, Mancebo J, Sirodot M, Hilbert G, Bengler C, Richecoeur J, Gainnier M, Bayle F, Bourdin G, Leray V, Girard R, Baboi L, Ayzac L; PROSEVA Study Group. Prone positioning in severe acute respiratory distress syndrome. N Engl J Med. 2013 Jun 6;368(23):2159-68. https://doi.org/10.1056/NEJMo a1214103. Epub 2013 May 20. PMID: 23688302

\section{5}

Thirty-day mortality externally validated prediction model of critically ill patients undergoing percutaneous dilational tracheostomy: two center cohort study

J. Nashashibi'; A. Miller ${ }^{2}$

${ }^{1}$ Internal Medicine D, Rambam Healthcare Campus, Haifa, Israel; ${ }^{2}$ Medical Intensive Care Unit, Rambam Healthcare Campus, Haifa, Israel

Correspondence: J. Nashashibi

Intensive Care Medicine Experimental 2021, 9(1): 001215
Introduction. Tracheostomy is a common procedure in critically ill patients; nearly $10 \%$ of patients requiring mechanical ventilation (MV) in the intensive care unit (ICU) receive a tracheostomy tube. Although mortality is high among these patients, risk stratification models after tracheostomy are lacking.

Objectives. The aim of our study was to develop and validate a 30-day mortality prediction model that could be used as a risk stratification tool in ICU patients undergoing percutaneous dilational tracheostomy (PDT).

Methods. Database driven retrospective cohort study using electronic medical records between the years 2009-2019 in two tertiary care university-affiliated hospitals: Rambam Health Care Campus (RHCC) and Beilinson Rabin Medical Center (BRMC) were used as derivation and validation cohorts, respectively. The main outcome was 30-day mortality after PDT in ICU patients. Independent factors included demographics, comorbidities, kidney function, albumin, lactate, hemoglobin, platelets, white blood cell count and partial pressure of carbon dioxide $\left(\mathrm{pCO}_{2}\right)$. Predictive score was developed and validated externally using multivariate logistic regression with the backward stepwise elimination method. Discrimination (c-statistic) and calibration of the model was assessed by area under the receiver operating characteristic (AUROC) curve and the Brier score, respectively.

Results. PDT was performed on 945 patients at RHCC between the years 2009-2019, and on 503 patients at BRMC general ICU between the years 2013-2018. Mortality at 30-days was 281 (28.7\%) and 110 (21.9\%), respectively. The c-statistic of the model was $0.80(95 \% \mathrm{Cl}$ $0.77-0.83)$ and $0.74(0.68-0.79)$ for the derivation (RHCC) and validation (BRMC) cohorts, respectively. A good calibration of the model was achieved in both cohorts, with Brier scores of 0.158 and 0.151. After adjusting for demographic, comorbidities, kidney function, albumin, $\mathrm{pCO}_{2}$ and complete blood counts; the only comorbidities correlated with 30-days mortality were diabetes mellitus (OR 1.52 with $95 \% \mathrm{Cl}$ 1.10-2.28) and chronic obstructive pulmonary disease (OR 1.82 with $95 \% \mathrm{Cl} 1.16-2.85)$

Conclusion. We have developed and externally validated a model for 30-day mortality after PDT in ICU patients with a good discrimination and calibration ability. This model could be used as a tool for risk-stratification in ICU patients undergoing PDT as those in a highrisk group might benefit from a more intensive care and vice versa. Further studies are required to evaluate whether patients at very high risk might benefit from a prolonged stay in the ICU or high dependency unit or whether the procedure might prove futile under certain circumstances.

\section{Reference(s)}

1. Cinotti R, Voicu S, Jaber $\mathrm{S}$, et al. Tracheostomy and long-term mortality in ICU patients undergoing prolonged mechanical ventilation. PLoS One 2019;14(10):e0220399.

2. Lone NI, Walsh TS. Prolonged mechanical ventilation in critically ill patients: epidemiology, outcomes and modelling the potential cost consequences of establishing a regional weaning unit. Crit Care 2011;15(2):R102.

\section{4}

Elderly patients admitted to the ICU due to COVID-19: characteristics and risk factors for mortality

E. Maneta'; G. Ntoumas ${ }^{1}$; S. Kokkoris ${ }^{1}$; A. Gkoufa' ${ }^{1}$;. Mizi ${ }^{1}$;. Sigala'; E. Dima ${ }^{1}$;T. Ntaidou'; A. Kanavou ${ }^{1}$; Y. Papachatzakis ${ }^{1}$; V. Markaki' ${ }^{1}$; D.

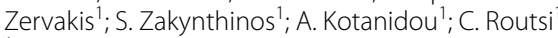

${ }^{1}$ Department of Intensive Care, Evangelismos Hospital, Medical School, National and Kapodistrian University of Athens, Athens, Greece

Correspondence: $C$. Routsi

Intensive Care Medicine Experimental 2021, 9(1): 001224

Introduction. Within the context of Coronavirus disease 2019 (COVID19) pandemic, elderly patients are at particularly high risk for severe disease, requiring intensive care unit (ICU) admission. However, sometimes, especially in insufficient hospital resources, advanced age is included in the triage criteria (1). While many studies on elderly adults 
with COVID-19 in non-ICU wards have been published, there is limited information about those admitted to the ICU (2).

Objectives. To provide information concerning clinical features and outcome of patients $\geq 70$ years old admitted to the ICU due to COVID-19.

Methods. Observational study, conducted in the COVID-ICUs at Evangelismos Hospital, Athens, Greece from March 19, 2020 to April 24,2021 . All consecutive patients $\geq 70$ years of age, with confirmed COVID-19, were enrolled. No age restrictions were applied during the study period concerning ICU admission because hospital capacity had not been overwhelmed by the COVID-19 pandemic. Clinical characteristics were compared between survivors and non-survivors.

Results. Out of 461 patients admitted to the COVID ICUs during the study period, $183(40 \%)$ patients were $\geq 70$ years old (range 70-102 years) and were included in this analysis. ICU mortality was $65 \%$ overall and $71 \%$ in the subgroup of patients with invasive mechanical ventilation. Non-survivors compared to survivors, were older (78 vs. 74 years, $p<0.001$ ), had higher illness severity scores (APACHE II score 20 vs. 14, $p<0.001$, SOFA score 9 vs $4, p<0.001$, Charlson comorbidity index 5 vs. $4, p<0.004)$, higher values of creatinine, troponin, lactate and d-dimers, suffered a more severe shock and required more frequently mechanical ventilation ( $100 \%$ vs. $67 \%$, $p<0.001)$ and continuous renal replacement therapy $(40 \%$ vs $10 \%$, $\mathrm{p}<0.001$ ). Regarding comorbidities, no statistically significant difference between survivors and non-survivors was found, except for the presence of chronic kidney disease $(18 \%$ vs. $2 \%, \mathrm{p}<0.01)$. Cox regression analysis showed that age remained an independent risk factor for mortality, after adjustment for confounding factors (OR 1.045; $\mathrm{Cl}$ : $1.02-1.07, \mathrm{p}=0.001)$.

Conclusion. When hospital capacities have not been overwhelmed, critically ill patients $\geq 70$ years old represent a high proportion of COVID-19 ICU patients and present high mortality. Severity of illness and age are independent risk factors for mortality.

\section{Reference(s)}

1. 1. LEM Haas et al. Intensive Care Med (2020) 24:321 2. M Dres et al. Ann Intensive Care (2021) 11:77

\section{7}

\section{Burnout experience in the ICU staff during COVID-19 pandemic} in Ukraine

M. Grynovska'; A. Wong ${ }^{2}$

${ }^{1}$ Anesthesia and Intensive Care, Ivano-Frankivsk National Medical

University and Regional University Teaching Hospital, Ivano-Frankivsk, Ukraine; ${ }^{2}$ Critical care, Royal Surrey County Hospital, Guildford, United Kingdom

Correspondence: M. Grynovska

Intensive Care Medicine Experimental 2021, 9(1): 001227

Introduction. The COVID-19 outbreak has resulted in unprecedented healthcare crisis with high prevalence of psychological distress in healthcare providers. $1 \mathrm{lt}$ is well recognized that the pandemic is puttting critical care under extreme pressure. 2 The ICM workforce are particularly susceptible to burnout, rating higher on stress, burnout (BO) and compassion fatigue indices. This may lead to poor patient safety outcomes.3 In Ukraine, specifically, the concept of BO in ICU is largely under recognized. We sought to document the unfolding impact of burnout on ICU staff in the times of pandemic.

Objectives. To assess the burnout experience and identify associated risk factors in ICU staff facing COVID-19 outbreak

Methods. The focus of the study was the ICU staff in several different hospitals in Ukraine. The final sample size was nurses $(n=139)$ and doctors $(n=162)$. Data collection was performed with the help of the Maslach Burnout Inventory (MBI) survey. The BO rate was assessed across various ICU types, seniority, gender, age groups, university affiliation and individual ethical climate rating.

Results. The response rate was $70 \%$. The overall prevalence of BO in ICUs was $84.5 \%$ (nurses $88 \%$, doctors $81 \%$ ). There was no difference in $\mathrm{BO}$ subscales between doctors and nurses $(95 \% \mathrm{Cl}, \mathrm{P}>0,05)$. We also found no statistically significant difference in the incidence of $\mathrm{BO}$ between doctors and nurses $(P>0,05)$. Our study showed no difference $(P>0,05)$ in the incidence of $B O$ in doctors across ICU type, seniority level, gender or age group. In contrast, there was a difference across university affiliation and ethical climate $(P<0.005)$. In nurses we found statistically significant difference in the incidence of $\mathrm{BO}$ across seniority and ICU type $(P<0,05)$.

Conclusion. BO prevalence has notably increased in both ICU nurses and doctors, compared to previous survey results, which reflects the pandemic toll, but also an increase in BO awareness among ICM community. Our findings indicate that doctors and nurses are equally at risk of $\mathrm{BO}$. The study showed a clear association between the incidence of $\mathrm{BO}$ and academic commitments, and low ethical climate rating in doctors. With regard to nurses, seniority and the ICU type proved to be a factor-possibly due to shortage of staff and logistic support in the respective units. Limitations of our study include a relatively small number of respondents that prevents from drawing conclusions in the context of a country/healthcare system. Further research is warranted in hospitals across Ukraine to determine BO rates and risk factors. We hope obtained data will contribute to early recognition of BO.

\section{Reference(s)}

4.Elbay RY eat al., Psychiatry Res. 2020; 290:113130.

3. Hall LH et al., PLoS One.2016;11(7):e0159015

2. Lai J et al., JAMA Netw Open. 2020;3(3):e203976.

1. Azoulay et al. Ann. Intensive Care (2020) 10:110

5. I would like to thank ESICM NEXT Mentoriship Program and my mentor Adrian Wong for supervision and guidance.

\section{4}

Quality improvment for implementing a new therapy in COVID-19 ICU; tocilizumab for everyone

M. Abusayed ; N. Navaneetham, ; A. Salama, ${ }^{1}$; S. Brooks, ; ${ }^{1}$ S. Ahmed, ${ }^{1}$; A.

Garg,

${ }^{1}$ Deparment of Anaesthetics and Critical Care, Walsall Healthcare NHS

Trust, Walsall, United Kingdom

Correspondence: M. Abusayed

Intensive Care Medicine Experimental 2021, 9(1): 001234

Introduction. Tocilizumab (TOC) is an IL- 6 inhibitor that was introduced in our ICU to treat patients with COVID-19 based on the REMAPCAP trial [1]. TOC should be administered in the first $24 \mathrm{~h}$ from ICU admission and respiratory support [1]. The strict inclusion and exclusion criteria and the short administration time window form a challenge for the ICU team to adhere to the prescription guidelines. We conducted a Quality Improvement Project (QIP) to improve the prescription and administration of tocilizumab to COVID ICU patients based on the REMAP-CAP protocol.

Objectives. The main objective was to administer Tocilizumab to all eligible COVID-19 ICU patients within $24 \boldsymbol{h}$ of admission, in addition, we aimed to ensure all patients administered Tocilizumab were screened for other infections as per protocol.

Methods. The Standard Operation Procedure (SOP) was based on strict REMAP-CAP trial inclusion and exclusion criteria, along with a few local modifications. A clear flowchart was included in this SOP, containing screening and assessing patients according to the guidelines. The QIP was conducted between January to March 2021 in COVID Wave 2 of the UK for patients admitted to our ICU in a District General Hospital. We collected patient data for admission details, eligibility for Tocilizumab, details of prescription, viral screening and hospital outcome. The data were collected using REDCap ${ }^{\mathrm{TM}}$, a secure web application used by various research studies. No ethical approval or consent was required for this QIP since the SOP was approved by hospital administration as the standard of care for Covid ICU patients. We used Plan Do See Act (PDSA) cycles to guide our improvement. We included pharmacists and senior ICU nurses as stakeholders for the MDT approach.

Results. We admitted a total of 70 patients to our ICU during this period; 43 patients $(61.4 \%)$ of them were eligible for TOC. From the eligible patients, 38 patients $(88.4 \%)$ received the medication, and 5 patients $(11.6 \%)$ missed the administration window. We found 27 
patients $(38.5 \%)$ were not eligible for TOC. The most common exclusion cause was high procalcitonin levels (14 patients, $51.9 \%$ ) followed by high ALT 5 (5 patients, 18.5\%). We conducted 2 PDSA cycles and several micro-PDSA cycles to guide improvement: 1-PDSA-1: SOP created and education provided in form of a webinar among local staff within the ICU team. 2-PDSA-2: We added admission time, TOC status and Procalcitonin levels to our handover sheets. Daily reminders and discussions during handover acted as micro-PDSA cycles. The impact of the PDSA cycles is shown in the run chart (Figure 1). Changing the culture within ICU was the main factor for improvement.

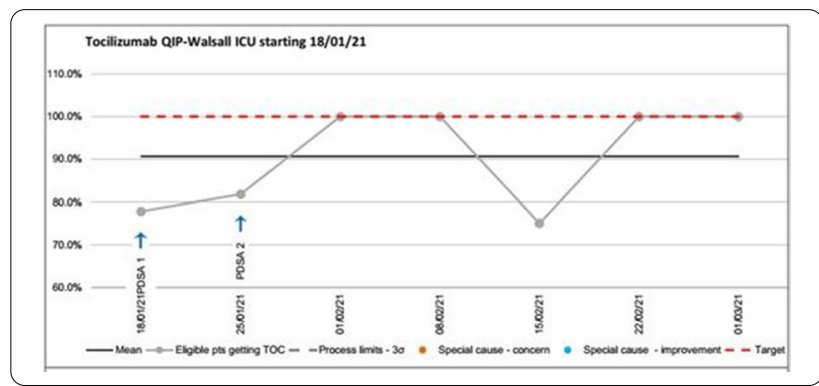

\section{Figure 1 Run chart for the QIP}

During mid-February 21, our Covid admissions had reduced significantly, and an eligible patient was missed. Viral screening as part of the SOP was done in only $9(23.7 \%)$ of patients given TOC. This may have been related to poor buy-in from the ICU staff. Although patients who got TOC showed less mortality (Table 1), it was not found to be statistically significant in this small sample.

\begin{tabular}{lll}
\hline $\begin{array}{l}\text { TOC prescribed Vs discharge } \\
\text { status }\end{array}$ & \multicolumn{2}{l}{$\begin{array}{l}\text { Discharged alive from the } \\
\text { hospital? }\end{array}$} \\
\cline { 2 - 3 } Was TOC prescribed? & No & Yes \\
\hline No & $(59 \%) 19$ & $13(41 \%)$ \\
Yes & $15(39 \%)$ & $23(61 \%)$ \\
\hline
\end{tabular}

\section{Table 1. Mortality outcome}

Conclusion. $-1-60 \%$ of patients were eligible for TOC. Of those, $88 \%$ received TOC within $24 \mathrm{~h}$ of admission -2- High PCT was the main reason for not being eligible for TOC -3-Conducting two PDSA cycles including education and modifying the handover sheets assisted in reaching our target -4-MDT approach supported the effective implementation of a new line of treatment during a difficult pandemic time.

\section{Reference(s)}

1. 1) New England Journal of Medicine, 2021. Interleukin-6 Receptor Antagonists in Critically III Patients with Covid-19. 384(16), pp.1491-1502.

\section{7}

COVID-19 pandemic in a general ICU in the North East of England - a Population Descriptor

J. Thompson ${ }^{1}$; J. Jones, ${ }_{1}^{\text {; }}$ A. Roy, ${ }_{1}^{1}$ : P. Mcandrew, ; A. Rostron,

'Integrated critical care unit, Sunderland Royal Hospital, Sunderland, United Kingdom

Correspondence: J. Thompson

Intensive Care Medicine Experimental 2021, 9(1): 001247

Introduction. Patients admitted to ICCU in Sunderland had an increased mortality rate $(44.6 \%)$ compared to national data $(39.7 \%)$ [1]. The cause of this is unclear but likely related to social and health inequalities.

Methods. Data was collected from 112 patients admitted to ICCU with PCR positive/clinically suspected COVID-19 infection admitted from $19 / 09 / 20$ to $31 / 01 / 21$. This was compared against national
Intensive Care National Audit \& Research Centre (ICNARC) data [1] Social deprivation quintile scores were obtained from English indices of deprivations webtool (https://tinyurl.com/y3vqql3q) and compared.

Results. Patient demographics were similar to those in the national dataset (Table 1), with key differences seen in support and outcomes. A significant proportion of patients had cardiometabolic risk factors: $50.4 \%$ were obese, $48.4 \%$ were hypertensive and $33.6 \%$ were diabetic. All of these are known to be significant risk factors for worse outcomes in COVID-19 patients [2].

COVID-19 has worse outcomes in socially deprived areas [3], Sunderland and the surrounding area is known to be an area of high social deprivation [4]: $50.4 \%$ of the cohort were in the lowest quintile for social deprivation compared with $29.5 \%$ nationally.

Table 1. Demographics and mortality rates for Sunderland ICCU and national critical care patients

\begin{tabular}{lll}
\hline & SRH & National \\
\hline Age (Median (IQR)) & $63(55,66)$ & $60(51,67)$ \\
Male:Female (\%) & $65: 35$ & $66: 34$ \\
APACHE II Score (Median (IQR)) & $14(11,17)$ & $14(11,17)$ \\
ARS* (Mortality) & $63.1 \%(67.6 \%)$ & $52.2 \%(59.1 \%)$ \\
BRS** (Mortality) & $29.5 \%(12.1 \%)$ & $44.7 \%(18.6 \%)$ \\
BMI $\geq 30$ (Mortality) & $50.4 \%(51.0 \%)$ & $47.8 \%(34.8 \%)$ \\
\hline
\end{tabular}

*ARS-Advanced Respiratory Support provided

**BRS-Basic Respiratory Support provided

Conclusion. The data proves useful as a population descriptor, with our patient cohort having greater cardiometabolic risk and being from more socially deprived postcodes which may explain observed increased mortality.

Reference(s)

1. 1 Intensive Care National Audit \& Research Centre. ICNARC report on COVID-19 in critical care: England, Wales and Northern Ireland 26 February 2021. 101 p. 2021.

2. 2 Richarson et al. Presenting Characteristics, Comorbidities, and Outcomes Among 5700 Patients Hospitalized With COVID-19 in the New York City Area. JAMA.2020;323(20):2052-2059.

3. 3 Burström et al. Social determinants of health and inequalities in COVID19. Eur. J. Public Health. 2020; 30(4):617-618.

4. 4 Office for National Statistics UK. The English Indices of Deprivation 2019. 31 p. 2019

001249

Long term morbidity and mortality in covid patients discharged from hospital with or without steroid as discharge medication

P. Routray '; S. Samal'; D. Mishra

${ }^{1}$ critical care, care hospitals, BHUBANESWAR, India

Correspondence: P. Routray

Intensive Care Medicine Experimental 2021, 9(1): 001249

Introduction. Steroid are main stay of treatment along with anticoagulants in Moderate to severe Covid 19 patients. Ideally steroid should be stopped as patients clinical and inflammatory markers stabilizes. Patients requiring Oxygen have shown significant benefit if steroids are started early.

But during discharge the same steroid should be abruptly stopped or continued remain area of dispute where clinicians remain divided in their opinion.

Objectives. $\cdot$ To find out whether long term steroid on discharge are contributing towards 90 day morbidity and mortality in post covid pneumonia.

Methods. •In our hospital, Covid patients are admitted under four physicians out of which 2 physician have been giving steroid on discharge to Covid Pneumonia while 2 physician were discharging without steroid. 
- Retrospectively we will analyse patients who are discharged from May 2020 to october 2020. All patients are divided into 2 groups. One group who are discharged with a protocolized steroid tapering dose and other group without any steroid dose.

All patients will be followed for 90 days. And patients are seen for secondary outcomes like

1. Mortality.

2. Readmission in hospital.

3. Secondary Infection requiring antibiotics and visit to OPD.

4. Psychological depression.

5. Uncontrolled Diabetes Mellitus requiring change in medications.

6. Fractures

Results. We followed up Moderate to severe Covid 19 patients 243 patients in nonsteroid arm (steroid was abruptly stopped when oxygen requirement caeses) and 232 patients in protocolized steroid tapering regimen of 15 days (Three days each of decreasing dose of steroid starting from Methylprednisolone $16 \mathrm{mg}$ twice daily) for 90 days post discharge.

We found there is no statistically significant difference in Mortality (4 in nonsteroid arm and 3 in non steroid arm), readmssion in hospital ( 7 in non steroid arm and 5 in steroisd arm), secondary infection requiring antibiotics (5 in non steroid arm vs 4 in steroid arm), psychological depression, Fractures (0). Only there is slight increase in uncontrolled Diabetes Mellitus ( 28 in steroid arm compared to 2 in non steroid arm) Conclusion. Adding a protocolized steroid regimen during discharge gives rise to same outcome to those patients where steroid was abruptly stopped when oxygen requirement caeses. Rather it increases chances of uncontrolled diabetes mellitus requiring increase in antidiabetic dose. So it is appropriate to stop steroid whenever patient is stable and there is no oxygen requirement. Adding a tappering dose of steroid extending way beyond discharge does not have any advantage. Need large Randomized Controlled trials to confirm our findings in this study.

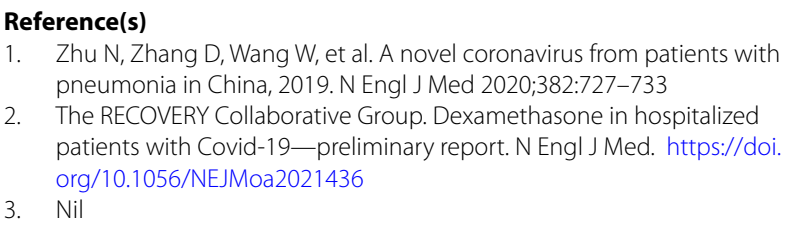

001251

The Critical Care Resources Adaptation to the Covid-19 Pandemic in Madrid

M. Sanchez Garcia'; M. Covid-Icu Group ${ }^{2}$

${ }^{1}$ Critical Care, Hospital Clinico Universitario San Carlos, Madrid, Spain;

${ }^{2}$ Intensive care units, Hospitals in the Province of Madrid, Madrid, Spain

Correspondence: M. Sanchez Garcia

Intensive Care Medicine Experimental 2021, 9(1): 001251

Introduction. The SARS-CoV-2 pandemic has put ICUs units under the extreme pressure of a large numbers of patients with severe respiratory failure.

Objectives. Describe the adaptation of intensive care the the pandemic surge in Madrid.

Methods. We performed a retrospective observational study of the clinical characteristics of all consecutive critically ill Covid-19 patients admitted in March and April 2020 to 54 ICUs in the Province of Madrid, the expansion of total ICU bed capacity and the process of relocation of patients between centers. Statistical analysis included independent predictors of mortality and propensity score matching of transferred and non-transferred cases.

Results. Total ICU capacity enlarged from 647 to 1942 to admit 3,192 patients. On duty staff increased from 61 intensivists to a multidisciplinary team of 202. 2,576 available Covid-19 cases had a median Apache II score of 15 (interquartile range [IQR], 11 to 19), median age was 63 (IQR, 55 to 70 ) years, $72.3 \%$ were male, and $44.2 \%$ died in the ICU. Emergency Medical Services transferred 197 patients with ARDS, 86.4\% under invasive mechanical ventilation with median $\mathrm{FiO}_{2} 0.6$ (IQR
0.5 to 0.8 ), PEEP 12 (IQR 10 to 14 ) $\mathrm{cmH} 2 \mathrm{O}$, a noradrenaline infusion in $60 \%$, and $18.7 \%$ suffered events needing medical intervention. Independent variables for ICU mortality were Apache II score (Odds ratio (OR) $1.061 ; 97.5 \% \mathrm{Cl}, 1.041-1.082$, per point increment), age (OR 1.069 ; $97.5 \% \mathrm{Cl}, 1.056-1.084$. per year increment), BMI (OR 1.039; $97.5 \% \mathrm{Cl}$, 1.015-1.063, per unit increment), duration of pre-ICU hospital stay (OR $1.035 ; 97.5 \% \mathrm{Cl}, 1.007-1.064$, per day increment), prone positioning (OR 1.305; $97.5 \% \mathrm{Cl}, 1.026-1.661$ ), and days from start of the epidemic outbreak on March 2, 2020 to date of hospital admission (OR 0.721; $97.5 \% \mathrm{Cl}, 0.632-0.819$, per 10 days increment). Mortality and length of stay were similar in transferred and non-transferred patients.

Conclusion. The explosive increase in demand associated with the SARS-CoV-2 required an immediate increase of ICU capacity and relocation of patients. Transfer of critically ill Covid-19 patients may be considered an additional measure of adaptation to pandemic surge. ICU admission should be attempted as soon as possible.

\section{Reference(s)}

1. None.

001258

Albumin as a prognostic factor in critically ill COVID-19 patients

D. Adrião ${ }^{1}$; P. Oliveira'; D. Miranda,

${ }^{1}$ Department of Intensive Care, Centro Hospitalar Vila Nova de Gaia/

Espinho, Vila Nova de Gaia, Portugal

Correspondence: D. Adrião

Intensive Care Medicine Experimental 2021, 9(1): 001258

Introduction. COVID-19 is the most threatening pandemic outbreak in modern history [1] and the identification of high-risk patients for clinical deterioration is essential. Hypoalbuminemia has been associated with mortality across numerous clinical settings [2], but there is little data examining the association between serum albumin levels and prognosis in critically ill COVID-19 patients.

Objectives. To determine whether serum albumin concentration reliably predicts adverse outcomes in critically ill COVID-19 patients.

Methods. Observational retrospective study of serum albumin concentration and outcomes of COVID-19 patients admitted to a polyvalent intensive care unit (ICU) of a Portuguese tertiary hospital between March 15th 2020 and March 14th 2021. Asymptomatic carriers of SARS-CoV-2 were excluded. Serum albumin levels were obtained within $24 \mathrm{~h}$ of ICU admission and hypoalbuminemia was defined as albumin $<3.5 \mathrm{~g} / \mathrm{dL}$.

Results. A total of 237 patients were included. Median age was 65 [58; 71] years, 168 (70.9\%) were men and median APACHE II and SAPS II scores were $16[13 ; 20]$ and $34[28 ; 43]$, respectively. Two hundred and six $(86.9 \%)$ patients had comorbidities, mainly arterial hypertension $(n=148,62.4 \%)$, diabetes $(n=80,33.8 \%)$, obesity $(n=80,33.8 \%)$, chronic respiratory disease $(n=35,14.8 \%)$, coronary heart disease $(n=32,13.5 \%)$ and chronic kidney disease $(n=18,7.6 \%)$. Median ICU length of stay was $11[4.9 ; 19.5]$ days and the mortality rate was $24.9 \%$ $(n=59)$. Seven patients died after ICU discharge. Sixty-two $(26.2 \%)$ patients were submitted to non-invasive ventilation, $134(56.5 \%)$ to high flow nasal cannula and $155(65.4 \%)$ patients required mechanical ventilation (median duration of 13 days). Hypoalbuminemia was found in $164(69.2 \%)$ patients and mean serum albumin on admission was $3.1 \pm 0,44 \mathrm{~g} / \mathrm{dL}$. ICU mortality was higher in patients with albu$\min <3.0 \mathrm{~g} / \mathrm{dL}(p=0.048)$. Only severe hypoalbuminemia $(<2.5 \mathrm{~g} / \mathrm{dL})$ was associated with in-hospital mortality $(p=0.024)$. No difference in ICU length of stay (17.1 vs 16.0 days, $p=0.166$ ) or duration of mechanical ventilation (14.0 vs $13.0, p=0.643)$ was found between patients with hypoalbuminemia and normal albumin.

Conclusion. Hypoalbuminemia has been described as a negative prognostic factor in several diseases [1,2]. Because of the broad spectrum of COVID-19 presentation, it is important to determine predictors of worse outcomes [3]. We demonstrate the association of mild and severe hypoalbuminemia and mortality in critically ill COVID-19 patients. Further studies are needed to clarify if albumin levels may be useful to evaluate the response to treatment and if hypoalbuminemia should be corrected. 
Reference(s)

1. 1. Paliogiannis P, Mangoni AA, Cangemi M, Fois AG, Carru C, Zinellu A. Serum albumin concentrations are associated with disease severity and outcomes in coronavirus 19 disease (COVID-19): a systematic review and meta-analysis. Clin Exp Med. 2021 Jan 28:1-12. https://doi.org/10. 1007/s10238-021-00686-z. Epub ahead of print. PMID: 33511503; PMCID PMC7842395.

2. 2. Akirov A, Masri-Iraqi H, Atamna A, Shimon I. Low Albumin Levels Are Associated with Mortality Risk in Hospitalized Patients. Am J Med. 2017 Dec;130(12): 1465.e11-1465.e19. https://doi.org/10.1016/j.amjmed.2017. 07.020. Epub 2017 Aug 9. Erratum in: Am J Med. 2020 May;133(5):646 PMID: 28803138.

3. 3. Aziz M, Fatima R, Lee-Smith W, Assaly R. The association of low serum albumin level with severe COVID-19: a systematic review and meta-analysis. Crit Care. 2020 May 26;24(1):255. https://doi.org/10.1186/s13054-02002995-3. PMID: 32456658: PMCID: PMC7249975.

\section{1}

1-year outcomes in patients with and without treatment limitations set on admission to critical care: a 2-year dual-centre observational study

M. Yakubi ; A. Devlin, ${ }^{2}$;. Pratt, ${ }^{3}$; L. Hodgson ${ }^{4}$

${ }^{1}$ Anaesthetic Trainee, Worthing Hospital, University Hospitals Sussex NHS Foundation Trust, Worthing, United Kingdom; ${ }^{2}$ Internal medicine trainee, Worthing Hospital, University Hospitals Sussex NHS Foundation Trust, Worthing, United Kingdom; ${ }^{3}$ Consultant in critical care and anaesthesia, Worthing Hospital, University Hospitals Sussex NHS Foundation Trust, Worthing, United Kingdom; ${ }^{4}$ Consultant in Critical Care and Respiratory Medicine, Worthing Hospital, University Hospitals Sussex NHS Foundation Trust, Worthing, United Kingdom

Correspondence: $M$. Yakub

Intensive Care Medicine Experimental 2021, 9(1): 001261

Introduction. Intensivists frequently navigate patients and their families through complex decisions about appropriate levels of care for the individual. 1 We aim to compare longer-term outcomes of patients with and without treatment limitations placed at admission to critical care.

Methods. Retrospective observational study including all critical care admissions across two sites over a two year period in Sussex UK (2018-20). We identified if treatment limitations had been set on admission to critical care after consultant review within the first $24 \mathrm{~h}$ and followed-up subsequent outcomes.

Results. Of 3781 admissions, $87 \%(n=3295)$ were for full escalation with $13 \%(n=486)$ having treatment limitations placed. $32 \%$ of the overall cohort received invasive positive pressure ventilation (IPPV), $14 \%$ non-invasive ventilation (NIV) and 7\% renal replacement therapy (RRT). Median clinical frailty scale was 3 (IQR 2-5). Patients with treatment limitations had significantly higher critical care $(29 \%$ vs $9 \%$ $\mathrm{P}<0.001$ OR 0.25 (95\% Cl 0.20-0.32)), in-hospital (45\% vs $13 \% \mathrm{P}<0.001$ OR 0.19 (0.15-0.23)), 90-day (52\% vs $16 \% \mathrm{P}<0.001$ OR $0.18(0.15-0.22)$ ) and 1 -year mortality (67\% vs $25 \% \mathrm{P}<0.001$ OR $0.17(0.13-0.21))$. Of patients who survived to hospital discharge, those for full escalation were more likely to be discharged directly home ( $81 \%$ vs $70 \%)$. The CFS was significantly higher in those who had treatment limitations (3 (IQR 2-4) vs 6 (4-7), $P<0.001$ ).

Patients for full escalation were more likely to receive IPPV (34\% vs $21 \%, \mathrm{P}<0.001$, OR $1.96(1.56-2.47))$, but received less non-invasive ventilation $(14 \%$ vs $20 \%, P<0.001$, OR $0.61(0.48-0.78))$, with similar RRT delivery (6\% vs $8 \%, P=0.189$, OR $0.79(0.55-1.13)$ ).

Conclusion. This study has shown that a significant proportion of patients are admitted to critical care with limitations placed. Importantly we found that although mortality was higher than those without limitations, a significant proportion of patients do survive to discharge home and $33 \%$ were alive at least one year after their critical care admission.

There is evidence that intensivists are increasingly instituting limitations of treatment though there is significant variability between and within countries, and even individual intensivists within the same critical care unit in decision-making.2,3
This observational study adds to this literature by reporting longerterm outcomes of patients with treatment limitations compared to those for full escalation. A shortcoming of the study is its retrospective nature and future study could further investigate the quality of life that such patients report in the longer-term.

\section{Reference(s)}

1. 1-Sprung, C. L et al. (2014). Seeking worldwide professional consensus on the principles of end-of-life care for the critically III: The consensus for worldwide end-of-life practice for patients in intensive care units (WELPICUS) study. American Journal of Respiratory and Critical Care Medicine, 190(8), 855-866.

2. 2-Mark, N. M et al. (2015). Global variability in withholding and withdrawal of life-sustaining treatment in the intensive care unit: a systematic review. Intensive Care Medicine, 41(9), 1572-1585.

3. 3-Guidet, B et al. (2018). Withholding or withdrawing of life-sustaining therapy in older adults ( $\geq 80$ years) admitted to the intensive care unit. Intensive Care Medicine, 44(7), 1027-1038.

\section{5}

Three months post COVID: respiratory and psychological

\section{sequelae}

F. Essafi ${ }^{1}$;I. Sdiri ${ }^{2}$;I. TALIK ${ }^{3} ;$ K. Benismail ${ }^{4}$; A. Silini ${ }^{3} ;$ K. Moez $^{3}$; T. Merhabene ${ }^{2}$

${ }^{1}$ intensive Care Unit, Regional HospitalZaghouan, Zaghuan, Tunisia;

${ }^{2}$ Réanimation médicale, ICU of Zaghouan Regional hospital, Tunis, Tunisia;

${ }^{3}$ Réanimation médicale, ICU of Zaghouan Regional hospital, Tunis, Tunisia;

${ }^{4}$ Réanimation médicale, ICU of Zaghouan Regional hospital, Zaghouan,

Tunisia

Correspondence: F. Essafi

Intensive Care Medicine Experimental 2021, 9(1): 001265

Introduction. Lessons from past viral epidemics reveal that, after recovery, patients with viral pulmonary infections can suffer from irreversible pulmonary dysfunction and demonstrate residual imaging or functional abnormalities. The long-term sequelae of coronavirus disease 2019 (COVID-19) are still unknown.

Objectives. During the recovery period, our study aims to evaluate changes in pulmonary function, computed tomography (CT) and psychological impact in COVID-19 patients.

Methods. It was a prospective observational 3-month follow-up study that evaluated respiratory symptoms, lung function, and high resolution CT-scan of survivors from COVID-19 after discharge from ICU Zaghouan's regional hospital, TUNISIA. Psychological effect was evaluated by assessing the Hospital anxiety and depression scale (HADs) and post-traumatic stress test (PTST). Lung function was explored by 6-min walk test. Desaturation was defined as a degression of pulse oximetry oxygen by $2 \%$. Dyspnea was measured according to NHYA classification.

Results. Thirty-three survivors were enrolled; they were checked between 8 and 13 weeks (mean 11.8 weeks) after hospital discharge. Twenty-six participants were previously hospitalized in ICU among them three had requiring invasive mechanical ventilation and seven were managed in hospital wards.

New breathlessness was the most common reported symptom for $13 / 33$. Middle dyspnea stage was 2 . Mean oximetry oxygen saturation was $97 \%$ on air room at rest. We reported an oxygen desaturation during the 6-min walk test in 10 patients. The second most common symptoms were asthenia and psychological distress. CT control revealed total disappearance of lesions on 15/33 and abnormalities sequalae on $18 / 33$. Founded features were mainly residual ground glass opacities (18/18), reticular and linear opacities (1/18), residual crazy paving pattern (5/18), distortions parenchymatous with traction bronchiectasis (1/18) and parenchymal fibrotic bands (1/18) Functional respiratory exploration showed a restrictive syndrome for 3 patients.

In all patients, psychological 3-month check-up was carried out. Depressive syndrome was found in 2 patients with a HAD scale of 13 and 15 respectively. Anxiety was diagnosed in 5 patients with HAD scale more than 13. Sever post-traumatic stress was diagnosed in 3 patients with test score between 41 and 44, it was associated to 
anxiety in two patients and depression in one. Only one patient did not return to his usual activities at three months.

Conclusion. COVID-19 infection is associated with several respiratory and psychological sequelae that can be disabling. Prolonged followup is recommended in these patients. There is a real need for rehabilitation services to manage COVID-19 survivors.

\section{0}

\section{Obesity as a severity factor in COVID-19 patientes admitted} to the ICU

A. Nogales Gomez' ;. Fernández Moreno'; G. ÁVila Martín'; L. González Morales $^{3}$; AC. Martin Guerrero ${ }^{2}$

'Nurse. Intensive Care Unit, Hospital Nuestra Señora del Prado (SESCAM), Talavera de la Reina, Spain; ${ }^{2}$ Phd, Unidad de apoyo a la investigación. Gerencia de atención integrada de Talavera (SESCAM), Talavera de la Reina, Spain; ${ }^{3}$ Md, U. Docente de Medicina Preventiva y Salud Pública. Gerencia atención integrada Talavera (SESCAM), Talavera de la Reina, Spain

Correspondence: A. Nogales Gomez

Intensive Care Medicine Experimental 2021, 9(1): 001270

Introduction. WHO declared COVID-19 illness as a pandemic in March 2020 (1). Arterial hypertension, type 2 diabetes and dyslipidemia have been observed in seriously ill patients affected by SARS-Cov-2 (2-6). The relatitonship between obesity and severity of COVID-19 disease is not clear. Several studies showed no direct asociation between severity of the illness produced by SARS-Cov-2 and obesity (7). However, other studies showed a higher body mass index in critical pacients with COVID-19 (3), suggesting that obesity may be a risk factor for admission to intensive care units.

Objectives. To evaluate obesity as a severity factor for COVID-19 patients admitted in the Intensive Care Unit (ICU) at Hospital General Universitario Nuestra Señora del Prado in Talavera de la Reina.

Methods. A retrospective observational study was carried out in COVID-19 patients admitted to the intensive care unit during the year 2020. Data was recorded from patients clinical history. The status of the patient at the discharge from ICU (alive/dead) and the days spent in ICU were used to quantify the severity of disease. Obesity through the body mass index was considered as an independent variable. Age, sex, smoking habit, previous diseases (arterial hyperpressure, diabetes mellitus and respiratory diseases) were used as covariates. A logistic regression adjusted by the covariates was performed to analyse the relation between obesity and the fatal outcome. It was considered statistical significance $p<0,05$. The study was approved by the ethics committee.

Results. A total of 106 patients were included. $64,2 \%$ were male with an average age of $59,3 \pm 12,5$ years and $49,1 \%$ were obese. Median stay at ICU was 13 days and COVID-19 patients mortality was $49,1 \%$.

$42,3 \%$ of obese patients and $55,6 \%$ of overweight and normal weight patients died. No statistical differences were found between both groups $(P=0,173)$. Obesity adjusted by covariates was not found to be a risk factor associated to a fatal outcome (OR: 0,93; IC 95\%: 0,36-2,39). Regarding the lenth of stay in the ICU, the median days of stay of obese patients was 14 days, while in overweigh and normal weigh patients it was 13 days, with no differences between groups $(P=0,481)$.

Conclusion. There is no relationship between obesity and mortality or length of stay in the ICU in the sample. It could be due to the patient profile admitted in the ICU during the period of study.

\section{Reference(s)}

1. 1. Organización Mundial de la Salud La OMS caracteriza a COVID-19 como una pandemia. 11 de marzo de 2020. Acceso 7 de junio de 2020 https://www.who.int/emergencies/diseases/novel-coronavirus-2019/ events-as-they-happen

2. 2. Ryan DH, Ravussin E, Heymsfield S. COVID 19 and the Patient with Obesity - The Editors Speak Out. Obesity. 2020;28(5):847

3. 3. Ramanathan K, Antognini D, Combes A, Paden M, Zakhary B, Ogino M, et al. Epidemiology, clinical course, and outcomes of critically ill adults with COVID-19 in New York City: a prospective cohort study. The Lancet. 2020;395:1763-70

4. 4. Grasselli G, Zangrillo A, Zanella A, Antonelli M, Cabrini L, Castelli A, et al. Baseline characteristics and Outcomes of 1591 patientes infected with SARS-CoV-2 admitted to ICUs of the Lombardy Region, Italy. JAMA. 2020;323(16): 1574-81

5. 5. Barrasa H, Rello J, Tejada S, et al. SARS-CoV-2 in Spanish Intensive Care Units: Early experience with 15-day survival in Vitoria. Anaesth Crit Care Pain Med. 2020;39(5):553-561.

6. 6. Simonnet A, Chetboun M, Poissy J, Raverdy V, Noulette J, Duhamel A, et al. High prevalence of obesity in severe acute respiratory syndrome coronavirus-2 (SARS-CoV-2) requiring invasive mechanical ventilation. Obesity. 2020;00(00):1-5

7. 7. Hajifathalian K, Kumar S, Newberry C, Shah S, Fortune B, Krisko T, et al. Obesity is associated with worse outcomes in COVID-19: Analysis of Early Data From New York City. Obesity. 2020;0-2.

8. Does not apply.

\section{6}

Prevalence of Burnout Syndrome: A cross-sectional study in healthcare professionals in Chile during COVID-19 pandemic

L. Alegria Vargas ${ }^{1}$; N. Navarro'; P. Repetto ${ }^{2}$; D. Acuña ${ }^{3}$

${ }^{1}$ Departamento de medicina intensiva, Pontificia Universidad Católica de Chile, Santiago, Chile; ${ }^{2}$ Escuela de psicología, Pontificia Universidad Católica de Chile, Santiago, Chile; ${ }^{3}$ Sociedad chilena de medicina intensiva, Sociedad Chilena de Medicina Intensiva, Santiago, Chile

Correspondence: L. Alegria Vargas

Intensive Care Medicine Experimental 2021, 9(1): 001276

Introduction. The coronavirus disease pandemic 2019 (Covid-19) has meant the greatest health crisis in recent times, without discriminating between countries, socioeconomic status or ethnic groups (1). Healthcare professionals may be one of the most affected groups during the pandemic, resulting in being exposed to three major types of stressors: (1) risk of infection from exposure to cases; (2) job demands, shift and workplace changes, new requirements regarding work methods and an increased pressure to correctly complete the different tasks the job ensues, especially the care of patients; and (3) social changes, which are related to modifications in routine, in their family and interpersonal relationships, among others $(2,3,4)$. In this scenario, understanding the health-related consequences of COVID-19 outbreak on frontline Chilean healthcare workers is urgent to provide timely interventions to protect their health.

Objectives. The main objective of this study is to assess the prevalence of burn-out syndrome in healthcare workers in intensive care units (ICUs), directly involved in the care of patients with COVID-19 during the pandemic in Chile.

Methods. This is a Cross-sectional, online survey-based study. Sampling was performed between dec 8th and 19th 2020. The survey collected demographic data and questions regarding participants working position since the pandemic outbreak. Chilean healthcare workers working on the original and expanded ICUs were eligible. Burn-out syndrome was assessed by the Maslach Burnout InventoryMedical Personnel.

Results. Of the 1.743 eligible responding participants, $73 \%$ were women. $60.7 \%(n=1,195)$ of the participants were between 30 and 49 years of age, most of the respondents were single $(n=1,079)$ and $43.3 \%$ declared they had at least one child $(n=853)$. Most of the participants were nurses $(43.2 \%)$, from original intensive care units $(62.4 \%)$ and worked in public centers. $78.7 \%$ of those surveyed express concern for their mental health due to the pandemic, $23 \%$ have received some type of mental health care during this time, while $13.4 \%$ had a medical license for this reason. Regarding the use of drugs to treat mental health symptoms, $26 \%$ of the respondents state that they are using pharmacological treatment. Prevalence of burnout syndrome was $87 \%$, higher in women $(73.2 \%, p=0,03)$, nurses $(43.2 \%, \mathrm{p}<0.0001)$, singles workers $(54.8 \%, \mathrm{p}=0.02)$, and without children $(56.7 \%, p<0.0001)$. More burn-out were those between 30 and 39 years old $(44.5 \%, p<0.0001)$ and those between 1 and 10 years of professional experience $(58.9 \%, \mathrm{p}<0.0001)$. Multivariable logistic 
regression analysis revealed that being a woman (OR $0.72 ; 95 \% \mathrm{Cl} 0.53$ to $0.97, \mathrm{p}=0.03)$, being under 39 years old (OR $0.61 ; 95 \% \mathrm{Cl} 0.53$ to $0.76, p<0.0001)$, being under 10 years of professional experience (OR $0.76 ; 95 \% \mathrm{Cl} 0.69$ to 0.84 ) and to have no previous ICU experience (OR $0.86 ; 95 \% \mathrm{Cl} 0.79$ to 0.9 ) were associated with higher risk of burn-out syndrome.

Conclusion. This survey study of healthcare professionals reported high rates of burnout syndrome. In this scenario, it is key to pay attention to aspect of the work organization that may affect the well-being of health personnel, decompress emotional exhaustion and generate spaces to share daily experiences, therefore communicating the suffering derived from work.

\section{Reference(s)}

1. 4. Ruiz-Fernández MD, Ramo-Pichardo J, Ibáñez-Masero O, CabreraTroya J, et al. Compassion fatigue, burnout, compassion satisfaction and perceived stress in healthcare professionals during the COVID-19 health crisis in Spain. J Clin Nurs. 2020:29:4321-4330

2. 3. Zhang Y, Wang Ch, Pan W, Zheng J, Gao J, et al. Stress, Burnout, and Coping Strategies of Frontline Nurses During the COVID-19 Epidemic in Wuhan and Shanghai, China. Front. Psychiatry, 26 October 2020

3. 2. Cipolotti L, Chan E, Murphy P, van Harskamp N, Foley J. Factors contributing to the distress, concerns, and needs of UK Neuroscience health care workers during the COVID-19 pandemic. Psychol Psychother. $2020 \mathrm{Ju}$ $16 ; \mathrm{e} 12298$

4. 1. Fei Zhou, Ting Yu, Ronghui Du, Guohui Fan, Ying Liu, et al. Clinical course and risk factors for mortality of adult in patients with COVID19 in Wuhan, China: a retrospective cohort study. Lancet. 2020 Mar 28:395(10,229):1054-1062.

\section{9}

Prevalence of critically ill patients in General Medical Wards and Difference in Mortality rate in critically ill patients admitted to Medical Intensive Care Units and General Medical Wards in a Tertiary University Hospital

N. Kongpolprom ${ }^{1}$; A. Wannakittirat,

${ }^{1}$ Department of Internal Medicine, Faculty of Medicine, Chulalongkorn University, King Chulalongkorn Memorial Hospital, Bangkok, Thailand

Correspondence: N. Kongpolprom

Intensive Care Medicine Experimental 2021, 9(1): 001279

Introduction. Nowadays, Thai society is approaching an aging society. The universal coverage policy of the government provides better care to Thai people than in the past healthcare system. Regardless of socioeconomic status, all Thai people can access medical care. In addition, the current Thai public health system has modern equipment. The advanced and effective treatment makes the Thai population live longer. As a result, there is a need to develop a highly efficient system for treatment, transfer, and hospital admissions. However, several hospitals have a shortage of medical personnel and medical supplies. The hospital beds, particularly ICU beds are insufficient to the demand due to the increasing numbers of patients.

Objectives. Our study aimed to determine the number of critically il patients newly admitted in general medical wards who needed ICU since the initial admission and compare mortality and other outcomes between the critically ill patients able to transfer to treat in the medical ICU and the patients unable to transfer to the ICU. In addition, the prioritization for ICU admission in the real practice was evaluated.

Methods. This prospective cohort study was descriptive andanalytic. We recruited a total of 136 newly admitted patients from August 2019 to September 2019. Data were obtained from the patient interview and the IPD chart review. Baseline characteristics, indications for ICU admission (according to Guidelines for intensive care unit admission by Society of Critical Care Medicine (1999)), severity scores, ventilatorfree days (VFD), and mortality was recorded. The continuous variables presented as mean $\pm S D$ and the categorical variables presented as number or percentage. Independent t-test was used to compare the continuous variables, while Chi-square tests or Fisher's exact tests were used to compare the categorical variables. Multiple logistic regression was used to analyze the association between the variables and the mortality rates at the 7 th day and 28th day.

Results. Among a total of 136 eligible patients, 51 patients (37.5\%) met the ICU admission criteria. However, only 15 (29.4\%) patients were able to transfer to treat in the medical ICU. Moreover, among patients transferred to the ICU, the delays (at least $24 \mathrm{~h}$ after the patients met the ICU admission criteria) occurred in nine cases (60\%). In the comparison of patients in the ICU-indicated group and patients without the indication for ICU admission, there were significant differences of baseline characteristics, including age, Charlson Comorbidity Index Nutrition Status by Nutritional Alert Form, Barthel Index, SOFA และ APACHE II on the admission date, and clinical outcomes, including the mortality rates at 7th day and 28th day, length of hospital stay, and ventilator-free day in 7 days. The factors significantly associated with the 7-day the mortality rate was Combined Age Charlson Comorbidity Index $(p=0.002)$, Nutrition status $(p=0.045)$, Barthel Index $(p=0.013)$, SOFA score $(p=0.003)$ and the patient with indication for ICU admission at the beginning $(p=0.028)$

Conclusion. Among the newly admitted patients in the general medical wards, 37.5\% obviously needed the ICU admission at the beginning. For the ICU prioritization in the real practice, the patients with Priority 1 were the first group transferred to the ICU, followed by the patients with Priority 2 and 3, which was the appropriate management. The critically ill patients able to transfer to treatment in the medical ICU tended to more survive than the patients unable to transfer for ICU admission.

\section{Reference(s)}

1. None.

\section{4}

Trust Standardisation, cost saving and ergonomic restructuring of emergency bags in Critical Care

S. Hughes'; A. Choyce ${ }^{2}$; J. Bower ${ }^{2}$; M. Martin, ${ }^{2}$

${ }^{1}$ Critical Care, NHS Glouestershire, Gloucester, United Kingdom; ${ }^{2} \mathrm{ICU}$

Gloucestershire NHS Trust, gloucester, United Kingdom

Correspondence: $\mathrm{S}$. Hughes

Intensive Care Medicine Experimental 2021, 9(1): 001284

Introduction. Trust Standardisation, cost saving and ergonomic restructuring of emergency bags in Critical Care

Objectives. To improve familiarity with contents, optimise adherance to our weekly 'kit check', reduce risk of manual handling injuries and increase efficiency when providing critical care in the emergency hospital environment.

Methods. We created a survey for all new trainees to critical care asking questions regarding selected key contents of our current emergency bags. In addition, we performed random spot checks to ensure adherance to correct restocking (even when bags had tag seals applied).

We went on to source two new transfer bags- one to contain our capnography, intraosseous 'gun', drug and videolaryngoscopy bags, and a 2nd 'slimmed down' version of our currentlly heavy and at times inadequately restocked emergency

This progressed to creating an online 'kit check' form using Google forms, incorporating a QR code for ease of use whilst restocking.

To create our ideal emergency bag content list, we met with key stakeholders- consultant transfer lead, senior nursing staff, trainees and ACCPs.

Finally, we created a simulation session for new to ICU trainees, timing speed of attendance and preparation of key equipment and drugs for a Rapid Sequence Intubation comparing old and new emergency bags.

Results. A survey of trainees new to critical care revealed confusion and knowledge deficit in many of the emergency bag key components. A random spot check of bag contents showed key components missing on more than one occasion- a gum elastic bougie being one example.

Our new emegency bag restructuring showed improved knowledge and confidence amongst trainees due to the pictorial aids and 
locations of items to re-stock after use. The creation of QR codes has also significantly improved adherance to our weekly bag 'kit' checks, which has shown the additional benefit of easy data sourcing for future audit purposes.

A weight reduction of several kilograms was noted comparing old and new emergency bags which was gratefully received by trainees.

A simulation comparing speed of preparation for a bedside rapid sequence intubation with the old bags versus new was carried out. This demonstrated significantly reduced speed of preparation with the new bags, improved familiarity with key emergency items, and comfort and speed of attendance at the simulation centre.

Conclusion. This cross-site quality improvement project has shown that with collaborative working between Advanced Critical Care Practitioners, FICM trainees, consultants and senior nursing staff- a significant improvement in delivery of prompt, efficient emergency critical care at the bedside can be provided. Further to this project, we are now modifying our inter and intrahospital transfer bags in addition to creating a 'Hot Admission Trolley'. We would encourage other units to consider what items in their emergency critical care bags are deemed essential, and in addition to the manual handling concerns of some extremely heavy bags, the benefits to trainee confidence in kit familiarity and patient safety regarding restocking and knowledge of key equipment items.

\section{Reference(s)}

1. The Intensive Care Society. Guidelines for the transport of the critically il adult. 3rd ed. 2011. Appendix 6, Supplementary equipment for use during transport.

2. Manual handling at work-a brief guide. Health and Safety England 2020 .

3. Guidance for safer handling during cardiopulmonary resuscitation in healthcare settings. Working Group of the Resucitation Council UK

\section{8}

Prognostic value of white blood cells, C-reactive protein, and procalcitonin in critically ill COVID-19 patients

D. Adrião ${ }^{1}$; P. Oliveira' ${ }^{1}$; D. Miranda, ${ }^{1}$

'Department of Intensive Care, Centro Hospitalar Vila Nova de Gaia/

Espinho, Vila Nova de Gaia, Portugal

Correspondence: D. Adrião

Intensive Care Medicine Experimental 2021, 9(1): 001288

Introduction. The inflammatory response plays a critical role in COVID-19, yet no reliable indicators are available to predict disease severity and progression. The added value of biomarkers, such as procalcitonin (PCT), C-reactive protein (CRP) and white blood cells (WBC), as adjuncts for predicting the outcome of critically ill patients with COVID-19 is in question.

Objectives. To determine the ability of WBC, CRP and PCT to predict adverse outcomes in critically ill COVID-19 patients.

Methods. Observational retrospective study of COVID-19 patients admitted to a polyvalent intensive care unit (ICU) of a Portuguese tertiary hospital between March 15th 2020 and March 14th 2021. Asymptomatic carriers of SARS-CoV-2 were excluded. WBC, CRP, and PCT levels were collected within $24 \mathrm{~h}$ of ICU admission. PCT $>0.5 \mathrm{ng} / \mathrm{mL}$ was considered elevated.

Results. During the study period, 237 patients were included. Median age was $65[58 ; 71]$ years, $168(70.9 \%)$ were men and median APACHE II and SAPS II scores were $16[13 ; 20]$ and 34 [28; 43], respectively. Arterial hypertension $(n=148,62.4 \%)$, diabetes $(n=80,33.8 \%)$, obesity $(n=80,33.8 \%)$, respiratory disease $(n=35,14.8 \%)$, coronary heart disease $(n=32,13.5 \%)$ and chronic kidney disease $(n=18,7.6 \%)$ were the most common comorbidities. The median ICU length of stay was 11 [4.9; 19.5] days and the ICU mortality was $24.9 \%(n=59)$. The overall in-hospital mortality was $27.8 \%(n=66)$. Sixty-two $(26.2 \%)$ patients were submitted to non-invasive ventilation, $134(56.5 \%)$ to high flow nasal cannula and 155 (65.4\%) patients required mechanical ventilation (median duration of 13 days). PCT was only available in 160 patients. Median levels of WBC, CRP, and PCT on admission were 7.9 $[5.8 ; 11.1] \mathrm{g} / \mathrm{L}, 14.2[7.1 ; 22.5] \mathrm{mg} / \mathrm{dL}$ and $0.25[0.1 ; 1.0] \mathrm{ng} / \mathrm{mL}$, respectively. Non-survivors had significantly higher levels of WBC (10.4 vs
$7.6 \mathrm{~g} / \mathrm{L}, \mathrm{p}<0.001)$ and CRP (15.5 vs $12.2 \mathrm{mg} / \mathrm{dL}, \mathrm{p}<0.001)$ compared to survivors. $P C T>0.5 \mathrm{ng} / \mathrm{mL}$ was significantly associated with ICU mortality $(p=0.002)$, in-hospital mortality $(p=0.004)$ and the need for mechanical ventilation $(p=0.03)$.

Conclusion. WBC, CRP and PCT levels have a significant association with worse outcomes in critically ill COVID-19 patients. Additional investigation is needed to clarify if elevated PCT observed in COVID19 patients could be due either to bacterial co-infection, which is itself causing increased severity and driving systemic sepsis, or as a direct marker of a more severe viral infection.

\section{Reference(s)}

1. Liu F, Li L, Xu M, Wu J, Luo D, Zhu Y, Li B, Song X, Zhou X. Prognostic value of interleukin-6, C-reactive protein, and procalcitonin in patients with COVID-19. J Clin Virol. 2020 Jun;127:104370. https://doi.org/10.1016/j.jcv. 2020.104370. Epub 2020 Apr 14. PMID: 32344321; PMCID: PMC7194648.

2. Ponti G, Maccaferri M, Ruini C, Tomasi A, Ozben T. Biomarkers associated with COVID-19 disease progression. Crit Rev Clin Lab Sci. 2020 Sep;57(6):389-399. https://doi.org/10.1080/10408363.2020.1770685. Epub 2020 Jun 5. PMID: 32503382; PMCID: PMC7284147.

3. Hu R, Han C, Pei S, Yin M, Chen X. Procalcitonin levels in COVID-19 patients. Int J Antimicrob Agents. 2020 Aug;56(2):106051. https://doi.org/ 10.1016/j.ijantimicag.2020.106051. Epub 2020 Jun 10. PMID: 32534186; PMCID: PMC7286278.

\section{8}

\section{Combating Noise in the ICU (The effect on healthcare personnel)}

M. Fakher ${ }^{1}$; M. Mostafa ${ }^{2}$;. Khaled ${ }^{2}$; S. Maichel ${ }^{2}$

${ }^{1}$ Critical care, Cairo University, Faculty Of Medicine, Kasr Al Ainy, cairo,

Egypt; ${ }^{2}$ Critical care, cairo university, Cairo, Egypt

Correspondence: M. Fakher

Intensive Care Medicine Experimental 2021, 9(1): 001298

Introduction. Working in an intensive care unit (ICU) can be especially stressful because of the high patient morbidity and mortality, challenging daily work routines, and regular encounters with traumatic and ethical issues. This level of nearly continuous and excessive stress can rapidly accelerate when caregivers perceive that there is insufficient time or limited resources to properly care for patients. Until recently, the critical care community was relatively unaware of the harmful effects of working in a stressful ICU environment, including the development of BOS and other psychological disorders (Boone, 2012).

Objectives. This study aimed Tto assess the impact of noise reduction on ICU healthcare personnel (nurses and physicians).

Methods. The study was conducted for six months during which the sound levels were measured continuously using NIOSH (The National Instiute for Occupational Safety and Health) Sound Level Meter (SLM) app for iOS devices in the general ICU and CCU. In the present study, noise was measured over three days: Thrusday (a working day), Friday (a weekend day without noise-reducing intervention) and Saturday (a weekend day with noise-reducing intervention).We calculated Time weight average (TWA) of noise measurement for each working shift (Morning, Afternoon, and Night)As well as for the entire day for each of the sampled days (Thrusday, Friday, and Saturday) in addition we collected the following data from every eligible participants (Celestina et al., 2018).

Medical staff personnel (nurses \& physicians) in charge of each day were assessed by burnout quesionnaire (mbi-hss scoring) as well depression questionnaire (Beck Depression Inventory) on working day with no noise intervention for three months. These same medical staff personnel reassessed with the same questionnaire after 3 months on working days with noise intervention (white sound/music) applied.

Results. The post-intervention burnout level was significantly less than the pre-intervention level, particularly in Emotional Exhaustion and Personal Accomplishment dimensions of the burnout scale (Table 1). However, the total depression score did not show statistically significant difference between pre- and post-intervention levels Table (1) 


\begin{tabular}{|c|c|c|c|}
\hline & $\begin{array}{l}\text { PRE-INTERVEN- } \\
\text { TION }\end{array}$ & $\begin{array}{l}\text { POST INTERVE- } \\
\text { TION }\end{array}$ & P val \\
\hline \multicolumn{4}{|l|}{ Burnout subscale } \\
\hline $\begin{array}{l}\text { Emotional Exh- } \\
\text { huastion }\end{array}$ & 31.0.土11.6 & $27.2 \pm 11.1$ & 0.004 \\
\hline Depersonaliztion & $13.2 \pm 6.2$ & $12.5 \pm 6.3$ & 0.418 \\
\hline $\begin{array}{l}\text { Personal Acom- } \\
\text { plishment }\end{array}$ & $33.3 \pm 11.1$ & $23.5 \pm 10.2$ & 0.0001 \\
\hline $\begin{array}{l}\text { Total depression } \\
\text { score }\end{array}$ & $19.4 \pm 11.7$ & $19.6 \pm 11.6$ & 0.76 \\
\hline
\end{tabular}

Conclusion. Noise reduction improved healthcare personnel burnout yet depression symptoms was not affected.

\section{Reference(s)}

1. Celestina, M., Hrovat, J., \&Kardous, C.A. (2018).Smartphone-based sound level measurment app: Evaluation of compliance.Applied Acoustics, 139, $119-128$

2. Boone, S. (2012). Burnou1. Boone S. Burnout and Satisfaction With WorkLife Balance Among US Physicians Relative to the General US Population < alt-title > Burnout and Satisfaction With Work-Life Balance $<$ /alttitle $>$. Archives of Internal Medicine. 2012. p. 1.t and Satisfactio. Archives of Internal Medicine.

\section{7}

\section{Hospital Mortality in ICU COVID-19 Patients-what are the true} risk factors?

P. Campos'; D. Guerra²; MJ. Ferreira Da Silva'; M. Tavares³; I. Aragão'; R. Antunes ${ }^{1}$

${ }^{1}$ Unidade de cuidados intensivos polivalente, Centro Hospitalar e Universitário do Porto, Porto, Portugal; ${ }^{2}$ Serviço de doenças infeciosas, Centro Hospitalar e Universitário do Porto, Porto, Portugal; ${ }^{3}$ Serviço de cuidados intensivos, Centro Hospitalar e Universitário do Porto, Porto, Portugal

Correspondence: P. Campos

Intensive Care Medicine Experimental 2021, 9(1): 001307

Introduction. The novel coronavirus disease (COVID-19) progresses to critical illness in approximately $10 \%$ of confirmed cases. Acute respiratory distress syndrome (ARDS) has been diagnosed in about $40 \%$ to $96 \%$ of patients admitted in intensive care unit (ICU). Some patients also develop multiorgan failure, with a high mortality rate.

Reported factors associated with mortality were older age, male sex, chronic arterial hypertension, cardiovascular disease, stroke, asthma, chronic obstructive pulmonary neutrophilia, higher lactate dehydrogenase value, organ disfunction, coagulopathy, higher D-dimer value and invasive mechanical ventilation (IMV).

The understanding of indicators for mortality in critical ill patients is crucial for strategic planning and adequate resource allocation.

Objectives. This study aimed to identify the risk factors associated with hospital mortality in the COVID-19 patients admitted to the ICU.

Methods. Retrospective and observational study conducted at a tertiary hospital, from March to May 2020. All patients with confirmed COVID-19 admitted in the ICU were included. Patients under 18 years of age were excluded. A univariate logistic regression analysis for risk factors of hospital mortality in ICU COVID-19 patients was performed.

Results. Of 49 patients included, the median age was 67 years [interquartile range (IQR) 57-71], and 31 (63.3\%) were men. The median Simplified Acute Physiology Score II (SAPS II) of survivors was 34 [IQR 24-41] and that of non-survivors was 43 [IQR 37-46]. As for Sequential Organ Failure Assessment (SOFA) scores, the median in survivors was 6 [IQR 3-9] and 7 [IQR 6-9] in non-survivors. IMV was required in 38 (77.6\%) patients and ARDS developed in 37 (75.5\%). The median time from symptoms onset to ICU admission was seven [IQR 7-10] days; median length of ICU stay was 10 [IQR 5-26] days; and median length of IMV was 12 [IQR 7-23] days. ICU mortality was $42.9 \%$ with overall hospital mortality being $46.9 \%$.
When conducting the univariate analysis, the factors independently associated with hospital mortality were older age [Odds Ratio (OR) 1.087; 95\% Confidence Interval (Cl) 1.017-1.160, $p=0.013$, higher SAPS II [OR 1.081; $95 \% \mathrm{Cl} 1.017-1.150, p=0.012$ ], arterial hypertension [OR 4.889; $95 \% \mathrm{Cl} 1.157-20.665, p=0.031]$, infection at ICU admission [OR 6.400; $95 \% \mathrm{Cl} 1.195-34.285, p=0.030$ ], bacterial co-infection during ICU stay [OR $13.750 ; 95 \% \mathrm{Cl} 1.595-118.531, p=0.017]$, higher lactate values [OR 3.630; $95 \% \mathrm{Cl} 1.071-12.300, p=0.038$ ], need of IMV [OR 5.559; 95\% Cl 1.057-29.243, $p=0.043$, need of higher fraction of inspired oxygen [OR 1.064; $95 \% \mathrm{Cl} 1.003-1.129, p=0.038$ ], ARDS [OR $6.562 ; 95 \% \mathrm{Cl} 1.258-34.227, p=0.026]$, need of neuromuscular block [OR 4.889; 95\% Cl 1.157-20.665, $p=0.031$ ], need of prone position [OR 6.667; $95 \% \mathrm{Cl} 1.585-28.040, p=0.010]$, acute kidney injury (AKI) [OR $6.333 ; 95 \% \mathrm{Cl} 1.501-26.732, p=0.012]$, shock [OR 5.559; $95 \% \mathrm{Cl} 1.057-$ $29.243, p=0.043]$, hepatic disfunction [OR $5.600 ; 95 \% \mathrm{Cl} 1.024-30.615$, $p=0.047$ ] and higher number of organ disfunctions [OR $2.724 ; 95 \% \mathrm{Cl}$ $1.278-5.810, p=0.009]$.

Conclusion. The mortality rate in critically ill COVID-19 patients in our study population was high. The risk factors of hospital mortality in our study population appear to be patient characteristics, organ disfunction and co-infection related.

\section{Reference(s)}

1. P. Garcia et al.: Prognostic factors associated with mortality risk and disease progression in 639 critically ill patients with COVID-19 in Europe: Initial report of the international RISC-19-ICU prospective observational cohort; EClinicalMedicine 25 (2020) 100449

2. G. Grasselli et al.; Risk Factors Associated With Mortality Among Patients With COVID-19 in Intensive Care Units in Lombardy, Italy; JAMA Intern Med. 2020 Oct 1; 180(10):1345-1355.

\section{9}

Blood transfusion practice in the ICU—time to update training and education

J. Martins ${ }^{1}$; A. Silva ${ }^{1}$; HC. Pinheiro ${ }^{2}$;CC. Dias ${ }^{3}$; IJ. Pereira ${ }^{4}$; GC. Campello ${ }^{1}$ Intensive Care Medicine Service, Centro Hospitalar Tâmega e Sousa, Penafiel, Portugal; ${ }^{2}$ Internal Medicine, Centro Hospitalar Tâmega e Sousa, Penafiel, Portugal; ${ }^{3}$ Department of Community Medicine, Information and Health Decision Sciences (medcids), Center for Health Technology and Services Research (CINTESIS), Porto, Portugal; ${ }^{4}$ Intensive care medicine service, centro hospitalar de vila nova de gaia-espinho, CRITICALMED_CINTESIS—Center for Health Technology and Services Research, Porto, Portugal

Correspondence: J. Martins

Intensive Care Medicine Experimental 2021, 9(1): 001319

Introduction. Anemia is highly prevalent in critical care patients [4] and is independently associated with higher mortality and morbidity $[1,2]$. Although red blood cell (RBC) transfusions may be lifesaving, it is not an innocuous intervention. Recommendations point towards transfusion practices gradually more restrictive [2,3].

Objectives. To characterize the clinical practice regarding RBC transfusions in a cohort of critically ill patients with anemia during their stay in the intensive care unit (ICU), and to evaluate if the practice follows the current guidelines and its impact on mortality and length of hospital stay (LOS).

Methods. Observational retrospective study on patients admitted in a mixed ICU submitted to RBC transfusion, in a tertiary hospital, between January 1st and December 31st 2019. Collected demographic, clinical, transfusional and mortality data. The SPSS statistical program v. 25.0 was used. When adequate we performed the Student T test and the Mann Whitney test; the Chi-squared test and the Fisher's exact test. We also performed a logistic regression and linear regression models. The significance level used was 0,05.

Results. During the referral period, 330 patients were admitted, of which, $27.9 \%$ received at least one RBC transfusion. In the first transfusion, $77 \%$ of the patients had a hemoglobin $(\mathrm{Hb})$ value $>7 \mathrm{~g} / \mathrm{dL}$. When considering the total number of transfusions, $44,6 \%$ of the RBC transfusions were inappropriate. When analyzing transfusion with a 
pre-transfusion $\mathrm{Hb}>7 \mathrm{~g} / \mathrm{dl}$, and taking into account the clinical reason for transfusion, 58,6\% were inappropriate.

The logistic regression model showed that RBC transfusion even when adjusted for the clinical reason for transfusion did not have an impact on mortality. Only SAPS II had an impact on mortality, $p \leq 0.05$, OR 1.06, IC $[1.02 ; 1.10]$

There was no statistical association between the number of RBC transfusions and the survival in ICU $(p=0,333)$, at hospital discharge $(p=0,40)$, after 60 days $(p=0,92)$ and 1 year $(p>0,99)$.

Finally, through a linear regression model, we verified that patients who had ischemic heart disease and required a RBC transfusion were significatively more likely to have an increased length of stay, with an OR 5.25, ( $\leq<0.04)$, IC [0.22; 10.27].

Conclusion. In our cohort only $27,9 \%$ of the patients received RBC transfusion, which is in agreement with the literature [1].

Although not associated with higher mortality, $44,6 \%$ of the transfusions were considered inappropriate if we consider recommendations from the European Society of Intensive Care Medicine $[2,8]$. These data made us aware of the need to update training and education of our staff in order to avoid unnecessary transfusions.

Ischemic heart disease was the only variable influencing the LOS. This may be explained by the fact that anemia is associated with worse outcomes in patients with cardiovascular disease, and heart failure is associated with increased rate of hospitalizations and poor prognosis $[6,7]$.

\section{Reference(s)}

1. 8. Spahn DR, Bouillon B, Cerny V, Duranteau J, Filipescu D, et al. (2019). The European guideline on management of major bleeding and coagulopathy following trauma: fifth Editions. Critical Care, 23:98. https://doi.org/10. 1186/s13054-019-2347-3

2. 7. Docherty AB; Walsh TS. (2017). Anemia and blood transfusion in the critically ill patient with cardiovascular disease. Crit Care 21, 61. https:// doi.org/10.1186/s13054-017-1638-9

3. 6. Ezekowitz JA, McAlister FA, Armstrong PW (2003). Anemia is common in heart failure and is associated with poor outcomes: insights from a cohort of 12065 patients with new-onset heart failure. Circulation, 107:223-5. https://doi.org/10.1161/01.CIR.0000052622.51963.FC

4. 5. Vincent JL, Sakr Y, Sprung C, Harboe S, \& Damas P. (2008). Are blood transfusions associated with greater mortality rates? Results of the sepsis occurrence in acutely ill patients study. Anesthesiology, 108(1), 31-39. https://doi.org/10.1097/01.anes.0000296070.75956.40

5. 4. Chornenki, NLJ, James TE, Barty R, Liu Y, Rochwerg B, et al. (2020). Blood loss from laboratory testing, anemia, and red blood cell transfusion in the intensive care unit: a retrospective study. Transfusion, 60(2), 256-261. https://doi.org/10.1111/trf.15649

6. 3. Rasmussen L, Christensen S, Lenler-Petersen P, Johnsen SP. (2010). Anaemia and 90-day mortality in COPD patients requiring invasive mechanical ventilation. Clin Epidemiol, 3:1-5. https://doi.org/10.1513/ AnnalsATS.201708-6870C

7. 2. Vlaar AP, Oczkowski S, Bruin S, Wijnberge M, Antonelli M, Aubron C, et al. (2020). Transfusion strategies in non-bleeding critically ill adults: a clinical practice guideline from the European Society of Intensive Care Medicine. Intensive Care Med, 46:673-696. https://doi.org/10.1007/ s00134-019-05884-8

8. 1. Vincent JL, Jaschinski U, Wittebole X, Lefrant JY, Jakob SM, Almekhlafi GA, et al. (2018). Worldwide audit of blood transfusion practice in critically ill patients. Crit Care, 22(102):1-9. https://doi.org/10.1186/ s13054-018-2018-9

9. Acknowledgment to: Cristina Granja, M.D., PhD. Anaesthesiology Department, São João University Hospital Center, Porto. Associate Professor, Surgery and Physiology Department, Faculty of Medicine of Porto. Principal Investigator CRITICALMED_CINTESIS
001323

Predictive Performance of Hospital Mortality Scores in the ICU COVID-19 Patients

P. Campos; D. Guerra²; MJ. Ferreira Da Silva'; M. Tavares³; I. Aragão1; R.

Antunes

${ }^{1}$ Unidade de cuidados intensivos polivalente, Centro Hospitalar e

Universitário do Porto, Porto, Portugal; ${ }^{2}$ Serviço de doenças infeciosas, Centro Hospitalar e Universitário do Porto, Porto, Portugal; ${ }^{3}$ Serviço de cuidados intensivos, Centro Hospitalar e Universitário do Porto, Porto,

Portugal

Correspondence: $P$. Campos

Intensive Care Medicine Experimental 2021, 9(1): 001323

Introduction. The novel coronavirus disease (COVID-19) pandemic rapidly spread around the world and become a global public health threat. Most patients have mild to moderate respiratory or digestive symptoms; however, some may present severe disease, developing respiratory failure, acute respiratory distress syndrome, and multiple organ failure, culminating in death. The application of scoring systems can facilitate effective evaluation by emergency or critical care physicians to screen severe patients.

Modified Early Warning Score (MEWS) and Rapid Emergency Medicine Score (REMS) are used in the emergency department to assess the risk of admission to intensive care and mortality. The Acute Physiology and Chronic Health Evaluation II (APACHE II), Simplified Acute Physiology Score II (SAPS II) and Sequential Organ Failure Assessment (SOFA) are used on admission to intensive care to assess the severity of the disease and estimate the associated hospital mortality. Confusion, Urea, Respiratory Rate, Blood Pressure and Age Above or Below 65 Years (CURB-65) is a score used to assess severity and estimate mortality in patients with pneumonia.

Due to the particularities of the COVID-19 patients, these score's use and validity in this population are still unknown

Objectives. To assess and compare the performance of different predictive systems of hospital mortality in COVID-19 patients admitted to the intensive care unit, namely the APACHE I, SAPS II, MEWS, REMS, CURB-65 and SOFA.

Methods. Retrospective and observational study conducted in a tertiary hospital, from March to May 2020. All patients with confirmed COVID-19 admitted in the ICU were included. Patients under 18 years of age were excluded. Statistical analysis, including receiver operating characteristic (ROC) curve, was performed.

Results. A total of 49 patients were included. Twenty-one (42.9\%) died during ICU stay and 23 (46.9\%) died during hospital stay.

The median REMS of survivors was 8 [Interquartile range (IQR) 6-10] and 9 in non survivors [IQR 9-11]; MEWS medians were 5 [IQR 2-6] and 5 [IQR 3-6]; APACHE II medians were 14 [IQR 11-15] and 17 [IQR 13-22]; SAPS II medians were 34 [IQR 24-41] and 43 [IQR 37-46]; SOFA medians were 6 [IQR 3-9] and 7 [IQR 6-9]; and CURB-65 medians were 3 [IQR 2-3] and 3 [IQR 2-3], respectively.

The area under the ROC curve for the REMS, MEWS, APACHE II, SAPS II, SOFA and CURB-19 in predicting mortality were 0.687 [95\% Confidence Interval $(\mathrm{Cl}) \quad 0.525-0.848], 0.560$ [95\% Cl $0.389-0.730], 0.662$ [95\% Cl 0.499-0.824], 0.749 [95\% Cl 0.605-0.893], 0.634 [95\% Cl 0.470$0.798]$ and 0.581 [95\% Cl 0.413-0.749], respectively.

Conclusion. The SAPS II score demonstrated an acceptable discriminatory power in predicting hospital mortality in patients with COVID19. However, all other compared scores had a poor performance.

\section{Reference(s)}

1. X. Zou et al.; Acute Physiology and Chronic Health Evaluation II Score as a Predictor of Hospital Mortality in Patients of Coronavirus Disease 2019; Crit Care Med. 2020 Aug; 48(8): e657-e665.

2. H. Hu et al.; Comparing Rapid Scoring Systems in Mortality Prediction of Critically III Patients With Novel Coronavirus Disease; ACADEMIC EMERGENCY MEDICINE 2020; 00:1-8. 
001327

Risk factors for mortality in COVID-19 critically ill patients

I. TALIK'; B. Ben Dhia'; IK. Ben²; A. Hadded'; M. Kaddour'; T. Merhabene ${ }^{3}$ ${ }^{1}$ Intensive Care Unit, Zaghouan Hospital, Zaghouan, Tunisia; ${ }^{2}$ Réanimation médicale, ICU of Zaghouan Regional Hospital, Tunis, Tunisia; ${ }^{3}$ Réanimation médicale, ICU of Zaghouan Regional hospital, Tunis, Tunisia

Correspondence: I.TALIK

Intensive Care Medicine Experimental 2021, 9(1): 001327

Introduction. Coronavirus disease 2019 (COVID-19) becomes a global pandemic in 2020. Many studies have revealed several risk factors associated with worse prognosis in patients with COVID-19, those associated with death in critically ill class needs to be fully elucidated.

Objectives. Therefore, we analyzed clinical characteristics and laboratory data of ICU COVID 19 patients to identify risk factors associated with death.

Methods. We conducted a retrospective, single-center study including all patients hospitalized in the ICU of Zaghouan hospital (Tunisia), with a confirmed diagnosis of COVID-19, from 23 th March 2020 to 31th January 2021. The demographic, clinical, laboratory and chest imaging data were retrospectively collected and analyzed. Student's t-test and Chi-square test or Fisher's exact test were used to compare the continuous and categorical variables, respectively. The logistic regression model was employed to ascertain the risk factors of mortality.

Results. 121 patients were enrolled, including 53 dead (43.8\%) and 68 survivors. median age was $64 \pm 12$ years (IQR, $56-70)$, sex ratio $=1.5$, Medians of SAPS II and APACHE II scores were respectively $26 \pm 8$ and $8 \pm 3$. fifty-one percent of patients were blood group A. Hypertension $(48 \%)$ and diabetes (35\%) were the most frequent underlying diseases. fifty patients (41\%) required invasive mechanical ventilation (IMV). All patients have received corticostroids, Azithromycin, beta-lactam, Anticoagulation and vitamin therapy at admission. During hospitalization, $40 \%$ of cases had developed at least one episode of nosocomial infection $(\mathrm{NI})$ and $30 \%$ secondary acute kidney injury (AKI), defined according to KDIGO criteria. Median length of hospital stay was 8 (IQR,6-14.5) days.

We found that IMV (OR 47, 74 IC 95\% [4, 23-537, 94], p=0, 002), foci of condensation on chest computed tomography (OR 8, 59 IC 95\% [1, $33-55,37], p=0,024)$ and blood group A (OR 5, 49 IC 95\% [1, 14-26, $41], p=0,034)$, were associated with increased odds of mortality.

Conclusion. Mortality in COVID-19 was not rare in critically ill patients and its main risk predictor was IMV requirement. Non-invasive respiratory support strategies may have survival benefits.

\section{7}

Critical Care Transfers of COVID-19 patients: Strengthening the Network

F. Barros'; LM. Ladeira ${ }^{1}$; J. Lourenço'; F. Rato

'Departamento de emergência médica, Instituto Nacional de Emergência Médica, Lisboa, Portugal

Correspondence: F. Barros

Intensive Care Medicine Experimental 2021, 9(1): 001337

Introduction. Portugal had an incidence peak of COVID-19 in February 2021, with the highest level of transmission and positive cases since the beginning of the SARS-CoV-2 pandemic. The number of hospital admissions raised-remarkably in intensive care-therefore challenging the regular capacity. Most hospitals doubled the number of ICU beds in spaces previously allocated to emergency or operating rooms.

Demand for intensive care was not uniform across the country. Epidemic outbreaks took place in different regions; this demand heterogeneity opened the possibility of balancing the load of patient admissions such that excessive demand was re-routed to places with spare capacity.

INEM is the National Institute for Prehospital Medical Care and was under great stress to match the needs of hospital transferences. INEM adapted to manage transferences between hospitals throughout the country. A commission (CARNMI) created to supervise the management of ICU transfers. INEM coordinated the transferences in close contact with the CARMNI. This commission had permanent access to the national capacity of ICU beds and directly articulated with ICU directors, planning of needs. INEM integrated this network, catalysing a capacity of 8 nurse-staffed ambulances in the country, specially allocated to these transports.

Objectives. It is essential to organize nationally a network to respond to a catastrophic increment of patients. This pandemic showed that management of ICU beds is directly linked to the national networking capacity, not only between ICUS but also with the operational partners as critical patient transferences involve many professionals, equipment, and transportation. Prehospital medicine must have its capacity incremented in close relation to intensive care needs.

Methods. Data from INEM activity records were retrospectively analyzed. Namely the inter-hospital transportations organized regionally with CARNMI during the month of February - that were strategically supported by a crisis room that has been operating since the beginning of the pandemic in INEM.

Results. During February, with Portugal under major restrictive measures of lockdown INEM received 85.011 calls and dispatched 79.387 ambulances. 173 secondary transports were made. During that period thirty-two patients were transferred between ICUs of different regions. Median age was 64 years, and $62.2 \%$ were male. Almost the total transferred cases $(90.6 \% ; n=29)$ were COVID positive, $40.6 \%(n=13)$ were receiving mechanical ventilation $34.4 \%(n=11)$ were on non-invasive ventilation and $18.8 \%(n=6)$ need high oxygen support.

Conclusion. This response is a testament to the power and benefits of a regionally networked approach to critical events, showing the importance of a multidisciplinary approach in care patients. Lessons learned may benefit other healthcare providers, managers, and policymakers to prepare for future calamities.

\section{Reference(s)}

1. Barba R, Rosado C, Pardo-Moreno JV, Rey-Biel J, (2020). Managing People, Roles, and Resources During Covid-19 Surge. NEJM Catalyst Published Online First: 18 May 2020. https://catalyst.nejm.org/doi/full/10.1056/cat. 20.0152

2. James RH, Doyle CP, Cooper DJ, (2020). Descriptive record of the activity of military critical care transfer teams deployed to London in April 2020 to undertake transfer of patients with COVID-19. BMJ Mil Health Published Online First: 28 December 2020. https://doi.org/10.1136/bmjmi litary-2020-001619

3. Pett E, Leung HL, Taylor E, et al. Critical care transfers and COVID-19: Managing capacity challenges through critical care networks. Journal of the Intensive Care Society. Published Online First: 16 December 2020 https:// doi.org/10.1177/1751143720980270

4. Ma X., \& Vervoort D. (2020). Critical care capacity during the COVID-19 pandemic: Global availability of intensive care beds. Journal of critical care, 58, 96-97. https://doi.org/10.1016/j.jcrc.2020.04.012

5. Lacasa L, Challen R, Brooks-Pollock E, Danon L (2020) A flexible method for optimising sharing of healthcare resources and demand in the context of the COVID-19 pandemic. PLoS ONE 15(10): e0241027. https://doi. org/10.1371/journal.pone.0241027

6. Carenzo L, Costantini E, Greco M, Barra FL, Rendiniello V, Mainetti M, et al. Hospital surge capacity in a tertiary emergency referral centre during the COVID-19 outbreak in Italy. 2020

7. We thank Força Aérea Portuquesa (Portuquese Air Force) and INEM's Sala de Situação Nacional (Crisis coordination cell) for useful work and data.

001349

Optimising usage of critical care services: The case for enhanced surgical care units: A single centre experience

M. Abbas ${ }^{1}$; J. Dixon ${ }^{2}$

${ }^{1}$ Intensive Care Unit, St Helier Hospital, Sutton, United Kingdom;

${ }^{2}$ Intensive Care Unit St Helier Hospital, Epsom and St Helier University

Hospitals, London, United Kingdom

Correspondence: $M$. Abbas

Intensive Care Medicine Experimental 2021, 9(1): 001349

Introduction. Intensive Care Units (ITUs) are the backbone of the healthcare system. In the majority of UK district general hospitals, emergency admissions and many elective surgical procedures are 
dependent on the availability of ITU beds. We have previously demonstrated that inadequate patient flow into and out of ITU increases mortality (Ref 1,2). In our hospital, over 300 patients are admitted annually to ITU following major elective surgery (General Surgery, Gynaecological, Urology, and Orthopaedic). Some require more frequent monitoring than available in standard wards, but not all require organ support. In this current study we looked at the organ support required by elective surgical admissions in ITU.

Methods. In this retrospective study we looked at the utilisation of ITU beds by various surgical specialities. We recorded their general demographics, indication for ITU admission, ITU management, and length of stay in ITU. We looked at all elective surgical admissions to ITU between 01 April 2019 to 30 March 2020.

Results. A total of 343 elective surgical admissions occurred during this time with only $98(28.5 \%)$ patients required either organ support or invasive monitoring [Table 1]. Two hundred and three patients were deemed to no longer require ITU level care within $24 \mathrm{~h}$ [Table 2]. There were significant delays in stepping-down patients from ITU to a surgical wards: $141 / 343(41 \%)$ were stepped-down within $4 \mathrm{~h}$ of the decision; 157 (59\%) were stepped-down after $4 \mathrm{~h}$. Table 1: Care received according to surgical group

\begin{tabular}{|c|c|c|c|c|}
\hline & $\begin{array}{l}\text { General } \\
\text { surgery }\end{array}$ & Gynaecology & Orthopaedics & Urology \\
\hline Total & 231 & 13 & 18 & 81 \\
\hline $\begin{array}{l}\text { Standard monitor- } \\
\text { ing }\end{array}$ & 144 & 11 & 18 & 72 \\
\hline \multicolumn{5}{|c|}{ Organ support/Invasive monitoring } \\
\hline BRS & 6 & 1 & 0 & 3 \\
\hline ACVS & 1 & 0 & 0 & 0 \\
\hline $\mathrm{BCVS}+\mathrm{CVC}$ & 80 & 1 & 0 & 6 \\
\hline A-line only & 132 & 5 & 13 & 46 \\
\hline
\end{tabular}

Table 2: Discharges from HDU to wards

\begin{tabular}{lllll}
\hline & $\begin{array}{l}\text { General } \\
\text { surgery }\end{array}$ & $\begin{array}{l}\text { Gynaecol- } \\
\text { ogy }\end{array}$ & $\begin{array}{l}\text { Orthopae- } \\
\text { dics }\end{array}$ & Urology \\
\hline Within $4 \mathrm{~h}$ & 95 & 6 & 10 & 30 \\
$>4 \mathrm{~h}$ & 136 & 7 & 8 & 51 \\
$>24 \mathrm{~h}$ & 36 & 0 & 1 & 8 \\
$\begin{array}{c}\text { Mean } \pm \text { SD } \\
\text { (hours) }\end{array}$ & $10.3 \pm 12.2$ & $6.1 \pm 5.1$ & $6.6 \pm 7.0$ & $11.8 \pm 30.0$ \\
$\begin{array}{c}\text { Range } \\
\text { (hours) }\end{array}$ & 0.0 to 79.5 & 0.8 to 20.5 & 1.1 to 25.0 & 0.0 to 266.0 \\
\hline
\end{tabular}

Conclusion. The number of elective surgical patients requiring organ support is likely to be an over-estimate. Some patients require central venous or arterial catheters but do not require vasopressors. Many patients that were deemed to require ITU care beyond $24 \mathrm{~h}$ only required frequent monitoring. This study demonstrates the need for dedicated enhanced post-operative surgical care units where an intermediate level of care where no organ support is provided, but a higher level of observation, monitoring, and interventions can be provided than on a general ward. Surgical enhanced care units have been recognised by the Faculty of Intensive Care Medicine and the Royal College of Surgeons as an efficient way to utilise resources and may result in improved quality of care with less variation, reduced cancellation of elective surgery, and provide cost savings. In addition, dedicated enhanced surgical care units frees up beds in ITU for emergency patients who require organ support.

\section{Reference(s)}

1. 2. Having an empty bed in ICU improves mortality: A retrospective cohort study: M Abbas, A Dowdell, P Sarkar, J Camilleri, R Kilvington, J Dixon. ESICM EuroAsia 2020

2. 1. Delayed step down from ICU is associated with delayed admissions to ITU and increase mortality. Madiha Abbas, P Sarkar, J Camilleri, R Kilvington, John Dixon. ESICM EuroAsia 2019

\section{8}

Long-term mental health impairments post severe COVID-19

L. Alegria Vargas'; P. Repetto ${ }^{2}$; M. Henriquez ${ }^{2}$; M. Bustamante ${ }^{2}$

'Departamento de medicina intensiva, Pontificia Universidad Católica de Chile, Santiago, Chile; ${ }^{2}$ Escuela de psicología, Pontificia Universidad

Católica de Chile, Santiago, Chile

Correspondence: L. Alegria Vargas

Intensive Care Medicine Experimental 2021, 9(1): 001358

Introduction. Survivors of ICU stay commonly experience long-lasting mental health impairments (1). Additionally, COVID-19-related changes in the hospital environment may pose an increased risk of adverse psychological symptoms. For example, reduced access to family members, pleasurable activities, and rehabilitation may result in anxiety and demoralization in patients. Such stressors may have important implications for psychological outcomes in survivors of COVID-19 (2).

Objectives. The primary purpose of this study is to observe and describe psychological sequelae at three months post-discharge in patients admitted with respiratory failure due to Covid-19 to a polyvalent ICU of a health center.

Methods. Prospective, observational study, carried out in the Intensive Care Unit at Hospital Clínico UC-CHRISTUS, between May and August 2020. Patients with severe COVID-19 and mechanical ventilation for over $48 \mathrm{~h}$. Participants were recruited and underwent followup approximately three months following discharge. We assessed anxiety and depression symptoms, and post-traumatic stress disorder (PTSD) using validated measures.

Results. A total of 27 patients completed the follow-up. The median age was 58 years, and the median number of days on mechanical ventilation was 20 days. The mean Sequential Organ Failure Score and the Acute Physiology were $5.2 \pm 2.5$ and $11 \pm 7.1$, respectively. Median ICU and hospital length of stay were 26 days and 36 days, respectively. Table 1 shows demographic information and relevant clinical outcomes of the patients. At follow-up at three months, we found anxiety to be the most prevalent disorder $(30,8 \%)$ that persisted overtime. Risk for anxiety was higher among those who spent 20 days or more in mechanical ventilation.

Conclusion. This was a single center case series. Our study suggests a high prevalence of anxiety symptoms over other psychological sequelae. Careful implementation of evidence-based critical care interventions combined with robust rehabilitation programs that begin in the ICU and continue after discharge are necessary to reduce future mental health problems.

\section{Reference(s)}

1. 2. Hosey M, Needham D. Survivorship after COVID-19 ICU stay. Nat Rev Dis Primers. 2020 Jul 15:6(1):60.

2. 1. Cuiyan Wanga, Riyu Pana, Xiaoyang Wana, Yilin Tana, Linkang Xu, et al. A longitudinal study on the mental health of general population during the COVID-19 epidemic in China. Brain Behav Immun. 2020 Jul;87:40-48. 
001364

Computed Tomography Findings and inflammatory biomarquers in ICU 2019

Novel Coronavirus patients

A. Meftah, ${ }^{1}$;Y. Touil, ${ }^{2}$; E. Abid, ${ }^{3}$; S. Ben Nessira ${ }^{3} ;$ F. Daly, ${ }_{1}^{2}$; A. Trifir $;$ S.

Abdellatif, ${ }^{2} ;$ LS. Ben ${ }^{2}$

${ }^{1}$ Hospital la rabta, Intensive Care Unit, Tunis, Tunisia; ${ }^{2}$ Intensive Care Unit, Hospital la Rabta, Tunis, Tunisia; ${ }^{3}$ Hospital la rabta, Intensive Care Medcine, Tunis, Tunisia

Correspondence: $Y$. Touil

Intensive Care Medicine Experimental 2021, 9(1): 001364

Introduction. Coronavirus disease 2019 (COVID-19) is a highly infectious respiratory disease that has emerged by a new coronavirus. COVID-19 can lead to acute respiratory distress syndrome in human beings. Chest computed tomography (CT) scan plays a crucial role in the early diagnosis of SARS-CoV-2 new cases. Typical radiologic images of COVID-19 demonstrated clear destruction of the pulmonary parenchyma, including interstitial inflammation and extensive consolidation.

Objectives. The aim of this study is to evalute the Severity of lung disease on chest CT scan and to determine the relationship between CT scores and inflammatory biomarquers.

Methods. All patients admitted to ICU of Rabta Universitary Hospital between September, 2020 and February, 2021, with real-time fluorescence polymerase chain reaction (RT-PCR)-confirmed COVID-19 and underwent chest $\mathrm{CT}$, were included in this study. Laboratory parameters and $\mathrm{CT}$ findings, including overall lung total severity score based on inflammatory lung lesions degree and rated from 0 to 20 , were retrospectively collected from medical records. It has to be noted that inflammatory parameters were collected the day of CT scan realisation. Results. A total of 30 patients (median age, 57 years [47-66]) were evaluated including 23 males $(76,7 \%)$ and 7 females $(23,3 \%)$. The median BMI (Body Mass Index) was 27,7 [25-31]. Twelve patients (40\%) were intubated for pulmonary distress. Total rate of death was 10 . The mean day of CT scan realisation corresponded to the 11th day of evolution [7-15].

Of 30 patients:

27 had involvement of more than two lobes at chest CT.

12 had only ground glass opacities (GGO) and 16 had consolidation with GGO.

12 had crazy paving pattern.

$17 \mathrm{had}$ a peripheral distribution of the disease.

None presented with a normal CT scan.

In 27 cases lung cavitations, discrete pulmonary nodules, pleural effusions, fibrosis, emhysema or lymphadenopathy were present.

Median total lung severity CT score was 10 [6,75-13,25].

Median neutrophil count was 8095 [4467], white blood cell $=9495$

[6035-11625], lymphocyte count $=950[710-1295]$ and C-reactive protein (CRP) $=140$ [87-188].

Total lung severity CT score wasn't correlated to inflammatory parameters $(P>0,1)$. No age difference occurred regarding $C T$ score.

Discussion. Little is known about the potential association between the CT images reflecting the lung disease's degree and other parameters especially inflammatory biomarquers. In this study, total lung CT score wasn't correlated to inflammatory parameters for many reasons. First of all, it is a small sample. Moreover, we had only one CT scan for each patient so it wasn't possible to compare the evolution of CT findings to laboratory parameters. Finally, the date of CT scan corresponded to a late stage of infection and not at the beginning of symptomatology.

Conclusion. Despite the important lung damage caused by 2019 novel coronavirus in CT our study showed no correlation between total lung severity score and inflammatory parameters. Thus, larger cohort studies are needed to adjust these findings.
001370

Outcomes of COVID 19 patients admitted to a critical care unit in Wales during the 'second wave'

V. Maples, ${ }^{1}$

'School of Medicine, Cardiff University, Cardiff, United Kingdom

Correspondence: $\vee$. Maples

Intensive Care Medicine Experimental 2021, 9(1): 001370

Introduction. As of 15th April 2021, over 140 million cases of COVID 19 have been confirmed worldwide (1). In most cases of COVID 19, individuals are either asymptomatic or have a mild flu-like illness (2) with symptoms including fever, cough and fatigue (3). However, many individuals require hospital-based care and approximately $5 \%$ of patients require critical care admission (4).

A common indication for critical care admission with COVID 19 is for ventilatory support (5). Some hospitals also admit patients to critical care for other forms of ventilatory support including Continuous Positive Airway Pressure (CPAP) or High Flow Nasal Oxygen (HFNO). Other indications for critical care admission can include septic shock or acute organ dysfunction (5).

Unfortunately, COVID 19 infection has a high mortality especially when the patient requires hospitalisation and critical care. Although estimates vary, the mortality rate for individuals infected with COVID 19 is between $0.5 \%-4 \%$ (4). Critical care patients have the highest mortality with rates documented as high as $22-62 \%(6,7)$.

Objectives. This paper describes the patient demographics and pertinent medical background related to a cohort or patient admitted to 1 critical care unit in Wales between 1st January and 6th March 2021 as well as comparing these factors between the patients who survived and who died.

Methods. The clinical cohort involved in this study is 41 patients with COVID-19 admitted into 1 critical care unit in Wales between 1st January and 6th March 2021. Data was collected via reading the electronic medical records of the patients.

Patient demographics analysed in this report are in line with the usual demographic information collected on critical care patients, as identified by the ICNARC report (8). Clinical information collected regarding ventilatory support and medications was based on a UKwide clinical audit by ICNARC.

Results. Mean age was 56.41 (SD 11.26) years, $75.61 \%$ were male, $73.14 \%$ were white, $46.34 \%$ were from the most deprived areas, $58.53 \%$ were obese and $51.22 \%$ had at least 1 documented comorbidity. $41.46 \%$ survived critical care admission.

There was no significant difference in the number of days an individual had been suffering from COVID 19 prior to presenting to hospital $(p=0.6683)$, the length of hospital admission prior to transfer to critical care $(p=0.0939)$, the length of stay in critical care $(p=0.4216)$ or the length of hospital stay $(p=0.2153)$.

Conclusion. From analysis of patient characteristics, clinical processes, treatment delivered to patients and their outcomes from the care unit, over a 3-month period, has shown similar trends as seen elsewhere in the United Kingdom (8). It was encouraging to see the high percentage of patients given evidence-based treatments, such as dexamethasone (9) and IL-6 inhibitors (10). While acknowledging the limitations of the size of the patient cohort and the completeness of the data set, this paper is beneficial in identifying areas to target to improve patient outcomes going forward.

\section{Reference(s)}

1. (1) WHO Coronavirus (COVID-19) Dashboard. Covid19.who.int. 2021. [accessed 15 Apr 2021] Available from: https://covid19.who.int.

2. (2) Verity R, Okell L, Dorigatti I, Winskill P, Whittaker C, Imai N et al. Estimates of the severity of coronavirus disease 2019: a model-based analysis. The Lancet Infectious Diseases. 2020;20(6):669-677. https://doi. org/10.1016/s1473-3099(20)30243-7. 
3. (3) Sun J, Chi C, Li C, Buys N, Yin C. Clinical Characteristics of 92,800 Patients with Confirmed Coronavirus Disease (COVID-19) Infection: A Systematic Review and Meta-Analysis. SSRN Electronic Journal. 2020;. https://doi.org/10.2139/ssrn.3677878.

4. (4) Wu Z, McGoogan J. Characteristics of and Important Lessons From the Coronavirus Disease 2019 (COVID-19) Outbreak in China. JAMA. 2020;323(13):1239. https://doi.org/10.1001/jama.2020.2648.

5. (5) Murthy S, Gomersall C, Fowler R. Care for Critically III Patients With COVID-19. JAMA. 2020;323(15):1499. https://doi.org/10.1001/jama.2020 3633

6. (6) Wang D, Hu B, Hu C, Zhu F, Liu X, Zhang J et al. Clinical Characteristics of 138 Hospitalized Patients With 2019 Novel Coronavirus-Infected Pneumonia in Wuhan, China. JAMA. 2020;323(11):1061. https://doi.org/ 10.1001/jama.2020.1585.

7. (7) Yang X, Yu Y, Xu J, Shu H, Xia J, Liu H et al. Clinical course and outcomes of critically ill patients with SARS-CoV-2 pneumonia in Wuhan, China: a single-centered, retrospective, observational study. The Lancet Respiratory Medicine. 2020;8(5):475-481. https://doi.org/10.1016/s22132600(20)30079-5.

8. (8) Intensive Care National Audit and Research Centre. ICNARC report on COVID-19 in critical care: Wales_-26 March 2021.

9. (9) Horby P, Lim W, Emberson J, Mafham M, Bell J, Linsell L et al. Effect of Dexamethasone in Hospitalized Patients with COVID-19 - Preliminary Report. 2020; https://doi.org/10.1101/2020.06.22.20137273

10. (10) RECOVERY TRIAL. Recoverytrial.net. 2021. [accessed 25 Apr 2021] Available from: https://www.recoverytrial.net/files/recovery-press-relea se-tocilizumab_final.pdf.

11. I would like to thank Dr Babu Muthuswamy for his support with the project.

\section{8}

Major vascular injuries during laproscopy. Two rare case reports

S. Nissar ${ }^{1}$; S. NAWAZZ2; A. Sumayya2 ; C. Arshad'; MA. Syed ${ }^{3}$

${ }^{1} \mathrm{Sicu}$, Hamad Medical Corporation, Doha, Qatar; ${ }^{2}$ Medical Education,

Hamad Medical Corporation, Doha, Qatar; ${ }^{3}$ General surgery, Hamad

Medical Corporation, Doha, Qatar

Correspondence: S. NAWAZ

Intensive Care Medicine Experimental 2021, 9(1): 001398

Introduction. Laproscopic surgeries are less invasive than open procedures and have become common due to faster recovery and discharge from hospital. The basic risk difference in laparotomy and laparoscopy is that the initial steps of Veress needle or trocar are inserted blindly during laparoscopic surgeries, which may cause major vascular, bowel or urinary tract injuries. Although these entry or initial injuries are rare; they can cause life threatening hemorrhage or sepsis. We report two cases of vascular laparoscopic entry injuries.

Case1: A young male patient was transferred from a private hospital in hemorrhagic shock after trocar insertion for laparoscopic appendectomy. We resuscitated the patient with massive blood transfusion protocol and vasopressors. Immediately he was taken for exploratory laparotomy which revealed abdominal aortic and small bowel injury. The aortic and small bowel injuries were repaired and abdomen was closed in stages. Post operatively the patient had acute renal failure which required dialysis and disseminated intravascular coagulopathy which required supportive care. He improved and his trachea was extubated on day 5. Supportive care was continued, and the patient was transferred to ward on day 15 . From there he was discharged home and follow up in the outpatient clinic did not reveal any complications.

Case2: A 49 year old female patient had trocar injury to the right common iliac artery during laparoscopic oophorectomy which required 1:1:1 massive blood transfusion protocol resuscitation and vascular repair had to be performed. Post operatively she was transferred to surgical intensive care unit for resuscitation and care. She was extubated after $24 \mathrm{~h}$ and transferred to the ward soon thereafter. She was discharged home without any abnormalities.

Objectives. The objective of our case reports is to identify and highlight management of major vascular injuries during laparoscopy and their management in ICU.

Methods. These are retrospective case reports.
Results. Both patients survived without long term complications following major vascular injuries during laproscopy which lead to massive intraperitoneal bleeding.

Conclusion. Laparoscopic entry injuries are rare, but can be life threatening and can cause multiple organ dysfunctions. Early recognition, aggressive resuscitation and appropriate management of laparoscopic entry injuries is vital for better outcome.

\section{Reference(s)}

1. 1. Krishnakumar S, Tambe P. Entry complications due to trocher insertion in laparoscopic surgery. J Gynecol Endosc Surg 2009; 1(1):4-11

2. 2. Asfour V, Smythe E, Attia, R. Vascular injury at laparoscopy: a guide to management. Journal of obstetrics and gynaecology: the journal of the Institute of Obstetrics and Gynaecology 2018; 38(5), 598-606.

3. 3. Brierley G, Arshad I, Shakir F, Visvathanan D, Arambage K. Vascular injury during laparoscopic gynaecological surgery: a methodological approach for prevention and management. The Obstetrician \& Gynaecologist 2020; 2: $1-8$

4. 4. Fuller J, Ashar BS, Carey-Corrodo J. Trocar associated injuries and fatalities: an analysis of 1399 reports to the FDA. J Mini Invasive Gynecol 2005; 12(4):3002-7

5. 5. Harkki-siren P, Sjoberg J, Kurki T. Major complications of laparoscopy: A follow -up Finnish study. Obstet Gynecol 1999; 94:94,098

6. 6. Chandler JG, Corson SL, Way LW. Three spectra of laparoscopic entry access injuries. J Am Coll Surg 2001; 192:478-91

001410

Fluid overload phenotypes in critical illness-a machine learning approach

CA. Pfortmueller ${ }^{1}$; A. Messmer ; ; M. Moser ${ }^{1}$; P. Zuercher, ${ }^{1}$; JC. Schefold, ${ }^{1}$; M. Mueller,

${ }^{1}$ Department of Intensive Care Medicine, Inselspital, Bern University

Hospital, University of Bern, Bern, Switzerland

Correspondence: C.A. Pfortmueller

Intensive Care Medicine Experimental 2021, 9(1): 001410

Introduction. Awareness for the detrimental effects of fluid overload (FO) in the critically ill has risen considerably during the last decade and strategies to minimize FO were developed. Problematically, current research on FO does not take in to account the extreme heterogeneity of critical illness and no effort was undertaken to identify subgroups of patients particularly prone to FO in intensive care so far. Thus, the aim of this retrospective cohort study was to derive specific "FO phenotypes" by using machine learning techniques.

Methods. In this retrospective single center study we included all adult ( $<16$ years) intensive care (ICU) patients with a minimum LOS of 3 days. Patients with insufficient data to compute FO (fluid data, weight) were excluded. Data was analyzed by univariate and multivariate logistic regression (MV), fast and fugal trees (FFT) and a random forest (RF) model.

Results. 1772 patients were included in our analysis, thereof $21.8 \%$ $(n=387)$ had FO at ICU day3. The random forest model had the highest AUC (0.85) followed by logistic regression $(0.81)$ and FFT (0.75). The most important predictors identified in all 3 models were lactate at admission, admission after major surgery and bicarbonate at admission. Sepsis/septic shock and female sex were identified as risk factors and non-traumatic neurological disease as a protective factor in the MV and RF analysis. Renal factors (chronic kidney disease at admission and creatine at admission) and disease severity were identified only in the RF model and had a significantly lower importance.

Conclusion. The FO phenotype consists of patients admitted after major surgery or with sepsis/septic shock with high lactate and low bicarbonate. Renal factors and disease severity at admission have some influence, but are of minor importance. 
001415

Long-term cognitive impairment in COVID-19 ARDS survivors: a multicentre, prospective cohort study

C. Bouts ${ }^{1}$; B. Janssen ${ }^{2}$; S. Thiessen ${ }^{1}$; X. Willaert ${ }^{1}$; W. Boer ${ }^{1}$; K. Engelen ${ }^{1}$; T. Fivez, ; M. Vander Laenen, ; A. Creemers ${ }^{1}$; G. Vingerhoets ${ }^{2}$; D. Mesotten ${ }^{1}$ Department of Anaesthesiology, Intensive Care Medicine, Emergency Medicine and Pain Therapy, Ziekenhuis Oost-Limburg, Genk, Belgium;

${ }^{2}$ Department of Psychology, Universiteit Gent, Ghent, Belgium

Correspondence: $C$. Bouts

Intensive Care Medicine Experimental 2021, 9(1): 001415

Introduction. COVID-19 can lead to respiratory failure and acute respiratory distress syndrome (ARDS) (1). ARDS is frequently complicated by long-term consequences and recent articles have suggested that COVID-19 survivors also suffer from these complications $(2,3)$. It is known that cognitive impairment in ARDS survivors ranges from 70 to $100 \%$ at hospital discharge and is still present in 46 to $80 \%$ of these patients one year after discharge $(2,4)$. However the long-term cognitive impairment in COVID-19 ARDS survivors has not been thoroughly investigated.

Objectives. We conducted a small prospective study (clinicaltrials. gov: NCT04593069) to investigate the long-term cognitive impairment in COVID-19 ARDS survivors. We hypothesized that COVID19 ARDS patients perform worse on cognitive tasks 6 months after admission in comparison to reference values of a healthy population.

Methods. We assessed the cognitive function at 6 months after discharge in COVID-19 ARDS survivors, admitted to the ICU during the 2020 spring outbreak (March to June) in two Belgian hospitals. The Repeatable Battery for the Assessment of Neuropsychological Status (RBANS) and Trail Making Test (TMT) were used to measure cognitive and executive functions, respectively (4). The Short Form of the Informant Questionnaire on Cognitive Decline in the Elderly (short IQCODE) evaluated cognitive impairment to assess patients not able to perform the RBANS and TMT (5).

Results. A total of 117 patients were admitted to the ICU with COVID19 ARDS. Of the 85 survivors, we obtained the short IQCODE in 67 patients. Among these patients, we measured the RBANS and TMT in 45 patients. The remaining patients were not able to take these tests due to a language barrier. Patient characteristics are shown in Table 1. COVID-19 survivors had lower cognition scores than expected. The mean score of the short IQCODE $(n=67)$ was 3.4 (SD 0.4), suggestive of cognitive dysfunction. The total RBANS score $(n=45)$ was on average minus 1 standard deviation below the age-corrected reference mean (Figure 1). Interestingly, executive function as measured by the task-switching part B of the TMT $(n=45)$ was also decreased, with a median score of 40 (IQR 10-65).

Conclusion. Our results suggest that COVID-19 ARDS negatively affects long term cognitive function, which can be a huge burden on patients, care givers and society, necessitating larger trials for further investigations.

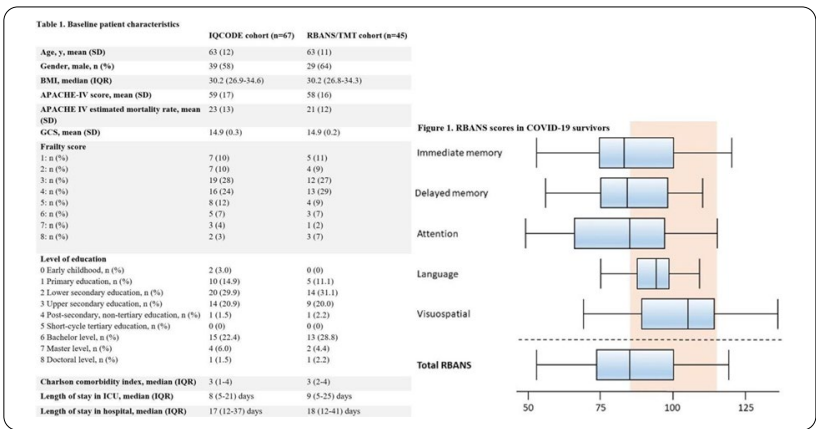

Reference(s)

1. (1) Grasselli G, Tonetti T, Protti A et al. Pathophysiology of COVID-19-associated acute respiratory distress syndrome: a multicentre prospective observational study. Lancet Respir Med. 2020; 8: 1201-1208.

2. (2) Herridge M, Moss M, Hough C et al. Recovery and outcomes after the acute respiratory distress syndrome (ARDS) in patients and their family caregivers. Intensive Care Medicine. 2016; 42: 725-738.

3. (3) Huang C, Huang L, Wang Y et al. 6-month consequences of COVID-19 in patients discharged from hospital: a cohort study. Lancet. 2021; 397: 220-32.

4. (4) Pandharipande P, Girard T, Jackson J et al. Long-Term Cognitive Impairment after Critical Illness. N Engl J Med. 2013, 369: 1306-1316.

5. (5) Jorm A. The Informant Questionnaire on Cognitive Decline in the Elderly (IQCODE). International Psychogeriatrics. 2004, 16: 275-93.

\section{5}

Epidemiological characteristics, baseline clinical features and outcomes of critically ill patients treated in a COVID-19 tertiary center in continental Croatia

A. Šribar ; D. Tipura ${ }^{1}$; M. Pražetina ${ }^{1}$; Ž. Drmić, ${ }^{1}$; M. ČUčković, ${ }^{1} ;$ A. Kukoč ; M.

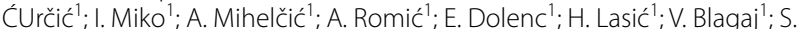
Hleb $^{1} ;$ H. Almahariq ${ }^{1}$; J. Peršec

${ }^{1}$ Clinical Department of Anesthesiology, Resuscitation and Intensive Care Medicine, Clinical Hospital Dubrava, Zagreb, Croatia

Correspondence:Correspondence: A. Šribar

Intensive Care Medicine Experimental 2021, 9(1): 001425

Introduction. On Feb 26th 2020. First case of COVID-19 was documented in Croatia. In order to contain the spread of the SARS-CoV-2 virus while maintaning the continuity of healthcare, the Ministry of Health and National crisis management made a resolution to repurpose the University Hospital Dubrava into a Primary Respiratory Center (PRIC) for patients with confirmed COVID-19 infection. Although scientific knowledge of COVID-19 increases daily, limited information is available in early identification of individuals who are at risk of developing severe symptoms. Certain demographic features and clinical characteristics among patients who were likely to develop severe symptoms of COVID-19 and thus require mechanical ventilation have been previously described. Published data worldwide reported high mortality rates ranging from 40 to $97 \%$ in those requiring mechanical ventilation. By Feb 1st 2021. 692 critically ill patients were treated in PRIC intensive care units (PRIC-IC).In this paper we present their epidemiological characteristics, baseline clinical features and outcomes.

Methods. 692 critically ill COVID-19 patients were included in this retrospective observational study. Recorded variables were: basic demographic characteristics (gender, age), laboratory parameters at ICU admission: white blood cell count (WBC, $\times 109 / \mathrm{L})$, neutrophil and lymphocyte percentage in $\mathrm{WBC}, \mathrm{PaO}_{2} / \mathrm{FiO}_{2}$ ratio $(\mathrm{mmHg})$, serum $\mathrm{D}$-dimer $(\mathrm{mg} / \mathrm{L})$, serum lactate $(\mathrm{mmol} / \mathrm{L})$, serum ferritin $(\mu \mathrm{g} / \mathrm{L})$, serum procalcitonin $(\mathrm{PCT}, \mathrm{ng} / \mathrm{ml})$, serum C-reactive protein (CRP, $\mathrm{mg} / \mathrm{L})$, serum IL-6 $(\mathrm{pg} / \mathrm{ml})$, and glomerular filtration rate $\left(\mathrm{ml} / \mathrm{min} / 1.73 \mathrm{~m}^{2}\right)$, organizational aspects (patient admitted to the ICU from other departments of PRIC UH Dubrava or admitted directly from ICUs in other hospitals in continental Croatia), basic anthropomorphic characteristics (body mass index-BMI, $\mathrm{kg} / \mathrm{m}^{2}$ ), presence of major comorbidities (arterial hypertension, diabetes mellitus, congestive heart failure defined as NYHA status $>\mathrm{II}$, chronic kidney disease defined as glomerular filtration rate $<60 \mathrm{ml} / \mathrm{min} / 1.73 \mathrm{~m}^{2}$ calculated by using CKD-EPI formula and chronic hematologic disorders), Charlson comorbidity index (CCI), sequential organ failure assessment (SOFA) score, duration of COVID19 disease before ICU admission, duration of ICU stay, mechanical ventilation, high-flow nasal oxygen therapy and ICU and in-hospital mortality rate. 
Results. Median age was 72 (64-78) years, and there were $67.1 \%$ males and $32.9 \%$ females. Median patient body mass index was $29.1 \mathrm{~kg} / \mathrm{m}^{2}$, $32.6 \%$ patients had diabetes mellitus, $71.2 \%$ had arterial hypertension, $19.1 \%$ had congestive heart failure, $12.6 \%$ had chronic kidney disease, and $10.3 \%$ patients had hematological disorders. Median number of comorbidities was 3 and median CCl was $5.61 .8 \%$ patients received high flow nasal oxygen (HFNO), and $80.5 \%$ received mechanical ventilation (MV). Survivors had significantly lower age, number of comorbidities, CCl, SOFA score, serum ferritin, CRP, D-dimer, PCT. IL-6, lactate, WBC and neutrophil counts; while their lymphocyte counts, $\mathrm{PaO}_{2} / \mathrm{FiO}_{2}$ ratio and GFR were higher at admission. Duration of HFNO was 3 (1-6) days and duration of MV was 7 (3-12) days. 36 (5.2\%) patients only received supplemental oxygen during ICU stay, and 6 patients $(0.9 \%)$ received extracorporeal membrane oxygenation support. 41 patients (5.9\%) received renal replacement therapy (RRT), of those patients 16 received intermittent hemodialysis (IHD), 18 received continuous renal replacement therapy (CRRT), 2 received both IHD and CRRT and 5 patients were dialysed due to end-stage renal disease and need for chronic renal replacement therapy. Length of ICU stay was 9 (4-14) days. Median survival for mechanically ventilated patients was 11 days, and 24 days for patients that were not mechanically ventilated. For patients with bacterial superinfections median survival was 13 days and 8 days for those without bacterial superinfections.

Conclusion. Identified parameters that differentiate survivors from nonsurvivors are in general agreement with published data. Further multivariate analyses are warranted to isolate individual mortality risk factors.

\section{Reference(s)}

1. Richardson S, Hirsch JS, Narasimhan M, Crawford JM, McGinn T, Davidson KW, et al. Presenting Characteristics, Comorbidities, and Outcomes Among 5700 Patients Hospitalized With COVID-19 in the New York City Area. JAMA. 2020 May 26;323(20):2052

2. Auld SC, Caridi-Scheible M, Blum JM, Robichaux C, Kraft C, Jacob JT, et al. ICU and Ventilator Mortality Among Critically III Adults With Coronavirus Disease 2019*. Critical Care Medicine. 2020 Sep:48(9):e799

3. Roedl K, Jarczak D, Thasler L, Bachmann M, Schulte F, Bein B, et al. Mechanical ventilation and mortality among 223 critically ill patients with coronavirus disease 2019: A multicentric study in Germany. Australian Critical Care. 2021 Mar 1:34(2):167-75.

4. Peršec J, Šribar A, Kereš T. Organizational aspects of intensive care unit resource allocation in a primary respiratory intensive care center specialized for the treatment of SARS-COV-2 patients. Croat Med J. 2020 Jun;61 (3):304-5

5. Myers LC, Parodi SM, Escobar GJ, Liu VX. Characteristics of Hospitalized Adults With COVID-19 in an Integrated Health Care System in California. JAMA. 2020 Jun 2;323(21):2195-8

6. Wang D, Hu B, Hu C, Zhu F, Liu X, Zhang J, et al. Clinical Characteristics of 138 Hospitalized Patients With 2019 Novel Coronavirus-Infected Pneumonia in Wuhan, China. JAMA. 2020 Mar 17:323(11):1061

7. Grasselli G, Zangrillo A, Zanella A, Antonelli M, Cabrini L, Castelli A, et al. Baseline Characteristics and Outcomes of 1591 Patients Infected With SARS-CoV-2 Admitted to ICUs of the Lombardy Region, Italy. JAMA. 2020 Apr 28:323(16):1574-81

8. Wu C, Chen X, Cai Y, Xia J, Zhou X, Xu S, et al. Risk Factors Associated With Acute Respiratory Distress Syndrome and Death in Patients With Coronavirus Disease 2019 Pneumonia in Wuhan, China. JAMA Intern Med. 2020 Mar 13

\section{6}

Mortality prediction in COVID-19: external validation of the $4 \mathrm{C}$ mortality model and exploration of frailty a prospective 1-year dual-centre observational cohort study

A. Pantelides'; L. Hodgson ' ; A. Hunter' ; E. Owen²; A. Khushal' ${ }^{3}$; S. Dooris G. Mcgrath

${ }^{1}$ Intensive Care, Worthing Hospital, Worthing, United Kingdom;

${ }^{2}$ Medicine, Brighton \& Sussex Medical School, Falmer, United Kingdom;

${ }^{3}$ Medicine, Gloucestershire Hospitals NHS Foundation Trust, Gloucester, United Kingdom; ${ }^{4}$ Emergency Department, Worthing Hospital, Worthing, United Kingdom

Correspondence: $L$. Hodgson

Intensive Care Medicine Experimental 2021, 9(1): 001436
Introduction. Following the first wave of the ongoing COVID-19 pandemic the $4 \mathrm{C}$ mortality score reported accurate prediction of early mortality. This study aimed to explore whether establishing whether frailty was an additional predictor and report 1-year outcomes of patients admitted in the first wave to two UK hospitals.

Methods. This prospective observational cohort study included all adults ( $\geq 18$ years) admitted to hospital with confirmed COVID-19 between 1st March and 31st May 2020. Data collection was from electronic capture of routine data on admission, including the clinical frailty scale (CFS). Primary outcomes were in-hospital and 1-year mortality.

Results. 297 patients with COVID-19 were included. 58\% $(n=173)$ were male, $38 \%(n=114)$ died in hospital. $54 \%$ had a CFS of $>5$. In-hospital mortality was significantly associated with frailty $(p=0.003)$. Mortality ranged from $19.1 \%$ in those rated a CFS of $1-2$, $31.9 \% 3-4,42.9 \% 5-6$ and $45.1 \%$ 7-8 points. Area under the receiver operating characteristic curve (AUC) was 0.79 (95\% Cl $0.74-0.85)$ for the 4C mortality score compared to $0.66(0.56-0.69)$ for the CFS. Using logistic regression on backward stepwise analysis, of the eight variables of the $4 C$ model and CFS, the only retained variables were CRP, oxygen saturation, urea and CFS. A further 24 patients died over the year from admission giving a 1 -year mortality of $46 \%$. Of the survivors to hospital discharge $31 \%$ were re-admitted during the year of follow-up.

Conclusion. In this population of patients admitted to hospital with COVID-19, frailty was an independent predictor of mortality. Further exploration of the use of frailty in COVID prediction models is desirable.

\section{4}

Opening visitation policy during COVID-19 is safe and increases patient and family satisfaction

L. Fernandez Ruiz'; A. Carranza Pinel'; ; A. Aranda León ${ }^{1}$; M. Molina Rodriguez ${ }^{1}$; C. Mingorance Garvi ${ }^{1}$; M. Arias Diaz ; G. Heras La Calle 'Intensive Care Unit, Hospital Santa Ana, Motril, Spain

Correspondence: $L$. Fernadez Ruiz

Intensive Care Medicine Experimental 2021, 9(1): 001444

Introduction. The onset of the SARS-COV2 pandemic in 2020 made it necessary to change the usual organisation of ICUS worldwide, including the visiting policies. In the first wave of the pandemic, visits were completely suspended in most Spanish ICUs, leading to patient and family dissatisfaction, as well as eliminating the benefits that family visits have on the evolution of the critically ill patient. Since the second wave, some ICUs eliminated this restriction according to the recommendations published on June 2020 by the Spanish Societies of Intensive Care (SEMICYUC and SEEIUC) and the Pan-American and Iberian Federation (FEPIMCTI), allowing a more flexible visitation policy with different safety recommendations. The ICU of Hospital Santa Ana de Motril, Granada (Spain), opted for this attitude, considering it beneficial for the patient and families.

Objectives. The aim of the study of this study is to compare the visiting regime established in the first wave and the new one established in the following waves, and its influence on the number of infections in relatives and the satisfaction of patients and their relatives.

Methods. Retrospective descriptive study. We collected demographic data, the visiting regime adopted in each of the waves, the number of infections in family members and the degree of satisfaction achieved. Results. The number of patients admitted in the ICU of Hospital Santa Ana de Motril was 9 patients in the first wave (from March to July 2020) and 89 in the following waves until May 2021. In the first wave, a closed visiting policy was adopted with absolute restriction of visits except in terminal situations. In the second wave, a flexible regime was adopted according to the recommendations of the Pan-American and Iberian Federation, allowing a family member to visit twice a day with several safety recommendations, including the instruction in the use of Personal Protection Equipment (PPE) for families. No contagion was recorded in family members attending the visits. The degree of satisfaction achieved was $100 \%$. 
Conclusion. In our experience, opening visitation policy during COVID-19 pandemic is safe and increases patient and family satisfaction.

\section{Reference(s)}

1. 1. Plan de desescalada para los servicios de medicina intensiva tras la pandemia producida por la COVID-19. June 2020. SEMICYUC, SEEIUC, FEPIMCT

001446

Psycological effects of video calls in caregivers of patients admitted in intensive care unit (ICU): a feasibility prospective pilot study

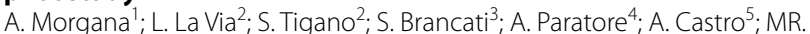
Valenti ${ }^{6}$; F. Oliveri'; F. Sanfilippo ${ }^{6} ;$ M. Astuto $^{6}$

'School of Anaesthesia and Intensive Care, Magna Græcia University, Catanzaro, Italy; ${ }^{2}$ School of Anaesthesia and Intensive Care, University Hospital "G. Rodolico", Catania, Italy; ${ }^{3}$ Department of Surgery and Surgical Specialties, University of Catania, Catania, Italy: ${ }^{4}$ Responsible for "Osiride" Project, University Hospital "G. Rodolico", Catania, Italy; ${ }^{5}$ Anesthesiology and Intensive Care, University Hospital "G.Rodolico", Catania, Italy;

${ }^{6}$ Department of Anesthesiology and Intensive Care, University Hospital "G. Rodolico", Catania, Italy

Correspondence: L. La Via

Intensive Care Medicine Experimental 2021, 9(1): 001446

Introduction. The Sars-Cov2 pandemic outbreak originated in China in 2019 and has so far resulted in over 164 million of people infected and over 3 million deaths, with a high number of hospital and intensive care unit admissions. There has been an inevitable and considerable detachment between patients and their families in order to prevent further diffusion of the pandemic infection. The impossibility to visit his/her own relative has negative effects on anxiety, depression, and post-traumatic stress disorder (PTSD). Video-calls between patients and their families may be used to blunt the negative effects of this detachment. The effect of such strategy on the levels of anxiety, depression, and PTSD are unclear.

Methods. We conducted a prospective study at the Covid-19 ICU in the University Hospital Policlinico "G. Rodolico", Catania (Italy). We evaluated the effects of video-calls performed weekly (OSIRIDE ${ }^{\circledR}$ project) on the incidence of depression, anxiety and PTSD on caregivers of Covid-19 ICU patients. Data were collected with validated questionnaires filled online by caregivers: Impact of Event Scale (Revised IES-R), Center for Epidemiologic Studies Depression Scale (CES-D) and Hospital Anxiety and Depression Scale (HADS). Caregivers were included if they answered the questionnaires both before and a week after the video-call.

Results. 12 caregivers of 11 patients were included. Nine of these patients were successfully discharged from ICU. Table 1 shows both the ventilatory strategy and the mean results of questionnaires on depression, anxiety and PTSD of the included caregivers collected at two time-points: before and one week after the video-call. None of the tests showed statistically significant variations in the questionnaire results.

\begin{tabular}{llllll}
\hline $\begin{array}{l}\text { Strategia } \\
\text { ventilatoria }\end{array}$ & $\mathbf{N}=\begin{array}{l}\text { Question- } \\
\text { ario }\end{array}$ & $\begin{array}{l}\text { PRE Vide- } \\
\text { ocall }\end{array}$ & $\begin{array}{l}\text { POST Vide- } \\
\text { ocall }\end{array}$ & P value \\
\hline $\begin{array}{c}\text { NIV pre-NIV } \\
\text { post }\end{array}$ & $\mathbf{5}$ & CES-D & $15.3 \pm 7.8$ & $18.3 \pm 8.0$ & 0.13 \\
$\begin{array}{c}\text { NIV pre-ETT } \\
\text { post }\end{array}$ & $\mathbf{1}$ & IES-R & $17.1 \pm 9.8$ & $18.2 \pm 9.9$ & 0.45 \\
$\begin{array}{c}\text { NIV pre- } \\
\text { HFNO }\end{array}$ & $\mathbf{3}$ & HADS-A & $8.2 \pm 2.2$ & $7.2 \pm 3.9$ & 0.37 \\
$\begin{array}{c}\text { post } \\
\text { HFNO } \\
\text { pre-HFNO } \\
\text { post }\end{array}$ & $\mathbf{2}$ & HADS-D & $9.0 \pm 0.8$ & $7.4 \pm 3.5$ & 0.15 \\
\hline
\end{tabular}

$\mathrm{NIV}=$ noninvasive ventilation, $\mathrm{ETT}=$ endotracheal tube, $\mathrm{HFNO}=\mathrm{High}-$ flow nasal oxygen

Conclusion. A preliminary analysis of this pilot study demonstrates the feasibility of implementing a communication strategy with videocalls between covid-19 patients and their caregivers. Explorative analysis on a reduced sample size did not show differences in the levels of anxiety, depression and PTSD. Studies on larger populations are needed to confirm these results.

\section{8}

Prognostic Value OF Lactate In Predicting Intensive Care Unit Mortality

CE. Ben Maaouia'; F. Haddad'; A. Ben Souissi' ; C. Ben Miled'; H. Arfaoui'; S. Naimi ${ }^{1}$; MS. Mebazaa'

${ }^{1}$ Anesthesiology and Intensive Care, Mongi Slim University Hospital, La

Marsa, Tunis, Tunisia, Tunis, Tunisia

Correspondence: C.E. Ben Maaouia

Intensive Care Medicine Experimental 2021, 9(1): 001448

Introduction. Many prognostic indices have been developed to assess clinical status and predict the probability of death in the intensive care unit (ICU) but none have perfect sensitivity or specificity. Lactates have been always seen as a metabolic waste and therapeutic dead end, is now considered as a powerful prognostic tool in ICU. The aim of our study was to evaluate the prognostic value of admission lactate in patients admitted to ICU. Many prognostic indices have been developed to assess clinical status and predict the probability of death in the intensive care unit (ICU) but none have perfect sensitivity or specificity. Lactates have been always seen as a metabolic waste and therapeutic dead end, is now considered as a powerful prognostic tool in ICU. The aim of our study was to evaluate the prognostic value of admission lactate in patients admitted to ICU.

Methods. An analytic, prospective study was carried out in the ICU, over a 12 mounths period, including 135 patients admitted in the ICU for a hospitalization stay longer than $24 \mathrm{~h}$. Arterial bood lactate (ABL) was measured in ICU admission ( $\mathrm{H0})$, then $6 \mathrm{~h}(\mathrm{H} 6), 12 \mathrm{~h}(\mathrm{H} 12), 24 \mathrm{~h}$ $(\mathrm{H} 24)$ and $48 \mathrm{~h}(\mathrm{H} 48)$ after admission. Lactate superior to $2 \mathrm{mmol} / \mathrm{l}$ defined hyperlactatemia. Prognostic scores were calculated $24 \mathrm{H}$ since the admission. We also recorded biological data, hemodynamic parameters and the evolution during the stay in intensive care. Primary endpoint was ICU mortality. The final step in the statistical study was to perform a multivariate analysis.

Results. The average age was $47.22 \pm 16.88$ years. ICU mortality was $48 \%$. The mean $A B L$ on admission was $3.05 \pm 2.63 \mathrm{mmol} / \mathrm{l}$, higher in the in the dead group with a statistically significant difference $(p<10-3)$. Patients with hyperlactatemia on arrival were clinically more serious with significantly higher prognostic scores. Prognostic value of lactate on admission was less powerful than severity indices in this study but remain excellent with an AUC $>0.7$ defining "cut-off» values with a good sensitivty and specificty. In multivaritate analysis, initial lactate $>2 \mathrm{mmol} / \mathrm{l}$ was found to be an independent predictive factor of ICU mortality with an Odd Ratio [IC 95\%] =1.16 [1.07-3.6] $\mathrm{p}=0.04$. A derived score including lactate $>34$ showed the best sensitivity and specificity to predict ICU mortality $(84.6 \%$ and $75.5 \%$ respectively).

Conclusion. Monitoring lactatemia in ICU could allow better identification of patients at high risk of death and the reassessment of therapeutic efficacy.

001463

Referral to an inpatient rehabilitation facility evaluation

of covid-19 patients after icu stay: a prospective cohort study assesing functional status $\mathbf{3}$ months after hospital discharge

C. Díaz-Rodríguez'; D. Pérez-Torres²; I. Canas-Pérez²; AM. Prieto-Lamo ${ }^{2}$;. Cuenca-Rubio²; MM. García-Garcíaª; R. Herrán-Monge²; JÁ. Berezo-Garcíaª LM. Tamayo-Lomas'; PM. Enríquez-Giraudo ${ }^{2}$

${ }^{1}$ Department of Intensive Care Medicine, Hospital Universitario Río Hortega, Valladolid, Spain; ${ }^{2}$ Servicio de medicina intensiva, Hospital Universitario Río Hortega, Valladolid, Spain

Correspondence: C. Díaz-Rodríguez

Intensive Care Medicine Experimental 2021, 9(1): 001463 
Introduction. Advances in critical care medicine have resulted in a growing population of critical illness survivors. However, a great number of them experience impairment in cognition, mental health and physical function, termed post-intensive care syndrome (PICS). Many patients who have recovered from severe COVID-19 suffer from PICS and require further rehabilitation services after ICU and hospital discharge, some of which involving admission to a longterm acute care facility (LTAC) or an inpatient rehabilitation facility (IRF).

Methods. We prospectively enrolled PCR-confirmed COVID19 patients admitted to the ICU of a university hospital in Spain between March and May 2020 (corresponding to the first epidemic wave of COVID-19 in the country) and the follow-up three months after hospital discharge. Clinical, epidemiological and outcome data were recorded. We created a multidisciplinary critical care followup clinic leaded by intensivists and also teamed by pulmonologists and nurses. We assessed functional status and PICS three months after hospital discharge by performing a protocolized scale-based evaluation of the physical, psychological and cognitive dimensions (Barthel, MoCA, HAD, PTSD and SF12), estimating muscle strength by handheld dynamometry, and measuring respiratory function with spirometry and diffusion. Results are expressed as frequency (\%), mean \pm standard deviation or median (interquartile range), as appropriate. Association between each risk factor and referral to an IRF was assessed with Student T or Mann-Whitney $U$ tests, as appropriate.

Results. We included 37 patients, from 50 eligible survivors to hospital discharge, $70 \%$ male, aged $53 \pm 12$, BMI $27(25-32) \mathrm{kg} / \mathrm{m}^{2}$, Barthel index 100 points $100 \%$, APACHE-II $14(11-16)$ points. Past medical history: $24 \%$ dyslipidaemia, $19 \%$ hypertension, $13 \%$ asthma or chronic obstructive pulmonary disease, $8 \%$ diabetes. ICU length of stay (LOS) 20 (12-36) days and hospital LOS $40 \pm 22$ days.

$100 \%$ of the patients fulfilled Berlin criteria of ARDS. 95\% needed mechanical ventilation (MV), during 18 (10-29) days, the remainder $5 \%$ was managed with high-flow oxygen therapy. $92 \%$ of the patients underwent neuromuscular blockade (NMB), with a mean of $8 \pm 5$ days. Length of sedation was $18 \pm 10$ days ( $92 \%$ of which received midazolam). $78 \%$ required prone position ventilation, with a median of 2 (1-4) sessions.

$11 \%$ of the patients underwent extracorporeal life support. $24 \%$ of the patients required tracheostomy during the weaning process.

Complications during ICU stay included ICU-acquired weakness (65\%), delirium (38\%), pressure injuries (31\%), shock (22\%), ventilator-associated pneumonia (19\%) and deep vein thrombosis (16\%). 59\% of our patients received physiotherapy, starting after $24 \pm 12$ days, and only $5 \%$ benefited from family visits.

$30 \%$ were referred to an IRF for further recovery after discharge. For each potential risk factor, we compared the group of patients who were referred to an IRF vs the group of patients who were not: age $66 \pm 2$ vs $51 \pm 2$ years old, $p<0.01$; ICU LOS 46(17-77) vs 19(10-35) days $p=0.02$; hospital LOS $63 \pm 10$ vs $34 \pm 3$ days, $p<0.01$; days of MV $33 \pm 8$ vs $19 \pm 3, p=0.03$; and days of sedation $24 \pm 6$ vs $16 \pm 2$, $\mathrm{p}=0.07$.

Three-month after-discharge visit in the outpatient critical care followup clinic: BMI $25(23-31) \mathrm{kg} / \mathrm{m}^{2}, 100 \%$ Barthel index 100 points, 39\% alopecia, 30\% insomnia, 3\% ICU-acquired swallowing disorders. $76 \%$ had visited their General Practitioner, whereas $16 \%$ had visited the Emergency Department. 51\% were under physiotherapy follow-up. $19 \%$ were still on sick leave. $35 \%$ still had impaired functional respiratory tests. $62 \%, 22 \%$ and $10 \%$ experienced alterations of physical, psychological and cognitive dimensions, respectively (68\% experienced alterations of at least one dimension). $32 \%$ were referred to other specialists for further follow-up.
Conclusion. In our series, patients with longer courses of mechanical ventilation and more prolonged ICU and hospital LOS, were significantly associated with referral to an IRF.

At three-month after-discharge visit, 1/3 patients needed referral to other specialists.

\section{Reference(s)}

1. Chopra V, Flanders SA, O'Malley M, et al. Sixty-Day Outcomes Among Patients Hospitalized With COVID-19. Ann Intern Med 2020.

e-Posters: ICU information systems

\section{3}

Automatic real-time analysis and interpretation of arterial blood gas sample for Point-of-Care testing: clinical validation

S. Rodríguez-Villar ${ }^{1}$; P. Poza ${ }^{2}$; S. Freigang ${ }^{3}$; I. Zubizarreta-Ormazabal ${ }^{4}$ ${ }^{1}$ Critical Care, King's College Hospital NHS Trust Foundation, London, United Kingdom; ${ }^{2}$ Surgical Intensive Care Unit. Toledo., Anaesthesia Department. Virgin Health Hospital, Toledo, Spain; ${ }^{3}$ Department of Neurosurgery, Neurosurgical Intensive Care Unit., Medical University of Graz. Graz, Austria., Graz, Austria; ${ }^{4}$ Anaesthesia department, Marqués de Valdecilla University Hospital, Santander, Spain

Correspondence: S. Rodríguez-Villar

Intensive Care Medicine Experimental 2021, 9(1): 000033

Introduction. Point-of-care arterial blood gas (ABG) is a blood measurement test and a useful diagnostic tool that assists with treatment and therefore improves clinical outcomes. However, numerically reported test results to make rapid interpretation difficult or open to interpretation. The arterial blood gas algorithm (ABG-a) is a new digital diagnostics solution that can provide clinicians with a real-time interpretation of preliminary data on safety features, oxygenation, acid-base disturbances and renal profile.

Objectives. The main aim of this study was to clinically validate the algorithm against senior experienced clinicians, for acid-base interpretation, in a clinical context.

Methods. We conducted a prospective international multicentre observational cross-sectional study. 346 sample sets and 64 inpatients eligible for ABG met strict sampling criteria. Agreement was evaluated using Cohen's kappa index, diagnostic accuracy was evaluated with sensitivity, specificity, efficiency or global accuracy and positive predictive values (PPV) and negative predictive values (NPV) for the prevalence in the study population.

Results. The concordance rates between the interpretations of the clinicians and the ABG-a for acid-base disorders were an observed global agreement of $84,3 \%$ with a Cohen's kappa coefficient $0.81 ; 95 \%$ $\mathrm{Cl} 0.77$ to $0.86 ; \mathrm{p}<0.001$. For detecting accuracy normal acid-base status the algorithm has a sensitivity of $90.0 \%(95 \% \mathrm{Cl} 79.9$ to 95.3$)$, a specificity $97.2 \%$ (95\% Cl 94.5 to 98.6$)$ and a global accuracy of $95.9 \%$ ( $95 \% \mathrm{Cl} 93.3$ to 97.6$)$. For the four simple acid-base disorders, respiratory alkalosis: sensitivity of 91.2 (77.0 to 97.0$)$, a specificity 100.0 (98.8 to 100.0) and global accuracy of 99.1 (97.5 to 99.7); respiratory acidosis: sensitivity of 61.1 (38.6 to 79.7), a specificity of 100.0 (98.8 to 100.0) and global accuracy of 98.0 (95.9 to 99.0); metabolic acidosis: sensitivity of 75.8 (59.0 to 87.2 ), a specificity of 99.7 (98.2 to 99.9 ) and a global accuracy of 97.4 (95.1 to 98.6 ); metabolic alkalosis sensitivity of 72.2 (56.0 to 84.2), a specificity of 95.5 (92.5 to 97.3 ) and a global accuracy of 93.0 (88.8 to 95.3); the four complex acid-base disorders, respiratory and metabolic alkalosis, respiratory and metabolic acidosis, respiratory alkalosis and metabolic acidosis, respiratory acidosis and metabolic alkalosis, the sensitivity, specificity and global accuracy was also high For normal acid-base status the algorithm has PPV 87.1 (95\% Cl 76.6 
to 93.3 ) \%, and NPV 97.9 ( $95 \% \mathrm{Cl} 95.4$ to 99.0 ) for a prevalence of 17.4 (95\% Cl 13.8 to 21.8$)$. For the four-simple acid-base disorders and the four complex acid-base disorders the PPV and NPV were also statistically significant.

Conclusion. The ABG-a showed very high agreement and diagnostic accuracy with experienced senior clinicians in the acid-base disorders in a clinical context. The method also provides refinement and deep complex analysis at the point-of-care that a clinician could have at the bedside on a day-to-day basis. The ABG-a method could also have the potential to reduce human errors by checking for imminent life-threatening situations, analysing the internal consistency of the results, the oxygenation and renal status of the patient.

\section{Reference(s)}

1. Rodríguez-Villar S, Do Vale BM, Fletcher HM. The arterial blood gas algorithm: proposal of a systematic approach to analysis of acid-base disorders. Revista Española de Anestesiología y Reanimación. Rev Esp Anestesiol Reanim. 2020 Jan;67(1):20-34

2. D'Orazio P, Fogh-Andersen N, Okorodudu A, Shipp G, Shirley T, Toffaletti J. Critical Care Chapter 5. In: Nichols JH, editor. Evidence Based Practice for Point-of-Care Testing: A National Academy of Clinical Biochemistry Laboratory Medicine Practice Guideline. Washington DC: AACC Press; 2006. pp. 30-43.

3. Adrogué HJ, Madias NE. Secondary responses to altered acid-base status: the rules of engagement. J Am Soc Nephrol. 2010 Jun;21 (6):920-3. https://doi.org/10.1681/ASN.2009121211. Epub 2010 Apr 29. PMID: 20431042.

4. Prause G, Ratzenhofer-Komenda B, Offner A, Lauda P, Voit H, Pojer H. Prehospital point of care testing of blood gases and electrolytes -an evaluation of IRMA. Crit Care. 1997; 1:79-83.

\section{0}

Assessing the utility of a wearable device to monitor critically unwell patients within a critical care outreach team: A service evaluation

T. Trencher ; R. Ambrus ${ }^{2}$; F. Baldwin ${ }^{3}$

${ }^{1}$ Brighton \& Sussex Medical School, Brighton, United Kingdom; ${ }^{2}$ Critical Care Outreach Team, Brighton \& Sussex University Hospitals Nhs Trust, Brighton, United Kingdom; ${ }^{3}$ ntensive Care Department, Brighton \& Sussex University Hospitals Nhs Trust, Brighton, United Kingdom

\section{Correspondence: $T$. Trencher}

Intensive Care Medicine Experimental 2021, 9(1): 000480

Introduction. The ability to closely monitor patient vital signs is a key aspect of the management of patients requiring critical care. To theThe detection of changes in vital signs in real time can allow for more rapid review and interventions to be made.

The current COVID-19 pandemic has highlighted the importance of detecting the deteriorating patient and the possibility of remote monitoring.use of a wearable wireless monitoring device. In this study, the device evaluated was the Caretaker ${ }^{\circledR} 4$ (CT4) from Caretaker Medical ${ }^{\circledR}$ We performed a staff evaluation of the CT4 device within a Critical Care Outreach Team (CCOT) with a view to augmenting the existing monitoring practices.

Objectives. 1. To evaluate and consider the responses of members of the CCOT after trialling the device.

2. Consider how the experience of members of the CCOT in using this device would impact on their willingness to use this device in the future.

Methods. This evaluation was conducted by nurses of the CCOT from based at the Royal Sussex County Hospital, Brighton, UK. Team members interacted with the CT4 by attaching, calibrating, recording observations, detaching, cleaning and storing the device. Staff evaluations were recording using a survey via the Qualtrics ${ }^{\circledR}$ platform.

Results. Ordinal survey data collected will were analysed using non-parametric descriptive statistics. Survey comments wierell be reviewed by thematic analysis. Of the 19 CCOT nursing staff $12(63.2 \%)$ responses were recorded. Of the 12 respondents, 10 (83.3\%) stated that they would use the CT4 on their patients in future.

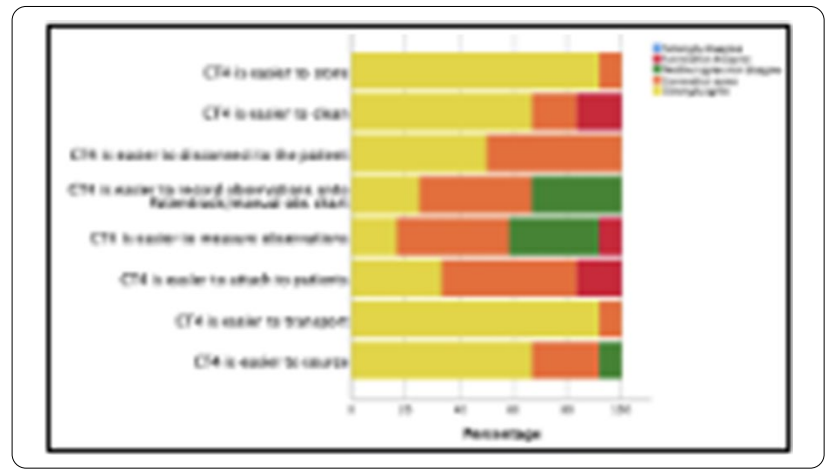

From the 96 recorded survey data points 81 (84.4\%) either strongly or somewhat agreed that the CT4 was easier to use.

Conclusion. The device (CT4) was being evaluated to assess whether the CCOT would use CT4 to monitor patients within their service, based on perceived usability. Use of the CT4 was positively received by the team in all areas assessed. Implementation of the device alongside the existing monitoring could be trialled with patient outcomes measured. This further work however would bewas limited in scope due to the current impact of the COVID-19 pandemic on critical care services. Use of this style of monitoring has potential use in other scenarios such as transferring critical care patients and remote monitoring of patients recently discharged from the Intensive Care Unit (ICU).

\section{4}

One-day cost-effectiveness of covid-19 patient's management in intensive care unit

S. AYED'; D. Jarraya ${ }^{2}$; A. Jamoussi ; E. Rachdi'; F. Jarraya ${ }^{1}$; J. Benkhelil' ${ }^{1}$ Intensive Care, Hôpital Abderrahmen Mami de pneumo-phtisiologie, Ariana, Tunisia; ${ }^{2}$ Pharmacy Department, Hôpital Abderrahmen Mami de pneumo-phtisiologie, Ariana, Tunisia

Correspondence: S. AYED

Intensive Care Medicine Experimental 2021, 9(1): 000954

Introduction. The coronavirus disease 2019 (COVID-19) has spread from China to the whole of the world leading to a great threat to public health. COVID19 epidemic has led to an increased cost of medical care thus intensive care unit (ICU) costs account for a great part of hospital's expenses.

Objectives. The purpose of this study was to analyze the cost effectiveness of one-day ICU management of COVID19 patients.

Methods. We performed a prospective cross-sectional study in the 20-bed medical ICU of Abderrahmen Mami hospital dedicated to manage COVID19 patients. We evaluated patient's resource utilization by a review of medical prescriptions and bedside checklists. Laboratory investigation and diagnostic imaging costs were calculated. Medical and nursing staffing costs were obtained from the human resources department even for the additional staff including ward clerk, secretary, pharmacist, dietician and physiotherapists. The hospital pharmacy supplied drug and devices costs. Maintenance and laundry were also calculated. Total day cost was calculated in Tunisian dinar (DT) with the amount in EUROS (one euro=3.294 DT).

Results. On the study-day, 17 patients were hospitalized with ICU occupancy of $85 \%$. Total cost was calculated to 36685 DT 584 equivalent to 11137 Euros 092 . Total cost by patient was calculated to 2157 DT 975 (=655 Euros). Drug cost was evaluated to 9429 DT 559 (=2 862 Euros 646). Devices cost was 3149 DT 003 (=955 Euros 981). Laboratory investigations and diagnostic imaging costs were evaluated respectively to 1804 DT 100 (=547 Euros 692) and 513 DT 900 (=156 Euros 010). Medical and nursing staffing costs including the night shifts were calculated respectively to 1250 DT 800 (=379 Euros 720) and 1155 DT 730 (=350 Euros 859). Two Computers and two printers were used to manage patients in ICU with a cost of 4000 DT (=1 
214 Euros 329). Laundry and sterilization products costs were respectively of 1440 DT (=437 Euros 158) and 782 DT 550 (=237 Euros 568). Patients paid a package of 60 DT 000 (=18 Euros 214) for one ICU-day management and COVID19 patient's management in Tunisia was decreed totally gratis from the beginning of the epidemic

Conclusion. This study highlights the expensive cost of one-day COVID19 patient's management in ICU compared to the governmental decision decreed in Tunisia. The highest cost was for drugs and the lowest was for medical and nursing staffing.

\section{5}

\section{Psychological impact of Covid-19 on healthworkers}

K. Benismail ${ }^{1}$; I. Sdiri'; B. Wala ${ }^{1}$; S. Dorgham ${ }^{1}$; T. Merhabene ${ }^{1}$; K. Moez ${ }^{1}$ Réanimation médicale, ICU of Zaghouan Regional hospital, Zaghouan, Tunisia

Correspondence: I.K. Ben

Intensive Care Medicine Experimental 2021, 9(1): 000955

Introduction. Multiple studies showed that pandemic Coronavirus disease 2019 (COVID-19) has profound psychological and social effects which persist for long period. This studies indicate that most manifestations such as distress, anxiety, fear of contagion, depression and insomnia are more frequent among health care professionals wich are in the front line in fighting this disease.

Objectives. To assess psychological stress of healthcare workers caused by the COVID-19 pandemic.

Methods. It was a descriptive survey conducted on Zaghouan hospital's healthworkers and composed of 4 parts collecting first participant's demographic characteristics and then assessing burnout level; signs of depression, stress and anxiety and finally existence of peri-traumatic stress. Our survey employed the Copenhagen burn out inventory (CBI) known to be a good instrument for investigating job burnout and the DASS-21 which offers adequate levels of validity for assessing the stress, anxiety, and depression. And finally, the Impact of event scale-revised (IES-R) which show reliability to describe traumatic stress.

Results. 22 workers were enrolled; mean age was $29 \pm 8$ years [21-54] and sex ratio was 1.17. All healthworkers were affected on COVID-19 units: 16 in ICU, one in emergency department and 4 in others wards. 9 were nurses, 8 interns and 5 medical assistants. Only 2 had exclusive night shift. 4 work $36 \mathrm{~h}$ per week, 10 work $42 \mathrm{~h}$ per week, 7 work $48 \mathrm{~h}$ per week, one work $50 \mathrm{~h}$ per week. 16 workers had received COVID-19 vaccin.

13 workers had positive $\mathrm{CBI}$ burnout, underline physical and psychological fatigue and exhaustion. Our study showed considerable variation of burnout levels between status, with the highest level among nurses and the lowest among chief doctors. 16 participants presented depression and anxiety with different degrees. The severity of mental symptoms was influenced by age, gender, occupation, specialization, type of activities performed and proximity to COVID-19 patients. In the subgroup of workers presented psychological impact, we identify the female sex as a risk factor,the nomber of patients occupied by the healthworker, an exclusive night work and the nomber of hours influence in the appearance of anxiety and depression.

Conclusion. Considering the frequency of mental symptoms occurring in healthcare workers, Community mental health service systems, online mental health services, telemedicine, and other measures for healthworkers with mental disorders may play a vital role to facilitate coping during the pandemic.

\section{0}

Quality assessment of a cloud-based ICU registry network in 7 Asian countries

L. Pisani' ; T. Rashan²; M. Shamal'; A. Ghose ${ }^{4}$; B. Kumar 5 ; S. Tripathy'; D. Aryall'; M. Hashmi $^{8}$; B. Nor ${ }^{9}$; Y. Lam Minh ${ }^{10}$; A. Dondorp ${ }^{11}$; R. Haniffa ${ }^{2}$; A. Beane $^{2}$

'Mahidol University, Mahidol Oxford Research Unit (MORU), Bangkok, Thailand; ${ }^{2}$ Crit Care Asia Network, NICS MORU, Colombo, Sri Lanka;

${ }^{3}$ Afghanistan icu registry, Crit Care Asia Network, Kabul, Afghanistan;

${ }^{4}$ Department of Medicine, Chittagong Medical College, Chittagong,
Bangladesh; ${ }^{5}$ Crit Care Asia Network, Indian Registry of IntenSive care, Chennai, India; ${ }^{6}$ Anesthesia and Critical Care, All India Institute of Medical Sciences, Bhubaneswar, Bhubaneswar, India; ${ }^{7}$ Department of Critical Care and Anesthesia, Nepal Mediciti Hospital, Lalitpur, Nepal; ${ }^{8}$ Department of Critical Care, Ziauddin University, Karachi, Pakistan; ${ }^{9}$ Department of Anaesthesiology and Intensive care, International Islamic University Malaysia, Kuala Lumpur, Malaysia; ${ }^{10} \mathrm{Crit}$ Care Asia network, Oxford University Clinical Research Unit, Ho Chi Minh City, Vietnam; ${ }^{11}$ Mahidol Oxford Tropical Research Unit, Mahidol University, Bangkok, Thailand

Correspondence: L. Pisani

Intensive Care Medicine Experimental 2021, 9(1): 001030

Introduction. For the CRIT CARE ASIA network

The value of medical registries strongly depends on the quality and curation of the data collected. Efforts to build robust registries of critically ill patients are increasing in low and middle income countries. The Crit Care Asia (CCA) federated registry platform enables ICUs in several Asian countries to collect data on consecutive admissions and process data for aggregate and unit-level analysis [1].

Objectives. We aimed to systematically evaluate the quality of a multinational ICU network of registries established in seven Asian middle income countries, namely Afghanistan, Bangladesh, India, Malaysia, Nepal, Pakistan and Vietnam.

Methods. The registry core dataset infrastructure and outputs were evaluated according to 10 standardized criteria proposed by the Directory of Clinical Databases (DoCDat): four relating to registry coverage and six relating to reliability and validity of the data [2]. Each item is rated on a scale of 1 to 4 , with level one representing the least rigorous methods and Level 4 representing the most rigorous. Two external reviewers and three members of the registry network performed the assessment independently and scores were averaged for reporting purposes. Data from 33 core variables collected in a pre-specified period of 6 months (from June to December 2020) was used as the evaluation case study.

Results. More than 20.000 patient episodes from 97 ICUs were used in the assessment. The average performance of the registries using the DoCDat criteria is shown in Table, and compared to the average evaluation of other existing DoCDat databases. The median score achieved by the registries across all criteria was 3.4 (minimum 1.4 maximum 4). The geographic spread inside each country was heterogeneous and thus a low score was achieved for this criterion. Recruitment completeness was above $95 \%$ in all ICUs that reported census data. Missingness of data was below $5 \%$ and the whole core data set was collected as raw data. Explicit rules were provided for data capture and international coding systems used to map comorbidities, diagnostic categories and operative procedures. Data was validated internally using completeness, range and consistency checks. Free text fields were kept to a minimum and only to support predetermined menus.

Table: Aggregate assessment of the registries in the 7 countries according to the Directory of Clinical Databases (DocDat) criteria.

\begin{tabular}{|c|c|c|c|}
\hline & Crit Care & Asia score\# & $\begin{array}{l}\text { Average } \\
\text { of DoCDat } \\
\text { data- } \\
\text { bases* }\end{array}$ \\
\hline A. Representativeness of country & $1.4(3-4)$ & உロロ & $3(2-4)$ \\
\hline B. Completeness of recruitment & $2.6(2-3)$ & $\mathbf{\square} \mathbf{\square}$ & $3(1-4)$ \\
\hline C. Variables included & $3.4(3-4)$ & $\mathbf{0} \mathbf{\square}$ & $3(2-4)$ \\
\hline D. Completeness of data & $3.8(3-4)$ & $\square \square \square$ & $2(1-3)$ \\
\hline E. Collection of raw data & $3.8(3-4)$ & 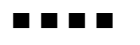 & $4(4-4)$ \\
\hline F. Explicit definitions & $4(4-4)$ & 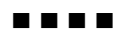 & $2(1-4)$ \\
\hline G. Explicit rules & $3.8(3-4)$ & $\square \square \square$ & $3(1-4)$ \\
\hline H. Reliability of coding & $4(4-4)$ & घav & $1(1-4)$ \\
\hline I. Independence of observations & $3.8(3-4)$ & 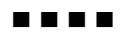 & $4(2-4)$ \\
\hline J. Data validation & $3.4(3-4)$ & $\mathbf{\square} \mathbf{\square}$ & $3(3-4)$ \\
\hline
\end{tabular}


\# Aggregate score of 5 independent reviewers, displayed as average (minimum and maximum scores attributed by individual scorers).

*Extracted from Stow et al. Jou Crit Care 2006;21:133-134

Conclusion. The federated ICU registries in Afghanistan, Bangladesh, India, Malaysia, Nepal, Pakistan and Vietnam performed equally or above the average against the DoCDat criteria assessing data structure, reliability and validity. Increasing the geographic representativeness was flagged as a potential improvement. This comprehensive evaluation enables the high quality data derived from these national registries to be used for observational research, quality improvement initiatives, service evaluation and registry embedded clinical trials.

\section{Reference(s)}

1. 2. Black N, Payne M. Directory of clinical databases: improving and promoting their use. Qual Saf Health Care. 2003;12(5):348-352

2. 1. CRIT CARE ASIA. Establishing a critical care network in Asia to improve care for critically ill patients in low- and middle-income countries. Crit Care Lond Engl. 2020;24(1):608

3. The Crit Care Asia network is supported by the Wellcome Trust (Grant number 215522)

\section{3}

A reliable artificial intelligence models to predict potassium leve from electronic health records in intensive care unit

Z. Hajjej'; W. Sellami'; A. Gorbel' ${ }^{2}$; N. Nouri ${ }^{2}$; M. Ferjani

'Department of Anesthesiology and Critical Care Medicine, Ir12dn01, Military Hospital, Université de Tunis El manar, Faculté de Médecine de tunis, Tunis, Tunisia; ${ }^{2}$ Medilsys, SA, Tunis, Tunisia

Correspondence: Z. Hajje

Intensive Care Medicine Experimental 2021, 9(1): 001183

Introduction. Currently, the detection of dyskalemia is based on laboratory tests. Point-of-care blood testing provides rapid analysis of electrolyte levels, however, the accuracy in its performance may not be as trustworthy as that from a central laboratory.

Objectives. Our study aimed to develop a machine learning algorithm based prediction tool for serum potassium concentration in critically ill patients and to evaluate the logic and performance of this model.

Methods. Medilsys developed four potassium predictor models (MPP) as following: (i) potassium value predictor (PVP): predicts Kalemia value per hour, (ii) potassium trend predictor (PTP): predicts real-time patient kalemia trend (increase; decrease), (iii) hyperkalemia predictor (HEP): predicts hyperkalemia status, (iv) hypokalemia predictor (HOP): predicts hypokalemia status. The prediction models were an «XG Boost classifier». Experiments were conducted on the Medical Information Mart for Intensive Care III (MIMIC-III) data base (validation set). Data from patients admitted to the intensive care unit between July 2019 and December 2019 were included in the training, called preproduction set; and those admitted between December 2020 and March 2021 were included in the test set to assess model performance, called post production set. The required data that included patients' information, admissions, chart and laboratory information's were extracted from the electronic medical record (computerized record, Medilsys). Area under the ROC curves, mean absolute error and balance accuracy were used to evaluate the performance of our models.

Results. We ran our models in different datasets and we found that all them were in close agreement. The AUCs of our models were $0.903,0.914,0.958$, and 0.976 in the detection of hypokalemia, severe hypokalemia, hyperkalemia and severe hyperkalemia, respectively. In terms of balance accuracy, potassium predictor model's performances were higher than 0.9 . Tested on the post production data set, the performance of the models remains likewise high up to $3 \mathrm{~h}$. MAE was low for each model and in each database set (less than 0.5).

Conclusion. A machine learning algorithm models based on electronic medical records may help physicians promptly recognize dyskalemias and thereby potentially mitigate the risk of cardiac events.

\section{Reference(s)}

1. We gratefully acknowledge «Medilsys», a medical systems and services company founded in 2015, aiming to provide intensive care units with innovative clinical information systems and to enhance all of care quality and clinical research.

\section{3}

Evaluation of hidden hospital mortality in an ICU unit, a 3 year period study

H. Santos, ; R. Amaral ${ }^{1}$; B. Costa, ; S. Carvalho, ${ }^{1}$; M. Contente, ; J. Carvalho, ; C. Carvalho, ${ }^{1}$; L. Gonçalves,

${ }^{1}$ Intensive Medicine, Centro Hospitalar De Trás-Os-Montes E Alto Douro,

E.P.E., Vila Real, Portugal

Correspondence: $\mathrm{R}$. Amaral

Intensive Care Medicine Experimental 2021, 9(1): 001363

Introduction. Evaluation of hidden hospital mortality in an ICU unit, a 3 year period study

Objectives. One main purpose of our practice is to get patients alive, not just from the ICU but obviously from the hospital, and with good quality of life.

We pretend to evaluate the hidden hospital mortality of our unit and the characteristics of this patients, in a 3 years period.

Methods. This is a retrospective study made in Centro Hospitalar Trásos-Montes e Alto Douro's ICU unit, between 1st of January 2016 to 31st December 2018.

Were included all patients discharged from the ICU and deceased in the ward, without a decision to limit of care.

We assess demographic characteristics, comorbidities and severity scores. Admission diagnostic was divided in major categories based on Knauss classification, with trauma and sepsis recorded in a separate category. Length of ICU and hospital stay was evaluated.

Results. Between this period, we looked at 1928 ICU admissions, with an ICU mortality of $11,6 \%$. There were 1665 ICU survivals without end of care decisions.

The hidden mortality was $6,1 \%$. This population had a mean age of $69,9 \pm 11,3$ years with a male predominance of $61,4 \%$. Main comorbidities were pulmonary disease $(42,6 \%)$ and any kind of cardiac disease (55,4\%). 28,7\% had oncological history.

APACHE II was $25,1 \pm 9,2$, SAPS II $54,97 \pm 19,1$, with an initial SOFA of 9,3 and a final SOFA of 8,0

We found that $67,3 \%$ were admitted for a medical issue, with urgent surgery present in $26,7 \%$ of patients. Septic shock was found in $40,6 \%$ and sepsis in $14,9 \%$ of patients. Trauma was present in $13,4 \%$

The length of ICU stay was $7 \pm 9,9$ days and the hospital stay was $20 \pm 26,4$ days

Conclusion. With this study, we want to highlight the need to realize what happens next to the ICU.

As showed in previous studies, presence of infection with sepsis and septic shock is a major concern, not only on the immediate mortality but also on the long term outcome.

Patient background is major concern in ICU outcome.

\section{Reference(s)}

1. Kim, Do Yeun MD, PhDa; Lee, Mi Hyun MSb,c; Lee, Sung Yeon MDb; Yang, Bo Ram PhDd; Kim, Hyun Ah MD, PhDb,c, * Survival rates following medical intensive care unit admission from 2003 to 2013, Medicine: September 2019-Volume 98-Issue 37-p e17090 https://doi.org/10. 1097/MD.0000000000017090

2. Shmueli, A., Baras, M. \& Sprung, C.L. The effect of intensive care on inhospital survival. Health Serv Outcomes Res Method 5, 163-174 (2004). https://doi.org/10.1007/s10742-006-6826-z

3. Andersen, F.H., Flaatten, H., Klepstad, P. et al. Long-term survival and quality of life after intensive care for patients 80 years of age or older. Ann. Intensive Care 5, 13 (2015). https://doi.org/10.1186/s13613-015-0053-0 
4. Aguiar-Ricardo I, Mateus H, Gonçalves-Pereira J. Hidden hospital mortality in patients with sepsis discharged from the intensive care unit. Mortalidade oculta em pacientes sépticos após alta da unidade de terapia intensiva. Rev Bras Ter Intensiva. 2019;31(2):122-128. Published 2019 Jun 10. https://doi.org/10.5935/0103-507X.20190037

e-Posters: Imaging in Intensive Care

\section{0}

Point of care echocardiography in a district general hospital during the COVID 19 pandemic: Does it change management?

A. Balu'; K. Haiba ${ }^{2}$

${ }^{1}$ Department of Intensive Care Medicine, Queen Elizabeth Hospital Birmingham, Birmingham, United Kingdom; ${ }^{2}$ Anaesthetics and Critical Care, Birmingham City Hospital, Birmingham, United Kingdom

Correspondence: A. Balu

Intensive Care Medicine Experimental 2021, 9(1): 000050

Introduction. Focused intensive care echocardiography (FICE) is growing in popularity. The uptake or prevalence of its use in our department is unknown and burden on departmental echocardiography technicians remains high. We aimed to characterise the usage of point of care echocardiography and examine the sequelae of these scans at our institution during the first wave of the pandemic.

Methods. A retrospective evaluation of 235 patients was conducted over the period April 20th 2020 to August 5th 2020. Case review of the electronic notes was performed using a prepared electronic proforma. Results. FICE scans were performed in 69/235 admissions (29.3\%), $41 / 235$ patients (17.4\%) only had departmental transthoracic or transoesophageal echocardiograms. 54/69 (78.2\%) patients who had FICE scans also had departmental echocardiograms. Changes in management were initiated in $35 / 69$ (50.7\%) of the FICE subgroup compared to $8 / 41(19.5 \%)$ in the departmental scan group only. Discrepancies between FICE scans and departmental scans were identified in 3/54 patients (5.55\%). Additional diagnostic information was attained in $7 / 41$ (17.1\%) of follow-up departmental scans.

Conclusion. FICE uptake is reasonable (29.3\%) in our department and this paper supports previous literature showing that it is safe and feasible [1]. FICE scans led to a change in management in a similar proportion of patients $(50.7 \%)$ compared to other published literature $[1,2]$. Discrepancies exist in $5.5 \%$ of the case mix and clinicians need to be cautious in conclusions garnered from FICE scans. We aim to reduce this with departmental quality assurance days reviewing $10 \%$ of scans performed on a monthly basis.

\section{Reference(s)}

1. 2) Hall $D$, Jordan $H$, Alam $S$, Gillies M. The impact of focused echocardiography using the Focused Intensive Care Echo protocol on the management of critically ill patients, and comparison with full echocardiographic studies by BSE-accredited sonographers. Journal of the Intensive Care Society. 2017; 18(3): 206-211

2. 1) Vieillard-Baron A, Millington S, Sanfilippo F, Chew M, Diaz-Gomez J, McLean A et al. A decade of progress in critical care echocardiography: a narrative review. Intensive Care Medicine. 2019; 45(6): 770-788

\section{8}

Routine advanced echocardiography in the evaluation of cardiovascular sequelae of COVID19 survivors with elevated cardiovascular biomarkers

P. Jordan Marchite'; E. Ródenas Alesina ${ }^{1}$; M. Bach Oller ${ }^{1}$; L. Herrador Galindo ; C. Badia Molins'; J. Bañeras Rius'; J. Rodríguez-Palomares'; M. García de Acilu²; F. Clau-Terré3; I. Ferreira-González ${ }^{1}$

${ }^{1}$ Cardiology, Hospital Universitari Vall d'Hebron, Barcelona, Spain; ${ }^{2}$ Critical Care Department, Vall d'Hebron University Hospital, Vall d'Hebron Research Institute, Barcelona, Spain; ${ }^{3}$ Anesthesiology, Hospital Universitari Vall d'Hebron, Barcelona, Spain

Correspondence: P. Jordan Marchite

Intensive Care Medicine Experimental 2021, 9(1): 000098
Introduction. COVID19 has been related to elevated cardiovascular biomarkers (CVB) and biventricular dysfunction during hospitalization. We sought to determine, using advanced echocardiography, the prevalence and type of cardiovascular sequelae after COVID19 infection with marked elevation of CVB, and their prognostic implications.

Methods. All patients admitted from March 1st to May 25th, 2020 to a tertiary referral hospital were included. Those with cardiovascular diseases, death during admission or the first 30 days after discharge were excluded. Patients with hs-Tnl $>45 \mathrm{ng} / \mathrm{L}$, NT-proBNP $>300 \mathrm{pg} / \mathrm{ml}$, and D-dimer $>8000 \mathrm{ng} / \mathrm{ml}$ were matched with COVID controls (three biomarkers within the normal range) based on intensive care requirements and age, and separately analyzed.

Results. From a total of 2025 hospitalized COVID19 patients, 80 patients with significantly elevated CVB and 29 controls were finally included. No differences in baseline characteristics were observed among groups, but elevated CVB patients were sicker. Follow-up echocardiograms showed no differences among groups regarding LVEF or RV diameters, but TAPSE was lower if hs-Tnl or D-dimer were elevated. Hs-Tnl patients also had lower global myocardial work and global longitudinal strain. The presence of an abnormal echocardiogram was more frequent in the elevated CVB group compared to controls ( 23.8 vs $10.3 \%, P=0.123$ ) but mainly associated with mild abnormalities in deformation parameters. Management did not change in any case and no major cardiovascular events except deep vein thrombosis occurred after a median follow-up of 7 months.

Conclusion. Minimal abnormalities in cardiac structure and function are observed in COVID19 survivors without previous cardiovascular diseases who presented a significant CVB rise at admission, with no impact on patient management or short-term prognosis. These results do not support a routine screening program after discharge in this population.

\section{4}

Evaluation of Gastric Emptying In Critically III Patients Using Electrical Impedance Method

A. Basher'; M. Moniruzzaman²; M. Islam²; EA. Chowdhury'; MB. Paul ${ }^{2}$; IH. Chowdhury $^{3}$; A. Akhtaruzzaman ${ }^{3}$; K. Siddique-E Rabbani5 ${ }^{4}$

${ }^{1}$ Dept. of Anaesthesia, Analgesia and Intensive Care Medicine, bangabandhu sheikh mujib medical un, Resident, Dhaka, Bangladesh;

${ }^{2}$ Department of Biomedical Physics Dhaka University, Research fellow,

Dhaka, Bangladesh; ${ }^{3}$ Dept. of Anaesthesia, Analgesia and Intensive Care

Medicine, bangabandhu sheikh mujib medical un, Professor, Dhaka,

Bangladesh; ${ }^{4}$ Department of Biomedical Physics Dhaka University, Profes-

sor, Dhaka, Bangladesh

Correspondence: A. basher

Intensive Care Medicine Experimental 2021, 9(1): 000154

Introduction. Nasogastric feeding is commonly used to deliver enteral feed in critically ill patients, but current methods used to assess tolerance based on measurement of gastric residual volume have been criticised. A new approach of gastric emptying time measurement and evaluation has been developed using Electric impedance Method (EIM). EIM is a simple and non-invasive tool that measures gastric emptying by monitoring changes in gastric electrical impedance when a meal progressively empties from the stomach.

Objectives. The study aim is to establish whether EIM is useful for measuring gastric emptying during nasogastric (NG) infusion comparing the results with naso-gastric suction.

Methods. A computerised instrument for recording and measuring using EIM was designed and developed locally by us. About 150$200 \mathrm{ml}$ of enteral feed was given by NG tube and gastric emptying was measured using EIM in each of twenty critically ill patients. Gastric residual volume (GRV) was measured by naso-gastric suction before next feeding. Patient's clinical data were recorded.

Results. Gastric emptying curves were obtained in 20 patients by EIM. Visual examination of the curves showed reasonable agreement. Patterns of filling and emptying were almost the same in all subjects but emptying time varied between individuals that correlated well with GRV in 16 patients. Only in four patients the emptying times did not 
match with GRV. Therefore, this study showed that the measurement of gastric volume by noninvasive and hazard free electrical impedance method has a high specificity (90\%) and an efficacy of $80 \%$. The study also revealed that there were significant changes of gastric emptying time due to different body state. Patient age, temperature, hematocrit, mean arterial pressure (MAP) and increased serum creatinine were associated with delayed gastric emptying.

Conclusion. Application of EIM among ICU patient to observe gastric emptying was not done earlier. EIM seems to be capable of measuring stomach content satisfactorily during feeding; it also shows patterns of gastric emptying over time. With further development EIM could become a standard tool for monitoring gastric emptying in patients at risk of gastroparesis.

\section{4}

Transesophageal Lung Ultrasound (TELUS) In Adult Cardiac Surgery: Clinical Significance and Predictive Performance For Postoperative Respiratory Events (PORE)

M. Sausse 1; P. Burtin²; E. Lorne'; JY. Bigeon²; M. Lalande'; P. Sentenac

${ }^{1}$ ANESTHESIE CHIRURGIE CARDIAQUE, Clinique du Millénaire, Montpellier,

France; ${ }^{2}$ Anesthésie et Réanimation, Clinique du Millénaire, Montpellier,

France

Correspondence: M. Sausse

Intensive Care Medicine Experimental 2021, 9(1): 000224

Introduction. Postoperative respiratory events (PORE) are associated with clinical outcome after surgery and specifically after cardiac surgery (CS) $(1,2)$. Transesophageal Lung Ultrasound (TELUS) has been described recently (3) in intensive care for the diagnosis of respiratory failure. Nevertheless the clinical significance of TELUS is underevaluated to date. This report is an intermediate analysis of the TELUSPORE database.

Objectives. The TELUSPORE project seeks to describe the incidence and severity of the alteration of the TELUS imaging before and after CPB in adult cardiac surgery and the relation of these changes to the occurrence of PORE (NCT04760340).

Methods. This is a single center, prospective observational study. Patients with CS and CPB and TEE monitoring are included (except for video assisted (S). TELUS imaging is performed bilateraly in 6 zones (anterior, lateral, posterior) before and after CPB. A transthoracic lung ultrasound (LUS) is performed at the arrival in ICU. A conventional LUS scoring is applied to all zones (range 0-18). Comorbidities, demographic, procedural, hemodynamic and biological data are recorded. PORE are defined as pneumonia, atelectasis, pneumothorax, pleural effusion or drainage, ARDS, reintubation, tracheotomy, NO inhalation, pulmonary embolism, sternal wound dehiscence, ICU re-admission. Non respiratory complications during hospital stay are also recorded. Two groups (PORE vs NOPORE) are defined according to the occurence of at least 1 PORE and compared using Student t test, Khi 2, Fisher exact and Mann Whitney test. ROC curves are built for preCPB and postCPB TELUS and postop LUS scores. A $p \leq 0.05$ is considered significant.

Results. 72 patients are included, 40 PORE occur in 14 patients (19.4\%) with 3 deaths. TELUS score is impaired after CPB $(p=0,03)$, as $\mathrm{P} / \mathrm{F}$ ratio (preCPB $284 \mathrm{mmHg}$ vs postCPB $279 \mathrm{mmHg} ; \mathrm{p}=0.03$ ). There is no difference between groups for comorbidities, demographic, procedural, Euroscore and LVEF data. PORE group has a postCPB TELUS score higher $(10.57 \pm 2.3$ vs $8.91 \pm 1.65 ; \mathrm{p}=0,003)$, $\mathrm{CPB}$ and aortic cross clamping time higher (respectively $90.35 \mathrm{~min}$ vs $72.05 ; p=0.03$ and 69.35 min vs $52.89 ; p=0.011$ ) than group NOPORE. Troponin peak value ( $1179 \mathrm{ng} / \mathrm{L}$ vs $357 ; \mathrm{p}=0.0014)$, transfusion requirement $(50 \%$ vs $5.17 \% ; p=0.0002)$, time to extubation (16.47 h vs $5.3 ; p=0.0045)$, ICU stay ( 13.35 days vs $2.19 ; p=0.0001$ ) and hospital stay ( 22 days vs $10.9 ; p=0.0006$ ) are higher in group PORE. Figure shows the ROC curves for PreCPB and postCPB TELUS and LUS scores for the occurrence of at least 1 PORE.

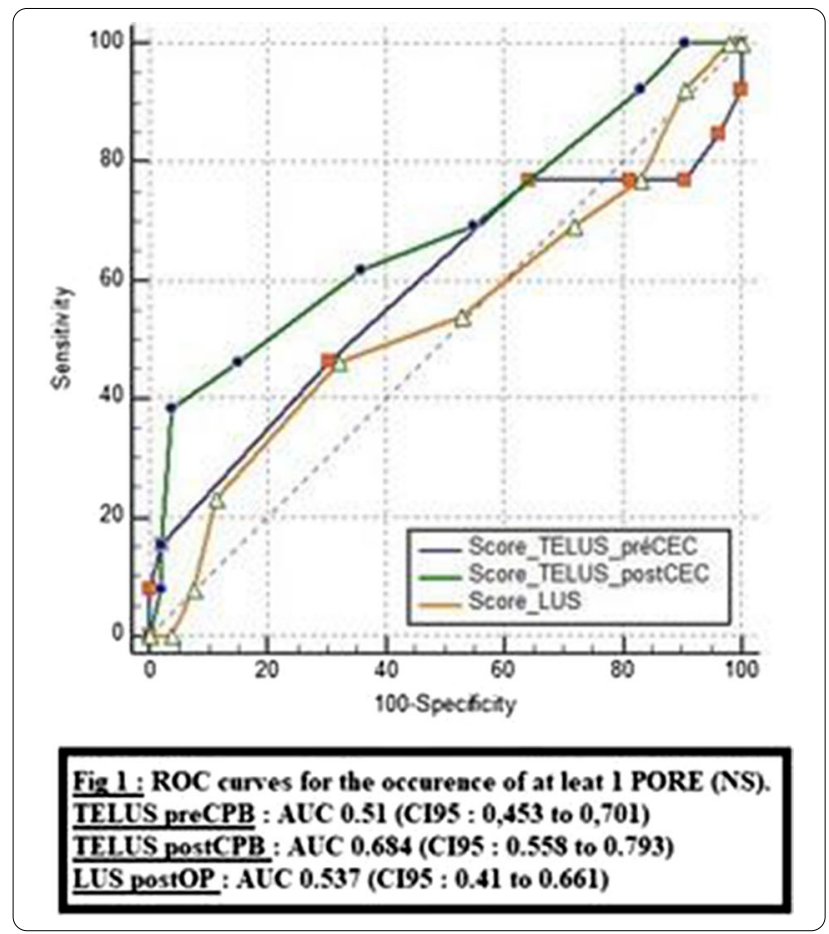

Conclusion. To our knowledge, this is the only study to date investigating the clinical usefulness of TELUS imaging in the perioperative care specifically in CS. Our three main findings are

TELUS score is impaired postCPB and parallels the $\mathrm{P} / \mathrm{F}$

Patients with PORE have a significantly higher TELUS post $\mathrm{CPB}$ suggesting that structural changes and lung de-aeration captured by TELUS occur during CPB.

PostCPB TELUS imaging shows the highest predictive value for PORE occurrence when compared to preCPB TELUS and postoperative LUS. Sensitivity and specificity of the postCPB TELUS score should be tested for more restrictive or specific definition of PORE.

Reference(s)

1. 1) Anesth Analg, 2021;132:172-181 2) J Cardiothorac Vasc Anesth, 2015; 29: 570-575 3) Can J Anest 2016; 63:1266-12,761,276

\section{6}

Lung ultrasound and chest X-Ray in comparison to Chest CT in adult mechanically Ventilated ICU patients, Observational trial A. Al Tayar'; E. Abdelshafey; M. Fawzy²; M. Rashwan

${ }^{1}$ ICu, SFHD, Dammam, Saudi Arabia; ${ }^{2} \mathrm{ICU}, \mathrm{ICU}$, Dammam, Saudi Arabia Correspondence: A. Al Tayar

Intensive Care Medicine Experimental 2021, 9(1): 000246

Introduction. Lung ultrasonography (LUS) is being increasingly utilized in Intensive care unit(ICU) settings. Nevertheless ICU physicians are still depending on portable chest X-ray (CXR) for diagnosing common Lung pathologies. 
Objectives. We aimed to compare using bedside LUS versus portable CXR for detection of 5 main lung pathologies in ICU ventilated patients

Methods. Adult ventilated patients in single center randomly planned for plain axial CT imagingof chest for a reason determined by treating team and not for the purpose of the study, were screened $1 \mathrm{~h}$ before planned CT using standard portable CXR and standard LUS imaging for presence of any imaging signs of 5 major thoracic pathologies:pneumothorax, pleural effusion, consolidation, collapse and pulmonary edema.CXR was read by 2 different experienced intensivist and any contradiction was solved by the opinion of third intensivist. LUS was performed by experienced intensivist who was blind to CXR result. CT was read by consultant radiologist and was blind to CXR and LUS findings. CT was considered as gold standard for diagnosis. Results. 110 LUS exam of RT and Lt Sides of the chest were compared to chest CT and CXR in which CT detected 9 cases positive of pneumothorax, 58 cases positive of pleural effusion, 69 cases positiveof consolidation, 61 cases of lung collapse and 25 case of pulmonary edema. There was a very good agreement for LUS with CT in pneumothorax with sensitivity, specificity and accuracy of 100,97 and $97 \%$ respectively while it was 66,88 and 86 respectively for CXR and with fair agreement to CT.In pleural effusion there was total agreement with $C T$ and it was $100 \%$ for sensitivity, specificity and accuracy compared to only 55, 62 and $68 \%$ respectively for CXR. Regarding consolidation and collapse there was a very good agreement and high accuracy for LUS compared to CXR, with sensitivity and specificity $100,97 \%$ compared to $34 \%$ and $59 \%$ respectively.Accuracy of LUS in pulmonary edema was higher also with $96 \%$ compared to $81 \%$ for CXR.

Conclusion. LUS detected all thoracic 5 main pathologies with high accuracy $(96.36-100 \%)$ and very good agreement with CT $(0.842-1)$ that was much better than CXR which showed much lower accuracy (59.09-86.36\%) and fair to moderate agreement to CT (0.279-0.501).

\section{7}

Covid-19 patients lung ultrasound follow-up after discharge: a series of 35 patients from Ospedale Civile di Vigevano, Italy T. Persiano ${ }^{1}$; F. Grulli ${ }^{1}$; P. Cornara ${ }^{2}$; F. Bonomi ${ }^{3}$; ST. Mediani ${ }^{4}$; A. Casazza ${ }^{3}$; L. Carnevale $^{3}$

'Scuola di specialità medicina di emergenza e urgenza, The University of Pavia, Pavia, Italy; ${ }^{2}$ Scuola di specialità anestesia e rianimazione, The University of Pavia, Pavia, Italy; ${ }^{3}$ Anestesia e rianimazione vigevano, ASST di Pavia, ospedale di Vigevano, Vigevano, Italy; ${ }^{4}$ Uoc cure palliative, ASST di Pavia, ospedale di Vigevano, Vigevano, Italy

Correspondence: A. Casazza

Intensive Care Medicine Experimental 2021, 9(1): 000337

Introduction. Lung ultrasound proved to be a very useful tool in order to assess lung involvement in COVID-19 patients. It can be standardised using LUS (lung ultrasound score), that analyse 12 ultrasound scans ( 6 for each hemithorax) to assign a score from 0 to 3 for each scan, for a maximum of 36 points.

Objectives. We wanted to evaluate LUS findings evolution in discharged, clinically recovered, patients after SARS-Cov2 pneumonia.

Methods. We analysed 35 patients admitted to our hospital during first pandemic wave in spring 2020: 27 males (average age 59,5 yo), 8 females (average age 57,1 yo). Six patients were mechanically ventilated, 16 received helmet CPAP ventilation and 14 needed conventional oxygen supplementation through low flow nasal cannulas or Venti-mask or high flux reservoir mask.

All patients had Covid-19 diagnosis based on nasopharyngeal swab positivity and were admitted to hospital with pneumonia. LUS was performed during hospitalization (hLUS) and, later, we evaluated patients in two follow-up (FUP) medical examinations at 15-30 and 45-60 days after discharge; in both FUP examinations we performed furthermore a short walking test ( $180 \mathrm{~s}$ of brisk walk) and we recorded mMRC (modified Medical Research Council) score to evaluate dyspnea degree. Blood gas analysis in air was performed only during first FUP examination.

Results. Average LUS during hospitalization was 17,5 for mechanically ventilated patients, 13,8 for CPAP patients and 10,1 for patients in conventional oxygen therapy. Average in hospital LOS was 31 days: 52 days for mechanically ventilated, 32 days for CPAP and 20,8 days for conventional oxygen therapy patients.

Average LUS for mechanically ventilated patients was 10,3 at the first FUP, and 8,2 at the second, for CPAP patients we observed an improvement of the average LUS from 7,2 at the first FUP to 4,3 at second FUP. Patients with conventional oxygen supplementation showed an average LUS of 5,8 at the first FUP and 2,7 at the second FUP examination. Using linear regression we found that LUS improves 6,25\% every ten days after discharge and improves $6,37 \%$ every ten days from the first lung ultrasound during hospitalization to the last follow up.

Walking test was positive in $8 / 35$ patients at first FUP and in $4 / 34$ at the second.

mMRC was more than 1 in 10/35 patients at first FUP, but only in 2/34 at the second medical examination.

Blood gas analysis showed average $\mathrm{pO}_{2}$ of $87,8 \mathrm{mmHg},(\mathrm{p} / \mathrm{F}=418)$.

All the patients reported that during their hospitalization, they suffered from loneliness because of the distance of their families and they reported as the main positive aspect the proximity of medical and nursing staff.

Conclusion. In this small study we tried to define the resolution rate of pathological lung ultrasounds findings in discharged, clinically recovered, Covid-19 patients. Even if we have a small cohort of patients, our data suggest that lungs require several weeks to reach total or partial resolution, even if patients have been judged clinically recovered and discharged. LUS seems to be a useful non-invasive tool for Covid-19 patients' FUP.

\section{Reference(s)}

1. Lichtenstein DA. Lung ultrasound in the critically ill. Curr Opin Crit Care. 2014 Jun 20(3):315-322.

2. Peng QY, Wang XT, Zhang LN; Chinese Critical Care Ultrasound Study Group (CCUSG). Findings of lung ultrasonography of novel coronavirus pneumonia during the 2019-2020 epidemic. Intensive Care Med. 2020 May:46(5):849-850

\section{8}

Lung ultrasound findings in patients with COVID-19 Pneumonia and cardiac dysfunction

J. Leote'; T. Judas, ${ }^{2} ;$ AL. Broa ${ }_{1}^{2} ;$ M. Lopes, ${ }_{1}^{3} ;$ F. Abecassis, ${ }^{2} ;$ I. Pintassilgo, ${ }_{1}^{2} ;$ A. Gonçalves, ; F. Gonzalez,

${ }^{1}$ Intensive Care, Hospital Garcia de Orta, Almada, Portugal; ${ }^{2}$ Internal Medicine, Hospital Garcia de Orta, Almada, Portugal; ${ }^{3}$ Pneumology, Hospital Garcia de Orta, Almada, Portugal; ${ }^{4}$ Radiology, Hospital Garcia de Orta, Almada, Portugal

\section{Correspondence: $\mathrm{J}$. Leote}

Intensive Care Medicine Experimental 2021, 9(1): 000378

Introduction. The severe acute respiratory syndrome coronavirus discovered in December 2019 (SARS-CoV 2) in China, transformed the modern world during last year. Lung ultrasound (LUs) has been used to diagnose and monitor COVID-19 pneumonia. Additionally, SARSCoV 2 infection can develop with cardiac dysfunction (CD), which can also be diagnosed through ultrasound imaging.

Objectives. Our study aims to understand if LUS findings in COVID19 patients are influenced by cardiac dysfunction detected by echocardiography.

Methods. Adult patients with a positive real-time polymerase chain reaction for SARS-COV 2 collected in nasal and pharyngeal swab specimens were recruited from June to September 2020 in a tertiary hospital. Clinical, laboratory, and chest imaging including chest X-ray, LUS, and Focus echocardiography was performed on the D1, D5, D10, D15. The LUS score was calculated based on pleural and B-lines characteristics and the presence of consolidations. Spearman and Pearson's correlation was used for patients' baseline characteristics. A subgroup analysis was made by comparing variables between patients with cardiac dysfunction (CD) and without cardiac dysfunction (wCD) using independent t-tests. Cardiac dysfunction was defined as left ventricular systolic dysfunction or right ventricular dilatation, by eyeballing. 
Results. Fifty-one patients ( 28 women) were included (mean age of $62 \pm 17$ years old). Three cardiovascular risk factors were present in 35\% of the patients. Thirty patients were admitted to the $\mathrm{UCl}$ due to acute respiratory failure. Eight were on mechanical ventilation, nine were on vasopressor therapy and eight died within 30 days after hospital discharge. Thirty-one patients had three evaluations and 13 completed four evaluations, in a total of 141 evaluations. The median hospital stay was 14 days. Chest $x$-ray was suggestive of COVID-19 pneumonia in twenty-four patients (47\%).

On admission, 22 patients (43\%) showed CD, of whom seven received mechanical ventilation support and one died. The past medical history of hypertension, diabetes, obesity and ischemic heart disease was similar in both groups. Overall LUS score at admission predicted $(r=0.315, F(1,49)=5.39, p=0.002)$ the ratio of arterial oxygen partial pressure $\left(\mathrm{PaO}_{2}\right)$ to fractional inspired oxygen $\left(\mathrm{FiO}_{2}, \mathrm{P} / \mathrm{F}\right)$ and correlated with the hospital length of stay. The following equation was produced: $\mathrm{P} / \mathrm{F}=295,423+(-2.211 \times$ LUs score $)$; with a coefficient of -2.211 with $95 \% \mathrm{Cl}$ of $-4,124$ to $-0,299$. Patients with CD showed a lower $\mathrm{P} / \mathrm{F}$ ratio from admission until the third evaluation with a greater difference on the second evaluation (mean of $189,95 \% \mathrm{Cl} 152-224$, vs $275,232-318)$. LUs score was similar in both groups. $(33,95 \% \mathrm{Cl} 26-39$ vs. $26,22-31, p=0.1$ ). LUs findings showed more confluent B-lines on the lower quadrants of the right hemithorax in the CD group $(p=0.08)$ Patients that received mechanical ventilation presented, at admission, higher LUs score (mean of $44,95 \% \mathrm{Cl} 34-55$ vs $26,95 \% \mathrm{Cl} 22-30$; $\mathrm{p}<0.003$ ) and a high total number of subpleural consolidations in the upper quadrants of the right hemithorax $(p=0.002)$.

Conclusion. LUS findings in patients with COVID-19 pneumonia correlate with respiratory failure severity predicting the $\mathrm{P} / \mathrm{F}$ ratio. Cardiac dysfunction in COVID-19 may contribute to a worse P/F ratio. Without influencing the LUS score. Involvement of the upper quadrants of the right hemithorax is associated with mechanical ventilation.

\section{Reference(s)}

1. The work was funded by RESEARCH 4 COVID-19 (number 101) from Fundação para a Ciência e Tecnologia; and partially by a public petition organized by J.L.

\section{7}

Use of point of care lung ultrasound in the diagnosis and assessment of patients with COVID-19

B. Saeed, ; B. Johnston ${ }^{2}$; A. Waite, ${ }^{2}$; O. Hamilton, ${ }^{2}$; S. Mulla, ${ }^{2}$;. Welters ${ }^{2}$

${ }^{1}$ Medicine, University of Liverpool, Liverpool, United Kingdom; ${ }^{2}$ Intensive Care Unit, Royal Liverpool University Hospital, Liverpool, United Kingdom Correspondence: $\mathrm{B}$. Johnston

Intensive Care Medicine Experimental 2021, 9(1): 000577

Introduction. Chest X-ray is characteristically used as the first line imaging modality in patients who are suspected of having COVID 19. It has been seen that lung ultrasound scan (USS) are more accurate when compared to Chest X-ray when evaluating lung disorders-specifically pneumonia, oedema, pneumothoraxes and effusions. The aims of the study were to describe the applicability of a Lung ultrasound protocol in a pandemic situation, investigate the interrater variability between a novice and an expert scorer, and finally, explore correlations between markers of disease severity, inflammation and Lung ultrasound scores.

Methods. A retrospective study was undertaken on patients at the Royal Liverpool University Hospital (RLUH) between 30thMarch 2020 and 8th May 2020. Information that included patient demographics, laboratory findings and Imaging were all recorded using the Patient Electronic Notes System. Spearman's correlation used to assess correlation between lung USS and variables. Modified Soldati Scoring System used to compare Lung USS scores. Intraclass Correlation Coefficient using a two-way mixed model based on absolute agreement was used when assessing scorer agreement.

Results. 15 patients were included in the Lung USS scoring assessment and 11 in correlation studies once criteria had been applied. 51 lung USS were performed with a total of 408 regions scanned which included 198 Anterior, 181 Lateral and 29 posterior regions Moderate positive correlation between USS and D-dimer $(r=0.607)$, Ferritin $(r=0.603)$, RALES score $(r=0.539)$ and Lactate $(r=0.500)$. Moderate negative correlation for lymphocyte count $(r=-0.593)$. No significant findings between USS scores and markers of disease severity or respiratory failure and inflammation amongst the 11 patients aside from $d$ dimer $(p<0.05$ Spearman's correlation). The Interclass correlation (ICC) for inter-rater reliability was 0.944 with a $95 \% \mathrm{Cl}$ from 0.846 to $0.975(p<0.001)$.

Conclusion. High inter-rater reliability exists between novice and experienced Lung USS scorers. We could not correlate Lung USS scores with markers of disease severity or respiratory failure and inflammation aside from d-dimer. A standardised approach to performing and scoring Lung USS is needed. The recruitment of more novice and experienced scorers will increase the reliability of the interrater correlation. A larger sample size is necessary for future studies.

\section{3}

Chest ct scan findings in covid 19 pneumonia: correlation with clinical severity

H. Maamouri ${ }^{1}$; A. Ben Jazia²; M. Fatnassi'; F. Haifa ${ }^{3}$; N. Brahmi ${ }^{3}$

${ }^{1}$ Medical ICU, Hôpital Abderrahmen Mami de pneumo-phtisiologie,

Ariana, Tunisia; ${ }^{2}$ ntensive Care, Centre d'assistance médicale urgente-

CAMU, Tunis, Tunisia; ${ }^{3}$ Intensive Care Unit, Centre d'assistance médicale urgente-CAMU, Tunis, Tunisia

Correspondence: $\mathrm{H}$. Maamouri

Intensive Care Medicine Experimental 2021, 9(1): 000663

Introduction. The COVID 19 pandemic is a global health crisis. Chest computed tomography scan (CT) plays a key role in the diagnosis of COVID 19 pneumonia. The extent of CT lesions may be indicative of clinical severity. The aim of this study was to assess the correlation between the extent of pulmonary involvement on $\mathrm{CT}$ and clinical severity.

Methods. We conducted a prospective study during a 3 month period in our ICU. We included patients with COVID 19 pneumonia confirmed wit RT-PCR who underwent a chest CT. Clinical severity was asesed based on progression to Acute Respiratory Distress Syndrome (ARDS) defined by the criteria of Berlin. The CT was described using visual quantification as a percentage of the lung parenchyma affected based on the criteria of the French Society of Radiology.

Results. During the study period 36 patients were included. The mean age was $62 \pm 9$ with male predominance $(85 \%)$. The most common comorbidities were hypertension (44\%) and diabetes (42\%). Non invasive ventilation was required for $72 \%$ of patients and invasive mechanical ventilation was necessary in $42 \%$ of cases. Progression to ARDS was note in $69 \%$ of cases. The median $\mathrm{PaO}_{2} / \mathrm{FiO}_{2}$ ratio was 161 [100258]. Mortality rate was of $31 \%$. Bilateral and peripherical ground-glass opacietis with consolidation were the most common chest $C T$ findings. Pulmonary embolism was found in $11 \%$ cases. The extent of pulmonary involvement was less than $25 \%$ in $19 \%$ of cases, between 25 and $50 \%$ in $42 \%$ of cases and greater than or equal to $50 \%$ in $39 \%$ of cases. A statistically significant association was observed between the extent of pulmonary involvement and progression to ARDS $(p=0.028)$ and use of non invasive ventilation $(p=0.022)$. On multivariate analysis, the independent predictor of mortality was the occurrence of acute renal failure. The extent of pulmonary involvement was not a predictor of mortality.

Conclusion. The extent of pumonary involvement on chest $\mathrm{CT}$ in COVID 19 pneumonia was significantly associated with progression to ARDS. However, it was not predictive of mortality.

\section{5}

Implementation of portable head-CT imaging for severely acute brain injured patients in a French ICU: a pilot observational study Y. Launey ${ }^{1}$; C. Mycinski'; F. Eugène ${ }^{2}$; J. Ferré2; P. Seguin ${ }^{3}$; J. Gauvrit ${ }^{2}$

${ }^{1}$ Anesthesia and Critical Care Medicine, CHU Rennes-Pontchaillou Hospital, Rennes, France; ${ }^{2}$ Neuroradiology_Department of Medical Imaging, CHU Rennes_-Pontchaillou Hospital, Rennes, France; ${ }^{3}$ Anaesthesiology and Surgical Intensive Care Unit, Pontchaillou University Hospital Center, Rennes, France

Correspondence: $Y$. Launey

Intensive Care Medicine Experimental 2021, 9(1): 000835 
Introduction. Head-CT-scanning is a cornerstone procedure during the management of patients admitted for acute brain injury (ABI) in intensive care unit (ICU). But, intrahospital transfer for these procedure may cause severe adverse events potentially worsening the brain injuries [1]. As previously reported, portable head-CT (pCTH) is a scanner used in several countries that may facilitate $\mathrm{PCTH}$ performance in safer conditions for the patients and enhance the costeffectiveness ratio $[2,3]$.

Objectives. The objective of this study was to evaluate the feasibility and safety of PCTH in the French healthcare system for ICU patients admitted for ABI.

Methods. This was a prospective before-after study of patients admitted in ICU for severe $A B I$ including traumatic brain injury, subarachnoid haemorrhage, intracranial haemorrhage or ischemic stroke from June 2019 to January 2021 in a university hospital. A written inform consent of patients or her/his relative were obtained as required by Research Ethics Board. The main primary endpoint was the time duration to perform pCTH compared to conventional head CT-scanner (cCTH). Collected data during CT-scanning periods including demographics and clinical characteristics, intracranial and arterial blood pressures patterns, ventilation parameters and adverse events occurrence were recorded and compared between the 2 periods. We estimated the cost of personal over one year. Nonparametric tests were used for the statistical analyses.

Results. Forty-five patients were included ( 25 in the pCTH group and 21 in the $\mathrm{CCTH}$ group). For reasons of device malfunction or monitoring failure, 22 patients were analysed in PCTH group and 20 in the CCTH group. The median (interquartile) time duration to perform pCTH was 29 (25-48) minutes compared to 30 (25-36) minutes in cCTH group, $(p=0.91)$. A significant longer duration emerged for the first $8 \mathrm{pCTH}$ procedures compared to the 12 following: $53 \mathrm{~min}$ $(36-48)$ versus $25 \mathrm{~min}(18-28)$ respectively $(p<0.01)$ No significant differences were observed in adverse events occurrence. A trend in higher ventilator mishaps was observed in CCTH group: 6 ventilator disconnection vs 1 in PCTH group. No differences in ventilator-associated pneumonia or in-ICU mortality were observed in this study. The attempt to quantify the cost of staff mobilized during scanning assessed a gain of 2700 euros over 1 year. These results may only be applied to our ICU.

Conclusion. The PCTH seems feasible in ICU of the French healthcare system, with similar time of procedure and without increasing adverse events. Short training period may be needed as the time duration of pCTH decreased over time. Financial cost savings might be significant when using such a device. Further larger studies in French ICUs are needed to confirm the results reported by North-American healthcare system

\footnotetext{
Reference(s)

1. [1] Martin M, et al. Neurocrit Care. 2017 Feb;26(1):87-95

2. [2] Rumboldt Z, et al. AJNR Am J Neuroradiol. 2009 Oct;30(9):1630-6.

3. [3] Masaryk T, et al. Radiol Manage. 2008 Mar-Apr;30(2):50-4
}

\section{1}

Prevalence and limitations of point-of-care echocardiography use among European Intensivists and Anaesthesiologistspreliminary results

M. Zawadka'; A. Janiszewska²; I. Jammer ${ }^{3}$; A. Wong ${ }^{4}$

${ }^{1}$ 2nd Department of Anaesthesia and Intensive Care, Medical University

of Warsaw, Polish National Agency for Academic Exchange), Warsaw,

Poland: ${ }^{2} 2$ nd Department of Anaesthesia and Intensive Care, Medical

University of Warsaw, Warszawa, Poland; ${ }^{3}$ Perioperative and Intensive Care

Medicine, Haukeland University Hospital, Bergen, Norway; ${ }^{4}$ King's Critical

Care, King's College Hospital, London, United Kingdom

Correspondence: M. Zawadka

Intensive Care Medicine Experimental 2021, 9(1): 000971
Introduction. Although there is widespread acceptance and expansion on the use of point-of-care echocardiography amongst European Intensivists and Anesthesiologist, published data suggest significant variations in implementation/use and barriers despite guidelines published over a decade ago.

Objectives. The primary objective is to determine the prevalence of point-of-care echocardiography use among European Intensivists and Anaesthesiologists. The secondary objectives of the study are: to determine factors associated with the use and education of point-ofcare echocardiography; to identify the major clinical applications of echocardiography; to identify factors that limit the implementation of echocardiography.

Methods. The study is designed as a cross-sectional survey. The SurveyMonkey hosting platform is used to collect responses. Participants were asked to complete a 25 -item questionnaire; most variables were discrete in nature. Questionnaires were distributed to participants via European Society of Intensive Care Medicine and national societies. Subsequently, the survey was distributed via social media and direct email contact with anaesthesia and critical care departments. Separate tickets were used to allow tracing source of the replies (social media, national societies).

Results. Over a continuous three-month period (March-May 2021) we collected 341 responses. Reminders were sent using various platforms to improve response rate. Preliminary results indicate that $87.5 \%$ of participants use bedside echocardiography. Of those using echocardiography, the majority of participants reported competency in basic critical care echocardiography as defined by the consensus statement. Limiting factors were: lack of experience/skill, lack of formal qualifications, insufficient time, not having a mentor. In most cases, echocardiography is not part of the training ( $62.30 \%$ of respondents) and is more commonly performed in the ICU than in the perioperative setting. Knowledge of echocardiography is mostly acquired from colleagues and online resources. Inferior vena cava collapsibility/ distensibility remains the most commonly use ultrasound examination for haemodynamic management. Over one hundred participants described clinical situations in which echocardiography significantly changed patient management.

Conclusion. The preliminary data presented indicate the need for formal training. We believe that the final results of this ongoing study will provide insight into the true prevalence of bedside echocardiography and answer the question of how the specific training and accreditation programme should be designed in the future. We recognise that our responses may not be representative because of a sample selection bias.

\section{Reference(s)}

1. Galarza L, Wong A, Malbrain MLNG. The state of critical care ultrasound training in Europe: A survey of trainers and a comparison of available accreditation programmes. Anaesthesiol Intensive Ther. 2017:49(5):382386. https://doi.org/10.5603/AIT.a2017.0075. Epub 2017 Dec 1. PMID: 29192421.

2. Zdravkovic M, Berger-Estilita J, Sorbello M, Hagberg CA. An international survey about rapid sequence intubation of 10,003 anaesthetists and 16 airway experts. Anaesthesia. 2020 Mar;75(3):313-322. https://doi.org/10. 1111/anae 14867. Epub 2019 Oct 30. PMID. 31667827.

3. Mayo PH, Beaulieu Y, Doelken P, Feller-Kopman D, Harrod C, Kaplan A, Oropello J, Vieillard-Baron A, Axler O, Lichtenstein D, Maury E, Slama M, Vignon P. American College of Chest Physicians/La Société de Réanimation de Langue Française statement on competence in critical care ultrasonography. Chest. 2009 Apr;135(4):1050-1060. https://doi.org/10. 1378/chest.08-2305. Epub 2009

4. We thank the European Society of Intensive Care Medicine for endorsement of this study. 
001013

Restricted versus Routine bedside Chest X-ray in ICU, time to restrict: an observational study

A. Al Tayar ${ }^{1}$; E. Abdelshafey ${ }^{1}$; M. Rashwan ${ }^{1}$; M. Fawzy²; E. Fares ${ }^{3}$; A. Abujaber $^{3}$; H. Bin Ghezala ${ }^{3}$

${ }^{1} \mathrm{ICU}$, SFHD, Dammam, Saudi Arabia; ${ }^{2} \mathrm{CCU}, \mathrm{ICU}$, Dammam, Saudi Arabia;

${ }^{3}$ Icu, Security Forces Hospital Dammam, Dammam, Saudi Arabia

Correspondence: A. Al Tayar

Intensive Care Medicine Experimental 2021, 9(1): 001013

Introduction. Routine chest X-ray is still commonly practiced in many intensive care units (ICU) especially for ventilated patients in spite of low sensitivity, specificity, negative and positive predictive values. Lung ultrasonography (LUS) use is increasing nowadays and can be considered as good alternative with many evidence based reported advantages. We aimed at comparing restricted CXR practice to the usual routine CXR in our patients.

Objectives. We aimed at comparing restricted CXR practice to the usual routine CXR in our ICU ventilated adult patients. We proposed that restricted CXR practice would be associated with many advantages and relying on LUS may be better and safer

Methods. After approval of local hospital administration and ethics committee a quality project of restricting CXR to ICU cases that intensivist would see a strong indication including but not limited to medicolegal issues and replacing it with LUS (for 6 months from October 2020 to March 2021) and to compare that previous 6 months (from March 2020 to August 2020) in which there was daily routine CXR is a prevailing practice, excluding month of September as transition period between two periods. The comparison included number of CXR, radiation exposure dose, CXR cost, ICU acquired infections, ICU mortality and length of ICU stay.

Results. A total of 192 admissions and 941 patient days in routine period compared to 135 admissions and 649 patient days in restriction period with significant reduction of number of CXR from 732 to 219 with ratio of Xray /patient days reduced from $77.8 \%$ to $33.7 \%$ with $P$ value $<0.001$ with ultimate reduction of radiation dose from 146.4 to 43.8 rad and CXR cost reduction from 109,800 to 32,850 SAR.

No significant change observed between both periods regarding procedures done in ICU as tracheostomy, bronchoscopy, central line and chest tube insertions ( $p$ value of $0.193,0.193,0.253$ and 0.252 respectively)

VAP and CAUTI remained at zero levels in both periods, however 3 cases of CLABSI seen in restriction period which showed no significant difference as $p$ value of 0.068 .

Mortality ratio was not significantly different in both periods $(4.2 \%$ in routine period vs $7.4 \%$ in restriction period with $p$ value of 0.206 while average length of stay decreased significantly from $6.94 \pm 1.47$ days in routine period to $6.33 \pm 1.33$ in restriction period with $P$ value $<0.001$

Conclusion. Decision to restrict CXR orders in ICU with staff well trained in LUS resulted in significant reduction of number of Chest $X$ rays without significant effect on number of procedures, complication rates and outcome indicators with observed significant reduction of length of stay, cost and radiation exposure.

\section{Reference(s)}

1. Special thanks hospital administrators and to all ICU doctors, nurses and technicians who supported the project.

\section{2}

CELUS detects peripheral embolic lesions in COVID-19 patients

JA. CALDERON'; L. Zieleskiewicz²; M. Ibarra33; A. Lopez ${ }^{4}$; P. Aguilera

${ }^{1}$ Departamento de terapia intensiva. hospital general de zona número 4., MEXICAN SOCIAL SECURITY INSTITUTE, Monterrey, Mexico; ${ }^{2}$ Service d'anesthésie et de réanimation, Hospitals Academics De Marseille, Marseille, France; ${ }^{3}$ Departamento de terapia intensiva., Hospital Civil de Guadalajara Fray Antonio Alcalde, Guadalajara, Mexico; ${ }^{4}$ Departamento de terapia intensiva, Hospital Civil de Guadalajara Fray Antonio Alcalde, Guadalajara, Mexico; ${ }^{5}$ Departamento de terapia intensiva, INSTITUTO
JALISCIENSE DE CANCEROLOGIA, Guadalajara, Mexico

Correspondence: J.A. CALDERON

Intensive Care Medicine Experimental 2021, 9(1): 001052

Introduction. In patients with SARS-COV2, various coagulation abnormalities have been demonstrated, as well as systemic microcirculatory disorders which produce an increase in thrombotic events linked to a poor prognosis. (1)

Contrast Enhanced Lung Ultrasound (CELUS) identifies embolic consolidations by demonstrating the absence of enhancement to the Contrast administration (2), allowing to distinguish and differentiate pulmonary infarction from other common etiologies (3-7) in critically ill patients with severe COVID-19 pneumonia such as consolidated pneumonia or atelectasis.

Objectives. This study demonstrates the perfusion patterns of peripheral pulmonary lesions from three COVID-19 pneumonia patients by using CELUS showing no enhancement to contrast administration therefore suggesting avascular pattern.

Methods. Three patients with SARS-COV2 disease confirmed by RT-PCR and presence of subpleural, hypoechoic lesions identified by Mode B Ultrasound were selected. SonoVue/Lumason ${ }^{\mathrm{TM}} 2.4, \mathrm{~mL}$ via venous line was given followed by a bolus of normal saline. The enhancement was continuously observed during $120 \mathrm{~s}$. Mechanical Index was set at 0.3 in order to minimize microbubble disruption.

Results. Absent enhancement on CEUS was observed during full ultrasound examination of three confirmed COVID-19 pneumonia patients, being highly suggestive of embolic consolidation.

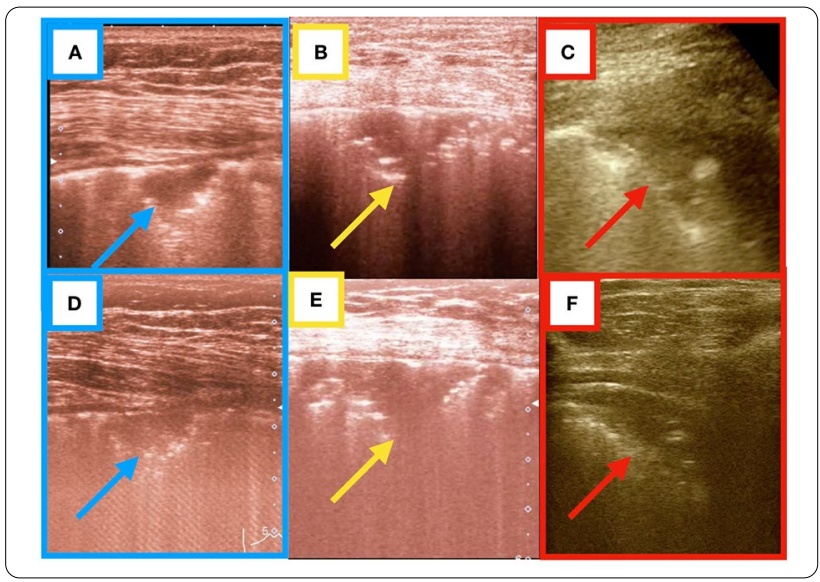

Fig. 1 Brightness-mode ultrasound images of subpleural consolidations (arrows) from three different patients with SARS-COV-2 pnemonia. $A, B$ and $C$ images correspond to prior contrast administration. (mechanical index $=1.3$ ) D, E and F images correspond to the same subpleural consolidations after sulfur hexafluoride microbubbles (SonoVue) contrast administration showing absent enhancement on CEUS. Mechanical index $=0.3$ was used at this time in order to minimize microbubble disruption

Conclusion. The use of CELUS in patients with COVID-19 pneumonia can help identify hypoechoic areas caused by micropulmonary infarction that are difficult to visualize with other imaging methods, which could potentially be useful to guide management and determine prognosis, which warrants future research.

\section{Reference(s)}

1. 7.-Trenker C, Apitzsch JC, Pastor S, Bartelt S, Neesse A, Goerg C. Detection of peripheral embolic consolidations using contrast-enhanced ultrasonography in patients with no evidence of pulmonary embolism on computed tomography: a pilot study. J Clin Ultrasound. 2017:45(9):575-579. 
2. 6.-Grillet F, Behr J, Calame P, Aubry S, Delabrousse E. Acute pulmonary embolism associated with COVID-19 pneumonia detected by pulmonary CT angiography. Radiology. 2020;296(3):E186-E188.

3. 5.-Dolhnikoff M, Duarte-Neto AN, de Almeida Monteiro RA, et al. Pathological evidence of pulmonary thrombotic phenomena in severe COVID19. J Thromb Haemost. 2020;18(6):1517-1519.

4. 4.-Sakr, Y., Giovini, M., Leone, M., Pizzilli, G., Kortgen, A., Bauer, M., .. \& \& Antonucci, E. (2020). Pulmonary embolism in patients with coronavirus disease-2019 (COVID-19) pneumonia: a narrative review. Annals of intensive care, 10(1), 1-13.

5. 3.-Bartelt S, Trenker C, Görg C, Neesse A. Contrast-enhanced ultrasound of embolic consolidations in patients with pulmonary embolism: a pilot study. J Clin Ultrasound. 2015;44(3):129-135)

6. 2.-Sartori S, Postorivo S, Di Vece F, Ermili F, Tassinari D, Tombesi P. Contrastenhanced ultrasonography in peripheral lung consolidations: What's its actual role? World J Radiol 2013; 5(10): 372-380

7. 1.-Dolhnikoff M, Duarte-Neto AN, de Almeida Monteiro RA, et al. Pathological evidence of pulmonary thrombotic phenomena in severe COVID19. J Thromb Haemost. 2020;18(6):1517-1519.

8. NOT APPLICABLE.

\section{1}

\section{Contribution of chest computed tomography during acute} infiltrative lung diseases management in ICU

A. Jamoussi ${ }^{1}$; R. Khayati'; M. Affes ${ }^{2}$; M. Attia ${ }^{2}$; S. AYED ${ }^{1}$; E. Rachdi ${ }^{1}$; F. JARR

AYA $^{1}$; H. Neji ${ }^{2}$; S. Hantous ${ }^{2}$; M. Besbes ${ }^{1}$; J. Ben Khelil ${ }^{1}$

${ }^{1}$ Medical ICU, Hôpital Abderrahmen Mami de pneumo-phtisiologie,

Ariana, Tunisia; ${ }^{2}$ Medical Imaging, Hôpital Abderrahmen Mami de

pneumo-phtisiologie, Ariana, Tunisia

Correspondence: A. Jamoussi

Intensive Care Medicine Experimental 2021, 9(1): 001141

Introduction. Acute infiltrative lung disease is a heterogeneous group of respiratory disorders characterized by diffuse parenchymal lung involvement, affecting the interstitial sector and/or the distal airways. Their etiological diagnosis remain problematical because of the huge number of conditions in question.

Objectives. The purpose of our study was to assess the role of computed tomography (CT) in the etiological diagnosis of acute infiltrative lung diseases and in their management in intensive care unit.

Methods. It was a retrospective study over a period of five years and 6 months including patients who were admitted in the intensive care unit for an acute infiltrative lung disease and who underwent chest CT. Our diagnostic approach on the CT was based on the analysis of patterns or the association of elementary lesions which may be characteristic of some diseases. We have, then, compared our diagnosis hypothesis to the final diagnosis.

Results. Our study included 70 patients with a mean age of 51 years. Infection was the main cause present in $50 \%$ of cases (including $31 \%$ of viral infections), followed by alveolar hemorrhage $(15,7 \%)$, then acute pulmonary edema $(12,8 \%)$ and acute exacerbations of fibrotic interstitial lung diseases (8.5\%). The correct diagnosis was made by CT scan in $82.8 \%$ of the cases, with a very high level of confidence in $48.5 \%$ of the cases. Substantial agreement existed between the diagnosis suggested by the CT and the final diagnosis retained in intensive care unit (kappa $=0,61$ and $p<0,001$ ). The Infection was suggested on CT scan in 32 among 35 patients. A correct diagnosis was made in 10 out of 11 patients with alveolar hemorrhage, 7 of 8 patients with acute pulmonary edema and 5 among 6 cases of acute exacerbations of fibrotic interstitial lung diseases. The most frequent elementary signs were the ground glass opacities (88\%) and the consolidations (78.6\%). A central distribution of the lesions was correlated to the diagnosis of alveolar hemorrhage $(p=0.01)$. Interlobular septal thickening $(p=0.04)$ and bronchovascular thickening $(p=0.017)$ were significantly more common in acute pulmonary edema than in the other causes of acute infiltrative lung disease.

Conclusion. In front of an acute infiltrative lung disease in intensive care unit, elementary CT scan signs and their distribution integrated to anamnestic, clinical and paraclinical findings often contributed to build the right diagnosis.

\section{9}

A chest CT-based mortality scale for alveolar hemorrage in ICU

A. Jamoussi ; S. Jazzar ${ }^{2}$; M. Attia ${ }^{2}$; S. AYED ${ }^{1}$; E. Rachdi ${ }^{1}$; F. JARRAYA ${ }^{1}$; M.

Affes $^{2}$; H. Neji ${ }^{2}$; S. Hantous ${ }^{2}$; M. Besbes ${ }^{1}$; J. Ben Khelil ${ }^{1}$

${ }^{1}$ Medical ICU, Hôpital Abderrahmen Mami de pneumo-phtisiologie,

Ariana, Tunisia; ${ }^{2}$ Medical Imaging, Hôpital Abderrahmen Mami de

pneumo-phtisiologie, Ariana, Tunisia

Correspondence: A. Jamoussi

Intensive Care Medicine Experimental 2021, 9(1): 001189

Introduction. Alveolar hemorrhage $(\mathrm{AH})$ is a life-threatening emergency that may need to be managed in ICU. The chest CT is able to suggest $\mathrm{AH}$ diagnosis.

Objectives. We aimed to establish a $\mathrm{AH}$ mortality scale based on clinical and chest CT findings.

Methods. It was a retrospective study conducted in partnership between intensive care ward and medical imaging department of Abderrahmen Mami hospital of Ariana, Tunisia. It included patients admitted to ICU between January 2009 and December 2018 having an $\mathrm{AH}$ confirmed with bronchoalveolar lavage performed after a chest CT. A mortality scale was established based on clinical and chest CT findings.

Results. We included 56 patients with mean age 48.6 years. In-hospital mortality was $34 \%$. Crazy paving pattern and an extent of damage to 6 pulmonary areas or more were related to a lower ratio $\mathrm{PaO}_{2} /$ $\mathrm{FiO}_{2}$, more frequent need to invasive ventilation and a higher mortality rate. The $\mathrm{AH}$ mortality scale established included 3 parameters: $\mathrm{PaO}_{2} / \mathrm{FiO}_{2}<100 \mathrm{mmHg}$ at admission (9 points), extent of CT damage imaging $>=6$ areas ( 4 points) and crazy paving pattern ( 3 points). The threshold was of 7 points.

Conclusion. The proposed $\mathrm{AH}$ mortality scale here above is easy to calculate, it alerts clinicians to patients with higher risk of death. External validity in mandatory to enhance this score.

\section{8}

Ultrasound measurements of the flow rate through the brachial artery, correlation with inflammatory markers, and the occurrence of edema in postoperative surgical patients in the ICU

A. Peserovic ; K. Kedacic 2 ; S. Kvolik ${ }^{2}$

${ }^{1}$ Anesthesiology and ICU, Medical Faculty, Osijek, Croatia; ${ }^{2}$ Anesthesiology and ICU, Osijek University Hospital; Medical Faculty, Osijek, Croatia

Correspondence: $\mathrm{S}$. Kvolik

Intensive Care Medicine Experimental 2021, 9(1): 001278

Introduction. Ultrasound measurements of the velocity of flow through large blood vessels showed an association between the velocity of flow through the brachial artery and survival in postoperative surgical patients.

Objectives. The aim of this study was to measure whether the flow rate through the brachial artery and inflammatory markers were associated with the development of tissue edema in the surgical intensive care unit (ICU) patients.

Methods. After the ethics committee approval was obtained, a total of 61 postoperative patients or their caregivers signed informed consent for the study participation. The study was performed using a Mindray T7 ultrasound machine with linear probe settings at $50 \mathrm{~mm}$ depth and $40 \mathrm{~dB}$ gain that did not change during the study. The probe was placed on vascular ultrasound measurement. The diameter of the brachial artery in the middle of the upper arm and the flow rate through the artery was measured on postoperative days 1 and 2 . The presence of the edema was measured above the 3rd metacarpal bone as the distance from the skin surface to the periosteum. An average of three assessments was shown. Patients' age, BMI, type of admission; white blood cells, CRP, and procalcitonin were also registered. The Mann-Whitney $U$ test and Pearson correlation were used for statistical analysis. 
Results. The study included 33 men and 28 women, mean age $62 \pm 13$ years, and BMI $27 \pm 4.4 \mathrm{~kg}$. The median ICU stay was 1 day [1-5 days], and the median length of mechanical ventilation (MV) was 16 [5- to 90] hours. A statistically significant correlation was observed between velocity flow and artery width $(r=0792, \mathrm{P}<0.001)$; flow rates, and edema $(r=0.324, \mathrm{P}=0.02)$, edema and PCT $(r=0.570, \mathrm{P}=0.027)$. Artery diameter, flow rate and edema increased on the second day compared to the first, although the difference was not statistically significant ( $P>0.05$ for all measurements). The formation of edema was significantly higher in non-survivors ( $M W U$ test, $P=0.003$ ).

Conclusion. The ultrasound was proven as a useful tool in the ICU. The presence of edema is measurable by ultrasound and showed correlation with flow rate and indicators of inflammation. A larger study should confirm the usefulness of these data in the early prediction of the development of inflammation.

\section{Reference(s) \\ 1. This study was supported by institutional grant IP-18-21, by Medical faculty Osijek, Croatia. \\ 2. Malheiro LF, et al. J Clin Monit Comput. 2020:1-13. https://doi.org/10 1007/s10877-020-00586-9.}

\section{8}

What's the contribution of chest computed tomography in the management of COVID-19 pneumonia?

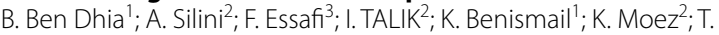
Merhabene $^{4}$

'Réanimation médicale, ICU of Zaghouan Regional hospital, Zaghouan, Tunisia; ²Réanimation médicale, ICU of Zaghouan Regional hospital,

Tunis, Tunisia; ${ }^{3}$ intensive care unit, Regional HospitalZaghouan, Zaghuan, Tunisia; ${ }^{4}$ Réanimation médicale, ICU of Zaghouan Regional hospital, Tunis, Tunisia

\section{Correspondence: F. Essafi}

Intensive Care Medicine Experimental 2021, 9(1): 001318

Introduction. COVID-19 pandemic is an unprecedented global health crisis that started in December 2019. Chest computed tomography (chest CT) has demonstrated its valuable contribution even in pneumonia diagnosis establishing or prognosis scoring.

Objectives. The aim of our study was to evaluate the contribution of chest CT in early COVID 19 diagnosis and look for eventual correlation between clinical outcome and chest CT findings.

Methods. This study was performed at the zaghouan's hospital ICU, a 10-bed tertiary ICU in Tunisia. We reviewed retrospectively all data of confirmed COVID-19 patients hospitalized between September 2020 and April 2021. Were excluded those who did not have a chest CT on admission.

Were collected epidemiological, clinical and chest CT findings. Factors associated with in hospital mortality were evaluated through binary logistic regression models.

Results. During the study period, 192 COVID-19-related pneumonia were admitted, 172 met the inclusion criteria. The mean age was 61.6 years \pm 13 [28-92] and sex ratio was 1.5. Mean SAPS II and APACHE II scores were respectively $27 \pm 9$ and $7 \pm 4 . \mathrm{PaO}_{2} / \mathrm{FiO}_{2}$ ratio at admission was $154 \pm 94$. The median time of symptoms at admission was $9 \pm 4.3$ days [1-20]. The positive diagnosis was made respectively through: rapid antigenic test in 16 patients, RT-PCR in 156 patients and detection of $\mathrm{lgG} / \mathrm{lgM}$ antibodies in 1 patient. All patients received the same treatment protocol.

The predominant pattern of abnormality observed in chest CT was bilateral opacities in 161 patients (83.9\%), mixed sub pleural and peribronchovascular in 175 patients $(75.5 \%)$, ground-glass opacification in 160 patients (83.3\%), crazy paving in 119 patients (62\%). Lung extension was estimated less than $20 \%$ in 12 patients $(7 \%)$, between $20-50 \%$ in 41 patients (24\%), between $50-75 \%$ in 57 patients $(33 \%)$ and more than $75 \%$ in 62 patients (36\%). Lung extenstion more than $50 \%$ and organizing consolidation in both lower lobes were associated with severe acute respiratory distress syndrome at admission $(p=0.04$; $\mathrm{p}=0.025$ ).

Chest CT has allowed a faster diagnosis comparatively to the virological diagnosis with a median difference of 2.4 days [0-15]. Mean Length of ICU stay was significantly longer in patients who had crazy paving on chest CT $10.8 \pm 10$ vs $8 \pm 6$ days $(p=0.01)$.

ICU mortality was significantly associated with crazy paving $(p<0.005)$ condensation areas $(p=0.038)$, predominance in the posterior lobes $(p=0.029)$, lung abnormality in favor of another infection $(p=0.003)$, and CT extent $(p=0.008)$. However previous lung disease was not correlated with poor prognosis $(p=0.263)$.

Conclusion. Chest computed tomography (CT) plays a pivotal role not only in the early detection and diagnosis, especially for false negative RT-PCR and late results, but also in monitoring the clinical course and in evaluating the disease severity.

001377

The state of critical care ultrasound training during the COVID-19 pandemic in Ukraine

M. Grynovska1; A. Wong ${ }^{2}$

${ }^{1}$ Anesthesia and Intensive Care, Ivano-Frankivsk National Medical University and Regional University Teaching Hospital, Ivano-Frankivsk, Ukraine; ${ }^{2}$ Critical Care, Royal Surrey County Hospital, Guildford, United Kingdom

Correspondence: M. Grynovska

Intensive Care Medicine Experimental 2021, 9(1): 001377

Introduction. Ultrasound (US) is increasingly recognized as an essential tool in the ICU setting. The safety and utility of bedside ultrasonography by an appropriately trained ICU physician has now been well demonstrated.1,2 The advantages of the POCUS use during the pandemic in critically ill patients include immediate bedside diagnosis, reduced exposure of sonographers to SARS-CoV-2 and conservation of PPE. However, there is a wide range of skill among intensivists in the performance and interpretation of ultrasound. 3 We sought to investigate the evolution of ICU physicians' CCUS skills and competencies during COVID-19 pandemic in our country.

Objectives. To summarize knowledge on the use of CCUS and attitudes towards itsutilization in the the times of pandemic.

Methods. Our study focused on the ICU physicians $(n=162)$ across different hospitals in Ukraine. Data collection was performed with the help of an electronic survey.

Results. The response rate of the survey was $71 \% .62 \%$ of the respondents were young specialists. The overwhelming majority $(88 \%)$ recognized POCUS an invaluable diagnostic tool in the ICU setting. $68 \%$ were aware of the locally organized training programs, $66 \%$ have joined CCUS training program within their instution since the outbreak of COVID-19. More than half (52\%) of the respondents had no previous experience in CCUS. The most useful CCUS modalities reported were pleural/lung ultrasound and TTE/TEE. All of the respondents recognized the existence of barriers to CCUS training, citing lack of formal training and financial costs as the most significant ones. Although in Ukraine CCUS still remains the prerogative of the on-call radiologists, it was not perceived as a training barrier.

Conclusion. While Ukraine is lacking a formal CCUS training program, there is an unmet demand for it in the ICU. During the pandemic we observed increased recognition of the clincal untility of POCUS by ICU physicians. There has also been a significant increase in locally organised CCUS training courses. Our survey demonstrates a prevailing lack of US skills among the young ICU physicians. There is a general consensus on the list of core US modalities that should be incorporated in such training as well as on the barriers that prevent ICU physicians from mastering them. The young demographics of the study aso highlightes the potential for international training programs and 
initiatives. Due to the limited number of respondents in our survey, further study across hospitals is required to fill in the knowledge gap on the country-specific state of CCUS training. In future we hope to start a cross-country conversation on the challenges of CCUS standards implementation and the possible ways forward.

\section{Reference(s) \\ 1. I would like to thank ESICM NEXT Mentorship and my mentor Adrian Wong for supervision and guidance. \\ 2. 3. Yuriditsky E, et al., Echocardiography. 2021;38:446-449. \\ 3. 2. International expert statement on training standards for critical care ultrasonography. Intensive Care Medicine 2011; 37: 1077-83 \\ 4. 1. Beaulieu Y, et al. Chest. 2005 Sep;128 (3):1766-81.}

e-Posters: Infections and prevention

\section{9}

Determination of side effects seen in healthcare workers after COVID-19 vaccine in Türkiye

C. Balci'; O. Ak, ${ }^{2}$; DP. Renders ${ }^{3}$; E. Yildiz

${ }^{1}$ Anaesthesiology and Reanimation, Healty Science Un., Kütahya, Turkey; ${ }^{2}$ Infection, Healty Science Un., Kütahya, Turkey; ${ }^{3}$ Microbiology, Healty Science Un., Kütahya, Turkey

Correspondence: $C$. Balci

Intensive Care Medicine Experimental 2021, 9(1): 000039

Introduction. The COVID-19 pandemic caused by the severe acute respiratory syndrome coronavirus 2 (SARS-CoV-2) has led to high morbidity and mortality worldwide. Healthcare workers, the elderly (over 60 years old), and those with underlying health problems are at particularly high risk. To date, serious side effects have not been encountered in both clinical studies conducted for COVID-19 vaccines and in current vaccines. Side effects seen after vaccination are mild side effects such as fatigue, headache, fever, chills, muscle/joint pain, vomiting, diarrhea, pain in the area where the vaccine was applied, redness, swelling. These normal vaccine reactions are usually mild and subside after a few days. They show that the vaccine works because it stimulates the immune system and the body creates antibodies against the "fake" infection only by the vaccine. However, despite the rare occurrence of allergic reactions, discomfort may be felt after the vaccine administration. For this reason, it was recommended that the person be followed up in the vaccination outpatient clinic for 15 to 30 min after vaccination.

Objectives. CoronaVac, an inactivated vaccine candidate against COVID-19, is an inactive vaccine developed by Beijing-based Sinovac Life Sciences company. With this study, we wanted to determine the side effects that may be seen in healthcare workers after vaccination in our hospital.

Methods. A pre-vaccination questionnaire was conducted to the healthcare professionals who came to have the second dose vaccine after the first dose of vaccine administration. Age, gender and comorbidities, and presence of allergies were recorded. It was recorded what the side effect was and how long it lasted and whether he went to the health institution.

Results. 299 healthcare professionals completed the post-vaccination questionnaire. Average age was 35 (min: 21, max: 74.139 were men, 160 were women. The most common complaints after vaccination were $55.5 \%$ pain and redness in the vaccine area, $44.4 \%$ headache, $34.7 \%$ muscle and joint pain, respectively $33.3 \%$ had complaints of fatigue, $6.9 \%$ fever, $5.5 \%$ nausea and vomiting, $2.7 \%$ diarrhea, $1.38 \%$ tremor, syncope and disorientation, loss of taste and smell, ear pain. The average duration of complaints is 1.07 days (min: $1 \mathrm{~h}$, max: 30 days).

Conclusion. No serious side effects were observed after the first dose of COVID-19 vaccine administered to healthcare professionals in our hospital. Side effects seen were mild symptoms that could be seen after vaccination and most of them disappeared without treatment.
000078

Prevalence and predictive value for poor outcome of overweight and obesity in critically ill COVID-19 patients: a retrospective cohort study

B. Byloos ${ }^{1}$; K. Van Halem²; I. Callebout '; J. Dubois'; P. Messiaen²; B. Stessel ${ }^{3}$ ${ }^{1}$ Anesthesiology and Intensive Care, Virga Jessa, Hasselt, Belgium; ${ }^{2}$ Infectious diseases and immunity, Virga Jessa, Hasselt, Belgium; ${ }^{3}$ Anesthesiology and Intensive Care, Virga Jessa and Hasselt University, Hasselt, Belgium

\section{Correspondence: B. Byloos}

Intensive Care Medicine Experimental 2021, 9(1): 000078

Introduction. A systematic review pooling 75 studies, showed that obese COVID-19 patients are more likely to be hospitalized, to be admitted to Intensive Care Unit (ICU) and die (1). The weekly ECDC COVID-19 surveillance reports however fail to demonstrate a clear link between obesity and disease severity in a European population. Our aim was to evaluate the distribution of Body Mass Index (BMI) categories in a mono-center dataset of hospitalized COVID-19 patients and assess the association between body weight and poor outcome after COVID-19 infection.

Methods. All patients aged 16 years or older, admitted to the JESSA hospital, Hasselt, Belgium for at least $24 \mathrm{~h}$ with laboratory-confirmed diagnosis of COVID-19 between March 16th and May 2th 2020, were included in the study. We collected data on patient characteristics, comorbidities, and outcomes including disease severity, need for $I C U$, length of hospital stay, acute kidney injury, venous thromboembolism and 1-month mortality. Disease severity score (I to IV) was based on the WHO classification of clinical Severity of Confirmed COVID-19 (2).

Results. In total, 331 COVID-19 patients were admitted to the Hospital from March 16th until May 2th 2020. In total, $41.3 \%$ of all patients were overweight and $23.3 \%$ obese. An overview of outcome parameters stratified for BMI distribution is presented in Table 1. A statistical significant association between BMI distribution and disease severity or poor outcome was not detected in our cohort. Table 1. Overview of disease severity and outcomes, in total group and stratified for BMI distribution. Data are presented as mean \pm standard deviation or as numbers (frequencies). A $p$-value $<0.05$ is considered statistically significant (Chi-square test).

\begin{tabular}{|c|c|c|c|c|c|c|c|}
\hline & Total Group & $\begin{array}{l}\text { Under- } \\
\text { weight } \\
\mathrm{N}=5\end{array}$ & $\begin{array}{l}\text { Normal } \\
\text { weight } \\
\mathrm{N}=91\end{array}$ & $\begin{array}{l}\text { Overweight } \\
\mathrm{N}=112\end{array}$ & $\begin{array}{l}\text { Moderate } \\
\text { obesity } \\
N=46\end{array}$ & $\begin{array}{l}\text { Severe obe- } \\
\text { sity } n=17\end{array}$ & $\mathrm{p}$-value \\
\hline $\begin{array}{l}\text { ICU stay } \\
\text { (num- } \\
\text { ber of } \\
\text { patients) }\end{array}$ & & $0(0.0 \%)$ & $24(26.4 \%)$ & $24(21.4 \%)$ & $12(26.1 \%)$ & $5(29.4 \%)$ & 0.62 \\
\hline LOSICU & $3.7 \pm 11.17$ & $0.00 \pm 0.00$ & $5.03 \pm 12.57$ & $2.87 \pm 7.27$ & $6.52 \pm 16.73$ & $6.65 \pm 19.97$ & 0.95 \\
\hline $\begin{array}{l}\text { LOS Hos- } \\
\text { pital }\end{array}$ & $12.68 \pm 14.04$ & $10.80 \pm 9.86$ & $16.27 \pm 17.60$ & $10.65 \pm 9.40$ & $15.61 \pm 18.55$ & $15.06 \pm 18.65$ & 0.858 \\
\hline \multicolumn{2}{|c|}{ Type COVID-19* } & & & & & & 0.09 \\
\hline । & & $2(40.0 \%)$ & $20(22.0 \%)$ & $25(22.3 \%)$ & $5(10.9 \%)$ & $2(11.8 \%)$ & \\
\hline$\|$ & & $2(40.0 \%)$ & $24(26.4 \%)$ & $12(10.7 \%)$ & $7(15.2 \%)$ & $4(23.5 \%)$ & \\
\hline III & & $0(0.0 \%)$ & $26(28.6 \%)$ & $34(30.4 \%)$ & $17(37.0 \%)$ & $6(35.3 \%)$ & \\
\hline IV & & $1(20.0 \%)$ & $21(23.1 \%)$ & $41(36.6 \%)$ & $17(37.0 \%)$ & $5(29.4 \%)$ & \\
\hline \multicolumn{4}{|c|}{ Venous thromboembolism } & & & & 0.069 \\
\hline $\mathrm{PE}$ & $32(9.7 \%)$ & $0(0.0 \%)$ & $8(8.8 \%)$ & $11(9.8 \%)$ & $8(17.4 \%)$ & $1(5.9 \%)$ & \\
\hline DVT & $13(3.9 \%)$ & $0(0.0 \%)$ & $6(6.6 \%)$ & $4(3.6 \%)$ & $2(4.3 \%)$ & $1(5.9 \%)$ & \\
\hline Both & $1(0.3 \%)$ & $0(0.0 \%)$ & $0(0.0 \%)$ & $0(0.0 \%)$ & $0(0.0 \%)$ & $1(5.9 \%)$ & \\
\hline AKI & $40(12.1 \%)$ & $0(0.0 \%)$ & $15(16.7 \%)$ & $9(8.0 \%)$ & $8(17.4 \%)$ & $4(23.5 \%)$ & 0.16 \\
\hline $\begin{array}{l}1 \text { month } \\
\text { mortal- } \\
\text { ity }\end{array}$ & $72(21.8 \%)$ & $1(20.0 \%)$ & $18(19.8 \%)$ & $24(21.4 \%)$ & $13(28.3 \%)$ & $4(23.5 \%)$ & 0.19 \\
\hline
\end{tabular}

Conclusion. Overweight and obese individuals seem over-represented in this cohort of hospitalized COVID-19 patients. However, we couldn't detect an association between body weight and poor outcome in this dataset. 
Reference(s)

1. 1. Popkin BM, Du S, Green WD, Beck MA, Algaith T, Herbst CH, et al. Individuals with obesity and COVID-19: A global perspective on the epidemiology and biological relationships. Obes Rev. 2020;21(11):e13128.

2. 2. WHO Clinical Management of COVID-19 Interim guidance, 27 may 2020 WHO/2019-nCoV/clinical/2020.5. Available at https://www.who.int/ publications/i/item/clinical-management-of-covid-19.

\section{0}

Vitamin C kinetics in critically ill patients with severe SARS-CoV-2 pneumonia

L. Chiscano ${ }^{1}$; C. Palmada'; A. Ruiz-Sanmartin'; S. Camós Anguila²; R. Ferrer Roca'; O. Roca'; R. Ferrer Roca'; JC. Ruiz-Rodríguez

'Intensive Care Department, Vall d'Hebron University Hospital, Barcelona, Spain; ${ }^{2}$ Clinical Biochemistry Service, Hospital Vall d'Hebron, Barcelona,

Spain

Correspondence: L. Chiscano

Intensive Care Medicine Experimental 2021, 9(1): 000180

Introduction. Vitamin $\mathrm{C}$ is an antioxidant with anti-inflammatory and immune-supportive properties. Its levels are decreased in patients with sepsis-related acute respiratory distress syndrome (ARDS). Moreover, a significant number of patients with severe acute respiratory syndrome Coronavirus-2 (SARS-CoV-2) disease developed ARDS. Therefore, we hypothesized that ARDS Coronavirus disease 2019 (COVID-19) patients may present vitamin C deficiency.

Objectives. Study of plasma concentrations of vitamin $C$ analyzed sequentially in patients requiring admission to the ICU due to respiratory failure secondary to SARS-CoV-2.

Methods. Single-center, observational, prospective study. Patients admitted to the ICU for severe SARS-CoV-2 pneumonia. Plasma vitamin $C$ concentrations were measured on days 1,5 and 10 of admission to the ICU. Demographic variables, severity (APACHE II), organ dysfunction (SOFA), mortality (ICU and hospital), variables related to the treatment of SARS-CoV-2 pneumonia and organic support measures have been analyzed. Acute respiratory distress syndrome (ARDS) was defined following the Berlin definition[i]. The plasma concentration of vitamin $C$ was analyzed by high performance liquid chromatography (HPLC), whose detection limit is $1.5 \mathrm{mg} / \mathrm{L}$. We define plasmatic levels of vitamin C following the next standard[ii]: deficiency: $<2 \mathrm{mg}$ / Li low plasma concentration: 2-5 mg/L; normal plasma concentration: $>5 \mathrm{mg} / \mathrm{L}$. The data have been expressed as frequency (\%) if they are categorical and median (interquartile range) or mean (standard deviation) if they are quantitative. The study was approved by the Clinical Research Ethics Committee of our center (PR (AG) 270/2020).

i. Ranieri V, Rubenfeld G, Thompson B, Ferguson N, Caldwell E, Fan E Camporota L, Slutksy A. Acute Respiratory Distress Syndrome: The Berlin Definition. JAMA 2012 307 (23): 2526-2533.

ii. Hampl J, Taylor C, Johnston. Vitamin C deficienciy and depletion in the United States: the Third National Health and Nutrición Examination Survey, 1988 to 1994. Am J Public Health. 2004; 94 (5): 870-5

Results. Fifteen patients were included [10 (67.0\%) men, age 60.93 (7) years, SOFA 3 (1-4) and APACHE II 15 (8-24)]. One patient presented shock and initial bacterial coinfection was documented in $2(13.33 \%)$ patients. All patients required high-flow oxygen therapy and during evolution, 7 (46.7\%) required mechanical ventilation for 26 (11.2) days. Mortality in ICU and hospital was $27 \%$. The rest of the clinical characteristics are described in Table 1 and 2. During the study period, all patients presented undetectable concentrations of vitamin $C$ except for 2 patients who, based on undetectable initial concentrations, presented concentrations in the low range from 5th day, coinciding with discharge from the ICU.

Conclusion. In our cohort of patients, vitamin C concentrations were undetectable in the first $24 \mathrm{~h}$ after admission to the ICU and remained that way throughout the study period in most of the patients.

\section{Reference(s)}

1. [ii] Hampl J, Taylor C, Johnston. Vitamin C deficienciy and depletion in the United States: the Third National Health and Nutrición Examination Survey, 1988 to 1994. Am J Public Health. 2004; 94 (5): 870-5

2. [i] Ranieri V, Rubenfeld G, Thompson B, Ferguson N, Caldwell E, Fan E Camporota L, Slutksy A. Acute Respiratory Distress Syndrome: The Berlin Definition. JAMA. 2012; 307 (23): 2526-2533.

3. The authors have no conflicts of interest to declare. No funding

000182

Cytokine hemoadsorption as a rescue therapy of patients with SARS-CoV-2 pneumonia with severe respiratory failure and hypercytokinemia

L. Chiscano'; C. Palmada ; A. Ruiz-Sanmartin'; M. Pérez-Carrasco ${ }^{1}$; J. Perurena²; M. Hernandez-Gonzalez²; R. Ferrer Roca ${ }^{1}$; JC. Ruiz-Rodríguez ${ }^{1}$ ${ }^{1}$ Intensive Care Department, Vall d'Hebron University Hospital, Barcelona, Spain; ${ }^{2}$ Immunology Service, Vall d'Hebron University Hospital, Barcelona, Spain

Correspondence: $L$. Chiscano

Intensive Care Medicine Experimental 2021, 9(1): 000182

Introduction. There is a subgroup of patients who develop a hyperinflammatory response and the arrest of these patients is extremely important[i] as they can benefit from specific therapies. The capability of blood purification therapy in removing cytokines may be then a promising therapeutic tool[ii] [iii].

Objectives. Our purpose was to study the clinical utility of cytokine hemoadsorption (HA) in SARS-CoV-2 pneumonia with severe acute respiratory failure and hypercytokinemia.

Methods. Single-center, observational and prospective study of patients with severe SARS-CoV-2 pneumonia who were treated with cytokine HA with Cytosorb ${ }^{\circledR}$ (Cytosorbents Europe, Berlin, Germany). The inclusion criteria were refractory acute respiratory failure $\left(\mathrm{PaO}_{2} /\right.$ $\mathrm{FiO}_{2}$ ratio < 100) with poor response to prone associated with a hyperinflammatory state manifested in the form of hypercytokinemia (IL-6>1000 pg/mL), hyperferritinemia and elevation of D-dimer. The clinical and analytical characteristics of the patients with main interest on the inflammation markers (IL6, IL10, CRP) and oxygenation $\left(\mathrm{PaO}_{2} / \mathrm{FiO}_{2}\right.$ ratio) were studied; variables related to treatment, organ support, severity (APACHE II), organ dysfunction (SOFA), mortality (ICU and hospital). Acute respiratory distress syndrome (ARDS) was defined following the Berlin definition[iv]. Data are expressed as frequency (\%) if they are categorical and median (interquartile range) or mean (standard deviation) if they are quantitative. The statistical tests were the Wilcoxon test and the Mann Whitney $U$ test. The study was approved by the Clinical Research Ethics Committee of our center (PR (AG) 270/2020) and the need for informed consent was waived.

Results. A total of 343 patients were admitted to the ICU due to SARS-Cov-2 infection. Of these, six patients [5 (83.3\%) men, 57 (10.5) years, SOFA 5 (1.4), APACHE II 19.5 (6)] received treatment with HA of cytokines. All patients presented criteria for severe ARDS, all required prone position and were on mechanical ventilation for 15.2 (7.2) days. One session of 16 (9) hours duration was performed with a significant reduction of IL-6 [(IL-6 pre-HA 17,367.5 (4538.7-22,532.0) pg/ml, IL-6 post-HA $2403.0(917-3724) \mathrm{pg} / \mathrm{ml}, \mathrm{p}=0.043]$ and an improvement in oxygenation $\left[\mathrm{PaO}_{2} / \mathrm{FiO}_{2}\right.$ ratio pre-HA 103 (18.4), $\mathrm{PaO}_{2} / \mathrm{FiO}_{2}$ ratio postHA 221.75 (20.9), $p=0.029$ ] and in organ dysfunction [SOFA pre-HA 9 (4.75) and SOFA post-HA 7.7 ( 5.4), $p=0.046]$. Inflammatory markers (CRP, DD, Ferritin) also improved significantly. Mean stay in ICU was 17.2 (8.0) days. Mortality in ICU and in-hospital was $33.7 \%$.

Conclusion. In our cohort of patients with SARS-CoV-2 pneumonia with severe acute respiratory failure and hypercytokinemia, cytokine $\mathrm{HA}$ has induced a reduction in the hyperinflammatory state and an improvement in organ dysfunction. The cytokine hemoadsorption could be a safe and effective rescue therapy of patients with severe and refractory COVID-19 ARDS. 
Reference(s)

1. [iv] Ranieri V, Rubenfeld G, Thompson B, Ferguson N, Caldwell E, Fan E, Camporota L, Slutksy A. Acute Respiratory Distress Syndrome: The Berlin Definition. JAMA. 2012; 307 (23): 2526-2533.

2. [iii] S. Bucciarelli, G. Espinosa, R. Cervera, D. Erkan, J.A. Gomez-Puerta, M. Ramos-Casals, et al., Mortality in the catastrophic antiphospholipid syndrome: causes of death and prognostic factors in a series of 250 patients, Arthritis Rheum. 2006; 54 (8) 2568-2576

3. [ii] V. Schwindenhammer, T. Girardot, K. Chaulier, A. Gregoire, C. Monard, L. Huriaux, et al., oXiris(R) use in septic shock: experience of two French centres, Blood Purif. 47 (Suppl. 3) (2019) 1-7.

4. [i] Mehta P, McAuley D, Brown M, Sanchez E, Tattersall R, Manson J, et al. COVID-19: consider cytokine storm syndromes and immunosupresion. Lancet. 2020; 395 (10229): 1033-1034.

5. The authors have no conflicts of interest to declare. No funding

\section{6}

Tension Pneumomediastinum: Another life threatening condition in COVID19 Patients

N. Shaikh ${ }^{1}$; E. Jamal ${ }^{2}$; AD. Hani ${ }^{3}$;. Mustafa ${ }^{4}$; M. Arif ${ }^{4} ;$ K. Muhmmad $^{5}$

${ }^{1}$ Hamad Medical Corporation, Doha, Qatar; ${ }^{2}$ Surgical Intensive Care Unit, Hamad Medical Corporation, Doha, Oatar ${ }^{3} \mathrm{Hmgh}$ Intensive Care, Hamad Medical Corporation, Doha, Qatar; ${ }^{4} \mathrm{Hmgh}$ ICU, Hamad Medical Corporation, Doha, Qatar; ${ }^{5}$ Dirctor hmgh Intensive Care Units, Hamad Medical

Corporation, Doha, Qatar

Correspondence: N. Shaikh

Intensive Care Medicine Experimental 2021, 9(1): 000216

Introduction. Tension penumomediastinum (TPM) is a rare but potentially fatal clinical entity. Causes of TPM include barotrauma to lungs, trauma to esophagus, tracheobronchial tree, or alveoli. This leads to leakage of air into the mediastinal cavity and increased pressure on thoracic vessels, respiratory tract, and the heart. Literature related to COVID19 patients developing TPM due to invasive ventilation is relatively rare.

Objectives. A series of five cases of COVID19 complicating into ARDS and developing TPM.

Methods. All patients admitted to COVID-19 intensive care units with tension pneumomediastinum were included retrosepectively. Patients demographic data, diagnossis, complicated into tension mediastinum and management were recorded.

Results. All patients were over 50 years and male. They were admitted with primary diagnosis of deteriorating COVID-19 pneumonia. Most of them developed secondary bacterial and fungal infections complicating into severe ARDS requiring intubation and mechanical ventilation. Three patients required reintubations. One patient had tracheal injury. Three of them developed cardiac arrest due to TPM. Diagnosis of TPM was made with clinical signs followed by thoracic computer tomography (CT) for all the patients. Two patients required decompression with chest drains, whereas three patients were managed conservatively, and one patient received ECMO (extracorporeal membrane oxygen therapy) therapy. Most of the patients had underlying health conditions like diabetes mellitus. Three patients survived and two died.

Conclusion. COVID19 pneumonia patients with ARDS requiring invasive ventilation and prone positioning can complicate into TPM. Early recognition is vital in management as it may lead to serious complications as cardiac arrest.

\section{Reference(s)}

1. Wali A, Rizzo V, Bille A, Routledge T, Chambers A. Pneumomediastinum following intubation in COVID-19 patients: a case series. Anaesthesia2020; 75: 1076-1081

2. None.

\section{9}

Effect of Antimicrobial Stewardship Programs on the pediatric patients of ENVIN-HELICS data base

E. FRESAN RUIZ ; I. Jordan Garcia ${ }^{2}$; AC. Izurieta Pacheco ${ }^{3}$; A. Covas Esteva ${ }^{3}$; S. Bobillo ${ }^{4}$; JC. De Carlos Vicente ${ }^{5}$; M. Slocker Barrio ${ }^{6}$; L. Herrera ${ }^{6}$; L. Pinos ${ }^{7}$; S. Uriona ; C. Zazo Sanchidrian ${ }^{8}$; C. Calvo Monge ${ }^{7}$; A. Coca Perez ${ }^{10}$; ME. Valeron Lemaur ${ }^{11}$; B. De Miquel Laviser ${ }^{12}$; M. Ortiz Pallares ${ }^{13}$; S. Sanchez Perez $^{14}$; S. Brio Sanagustin ${ }^{15}$; JS. Leon Gonzalez ${ }^{16}$; P. Envin-Helics Study Group

${ }^{1}$ PICU, Hospital Sant Joan de Déu Barcelona, Esplugues de Llobregat, Spain; ${ }^{2} P i c u$, H Sant Joan de Déu Barcelona, Barcelona, Spain; ${ }^{3}$ Pediatric Department, Hospital Sant Joan de Déu Barcelona, Esplugues de Llobregat, Spain; ${ }^{4} \mathrm{Picu}, \mathrm{H}$ Sant Joan de Déu, Barcelona, Spain; ${ }^{5} \mathrm{Picu}$, Hospital Universitari Son Espases, Palma, Spain; ${ }^{6}$ Picu, Gregorio Marañón Hospital, Madrid, Spain; ${ }^{7}$ Administration envin-helics registry, Hospital Universitari Vall d'Hebron, Barcelona, Spain; ${ }^{8} \mathrm{Picu}$, Hospital Alicante, Alicante (Alacant), Spain; ${ }^{9} \mathrm{Picu}$, Hospital universitario de Donostia- Edificio Amara, Donostia, Spain; ${ }^{10} \mathrm{Picu}$, Hospital Ramón y Cajal, Madrid, Spain; ${ }^{11} \mathrm{Picu}$, C.H.U. Insular-Materno Infantil, Las Palmas de Gran Canaria, Spain; ${ }^{12}$ Picu, Hospital Clinico Universitario San Carlos, Madrid, Spain; ${ }^{13}$ Picu, Álvaro Cunqueiro Hospital, Vigo, Spain; ${ }^{14} \mathrm{Picu}$, Hospital Parc Taulí de Sabadell, Sabadell, Spain; ${ }^{15} \mathrm{Picu}$, Hospital de la Santa Creu i Sant Pau, Barcelona, Spain; ${ }^{16}$ Picu, Hospital Nuestra Señora de Candelaria, Santa Cruz de Tenerife, Spain

Correspondence: E. FRESAN RUIZ

Intensive Care Medicine Experimental 2021, 9(1): 000219

Introduction. Suspected healthcare-associated infections (HAI) require immediately empiric antibiotic $(A B)$ treatment with broad spectrum $A B$. The implementation of Antimicrobial Stewardship Programs (ASP) seems to be a useful way to improve $A B$ management, also in $\mathrm{HAl}$.

Objectives. The objectives are to describe the use of $A B$ during the last year of the Spanish Registry Pediatric-ENVIN-HELICS, and also to compare the evolution of $\mathrm{AB}$ used for $\mathrm{HAl}$ in Pediatric Intensive Care Units (PICU) after a progressive ASP implementation.

Methods. Multicenter, prospective and observational study of infections treated in 26 Spanish PICU, from April to June of 2013-2019. The ENVIN diagnostic criteria adapted to pediatrics were used, based on European Centre of Prevention and Disease Control (ECDC) recommendations. Data was collected from the Pediatric-ENVIN data base ASP were consensuated and recommended, with progressive implementation along these years. SPSS ${ }^{\circledR} 21$ program was used for the statistical analysis.

Results. The total number of patients included in 7 years was 10,469 , 1,752 in 2019 . The $A B$ use rate was $73 \%(n=1,275)$, with a decrease of $5 \%(p>0.05)$ compared to 2013. The number of $A B$ was 1.42 per patient and 1.96 per patient treated with $A B$. The most frequent indication was prophylaxis $(36.2 \%, n=904)$, followed by suspected community infection $(\mathrm{Cl})(31.6 \%, \mathrm{n}=788)$; and suspected $\mathrm{HAl}(31.5 \%$, $n=784)$. The $A B$ indication was empirical in $83.7 \%(n=1316)$ of the prescriptions.

The most used $A B$ for surgical prophylaxis was Cefazolin (56.3\%). In suspected Cl: Cefotaxime (22.7\%). For suspected outside-PICU HAI Vancominycin (13.7\%) and Meropenem (12.9\%); and for suspected inside-PICU HAl: Vancomycin (18.3\%) and Piperacillin-tazobactam (14.8\%). A decrease in the prescription of meropenem for $\mathrm{HAl}$ was observed in 2019 compared to the previous year: $5 \%$ for outsidePICU and $1.7 \%$ for inside-PICU ( $p>0.05)$. Empirical $A B$ treatment was inadequate in $12.2 \%(n=160)$. The early suspension rate was $18.1 \%$ $(n=284)$, which increased $3.9 \%(p<0.001)$ compared to the first year of the registry. Results showed that $15 \%$ of the $A B$ prescribed were changed $(n=234)$. Out of those changed, $45.3 \%(n=106)$ were deescalated; this rate was $18.6 \%$ higher compared to $2013(p<0.001)$. AB modification due to adverse events was $7.5 \%(n=95)$, with a decreasing trend $(0.6 \%, p>0.05)$. Other causes for change were toxicity $(4.3 \%$, $\mathrm{n}=10)$ and new antimicrobial resistances $(0.4 \%, \mathrm{n}=1)$. 
Conclusion.

1. The $A B$ use rate continues to be high in patients from the ENVIN registry (73\%) with almost $2 A B$ per patient with $A B$, with an upward trend in 2019.

2. This last year of the registry a decrease in the use of carbapenems was observed for the first time in the years of the ENVIN registry, both for inside and outside-PICU HAl.

3. The improvement on the antibiotic policy has been made effective through an increase in the rate of de-escalation and the maintenance of a high early suspension rate.

4. The modification due to an adverse event has also decreased in the recent years, accompanied by a decrease in new antimicrobial resistances.

\section{6}

MULTIRESISTANT MICROORGANISMS IN SARS COV-2 PATIENTS ADMITTED TO THE ICU

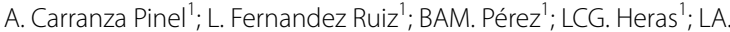
Aranda

${ }^{1}$ Intensive Care Unit, Regional Hospital Santa Ana de Motril, Motril, Spain Correspondence: A. Carranza Pinel

Intensive Care Medicine Experimental 2021, 9(1): 000226

Introduction. The emergence of SARS CoV-2 has posed a challenge in the monitoring and control of infections and colonization by multiresistant microorganisms (MMR) in patients admitted to intensive care units. Current guidelines for the management of SARS CoV-2 patients do not include recommendations on performing surveillance cultures or control measures to avoid nosocomial MMR infections in these patients.

Objectives. The main objective of our study is to relate the appearance of MR, colonization and/or infection, in SARS CoV-2 patients. To do this, we identified, among other variables, antibiotics used during their stay in the ICU and days of treatment with corticosteroids.

Methods. This is a descriptive retrospective study of patients diagnosed with SARS CoV-2 pneumonia who have required admission to a polyvalent ICU and treatment. Collect a year of pandemic in an ICU that has doubled its number of beds to care for all patients. MMR has been followed by colonization cultures of axillary, pharyngeal and perianal exudates on a weekly basis. The treatment of the pathology has followed the protocols and consensus of the scientific societies (SEMICYUC and ESICM). Data are expressed with the median interquartile range for quantitative variables, qualitative variables were expressed in frequencies and percentages.

Results. 64 patients of which $70.3 \%$ male (45), 29.68\% female (19). Average age is 61.1 years $\{30-79\}$. The most frequent personal antecedents collected were HBP $53.12 \%$, obesity $48.43 \%$ and ex-smoker $37.5 \%$. $78.12 \%(50)$ of the patients required mechanical ventilation, the rest were treated with high-flow oxygen therapy. $59.37 \%$ (38) of the patients have benefited from prone therapy. 39\% required tracheostomy (25). The mean stay in the ICU was 22.3 days $\{1-93\}$. The mean hospital stay of 31.74 days $\{3-103\}$. Mortality in the ICU has been $28.12 \%$ (18). The mean number of days of IV corticosteroids was 20.67 days. $46.8 \%$ (30) presented in MMR colonization cultures. MMR infection 39\% (25) being the most frequent pneumonia associated with mechanical ventilation $31.2 \%$ (20) followed by bacteraemia $28.1 \%$ (18). The patients colonized by MMR were $35.9 \%$ (23). The most frequent MMR both in infection and in colonization was Pseudomonas aeruginosa in $63.3 \%$ (19), Klebsiella Pneumoniae ESBL $26.6 \%$ (8), the rest Enterococcus spps faecium, Enterobacter Cloacae, E. Coli, MRSA. Only nine patients did not receive empirical antibiotics upon admission to the ICU (14\%). The rest received antibiotics. The most used was ceftriaxone $2 \mathrm{~g}$ every $24 \mathrm{~h}$ in $75 \%$ (48).

Conclusion. As reflected in recent medical literature, CoV-2 patients admitted to ICUs are more susceptible to colonization and infection by MMR compared to other pathologies. This may be due to the prolonged stay (mechanical ventilation, central catheters) and the use of empirical antibiotic therapy and IV corticosteroids. It would be important and necessary more studies including other areas of hospitalization.
000227

Intrapulmonary Pharmacokinetics of high doses of tigecycline in patients with Ventilator-Associated Pneumonia (VAP) MP. Almyroudi'; G. Dimopoulos ${ }^{1}$; I. Kapralos²; O. Apostolopoulou'; A. Flevari'; DP. Nicolau ${ }^{3}$; A. Dokoumetzidis ${ }^{2}$

${ }^{1}$ Department of Critical Care, Attikon University Hospital, National and Kapodistrian University of Athens, Athens, Greece; ${ }^{2}$ Department of Pharmacy, National and Kapodistrian University of Athens, Athens, Greece: ${ }^{3}$ Center for Anti-infective Research \& Development, Hartford Hospital, Hartford, United States of America

Correspondence: M.P. Almyroudi

Intensive Care Medicine Experimental 2021, 9(1): 000227

Introduction. Tigecycline is a glycylcycline, primarily bacteriostatic drug that is commonly used for infections by multidrug resistant bacteria. However, it is not approved for ventilator associated pneumonia (VAP), as increased mortality has been reported in patients with VAP treated with the conventional dose (100 mg loading dose followed by $50 \mathrm{mg}$ every $12 \mathrm{~h}$ ). (1) However improved rates of clinical cure have been observed with an off-label higher dose. (2) (3) It is speculated that inadequate dose resulting in low serum and epithelial lining fluid (ELF) levels, along with increasing Minimum Inhibitory Concentration (MIC) of the pathogens in VAP lead to failure to reach the Area Under the Curve (AUC)/MIC target, especially when pharmacokinetic (PK) features are altered, as in critically ill patients.

Objectives. The purpose of the study was to evaluate the intrapulmonary PK of off-label high tigecycline dose in critically ill patients with VAP.

Methods. Nine mechanically ventilated patients with VAP received tigecycline intravenously (200 mg loading dose followed by $100 \mathrm{mg}$ every $12 \mathrm{~h}$ ). After at least five doses, to achieve steady state, blood samples were collected at $0 \mathrm{~h}, 30 \mathrm{~min}, 1,2,4,6$ and $12 \mathrm{~h}$ after the start of infusion and at the next day at the time of the bronchoalveolar lavage (BAL). Two bronchoscopies with BAL were performed at each patient in two consecutive days at 2, 4,6 or $12 \mathrm{~h}$ after the start of infusion. Tigecyline concentrations in plasma and BAL fluid were determined by liquid chromatography and in ELF with urea-dilution method. The urea concentrations were analyzed by an enzymatic assay. Plasma and ELF concentration data were simultaneously analyzed according to the population PK approach methodology implemented in Monolix (version 2019R1. Antony, France: Lixoft SAS, 2019). The ELF:plasma penetration ratio was a parameter of the model.

Results. $56 \%$ of patients were men and the mean age, Body Mass Index and APACHE II score were $69 \pm 11.86$ years, $27 \pm 4.4 \mathrm{~kg} / \mathrm{m}^{2}$ and 21 respectively. A two-compartment PK model was found to best describe the concentration-time course of tigecycline. The estimated population mean PK parameters (relative standard errors\%) were: Clearance $11.64 \mathrm{~L} / \mathrm{h}(54 \%)$, volume of distribution in the central compartment $79.01 \mathrm{~L}(37 \%)$, volume of distribution in the peripheral compartment 92.95 L (17\%), inter-compartmental clearance $62.81 \mathrm{~L} / \mathrm{h}$ (34\%) and ELF penetration ratio $2.41(40 \%)$. The maximum concentration, minimum concentration, plasma AUC0-12, free plasma AUC0-12 and ELF AUCO12 were $1.99 \pm 1.82 \mu \mathrm{g} / \mathrm{ml}, 0.81 \pm 1.27 \mu \mathrm{g} / \mathrm{ml}, 12.89 \pm 17.25 \mu \mathrm{g}^{*} \mathrm{~h} / \mathrm{ml}$, $3.24 \pm 3.09 \mu \mathrm{g}^{*} \mathrm{~h} / \mathrm{ml}$ and $7.13 \pm 2.61 \mu \mathrm{g}^{*} \mathrm{~h} / \mathrm{ml}$ respectively.

Conclusion. In conclusion increased plasma and ELF AUCO-12 were achieved with the high tigecycline dose, while the penetration ratio into ELF indicates a sufficient intrapulmonary disposition. Based on these observations enhanced AUC/MIC ratios are expected, supporting the effectiveness of high tigecycline dose in VAP.

\section{Reference(s)}

1. 1. Antonio T Freire, Vasyl Melnyk, Min Ja Kim et al. Comparison of tigecycline with imipenem/cilastatin for the treatment of hospital-acquired pneumonia. Diagn Microbiol Infect Dis. 2010 Oct;68(2):140-51. https:// doi.org/10.1016/j.diagmicrobio.2010.05.012. 2. Gennaro De Pascale, Luca Montini, Mariano Pennisi et al. High dose tigecycline in critically ill patients with severe infections due to multidrug-resistant bacteria. Crit Care. 2014 May 5;18(3):R90. https://doi.org/10.1186/cc13858. 3. Julio Ramirez, Nathalie Dartois, Hassan Gandjini et al. Randomized phase 2 trial to evaluate the clinical efficacy of two high-dosage tigecycline regimens 
versus imipenem-cilastatin for treatment of hospital-acquired pneumonia. Antimicrob Agents Chemother. 2013 Apr;57(4):1756-62. https://doi org/10.1128/AAC.01232-12

\section{6}

Antibiotic multiresistance and immunosuppressor treatment in patients admitted to the Intensive Care Unit for Covid-19 pneumonia

C. Rodriguez Mejias'; MDM. Molina Morales'; S. Ramiro González; I. Guzman Adum

${ }^{1}$ Intensive Care Unit, Virgen de las Nieves Hospital, Granada, Spain

Correspondence: S. Ramiro González

Intensive Care Medicine Experimental 2021, 9(1): 000256

Introduction. The patient admitted to the ICU for respiratory infection by SARS-CoV 2 (COVID-19) due to its severity, prolonged hospitalization and concomitant immunosuppressive treatment, represents a risk scenario for infections by multi-resistant microorganisms.

Objectives. Describe the pattern of multiresistant microorganisms isolated in COVID-19 patients admitted to our ICU and the immunosuppressive treatment received.

Methods. Retrospective descriptive study carried out with data collected from patients admitted for COVID-19 in the period from March 13, 2020 to June 30, 2020 in the ICU of the Virgen de las Nieves Hospital in Granada, Spain. The clinical-demographic variables of the patients, severity on admission, immunosuppressive treatment, and isolated multiresistant microorganisms are described.

Results. A total of 59 patients were included, $79.66 \%$ being men, with a mean age of $60.14 \pm 11.18$ years and with APACHE II at admission of $14.14 \pm 6.31$. Corticosteroid treatment was received by $61.01 \%$ of the patients during their hospital stay, with highly variable doses and duration of treatment, with methylprednisolone and hydrocortisone being the most widely used. Other immunosuppressants were administered to $40 \%$ of the patients, the most prescribed being tozilizumab (21 patients) in a single $600 \mathrm{mg}$ dose. Only 33\% of the patients did not receive any immunosuppressive treatment.

Multiresistant isolates were detected in $16.94 \%$ of the cases, of which $85.17 \%$ developed an active infectious process. The most frequent microorganisms were extended-spectrum betalactamase-producing enterobacteria (50\%), followed by multiresistant Pseudomonas (25\%), gram negative bacilli producers carbapenemases $(21.43 \%)$ and methicillin-resistant Staphylococcus aureus (7.14\%).

Conclusion. The data obtained in patients admitted for COVID-19 pneumonia in our ICU reflect a high number of multiresistant microorganisms isolation, having received concomitant immunosuppressive treatment in most cases.

\section{6}

Three-pronged strategy for COVID-19 prevention in medical personnel and patients in a hospital setting during an outbreak T. Abe ${ }^{1}$; H. Watanabe ${ }^{2}$; H. Iriyama1; A. Komori ${ }^{1}$; T. Kainoh ${ }^{1}$; K. Inaba ${ }^{1}$; K. Narahashi ${ }^{1}$; Y. Tokuda, ${ }^{3}$

${ }^{1}$ Department of Emergency and Critical Care Medicine, Tsukuba Memorial Hospital, 1187-299 Kaname, Tsukuba, Ibaraki, Japan, Tsukuba, Japan; ${ }^{2}$ Department of Respiratory Medicine, Tsukuba Memorial Hospital,

1187-299 Kaname, Tsukuba, Ibaraki, Japan, Tsukuba, Japan; ${ }^{3}$ Department of Medicine, Muribushi Project for Okinawa Residency Programs, Naha,

Okinawa, Japan, Japan

Correspondence: T. Abe

Intensive Care Medicine Experimental 2021, 9(1): 000266
Introduction. A clustered COVID-19 outbreak associated with medical personnel occurred in a hospital in Japan in February 2021. COVID-19 vaccination programs had not been initiated in the hospital before this outbreak. Thus, it was urgent to formulate a new strategy for epidemic prevention to achieve zero tolerance for in-hospital transmission.

Objectives. This study aims to evaluate whether the COVID-19 vaccination, in addition to conventional preventive measures against the spread of infection, is effective and safe as a preventive measure in a hospital during a nosocomial COVID-19 outbreak.

Methods. This was a prospective observational study examining the effectiveness and safety of the new COVID-19 prevention strategy. It was conducted form March 8, 2021 to May 8, 2021 in the Tsukuba Memorial Hospital, a care mix community hospital with 487 beds located in the middle region of Japan. First, conventional infection prevention and control measures (e.g., contact tracing, quarantine of exposed persons, and isolation of persons with suspected COVID-19) were conducted. Second, medical personnel in the hospital underwent a universal polymerase chain reaction (PCR) screening for COVID19 before starting the COVID-19 vaccination. Then, they received the COVID-19 vaccine as a new preventive measure for the COVID-19 outbreak. The primary endpoint was adverse reactions of vaccination. Secondary endpoints were adverse events, safety endpoints, and successful containment of the COVID-19 infection (UMIN000043534).

Results. This study included 1,130 medical personnel and staff, including physicians, nurses, and office workers. There were 26 medical personnel and 34 patients who had outbreak-related COVID-19 in the hospital by March 8, 2021. Medical personnel and staff were confirmed to have negative PCR tests of COVID-19 before receiving vaccinations. No severe adverse reactions or events related to both the first and second vaccinations were noted. Those with mild adverse reactions (fever [>37.4], 33 [3\%]; fatigue, 323 [29\%]; headache, 213 [19\%]; chills, 76 [7\%]; and muscle pain, 541 [49\%]) were noted in the first vaccination. Two nurses were diagnosed with COVID-19 after the vaccination during 1 month observation period. No patient had a nosocomial COVID19 infection in the hospital during 1 month after the vaccination but one patient were diagnosed with COVID-19 after his discharge.

Conclusion. The three pillars of strategies were (1) conventional infection prevention and control measures, such as case identification and contact tracing; (2) universal screening tests; and (3) rapid introduction of vaccinations, which could be performed safely and effectively during the nosocomial COVID-19 outbreaks.

000273

Changes in migobiology findings from blood cultures during the past 15 years in ICU patients

A. Vakalos ${ }^{1}$; G. Papageorgiou ${ }^{1}$

${ }^{1}$ Icu, Xanthi General Hospital, Xanthi, Greece

Correspondence: A. Vakalos

Intensive Care Medicine Experimental 2021, 9(1): 000273

Introduction. Knowledge of the bacterial flora of an ICU department is indicated to the rational formulation of infection control policy.

Objectives. The aim of our retrospective observational study was to record the percentage of positive blood cultures per pathogenic micro-organism per year and overall, in mixed ICU served in community hospital.

Methods. From January 2016 to September 2020, 1454 patients admitted to our ICU and included to the study. Mean age 66.6 years, length of stay 11.5 days, APACHE II score 21.7, standardized mortality ratio: 0.73 . Using the department's database we looked for the six main pathogenic micro-organisms isolated in blood cultures as a percentage of all positive blood cultures, per year and overall. 
Results.

\begin{tabular}{lrlllll}
\hline$\%$ & AC. B & PS. A & KI.P & Pr. M & En. F & St. A \\
\hline 2006 & 35.0 & - & 15.0 & - & 5.0 & 5.0 \\
2007 & 37.9 & 13.8 & 3.4 & - & 6.9 & - \\
2008 & 4.0 & 4.0 & 16.0 & - & - & 4.0 \\
2009 & 28.8 & 21.2 & 15.4 & - & 1.9 & 5.8 \\
2010 & 27.3 & 18.2 & 20.8 & 1.3 & 3.9 & - \\
2011 & 31.3 & 8.3 & 22.9 & 2.1 & 4.2 & - \\
2012 & 40.0 & 18.8 & 2.4 & 8.2 & 4.7 & - \\
2013 & 38.2 & 5.5 & 10.9 & - & 1.8 & 1.8 \\
2014 & 22.9 & 8.4 & 8.4 & 1.2 & 2.4 & 2.4 \\
2015 & 26.7 & 13.3 & 4.4 & 8.9 & 2.2 & - \\
2016 & 12.9 & 22.6 & 3.2 & - & 3.2 & 3.2 \\
2017 & 12.2 & 9.1 & 4.5 & 4.5 & - & 13.6 \\
2018 & 5.4 & 27 & 2.7 & 8.1 & 5.4 & - \\
2019 & 14.3 & 35.7 & 7.1 & - & - & - \\
2020 & 25.0 & 12.5 & 4.2 & 4.2 & 4.2 & - \\
SUM & 26.6 & 14.8 & 10.0 & 2.9 & 4.2 & 1.8 \\
\hline
\end{tabular}

Conclusion. According to our data, Gram negative microorganisms are the predominant flora isolated from blood cultures, dominated by Acinetobacter baumanni. In order to evaluate the difference over time, we need more data according to the incidence of infection, the variability of the length of stay and the variability of the percentage of the post anesthesia care patients.

\section{9}

Diagnostic accuracy of Bio Fire Film Array Pneumonia Panel for detection of viral and bacteria pathogens in lower respiratory specimens

AC. Dinis' ${ }^{1}$; S. Narciso ${ }^{1}$; C. Nuno ${ }^{1}$; T. Oliveira ${ }^{1}$; L. Caley ${ }^{1}$; T. Pereira ${ }^{1}$; AM. Neto Real $^{1}$

Intensive Medicine, Centro hospitalar médio Tejo, Abrantes, Portugal

\section{Correspondence: A.C. Dinis}

Intensive Care Medicine Experimental 2021, 9(1): 000299

Introduction. Pneumonia is one of the most common cause of hospitalization all over the world. (1)

For bacterial pneumonia delay in effective antimicrobial therapy is associated with increased mortality and length of hospital stay. $(2,3)$ However the extensive misuse of antibiotics has led to a worrisome rise in the numbers of antibiotic-resistent infections. (4)

Rapid diagnostic testing for pneumonia has the presume potential to guide clinical decisions in reducing the use of broad-spectrum antibiotics. These benefits should be dependent upon the accuracy of these test. $(5,6)$

Objectives. In order to evaluate the diagnostic yield and accuracy of the BioFire FilmArray pneumonia panel (BFPP) for pathogens identification in lower respiratory tract specimens, we conduct this retrospective study in a intensive care unit (ICU).

Methods. In this study were included all patients $(n=104)$ admitted in our ICU with pneumonia who underwent BFPP test, between December 2019 and March 2021.

Lower respiratory tract samples were collected and analysed simultaneously with BFPP and microbiological culture, as part of standard-ofcare testing.

The BFPP panel includes for 18 bacteria and 8 virus, that commonly cause pneumonia as well 7 antibiotic resistance genes, identified from tracheal aspirate or bronchial alveolar lavage (BAL) specimens.

Results. This study included 104 patients, with an average age of 62 years. We present the demografhic and clinical characteristics in chart 1.

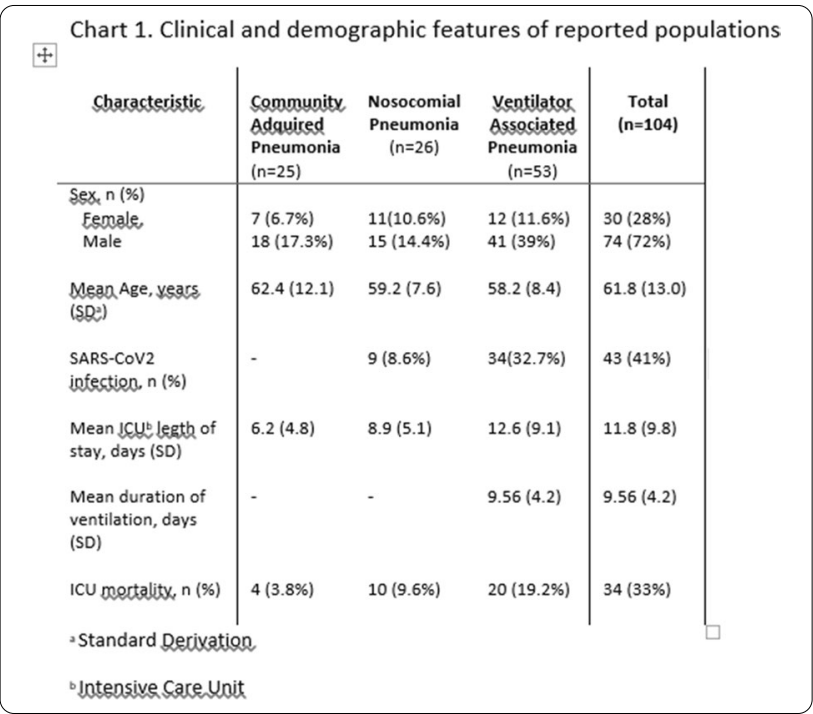

The BFPP was positive in $111 / 125$ (88\%) samples. The most common bacteria detected were Staphylococcus aureus (19\%), Klebsiella pneumonia (14\%) and Haemophilus influenza (10\%).

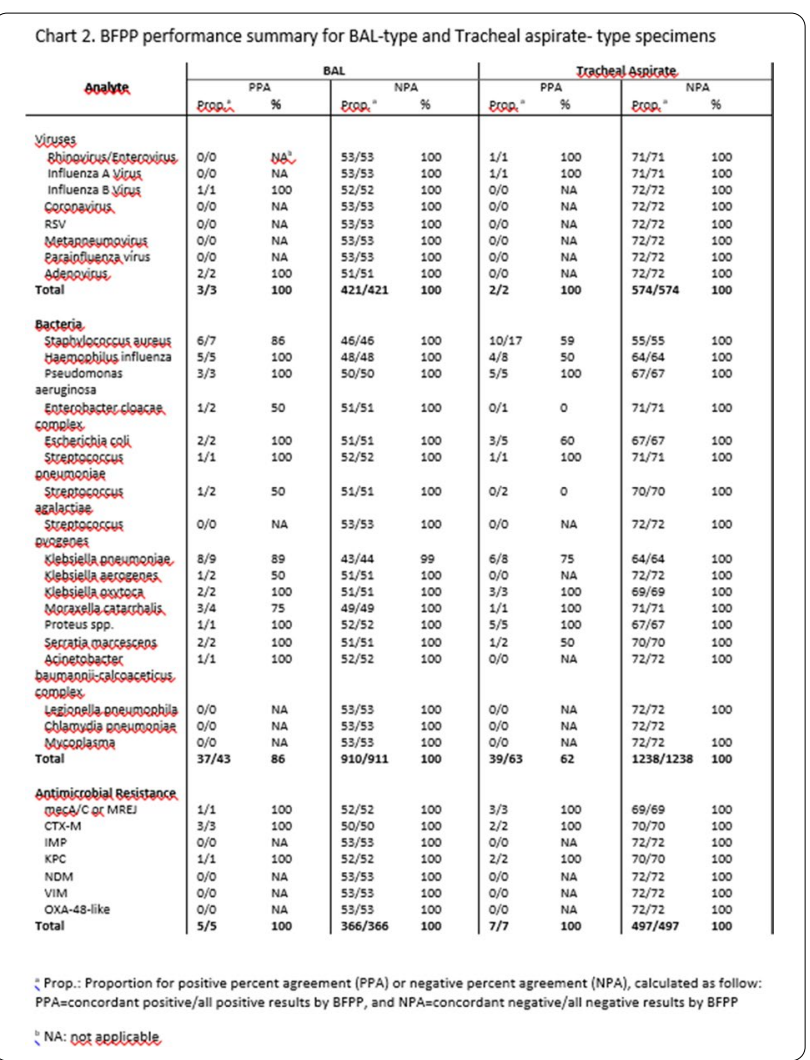

Overall, there was strong correlation between BFPP and culture for detection of bacteria. In fact, most bacteria (76/106 [72\%]) detected by BFPP were also identified in culture.

The remaining samples were not validated as positive due to their laboratory cut-off value. 
In other hand, it is relevant that only one negative BFPP had an isolated agent in the cultural exam.

Conclusion. Real-time specimen analysis with BFPP has the potential to identify bacterial and viral pathogens and theirs resistance markers, faster than traditional culture-based methods.

In fact, the BFPP is a rapid and accurate method for detection of pathogens from lower respiratory tract infections with a $72 \%$ sensitivity and $100 \%$ specificity, in our studied population.

\section{Reference(s)}

1. 6. BioFire Diagnostics.2019. FilmArray pneumonia panel instructions for use

2. 5. Collins ME, Popowitch EB, Miller MB. 2020. Evaluation of a novel multiplex PCR panel compared to quantitative bacterialculture for the diagnosis of lower respiratory tract infections.J Clin Microbiol58:e02013-19.

3. 4. O’Neill J. 2016.Tackling drug-resistant infections globally: final reportand recommendations. Review on Antimicrobial Resistance, London, United Kingdom

4. 3. Meehan TP, Fine MJ, Krumholz HM, Scinto JD, Galusha DH, Mockalis JT, et al. 2007. Quality of care,process and outcomes in patients wih pneumonia. JAMA 278:2080-2084

5. 2. Kuti EL, Patel AA, Coleman Cl. 2008. Impacto f inappropriate antibiotic therapy on mortality in patients with ventilator-associated pneumonia and blood stream infection:a meta-analysis. J Crit Care 23:91-100.

6. 1. WHO. 2019. World health statistics 2019: monitoring health for the SDGs, sustainable development goals. World Health Organization, GEneva, Switzerland.

\section{2}

\section{Colonization by multidrug resistant pathogens in Intensive Care} Unit patients during COVID-19 pandemic

ML. Cantón Bulnes '; J. Garnacho Montero

${ }^{1}$ Intensive Care, Hospital Universitario Virgen Macarena, Sevilla, Spain

Correspondence: M.L. Cantón Bulnes

Intensive Care Medicine Experimental 2021, 9(1): 000362

Introduction. The COVID-19 pandemic has increased the care burden and has necessitated changes in workflows in intensive care units (ICU). These factors can contribute to a decreased adherence to infection prevention and control measures and, combined with a high antimicrobial selection pressure, may facilitate the emergence of multidrug resistant bacteria (MDRB). Despite all the difficulties, in our unit we continue with the active surveillance programmes for identification MDRB, as we were doing before the start of the pandemic.

Objectives. The aim of this work is to study the impact of COVID-19 on MDRB colonization in patients admitted to ICU in a tertiary hospital.

Methods. We conducted a prospective observational study carried out in a polyvalent ICU, using the ENVIN (National Nosocomial Infection Surveillance Study) registry. All patients admitted between March 14st 2020 (first patient COVID admitted to ICU) and December 31st 2020 were included. We perform nasal, axillary, tracheal aspirate if the patient was intubated and rectal surveillance cultures upon ICU admission and weekly thereafter, according to the definition provided in "Resistencia Zero" program. Colonization by MRSA (Methicilin resistant Staphylococcus aureus), ESBL (Extended spectrum betalactamase Enterobacteriaceae), CRE (Carbapenem resistant Enterobacteriaceae), multiresistant Pseudomona, multiresistant Acinetobacter baumannii and VRE (vancomycin resistant Enterococcus) is indicated at the room door and requires health care workers to wear gloves and gown before entry.

We compared the rate of MDRB colonization of COVID-19 patients with patients admitted for others pathologies.

Demographic data and severity scores were collected at ICU admission. We collected the following data during the exposure time: antimicrobial use, presence of central venous catheter, urinary tract catheter and mechanical ventilation.

Statistical analysis: categorical variables are reported as count (\%), continuous data as median [interquartile range]. We compared patients cohort using Student's T-test, Mann-Whitney test, $x^{2}$ test or Fisher's exact test, as appropriate.
Results. We identified 695 patients admitted to ICU during the study period. COVID-19 was diagnosed in 121 out of 695 patients. Table 1 shows demographic characteristics and risk factors for infections. The incidence rate of MDRB colonization at admission was $1.6 / 100$ patients COVID-19 and 4.7/100 patients no-COVID 19 and during ICU stay was $8.3 / 100$ patients COVID-19 and $1.2 / 100$ patients no-COVID 19. The isolated MDRB that were mostly BLEE Enterobacteriaceae. No VRE were isolated. Table 2 shows the incidence rate of colonization per microorganism.

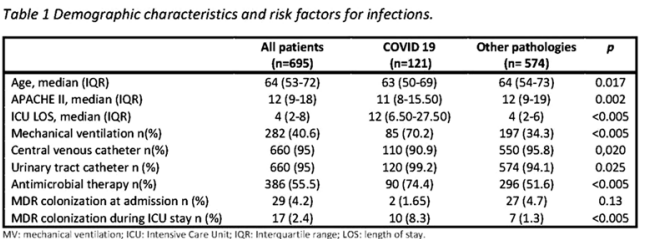

Table 2 incidence rate of colonization per microorganism.

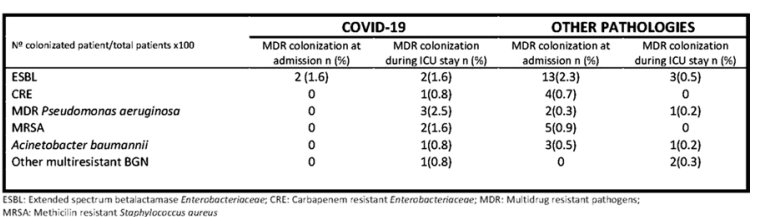

Conclusion. The rate of MDRB acquisition during ICU stay was higher in patients with COVID-19 than in other admissions during the same time period although both groups of patients are imbalanced regarding severity of illness, need of mechanical ventilation and length of stay.

\section{Reference(s)}

1. 3. Brusselaers $N$, Vogelaers $D$, Blot $\mathrm{S}$ : The rising problem of antimicrobial resistance in the intensive care unit. Ann Intensive Care 2011, 1:47.

2. 2. Rawson TM, Moore LSP, Castro-Sanchez E, Charani E, Davies F, Satta G, Ellington MJ, Holmes AH: COVID-19 and the potential long-term impact on antimicrobial resistance. J Antimicrob Chemother 2020

3. 1. Montero JG, Lerma FÁ, Galleymore PR, Palomar Martínez M, Rocha LÁ, Gaite FB, et al. Combatting resistance in intensive care: the multimodal approach of the Spanish ICU Zero Resistance program. Crit Care 2015; 19 (1): 114

\section{9}

Healthcare-associated infections in COVID-19 patients in the Intensive Care Unit: Description of early and late onset infections, utility of blood tests and empirical antimicrobial therapy on admission, and influence on length of stay and mortality

D. Pérez-Torres ${ }^{1}$; C. Cuenca-Rubio ${ }^{1}$; C. Díaz-Rodríguez ${ }^{1}$; I. Canas-Pérez ${ }^{1}$. V. Fraile-Gutiérrez'; A. Martín-Luengo'; P. Blanco-Schweizer'; PM.

Enríquez-Giraudo'; LM. Tamayo-Lomas'; JÁ. Berezo-García

${ }^{1}$ Servicio de medicina intensiva, Hospital Universitario Río Hortega, Val-

ladolid, Spain

Correspondence: D. Pérez-Torres

Intensive Care Medicine Experimental 2021, 9(1): 000369

Introduction. Healthcare-associated infections (HAI) represent one of the most relevant complications of critically ill COVID-19 patients. Knowledge of early and late onset microbiology of these HAls allows the clinician to prescribe antibiotics more judiciously. The utility of blood tests to predict the development of $\mathrm{HAl}$ and the administration of a severe-CAP-like empirical antimicrobial therapy (EAT) course to prevent the development of HAI remain controversial.

Methods. We conducted a retrospective observational single-centre study in the ICU of a University Hospital in Spain, over 3 months 
(first epidemic wave of COVID-19 in the country). All the patients who were admitted to the ICU with PCR-confirmed COVID-19 were included. Demographics, first blood tests and HAI during ICU stay were retrieved from the electronic medical record. Data are expressed as mean $\pm S D$, median (IQR) or frequency (\%). Chi-square, Student's T and Mann-Whitney U tests were applied as appropriate. Results. We included 87 patients, $77 \%$ male, aged $60 \pm 12$, APACHEII 16 (13-16), mechanical ventilation 93\%, ICU stay 20 (9-36) days, hospital mortality $37 \%$ ( $31 \%$ in patients with HAI vs $51 \%$ in patients without HAl, $p=0.06$; time-to-event 24 [14-40] vs 10 [6-17], $\mathrm{p}<0.01)$.

During the first week of ICU stay, 32 episodes of HAI occurred in 25 patients (29\%), caused by Gram-positive microorganisms (63\%), fungi (19\%), Gram-negative microorganisms (12\%) and virus (6\%). The microorganisms responsible for these HAls are presented below:

- Bloodstream infection (BSI) (15): S. epidermidis (5), E. faecium (3), E. faecalis (1), Staphylococcus spp (1), Streptococcus spp (1), and cytomegalovirus (1).

- Ventilator-associated pneumonia (VAP) (14): MSSA (3), E. faecalis (2), MDR P. aeruginosa (2), MDR A. baumanii (2), Aspergillus (2), Streptococcus spp (1), E. faecium (1), and cytomegalovirus (1).

- Urinary-tract infection (UTI) (2): E. faecalis (1) and Candida spp (1).

- Catheter-related BSI (CRBSI) (1): Candida spp (1).

Multifocal colonization by Candida spp occurred in $31 \%$ of patients within the first week.

Results of first blood tests were compared in patients who developed HAl vs those who did not: leukocyte count $8(5-14)$ vs $7(6-9) \times 10^{3} / \mu \mathrm{L}$, $\mathrm{p}=0.92$; lymphocyte count $0.7(0.3-1)$ vs $0.7(0.5-1) \times 10^{3} / \mu \mathrm{L}, \mathrm{p}=0.58$; $C$ reactive protein $123(63-299)$ vs $114(58-173) \mathrm{mg} / \mathrm{L}, \mathrm{p}=0.41$; procalcitonin $0.2(0.1-0.4)$ vs $0.2(0.1-0.3) \mathrm{ng} / \mathrm{mL}, \mathrm{p}=0.73$.

Administration of EAT had the potential to cover $12.5 \%$ of HAls within the first week in ICU.

During the whole ICU stay, 200 episodes of HAl occurred in 59 patients (67\%):

- 65 BSI (40 bacteraemia of unknown origin [BUO], 19 catheterrelated BSI, 6 secondary BSI): S. epidermidis (28\%), E. faecalis (17\%), E. faecium (17\%), Candida spp (15\%), S. aureus (5\%, of which 33\% MRSA), Staphylococcus spp (5\%), MDR A. baumanii (3\%), cytomegalovirus (3\%), other (7\%).

- 59 respiratory-tract infections (36 VAP and 23 VA tracheobronchitis[VAT]): P. aeruginosa (24\%, of which 35\% MDR), MDR A. baumanii (19\%), S. aureus (15\%, of which $11 \%$ MRSA), E. faecalis (7\%), S. epidermidis (5\%). S. maltophilia (5\%), Aspergillus spp (3\%), E. cloacae (3\%), E. faecium (3\%), K. pneumonia $(3 \%)$ and other (13\%).

- 36 multifocal fungal colonization: C. albicans (86\%) and other $(14 \%)$.

- 21 CRI without bacteraemia: S. epidermidis (29\%, of which $17 \%$ linezolid-resistant), Candida spp (29\%), E. faecalis (15\%) and other (27\%).

- 11 catheter-associated UTI (CAUTI): E. faecalis (27\%), E. faecium (18\%), Candida spp (18\%), MDR A. baumanii (18\%) and other (19\%).

- 8 episodes of other source infection.

Length of stay, expressed in days, was compared in patients who developed HAI vs those who did not: $35 \pm 24$ vs $9 \pm 7, p<0.01$. By source of infection: BUO $46 \pm 30$ vs $18 \pm 14, p<0.01$; CRBSI $43 \pm 26$ vs $23 \pm 21, p<0.01$; VAP $43 \pm 31$ vs $20 \pm 17, p<0.01$; VAT $54 \pm 34$ vs $19 \pm 15, p<0.01$; and CAUTI $42 \pm 27$ vs $24 \pm 22, p=0.04$.

Conclusion. Microbiology of early and late onset HAls in COVID-19 patients differ from that of other critically ill patients. Blood tests and EAT on admission are not useful to predict nor prevent HAls.
000443

Analysis of nosocomial infections in patients admitted to the ICU during the COVID-19 era

R. Fernández Fernández'; P. Fernandez Morales²; ME. Yuste Ossorio ${ }^{3}$ ${ }^{1}$ \{street_address\}, Granada, Spain; ${ }^{2}$ Intensive Care Unit, Hospital Universitario Clínico San Cecilio, Granada, Spain; ${ }^{3}$ Intensive Care Unit, Hospital Universitario San Cecilio, Granada, Spain

Correspondence: R. Fernández Fernández

Intensive Care Medicine Experimental 2021, 9(1): 000443

Introduction. Now just a year ago our units began to admit patients with respiratory failure in the context of the SARS-CoV-2 pandemic. In a short period of time they had to expand the number of critical beds and adapt human resources to this new situation. The own conditions of COVID-19 patients and the enormous healthcare burden that had to be faced during the first months of the pandemic affected and changed the usual work dynamics in the units. The information contained in this work is the result of an important effort made during a period of time in which ICUs have continued to be under high pressure care, but with out a doubt it has been an effort that has been worthwhile because it provides us with data important information on the impact of HAls in this group of patients, and highlights the need to recover prevention programs (Project Zero) as soon as possible, as well as the strategies to adapt them to the situation generated by the pandemic.

Objectives. To analyze nosocomial infections presented by patients admitted in the first and second period with the highest prevalence of COVID-19 and to study the differences between them.

Methods. Retrospective descriptive observational study. Data from ICU admissions (cardiological, medical: multipurpose, COVID, trauma)from 03-01-20 to 06-30-20 and from 09-01-20 to 12-31-20, analyzing density of incidence (DI): ventilator-associated pneumonia (VAP), bacteraemiacatheter-associated (BAC) and urinary tract infections related to bladder catheter(ITUSV). Work teams have been formed mainly by intensivists (14 adjuncts and 5 residents) and anesthetists, presenting a ratio doctor/patient 1/11. Patients have been treated in our facilities (coronary, COVID) and in Resuscitation (general and COVID). The nursing team and auxiliary personnel in first wave were ICU and Anesthesia and Resuscitation personnel with experience in critical patients and familiar with Zero programs. In the second wave was ICU staff and staff with little experience in patients Critics and Zero Programs.The data has been taken from ENVIN-HELICS. Demographic variables are collected, APACHE II, days of ICU stay and mortality.

Results. Flrst period: NAVM: incidence density: 2.81/2: 7.01, BAC: 2.96/5.73, ITUSV: 7.35/8.05.

First period: APACHE $13.97+-7.90$; days stay $7.19+-10.92$, mortality $11.33 \%$.

Medical: N 187 46.63\%, Surgical: N 55 13.72\%, Traumatological: N 13 $3.24 \%$, Coronary: N $14636.41 \%$.

Second period: APACHE $13.74+-8.90$; days stay $7.08+-9.38$, mortality $15.65 \%$.

Medical 254 51.94\%, Surgical 47 9.61\%, Traumatological 9 1.84\%, Coronary $17936.61 \%$.

Conclusion. The most important data is that in a pandemic situation, nosocomial infections far exceed the incidence that we have previously presented, where we had quite satisfactory data (in all of 2019 the DI of BAC was 1.5, ITUSV 2.28, NAMV 3, 6 with APACHE II 12.68 , days of stay 4.56 , mortality $9.13 \%$ ). DI was higher in all infections studied in the second period, possibly due to the inexperienced nursing staff in both the Zero programs and ICU work. Perhaps it would be interesting to carry out a training similar to the one that was implemented at the beginning of our Zero programs.

\section{7}

Study of multidrug resistance and antibiotic therapy used in the ICU between the first and second period with the highest prevalence of COVID-19

R. Fernández Fernández'; P. Fernandez Morales²; ME. Yuste Ossorio ${ }^{3}$

${ }^{1}$ \{street_address\}, Granada, Spain; ${ }^{2}$ Intensive care unit, Hospital Universitario Clínico San Cecilio, Granada, Spain; ${ }^{3}$ Intensive care unit, Hospital Universitario San Cecilio, Granada, Spain

Correspondence: R. Fernández Fernández

Intensive Care Medicine Experimental 2021, 9(1): 000447 
Introduction. Now just a year ago our units began to admit patients withrespiratory failure in the context of the SARS-CoV-2 pandemic. In ashort period of time they had to expand the number of critical beds and adapt thehuman resources to this new situation. The own conditions of COVID-19 patients andthe enormous healthcare burden that had to be faced during the first months of thepandemics affected and changed the usual work dynamics in the units. Presumably this has affected the flora of our units and therefore the antimicrobials used

Objectives. Analyze the differences between the first and second period of COVID-19 regarding multi-resistant germs isolated in our unit andantibiotic therapy used.

Methods. Retrospective descriptive observational study. The data has been collected from all patients admitted to the ICU (medical patients:polyvalent and COVID, surgical, coronary and trauma) from $1-03-20$ to $06-30-20$ and from $09-01-20$ to $12-31-20$. N 890. The data has been extracted from the registry ENVIN- HELICS.Total patients with antibiotics: 217 , Total antibiotics used: 427

Results. Descriptive profile of our patients:

First period: APACHE $13.97+-7.90$; days stay $7.19+-10.92$, mortality $11.33 \%$.

Second period: APACHE $13.74+-8.90$; days stay $7.08+-9.38$, mortality $15.65 \%$

The most used antibiotics in the first period have been: Meropenem 18.11\%/6.38 days, Linezolid 14.96\%/5.21 days, Ciprofloxacin $7.09 \% / 7$ days an dAmikacin 6.3\%/3.75 days. This is directly related to germs most frequently isolated: multiresistant $\mathrm{GNBN}=3$, (Previous 2/ During income 1) Extended Spectrum Betalactamase $N=11$, (Previous 5/During 6) Metallobetalactamases (VIM, KPC, etc.) $\mathrm{N}=1$ (Previous 0/ During 1), PseudomonasMulti-resistant $\mathrm{N}=3$ (Previous 1/During 2), MRSA N $=3$ (Previous 2/During 1).

Second: Meropemen $14.29 \% 5.76$ days, Linezolid 13.58\% 5.69 days, Daptomycin $10.77 \% \quad 6.27$ days, Piperacillin-tazobactam $6.79 \%$ 5.42 days, Levofloxacin $6.09 \% 7.82$ days, Ciprofloxacin $5.39 \%$ 7.22 days, Cotrimoxazole $4.22 \% 8.94$ days, Ceftazidime-avibactam $3.51 \% 6.20$ days. Germs: BGNMultiresistant $\mathrm{N}=4$ (Previous 1/During 3), Extended Spectrum Betalactamase $N=9$, (Previous 2/During 7), Metallobetalactamases (VIM, KPC, etc.) $\mathrm{N}=9$ (Previous 0/During 9), Multiresistant Pseudomonas $\mathrm{N}=1$ (Previous 0 / During 1), MRSA N $=1$ (Previous 1 / During 0)

Conclusion. The multidrug resistance profile is very similar, as is the treatment antibiotics, the most widely used have been meropenem and linezolid, with a durationless than 1 week. In the second period, more quantity of antibiotics, keeping them less than 7 days. Antibiotic therapy is fineoriented taking into account the flora of our unit, the duration has alsobeen adequate, in no case have we exceeded 1 week in duration. The ESBLand GNB, have been isolated prior to admission to the ICU, which indicates that theZero Resistance program can help us.

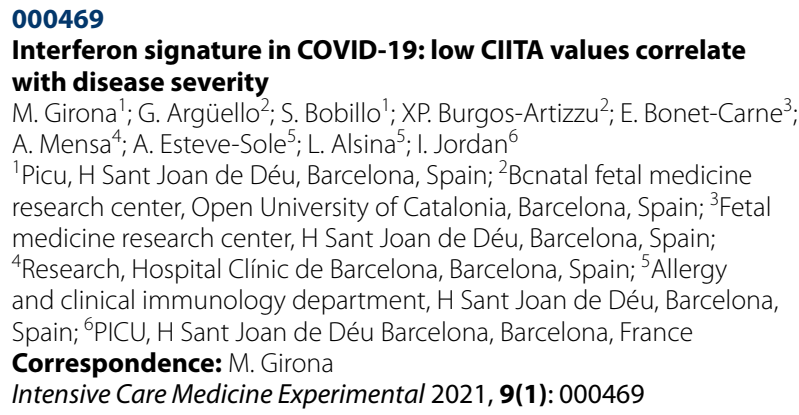

Introduction. The novel severe acute respiratory syndrome coronavirus 2 (SARS-CoV-2) has caused a pandemic with 130,459,184 cases and 2,842,325 deaths. It is unclear why SARS-CoV-2 affects differently depending on the age of the patient, and why the affection ranges from asymptomatic patients to severe disease and even death. When a viral infection is detected by the innate immune sensors, Interferon (IFN) response is activated, and it seems that patients with COVID-19 might have a low or delayed IFN response.

Objectives. The main aim of the study was to analyse the acute IFN response in children and adults infected with SARS-CoV-2, so as to identify differences between them. Secondary objectives were to compare the immune responses depending on the severity of the disease: asymptomatic/mild symptoms, moderate disease, and severe disease.

Methods. Prospective and observational study, performed in a paediatric tertiary hospital. A sample of adults and children with asymptomatic, mild, moderate (admission to hospital), and severe disease (admission to intensive care unit), of which it was possible to perform IFN signature transcriptomic analysis on admission were included. For the patients, $z$-score measure for the 28 genes that define the IFN signature used for interpheronopathies diagnosis plus 4 other genes (CIITA, CXCL9, IFNA2, STAT1) were analysed.

Results. In total, 72 patients were included: 38 (52.7\%) children and $34(47.3 \%)$ adults. Of them, $18(25 \%)$ were asymptomatic, $16(22.2 \%)$ had mild disease, $10(13.9 \%)$ moderate disease, and 28 (38.9\%) severe disease.

All IFN genes showed a positive correlation, except for CIITA. Therefore, two genes were selected because they better correlated with the disease severity: SOCS1 (representative of IFN genes) and CIITA (inverse correlation), and a decision tree for severity classification was created. Low RNA levels of CIITA and high values of SOCS1 were indicative of severe disease.

In children, the decision tree classified properly $33 / 38$ (86.8\%) observations. Patients with severe disease showed lower CIITA $(-1.77$ vs. -0.29 , $p<0.001)$ and higher SOCS1 values ( 3.28 vs. $-0.36, p=0.002$ ).

In adults, the tree classified properly $27 / 34$ observations (79\%). Patients with severe disease showed lower CIITA ( -2.47 vs. -1.29 , $p=0.006)$ and higher SOCS1 values ( 1.28 vs. $-1.13, p=0.021)$.

Moreover, CIITA values were significantly lower in adults than in children $(-1.76$ vs. $-1.36, p=<0.037)$, and SOCS1 values tended to be lower in adults than in children $(-0.54$ vs. $0.16, p=0.174)$, although not researching statistical significance.

Conclusion. This is the first study that compares the IFN signature in children and in adults with COVID-19. IFN response is different in children and adults; what is more, it is related to the severity of COVID-19 disease. Particularly, CIITA shows a unique pattern: low values of CIITA correlate with more severe disease and adults have lower CIITA values than children.

\section{Reference(s)}

1. Bastard P, Rosen LB, Zhang Q, Michailidis E, Hoffmann H, Zhang Y, et al. Auto-antibodies against type I IFNs in patients with life-threatening COVID-19. Science (80-) [Internet]. 2020 Oct 23;370(6515):eabd4585. Available from: https://www.sciencemag.org/lookup/doi/10.1126/scien ce.abd4585

2. Zhang Q, Bastard P, Liu Z, Le Pen J, Moncada-Velez M, Chen J, et al. Inborn errors of type I IFN immunity in patients with life-threatening COVID-19. Science (80-) [Internet]. 2020 Oct 23;370(6515):eabd4570. Available from: https://www.sciencemag.org/lookup/doi/10.1126/science.abd4570

3. Hadjadj J, Yatim N, Barnabei L, Corneau A, Boussier J, Smith N, et al. Impaired type I interferon activity and inflammatory responses in severe COVID-19 patients. Science (80-) [Internet]. 2020 Aug 7;369(6504):718-24. Available from: https://www.sciencemag.org/lookup/doi/10.1126/scien ce.abc6027

4. Park A, Iwasaki A. Type I and Type III Interferons - Induction, Signaling, Evasion, and Application to Combat COVID-19. Cell Host Microbe [Internet]. 2020 Jun;27(6):870-8. Available from: https://doi.org/10.1016/j. eclinm.2020.100527

5. Ivashkiv LB, Donlin LT. Regulation of type I interferon responses. Nat Rev Immunol [Internet]. 2014 Jan 23;14(1):36-49. Available from: http://dx.doi. org/10.1038/nri3581 
6. Anastassopoulou C, Gkizarioti Z, Patrinos GP, Tsakris A. Human genetic factors associated with susceptibility to SARS-CoV-2 infection and COVID19 disease severity. Hum Genomics [Internet]. 2020 Dec 22;14(1):40. Available from: https://humgenomics.biomedcentral.com/articles/10. 1186/s40246-020-00290-4

7. Ellinghaus D, Degenhardt F, Bujanda L, Buti M, Albillos A, Invernizzi P, et al. Genomewide Association Study of Severe Covid-19 with Respiratory Failure. N Engl J Med [Internet]. 2020 Oct 15;383(16):1522-34. Available from: http://www.nejm.org/doi/10.1056/NEJMoa2020283

\section{4}

Role of determining percentage of deoxyribonucleic acid in biofilms amongst severe respiratory infections

V. Ziamko

${ }^{1}$ Viciebsk State Order of Peoples' Friendship Medical University, Viciebsk, Belarus

Correspondence: $\vee$. Ziamko

Intensive Care Medicine Experimental 2021, 9(1): 000474

Introduction. It has been known about possibility of microorganisms to create specific multi-layered structures called biofilms. Non-cellular deoxyribonucleic acid actively participates in regulation of properties of biofilms. Thus, in biofilms transfer of genetic information including genes responsible for sensitivity to antibacterial drugs occurs much more often than in single-living bacterial cells. However, despite the involvement of extracellular deoxyribonucleic acid in adhesive processes and intercellular interactions, its role has not been fully understood.

Objectives. To analyse if there is correlation between severety of bacterial infection biofilm formation and percentage of deoxyribonucleic acid in microbial community.

Methods. 238 isolates isolated from sputum and pharynx of 175 patients during 2016-2019 were studied. Patients were divided into two groups: the 1 st group of 139 people $(79,4 \%)$ had severe respiratory infections, the 2 nd of 36 people $(20,6 \%)$-respiratory infections of moderate severity.

Results. A method was developed for determining percentage of deoxyribonucleic acid in microbial community using $4^{\prime} 6$-diamidino2-phenylindole dihydrochloride. Average age of the 1st group was higher than the second $(p<0,05)$. Pseudomonas aeruginosa had the largest mass of biofilm and percentage of deoxyribonucleic acid in group $1,48,25[30,5-70,1] \mathrm{mcg} / \mathrm{ml}$ and $5,21[2,17-7,67] \%, p=0,04$. A strong relationship was found between percentage of deoxyribonucleic acid in Pseudomonas aeruginosa and severity of disease, $r=0,73$, $p<0,05$. The incidence of adverse outcomes in isolating antibiotic resistant isolates was higher than in antibiotic sensitive $(p<0,05)$. Analysis of results made it possible to propose fatal outcome when mass of microbial biofilm is $>47,5 \mathrm{mcg} /$ well and percentage of deoxyribonucleic acid is $>2,33 \%(p<0,01)$.

Conclusion. Method for determining percentage of deoxyribonucleic acid in biofilm has been proposed. With age there is a decrease in immune system which contributes to adherence of more pathogenic, antibiotic resistant microflora which has high biofilm weight and deoxyribonucleic acid percentage leading to disease progression and death.

\section{Reference(s)}

1. Microbiota diversity and gene expression dynamics in human oral biofilms / A. Benitez-Paez [et al.] // BMC Genomics. 2014. Vol. 15. P. 311-323.

2. Fernández-Barat, L. Biofilms in ventilator-associated pneumonia/L. Fernández-Barat, A. Torres // Future Microbiology. 2016. Vol. 11. P. 1599-1610
000482

Determination of the characteristics of invasive fungal infection in patients admitted for SARS-CoV-2 pneumonia in an Intensive Care Unit

V. Martínez de Pinillos Sánchez ${ }^{1}$; I. De Dios Chacón ${ }^{\text {; D. Darcía Huertas }}{ }^{1}$

${ }^{1}$ Intensive Care Unit, University Hospital Complex of Granada, Granada, Spain

Correspondence: $\vee$. Martínez de Pinillos Sánchez

Intensive Care Medicine Experimental 2021, 9(1): 000482

Introduction. The SARS-CoV2 pandemic has motivated a change in the profile of the patient admitted to the Intensive Care Units during 2020. The intensivists have faced, not only severe acute respiratory failure/adult respiratory distress syndrome and shock but to a series of complications that have been more frequent in patients with severe SARS-CoV2 pneumonia, such as the appearance of thrombotic phenomena. In addition, the physical barrier posed by individual protection equipment, and the constant appearance of information about this emerging pathology, has made us combat and try to prevent, perhaps more aggressively, the infections that appeared and that have motivated in many cases the worsening of patients and even their death. The conjunction in these patients of high severity and complexity, the more frequent use of broad-spectrum antimicrobials, and the addition of immunosuppressive drugs in the treatment (apart from other factors intrinsically present in ICU patients, such as venous catheters or parenteral nutrition), may have led to a high incidence of fungal infection in these patients.

Objectives. The main objective of the study is to determine the frequency of invasive fungal infection (IFI) in patients admitted to our unit with SARS-CoV2 pneumonia. IFI was defined as the appearance of invasive candidiasis (candidemia or infection with multiple organ involvement), and invasive pulmonary aspergillosis (IPA). In addition, the relationship of IFI with the presence of multi-resistant microorganisms (MROs), with the number of broad-spectrum antimicrobials used, and with the severity of the patient in the first $24 \mathrm{~h}$ of admission (measured using the APACHE II scale) was analyzed. The data were extracted from the ENVIN-HELICS registry.

Methods. This is a retrospective descriptive study. Data were extracted from the 201 patients admitted to our Intensive Care Unit (multipurpose profile), belonging to a third-level hospital, since the beginning of the COVID-19 pandemic. The variables analyzed were: age, days of stay in the ICU, APACHE II, need for invasive mechanical ventilation, death data, and the relationship of IFI with the use of broad-spectrum antimicrobials and with the appearance of multi-resistant microorganisms (MROs).

Results. The mean age of the patients was 61.18 years $( \pm 13.23)$, and the mean stay was 22.41 days \pm 16.82 . The mean severity score, measured by the APACHE II scale, was $13.77 \pm 6.37$. Invasive mechanical ventilation required $78.6 \%$ of the patients. A total of 78 patients (38.8\%) died, of which $18.05 \%$ occurred once they were discharged from the ICU. Among the patients who presented invasive fungal infection, the mean stay was $39.19 \pm 24.48$, the mean APACHE II score was $13.33 \pm 5.07$. All patients with IFI received invasive mechanical ventilation, and mortality in this group was $52.63 \%$. In our series of patients, the overall incidence of fungal infection was $14.5 \%$, with invasive fungal infection representing $10.5 \%$ of the total, and localized fungal infection, $4 \%$ of the total. Among the patients who presented IFI, $52.17 \%$ did so in the form of candidemia, and $47.83 \%$ in the form of IPA. Of the total number of patients, $1 \%$ presented concomitant candidemia and invasive pulmonary aspergillosis. All patients with IFI received some broad-spectrum antimicrobial at some point during their stay. Of the total number of patients with IFI, $34.78 \%$ also detected MROs infection.

Conclusion. Invasive fungal infection is an emerging problem in Intensive Care Units, which mainly conditions an increase in the number of days spent in the ICU. In our series, we observed that the severity of the patients, measured by the APACHE II score, did not differ 
much between the patients who developed IFI compared to those who did not. During the SARS-CoV2 pandemic, an incidence of IFI was identified in our unit slightly higher than that described in the same period in previous years, which may be due to longer stays, use of broad-spectrum antimicrobials... We must suspect the appearance of IFI in patients admitted for severe SARS-CoV2 infection in these cases, try to identify them early and start treatment as soon as possible. In critically ill patients admitted to Intensive Care Units, the early identification of IFI is complex, but it is essential to start treatment as soon as possible, being a variable that has been associated with a better prognosis and longer survival.

\section{0}

Coinfection in patients admitted to the ICU with covid-19, a retrospective study

D. García Huertas ${ }^{1}$ :V. Martínez de Pinillos Sánchez ${ }^{1}$; I. de Dios Chacón ${ }^{1}$

${ }^{1}$ ICU, University Hospital Complex of Granada, Granada, Spain

Correspondence: D. García Huertas

Intensive Care Medicine Experimental 2021, 9(1): 000490

Introduction. The last year, the main cause of admission for pneumonia to the ICU of Virgen de las Nieves Hospital has been the coronavirus disease (covid-19). In most patients, pneumonia has been caused only by this virus, but in some cases, concomitant infections have been detected, which can play an important role in the development of the disease and in treatment.

Objectives. To investigate the incidence of microbial coinfection in critically ill patients with confirmed severe acute respiratory syndrome coronavirus 2 (SARS-CoV-2) during the months of the pandemic.

Methods. A retrospective study of patients admitted to the ICU for covid- 19 confirmed by PCR was designed. Patients were included from the first wave of the pandemic (March 2020) to February 28, 2021. The search for coinfection was performed using respiratory viral PCR panels, sputum culture or bronchial aspirate on admission, serology of atypical bacteria and pneumococcal and Legionella urinary antigens.

Results. The study included 371 patients, in 35 of which $(9,43 \%)$ coinfection was detected. The most frequent causative microorganisms were atypical bacteria, with positive serology found in 19 of the 35 patients (54\%): 7 Chlamydophila pneumoniae, 7 Mycoplasma pneumoniae, 3 Mycoplasma and Chlamydophila, 2 Coxiella burnetti. The rest of bacterial isolates accounted for $26 \%$ (9 patients), in 3 cases Streptococcus pneumoniae urinary antigens were positive and in the other cases, there was bacterial isolation in sputum or bronchial aspirate: 3 methicillin-sensitive Staphylococcus aureus (MSSA), 1 Corynebacterium pseudodyphtheriticum, 1 Haemophilus influenzae, 1 MSSA and Neisseria meningitidis. In 7 patients (20\%), PCR of other pneumotropic viruses was positive (2 cytomegalovirus, 1 influenza $A$, 1 metapneumovirus, 1 Human Herpes Simplex virus, 1 rhinovirus, 1 Ebstein-Barr virus). In the subsidiary microorganisms of treatment, the corresponding antibiotic or antiviral therapy was used.

Conclusion. Patients admitted to the ICU for SARS-CoV-2 pneumonia may also present infection by other entities that can modify the clinical course of the disease and some of them have specific treatment. Therefore, at the time of admission, an active search for coinfection should be carried out to understand possible changes in the evolution and to establish the appropriate treatment.

\section{6}

Hyponatraemia in COVID-19 critically ill patients

S. Fratino ${ }^{1}$; K. Kaefer ${ }^{1}$; A. Cudia' ; R. Rozenblum ${ }^{1}$; P. Foulon'; D. De Backer ${ }^{1}$ ${ }^{1}$ Department of intensive care, CHIREC Hospitals, Université Libre de

Bruxelles, Brussels, Belgium

Correspondence: $\mathrm{S}$. Fratino

Intensive Care Medicine Experimental 2021, 9(1): 000496

Introduction. According to previous studies, hyponatraemia has been reported in COVID-19 patients. However, its incidence and clinical impact have not been established $(1,2,3)$.

Objectives. To assess the incidence of hyponatraemia in critically ill COVID-19 patients and its association with morbidity and mortality.
Methods. This retrospective cohort study included all consecutive critically ill patients with a positive diagnosis of SARS-Cov-2 pneumonia (positive nasopharyngeal or bronchial PCR and/or CT Scan images suggestive for COVID-19 infection) admitted between March 2020 and March 2021 in our Intensive Care Department.

Hyponatraemia was defined as serum sodium $<135 \mathrm{mmol} / \mathrm{l}$; we considered the first sodium value available from the date of hospitalisation (hospital or ICU admission) after correction for the blood glucose value according to published guidelines (4). Chronic hyponatraemia and pregnant patients were excluded. We collected demographic, clinical and laboratory data at hospital and ICU admission and during the ICU stay in order to compare hyponatraemia with nonhyponatraemia patients.

Results. 227 patients were screened. After exclusion of one patient for chronic hyponatraemia and 3 patients for pregnancy, 223 patients were enrolled in the study. Hyponatraemia was observed in $64(28 \%)$ patients.

Most of the baseline characteristics were similar in hyponatraemic and non-hyponatraemic patients. Of note, lymphocyte count was lower in hyponatraemia patients compared with non-hyponatraemia patients, both at hospital admission (760[542-1113] vs 990[700-1320] $p=0,03)$ and at ICU admission (810 [570-1040] vs $920[680-1375] p=0,06)$. The other laboratory variables were similar in the two groups, as well as demographics and clinical features.

During ICU stay, endotracheal intubation was performed in $71 \%$ of hyponatraemia patients compared to $55 \%$ of non-hyponatraemia patients. Even though creatinine levels and creatinine clearance were similar at admission in the 2 groups, the incidence of acute kidney injury during ICU stay was higher in hyponatraemia patients compared with non-hyponatraemia patients ( $48 \%$ vs $34 \%, p=0,03)$.

There was no significant difference in ICU mortality between the two groups ( $25 \%$ in hyponatraemia patients vs $19 \%$ in non-hyponatraemia patients, $p=0.32$ )

Conclusion. In this cohort, hyponatraemia was present in one third critically ill COVID-19 patients.

Hyponatraemic patients tended to be more severe with higher incidence of intubation and acute kidney injury, even though mortality did not differ.

\section{Reference(s)}

1. 4. Spasovski G., Raymond V., Allolio B., Annane D., Ball S., Bichet D., Decaux G.,Fenske W., Hoorn E.J., Ivhai C.,Joannidis M.,Soupart A.,Zietse R.,Haller M., van der Veer S.,Van Bisesen, Nagler E on behalf of the Hyponatraemia Guide Development Group. Clinical practice guideline on diagnosis and treatment of hyponatraemia. European Journal of Endocrinology (2014) 170,G1-G47.

2. 3. Lippi G, South AM, Henry BM (2020) Electrolyte imbalances in patients with severe coronavirus disease 2019 (COVID-19). Ann Clin Biochem.

3. 2. Post A, Dullaart RPF, Bakker SJL (2020) Is low sodium intake a risk factor for severe and fatal COVID-19 infection? Eur J Intern Med 75:109.

4. 1. Aggarwal, S.; Garcia-Telles, N.; Aggarwal, G.; Lavie, C.; Lippi, G.; Henry, B.M. Clinical features, laboratory characteristics, and outcomes of patients hospitalized with coronavirus disease 2019 (COVID-19): Early report from the United States. Diagnosis 2020, 7, 91-96.

000503

Infection and colonization of Stenotrophomonas maltophilia in $\mathbf{2 0 2 0}$ on an Intensive Care Unit (ICU) of a third level hospital: a descriptive study in Covid 19 and non-Covid19 patients I. Guzman Adum¹; CM. Rodríguez Mejías'; J JP. Valencia Quintero ${ }^{1}$; CJF.

Machado

${ }^{1}$ Medicina intensiva, Hospital Universitario Virgen de las Nieves, Granada,

Spain

Correspondence: I. Guzman Adum

Intensive Care Medicine Experimental 2021, 9(1): 000503

Introduction. Stenotrophomona maltophilia is a cosmopolitan and ubiquitous bacterium and an increasingly prevalent cause of nosocomial opportunistic infections, life-threatening diseases in 
immunocompromised haematology-oncology patients and pulmonary infections in chronic respiratory patients. This pathogen remains an important problem in the ICU due to its association with high morbidity and mortality in debilitated individuals and its remarkable intrinsic and acquired multidrug resistance.

Objectives. To describe de demographic characteristics, comorbidities, immunologic status and mortality in patients with and without Covid19, colonized or infected with S. maltophilia in the ICU of a third level hospital. Methods. Observational, descriptive and retrospective study that includes the 25 patients admitted in the ICU from January to December 2020 that were colonized or infected with S.maltophilia during this period. A database was created in SPSS with demographic characteristics, comorbidities, APACHE II, SOFA score, mortality and cause of death. They were evaluated in terms of descriptive statistics.

Results. The mean age of all the included patients was 61.48 years (SD 13.54 ), $28 \%$ were women and $72 \%$ men. $22.2 \%$ of non-Covid patients were diabetic and $77.8 \%$ weren't $6.3 \%$ of Covid patients had diabetes and $93.8 \%$ didn't. Obesity was more frecquent in the non-Covid group (55.6\% vs $12.5 \%$ ). Only $12.5 \%$ of the patients were inmunodepressed in the Covid group and non in the non-Covid. Hypertension was eaqually distributed in both groups. The SOFA score at admission was 9.11 (SD 3.6) in non-Covid patients and 4.94 (SD 2.38) in Covid patients. APACHE II score was 20.89 (11.5) in non-Covid and 9.75 (4.97) in Covid patients. In this study most of the patients received IMV (88.9\% of non-Covid and 93.8\% of Covid). Regarding the medication: corticosteroids were given to all patients in the Covid group and only in $22.2 \%$ of the non-Covid patients; Ritonavir/Lopinavir, azitromicine, aceticisteine and hydroxicloroquine only in $31.3 \%$ of Covid patients. Tocilizumab, remdesivir, anakinra, rivabirine, INF B had only 1 patient in the Covid group. $20 \%$ of all cases were colonizations ( $44.4 \%$ in non Covid, $12.5 \%$ in Covid group) and $80 \%$ infections (55.6\% and $93.8 \%$ respectively); $52 \%$ in bronquial aspirate, $40 \%$ in colonization studies (exhudates) and $8 \%$ in hemocultives. All the patients had another bacteria identified. The total mortality was $36 \%$ (22.2\% in non-Covid and $43.8 \%$ in Covid) and the most frecquent cause of death was septic shock with multiorgan failure (16\%).

Conclusion. - Even though most of the patients weren't previously inmunosupressed, everey patient in the Covid group received high dose and prolonged therapy with steroides.

- Infections were significantly more frecquent in the Covid group ( $p$ 0.02 ) and colonizations in the non-Covid patients. All patients were infected/colonized by other bacteria.

- The Covid group had a higher all-cause mortality ( $p$ 0.05), which didn't correlate with the SOFA and APACHE II scores (both higher in the non-Covid group), probably due to the higher infection rate.

\section{Reference(s)}

1. Expert Rev Anti Infect Ther. 2019 Nov;17(11):877-893. https://doi.org/10. 1080/14787210.2019.1685875. Epub 2019 Nov 1.

2. Front Microbiol. 2017 Nov 30;8:2276. https://doi.org/10.3389/fmicb.2017. 02276. eCollection 2017

3. Trends Microbiol. 2018 Jul;26(7):637-638. https://doi.org/10.1016/j.tim. 2018.04.006. Epub 2018 May 10.

\section{3}

Vaccine hesitancy and distrust: Concerns of healthcare workers of Pakistani origin

MMA. Zaki'; SW. Siddiqui'; M. Zafar ${ }^{3}$

${ }^{1}$ Department of Anaesthesia and Critical Care, London North West

University Healthcare NHS Trust, London, United Kingdom; ${ }^{2}$ Information, Communication and Technology, Director General Health Services Punjab, Lahore, Pakistan; ${ }^{3}$ Department of Anaesthesia and Critical Care, King Edward Medical University, Lahore, Lahore, Pakistan

Correspondence: M.M.A. Zaki

Intensive Care Medicine Experimental 2021, 9(1): 000513

Introduction. Vaccine hesitancy remains a barrier against containing the spread of Covid-19 [1]. Ironically rapid development of Covid19 vaccines has raised distrust and safety concerns among general population [2]. Healthcare workers, despite being first in line to be vaccinated, have expressed concerns regarding efficacy and safety of vaccines $[\mathbf{3 , 4}]$.

Objectives. Purpose of this survey was to explore reasons for hesitancy and distrust in Covid-19 vaccine expressed by Healthcare workers of Pakistani origin.

Methods. A questionnaire designed on google.forms was sent to $\mathbf{1 4 0}$ randomly selected healthcare workers of Pakistani origin. Questions were designed to ascertain proportions of vaccine reluctant individuals and exploring causes of vaccine hesitancy, highlighting safety concerns Results. 139 responses were collected. 127 (91\%) respondents reported being at high risk of contracting Covid-19 from possible patient contact. 36 (26\%) had either tested PCR positive for Covid19 or had a raised antibody level $(\mathbf{1 0 \%}) .101(\mathbf{7 3} \%)$ respondents had already been vaccinated against Covid-19. Of the 38 (27\%) unvaccinated individuals $19(\mathbf{1 4 \%})$ had refused vaccination entirely.110(81\%) respondents pointed out distrust among healthcare workers regarding vaccine safety and efficacy. $94(69 \%)$ believed rapid development of vaccines and lack of evidence regarding efficacy has compromised overall safety. $36(\mathbf{2 6 \%})$ believed vaccines will have long term adverse effects while $22(\mathbf{1 6 \%})$ believed published data was falsified by manufacturers.

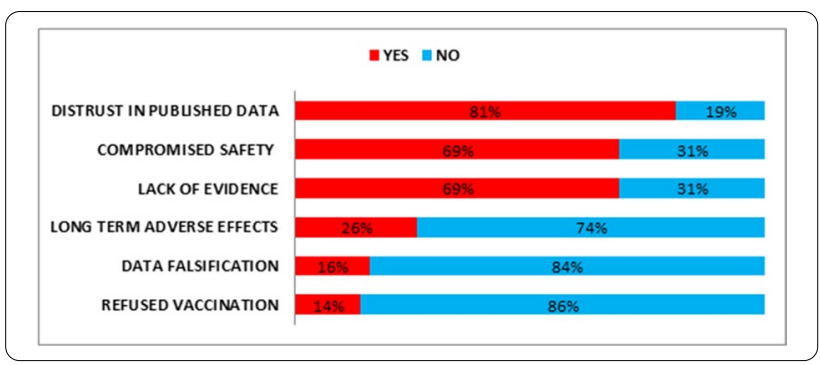

Conclusion. Fears related to quality control and side effect profile of Covid-19 vaccine can hamper efforts to control spread of disease and dissemination of vaccine. There is still opportunity to educate and inform. We hope to further explore and address these concerns.

Reference(s)

1. 4. Shekhar R, Sheikh AB, Upadhyay S, Singh M, Kottewar S, Mir H, Barrett E, Pal S. COVID-19 vaccine acceptance among health care workers in the United States. Vaccines. 2021 Feb;9(2):119.

2. 3. Verger P, Scronias D, Dauby N, Adedzi KA, Gobert C, Bergeat M, Gagneur A, Dubé E. Attitudes of healthcare workers towards COVID-19 vaccination: A survey in France and French-speaking parts of Belgium and Canada, 2020. Eurosurveillance. 2021 Jan 21;26(3):2,002,047

3. 2. Dror AA, Eisenbach N, Taiber S, Morozov NG, Mizrachi M, Zigron A, Srouji S, Sela E. Vaccine hesitancy: the next challenge in the fight against COVID-19. European journal of epidemiology. 2020 Aug;35(8):775-9.

4. 1. Verger P, Dubé E. Restoring confidence in vaccines in the COVID-19 era.

5. No conflict of interest to declare

\section{4}

Analysis of last 10 years Pseudomonas Aeruginosa Infections in ICU Department Of Burgos University Hospital

AO. Badallo"; VM. Del²; S. Ossa, ; R. Vara Arlanzón ${ }^{1} ;$ D. Iglesias²; E. Martínez Barrio $^{2}$; A. Larrosa, ${ }^{2}$; A. Ruiz, ; JA. Fernández Ratero²

${ }^{1}$ Intensive Care, Hospital Universitario de Burgos, Burgos, Spain; ${ }^{2}$ Intensive Care, Hospital Universitario de Burgos, Avenida Islas Baleares, Burgos, Spain, Burgos, Spain

Correspondence: A.O. Badallo

Intensive Care Medicine Experimental 2021, 9(1): 000524

Introduction. Pseudomonas aeruginosa (P.aerug) is classically known as a microorganism frequently isolated in Intensive Care Units (ICU). Lately, it has been overtaken by Escherichia coli, although P. aerug continues being the second causal agent with $13 \%$ of device-related infections. 
P. aerug. is characterized by remarkable intrinsic resistance to antibiotics and the ability to develop resistance, nearly against all available antibiotics through mutations selection. These strains suppose a real challenge to physicians mostly in empiric treatments, but also in targeted treatments, due to limited alternative therapeutics.

Objectives. To describe Pseudomonas aeruginosas (P. aerug) infections during 10 years in our ICU Department.

Methods. Observational prospective study involving patients admitted in our 24 bed ICU that were registered in ENVIN-HELICS data base during April, May and June from 2009 to 2019.

Results. 2.702 patients were analysed with a mean stay of 21,12 days. 502 ICU infections were recorded. Most common infection was urinary tract catheter infection (UTCI) 24,5\%, secondly tracheobronchitis 9,3\% and surgical wound infection $7,7 \%$. P.aerug was the infective agent in $12,5 \%$ of all the infections.

There were observed 184 medical device-related infections, from which 123/184 (66,84\%) were UTCI, 31/184 $(16,84 \%)$ were ventilatorassociated pneumonia (VAP), 30/184 (16,3\%) catheter-related bacteriemia (CRB). P. aerug was observed in $27 / 123(22,14 \%)$ UTCI, $5 / 31$ $(16,67 \%)$ VAP, $1 / 30$ (3,33\%) CRB.

Sensitivity to specific antimicrobials of $P$. aerug: Data Table 1.

Data table 1.
\begin{tabular}{|l|l|l|l|l|}
\hline Sensitivity & Overall \% & UTI \% & VAP $\%$ & CRB $\%$ \\
\hline Amikacin & 80,5 & 91,6 & 77 & 75 \\
\hline Cefepime & 68,6 & 91,6 & 53,8 & 75 \\
\hline Ceftazidime & 74,6 & 91,6 & 53,8 & 75 \\
\hline Ciprofloxacin & 56,7 & 75 & 30,7 & 25 \\
\hline Colistin & 96,7 & 100 & 100 & 100 \\
\hline Imipenem & 46,3 & 75 & 23,1 & 25 \\
\hline Levofloxacin & 56 & 75 & 38,5 & 25 \\
\hline Meropenem & 49,2 & 75 & 30,8 & 25 \\
\hline $\begin{array}{l}\text { Piperacilin- } \\
\text { tazobactam }\end{array}$ & 68,6 & 100 & 53,8 & 75 \\
\hline
\end{tabular}

About Multidrug-resistant (MDR) P.aerug, 3,81\% of all patients suffered colonization or infection of this bacteria. The acquisition rate of P.aerug. MDR during ICU hospitalization is 2,2\% and 2,9 cases per 1.000 day stay.

A $12,5 \%$ of the colonised patients developed a P. aerug MDR infection or what is the same, $0,48 \%$ of the patients and 0,61 cases per 1.000 day stay.

Overall mortality of ICU infection was $36,3 \%, 58,6 \%$ in VAP, $27,5 \%$ for UTCl infections and $25 \%$ in CRB.

Conclusion. P. aerug causes $12,5 \%$ of ICU infections, being UTCI the most frequent.

Our P.aerug sensitivity chart is marked by high sensitivity to Colistin and low to Carbapenem. It depends on the focus of infection, where UTI present better sensitivity and VAP more resistance, fact that is correlated to device-related infections' overall mortality.

P. aerug MDR appears in 3,81\% of the patients, although only $12,5 \%$ presents infection, considering it a low rate.

\section{Reference(s)}

1. 8. Epidemiology of antibiotic resistance in Pseudomonas aeruginosa. Implications for empiric and definitive therapy. Ruiz-Garbajosa P1, Cantón R. Rev Esp Quimioter. 2017 Sep;30 Suppl 1:8-12.

2. 4. Magiorakos AP, Srinivasan A, Carey RB et al. Multidrug-resistant, extensively drug-resistant and pandrug-resistant bacteria: an international expert proposal for interim standard definitions for acquired resistance. Clin Microbiol Infect 2012; 18(3):268-281. https://doi.org/10.1111/j.14690691.2011.03570.x

3. 3. Antibiotic selection in the treatment of acute invasive infections by Pseudomonas aeruginosa: Guidelines by the Spanish Society of Chemotherapy. Mensa J1, Barberán J, Soriano A, Llinares P, Marco F, Cantón R, Bou G, González Del Castillo J, Maseda E, Azanza JR, Pasquau J, GarcíaVidal C, Reguera JM, Sousa D, Gómez J, Montejo M, Borges M, Torres A, Alvarez-Lerma F, Salavert M, Zaragoza R, Oliver A. Rev Esp Quimioter. 2018 Feb;31(1):78-100.
4. 2. Invasive device-associated infections caused by Pseudomonas aeruginosa in critically ill patients: evolution over 10 years. F. Álvarez-Lerma, P. Olaechea-Astigarraga, M. Palomar-Martínez, M. Catalan, X. Nuvials, R. Gimeno, M.P. Gracia-Arnillas,I. Seijas-Betolaza, ENVIN-HELICS Study Group. Journal of Hospital Infection 100 (2018) e204ee208.

5. 1. Estudio Nacional de Vigilancia de Infección en Servicios de Medicina Intensiva. Informes anuales. http://hws.vhebron.net/envin-helics/

\section{4}

ICU mortality in patients with COVID-19 pneumonia: incidence, causes and patients characteristics

N. Cruza Leganés'; J. Martin Rasero'; LM. Vega ${ }^{1}$; F. Arbol Linde'; A. Simón Martin ${ }^{1}$; MA. Taberna Izquierdo

${ }^{1}$ Intensive Care Unit, General Hospital Nuestra Señora del Prado, Talavera de la Reina, Spain

Correspondence: N. Cruza Leganés

Intensive Care Medicine Experimental 2021, 9(1): 000534

Introduction. The main reason for admission of COVID patients to the ICU is severe respiratory failure, although other organs may be affected to a greater or lesser extent, and it is interesting to know the characteristics of those patients who die and the final cause of death.

Objectives. To evaluate the mortality of patients admitted to our ICU with COVID pneumonia (PNCOV), its incidence and causes, and to describe characteristics and evolution of patients who die compared to those who live.

Methods. A retrospective descriptive observational study of all patients admitted to our ICU with PNCOV during the first and second wave of the pandemic, from March 13 to December 21, 2020. We analyze characteristics and evolution of patients who died and those who lived, incidence and causes of mortality.

Results. During the study period, 122 patients with PNCOV were admitted to our ICU, of whom $33(27,04 \%)$ died. The average age of those who dies was $65,15(95 \% \mathrm{Cl}, 62,3-68)$ years old, and that of those lived was $57,11(95 \% \mathrm{Cl}, 54,57-59,65)$. Those who died, 21 $(63,63 \%)$ were male, and $67,41 \%$ of those who lived too. Those death had a mean of $2,39(95 \% \mathrm{Cl} 1,83-2,95)$ comorbidities and $18(54,54 \%)$ had hypertension, with $1,52(95 \% \mathrm{Cl}, 1,25-1,79)$ and $44,94 \%$, respectively, in those who lived. The mean APACHE II score of the deceased was $18,33(95 \% \mathrm{Cl}, 16,19-20,47)$ and $14,72(95 \% \mathrm{Cl}, 13,67-15,77)$ in the living. Twelve $(36,36 \%)$ of those who died were admitted directly to the ICU from the emergency room, and $21(23,60 \%)$ of those who lived. Admission $\mathrm{PaO}_{2} / \mathrm{FiO}_{2}$ mean of the deceased was $115,97(95 \% \mathrm{Cl}$, $28-136,66)$, and $114,82(95 \% \mathrm{Cl} ; 102,22-127,42)$ in the living. Mechanical ventilation (MV) was required in the first $24 \mathrm{~h}$ in 26 patients $(78,79 \%)$ of the deceased, and 49 patients $(55,05 \%)$ of the living, with $23,22(95 \% \mathrm{Cl} 16,77-29,67)$ days of $\mathrm{MV}$ and $17,92(95 \% \mathrm{Cl}, 14,92-20,92)$ days respectively. Nineteen $(57,57 \%)$ deceased patients had at least one episode of nosocomial infection, and living patients $32(35,95 \%)$. The mean ICU stay in both groups is: $25,06(95 \% \mathrm{Cl}, 18,01-32,11)$ and $17,72(95 \% \mathrm{Cl} 14,44-21)$ days respectively. Those patients were treated with: methylprednisolone $17(51,51 \%)$ of the deceased and $46(51,68 \%)$ of living; dexamethasone: $15(45,45 \%)$ and $39(43,82 \%)$ respectively; anakinra: $5(15,15 \%)$ of the deceased and $19(21,34 \%)$ of the living; tocilizumab: $5(15,5 \%)$ and $19(21,34 \%)$ respectively.

Regarding the cause of death, $15(45,45 \%)$ patients died due to unfavorable evolution of the COVID-19 infection [10/15 $(66,66 \%)$ due to multiorgan failure and $5 / 15$ (33,33\%) due to refractory hypoxemia], 15 $(45,45 \%)$ due to septic shock secondary to ICU acquired infection, and $3(9,09 \%)$ due to other causes ( 1 mesenteric ischemia, 1 cardiogenic shock, 1 pneumothorax).

Conclusion. ICU mortality in patients admitted for PNCOV is $27,04 \%$. The causes of death are mainly: multiorgan failure, refractory hypoxemia and septic shock secondary to nosocomial infection 


\section{8}

Experience of inhalation use of antibacterial medicines in treatment of severe pneumoniae

V. Ziamko

'Viciebsk State Order of Peoples' Friendship Medical University, Viciebsk, Belarus

Correspondence: $\vee$. Ziamko

Intensive Care Medicine Experimental 2021, 9(1): 000558

Introduction. The main problem in treatment of nosocomial pneumonia is a poor penetration of intravenous antibacterial drugs into lungs: the approaches that have developed over the years for the predominant use of parenteral antibacterial drugs do not allow creating an adequate bactericidal concentration in the lung tissue and lead to an increase in the resistance of microorganisms.

Objectives. To study effectiveness of inhaled use of antibacterial drugs in the treatment of severe pneumoniae.

Methods. Comprehensive examination and treatment of 10 patients with severe bilateral polysegmental pneumonia was performed. Mean age of patients-49,5 $\pm 19,7$ years. Proposed method of administration of inhaled antibiotics to a patient breathing spontaneously through a tracheostomy or endotracheal tube is based on the introduction of an antibiotic into a tracheostomy or endotracheal tube into carina area of the trachea through an aspiration catheter.

Results. Method was used as a supplement to complex intensive therapy of patients receiving intravenous antibacterial drugs. After isolation of $P$. aeruginosa colistate was added at a dose of $2 \mathrm{mln}$. ME 3 times a day inhaled into tracheostomy tube according to proposed method of administration of inhaled antibiotics for treatment of acute respiratory inflammatory diseases to main scheme of antibacterial therapy. Temperature dynamics, laboratory data, $\mathrm{X}$-ray picture and microbiological analysis were evaluated as efficiency of the applied method. Positive X-ray dynamics, temperature $(p=0,017)$ and $\operatorname{ESR}(p=0,011)$ decrease, normalized leukocyte count $(p=0,009)$, leukocyte formula $(p=0,046)$ and negative results of microbiological sputum examination were observed in 6 days.

Conclusion. First experience of administration of inhaled antibiotics showed a rather high efficiency of its use in treatment of severe pneumoniae.

\section{Reference(s)}

1. 5. Spencer S., Felix L., Milan S. et al. Oral versus inhaled antibiotics for bronchiectasis (Review). Cochrane Database of Systematic Reviews 2018, Issue 3. Art. No.: CD012579 https://doi.org/10.1002/14651858.CD012579. pub2.

2. Smith S., Rowbotham N. Regan K. Inhaled anti-pseudomonal antibiotics for long-term therapy in cystic fibrosis. Cochrane Database of Systematic Reviews 2018, Issue 3. Art. No.: CD001021. https://doi.org/10.1002/14651 858.CD001021.pub3.

\section{0}

\section{Nosocomial infection in patients admitted to our ICU} with COVID-19 pneumonia

N. Cruza Leganés'; LM. Vega'; J. Martin Rasero'; F. Arbol Linde'; A. Simón Martin ${ }^{1}$; MA. Taberna Izquierdo

${ }^{1}$ Intensive Care Unit, General Hospital Nuestra Señora del Prado, Talavera de la Reina, Spain

Correspondence: $\mathrm{N}$. Cruza Leganés

Intensive Care Medicine Experimental 2021, 9(1): 000570

Introduction. Patients with COVID pneumonia (PNCOV) may be more susceptible to infections due to the immune alterations produced by the virus and by the treatments used.

Objectives. To analyze ICU-acquired infections in patients admitted with PNCOV during the first and second waves of the pandemic, characteristics and evolution of these patients.

Methods. A retrospective descriptive observational study on nosocomial infection (NI) during their stay in the ICU of all patients admitted to our ICU with PNCOV from March 13 to December 21, 2020. The following are analyzed: number and kind of infections, situation on admission to the ICU and evolution.

Results. During the study period, 122 patients were admitted with PNCOV, of whom $51(41,8 \%)$ presented at least one episode of NI during their stay in the ICU, with a total of 112 episodes, and a mean of 2,2 (95\% Cl 1,72-2,68) episodes per patient: 44 (39,29\%) episodes of ventilator-associated pneumonia (VAP); $28(25 \%)$ urinary tract infections (UTI); $26(23,21 \%)$ catheter bacteremias (CB); $13(11,6 \%)$ primary bacteremias (BP), and 1 (0,89\%) endocarditis. In 109/112 (97,32\%) the causal germ was identified (culture, galactomannan). VAP were mainly caused by Gram-negative bacilli (GNB) $(28 / 44,63,64 \%)$, especially Acinetobacter Baumannii (15/28, 53,57\%) and Pseudomona Aeruginosa $(8 / 28,28,57 \%)$, followed by Aspergillus (14/44, 31,82\%). UTIs were caused by Enterococcus (15/28, 53,57\%), GNB (9/28, $32,14 \%)$, especially Pseudomona Aeruginosa (7/9, 77,77\%), and Candida $(9 / 28,32,14 \%)$. CB due to coagulase-negative Staphylococcus $(15 / 26,57,69 \%)$, Acinetobacter baumannii $(5 / 26,19,23 \%)$ and Pseudomona aeruginosa $(4 / 26,15,38 \%)$. BPs were more frequently Gram-positive $(8 / 13,61,53 \%)$, especially coagulase-negative Staphylococcus $(5 / 8,62,5 \%)$, and endocarditis due to Staphylococcus Aureus. On admission to the ICU, these patients who presented $\mathrm{NI}$ had a mean APACHE II score $16,1(95 \% \mathrm{Cl}, 14,61-17,59)$ and mean $\mathrm{PaO}_{2} / \mathrm{FiO}_{2}$ 111,84 (95\% Cl, 96,14-127,54); 50 (98,04\%) received corticosteroids, $12(23,53 \%)$ anakinra and $9(17,65 \%)$ tocilizumab; 41 (80,39\%) required invasive mechanical ventilation (IMV) in the first $24 \mathrm{~h}$, and $50(98,04 \%)$ needed IMV the following 4 days, with a duration of MV of 27,41 days $(95 \% \mathrm{Cl}, 26,78-37,72)$ and $37,25 \%$ mortality.

Conclusion. Patients admitted in our ICU with PNCOV present a high incidence of $\mathrm{NI}$, the most frequent being VAP, which is mainly caused by Acinetobacter baumannii, followed by Aspergillus and Pseudomonas aeruginosa. Gram-positive bacteria were the most frequently implicated in UTIs and bacteremias, both BP and CB. Most patients required IMV in the first $24 \mathrm{~h}$ of admission and presented $37,25 \%$ mortality.

\section{5}

Prevalence and incidence of MRSA at admission in an ICU

T. Oliveira ${ }^{1}$;T. Pereira' ; AC. Dinis ${ }^{1}$; C. Nuno ${ }^{1}$; S. Narciso ${ }^{1}$; L. Caley ${ }^{1}$; AM. Neto Real'

${ }^{1}$ Intensive Medicine, Centro Hospitalar Médio Tejo, Abrantes, Portugal

Correspondence: T. Oliveira

Intensive Care Medicine Experimental 2021, 9(1): 000595

Introduction. Staphylococcus aureus is part of the normal skin and nasal microbiota, and around $30 \%$ of the healthy adult population is colonized mainly in the nasopharyngeal cavity.

Methicillin-resistant Staphylococcus aureus (MRSA) first emerged after methicillin was introduced to combat penicillin resistance in 1959.

Portugal has one of the highest incidences of MRSA in Europe-more than $50 \%$ in 2012.

It is a major human pathogen causing a wide range of communityonset and nosocomial infections with significant morbidity and mortality. For this reason, nasal swabs, perianal and oropharyngeal samples should be a part of the surveillance cultures at admission in an Intensive Care Unit (ICU).

Objectives. Retrospective observational study that aims to characterize and evaluate the population admitted in an ICU between 2019 and 2020 with positive MRSA colonization at admission screening.

Methods. Retrospective observational study that aims to characterize and evaluate the population admitted in an ICU between 2019 and 2020 with positive MRSA colonization at admission screening.

Results. Between January 2019 and December 2020 we admitted a total of 64 patients with positive nasal and perianal swabs for MRSA in our ICU, of whom 26 where females and 38 males between the ages of 33 to 96 (average 78 years old).

All had risk factors for MRSA: 39 (61\%) where under immunosuppressive therapy (we included patients with Diabetes Mellitus), 16 (25\%) used antibiotherapy during the previous 6 months, $5(8 \%)$ where on a program of chronic hemodialysis and $10(16 \%)$ had a long term catheter. 15 patients $(23 \%)$ where institutionalized and $25(39 \%)$ had previous hospitalization for more than 5 days. 
From the total of 64 patients, 7 (11\%) developed positive blood cultures for MRSA, 46 (72\%) had negative blood cultures at admission and 11 (17\%) positive blood cultures for other microorganisms. About 3 patients (5\%) had a second positive blood culture for MRSA during their stay in the ICU, other $3(5 \%)$ had positive bronchial cultures for MRSA and one patient developed a septic shock from a soft tissue infection.

The mortality rate in the patients with bacteremia was $45 \%$ and was $100 \%$ in those patients that had a positive 2 nd blood culture.

Those with a positive respiratory culture had a mortality rate of $67 \%$.

More than half of this patients (55\%) who developed infection had a hospital stay of $>48 \mathrm{~h}$ previous to the admission to the ICU and the remaining $45 \%$ of $<48 \mathrm{~h}$

Conclusion. It is clear that patients who are colonized with MRSA and develop positive cultures show worse outcomes. Most (61\%) developed nosocomial infection.

Immunosuppressive therapy, can be considered an important risk factor for colonization with MRSA.

Reference(s)

1. Anthony D. Harris, et al. Targeted Surveillance of Methicillin-Resistant Staphylococcus aureus and Its Potential Use To Guide Empiric Antibiotic Therapy, ANTIMICROBIAL AGENTS AND CHEMOTHERAPY, Aug. 2010, p. 3143-3148

2. https://www.dgs.pt/estatisticas-de-saude/estatisticas-de-saude/publi cacoes/portugal-controlo-da-infecao-e-resistencia-aos-antimicrobianosem-numeros-2014-pdf.aspx

\section{3}

Type I anti-interferon autoantibodies in critically ill patients with severe SARS-CoV-2 pneumonia: pilot study

L. Chiscano'; I. Bajaña'; C. Palmada'; M. Garcia-Parra²; A. Parra-Martínez². M. Pérez-Carrasco'; P. Soler-Palacin³ ; R. Colobran²; R. Ferrer Roca'; JC. Ruiz-Rodríguez

'Intensive Care Department, Vall d'Hebron University Hospital, Barcelona, Spain; ${ }^{2}$ Immunology Service, Vall d'Hebron University Hospital, Barcelona, Spain; ${ }^{3}$ Pediatric Infectious Diseases and Immunodeficiencies Unit, Vall d'Hebron University Hospital, Barcelona, Spain

Correspondence: $L$. Chiscano

Intensive Care Medicine Experimental 2021, 9(1): 000603

Introduction. Immunomodulation is complex, and timing of the treatments is critical. Type linterferons, especially IFN-beta, have been appointed as potential leadingtherapeutics to tackle severe COVID-19 and are currently being evaluated.

Objectives. To study the incidence of anti-interferon type 1 antibodies (Ab-anti-IFN1) inpatients with pneumonia severe due to SARS-CoV-2 admitted to the ICU and its relationship with morbidity and mortality.

Methods. Prospective observational study of a cohort of patients admitted to the ICU forsevere pneumonia for SARS-CoV-2 in the period from November 11 to December13, 2020. Inclusion criteria: ICU admission for acute respiratory failure secondaryto severe SARSCoV-2 pneumonia. Do not there were exclusion criteria.Demographic variables, severity scales (APACHE II), organ dysfunction scale (SOFA), invasive mechanical ventilation (IMV) requirement, inflammatoryparameters (CRP, IL-6, D-dimers, ferritin and leukocytes) and hospital mortality.The Blood samples for analysis were obtained during the first 5 days of admission. The Determination of Ab-anti-IFN1 was carried out using the ELISA technique and, specifically, determined the existence of anti-IFN-alpha2 (a2) and anti-IFN-omega(w) Ab. The data is expressed as frequency (\%) if they are categorical and mean (standard deviation) or median (25-75th percentile) if they are quantitative. Statistical tests: Student's t and Chi-square. The study was approved by theCommittee Clinical Research Ethic (PR (AG) 270/2020) with exemption of informedconsent.

Results. Eighty-five patients were included $[61(71.8 \%)$ men, age 58.5 (11.0) years, SOFA5.0 (3.19) and APACHE II 17.2 (6.5)]. Sixty (70.6\%) patients required IMV. Hospital mortality was $22.4 \%$. The Ab-antiIFN1 were positive in 10 patients $(11.8 \%)$ [9 (90.0\%) men, age 54.8
(16.0) years, SOFA 6.6 (4.8) and APACHE II17.3 (4.4)]: nine of them corresponded to Ab-anti-IFN-a2 (10.6\% of all patients) and $3(3.5 \%)$ to Ac-anti-IFN1- $\omega$ (the 3 patients also presented positivity to AcantiIFN-a2). As a function of the positivity of the Ab-anti-IFN1, there were nosignificant differences in none of the variables studied (age, gender, APACHE II,SOFA, CRP, IL-6, D-dimer, ferritin, leukocytes, requirement for mechanicalventilation and mortality).

Conclusion. In our series, the incidence of Ab-anti-IFN1 was $11.8 \%$ and mostly correspondedto Ac-anti-IFN-a2. Although the results obtained must be interpreted with cautiongiven the scarce representation of positive patients, the existence of Ab-anti-IFN1does not seem to be associated with higher morbidity and mortality.

\section{4}

Prophylactic aspergillus treatment in severe COVID19 patients prevents IPA and mechanical ventilation

J. De Kleijn'; G. Kadijk-Dohmen'; M. Wegdam-Blans²; K. Verduin²; S.

Wessels-Basten ${ }^{3}$; S. Sanders ${ }^{3}$; M. Buise ${ }^{1}$; P. Van De Berg ${ }^{1}$; M. Kamps

${ }^{1}$ Intensive care, Catharina Ziekenhuis, Eindhoven, Netherlands: ${ }^{2}$ Micro-

biology, Stichting Pamm, Veldhoven, Netherlands; ${ }^{3}$ Pharmacy, Catharina

Ziekenhuis, Eindhoven, Netherlands

Correspondence: $\mathrm{M}$. Kamps

Intensive Care Medicine Experimental 2021, 9(1): 000624

Introduction. Aspergillus fumigatus can cause invasive pulmonary aspergillosis (IPA), a severe infection of the lungs with a high mortality rate. $(1,2)$ During the months September and October 2020, we were confronted with a high incidence of IPA in ICU patients treated for severe COVID19. Trying to reduce these infections, voriconazole in a prophylactic dose was administered to all admitted patients. In this retrospective study we have analysed the effect of voriconazole as prophylactic treatment on the incidence of IPA as well as on duration of intubation and ICU stay.

Methods. This retrospective study was conducted in Catharina hospital Eindhoven, the Netherlands. Patients, who were admitted to the ICU between September 2020 and March 2021 with respiratory insufficiency due to COVID-19 disease, were included. Patients admitted before November 2020 did not receive voriconazole prophylaxis (group A), patients admitted from November 2020 were treated with voriconazole prophylaxis $200 \mathrm{mg}$ on a daily dose for seven days (group B). IPA was considered proven when galactomannan (cut-off value of $0,80 \mathrm{OD}$ ) as well as Aspergillus culture were positive from bronchial lavage. IPA was considered probable when only galactomannan was positive.

Baseline characteristics, including age, gender, duration of stay and intubation, were extracted from the patient record files.

Results. In the study period 143 patients were treated on the ICU for COVID-19 infection. 117 Patients were included, from which 38 patients received standard care without voriconazole prophylaxis (group $A, n=38$ ) and 79 patients received standard care supplemented with voriconazole prophylaxis (group $B, n=79$ ). 26 Patients were excluded because of another primary diagnosis than respiratory failure due to COVID19, transfer to another hospital or history of chronic pulmonary aspergillosis.

There were no differences in baseline characteristics between both groups.

In group A $47 \%$ of the patients (18/38) underwent bronchoscopy versus $34 \%(27 / 79)$ in group B ( $p$ 0.17). The incidence of probable IPA in group A was $33 \%(6 / 18)$ and the incidence of proven IPA 17\% (3/18), while in group $B$ none of the patients developed a probable or proven IPA (p 0.001)

In group A $82 \%(31 / 38)$ of the patients were intubated versus $54 \%$ of the patients (43/79) in group $B(p 0.004)$

Patients in group A received more days of mechanical ventilation during admission at the ICU, 9 days (IQR 5-14) versus 3 days (IQR 0-14) in group B ( $p$ 0.023)

Patients in group A had a median stay at the ICU of 12 days (IQR $8-21)$, where patients in group B were admitted to the ICU for 9 days (IQR 6-20, p 0.174). There was no significant difference in mortality between both groups. In group A 37\% (14/38) of the patients died versus $34 \%(27 / 79)$ in group B (p 0.777). 
Conclusion. Our study showed a preventive effect of voriconazole prophylaxis on the occurrence of IPA in patients admitted to the ICU with COVID19. It also showed lower risk for intubation and a shorter duration of mechanical ventilation.

\section{Reference(s) \\ 1. Bartoletti M et all; PREDICO study group. Epidemiology of invasive pul- monary aspergillosis among COVID-19 intubated patients: a prospective study. Clin Infect Dis. 2020 Jul 28:ciaa1065. https://doi.org/10.1093/cid/ ciaa1065. Epub ahead of print. PMID: $32719848 ;$ PMCID: PMC7454393 \\ 2. Lai CC, Yu WL. COVID-19 associated with pulmonary aspergillosis: A litera- ture review. J Microbiol Immunol Infect. 2021 Feb;54(1):46-53. https:// doi.org/10.1016/j.jmii.2020.09.004. Epub 2020 Sep 24. PMID: 33012653; PMCID: PMC7513876 \\ 3. None.}

\section{1}

Differences in mortality and nosocomial infection in patients treated with corticosteroids during the COVID-19 pandemic: dexamethasone versus methylprednisolone; in addition to Tocilizumab and Anakinra

J. Martin Rasero'; N. Cruza Leganés'; LM. Vega'; F. Arbol Linde'; E.

Humanes ${ }^{2}$; MA. Taberna Izquierdo'; A. Simón Martin

${ }^{1}$ Intensive Care Unit, General Hospital Nuestra Señora del Prado, Talavera de la Reina, Spain; ${ }^{2}$ Emergencies, General Hospital Nuestra Señora del

Prado, Talavera de la Reina, Spain

Correspondence: J. Martin Rasero

Intensive Care Medicine Experimental 2021, 9(1): 000661

Introduction. The pandemic due to COVID-19, has put the world of medicine and within this, the ICUs, in check, due to the high number of patients in a short period of time and the uncertainty of not knowing how to treat them. Due to the lack of knowledge, protocols changed almost daily, until the cornerstone that tipped the balance towards improved survival, corticosteroids, was found.

Objectives. To compare the differences between treatment with Dexamethasone $20 \mathrm{mg} / 24 \mathrm{~h}$ for 5 days and Methylprednisolone $500 \mathrm{mg} / 24 \mathrm{~h}$ for 5 days. Additionally some of the patients of each group received complementary treatment with Anakinra or Tocilizumab. As outcome variables we have used: number of nosocomial infections and number of deaths. All this in patients with severe COVID-19 pneumonia who required admission to ICU and mechanical ventilation (MV).

Methods. A retrospective observational cohort study of patients admitted with severe COVID-19 pneumonia in our ICU between 03/14/2020 and $12 / 21 / 2020$. During the study period, 122 patients were admitted, excluding those who took a different corticosteroid regimen and those who did not require MV during their admission. The analysis was performed with ANOVA test for a single value, in which we wanted to calculate whether there was a significant difference between dexamethasone $20 \mathrm{mg} / 24$ for 5 days and methylprednisolone $500 \mathrm{mg} / 24 \mathrm{~h}$ for 5 days. The reason for using one treatment or the other is based on literature at that time. During the first and the beginning of the second wave, patients were treated with methylprednisolone and later, based on data from new publications, this was replaced by dexamethasone. The patients whose diagnostic tests did not depict bacterial coinfeccion also recived treatment with Anakinra, if IL-6 levels where less than $40 \mathrm{pg} / \mathrm{ml}$, or Tocilizumab, if IL-6 levels where higher than $40 \mathrm{pg} / \mathrm{ml}$.

Results. During the study 122 patients were admitted to our ICU (total included 76 patients). The group treated with dexamethasone had 39 patients, with a mean age of $60.74\left(\mathrm{Cl} \% \%\right.$ 56.8-64.68), $\mathrm{PaO}_{2} / \mathrm{FiO}_{2}$ at admission of 99.62 (CI95\% 86.9- 112.33) and APACHE II of 15.46 (Cl95\% 14.08-16.85). The methylprednisolone group has 37 patients, with a mean age of $58.95(95 \% \mathrm{Cl} 55.71-62.79), \mathrm{PaO}_{2} / \mathrm{FiO}_{2}$ of $109.97(95 \% \mathrm{Cl} 90.62-$ 129.32) and APACHE II score 18.38 (95\%Cl 16.32-20.44). In the first group, 6 patients underwent complementary therapy with Tocilizumab and 11 with Anakinra. In the second group, 13 patients received Tocilizumab as well and 6 received Anakinra. As for nosocomial infection, the mean in both groups was: dexamethasone 1.44 cases per patient (C195\% - 2.29 to $5.16, \mathrm{p}=0.6765)$ and methylprednisolone 1.24 cases per patient $(\mathrm{Cl} 95 \%$
- 1.57 to $4.05, p=0.6765)$. In total, there were 14 deaths in both groups combined. In the group treated with dexamethasone the mean number of deaths was $35.8 \%(95 \% \mathrm{Cl} 0.21-0.51, \mathrm{p}=0.479)$ and in the group treated with methylprednisolone $37.8 \%$ ( $95 \% \mathrm{Cl} 0.22-0.54, \mathrm{p}=0.479)$.

Conclusion. Therefore, we cannot conclude that one treatment is better than the other in terms of mortality and incidence of nosocomial infection in both groups.

\section{3}

Sphingomonas Paucimibilis infections: An Indian case series

J. Nikhilesh'; V. Joshi'; G. Ghosh ${ }^{3}$; S. Khare ${ }^{4}$

${ }^{1}$ Dept of Critical Care Medicine, CHL Hospital, Indore, India; ${ }^{2}$ Dept of Critical Care Services, Shalby Hospitals, Indore, Madhya Pradesh, India, India;

${ }^{3}$ Dept of Microbiology, CHL Hospital, Indore, India; ${ }^{4}$ Dept of pathology,

CHL Hospital, Indore, India

Correspondence: J. Nikhilesh

Intensive Care Medicine Experimental 2021, 9(1): 000683

Introduction. Background: Sphingomonas Paucimobilis is an aerobic nonfermentative motile bacteria found in soil and water and is rarely associated with fatal infections. It has been linked to health care infections and this communication attempts to delineate and document a case series on the same.

Objectives. To document prevalence of Sphingomonas paucimobilis infections across different healthcare settings and document association with various co-morbidities across different subsets of patients.

Methods. Setting: Multidiscliplinary tertiary care medical surgical Intensive Care Units (ICUs) of two hospitals.

Study Module: Consecutive patients who had culture positive samples with Sphingomonas Paucimobilis were analyzed and data was collected with a focus on demographic variables, Length of Stay (LOS), SOFA scores at the time of sending cultures and antibiotics used as per cultures. Discharge from ICU or death was considered as end point of study. Results. Fourteen patients were included ( $n=14, M: F-9: 5)$ for a study duration dated March 20-March 21. Age of the entire cohort was $54.2 \pm 10.1$ years. LOS in ICU was $9.2 \pm 2.8$ days. In hospital mortality was $7.2 \%(n=1)$. SOFA scores for the entire cohort were $12.3 \pm 4.3$. In terms of prevalence of risk factors only diabetics revealed a major association of $42.9 \%(n=6)$. The antibiotics used for above infections as per culture reports included Levofloxacin $28.6 \%(n=4)$, Piperacillin Tazobactam 28.6\% $(n=4)$,Cefoperazone Sulbactum 14.3\% $(n=2)$ and Imipenem Cilastatin $28.6 \%(n=4)$. The infections commonly documented with above included Urinary tract infections $28.6 \%(n=4)$, Skin and superficial tissue infections $28.6 \%(n=4)$,Central line associated blood stream infections $21.4 \%(n=3)$, blood stream bacteremia $14.3 \%(n=2)$ and Peritonitis $7.2 \%(n=1)$.

Conclusion. It remains an unusual, rare and opportunistic pathogen with a potential of causing nonfatal infections across different subgroups of patients in ICUs. Environmental contamination remains a very likely possibility in the spread of this infection. This communication demonstrates a prevalence of the same along with a higher likelihood of diabetics acquiring these infections. A larger case series would enhance our understanding of this bacterium which remains a potential healthcare problem.

\section{9}

Peak C-reactive protein (CRP) and trough lymphocyte levels as predictors of mortality in SARS-CoV-2 ventilated ICU patients (comparisons between two pandemic surges)

R. Sterry-Blunt ; M. Mazalkov ; M. Davies

${ }^{1}$ Intensive care medicine, North West Anglia NHSFT, Peterborough,

United Kingdom

Correspondence: $\mathrm{R}$. Sterry-Blunt

Intensive Care Medicine Experimental 2021, 9(1): 000709

Introduction. We aim to establish if there is a significant difference in peak C-reactive protein (CRP) or trough lymphocyte count between ventilated SARS-CoV-2 survivors and non-survivors on the intensive care unit at a District General Hospital; and, whether there is a significant difference in these parameters between the pandemic waves in 
the UK. If a significant link with mortality is found, these markers may be useful in prognostication; counselling patients and families, and; influencing clinical decisions.

Methods. Data were collected retrospectively for patients who were admitted to ICU with SARS-CoV-2 and who received mechanical ventilation between March-May 2020 and October 2020-February 2021. Patients were grouped into 'non-survivors' and 'survivors' (survival to ICU discharge). Peak CRP and trough lymphocyte counts were obtained. Medians and quartile 1-3 ranges were calculated. Mann-Whitney $U$ test was used for statistical analysis with Bonferroni correction factor of 4 for multiple comparisons. Therefore, statistical significance of $5 \%$ was reduced to $p$-value of $\underline{0.0125}$ as a cut-off. We compared survivors versus non-survivors and their counterparts between the waves.

Results. Data were analysed for a total of 34 patients during the first wave and 39 patients in the second wave. There was no difference in mean age among the groups. For the first and second waves, mortality rate for ventilated patients was $41 \%(n=14)$ and $44 \%(n=17)$ respectively. Results for survivors versus non-survivors as below:

\begin{tabular}{|c|c|c|c|c|c|}
\hline \multicolumn{3}{|c|}{ Survivors } & \multicolumn{3}{|c|}{ Non-survivors } \\
\hline Wave $\mathrm{N}$ & Peak CRP & $\begin{array}{l}\text { Trough lym- } \\
\text { phocytes }\end{array}$ & Wave $\mathrm{N}$ & Peak CRP & $\begin{array}{l}\text { Trough lym- } \\
\text { phocytes }\end{array}$ \\
\hline 1 & $\begin{array}{l}318.5 \\
\quad(257-356)\end{array}$ & $\mathbf{0 . 6}(0.5-0.8)$ & 1 & $\begin{array}{l}347 \\
(288-455)\end{array}$ & $0.55(0.4-0.7)$ \\
\hline 2 & $\begin{array}{l}234 \\
(150-270)\end{array}$ & $\mathbf{0 . 5}(0.3-0.6)$ & 2 & $\begin{array}{l}333 \\
(244-388)\end{array}$ & $\mathbf{0 . 3 5}(0.3-0.5)$ \\
\hline
\end{tabular}

Table 1: medians and quartiles (1-3) of peak CRPs and trough lymphocytes for survivors versus non-survivors of the first and second waves. Regarding the trough lymphocyte counts, there was no statistical difference between any of the groups correspondingly. Regarding the peak CRP levels: there was a trend towards the second wave survivors having lower peak CRPs as compared with the second wave non-survivors $(p-0.021)$ or the first wave survivors $(p-0.017)$, but these have not reached the target $\mathrm{p}$-value.

Conclusion. The trend of the second wave SARS-CoV-2 survivors towards lower peak CRP could have reached the statistical significance if 10 patients' data ( $10 \%$ mortality) were not rendered unavailable due to non-clinical transfers. This is plausible as there was a ubiquitous use of steroids during the second wave secondary to recommendations of the Recovery trial. 'CRP responsiveness' to steroids was linked to improved survival previously. Finally, in our small cohort, peak CRP does not clearly differentiate between survivors and non-survivors. Therefore, other clinical parameters need to be considered for this purpose in larger reviews.

\section{Reference(s)}

1. Ahnach, M., Zbiri, S., Nejjari, S., Ousti, F., Elkettani, C. (2020). C-reactive protein as an early predictor of COVID-19 severity. J Med Biochem.39(4): 500-507

2. Cui, Z, Merritt, Z, Assa, A., Mustehsan, H., Chung, E, Liu, Sichen.,Kumthekar, A., Ayesha, B., McCort, M., Palaiodimos, L., Baron, S., Averbukh, Y., Southern, W., Aora, S. (2021). Early and Significant Reduction in C-Reactive Protein Levels After Corticosteroid Therapy Is Associated With Reduced Mortality in Patients With COVID-19. J. Hosp. Med 16(3):142-148.

3. Potempa, L.A., Rajab, I.M., Hart, P.C., Bardon, J., Fernandez-Botran, R. (2020). Insights into the Use of C-Reactive Protein as a Diagnostic Index of Disease Severity in COVID-19 Infections. Am J Trop Med Hyg.103(2): 561-563.

4. Smilowitz, N.R., Kunichoff, D., Garshick, M., Shah, B., Pilinger, M., Hochman, J.S., Berger, J.S. (2021). C-reactive protein and clinical outcomes in patients with COVID-19.European Heart Journal. ehaa1 103, https://doi.org/10. 1093/eurheartj/ehaa1103.

5. The WHO Rapid Evidence Appraisal for COVID-19 Therapies (REACT) Working Group. (2020). Association Between Administration of Systemic
Corticosteroids and Mortality Among Critically III Patients With COVID-19: A Meta-analysis. JAMA. 324(13):1330-1341.

\section{0}

Factors associated with mortality in COVID-19: a retrospective cohort study in low-middle income country of Latin America R. Santos Oliveira Jr' ; M. Pina ; R. Menezes, ${ }^{2}$; I. Ferreira, ${ }^{3}$; Q. Oliveira, ${ }^{4}$; R. Vieira, ; B. Darzé, ; C. Cunha, ; L. Amorim, ; ;. Vicente, ; J. Ramos, ; E. Darzé, ; L. Ritt, ; NM. Filgueiras Filho ${ }^{9}$

${ }^{1}$ Research, Teaching and Communication Center, Hospital da Cidade, Salvador, Brazil; ${ }^{2}$ Inflammation and Biomarkers Laboratory, Oswaldo Cruz Foundation, Salvador, Brazil; ${ }^{2}$ Life Sciences Department, University of State of Bahia, Salvador, Brazil; ${ }^{4}$ Research center, Hospital Cárdio Pulmonar, Salvador, Brazil; ${ }^{5}$ Critical care, Hospital e Clínica São Roque, Ipiaú, Brazil; ${ }^{6}$ School of medicine, BAHIANA — School of Medicine and Public Health, Salvador, Brazil; ${ }^{7}$ Board of Directors, Hospital Cárdio Pulmonar, Salvador, Brazil; ${ }^{8}$ Training, Teaching and Research Manager, Hospital Cárdio Pulmonar, Salvador, Brazil; ${ }^{9}$ Medicine, Salvador University, Campus Teacher Barros, Salvador, Brazil

Correspondence: N.M. Filgueiras Filho

Intensive Care Medicine Experimental 2021, 9(1): 000710

Introduction. The COVID-19 pandemic remains active, being responsible for an increase in admissions in intensive care units (ICU) and deaths worldwide. Thus, understanding the factors associated with mortality in COVID-19 patients is of vital importance for healthcare workers to improve the management and disease outcomes.

Objectives. To describe the epidemiological and clinical profile and evolutionary findings of patients with COVID-19 in multiple centers in developing country of Latin America, and to analyze factors associated with mortality.

Methods. Multi-center, retrospective cohort, which evaluated admitted patients due COVID-19 from 6 tertiary care hospitals in Brazil, from March 2020 to October 2020. Patients were compared based on clinical outcome. We collected demographic, clinical and laboratorial, therapeutic, complications and outcomes data. Results were described using mean \pm standard deviation or frequency (percentage). Differences between continuous variables were analyzed using T-test, while categorical variables were assessed using Chi2 or Fisher exact test. To assess mortality, a binary logistic regression was performed, which included the characteristics that showed a possible association with the outcome in univariate analysis $(p<0.1)$. ROC curves were used to determine the ideal cutoff values for continuous variables. This study was approved by the ethical and research committee.

Results. There were 430 patients included in the analysis. $257(60 \%)$ were male with an average age of $63 \pm 18$ years, and mean BMI was $29 \pm 6 \mathrm{~kg} / \mathrm{m}^{2} .53 .5 \%$ had a history of hypertension, $27.4 \%$ diabetes mellitus, $7.9 \%$ previous ischaemic heart disease, $9.3 \%$ chronic kidney disease, $39.8 \%$ obesity. Ground-glass opacity was the most common finding in lung CT scan (78\%). Need for admission in ICU occurred in 201 patients $(46.7 \%)$, and mean time between symptom onset and admission on ICU were $7 \pm 6$ days. Regarding life-support measures in ICU, mechanical ventilation was required in $44.2 \%$ of patients, proneposition $34.3 \%$, vasopressor therapy $45 \%$, and $23.3 \%$ underwent dialysis. Global mortality rate was $16.3 \%$, while at ICU the rate was $31.8 \%$. In the multivariate analysis, we identified the following variables related to mortality: age $>70$ years old (odds ratio [OR], $7.65 ; 95 \%$ confidence interval $[\mathrm{Cl}], 2.05-28.5 ; \mathrm{p}<0.002)$, absence of myalgia (OR 10.1; $95 \% \mathrm{Cl}$ $1.56-64.1 ; p<0.01$ ), asthenia (OR $4.1 ; 95 \% \mathrm{Cl} 1.14-15.3 ; p<0.03$ ), hypertension (OR $6.1 ; 95 \% \mathrm{Cl} 1.25-29.7 ; \mathrm{p}<0.02$ ), and lactate $>1.08 \mathrm{mg} / \mathrm{dL}$ (OR 2.62; 95\% Cl 2.62-61.2; $\mathrm{p}<0.002$ ).

Conclusion. This cohort in Brazil's Northeastern region has shown that early and easy detectable risk factors such as age, hypertension, lactate, asthenia and absence of myalgia are independently associated with mortality. Our findings could promote the development of an accessible and simple score in COVID-19 that can be useful in predicting mortality in these patients. 
Reference(s)

1. World Health Organization. COVID-19 Weekly Epidemiological Update 22. World Heal Organ. 2020;(December):1-3. https://www.who.int/docs/ default-source/coronaviruse/situation-reports/weekly_epidemiological_ update_22.pdf

2. Petrilli CM, Jones SA, Yang J, et al. Factors associated with hospital admission and critical illness among 5279 people with coronavirus disease 2019 in New York City: Prospective cohort study. BMJ. 2020;369. https:// doi.org/10.1136/bmj.m1966

3. Booth A, Reed AB, Ponzo S, et al. Population risk factors for severe disease and mortality in COVID-19: A global systematic review and meta-analysis. PLoS One. 2021;16(3):e0247461. Published 2021 Mar 4. https://doi.org/ 10.1371/journal.pone.0247461

4. No funding has been required.

\section{5}

Tocilizumab Association with Emerging Multi-drug Resistance Organisms and Mortality in critically ill patients with Coronavirus disease 2019 (COVID-19): A Multicenter, Retrospective Cohort Study

O. Aljuhani ${ }^{1}$; K. Al Sulaiman²; A. Adel ${ }^{3}$; K. Eljaaly ${ }^{4}$; A. Haytham³ ; A. Mohammed ${ }^{3}$;AD. Bodoor ; A. Amina ${ }^{4}$

${ }^{1}$ Faculty of Pharmacy, Pharmacy Practice, King Abdulaziz University, Jeddah, Saudi Arabia; ${ }^{2}$ Pharmaceutical Care Department, King Abdulaziz Medical City, Riyadh, Saudi Arabia; ${ }^{3}$ Department of Anesthesia and Intensive Care, King Abdulaziz University Hospital, Jeddah, Saudi Arabia; ${ }^{4}$ Pharmacy Practice Department, Faculty of Pharmacy, King Abdulaziz University, Jeddah, Saudi Arabia; ${ }^{5}$ Pharmaceutical Care Department, King Abdulaziz University Hospital, Jeddah, Saudi Arabia

Correspondence: $\mathrm{O}$. Aljuhani

Intensive Care Medicine Experimental 2021, 9(1): 000755

Introduction. Tocilizumab is an IgG1 class recombinant humanized monoclonal antibody directly working on the receptor interleukin-6 (IL-6) (9). Several RCTs have evaluated tocilizumab safety and efficacy in patients with COVID-19. Even though tocilizumab was associated with a lower incidence of serious infections (11), new evidence has emerged about the increased risk of new Delhi Metallo-Beta-Lactamase-Producing Carbapenem-Resistant Enterobacteria (NDM-CRE) acquisition in patients with COVID-19 (13). The safety of tocilizumab in patients with COVID-19 in relation to the risk of emerging resistant coinfections and its effect on mortality was not adequately assessed in any previous studies.

Objectives. To determine the association of treatment with tocilizumab and the incidence of emerging multidrug-resistant infections in critically ill patients with COVID-19.

Methods. A multicenter, retrospective, cohort study conducted at wo governmental tertiary hospitals in Saudi Arabia. Critically ill patients who were admitted to an ICU with positive COVID-19 PCR test between March 1st, 2020 to January 31st, 2021. Patients who recived tocilizumab comapred to no tocilizumab group.

Results. A total of 738 patients met our inclusion criteria and were included in the analysis. Of these 262 (35.5\%) received tocilizumab and $476(64.5 \%)$ were included in the control group. Patients who received tocilizumab were not associated with higher secondary infection (OR 1.34; $95 \% \mathrm{Cl} 0.91-1.94 \mathrm{p}=0.13$ ), resistant organisms (OR 1.00; $95 \%$ $\mathrm{Cl} 0.51-1.98 \mathrm{p}=0.99)$ nor CRE detection (OR $0.67 ; 95 \% \mathrm{Cl} 0.29-1.54$ $\mathrm{p}=0.34$ ). In a multivariable logistic regression adjusting for age, baseline SOFA scores, $\mathrm{PaO}_{2} / \mathrm{FiO}_{2}$ ratio, systemic corticosteroid use there was no difference in in-hospital mortality (OR 1.18; 95\% Cl 0.79-1.76 $\mathrm{p}=0.42$ ) or 30 -day ICU mortality (OR $0.96 ; 95 \% \mathrm{Cl} 0.65-1.43 \mathrm{p}=0.85$ ). However, among the non-mechanically ventilated patients with $24 \mathrm{~h}$ of ICU admission, there was a significant difference in the prevalence of respiratory failure requiring $\mathrm{MV}$ between the two groups (OR 2.27; $95 \% \mathrm{Cl} 1.05-4.89 \mathrm{p}=0.03$ ).

Conclusion. Tocilizumab use in COVID-19 critically ill patients was not associated with increased incidence of secondary infections, the emergence of resistant organisms, or mortality benefits but led to an increased risk of respiratory failure requiring mechanical ventilation.
Further randomized clinical and interventional studies are required to confirm our findings.

\section{Reference(s)}

1. 1. COVID-19 Dashboard by the Center for Systems Science and Engineering (CSSE) at Johns Hopkins University. Retrieved 1 February 2021. 2. Wiersinga WJ, Rhoades A, Cheng A, Peacock S, Prescott H. Pathophysiology, transmission, diagnosis, and treatment of coronavirus disease 2019 (COVID-19): a review. JAMA 2020;324:782e93. 3. Clinical features of patients infected with 2019 novel coronavirus in Wuhan, China. PubmedID 31,986,264 4. Chen X, Zhao B, Qu Y, Chen Y, Xiong J, Feng $Y$, et al. Detectable serum SARSCoV-2 viral load (RNAaemia) is closely correlated with drastically elevated interleukin 6 (IL-6) level in critically ill COVID-19 patients. Clin Infect Dis 2020;71:1937e42. https://doi.org/ 10.1093/cid/ciaa449. 5. Rosasl, BräuN, WatersM, et al. Tocilizumab in hospitalized patients with COVID-19 pneumonia. February 25, 2021 https://doi.org/10.1056/NEJMoa2028700 6. SalamaC, HanJ, YauL, et al. Tocilizumab in patients hospitalized with Covid-19 pneumonia. N Engl J Med. 2021;384:20-30. [Crossref], [PubMed], [Web of Science ${ }^{\circledR}$ ], [Google Scholar] 7. SalvaraniC, DolciG, MassariM, et al. Effect of tocilizumab vs. standard care on clinical worsening in patients hospitalized with COVID-19 pneumonia: a randomized clinical trial. JAMA Intern Med. 2021;181:24-31. [Crossref], [PubMed], [Web of Science ${ }^{\circledR}$ ], [Google Scholar] 8. McCreary EK, Pogue JM. Coronavirus disease 2019 treatment: a review of early and emerging options. Open Forum Infect Dis. 2020;7(4):ofaa1 05. 9. Sebba A. Tocilizumab: the first interleukin-6-receptor inhibitor. Am J Health Syst Pharm. 2008 Aug 1;65(15):1413-8. https:// doi.org/10.2146/ajhp070449. PMID: 18653811. 10. HermineO, MarietteX, TharauxPL, et al. Effect of tocilizumab vs. usual care in adults hospitalized with COVID-19 and moderate or severe pneumonia: a randomized clinical trial. JAMA Intern Med. 2021:181:32-40. [Crossref], [PubMed]. [Web of Science ${ }^{\circledR}$ ], [Google Scholar] 11. StoneJH, FrigaultMJ, Serling-BoydNJ, et al. Efficacy of tocilizumab in patients hospitalized with Covid-19. N Engl J Med. 2020;383:2333-2344. [Crossref], [PubMed], [Web of Science ${ }^{\circledR}$ ], [Google Scholar] 12. Tocilizumab in patients with severe COVID-19: a retrospective cohort study. PMID: 32835257 13. Porretta AD, Baggiani A, Arzilli G, Casigliani V, Mariotti T, Mariottini F, Scardina G, Sironi D, Totaro M, Barnini S, Privitera GP. Increased Risk of Acquisition of New Delhi Metallo-Beta-Lactamase-Producing Carbapenem-Resistant Enterobacterales (NDM-CRE) among a Cohort of COVID-19 Patients in a Teaching Hospital in Tuscany, Italy. Pathogens. 2020 Aug 5;9(8):635. https://doi. org/10.3390/pathogens9080635. PMID: 32764228; PMCID: PMC7459993. 14. Aleidan FAS, Alkhelaifi H, Alsenaid A, et al. Incidence and risk factors of carbapenem-resistant Enterobacteriaceae infection in intensive care units: a matched case-control study [published online ahead of print, 2020 Sep 20]. Expert Rev Anti Infect Ther. 2020:1-6. 15. The Clinical and Laboratory Standards Institute (CLSI) M60 ED2:2020 edition. (Accessed 02/09/2021) 16. Lin CY, Chen YC. Acute kidney injury classification: AKIN and RIFLE criteria in critical patients. World J Crit Care Med. 2012;1 (2):4045. Published 2012 Apr 4. https://doi.org/10.5492/wjccm.v1.i2.40 17. ICD-ICD-10-CM — International Classification of Diseases, Tenth Revision, Clinical Modification. Cdc.gov. https://www.cdc.gov/nchs/icd/icd10 $\mathrm{cm} . h t m$. Published 2021. Accessed January 29, 2021. 18. Magiorakos AP, Srinivasan A, Carey RB, et al. Multidrug-resistant, extensively drug-resistant and pandrug-resistant bacteria: an international expert proposal for interim standard definitions for acquired resistance. Clin Microbiol Infect. 2012;18(3):268-281. https://doi.org/10.1111/j.1469-0691.2011.03570.x 19. Ignatius EH, Wang K, Karaba A, Robinson M, Avery RK, Blair P, Chida N, Jain T, Petty BG, Siddiqui Z, Melia MT, Auwaerter PG, Xu Y, Garibaldi BT. Tocilizumab for the Treatment of COVID-19 Among Hospitalized Patients: A Matched Retrospective Cohort Analysis. Open Forum Infect Dis. 2020 Dec 28;8(1):ofaa598. https://doi.org/10.1093/ofid/ofaa598. PMID: 33537364; PMCID: PMC7798657. 20. Rajendram P, Sacha GL, Mehkri O, Wang X, Han X, Vachharajani V, Duggal A. Tocilizumab in Coronavirus Disease 2019-Related Critical IIIness: A Propensity Matched Analysis. Crit Care Explor. 2021 Jan 13;3(1):e0327. https://doi.org/10.1097/CCE.00000 00000000327. PMID: 33490955; PMCID: PMC7808529. 21. Campochiaro C, Della-Torre E, Cavalli G, et al. Efficacy and safety of tocilizumab in severe COVID-19 patients: a single-centre retrospective cohort study. Eur J Intern Med 2020; 76:43-9 22. Gupta S, Wang W, Hayek SS, et al. Association 
between early treatment with tocilizumab and mortality among critically ill patients with COVID-19. JAMA Intern Med 2020; e206252. 23. Soheila Rezaei, Behzad Fatemi, Zahra Karimi Majd, Hossein Minaei, Mohammad Peikanpour, Nassim Anjidani, Ali Taheri, Farzaneh Dastan \& Reza Mosaed (2021) Efficacy and safety of Tocilizumab in severe and critical COVID-19: A Systematic Review and Meta-Analysis, Expert Review of Clinical Immunology, https://doi.org/10.1080/1744666X.2021.1908128 24. Guaraldi G, Meschiari M, Cozzi-Lepri A, et al. Tocilizumab in patients with severe COVID-19: a retrospective cohort study. Lancet Rheumatol 2020; 2:e474-84. 25. Horby PW, Pessoa-Amorim G, Peto L, Brightling CE, Sarkar R, Thomas K et al. Tocilizumab in patients admitted to hospital with COVID-19 (RECOVERY): preliminary results of a randomised, controlled, open-label, platform trial. medRxiv. 2021:2021.02.11.21249258. https:// doi.org/10.1101/2021.02.11.21249258. 26. Veiga VC, Prats JAGG, Farias DLC, et al. Effect of tocilizumab on clinical outcomes at 15 days in patients with severe or critical coronavirus disease 2019: randomised controlled trial. BMJ. 2021;372:n84. Published 2021 Jan 20. https://doi. org/10.1136/bmj.n84 27. Rossotti R, Travi G, Ughi N, et al. Safety and efficacy of anti-il6-receptor tocilizumab use in severe and critical patients affected by coronavirus disease 2019: a comparative analysis. J Infect. 2020;81(4):e11-e17 28. https://www.cdc.gov/coronavirus/2019-ncov/ long-term-effects.html.Accessed on 21April,2021. 29. Price, Christina C et al. "Tocilizumab Treatment for Cytokine Release Syndrome in Hospitalized Patients With Coronavirus Disease 2019: Survival and Clinical Outcomes." Chest vol. 158,4 (2020): 1397-1408. https://doi.org/10.1016/j. chest.2020.06.006 30. Atallah, B., El Nekidy, W., Mallah, S.I. et al. Thrombotic events following tocilizumab therapy in critically ill COVID-19 patients: a Façade for prognostic markers. Thrombosis J 18, 22 (2020). https://doi. org/10.1186/s12959-020-00236-9 31. Bikdeli B, Madhavan MV, Jimenez D, Chuich T, et al. Global COVID-19 Thrombosis Collaborative Group, Endorsed by the ISTH, NATF, ESVM, and the IUA, Supported by the ESC Working Group on Pulmonary Circulation and Right Ventricular Function. COVID-19 and Thrombotic or Thromboembolic Disease: Implications for Prevention, Antithrombotic Therapy, and Follow-Up: JACC State-of-theArt Review. J Am Coll Cardiol. 2020 Jun 16;75(23):2950-2973. https:// doi.org/10.1016/j.jacc.2020.04.031. Epub 2020 Apr 17. PMID: 32,311,448; PMCID: PMC7164881

\section{3}

Severe Covid-19 infection in migrant population in Valencia, Spain

E. Sancho Ferrando ; R. Fortea Carrasco'; R. Martín Latorre'; S. Sancho Chinesta' ; F. ÁLvarez Cebrián

${ }^{1}$ Intensive Care Unit, Hospital Universitario y Politécnico La Fe, Valencia, Spain

Correspondence: E. Sancho Ferrando

Intensive Care Medicine Experimental 2021, 9(1): 000783

Introduction. Since the Covid-19 pandemic started, many clinical characteristics have been identified as risk factors. Other circumstances as socio-economic disparities and vulnerability that usually have an impact in health, seem to be also determinant in the virus spread. Some studies pointed that migrant population have higher coronavirus infection rates. Moreover, underprivileged socioeconomic and living conditions may increase Covid-19 mortality risk.

Objectives. The aim of this study is to describe and analyse the impact of being migrant in terms of intensive care unit (ICU) admission and clinical outcome in a population from Valencia, Spain.

Methods. This is a retrospective observational study. We recorded data from 2173 Covid- 19 adult patients admitted to a tertiary reference hospital in Valencia between 14th March 2020 and 21th February 2021 . The statistics were performed using RStudio software. After testing data for normality, Chi-squared test was applied to categoric variables and Mann-Whitney test for quantitative variables.

Results. Thirty-one percent of all hospitalized patients were immigrant (46 different countries). Mortality rate was $6 \%$ for migrant patients and $17 \%$ for Spanish patients admitted to hospital, with an odds ratio adjusted by age of 1 , showing no differences in the risk of dying. A total of 313 patients required admission in a critical care unit. We only included in the analysis 222 critically ill patients admitted to the medical ICU. Between these 222 critically ill patients, migrant population represented $38 \%$, significantly higher rate of migrant patients than between hospitalized patients, where it was $31 \%(p=0.02)$.

There were no differences in terms of gender or length of stay between both groups in ICU. Simplified Acute Physiology Score III (SAPS III) was higher for Spanish patients 53 points (Cl 46-62) vs 50 points $(\mathrm{Cl} 45-57)$ for migrants $(p=0.03)$. Spanish population was older 64 vs 55 years $(p<0.001)$. ICU mortality rates were $27 \%$ and $26 \%$ for migrant population and local population respectively. Nevertheless, when adjusted by SAPS III, ICU mortality risk was higher for migrant people, with an odds ratio of 2.04 ( $\mathrm{Cl} 0.94-4.50, \mathrm{p}=0.073$ ).

Conclusion. A high percentage of migrant population (31\%) was admitted to a tertiary hospital in Valencian Community where, in February 2021 , only $10 \%$ of immigrants were censed. Migrant population had higher risk to critically Covid-19 infection and despite lower age at ICU admission, had higher risk of dead.

\section{Reference(s)}

1. Shah GH, Shankar P, Schwind JS, Sittaramane V. The Detrimental Impact of the COVID-19 Crisis on Health Equity and Social Determinants of Health. J Public Health Manag Pract 2020;26:317-9. https://doi.org/10.1097/PHH. 0000000000001200.

2. Rostila M, Cederström A, Wallace M, Brandén M, Malmberg B, Andersson G. Disparities in COVID-19 deaths by country of birth in Stockholm, Sweden: A total population based cohort study. Am J Epidemiol 2021. https://doi.org/10.1093/aje/kwab057.

3. Paremoer L, Nandi S, Serag H, Baum F. Covid-19 pandemic and the social determinants of health. BMJ 2021;372:n129. https://doi.org/10.1136/bmj. n129.

000798

Evaluation of central line complications in COVID patients admitted to intensive care at the Queen Elizabeth Hospital Birmingham

A. Barrios ${ }^{1}$; P. Brown ${ }^{2}$; L. Taylor ${ }^{2}$

${ }^{1}$ Anaesthetics, Queen Elizabeth Hospital Birmingham, Birmingham, United Kingdom; ${ }^{2}$ Faculty of medicine, University of Birmingham, Birmingham, United Kingdom

Correspondence: A. Barrios

Intensive Care Medicine Experimental 2021, 9(1): 000798

Introduction. Central venous cannulation is necessary for the clinical management of the majority of COVID 19 patients admitted to intensive care (ICU). Patients critically ill with COVID 19 presented to ICU at the Qeen Elizabeth Hospital Birmingham (QEH) in surges that ocurred in April, October, November, December 2020 and January 2021. During the surges our ICU capacity was increased to $200 \%$. Physical proximity between patients made standard ICU processes difficult including infection prevention and control.

Objectives. We sought to identify complications associated with central venous catheters (lines) in COVID 19 patients admitted to ICU at QEH.

Methods. Using local Intensive Care National Audit and Research Centre data we identified all COVID 19 patients admitted to ITU at QEH between 01/03/2020 and 01/03/2021.

Using the Prescribing, Information and Communication System at QEH we determined the number of lines each patient had inserted, the number of days lines were in place for, complications at insertion and the reason lines were removed.

DEFINITIONS

CENTRAL LINE CONFIRMED INFECTION

Positive cultures in a central line.

Evidence of sepsis/septic shock.

Action by the clinical team in reference to the central line-e.g. change in antibiotics, removal of the central line.

CENTRAL LINE SUSPECTED INFECTION

Absence of positive cultures in a central line.

Evidence of sepsis/septic shock. 
Action by the clinical team in reference to the central line-e.g. change in antibiotics, removal of the central line.

MECHANICAL INDICATION FOR REMOVAL OF A CENTRAL LINE

Mechanical problems-e.g. impossible to run dialysis machine, malposition, rupture of the line, haemorrhage, blockage of the line. INDICATION CEASED

Clinical team's decision the line was not needed with no evidence of sepsis.

We did not study reasons for removing central lines that were in-situ when patients died or when patients were transferred out of ITU.

Results. Four hundred and fifty six patients had 1426 lines inserted. Complications at insertion were arterial puncture 2.1 per thousand, haematoma 0.7 per thousand and pneumothorax 0.7 per thousand. Two hundred and seventy eight lines were not studied for removal indication because of patient death (219 lines) and patient transfer (59 lines). Out of 1148 lines studied for removal indication, 25\% were removed because indication ceased but $4.97 \%$ were removed due to infection confirmed, $61.32 \%$ due to infection suspected and $8.71 \%$ due to mechanical failure. The percentage number of lines removed due to infection confirmed and infection suspected increased during the surge months to $14.04 \%$ and $10.09 \%$ in April $2020,8.77 \%$ and 10.09\% in December 2020 and $45.61 \%$ and $42.61 \%$ in January 2021 respectively.

Conclusion. At QEH, for COVID 19 patients who needed ICU, complications at central line insertion were very few. The minority of lines were removed due to indication ceased. The majority of lines were removed due to infection confirmed and infection suspected. We identified a trend towards infection concerns with lines increasing when our ICU increased its capacity in order to accomodate the surges in COVID 19 patients. Further research is warranted to identify the reasons line infections seem to have been more frequent during the surges in the COVID 19 pandemic in our ICU.

\section{Reference(s)}

1. Mermel, Leonard A., et al. "Clinical practice guidelines for the diagnosis and management of intravascular catheter-related infection: 2009 Update by the Infectious Diseases Society of America." Clinical infectious diseases 49.1 (2009): 1-45

\section{0}

The use of delta neutrophil index as diagnostic predictors of strangulated mechanical bowel obstruction in the surgery department

J. Fuss' ${ }^{1}$; A. Voloboyeva ${ }^{2}$; V. Polovyj ${ }^{3}$

'Department of Surgery, Regional Hospital of Pustomyty, Lviv, Ukraine;

${ }^{2}$ Department of Anestesiology, Communal Municipal Clinical Hospital 8 L'viv, Ukraine; ${ }^{3}$ Department of Surgery, Bukovinian State Medical University, Chernivtsi, Ukraine

Correspondence: J. Fuss

Intensive Care Medicine Experimental 2021, 9(1): 000840

Introduction. The issues of diagnosis and surgical treatment of patients with strangulated mechanical bowel obstruction (SMBO) are among the most difficult in urgent surgery [1]. This is due to the steady increase in the number of patients with this pathology, late admission to the hospital, diagnostic errors at the prehospital stage, a large number of postoperative complications, high mortality, which is $20-50 \%$ and does not tend to decrease [2].

The delta neutrophil index (DNI) is a novel inflammatory marker that measures the proportion of circulating immature granulocytes [3]. Since infectious conditions are associated with increased levels of immature granulocytes, several researchers have investigated whether this level can be used to predict the development of sepsis [4].

Objectives. Assess the predictive role of DNI as diagnostic predictors of strangulated mechanical bowel obstruction surgery department (SD).

Methods. This study was conducted in hospitals in Lviv and Pustomyty. The study included 258 patients with SMBO, including 167 men and 91 women. The age of patients ranged from 18 to 76 years and averaged $45.4 \pm 3.2$ years. All patients were divided into 2 groups depending on the form of mechanical obstruction: Group I $(n=154$; 59.7\%)-strangulation obstruction; Group II $(n=104 ; 40.3 \%)-$ obstructive obstruction.

The following information was obtained from patient medical records: age, sex, abdominal physical examination, cause and sites of obstruction, CT findings, and presence of strangulation. White blood cell (WBC), neutrophil count, C-reactive protein (CRP), and DNI were measured upon SD arrival. We calculated DNI from the leukocyte differentials using the following formula: $\mathrm{DNI}=$ (leukocyte subfraction assayed in the MPO channel by cytochemical reaction) - (leukocyte subfraction counted in the nuclear lobularity channel by the reflected light beam). Results. Common initial symptoms at SD presentation were abdominal pain $(99,8 \%)$, no passage of gas $(79.2 \%)$, and vomiting (54.6\%). 15 patients $(10.6 \%)$ were suspected to have strangulation of the bowel based on $\mathrm{CT}$ and emergency surgery was performed in 54 patients (14.4\%).

The median initial DNI value was the only factor that was significantly higher in the first group compared with the second group ( $0 \%$ vs $3.5 \%, P=0.003$ ).

In this study, of 57 patients in the I group, 5 (20\%) had strangulated bowel, as confirmed during surgery, but had no findings of strangulation on CT (i.e., false-negative CT finding). The median initial DNI of these patients was $15 \%$. Of the 83 patients in the second group, 5 also had confirmed nonstrangulated bowel but had findings of strangulation on $\mathrm{CT}$ (i.e., false-positive $\mathrm{CT}$ finding). The median initial DNI of these patients was $0 \%$.

Conclusion. In conclusion, initial DNI, which was measured in the $\mathrm{SD}$, was found to be significantly higher in the first group than in the second group. Initial DNI might be a useful additional parameter that could improve the prediction accuracy of CT.

\section{1}

Acute perforated ulcers of small intestine-cause of postoperative sepsis

J. Fuss ${ }^{1}$; A. Voloboyeva ${ }^{2}$; V. Polovyj ${ }^{3}$

${ }^{1}$ Department of Surgery, Regional Hospital of Pustomyty, Lviv, Ukraine 2Department of Anestesiology, Communal Municipal Clinical Hospital 8, L'viv, Ukraine; ${ }^{3}$ Department of Surgery, Bukovinian State Medical University, Chernivtsi, Ukraine

Correspondence: J. Fuss

Intensive Care Medicine Experimental 2021, 9(1): 000841

Introduction. The evolution of surgery has led to an increase in operative activity and complexity of surgical interventions, resulting in more postoperative complications that surgeons have not encountered before [1.2]. One such complication is acute perforated small bowel ulcer (APSBU) [3.4]. The problem of formation of ulcers of the upper gastrointestinal tract is known to all surgeons, as for small bowel ulcers-the problem is little covered in the modern literature [5].

Objectives. study of the main clinical and morphological features of APSBU, which arise as a complication after operations on the abdominal cavity.

Methods. The study included 56 patients who underwent surgical treatment in hospitals in Lviv and Pustomyty in period from 2015 to 2019. Patients underwent surgery on abdominal organs, the postoperative period of which was complicated by the development of APSBU. The mean age of patients was $72.8 \pm 9.7$ years.

Results. The structure of primary nosology was dominated by malignant neoplasms of different localization: cancers that required radical surgery occurred in $72.4 \%$, and most often surgery was performed for various forms of rectal cancer-27.6\% (18 patients). The second group by number were patients with acute adhesive small bowel obstruction of $20.9 \%$ (11 patients), whose primary operation was adhesiolysis. In $12.9 \%$ of cases (8 patients), resection of the colon was performed due to the development of complications. A clamped hernia of the anterior abdominal wall was also observed in $6.5 \%$.

Less than half of the patients had direct APSBU before the first relaparotomy -26 cases $(41.9 \%)$. The rest of the patients initially developed 
other complications that required relaparotomy: in $12.9 \%$ of casesearly adhesive postoperative obstruction, in $11.3 \%$ of cases-failure of the intestinal anastomosis, with the same frequency were patients with programmed abdominal rehabilitation on the background of early rehabilitation secondary peritonitis.

Speaking of the timing of the first APSBU after the primary operation, it should be noted a fairly wide range: from 2 to 28 days postoperatively, but in more than half of cases (53.2\%) the first perforation appears within 4 to 10 days. The formation of ulcers in the small intestine, as a rule, was multiple and recurrent.

In general, discussing the relationship of this complication with peritonitis and sepsis, it should be emphasized that in $65.3 \%$ of patients the first perforation of the small intestine developed on the background of peritonitis, and 27 patients (43.6\%) had diffuse serous fibrinous peritonitis. against the background of various forms of intestinal obstruction, $11(14.6 \%)$ had intestinal perforation, and in 5 patients $(8.1 \%)$ with the development of isolated peritonitis in the form of an abscess, and in 4 patients $(6.5 \%)$ with the development of diffuse fecal peritonitis, 2 (3.2\%) patients had widespread purulent peritonitis.

Conclusion. APSBU is especially dangerous due to the unpredictability of their occurrence and the high frequency of adverse effects. APSBU is a complication of various diseases that can occur from 2 to 28 days after surgery and can be multiple and recurrent.

\section{5}

\section{Clinical impact of empiric antibiotic therapy in severe COVID-19} patients at ICU admission: is really there something to treat?

JP. Cidade ${ }^{1}$; V. Costa, ${ }^{1}$; S. Brito ; B. Valerio, ; D. Nora ${ }^{1}$; C. Tapadinhas, ${ }^{1}$; P. Povoa ${ }^{1}$; L. Coelho, ${ }^{1}$; A. Tralhão ${ }^{1}$; C. Paulino, ; R. Morais, ; ;. Morais, ; ;. Fidalgo, ; P. Moniz, ${ }^{1}$ Polyvalent Intensive Care Unit, Hospital São Francisco De Xavier, Lisboa, Portugal

\section{Correspondence: J.P. Cidade}

Intensive Care Medicine Experimental 2021, 9(1): 000855

Introduction. COVID-19 is a new and complex form of hypoxemic acute respiratory failure, with several pathological pathways, leading to frequent progression to acute respiratory distress syndrome, multiorgan failure and ICU admission. Bacterial co-infections in COVID-19 patients, albeit what was initially thought, have been recognized as a rare event, in contrast to bacterial coinfection rates previously found in influenza infections (rates of approximately $20 \%$ ) [1-3]. This renders empiric antibiotic therapy (EAT), that was initially recommended [4], of limited value and possibly leading to antibiotic pressure and emergence of drug resistance. Furthermore, considering that the impact in clinical outcomes of bacterial co-infections in COVID-19 patients are still uncertain, the significance of EAT remains to be determined [5-6]. Objectives. This is a single center study aiming to describe the proportion of patients with severe COVID-19 and EAT at admission in ICU, and describe its clinical outcomes and mortality rate.

Methods. We performed a retrospective cohort study including all patients with COVID-19 diagnosed using clinical and radiologic criteria with a SARS-CoV-2 positive RT-PCR test. Patients were divided in 2 groups according to the presence of empiric antibiotic therapy: "EAT" and "control" groups. Qui-square test was used for categorical variables and Kruskal-Wallis and logistic regression were used on continuous variables for statistical assessment of outcomes between groups. Kaplan-Meier survival curve and log-rank test were also obtained.

Results. 110 patients (mean age 63 years, $73.6 \%$ males) were included in the analysis ( $\mathrm{N}=52$ EAT group; $\mathrm{N}=58$ control group), between March and September of 2020. Bacterial coinfection was documented in four patients in each group $(p=0.87)$. EAT was maintained for 5.4 days, in average. SOFA score at ICU admission $(p=0.06)$, C-reactive protein $(p=0.9)$ and procalcitonin $(p=0.7)$ serum levels at admission were not different between EAT and control groups. Similarly, ventilator-free days and vasopressor-free days were also not different between groups $(p=0.86)$. A trend to lower rate of nosocomial infections was observed in EAT group when compared to controls, but without statistical difference (14 events vs 26 events, respectively, $p=0.051$ ). Mortality rate and ICU length of stay were found to be similar in both groups $(p=0.4$ and $p=0.21$, respectively). Log rank test of Kaplan-Meier survival curves and Cox-regression analysis of survival were also not different between EAT and control groups $(p=0.422$ and $\mathrm{p}=0.424$, respectively) (Figure 1 ).

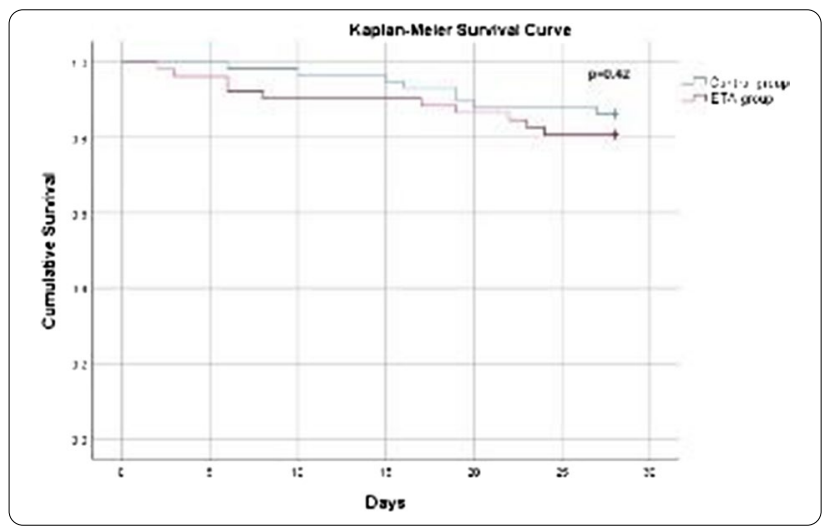

Conclusion. In severe COVID-19 patients, the use of empiric antibiotic therapy at ICU admission does not result in an improvement of clinical outcomes or survival. As result, we propose a more rationale use of empiric antibiotic therapy in these patients.

\section{Reference(s)}

1. 6-Lai CC, Wang CY, Hsueh PR. Co-infections among patients with COVID-19: The need for combination therapy with non-anti-SARS-CoV-2 agents? J Microbiol Immunol Infect. 2020 Aug;53(4):505-512. https:// doi.org/10.1016/j.jmii.2020.05.013. Epub 2020 May 23. PMID: 32482366; PMCID: PMC7245213.

2. 5-Silva DL, Lima CM, Magalhães VCR, Baltazar LM, Peres NTA, Caligiorne RB, Moura AS, Fereguetti T, Martins JC, Rabelo LF, Abrahão JS, Lyon AC, Johann S, Santos DA. Fungal and bacterial coinfections increase mortality of severely ill COVID-19 patients. J Hosp Infect. 2021 Apr 11:S01956701(21)00151-1. https://doi.org/10.1016/j.jhin.2021.04.001.

3. 4-Alhazzani W, Møller MH, Arabi YM, Loeb M, Gong MN, Fan E, Oczkowski S, Levy MM, Derde L, Dzierba A, Du B, Aboodi M, Wunsch H, Cecconi M, Koh Y, Chertow DS, Maitland K, Alshamsi F, Belley-Cote E, Greco M, Laundy M, Morgan JS, Kesecioglu J, McGeer A, Mermel L, Mammen MJ, Alexander PE, Arrington A, Centofanti JE, Citerio G, Baw B, Memish ZA, Hammond N, Hayden FG, Evans L, Rhodes A. Surviving Sepsis Campaign: Guidelines on the Management of Critically III Adults with Coronavirus Disease 2019 (COVID-19). Crit Care Med. 2020 Jun;48(6):e440-e469. https://doi.org/10.1097/CCM.0000000000004363. PMID: 32224769; PMCID: PMC7176264

4. 3-attorini L, Creti R, Palma C, Pantosti A; Unit of Antibiotic Resistance and Special Pathogens; Unit of Antibiotic Resistance and Special Pathogens of the Department of Infectious Diseases, Istituto Superiore di Sanità, Rome. Bacterial coinfections in COVID-19: an underestimated adversary. Ann Ist Super Sanita. 2020 Jul-Sep;56(3):359-364. https://doi. org/10.4415/ANN_20_03_14.PMID: 32959802.

5. 2-Martin-Loeches I, J Schultz M, Vincent JL, Alvarez-Lerma F, Bos LD, Solé-Violán J, Torres A, Rodriguez A. Increased incidence of co-infection in critically ill patients with influenza. Intensive Care Med. 2017 Jan;43(1):4858. https://doi.org/10.1007/s00134-016-4578-y. Epub 2016 Oct 5. PMID: 27709265.

6. 1-Langford BJ, So M, Raybardhan S, Leung V, Westwood D, MacFadden $D R$, Soucy JR, Daneman N. Bacterial co-infection and secondary infection in patients with COVID-19: a living rapid review and meta-analysis. Clin Microbiol Infect. 2020 Dec;26(12):1622-1629. https://doi.org/10. 1016/j.cmi.2020.07.016. Epub 2020 Jul 22. PMID: 32711058; PMCID: PMC7832079. 


\section{4}

Urinary catheter securement: safety profile and prevention of urinary tract infection. Final results from a randomized $c$ N. Calpe ; L. Wennberg, ${ }^{2}$; G. Remei ${ }^{3}$; G. Thais ${ }^{4} ;$ V. Arminda ${ }^{3}$; M. Llauradó, ${ }^{2}$ ${ }^{1}$ Intensive Care Unit, Hospital Universitari General de Catalunya; Universitat Internacional de Catalunya, Barcelona, Spain; ${ }^{2}$ Nursing department, UIC Barcelona -Universitat Internacional de Catalunya, Barcelona, Spain; ${ }^{3}$ Intensive Care Unit, Hospital Universitari General de Catalunya, BarceIona, Spain; ${ }^{4}$ intensive Care Unit, Parc de Salut Mar, Barcelona, Spain Correspondence: N. Calpe

Intensive Care Medicine Experimental 2021, 9(1): 000864

Introduction. Urinary catheter securement is recommended as part of care bundles for general Urinary Catheter (UC) care and for Catheter Associated Urinary Tract Infection (CAUTI) prevention (1,2). However prevalence studies show a low prevalence of catheter securement (3). Moreover, the relationship between this care and CAUTI and its safety have not been studied individually.

Objectives. The purpose of this study was to determinate the impact of securing urinary catheters on the rate of CAUTI and to analyse its safety profile.

Methods. Randomized, non-blinded multicentre clinical trial conducted in two general Intensive Care Units of two tertiary hospitals in Spain. Inclusion criteria: Age > 18, UC inserted in Intensive Care Unit or in the Operating room, informed consent. Exclusion criteria: Securement not possible, urinary tract infection at the admission, UC plan to be used and anticipated ICU stay for $48 \mathrm{~h}$ or more. Participants were randomized into the control group (CG), to which the $U C$ received traditional care, and the experimental group (EG), to which the UC was secured to the upper half thigh. Before securing, a barrier film was applied to protect the thigh skin from adhesive lesions. Protocols from both sites were equivalent. Data were obtained twice a day until study ends, including: patient's comfort, skin condition, properly done securement and sings of CAUTI. Study end was defined as: CAUTI diagnosis, $48 \mathrm{~h}$ after catheter removal, $48 \mathrm{~h}$ after ICU discharge or after 30 days of catheter. If CAUTI was suspected a urine sample was collected. A descriptive and bivariate analysis performed with SPSS 21 program. IRB approval from both sites was obtained.

Results. Three hundred and fifty patients were included, 196(56\%) males. Median age 63(53-72) years. Allocation: 169(48.29\%) IG, $181(51.71 \%)$ CG. Twenty-one CAUTI were diagnosed, nineteen of them from CG $(p<0.05)$. UC securement was independently related with CAUTI (RR $=11.112,95 \% \mathrm{Cl} 3.064,71.747)$. Survival analyses showed that survival was better in IG $(p=0.006)$ (HR $0.130,95 \% \mathrm{Cl} 0.030$, 0.565 ) Forty-two patients suffered urinary meatus injuries, thirty-four (80.95\%) from CG $(R R=1.702,95 \% \mathrm{Cl} 1.411,2.054)$. Extra securement changes were needed in patients with RASS $>0(\mathrm{RR}=2.127,95 \%$ IC $1.334,3.391)$. Twenty-six (1.06\%) securement-related skin injuries were found.

Conclusion. The results support that urinary catheter securement plays a role in decreasing CAUTI and it seems to be a safe practice. Securing urinary catheters is an easy nurse care that can imply great benefits for patients and institutions.

\section{Reference(s)}

1. Appah Y, Hunter KF, Moore KN. Securement of the Indwelling Urinary Catheter: A Prevalence Study. J Wound Ostomy Continence Nurs [Internet]. 2015;43(2):173-7. Available from: http://www.ncbi.nlm.nih.gov/ pubmed/26418849

2. Grupo de trabajo ITU-ZERO. Prevención de la infección de la sonda uretral en los pacientes críticos ingresados en las unidades de cuidados intensivos " PROYECTO ITU-ZERO." Madrid: SEMIYUC; 2018.

3. Sampathkumar P, Barth JW, Johnson M, Marosek N, Johnson M, Worden W, et al. Mayo Clinic reduces catheter-associated urinary tract infections through a bundled 6-C approach. Jt Comm J Qual Patient Saf. 2016:42(6):254-61.

4. This project has been granted by the Sociedad Sociedad Española de Enfermería Intensiva y Unidades Coronarias (SEEIUC) as the best research project in nursing. 2019

\section{5}

Characteristics, interventions and outcomes of COVID-19 ICU patients in Denmark during the first 2 waves of the SARS-CoV-2 pandemic-A nationwide, observational study

N. Haase ${ }^{1}$; LM. Poulsen ${ }^{2}$; S. Christensen ${ }^{3}$; AC. Brøchner ${ }^{4}$; BS. Rasmussen ${ }^{5}$; A Perner ${ }^{6}{ }_{i .}$. For The National Covid-19 In Icu Database Investigators ${ }^{7}$ ${ }^{1}$ Department of Intensive Care, Copenhagen University Hospital, Rigshospitalet, Copenhagen, Denmark; ${ }^{2}$ Department of Anaesthesiology, Intensive Care Unit, Zealand University Hospital, Køge, Denmark; ${ }^{3}$ Department of Anaesthesia and Intensive Care Medicine, Aarhus University Hospital, Aarhus, Denmark; ${ }^{4}$ Department of Anesthesiology and Intensive Care, Kolding Hospital, Kolding, Denmark; ${ }^{5}$ Department of Anesthesiology and Intensive Care, Aalborg University Hospital South, Aalborg, Denmark; ${ }^{6}$ Department of Intensive Care, Rigshospitalet, København, Denmark; ${ }^{7}$ Department of Anesthesiology and Intensive Care, Rigshospitalet, København, Denmark

Correspondence: $\mathrm{N}$. Haase

Intensive Care Medicine Experimental 2021, 9(1): 000865

Introduction. The SARS-CoV-2 pandemic hit Denmark in two waves in Spring 2020 and during winter 2020-21, respectively. We previously described the entire national cohort of patients with COVID-19 presenting to intensive care in Denmark during the first pandemic wave (1). It was unknown whether characteristics, use of organ support or outcomes would change during the continuous course of the pandemic.

Objectives. To describe Danish patients admitted to intensive care with COVID-19 with regard to characteristics, use of organ support and outcomes on a national level and to compare patients admitted early vs late during the pandemic.

Methods. Observational retrospective study with manual identification of all patients with COVID-19 admitted to Danish intensive care units (ICU) from 10th March 2020 (index patient) to 31st March 2021. Baseline characteristics, use of organ support and outcomes were extracted by hand from patient notes. Based on curves of ICU-admittance in Denmark, we arbitrarily defined two pandemic waves, the first from 10th March 2020 to 20th May 2020 and the second from 1st November 2020 to 28th February 2021. Descriptive statistics, Chi-square testing and non-parametric tests were used where appropriate.

Results. In the overall study period 1193 patients with COVID-19 were admitted to a Danish ICU; 326 were admitted during the 1st wave and 659 during the 2 nd. The average patient was a $68(58-75)$ years (median, IQR) old male with hypertension and a BMI of 28 (25-33) presenting to ICU 9 days after the initial COVID-19 symptom (Table 1). Overall, $65 \%$ of patients were mechanically ventilated for $13(6-23)$ days, $16 \%$ were treated with renal replacement therapy and $4 \%$ with ECMO. Length of ICU and hospital stay was 11 (5-21) and 17 (10-29) days respectively. Overall, hospital mortality was 35\% (95-Cl 33-38), but $6 \%$ were still admitted.

Baseline characteristics did not change from wave 1 to wave 2, but the fraction of all patients admitted to hospital who came to ICU went down from $13 \%$ to $7,5 \%$. From the 1 st wave 1 to the 2 nd, there was a marked decrease in the use of mechanical ventilation and renal replacement therapy from 81 to $61 \%$ and $26 \%$ to $13 \%$, respectively. ICU length of stay was 3 days shorter during wave 2 . Hospital mortality was similar during the two waves, $37 \%$ and $38 \%$, respectively (Table 2 ). Conclusion. In this nationwide cohort of ICU patients with COVID-19, hospital mortality was relatively low despite high age and substantial use of organ support. From wave 1 to 2, we observed a lower fraction of hospitalized patients with COVID-19 admitted to ICU and less use of organ support, but mortality remained the same.

\section{Reference(s)}

1. Haase et al. Characteristics, interventions, and longer term outcomes of COVID-19 ICU patients in Denmark-A nationwide, observational study. Acta Anaesthesiol Scand. 2021 Jan;65(1):68-75. https://doi.org/10.1111/ aas.13701

2. The study was funded by the participating intensive care units and The Danish Clinical Quality Program (RKKP) 
000873

Multidrug-resistant bacteria, fungal microorganism

and nosocomial bacterial infections in patients

with severe pneumonia by SARS-COV2 in relation to use

of immunomodulators, long-term and high dose corticosteroids

and prolonged-course antibiotic therapy

E. Recart Batrakova ${ }^{1}$; D. Mora, ; I. Villa, ; M. Dominguez, ; A. Ruíz, ${ }^{1}$; P.

Domínguez, ; E. Pino,

${ }^{1}$ Critical Care Unit, Hospital Universitario Juan Ramón Jiménez, Huelva,

Spain

Correspondence: E. Recart Batrakova

Intensive Care Medicine Experimental 2021, 9(1): 000873

Introduction. Since the beginning of the COVID-19 pandemic, we have faced uncertainty, given the lack of scientific evidence in this field. We have had to use many aggressive treatments whose effects can be harmful and without being clear about their impact on the evolution of this disease. At the same time, we notice a growing emergence of nosocomial infections, fungal microorganism and multidrugresistant bacteria in this population.

Objectives. Evaluate if the use of immunomodulators (Tocilizumab, Interferon and Sarilumab), long-term use of corticosteroids ( $>7$ days), corticosteroids in high doses (boluses of Methylprednisolone $>250 \mathrm{mg}$ ) and prolonged-course antibiotic therapy, may have a direct relationship with emergence of multidrug-resistant bacteria, fungal microorganism and nosocomial bacterial infections in patients with COVID-19.

Methods. Descriptive and analytical observational study of a retrospective cohort of patients with severe pneumonia due to SARS-CoV2, admitted to our intensive care unit, between March 2020 to April 2021. We have collected data from all patients, successively admitted to our ICU, since the onset of the Pandemic. We have used SPSS statistical software for statistical analysis, median description for continuous data and RR calculation for associations with $95 \% \mathrm{Cl}$ for statistical significance.

Results. The cohort consists of 119 patients, of which $65,5 \%$ are men, with median age of $66(20-81)$ years. The median stay at the hospital was 23 (4-138) days, at ICU was $13(1-128)$ days and the median of mechanical ventilation was $5(0-121)$ days. Median use of antibiotherapy until the appearance of the first multidrug-resistant bacterium or fungal microorganism was $6(0-68)$ days, being its use by $>5$ days at $52,9 \%$ of patients. The median of corticosteroids use was $11(0-65)$ days and 2 (0-6) bolus of Metilaprednisolone $>250 \mathrm{mg}$ have been received. Use of immunomodulator was at $73,1 \%$ of cases. Presence of multidrug-resistant bacteria or fungal microorganism in respiratory sample, blood, urine, rectal or nasal exudate was $29,4 \%$, fungal microorganism in respiratory sample or blood was $21,8 \%$ and nosocomial bacterial infection was $41,1 \%$. Global mortality was $30,2 \%$, being this $38,2 \%$ in patients who have presented a multidrug-resistant bacteria or fungal microorganism and $48,9 \%$ in patients who developed a nosocomial bacterial infection. There has been a significant association of use of antibiotherapy by $>5$ days for both group, who have had a multidrug-resistant bacteria or fungal micro-organism $(52,3 \%$ vs $25 \%$ RR $2,09(1,25-3,49)$, as those that developed a nosocomial bacterial infection $(53,9 \%$ vs $26,7 \%$ RR $2,01(1,23-3,28)$, being also a significantly higher mortality in both groups, $38,2 \%$ vs $19,4 \%$ RR 1, 97 $(1,08-3,56)$ and $40,6 \%$ vs $17,1 \%$ RR $2,85(1,58-5,14)$, respectively. No significant association of use of immunomodulator, long-term use of corticosteroids and bolus of Metilprednisolona has been seen in any of the groups. But, it should be notice, a trend of possible influence of prolonged use of corticosteroids $(43,7 \%$ vs $30,4 \%$ RR $1,43(0,74-2,77)$ and bolus of high dose $(42,3 \%$ vs $38,2 \%$ RR $1,10(0,67-1,81)$ in development of nosocomial bacterial infection, although not significant.

Conclusion. The use of immunomodulators and corticosteroids, in patients with severe SARS-COV2 pneumonia, wasn't associated with multi-resistant bacteria, fungal microorganism or nosocomial bacterial infections. However, the use of corticosteroids presents a non-statistical risk of nosocomial bacterial infections. Finally, being the coinfection rate in Covid-19 by bacteria, according to literature, low (3.5-14.3\%), we are using prolonged antibiotherapy in $52 \%$ of patients. That point is important, given that it is significantly associated with both, multi-resistant bacteria or fungal and development of nosocomial bacterial infections, and being a higher mortality in these groups of patients. In this way, may be, we must replant the use of coritcoides at high doses and prolonged-course, and in the same way, with empirical antibiotherapy.

\section{Reference(s)}

1. Bradley J. Langford, Miranda So, Sumit Raybardhan, Valerie Leung, JeanPaul R. Soucy, Duncan Westwood, Nick Daneman, Derek R. MacFadden. Antibiotic prescribing in patients with COVID-19: rapid review and meta-analysis.

2. P. Fernández, L. Moreno, G. Yagüe, E, Andreu, R. Jara, M. Segovia. Colonización por microorganismos multirresistentes en pacientes de UCI durante la pandemia de la COVID-19. Medicina Intensiva 2021;45:313-319

3. Andrew R.Mahoney, Mohammad Moein Safaee, William M Wues, Doris Rusic, Marino Volovic, Josipa Bukic, Dario Leskur, Ana Seselja Perisin, Marko Kumric, Dinko Martinovic, Ana Petric, Darko Modun, Josko Bozik. Implication of COVID-19 pandemic on the emergence of antimicrobial resistence: adjusting the response to the future outbreaks. Life 2021: 11(220):1-15

4. Soumya Ghosh, Charné Broman, Mai M. Zafer. Antimicrobial resistence treats in the emerging COVID-19 pandemic: where do we stand? Journal of infection and public health 2021;14:555-560

\section{8}

The use of Procalcitonin to Improve Antibiotic Stewardship in Intensive Care during the COVID Pandemic

D. Goold'; S. Gopal

${ }^{1}$ Critical Care, The Royal Wolverhampton NHS Trust, West Midlands, United Kingdom

\section{Correspondence: D. Goold}

Intensive Care Medicine Experimental 2021, 9(1): 000908

Introduction. The 2019 SARS-CoV-2 pandemic created significant pressures on intensive care services. One of the many challenges was rationalising the use of antibiotics in patients with suspected secondary bacterial infection due to the majority of patients showing diffuse pulmonary infiltrates and raised inflammatory markers. Procalcitonin is a biomarker that, if measured at a level greater than $0.5 \mathrm{ng} /$ $\mathrm{ml}$ in acutely unwell patients suggests a bacterial infection is present (Schuetz et al. 2019). In April 2020 NICE and local guidance did not advocate its routine use but encouraged centres to participate in data collection (National Institute for Health and Care Excellence 2020).

Objectives. We undertook a quality improvement project to ascertain the benefits of procalcitonin with regards to its antibiotic stewardship in COVID 19 patients.

Methods. A retrospective analysis of 80 COVID-19 patients in Newcross Hospital Wolverhampton UK between March and June 2020 was performed. Patients were stratified into whether they had a procalcitonin measurement during their ICU Stay. Primary comparisons were made with regards to the duration of days during ICU admission when patients were on antibiotics.

Results. Routine procalcitonin levels were performed from April 2020 , leading to 56 patients having procalcitonin measurements and 24 without. There was no statistical difference in the median (IQR) age [62 (16) vs 64.5 (16), $p=0.56$ ]; APACHE II scores [17.0 (8) vs 16.5 (6), $p=0.49$ ] and number of days that patients were on antibiotics [11 (11) vs 7.5 (11), $p=0.29$ ] between those who did and did not have a procalcitonin measurement respectively. However patients who had a procalcitonin measurement had a significantly longer ICU median (IQR) stay [20 (15) vs $10(11), \mathrm{p}<0.01]$ and when comparisons were made for the percentage of days during an ICU stay when a patient was on antibiotics, those who had a procalcitonin measurement had significantly fewer percentage of days on antibiotics [59.5\% (49.25\%) vs $100 \%(28.7 \%) p<0.001]$. 


\begin{tabular}{|c|c|c|c|}
\hline & $\begin{array}{l}\text { Had a Procalci- } \\
\text { tonin Measure- } \\
\text { ment }\end{array}$ & $\begin{array}{l}\text { Didn't have a } \\
\text { Procalcitonin } \\
\text { Measurement }\end{array}$ & Significance \\
\hline Number & 56 & 24 & - \\
\hline Age, Years + (IQR) & $62(16)$ & $64.5(16)$ & $p=0.56^{*}$ \\
\hline $\begin{array}{l}\text { APACHE } \| \\
\text { Score + (IQR) }\end{array}$ & $17.0(8)$ & $16.5(6)$ & $p=0.49^{*}$ \\
\hline $\begin{array}{l}\text { Number of days } \\
\text { on antibiot- } \\
\text { ics + (IQR) }\end{array}$ & $11(11)$ & $7.5(11)$ & $p=0.298^{*}$ \\
\hline $\begin{array}{l}\text { Length of Stay } \\
\text { (Days) }+(\text { (QR) }\end{array}$ & $20(15)$ & $10(11)$ & $p<0.01^{*}$ \\
\hline $\begin{array}{l}\text { \% of days during } \\
\text { ICU admission } \\
\text { on antibiot- } \\
\text { iCS + (IQR) }\end{array}$ & $59.5 \%(49.25 \%)$ & $100 \%$ (28.7\%) & $P<0.001^{*}$ \\
\hline \multicolumn{4}{|c|}{$\begin{array}{l}\text { *Comparisons were made with the Mann Whitney U Test + Date is pre- } \\
\text { sented with Median values and Inter Quartile Range in brackets }\end{array}$} \\
\hline
\end{tabular}

Conclusion. Routine measurement of procalcitonin levels were associated with a significant reduction in the duration of antibiotic use in our intensive care unit. Further rigorous studies are required to investigate the potential benefit of procalcitonin on a broader scale for general ICU admissions with regards to antibiotic resistance and cost efficiency.

\section{Reference(s)}

1. Nil.

2. Schuetz, P, Beishuizen, A, Broyles, M, Ferrer, R, Gavazzi, G, Gluck, EH, González Del Castillo, J, Jensen, J-U, et al. 2019. Procalcitonin (PCT)guided antibiotic stewardship: an international experts consensus on optimized clinical use. Clinical chemistry and laboratory medicine: CCLM / FESCC. 57(9):1308-1318.

3. National Institute for Health and Care Excellence. 2020. COVID-19 rapid guideline: antibiotics for pneumonia in adults in hospital. https://www. nice.org.uk/guidance/ng173 (Accessed 13 May 2021).

\section{5}

\section{Viral and fungal infections in critically ill COVID-19 patients}

K. Bentoumi ; A. Cudia'; P. Foulon ${ }^{1}$; K. Kaefer ${ }^{1}$; S. Fratino'; D. De Backer ${ }^{1}$

'Department of intensive care, CHIREC Hospitals, Université Libre de Bruxelles, Brussels, Belgium

Correspondence: D. De Backer

Intensive Care Medicine Experimental 2021, 9(1): 000915

Introduction. Preliminary reports suggest that viral reactivation and aspergillus infections may occur in critically ill COVID-19 patients (1). Objectives. To evaluate the incidence and potential clinical complications of viral and fungal infections in critically ill COVID-19 patients.

Methods. This retrospective cohort study included all consecutive critically ill patients with a positive diagnosis of SARS-Cov-2 pneumonia (positive nasopharyngeal or bronchial PCR and/or CT Scan images suggestive for COVID-19 infection) admitted between March and December 2020 in our Intensive Care Department.

Viral infection/reactivation was identified as a positive multiplex PCR on broncho-alveolar lavage or ELISA serologic test. Aspergillosis was detected by a positive galactomannan or PCR on bronchoalveolar lavage.

Data are presented as median (interquartile range) or number (percentage) and compared with Mann-Whitney or Fischer exact test.

Results. 202 patients were included. Viral infection/reactivation was observed in 51 patients (25\%). Among the virus observed, cytomegalovirus, and herpes virus (HSV 1 and 2 and HHV6) were the commonest. Aspergillosis was observed in 18 patients (9\%), 14 (78\%) of which also presented viral infection/reactivation.
There were no differences in age, comorbidities, use of immunosuppressive drugs before hospital admission and physiologic variables on ICU admission between patients without and with viral infection/reactivation or aspergillosis.

Compared to patients without viral infection/reactivation, patients with viral infection/reactivation had an increased duration of mechanical ventilation (38 $(18-60)$ vs $18(14-31)$ days, $p<0.001)$ and longer ICU length of stay (47 (25-70) vs $20(11-38), p<0.001)$, even though mortality was not different ( 33 vs $22 \%, p=0.20$ ).

Aspergillus infection was also associated with increased duration of mechanical ventilation (45 (25-62) vs $18(14-31)$ days, $\mathrm{p}<0.001)$ and ICU length of stay $(54(37-100)$ vs $20(11-38), p<0.001)$ even though mortality was not different ( 29 vs $22 \%, p=0.20$ ). Interestingly, there was no difference in duration in mechanical ventilation, ICU length of stay and mortality between patient with viral infection coinfected or not by aspergillus.

Conclusion. Viral infection/reactivation and aspergillosis are frequent in severe COVID patients. Viral or aspergillus coinfections are associated with prolonged mechanical ventilation and ICU stay.

\section{Reference(s)}

1. 1. Van Biesen S, Kwa D, Bosman RJ, Juffermans NP. Detection of Invasive Pulmonary Aspergillosis in COVID-19 with Non-directed Bronchoalveolar Lavage. Am J Respir Crit Care Med. 2020;202:1171-3

\section{8}

Impact of age, sex and ethnicity on specific and non-specific host responses to the SARS-CoV-2 infection

A. Kulkarni ${ }^{1} ;$ T. Snow ${ }^{2}$; N. Arulkumaran ${ }^{2}$; M. Singer ${ }^{2}$

${ }^{1}$ Dept of Critical care, University college london, London, United

Kingdom; ${ }^{2}$ Bloomsbury Institute of Intensive Care Medicine, University

College London, London, United Kingdom

Correspondence: A. kulkarni

Intensive Care Medicine Experimental 2021, 9(1): 000918

Introduction. As the pathobiology of COVID-19 is multifactorial, an integrated approach combining patient demographics, routine biochemistry, and markers of specific and non-specific host immune response may provide important insights. 1

Objectives. Differences in immune responses to COVID-19 may underpin sex-specific outcome differences. To understand whether this process might be a contributor to the pathophysiology, we examined sex differences in physiology, viral loads, SARS-CoV-2-specific antibody titres, and plasma cytokines on hospital admission in patients who had not received immunomodulatory therapies.

Methods. Demographic, clinical data, treatments and WHO COVID-19 severity scale3 outcome data were collected from adult patients positive for COVID-19 within 5 days of hospital admission $(n=86)$. Comparison was made against healthy $(n=7)$ and infected asymptomatic $(n=11)$ volunteers. Serum samples were analysed for inflammatory mediator levels, SARS-CoV-2 antibody levels and pseudotype neutralisation assays.

Results. Of 86 patients admitted, 44 patients had mild disease, 22 progressed to critical illness, and 20 subsequently died in hospital. Patients who did not seroconvert at presentation had higher mortality than those who did $(p=0.025)$. Significant differences in serum cytokine levels were seen between survivors and non-survivors for IL-6 $(p=0.020), I L-10(p=0.032)$, and IP-10 $(p=0.030)$. Notably, male patients had higher cytokine levels than females but no differences were seen with age or ethnicity. Advancing age (adjusted Hazard Ratio 1.064 (1.019-1.112); $\mathrm{p}=0.005)$, lower $\mathrm{SpO2}: \mathrm{FiO}_{2}$ ratio (adjHR 0.994 (0.990-0.998); $p=0.004)$, and days from symptom onset to hospital presentation (adjHR $0.85(0.728-0.993) ; p=0.004)$ were independent predictors of mortality. (See Table 1) 


\begin{tabular}{|c|c|c|c|c|}
\hline & Total $(n=86)$ & $\begin{array}{l}\text { Female } \\
(n=30)\end{array}$ & Male $(n=56)$ & $\begin{array}{l}\text { p-value (Male } \\
\text { vs. Female) }\end{array}$ \\
\hline Age (yrs) & $61(48-73)$ & $59(42-72)$ & $64(49-73)$ & 0.517 \\
\hline $\begin{array}{l}\text { Symptoms to } \\
\text { hospital (days) }\end{array}$ & $7(4-11)$ & $7(3-12)$ & $7(4-11)$ & 0.771 \\
\hline $\mathrm{SpO} 2: \mathrm{FiO}_{2}$ ratio & $438(378-462)$ & $454(414-464)$ & $433(376-457)$ & 0.133 \\
\hline RR & $26(20-32)$ & $26(19-32)$ & $26(20-35)$ & 0.685 \\
\hline Temperature $\left({ }^{\circ} \mathrm{C}\right)$ & $37.5(36.9-38.4)$ & $37.5(36.8-38.2)$ & $37.6(37.0-38.5)$ & 0.544 \\
\hline Ct value & $37(32-40)$ & $38(33-40)$ & $37(32-39)$ & 0.753 \\
\hline $\begin{array}{l}\text { Diabetes mellitus } \\
(\%)\end{array}$ & $18(21 \%)$ & $5(17 \%)$ & $13(24 \%)$ & 0.452 \\
\hline Hypertension (\%) & 30 (35\%) & $10(33 \%)$ & 20 (36\%) & 0.780 \\
\hline $\begin{array}{l}\text { Creatinine } \\
(\mu \mathrm{mol} / \mathrm{L})\end{array}$ & $88(68-114)$ & 68 (59-89) & $97(81-125)$ & 0.001 \\
\hline CRP (mg/L) & $114(52-197)$ & $74(32-157)$ & $126(68-214)$ & 0.030 \\
\hline $\begin{array}{l}\text { Lymphocyte } \\
\text { count }\end{array}$ & $0.93(0.62-1.36)$ & $1.17(0.64-1.84)$ & $0.81(0.56-1.25)$ & 0.064 \\
\hline Neutrophil count & $6.42(4.40-9.08)$ & $\begin{array}{c}6.62(4.47- \\
11.00)\end{array}$ & $6.30(4.09-9.08)$ & 0.412 \\
\hline $\begin{array}{l}\text { Antibiotic use } \\
\text { (\%) }\end{array}$ & $62(72 \%)$ & $21(70 \%)$ & $41(75 \%)$ & 0.625 \\
\hline Antiviral use (\%) & $2(2 \%)$ & $0(0 \%)$ & $2(4 \%)$ & 0.286 \\
\hline CPAP (\%) & $34(40 \%)$ & $9(30 \%)$ & $25(46 \%)$ & 0.165 \\
\hline $\begin{array}{l}\text { Mechanical ven- } \\
\text { tilation (\%) }\end{array}$ & $13(15 \%)$ & $3(10 \%)$ & $10(18 \%)$ & 0.317 \\
\hline Vasopressors (\%) & $14(15 \%)$ & $3(10 \%)$ & $11(20 \%)$ & 0.235 \\
\hline RRT (\%) & $3(3 \%)$ & $1(3 \%)$ & $2(4 \%)$ & 0.942 \\
\hline WHO < 6 (\%) & $43(50 \%)$ & $19(63 \%)$ & $24(44 \%)$ & 0.113 \\
\hline $\begin{array}{l}\text { Hospital mortal- } \\
\text { ity }\end{array}$ & $20(23 \%)$ & $5(17 \%)$ & $15(27 \%)$ & 0.271 \\
\hline
\end{tabular}

Table 1: Demographics and outcomes.

Conclusion. Many cytokines were more pronounced in males, explaining some of the marked sex variances in treatment responses noted in COVID-19 RCTs. Age and aggressive respiratory illness trajectory but not seroconversion on hospital admission were mortality risk factors.

\section{Reference(s)}

1. 1. loannou GN, Locke E, Green P, et al. Risk Factors for Hospitalization, Mechanical Ventilation, or Death Among 10,131 US Veterans With SARSCoV-2 Infection. JAMA Netw Open. 2020;3(9):e2022310.

2. 2.WHO_Working_Group_on_the_Clinical_Characterisation_and_Management_of_COVID-19_infection. A minimal common outcome measure set for COVID-19 clinical research. Lancet Infect Dis. 2020;20(8):e192-e197.

3. UCLH Biomedical Research Centre, UCL Coronavirus Response Fund, and MRC UKRI.

\section{4}

Safe use of FFP2 masks: The current certification method has no predictive value for the fit and safety of face masks when used by health care workers and is disproportionately unsafe for women

S. Galli $i^{1}$

${ }^{1}$ Anesthesiology, HMC Westeinde, Den Haag, Netherlands

Correspondence: S Galli

Intensive Care Medicine Experimental 2021, 9(1): 000924

Introduction. The COVID-19 pandemic has created an unprecedented and persistent demand for PPE as despite vaccination efforts there is still a lot of uncertainty about its protection against current and future variants. [1] When a new supplier of FFP2 mask was needed in March of 2021, a Dutch hospital performed preliminary tests on a sample of 33 Health Care Workers (HCWs) within the hospital to make an informed decision between 7 masks. This study performed quantitative fit tests to rank the masks, but also to compare them to the currently used FFP2 mask, surgical face masks and the compliance to EN149.

Methods. All masks were produced and certified in Western countries and obtained directly from the producer. Also, 4 of the 7 tested masks were approved by the infection prevention service before the testing started. We chose to perform quantitative fit tests because of the absence of available Total Inward Leakage Test (TILT) facilities and the ability to get an objective and exact measurement of the performance of a mask, contrary to the qualitative fit test. An external company used a Quantitative Method Ambient Particle Counting (APC) with a TSI PortaCount model 8048 according to the Health and Safety Executive (HSE) INDG 479 to determine fit factor during 7 exercises by dividing the concentration of particles outside and inside the mask. This is then converted $(100 /$ fit score $=$ leakage $\%)$ and compared with the EN149: "at least 8 out of the 10 individual wearer arithmetic means for the total inward leakage shall be not greater than: $22 \%$ for $F F P 1,8 \%$ for FFP2, 2\% for FFP3." [2]

Results. In 5 of the 7 tested masks (71\%) the norms for the EN149 was not met, with a fail rate of $23-82 \%$. A surprising amount of these masks failed to even pass the FFP1 threshold of $22 \%$ leakage, which means that in $14-50 \%$ of HCWs these FFP2 masks actually performed more like a surgical mask that had an average leakage of $25 \%$ in our study. Furthermore, HCWs wearing a failing brand had a $22 \%$ risk of having a fit score of 1 during at least one exercise. This means $100 \%$ leakage, with no measurable concentration difference whatsoever between inside and outside the FFP2 mask. If the mask passed the EN149 norm, this risk was diminished to 1,7\%. Although both men and women are affected with poor fitting masks, woman are up to $90 \%$ more likely to fail the fittest, depending on the type of mask. Furthermore, we found from the questionnaires that if we were to rely solely on fit-checking alone, a self-test advised among others by NHS selfassessment guidelines, HCWs would not have detected a little over half the failing masks.

Conclusion. This failing quality among FFP2 masks and the strong gender bias is not unprecedented in the literature. [3] [4] [5]. But this study shows to an ever greater extend exactly how unsafe the masks are for HCWs and especially women, despite certification. One factor is that the EN149 was originally developed in the ' 90 for (burly) men in various industries and up to this day does not take into consideration that HCWs consist for up to $85 \%$ of women. [6] With one producer stating that one notified body is known to use only firefighters as test subjects. During conversations with several notified bodies we also found out that for the sake of productivity it is a longstanding industry standard to perform the TILT solely on a manikin, a Sheffield head that comes in one size only. This could account also for the male failing rate as we found that $74 \%$ of the lowest fit scores were obtained during movement.

A study analysed 5148 intubation of COVID-19 patients and found that female HCWs had a higher risk of contamination, with a hazard ratio of 1.36 [7]. This has prompted the question whether the gendered PPE design can account for high infection rates in female healthcare workers following intubation, as there is no such biological distinction in the general population [8]. The extend of the genderd leakage found in this study supports this theory.

The certification requirements must be addapted to include mandatory fit tests on a variety of users. Meanwhile the safety of masks can be assessed by performing quantitative fit tests. If time of money is limited, a real-life check in $30 \mathrm{~s}$ while bending over, talking and moving the head up and down will detect $98 \%$ of failing masks in this study. Information to compare mask types can be found on a webpage where others may also choose to add their test results in order to help share and expand knowledge and keep each other safe: https://www.reusablemasks.nl/testresults-of-ffp2-masks.

\section{Reference(s)}

1. [1] Get Us PPE: Shortage Index, February 2021

2. [2] Wilkinson IJ, Pisaniello D, Ahmad J, Edwards S. Evaluation of a largescale quantitative respirator-fit testing program for healthcare workers: 
survey results. Infection Control and Hospital Epidemiology 2010; 31: 918- 25.

3. [3] Ascott, A., Crowest, P., de Sausmarez, E., Khan, M., \& Chakladar, A. (2021). Respiratory personal protective equipment for healthcare workers: impact of sex differences on respirator fit test results. British journal of anaesthesia, 126(1), e48-e49. https://doi.org/10.1016/j.bja.2020.10.016

4. [4] Regli, A., Sommerfield, A., \& von Ungern-Sternberg, B. S. (2021). The role of fit testing N95/FFP2/FFP3 masks: a narrative review. Anaesthesia, 76(1), 91-100. https://doi.org/10.1111/anae.15261

5. [5] Lee, K., Slavcev, A., \& Nicas, M. (2004). Respiratory protection against Mycobacterium tuberculosis: quantitative fit test outcomes for five type N95 filtering-facepiece respirators. Journal of occupational and environmental hygiene, 1(1), 22-28. https://doi.org/10.1080/154596204902500 26

6. [6] In een derde van beroepen op hoogste niveau is meerderheid vrouw. CBS 2019

7. [7] El-Boghdadly, K., Wong, D., Owen, R., Neuman, M. D., Pocock, S., Carlisle, J. B., Johnstone, C., Andruszkiewicz, P., Baker, P. A., Biccard, B. M., Bryson, G. L., Chan, M., Cheng, M. H., Chin, K. J., Coburn, M., Jonsson Fagerlund, M., Myatra, S. N., Myles, P. S., O'Sullivan, E., Pasin, L., ... Ahmad, I. (2020). Risks to healthcare workers following tracheal intubation of patients with COVID-19: a prospective international multicentre cohort study. Anaesthesia, 75(11), 1437-1447. https://doi.org/10.1111/anae. 15170

8. [8] Turner, M. C., \& Marshall, S. D. (2021). Can gendered personal protective equipment design account for high infection rates in female healthcare workers following intubation?. Anaesthesia, 76(1), 132-133. https:// doi.org/10.1111/anae.15206

\section{9}

Procalcitonin as decisive factor in antibiotic prescription at admission of severe COVID-19 patients in ICU: a prospective cohort study

F. Cunha'; J. Caldas²; C. Silva²; A. Martins²; A. Silva-Pinto²; AS. Santos, ${ }^{2} ;$ S. Xerinda, ${ }^{2}$; A. Ferreira ${ }^{2}$; P. Figueiredo, ${ }^{2}$; A. Sarmento, ${ }^{2}$, L. Santos, ${ }^{2}$

'Infectious Diseases Department, Centro Hospitalar e Universitário de Coimbra, Coimbra, Portugal; ${ }^{2}$ Serviço de doenças infecciosas, Centro Hospitalar e Universitário de São João, Porto, Portugal

Correspondence: F. Cunha

Intensive Care Medicine Experimental 2021, 9(1): 000929

Introduction. Coronavirus disease 2019 (COVID-19) has been declared a pandemic in March 2020. Procalcitonin (PCT) is considered useful in predicting the likelihood of bacterial infection and is an emerging prognostic marker in COVID-19. Whether PCT can help the clinician in the decision of prescribing antibiotics at admission in COVID-19 patients is still unclear. An ICU protocol was developed and applied since early pandemic to clarify the potential role of PCT in antibiotic prescription.

Methods. We conducted a prospective cohort study in an ID-ICU of a tertiary hospital in Portugal. All consecutive critical COVID-19 patients admitted between April and December 2020 were included. Patients' demographic characteristics, comorbidities and clinical data were collected from individual medical records.

Results. During the study period, 119 patients with severe COVID19 were admitted to the ID-ICU. Of these, 98 patients had PCT values available for analysis. Sixty-five percent were male and mean age was 65.4 years. The most frequent comorbidities detected were hypertension $(65.3 \%)$, dyslipidaemia (53.1\%), diabetes (39.8\%), obesity (26.5\%) and tobacco use (25.5\%). The severity of the disease at admission was assessed through SAPS II (median 33.5, IQR 30.0-38.0), SAPS III (median 55, IQR 49.0-59.6) and APACHE II (median 18, IQR 16.0-18.6). Ninety-five patients (96.9\%) developed respiratory failure, from which $67.4 \%$ needed mechanical ventilation. Cardiovascular, renal and hepatic dysfunctions were also frequent $(37.8 \%, 36.7 \%$ and $33.6 \%$, respectively).

PCT levels were assessed during the first $72 \mathrm{~h}$, median values were: $0.24 \mathrm{mg} / \mathrm{L}(\mathrm{IQR}, 0.08-0.62)$ at admission, $0.16 \mathrm{mg} / \mathrm{L}$ (IQR, $0.08-0.43)$ at $24 \mathrm{~h}, 0.18 \mathrm{mg} / \mathrm{L}(\mathrm{IQR}, 0.07-0.5)$ at $48 \mathrm{~h}$ and $0.16 \mathrm{mg} / \mathrm{L}$ (IQR, 0.07-0.32) at $72 \mathrm{~h}$. The highest PCT (PCTmax) levels were less than $0.1 \mathrm{mg} / \mathrm{L}$ in $26 / 98$ (26.5\%) patients and greater than $0.5 \mathrm{mg} / \mathrm{L}$ in 35/98 (35.7\%) patients, suggesting low and high probability of bacterial infection, respectively. Bacterial co-infection was detected in 19/98 (19.4\%) patients at admission, mostly pneumonia (94.7\%). The majority of cases with PCTmax levels $<0.1 \mathrm{mg} / \mathrm{L}$ did not underwent antibiotic treatment (84.6\%). In a logistic regression model, the decision against antibiotic prescription showed a significant relationship with PCTmax level (OR $0.5,95 \% \mathrm{Cl} 0.36-0.85, \mathrm{p}=0.007)$, but not with CRP levels (OR $0.99,95 \% \mathrm{Cl} 0.98-1.0)$.

Conclusion. Multiorgan dysfunction syndrome was frequent in COVID-19 patients admitted in ICU. Bacterial co-infection at admission was detected in about $20 \%$ of our population. Low procalcitonin levels supports the decision of not prescribing antibiotics in severe COVID19 patients at admission, even in the presence of elevated inflammatory markers, such as C-reactive protein.

\section{1}

Bacterial microbiological confirmed ventilator associated pneumonia in severe patients with SARS-CoV-2

A. Estella ${ }^{1}$; A. Garrino. ${ }^{2}$; I. Blanco Saez ${ }^{2}$

${ }^{1}$ University Hospital Of Jerez., Intensive Care Unit. Medicine Department Cadiz University. IniBICA, Jerez, Spain; ${ }^{2}$ University Hospital Of Jerez., Intensive Care Unit, Jerez, Spain

Correspondence: A. Estella

Intensive Care Medicine Experimental 2021, 9(1): 000961

Introduction. The difficulty during pandemic to implementation measures to prevent Health care related infections, with prolonged ICU lenght of stay of severe SARS-CoV-2 patients treated with inmunomodulatory drugs have been able to influence in an increase in rates of Ventilator associated pneumonia (VAP).

Objectives. To asses the incidence of VAP and to analyze outcome of this group of patients.

Methods. Retrospective, single-center case series of consecutive critically ill patients admitted in ICU of a community hospital with severe pneumonia caused by SARS-CoV-2. Time of study was one year. Patients with pulmonary aspergillosis and Non ventilated patients were excluded. VAP was define according to clinical suspicion plus bacterial positive cultures in bronchoalvelar lavage samples. Clinical features, ICU length of stay, microbiologic results and mortality were collected. Statistical analysis: Data were analyzed by IBM SPSS 24.

Results. 97 consecutive mechanically ventilated patients were included, to compare the two groups according to have VAP we have excluded the group of patients with pulmonary aspergillosis and other fungal isolates (20 patients) (Table 1).

Comparative table according microbiological confirmed VAP

\begin{tabular}{|c|c|c|c|}
\hline $\begin{array}{l}\text { SARS COV } 2 \\
\text { Pneumonia }\end{array}$ & $\begin{array}{l}\text { All patients } \\
\mathrm{n}: 97\end{array}$ & $\begin{array}{l}\text { Patients with- } \\
\text { out confirmed } \\
\text { VAP n:50 }\end{array}$ & $\begin{array}{l}\text { Patients with } \\
\text { confirmed VAP } \\
\text { n: } 27\end{array}$ \\
\hline Age (years) & $63,71 \pm 9,6$ & $61,58 \pm 10$ & $64,7 \pm 7,6$ \\
\hline $\begin{array}{l}\text { Gender (male/ } \\
\text { female) }\end{array}$ & $70 / 27$ & $34 / 16$ & $20 / 7$ \\
\hline $\begin{array}{l}\text { Prone Mechani- } \\
\text { cal ventilation } \\
(\%)\end{array}$ & $74,2 \%$ & $56 \%$ & $88,9 \%$ \\
\hline $\begin{array}{l}\text { APACHE II at } \\
\text { admission }\end{array}$ & $12,53 \pm 6,7$ & $12,2 \pm 6$ & $13,04 \pm 7,3$ \\
\hline $\begin{array}{l}\text { SOFA at admis- } \\
\text { sion }\end{array}$ & $5,86 \pm 2,9$ & $5,4 \pm 3,1$ & $6,67 \pm 2,9$ \\
\hline $\begin{array}{l}\text { ICU lenght of } \\
\text { stay (days) }\end{array}$ & $22,08 \pm 17,5$ & $16,9 \pm 15,2$ & $34,74 \pm 23,3$ \\
\hline Tracheostomy & $46,4 \%$ & $20 \%$ & $81,5 \%$ \\
\hline
\end{tabular}


Comparative table according microbiological confirmed VAP

\begin{tabular}{llll}
\hline $\begin{array}{l}\text { SARS COV 2 } \\
\text { Pneumonia }\end{array}$ & $\begin{array}{l}\text { All patients } \\
\text { n:97 }\end{array}$ & $\begin{array}{l}\text { Patients with- } \\
\text { out confirmed } \\
\text { VAP n:50 }\end{array}$ & $\begin{array}{l}\text { Patients with } \\
\text { confirmed VAP } \\
\text { n: 27 }\end{array}$ \\
\hline Haemofiltration & $32 \%$ & $18 \%$ & $44,4 \%$ \\
Mortality (\%) & $63,91 \%$ & $38 \%$ & $66,7 \%$ \\
\hline
\end{tabular}

Table 2 shows bacterial etiology of VAP.

\begin{tabular}{|c|c|}
\hline Gram-negative bacteria & Gram positive bacteria \\
\hline Klebsiella pneumoniae n: 3 & $\begin{array}{l}\text { Staphylococcus aureus methicillin } \\
\text { resistant } \mathrm{n}: 2\end{array}$ \\
\hline Stenotrophomonas maltophilia n:3 & $\begin{array}{l}\text { Staphylococcus aureus methicillin } \\
\text { sensible } n: 2\end{array}$ \\
\hline Pseudomonas aeruginosa n:2 & Other Gram positives: 2 \\
\hline \multicolumn{2}{|l|}{ Escherichia coli n:2 } \\
\hline \multicolumn{2}{|l|}{ Acinetobacter baumannii n:1 } \\
\hline \multicolumn{2}{|l|}{ Other Gram negatives n:6 } \\
\hline Polimicrobial n: 4 & \\
\hline
\end{tabular}

\section{Conclusion.}

- A high incidence of Ventilator associated pneumonia was observed in critically ill patients wit severe SARS-CoV-2 pneumonia.

- Ventilator associated pneumonia was associated with increased morbidity and mortality, most cases were caused by Gram negative.

\section{Reference(s)}

1. Rouzé A et al.; coVAPid study Group. Relationship between SARS-CoV-2 infection and the incidence of ventilator-associated lower respiratory tract infections: a European multicenter cohort study. Intensive Care Med. 2021 Feb;47(2):188-198. https://doi.org/10.1007/s00134-020-06323-9.

2. No

\section{6}

Community-acquired pulmonary bacterial co-infection in severe patients with SARS-CoV-2

A. Estella'; A. Garrino. ${ }^{2}$; I. Blanco Saez ${ }^{2}$

${ }^{1}$ University Hospital of Jerez., Intensive Care Unit. Medicine Department Cadiz University, INiBICA, Jerez, Spain; ${ }^{2}$ University Hospital of Jerez., Intensive Care Unit, Jerez, Spain

Correspondence: A. Estella

Intensive Care Medicine Experimental 2021, 9(1): 000966

Introduction. Several studies documented a low incidence of bacterial co-infection in patients with severe SARS-CoV-2 pneumonia compared with other viral pneumonia. Despite in medical wards incidence is low, in Intensive Care Unit may be higher.

Objectives. The aim of the present study is to asses the incidence of pulmonary bacterial co-infection in critically ill patients.

Methods. Retrospective, single-center case series of consecutive critically ill patients admitted in ICU of a community hospital with severe pneumonia caused by SARS-CoV-2. Time of study was one year. Bacterial co-infection was define according to positive cultures in bronchoalvelar lavage samples performed within the first $48 \mathrm{~h}$ of ICU admission, or with positive urine Streptococcus pneumoniae or Legionella pneumophila antigen detection.

Medical history, age, ICU length of stay, procalcitonin and C-reactive protein levels, microbiologic results and mortality were collected. Statistical analysis: Data were analyzed by IBM SPSS 24 and quantitative variables were expressed as a mean \pm standard deviation.
Results. 128 patients were included, most of them received previous ICU admission antibiotic therapy $(67,9 \%)$. Table 1 shows clinical characteristics of patients. Microbiologic confirmation was obtained in 11 patients, 8,5\%. Culture of bronchoalveolar lavage identified 6 Streptococcus pneumoniae, in one case with urine Streptococcus pneumoniae antigen detection negative, 4 Staphylococcus aereus and one Haemophilus Influenzae. All patients with bacterial coinfection were intubated and underwent invasive mechanical ventilation. There were not differences in mortality.

\begin{tabular}{lll}
\hline \multicolumn{2}{l}{ Clinical characteristics of patients } & \\
\hline SARS COV 2 Pneumonia & All patients n:128 & $\begin{array}{l}\text { Bacterial coinfection } \\
\mathbf{n}: \mathbf{1 1}\end{array}$ \\
\hline Age (years) & $62,8 \pm 10,4$ & $60,8 \pm 10,1$ \\
Gender (male/female) & $89 / 39$ & $7 / 4$ \\
Body mass index & $30,11 \pm 3,1$ & $28,45 \pm 2,9$ \\
Mechanical ventilation (\%) & $75,78 \%$ & $100 \%$ \\
APACHE Il at admission & $11,51 \pm 6,83$ & $16,8 \pm 6,6$ \\
SOFA at admission & $5,63 \pm 2,98$ & $8,1 \pm 3,7$ \\
ICU lenght of stay (days) & $18,67 \pm 17,61$ & $19,2 \pm 18,3$ \\
Leucocite count /\% & $10,333,7 \pm 5348,61$ & $12,855 \pm 5752$ \\
Neutrophil/\%Linf & $184,6 \% / 10,3 \%$ & $189,8 \% / 17,6 \%$ \\
PCR (mg/l) & $186,9 \pm 120,3$ & $205,6 \pm 71,4$ \\
PCT (ng/ml) & & \\
$<0,5 \mathrm{n} /(\%)$ & $82 / 64,06 \%$ & $5 / 45,45 \%$ \\
$0,5-1$ n/(\%) & $9 / 7,03 \%$ & $2 / 18,18 \%$ \\
$>$ 1 n/(\%) & $37 / 28,9 \%$ & $4 / 36,36 \%$ \\
Mortality (\%) & $50 \%$ & $54,5 \%$ \\
\hline
\end{tabular}

\section{Conclusion.}

- In our experience, bacterial co-infection in mechanically ventilated patients with SARS-CoV-2 pneumonia is higuer than incidence described in less severe patients admitted in medical wards.

- Despite previous antibiotic therapy, Gram-positive organisms were found in one in ten mechanically ventilated patients with SARS-CoV-2 pneumonia.

\section{9}

Differences in the attitudes and beliefs of healthcare workers, before and at the beginning of the COVID-19 pandemic BN. Santana-Lopez; ; MD. Bernat-Adell, ${ }_{1}^{2}$ Y YG. Santana-Padilla ${ }^{3}$; L. Santana-Cabrera ${ }^{4}$

${ }^{1}$ Intensive Care Unit, University Hospital of Gran Canaria Dr. Negrin, Las Palmas de Gran Canaria, Spain; ${ }^{2}$ Nursing Department, Jaume I University, Castelló de la Plana, Spain; ${ }^{3}$ Surgery unit, Complejo Hospitalario Universitario Insular Materno Infantil de Gran Canaria, Las Palmas de Gran Canaria, Spain; ${ }^{4}$ intensive Care Unit, Complejo Hospitalario Universitario Insular Materno Infantil de Gran Canaria, Las Palmas de Gran Canaria, Spain

Correspondence: B.N. Santana-Lopez

Intensive Care Medicine Experimental 2021, 9(1): 000979

Introduction. Before the start of the COVID 19 pandemic, it was estimated that, in the face of a hypothetical pandemic scenario such as the one we are experiencing, up to $50 \%$ of health professionals would not comply with their work responsibilities for their own protection or that of their family members.

Objectives. For this reason, we set ourselves the objective of comparing the attitudes and beliefs of healthcare workers (HCW) at two moments, before and during the COVID 19 pandemic. 
Methods. Descriptive, cross-sectional and comparative study carried out using a questionnaire-which was sent to healthcare professionals in 2017, and during the week of March 20-27, 2020.

Results. 2 samples of HCW, one studied in $2017(n=258)$ and another in March $2020(\mathrm{n}=969)$. In both, the majority are women (75.9\% \& $75.6 \%)$, aged between 30 and 50 years $(57.7 \%$ \& $53.1 \%)$ and living with their partner and children (37.2\% \& 43.1\%). On the other hand, nurses were the most represented professionals in both samples $(45.7 \%$ \& $52.0 \%)$

Regarding their beliefs, health professionals in 2020 were more confident that a vaccine would be developed to immunize them $(72.6 \%$ vs $14.7 \% p=0.000)$ as well as their access to it $(63.9 \%$ vs $52.7 \% p=0.000$ ). In 2017, HCW were more confident in having the appropriate protective equipment to prevent them from becoming infected $(45.7 \%$ vs $12.1 \% p=0.000)$.

Concerning attitudes, during a real health crisis situation, $\mathrm{HCW}$ seem more willing to take on tasks for which they haven't been trained/ educated $(42.0 \%$ vs $17.0 \% \mathrm{p}=0.000)$, they are predisposed to work more hours $(83.9 \%$ vs $54.6 \% p=0.000)$ and even do it in a different workplace $(71.8 \%$ vs $44.1 \% p=0.000)$.

However, before the pandemic, they considered a higher proportion that health professionals had a duty to care for the sick, even when there were high risks of infecting themselves or their family $(74 \%$ vs $63.4 \% p=0.001$ ), than all health personnel had to work during the pandemic $(78.2 \%$ vs $69.9 \% p=0.008)$ and that workers should be allowed to refuse to work with infected patients $(50.0 \%$ vs $31.7 \%$ $\mathrm{p}=0.000$ )

However, before the pandemic, they considered a higher proportion that HCW had a duty to care or treat infected patients, even when there were high risks of infecting themselves or their family $(74 \%$ vs $63.4 \% \mathrm{p}=0.001)$. In addition, they considered that all the HCW should be working during the pandemic $(78.2 \%$ vs $69.9 \% p=0.008)$ and that workers should be allowed to refuse to work with infected patients $(50.0 \%$ vs $31.7 \% \mathrm{p}=0.000)$

Conclusion.

1. HCW at the beginning of the pandemic were more confident in the development of a vaccine that would immunize them, but feared because they did not have the necessary protective equipment.

2. HCW, in a crisis situation, seem to be very committed to their work as they are willing to work longer hours, to do it in other work centers and to take on tasks for which they are not prepared/educated.

\section{Reference(s)}

1. 3. Santana López BN, Santana-Padilla YG, González-Martín JM, SantanaCabrera L. Attitudes and beliefs of Spanish healthcare professionals during the COVID-19 pandemic. Science Progress. 2021;104(2). https:// doi.org/10.1177/00368504211003775

2. 2. Santana-López BN, Santana-Padilla YG, Martin-Santana JD, et al. Creencias y actitudes de trabajadores sanitarios y estudiantes de enfermería de una región de España ante una pandemia de gripe. Rev Peru Med Exp Salud Publica 2019; 36(3):481-486. https://doi.org/10.17843/rpmesp. 2019.363.437

3. 1. Qureshi K, Gershon RRM, Sherman MF, et al. Health care workers' ability and willingness to report to duty during catastrophic disasters. J Urban Health 2005; 82(3): 378-88.

\section{4}

Antibiotic use in Swedish ICUs 2016-2018

F. Sjövalli'; M. Edström²; H. Hanberger ${ }^{3}$; S. Walter ${ }^{4}$

'Intensive and Perioperative Care, Skåne University Hospital, SUS, Malmö,

Malmö, Sweden; ${ }^{2}$ Department of Pharmacy, Linköping University,

Linköping, Sweden; ${ }^{3}$ Department of Infectious Diseases, Linköping

University, Linköping, Sweden; ${ }^{4}$ Dept of Cardiothoracic Anaesthesia and Intensive Care, Linkoping University Hospital, Linköping, Sweden

Correspondence: F. Sjövall

Intensive Care Medicine Experimental 2021, 9(1): 000984

Introduction. Overuse of antibiotics in the ICU is common (1). It has been estimated that up to $30 \%$ of all patients admitted to the ICU with presumed infection, and treated with antibiotics, are evaluated as not having an infection, or a very low likelihood thereof (2). Measuring antibiotic quantity is key in order to evaluate change or to compare antibiotic use in different settings and monitoring itself can lead to performance improvement and is an essential part of a antimicrobial stewardships (AMS) program.

Objectives. The purpose of the present investigation was to explore the use of antibiotics in Swedish ICUs in the years 2016-2018.

Methods. Daily Defined Doses (DDDs) of antimicrobials delivered to Swedish ICUs, 2016-2018, were retrieved from the Swedish pharmacies. From the Swedish Intensive Care Registry, we extracted data on number of patient admissions, occupied bed days and Simplified Acute Physiology Score (SAPS)-III.

Results. There was a similar rate of total DDDs per admission of (3.7, $3.7,3.8)$ and total DDDs per occupied bed days of $(1.1,1.2,1.2) 2016$ 2018 but with an approximate sevenfold difference of DDDs per occupied bed days $(0.6-4.1)$ between the ICUs.

The most used antibiotics were isoxazolyl penicillins (J01CF), penicillins with betalactamase-inhibitors, mainly piperacillin/tazobactam (J01CR), third and 4th generation cephalosporins (J01DD + DE) and carbapenems (J01DH). Together these four classes accounted for $63 \%$ of all antibiotic use. There was a similar proportion of DDDs of all antibiotic classes over the three years except for aminoglycosides (J01GB) and co-trimoxazole (J01EE) where the DDDs were significantly lower 2018 compared to 2016 ( $p=0.03$ and $p=0.04$ respectively).

The use of carbapenems had a moderate positive correlation with mean SAPS3 score $(r=0.6, p=0.01)$. This correlation was not present for penicillins with beta-lactamase inhibitors (J01CR).

Conclusion. Overall antibiotic use has remained similar in Swedish ICUs during the years 2016 - 2018. There is a large inter-ICU variation that is only in part explained by case-mix. The use of broad-spectrum antibiotics accounts for almost $50 \%$ of all DDDs.

\section{Reference(s)}

1. 2. Klein Klouwenberg PM, Cremer OL, van Vught LA, Ong DS, Frencken JF, Schultz MJ, et al. Likelihood of infection in patients with presumed sepsis at the time of intensive care unit admission: a cohort study. Crit Care. 2015;19:319.

2. 1. Denny KJ, De Waele J, Laupland KB, Harris PNA, Lipman J. When not to start antibiotics: avoiding antibiotic overuse in the intensive care unit. Clin Microbiol Infect. 2020;26(1):35-40.

3. Supproted by the Swedish Research Council 2019-05,908

\section{4}

New wave, new line policy? Effect of mandatory central venous catheter changes on blood stream infection in COVID-19 ventilated patients

E. Patterson, ; A. Parajuli, ${ }^{1}$; J. Carne, ; R. Hart ${ }^{1}$; C. McCue

1 Intensive care, Queen Elizabeth University Hospital, Glasgow, United

Kingdom

Correspondence: $\mathrm{C}$. McCue

Intensive Care Medicine Experimental 2021, 9(1): 000994

Introduction. Since the onset of the COVID-19 pandemic, there have been a number of studies examining bacterial super-infection in critically ill ventilated patients. Due to the typically prolonged periods of ICU admission, and excess capacity utilisation of critical care a presumption of increased hospital-acquired infection seems reasonable, a recent case-cohort study demonstrated increased risks of blood stream infection (BSI) in COVID-19 patients, particularly after 7 days in ICU (1). During the first wave, published literature suggested increased BSIs, particularly of Gram negative species, and association with central venous catheter (CVC) BSI and placement $>7$ days (2). We sought to determine our initial rates of CVCBSI in the first wave, and any significance on length of line placement or site. Following concerns raised in the literature of CVCBSI, we adopted a policy locally of mandatory CVC changes at 7 days in COVID-19 ventilated patients. Following this intervention we reviewed our adherence to the practice, and any effect seen on rates of line infection. 
Methods. We identified all ventilated COVID-19 patients admitted to our ICU within the first wave (March to May 2020) at our 24-bedded level 3 unit. We collected data on length and site of CVC placement; and patient length of stay from clinical information systems. Documented positive cultures were obtained from laboratory information systems, and reviewed for likely pathogenicity. Due to clinical governance nature of project; no formal ethical approval was required. Following the first wave, a unit policy of mandatory CVC line change at day 7 was introduced, and the same dataset collected on the second cohort (October 2020 to January 2021). We also assessed adherence to the new policy.

\section{Results.}

\begin{tabular}{llll}
\hline & Wave 1 & Wave 2 & p value \\
\hline Total number of patients & 41 & 102 & \\
Total number of CVCs & 76 & 234 & \\
Overall rates of BSI (\%) & 22 & 12 & 0.21 \\
$\begin{array}{ll}\text { BSI Pathogenic (\%) } \\
\text { Median length of line }\end{array}$ & 8 & 4 & \\
$\begin{array}{l}\text { placement (days) } \\
\text { \% of infected lines per }\end{array}$ & Femoral 10\%, & Femoral 9\%, & \\
$\begin{array}{l}\text { site of placement } \\
\text { \% lines }>\text { 7 days }\end{array}$ & IJ 24\% & IJ 12\% & \\
\hline
\end{tabular}

\begin{tabular}{llll}
\hline & $<=7$ days & $>$ 7 days & p value \\
\hline WAVE 1 & $4 \%$ & $4 \%$ & 0.67 \\
WAVE 2 & $2 \%$ & $2 \%$ & 0.73 \\
COMBINED & $5 \%$ & $5 \%$ & 0.79 \\
\hline
\end{tabular}

Overall following policy change we demonstrated a reduction in overall line infections (pathogenic and likely contaminants). Our rates of infection in the first wave are similar with published literature (3). The most common infection seen in wave 1 was Enterococcus faecium, and wave 2 Klebsiella pneumoniae. Interestingly in both first and second waves, we demonstrated no increased rates of CVCBSI in lines kept in longer than 7 days, suggesting other reasons for improved rates of infection in the second wave. Of note, higher rates of infection were seen in internal jugular (IJ) compared with femoral lines, though not statistically significant. Moderate adherence to line change policy was seen with overall reduction in median length of line placement.

Conclusion. In order to reduce CVCBSI in our second wave of COVID19 ventilated patients we successfully implemented a mandatory 7 day line change policy. We demonstrated a reduction in CVCBSI overall, but this is not explained by length of line placement. Possible explanations include staff familiarity with condition, improved PPE/hygiene practice and/or more manageable surge capacity. The increased rate of infection seen in IJ lines may be related to proning practices.

\section{Reference(s)}

1. 3. Rawson T.M., Moore L.S.P., Zhu N., et al. Bacterial and fungal co-infection in individuals with coronavirus: a rapid review to support COVID-19 antimicrobial prescribing. Clin Infect Dis. 2020

2. 2. Sturdy A., Basarab M., Cotter M., et al. Severe COVID-19 and healthcareassociated infections on the ICU: time to remember the basics? J Hosp Infect. 2020 Aug; 105(4): 593-595

3. 1. Buetti N, Ruckly S, de Montmollin E et al. COVID-19 increased the risk of ICU-acquired bloodstream infections: a case-cohort study from the multi centric OUTCOMEREA network. Intensive Care Med 47, 180-187 (2021)
001040

Outcomes and Predictors of Surgical Site Infection after Cardiac Surgery: A Prospective Analysis

DSN. Luiz Alves'; VDM. Ana Paula ${ }^{1}$; C. Najara' ; C. Beatriz²; FOC. Murilo ${ }^{3}$; L. Tomich

${ }^{1}$ Infectious diseases, Hospital de Doenças Tropicais, Goiânia, Brazil; ${ }^{2}$ Intensive care, Orion-Business \& Health Complex, Goiânia, Brazil; ${ }^{3}$ Intensive

care, Hospital de Doenças Tropicais, Goiânia, Brazil

Correspondence: $\mathrm{L}$. Tomich

Intensive Care Medicine Experimental 2021, 9(1): 001040

Introduction. Surgical Site Infections (SSIs) after cardiac surgery increases morbimortality. This complication could be reduced by knowing risk factors.

Methods. All patients who underwent cardiac surgery from 20162018 in a tertiary referral hospital in Brazil were included. All patients were visited in the postoperative period during hospitalization by the nursing team of the infection control commission for wound assessment, also telephone call contact for post-discharge surveillance were performed after 30 days, 90 days and 1 year after each surgical procedure. Standardized data collection forms, containing demographic, surgical and clinical information were completed. Variables were assessed by infection status using parametric and non-parametric tests when applicable. Logistic and linear regression were applied to access risk factors for SSIs.

Results. A total of 99 patients were followed, predominantly male $61(61.6 \%)$ with a median age of 60 years (16-89), $22(22.2 \%)$ had diabetes, $68(68.7 \%)$ had hypertension and $13(13.1 \%)$ had insufficiency cardiac congestive. Thereafter, 31(31.3\%) had postsurgical complications; length of mechanical ventilation was average in 1.2-day (1-6); the body mass index average was $26.14(15-40) ; 82(82.8 \%)$ and $12(12.1)$ patients used cefuroxime and cefazoline as surgical prophylaxis, respectively. We also found that $10(10.1 \%)$ had nosocomial pneumonia (4 were ventilator-associated pneumonia) and $18(18.2 \%)$ developed SSI, 9(50\%) were superficial incisional infections, 4(22,22\%) deep infections and $5(27.78 \%)$ were organ/space infections. Seven 9(38,9\%) patients had a microbiological diagnosis of SSI: Enterococcus faecalis (2), coagulase-negative staphylococci (1), Klebsiella pneumoniae (1), Pseudomonas aeruginosa (1), Streptococcus gallolyticus (1) and Streptococcus sanguinis (1). The average length of hospital stay was 10.9 (2-76) days. Other variables are showed in Table. As a risk factor for the SSI, we found that the mean ICU stay of 7.1 days in the group with infection was statistically higher than 3.6 days in the group without infection $(p<0.00)$, and patients with SSI had an 11 times higher chance of dying than patients without infection (OR 11.28; 1.88-67.59; $\mathrm{p}=0.009$ ).

\begin{tabular}{|c|c|c|c|c|c|c|}
\hline \multirow[t]{2}{*}{ VARIABLES } & \multirow[t]{2}{*}{ Total } & \multicolumn{3}{|l|}{ INFECTION } & \multirow[b]{2}{*}{$\mathrm{Cl} 95 \%$} & \multirow[b]{2}{*}{$p$} \\
\hline & & $\begin{array}{l}\text { No } \\
(n=81)\end{array}$ & $\begin{array}{l}\text { Yes } \\
(n=18)\end{array}$ & OR & & \\
\hline Age & $60(16-89)$ & $59(16-89)$ & $64(42-85)$ & - & & 0.802 \\
\hline $\begin{array}{l}\text { Average } \\
\text { length of } \\
\text { preopera- } \\
\text { tive hospi- } \\
\text { tal stay } \\
\text { (days) }\end{array}$ & $1.7(0-41)$ & $1.5(0-31)$ & $2.8(0-41)$ & - & & $<0.00$ \\
\hline $\begin{array}{l}\text { Extracor- } \\
\text { poreal } \\
\text { circula- } \\
\text { tion time } \\
\text { (min) }\end{array}$ & $\begin{array}{l}97.2 \\
\quad(15-190)\end{array}$ & $\begin{array}{l}99.9 \\
\quad(15-190)\end{array}$ & $\begin{array}{c}86.6(46- \\
180)\end{array}$ & - & & 0.578 \\
\hline $\begin{array}{l}\text { ICU length } \\
\text { of stay } \\
\text { (days) }\end{array}$ & $4.3(1-33)$ & $3.6(1-33)$ & $7.1(1-32)$ & - & & $<0.00$ \\
\hline Comorbidity & 85 (85.8\%) & $68(83.9 \%)$ & 17 (94.4\%) & 3.25 & $\begin{array}{r}0.39- \\
26.6\end{array}$ & 0.45 \\
\hline
\end{tabular}




\begin{tabular}{|c|c|c|c|c|c|c|}
\hline \multirow{2}{*}{ VARIABLES } & \multirow[t]{2}{*}{ Total } & \multicolumn{3}{|c|}{ INFECTION } & \multirow[b]{2}{*}{$\mathrm{Cl} 95 \%$} & \multirow[b]{2}{*}{$p$} \\
\hline & & $\begin{array}{l}\text { No } \\
(n=81)\end{array}$ & $\begin{array}{l}\text { Yes } \\
(n=18)\end{array}$ & OR & & \\
\hline $\begin{array}{l}\text { Adequate } \\
\text { antibiotic } \\
\text { prophy- } \\
\text { laxis }\end{array}$ & $84(84.8 \%)$ & $67(82.7 \%)$ & $17(94.4 \%)$ & 1.95 & $\begin{array}{r}0.58- \\
6.41\end{array}$ & 0.29 \\
\hline $\begin{array}{l}\text { Type of } \\
\text { surgery: } \\
\text { valve } \\
\text { change }\end{array}$ & $66(66.7 \%)$ & $52(64.2 \%)$ & $14(77.8 \%)$ & 1.95 & $\begin{array}{l}0.58- \\
6.48\end{array}$ & 0.40 \\
\hline $\begin{array}{l}\text { Elective } \\
\text { surgery }\end{array}$ & 90 (90.9\%) & 74 (91.3\%) & 16 (88.9\%) & 0.75 & $\begin{array}{r}0.14- \\
3.98\end{array}$ & 0.66 \\
\hline Death & $6(6 \%)$ & $2(2.5 \%)$ & $4(22.2 \%)$ & 11.28 & $\begin{array}{c}1.88- \\
67.59\end{array}$ & 0.009 \\
\hline
\end{tabular}

Conclusion. We found that the longer ICU stay significantly increases the probability of developing SSI carrying an increased risk factor of death. These findings should reinforce prevention strategies in order to reduce the disease burden.

\section{Reference(s)}

1. Lepelletier D, Bourigault C, Roussel JC, Lasserre C, Leclère B, Corvec S, Pattier S, Lepoivre T, Baron O, Despins P. Epidemiology and prevention of surgical site infections after cardiac surgery. Med Mal Infect. 2013 Oct;43(10):403-9. https://doi.org/10.1016/j.medmal.2013.07.003. Epub 2013 Aug 26. PMID: 23988675

2. Anderson DJ, Podgorny K, Berríos-Torres SI, Bratzler DW, Dellinger EP, Greene L, Nyquist AC, Saiman L, Yokoe DS, Maragakis LL, Kaye KS. Strategies to prevent surgical site infections in acute care hospitals: 2014 update. Infect Control Hosp Epidemiol. 2014 Jun;35(6):605-27. https:// doi.org/10.1086/676022. PMID: 24799638; PMCID: PMC4267723.

3. Andrade LS, Siliprandi EMO, Karsburg LL, Berlesi FP, Carvalho OLDF, Rosa DSD, Santos RPD. Surgical Site Infection Prevention Bundle in Cardiac Surgery. Arq Bras Cardiol. 2019 Jun;1 12(6):769-774. https://doi.org/10.5935/ abc.20190070. Epub 2019 Apr 8. PMID: 30970144; PMCID: PMC6636364.

4. Engelman DT, Ben Ali W, Williams JB, Perrault LP, Reddy VS, Arora RC, Roselli EE, Khoynezhad A, Gerdisch M, Levy JH, Lobdell K, Fletcher N, Kirsch M, Nelson G, Engelman RM, Gregory AJ, Boyle EM. Guidelines for Perioperative Care in Cardiac Surgery: Enhanced Recovery After Surgery Society Recommendations. JAMA Surg. 2019 Aug 1;154(8):755-766. https://doi.org/10.1001/jamasurg.2019.1153. PMID: 31054241

5. Figuerola-Tejerina A, Rodríguez-Caravaca G, Bustamante-Munguira J, María San Román-Montero J, Durán-Poveda M. Epidemiological Surveillance of Surgical Site Infection and its Risk Factors in Cardiac Surgery: A Prospective Cohort Study. Rev Esp Cardiol (Engl Ed). 2016 Sep;69(9):8428. English, Spanish. https://doi.org/10.1016/j.rec.2016.01.030. Epub 2016 May 5. PMID: 27155925

6. The authors would like to thank Alexander C. Lees, Senior Lecturer in Manchester Metropolitan University/UK, and Nárgila G. Moura for the English language review.

\section{3}

Carbapenemase mapping for burn patients in intensive care unit S. Zarrouk'; A. Mokline'; N. Ben Slimene'; H. Fredj'; M. Ben Saad'; AA. Messadi ${ }^{1}$

'Burn Intensive Care Unit, Centre De Traumtologie Et Des Grands Brulés, Ben Arous, Tunisia

Correspondence: N. Ben Slimene

Intensive Care Medicine Experimental 2021, 9(1): 001043

Introduction. The detection of carbapenemases in burn patients is of great benefit in identifying areas of carbapenemase carrying, implementing measures to isolate carriers, and assisting in the choice of antibiotic therapy in case of sepsis.

Objectives. The goal of our work is to map the portage of carbapenemases in burnt patients hospitalized in our intensive care unit.
Methods. Prospective study conducted at The Burn Resuscitation Service over the period (April 2018-December 2018). A PCR in the search for carbapenemse portage was performed in all patients admitted via emergency room or secondary transfer. The enzymes studied were VIM, NDM, OXA 48.

Results. During the study period, 221 patients were admitted, 121 had a PCR of which 53 were positive. PCR samples were realized 2 days after admission ( 3 days after burn). Seventeen patients had a single carbapenemase, 11 had two, and 25 had three. The enzymes detected were VIM in 41 patients, NDM in 41 patients, and OXA 48 in 32 patients. The distribution of these enzymes by region revealed the predominance of NDM/OXA48 in kairouan, and NDM/VIM in kasserine. The main risk factors studied in our patients were prior antibiotic therapy, invasive procedures (vascular catheterization and mechanical ventilation) and a history of stay in intensive care unit. Twenty-nine patients (55\%) had received prior antibiotic therapy; 30 patients $(57 \%)$ were under mechanical ventilation; 31 patients $(59 \%)$ had vascular catheterization and 11 patients (21\%) had a history of stay in intensive care units.

Conclusion. The main carrying areas for carbapenemases have been identified: kairouan, Kasserine.

The main risk factors present in our patients were prior antibiotic therapy, a stay in intensive care units and invasive procedures.

Efforts in terms of training regarding antibiotic prescriptions in burns are highly recommended.

\section{9}

Predictors of Intensive Care Unit Admission in Acute Febrile Illness: A Prospective Observational Study

S. Behera'; DK. Baidya'; S. Maitra²; RR. Bikash ${ }^{3}$; S. Rajeshwari ${ }^{4}$; V. Rewari ${ }^{3}$; P. Aggarwal ${ }^{5} ; \mathrm{N} . \mathrm{Wig}^{6}$

${ }^{1}$ Anaesthesiology, Pain Medicine \& Critical Care ${ }_{\text {" }}$ All India Institute Of Medical Sciences, New Delhi, NEW DELHI, India; ${ }^{2}$ Anaesthesiology, Pain Medicine \& Critical Care, All India Institute Of Medical Sciences, New Delhi, India; ${ }^{3}$ Anaesthesiology, Pain Medicine \& Critical Care, All India Institute of Medical Sciences, New Del, All India Institute OF Medical Sciences, New Delhi, New Delhi, India; ${ }^{4}$ Anaesthesiology, Pain Medicine \& Critical Care, All India Institute of Medical Sciences, New Delhi, India; ${ }^{5}$ Emergency Medicine, All India Institute of Medical Sciences, New Delhi, India; ${ }^{9}$ Medicine, All India Institute Of Medical Sciences, New Delhi, India

Correspondence: S. Behera

Intensive Care Medicine Experimental 2021, 9(1): 001069

Introduction. Acute febrile illness (AFI) often presents as undifferentiated fevers. Some AFI infections are more prevalent in tropical and subtropical regions; and are known as tropical fevers.A significant number of AFI patients require intensive care unit (ICU) admission. However, there is no established risk predictor of ICU admission of AFI patients.

Objectives. To identify the risk factors of ICU admission of Acute Febrile Illness patients admitted to Emergency Department (ED).

Methods. After ethics clearance and informed written consent patients presenting to ED with $\mathrm{AFI}$ and age $>12$ years were included in this prospective observational study. Exclusion criteria were: refusal to consent for study, pre-existing chronic organ dysfunction, presented to hospital for $>24 \mathrm{~h}$. Details of history, physical examination findings, and investigation reports, SOFA and qSOFA scores were noted for all recruited patients at admission. The entire study population was divided into two groups based on whether ICU admission required or not. Risk factors of need for ICU admission were identified on the basis of binary logistic regression analysis.

Results. In this prospective study, $\mathrm{n}=104$ patients were recruited; the median (IQR) age was $25(21-35)$ years and $58.7 \%$ cases were male. Most common diagnosis was Dengue $(36.5 \%)$ followed by Vivax malaria (20.2\%). Others were Scrub typhus (10.6\%), Falciparum malaria (9.6\%), Typhoid (6.7\%) and Leptospirosis (4.8\%). The aetiological diagnosis could not be established in $11.5 \%$ cases. Among the 104 patients, $52(50 \%)$ cases required ICU admission. A stepwise binary logistic regression model was consctructed with all variables associated with ICU admission at $\mathrm{p}<0.05$ and the model with 
lowest Akaike information criteria was selected. Stepwise regression revealed that a model consisting of qSOFA, serum lactate and serum albumin level could predict ICU admission.

\section{Regression analysis for ICUAdmission with selected variables in model}

\begin{tabular}{|c|c|c|c|c|c|}
\hline \multicolumn{2}{|l|}{$\begin{array}{l}\text { Dependent: } \\
\text { ICU_Admission }\end{array}$} & \multirow[t]{2}{*}{$\begin{array}{l}\text { Not } \\
\text { Required }\end{array}$} & \multirow[t]{2}{*}{ Required } & \multirow{2}{*}{$\begin{array}{l}\text { OR (univari- } \\
\text { able) } \\
18.32 \\
(7.56-60.97, \\
p<0.001)\end{array}$} & \multirow{2}{*}{$\begin{array}{l}\begin{array}{l}\text { OR (multi- } \\
\text { variable) }\end{array} \\
7.50 \text { (2.74- } \\
27.90 \\
p<0.001)\end{array}$} \\
\hline qSOFA & & & & & \\
\hline $\begin{array}{c}\text { S_Lactate_ } \\
\text { mmol_L }\end{array}$ & $\begin{array}{l}\text { Mean } \\
\text { (SD) }\end{array}$ & $1.5(0.8)$ & $4.2(3.2)$ & $\begin{array}{l}3.46 \\
\quad(2.10-6.47 \\
p<0.001)\end{array}$ & $\begin{array}{l}2.39 \\
(1.21-5.36 \\
p=0.019)\end{array}$ \\
\hline $\begin{array}{l}\text { S_Albumin_g_ } \\
\text { dL }\end{array}$ & $\begin{array}{l}\text { Mean } \\
\text { (SD) }\end{array}$ & $3.6(0.5)$ & $2.6(0.6)$ & $\begin{array}{l}0.06 \\
\quad(0.02-0.14 \\
p<0.001)\end{array}$ & $\begin{array}{l}0.19 \\
(0.04-0.78 \\
p=0.032)\end{array}$ \\
\hline $\begin{array}{r}\text { MODEL FIT: } \mathrm{X}^{2}( \\
\text { frame }=104, \\
\text { tic }=0.967, \mathrm{H}\end{array}$ & 6.73, & & $\begin{array}{l}\text { eudo- } \mathrm{R}^{2}= \\
\text { Missing }=\end{array}$ & 7 Number in $\mathrm{d}$ & \\
\hline
\end{tabular}

Final Risk Score $=1.33+(2.02 *$ qSOFA $)+(0.87 *$ Lactate $)+(-1.64 *$ Albumin)

At a cutoff of Final Risk Score $\geq 0.461$, it predicts ICU Admission with a sensitivity of $94 \%$, and a specificity of $92 \%$. The area under the ROC curve for predicting ICU admission was 0.967 (95\% Cl: 0.934-1).

Limitation: The tropical fever is a syndrome involving heterogeneous group of patients, and the risk factors of ICU admission may vary in different diseases.

Conclusion. Admission qSOFA, baseline serum lactate level with serum albumin level are useful predictor of ICU admission in AFI patients.

\section{Reference(s)}

1. 1. Rani RV, Sundararajan T, Rajesh S, Jeyamurugan T. A study on common etiologies of acute febrile illness detectable by microbiological tests in a tertiary care hospital. Int J Curr Microbiol Appl Sci 2016;5:670-4. 2. Crump JA. Time for a comprehensive approach to the syndrome of feve in the tropics.Transactions of the Royal Society of Tropical Medicine and Hygiene. 2014;108(2):61-2. 3.Steurer J, Held U, Spaar A, Bausch B, Zoller M, Hunziker R, Bachmann LM. A decision aid to rule out pneumonia and reduce unnecessary prescriptions of antibiotics in primary care patients with cough and fever. BMC Med. 2011;9:56. 4. The Indian Society of Critical Care Medicine Tropical Fever Group, Singhi S, Chaudhary D, Varghese GM, Bhalla A, Karthi N, et al. Tropical fevers: Management guidelines. Indian J Crit Care Med 2014; 18:62-9.

\section{1}

\section{General scores, commonly used in ICU, are inaccurate} in the context of covid-19

I. Ferreira, ${ }_{1}^{1}$; R. Menezes, ${ }^{2}$; M. Pina ${ }^{3}$; R. Santos Oliveira Jr ${ }^{3}$; G. Telles ${ }^{3} ;$ P. Pugas $^{3}$; L. Pamplona Neto ${ }^{4}$; S. Agareno ${ }^{5}$; A. Bruno ${ }^{2}$; NM. Filgueiras Filho ${ }^{6}$ ${ }^{1}$ Life Sciences Department, University of State of Bahia, Salvador, Brazil; ${ }^{2}$ Inflammation and Biomarkers Laboratory, Oswaldo Cruz Foundation, Salvador, Brazil; ${ }^{3}$ Research, Teaching and Communication Center, Hospital da Cidade, Salvador, Brazil; ${ }^{4}$ Critical care, Hospital da Cidade, Salvador, State of Bahia, Brazil, Brazil; ${ }^{5}$ Board of Directors, Hospital da Cidade, Salvador, Brazil; ${ }^{6}$ Medicine, Salvador University, Campus Teacher Barros, Salvador, Brazil

Correspondence: N.M. Filgueiras Filho

Intensive Care Medicine Experimental 2021, 9(1): 001081

Introduction. Changes in life expectancy, associated with an increase in the burden of comorbidities, have changed the profile of critically ill patients. This phenomenon intensified during the covid-19 pandemic when the scarcity of resources, the increase in demand and the lack of screening tools led many services to collapse. Thus, there was an emergency need to search for existing and validated prognostic tools that could be adapted to this new clinical context.

Objectives. To assess the accuracy of commonly used scores in determining ICU outcomes in critically ill COVID-19 patients.

Methods. Retrospective cohort of secondary data from medical records from a general intensive care unit from Brazil, which included all covid-19 infected patients between April 2020 to May 2021. Results were described using mean \pm standard deviation or frequency (percentage). To assess the accuracy of the Modified Frailty Index 11 it was used the area under the ROC curve, the optimal cut-off points for the continuous variables were determined with the Youden's criteria. To assess the model calibration, the Hosmer-Lemeshow test was used.

Results. There were included 195 patients, mean age was 59 \pm 15.1 , there was a prevalence of males $118(60.5 \%)$, and the overall ICU mortality was $23.1 \%$ with a mean unit length of stay of $12.3 \pm 12.5$ days. The mean Charlson comorbidity index was $1.0 \pm 1.4$ and it had an AUROC of $0.72(95 \% \mathrm{Cl} 0.65-0.78 ; \mathrm{p}<0.0001)$. The mean SAPS3 score was $51.4 \pm 11.9,53(48.6 \%)$ with an AUROC of $0.77(95 \% \mathrm{Cl} 0.71-0.83$ $\mathrm{p}<0.0001)$. Also, the mean MFI-11 score was $0.11 \pm 0.11$, and its area under roc curve was $0.73(95 \% \mathrm{Cl} 0.66-0.79 ; \mathrm{p}<0.0001)$. The ACIS, a score developed by our research group, had a mean value of $5.5 \pm 3.5$, and an AUROC of $0.73(95 \% \mathrm{Cl} 0.67-0.80 ; \mathrm{p}<0.0001)$. There were 99 $(50.7 \%)$ patients who needed mechanical ventilation and 69 (35.4\%), vasoactive drugs.

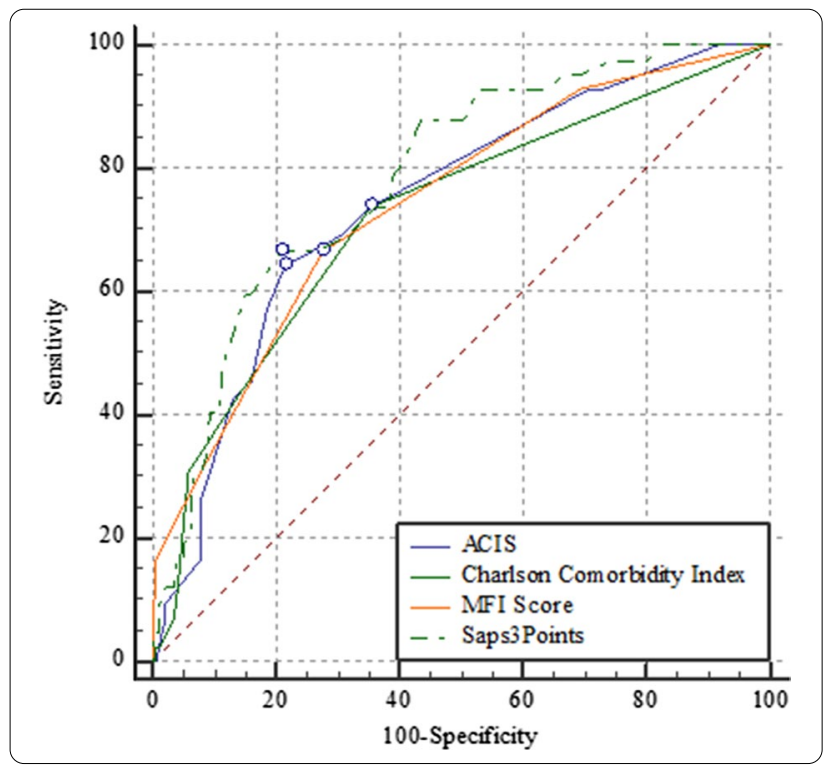

Conclusion. All scores analyzed in this study were fairly accurate, but insufficient to be used in the intensive care routine in the context of covid-19.

\section{Reference(s)}

1. Menezes RC, Ferreira IBB, Carmo TA, Telles GP, Pugas PLD, Otero ML, Arriaga MB, Fukutani KF, Neto LP, Agareno S, Filgueiras Filho NM, Akrami KM, Andrade BB. Are prognostic tools losing accuracy? Development and performance of a novel age-calibrated severity scoring system for critically ill patients. PLoS One. 2020 Nov 4;15(11):e0240793. https://doi.org/ 10.1371/journal.pone.0240793. PMID: 33147243; PMCID: PMC7641388.

2. Charlson ME, Pompei P, Ales KL, MacKenzie CR. A new method of classifying prognostic comorbidity in longitudinal studies: development and validation. J Chronic Dis. 1987:40(5):373-83. https://doi.org/10.1016/ 0021-9681(87)90171-8. PMID: 3558716

3. Metnitz PG, Moreno RP, Almeida E, Jordan B, Bauer P, Campos RA, lapichino G, Edbrooke D, Capuzzo M, Le Gall JR; SAPS 3 Investigators. SAPS 
3-From evaluation of the patient to evaluation of the intensive care unit. Part 1: Objectives, methods and cohort description. Intensive Care Med. 2005 Oct;31(10):1336-44. https://doi.org/10.1007/s00134-005-2762-6. Epub 2005 Aug 17. PMID: 16132893; PMCID: PMC1315314.

4. Farhat JS, Velanovich V, Falvo AJ, Horst HM, Swartz A, Patton JH Jr, Rubinfeld IS. Are the frail destined to fail? Frailty index as predictor of surgical morbidity and mortality in the elderly. J Trauma Acute Care Surg. 2012 Jun;72(6):1526-30; discussion 1530-1. https://doi.org/10.1097/TA.0b013 e3182542fab. PMID: 22695416.

\section{9}

Inhaled nebulised unfractionated heparin for the treatment of hospitalised patients with COVID-19: A multicentre case series of 98 patients

F. van Haren ${ }^{1}$; A. Vilaseca²; T. Smoot ${ }^{3}$; J. Laffey ${ }^{4}$; L. Van Loon'; A. Artigas5'; B. Dixon ${ }^{6}$; C. Page

${ }^{1}$ College of Health and Medicine, The Australian National University, Canberra, Australia; ${ }^{2}$ Service of Haematology and Haemostasis, San Camilo Clinic, DJl, Argentina; ${ }^{3}$ Critical care, Frederick Health Hospital, Frederick, United States of America; ${ }^{4}$ Anaesthesia and Intensive Care Medicine, School of Medicine, and Regenerative Medicine Instit, National University of Ireland Galway, Galway, Ireland; ${ }^{5}$ Department of Intensive Care Medicine, Corporacion Sanitaria Universitaria Parc Tauli, Barcelona, Spain; ${ }^{6}$ Critical Care, St Vincent's Hospital, Melbourne, Australia; ${ }^{7}$ Sackler Institute of Pulmonary Pharmacology, King's College, London, United Kingdom Correspondence: F. van Haren

Intensive Care Medicine Experimental 2021, 9(1): 001089

Introduction. There is a strong scientific rationale to investigate the therapeutic potential of inhaled nebulised unfractionated heparin (UFH) for patients with COVID-19. UFH has antiviral, anti-inflammatory, and anticoagulant properties relevant to SARS-CoV-2 infection. In pre-pandemic clinical trials, nebulised UFH limits lung injury progression and the development of ARDS and accelerates recovery in patients with, or at risk of, acute respiratory distress syndrome.

Objectives. To determine the safety and efficacy-potential of inhaled nebulised unfractionated heparin (UFH) in the treatment of hospitalised patients with COVID-19.

Methods. Retrospective, uncontrolled multicentre single-arm case series of hospitalised patients with laboratory-confirmed COVID19, treated with inhaled nebulised UFH (5000 IU 8-hourly, 10000 IU 4-hourly, or 25000 IU 6-hourly) for $6 \pm 3$ (mean \pm SD) days. Outcomes were APTT before treatment (baseline) and highest-level during treatment (peak), and adverse events including bleeding. Exploratory efficacy outcomes were oxygenation, assessed by $\mathrm{SpO}_{2}$ to $\mathrm{FiO}_{2}(\mathrm{~S} / \mathrm{F})$ ratio and $\mathrm{FiO}_{2}$, and the WHO modified ordinal clinical scale (MOCS).

Results. 98 patients were included. In patients on stable prophylactic or therapeutic systemic anticoagulant therapy but not receiving therapeutic UFH infusion, APTT levels increased from baseline of $34 \pm 10 \mathrm{~s}$ to a peak of $38 \pm 11 \mathrm{~s}(\mathrm{p}<0.0001)$. In 3 patients on therapeutic UFH infusion, APTT levels did not significantly increase from baseline of $72 \pm 20$ to a peak of $84 \pm 28 \mathrm{~s}(p=0.17)$. Two patients had serious adverse events: bleeding gastric ulcer requiring transfusion; thigh haematoma; both were on therapeutic anticoagulation. Minor bleeding occurred in 16 patients, 13 of which were on therapeutic anticoagulation. The $\mathrm{S} / \mathrm{F}$ ratio, the $\mathrm{FiO}_{2}$ and the MOCS worsened before and improved after commencement of inhaled UFH (change in slope, $\mathrm{p}<0.001$ ).

Conclusion. Inhaled nebulised UFH in hospitalised patients with COVID-19 was safe. Although statistically significant, inhaled nebulised UFH did not produce a clinically relevant increase in APTT (peak values in the normal range). Exploratory analyses showed a significant improvement in oxygenation. Urgent randomised evaluation of nebulised UFH in patients with COVID-19 is warranted and several studies are currently underway.

\section{Reference(s)}

1. Dixon B, Smith RJ, Campbell DJ, Moran JL, Doig GS, Rechnitzer T, Maclsaac CM, Simpson N, van Haren FMP, Ghosh AN, Gupta S, Broadfield EJC,
Crozier TME, French C, Santamaria JD. Nebulised heparin for patients with or at risk of acute respiratory distress syndrome: a multicentre, randomised, double-blind, placebo-controlled phase 3 trial. The Lancet Respiratory Medicine 2021.

2. van Haren FMP, Richardson A, Yoon H-J, Artigas A, Laffey JG, Dixon B, Smith R, Vilaseca AB, Barbera RA, Ismail TI, Mahrous RS, Badr M, De Nucci G, Sverdloff C, van Loon LM, Camprubi-Rimblas M, Cosgrave DW, Smoot TL, Staas S, Sann K, Sas C, Belani A, Hillman C, Shute J, Carroll M, Wilkinson T, Carroll M, Singh D, Page C. INHALEd nebulised unfractionated HEParin for the treatment of hospitalised patients with COVID-19 (INHALEHEP): Protocol and statistical analysis plan for an investigator-initiated international metatrial of randomised studies. British Journal of Clinical Pharmacology 2021: 1-17

3. van Haren FMP, Page C, Laffey JG, Artigas A, Camprubi-Rimblas M, Nunes Q, Smith R, Shute J, Carroll M, Tree J, Carroll M, Singh D, Wilkinson T, Dixon B. Nebulised heparin as a treatment for COVID-19: scientific rationale and a call for randomised evidence. Crit Care 2020; 24: 454

4. No funding was obtained for this work

\section{2}

Antibiotic Duration by Clinicians Varies significantly

from Recommended Evidence-based Guidelines

A. Ekanayaka'; T. Navabi ${ }^{2}$; L. Irshad ${ }^{2}$; M. Harris' ${ }^{2}$;. Whitehouse ${ }^{1}$

${ }^{1}$ Department of anaesthesia and critical care, Queen Elizabeth Hospital Birmingham, Birmingham, United Kingdom; ${ }^{2}$ School of medicine, University of Birmingham, Birmingham, United Kingdom

Correspondence: A. Ekanayaka

Intensive Care Medicine Experimental 2021, 9(1): 001102

Introduction. Evidence suggests short course antibiotic therapy is non-inferior and causes fewer adverse events than long course therapy in multiple settings [1,2]. We believed variation existed between clinicians in the antibiotic durations they prescribed. We performed a survey to assess disparities between clinical practice and recommendations.

Objectives. To assess current prescription practice and barriers to trialing short course antibiotic therapy of 2-3 day duration in common uncomplicated infections (community acquired pneumonia [CAP], cellulitis, intra-abdominal infection [IAI], urinary tract infection [UTI]).

Methods. An online survey was distributed to doctors, nurses and allied health professions who prescribe antibiotics.

Results. 157 responses were acquired in January 2021: 77\% specialist doctors, $19 \%$ doctors in training. $50 \%$ of respondents worked in Adult Critical Care. Prescription guidance was sought from local trust/ practice guidelines in $87 \%$ of respondents, $66 \%$ after consultation with microbiology and $67 \%$ from previous experience.

\section{Current Prescription Practice}

Current antibiotic prescriptions for common infections are shown in Table 1.

Table 1. Duration of antibiotic prescription (days) with diagnosis

\begin{tabular}{|c|c|c|c|c|c|c|}
\hline & CAP & $\begin{array}{l}\text { Aspira- } \\
\text { tion } \\
\text { Pneu- } \\
\text { monia }\end{array}$ & $|A|$ & $\begin{array}{l}\text { Uncom- } \\
\text { plicated } \\
\text { cellulitis }\end{array}$ & $\begin{array}{l}\text { Uncom- } \\
\text { plicated } \\
\text { UTI } \\
\text { (male) }\end{array}$ & $\begin{array}{l}\text { Uncom- } \\
\text { plicated } \\
\text { UTI } \\
\text { (female) }\end{array}$ \\
\hline $\begin{array}{l}\text { Recom- } \\
\text { mended } \\
\text { duration } \\
\text { (days) }\end{array}$ & 5 [3] & 0 & $4[4]$ & $5-7[5]$ & $7-10[6]$ & $3[7]$ \\
\hline $\begin{array}{l}\text { No of } \\
\text { Respond- } \\
\text { ents }\end{array}$ & 157 & 157 & 156 & 157 & 157 & 157 \\
\hline $\begin{array}{l}\text { Number com- } \\
\text { pliant with } \\
\text { Recom- } \\
\text { menda- } \\
\text { tions n (\%) }\end{array}$ & $\begin{array}{l}115 \\
(73.2)\end{array}$ & $13(8.7)$ & $3(1.9)$ & $129(82.2)$ & $48(34.3)$ & 115 (73.2) \\
\hline
\end{tabular}


Persistent symptoms despite antibiotics in CAP led to $5 \%$ of respondents prescribing a second course of the same antibiotic, $50 \%$ prescribing a different antibiotic and $15 \%$ stopping antibiotics. The remainder decide on their actions depending on patient condition, microbiology advice or laboratory test.

\section{Short (2-3 day) course Prescriptions}

$77 \%$ of respondents said that a 2-3 day course of antibiotics within a trial setting was satisfactory for managing CAP and $65.8 \%$ in response to Controlled IAI. Reluctance to trial this duration of treatment in the remainder was due to concern about perceived or lack of published evidence suggesting community equipoise.

In such a trial, $70 \%$ and $58 \%$ would be willing to follow study protocol with signs/symptoms of persistent CAP or intra-abdominal infection respectively. $17 \%$ would revert to usual practice with either diagnosis. The remainder would review treatment based on clinical condition or consult trial teams.

Triggers to cease antibiotics in controlled IAI included Inflammatory Markers (CRP/WCC/Procalcitonin), or advice from specialist services (Surgery/Microbiology).

Conclusion. Current duration of antibiotic prescription for uncomplicated infections is not in accordance with current recommendations in up to $98 \%$.

The majority of respondents had a desire for short-course 2-3 day prescriptions but concern about evidence base. Clinical trials to confirm safety and effectiveness of 2-3 day course antibiotics for uncomplicated infection with robust protocols and monitoring are required.

\section{Reference(s)}

1. 1. Onakpoya IJ, Walker AS, Tan PS, Spencer EA, Gbinigie OA, Cook J, et al. Overview of systematic reviews assessing the evidence for shorter versus longer duration antibiotic treatment for bacterial infections in secondary care. PLoS One. 2018;13(3):e0194858.

2. 2. Hanretty AM, Gallagher JC. Shortened Courses of Antibiotics for Bacterial Infections: A Systematic Review of Randomized Controlled Trials. Pharmacotherapy. 2018:38(6):674-87.

3. 3. NICE CG 138 https://www.nice.org.uk/guidance/ng138/chapter/ Recommendations\#treatment-for-adults-young-people-and-children

4. 4. Sartelli M et al. Management of intra-abdominal infections: Recommendations by the WSES 2016 consensus conference. World Journal of Emergency Surgery. 2017;12(1):22

5. 5. https://www.nice.org.uk/guidance/ng141/resources/visual-summarypdf-6908401837

6. 6. Kyriakidou KG et al. Short- versus long course antibiotic therapy for acute pyelonephritis in adolescents and adults: A meta-analysis of randomized controlled trials. Clinical Therapeutics. 008;30(10):1859-68

7. 7. https://www.nice.org.uk/guidance/ng109/chapter/recommendations 8. Nil.

\section{6}

Predictive factors of secondary infections in critically ill COVID-19 patients

M. zghidi' ; I. Ben Saida²; R. Chelbi'; MY. KALLALA'; K. Meddeb ${ }^{1}$; E.

Bedhiafi'; R. Toumi ${ }^{1}$; M. Boussarsar ${ }^{1}$

${ }^{1}$ Medical Intensive Care Unit, Farhat Hached University Hospital, Sousse, Tunisia; ${ }^{2}$ Farhat Hached University Hospital, Medical Intensive Care Unit, Université de Sousse, Faculté de Médecine de Sousse, UR Nํ LR12SP09. Heart Failure, Sousse, Tunisia

Correspondence: M. zghidi

Intensive Care Medicine Experimental 2021, 9(1): 001106

Introduction. COVID-19 is a respiratory illness caused by an emerged virus SARS-CoV-2. The clinical features of this disease can range from asymptomatic infection to severe pneumonia. Like other viral pathogens, SARS-CoV-2 may predispose severe patients to bacterial coinfection. Data on infectious complications in critically ill COVID-19 patients are scarce.

Objectives. To determine the frequency and predictive factors of secondary infections in critically ill COVID-19 patients.
Methods. This was a retrospective study of patients hospitalized with severe COVID-19 from March 10th, 2020 to January 10th, 2021, in a medical intensive care unit. Were considered secondary infections occurring $48 \mathrm{~h}$ after hospital admission until death or discharge. Univariate analysis and multivariable logistic regression were used to analyze the associated factors with secondary infections.

Results. During the study period, 113 patients with positive RT-PCR COVID-19 were included. Patients' characteristics were: mean age, $64 \pm 11$ years; male, 80(70.8\%); Median SAPS II on admission, 29[2436]; Median SOFA on admission, 3[2-4]; invasive mechanical ventilation (IMV), 67(60.4\%); Median IMV duration, 4[0-10]; vasopressors use, $63(54.3 \%)$ and steroids use, $96(86 \%)$. The median length-of-stay was at $10[6-14]$ days. The overall mortality rate was $56.6 \%$.

Overall, 40(35.4\%) patients developed secondary infections. Patients with secondary infections had significantly longer duration of IMV (10[6-14] vs $0[0-4]$ days, $p=0.000)$, longer length of ICU stay (13[10$16]$ vs $7[4-11]$ days, $p=0.000)$ and higher mortality rate ( $85 \%$ vs $41 \%$, $\mathrm{p}=0.000$ ).

Ventilator Associated Pneumonia was the most common nosocomial infection 22(19.5\%) followed by infected atelectasis 15(13.3\%) and Central-venous-catheter-infection 9(8\%). The most common organism was Acinetobacterbaumanni $(\mathrm{n}=16)$.

Risk factors independently associated with secondary infection were length-of- ICU stay (RR 1,15 95\%Cl [1.04-1.27]; $p=0.006$ ) and NIV failure (RR 7.8, 95\%Cl [1.56-39]; $p=0.012$ ).

Conclusion. Patients hospitalized with severe and critical COVID-19 pneumonia had a high Frequency of secondary infections. At multivariable analysis, length of ICU stay and NIV failure were identified as independent risk factors for secondary infections.

\section{7}

Profile of secondary infections and patterns of antibiotic use in critically ill COVID-19 patients

M. zghidi ${ }^{1}$; I. Ben Saida ${ }^{2}$; MY. KALLALA ${ }^{1}$; K. Meddeb ${ }^{1}$; E. Bedhiafi'; R. Toumi ${ }^{1}$; J. Mahmoud'; M. Boussarsar'

${ }^{1}$ Medical Intensive Care Unit, Farhat Hached University Hospital, Sousse,

Tunisia; ${ }^{2}$ Farhat Hached University Hospital, Medical Intensive Care Unit, Université de Sousse, Faculté de Médecine de Sousse, UR N LR12SP09.

Heart Failure, Sousse, Tunisia

Correspondence: M. zghidi

Intensive Care Medicine Experimental 2021, 9(1): 001107

Introduction. COVID-19 is a respiratory illness caused by a newly emerged viral pathogen, SARS-CoV-2 coronavirus. Some reports have shown that COVID-19 patients are predisposed to develop secondary infections. While co-infection is a common phenomenon in other viral illnesses, data regarding secondary infections in COVID-19 is scarce.

Objectives. To assess the profile of secondary infections and pattern of antibiotic use in critically ill COVID-19 patients.

Methods. This was a retrospective study of patients hospitalized with severe COVID-19 from March 10th, 2020 to January 10th, 2021, in a medical intensive care unit. Were assessed clinical and microbiological features of ICU-acquired infections.

Results. A total of 113 patients with confirmed SARS-CoV-2 were included. Invasive mechanical ventilation (IMV), 67(60.4\%); Mean IMV duration, $9 \pm 2$ days; Central-venous-catheter (CVC), 69(61\%); Mean CVC duration, $6 \pm 4$ days. The median length-of-stay was at 10[6-14] days. The overall mortality rate was $56.6 \%$.

Forty(35.4\%) patients developed 45 episodes of nosocomial infections. Infection occurred after a median of 3[2-6] days of ICU admission. Ventilator-associated-pneumonia 22(19.5\%), with density incidence rate estimated at $35 \mathrm{VAP} / 1000$ ventilator days, was the most common nosocomial infection, followed by infected atelectasis $15(13.3 \%)$ and Central-venous-catheter-infection 9(8\%) with density incidence rate estimated at $21 \mathrm{CLABSI} / 1000$ catheter days. Among 224 samples, the most predominant were blood samples $159(71 \%)$ followed by endotracheal aspirates $29(13 \%)$, central venous catheter cultures $21(9.3 \%)$ and others $15(6.7 \%)$. Among the bacterial isolates $(n=35)$, Acinetobacterbaumannii, 16(55.1\%) was the most common pathogen, 
followed by Klebsiella pneumoniae, 6(20.7\%), and Pseudomonas aeruginosa, 4(13.7\%).

Most patients with COVID-19 received antibiotics prior to ICU admission, $75(64.7 \%)$. During hospitalization, $64(55 \%)$ patients had received at least 2 antibiotics and 5(4.4\%) patients had received 5 antibiotics. The main antibiotics used were Imipinem, 50(44.2\%); Amikacin, 40(35.9); Vancomycin, 33(29.2\%) and Colimycin, 33(29.2\%). The median duration of antibiotics use was 7[3-10]days.

Conclusion. Secondary infections are common in critically ill COVID19 patients. Measures aiming at reducing secondary infection are necessary to provide adequate critical care to those patients.

\section{3}

Profile and predictors of outcome in covid-19 disease:

\section{a retrospective cohort study}

P. Parhad'; A. Jindal 2 ; A. Galhotra'; N. Nagarkar'; AK. Behera

${ }^{1}$ School of Public Health, All India Institute Of Medical Sciences, Raipur, Bhilai, India; ${ }^{2}$ Pediatrics, All India Institute of Medical Sciences, Raipur, India; ${ }^{3}$ Community and family medicine, All India Institute of Medical Sciences, Raipur, India; ${ }^{4}$ Otorhinolaryngology and head and neck surgery, All India Institute of Medical Sciences, Raipur, India; ${ }^{5}$ Pulmonology, All India Institute of Medical Sciences, Raipur, India

Correspondence: P. Parhad

Intensive Care Medicine Experimental 2021, 9(1): 001123

Introduction. Central India remains an epicenter of Covid-19 in the country. Profile and predictors are important aspects to correlate with comorbidity in Covid-19 patients. The assessment of these records are highly valuable to understand and characterize the outcomes of disease in different age groups.1-4

Objectives. To assess the clinical profile and predictors of outcome in COVID-19 patients.

Methods. We assessed Profile, Predictors \& Comorbidities in Covid19 patients admitted to All India Institute of Medical Sciences, Raipur (C.G) which is a tertiary care health care institute of Central India. This retrospective study was performed from 19th March 2020 to 31st December 2020. Data of 5552 subjects who were hospitalized for Covid-19 disease had been collected in this study using WHO validated case record form. The records of $<14$ years and Brought dead patients have been excluded from the study. Demographic, epidemiological, clinical profile, predictors, and outcome data were obtained. Analysis of age, sex, epidemiological factors, signs and symptoms, comorbidities, and laboratory parameters were evaluated. IBM SPSS version 26 was used for data analysis and Pearson's Chi-square test, Mann-Whitney $\mathrm{U}$ test, Univariate and Multivariate logistic regression models were fitted to quantify the risk and predictors of mortality from Covid- 19 .

Results. Five thousand five hundred and fifty two patients $>14$ years of age ( $<40 \mathrm{yrs}, 38.38 \%$ and $>40 \mathrm{yrs}, 61.62 \%)$ were included. $71.24 \%$ were males. Clinical findings elicited fever (41.30\%) and Cough $(40.20 \%)$ as the predominant symptoms in patients. Comorbidities elicited hypertension (29.70\%), chronic cardiac disease $(5.80 \%$, including congenital heart disease excluding hypertension), diabetes $(25.40 \%)$, chronic kidney disease $(4.34 \%)$, thyroid disorder $(5.39 \%)$, obesity, $(0.31 \%)$, cancer $(1.57 \%)$, liver disease $(0.95 \%)$, chronic neurological disease $(1.10 \%)$, asthma (1.87\%) respectively. $47.51 \%$ had one or the other Comorbidities. Five hundred fifty nine patients $(10.07 \%)$ died. In multivariate logistic regression model, Age ( $>40$ years, OR: $5.33,95 \% \mathrm{Cl} 1.70-16.70)$, Obesity $(102.53,7.02-1496.06)$, Leucocytosis (6.63, 3.25-13.49) Lymphopenia (10.16, 3.08-33.51), Thrombocytopenia $(3.93,1.77-8.70)$, and Ferritin levels $>1000 \mathrm{ng} / \mathrm{ml}(4.67,1.99-10.97)$ were found significantly associated with mortality.

Conclusion. Our findings suggest that individuals $>40$ years and Obese patients have an increased risk of in-hospital mortality following COVID-19, Hypertension and Diabetes are the main Comorbidities present. Also, Leucocyte count, Lymphocyte, Platelet count, and Ferritin levels could be considered high risk and poor prognostic predictors in COVID-19 patients.
Reference(s)

1. Schirmer A, Wijaya M, Chiu MH, Maess B, Gunter TC, Brain T, et al. Sc Co Sc. 2020;1-25.

2. Wang D, Yin Y, Hu C, Liu X, Zhang X, Zhou S, et al. Clinical course and outcome of 107 patients infected with the novel coronavirus, SARS-CoV-2, discharged from two hospitals in Wuhan, China. Crit Care. 2020;24(1):1-9.

3. Yang X, Yu Y, Xu J, Shu H, Xia J, Liu H, et al. Clinical course and outcomes of critically ill patients with SARS-CoV-2 pneumonia in Wuhan, China: a single-centered, retrospective, observational study. Lancet Respir Med [Internet]. 2020;8(5):475-81

4. Guan W, Ni Z, Hu Y, Liang W, Ou C, He J, et al. Clinical characteristics of coronavirus disease 2019 in China. N Engl J Med. 2020;382(18):1708-20.

\section{4}

Mediterranean Spotted Fever: A review of severe cases admitted to intensive care unit

A. Costa, ${ }^{1}$ : B. Costa ${ }^{2}$; R. Amaral ${ }^{2}$; H. Santos, ${ }^{2}$ S. Carvalho, ${ }^{2}$ : M. Contente, ${ }^{2}$ 1 Internal Medicine, Centro Hospitalar De Trás-Os-Montes E Alto Douro, E.P.E., Vila Real, Portugal: ${ }^{2}$ Intensive Medicine, Centro Hospitalar De

Trás-Os-Montes E Alto Douro, E.P.E., Vila Real, Portugal

Correspondence: R. Amaral

Intensive Care Medicine Experimental 2021, 9(1): 001144

Introduction. Mediterranean Spotted Fever (MSF) remains endemic in Mediterranean region with Portugal having one of the highest incidences. The incidence of severe cases of this global and seasonal zoonosis is increasing.

Objectives. This retrospective study aims to reevaluate the disease clinical picture of patients with MFS admitted to the intensive care unit (ICU) of a tertiary hospital in the North of Portugal between 2017-2019.

Methods. Laboratory confirmation were obtained (serological and/or through direct isolation through nucleic acid amplification test). Three seronegative cases were also included due to its typical clinical presentation and epidemiologic context.

Results. From 2017 to 2019 , out of the 30 patients admitted for MSF $9(30 \%)$ were in our ICU, between June and October, with September being the month with highest incidence. The mean age was 75 (51-87) years and $55.5 \%(5 / 9)$ were male. The most prevalent comorbidities were diabetes mellitus and arterial hypertension; two patients were alcoholic. The most common symptoms at admission were fever $(100 \%)$, confusion/obtundation $(77,7 \%)$, gastrointestinal symptoms $(44,4 \%)$, arthalgias and/or myalgias $(44,4 \%)$. Eight patients $(88,8 \%)$ had maculopapular erythema and a "tache noir" injury was identified in 5 $(55,5 \%)$. Only 4 had the classical triad (44\%). Regarding acute severity scores and organ dysfunction, the mean SOFA score was 11 (6-20) and mean APACHE score was 23 (14-42). All patients had hematological dysfunction and $7(77,7 \%)$ had associated coagulopathy; 6 needed vasopressor support $(66.6 \%), 5(55,5 \%)$ had to be invasively ventilated and 3 needed renal replacement therapy (33,3\%). One patient had disseminated intravascular coagulation and two had confirmed meningoencephalitis. Concerning antibiotic approach, all patients were treated with doxycycline for, at least, 7 days, combined with cephalosporins and/or quinolones due to serologic confirmation delay and, in two cases, due to disease recognition delay ( $>48 \mathrm{~h}$ ). Mortality rate was $22,2 \%$ with one patient having a fulminant course of disease (deceased in the first $24 \mathrm{~h}$ after admission).

Conclusion. Despite its rarity and frequent favourable evolution, it is not accurate to consider MSF as a benign disease since it can evolve with multiple organ failure and need of invasive organic support with higher mortality. Awareness of this disease in endemic regions is essential for an early diagnosis since initial manifestations may be nonspecific or atypical. As directed antibiotic therapy is the cornerstone of MFS treatment, empirical therapy should be initiated as soon as possible. Despite the impossibility to identify risk factors associated with mortality due to our small casuistic, we hope this study contributes to its prompt future diagnosis and adequate treatment. 
Reference(s)

1. Abdad MY, Abou Abdallah R, Fournier P-E, Stenos J, Vasoo S. A concise review of the epidemiology and diagnostics of rickettsioses: Rickettsia and Orientia spp. J Clin Microbiol 2018 56:e01728-17

2. De Vito A., Geremia N., Mameli S.M., Fiore V., Serra P.A., Rocchitta G., Nuvoli S., Spanu A., Lobrano R., Cossu A., Babudieri S., Madeddu G. Epidemiology, clinical aspects, laboratory diagnosis and treatment of rickettsial diseases in the mediterranean area during COVID-19 pandemic: a review of the literature. Mediterr J Hematol Infect Dis 2020, 12(1): e2020056

3. Meireles M, MAagalhães M, Guimas A, Febre Escaro-Nodular: Revisão Retrospetiva de Casos Hospitalizados e Fatores Preditores de Doença Severa. Acta Med Port 2015:28:624-631

4. Ivan Baltadzhiev I, Popivanova N, Zaprianov Z. Malignant forms of Mediterranean spotted fever: risk factors for fatal outcomes. b r a z j i n f e c t d is. 2016

5. Carvalho et al. Rickettsiosis: a rare challenge in ICU. Intensive Care Medicine volume 43, pages1514-1515 (2017)

6. Bagshaw R. et al. The Characteristics and Clinical Course of Patients with Scrub Typhus and Queensland Tick Typhus Infection Requiring Intensive Care Unit Admission: A 23-year Case Series from Queensland, Tropical Australia. Am J Trop Med Hyg 2020 Dec;103(6):2472-2477

7. Nicholson W, Paddock C. Rickettsial Diseases (Including Spotted Fever and Typhus Fever Rickettsioses, Scrub Typhus, Anaplasmosis, and Ehrlichioses). Travel-Related Infectious Diseases. Centers for Disease Control and Prevention

8. Direção Geral da Saúde. Doenças de notificação, clínica e laboratorial obrigatória. Diário da República n. 251/2019, 12,513-B/2019

9. Sousa R, Nóbrega S, Bacellar F, Torgal F. Sobre a realidade da febre escaronodular em Portugal. Acta Médica Portuguesa. 2003; 16: 429-436

\section{9}

\section{Bilateral mydriasis in COVID-19 patients with ARDS receiving} rocuronium

P. Kontou ${ }^{1}$; V. Soulountsi ${ }^{1}$; C. Theocharidou ${ }^{1}$; C. Giannaki ${ }^{1}$; S. Akritidou'; SC. Kotoulas $^{1}$; M. Bitzani ${ }^{1}$

${ }^{1} A^{\prime}$ ICU, G. Papanikolaou Hospital, Thessaloniki, Greece

Correspondence: P. Kontou

Intensive Care Medicine Experimental 2021, 9(1): 001159

Introduction. COVID-19 patients hospitalized in the intensive care unit (ICU) mainly present acute respiratory distress syndrome (ARDS) and are prone to various complications. They require deep sedation and muscle paralysis due to high respiratory drive.

Objectives. At a certain time period, some patients hospitalized in a 18-bed ICU that was exclusively for COVID-19 patients, suddenly presented bilateral dilated nonreactive pupils. We aimed to evaluate this condition and to find any causative effect.

Methods. Based on the fact that these patients received increased dose of thromboprophylaxis because of the high risk of thrombosis, we first investigated any possible cerebrovascular event. In case this was excluded, we reviewed the drugs that the patients were receiving and that could cause bilateral mydriasis.

Results. Seven patients were identified presenting bilateral dilated nonreactive pupils on December 2020 and on April 2021. Their mean age was $66 \pm 7$ years and the majority of them (6) were female. They were admitted to the ICU due to acute respiratory failure $\left(\mathrm{pO}_{2} / \mathrm{FiO}_{2}\right.$ $110 \pm 33$ ), with mean APACHE II and SOFA scores on admission $15 \pm 2$ and $7 \pm 2$ respectively. During the first few days of their hospitalization, $70 \%$ of them were placed in prone position. Mydriasis was noted at a mean time of $14 \pm 7$ days from admission. A transcranial doppler ultrasonography (TCD) was subsequently performed in all patients and 3 out of 7 underwent head computed tomography (CT). The results of all the above examinations were negative for any brain pathology. By meticulous investigation to the patients' medications, we noticed that all of them were receiving the neuromuscular blocker, rocuronium. Moreover, December and April were the 2 months that there was shortage of cisatracurium, which is the neuromuscular blocking agent that we routinely use and which had been replaced by rocuronium. The patients with mydriasis had been receiving rocuronium in continuous infusion for $5 \pm 3$ days. In three patients, rocuronium infusion was stopped and the pupils gradually decreased in size and became reactive to light. Unfortunately, all patients eventually died due to septic shock, after being hospitalized for $21 \pm 7$ days in the ICU.

Conclusion. Pupil monitoring is important in COVID-19 patients, who are at high risk for thrombotic events. Clinicians should pay special attention to all the drugs administered to these patients. Continuous infusion of rocuronium can cause bilateral fixed and dilated pupils and mislead clinicians.

\section{2}

Risk factors for hospital mortality among critically ill COVID-19 patients: a multicenter retrospective study

MMS. Santos ${ }^{1}$;. Cardoso ${ }^{2}$; IJ. Pereira ${ }^{1}$;. Aragão ${ }^{3}$; A. Silva-Pinto ${ }^{4}$; L. Santos, ${ }^{4}$; A. Sarmento, ; L. Azevedo ${ }^{5}$; C. Granja

${ }^{1}$ CINTESIS, Faculdade de Medicina da Universidade do Porto, Porto,

Portugal; ${ }^{2}$ Unidade de cuidados intensivos polivalente, Centro Hospitalar Universitário do Porto, Porto, Portugal; ${ }^{3}$ Unidade de cuidados intensivos polivalente, Centro Hospitalar e Universitário do Porto, Porto, Portugal, Portugal; ${ }^{4}$ Serviço de doenças infecciosas, Centro Hospitalar e Universitário de São João, Porto, Portugal; ${ }^{5}$ Department of Community Medicine, Information and Health Decision Sciences, Faculdade de Medicina da Universidade do Porto, Porto, Portugal

Correspondence: M.M.S. Santos

Intensive Care Medicine Experimental 2021, 9(1): 001172

Introduction. Almost $10 \%$ of COVID-19 patients in Portugal were critical $(1,2)$, with prolonged Intensive Care Unit (ICU) length of stay and high mortality.(3) The analysis of pre-ICU patients profile and treatment-related aspects may identify individuals at increased risk for post-ICU mortality (4).

Objectives. To assess risk factors of hospital mortality among adult COVID-19 critically ill patients.

Methods. All adult COVID-19 patients admitted at 3 ICU in Portugal, from March 2020 to February 2021, were included based on their medical records. Pre-ICU health state was defined by the Charlson Comorbidity Index (CCI) (5) score considered as low (up to 1) or high comorbidity $(\geq 2)$. Severity of critical illness was assessed by the predicted risk of hospital death (Simplified Acute Physiology Scores-2) $(6,7)$. The need of organ support during ICU stay defined organ dysfunction. Infection, sepsis and septic shock were classified based on clinical evaluation.

Results. Table 1. Characteristics of ICU-treatment and patients $(n=231)$.

\begin{tabular}{|c|c|}
\hline \multicolumn{2}{|l|}{ Sociodemographic and pre-ICU state of health } \\
\hline Age, year-median (IQR) (n) & $67.0(57.0-74.0)(231)$ \\
\hline Age $\geq 65 \mathrm{yr}-\mathrm{n} /$ total $\mathrm{n}(\%)$ & $135 / 231(58.4)$ \\
\hline Female-n/total n(\%) & $80 / 231(34.6)$ \\
\hline High comorbidity—n/total n(\%) & $163 / 206(79.1)$ \\
\hline Obesity-n/total n(\%) & $69 / 206(33.5)$ \\
\hline SAPS II-median (IQR) (n) & $33.0(22.0-44.0)(231)$ \\
\hline $\begin{array}{l}\text { Sepsis or septic shock at ICU admission-n/total } \\
\mathrm{n}(\%)\end{array}$ & 6/231 (2.6) \\
\hline \multicolumn{2}{|l|}{ Organ dysfunctions during ICU stay } \\
\hline $\begin{array}{l}\text { Need of invasive mechanical ventilation (IMV) - n/ } \\
\text { total } n(\%)\end{array}$ & $115 / 206(55.8)$ \\
\hline Need of vasopressor (VPS)—n/total n(\%) & $98 / 204(48.0)$ \\
\hline $\begin{array}{l}\text { Need of renal replacement therapy (RRT) —n/ } \\
\text { total } n(\%)\end{array}$ & $23 / 200(11.5)$ \\
\hline $\begin{array}{l}\text { Need of blood/blood products transfusion-n/ } \\
\text { total } n(\%)\end{array}$ & $29 / 200(14.5)$ \\
\hline Nosocomial infection—n/total n(\%) & $93 / 201(46.3)$ \\
\hline Sepsis_-n/total n(\%) & $14 / 200(7.0)$ \\
\hline
\end{tabular}




\begin{tabular}{ll}
\hline Septic shock—n/total n(\%) & $37 / 200(18.5)$ \\
Length of ICU stay, d-median (IQR) (n) & $9(3.0-19.0)(231)$ \\
$\begin{array}{l}\text { Length of hospital stay, d—median (IQR) (n } \\
\text { assessed) }\end{array}$ & $18(10.0-36.0)(203)$ \\
ICU mortality—n/total n(\%) & $42 / 231(18.2)$ \\
Hospital mortality—n/total /n(\%) & $60 / 224(26.8)$ \\
\hline
\end{tabular}

$\mathrm{IQR}=$ interquartile range (p25-p75).

Using a logistic regression forward stepwise selection method, we identified the incidence of septic shock during ICU-stay (Odds ratio $[O R]=10.25 ; p<0.001)$, high comorbidity at ICU admission $(O R=6.54$; $p=0.034)$ and age $>65$ years $(O R=2.64 ; p=0.050)$ as the best, independent and statistically significant predictors of hospital mortality. Variables not included in the model were SAPS II, obesity, need of IMV, RRT, VPS, blood/blood products transfusion and the incidence of nosocomial infection and sepsis at ICU stay.

Conclusion. A high hospital mortality rate was observed among critically ill adult COVID-19 patients. Age $>65$ years, septic shock and high comorbidity were identified as main predictors of hospital mortality. Further research on predictors of mortality among critically ill COVID19 patients could improve patient stratification and support decisions regarding clinical management.

\section{Reference(s)}

1. No reported grant.

2. 1. Mendes JJ, Mergulhão P, Froes F, Paiva JA, Gouveia J. Recommendations from the Sociedade Portuguesa de Cuidados Intensivos and Infection \& Sepsis Group for intensive care approach to COVID-19. Revista Brasileira de terapia intensiva. 2020;32(1):2-10

3. 2. Truffaut L, Demey L, Bruyneel AV, Roman A, Alard S, De Vos N, et al. Post-discharge critical COVID-19 lung function related to severity of radiologic lung involvement at admission. Respiratory research. 2021;22(1):29

4. 3. Serafim RB, Póvoa P, Souza-Dantas V, Kalil AC, Salluh JIF. Clinical course and outcomes of critically ill patients with COVID-19 infection: a systematic review. Clinical microbiology and infection: the official publication of the European Society of Clinical Microbiology and Infectious Diseases. 2021;27(1):47-54.

5. 4. Rosa RG, Falavigna M, Robinson CC, Sanchez EC, Kochhann R, Schneider D, Sganzerla D, Dietrich C, Barbosa MG, de Souza D, Rech GS, Dos Santos RDR, da Silva AP, Santos MM, Dal Lago P, Sharshar T, Bozza FA, Teixeira C; Quality of Life After ICU Study Group Investigators and the BRICNet. Early and Late Mortality Following Discharge From the ICU: A Multicenter Prospective Cohort Study. Crit Care Med. 2020 Jan;48(1):64-72.

6. 5. Charlson ME, Pompei P, Ales KL, Mackenzie CR. A new method of classifying prognostic comorbidity in longitudinal studies: development and validation. J Chronic Dis. 1987;40(5):373-83. https://doi.org/10.1016/ 0021-9681(87)90171-8.

7. 6. Knaus WA, Draper EA, Wagner DP, Zimmerman JE. APACHE II: a severity of disease classification system. Crit Care Med. 1985 Oct;13(10):818-29.

8. 7. Le Gall JR, Lemeshow S, Saulnier F (1993) A new simplified acute physiology score (SAPS II) based on a European/North American multicenter study. JAMA 270: 2957-2963.

\section{3}

The effect of administration time of Tocilizumab therapy on mortality in critically ill COVID-19 patients

S. Ugur, ; UD. Adanur ${ }^{1}$; C. Emral ${ }^{2}$; I. Erus ${ }^{1}$; S.. Keske $;$; N. Cakarr'; E. Senturk ${ }^{1}$

${ }^{1}$ Anesthesiology and Reanimation, Koc University, Istanbul, Turkey;

${ }^{2}$ Metavision, Qlinicu, Istanbul, Turkey; ${ }^{3}$ Infectious Diseases and Clinical

Microbiology, American Hospital, Istanbul, Turkey

Correspondence: U.D. Adanur

Intensive Care Medicine Experimental 2021, 9(1): 001173

Introduction.: COVID-19 has caused 2.95 million of death because of ARDS and hyperinflammation. IL-6 blockers(Tocilizumab) have also been tried in the treatment of hypoxic patients. There are conflicting results for the treatment of Tocilizumab, especially timing of treatment. We evaluated the effect of Tocilizumab administration timing in COVID-19 patients in ICU on mortality and mechanical-ventilation (MV) duration.

Methods. A retrospective series of 219 COVID-19 patients that administered to two intensive care units (VKV-Amerikan Hospital and Koc University Hospital) between March 2020-2021 were analyzed. Tocilizumab was administered to patients who needed oxygen therapy and had fever with increased C-reactive protein value. The outcome measures were MV duration and mortality. Patients were divided into four groups according to timing of administration. 1-Pre-ICU-group: received Tocilizumab in the ward. 2-HFNC-group: during ICU stay with High Flow Nasal Cannula (HFNC) 3-NIMV-group: during non-invasive ventilation in ICU. 4-MV group: received Tocilizumab while intubated. Results. 219 patients were included; (140 Male, 79 Female), 110 patients received Tocilizumab therapy.

\begin{tabular}{llll}
\hline & $\begin{array}{l}\text { Num- } \\
\text { ber of } \\
\text { patients }\end{array}$ & $\begin{array}{l}\text { Median MV- } \\
\text { duration(day) }\end{array}$ & Mortality-rate \\
\hline Pre-ICU & 45 & 4.2 & $\% 24$ \\
HFNC & 16 & 1 & $\% 0$ \\
NIMV & 27 & 5 & $\% 37$ \\
MV & 22 & 18,2 & $\% 50$ \\
Not received & 109 & 8,5 & $\% 39$ \\
\hline
\end{tabular}

Of these 110 Patients; 45 treated Tocilizumab therapy in pre-ICU period, 16 patients during HFNC, 27 patients during NIMV, and 23 patients while on MV. Overall mortality was $38.8 \%$ in patients who did not receive Tocilizumab and $28.8 \%$ in patients received Tocilizumab. Mortality was $24 \%$ in pre-ICU, $0 \%$ in HFNC, $37 \%$ in NIV, $50 \%$ in IMV. Secondary infection rate was recorded as $53.7 \%$ and $58.5 \%$ in patients who didn't receive and received Tocilizumab respectively.

Conclusion. Our series showed statistically significant results, prior to initiation of MV of Tocilizumab administration had mortality rate of $\% 18$, which lower than administration during mechanical ventilation $\% 42$. Furthermore, no statistically significant results could be shown in secondary infection rate.

001191

Profile and predictors of outcome in children with covid-19

disease: a prospective cohort study

S. Veena Sudeepthi ${ }^{1}$; A. Jindal ${ }^{1}$

${ }^{1}$ Pediatrics, All India Institute of Medical Sciences, Raipur, India

Correspondence: A. Jindal

Intensive Care Medicine Experimental 2021, 9(1): 001191

Introduction. Central India remains an epicenter of Covid-19 in the country. Profile and predictors are important aspects to correlate with comorbidity in Covid-19 patients. The assessment of these records are highly valuable to understand and characterize the outcomes of disease in children.

Objectives. To describe the clinical characteristics of children and adolescents admitted to tertiary care hospital with confirmed COVID-19.

Methods. We assessed Profile, Predictors \& Comorbidities in Covid19 patients admitted to All India Institute of Medical Sciences, Raipur (C.G) which is a tertiary care health care institute of Central India. This prospective study was performed from 19th March 2020 to 31st December 2020. Data of 292 subjects who were hospitalized for Covid-19 disease had been collected in this study using WHO validated case record form. The records of $>16$ years patients have been excluded from the study. Demographic, epidemiological, clinical profile, predictors, and outcome data were obtained. Analysis of age, sex, epidemiological factors, signs and symptoms, comorbidities, and laboratory parameters were evaluated. IBM SPSS version 26 was used for data analysis and Pearson's chi-square test, Mann-Whitney U test, 
Univariate and Multivariate logistic regression models were fitted to quantify the risk and predictors of mortality from Covid- 19 .

Results. Two hundred and ninety two patients $<16$ years of age (age range 2 days-15 years) were included. 178 were males. Clinical findings elicited fever (11.64\%), Cough (10.27\%) and running nose $(7.87 \%)$ as the predominant symptoms in patients. Comorbidities was seen in $11.98 \%$ of kids including malignancy $(2.05 \%)$, chronic pulmonary disorders (2.05\%), chronic neurological disorders $(1.71 \%)$, chronic haematological disorders (1.36\%), liver disease $(1.03 \%)$, diabetes $(0.34 \%)$, chronic kidney disease $(0.34 \%)$, obesity, (0.34\%), asthma (0.34\%), Immunodeficiency disorders (0.34\%) and IEM (0.34\%) respectively. Admission to the PICU was significantly associated with higher C-reactive protein, CPK-MB levels and platelet counts $(\mathrm{P}<.05$ for all). Patients in the PICU were more likely to require high-flow nasal cannula $(P=.0001)$ and Remdesivir was not used in any one. Severe sepsis and septic shock syndromes were observed in $9(6 \%)$ patients in the PICU. Acute respiratory distress syndrome was observed in 20 (83.3\%) PICU patients, all required invasive mechanical ventilation for a median of 5 days. Of the 24 patients in the PICU, 18 (75\%) were discharged home, and $6(25 \%)$ patients died. The median pediatric intensive care unit length-ofstay was 6 days. Patients with comorbidities were older and comorbidities were independently associated with the need for invasive mechanical ventilation (OR $4.2 ; 95 \% \mathrm{Cl}, 1.67-17.12 ; \mathrm{p}=0.01$ ).

In multivariate logistic regression model, Leucocytosis $(6.63,3.25-13.49)$ Lymphopenia (10.16,3.08-33.51), Thrombocytopenia (3.93,1.77-8.70), and Ferritin levels $>1000 \mathrm{ng} / \mathrm{ml}(4.67,1.99-10.97)$ were found significantly associated with mortality.

Conclusion. We describe a higher than previously recognized rate of severe disease requiring PICU admission in pediatric patients admitted to the hospital with COVID-19. Age less than 1 year was not associated with a worse prognosis. Our findings suggest that children having leukocytosis, lymphopenia, thrombocytopenia and high Ferritin levels could be considered high risk and poor prognostic predictors in COVID-19 patients.

\section{Reference(s)}

1. Yang X, Yu Y, Xu J, Shu H, Xia J, Liu H, et al. Clinical course and outcomes of critically ill patients with SARS-CoV-2 pneumonia in Wuhan, China: a single-centered, retrospective, observational study. Lancet Respir Med [Internet]. 2020;8(5):475-81. Available from: http://dx.doi.org/10.1016/ S2213-2600(20)30079-5

2. Guan W, Ni Z, Hu Y, Liang W, Ou C, He J, et al. Clinical characteristics of coronavirus disease 2019 in China. N Engl J Med. 2020;382(18):1708-20.

\section{5}

Differences between the first and second waves in the profile, management and mortality of critically ill COVID-19 patients MMS. Santos ${ }^{1}$; T. Cardoso ${ }^{2}$; IJ. Pereira'; I. Aragão ${ }^{3}$; A. Silva-Pinto ${ }^{4}$; L. Santos, ${ }^{4}$; A. Sarmento, ${ }^{4}$, L. Azevedo ${ }^{5} ;$ C. Granja ${ }^{6}$

${ }^{1}$ CINTESIS, Faculdade de Medicina da Universidade do Porto, Porto, Portugal; ${ }^{2}$ Unidade de cuidados intensivos polivalente, Centro Hospitalar Universitário do Porto, Porto, Portugal; ${ }^{3}$ Unidade de cuidados intensivos polivalente, Centro Hospitalar e Universitário do Porto, Porto, Portugal, Portugal; ${ }^{4}$ Serviço de doenças infecciosas, Centro Hospitalar e Universitário de São João, Porto, Portugal; ${ }^{5}$ Department of Community Medicine, Information and Health Decision Sciences, Faculdade de Medicina da Universidade do Porto, Porto, Portugal; ${ }^{6}$ Unidade de cuidados intensivos, Centro Hospitalar e Universitário de São João, Porto, Portugal

Correspondence: M.M.S. Santos

Intensive Care Medicine Experimental 2021, 9(1): 001205

Introduction. In Europe, first-wave COVID-19 patients apparently had higher in-hospital mortality than second-wave patients (1). Pre-ICU patient characteristics and treatment-related aspects may explain different risks for post-ICU mortality between the first and second waves (2).
Objectives. To assess differences in the profiles and management of patients with COVID-19 admitted at the Intensive Care Unit (ICU) in the two pandemic waves and their impact in all-cause hospital mortality. Methods. We retrospectively collected data from all adult patients with COVID-19 diagnosed in three hospitals in Porto, Portugal, from March 2020 to February 2021. Pre-ICU state of health was assessed using the Charlson Comorbidity Index (CCl) (3) considered as low (score 0 to 1 ) or high comorbidity $(\geq 2)$. Severity of critical illness was assessed by the predicted risk of hospital death (Simplified Acute Physiology Scores 2) (4). Sepsis or septic shock were considered according to medical evaluation. We considered the first COVID-19 wave spanned from March 2020 to May 2020, while the second wave spanned from October 2020 to February 2021.

Results.

Table 1. Characteristics of patients, treatment and outcomes.

\begin{tabular}{|c|c|c|c|c|}
\hline & $\begin{array}{l}\text { 1st Wave } \\
(n=104)\end{array}$ & $\begin{array}{l}\text { Period between } \\
\text { waves }(n=32)\end{array}$ & $\begin{array}{l}\text { 2nd Wave } \\
(\mathrm{n}=95)\end{array}$ & $\mathrm{P}^{*}$ \\
\hline \multicolumn{5}{|c|}{ Sociodemographic and Pre-ICU state of health } \\
\hline $\begin{array}{l}\text { Age, year-median } \\
\quad(I Q R)(n)\end{array}$ & $\begin{array}{l}68.0(59.2-76.0) \\
\quad(10-4)\end{array}$ & $\begin{array}{l}70.5(55.7-78.0) \\
\quad(32)\end{array}$ & $\begin{array}{l}66.0(56.0-72.0) \\
\quad(95)\end{array}$ & 0.045 \\
\hline $\begin{array}{l}\text { Age } \geq 65 \mathrm{yr}-\mathrm{n} / \text { total } \\
\mathrm{n}(\%)\end{array}$ & $63 / 104(60.6)$ & $22 / 32(68.8)$ & $50 / 95(52.6)$ & 0.233 \\
\hline $\begin{array}{l}\text { Female sex-n/total } \\
n(\%)\end{array}$ & $39 / 104(37.5)$ & $12 / 32(37.5)$ & 29/95 (30.5) & 0.300 \\
\hline $\begin{array}{l}\text { High comorbidity-n/ } \\
\text { total } n(\%)\end{array}$ & $77 / 92(83.7)$ & $18 / 24(75.0)$ & $68 / 90(75.6)$ & 0.200 \\
\hline Obesity—n/total n(\%) & 28/92 (30.4) & $6 / 24(25.0)$ & $35 / 90(38.9)$ & 0.310 \\
\hline $\begin{array}{l}\text { SAPS II-median (IQR) } \\
(\mathrm{n})\end{array}$ & $\begin{array}{l}35(22.0-45.0) \\
\quad(104)\end{array}$ & $\begin{array}{l}34.5(23.2-45.0) \\
\quad(32)\end{array}$ & $\begin{array}{l}30(22.0-39.0) \\
\quad(95)\end{array}$ & 0.074 \\
\hline $\begin{array}{l}\text { Sepsis or septic shock } \\
\text { at ICU admission-n/ } \\
\text { total n(\%) }\end{array}$ & $3 / 104(2.9)$ & $0 / 32(0)$ & $3 / 92(3.2)$ & 0.910 \\
\hline \multicolumn{5}{|c|}{ Treatment during ICU stay } \\
\hline $\begin{array}{l}\text { Need of invasive } \\
\text { mechanical ventila- } \\
\text { tion (IMV)—n/total } \\
\text { n(\%) }\end{array}$ & $57 / 91(62.6)$ & $12 / 24(50.0)$ & $46 / 91(50.5)$ & 0.100 \\
\hline $\begin{array}{l}\text { Need of vasopressor- } \\
\text { n/total } n(\%)\end{array}$ & $49 / 89(55.1)$ & $11 / 24(45.8)$ & 38/91 (41.8) & 0.074 \\
\hline $\begin{array}{l}\text { Need of renal replace } \\
\text { therapy—n/total n(\%) }\end{array}$ & 9/85 (10.6) & $1 / 24(4.2)$ & 13/91 (14.3) & 0.459 \\
\hline $\begin{array}{l}\text { Length of ICU stay, d- } \\
\text { median (IQR) (n) }\end{array}$ & $6(2-19)(104)$ & $10(5.2-21.2)(32)$ & $\begin{array}{l}9(5.0-19.0) \\
(95)\end{array}$ & 0.094 \\
\hline $\begin{array}{l}\text { ICU mortality, } n-n / \\
\text { total } n(\%)\end{array}$ & 23/104 (22.1) & $4 / 32(12.5)$ & 15/95 (15.8) & 0.343 \\
\hline $\begin{array}{l}\text { Hospital mortality, } n-n / \\
\text { total (\%) }\end{array}$ & 32/103 (31.1) & $7 / 31(22.6)$ & $21 / 90(23.3)$ & 0.230 \\
\hline
\end{tabular}

*Comparing only the first and second COVID-19 waves. IQR =interquartile range (p25-p75).

In the first wave, although non-significant, there was a trend for patients being older, having higher comorbidity index and higher disease severity. There was also a clear trend for higher IMV use, lower length stay and higher all-cause ICU and hospital mortality in the first wave.

Conclusion. There were clinically relevant differences between the first and second pandemic waves regarding ICU COVID-19 patient profile, management and mortality.

\section{Reference(s)}

1. 4. Le Gall JR, Lemeshow S, Saulnier F (1993) A new simplified acute physiology score (SAPS II) based on a European/North American multicenter study. JAMA 270: 2957-2963.

2. No reported grant.

3. 1. Domingo P, Pomar V, Mur I, Castellví I, Corominas H, de Benito N. Not all COVID-19 pandemic waves are alike. Clin Microbiol Infect. 2021 Apr 19:S1 198-743X(21)00,188-9. https://doi.org/10.1016/..cmi.2021.04.005. Epub ahead of print. 
4. 2. Rosa RG, Falavigna M, Robinson CC, Sanchez EC, Kochhann R, Schneider D, Sganzerla D, Dietrich C, Barbosa MG, de Souza D, Rech GS, Dos Santos RDR, da Silva AP, Santos MM, Dal Lago P, Sharshar T, Bozza FA, Teixeira C; Quality of Life After ICU Study Group Investigators and the BRICNet. Early and Late Mortality Following Discharge From the ICU: A Multicenter Prospective Cohort Study. Crit Care Med. 2020 Jan;48(1):64-72.

5. 3. Charlson ME, Pompei P, Ales KL, MacKenzie CR. A new method of classifying prognostic comorbidity in longitudinal studies: development and validation. J Chronic Dis. 1987;40(5):373-83. https://doi.org/10.1016/ 0021-9681(87)90171-8.

\section{3}

Outcomes of COVID-19 obese patients admitted to an Infectious Diseases Intensive Care Unit (ID-ICU)

C. Silva ${ }^{1}$; A. Martins, ; J. Caldas, ${ }_{1}^{1}$; F. Cunha, ${ }^{2}$; A. Silva-Pinto ${ }^{1}$; AS. Santos, ${ }_{1}^{1}$; S. Xerinda, ${ }^{1}$; A. Ferreira, ${ }^{1}$; P. Figueiredo, ${ }^{1}$; A. Sarmento, ${ }^{1}$; L. Santos, ${ }^{1}$

${ }^{1}$ Serviço de doenças infecciosas, Centro Hospitalar e Universitário de São João, Porto, Portugal; ' ${ }^{2}$ Serviço de doenças infecciosas, Centro Hospitalar e Universitário de Coimbra, Coimbra, Portugal

Correspondence: $C$. Silva

Intensive Care Medicine Experimental 2021, 9(1): 001233

Introduction. COVID-19 obese patients have higher rates of hospitalisation and Intensive Care Unit (ICU) admission. To date, studies on whether obesity influences the outcome of patients once admitted to ICUs have yielded equivocal results.

Objectives. We aimed to compare the characteristics and outcomes of obese and non-obese COVID-19 patients admitted to an Infectious Diseases ICU (ID-ICU) dedicated to COVID-19 critically ill care during 2020.

Methods. We have conducted a retrospective analysis on 117 out of 144 confirmed COVID-19 cases who were admitted to our ICU between March and December 2020 and for whom data on Body Mass Index (BMI) was available. Proven COVID-19 required a positive SARS-CoV-2 nucleic acid amplification test on a respiratory sample. In accordance with World Health Organization (WHO), obesity was considered if patient's BMI $\geq 30 \mathrm{~kg} / \mathrm{m}^{2}$.

Results. In the final analysis, 117 patients were included. Mean $( \pm S D)$ BMI was $28.6 \pm 5.8$ with 39 patients $(33.3 \%)$ being obese. Mean age $( \pm S D)$ was $59 \pm 16$ in obese patients and $65 \pm 14$ years in non-obese. Considering gender, among women $53.3 \%$ were obese and among men 20.8\% ( $<<0.001)$. Mean ( \pm SD) APACHE II, SAPS II and SAPS III scores were $16.37 \pm 5.58,30.68 \pm 15.87$ and $53.41 \pm 14.41$, respectively. Thirty- eight (97.4\%) obese patients had at least one comorbidity (other than obesity) with hypertension (71.8\%), dyslipidaemia (59\%) and diabetes mellitus (43.6\%) being the most frequent ones. Median time from symptom onset to ICU admission was 8 days in both obese and non-obese. Thirty-five obese patients (89.7\%) presented respiratory dysfunction, but cardiovascular, hepatic and renal dysfunction were also common $(28.2 \%, 41.0 \%$ and $28.2 \%$, respectively). Nineteen obese patients $(48.7 \%)$ received corticosteroids, 12 (30.8\%) hydroxychloroquine and $4(10.3 \%)$ remdesivir. None received tocilizumab. Twenty-two (57.9\%) obese and 20 (61.5\%) non-obese patients were treated with invasive mechanical ventilation (IMV). BMI of patients on IMV was not statistically different from patients treated with non-invasive ventilation $(95 \% \mathrm{Cl}-0.39 ; 3.98, \mathrm{p}=0.106)$. In our cohort, nosocomial infections occurred in $31.3 \%$ patients. IMV patients that had secondary bacterial infections had a higher mean $\mathrm{BMI}(95 \% \mathrm{Cl} 0.54$; $6.18, p=0.02$ ). In a multivariable analysis using logistic regression, BMI (OR 1.13, 95\% Cl 1.01-1.26) was independently associated with bacterial infections in IMV patients. Median time on IMV was 16 days (IQR, 9-22) in obese and 13 days (IQR, 8-18) in non-obese individuals $(p=0.231)$, and mortality $13.2 \%$ and $15.4 \%$, respectively $(p=0.969)$. Non-survivors BMI was not statistically different from survivors $(95 \% \mathrm{Cl}$ $-2.25 ; 3.85, \mathrm{p}=0.607)$.

Conclusion. In this cohort of critically ill patients with COVID-19, obesity was an independent risk factor for bacterial infection in IMV patients but was not associated with statistically significant differences in IMV needs, duration of mechanical ventilation and allcause mortality.

\section{7}

Increase in ventilator-associated pneumonia incidence during the COVID-19 pandemic

D. Mestre ${ }^{1}$; P. Fernandes ${ }^{1}$; A. Paula; D. Pinto ${ }^{1}$; F. Gil ${ }^{1}$; J. Vaz ${ }^{1}$

${ }^{1}$ Unidade de cuidados intensivos polivalente, Hospital José Joaquim

Fernandes, Beja, Portugal

Correspondence: D. Mestre

Intensive Care Medicine Experimental 2021, 9(1): 001257

Introduction. Ventilator-associated pneumonia (VAP) remains one of the most common nosocomial infections in the intensive care unit (ICU), contributing to increased mortality and morbidity and leading to prolonged duration of mechanical ventilation (MV) and ICU stay. The current data on correlation between SARS-CoV-2 pneumonia and incidence of VAP are limited and a wide range of incidence values has been reported.

Objectives. We intended to identify and compare VAP incidence in one ICU, using the same prevention strategies, before and during the corona virus disease (COVID-19) pandemic. We aimed to understand and determine the correlation between SARS-CoV-2 pneumonia and VAP incidence and also identify the most commonly VAP associated microbiological agents in COVID-19 patients.

Methods. In this retrospective study we analysed all admitted adult patients requiring MV for more than $48 \mathrm{~h}$ during two periods, between April 2019 and March 2020 (before our first COVID-19 patient admission) and between April 2020 and March 2021. For VAP diagnosis we used clinical, radiological and microbiological criteria.

Results. In the study's first period 88 patients were included, with a median age of 69 and predominantly male (61.4\%). The VAP incidence was $8 \%$ and the average duration of MV was 9 days, with VAP diagnosis on average at day 6 of MV. In the second period 90 patients were included (37 patients with SARS-CoV-2 pneumonia at admission, 53 patients admitted for other causes). The median age was 67, the patients were predominantly male (62.2\%). The VAP incidence was $34 \%$, higher than the incidence in the first period, the average duration of MV was 11 days with VAP diagnosis on average at day 8 of MV. During the second analysed period the VAP incidence in patients admitted for SARS-CoV-2 pneumonia was statistically superior than in patients requiring $M V$ for other causes $(p<0.001) .74 .2 \%$ of all VAP diagnosis in this period were related to patients with SARS-CoV-2 pneumonia. Among 37 patients admitted for SARS-CoV-2 pneumonia $62.2 \%$ had a VAP diagnosis, on average at day 9 of MV. In this group the median duration of MV was 16 days. The most commonly isolated microorganism in COVID-19 patients VAP was Klebsiella pneumoniae (35.5\%), followed by Staphylococcus aureus (25.8\%) and Enterobacter aerogenes (6.5\%).

Conclusion. VAP incidence increased in MV patients during the COVID-19 pandemic and it was related to SARS-CoV-2 pneumonia. Critically ill patients with SARS-CoV-2 pneumonia requiring MV had a superior VAP incidence than patients requiring MV for other causes. Gram-negative bacilli were the VAP leading causative pathogens in patients requiring MV due to SARS-CoV-2 pneumonia.

\section{Reference(s)}

1. Rouzé A, Martin-Loeches I, Povoa P, Makris D, Artigas A, Bouchereau M, Lambiotte F, Metzelard M, Cuchet P, Boulle Geronimi C, Labruyere M, Tamion F, Nyunga M, Luyt CE, Labreuche J, Pouly O, Bardin J, Saade A, Asfar P, Baudel JL, Beurton A, Garot D, loannidou I, Kreitmann L, Llitjos JF, Magira E, Mégarbane B, Meguerditchian D, Moglia E, Mekontso-Dessap A, Reignier J, Turpin M, Pierre A, Plantefeve G, Vinsonneau C, Floch PE, Weiss N, Ceccato A, Torres A, Duhamel A, Nseir S; coVAPid study Group. Relationship between SARS-CoV-2 infection and the incidence of ventilatorassociated lower respiratory tract infections: a European multicenter cohort study. Intensive Care Med. 2021 Feb;47(2):188-198.

2. Wicky, PH., Niedermann, M.S. \& Timsit, JF. Ventilator-associated pneumonia in the era of COVID-19 pandemic: How common and what is the impact?. Crit Care 25, 153 (2021). 
3. Papazian, L., Klompas, M. \& Luyt, CE. Ventilator-associated pneumonia in adults: a narrative review. Intensive Care Med 46, 888-906 (2020).

4. S. Ewig, E. Müller, E. Hecker. Ventilator-associated pneumonia: approaching the horizon. European Respiratory Journal Apr 2010, 35 (4) 725-727.

5. No funding was received for the study.

\section{1}

\section{Tocilizumab use in critically ill COVID-19 patients increases} the risk of central line associated blood stream infections (CLABSI) A. Harper ${ }^{1}$; SR. Ashok, ${ }^{2}$

${ }^{1}$ Anaesthetics and Intensive Care, Croydon University Hospital, Croydon, United Kingdom; ${ }^{2}$ Intensive care, Croydon University Hospital, Croydon, United Kingdom

Correspondence: A. Harper

Intensive Care Medicine Experimental 2021, 9(1): 001311

Introduction. Tocilizumab is a humanised monoclonal antibody against the Interleukin 6 (IL6) receptor. Following the RECOVERY and REMAP-CAP trials NHS England has commissioned tocilizumab for use in severe COVID-19 infection.1,2 IL6 influences various immune functions involving both innate and adaptive immunity, recognition of pathogens, immune cell differentiation, plasma cell growth and immunoglobulin production.

It has been established that tocilizumab causes immunosuppression and an increased risk of serious infection with long term use in rheumatoid arthritis. 3-5 However, the risk of secondary infections with its short-term use in critically ill COVID 19 patients is less certain.

Objectives. To compare the incidence of CLABSI between critically ill COVID-19 patients treated with tocilizumab and those who were not.

Methods. Retrospective analysis of patients admitted to critical care testing PCR positive for COVID 19 and ultimately requiring invasive ventilation. Patients treated with Tocilizumab $(8 \mathrm{mg} / \mathrm{kg})$ within $48 \mathrm{~h}$ of ITU admission were compared with those not treated. The primary outcome measure was incidence of CLABSI within the first 28 days of ITU admission. The centres for disease control and prevention (CDC) definition of CLABSI was used for this study.

Results. There were 50 patients identified meeting the inclusion criteria, of which 32 were treated with tocilizumab and 18 were not The risk of developing CLABSI was significantly higher in those who received tocilizumab compared to those who did not $(40.6 \%$ vs. $11.8 \%$; $p=0.037)$. The two groups showed no difference between the mean age (51.5 years vs. 50.4 years) or Charlson Comorbidity Index (1.9 vs. 1.6 points). Additionally, the mean APACHEll scores on admission were no different between the group treated with tocilizumab and the group who were not (12.2 points vs. 12.8 points). However, the group treated with tocilizumab had a mortality rate of $56.3 \%$ compared to $82.3 \%$ in the group not treated with tocilizumab although this was not statistically significant ( $p=0.068$ )

Conclusion. Tocilizumab use causes immune suppression. This likely increases the risk of CLABSI for critically ill patients with COVID-19 requiring invasive ventilation. It has, however, also been demonstrated to provide a significant survival benefit 1,2. For patients treated with tocilizumab in critical care clinicians should have increased vigilance for CLABSI, especially given that tocilizumab also supresses inflammatory markers such as c-reactive protein, neutrophil count and procalcitonin6.

\section{Reference(s)}

1. 6. Ivan Hariyanto T, Kurniawan A. Tocilizumab administration is associated with the reduction in biomarkers of coronavirus disease 2019 infection. J Med Virol. 2021 Mar;93(3):1832-1836.

2. 5. Grøn KL, Arkema EV, Glintborg B, Mehnert F, Østergaard M, Dreyer L, Nørgaard M, Krogh NS, Askling J, Hetland ML; ARTIS Study Group. Risk of serious infections in patients with rheumatoid arthritis treated in routine care with abatacept, rituximab and tocilizumab in Denmark and Sweden. Ann Rheum Dis. 2019 Mar;78(3):320-327.

3. 4. Hoshi D, Nakajima A, Inoue E, Shidara K, Sato E, Kitahama M, Seto Y, Tanaka E, Urano W, Ichikawa N, Koseki Y, Momohara S, Taniguchi A, Nishimoto $\mathrm{N}$, Yamanaka $\mathrm{H}$. Incidence of serious respiratory infections in patients with rheumatoid arthritis treated with tocilizumab. Mod Rheumatol. 2012 Feb;22(1):122-7.

4. 3. Pawar A, Desai RJ, Solomon DH, Santiago Ortiz AJ, Gale S, Bao M, Sarsour K, Schneeweiss S, Kim SC. Risk of serious infections in tocilizumab versus other biologic drugs in patients with rheumatoid arthritis: a multidatabase cohort study. Ann Rheum Dis. 2019 Apr;78(4):456-464.

5. 2. REMAP-CAP Investigators, Gordon AC et al. Interleukin-6 Receptor Antagonists in Critically III Patients with Covid-19. N Engl J Med. 2021 Feb 25:NEJMoa2100433. https://doi.org/10.1056/NEJMoa2100433. Epub ahead of print. PMID: 33631065; PMCID: PMC7953461.

6. 1. RECOVERY Collaborative Group, Horby PW, Pessoa-Amorim G, et al. Tocilizumab in patients admitted to hospital with COVID-19 (RECOVERY): preliminary results of a randomised, controlled, open-label, platform trial. medRxiv. 2021;preprint.

7. This research project was registered locally with Croydon University Hospitals research and development team. There are no conflicts of interest, grants or funding to declare.

\section{5}

\section{Bacterial co-infection and COVID-19 in ICU}

D. Guerra, ; P. Campos²; R. Antunes²; I. Aragão²; MJ. Ferreira Da Silva²

${ }^{1}$ Serviço de doenças infeciosas, Centro Hospitalar e Universitário do Porto, Porto, Portugal; ${ }^{2}$ Unidade de cuidados intensivos polivalente, Centro

Hospitalar e Universitário do Porto, Porto, Portugal

Correspondence: D. Guerra

Intensive Care Medicine Experimental 2021, 9(1): 001315

Introduction. Many airway infections result from a bacterial superinfection either subsequent to, or concurrent with, an ongoing upper respiratory tract viral infection.

The examples include the role of influenza virus in bacterial pneumonia [1] and the fact that "viral colds" predispose to bacterial rhinosinusitis in both adults and children [2].

Overall, the average bacterial co-infection in patients with severe influenza is estimated to be $20-30 \%$. Moreover, bacterial co-infection has been linked to disease severity and increased mortality [2].

Fearing the same outcomes in COVID-19 patients, many start empirical antibiotics, assuming co-infection. This raises concerns regarding antimicrobial resistance and treatment failure, particularly, in Intensive Care Unit (ICU) environment.

Objectives. Evaluate bacterial co-infections in COVID-19 patients, determine microbiological etiology, as well as clinical impact in COVID-19 natural evolution.

Methods. Descriptive, retrospective and observational study conducted in a tertiary hospital, from March to May 2020. All patients with confirmed COVID-19 admitted in ICU were included. Patients under 18 years of age were excluded. The statistical analysis was performed by using IBM-SPSS ${ }^{\circledR}, \mathrm{v} .27$.

Results. From 49 patients admitted at ICU due to severe COVID-19, 38 were assumed to have a bacterial co-infection and 13 even presented with more than a single co-infection.

Most co-infected patients were male $(n=23 ; 60.5 \%)$, with 68 years-old in median and only seven were active or past smokers. Smokers did not have an increased risk of co-infection [Odds ratio (OR) 2.258 (Confidence Interval-Cl-95\% 0.247-20.650, $p=0.471)]$.

Bacterial pneumonia was the most common infectious complication in ICU patients admitted due to COVID-19 affecting 34 (69.4\%) patients, followed by urinary tract infection in $13(26.5 \%)$ and bacteremia in five (10.2\%) patients.

Piperacillin/tazobactam was the most frequently used empirical antibiotic $(n=15 ; 39.5 \%)$, followed by amoxicillin/clavulanate $(n=11$; 28.9\%).

Microbial isolation was possible in 20 (52.6\%) cases and E. coli was the most frequently encountered pathogen $(n=9)$. Nine $(23.7 \%)$ patients had been infected by multi-resistant microorganisms including two (5.3\%) by carbapenemase-producing Enterobacteriaceae.

Overall, co-infection was associated with higher hospital mortality $(n=22 ; 57.9 \%)$ in patients admitted due to severe COVID [OR 13.750 (Cl 95\% 1.595-118.531, $p=0.017)]$. When conducting logistic regression, multivariate analysis determined that both age $(p=0.037$ OR 
1.004-1.149) and co-infection ( $p=0.033 \mathrm{mOR} 10.814 \mathrm{Cl} 1.209-96.767)$ were independent risk factors for mortality. While no patient without co-infection died in the ICU, co-infected patients presented a mortality of $55.3 \%(p=0.001)$

Conclusion. Bacterial co-infection is a common complication of viral airway infections which also seems to be true regarding severe COVID19. According to our data, smokers do not seem to have increased risk of bacterial co-infection once infected with SARS-CoV-2. Microbiologic findings revealed $23.7 \%$ of co-infections by multirresistant organisms. Finally, co-infection had a major role on hospital mortality.

\section{Reference(s)}

1. 2) Marom T, Alvarez-Fernandez PE, Jennings K, Patel JA, McCormick DP, Chonmaitree T. Acute bacterial sinusitis complicating viral upper respiratory tract infection in young children. Pediatr Infect Dis J. 2014;33(8):803808. https://doi.org/10.1097/INF.00000000000000278

2. 1) Bakaletz LO. Viral-bacterial co-infections in the respiratory tract. Curr Opin Microbiol. 2017;35:30-35. https://doi.org/10.1016/j.mib.2016.11.003

\section{5}

Superinfections in critically ill patients with severe covid-19: incidence and microbiological profile in the first and subsequent epidemic waves

L. López-Rodríguez'; D. Molina, ${ }^{2}$; D. Vasco ${ }^{3}$; D. Manzano, ; C. Jaramillo, ; O. Peñuelas ${ }^{5}$. JÁ Lorente,

${ }^{1}$ Universidad europea de madrid, Getafe University Hospital, Getafe, Spain; ${ }^{2}$ Department of Medical Microbiology, Getafe University Hospital, Getafe, Spain; ${ }^{3}$ Intensive Care Unit, Getafe University Hospital, Getafe, Spain; ${ }^{4}$ Intensive Care Unit, University Hospital of Getafe, Madrid, Spain; ${ }^{5}$ Ciberes, Getafe University Hospital, Getafe, Spain; ${ }^{6}$ Getafe University Hospital. universidad europea, CIBERES, Madrid, Spain

Correspondence: $\mathrm{O}$. Peñuelas

Intensive Care Medicine Experimental 2021, 9(1): 001325

Introduction. The incidence and microbiological profile of superin fections in patients with coronavirus disease (COVID)-19, as well as their time course from the first wave to subsequent waves is not well known.

Objectives. To describe the incidence and etiology of infections in patients with COVID-19 admitted to the ICU, and their change over time from the first wave (March 7, 2020 to April 30, 2020, period 1) to subsequent waves (May 1, 2020 to March 31, 2021, period 2).

Methods. We retrospectively analyzed prospectively collected data of patients admitted to the intensive care unit (ICU) of our institution with the diagnosis of COVID-19. Inclusion criteria was admission because of acute respiratory failure due to COVID-19, positive RT-qPCR test for SARS-CoV-2, and requirement of mechanical ventilation. Exclusion criteria were transfer to another hospital before ICU discharge. Information pertaining their demographic characteristics, results of cultures of diagnostic samples as well as diagnoses of infections (ventilator associated pneumonia [VAP]; primary [PriBact], secondary [SecBact] or catheter-related bacteremia (CathBact); urinary tract infection [UTI]), diagnosed according to commonly accepted criteria was collected (ref.1). Only the first episode of each infection was considered for analysis. The primary outcome variable was ICU mortality. Categorical and quantitative variables were compared by the Chi square test and the Student's $\mathrm{t}$ test, respectively. Data are $\mathrm{n}$ and percentage, or means \pm SEM. A $p$ value $<0.05$ was considered statistically significant. The study was approved by the IRB.

Results. Patients in period $1(\mathrm{n}=112)$ and period $2(\mathrm{n}=102)$ were $64.6 \pm 1.0$ vs. $63.9 \pm 1.3$ years of age $(p=0.152)$, SAPS III score $51.4 \pm 0.8$ vs. $53.7 \pm 0.9(p=0.354)$, male sex $79(70.5 \%)$ vs. $75(73.5 \%)$ $(p=0.650)$, ICU mortality $38(33.9 \%)$ vs. $33(32.4 \%)(p=0.995)$, hospital mortality $43(38.4 \%)$ vs. $35(34.3 \%)(p=0.885)$, respectively. The incidence of infections in periods 1 and period 2 were, respectively: patients with at least one episode of infection $53(47.3 \%)$ vs. $60(58.8 \%) \quad(p=0.101)$; VAP $12(10.7 \%)$ vs. $30(29.4 \%) \quad(p=0.001)$; CathBact $24(21.4 \%)$ vs. 20 (19.6) $(p=0.866)$; SecBact $8(7.1 \%)$ vs. 14 $(13.7 \%)(p=0.122)$; PrimBact 12 (10.7\%) vs. 8 (7.8\%) $(p=0.492)$; ITU 20
(17.9\%) vs. 25 (24.5\%) ( $p=0-245)$. The most common microorganisms (number of positive cultures / total number of isolates [percentage of isolates], during period 1 and period 2, respectively) were: VAP, P. aeruginosa $1 / 12(8 \%)$ vs. $8 / 30(27 \%)(p=0.191)$, and S. aureus $0 / 12(0 \%)$ vs. $6 / 30(20 \%)(p=0.579)$; CathBact, S. epidermidis $12 / 24(50 \%)$ vs. $14 / 20$ $(70 \%)(p=0.300)$; SecBact, E. faecalis 3/8 (38\%), 3/14 (21\%) $(p=0.752)$ PriBact, E. faecalis $6 / 12(50 \%)$ vs. $2 / 8(25 \%)(\mathrm{p}=0.514) ; \mathrm{ITU}, E$. faecalis $8 / 20(40 \%) 7 / 25(28 \%)(p=0.396)$.

Conclusion. Mechanically ventilated patients with COVID-19 admitted to the ICU during the first vs. subsequent epidemic waves did not differ in severity or ICU mortality. Significantly more cases of VAP were diagnosed during period 2 as compared to period 1, probably due to more frequent sampling. The most frequent microorganisms were $P$. aeruginosa and S. aureus in VAP, and E. faecalis in primary and secondary bacteremia. We could not prove based on our results a change in the microbiological profile form the first to subsequent epidemic waves.

\section{Reference(s)}

1. 1.- Zaragoza R, Ramírez P, López-Pueyo MJ. Infección nosocomial en las unidades de cuidados intensivos. Enferm Infecc Microbiol Clin 2014; 32(5): $320-327$

\section{6}

Association between cardiac troponin I levels and mortality in 100 patients admitted to the ICU of "Hospital Virgen de las Nieves (HUVN)" during the Covid19 pandemic

I. Guzman Adum '; L. Hidalgo Manchado ${ }^{1}$; R. Diaz Contreras ${ }^{1}$

${ }^{1}$ Intensive Care Unit, University Hospital Complex of Granada, Granada, Spain

Correspondence: I. Guzman Adum

Intensive Care Medicine Experimental 2021, 9(1): 001336

Introduction. Covid19 is a serious infective condition caused by the new coronavirus SARS-CoV- 2 , but less than $20 \%$ of the cases evolve into a severe critical illness characterized by interstitial pneumonia, which may turn into ARDS and multi organ failure. The role of any predisposing or prognostic factors are still in discussion; major cardiac complications are reported in several patients, possibly leading to death.

Objectives. To determine whether high sensitivity cardiac troponin I (hs-Tnl) levels, during ICU hospitalization, are predictive of mortality in patients with COVID-19. We believe that troponin can be a useful biomarker of disease progression and worse prognosis in COVID-19 patients.

Methods. We performed an observational, retrospective and descriptive study that includes 100 random patients admitted to the HUVN's ICU with Covid19 during the pandemic (October/2020 to December/2021) and had at least one cardiac troponin determination during the ICU admission period.

We collected and analyzed data with SPSS program in terms of descriptive statistics. Even with this small sample, the study's population follows a normal distribution, so we use the mean and standard deviation for quantitative variables and absolute and relative frequencies for qualitative variables.

Results. The mean age of the included patients was 61.27 (SD 13), $26 \%$ of them were women and $74 \%$ men. Regarding the most prevalent comorbidities: diabetes mellitus in $33 \%$, hypertension in $53 \%$, chronic kidney disease in $7 \%$, ischemic heart disease $11 \%$, chronic obstructive pulmonary disease in $8 \%$, obstructive sleep apnea in $9 \%$, and immunosuppression due to different etiologies in $8 \%$ of the cases. Total mortality in the ICU was $32 \%$, which corresponds to an APACHE II score at admission of 10.98 on average (SD 5.3), SOFA at admission 4.09 (SD 1.8). The highest cardiac troponin level during admission was in averaged 2870 but with a highly variable range, most of the data being below $500 \mathrm{pg} / \mathrm{ml}$. A mortality analysis was performed according to the level of troponins (categorized into intervals) and we found that the highest death rate was in the group of determinations below $500 \mathrm{pg} / \mathrm{ml}$ (it was also the largest group by far, 86 patients and 
mortality $32 \%)$. Hypothesis contrast tests were not performed due to the small number of patients in the other groups.

Conclusion. Even though it has being described in previous studies that an increase level of hs-Tnl is associated with elevated mortality and disease progression in patients with COVID-19, we were not able to address adequately this issue because of our limited sample sized and lack of patients with higher troponin levels. In terms of descriptive statistics, this study may suggest that other baseline characteristics have the most influence in the outcomes.

\section{Reference(s)}

1. Michela Salvatici, Barbara Barbieri, Sara Maria Giulia Cioffi, Emanuela Morenghi, Francesco Paolo Leone, Federica Maura, Giuseppe Moriello \& Maria Teresa Sandri (2020) Association between cardiac troponin I and mortality in patients with COVID-19, Biomarkers, 25:8, 634-640, https:// doi.org/10.1080/1354750X.2020.1831609

2. Faryal Khamis, Ziad Memish, Maher Al Bahrani, Samata Al Dowaiki, Nenad Pandak, Zakaryia Al Bolushi, Issa Al Salmi, Ibrahim Al-Zakwani. (2021) Prevalence and Predictors of in-Hospital Mortality of Patients Hospitalized with COVID-19 Infection. Journal of Infection and Public Health 35.

\section{8}

Predictive model of mortality in ICU patients with respiratory affection due COVID-19

M. Guerrero Marin ${ }^{1}$; M. Gordillo Resina ${ }^{2}$; AS. T ${ }^{1}$

${ }^{1}$ Medicina intensiva, Hospital of Jaen, Jaén, Spain; ${ }^{2}$ Medicina intensiva, Hospital of Jaén, Jaén, Spain

Correspondence: M. Guerrero Marin

Intensive Care Medicine Experimental 2021, 9(1): 001348

\section{Introduction.}

Objectives. Trying to build a predictive model admitted on ICU because of respiratory afection due COVID-19.

Methods. Survey of prospective cohorts of coronavirus patients admitted to ICU at the Hospital Universitario de Jaén, Hospital San Agustín de Linares, Hospital Infanta Margarita de Cabra y Hospital de la Serrania Ronda.

Continuous variables are expressed as median (25th percentile, 75th percentile) and qualitative variables with absolute and relative frequencies. The Mann Whitney $U$ was used for comparison of continuous variables, X2 for qualitative variables, and logistic regression for multivariate analysis. $\mathrm{P}<0.05$ was considered statistically significant (e.s).

Results. From the 73 studied patients, 72 were alive on the third day. We could have coticed that mortality was better predicted at this time than on the day of admission.

The average age was $62(58,71)$ years old, at the moment of the admittance APACHE II was $13(11,16)$ points, SOFA $6(3,8)$ points. Ont the third day SOFA was 7 (5,9 points) and the difference in SOFA between the first and third day was $0.5(0.3)$ points, $(p<0.001)$.

There was statistically significant relation with the number of lymphocites on the third day and with APACHE II at the admittance and with the SOFA on the third day, but not with the SOFA at the admittance.

An attempt was made to evaluate the relationship with mortality by categorizing these variables and to reach this, classification trees were used. $29.2 \%$ of the patients had a SOFA score $<6$ points on the third day and their mortality was $9.6 \%$ vs $51 \%$ of the 51 in which it was $>6$ points, $(p=0.001)$. On the third day, 27 patients had $<600$ lymphocytes on the third day and $66.7 \%$ died and $22.2 \%$ of the 45 who had $>600$ lymphocytes $(p<0.001) .16$ patients had an APACHE- $\|<11$ at admission and their mortality was $6.3 \%$ and in 56 it was $>11$ points and their mortality $48.2 \%(p=0.002)$.

The model with logistic regression was: SOFA on the third day $>6$ points vs $<6$ points OR: 11.66 (1.97-68.95) and Lymphocytes (less than or equal to 600 vs more than 600) OR: 7.34 (2.02-26.61) and APACHE II (greater than or equal to 11 points vs less than 11 points) OR: 15.46 (1.53-155.97); constant: -5.59 . The area under the ROC curve for this model was $0.852(0.765-0.938)$ and correctly classifies $79.17 \%$ of the patients.
Patients with SOFA on the third day greater or equal to 6, APACHE II at the admittance greater or equal to 11 points and les or equal to 600 lymphocites on the third day $(\mathrm{N}=20)$ had higher predicted mortality, according to the described modelo of $80.96 \%$, being the observed mortality the $80 \%$. And there were 7 patients withouth any of that features (SOFA on the third day $<6$ points, APACHE-II at the admittance $<11$ points and lymphocites on the third day $>600$ ) and in these one the predicted mortality was $3.7 \%$ and the observe done $0 \%$.

Conclusion. In patients admitted on ICU, mostly of those with respitatory pathology due COVID-19 on the third day were alive. In that momento it is posible to build a predictive model given the APACHE-II on admission, the SOFA on the third day and the number of lymphocytes. The model has high discrimination and is easy to apply.

\section{4}

Comparison of the results of pathogenic microorganisms isolation from ICU patients by standard methods of bacterial detection and MALDI-TOF mass spectrometry

V. Grnja' ; L. Jedud, 2; I. Kovacevic ${ }^{3}$; M. Bogdan ${ }^{4}$; Z. Debeljak'; D.

Drenjancevic ${ }^{4}$; S. Kvolik ${ }^{6}$

${ }^{1}$ Anesthesiology and ICU, Medical Faculty, Osijek, Croatia; ${ }^{2}$ Anesthsiology and icu, Medical Faculty, Osijek, Croatia; ${ }^{3}$ Anesthesiology and ICU, Medical Faculty, Osijek, Croatia; ${ }^{4}$ Department of microbiology, Osijek Univer-

sity Hospital; Medical Faculty, Osijek, Croatia; ${ }^{5}$ Department for Clinical and Laboratory Diagnostics, Osijek University Hospital; Medical Faculty, Osijek, Croatia; ${ }^{6}$ Anesthesiology and ICU, Osijek University Hospital; Medical Faculty, Osijek, Croatia

Correspondence: $\mathrm{S}$. Kvolik

Intensive Care Medicine Experimental 2021, 9(1): 001354

Introduction. Bacterial identification using standard bacterial cultivation can be significantly improved by applying matrix-assisted laser desorption ionization-time of light mass spectrometry (MALDI-TOF MS). This new technology makes it possible to quickly identify bacteria by measuring the mass and charge of various molecules, components of a bacterial cell. Conventional diagnostic methods are slower and more demanding, and it is possible that this method significantly speeds up and facilitates the process of identifying bacteria, gaining thus more positive results.

Objectives. During the COVID-19 pandemic, a large number of doctors and nurses from the surgical intensive care unit were directed towards the treatment of COVID-19 patients and were replaced by new staff. Rapid staff changes may contribute to an increased incidence of infections in the ICU. Simultaneously with the onset of the pandemic, in the tertiary hospital in Osijek, Croatia, microbiological diagnostics with MALDI-TOF technology began. More accurate diagnostics, and increased staff mobility within the ICU can contribute to an increased number of positive samples.

Methods. The ethics committee approval was obtained for this retrospective study. During 2019 and the first three months of 2020, a $3891 \mathrm{MB}$ sample was sent for MB analysis from the intensive care unit, i.e. skin swabs, wounds swabs, tracheal aspirates, urine and blood cultures, and other samples. In the period after April 2020, MALDI-TOF technology was introduced into microbiological diagnostics, which analyzed 1838 microbiological samples. The aim of this study was to compare the frequency of isolation of pathogenic bacteria and ICU mortality in these 2 periods.

Results. The ratio of emergency patients increased from $44.6 \%$ in 2019 to $66.9 \%$ in 2020. During the 2019 year out of 3891 samples were taken, the number of sterile samples was $74.6 \%$ in 2019 . In the 2020 year, out of 1838 samples, $75.6 \%$ were sterile (n.s. $P=0.41$ ). The total number of Acinetobacter Baumanii isolates decreased from 290 $(7.4 \%)$ to $106(5.8 \%, P=0.019)$, Pseudomonas strains increased from $3.0 \%$ to $4.2 \%(P=0.024)$, and Proteus sp. fell from $1.56 \%$ to $0.87 \%$ in $2020, P=0.032$ ). The total number of Staphylococcus aureus strains isolates decreased from 2.9 to $2.2 \%$. A small number of bacteria previously not recognized were isolated using MALDI-TOF, such as Delftia acidovorans, and Leclercia adecarboxylata. Mortality increased from $14 \%$ to $17.9 \%$ in the ICU during the pandemic period. 
Conclusion. The use of MALDI-TOF did not confirm a higher ratio of pathogenic bacteria in isolates taken from surgical ICU patients after the onset of the COVID-19 pandemic. Mortality in ICU was increased but was not associated with microbiological isolates. It was probably due to other comorbid patients' conditions, and most likely due to a higher ratio of emergency patients.

\section{Reference(s)}

1. Fujimoto K, et. al. J Occup Health. 2021 Jan;63(1):e12232.

2. IP-18-2020, Medical Faculty Osijek, Croatia.

3. European Union grant; Operational Program Competitiveness and Cohesion, Grant Agreement No. KK.01.1.1.02.0015

\section{6}

Bacterial secondary infection in patients with SARS-CoV-2 severe infection in a university Portuguese ICU

R. Pinto Medeiros ${ }^{1}$; N. A. ${ }^{2}$; V. A. ${ }^{3}$; R. R. ${ }^{4}$; C. H. ${ }^{5}$; G. N. ${ }^{1}$; A. M. ${ }^{6}$; M. A. ${ }^{2}$

${ }^{1} \mathrm{SCl}-1$, Hospital Geral de Santo António, Porto, Portugal; ${ }^{2} \mathrm{~S} \mathrm{Ci}-1$, Hospital Geral de Santo António, Porto, Portugal, Porto, Portugal; ${ }^{3}$ Sci-1, Hospital Geral de Santo António, Porto, Portugal, Porto, Portugal; ${ }^{4}$ Oncology department, Hospital Geral de Santo António, Porto, Portugal; ${ }^{5}$ Microbiology department, Hospital Geral de Santo António, Porto, Portugal; ${ }^{6}$ Sci- 1, Hospital Geral de Santo António, Porto, Portugal, Porto, Portugal

Correspondence: R. Pinto Medeiros

Intensive Care Medicine Experimental 2021, 9(1): 001366

Introduction. The prevalence rate of bacterial secondary infections among critically ill patients with SARS-CoV- 2 infection is scarce. Viral pneumonia is known to be related with bacterial nosocomial infection.1

Objectives. Assess the rate of bacterial secondary infection and report the most common microorganisms involved in patients admitted to the ICU for severe SARS-CoV-2 pneumonia.

Methods. Monocentric, descriptive observational study. Demographics variables and the prevalence of bacterial secondary infection (bloodstream, urinary and respiratory) admitted in our ICU from 1st September 2020 to 15 th January of 2021 were registered. We describe the results of microbiological data (blood, urine and respiratory secretions) along with the resistance phenotypes.

Results. A total of 186 patients with confirmed SARS-CoV-2 were admitted at the ICU; 91 patients had a diagnostic of secondary infection. In 12 patients it was not possible to isolate a microbiological agent. As for isolated microorganisms, the most common was Enterobacteriaceae (almost 50\%) in which E. coli was the most prevalent, followed by Klebsiella pneumoniae. The least common isolated microorganisms were Pseudomonas aeruginosa (9\%) and Acinetobacter baumannii (3\%). The mechanisms of resistance in each microbiological isolation were determined.

Conclusion. We report a prevalence of $54.3 \%$ bacterial secondary infection in patients with severe SARS-CoV-2 pneumonia on the ICU, mostly related with enterobacteria.Further larger studies are needed to assess the real prevalence and the risk factors predictors of secondary infection, together with its prognostic impact on critically ill patients with severe SARS-CoV-2 pneumonia.

\section{Reference(s)}

1. Gupta RK, George R, Nguyen-Van-Tam JS. Bacterial pneumonia and pandemic influenza planning. Emerg Infect Dis. 2008 Aug;14(8):1187-92. https://doi.org/10.3201/eid1408.070751. PMID: 18680640; PMCID: PMC2600366.

\section{6}

Burden of comorbidities and demographics in the Swedish critical care cohort: a comparison of COVID-19 to sepsis and acute respiratory distress syndrome

B. Ahlström¹; R. Frithiof²; IM. Larsson ; G. Strandberg²; M. Lipscey, ; M Hultström²

${ }^{1}$ Anesthesiology and Intensive Care, Department of Surgical Sciences, Uppsala University, Uppsala, Sweden; ${ }^{2}$ Anesthesiology and Intensive Care,
Department of Surgical Sciences, Uppsala University Hospital, Uppsala, Sweden

Correspondence: $\mathrm{B}$. Ahlström

Intensive Care Medicine Experimental 2021, 9(1): 001386

Introduction. Coronavirus disease 2019 (COVID-19), caused by severe acute respiratory syndrome coronavirus 2 (SARS-CoV-2), has overwhelmed intensive care units (ICUs) worldwide since late 2019. Severe COVID-19 is associated with several pre-existing comorbidities and demographic factors $(1,2)$. Similar factors are linked to critical sepsis and acute respiratory distress syndrome (ARDS) $(3,4)$. We hypothesized that age and comorbidities are more generically linked to critical illness than a specific disease state.

Methods. This cohort study used the Swedish intensive care registry to identify all consecutive adult ICU COVID-19 patients in the spring 2020. We also identified all adult ICU patients with non COVID-19 sepsis (i.e. severe sepsis and septic shock) and ARDS in 2011-2016. Data on comorbidities were retrieved from the national in-patient database. The relative importance of risk factors for ICU admission was assessed with logistic regression and the relative importance for ICU mortality was evaluated using the interaction with disease cohort in Cox proportional hazards regression.

Results. The study included 1986 COVID-19, 22,110 sepsis and 2776 ARDS adult ICU patients. Female sex and several comorbidities had a stronger association with sepsis and ARDS in ICU admission, whereas type 2 diabetes mellitus and asthma had a stronger association with COVID-19. In a Cox model on ICU mortality in sepsis and COVID-19 there were significant interactions with cohort for age, sex, asthma and cancer. In the model on ICU mortality in ARDS and COVID-19 significant interactions with cohort were found for (a modified) Simplified Acute Physiology Score 3, asthma and cancer.

Conclusion. The burden of age and comorbidity was more strongly associated with ICU admission in sepsis and ARDS than in COVID-19. However, most comorbidities showed similar importance for ICU mortality between cohorts.

\section{Reference(s)}

1. 1. Ahlström B, Frithiof R, Hultström M, Larsson IM, Strandberg G, Lipcsey $M$. The swedish covid-19 intensive care cohort: Risk factors of ICU admission and ICU mortality. Acta Anaesthesiol Scand. 2021;65:525-33.

2. 2. Grasselli G, Greco M, Zanella A, Albano G, Antonelli M, Bellani G, et al. Risk Factors Associated With Mortality Among Patients With COVID-19 in Intensive Care Units in Lombardy, Italy. JAMA Intern Med. 2020:180(10):1345-55.

3. 3. Strandberg G, Walther S, Agvald Öhman C, Lipcsey M. Mortality after Severe Sepsis and Septic Shock in Swedish Intensive Care Units 2008-2016-A nationwide observational study. Acta Anaesthesiol Scand. 2020;64(7):967-75.

4. 4. Angus DC, van der Poll T. Severe sepsis and septic shock. N Engl J Med. 2013:369(9):840-51

5. Centre for Clinical Research at Region Dalarna, Sweden funded this research

6. Uppsala University Hospital research fund, Uppsala, Sweden funded this research.

\section{8}

Real-life analysis of Ceftazidime-avibactam use in 5 French ICUs from the OUTCOMEREA study group

JF. Timsit ${ }^{1}$; Q. Staiquly ${ }^{2}$; J. Reignier ${ }^{3}$; C. Cerf ${ }^{4}$;V. Laurent ${ }^{5}$; S. Ruckly, ; B. Mourvillier?

${ }^{1}$ Intensive Care Medicine Department, Assistance Hopitaux Publique de Paris, Paris, France; ${ }^{2}$ Paris, Bichat-Claude Bernard Hospital, Paris, France; ${ }^{3}$ Médecine Intensive réanimation, Nantes University Hospital Hotel-Dieu, Nantes, France; ${ }^{4}$ Haut de seine, ICU, Hospital Foch, Suresnes, France; ${ }^{5} \mathrm{ICu}$, CH, Le Chesnay-Rocquencourt, France; ${ }^{6}$ Umr 1137, INSERM, Paris, France; ${ }^{7}$ Médecine intensive et réanimation polyvalente, CHU Reims-Champagne Clinic, Reims, France

Correspondence: J.F. Timsit

Intensive Care Medicine Experimental 2021, 9(1): 001408 
Introduction. Ceftazidime-avibactam (CAZ-AVI) is used for the treatment of extensively drug resistant gram negative bacterias. It is also effective against class $\mathrm{A}$ (bla-KPC) and D (bla-OXA 48) carbapenemases enterobacterales and MDR $P$ aeruginosa. However, its use, in routine practice, in severe ICU patients has not been completely described in countries where $\mathrm{KPC}$ is very rare.

Objectives. To described indications, dose, and outcome of ICU patients treated with ceftazidime avibactam in ICU patients registered in the OUTCOMEREA French database.

Methods. Descriptive analysis of the treatment given and outcomes. Results. From Sept:2017 to May 2020, 27 (male 16/Female 11; median age 58, SAPS II 36; Immunocompromized 7) out of 1462 patients (1.8\%) from 5 ICUs were treated with CAZAVI after 15 days $[5 ; 35]$ of ICU stay and 13 days in median $[4 ; 33]$ of mechanical ventilation. SOFA score was 5 [3; 8]. Acute kidney injury with a Kdigo 3 was present in 17/27 patients. Patients were treated for VAP (17), IAI (5), UTI (2) with positive bloodcultures in 5 cases. The treatment was directed against $P$ aeruginosa (9), ESBL (8), enterobacterales resistant to carbapenems without carbapenemases (1), bla-NDM enterobacterales (9), Bla-OXA48 enterobacterales (5) with multiple etiological agents in 6 cases. The treatment was immediately documented in 23/27 cases and empirically given based on previous colonization with extensively drug-resistant bacteria (XDR) in 4 cases.

CAZAVI was given for a median time of $8[2 ; 14]$ days. Patients were under extra-renal epuration in 13 cases and it was stopped for deescalation in 9 (33\% cases). Monotherapy was decided in 5 cases and bi or tri antibiotic therapy in 22 cases.

Clinical success was obtained 16 cases $(59 \%)$ with microbiological success in 22 cases (82\%). Proven superinfections was observed in 4 cases (Achromobacter 1 ; XDR A. baumannii 1 ; $E$ faecalis $1 ; P$ aeruginosa 1 ). 8 proven infections with bla-NDM enterobacterales were treated with the combination CAZAVI + aztreonam with 8 microbiological eradications. 6 patients with clinical success survived and 2 patients died.

Overall, 13/27 patients ultimately died in the hospital.

Conclusion. CAZAVI is used according to recommendations for proven or suspected infections due to XDR enterobacterales or $\mathrm{P}$ aeruginosa in severe ICU patients with a $59 \%$ clinical success rate. The association of CAZAVI with aztreonam was effective in therapy of metalobetalactamase-producer enterobacterales severe infections in critically ill patients.

\section{Reference(s)}

1. The study received a research grant from Pfizer France

\section{0}

Single versus multiple doses of Tocilizumab in critically ill patients with Coronavirus disease 2019 (COVID-19): A Two-center, Retrospective Cohort Study

K. Al Sulaiman'; O. Aljuhani²; K. Bin Salah ${ }^{3}$; K. Ghazwa ${ }^{4}$; K. Eljaaly ${ }^{5}$; A. Kharbosh ${ }^{6}$; A. Alshehri ${ }^{7}$ F. Al Harbi ${ }^{8}$

${ }^{1}$ King Abdulaziz Medical City, Riyadh, Saudi Arabia; ${ }^{2}$ Pharmaceutical care, King Abdulaziz University, Jeddah, Saudi Arabia; ${ }^{3}$ College of pharmacy, King Saud bin Abdulaziz University for Health Sciences, Riyadh, Saudi Arabia; ${ }^{4}$ College of pharmacy, Princess Nourah Bint Abdul Rahman University, Riyadh, Saudi Arabia; ${ }^{5}$ College of pharmacy, King Abdulaziz University, Jeddah, Saudi Arabia; ${ }^{6}$ College of pharmacy, Taif University, Taif, Saudi Arabia; ${ }^{7}$ Pharmaceutical Care, King Abdulaziz Medical City, Riyadh, Saudi Arabia; ${ }^{8}$ College of Medicine, King Saud bin Abdulaziz University for Health Sciences, Riyadh, Saudi Arabia

Correspondence: $\mathrm{K}$. Al Sulaiman

Intensive Care Medicine Experimental 2021, 9(1): 001440
Introduction. In COVID-19 patients, increased IL-6 levels have been associated with poor disease prognosis. The use of tocilizumab shown to be effective in treating COVID-19 with varying success. This study aims to evaluate the effectiveness and safety of using a single dose of tocilizumab compared with multiple doses in critically ill COVID-19 patients.

Methods. This study is a two-center, retrospective cohort, in which patients who received tocilizumab and were admitted to the ICU at two tertiary hospitals from March 1st, 2020, until January 31st, 2021 were included. Patients were divided into two groups based on the number of doses of tocilizumab they received. Furthermore, we gathered additional data from the patients, such as but not limited to demographic data, vital signs, and laboratory markers. Multivariable logistic and generalized linear regression were used. We considered a $P$ value of $<0.05$ statistically significant.

Results. Two hundred sixty-one patients were included in this study; $72.4 \%$ received a single dose of tocilizumab, while the rest $(27.6 \%)$ had received multiple doses. Most of the patients were male, with an average age of 59.2. After adjusting for possible confounders, the ICU mortality within 30 days $(\mathrm{OR} 0.83 ; 95 \% \mathrm{Cl} 0.33-2.08 \mathrm{p}=0.69)$ and in hospital mortality (OR $0.64 ; 95 \% \mathrm{Cl} 0.26-1.60 \mathrm{p}=0.35)$ were not significantly different between the two groups. On the flip side, patients who received multiple doses of tocilizumab have higher odds of secondary infection compared with a single dose (OR 3.06; 95\% Cl 1.18-7.89 $\mathrm{p}=0.02$ ).

Conclusion. Multiple doses of tocilizumab were not associated with a statistically significant difference in ICU and hospital mortality in critically ill patients infected with COVID-19. In contrast, it was associated with higher odds of secondary infections compared to a single dose.

e-Posters: Sepsis

000028

Significance of lactate clearance in septic shock patients with high bilirubin levels

N. Takahashi'; N. Taka-Aki'; K. Walley², R. James²

${ }^{1}$ Department of Emergency and Critical Care Medicine, Chiba University

Graduate School of Medicine, Chiba, Japan; ${ }^{2}$ Centre for heart lung inno-

vation, University of British Columbia, Vancouver, Canada

Correspondence: N. Takahashi

Intensive Care Medicine Experimental 2021, 9(1): 000028

Introduction. Lactate clearance is affected by hepatic function. However, it is unclear whether the association between hepatic dysfunction and lactate clearance can act as a prognostic marker of clinical outcomes in patients with septic shock. We aimed to evaluate the association between lactate clearance and mortality in two cohorts of septic shock patient who had hepatic dysfunction based on their total serum bilirubin levels (TBIL).

Methods. Lactate clearance which at $24 \mathrm{~h}$ after the onset of septic shock was analyzed using two cohorts, sub-categorized into two groups based on TBIL: $<2 \mathrm{mg} / \mathrm{dL}$ and $\geq 2 \mathrm{mg} / \mathrm{dL}$.

Blood lactate clearance (\%) was calculated using the following formula:

Lactate clearance $(\%)=($ Lactate day 1 - Lactate day2) / Lactate day $1 * 100$.

Results. In the derivation cohort, lactate clearance was lower in nonsurvivors than in survivors with $\mathrm{TBIL} \geq 2 \mathrm{mg} / \mathrm{dL}$, while there was no significant difference in lactate clearance between non-survivors and survivors with TBIL $<2 \mathrm{mg} / \mathrm{dL}$. Multivariate logistic regression analysis revealed that increased lactate clearance was significantly associated with decreased 28-day mortality in the $\mathrm{TBIL} \geq 2 \mathrm{mg} / \mathrm{dL}$ group $(10 \%$ 
lactate clearance, adjusted odds ratio [OR]: $0.88,95 \%$ confidence interval $(\mathrm{Cl}): 0.80-0.97, \mathrm{P}=0.0075$ ), Creatinine level $\geq 2 \mathrm{mg} / \mathrm{dL}$ group (adjusted OR: $0.88,95 \% \mathrm{Cl}: 0.81-0.95, \mathrm{P}=0.00069)$ and APACHE II score $\geq 35$ group (adjusted OR: $0.93,95 \% \mathrm{Cl}: 0.87-0.98, \mathrm{P}=0.013$ ). In the validation cohort, lactate clearance was lower in non-survivors than in survivors with TBIL $\geq 2 \mathrm{mg} / \mathrm{dL}$, while no significant difference in lactate clearance was observed between non-survivors and survivors with TBIL $<2 \mathrm{mg} / \mathrm{dL}$. Increased lactate clearance was significantly associated with decreased 28-day mortality in the TBIL $\geq 2 \mathrm{mg} / \mathrm{dL}$ group (10\% lactate clearance, adjusted OR: $0.89,95 \% \mathrm{Cl}: 0.83-0.96, \mathrm{P}=0.0038$ ) and the association was just about significant in APACHE II score $\geq 35$ group (adjusted OR: 0.86 , $95 \% \mathrm{Cl}: 0.74-1.00, \mathrm{P}=0.051$ ).

Conclusion. In conclusion, increased lactate clearance in septic shock patients with hepatic dysfunction (TBIL $\geq 2 \mathrm{mg} / \mathrm{dL}$ ) or high severity (APACHE II score $\geq 35$ ) was associated with decreased 28-day mortality.

\section{0}

\section{Lactate/albumin ratio predicts mortality in septic ICU patients}

R. Meireles ${ }^{1}$; M. Ribeiro ${ }^{1}$; E. Lafuente ${ }^{1}$

${ }^{1}$ Intensive care unit, Centro Hospitalar do Tâmega e Sousa, Penafiel, Portugal

Correspondence: $\mathrm{R}$. Meireles

Intensive Care Medicine Experimental 2021, 9(1): 000030

Introduction. Many biomarkers are often promoted with prognostic value in septic patients [1] but none is clearly superior to clinical scores [2]. Nevertheless, other authors have shown lactate/albumin ratio to be a promissing predictor [3].

Objectives. The aim of this study was to evaluate the performance of different biomarkers as predictive factors of ICU mortality and 28-day mortality in septic patients admited in ICU.

Methods. We conducted a prospective cohort study in an intensive care unit, enrolling all consecutive patients admited with sepsis as a primary diagnosis during a period of 6 months. Data were collected from 66 patients including C-reactive protein, IL-6, cholesterol, lactate and lactate/albumin ratio (L/A) at admission and after 24 and $72 \mathrm{~h}$. ICU mortality and 28-day mortality were considered as primary outcomes. SAPS II at admission was regarded as comparison. IBM SPSS 27 was used for inferencial statistics and MEDCALC for ROC curve AUC calculation and comparisons, with $95 \% \mathrm{Cl}$. T-test was used for parametric variables and Chi-squared (Fisher exact test or Pearson) for non parametric. Significant variables were included in a binary logistic regression model.

Results. Independent predictors of ICU mortality: IL- 6 at admission $(p<0.01), 24 \mathrm{~h}(\mathrm{p}<0.01)$ and $72 \mathrm{~h}(\mathrm{p}<0.01)$; lactates at admission $(\mathrm{p}<0.01), 24 \mathrm{~h}(\mathrm{p}<0.01)$ and $72 \mathrm{~h}(\mathrm{p}<0.01)$; serum albumin at admission $(p<0.01)$ and $24 \mathrm{~h}(\mathrm{p}<0.01)$; and L/A at admission $(\mathrm{p}<0.01)$ and $24 \mathrm{~h}$ $(p<0.01)$. SAPS II also significantly correlated $(p<0.01)$.

Independent predictors of 28-day mortality: IL- 6 at admission $(p<0.01)$, $24 \mathrm{~h}(\mathrm{p}<0.01)$ and $72 \mathrm{~h}(\mathrm{p}<0.01)$; lactates at admission $(\mathrm{p}<0.01), 24 \mathrm{~h}$ $(p<0.01)$ and $72 \mathrm{~h}(\mathrm{p}<0.01)$; cholesterol levels at $24 \mathrm{~h}(\mathrm{p}<0.02)$ and $72 \mathrm{~h}$ $(p<0.01)$; serum albumin at admission $(p<0.01)$ and $24 \mathrm{~h}(\mathrm{p}<0.01)$; and L/A at admission $(p<0.01)$ and $24 \mathrm{~h}(\mathrm{p}<0.01)$. SAPS II also significantly correlated $(p<0.01)$.

Binary regression excluded the other variables, remaining only L/A predicting, for each one added point, a 2.4 -fold increase in ICU mortality $(p<0.01)$ and a 2.1 -fold increase in 28-day mortality $(p<0.01)$.

ROC-AUC was calculated for values at admission regarding ICU mortality: SAPS II 0.907 (0.810-0.965), L/A 0.929 (0.839-0.978), Lactate 0.909 (0.812-0.966), IL-6 $0.836(0.724-0.915)$. ROC-AUC for 28-day mortality for values at admission: SAPS II $0.786(0.665-0,880)$, L/A 0.811 (0.693-0.899), Lactate $0.773(0.650-0.869)$, IL-6 $0.693(0.564-0.803)$ and Cholesterol $0.674(0.545-0.787)$. None of the ROC curves were significantly different in pairwise comparison ( $p>>0.1)$ except between IL- 6 and $L / A(p<0.05)$. L/A ratio cut-off (best discriminator) value for ICU mortality was 2.12 above which mortality significantly increases. No clear cut-off was found for 28-day mortality (Fig. 1).

C-reactive protein at any time did not significantly correlate with mortality $(p>0.05)$ neither age $(p>0.4)$, sex $(p>0.2)$ or source of infection $(p>0.35)$.

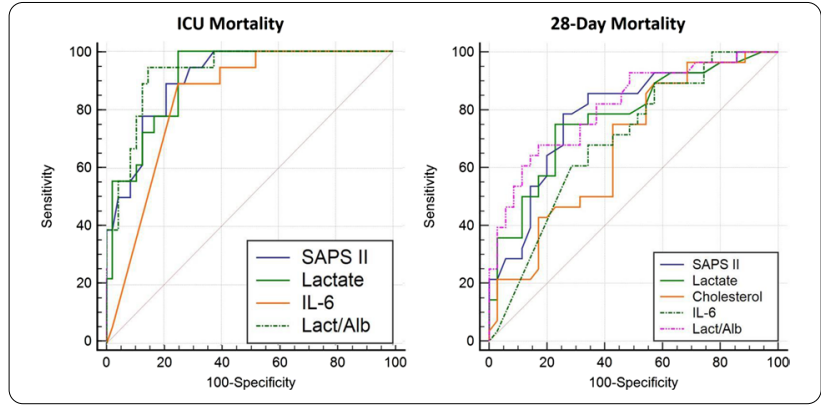

Conclusion. In our sample L/A ratio showed to be the best biomarker to predict ICU and 28-day mortality for sepsis of different aetiologies and is comparable (even superior) to SAPS II.

\section{Reference(s)}

1. [3] Wang B et al. Correlation Of Lactate/Albumin Ratio Level To Organ Failure And Mortality In Severe Sepsis And Septic Shock. Journal Of Critical Care. 2015; 30: 271-275

2. [1] Castelli GP et al. Procalcitonin And C-Reactive Protein During Systemic Inflammatory Response Syndrome, Sepsis And Organ Dysfunction. Critical Care. 2004; 8: R234-242

3. [2] Pettilae $V$ et al. Predictive value of procalcitonin and interleukin 6 in critically ill patients with suspected sepsis. Intensive Care Medicine. 2002; 28: $1220-1225$

000041

The utility of Sequential Organ Failure Assessment (SOFA) score for mortality prediction in kidney transplant recipients with urinary sepsis

T. Królicki'; A. Królicka²; K. Bardowska'; O. Mazanowska ${ }^{1}$; P. Poznański ${ }^{1}$; K. Letachowicz'; M. Krajewska'; D. Kamińska'

'Department of Nephrology and Transplantation Medicine, Wroclaw Medical University, Wrocław, Poland; ${ }^{2}$ Medical faculty, Wroclaw Medical University, Wrocław, Poland

Correspondence: T. Królick

Intensive Care Medicine Experimental 2021, 9(1): 000041

Introduction. Clinical scores such as Sequential-Organ-Failure-Assessment (SOFA) score used for quantitative evaluation of organ failure are rarely reported in studies including kidney transplant recipients (KTRs). According to the Third International Consensus Definitions for Sepsis and Septic Shock the rise of SOFA score (delta-SOFA) of 2 points or more, irrespective of whether it is calculated before or after hospital admission, identifies infectious patients with high mortality risk [1]. This has not yet been validated in KTRs with urinary sepsis (US).

Objectives. To assess validity and optimal cut-off values of SOFA score derivatives for mortality prediction in KTRs with diagnosed US.

Methods. 101 KTRs with confirmed urinary sepsis treated in a high volume transplantation center in 2014-2019 were retrospectively included in the study. Incidence of organ injury or failure, SOFA scores and 28-days mortality were registered. Sensitivity and specificity analysis was performed using ROC analysis. Following SOFAscore derivatives were analysed: baseline SOFA score prior to the disease (baseSOFA), SOFA score on admission to hospital (admSOFA), the maximal SOFA score values during hospitalisation (maxSOFA), the difference between admission and baseline SOFA score (admbaseSOFA), the difference between maximal and baseline SOFA score (max-baseSOFA) and the difference between maximal and admission SOFA score (max-admSOFA). All subjects received optimal care, compliant with local standards.

Results. The 28-days mortality in patients with US equalled $8 \%$. The prevalence of organ dysfunctions was following: AKI-75\%, acute thrombocytopenia-32\%, respiratory failure-26\%, acute liver 
injury $-10 \%$ and vasopressor use-6\%. Median GCS score equalled 14 (IQR: 13-15). Renal replacement therapy was triggered in $17 \%$ of the cases. Following SOFA values were observed in the nonsurvivor and survivor groups [median, IQR]: baseSOFA: $1(0-1,5)$ vs 1 (0-1); admSOFA: $5,5(4-10,5)$ vs 4 (3-5); maxSOFA: $11(8,5-14)$ vs $4(3-5)$; max-admSOFA: $3(2-5,5)$ vs $0(0-0)$ : max-baseSOFA: 10,5 (8-12) vs 3 (2-4). In ROC analysis the best discrimanative properties for 28-days mortality prediction were found for following SOFA derivatives (at appropriate cut-offs; with following sensitivity and specificity): maxSOFA $\geq 7: 100 \% \& 82,2 \%$, max-admSOFA $\geq 2: 87,5 \%$ \& $92,5 \%$ and max-baseSOFA $\geq 6: 100 \%$ \& $87 \%$ respectively. Both admSOFA and adm-baseSOFA showed to be statistically inferior to the above mentioned parameters.

ROC analysis for 28-days mortality prediction of investigated SOFA score derivatives

\begin{tabular}{llllll}
\hline & Youden point & AUC & AUC 95\% Cl & SE & p-value \\
\hline baseSOFA & n.a & 0,5048 & $0,029-0,720$ & 0,11 & 0,9654 \\
admSOFA & $\geq 12$ & 0,7184 & $0,500-0,937$ & 0,11 & 0,0504 \\
$\begin{array}{c}\text { adm- } \\
\text { baseSOFA }\end{array}$ & $\geq 4$ & 0,7609 & $0,583-0,938$ & 0,09 & 0,0040 \\
$\begin{array}{c}\text { maxSOFA } \\
\text { max-adm- }\end{array}$ & $\geq 7$ & 0,9644 & $0,925-1,000$ & 0,02 & $<0,0001$ \\
$\begin{array}{c}\text { SOFA } \\
\begin{array}{c}\text { max- } \\
\text { baseSOFA }\end{array}\end{array}$ & $\geq 6$ & 0,9059 & $0,756-1,000$ & 0,08 & $<0,0001$ \\
\hline
\end{tabular}

Conclusion. Maximal- and delta-SOFA values remain valid and strong predictors of death in KTRs with urinary sepsis, presenting comparable prognostic accuracy as observed in the ICU population [2]. However, the rise of SOFA score of $\geq 2$ points, which predicts increased mortality in general, septic, non-transplant patients, remains valid in KTRs with US only in the in-hospital setting. The rise of SOFA score prior to admission (adm-baseSOFA) showed lower prognostic precision with a higher optimal cut-off of $\geq 4$ points in this patient group-similar to prior studies [3]. This may be attributed to high allograft vulnerability to hemodynamic and immunologic factors occuring even in healthy KTRs without infections.

\section{Reference(s)}

1. The study was supported by the Wroclaw Medical University statutory funds (SUB.C160.19.055).

2. 1. Singer M, Deutschman CS, Seymour CW, Shankar-Hari M, Annane $D$ Bauer M, Bellomo R, Bernard GR, Chiche JD, Coopersmith CM, Hotchkiss RS, Levy MM, Marshall JC, Martin GS, Opal SM, Rubenfeld GD, van der Poll T, Vincent JL, Angus DC. The Third International Consensus Definitions for Sepsis and Septic Shock (Sepsis-3). JAMA. 2016 Feb 23;315(8):801-10. https://doi.org/10.1001/jama.2016.0287. PMID: 26903338; PMCID: PMC4968574

3. 2. Moreno R, Vincent JL, Matos R, Mendonça A, Cantraine F, Thijs L, Takala J, Sprung C, Antonelli M, Bruining H, Willatts S. The use of maximum SOFA score to quantify organ dysfunction/failure in intensive care. Results of a prospective, multicentre study. Working Group on Sepsis related Problems of the ESICM. Intensive Care Med. 1999 Jul;25(7):686-96. https://doi. org/10.1007/s001340050931. PMID: 10470572

4. 3. Chen YX, Li R, Gu L, Xu KY, Liu YZ, Zhang RW. Prognostic Performance of SOFA, QSOFA, and SIRS in Kidney Transplant Recipients Suffering from Infection: A Retrospective Observational Study. Adv Ther. 2020 Mar:37(3):1100-1113. https://doi.org/10.1007/s12325-020-01225-0. Epub 2020 Jan 24. PMID: 31981104.

\section{4}

Impact of Checklist for Early Recognition and Treatment of Acute Illness (CERTAIN) on treatment of critically ill septic patients in a low resource medical ICU

P. Kovacevic' ' S. Dragic' ; D. Momcicevic ${ }^{1}$; T. Kovacevic ${ }^{1}$; B. Zlojutro'; S. Topolovac'; M. Jandric

${ }^{1} \mathrm{MICU}$, University Clinical Centre of the Republic Srpska, Banja Luka,

Bosnia and Herzegovina

Correspondence: P. Kovacevic

Intensive Care Medicine Experimental 2021, 9(1): 000054

Introduction. Treatment of sepsis and septic shock can be a challenge even for intensive care units (ICU) in high income countries but it is especially difficult for ICUs with limited resources.

Objectives. To evaluate the impact of CERTAIN (CERTAIN: Checklist for Early Recognition and Treatment of Acute Illness) on treatment of critically ill septic patients in low resource medical ICU.

Methods. In a before-and-after study design, we compared clinical outcomes, processes and complications (hospital acquired infections) one year before and two years after (2016 and 2017) introduction of CERTAIN. Results. A total of 125 patients with sepsis were prospectively identified over a 3 year period. Mean patient age, gender distribution, number of patients on mechanical ventilation [33 $(76.7 \%)$ vs $42(84 \%)$ vs $24(75 \%))$ and vasopressor use $(23(53.5 \%)$ vs $34(68 \%)$ vs $24(75 \%)]$ were similar before (2015) and two years after (2016 and 2017) the implementation of CERTAIN checklist. Severity of illness (SAPS II score) was higher after the implementation. The checklist was incorporated in the daily practice with $100 \%$ adherence to its use. The duration of mechanical ventilation $(5.3 \pm 5.3$, vs $4.2 \pm 3.6$ vs $3.7 \pm 5.5)$, antibiotic treatment ( $8.2 \pm 5.4$ vs $6.9 \pm 4.1$ vs $5.8 \pm 5.6)$, central venous catheter use $(6.2 \pm 5.7$ vs $5.7 \pm 4.6$ vs $4.2 \pm 6.1)$, ICU stay $(8.4 \pm 5.4$ vs $7.1 \pm 4.1$ vs $5.8 \pm 5.6)$ and the incidence of nosocomial infection (33.3\% vs $30 \%$ vs $12.5 \%$ ) decreased in the period after the onset of the intervention but the results did not reach statistical significance. When adjusted for baseline characteristics, CERTAIN was not associated with hospital mortality (odds ratio $0.88,0.38$ to 2.04 ).

Conclusion. CERTAIN was readily adopted in the ICU workflow and was associated with improvement in treatment of critically ill patients with sepsis.

\section{Reference(s)}

1. 1. Singer M, Deutschman CS, Seymour CW, Shankar-Hari M, Annane D, Bauer $M$, et al. The third international consensus defnitions for sepsis and septic shock (sepsis-3). JAMA 2016; 315: 801-10. 2. Kwizera A, Baelani I, Mer M, Kissoon N, Schultz MJ, Patterson AJ, et al. The long sepsis journey in low- and middle-income countries begins with a first step... but on which road? Crit Care 2018; 22: 64. 3. GBD 2015 Mortality and Causes of Death Collaborators. Global, regional, and national life expectancy, all-cause mortality, and cause-specific mortality for 249 causes of death, 1980-2015: a systematic analysis for the Global Burden of Disease Study 2015. Lancet 2016: 388:1459-544. 4. Reinhart K, Daniels R, Kissoon N, Machado FR, Schachter RD, Finfer S.. Recognizing sepsis as a Global Health Priority—a WHO Resolution. N Engl J Med 2017; 377: 314-7. 5. Dellinger RP, Levy MM, Rhodes A, Annane D, Gerlach H, Opal SM, et al. Surviving Sepsis Campaign: international guidelines for management of severe sepsis and septic shock, 2012. Intensive Care Med 2013; 39: 165-228. 6. Rhodes A, Evans LE, Alhazzani W, Levy MM, Antonelli M, Ferrer $R$, et al. Surviving Sepsis Campaign: International guidelines for management of sepsis and septic shock: 2016. Crit Care Med 2017; 45: 486-552. 7. Vukoja M, Riviello ED, Schultz MJ. Critical care outcomes in resource-limited settings. Curr Opin Crit Care 2018; 24: 421-427. 8. Thiéry G, Kovacević P. Straus S, Vidovic J, Iglica A, Festic E, et al. From mechanical ventilation to intensive care medicine: a challenge for Bosnia and Herzegovina. Bosn J Basic Med Sci 2009 Oct;9 Suppl 1:69-76. 9. MacFie CC, Baudouin SV Messer PB. An integrative review of drug errors in critical care. J Intensive Care Soc 2016;17:63-72. 10. Haynes AB, Weiser TG, Berry WR, Lipsitz SR, Breizat AH, Dellinger EP, et al. A surgical safety checklist to reduce morbidity and mortality in a global population. N Engl J Med 2009;360:491-9. 11. Gawande A. The Checklist Manifesto: How to Get Things Right: Picador; Reprint edition (January 4, 2011). New York: Picador Publishers; 201112. 
Shao M, Kashyap R, Niven A, Barwise A, Garcia-Arguello L, Suzuki R, et al. Feasibility of an International Remote Simulation Training Program in Critical Care Delivery: A Pilot Study. Mayo Clin Proc Innov Qual Outcomes 2018; 2: 229-233. 13. Sevilla-Berrios R, O'Horo JC, Schmickl CN, Erdogan A, Chen X, Garcia Arguello LY, et al. Prompting with electronic checklist improves clinician performance in medical emergencies: a high-fidelity simulation study. Int J Emerg Med 2018;11:26. 14. Barwise A, GarciaArguello L, Dong Y, Hulyalkar M, Vukoja M, Schultz MJ, et al. Checklist for early recognition and treatment of acute illness (CERTAIN): Evolution of a content management system for point-of-care clinical decision support. BMC Med Inform Decis Mak 2016;16:127. 15. Kilickaya O, Bonneton B, Gajic O. Structured Approach to Early Recognition and Treatment of Acute Critical IIIness. In: Vincent JL. Annual Update in Intensive Care and Emergency Medicine 2014; 2014:689-703. 16. Weiss CH, Moazed F, McEvoy CA, Singer BD, Szleifer I, Amaral LA et al. Prompting physicians to address a daily checklist and process of care and clinical outcomes: a single-site study. American Journal of Respiratory and CriticalCare Medicine 2011;184:680. 17. Kogan A, Pennington KM, Vallabhajosyula S, Dziadzko M, Bennett CE, Jensen JB, et al. Reliability and Validity of the Checklist for Early Recognition and Treatment of Acute Illness and Injury as a Charting Tool in the Medical Intensive Care Unit. Indian I Crit Care Med. 2017;21:746-750. 18. Kovacevic P, Dragic S, Kovacevic T, Momcicevic $D$, Festic E, Kashyap R, et al.: Impact of weekly case-based tele-education on quality of care in a limited resource medical intensive care unit. Crit Care 2019; 23: 220

2. Sincere thanks to Dr Ognjen Gajic and Dr Emir Festic for providing and implementing the CERTAIN project and tele education in MICU University Clinical Center of Republika Srpska. This research article raised form these projects.

\section{3}

Impact of Polymyxin B Hemoperfusion on Septic Shock Pateints with Elevated Endotoxin Activity Levels

JC. Yoon ${ }^{1}$; EY. Kim ${ }^{2}$

'Department of Surgery, The Catholic University of Korea Seoul St. Mary's Hospital, Seoul, Republic of Korea; ${ }^{2}$ Division of Trauma and Surgical Critical Care, Department of Surgery, The Catholic University of Korea Seoul St. Mary's Hospital, Seoul, Republic of Korea

Correspondence: E.Y. Kim

Intensive Care Medicine Experimental 2021, 9(1): 000213

Introduction. We sought to evaluate the clinical implication of endotoxin levels on gram-negative bacilli (GNB) induced septic shock patients with Polymyxin B hemoperfusion (PMX-HP) treatment.

Methods. From January 2019 to December 2020, 60 patients who received abdominal surgery were enrolled assessing endotoxin activity assay (EAA) levels and Sequential Organ Failure Assessment (SOFA) scores immediately after intensive care unit admission (baseline), 24, and $48 \mathrm{~h}$ post baseline. Receiver operating characteristic (ROC) curves were used to evaluate the diagnostic ability of EAA with GNB induced septic shock and patients were stratified in two groups by the cut-off value (high EAA vs low EAA). Changes of EAA levels and SOFA scores were compared in each group to identify the effect of PMX-HP treatment. Logistic regression was performed to identify the clinical impact of PMX-HP on in-hospital death.

Results. Among the 31 high risk patients (EAA level>0.54), 16 patients $(16 / 31,51.6 \%)$ received PMX-HP treatment and revealed significant decrease of delta EAA levels ( 0.72 to 0.38 vs 0.71 to 0.59 . $p=0.01)$. SOFA scores also showed significant improvement with PMX-HP treatment (12.6 to 8.9, $p=0.007$ ), however, patients with conventional treatment failed to show statistical significance (8.2 to $6.2, p=0.12) .14$ in-hospital death occurred $(14 / 31,45.2 \%)$, and with adjusted logistic regression, PMX-HP treatment had a protective effect in in-hospital death (OR0.038, $p=0.028$ ). In 29 low risk patients (EAA level $\leq 0.54)$, seven patients $(7 / 29,24.1 \%)$ received PMX-HP treatment and showed significant decrease of delta EAA levels $(0.46$ to 0.16 vs 0.34 to $0.43, p<0.001)$. However, SOFA scores showed no improvements whether receiving PMX-HP treatment or not.[c1] PMX-HP treatment also failed to identify relationship with in-hospital death.
Conclusion. PMX-HP treatment accelerates endotoxin removal and might be a useful option to improve organ impairment and inhospital death in GNB induced septic shock patient with EAA level exceeding 0.54 .

\section{8}

A Novel Approach of a Metabolome Wide Association Study to Investigate Metabolic Derangement in Septic Shock Mortality RJND. Chang ${ }^{1}$; H. Hussain ${ }^{1}$; A. Gordon ${ }^{1}$; B. Jimenez ${ }^{2}$; DB. Antcliffe ${ }^{1}$ ${ }^{1}$ Division of Anaesthetics, Pain Medicine and Intensive Care, Department of Surgery and cancer, Imperial College London, London, United Kingdom; ${ }^{2}$ National Phenome Centre, department of Metabolism, Digestion and Reproduction, Imperial College London, London, United Kingdom Correspondence: R.J.N.D. Chang

Intensive Care Medicine Experimental 2021, 9(1): 000228

Introduction. Sepsis kills 11 million people a year and improving its treatment is a World Health Organisation priority. We hypothesise that through better understanding of metabolic derangement associated with septic shock, we could eventually track metabolic changes to predict clinical course.

Objectives. To apply the novel technique of a Metabolome Wide Association Study (MWAS) to identify metabolites that differentiate septic shock non-survivors from survivors.

Methods. 155 patients from the VANISH trial (1), contributed serum samples, within $6 \mathrm{~h}$ of the onset of shock. Metabolic profiles were generated by $1 \mathrm{H}$-nuclear magnetic resonance with a spin-echo experiment using a 1D-Carr-Purcell-Meiboom-Gill presat pulse sequence. (2) The dataset of 13,911 features, which correspond with roughly 80 metabolites, were analysed using the MWASTools metabolomics toolkit (3) which applies a logistic regression model to the dataset. The Benjamini-Hochberg correction was applied to reduce the false discovery rate (FDR). The variables of age, sex, BMI, immune status, ethnicity, ischaemic heart disease, diabetes, cirrhosis and immune status were assessed as potential confounders based on a literature search via a standard change-in-estimate criterion. A Manhattan-like Skyline plot (Figure 1.) was used to identify which of the metabolic features were associated with sepsis mortality.

Results. 1,012 features were different between septic shock survivors and non-survivors, prior to FDR correction. From these we were able to identify 17 metabolites all of which were of higher concentrations in non-survivors compared to survivors: valine, isoleucine, isobutyrate, 3-hydroxybutyrate, lysine, glutamate, glutamine, methionine, creatinine, formate, alanine, lactate, tyrosine, phenylalanine, 1-methylhistidine, glycine, and fumaric acid. The differences were however not statistically significant after correction, which is most likely due to the relatively small number of samples available for the high number of variables used in the study. None of the variables assessed as confounders were included in the logistic regression model, as they did not meet the standard change-in-estimate criterion of a $10 \%$ difference in odds ratio when included in the model. We have overlayed the spectra of 8 of the 17 metabolites on the skyline plot (Figure 1.) to illustrate our metabolite identification pipeline.

Conclusion. The MWAS approach shows promise to identify metabolic derangement in sepsis. We hypothesize the identified metabolites from these preliminary findings are indicative of the physiological dysregulation which occurs in septic shock, namely; mitochondrial dysfunction, protein catabolism, oxidative stress and hepatorenal dysfunction. This study helps to improve our understanding of the baseline metabolic changes between septic shock survivors and nonsurvivors and could help us explore how metabolic trajectories could alter over time in ICU. 


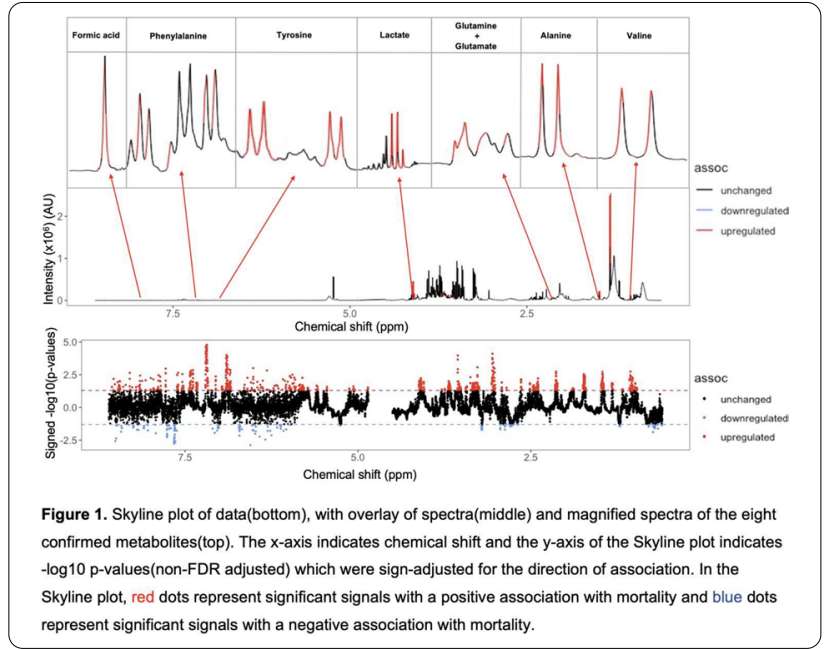

\section{Reference(s)}

1. 3. Rodriguez-Martinez A et al. MWASTools: an R/bioconductor package for metabolome-wide association studies. Bioinformatics. 2018;34(5):8902. https://doi.org/10.1093/bioinformatics/btx477.

2. 2. Dona AC et al. Precision high-throughput proton NMR spectroscopy of human urine, serum, and plasma for large-scale metabolic phenotyping. Anal Chem. 2014;86(19):9887-94. https://doi.org/10.1021/ac5025039.

3. 1. Gordon AC et al. Effect of Early Vasopressin vs Norepinephrine on Kidney Failure in Patients With Septic Shock: The VANISH Randomized Clinical Trial. JAMA 2016; 316(5):509-18. https://doi.org/10.1001/jama. 2016.10485

4. This abstract is independent research funded by the National Institute for Health Research (NIHR) Imperial Biomedical Research Centre (BRC). Infrastructure support was provided by the NIHR Imperial Biomedical Research Centre (BRC). ACG is an NIHR Research Professor (RP-2015-06-018)

\section{7}

S100A12 and Calprotectin are potential biomarkers of pancreatitis in septic patients

V. Pavel'; P. Mester ' ;. Gülow' ; C. Kunst ${ }^{1}$; E. Aschenbrenner ; M. Müller-Schilling ${ }^{1}$; S. Schmid

'Internal Medicine I, University Hospital Regensburg, Regensburg, Germany

Correspondence: $\mathrm{V}$. Pavel

Intensive Care Medicine Experimental 2021, 9(1): 000317

Introduction. Despite great advances in critical care medicine over the past years, the mortality rate of acute pancreatitis has persisted at about $10 \%$. Acute pancreatitis is confirmed by medical history, physical examination, and typically a blood test for digestive enzymes of the pancreas. In some cases when blood levels are not elevated and diagnosis is still in question, abdominal imaging might be performed. Although great advances had been made, the diagnosis of acute pancreatitis can be difficult.

Objectives. The aim of the study was to test the potential of S100A12 and Calprotectin for the diagnosis of pancreatitis in septic patients.

Methods. We evaluated serum levels of calprotectin and S100A12 in patients with different causes of systemic inflammatory response syndrome (SIRS) or sepsis. The study began in august 2018 and is still running. Blood samples were collected within $24 \mathrm{~h}$ after admission to the intensive care unit at the University Hospital Regensburg, Germany. Until now 79 blood samples from patients with different causes of sepsis were included in the study. 13 patients had acute pancreatitis. Blood samples from patients with different causes of sepsis were analyzed by Enzyme-linked Immunosorbent Assay's (ELISA). We compared our values with the normal values of calprotectin and S100A12 given in the literature.

Results. $65 \%$ of the patients with SIRS or sepsis in our cohort had elevated serum calprotectin values. Interestingly, there was a relevant difference between patients with pancreatitis and other patients with SIRS or sepsis. Furthermore, we could observe a good association with the severity of pancreatitis (Atlanta classification). Patients with necrotizing pancreatitis had higher calprotectin levels than patients with mild forms.

Comparing with the normal values given in the literature, levels of S100A 12 in our group were only elevated in $25 \%$ of the SIRS/sepic patients. However, the S100A12 protein was a very good marker for pancreatitis. Comparing with the other patients with SIRS/sepsis we saw a statistically relevant difference. Likewise, patients with necrotizing pancreatitis had higher levels comparing with the milder forms. The difference was also statistically significant.

Conclusion. Although many standalone biochemical markers have been studied for early detection of pancreatitis and severity evaluation, C-reactive protein (CRP) remains the most frequently used marker. Though, CRP is an acute phase reactant that is not specific for pancreatitis. Procalcitonin (PCT) can be useful for detecting pancreatitis, but PCT is also not specific for this disease. Regarding Lipase and Amylase, the magnitude of the elevation of these markers does not predict disease severity in adults. Moreover, in an acute exacerbation of chronic pancreatitis neither enzyme may be elevated.

As our study demonstrated, Calprotectin and S100A12 are good biomarkers for pancreatitis. Also, for severity evaluation of pancreatitis the two studied proteins showed promising data.

\section{7}

Clinical characteristics, outcomes and risk factors for mortality on patients with sepsis in intensive care units in Vietnam S. Do ; C. Luong ; D. Pham²; N. Nguyen ${ }^{3}$; D. Huynh ${ }^{4}$; Q. Hoang ${ }^{5}$; C. Dao ${ }^{6}$; T. Le ${ }^{7}$; H. Buil ${ }^{8}$; H. Nguyen ${ }^{9}$; H. Hoang ${ }^{10}$; T. Le ${ }^{11}$; L. Nguyen ${ }^{12}$; P. Duong ${ }^{13}$; T. Nguyen ${ }^{14} ;$ Y. Vu ${ }^{15} ;$ G. Pham ${ }^{16} ;$ C. Nguyen ${ }^{1} ;$ T. Pham ${ }^{4} ;$ A. Nguyen ${ }^{17}$

${ }^{1}$ Center for Emergency Medicine, Bach Mai Hospital, Hanoi, Vietnam; ${ }^{2}$ Department of nutrition and food safety, Faculty of Public Health, Thai Binh University of Medicine and Pharmacy, Thái Bình, Vietnam; ${ }^{3}$ ntensive Care Unit, Vietnam-Czechoslovakia Friendship Hospital, Haiphong, Vietnam; ${ }^{4}$ Intensive Care Department, Cho Ray Hospital, Ho Chi Minh City, Vietnam; ${ }^{5}$ Emergency Department, Hue Central General Hospital, Hue, Vietnam; ${ }^{6}$ Department of intensive care, Bach Mai Hospital, Hanoi, Vietnam; ${ }^{7}$ Intensive Care Unit, 115 People's Hospital, Ho Chi Minh City, Vietnam; ${ }^{8}$ ntensive Care Unit, Bai Chay General Hospital, Quang Ninh, Vietnam; ${ }^{9}$ ntensive Care Unit, Da Nang Hospital, Da Nang, Vietnam;

${ }^{10}$ Emergency and Critical Care Department, Hanoi Medical University Hospital, Hanoi, Vietnam; ${ }^{11}$ Intensive CARE UNIT, Dong Da General Hospital, Hanoi, Vietnam; ${ }^{12}$ Intensive care unit, Saint Paul General Hospital, Hanoi, Vietnam; ${ }^{13}$ Intensive Care Unit, Can Tho Central General Hospital, Can Tho, Vietnam; ${ }^{14}$ Intensive Care Unit, Vinmec Times City International Hospital, Hanoi, Vietnam; ${ }^{15}$ Intensive Care Unit, Thai Nguyen Central General Hospital, Thai Nguyen, Vietnam; ${ }^{16}$ Emergency Department, Thanh Nhan General Hospital, Hanoi, Vietnam; ${ }^{17}$ Department of Emergency and Critical Care Medicine, Hanoi Medical University, Hanoi, Vietnam Correspondence: $C$. Luong

Intensive Care Medicine Experimental 2021, 9(1): 000327

Introduction. Sepsis is an important global health problem. Sepsis is the most common cause of in-hospital deaths and extracts a high economic and social cost. Sepsis is treatable, and timely implementation of targeted interventions improves outcomes. However, Vietnam is still struggling to provide either enough resources or adequate diagnostic and treatment strategies for patients with sepsis and septic shock in both local and central settings. Understanding the countryspecific etiologies and the disease risk and prognosis of sepsis and septic shock therefore are crucial for reducing mortality in Vietnam.

Objectives. To investigate the mortality rate and associated factors from sepsis in intensive care units (ICUs) in Vietnam. 
Methods. We did a multicenter cross-sectional, point prevalence study of patients ( $\geq 18$ years old) presenting with sepsis to 15 adult ICUs of 14 hospitals throughout Vietnam on the 4 days representing the different seasons of 2019. We collected data on demographics, clinical parameters, investigations and management of patients with sepsis, and data on sepsis bundle and ICU resource utilization, and compared these data between survival and non-survival during the hospital and ICU stay. We assessed factors associated with hospital and ICU mortality using logistic regression analysis.

Results. Of 252 eligible patients with sepsis, 162 (64.3\%) were male and the median age was 65 years (interquartile range, IQR: $52-76.75)$. Up to $40.1 \%(101 / 252)$ of these patients died in hospital and $33.3 \%(84 / 252)$ died in ICU. Among the total patients, $80.2 \%$ (202/252) were from ICUs with accredited training programs, $74.2 \%$ (187/252) from ICUs with a nurse to patient ratio of $1: 2$, and $65.5 \%$ $(165 / 252)$ from ICUs with an intensivist to patient ratio of $1: 5$ or fewer. At the time of ICU admission, the median Sequential Organ Failure Assessment (SOFA) score was 7 points (IQR: 4.75-10). Compliance to the 3 -h sepsis bundle was $44.8 \%(108 / 241)$ of patients. Non-surgical source control was provided for $31.1 \%(78 / 251)$ of patients, mechanical ventilation (MV) for $68.9 \%(173 / 251)$ and renal replacement therapy (RRT) for $40.2 \%(101 / 251)$. In the multivariable analysis, ICUs with accredited training programs (odds ratio, OR: $0.277 ; 95 \%$ confidence interval, $\mathrm{Cl}: 0.105-0.732$ ) and completion of antibiotics administration and lactate measurement of the 3-h sepsis bundle (OR: $0.088 ; 95 \% \mathrm{Cl}: 0.010-0.790$ ) were independently associated with death in hospital. In addition, MV (OR: 3.599; 95\% Cl: 1.119-11.579), RRT (OR: 2.794; 95\% Cl: 1.279-6.106) and non-surgical source control (OR: $0.405 ; 95 \% \mathrm{Cl}: 0.168-0.978)$ were also independently associated with death in ICU.

Conclusion. The management of sepsis in Vietnam needs to be enhanced in order to decrease mortality in patients with sepsis in ICUs by such measures as increasing resources in critical care settings; making accredited critical care training programs more available; improving infection prevention and control; and providing appropriate initial antibiotic treatment, as well as treating underlying illness and shock optimally in patients with sepsis.

\section{Reference(s)}

1. We thank all ED and ICU staff of participating hospitals for their support with this study. We also thank the staff of the Faculty of Public Health at Thai Binh University of Medicine and Pharmacy for their support and statistical advice. Finally, we thank Miss Phuong Thi Tran from the Center for Emergency Medicine of Bach Mai Hospital, Hanoi, Vietnam for her secretarial works with this study.

2. Dat VQ, Long NT, Giang KB, Diep PB, Giang TH, Diaz JV. Healthcare infrastructure capacity to respond to severe acute respiratory infection (SARI) and sepsis in Vietnam: A low-middle income country. Journal of critical care. 2017:42:109-15

3. Chinh LQ, Manabe T, Son DN, Chi NV, Fujikura Y, Binh NG, et al. Clinical epidemiology and mortality on patients with acute respiratory distress syndrome (ARDS) in Vietnam. PloS one. 2019;14(8):e0221114-e.

4. Rudd KE, Johnson SC, Agesa KM, Shackelford KA, Tsoi D, Kievlan DR, et al. Global, regional, and national sepsis incidence and mortality, 1990-2017: analysis for the Global Burden of Disease Study. Lancet. 2020;395(10219):200-11.

5. Liu V, Escobar GJ, Greene JD, Soule J, Whippy A, Angus DC, et al. Hospital deaths in patients with sepsis from 2 independent cohorts. Jama. 2014:312(1):90-2.

6. Torio CM, Moore BJ. National Inpatient Hospital Costs: The Most Expensive Conditions by Payer, 2013: Statistical Brief \#204. In: David Knutson, editor. Healthcare Cost and Utilization Project (HCUP) Statistical Briefs. Rockville (MD): Agency for Healthcare Research and Quality (US); 2006

7. Evans IVR, Phillips GS, Alpern ER, Angus DC, Friedrich ME, Kissoon N, et al. Association Between the New York Sepsis Care Mandate and In-Hospital Mortality for Pediatric Sepsis. Jama. 2018;320(4):358-67.

8. Rhodes A, Evans LE, Alhazzani W, Levy MM, Antonelli M, Ferrer R, et al. Surviving Sepsis Campaign: International Guidelines for Management of Sepsis and Septic Shock: 2016. Critical care medicine. 2017;45(3):486-552.
9. Seymour CW, Gesten F, Prescott HC, Friedrich ME, I washyna TJ, Phillips GS, et al. Time to Treatment and Mortality during Mandated Emergency Care for Sepsis. The New England journal of medicine. 2017;376(23):2235-44.

\section{8}

ASSOCIATION OF THROMBOSIS AND LEVELS OF INFLAMMATORY MARKERS IN PATIENTS WITH SEVERE ACUTE RESPIRATORY SYNDROME CORONAVIRUS-2 (SARS COV-2) WHO ARE

\section{WITH ANTICOAGULATION}

P. Lopez ; I. Chavez'; J. Aguirre Sánchez'; R. Chaires Gutierrez

1 Intensive Care Unit, The American British Cowdray Medical Center,

Mexico City, Mexico

Correspondence: P. Lopez

Intensive Care Medicine Experimental 2021, 9(1): 000328

Introduction. The endothelium plays a key role in the immune response, especially in critical conditions, such as sepsis, the endothelium being susceptible to injury and inflammation due to infections of bacterial and viral etiology. SARS CoV-2 infection causes sepsis, which leads to the release of inflammatory cytokines such as interleukin-2 (IL-2) and interleukin-6 (IL-6). These cytokines activate the coagulation system in a deregulated way with a hypercoagulable state, which conditions an elevation of inflammatory markers, that has a procoagulant action, affecting the lungs in an early phase, which later progresses to a systemic inflammatory response. A notable feature in patients with SARS CoV- 2 is the high incidence of clinically presenting acute thrombotic episodes, such as deep vein thrombosis, pulmonary embolism, or acute coronary syndromes. Histopathological studies report generalized microvascular thrombosis, this being the pathogenesis of organ dysfunction during SARS CoV-2 infection. Here comes the importance of administering pharmacological anticoagulation.

Objectives. To evaluate the association of thrombosis and levels of inflammatory markers in patients with SARS CoV-2 infection who are with anticoagulation.

Methods. A retrospective and longitudinal cohort study was carried out with adult patients who were admitted to the department of Critical Care COVID unit 'Dr. Mario Shapiro' with a diagnosis of SARS CoV-2 infection. Electronic records were reviewed to obtain clinical and demographic characteristics and corresponding inflammatory markers to associate thrombosis. A linear analysis of repetitive measures was carried out with the support of the SPSS program. Levels of inflammatory markers were measured at admission, on the 3rd, 7th and 10 th day.

Results. A sample of 50 patients was obtained, with the male gender predominant in $78 \% .20 \%$ of the sample had thrombosis. The statistically significant inflammatory markers were D dimer (DD) with a $P$ value of 0.013 and levels $>1000 \mathrm{ng} / \mathrm{mL}$, and C-Reactive Protein (CRP) with a P 0.030 and levels $>8 \mathrm{mg} / \mathrm{dL}$. On the other hand, ferritin obtained a P value of 0.055 and levels $>900 \mathrm{ng} / \mathrm{mL}$.

Conclusion. The significant increase in DD levels is associated with a higher incidence of thrombosis, like so a high CRP levels that indicates a greater degree of inflammation and increase the risk of thrombosis. Ferritin gave a borderline estimate, but not ruled out for the association with thrombosis. This can be replicated for future studies, taking into account a larger sample, extending the linear measurement time. Leukocytes, platelets, procalcitonin (PCT), and IL-6 lack predictive sensitivity for thrombosis in patients with SARS CoV-2 infection.

\section{Reference(s)}

1. Pons et al. The vascular endothelium in severe SARS-CoV-2 infection. Critical Care (2020) 24:353.

2. Iba et al. The unique characteristics of COVID-19 coagulopathy. Critical Care (2020) 24:360.

3. Cui et al. Prevalence of venous thromboembolism in patients with severe novel coronavirus pneumonia. J Thromb Haemost. 2020;18:1421-1424.

4. Pons et al. Inmune consequences of endothelial cells activation and dysfunction during sepsis. Crit Care Clin (2019).

5. None. 
000338

Fluid Responsiveness and Creatinine Increase in Patients with Septic Shock

I. Douglas' ${ }^{1}$ P. Alapat ${ }^{2}$; K. Corl ${ }^{3}$; M. Exline, ${ }^{4}$; L. Forni ${ }^{5}$; A. Holder, ${ }^{6}$; D. Kaufman,; A. Khan, ; M. Levy, ; G. Martin ${ }^{10}$; J. Sahatjian ${ }^{11}$; E. Seeley, ${ }^{12}$; W. Self, ${ }^{13} ;$ J. Weingarten, ${ }^{14} ;$ M. Williams, ${ }^{15} ; \mathrm{D}$. Hansell ${ }^{16}$

${ }^{1}$ Pulmonary and Critical Care, Denver Health, Denver, United States of America; ${ }^{2}$ Critical Care, Ben Taub General Hospital, Houston, United States of America; ${ }^{3}$ Critical Care, Rhode Island Hospital, Providence, United States of America; ${ }^{4}$ Critical Care, Ohio State University Hospital, Columbus, United States of America; ${ }^{5} \mathrm{CU}$, Royal Surrey County Hospital, Surrey, United Kingdom; ${ }^{6}$ Pulmonary and Critical Care, Emory University Hospital Midtown, Atlanta, United States of America; ${ }^{7}$ Critical Care, NYU School of Medicine, New York, United States of America; ${ }^{8}$ Critical care, Oregon Health \& Science University, Portland, United States of America; ${ }^{9}$ Division of pulmonary, Critical Care and Sleep Medicine, Warren Alpert School of Medicine at Brown University, Providence, United States of America; ${ }^{10}$ Pulmonary and Critical Care, Emory University, Atlanta, United States of America; ${ }^{11}$ Clinical Affairs, Cheetah Medical Inc., Newton, United States of America; ${ }^{12} \mathrm{Icu}$, University of California San Francisco Parnassus Campus, San Francisco, United States of America; ${ }^{13} \mathrm{ICu}$, Vanderbilt University, Nashville, United States of America; ${ }^{14} \mathrm{ICU}$, NewYork-Presbyterian Brooklyn Methodist Hospital, New York, United States of America; ${ }^{15} \mathrm{ICU}$, Methodist Hospital, Indianapolis, United States of America; ${ }^{16}$ Anesethesiology, Massachusetts general hospital, Boston, United States of America

Correspondence: $J$. Sahatjian

Intensive Care Medicine Experimental 2021, 9(1): 000338

Introduction. IV fluid treatment is a central component of septic shock resuscitation but in excess is associated with adverse effects. Dynamic measurement of stroke volume (SV) following a passive leg raise (PLR) is a safe and feasible method of rapidly predicting the effectiveness of fluid-induced augmentation of cardiac output. The FRESH study demonstrated that SV-guided dynamic assessment could alter the amount of IV fluid administered to patients with septic shock and improve patient outcomes.1 Additionally, the kidney is known to be vulnerable to injury during septic shock. The goal of this analysis was to evaluate the incidence of AKI in the FRESH patient population.

Methods. FRESH was a prospective, randomized global clinical trial in adults with septic shock comparing PLR-guided SV responsiveness (intervention) as a guide for fluid management versus usual care.1 Patients presented to the ER with sepsis associated hypotension and anticipated ICU admission. In the Intervention arm, patients were assessed for fluid responsiveness before any clinically driven fluid bolus or increase in vasopressors. If a patient's stroke volume increased by $\geq 10 \%$ in response to a PLR, they were considered fluid responsive and fluid was recommended as the first therapy. If a patient's stroke volume increased by $<10 \%$ then they were considered not to be fluid responsive and vasopressors were recommended as the first therapy. The protocol included reassessment and therapy as indicated by the PLR result.

Results. 83 patients were randomized to the Intervention arm and 41 patients to the Usual Care control arm. Patients in the treatment arm received less fluid over the $72 \mathrm{~h}$ treatment period (3354.2 \pm 2179.6$)$ compared to the control group (4721.3 $\pm 3319.1, p=0.007)$. Patients in the treatment arm displayed a statistically significant decrease in the need for kidney replacement therapy ( $5.1 \%$ vs $17.5 \%, p=0.04) 0.1$ An analysis of patient baseline and pre hospitalization creatinine levels over the course of hospitalization indicated that in addition to a decrease in the need for KRT, patients in the treatment arm exhibited a decrease in observed AKI status. In the treatment arm $25.9 \%$ of patients exhibited a twofold increase in creatinine over their true baseline creatinine value (KDIGO stage 2), compared to $48 \%$ of patients in the control arm $(p=0.04)$.

Conclusion. Physiologically-informed fluid and vasopressor resuscitation using PLR-induced SV change to guide personalized management of septic shock was associated with a lower requirement of administered fluid and a decreased need for KRT. An analysis of creatinine levels indicated a potential benefit to preserving kidney function and decreasing the rate of AKI. Administering fluid only when it is effective at improving perfusion (SV change $>10 \%$ ) may help preserve kidney function and importantly limit the deleterious effect of volume overload on renal function.

\author{
Reference(s) \\ 1. 1 Douglas I et al. Fluid Response Evaluation in Hypotension and Shock: A \\ Randomized Clinical Trial. CHEST 2020; 158(4):1431-1445 \\ 2. This study was supported by Cheetah Medical
}

\section{0}

Fluid Responsiveness and Vasopressor Use in Patients with Septic Shock

I. Douglas ${ }^{1}$; P. Alapat ${ }^{2}$; K. Corl ${ }^{3}$; M. Exline, ${ }^{4} ;$ L. Forni ${ }^{5}$; A. Holder, ; D. Kaufman,; A. Khan, ; M. Levy, ; G. Martin ${ }^{10}$; J. Sahatjiann ${ }^{11}$; E. Seeley, ${ }_{1}^{12}$;W. Self, ${ }^{13}$; J. Weingarten, ${ }^{14} ;$ M. Williams, ${ }^{15} ; \mathrm{D}$. Hansell ${ }^{16}$

${ }^{1}$ Pulmonary and Critical Care, Denver Health, Denver, United States of America; ${ }^{2}$ Critical care, Ben Taub General Hospital, Houston, United States of America; ${ }^{3}$ Critical Care, Rhode Island Hospital, Providence, United States of America; ${ }^{4} \mathrm{Critical}$ care, Ohio State University Hospital, Columbus, United States of America; ${ }^{5} \mathrm{ICU}$, Royal Surrey County Hospital, Surrey, United Kingdom; ${ }^{6}$ Pulmonary and critical care, Emory University Hospital Midtown, Atlanta, United States of America; ${ }^{7}$ Critical care, NYU School of Medicine, New York, United States of America; ${ }^{8}$ Critical care, Oregon Health \& Science University, Portland, United States of America; ${ }^{9}$ Division of pulmonary, critical care and sleep medicine, Warren Alpert School of Medicine at Brown University, Providence, United States of America; ${ }^{10}$ Pulmonary and Critical Care, Emory University, Atlanta, United States of America; ${ }^{11}$ Clinical Affairs, Cheetah Medical, Newton Center, United States of America; ${ }^{12} \mathrm{ICU}$, University of California San Francisco Parnassus Campus, San Francisco, United States of America; ${ }^{13}$ Icu, Vanderbilt University, Nashville, United States of America; ${ }^{14} \mathrm{ICU}$, NewYork-Presbyterian Brooklyn Methodist Hospital, New York, United States of America; ${ }^{15} \mathrm{ICU}$, Methodist Hospital, Indianapolis, United States of America; ${ }^{16}$ Anesethesiology, Massachusetts general hospital, Boston, United States of America

Correspondence: $\mathrm{J}$. Sahatjian

Intensive Care Medicine Experimental 2021, 9(1): 000340

Introduction. Fluid and vasopressors are central components of septic shock resuscitation but in excess are associated with adverse effects. Dynamic measurement of stroke volume (SV) following a small IV fluid bolus or passive leg raise (PLR) is a safe and feasible method of rapidly predicting the effectiveness of fluid-induced augmentation of cardiac output.The FRESH study demonstrated that SV-guided dynamic assessment could alter the amount of IV fluid administered to patients with septic shock and improve patient outcomes.1The goal of this analysis was to evaluate the use of vasopressors administration in the FRESH patient population.

Methods. FRESH was a prospective, randomized global clinical trial among adults with sepsis-associated hypotension comparing PLRguided SV responsiveness (intervention) as a guide for fluid management versus usual care.1 Patients presented to the Emergency Room with sepsis associated hypotension and anticipated ICU admission. In the Intervention arm, patients were assessed for fluid responsiveness before any clinically driven fluid bolus or increase in vasopressors. If the patient's stroke volume increased by $10 \%$ or more in response to a passive leg raise (PLR), they were considered fluid responsive and fluid was recommended as the first therapy. If the patient's stroke volume increased by less than $10 \%$ during PLR, then the patient was considered not to be fluid responsive and vasopressors were recommended as the first therapy. The protocol included reassessment and therapy as indicated by the PLR result. The control arm received usual care.

Results. 83 patients were randomized to the Intervention arm and 41 patients to the Usual Care arm. Patients in the treatment arm received less fluid over the $72 \mathrm{~h}$ treatment period $(3354.2 \pm 2179.6)$ compared 
to the control group $(4721.3 \pm 3319.1, p=0.007) 0.1 \mathrm{~A}$ similar number of patients in the treatment and control group were initiated on vasopressors post randomization (25\% treatment vs. $20.8 \%$ control, $\mathrm{p}=0.683$ ). Patients in the treatment group underwent vasopressor use for a similar period of time $(40.74 \mathrm{~h} \pm 51.23$ vs $55.64 \pm 87.43 \mathrm{~h}$, reduction of $-14.91, p=0.426$ ) Overall, the percentage of PLRs resulting in dose or frequency change in vasopressor was $20 \%$.

Conclusion. Physiologically-informed fluid and vasopressor resuscitation using PLR-induced SV change to guide personalized management of septic shock resulted in a lower decreased fluid balance. Although the treatment algorithm directed providers to consider vasopressors when a patient was not fluid responsive, there was no increase in vasopressor administration or increase in time on vasopressors in the treatment group. Administering fluid only when it is effective at improving perfusion (SV change $>10 \%$ ) may lead to overall more effective perfusion management, and does not increase the need for vasopressors and time on treatment. A personalized approach to fluid management may help improve patient overall patient outcome.

\section{Reference(s)}

1. Douglas I et al. Fluid Response Evaluation in Hypotension and Shock: A Randomized Clinical Trial. CHEST 2020; 158(4):1431-1445

2. This study was supported by Cheetah Medical

\section{8}

\section{Sepsis heterogeneity in different age groups}

M. Petrushin ; N. Kostritca²; M. Babaev ${ }^{3}$

${ }^{1}$ Icu, Tver Regional Hospital, Tver, Russia; ${ }^{2}$ Faculty of Medicine, Moscow State University, Moscow, Russia; ${ }^{3} \mathrm{CU}$, Petrovsky National Research Centre of Surgery, Moscow, Russia

Correspondence: M. Petrushin

Intensive Care Medicine Experimental 2021, 9(1): 000358

Introduction. Sepsis is a heterogeneous condition and there is a necessity to develop a personalized approach to treatment based on the study of prognostic and predictive mortality factors. Age is one of the most important negative prognostic factors.

Objectives. To identify the features of the course of sepsis in different age groups based on the analysis of clinical and laboratory data received within the first $72 \mathrm{~h}$ of hospitalisation.

Methods. The study included 51 patients ( 32 male, 19 female) aged from 19 to 81 years old with pneumonia complicated by sepsis (diagnosed by sepsis-3 criteria). Anamnestic data were collected from all patients and a dynamic assessment of the severity of organ dysfunctions was performed with standard clinical and laboratory parameters within $72 \mathrm{~h}$. Patients received therapy according to the accepted recommendations. All patients were divided into 4 age groups: group $1-18-44$ years old $(n=14)$, group 2-45-59 $(n=16)$, group 3-6070 years old $(n=17)$, group $4-71$ and more years old $(n=4)$. The SPSS program was used for statistical analysis.

Results. The mortality rate was $61 \%$. Median age was $56(46 ; 67)$ and $49(32 ; 62)$ years for non-survivals survivals respectively, and the age more than 56 years old was identified as a mortality prognostic factor $(p=0,036)$. Also, the following were identified as mortality predictors: Charlson comorbidity index (ICh), $p=0.001$ (with cut-off 3 points), SOFA score, $p=0.02$ (7 points); GFR, $p=0.003(52 \mathrm{ml} / \mathrm{min} / 1.73 \mathrm{~m} 2)$ at the time of admission. The groups differed in ICh $(p=0.000), C R P(p=0.008), M S I$ $(p=0.023)$, platelet count $(p=0.000), p O 2 a(p=0.006), B E(p=0.01)$, arterial blood lactate level $(p=0.000)$. In group 1 mortality was minimal (43\%). We observed grade 1 obesity (BMI $30.4 \mathrm{~kg} / \mathrm{m}^{2}$ ), significant increase of MSI by $36 \%$ (up to $1.5, p=0.007$ ) within $72 \mathrm{~h}$ and statically insignificant increase of PCT by $48 \%$ (up to $9.5 \mathrm{ng} / \mathrm{ml}$ ), lactate by $33 \%$ (up to $1.96 \mathrm{mmol} / \mathrm{l}$ ) and SOFA score by $38 \%$ (up to 11 points) in nonsurvivals. In group 2 the mortality rate was $63 \%$. We observed significant increase in PCT by $22 \%(5.6 \mathrm{ng} / \mathrm{ml})$ in non-survivals. In group 3 the mortality rate was $65 \%$. We observed significant SOFA score increase by $27 \%$ (9.5 points, $p=0.01)$ and platelet count decrease by $21 \%(150 * 103 / \mu \mathrm{L}$, $p=0.014)$ in non-survivals. Patients over 71 years old did not survive and were characterized by significant rise in CRP by $102 \%(174 \mathrm{mg} / \mathrm{L}$, $\mathrm{p}=0.039)$ and insignificant rise in PCT by $93 \%(7.2 \mathrm{mg} / \mathrm{L}), \mathrm{MSI}-17 \%$ (1.3), bilirubin level-56\% (17.8 $\mathrm{mg} / \mathrm{l})$ and lactate-35\% (1.75 $\mathrm{mmol} / \mathrm{l})$. Short-term mortality (up to 7 days) was observed in men (63\%) with obesity (BMI $30 \mathrm{~kg} / \mathrm{m} 2$ in $3(63 \%)$ and 1 age groups (31\%)) who were on mechanical ventilation and had septic shock with progressive organ dysfunction (SOFA 10 points).

Conclusion. The age group determines the course of sepsis, as well as predictive mortality factors, which can become the basis for an individual approach to sepsis therapy.

\section{9}

Sepsis in the Medical Intensive Care Unit: Outcome Prediction Models-a Prospective, Observational, Single-centre, Clinical Study

I. Klobučar ${ }^{1}$; V. Vucelić2 $;$ V. Degoricija ${ }^{3}$

${ }^{1}$ Department of Cardiology, Sisters of Charity University Hospital Centre, Zagreb, Croatia; ${ }^{2}$ Department of Medicine, Sisters of Charity University Hospital Centre, Zagreb, Croatia; ${ }^{3}$ Department of Medicine, sisters of charity university hospital centre, University of Zagreb School of Medicine, Zagreb, Croatia

Correspondence: I. Klobučar

Intensive Care Medicine Experimental 2021, 9(1): 000379

Introduction. Sepsis is a life-threatening organ dysfunction caused by a dysregulated host response to infection,[1] which has the unacceptably high mortality rate (11.9-39.5\%)[2] despite the huge progress in the intensive care medicine during the past years.

Objectives. The aim of the study was to test the prognostic value of the scores widely used in the intensive care medicine (APACHE II, LODS, SAPS II, SOFA) and to determine additional independent mortality risk factors for the patients with sepsis treated in the medical intensive care unit (ICU).

Methods. A prospective, observational study conducted in the medical ICU (Sisters of Charity University Hospital Centre, Zagreb, Croatia) between April 2017 and May 2018. All patients treated with the diagnosis of sepsis (according to Sepsis-3 criteria [1]) were enrolled in the study. Demographic data, vital signs, laboratory test results and scores were assessed at the time of the patients' admission to the ICU. The outcome was recorded at the end of the ICU treatment.

Results. A total of 116 patients with sepsis were enrolled in the study. The median age was 73.5 years (range 23-98) and 63 (54.3\%) were female. Forty-four patients (37.9\%) died during the ICU treatment, with $65.9 \%$ of these deaths occurring within the first 72 ICU-hours. The mean ICU length of stay for ICU survivors was 6 days (1-34) and 3 days (1-19) for ICU non-survivors.

All mean score values [APACHE II-25 (15-33) vs 16 (4-31), LODS-12 (4-14) vs 5 (1-14), SAPS II-56 (22-89) vs 37 (19-87), SOFA-8 (4-10) vs 4 (2-9)] were significantly higher in the ICU non-survivor group compared to the ICU survivors $(\mathrm{P}<0.001)$. ROC curves for all scores discriminating ICU outcome are shown in Figure 1.

The independent risk factors for ICU mortality, calculated using the multiple binary logistic regression, were: reduced mobility level (odds ratio [OR] 11.16, 95\% confidence interval [CI] 2.45-50.91), failure in early sepsis recognition in the emergency department (OR 6.59, 95\% Cl 1.09-39.75), higher SOFA score at admission (OR 2.37, 95\% Cl 1.59-3.52), and inappropriate antimicrobial treatment (OR 9.99, 95\% Cl 2.57-38.87). Taken together, these variables form a new predictive model for ICU mortality, which significantly improves the predictive power of the SOFA score alone $(A \cup R O C($ SOFA $)=0.894$ vs AU ROC $($ model $)=0.959$, Fig. 2 ). 

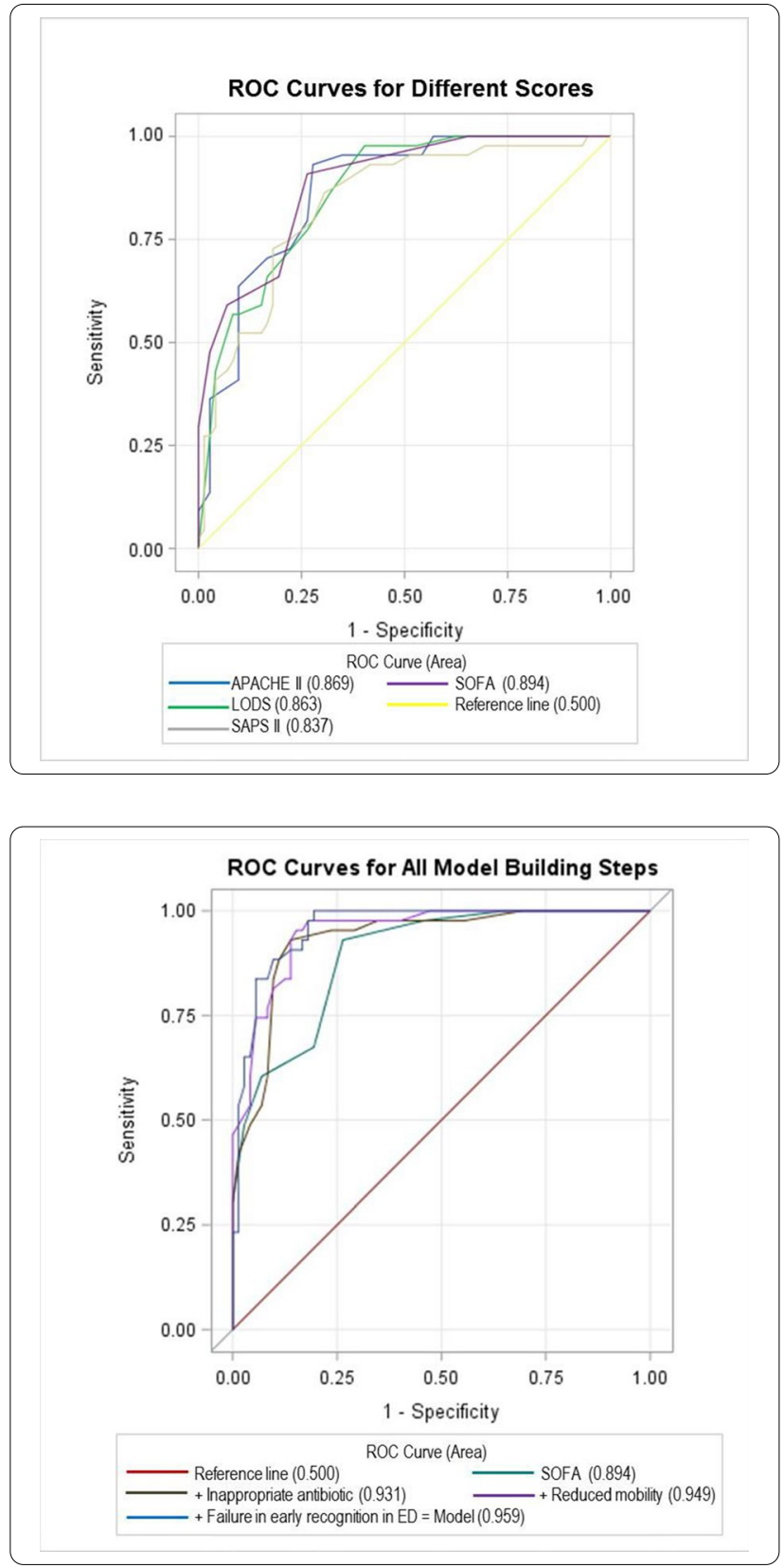

Conclusion. The ICU mortality rate for patients with sepsis was high in this study. The newly-formed ICU mortality predictive model for patients with sepsis, by adding three binary variables to the SOFA score, exceeds the predictive power of all other widely used scores in intensive care medicine. The limitations of the model are the unavailability of the information about the appropriateness of the antimicrobial treatment at the time of patients' admission to the ICU and the need for its validation on the bigger sets of patient data.

\section{Reference(s)}

1. 1 Singer M, Deutschman CS, Warren Seymour C, Shankar-Hari M, Annane D, Bauer M, et al. The Third International Consensus Definitions for Sepsis and Septic Shock (Sepsis-3). JAMA. 2016;315:801-810.

2. 2 Sakr Y, Jaschinski U, Wittebole X, Szakmany T, Lipman J, Namendys-Silva SA, et al. Sepsis in Intensive Care Unit Patients: Worldwide Data From the Intensive Care over Nations Audit. Open Forum Infect Dis. 2018;5:ofy313.

3. This research did not receive any specific grant from funding agencies in the public, commercial, or not-for-profit sectors.

\section{1}

Mortality risk factors in patients with nosocomial infection, in an ICU after long-term application of Selective Digestive Decontamination

C. Sánchez Ramírez'; RE. Morales Sirgado'; S. Hípola Escalada ${ }^{1}$; MA. Hernández Viera'; M. Cabrera Santana'; C. Rodriguez Mata'; ME. Gallardo Santos $^{1}$; CF. Lübbe Vázquez ${ }^{1}$; P. Saavedra-Santana² ${ }^{2}$ S. Ruiz-Santana ${ }^{1}$ ${ }^{1}$ Intensive Care Medicine, University Hospital of Gran Canaria Dr. Negrin, Las Palmas de Gran Canaria, Spain; ${ }^{2}$ Mathematics and Informatics Deparment, University of Las Palmas:, Las Palmas de Gran Canaria, Spain

Correspondence: $C$. Sánchez Ramírez

Intensive Care Medicine Experimental 2021, 9(1): 000381

Introduction. Recognition of mortality risk factors and early intervention with appropriate broad-spectrum antimicrobial administration in patients with nosocomial infection (NI) may significantly improve outcomes. Selective Digestive Decontamination (SDD) has been associated with reduced ICU mortality and acquired infection rates.

Objectives. To analyze mortality risk factors in patients with $\mathrm{NI}$ in an ICU after 8 years and 9 months of SDD application.

Methods. Patients with $\mathrm{NI}$ were prospectively included from October 1, 2010 to June 30, 2020 in a polyvalent ICU of 30 beds. SDD was applied for 8 years and nine months, from October 1, 2011 to June 30, 2020. Patients requiring mechanical ventilation for more than $48 \mathrm{~h}$, an enteral solution and a paste with colistin, tobramycin and nystatin every $8 \mathrm{~h}$ until discharge were applied. Also intravenous cefotaxime were administered during the first 4 days. Rectal and pharyngeal exudates were collected on admission and weekly. We used ENVIN NI criteria.The categorical variables were summarized in frequencies and percentages and the numerical variables as means and standard deviations or in medians and interquartile ranges. The percentages were compared with the $\mathrm{X}^{2}$ test or the Fisher exact test, the means with the t-test and the medians with the Wilcoxon test for independent data. A multidimensional logistic analysis was carried out. It was considered significant if $p \leq 0.05$.

Results. Of 8325 patients admitted, $253(35,38 \%)$ out of 715 patients with NIs died. In an univariate analysis we did no find statistically significant difference in ICU stay. Multiresistant (MR) Pseudomonas and MR Gram negative bacteria (GNB) NI were significantly higher in patients who died (Table 1). 


\begin{tabular}{|c|c|c|c|}
\hline & $\begin{array}{l}\text { Alive } \\
N=462\end{array}$ & $\begin{array}{c}\text { Death } \\
N=253\end{array}$ & $\mathbf{P}$ \\
\hline Age, years & $59.8 \pm 15.4$ & $63.7 \pm 13.5$ & $<0.001$ \\
\hline APACHE II score & $20.2 \pm 7.4$ & $24.1 \pm 7.6$ & $<0.001$ \\
\hline SOD & $369(79.9)$ & $208(82.2)$ & 0.448 \\
\hline Sex male & $303(65.6)$ & $165(65.5)$ & 0.535 \\
\hline Trauma patients & $70(15.2)$ & $12(4.7)$ & $<0.001$ \\
\hline Coronary arteria disease patent & $94(20.4)$ & $56(22.1)$ & 0.337 \\
\hline Emergency surgery & $111(24.0)$ & $58(22.9)$ & 0.427 \\
\hline Immunosupression & $31(6.7)$ & $47(18.6)$ & $<0.001$ \\
\hline Neutropenia & $8(1.7)$ & $18(7.1)$ & $<0.001$ \\
\hline Immurodepression & $4(0.9)$ & $1(0.4)$ & 0.661 \\
\hline Parenteral nutiton & $80(17.3)$ & $92(36.4)$ & $<0.001$ \\
\hline External venticuiar deviced & $57(12.3)$ & $8(3.2)$ & $<0.001$ \\
\hline RRT & $104(22.5)$ & $161(63.6)$ & $<0.001$ \\
\hline Malnutrion & $33(7.1)$ & $38(15.0)$ & 0.001 \\
\hline Diabetes mellitus & $121(26.2)$ & $108(42.7)$ & $<0.001$ \\
\hline COPD & $58(12.6)$ & $53(20.9)$ & 0.003 \\
\hline Renal faiure & $75(16.2)$ & $82(32.4)$ & $<0.001$ \\
\hline Cirrhosis & $16(3.5)$ & $21(8.3)$ & 0.009 \\
\hline Neoplasm & $27(5.8)$ & $42(16.6)$ & $<0.001$ \\
\hline VAP & $149(32.2)$ & $113(44.7)$ & $<0.001$ \\
\hline CRB & $173(37.5)$ & $81(32.0)$ & 0.334 \\
\hline Secondary bacteremia & $114(24.7)$ & $74(29.2)$ & 0.433 \\
\hline Urinary infection & $124(26.8)$ & $63(24.9)$ & 0.370 \\
\hline ATB 48 hours before admission & $106(22.9)$ & $85(33.6)$ & 0.005 \\
\hline Acinetobacter baumannii & $13(2.8)$ & $7(2.8)$ & 0.971 \\
\hline MRSA & $10(2.2)$ & $3(1.2)$ & 0.559 \\
\hline ESBL & $101(21.9)$ & $64(25.3)$ & 0.297 \\
\hline MR Pseudiomonas & $25(5.4)$ & $31(12.2)$ & 0.002 \\
\hline MR GNB & $16(3.5)$ & $20(7.9)$ & 0.009 \\
\hline Admission & & & $<0.001$ \\
\hline Medical & $303(65.6)$ & $210(83.3)$ & \\
\hline Scheduled surgery & $78(16.9)$ & $14(5.6)$ & \\
\hline Emergency surgery & $80(17.3)$ & $28(11.1)$ & \\
\hline Inflammatory response & & & $<0.001$ \\
\hline No sepsis & $17(3.7)$ & $8(3.2)$ & \\
\hline Sepsis & $137(29.7)$ & $37(14.6)$ & \\
\hline Septic shock & $308(66.7)$ & $208(82.2)$ & \\
\hline ICU days & $31.0\{19.0 .48 .0\}$ & $33.0(19.0-54.0\rangle$ & 0.218 \\
\hline
\end{tabular}

Table 2. Multivariate logistic regression for mortality

\begin{tabular}{ccc}
\hline & P & Odd-Ratio $(95 \% \mathrm{CI})$ \\
\hline APACHE II & $<.001$ & $1.057(1.032 ; 1.083)$ \\
Septic shock & $<.001$ & $2.762(1.929 ; 3.956)$ \\
Parenteral Nutrition & 0.002 & $1.912(1.274 ; 2.871)$ \\
RRT & $<.001$ & $4.264(2.976 ; 6.108)$ \\
Neoplasm & $<.001$ & $3.240(1.813 ; 5.790)$ \\
\hline
\end{tabular}

RRT: Renal replacement therapy

Independent mortality risk factors were: APACHE II Odds Ratio (OR) 1.057 (1.032; 1.083), renal replacement therapy OR: 4.264 (2.976; $6.108)$, septic shock OR: $2.762(1.929 ; 3.956)$, parenteral nutrition OR $1.912(1.274 ; 2.871)$ and neoplasm OR $3.240(1.813 ; 5.790)$ (Table 2).

Conclusion. In an ICU after long-term application of SDD, independently mortality risk factors in patients with NI were: APACHE II, renal replacement therapy, septic shock, parenteral nutrition and neoplasm. MR Pseudomonas and MR GNB NI were significantly higher in patients who died.
000389

Oxygen uptake and carbon dioxide production changes in mechanical ventilated sepsis patients: A prospective observational study

I. Hirayama' ${ }^{\text {; T. Asada }}{ }^{1}$; M. Yamamoto ${ }^{1}$; K. Tokunaga ${ }^{1}$; N. Hayase ${ }^{1}$; T. Hiruma ${ }^{1}$;Y. Ueda ${ }^{1}$; K. Doi

'Department of acute medicine, The University of Tokyo, Tokyo, Japan

Correspondence: I. Hirayama

Intensive Care Medicine Experimental 2021, 9(1): 000389

Introduction. Sepsis is a leading cause of morbidity and mortality in intensive care units (ICU) worldwide. Hemodynamic management of patients with sepsis had been recommended by evaluating lactate clearance and central venous oxygen saturation ( $\mathrm{ScvO2}$ ) normalization. Measurements of oxygen extraction (VO2) and carbon dioxide production (VCO2) by indirect calorimetry (IC) may provide additional information to understand the dynamic metabolic changes in sepsis.

Objectives. The purpose of this study was to evaluate the relationship between VCO2, VO2 changes and 28-day survival, using IC. VCO2 and VO2 were measured non-invasively and continuously.

Methods. Adult sepsis patients intubated in the ICU of our hospital between September 2019 March 2020 were enrolled prospectively. Patients were diagnosed based on definitions of Sepsis-3. Measurement by IC was initiated within $24 \mathrm{~h}$ after tracheal intubation and the values of VCO2 and VO2 were measured for $2 \mathrm{~h}$ continuously. The rate of change of VCO2 and VO2 over $2 \mathrm{~h}$ was defined as VCO2 slope and VO2 slope. CCM Express (MGC Diagnostics, Saint Paul, Minnesota) was used as IC. The relationship with each slope and 28-day survival was examined. In addition, the hourly lactate $\%$ change $(\% / \mathrm{hr})$ was also evaluated in comparison with VCO2 and VO2 slopes. Continuous variables were presented as median (interquartile range), and categorical variables were presented as percentages. Two-tailed $p<0.05$ was considered statistically significant for all tests. Results. The median age of the enrolled 34 patients was 73.0 (51.8-81.0) years old, and the cause of infection was pneumonia $(\mathrm{N}=22)$, urinary tract infection $(\mathrm{N}=3)$, biliary tract infection $(\mathrm{N}=4)$, and others $(\mathrm{N}=5) .26$ of 34 patients survived (76\%). APACHE II and SOFA scores were 25.0 (19.8-29.3) and $10.0(5.8-12.3)$, respectively. There were significant differences in $\mathrm{VCO}_{2}$ slope $(-1.412$ vs. $-0.446, p=0.012)$ and $\mathrm{VO}_{2}$ slope $(-2.098$ vs. -0.851 $\mathrm{p}=0.023)$ between non-survivors vs survivors. Of note, all the non-survivors showed negative slopes of $\mathrm{VCO}_{2}$ and $\mathrm{VO}_{2}$, whereas 17 of 26 survivors showed negative slopes. For these patients with negative slopes of $\mathrm{VCO}_{2}$ and $\mathrm{VO}_{2}, 17$ survivors had a median lactate \%change of $-2.4 \% / \mathrm{hr}$, while non-survivors had a median that of $2.6 \% / \mathrm{hr}(\mathrm{p}=0.023)$. On the other hand, the other 9 survivors showed a median lactate \%change of $1.7 \% / \mathrm{hr}$.

Conclusion. Non survivors in this study showed temporal decreases in $\mathrm{VCO}_{2}$ and $\mathrm{VO}_{2}$ together with lactate elevation. Monitoring the changes in $\mathrm{VCO}_{2}$ and $\mathrm{VO}_{2}$ in addition to lactate \%change evaluation may be useful in predicting the prognosis of sepsis.

Reference(s)

1. There was no funding source for this study.

2. Englert JA, Rogers AJ: Metabolism, Metabolomics, and Nutritional Support of Patients with Sepsis. Clin Chest Med 2016; 37(2):321-331

3. Squara P: Matching total body oxygen consumption and delivery: a crucial objective? Intensive Care Med 2004; 30(12):2170-2179

4. Hoeyer-Nielsen AK, Holmberg MJ, Grossestreuer AV, Yankama T, et al.: Association Between the Oxygen Consumption: Lactate Ratio and Survival in Critically III Patients With Sepsis. Shock 2020

5. Crouser ED: Mitochondrial dysfunction in septic shock and multiple organ dysfunction syndrome. Mitochondrion 2004; 4(5-6):729-741

\section{6}

Should we personalize catecholamin infusion according to transcranial doppler during the early phase of septic shock? A. Ben Souissi'; C. Ben Miled²; F. Haddad²; M. Machghoul2; M. Sbouil ${ }^{2}$;. Belhajyoussef2; MS. Mebazaa $^{3}$

${ }^{1}$ Anesthesiology and Intensive Care, Mongi Slim University Hospital, La Marsa, Tunis, Tunisia, Tunis, Tunisia; ${ }^{2}$ Anesthesiology Intensive Care Department, Mongi Slim University Hospital, La Marsa, Tunisia; ${ }^{3}$ Anesthesiology Intensive Care Department, Mongi Slim Universiry Hospital, La Marsa, Tunisia Correspondence: A. Ben Souissi

Intensive Care Medicine Experimental 2021, 9(1): 000426 
Introduction. Background:

In septic shock, the cerebral circulation is one of the first concerned by the redistribution of blood flow phenomenon. Cerebral perfusion can be assessed by Transcranial Doppler (TCD).

The Surviving Sepsis Campaign (SSC) guidelines give clear recommendations about the hemodynamic goals to achieve during the first hour of septic shock but remain incomplete regarding further goals (1).

Objectives. The main purpose of this study was to assess the contribution of TCD in hemodynamic management during the early phase of septic shock, by comparing a personalized «TCD-guided» hemodynamic goals to the standard SSC recommendations. The main outcome was 28 days mortality.

Methods. Fifty patients were included in this study, equally randomized into 2 groups.

Diagnosis of the septic shock was assessed according to Sepsis-3 consensus (2).

All patients were managed according to SSC guidelines. Concerning catecholamin infusion, in the first group, we aimed to maintain the same goals of MAP $\geq 65 \mathrm{mmHg}$ and negative arterial blood lactate level during the first $72 \mathrm{~h}$ after shock onset. In the second group, hemodynamic objectives and catecholamin infusion rate were determinated by the $T C D$, in order to obtain an optimized cerebral perfusion $(\mathrm{Pl}<1,3)$. For the 2 groups, TCD was performed within the first hour of resuscitation, and every $8 \mathrm{~h}$ during $72 \mathrm{~h}$. MCA velocities and PI were recorded every $8 \mathrm{~h}$, as well as other clinical data. Cerebral hypoperfusion was defined by meeting 2 out of 3 of these criteria: End diastolic velocity $(E D V)<20 \mathrm{~cm} / \mathrm{s}$, Mean flow velocity (MFV) $<35 \mathrm{~cm} / \mathrm{s}$ and/or PI $>1,3$. Sepsis associated encephalopathy was diagnosed using CAM-ICU score $>3$ or /and a GCS deterioration. Study was approved by the Ethics Committee of the Hospital. Statistical analysis was performed using SPSS 25. P-value $<0,05$ was considered statically significant.

Results. The 2 groups were comparable concerning demographic data, comorbidities, initial SOFA, APACHE II and SAPS II scores. The first TCD was performed within the first hour of resuscitation. Patients of the " Standard group» showed an increased mortality $(p=0,031)$ and worse neurological outcome with a higher rate of sepsis associated encephalopathy $(p<0,001)$. TCD-guided group patients experienced a lower rate of cerebral hypoperfusion $(p<0,001)$ and altered autoregulation $(p<0,001)$. TCD measurements improval was observed from the randomisation and along the study period. There was no difference between the groups regarding durations of mechanical ventilation, catecholamin infusion and ICU length of stay. Mean catecholamin infusion rate was also comparable.

Conclusion. In this study, the TCD-guided hemodynamic management during the early phase of septic Shock was associated with a lower rate of 28-days mortality and an improval of the neurological outcome.

\section{Reference(s)}

1. 1. Levy MM. Intensive Care Med 2018 2. Seymour CW.JAMA 2016.

\section{2}

Use of novel leukocyte cell population data in a medical intensive care unit setting - a retrospective study from Croatia

S. Šundalićí; A. Vujaklija Brajković'; I. Košuta'; I. Rako²; D. Rogić²; R.

Radonić'; j. Babel'

'Department of Internal Medicine, University Hospital Centre Zagreb,

Zagreb, Croatia; ${ }^{2}$ Department of Laboratory Diagnostics, University Hospital Centre Zagreb, Zagreb, Croatia

Correspondence: S. Šundalić

Intensive Care Medicine Experimental 2021, 9(1): 000432

Introduction. Sepsis is an important public health concern. According to the analysis of the Global Burden of Disease Study in 2017 20\% of all global deaths were sepsis-related (1). Sepsis is defined as lifethreatening organ dysfunction due to dysregulated host response to infection according to the Sepsis-3 criteria (2). Early diagnosis is key in the management of septic patients. Numerous biomarkers have been tested for early sepsis diagnosis and risk stratification. Technology advancements allow us to broaden the search to a panel of leukocyte cell population data (CPD), additional to the usual complete blood count, which could be a cost-effective option in sepsis management (3).

Objectives. To determine the use of leukocyte CPD in differentiating patients with sepsis, septic shock (Sepsis-3 definition) (2), and critically ill patients without infection. Also, we aimed to examine whether initial CPD values can predict intensive care unit (ICU) survival.

Methods. The study included patients hospitalized from 1 July 2018 till 28 February 2019 in the medical ICU at the University Hospital Centre Zagreb, Croatia. A complete blood count including CPD (Immature Granulocyte, IG\%, Total reactive lymphocytes, RE-LYMP, Antibody-synthesizing lymphocytes, AS-LYMP, Cytoplasmic granulation of neutrophils, NEUT-GI, Reactivity of neutrophils, NEUT-RI), C-reactive protein, and procalcitonin was measured. The CPD analysis was performed on the Sysmex XN-3000 hematologic analyzer. Statistical analysis was performed using SPSS for Windows version 23.0 (SPSS Inc, Chicago, IL, USA). Non-parametric tests were used for variables with the nonGaussian distribution. The level of statistical significance was chosen to be 0,05 .

Results. The study included 123 patients out of whom 48 had either sepsis or septic shock, defined as infection. Leukocyte CPD values were compared between patients with and without infection, with differences found in the IG $(p<0.001)$, AS-LYMP $(p<0.001)$ and NEUTRI $(p<0.001)$ values (Table 1$)$. The leukocyte CPD was unable to differentiate between sepsis and septic shock, with NEUT-RI values approaching significance $(p=0.07)$. Furthermore, IG count differentiated Gram-positive and Gram-negative sepsis $(p=0.009)$ and differed according to the infection site $(p=0.003)$. Logistic regression showed no significance in ICU survival for the tested CPD parameters, with NEUT-GI and NEUT-RI close to significance $(p=0.069 ; p=0.083)$.

Table 1. Descriptive statistics in the two patient groups.

\begin{tabular}{|c|c|c|c|}
\hline \multirow[t]{2}{*}{ Parameter } & \multirow{2}{*}{$\begin{array}{l}\text { Without infection } \\
\mathrm{n}=75 \\
\text { Median } \pm \text { IQR }\end{array}$} & \multirow{2}{*}{$\begin{array}{l}\text { Infection } \\
\mathrm{n}=48 \\
\text { Median } \pm \text { IQR }\end{array}$} & \multirow[b]{2}{*}{$p$} \\
\hline & & & \\
\hline Leukocytes (× 109/L) & $11.07 \pm 4.74$ & $16.55 \pm 16.55$ & NS \\
\hline IG (x 109/L) & $0.05 \pm 0.07$ & $0.19 \pm 0.87$ & $<0.001$ \\
\hline RE-LYMP (× 109/L) & $0.06 \pm 0$ & $0.06 \pm 0$ & NS \\
\hline AS-LYMP (x 109/L) & $0.00 \pm 0$ & $0.00 \pm 0$ & $<0.001$ \\
\hline NEUT-GI $(S I)^{*}$ & $151.2 \pm 6$ & $152.6 \pm 9$ & NS \\
\hline NEUT-RI (FI) * & $44.2 \pm 5$ & $56.2 \pm 17$ & $<0.001$ \\
\hline CRP (mg/L) & $3.3 \pm 8$ & $188.0 \pm 227$ & NS \\
\hline РCТ $(\mu \mathrm{g} / \mathrm{L})$ & NA & $8.0 \pm 49$ & \\
\hline
\end{tabular}

Conclusion. A significant difference in CPD values in critically ill patients with and without infection was shown. Even though no difference was observed in leukocyte CPD between patients with sepsis and septic shock, the IG count might be used to differentiate the source of infection and point toward Gram-negative sepsis. Furthermore, this pilot study might indicate an important role of the NEUT-GI and NEUT-RI values in predicting ICU survival for which further research is warranted.

\section{Reference(s)}

1. 1. Rudd KE, Johnson SC, Agesa KM, Shackelford KA, Tsoi D, Kievlan DR, et al. Global, regional, and national sepsis incidence and mortality, 1990-2017: analysis for the Global Burden of Disease Study. Lancet. 2020 Jan 18;395(10,219):200-11. 2. Singer M, Deutschman CS, Seymour CW, Shankar-Hari M, Annane D, Bauer M et al. The Third International Consensus Definitions for Sepsis and Septic Shock (Sepsis-3). JAMA. 2016 Feb 23;315(8):801-10. 3. Urrechaga E. Reviewing the value of leukocytes cell population data (CPD) in the management of sepsis. Ann Transl Med. 2020 Aug;8(15):953.

2. None. 
000439

Targeted Antithrombin Use for Sepsis with Coagulopathy: A Machine Learning-Based Approach to Estimate Heterogeneous Treatment Effects

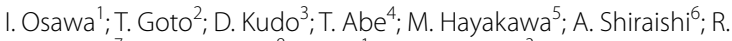

Uchimido $^{7}$; K. Yamakawa ${ }^{8}$; K. Doi ${ }^{1}$; S. Kushimoto ${ }^{3}$

${ }^{1}$ Department of Emergency and Critical Care Medicine, The University of Tokyo Hospital, Bunkyo City, Japan; ${ }^{2}$ Department of Clinical Epidemiology and Health Economics, The University of Tokyo, Bunkyo City, Japan; ${ }^{3}$ Division of Emergency and Critical Care Medicine, Tohoku University Graduate School of Medicine, Sendai, Japan; ${ }^{4}$ Department of Emergency and Critical Care Medicine, Tsukuba Memorial Hospital, Tsukuba, Japan; ${ }^{5}$ Department of Emergency Medicine, Hokkaido University Hospital, Sapporo, Japan; ${ }^{6}$ Emergency and Trauma Center, Kameda Medical Center, Kamogawa, Japan; ${ }^{7}$ Department of Intensive Care Medicine, Tokyo Medical and Dental University, Bunkyo City, Japan; ${ }^{8}$ Department of Emergency Medicine, Osaka Medical and Pharmaceutical University, Takatsuki, Japan Correspondence: I. Osawa

Intensive Care Medicine Experimental 2021, 9(1): 000439

Introduction. Although antithrombin (AT) was not shown to reduce mortality in patients with severe sepsis in a phase III randomized trial [1], several studies have since reported that AT may improve mortality in patients with septic coagulopathy [2-5]. Given that these studies only estimated the treatment effects of AT in certain prespecified patient groups, little is known about which patient subgroups are optimal treatment targets of AT. Therefore, we aimed to investigate the subpopulations of sepsis patients who should be targeted for AT, using a machine learning-based approach.

Methods. This was a multicenter registry-based retrospective cohort study to explore patient subgroups with high treatment effects of AT on 28-day mortality. We used two multicenter registries in Japan: (1) 3195 patients (aged $\geq 16$ years) with severe sepsis and septic shock based on the International Sepsis Definitions Conference criteria in 42 ICUs [6] (a derivation cohort) and (2) 1184 patients (aged $\geq 16$ years) with severe sepsis based on the Sepsis-2 criteria in 59 ICUs [7] (a validation cohort). The derivation cohort was split into a training and test set (50\% and $50 \%)$. We applied the causal forest approach-a machine learning-based approach to estimate heterogeneous treatment effects $[8,9]$ - to the training set with adjusting for potential confounders. In the test set, by applying the developed causal forest model, we identified subpopulations of those who are potential targets of AT. To confirm the consistency of our findings, we evaluated the treatment effects of AT in the obtained subpopulations of the validation cohort using inverse probability of treatment weighting.

Results. By applying the causal forest model developed using the training set to the test set, we found that $72 \%$ of sepsis patients in the test set had positive estimated treatment effects of AT (shaded area of the histogram in Figure 1). Patients with positive estimated treatment effects were more likely to have severe coagulopathy (e.g., higher APACHE2 and SOFA scores, a higher D-dimer level, and a lower AT activity) than those with negative estimated treatment effects. Patients with higher DIC scores (e.g., ISTH DIC score) were likely to have higher estimated treatment effects (Fig. 2). According to the heterogeneity in AT's treatment effects, we heuristically selected the potential treatment targets of AT as patients with ISTH DIC score $\geq 6$. Among the potential treatment targets (DIC score $\geq 6 ; 5.3 \%$ of the cohort) in the validation cohort, we found that AT use was significantly associated with a lower 28-day mortality (adjusted risk difference $-43.8 \%$; $95 \% \mathrm{Cl}$ -69.1 to $-25.3 \%$ ) after adjustment for potential confounders.

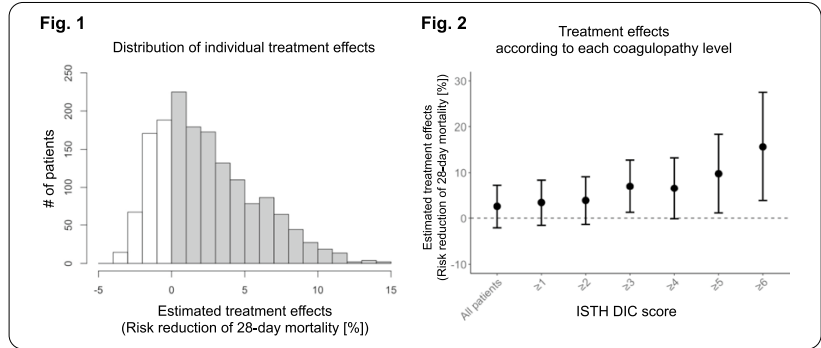

Conclusion. We found heterogeneity in the treatment effects of AT for sepsis with coagulopathy. Our findings suggest that certain subpopulations of patients with septic coagulopathy (e.g., ISTH DIC score $\geq 6$ ) may benefit considerably from targeted AT use.

\section{Reference(s)}

1. 2. Wiedermann CJ, Hoffmann JN, Juers M, et al. High-dose antithrombin III in the treatment of severe sepsis in patients with a high risk of death: efficacy and safety. Crit Care Med 2006; 34: 285-292

2. 3. Hoffmann JN, Wiedermann CJ, Juers M, et al. Benefit/risk profile of high-dose antithrombin in patients with severe sepsis treated with and without concomitant heparin. Thromb Haemost 2006; 95: 850-856

3. 4. Gando S, Saitoh D, Ishikura H, et al. A randomized, controlled, multicenter trial of the effects of antithrombin on disseminated intravascular coagulation in patients with sepsis. Crit Care 2013; 17: R297

4. 5. Hayakawa M, Kudo D, Saito S, et al. Antithrombin Supplementation and Mortality in Sepsis-Induced Disseminated Intravascular Coagulation: A Multicenter Retrospective Observational Study. Shock 2016; 46: 623-631

5. 6. Hayakawa M, Yamakawa K, Saito S, et al. Nationwide registry of sepsis patients in Japan focused on disseminated intravascular coagulation 2011-2013. Sci Data 2018; 5: 180243

6. 7. Abe T, Ogura H, Shiraishi A, et al. Characteristics, management, and in-hospital mortality among patients with severe sepsis in intensive care units in Japan: the FORECAST study. Crit Care 2018; 22: 322

7. 8. Wager S, Athey S. Estimation and Inference of Heterogeneous Treatment Effects using Random Forests. Journal of the American Statistical Association 2018; 113: 1228-1242

8. 9. Athey S, and Wager S. Estimating Treatment Effects with Causal ForestsAn Application. 20 Feb 2019, arXiv: 1902.07049

9. D.K., M.H., and S.K. received personal fees from Asahi Kasei Pharma Corporation. The other authors have no conflicts of interest to declare.

10. 1. Warren BL, Eid A, Singer P, et al. Caring for the critically ill patient. Highdose antithrombin III in severe sepsis: a randomized controlled trial. JAMA 2001; 286: 1869-1878 
000446

Use of biomarkers to improve 28-day mortality stratification in patients with SOFA score $<\mathbf{6}$

L. Chiscano ${ }^{1}$; JC. Ruiz-Rodríguez ; J. Baldirà2; A. Ruiz-Sanmartin ${ }^{1}$; R. Ferrer Roca $^{3}$; I. Comas ${ }^{4}$; J. Gonzalez ${ }^{5}$; R. Ferrer Roca ${ }^{1}$

${ }^{1}$ Intensive Care Department, Vall d'Hebron University Hospital, Barcelona, Spain; ${ }^{2}$ Intensive Care Department, Hospital de la Santa Creu i Sant Pau, Barcelona, Spain; ${ }^{3}$ Clinical Biochemistry Service, Hospital Vall d'Hebron, Barcelona, Spain; ${ }^{4}$ Clinical Analysis Service, Vall d'Hebron University Hospital, Barcelona, Spain; ${ }^{5}$ Microbiology Department, Vall d'Hebron University Hospital, Barcelona, Spain

Correspondence: L. Chiscano

Intensive Care Medicine Experimental 2021, 9(1): 000446

Introduction. The use of the SOFA score to stratify the risk of multiorgan failure and thus patient mortality is widely validated. At initial low values (SOFA score $<6$ ), the mortality risk is low although we can find patients in the subgroup of sepsis and septic shock with high mortality.

Objectives. The use of biomarkers like Midregional proadrenomedullin (MR-proADM), Procalcitonin (PCT), C-reactive protein (CRP) and lactate could help to improve 28-day mortality stratification in patients with SOFA score $<6$.

Methods. Retrospective study of patients activated by the inhospital sepsis code (CSG) at Hospital Universitari Vall d'Hebron within the emergency department (ED), hospital wards and intensive care unit (ICU) with a diagnosis of sepsis or septic shock and initial SOFA score $<6$ from April 2016 to July 2018. Blood samples were obtained with the activation of sepsis code and were stored in the Sepsis Bank until their analysis. Area under receiver operating characteristic (AUROC) curves, logistic and Cox regression analysis were used to assess performance. Mean was expressed in SD and median in IQR. The study has been approved by the ethics committee (PR(AG)333/2016, PR(AG)336/2016, PR(AG)11/2016) and informed consent was signed by patient or representatives.

Results. 296 patients were included, 271 alive (91.5\%). 184 (62.2\%) were male and had a mean of 63.18 (16.41) years. $185(62.5 \%)$ had sepsis and 111 (37.5\%) septic shock. The median of ICU length of stay was 4 days (2-10) and median of hospital length of stay 14 days (6.2530.98). The median of MR-proADM in non survivors was $2.53 \mathrm{nmol} / \mathrm{L}$ (1.7-4.97), PCT $2.31 \mathrm{ng} / \mathrm{mL}(0.7-8.63)$ and SOFA 5 (4-6). The mean of Lactate was $3.77 \mathrm{mmol} / \mathrm{L}$ (2.24) and CRP $149.35 \mathrm{mg} / \mathrm{L}$ (483.9).

The predictive ability of mortality of MR-proADM was low, with an AUROC of $0.56(0.39-0.73)$. Lactate had the highest capacity with AUROC $0.66(0.53-0.80)$, CRP $0.41(0.26-0.56)$. In the bivariate study, the combination of MR-proADM and Lactate had an OR $(95 \% \mathrm{Cl})$ 1.15 (0.93-1.36) and CRP and Lactate OR (95\% Cl) $1.20(0.98-1.45]$ in mortality. The combination of PCT and lactate had OR $(95 \% \mathrm{CI}) 1.15$ (0.97-1.36)

The predictive ability of MR-proADM in mortality within patients with community-acquired infection was 0.73 (0.61-0.84). The AUROC of lactate was $0.70(0.54-0.86)$ and SOFA $0.72(0.58-0.87)$. In the univariate study the SOFA OR $(95 \% \mathrm{Cl})$ was 2.04 (1.06-3.95). The bivariate study showed that the association of CRP and lactate had an OR (95\% Cl) 1.23 (0.90-1.68) for mortality. MR-proADM and Lactate OR $(95 \% \mathrm{Cl})$ 1.17 (0.87-1.57) and PCT and Lactate OR (95\% CI) $1.20(0.90-1.61)$. The other biomarkers' predictive performance was low.

Conclusion. The results suggest that community-acquired sepsis with initial SOFA score $<6$, MR-proADM could help to identify patients at risk of 28-day mortality. Therefore, initial measurement of these biomarkers could facilitate early treatment strategies after activation of a hospital sepsis code in patients with SOFA $<6$ and sepsis or septic shock.

\section{9}

\section{COVID 19: predictors of mortality in the Intensive Care Unit}

A. Alvarez ${ }^{1}$ : R Torcuato ${ }^{1}$ : M. Salgado ${ }^{1}$ : A Alonso ${ }^{1}$. I. Fernández ${ }^{2}$; A Ubeda ${ }^{1}$ Intensive Care Unit, Hospital Point Europe, Algeciras, Spain; ${ }^{2}$ Intensive Care Unit, Hospital Regional Universitario de Málaga (Carlos de Haya),
Málaga, Spain

Correspondence: A. Ubeda

Intensive Care Medicine Experimental 2021, 9(1): 000529

Introduction. In the pandemic situation due to COVID 19 in which we find ourselves, it is necessary to review and analyze all the information generated in the ICUs, in order to draw conclusions that may improve the management of these patients.

Objectives. To analyze factors related to mortality in patients admitted to intensive care units (ICU) with acute respiratory failure due to severe pneumonia caused by Covid19 infection.

Methods. Retrospective descriptive analysis was performed using a prospective cohort obtained from a 20-beds ICU collected between March 2020 and January 2021. Demographic outcomes, comorbidities, prognosis scores (APACHE and SOFA), treatment received, invasive mechanical ventilation (IMV) and ventilator parameters, acute kidney injury (AKI) and the need of any renal replacement therapy (RRT) Statistical analysis was performed: continuous outcomes (mean and standard deviation or median and interquartile range), categorical outcomes (percentages and frequencies). Comparison: Chi-square test (percentages) and T-student test (mean). Multiple logistic regression. Statistical significance was set at $p$-value $<0.05$.

Results. 74 patients were included, 56 males (75.7\%). Average age 65.6 ( \pm 12.4$)$. Prognosis scores: APACHE II $12.9( \pm 6.2)$, SOFA $4.2( \pm 2.3)$ Breathing support: IMV 29.7\%, non-invasive mechanical ventilation (NIMV) 43.2\%, high-flow nasal cannula oxygen (HFNC) 13.5\%. Mortality $63.5 \%$. Survivors vs. dead were compared: age (57.1 $[ \pm 12.4]$ vs. $70.6[ \pm 9.5], p<0.001)$, APACHE II $(9.3[ \pm 4.6]$ vs. $15.0[ \pm 6.1], p<0.001)$ SOFA (3.4 $[ \pm 2.2]$ vs. $4.6[ \pm 2.2], p=0.029)$. Previous treatment with angiotensin receptor blockers (ARBs) $(11.1 \%$ vs. $34.0 \%, p=0.030)$ Comorbidities: Chronic obstructive pulmonary disease (COPD) (3.7\% vs. $25.5 \%, p=0.024)$, high blood pressure (HBP) $(33.3 \%$ vs. $57.4 \%$ $\mathrm{p}=0.046)$. Mechanical ventilation during ICU's stay (85.2\% vs. $97.9 \%$ $p=0.056)$. Prone position (18.5\% vs. $72.3 \%, p<0.001)$. Blood test at ICU's admission: urea (47.1 [ \pm 28.8 ] vs. 64.1 [ \pm 32.6$], p=0.026)$. Blood test at 48 h: lactate dehydrogenase (LDH) (448.6 [ \pm 190.5$]$ vs. 747.8 $[ \pm 523.9], p=0.006)$, urea (55.4 [ \pm 23.1$]$ vs. $78.3[ \pm 48.7], p=0.008)$ protein-C reactive (PCR) (75.1 $[ \pm 74.5]$ vs. $158.9[ \pm 142.7], p=0.006)$. Procalcitonine $(P C T)(0.19[ \pm 0.42]$ vs. $0.72[ \pm 1.24], p=0.016)$. Respiratory status at $24 \mathrm{~h}: \mathrm{SpO} 2(97.8[ \pm 2.1]$ vs. $95.8[ \pm 3.1], \mathrm{p}=0.004)$. AKI (3.7\% vs. $40.4 \%, p=0.001)$. AKI AKIN III (3.7\% vs $29.8 \%, p=0.008)$. RRT (3.7\% vs. $34.0 \%, p=0.003$ ). Multiple logistic regression: age (OR 1.161 [IC 95\%, 1.068-1.262, p<0.001]), prone position (OR 18.597 [IC 95\%, 3.273-105.660, $\mathrm{p}=0.001]), \mathrm{LDH}$ at $48 \mathrm{~h}$ (OR 1.005 [IC 95\%, 1.001-1.009, $\mathrm{p}=0.007])$. AUROC: 0.944 (IC95\% 0.896-0.992).

Conclusion. In our ICU the mortality due to severe pneumonia related to COVID 19 infection was $63.5 \%$. Age, the requirement of prone position and higher $\mathrm{LDH}$ result at $48 \mathrm{~h}$ were associated to higher mortality.

\section{2}

Hyperdynamic left ventricular ejection fraction in septic ICU patients

M. Chotalia ${ }^{1}$; M. Ali ${ }^{2}$; M. Bangash,2 ; D. Parekh, ${ }^{3} ;$ J. Patel, ${ }^{2}$

${ }^{1}$ Institute of Inflammation and Ageing, University of Birmingham,

Birmingham, United Kingdom; ${ }^{2}$ Critical care, Queen Elizabeth Hospital Birmingham, Birmingham, United Kingdom; ${ }^{3}$ ttu, Queen Elizabeth Hospital

Birmingham, Birmingham, United Kingdom

Correspondence: M. Chotalia

Intensive Care Medicine Experimental 2021, 9(1): 000542

Introduction. The cause and prognosis of hyperdynamic left ventricular ejection fraction (HDLVEF) in critically ill patients with sepsis is unknown [1]

Methods. This was a retrospective cohort study of patients that received a transthoracic echocardiogram (TTE) within seven days of sepsis onset whilst admitted to the Queen Elizabeth Hospital Birmingham, UK between April 2016 and December 2019. The 90-day mortality rates of normal (55-70\%), depressed $(<55 \%)$ and hyperdynamic LVEF (> 70\%) were compared. The 90-day mortality rates of LVEF cohorts with varying vasoactive agent doses and choice were also 
compared. Multivariate logistic regression analysis was performed to determine the association of LVEF phenotypes with mortality and the association of clinical parameters with LVEF phenotypes.

Results. 1,014 patients met inclusion criteria and were aged 62 years (IQR 47-72), with mostly respiratory infections ( $n=557 ; 54.9 \%)$. Ninety-day mortality was $32.1 \%(n=325)$. Patients with HDLVEF had a higher mortality than depressed and normal LVEF cohorts $(58.9 \%$ $(n=103)$ vs. $34.0 \%(n=55)$ vs. $24.7 \%(n=167) ; p<0.0001$, respectively). After multivariate analysis, HDLVEF independently associated with mortality (OR 3.90 (2.09-7.40), whereas depressed LVEF did not (OR $0.62(0.28$ - 1.37)). Systemic vascular resistance (SVR) inversely associated with HDLVEF (OR 0.89 (95\% Cl 0.78-0.95), whereas age, frailty and ischaemic heart disease associated with depressed LVEF. Vasoactive agent choice and dose and systemic vascular resistance altered the mortality rate associated with LVEF cohorts (Figure 1).

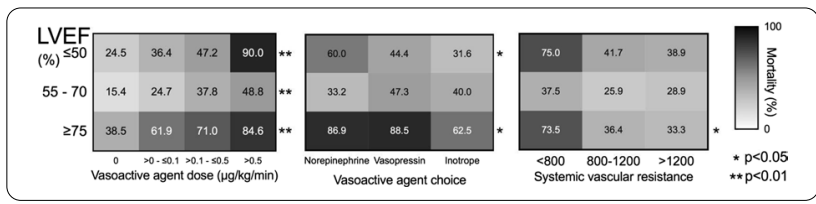

Conclusion. HDLVEF independently associated with mortality from sepsis whereas depressed LVEF did not. HDLVEF may reflect profound vasoplegia, whilst frailty and cardiovascular comorbidity may contribute to depressed LVEF in patients with sepsis.

\section{Reference(s)}

1. [1] Paonessa JR, Brennan T, Pimentel M, Steinhaus D, Feng M, Celi LA. Hyperdynamic left ventricular ejection fraction in the intensive care unit. Crit Care. 2015:19:288

2. None

\section{2}

Lipotoxicity is associated with worse outcomes in ICU patients with COVID-19

R. Cartin-Ceba ${ }^{1}$; B. Khatua, ${ }^{2}$; S. Trivedi, ${ }^{2}$; R. Smith, ${ }^{3}$;V. Singh, ${ }^{2}$

${ }^{1}$ Critical Care, Mayo Clinic Hospital, Phoenix, United States of America; ${ }^{2}$ Department of medicine, Mayo Clinic Hospital, Phoenix, United States of America; ${ }^{3}$ Mayo Clinic Alix School of Medicine, Mayo Clinic Building Scottsdale, Scottsdale, United States of America

Correspondence: R. Cartin-Ceba

Intensive Care Medicine Experimental 2021, 9(1): 000552

Introduction. Lipotoxicity refers to the local or systemic deleterious effects caused by increased levels of fatty acids (FAs), particularly unsaturated fatty acids (UFA) (1). Lipotoxicity has been well described in pancreatitis and recent growing literature provides evidence of the potential association of lipotoxicity and worse outcomes in COVID-19 patients (2). Approximately $30-40 \%$ of COVID-19 autopsies have shown pancreatitis and fat necrosis (3); and multiple observational studies have identified elevated lipase levels in COVID-19 patients without clinical evidence of pancreatitis (4). Furthermore, obesity is a well-known risk factor for both severe COVID19 and lipotoxic severe pancreatitis (5). While most UFAs exist conjugated to albumin, a small proportion of these are unbound, are biologically active, and have been shown to induce cell injury through mitochondrial toxicity (1).

Objectives. To determine the fatty acid profile and their biological activity in sera of COVID-19 patients with different disease severity as compared to ICU patients without COVID-19.

Methods. Prospective observational cohort study was conducted in a 30-bed multidisciplinary ICU during 2019 and 2020. Consecutive critically ill patients $\geq 18$ years admitted to the ICU during the study period were screened for inclusion in the study. Exclusion criteria: DNR/DNI and comfort care patients, ICU readmissions, and patients who had not agreed to the use of their medical records for research. Blood samples were obtained in the first $12 \mathrm{~h}$ after ICU admission. Serum fatty acids were analyzed by gas chromatography mass spectrometry and cytokines profile was measured using Luminex. All data are summarized as median [interquartile range (IQR)] or percentages. A p value $\leqslant 0.05$ was considered statistically significant.

Results. A total of 88 patients were included in the study, 39 of whom developed severe COVID-19 pneumonia. Although the median age in the COVID-19 group was lower: 55 (44-64) versus 67 (57-75), $p<0.0001$; the severity of disease based on APACHE IV scores was similar between the two groups: 52 (39-63) in the nonCOVID group versus 51 (34-69) in the COVID-19 group, $p=0.57$. The COVID-19 group also had a higher BMI: $30.2 \mathrm{~kg} / \mathrm{m} 2$ (26.8-35.8) versus 27.8 (25.1-32.5), $p=0.04$. Lipase levels without clinical evidence of pancreatitis were higher in the COVID group: $73 \mathrm{U} / \mathrm{L}$ (42-122) versus $21(11-46) p<0.0001$. Unbound FAs were higher in the COVID19 group: 4.8 uM (2.4-8.4) versus 3.4 (2.5-4.7), $\mathrm{p}=0.04$. From all FAs evaluated, only the UFA Linoleic acid (LA; $C 18: 2)$ was found to be significantly higher in the COVID-19 group: 113 uM (76-149) versus 83 (64-109), $p=0.002$. Pro-inflammatory cytokines were significantly higher in the COVID-19 group including IL-6 pg/mL (360 [67-641] versus 144 [33-357], $\mathrm{p}=0.006) ; \mathrm{IL}-8 \mathrm{pg} / \mathrm{mL}$ (42.2 [25.4-59.4] versus 26.5 [17.5-42.7], $p=0.01)$; and TNFa $\mathrm{pg} / \mathrm{mL}$ (40.4 [28.3-58.4] versus 13 [5.5-23.4], $\mathrm{p}<0.0001)$. Hospital and ICU outcomes were worse in the COVID-19 patients as compared to the non-COVID group, including a higher percentage of multiorgan failure development: $59 \%$ versus $35 \%, p=0.02$; higher hospital length of stay: 23 days (11-42) versus 7 (5-13), $p<0.0001$; and higher number of VTE events: $26 \%$ versus $2 \%, p=0.002$. Interventions such as renal replacement therapy and ECMO utilization were also seen more frequently in the COVID-19 group.

Conclusion. Our study showed that total unbound FAs and the UFA Linoleic acid were significantly increased in the sera of patients admitted to the ICU with severe COVID-19 as compared to ICU patients with no COVID-19. Patients with COVID-19 also presented higher levels of lipase and higher levels of pro-inflammatory cytokines as compared to non-COVID patients. Despite similar disease severity as evidenced by a similar APACHE IV score, COVID-19 patients presented worse outcomes including multiorgan failure development, longer hospital stay, and more venous thromboembolic events. The association of lipotoxicity in severe COVID-19 patients observed in this study, which mimics what is observed in pancreatitis, merits further investigation as a possible mechanistic process behind worse outcomes observed in these patients are compared to other similarly ill ICU patients.

\section{Reference(s)}

1. 5-Simonnet A, Chetboun M, Poissy J, et al. High Prevalence of Obesity in Severe Acute Respiratory Syndrome Coronavirus-2 (SARS-CoV-2) Requiring Invasive Mechanical Ventilation. Obesity (Silver Spring). 2020:28(7):1195-1199.

2. 4- Ahmed A, Fisher JC, Pochapin MB, et al. Hyperlipasemia in absence of acute pancreatitis is associated with elevated D-dimer and adverse outcomes in COVID 19 disease. Pancreatology. 2021.

3. 3-Hanley B, Naresh KN, Roufosse C, et al. Histopathological findings and viral tropism in UK patients with severe fatal COVID-19: a post-mortem study. Lancet Microbe. 2020;1 (6):e245-e253.

4. 2- El-Kurdi B, Khatua B, Rood C, et al. Mortality From Coronavirus Disease 2019 Increases With Unsaturated Fat and May Be Reduced by Early Calcium and Albumin Supplementation. Gastroenterology. 2020;159(3):1015-1018 e1014

5. 1-Navina S, Acharya C, DeLany JP, et al. Lipotoxicity causes multisystem organ failure and exacerbates acute pancreatitis in obesity. Sci Transl Med. 2011;3(107):107ra110 


\section{9}

\section{Bacterial co-infection and overinfection in patients} with SARS-COV2 pneumonia (COVID-19)

N. Rodrigo Castroviejo ; R. Beltrán Bernáldez'; G. Ferrigno²; C. Sanmartino Gonzalez ; M. Berenguer Rodriguez'; D. Monge Donaire ; LL. Beltran Martínez'; S. Cortés Díaz'; A. Marcos Gutiérrez' ; C. Tarancón Maján ${ }^{1}$ Intensive Care, Hospital Virgen De La Concha, Zamora, Spain; ${ }^{2}$ ntensive Care Unit, Hospital Virgen De La Concha, Zamora, Spain

Correspondence: $\mathrm{G}$. Ferrigno

Intensive Care Medicine Experimental 2021, 9(1): 000559

Introduction. Bacterial co-pathogens are commonly identified in viral respiratory infections and are important causes of morbidity and mortality. The prevalence of bacterial infection in patients infected with SARS-CoV-2 is not well understood (1)(2).

Objectives. To determine and evaluate the incidence of bacterial coinfection and/or superinfection with a diagnosis of sepsis in patients admitted to the Intensive Care Unit (ICU) for SARS COV2 Pneumonia (COVID19).

Methods. Retrospective study including patients admitted to the ICU with a diagnosis of SARS-COV-2 Pneumonia (COVID19). Data were collected during the pandemic period in our center from 08/27/2020 to $12 / 15 / 2020$. Demographic and epidemiological data and severity scales were analyzed. Statistical analysis of incidence is performed.

Results. 59 patients with the following baseline characteristics were included. The median age of the population included in the database was 64.4 (33-81) years, 81\% male, weight 80.6 (72.6-105.2) kg height $169.3(164.9-170.7) \mathrm{cm}$, with high severity as shown by a mean APACHE II score of 14.13 (11-18) points and SOFA score of 5 (3-8) points. The vast majority of the sample, $94.5 \%$ received antibiotic treatment with a mean duration of 7.4 days $( \pm 8.56 \mathrm{~d})$, however, diagnosis of sepsis with identified microbiological isolation only occurred in $19 \%$ of the sample. From the evaluation of the isolates, $56 \%$ presented only respiratory infection, being bacterial in $37 \%$ and mixed in $10 \%$ (positive fungical isolation); $12.5 \%$ presented only bacteremia; $6.2 \%$ presented simultaneous respiratory infection and bacteremia and $12.5 \%$ of the sample presented other isolates. In order of frequency, the germs isolated were: $43 \%$ Pseudomonas aeruginosa, of which $28.5 \%$ were MR, another $43 \%$ were S. maltophilia, 25\% Candida spp. species, equivalent in frequency to $19 \%$ were K. pneumoniae, S. epidermidis and E. coli. Less frequent isolates were E. faecalis, P. mirabilis, E. cloacae in $12.5 \%, 6.2 \%$ and $6.2 \%$ respectively. Of the total sample, intra-ICU mortality was $27 \%$, of those with sepsis diagnosis was $54.5 \%$.

Conclusion. Microbial overinfection is a serious complication in patients infected by SARS-COV-2 which leads to increased mortality and ICU stay (3). The most frequent superinfection is bacterial respiratory involvement. Bloodstream infections are a common occurrence and are frequently sustained by enterococci. In this context, measures to reduce bacterial infection are fundamental in order to provide appropriate critical care.

\section{Reference(s)}

1. 3. Bardi, T., Pintado, V., Gomez-Rojo, M. et al. Nosocomial infections associated to COVID-19 in the intensive care unit: clinical characteristics and outcome. Eur J Clin Microbiol Infect Dis 40, 495-502 (2021). https://doi. org/10.1007/s10096-020-04142-w

2. 2. Klein E.Y.,Monteforte B.,Gupta A.Jiang W., May L., Hsieh Y.-H.et al. The frequency of influenza and bacterial coinfection: a systematic review and meta-analysis.Influenza Other Respir Viruses. 2016; 10: 394-403

3. 1. Cox MJ, Loman N, Bogaert D, O'Grady J. Co-infections: potentially lethal and unexplored in COVID-19. Lancet Microbe 2020.S2666524720300094.

\section{4}

\section{COVID19 vs. Non-COVID19: clinical and epidemiological}

\section{differences in ICU}

M. Salgado '; A. Alvarez'; R. Torcuato'; A. Alonso'; I. Fernández²; A. Ubeda ${ }^{1}$ Intensive Care Unit, Hospital Point Europe, Algeciras, Spain; ${ }^{2}$ Intensive Care Unit, Hospital Regional Universitario de Málaga (Carlos de Haya),
Málaga, Spain

Correspondence: A. Ubeda

Intensive Care Medicine Experimental 2021, 9(1): 000574

Introduction. In the pandemic situation due to COVID 19 in which we find ourselves, it is necessary to review and analyze all the information generated in the ICUs, in order to draw conclusions that may improve the management of these patients.

Objectives. Analyze the differences observed between patients admitted in ICU with a diagnosis of Covid-19 and those without Covid diagnosis from the beginning of the pandemic until January 2021

Methods. Descriptive retrospective analysis on a prospective cohort performed in a 20-bed ICU between March 2020 and January 2021. 2 groups were compared: patients admitted with COVID-19 vs. admitted with non-COVID pathologies. Demographic variables, comorbidities, severity scores, received treatment, IMV, risk factors, infections in ICU, antibiotherapy, days in ICU, days in hospital and mortality. Statistical analysis: categorical variables (frequencies and percentages) and numerical variables (mean and standard deviation or median and interquartile range). Comparisons: X2 test (percentages), Student's t (means), and U-Mann-Whittney (medians). Statistical significance with $\mathrm{p}<0.05$.

Results. 412 patients were included. COVID-19 $(n=98)$ vs. non-COVID $(\mathrm{n}=314)$. Age $>80$ years: $27.6 \%$ vs $17.8 \%(\mathrm{p}=0.036)$. SAPS II $(28.6$ $[ \pm 11.3]$ vs. $34.6[ \pm 18.2], p<0.001)$. APACHE II $(12.8[ \pm 8.4]$ vs. 14.9 $[ \pm 8.7], p=0.040)$. Origin $(p<0.001)$ : ward $87.8 \%$ vs. $35.0 \%$, community $12.2 \%$ vs. $61.5 \%$. antibiotic therapy $48 \mathrm{~h}$ before admitted in ICU $(63.3 \%$ vs. $34.1 \%, p<0.001)$ RRT $(18.0 \%$ vs. $8.9 \%, p=0.016)$, Parenteral Nutrition $(4.4 \%$ vs. $13.7 \%, p=0.016)$, neutropenia $(1.1 \%$ vs. $6.7 \%, p=0.037)$ CVC ( $80.6 \%$ vs. $64.0 \%, p=0.002)$, IMV $(73.5 \%$ vs. $42.5 \%, p<0.001)$, Urinary catheter (UC) $(94.9 \%$ vs. $79.0 \%, p<0.001)$, tracheostomy $(26.7 \%$ vs. $3.3 \%, p<0.001)$. IMV's days: $10(4 ; 20)$ vs. $2(1 ; 6), p<0.001 . C V C ' s$ days: $10(4 ; 20)$ vs. $2(0 ; 6), p<0.001)$, UC's days: $13(7 ; 23)$ vs. $4(2 ; 7)$, $\mathrm{p}<0.001$. Infections during ICU admission $(50 \%$ vs. $18 \%, \mathrm{p}<0.001)$ Length of stay (days): ICU $(13[6 ; 22]$ vs. $3[2 ; 6], p<0.001)$, hospital (19 $[13 ; 29]$ vs. $9[5 ; 20], p<0.001)$. First antibiotic $(p<0.001)$ : ceftriaxone ( $81.0 \%$ vs. $28.6 \%)$. First infection ( $<<0.001)$ : NAVM $(7.7 \%$ vs. $4.0 \%)$, BAC $(15.4 \%$ vs. $3.3 \%)$, secondary bacteriemia( $11.5 \%$ vs. $6.3 \%)$. Mortality (64.7\% vs. $31.5 \%, \mathrm{p}<0.001)$

Conclusion. Patients admitted to the ICU due to COVID19 come from the hospitalization ward and present initially lower APACHE II and SAPS II scores. Greater use of prior antibiotic therapy, MV, CVC, UC and RRT was observed in patients with COVID19. The global incidence of infections in ICU and the incidence of NAVM, BRC and secondary bacteremia was higher in patients with COVID19. The mortality of COVID19 patients was double than in non-COVID patients.

\section{6}

Predictive model of mortality in ICU during the COVID-19 pandemic

M. Salgado ; R. Torcuato'; A. Alvarez; ; A. Alonso ${ }^{1}$; I. Fernández²; A. Ubeda'

${ }^{1}$ Intensive Care Unit, Hospital Point Europe, Algeciras, Spain; ${ }^{2}$ Intensive Care Unit, Hospital Regional Universitario de Málaga (Carlos de Haya),

Málaga, Spain

Correspondence: A. Ubeda

Intensive Care Medicine Experimental 2021, 9(1): 000576

Introduction. In the pandemic situation due to COVID 19 in which we find ourselves, it is necessary to review and analyze all the information generated in the ICUs, in order to draw conclusions that may improve the management of these patients.

Objectives. To analyze the factors associated with mortality in patients admitted to the ICU during the COVID 19 pandemic year.

Methods. Retrospective descriptive study carried out between January 2020 and January 2021. Demographic variables, comorbidities, diagnosis on admission, risk factors, APACHE II, SAPS II, infections during ICU stay, microorganisms isolated, antibiotic therapy, presence of shock, days of stay in ICU and hospital and mortality. Statistical analysis: categorical variables (frequencies and percentages) and 
numerical (mean and standard deviation or median and interquartile range). Comparisons: $\mathrm{X} 2$ test (percentages), Student's test (means) and U-Mann-Whitney (medians). Multivariate logistic regression. Statistical significance with $\mathrm{p}<0.05$.

Results. 396 patients were included. Comparisons: survivors 243 (61.4\%) vs. deads 153 (38.6\%): age (61.1 $[ \pm 16.6]$ vs. $67.7[ \pm 12.7]$, $\mathrm{p}<0.001)$, APACHE II (12.5 $[ \pm 7.8]$ vs. $17.7[ \pm 9.3], p<0.001)$, SAPS ॥ $(29.3[ \pm 15.8]$ vs. $40.1[ \pm 17.5], \mathrm{p}<0.001)$, chronic kidney disease $(9.5 \%$ vs. $20.9 \%, p<0.001)$, immunosuppression $(4.9 \%$ vs. $12.4 \%, p=0.007)$, COPD $(8.2 \%$ vs. $18.3 \%, p=0.003)$. Diagnosis on admission $(p<0.001)$ : heart disease $(13.7 \%$ vs. $34.2 \%)$, sepsis $(14.4 \%$ vs. $24.8 \%)$, acute respiratory failure ( $15.6 \%$ vs. $37.9 \%)$, COVID $(12.3 \%$ vs. $35.9 \%)$. Antibiotic the previous $48 \mathrm{~h}(32.1 \%$ vs. $54.9 \%, \mathrm{p}<0.001)$, renal replacement techniques (RRT) $(5.3 \%$ vs. $19.3 \%, p<0.001)$, neutropenia ( $3.3 \%$ vs. $9.2 \%$, $\mathrm{p}=0.013), \mathrm{CVC}(54.3 \%$ vs. $88.2 \%, \mathrm{p}<0.001)$, MV $(30.9 \%$ vs. $77.8 \%$, $\mathrm{p}<0.001)$, urinary catheter (UC) $(72 \%$ vs. $98 \%, p<0.001)$, tracheostomy $(2.6 \%$ vs. $15 \%, p<0.001)$. CVC days $(1[0 ; 5]$ vs. $5[2 ; 13], p<0.001)$, MV days $(0[0 ; 2]$ vs. $4[1 ; 11], p<0.001)$, UC days $(3[1 ; 6]$ vs. $6[2 ; 14]$, $\mathrm{p}<0.001)$, stay in the ICU $(3[2 ; 6]$ vs. $6[2 ; 14], \mathrm{p}<0.001)$.

Multivariate analysis: age (OR 1.033, 95\% Cl [1.014-1.053], $\mathrm{p}<0.001)$ APACHE II (OR 1.037, 95\% Cl [1.003-1.071], $p=0.030)$, COPD (OR 2.351 [1.131-4.885], $\mathrm{p}=0.022)$, COVID-19 (OR 3.621, 95\% Cl [1.931-6.789], $\mathrm{p}<0.001$ ), RRT (OR 2.386, 95\% CI [1.062-5.361], $p=0.035$ ), neutropenia (OR 2.959, 95\% Cl [1.085-8.066], $\mathrm{p}=0.034), \mathrm{MV}(\mathrm{OR} 5.044,95 \% \mathrm{Cl}$ [2.946-8.638], $\mathrm{p}<0.001$ ). AUROC: $0.828,95 \% \mathrm{Cl}$ [0.787-0.869]. Conclusion. COVID infection, age, APACHE II score, COPD, neutropenia, use of TDE and MV during the ICU stay were independent predictors of mortality.

\section{8}

Comparing Prognostic Significance Of Lactate Vs Venous-Arterial Carbon-dioxide Difference In Septic Shock Patients

M. Kumar'; S. Saigal'; S. KUMAR ${ }^{3}$

${ }^{1}$ Anaesthesiaology and Critical Care, AlIMS Hospital, Bhopal, India; ${ }^{2}$ Anesthesiology and Critical Acre, AIIMS BHOPAL, BHOPAL, India; ${ }^{3}$ Anesthesiology and critical acre, Rajendra Institute Of Medical Science, Ranchi, Ranchi, India

\section{Correspondence: S. KUMAR}

Intensive Care Medicine Experimental 2021, 9(1): 000588

Introduction. Septic shock causes deranged micro-circulation. Macrocirculatory markers are ineffective in guiding resuscitation. Carbondioxide has high diffusibility, hence reflect the rapid changes in micro-circulation effectively. Hence we compared the effect of lactate and Venous-arterial carbon-dioxide difference in mortality of septic shock patients.

Methods. A prospective single-centered observational study was done after obtaining the ethical committee approval, including 50 septic shock patients. Duration-October 2018 to November 2019. Patients meeting inclusion criteria were observed for lactate levels and venous-arterial carbon-dioxide difference at $0,6,12,24,48 \mathrm{~h}$ and observed their effect on 28 day mortality. We also measured the ratio of $\mathrm{Cv}-\mathrm{aCO} 2 / \mathrm{Da}-\mathrm{vO} 2$ among survivors(S) and non survivors (NS).

Results. Study data analysis was done using SPSS software v23 and R software. Our 50 patients (40NS \& 10S) showed significant difference $(p<0.005)$ in median values of SOFA \& APACHE II SCORES. The median values and the change of Lactate and $\mathrm{CO} 2$ difference over time was insignificant. Using Ridgeline plot for the $\mathrm{Cv}-\mathrm{aCO} 2 / \mathrm{Da}-\mathrm{vO} 2$ values the trend for Survivors $>1$ and Non-Survivors $<1$ was obtained.

Conclusion. We found that $\mathrm{CO} 2$ gap was $\leq 6$ (normal) in our septic shock patients. We further analysed the ratio of $\mathrm{CO} 2$ production to oxygen consumption and found that this ratio is $<1$ in survivors whereas it is $>1$ in non-survivors. We emphasize that these parameters derived from oxygen or $\mathrm{CO} 2$, when considered individually, are not, but when taken together, can help us distinguish the severity of shock and intervene timely. Complementing these with Lactate can be a better resuscitation protocol.
000637

Are inflammatory markers useful as prognostic markers in the evolution of patients with severe respiratory infection by SARS-Cov2?

JF. Martínez Carmona ${ }^{1}$; C. Joya Montosa ${ }^{\text {; }}$ G. Cazorla Barranquero ${ }^{1}$

${ }^{1}$ Intensive care medicine, Hospital Regional Universitario de Málaga, Málaga, Spain

Correspondence: J.F. Martínez Carmona

Intensive Care Medicine Experimental 2021, 9(1): 000637

Introduction. The approach and management of respiratory infection by SARS-Cov2 is complex, since the pathophysiology of this new viral infection is not yet fully understood. In clinical management, various acute phase reactants have been used as evolutionary markers, within this group it is worth highlighting: D-Dimer, Ferritin Serum and C-Reactive Protein, together with IL-6.

Objectives. To determine the prognostic capacity of the following inflammatory markers: D-Dimer, Ferritin serum, C-Reactive Protein.

Methods. 28 patients admitted to the ICU for severe respiratory infection due to SARS-Cov2 were included. The following variables are collected: age, sex, personal history, need for MV, ICU stay, ICU mortality, duration of MV, IL-6 upon admission, daily record of D-Dimer, Ferritin serum and C-Reactive Protein.

Results. Average age 63.67 years $\pm 7.76 .85 .7 \%$ males. $92.9 \%$ HBP. 42.9\% DM, 57.1\% Obesity (BMI > 30). 21.4\% immunosupressed. $64.1 \%$ required VMI. Medium duration MV 13.5 days. Median ICU stay 12.5 days. Mortality $46.4 \%$. We did not observe significant differences in the variations of D-Dimer between survivors vs non-survivors. Survivors had less variability in both Ferritin serum and C-Reactive Protein during their ICU stay than non-survivors, however, the differences were not statistically significant.

Conclusion. In our sample, we observed variations in the evolution of the different acute phase reactants between surviving vs non-surviving patients, however, these differences were not significant.

\section{0}

Mean arterial pressure is a major determinant of Acute kidney injury in early phase of septic shock: A retrospective observational study

M. Benlabed'; S. Benlabed²; R. Gaudy³ ; S. Aissaoui'; S. Nedjari'; A. Ladjouze $^{6}$

${ }^{1}$ Anesthesiology and Intensivecare, Lille University, Lille, France; ${ }^{2}$ Saint pierre hospital, Université Libre de Bruxelles, Bruxelles, Belgium; ${ }^{3}$ Anesthesiology and intensivecare, University of Lille, Lille, France; ${ }^{4}$ Anesthesiology, Algiers university, Algiers, Algeria, Algeria; ${ }^{5}$ Anesthesiology, Algiers university, Alger Centre, Algeria, Algeria; ${ }^{6}$ Anesthesiology, Algiers University, Algiers, Algeria

Correspondence: $M$. Benlabed

Intensive Care Medicine Experimental 2021, 9(1): 000660

Introduction. Some authors observed that increased hypotension exposure in septic shock would be associated with increased organ failure and mortality. Hypotension is defined as Mean arterial pressure (MAP) $<65 \mathrm{mmHg}$ and few data observed the association between hypotension and acute kidney injury in critically ill patients.

Objectives. So we hypothesized that duration of hypotension or increased number episodes of hypotension during the first $48 \mathrm{~h}$ of septic shock would be associated with an increased risk for AKI.

Methods. We conducted a retrospective observational study enrolling 30 patients who presented septic shock at the emergency department of an university hospital from May 2018 to May 2019.

We collected, analysed and compared the data of 2 groups of 15 patients: a first group of survivors and a second group of non survivors. Patients' baseline characteristics and disease severity were well matched between groups.Mean arterial pressure and CVP were monitored invasively.We recorded and analysed in the 2 groups: mean MAP, MAP time $<65 \mathrm{mmHg}$ to quantify hypotension, blood lactate level, SOFA score day 1 and day4, serum creatinine, urine output $\mathrm{ml} /$ $\mathrm{kg} / \mathrm{h}$ and the incidence of acute kidney injury. 
Results. Stastical analysis used Mann whitney test and results expressed as mean +-std deviation.

We observed first that Mean MAP, during the first $48 \mathrm{~h}$ was decreased significantly in the group of non survivors compared to the group of survivors respectively $62.3+-4.86$ vs $72+-4.93$. $p<0.0001$.

MAP time $<65 \mathrm{mmHg}$ expressed in percentage of total time monitoring was significantly increased in the group of non survivors compared to survivors during the first $48 \mathrm{~h}$ respectively $46+-5 \%$ vs $10+-1 \%$ $\mathrm{p}<0.0001$.

Blood lactate was significantly more elevated in non survivors than in survivors respectively $3.73+-0.35$ vs $2.5+-0.25$. $p<0.0001$.

Acute kidney injury according KDIGO criteria was significanly more frequent in non survivors than in the survivors respectively $35 \% \mathrm{~V} 11 \%$

\begin{tabular}{llll}
\hline \multicolumn{4}{l}{ Hemodynamic parameters and Incidence of AKI } \\
\hline PARAMETERS & SURVIVORS & NON SURVIVORS & P \\
\hline 48 h MEAN MAP mm HG & $72+-4.93$ & $62.3+-4.86$ & $\mathrm{P}<0.0001$ \\
48hMAP TIME $<65 \mathrm{~mm}$ & $10+-1 \%$ & $46 \%+-5 \%$ & $\mathrm{P}<0.0001$ \\
Hg \% & & & \\
SOFA score Day 4 & $5.6+-0.63$ & $7.86+-1.12$ & $\mathrm{P}<0.0001$ \\
Lactates Day1 mmol/l & $2.5+-0.27$ & $3.73+-0.35$ & $\mathrm{P}<0001$ \\
Creatinine Day4 mg/l & $14.4+-1.99$ & $24+-2.79$ & $\mathrm{P}<0.0001$ \\
Incidence of AKI & $\mathbf{1 1} \%$ & $\mathbf{3 5 \%}$ & \\
\hline
\end{tabular}

Conclusion. Our study confirm the association between duration of hypotension and the incidence of AKI in patients with shock.

\section{Reference(s)}

1. Izawa et al. early phase cumulative hypotension and severe stage progression in oliguric acute kidney injury with and without sepsis: an observational study

2. M.Varpula et al. hemodynamic variables related to outcome. Intensive Care Med 2005.31:1066-1071

\section{9}

Diastolic Shock Index-validation of a new prognostic tool in septic shock patients

A. Alexandre'; J. Colaço'; P. Povoa ${ }^{2}$

${ }^{1}$ Intensive Care Medicine Department, Hospital da Luz Lisboa, Lisboa, Portugal; ${ }^{2}$ Unidade de cuidados intensivos polivalentes, Hospital de São Francisco Xavier, Lisboa, Portugal

Correspondence: A. Alexandre

Intensive Care Medicine Experimental 2021, 9(1): 000719

Introduction. In septic shock patients, hypotension/hypoperfusion may arise from multiple causes: vasodilation, absolute and relative hypovolemia, septic myocardial dysfunction, and microcirculatory dysfunction.[1] The severity of peripheral vasodilation is of particular interest because it affects the need, dosage and timing of vasopressors introduction during treatment. Recently, Ospina-Tascón et al. [2] proposed the use of a new marker of vasodilation severity - the Diastolic Shock Index (DSI). The DSI is the ratio between heart rate (HR) and diastolic arterial pressure (DAP) and is suggested to represent the impact of DAP (mainly determined by vascular tone) on vasodilation, while reflecting the severity of circulatory dysfunction by accounting for HR. They postulated that evaluation of DSI during initial assessment could signal patients that would benefit from early vasopressors initiation during shock resuscitation. Additionally, they found that higher DSI values were associated with higher mortality.

Objectives. To validate DSI as prognostic marker of hospital mortality in septic shock patients.

Methods. We designed a cohort study using the Medical Information Mart for Intensive Care IV (MIMIC IV) v0.4 database [3,4]. Adult patients ( $\geq 18$ years-old) with septic shock (according to the SEPSIS-3 definition [5]) at intensive care unit (ICU) admission were selected. DSI was calculated as HR/DAP obtained just before (30 min timeframe) initiation of vasopressors. Logistic regression was used to evaluate the association of DSI with hospital mortality, both with crude and adjusted analysis for age, sex, lactate, SOFA and SAPS II scores. DSI's area under the receiver operating characteristic curve (AUROC) was calculated.

Results. We studied 3.088 patients admitted for septic shock. Sixty percent were males, median age was 67 (Interquartile Range [IQR]: 56-76) years and the hospital mortality during the studied admission was $31 \%$. At the first $24 \mathrm{~h}$ of ICU stay, the mean SAPS-II score was $49 \pm 16$ and the median SOFA score was 4 (IQR: $3-6$ ). The mean DSI before starting vasopressors was $2.1 \pm 0.7$. Increments in DSI were associated with higher hospital mortality, both in the crude (odd ratio [OR] 1.8; 95\% confidence interval [95\% Cl]: $1.6-2.0 ; p<0.001)$ and adjusted analysis (OR 1.5; $95 \% \mathrm{Cl}: 1.3-1.7 ; p<0.001$ ). The precision of DSI alone to predict hospital mortality was 0.60 , as calculated by AUROC, and 0.76 when included in the adjusted model.

Conclusion. Our findings reinforce the prognostic value of DSI as mortality predictor in septic shock patients. Its value as a severity marker of circulatory dysfunction with greater vasodilation and, as such, as a trigger to early initiation of vasopressors merits further research.

Reference(s)

1. 1. Vincent JL, De Backer D. Circulatory shock. N Engl J Med. 2013;369(18):1726-34.

2. 2. Ospina-Tascón, G.A., Teboul, JL., Hernandez, G. et al. Diastolic shock index and clinical outcomes in patients with septic shock. Ann. Intensive Care 10, 41 (2020).

3. 3. Johnson A, Bulgarelli L, Pollard T, Horng S, Celi L A, Mark R. MIMIC-IV (version 0.4). PhysioNet. 2020. Available from: https://doi.org/10.13026/ a3wn-hq05.

4. 4. Goldberger A, Amaral L, Glass L, Hausdorff J, Ivanov PC, Mark R, Mietus JE, Moody GB, Peng CK, Stanley HE. PhysioBank, PhysioToolkit, and PhysioNet: Components of a new research resource for complex physiologic signals. Circulation [Online]. 101 (23), pp. e215-e220.

5. 5. Singer M, Deutschman CS, Seymour CW, et al. The Third International Consensus Definitions for Sepsis and Septic Shock (Sepsis-3). JAMA. 2016;315(8):801-810. https://doi.org/10.1001/jama.2016.0287

6. None.

000722

Ventilation-associated pneumonia in patients with SARS COV2 pneumonia. Our flora, incidence and mortality

S. Moreno Cano'; A. Garrino Fernández'; A. Estella

${ }^{1}$ University hospital of jerez., Intensive care unit, Jerez, Spain

Correspondence: $\mathrm{S}$. Moreno Cano

Intensive Care Medicine Experimental 2021, 9(1): 000722

Introduction. Approximately $80 \%$ of the episodes of nosocomial pneumonia occur in patients with an artificial airway, which is called ventilation-associated pneumonia (VAP). VAP is the most frequent cause of mortality among nosocomial infections in the Intensive Care Units (ICU). COVID-19 patients frequently require prolonged invasive mechanical ventilation (MV) including prone positioning heavy sedation, and muscle blockers for several weeks, that increase secondary hospital-acquired infections and primarily VAP.

Objectives. To describe the incidence of bacterial or fungal VAP in patients with COVID-19 pneumonia, and the associated mortallity.

Methods. Retrospective descriptive study where critically ill patients with confirmed SARS-CoV-2 infection were included from March 1st 2020 to January 30th 2021. Bronchoalveolar lavage (BAL) were done to all of them when $\mathrm{MV}$ was required. Urinary antigen tests (UATs) for Streptococcus pneumoniae (SP) and Legionella pneumophila (LP), and influenza virus were tested in all the patients. Medical story, microbiological cultures, ICU lengh of stay and mortallity were analyzed by SSPS v18 Programme.

Results. 115 patient swere enrolled, median age was $63,6 \pm 10$ and $82(71 \%)$ were men. Median lenght of stay was 15 days [7-25]. 71 patients $(79 \%)$ required $M V$, in them, mortallity was $74 \%$. Empirical antibiotics were prescribed in $100 \%$, but suspended after $48 \mathrm{~h}$ 
except for microbiological isolation or increased biomarkers. Corticosteroids were prescribed in $85 \%$. VAP were diagnosed in $66 \%$ patients, $82 \%$ were bacterial and $45 \%$ fungal (Aspergillus spp). Coinfection bacterial-fungal was presented in $34 \%$. Pseudomonas Aeruginosa, Staphilococcus Aureus, Klebsiella Pneumoniae, Enterococcus Faecalis and Stenotrophomonas were the flora more founded.

Conclusion. Most of critically ill patients with confirmed SARSCoV-2 infection need MV, that was associated to high mortallity. A high incidence of bacterial and fungal VAP was observed.

\section{6}

Application of the spring constant and pressure phase plane methods to track changes in arterial elasticity during sepsis L. Pereira' ${ }^{1}$ U. wiersema'; S. Bihari

${ }^{1}$ Intensive care unit, Flinders Medical Centre, Bedford Park, Australia Correspondence: $L$. Pereira

Intensive Care Medicine Experimental 2021, 9(1): 000816

Introduction. The elastic properties of the arteries, with radial dilatation of the vessel wall, enables arterial pressure wave propagation and efficient transfer of blood flow and energy for organ perfusion(1-3). Chronic reduction of arterial elasticity is associated with increased cardiovascular events, hypertension and all-cause mortality (4). The effects of sepsis on arterial elasticity is not well characterised $(5,6)$. The spring constant method and the pressure phase plane method of analysis of the arterial pressure waveform may be used to track changes in arterial elasticity during sepsis and recovery from sepsis (7-9).

Objectives. We applied the spring constant and pressure phase plane methods to demonstrate/measure the changes in arterial elasticity during the course of illness in patients who presented with sepsis and hypotension.

Methods. We performed a prospective observational study in an adult medical/surgical intensive care unit in a tertiary teaching hospital, including 13 patients admitted with acute sepsis and hypotension with invasive radial arterial blood pressure monitoring. Data was acquired from the radial artery trace and analysed via the spring constant method and the pressure phase plane method, which are both direct calculations of arterial elasticity/stiffness. Measurements were taken at the time of admission, commencement of vasopressor and prior to discharge from the intensive care unit. Repeated measures ANOVA and paired T test was used to compare data acquired.

Results. Repeated measures ANOVA found significant differences between spring constant arterial elasticity values ( $p 0.033)$ and the pressure phase plane $K+$ stiffness analog values $(p 0.020)$ measured at time of admission, with vasopressor, and prior to discharge. Pearson correlation confirmed that neither the spring constant nor pressure phase plane $\mathrm{K}+$ findings were attributable to changes in mean arterial pressure, pulse pressure or heart rate. Paired T-test showed significance for changes in spring constant values between admission and vasopressor ( $\mathrm{p} 0.006)$, and those between vasopressor and discharge ( $p$ 0.041). A significant difference was also found between vasopressor and discharge pressure phase plane $K+$ stiffness analog values $(p$ 0.018 ).

Table 1. Spring Constant and Pressure Phase Plane (PPP) K+ and $\mathrm{K}$ - values at admission (T1), after commencement of vasopressor (T2), and at discharge from ICU (T3).

\begin{tabular}{cccccccc}
\hline $\begin{array}{l}\text { Vari- } \\
\text { able }\end{array}$ & $\begin{array}{l}\text { T1 Mean } \\
\text { (SD) }\end{array}$ & $\begin{array}{l}\text { T2 Mean } \\
\text { (SD) }\end{array}$ & $\begin{array}{l}\text { T3 Mean } \\
\text { (SD) }\end{array}$ & $\begin{array}{l}\text { ANOVA } \\
\text { P value }\end{array}$ & $\begin{array}{l}\text { T1-T2 } \\
\text { T test } \\
\mathbf{P} \\
\text { value }\end{array}$ & $\begin{array}{l}\text { T1-T3 } \\
\text { T test } \\
\text { Palue }\end{array}$ & $\begin{array}{l}\text { T2-T3 } \\
\text { T test } \\
\text { P value }\end{array}$ \\
\hline $\begin{array}{r}\text { Spring } \\
\text { Con- } \\
\text { stant } \\
(1 / 52)\end{array}$ & $\begin{array}{c}1418 \\
(384.3)\end{array}$ & $\begin{array}{c}968.2 \\
(522.5)\end{array}$ & $\begin{array}{c}1386 \\
(669.1)\end{array}$ & 0.033 & 0.006 & 0.773 & 0.041 \\
PPP K+ & $\begin{array}{c}1288 \\
(423.5)\end{array}$ & $\begin{array}{c}1143 \\
(406.4)\end{array}$ & $\begin{array}{c}1431 \\
(599.9)\end{array}$ & 0.020 & 0.133 & 0.096 & 0.018 \\
\hline
\end{tabular}

\begin{tabular}{llllllll}
\hline $\begin{array}{l}\text { Vari- } \\
\text { able }\end{array}$ & $\begin{array}{l}\text { T1 Mean } \\
\text { (SD) }\end{array}$ & $\begin{array}{l}\text { T2 Mean } \\
\text { (SD) }\end{array}$ & $\begin{array}{l}\text { T3 Mean } \\
\text { (SD) }\end{array}$ & $\begin{array}{l}\text { ANOVA } \\
\text { P value }\end{array}$ & $\begin{array}{l}\text { T1-T2 } \\
\text { T test } \\
\mathbf{P} \\
\text { value }\end{array}$ & $\begin{array}{l}\text { T1-T3 } \\
\text { T test } \\
\text { Palue }\end{array}$ & $\begin{array}{l}\text { T2-T3 } \\
\text { v test } \\
\text { P value }\end{array}$ \\
\hline PPP K- & $\begin{array}{r}286.1 \\
(119.4)\end{array}$ & $\begin{array}{c}229.7 \\
(91.58)\end{array}$ & $\begin{array}{c}243.2 \\
(88.00)\end{array}$ & 0.403 & 0.191 & 0.499 & 0.298 \\
& & & & & & \\
\hline
\end{tabular}

Conclusion. The spring constant and pressure phase plane methods provide a direct measurement of arterial elasticity, treating the arterial wall and blood as one system, and provide a novel way to track changes during sepsis and vasopressor use.

\section{Reference(s)}

1. 1. Lin Wang Y-Y, Chiu W-B, Jan M-Y, Bau J-G, Li S-P, Wang W-K. Analysis of transverse wave as a propagation mode for the pressure pulse in large arteries. J Appl Phys. 2007 Sep 15;102(6):064,702.

2. 2. Lin Wang Y-Y, Wang W-K. A hemodynamics model to study the collective behavior of the ventricular-arterial system. J Appl Phys. 2013 Jan 14;113(2):024,702.

3. 3. Lin Wang $Y-Y$, Wang W-K. Why the cardiovascular studies should start with the radial oscillation of arterial wall rather than from axial flow motion of blood. Int J Cardiol [Internet]. 2018 Jun 8; Available from: http://dx.doi.org/10.1016/j.ijcard.2018.06.019

4. 4. Vlachopoulos C, Aznaouridis K, Stefanadis C. Prediction of cardiovascular events and all-cause mortality with arterial stiffness: a systematic review and meta-analysis. J Am Coll Cardiol. 2010 Mar 30;55(13):1318-27.

5. 5. Vlachopoulos C, Dima I, Aznaouridis K, Vasiliadou C, loakeimidis N, Aggeli $C$, et al. Acute systemic inflammation increases arterial stiffness and decreases wave reflections in healthy individuals. Circulation. 2005 Oct 4;112(14):2193-200.

6. 6. Kazune S, Grabovskis A, Cescon C, Strike E, Vanags I. Association between increased arterial stiffness and clinical outcomes in patients with early sepsis: a prospective observational cohort study. Intensive Care Med Exp. 2019 May 16:7(1):26.

7. 7. Wei C-C. An innovative method to measure the peripheral arterial elasticity: spring constant modeling based on the arterial pressure wave with radial vibration. Ann Biomed Eng. 2011 Nov;39(11):2695-705.

8. 8. Chung CS, Strunc A, Oliver R, Kovács SJ. Diastolic ventricular-vascular stiffness and relaxation relation: elucidation of coupling via pressure phase plane-derived indexes. Am J Physiol Heart Circ Physiol. 2006 Nov;291(5):H2415-23.

9. 9. Wei C-C, Huang S-W, Bau C-T. Using the spring constant method to analyze arterial elasticity in type 2 diabetic patients. Cardiovasc Diabetol. 2012 Apr 25;11:39.

\section{2}

Biomarkers of sepsis in the diagnosis of stage of generalized peritonitis

J. Fuss ${ }^{1}$; A. Voloboyeva ${ }^{2}$

${ }^{1}$ Department of Surgery, Regional hospital of Pustomyty, Lviv, Ukraine ${ }^{2}$ Department of Anestesiology, Communal Municipal Clinical Hospital 8 L'viv, Ukraine

\section{Correspondence: J. Fuss}

Intensive Care Medicine Experimental 2021, 9(1): 000842

Introduction. The problem of generalizated peritonitis (GP), especially in the stage of abdominal sepsis, is one of the most relevant in modern surgery [1]. Unsatisfactory results of treatment of such severe pathology are associated with its high mortality-30-70\%. For effective prediction of the outcome of the disease, along with other diagnostic methods, biological markers are of particular importance $[2,3]$. The most important of these markers at this stage are presepsin (PSP), procalcitonin (PCT), C-reactive protein (CRP) [4].

Objectives. To evaluate the prognostic role of biomarkers of inflammation in determining the stage of development of GP.

Methods. This study was conducted in hospitals in Lviv and Pustomyty. The study included 128 patients with GP, including 85 men and 43 women. The age of patients ranged from 18 to 84 years and averaged $35.4 \pm 3.2$ years. All patients were divided into 3 groups depending on 
the stage of peritonitis: Group I $(n=83 ; 64.9 \%)$-initial stage; Group II $(n=23 ; 18 \%)$-toxic; Group III $(n=22 ; 17.1 \%)$-terminal.

The main nosologies that led to the development of GP were: in 74 (57.8\%)-perforation of the hollow organ, in $32(25 \%)$-acute intestinal obstruction, in $9(7 \%)$ cases-acute appendicitis, in $5(3,9 \%)$ cases-acute cholecystitis, in $3(2.4 \%)$ patients-pancreatic necrosis, in $3(2.4 \%)$ cases -intestinal perforation and in $2(1.5 \%)$ cases the causes of peritonitis were other diseases.

In order to determine the stage of development of the process and the presence of abdominal sepsis, the study of levels of presepsin (PSP), procalcitonin (PCT), C-reactive protein (CRP) in the blood was performed on the day of hospitalization.

Results. Our results showed that the highest rates of the studied biomarkers were observed in the group of patients in the terminal stage. Thus, the level of PSP in the blood of these patients was $1473.4 \pm 298.2 \mathrm{pg} / \mathrm{ml}$, the level of PCT $-18.0 \pm 2.9 \mathrm{ng} / \mathrm{ml}$, the level of CRP-284.5 $\pm 29.7 \mathrm{mg} / \mathrm{l}$ their maximum values were $1884.7 \mathrm{pg} /$ $\mathrm{ml}, 22.6 \mathrm{ng} / \mathrm{ml}, 312.4 \mathrm{mg} / \mathrm{l}$ and $329.7 \mathrm{pg} / \mathrm{ml}$, respectively. The lowest average value of PSP $345,0288 \pm 102,9112$, PCT $-1,6 \pm 0,8 \mathrm{ng} /$ $\mathrm{ml}, \mathrm{CRP}-89,5 \pm 31,2 \mathrm{mg} / \mathrm{l}$, as well as the minimum concentration of PSP- $56.1 \mathrm{pg} / \mathrm{ml}$, PCT-0.1 ng/ml, CRP- $10.6 \mathrm{mg} / \mathrm{l}$ were detected in patients with GP in the reactive stage. There was a strong statistical significance of the differences $(p<0,001)$.

In the study of the concentration of PSP it was found that its maximum values were observed in the terminal stage, with no statistical significance of differences between groups. Our data confirm the opinion of other scientists that in the presence of sepsis, including its abdominal form, there is a significant increase in the level of presepsin in the serum.

Conclusion. Thus, the studies revealed a statistically significant correlation between the severity of diffuse peritonitis and the level of presepsin in the blood, which allows it to be used effectively in the diagnosis of abdominal sepsis.

\section{2}

Early Intubation and patient-centered outcomes in septic shock: a secondary analysis of a prospective multicenter study

R. Mellado Artigas'; BL. Ferreyro, ${ }^{2}$; C. Ferrando, ; C. Darreau, ${ }_{1}^{3} ;$ F. Martino, ${ }_{1}^{4} ; \mathrm{S}$. Jacquier, ${ }^{5}$; A. Delbove, ; L. Brochard, ; N. Lerolle,

${ }^{1}$ Surgical Intensive Care Unit, Hospital Clínic de Barcelona, Barcelona, Spain; ${ }^{2}$ Intensive Care Unit, Sinai Health System, Toronto, Canada; ${ }^{3}$ Medical and Surgical Intensive Care Unit, Le Mans Hospital, Le Mans, France; ${ }^{4}$ Medical and Surgical Intensive Care Unit, Guadaloupe University Hospital, Les Abymes, Guadeloupe; ${ }^{5}$ Medical intensive care, Tours University Hospital, Tours, France; ${ }^{6}$ Medical Intensive Care Unit, Nantes University Hospital, Nantes, France; ${ }^{7}$ Interdepartmental Division of Critical Care, University of Toronto, Toronto, Canada; ${ }^{8}$ Faculty of Medicine, University of Angers, Angers, France

Correspondence: R. Mellado Artigas

Intensive Care Medicine Experimental 2021, 9(1): 000852

Introduction. Despite the tremendous benefits associated with mechanical ventilation, its use is associated with complications. The exact time to switch to invasive mechanical ventilation is not known.

Objectives. We assessed the effect of intubation in septic shock patients in the first $8 \mathrm{~h}$ after vasopressor start, as compared to nonearly intubated controls on in-hospital mortality, intensive care and hospital length of stay among survivors and non-respiratory organ dysfunction at $24 \mathrm{~h}$.

Methods. Secondary analysis of a multicenter prospective registry. To adjust for measured confounding, overlap weighting based on the propensity score was carried out. This statistical tool creates a pseudopopulation with identical covariate distribution by weighting treated subjects by the inverse of their propensity score and control individuals by their propensity score.

In-hospital mortality was analyzed in a time-to-event fashion while length of stay was assessed as a median difference using non-parametric bootstrapping. SOFA was compared with linear regression.
Results. 735 patients (137 intubated in the first 8 h) were evaluated. After adjustment, a population with 53 patients on each group showed excellent adjustment. In-hospital mortality occurred in 15 $(28 \%)$ and in $22(41 \%)$ and $15(28 \%)$ subjects in the control and early intubation groups (hazard ratio $1.60,95 \% \mathrm{Cl} 1.00-2.54, \mathrm{p}=0.05$ ). Among survivors, control subjects spent a median of 5 days in intensive care while early intubated individuals displayed a median length of stay of 10: (difference 5 days, $95 \% \mathrm{Cl} 2-8$ days). However, when considering hospital stay, differences were not significant: 20 versus 23 days (difference 1 day, $95 \% \mathrm{Cl}-3$ to 10 days). SOFA score at $24 \mathrm{~h}$ was not significantly different between groups ( 7.3 vs 8.0 points, mean difference $0.74,95 \% \mathrm{Cl}-0.04$ to $1.48, \mathrm{p}=0.06$ ).

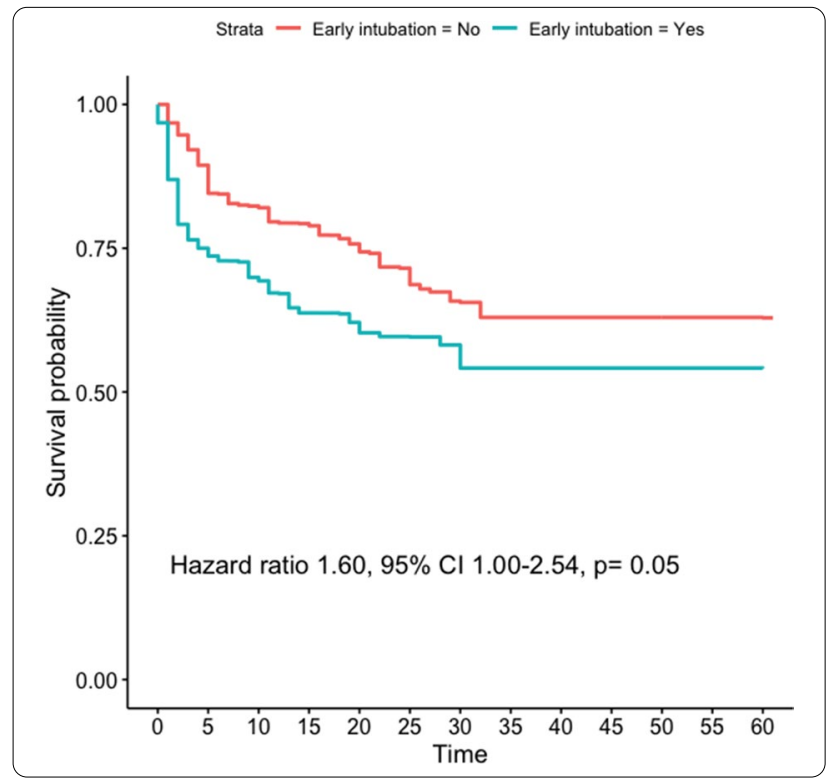

Conclusion. Early intubation did not improve outcomes in this weighted cohort of septic shock patients. There were large differences between intubated and non-early intubated subjects at baseline which advise for using these results to infer treatment estimates only in a subset of early intubated patients.

\section{Reference(s)}

1. Desai RJ, Franklin JM. Alternative approaches for confounding adjustment in observational studies using weighting based on the propensity score: A primer for practitioners. BMJ. 2019;367. https://doi.org/10.1136/bmj. 15657

2. Papoutsi E, Giannakoulis VG, Xourgia E, Routsi C, Kotanidou A, Siempos II. Effect of timing of intubation on clinical outcomes of critically ill patients with COVID-19: a systematic review and meta-analysis of non-randomized cohort studies. Crit Care. 2021;25(1):121. https://doi.org/10.1186/ s13054-021-03540-6

3. Mellado-Artigas R, Ferreyro B, Angriman F. High-flow nasal oxygen in patients with COVID-19-associated acute respiratory failure. Crit Care. Published online 2021.

4. Bellani G, Laffey JG, Pham T, et al. Noninvasive Ventilation of Patients with Acute Respiratory Distress Syndrome: Insights from the LUNG SAFE Study. Am J Respir Crit Care Med. 2017;195(1):67-77. https://doi.org/10.1164/ rccm.201606-13060C

5. Dumas $\mathrm{G}$, Lemiale V, Rathi N, et al. Survival in Immunocompromised Patients Ultimately Requiring Invasive Mechanical Ventilation: A Pooled Individual Patient Data Analysis. Am J Respir Crit Care Med. Published online March 22, 2021. https://doi.org/10.1164/rccm.202009-3575oc

6. Demoule A, Girou E, Richard JC, Taille S, Brochard L. Benefits and risks of success or failure of noninvasive ventilation. Intensive Care Med. 2006;32(11):1756-1765. https://doi.org/10.1007/s00134-006-0324-1 
7. Kangelaris KN, Ware LB, Wang CY, et al. Timing of intubation and clinical outcomes in adults with acute respiratory distress syndrome. Crit Care Med. 2016:44(1):120-129. https://doi.org/10.1097/CCM.0000000000 001359

8. Ricard JD, Roca O, Lemiale V, et al. Use of nasal high flow oxygen during acute respiratory failure. Intensive Care Med. Published online 2020 https://doi.org/10.1007/s00134-020-06228-7

9. Darreau C, Martino F, Saint-Martin M, et al. Use, timing and factors associated with tracheal intubation in septic shock: a prospective multicentric observational study. Ann Intensive Care. 2020;10(1):62. https://doi.org/ 10.1186/s13613-020-00668-6

\section{4}

\section{Septic shock 3.0 in severe COVID19 patients: are we missing} something clinically important?

JP. Cidade ${ }^{1}$; V. Costa, ${ }_{1} ;$ R. Morais, ${ }_{1}^{1}$; P. Moniz, ${ }_{1}$; L. Morais, ${ }_{1}$; P. Fidalgo, ${ }_{1}^{1}$;

S. Brito ${ }^{1}$; A. Tralhão ${ }^{1}$; C. Paulino, ; D. Nora ${ }^{1}$; B. Valerio, ${ }^{1}$; L. Coelho, ; C. Tapadinhas, ${ }^{1}$; P. Povoa ${ }^{1}$

${ }^{1}$ Polyvalent Intensive Care Unit, Hospital São Francisco De Xavier, Lisboa, Portugal

Correspondence: J.P. Cidade

Intensive Care Medicine Experimental 2021, 9(1): 000854

Introduction. COVID19 can be associated with life-threatening organ dysfunction due to septic shock, frequently requiring ICU admission, respiratory and vasopressor support. Therefore, clear clinical criteria are pivotal to early recognition of patients more likely to have poor outcomes, needing prompt organ support. Although most patients with severe COVID19 meet the Sepsis-3.0 criteria for septic shock, it has been increasingly recognized that, in this population, hyperlactatemia is frequently absent, possibly leading to an underestimation of illness severity and mortality risk.

Objectives. This study aimed to identify the proportion of patients with COVID19 with hypotension despite adequate volume resuscitation, needing vasopressors to have a MAP $>65 \mathrm{mmHg}$, with and without hyperlactatemia, in ICU, and describe its clinical outcomes and mortality rate.

Methods. We performed a single-center retrospective cohort study. All adult patients admitted to ICU with COVID19 were eligible and were further divided in 3 groups according to hyperlactatemia (lactate $>2 \mathrm{mmol} / \mathrm{L}$ ) and persistent hypotension with vasopressor therapy requirement: (1) sepsis group (without both criteria), (2) vasoplegic shock (with persistent hypotension with vasopressor therapy requirement without hyperlactatemia) and (3) septic shock 3.0. COVID19 was diagnosed using clinical and radiologic criteria with a SARS-CoV-2 positive RT-PCR test. Qui-square test was used for categorical variables and Kruskal-Wallis and logistic regression were used on continuous variables for statistical assessment of outcomes between groups. Kaplan-Meier survival curve and log-rank test were also obtained.

Results. 103 patients (mean age 62 years, 71\% males) were included in the analysis ( $N=45$ sepsis, $N=25$ vasoplegic shock; $N=33$ septic shock 3.0). SOFA score at ICU admission and ICU length of stay were different between groups $(p<0.001)$. Ventilator-free days and vasopressor-free days were also different between sepsis vs vasoplegic shock and septic shock 3.0 groups (both $p<0.001$ and $p<0.001$, respectively), and similar in vasoplegic vs septic shock 3.0 groups ( $p=0.387$ and $p=0.193$, respectively). Mortality was significantly higher in vasoplegic shock and septic shock 3.0 when compared with sepsis group $(p<0.001)$ without difference between the former two groups $(p=0.595)$. Log rank test of Kaplan-Meier survival curves were also different $(p=0.007)$ (Fig. 2$)$. Logistic regression identified the maximum dose of vasopressor therapy used (OR 1.065; CI 95\%: 1.023$1.108, \mathrm{p}=0.02$ ) and serum lactate level (OR 1.543; Cl 95\%: 1.069-2.23, $p=0.02$ ) as the major explanatory variables of mortality rates.

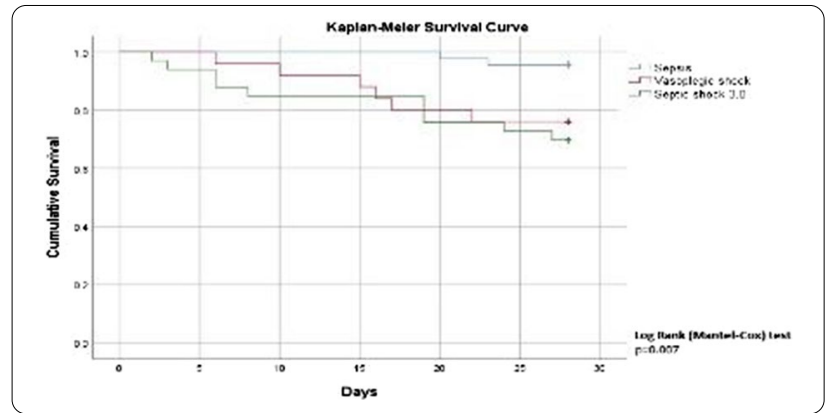

Conclusion. In severe COVID19 patients, the Sepsis 3 criteria of septic shock may exclude patients with a similarly high risk of poor outcomes and mortality rate, that should be equally approached.

\section{Reference(s)}

1. 2. Bone RC, Balk RA, Cerra FB, Knaus WA, et al. Definitions for sepsis and organ failure and guidelines for the use of innovative therapies in sepsis. The ACCP/SCCM Consensus Conference Committee. American College of Chest Physicians/Society of Critical Care Medicine. Chest. 1992;101(6):1644-55

2. 1. Ranzani OT, Monteiro MB, Noritomi DT; Grupo de Cuidados Críticos Amil. Reclassifying the spectrum of septic patients using lactate: severe sepsis, cryptic shock, vasoplegic shock and dysoxic shock. Rev Bras Ter Intensiva. 2013 Oct-Dec;25(4):270-8. https://doi.org/10.5935/0103-507X. 20130047.

\section{7}

Cytokine trajectories in response to drug treatment in septic shock

C. Cleasby ; J. Ward ${ }^{2}$; T. Marshall ${ }^{1}$; F. Al-Beidh ${ }^{3} ;$ A. Gordon ${ }^{4}$; DB. Antcliffe ${ }^{2}$

${ }^{1}$ Section of Anaesthetics, Pain Medicine and Intensive Care, Imperial

College London, London, United Kingdom; ${ }^{2}$ Chelsea and Westminster

Hospital, Imperial College London, London, United Kingdom; ${ }^{3}$ Section of Anaesthetics, Pain Medicine and Intensive Care, Imperial College Londom, London, United Kingdom; ${ }^{4}$ Section of Anaesthetics, Pain Medicine, and Intensive Care, Imperial College London, London, United Kingdom

Correspondence: $C$. Cleasby

Intensive Care Medicine Experimental 2021, 9(1): 000877

Introduction. Vasopressors are required in septic shock to maintain patients' mean arterial pressure (MAP). Noradrenaline is the first line vasopressor, however, it has been suggested vasopressin may provide some advantages by suppressing inflammatory mediators (1). Hydrocortisone is an adjunctive treatment in shock, if vasopressors cannot maintain MAP. Glucocorticoids are anti-inflammatory and they, too, can cause immune modulation.

Objectives. To examine the effects of noradrenaline versus vasopressin, and hydrocortisone versus placebo, on cytokine trajectories over the first $96 \mathrm{~h}$ of septic shock.

Methods. 19 cytokines were measured in the plasma of 196 patients who were enrolled in the VANISH clinical trial (2). Plasma samples were taken at up to 4 timepoints during a patient's ICU admission. Patients in this trial were randomized to four treatment groups (noradrenaline and hydrocortisone, noradrenaline and placebo, vasopressin and hydrocortisone and vasopressin and placebo). Mixed model analyses were used to compare how cytokines varied over time and by study drugs. The models were controlled for in-subject variability by including each patient as a random intercept. Vasopressin and noradrenaline were compared in the first analysis and hydrocortisone to placebo in the second. There was a decreased requirement for renal replacement therapy (RRT) in the vasopressin group compared to noradrenaline in VANISH $(25.4 \%$ vs $35.3 \%$, Odds ratio $=0.40$ [95\% Cl, $0.20-0.73])$ (1) and haemofiltration is thought to filter cytokines out of the circulation. Due to this, RRT could confound the causal pathway therefore 
the analysis of noradrenaline versus vasopressin was repeated adjusting for this.

\section{Results.}
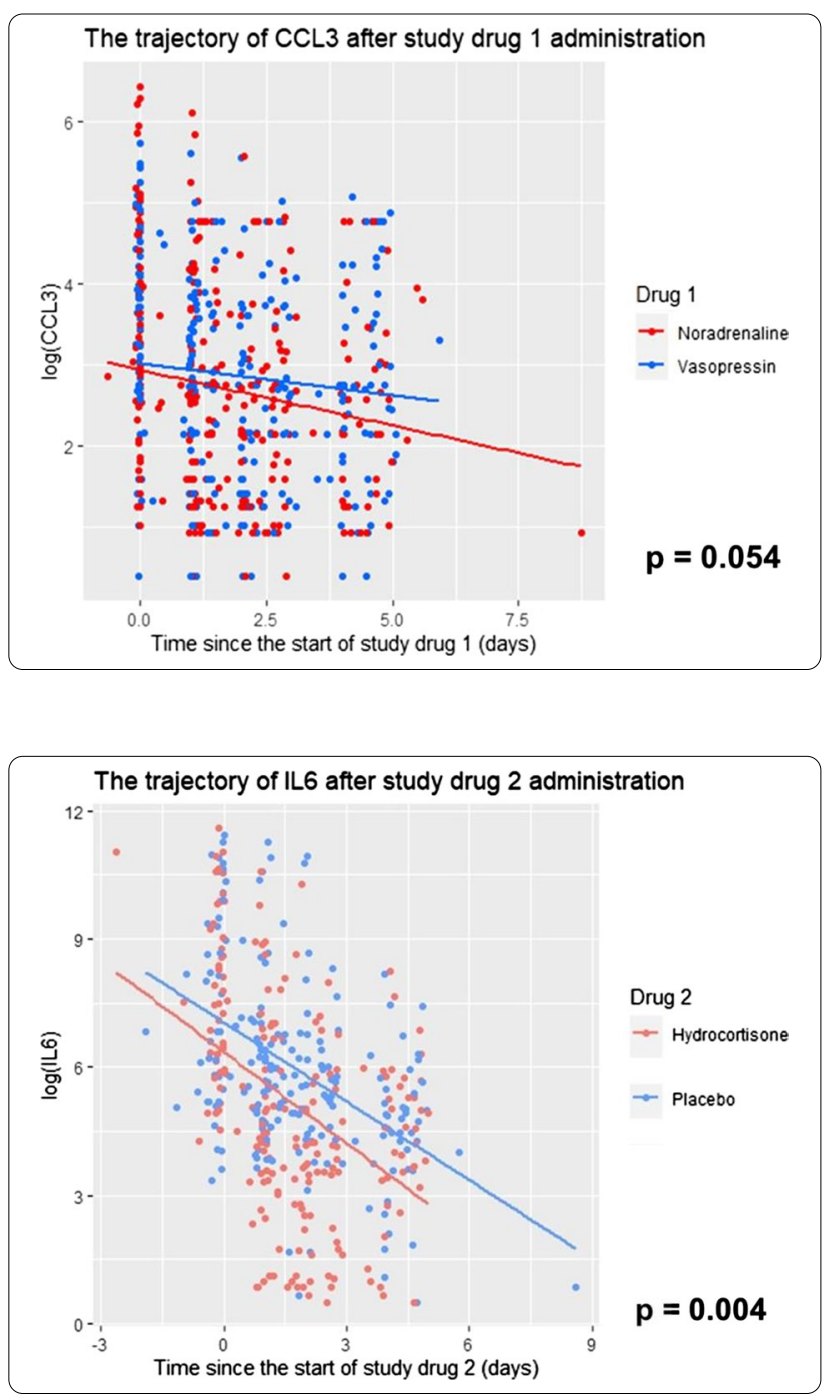

Most cytokines decreased over time after the onset of septic shock. Statistically significant differences were seen in IL17 $(p=0.016)$ (median decrease of $1.88 \mathrm{pg} / \mathrm{ml}$ in noradrenaline and $0.50 \mathrm{pg} / \mathrm{ml}$ in vasopressin) and IL12p70 ( $\mathrm{p}=0.042)$ (median decrease of $1.32 \mathrm{pg} /$ $\mathrm{ml}$ in noradrenaline vs $0.01 \mathrm{pg} / \mathrm{ml}$ in vasopressin). Although significant, these differences were small; however, CCL3 suggested a trend to greater differences but failed to reach statistical significance $(p=0.054)$ (median decrease of $7.9 \mathrm{pg} / \mathrm{ml}$ in noradrenaline vs $22.69 \mathrm{pg} / \mathrm{ml}$ in vasopressin). When adjusted for RRT, these three cytokines became less significant. Hydrocortisone led to a significant decrease of IL6 $(p=0.004)$ (median decline $1773 \mathrm{pg} / \mathrm{ml}$ with hydrocortisone vs $1019 \mu \mathrm{l} / \mathrm{ml}$ in placebo) and IL12p70 ( $p=0.025)$ (median decrease of $0.7 \mathrm{pg} / \mathrm{ml}$ in hydrocortisone vs $0.34 \mathrm{pg} / \mathrm{ml}$ in placebo).
When adjusted for multiple comparisons, all $p$ values failed to reach significance.

Conclusion. Although there were some statistically significant differences in the decrease in cytokines between noradrenaline and vasopressin, these were small and of questionable clinical meaning. Hydrocortisone had the greatest effect on IL6 suggesting IL6 levels could be used to stratify patients to receive hydrocortisone treatment in future trials.

\section{Reference(s)}

1. (2) Gordon AC et al. Effect of Early Vasopressin vs Norepinephrine on Kidney Failure in Patients With Septic Shock: The VANISH Randomized Clinical Trial. JAMA 2016:316(5):509-18

2. (1) Russell JA et al. Vasopressin Compared with Norepinephrine Augments the Decline of Plasma Cytokine Levels in Septic Shock. American Journal of Respiratory and Critical Care Medicine. 2013; 188 (3): 356-364.

3. This abstract is independent research funded by the National Institute for Health Research (NIHR) Imperial Biomedical Research Centre (BRC). ACG is an NIHR Research Professor (RP-2015-06-018)

000938

Circulating bioactive adrenomedullin is associated with mortality, organ failure and intensive care in sepsis patients in the emergency department

OHM. Lundberg ${ }^{1}$; M. Rosenquist ${ }^{2}$; K. Bronten, ${ }^{3}$; H. Friberg ${ }^{4}$; O. Melander, ${ }^{3}$ ${ }^{1}$ Department of Intensive and Perioperative Care, Skåne University Hospital, SUS, Malmö, Malmö, Sweden; ${ }^{2}$ Department of Infectious Diseases, Skåne University Hospital, SUS, Malmö, Malmö, Sweden; ${ }^{3}$ Department of Internal Medicine, Skåne University Hospital, SUS, Malmö, Malmö, Sweden; ${ }^{4}$ Department of Clinical Sciences, Intensive and Perioperative Care, Lund University, Lund, Sweden

Correspondence: O.H.M. Lundberg

Intensive Care Medicine Experimental 2021, 9(1): 000938

Introduction. Sepsis patients with increased risk of poor outcome are difficult, yet important, to identify. Adrenomedullin is a vasoactive hormone which could be of prognostic and potentially therapeutic value.

Objectives. The primary aim of this study was to investigate the association of circulating bioactive adrenomedullin (bio-ADM) levels among sepsis patients in the emergency department (ED) with mortality. Secondary aims were to assess whether bio-ADM could improve the prognostic capabilities of a mortality prediction model, investigate the association of bio-ADM with multi organ failure (MOF), ED discharge and intensive care unit (ICU) admission.

Methods. In this retrospective observational study, adult sepsis patients treated in the ED between 2013 and 2015 had bio-ADM levels analyzed. Un-adjusted and adjusted odds ratios (OR) with $95 \% \mathrm{Cl}$ for log-2 transformed bio-ADM were calculated. The primary outcome was 28-day mortality. Secondary outcomes were development of MOF, ED discharge and admission to the ICU.

Results. Bio-ADM in 594 sepsis patients was analyzed of whom 51 died within 28 days, 34 developed severe MOF, 67 were discharged from the ED and 28 were admitted to the ICU. The median bio-ADM $(\mathrm{pg} / \mathrm{mL})$ among non-survivors was 63 , survivors 36 , patients with severe MOF 81 and patients admitted to ICU 77.

The association of elevated bio-ADM and mortality resulted in an un-adjusted $\mathrm{OR}$ of 2.30 (95\\% Cl 1.74-3.04), whereas the multivariate adjusted $\mathrm{OR}$ was 2.50 (95\\% Cl 1.77-3.54). The addition of bioADM to a prognostic mortality model resulted in a $7 \%$ improvement $(p=0.009)$

Increasing bio-ADM was associated with severe MOF and ICU admission with multivariate adjusted ORs of 3.45 (95\% 2.24-5.30) and 1.75 
(95\% 1.11-2.76), respectively. Corresponding OR for ED discharge was $0.45(95 \% 0.31-0.65)$.

Conclusion. Increasing levels of bio-ADM are associated with mortality, MOF, ICU admission and inversely to discharge in sepsis patients in the ED. Bio-ADM may be useful in early identification of the sickest sepsis patients.

\section{5}

\section{Sepsis Resuscitation: Less is More?}

M. Gorski'; N. Puri, ; R. Cole, ${ }^{1}$; S. Patel,

${ }^{1}$ Critical Care Medicine, Cooper University Health Care, Camden, NJ, United States of America

Correspondence: M. Gorski

Intensive Care Medicine Experimental 2021, 9(1): 000945

Introduction. Sepsis is a continuum stemming from a systemic inflammatory response (SIRS). The sepsis SIRS response can progress to multi-organ failure. The medical community has developed guidelines aimed at reducing Sepsis mortality which emphasized early recognition and protocolized management. A pervasive protocol is the Early Goal-Directed Therapy (EGDT) which highlights the optimization of hemodynamic parameters using methods such as fluid administration (1). However, recent research has questioned the benefit and possible harm of liberal volume administration for sepsis $(2,3,4)$. Still, a common counter-argument for this finding is higher acuity patients require more volume. The fluid and mortality connection is burdened with the classic correlation versus causation conundrum. We aim to determine a causal relationship between volume overload and sepsis mortality using the causal analysis technique Targeted Maximal Likelihood Estimator (TMLE). TMLE limits confounding bias in observational data by generating a pseudo-population that models counterfactual outcomes (5).

Objectives. Utilizing TMLE, we will match patients using Apache IV score as our severity of illness surrogate to determine the effect of fluid balance on mortality outcomes.

Methods. We extracted data from the eICU Collaborative Research Database. We limited our dataset to patients with a diagnosis of Sepsis. A total of 17,091 patients were extracted. Exploratory data analysis performed in Python. Three features extracted were net total fluid balance, Apache IV, and Mortality. We binarized net total balance using Feature-Engine equal width discretiser. We used TMLE with an ensemble of machine learning algorithms as the primary model. The machine learning ensemble included Logistic Regression, a Neural Network, Naive Bayes, and XGboost. The analysis was performed on patient net fluid balance as the primary exposure. The covariate included is the Apache IV score. The primary outcome was mortality. Our primary metric was Average Treatment Effect (ATE). Secondary metrics were Odds Ratio and Risk Ratio.

Results. Net total fluid balance and Apache IV scores were both noted to be statistically different for the mortality groups. Using TMLE, mortality analysis demonstrated an ATE (RD) of $0.048,95 \% \mathrm{CI}[0.04,0.056]$, risk ratio $(\mathrm{RR}) 1.62,95 \% \mathrm{Cl}[1.488,1.768]$ and odds ratio (OR) of 1.711 $[1.556,1.881]$.

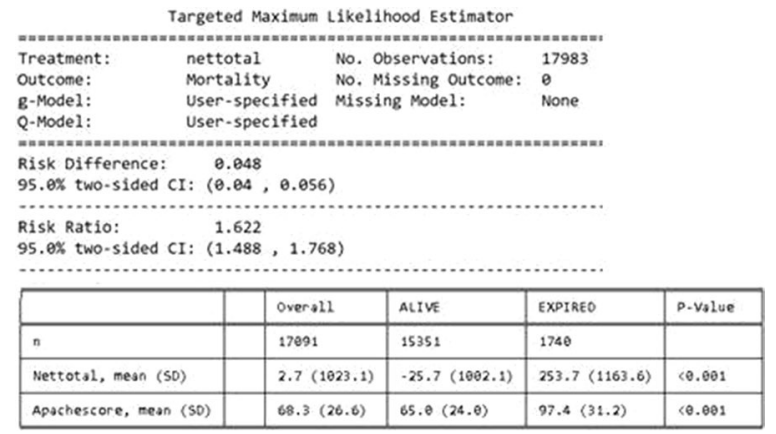

Conclusion. Using TMLE, there appears to be a causal relationship between higher fluid balances and worse mortality outcomes in our sepsis cohort; this relationship should be evaluated further via large and prospective studies.

\section{Reference(s)}

1. 1. Rivers, Emanuel, et al. "Early Goal-Directed Therapy in the Treatment of Severe Sepsis and Septic Shock." New England Journal of Medicine, vol. 345, no. 19, 2001, pp. 1368-1377.

2. 2. Mouncey, Paul R., et al. "Trial of Early, Goal-Directed Resuscitation for Septic Shock." New England Journal of Medicine, vol. 372, no. 14, 2015, pp. 1301-1311.

3. 3. Peake, Sandra L., et al. "Goal-Directed Resuscitation for Patients with Early Septic Shock." New England Journal of Medicine, vol. 371, no. 16, 2014, pp. 1496-1506.

4. 4. Yealy, Donald M., et al. "A Randomized Trial of Protocol-Based Care for Early Septic Shock." New England Journal of Medicine, vol. 370, no. 18, 2014, pp. 1683-1693.

5. 5. Van der Laan, MJ. and Rubin, D. Targeted Maximum Likelihood Learning. U.C. Berkeley Division of Biostatistics Working Paper Series. Working Paper 213. Published October 2006.

6. 6. Tigabu, Bereket M., et al. "Fluid volume, fluid balance and patient outcome in severe sepsis and septic shock: A systematic review." Journal of Critical Care, vol. 48, 2018, pp. 153-159.

\section{0}

Bioimpedance: an objective measure for fluid status in patients with septic shock?-A prospective observational study

J. Madsen ${ }^{1}$; T. Itenov' ${ }^{1}$ M. Mestle, ${ }^{1}$

${ }^{1}$ Department of Anaesthesiology and Intensive Care, Nordsjaellands

Hospital, Hilleroed, Denmark

Correspondence: J. Madsen

Intensive Care Medicine Experimental 2021, 9(1): 000970

Introduction. Fluid resuscitation is vital in the initial treatment of septic shock (1). However, the ongoing assessment of fluid status during acute resuscitation and in the aftermath relies on a composite of heterogeneous surrogate measures (2). Bioelectrical impedance analysis (BIA) is attractive for measures of fluid status because it is non-invasive, easy to use, quick, cheap, and safe (3-7). BIA measures impedance by sending a weak unnoticeable current through the body. A systematic review, currently in press, states that high-quality studies with adequate analysis are needed in order to judge the accuracy of BIA in ICU populations.

Objectives. The aim of this study was to determine if BIA is a precise and reproducible method for measuring fluid overload in patients with septic shock in the ICU.

Methods. A single-center prospective observational cohort study. The inclusion period was 1-12-2019-30-4-2021. We included adult ICU patients that met the criteria of septic shock according to the sepsis-3 guidelines (8) for a maximum of $24 \mathrm{~h}$. Two BIA devices were evaluated. The body composition monitor (BCM) from Fresenius measured impedance with a spectrum of frequencies from 50 to $1000 \mathrm{kHz}$. The Bioscan iTouch (MBS) from Maltron measured impedance at four frequencies; 5, 50, 100, and $200 \mathrm{kHz}$. Both devices had whole-body outcomes and electrodes placed ipsilateral, dorsally on the hand and foot. Results were the agreement between measurements of the left and right side of the patient, as well as measurements done one hour apart. The results were presented as Bland Altman plots with standard deviations (SD) of the mean difference and $95 \%$ Limits of Agreements (LoA).

Results. 49 severely ill patients were included. Table 1. MBS measured fluid overload on the left and right side showed a mean difference of $0.3 \mathrm{~L}$ with SD $2.7 \mathrm{~L}$ and $95 \% \mathrm{LoA}$ of $-4.0 \mathrm{~L}-4.7 \mathrm{~L}$. Fluid overload of MBS with one hour apart had a mean difference of $0.2 \mathrm{~L}$ and $\mathrm{SD}$ of $1.3 \mathrm{~L}$ and a $95 \%$ LoA of $-2.4 \mathrm{~L}-2.8 \mathrm{~L}$. BCM measured fluid overload comparing the left and right sides showed a mean difference of $0.5 \mathrm{~L}$ with SD $3.3 \mathrm{~L}$ and 95\% LoA - 6.2 L-7.2 L. BCM measured one hour apart gave a mean difference of $0.3 \mathrm{~L}$, SD 1.1L, and 95\% LoA - 4.1 L-3.6 L. Figure 1. Outcomes of total body water and raw data in the form of impedance show similar wide $95 \%$ LoA for both devices. No systematic bias was observed. 


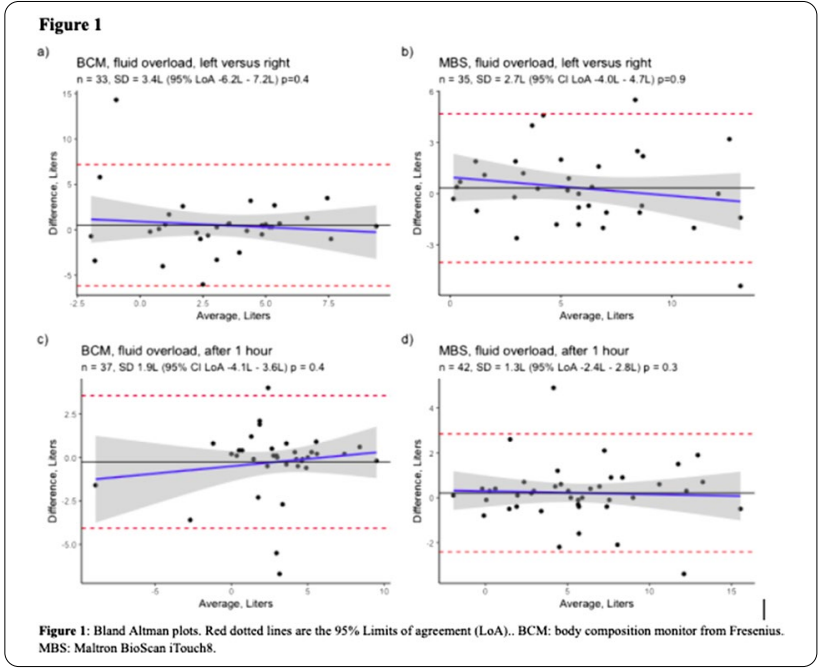

Table 1 Baseline

n

\section{Demographics}

$\begin{array}{lr}\text { Gender, male (\%) } & 47 \% \\ \text { Race } & 100 \% \text { caucasien } \\ \text { Age, years } & 71(63-78) \\ \text { Weight, kg } & 81(72-90) \\ \text { Height, metres } & 1.7(1.6-1.8) \\ \text { BMI } & 27(24-31)\end{array}$

\section{IIIness severity}

\section{Site of infection:}

Pulmonary: $55 \%$
Abdominal: $27 \%$
Other $18 \%$
$58(32-76)$
$10(8-13)$
$76 \%$
$14 \%$
$160(99-268)$
$171(95-257)$
$5.5(2.3-9.0)$
$4.2(2.7-6.2)$

SAPS 3

SOFA

Mechanical ventilation

Hemodialysis

CRP, mg/L

Creatinine

Cumulative fluid balance, $\mathrm{L}$

IV fluids last $24 \mathrm{hrs}, \mathrm{L}$

Table 1 if otherwise not stated results are given as median (inter quartile range).

Conclusion. We found that BIA on ICU patients with septic shock for a maximum of $24 \mathrm{~h}$ had a high degree of agreement, but with wide $95 \%$ LoA on all outcomes. Therefore we conclude that BIA measured fluid status is not sufficiently accurate in this population and setting.

Reference(s)

1. 1. Rhodes A, Evans LE, Alhazzani W, Levy MM, Antonelli M, Ferrer R, et al. Surviving Sepsis Campaign: International Guidelines for Management of Sepsis and Septic Shock: 2016. Intensive Care Med. 2017 Mar:43(3):304-77.

2. 2. Larsson A f., Rubertsson S, Perner A. Intensiv medicin. Kbh.: FADL; 2008. 730 sider, illustreret i farver.

3. 3. Mulasi U, Kuchnia AJ, Cole AJ, Earthman CP. Bioimpedance at the bedside: current applications, limitations, and opportunities. Nutr Clin Pract. 2015 Apr;30(2):180-93.

4. 4. Rochwerg B, Cheung JH, Ribic CM, Lalji F, Clarke FJ, Gantareddy S, et al. Assessment of Postresuscitation Volume Status by Bioimpedance Analysis in Patients with Sepsis in the Intensive Care Unit: A Pilot Observational Study. Can Respir J. 2016;2016:8671742.

5. 5. Dewitte A, Carles P, Joannes-Boyau O, Fleureau C, Roze H, Combe C, et al. Bioelectrical impedance spectroscopy to estimate fluid balance in critically ill patients. J Clin Monit Comput. 2016 Apr;30(2):227-33.

6. 6. Jones SL, Tanaka A, Eastwood GM, Young H, Peck L, Bellomo R, et al. Bioelectrical impedance vector analysis in critically ill patients: a prospective, clinician-blinded investigation. Crit Care. 2015 Aug 12;19:290.

7. 7. Samoni S, Vigo V, Reséndiz LIB, Villa G, De Rosa S, Nalesso F, et al. Impact of hyperhydration on the mortality risk in critically ill patients admitted in intensive care units: comparison between bioelectrical impedance vector analysis and cumulative fluid balance recording. Crit Care. 2016 Apr $8 ; 20: 95$

8. 8. Singer M, Deutschman CS, Seymour CW, Shankar-Hari M, Annane D, Bauer M, et al. The Third International Consensus Definitions for Sepsis and Septic Shock (Sepsis-3). JAMA. 2016;315(8):801-10.

9. Grant acknowledgement: Department of anesthesiology and intensive care, Copenhagen University Hospital-North Zealand, Copenhagen Denmark. Department of clinical medicine, University of Copenhagen, Denmark. Maltron and Fresenius made their BIA devices available during inclusion.. This was free of charge. Neither company had influence on design or outcomes of the study.

000993

Association of Red Cell Distribution Width (RDW) in septic patients: A multi-center retrospective analysis

R. Rezar'; D. Dankl²; B. Mamandipoor ${ }^{3}$; V. Osmani ; B. Wernly ${ }^{2}$

${ }^{1}$ Cardiology and Intensive Care, Paracelsus Medical University Salzburg,

Salzburg, Austria; ${ }^{2}$ Anesthesiology and Intensive Care, Paracelsus Medical University Salzburg, Salzburg, Austria; ${ }^{3}$ E-health Research Group, Fondazione Bruno Kessler Research Institute, Trento, Italy

Correspondence: R. Rezar

Intensive Care Medicine Experimental 2021, 9(1): 000993

Introduction. Sepsis is a clinical condition with ongoing high mortality despite advances in modern critical care. Prognostication and risk stratification are challenging, thus simple decision-aiding tools required. The Red Cell Distribution Width (RDW) is a parameter that is given by default in every blood count. It is calculated via the standard deviation of the Mean Corpuscular Volume (MCV) of a patients erythrocytes. Overall, the RDW is an indicator of anisocytosis, and can be altered in various (especially hematological) diseases. Various authors have shown changes in RDW to be associated with different acute and chronic diseases entities. This study aims to show an association of RDW with mortality in patients with sepsis in a large multi-center intensive care cohort.

Methods. In this study, 16,423 patients with sepsis from the multicenter eICU Collaborative Research Database were analyzed retrospectively. RDW was used as primary exposure as a continuous and a dichotomized variable ( $\leq 15 \%$ vs $>15 \%$ ). The primary end point was mortality, whereas different intensive care measures, development of acute kidney injury (AKI), length of stay, and ICU mortality were used as secondary end points. Univariate and multivariable regression analyses were performed to fit regression models with hospital mortality as random effect and RDW as fixed effect and two multivariable models adjusted for the Sequential Organ Failure Assessment (SOFA) and Acute Physiology And Chronic Health 
Evaluation (APACHE) IV scores. Furthermore, a sensitivity analysis was performed and a prediction model for hospital mortality calculated.

Results. Higher RDW ( $>15 \%)$ was associated with higher patient age ( $>65$ years; $57 \%$ vs. $50 \% ; p<0.001)$, higher prevalence of septic shock $(21 \%$ vs. $14 \%$; $p<0.001)$, higher SOFA ( 6 pts. vs. 5 pts. $p<0.001$ ) and APACHE IV scores (69 pts. vs. 60 pts.; $p<0.001)$. Secondary endpoints also occurred significantly more often in this group (mechanical ventilation: $23 \%$ vs. $20 \%, p<0.001$; vasopressors: $35 \%$ vs. $26 \%, p<0.001$; AKI: $7 \%$ vs. $5 \%, p<0.001$ ). RDW was associated as a continuous variable with hospital (aOR $1.1895 \% \mathrm{Cl}$ $1.16-1.20 ; p<0.001)$ and ICU mortality (aOR $1.1695 \% \mathrm{Cl} 1.14-1.18$; $\mathrm{p}<0.001$ ), a finding that remained significant after adjustment for SOFA- and APACHE IV-score. Also, as a dichotomized variable (RDW $>15 \%)$, there was a significant association with hospital mortality ( $20 \%$ vs. $9 \%$; aOR $2.6395 \% \mathrm{Cl} 2.38-2.90 ; \mathrm{p}<0.001)$ even after multivariable adjustment. This finding remained significant in the sensitivity analysis. Regarding the prediction model, the area under the curve (AUC) for RDW alone was $0.65(95 \% \mathrm{Cl} 0.64-0.66)$, and thus lower than for SOFA- and APACHE-IV scores (AUC 0.76 vs. 0.77). Yet, in a combined model with baseline lactate a comparable predictive value (AUC $0.75 ; p=0.05$ ) was shown. The optimal cut-off value for RDW as predictor of hospital mortality was found to be $16 \%$.

Conclusion. In this retrospective multi-center study, a higher RDW was shown to be associated hospital mortality in septic patients. This finding remained persistent after multivariable regression analysis and extensive sensitivity analysis. RDW is a simple, easy-to-obtain, ubiquitously available, and inexpensive tool that can be used early as a risk predictor in septic patients. A combination with lactate can further increase its predictive value and thus even compete with the much more complex SOFA- and APACHE-IV scores.

\section{7}

Monitoring of the procalcitonine in burned patients: Does it reduce the duration of the antibiotic treatment?

N. Ben Slimene ; A. Mokline ; S. Zarrouk'; H. Fredj ${ }^{1}$; M. Ben Saad ${ }^{1}$; AA.

Messadi $^{1}$

${ }^{1}$ Burn intensive care unit, Centre De Traumtologie Et Des Grands Brulés,

Ben Arous, Tunisia

Correspondence: N. Ben Slimene

Intensive Care Medicine Experimental 2021, 9(1): 001037

Introduction. The procalcitonin, a precursor of calcitonin, has been most widely studied to guide antibiotic prescription.

Objectives. The objective of this study was to evaluate the efficacity of procalcitonine-guided antibiotic discontinuation in burned septic patients to reduce the duration of antibiotic treatment.

Methods. We conducted a retrospective and analytic study in Intensive Burn Care Department in the Burn and Trauma Center of Ben Ben Arous, including septic patients. Patients were randomly assigned to a procalcitonin group in which stopping or changing the antibiotics was based on the procalcitonin levels, and a control group. The primary end-point was antibiotic duration. Hospital mortality and ICU stays were also studied.

Results. The two groups (60 patients in the procalcitonin group and 60 patients in the control group) had similar demographic and clinical characteristics. In the per-protocole analysis, the median duration of antibiotic treatment for sepsis was 4 days shorter in the procalcitonin group (5.51 days in the PCT group vs 9.14 days in the control group, $\mathrm{p}=0.027)$.

ICU stays were shorter in the procalcitonin group with a tendency to a significant difference between the two groups $(p=0.053)$.

However, hospital mortality was the same in both groups.

Conclusion. Procalcitonin-guided antibiotic discontinuation in burned septic patients could reduce the duration of antibiotic use but its impact on survival remains less certain.
001041

Frequency, baseline risk distribution and outcome in septic patients with severe hyperlactatemia in a retrospective multi-center analysis

R. Rezar' ; C. Seelmaier'; V. Osmani²; B. Mamandipoor ${ }^{2}$; B. Wernly

${ }^{1}$ Cardiology and Intensive Care, Paracelsus Medical University Salzburg, Salzburg, Austria; ${ }^{2}$ E-health research group, Fondazione Bruno Kessler

Research Institute, Trento, Italy; ${ }^{3}$ Anesthesiology and intensive care, Paracelsus Medical University Salzburg, Salzburg, Austria

Correspondence: R. Rezar

Intensive Care Medicine Experimental 2021, 9(1): 001041

Introduction. Where lactate comes from and where it goes is not always clear in every individual case. The original concept of tissue hypoxia does not always apply, nor is it by itself explanatory, as it is a too simple approach for a likewise complex dynamic. Especially in sepsis many vital processes like respiratory and perfusion dynamics, renal integrity, balance of coagulation and of course immunomodulatory pathways are simultaneously impaired. Here, a precise assignment of which problem is leading is often not possible. Nevertheless, in many cases hyperlactatemia is present, which is influenced by all these processes. Elevated lactate concentrations and also a limited negative delta-lactate have been correlated multiply in various acute disease entities in the past, whereby lactate is of particular importance in the assessment of the course and prognosis of sepsis. Excessive mortality rates have been shown in patients with extreme hyperlactatemia. This retrospective analysis from the large-scale elCU database is intended to provide further evidence here.

Methods. A total of 10,724 septic patients from the multicenter elCU Collaborative Research Database were included in this analysis. Patients were divided into three groups, namely: (1) lactate $<5 \mathrm{mmol} / \mathrm{l}$, (2) $\geq 5 \mathrm{mmol} / \mathrm{l}$ and $<10 \mathrm{mmol} / \mathrm{l},(3) \geq 10 \mathrm{mmol} / \mathrm{l}$. The primary endpoint was hospital mortality, secondary endpoints were vasopressor use, mechanical ventilation, ICU mortality and acute kidney injury (AKI). A multilevel logistic regression analysis was performed; in the baseline model, hospital was used as a random effect; the second model was adjusted for Acute Physiology And Chronic Health Evaluation (APACHE) IV score. Furthermore, a stratified sensitivity analysis was performed.

Results. In the following, continuous data are given as median values. Patients in the maximum lactate group tended to be younger (65 vs. 67 yrs. in both other groups, $p=0.041$ ), whereas no difference was found in respect of sex (male sex; group 3: 48\%; group 2: $52 \%$; group 1: $51 \%, p=0.39$ ). Regarding ethnicity, more patients in the severe hyperlactatemia group were African American (12 vs. 11 vs. $9 \%, p=0.030$ ), and less patients Caucasian ( 75 vs. 75 vs. $80 \%$, $p=0.030$ ). Individuals with higher lactate values showed higher sequential organ failure assessment (SOFA; 12 vs. 10 vs. 6 pts., $\mathrm{p}<0.001)$ and APACHE IV scores $(113$ vs. 90 vs. 66 pts., $p<0.001)$. Furthermore, hemoglobin values ( 9.7 vs. 10.5 vs. $10.3 \mathrm{~g} / \mathrm{dl}, \mathrm{p}<0.001$ ) were lower in group 3 compared with the other two groups. Thrombocyte counts ( 137 vs. 150 vs. $183 \mathrm{G} / \mathrm{l}, \mathrm{p}<0.001$ ) were lower in group 3 and 2 compared with group 1, whereas a trend but no statistically significant difference was found for higher leucocyte counts in groups 3 and 2 ( 14.1 vs. 14.1 vs. $13.3 \mathrm{G} / \mathrm{l}, \mathrm{p}=0.063$ ). Also, serum creatinine was higher in the higher lactate groups ( $2.8 \mathrm{vs} .2 .1 \mathrm{vs.} 1.3 \mathrm{mg} /$ $\mathrm{dl}, \mathrm{p}<0.001)$. In patients with severe hyperlactatemia significantly more patients had a gastrointestinal $(24$ vs. 20 vs. $13 \%, p<0.001)$ or unknown focus ( 18 vs. 13 vs. $11 \%, p<0.001$ ) of infection than in the other groups. Significant differences were observed for the primary ( 79 vs. 43 vs. $13 \%, p<0.001$ ), as well as all secondary endpoints (ICU mortality: 70 vs. 32 vs. $8 \%, p<0.001$; septic shock: 68 vs. 52 vs. $13 \%$, $p<0.001$; mechanical ventilation: 59 vs. 41 vs. $22 \%, p<0.001$; vasopressor use: 69 vs. 54 vs. $33 \%, p<0.001$; AKI: 22 vs. 14 vs. $6 \%$; ICU length of stay: 30 vs. 68 vs. 59 h.; all $p<0.001$ ). When comparing survivors and non-survivors in the severe hyperlactatemia group, they were younger ( 60 vs. 66 yrs., $p<0.001$ ), showed lower APACHE IV (98 vs. 116 pts., $p<0.001$ ) but not SOFA scores (both 12 pts., $p=0.22$ ) tended to have lower serum creatinine ( $2.6 \mathrm{vs} .2 .8 \mathrm{mg} / \mathrm{dl}, \mathrm{p}=0.46)$ but higher Hb-values (10.2 vs. $9.6 \mathrm{~g} / \mathrm{dL}, \mathrm{p}=0.052$ ). No difference was 
found for leucocyte counts ( 14.1 vs. $14.2 \mathrm{G} / \mathrm{l}, \mathrm{p}=0.095$ ), but again lower platelet counts were observed in non-survivors (128 vs. 156 $G / I, p=0.020$ ). In this subgroup analysis no difference was shown for ethnicity $(p=0.92)$ or focus of infection $(p=0.14)$. For all secondary endpoints except from ICU length of stay (survivors: $117 \mathrm{~h} . ;$ survivors: $26 \mathrm{~h} ., \mathrm{p}<0.001$ ) only trends but no statistically significant differences were observed.

Conclusion. This analysis of a large multi-center cohort of patients with sepsis shows excess mortality in patients with severe hyperlactatemia (lactate $>10 \mathrm{mmol} / \mathrm{l}$ ). The detailed subgroup- characterization is intended to be thesis-generating for future studies.

\section{5}

Analysis of the results after the implementation of the ultrasound-guided postmortem biopsy protocol of COVID 19 patients

G. Armenta López ; AA. Barrero ${ }^{\text {; } ~ J L . ~ G a r c i ́ a-G a r m e n d i a ² ; ~ F C . ~ M a r t i n e z ~}{ }^{1}$

${ }^{1}$ Intensive Care, Hospital San Juan de Dios del Aljarafe, Seville, Spain; ${ }^{2} \mathrm{cu}$, Hospital San Juan de Dios del Aljarafe, Bormujos, Sevilla, Spain

Correspondence: $\mathrm{G}$. Armenta López

Intensive Care Medicine Experimental 2021, 9(1): 001175

Introduction. Given the biological risk involved in performing autopsies in patients with SARS-COV-2 infection can only be carried out in certain centers. Due to the interest in obtaining postmortem studies of this new disease, we carry out sampling of different organs, guided by ultrasound.

Objectives. To analyze the results of a protocol of multiple ultrasound-guided post-mortem biopsies (echopsy) in COVID-19 patients.

Methods. Informed consent was requested from the family and the pertinent security measures were taken and authorized by the ethics committee. The demographic characteristics of the patients, the histological samples sought, and the histological characteristics related to the evolution of the case are described. Data are shown as mean and percentages.

Results. All the families questioned agreed to carry out the echopsy. The technique delayed the transfer of the corpse $90 \mathrm{~min}$. Samples were obtained from 12 patients, of which $82 \%$ were male with a mean age of 64.54 years and 38.1 days of stay in the ICU. They were 33.54 days in Mechanical Ventilation. 100\% were treated with high doses of corticosteroids and $80 \%$ were anticoagulated.

As a result, 27 lung samples were obtained, the findings of which we show in Table 1.14 liver samples, 7 of muscle, 5 of heart, 4 kidney and 1 thyroid were also studied. We observed: nonspecific inflammatory myopathy, myocarditis, sinusoidal lymphocytic infiltrate in the liver of uncertain origin and absence of relevant findings in renal and thyroid samples.

Table 1. Lung biopsy findings

\begin{tabular}{lc}
\hline Findings & No of samples (N: 27) \\
\hline Diffuse alveolar damage & $15(55,5 \%)$ \\
Aspergillomas & $3(11 \%)$ \\
Acute penumonia & $2(7,40 \%)$ \\
Obstructive pneumonia & $18(66,6 \%)$ \\
Fibroblastic bodies & $8(29,62 \%)$ \\
Microthrombi & $14(60 \%)$ \\
Fibrosis & $23(85 \%)$ \\
Pneumocyte hyperplasia & $11(40,74 \%)$ \\
\hline
\end{tabular}

Conclusion. The echopsy performed in the ICU is a safe, fast and more acceptable alternative for the family. The results may be relevant for a better understanding of SARS-COV2.

\section{Reference(s)}

1. $\mathrm{s}$

001195

Difference in the inflammatory response of COVID-19 positive patients in ICU between the first and second waves of the COVID-19 pandemic

W. Tuckwell ${ }^{1}$; E. Harte ${ }^{1}$; N. Wetherall ${ }^{1}$; S. Ramachandran ${ }^{1}$; S. Price ${ }^{1} ; H$.

Breen ${ }^{1}$; O. Richards' ${ }^{1}$;. Cheema ${ }^{1}$; C. Killick'; C. King ${ }^{1}$; T. Szakmany

${ }^{1}$ Department of Anaesthesia, Intensive Care and Pain Medicine, Cardiff

University, Cardiff, United Kingdom

Correspondence: T. Szakmany

Intensive Care Medicine Experimental 2021, 9(1): 001195

Introduction. Elevated levels of C-reactive protein (CRP) and Procalcitonin (PCT) have been associated with severe COVID-19, which suggests that these can be used as biomarkers for disease prognosis.

Objectives. The objective of this study was to determine if there was a difference in the longitudinal inflammatory response measured by CRP and PCT levels between the first COVID-19 wave and the second COVID-19 wave of ICU patients.

Methods. Single centre retrospective observational study carried out in Aneurin Bevan University Health Board in Wales, UK. Patients were enrolled during the first and second wave of COVID19 pandemic (first wave: 9th March 2020 to 5th June 2020, second wave: 17th November 2020 to 15 th March 2021).

During the study, consecutive patients admitted to ICU were screened daily and recruited to the study if they fulfilled all inclusion criteria: age $\geq 18$-years old; SARS-CoV-2 infection confirmed by positive RT-PCR. Data were collected on patient demographics, laboratory results and management strategies during the ICU stay. Patients were followed-up until discharge from the hospital. The inflammatory response was evaluated using the CRP and PCT levels on day of admission (Day 0), Day 3, 7, 10, 14, 21, 28, respectively.

For statistical analsysis Fisher's exact test, Mann-Whitney $\mathrm{U}$ test and multivariate mixed general linear model was used.

Results. 178 patients were admitted with a positive SARS-CoV-2 PCR test and recruited into the study. 65 patients were admitted during the first wave and 113 patients during the second wave (Table 1).

\begin{tabular}{llll}
\hline Table 1 & $\begin{array}{l}\text { First wave } \\
(\mathbf{n = 6 5 )}\end{array}$ & $\begin{array}{l}\text { Second wave } \\
(\mathbf{n = 1 1 3})\end{array}$ & $\mathbf{p}$-value \\
\hline Age (years) & $57(51-63)$ & $61(53-67)$ & 0.024 \\
Sex (Male/Female, n) & $43 / 22$ & $30 / 83$ & 0.310 \\
Dexamethasone (n, \%) & $14(21.5 \%)$ & $113(100 \%)$ & 0.001 \\
IL-6R. IL-1R inhibitors (n, \%) & $2(3.1 \%)$ & $75(66.4 \%)$ & 0.001 \\
\hline
\end{tabular}

CRP levels showed significant rise on Day 3 and levelling off after Day 7 in the first wave. In the second wave CRP levels were significantly lower on admission and continued to decrease further. Median PCT levels were below the $0.5 \mathrm{ng} / \mathrm{mL}$ threshold on admission in both waves. 

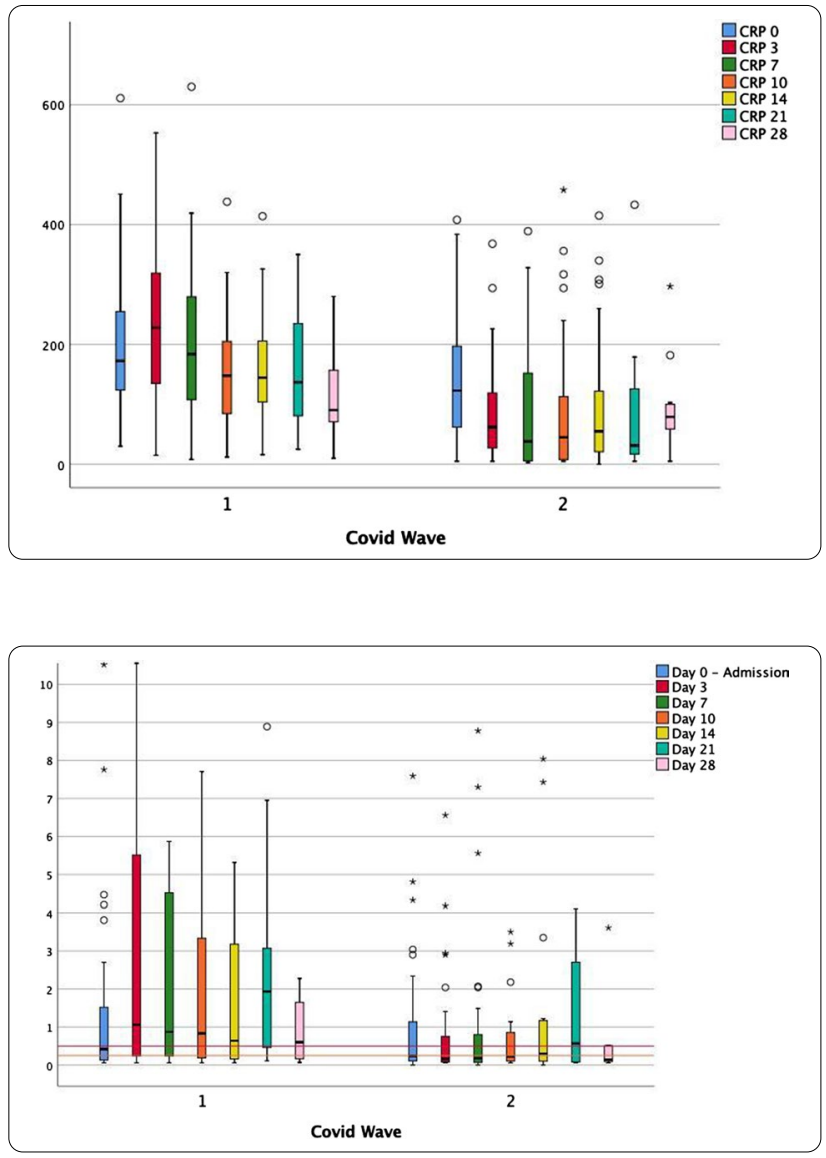

Conclusion. Commonly measured inflammatory markers such as CRP and PCT had markedly different longitudinal kinetics up until day 28 of ICU stay between the first and second wave of the pandemic. It's unclear if these common markers of infections could be effectively used for these purposes in COVID19 patients treated with evidence based therapies.

\section{2}

\section{Gut Lymph Purification in Rats with Acute Lung Injury Caused} by Gut Ischemia Reperfusion Injury

W. Zhang

${ }^{1}$ Critical Care Medicine, Affiliated Hospital of Zunyi Medical University, Zunyi, China

Correspondence: $W$. Zhang

Intensive Care Medicine Experimental 2021, 9(1): 001222

Introduction. It is unclear whether removing the danger-associated molecular patterns (DAMPs) of gut lymph (GL) in the rats of gut ischemia-reperfusion injury (GIRI) model may reduce the distant organ lung injury.

Objectives. To determine whether oXiris gut lymph purification (GLP) may remove the DAMPs of GL in therats' model of acute lung injury (ALI) caused by GIRI.

Methods. The experimental rats were divided into four groups: Sham group, GIRI group, GIRI+gut lymphdrainage (GLD) group, and GIRI+GLP group. After successful modeling, the lung tissue samples of rats ineach group were taken for hematoxylin-eosin (HE) staining and detection of expression levels of apoptoticindexes. The level of DAMPs was detected in blood and lymph. We observed its microstructure of type Ilalveolar epithelial cells (AECII), and detected the expression level of apoptosis indexes.

Results. GIRI-induced destruction of alveolar structure, thickened alveolar walls, inflammatory cell infiltration emerged in the GIRI group, HMGB-1 and IL-6 levels significantly increased, and HSP70 and IL-10 levels reduced in lymph and serum. Compared with GIRI group, the lung tissue damage in GIRI + GLP group significantly improved, the expression level of HMGB-1 and IL- 6 in the lymph and serum reduced, and HSP70 and IL-10 increased. The organelle structure of AECII in $\mathrm{GIRI}+\mathrm{GLP}$ group was significantly improved compared with the GIRI group.

Conclusion. oXiris GLP blocks the key link between DAMPs and mononuclear phagocyte system to inhibit inflammation and cell apoptosis, thereby reducing ALI induced by GIRI.

\section{Reference(s)}

1. 1. Assimakopoulos SF, Triantos C, Thomopoulos K, Fligou F, Maroulis I, Marangos M, Gogos CA. Gut-origin sepsis in the critically ill patient: pathophysiology and treatment. Infection 2018; 46: 751-760. 2. Carrico CJ, Meakins JL, Marshall JC, Fry D, Maier RV. Multiple-organ-failure syndrome. Arch Surg 1986: 121: 196-208. 3. Deitch EA, Xu D, Kaise VL. Role of the gut in the development of injury- and shock induced SIRS and MODS: the gut-lymph hypothesis, a review. Front Biosci 2006; 11: 520-528. 4. Varela JE, Cohn SM, Diaz I, Giannotti GD, Proctor KG. Splanchnic perfusion during delayed, hypotensive, or aggressive fluid resuscitation from uncontrolled hemorrhage. Shock 2003; 20: 476-480. 5. Nagpal R, Yadav H. Bacterial Translocation from the Gut to the Distant Organs: An Overview. Ann Nutr Metab 2017; 71 Suppl 1:11-16. 6. Balzan S, de Almeida Quadros C, de Cleva R, Zilberstein B, Cecconello I. Bacterial translocation: overview of mechanisms and clinical impact. J Gastroenterol Hepatol 2007; 22: 464-471. 7. Zhao W, Zhou S, Yao W, Gan X, Su G, Yuan D, Hei Z. Propofol prevents lung injury after intestinal ischemia-reperfusion by inhibiting the interaction between mast cell activation and oxidative stress. Life Sci 2014; 108: 80-87. 8. Groeneveld AB, Raijmakers PG, Rauwerda JA, Hack $\mathrm{CE}$. The inflammatory response to vascular surgery-associated ischaemia and reperfusion in man: effect on postoperative pulmonary function. Eur J Vasc Endovasc Surg 1997; 14: 351-359. 9. Watson AJ, Hughes KR. TNFa-induced intestinal epithelial cell shedding: implications for intestinal barrier function. Ann N Y Acad Sci 2012; 1258: 1-8. 10. Moon HG, Cao Y, Yang J, Lee JH, Choi HS, Jin Y. Lung epithelial cell-derived extracellular vesicles activate macrophage-mediated inflammatory responses via ROCK1 pathway. Cell Death Dis 2015; 6: e2016. 11. Wohlauer MV, Moore EE, Harr J, Eun J, Fragoso M, Banerjee A, Silliman CC. Cross-transfusion of postshock mesenteric lymph provokes acute lung injury. J Surg Res 2011; 170: 314-318. 12. Zhang YM, Zhang SK, Cui NQ. Intravenous infusion of mesenteric lymph from severe intraperitoneal infection rats causes lung injury in healthy rats. World J Gastroenterol 2014; 20: 4771-4777. 13. Zhao ZG, Zhu HX, Zhang LM, Zhang YP, Niu CY. Mesenteric lymph drainage alleviates acute kidney injury induced by hemorrhagic shock without resuscitation. ScientificWorldJournal 2014; 2014: 720,836. 14. Sambol JT, Lee MA, Caputo FJ, Kawai K, Badami C, Kawai T, Deitch EA, Yatani A. Mesenteric lymph duct ligation prevents trauma/hemorrhage shock-induced cardiac contractile dysfunction. Journal of applied physiology (Bethesda, Md: 1985) 2009; 106: 57-65. 15. Sambol JT, White J, Horton JW, Deitch EA. Burn-induced impairment of cardiac contractile function is due to gut-derived factors transported in mesenteric lymph. Shock 2002; 18: 272-276. 16. He GZ, Zhou KG, Zhang R, Wang YK, Chen XF. Impact of intestinal ischemia/reperfusion and lymph drainage on distant organs in rats. World J Gastroenterol 2012; 18: 7271-7278. 17. Zhang Y, Zhang S, Tsui N. Mesenteric lymph duct drainage attenuates acute lung injury in rats with severe intraperitoneal infection. Inflammation 2015; 38: 1239-1249. 18. Tong H, Chen R, Yin H, Shi X, Lu J, Zhang M, Yu B, Wu M, Wen Q, Su L. Mesenteric Lymph Duct Ligation Alleviating Lung Injury in Heatstroke. Shock 2016; 46: 696-703. 19. Zhang LM, Jiang L, Zhao ZG, Niu CY. Mesenteric lymph duct ligation after hemorrhagic shock enhances the ATP level and ATPase activity in rat kidneys. Ren Fail 2014; 36: 593-597. 20. de Jong PR, González-Navajas JM, Jansen NJ. The 
digestive tract as the origin of systemic inflammation. Crit Care 2016; 20: 279. 21. Druml W. [Intestinal cross-talk: The gut as motor of multiple organ failure]. Med Klin Intensivmed Notfmed 2018; 113: 470-477. 22. Tolle LB, Standiford TJ. Danger-associated molecular patterns (DAMPs) in acute lung injury. J Pathol 2013; 229: 145-156. 23. Vourc'h M, Roquilly A, Asehnoune K. Trauma-Induced Damage-Associated Molecular PatternsMediated Remote Organ Injury and Immunosuppression in the Acutely III Patient. Front Immunol 2018; 9: 1330. 24. Li Y, Cao Y, Xiao J, Shang J, Tan Q, Ping F, Huang W, Wu F, Zhang H, Zhang X. Inhibitor of apoptosisstimulating protein of $\mathrm{p} 53$ inhibits ferroptosis and alleviates intestinal ischemia/reperfusion-induced acute lung injury. Cell Death Differ 2020; 27: 2635-2650. 25. Livak KJ, Schmittgen TD. Analysis of relative gene expression data using real-time quantitative PCR and the 2(-Delta Delta C(T)) Method. Methods 2001; 25: 402-408. 26. Li Y, Feng D, Wang Z, Zhao Y, Sun R, Tian D, Liu D, Zhang F, Ning S, Yao J, Tian X. Ischemia-induced ACSL4 activation contributes to ferroptosis-mediated tissue injury in intestinal ischemia/reperfusion. Cell Death Differ 2019; 26: 2284-2299. 27. Liu J, Chen T, Lei P, Tang X, Huang P. Exosomes Released by Bone Marrow Mesenchymal Stem Cells Attenuate Lung Injury Induced by Intestinal Ischemia Reperfusion via the TLR4/NF-KB Pathway. Int I Med Sci 2019; 16: 1238-1244. 28. Andersen K, Kesper MS, Marschner JA, Konrad L, Ryu M, Kumar Vr S, Kulkarni OP, Mulay SR, Romoli S, Demleitner J, Schiller P, Dietrich A, Müller S, Gross O, Ruscheweyh HJ, Huson DH, Stecher B, Anders HJ. Intestinal Dysbiosis, Barrier Dysfunction, and Bacterial Translocation Account for CKD-Related Systemic Inflammation. J Am Soc Nephrol 2017; 28: 76-83. 29. Zeni F, Freeman B, Natanson C. Anti-inflammatory therapies to treat sepsis and septic shock: a reassessment. Crit Care Med 1997; 25: 1095-1100. 30. Liu H, Zhao ZG, Xing LQ, Zhang LM, Niu CY. Postshock mesenteric lymph drainage ameliorates cellular immune function in rats following hemorrhagic shock. Inflammation 2015; 38: 584-594.

2. This study was supported by the Science and Technology Support Plan of Guizhou Province in 2019 (Qian Ke He Support [2019] 2834), the Science and Technology Plan of Guizhou Province in 2020 (Foundation of Guizhou Science and Technology Cooperation [2020]1Z061), the National Natural Science Foundation of China No. 82001156, and the Education and Teaching Reform Project of Zunyi Medical University.

\section{7}

Neuroinflammatory biomarkers in critically ill patients with COVID-19

S. Kokkoris ${ }^{1}$; E. Stamataki '; G. Emmanouil'; C. Psachoulia' ${ }^{1}$ A. Maragouti';

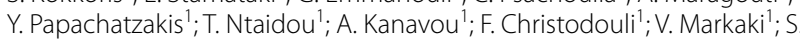
Malachias ${ }^{1}$; K. Glynos ${ }^{1}$; A. Kotanidou'; C. Routsi

'Department of Intensive Care, Evangelismos Hospital, Medical School, National and Kapodistrian University of Athens, Athens, Greece

Correspondence: $C$. Routsi

Intensive Care Medicine Experimental 2021, 9(1): 001237

Introduction. Coronavirus disease 2019 (COVID-19) caused by the severe acute respiratory syndrome coronavirus 2 (SARS-CoV-2) causes not only pneumonia leading to acute respiratory failure but also may affect several organs, including the brain, especially in patients with severe clinical manifestations (1). S100 calcium binding protein B (S100B) and neuron-specific enolase (NSE) are known serum biomarkers of neurologic injuries and they have been found elevated in sepsis related encephalopathy (2). However, information concerning their possible implication in the course of severe COVID-19 so far is lacking. Objectives. We evaluated whether S100 B and NSE are increased in patients with severe COVID-19 and their correlation with other variables and with clinical outcome.

Methods. In critically ill patients admitted to the ICU with acute respiratory failure due to confirmed COVID-19, blood samples were obtained within $24 \mathrm{~h}$ of their ICU admission to measure serum S-100B and NSE as well as interleukin-6 (IL-6). APACHE II and SOFA scores were recorded in all patients. Spearman's correlations were performed between S100B, NSE, IL- 6 and other routine laboratory and clinical variables. Patients with known neurologic conditions were excluded. Results. Fifty-two critically ill, mechanically ventilated patients (mean age $65 \pm 14$ years, 40 males) were included. S-100B and NSE levels were increased (above the laboratory cutoff of $0.14 \mu \mathrm{g} / \mathrm{L}$ and $15 \mu \mathrm{g} / \mathrm{L}$ respectively) in $21 / 52(40 \%)$ and $44 / 52$ (85\%) patients, respectively. $\mathrm{S} 100 \mathrm{~B}$ value was higher in the presence of shock compared to that without [0.15 (IQR 0.14) vs 0.10 (IQR 0.11), $p=0.023]$. There was a positive correlation between S100B and NSE $(r=0.283, p=0.047)$, IL-6 $(r=0.35, p=0.027)$, arterial lactate $(r=0.565, p=0.000)$, HCO3 $(-0.31$, $p=0.048)$, SOFA score $(r=0.39, p=0.04)$ and APACHE II score $(r=0.40$, $p=0.03$ ), whereas a correlation between NSE and any of these parameters was not observed. IL- 6 was significantly correlated with C-reactive protein $(r=0.349, p=0.025)$ and SOFA score $(r=0.419, p=0.06)$. Higher S-100B levels were associated with higher ICU mortality [0.15 (IQR 0.51) versus 0.11 (IQR 0.13), $\mathrm{p}=0.034$ ].

Conclusion. Biomarkers of neurologic injury are frequently increased in critically ill patients with acute respiratory failure due to COVID-19, in the absence of an apparent brain damage. They are positively associated with the presence of shock, with inflammatory indices, with markers of tissue perfusion and with more severe organ dysfunction. Studies to examine whether these biomarkers are associated with specific neurological findings are needed.

\section{Reference(s)}

1. 2.Nguyen DN et al. Elevated serum levels of S-100B protein and neuronspecific enolase are associated with brain injury in patients with severe sepsis and septic shock. Crit Care Med 2006; 34:1967-1974

2. 1. Vargas $\mathrm{G}$ et al. Severe acute respiratory syndrome coronavirus 2 (SARS-CoV-2) and glial cells: Insights and perspectives. Brain, Behavior, \& Immunity-Health 2020, 100,127

\section{2}

The temporal evolution of laboratory characteristics in critically ill patients with COVID-19

S. Kokkoris'; A. Kanavou'; D. Katsaros ${ }^{1}$; S. Karageorgiou'; A. Maragouti ${ }^{1}$;

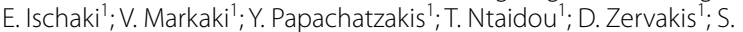

Zakynthinos ${ }^{1}$; A. Kotanidou' ${ }^{1}$ C. Routsi ${ }^{1}$

${ }^{1}$ Department of Intensive Care, Evangelismos Hospital, Medical School, National and Kapodistrian University of Athens, Athens, Greece

Correspondence: $C$. Routsi

Intensive Care Medicine Experimental 2021, 9(1): 001252

Introduction. Severe COVID-19 caused by the SARS-CoV-2 is mainly characterized by acute hypoxemic respiratory failure, requiring admission to the intensive care unit (ICU), frequently followed by sepsis and multi-organ failure. Certain laboratory findings including typical hematological abnormalities and impaired inflammatory and other biomarkers have been identified. While much information has been given concerning the clinical features over the course of the disease during the ICU stay, the available information on the evolution of laboratory abnormalities over time is limited.

Objectives. To present the temporal evolution of the most important laboratory findings during the course of the disease in critically ill patients with severe COVID-19.

Methods. We recorded retrospectively the main laboratory data, which are routinely daily tested, from all confirmed COVID-19 patients with acute hypoxemic respiratory failure, consecutively admitted to our ICU between March 19, 2020 and April 10, 2021, during their course of critical illness. For analysis, laboratory values of lymphocytes, platelets, fibrinogen, $\mathrm{C}$ - reactive protein (CRP), lactate dehydrogenase (LDH) and serum albumin at Days 1, 3, 5, 10, 15 and 21 post ICU admission were used; they were compared between survivors and non-survivors with repeated measures ANOVA.

Results. A total of 410 patients [mean $( \pm S D)$ age $66 \pm 14$ years, SOFA score $6 \pm 3$, APACHE II score $15 \pm 6,70 \%$ males] were included. Crude ICU mortality was $45 \%$. On ICU admission, the most frequent laboratory abnormalities were lymphopenia (lymphocyte count $\leq 1,100$ cells $/ \mu \mathrm{L}$ ) in $70 \%$ of the patients and elevated serum inflammatory markers i.e., CRP and fibrinogen $[14( \pm 10) \mathrm{mg} / \mathrm{dl}$ and $609( \pm 172)$ 
$\mathrm{mg} / \mathrm{dl}$, respectively] and elevated lactate dehydrogenase (LDH) [549 $( \pm 506) \mathrm{IU} / \mathrm{L}]$. Although abnormal, initial laboratory parameters did not differ significantly between survivors and non-survivors.

Over the follow up period of 21 days, lymphocyte count was further decreased on Day 3, though non-significant difference was observed between the two groups. Platelets, significantly were decreased over time in non survivors. CRP was significantly decreased on Day $3 \mathrm{com}-$ pared to the admission value in survivors; also, compared to nonsurvivors, CRP levels were significantly lower in survivors over time. Fibrinogen levels further increased after the 5th Day in non-survivors, whereas LDH values did not change significantly either over time or between the groups of survivors and non-survivors. Albumin levels significantly decreased over time, but significantly more in non-survivors during the course of the illness.

Conclusion. Among critically ill patients with severe COVID-19, the inflammatory markers and other typical laboratory abnormalities on ICU admission remain abnormal over the ICU course in both survivors and non-survivors and further deteriorate in non-survivors.

\section{3}

Cumulative fluid Balance Impact on Mortality in Septic Shock: A Retrospective Observational Study

SB. Mishra

${ }^{1}$ Department of Critical Care Medicine, IMS and SUM Hospital, Bhubaneswar, India

Correspondence: S.B. Mishra

Intensive Care Medicine Experimental 2021, 9(1): 001253

Introduction. Previous studies demonstrated that extensive fluid loading and consequently positive fluid balances during sepsis resuscitation are associated with adverse outcome. The association isn't established yet.

Objectives. We wanted to see the impact of fluid balance in the first seven days on mortality in septic shock.

Methods. A retrospective observational cohort study.Medical ICU of one university-affiliated hospital in Bhubaneswar, Odisha, India. Adult patients admitted with septic shock who survived for at least 7 days were included over a period of 2 years. Cumulative Fluid balance was calculated in the first seven days.

Results. Total 2356 patients were screened out of which 710 were included in the trial. 131 patients died. The mortality rate was $18.45 \%$. Mixed-effects logistic regression modelling, adjusted for possible confounders, showed that cumulative fluid balance in the 7 days was an independent predictor of ICU mortality: odds ratio 4.23 (2.90-5.33) per $10 \mathrm{~mL} / \mathrm{kg}$ increase in daily fluid balance.

Conclusion. A higher fluid balance in the first 7 days after the septic shock was associated with increased mortality. This shows the importance of implementing negative fluid management strategies after initial hemodynamic resuscitation. Prospective interventional studies are needed to confirm our results.

\section{Reference(s)}

1. van Mourik N, Geerts BF, Binnekade JM, Veelo DP, Bos LDJ, Wiersinga WJ, van der Poll T, Cremer OL, Schultz MJ, Vlaar APJ. A Higher Fluid Balance in the Days After Septic Shock Reversal Is Associated With Increased Mortality: An Observational Cohort Study. Crit Care Explor. 2020 Sep 23;2(10):e0219. https://doi.org/10.1097/CCE.0000000000000219. PMID: 33063027: PMCID: PMC7523767. Boyd JH, Forbes J, Nakada TA, Walley KR, Russell JA. Fluid resuscitation in septic shock: a positive fluid balance and elevated central venous pressure are associated with increased mortality. Crit Care Med. 2011 Feb;39(2):259-65. https://doi.org/10.1097/CCM Ob013e3181feeb15. PMID: 20975548.

2. All Our Nursing Staff.
001268

Association between left ventricular systolic function parameters and myocardial injury, organ failure and mortality in critically ill patients with septic shock

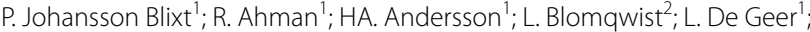
J. Engvall'; M. Astrom Aneq ${ }^{3}$; M. Chew

Department of Anaesthesiology and Intensive Care, Biomedical and Clinical Sciences, Linköping University, Linköping, Sweden; ${ }^{2}$ Department of Anaesthesiology and Intensive Care, Skåne University Hospital Malmö, Malmö, Sweden; ${ }^{3}$ Department of Clinical Physiology, Biomedical and Clinical Sciences, Linköping University, Linköping, Sweden

Correspondence: P. Johansson Blixt

Intensive Care Medicine Experimental 2021, 9(1): 001268

Introduction. Recent studies indicate that left ventricular longitudinal strain (LVLS) may be a more sensitive indicator of deteriorating left ventricular systolic function than traditional echocardiographic parameters such as left ventricular ejection fraction (LVEF) and may be associated with poorer outcomes $(1,2)$. Newer parameters such as mitral annular plane systolic excursion (MAPSE) and left ventricular longitudinal wall fractional shortening (LWFS) have been sparsely investigated $(3,4)$.

Objectives. Evaluate the association between 5 traditional and novel echocardiographic parameters of LV systolic function (LVEF, peak tissue Doppler velocity at the mitral valve (s'), LVLS, MAPSE and LWFS) and clinical outcome in patients admitted to ICU with septic shock. The primary outcome was myocardial injury, defined as $\mathrm{hsTnT} \geq 45 \mathrm{ng} / \mathrm{L}$. Secondary outcomes were organ-support free days, 30 - and 180-day mortality.

Methods. 139 TTE studies from 3 echocardiographic data repositories of patients admitted to ICU with septic shock were included of which 91 were suitable for LVLS analysis using speckle tracking Measurements were made within $12 \mathrm{~h}$ of study enrollment. The relationships between the echocardiographic parameters and clinical outcomes were evaluated using Mann-Whitney tests, binary and linear regression as well as multivariable analysis.

Results. LVLS, MAPSE and LWFS but not LVEF or $s^{\prime}$ were associated with myocardial injury. This relationship remained after adjustment for APACHE II score, preexisting cardiovascular disease and age. None of the systolic function parameters were associated with organ-support free days or mortality at 30 or 180 days.

Conclusion. All LV systolic function echocardiographic parameters except LVEF and s' were associated with myocardial injury. However this did not translate to an association with clinical outcomes such as the need for organ support and short- and mid-term mortalities.

\section{Reference(s)}

1. 1. Dalla K, Hallman C, Bech-Hanssen O, Haney M, Ricksten S-E. Strain echocardiography identifies impaired longitudinal systolic function in patients with septic shock and preserved ejection fraction. Cardiovasc Ultrasound 2015:13:30

2. 2. Sanfilippo F, Corredor C, Fletcher N, Tritapepe L, Lorini FL, Arcadipane A et al. Left ventricular systolic function evaluated by strain echocardiography and relationship with mortality in patients with severe sepsis or septic shock: a systematic review and meta-analysis. Crit Care 2018;22:183

3. 3. Bergenzaun L, Ohlin H, Gudmundsson P, Willenheimer R, Chew MS. Mitral annular plane systolic excursion (MAPSE) in shock: a valuable echocardiographic parameter in intensive care patients. Cardiovasc Ultrasound 2013:11:16

4. 4. Johansson Blixt P, Chew MS, Åhman R, de Geer L, Blomqwist L, Åström Aneq $M$, Engvall J, Andersson $\mathrm{H}$. Left ventricular longitudinal wall fractional shortening accurately predicts longitudinal strain in critically ill patients with septic shock. Ann Intensive Care 2021;11:52

5. Linköping University LIO-900161.

6. Linköping University LIO-935252.

7. Region Östergötland ALF grant SC-2019-00,155-12. 
001272

Epidemiology of sepsis and sepsis mortality in a pediatric intensive care unit

T. Tavladaki ${ }^{1}$; A. Spanaki ${ }^{2}$; E. Vasilaki ${ }^{3}$; E. Geromarkaki ${ }^{3}$; M. Miliaraki ${ }^{1}$; E. Dardamani4 ${ }^{4}$ : S. $\|$ lia ${ }^{2}$

${ }^{1}$ Picu, University Hospital, Medical School, University of Crete, Heraklion, Greece; ${ }^{2}$ Pediatric Intensive Care Unit, University Hospital, Medical School, University of Crete, Heraklion, Greece; ${ }^{3} \mathrm{Picu}$, University of Crete, Heraklion, Greece; ${ }^{4}$ Paediatric Intensive Care Unit, University General Hospital of Heraklion, Heraklion, Greece

Correspondence: $T$. Tavladaki

Intensive Care Medicine Experimental 2021, 9(1): 001272

Introduction. Sepsis has been known as a serious condition since the time of Hippocrates, the debate over what sepsis represents and how it should be delimited continues to this day.

Objectives. The purpose of our study was to determine the epidemiology of sepsis and death from that in Pediatric Intensive Care Unit (PICU).There are few other studies evaluating the sepsis mortality in PICUs in our country.

Methods. In the observational study we retrospectively identified six pediatric patients died during hospitalization in the PICU during the last five years (1st January 2016-31st March 2021).The study was conducted in our seven bed pediatric Intensive Care Unit in an urban university hospital responsible for children not only from our hospital but from all the Southern islands of our country. The data collected include demographic profile, diagnosis of sepsis and cause of death.

Results. From 758 PICU admissions, during this five years period, 213 were day care clinic patients and were excluded. Of the 545 remaining admissions, 29 died (5,32\%). The leading causes of death were sepsis $(31 \%)$,trauma $(17 \%)$, neurological disorders (17\%), and malignancy (14\%).As considering septic patients median age was 6 years. Female had higher preponderance $58 \%$ compared to male $42 \%$.Infant presented sepsis in 12,5\%, children preschool and school children $70 \%$ and adolescence the rest $14 \%$. Mortality rate was $16 \%$; infants comprising $50 \%$ and adolescence the rest $50 \%$. The majority of adolescence died had malignancy as underling disease.

Conclusion. Septic shock is the leading cause of death in pediatric population. Impressingly although precschool and school children present sepsis more frequently infants and adolescence especially due to malignancy have higher mortality rate.

\section{6}

\section{MiR-315-5p is involved with Wingless/Wnt pathway in sepsis} response using Drosophila model

J. Soh ${ }^{1}$; K. Laudanski,

${ }^{1}$ Neurology, University of Pennsylvania, Philadelphia, United States

of America; ${ }^{2}$ Anesthesiology, University of Pennsylvania, Philadelphia,

United States of America

Correspondence: J. Soh

Intensive Care Medicine Experimental 2021, 9(1): 001296

Introduction. Sepsis is a systemic reaction involving a sequence of responses by the immune system. In mammals, this process is highly regulated and continues to remain an area of active research. As new protocols are implemented and with the recent events in the world related to COVID pandemic, there has never been a more evident and clear need to better understand the interplay between host and infection.

Methods. Drosophila larvae were exposed to either sham surgery or injection of EColi pellet. After incubation time of 7, 14 and 21 days, samples were subsequently studied for RNA-Seq evaluation.

Results. RNA-Seq data obtained from various time points revealed marked changes in expression profile at day 14 and day 21 samples. Genes expressed in these groups were involved in various pathways. Of note, analysis of these genes suggest a role for a microRNA 315-5p to play a role in the $\mathrm{Wg} / \mathrm{Wnt}$ pathway.

Conclusion. Prolonged septic state suggest a role for dynamic transcriptional changes in Drosophila larvae model. The role of $\mathrm{Wg} / \mathrm{Wnt}$ pathway in management of sepsis is of interest. Additional role of miR315-5p in modulating a response in sepsis suggest networks of genes is involved in addition to $\mathrm{Wg} / \mathrm{Wnt}$.

\section{Reference(s)}

1. Drosophila macrophages siwtch to aerobic glycolysis to mount effective antibacterial defense. Krejcova G, et al. Elife. 2019

2. Cost of surviving sepsis: a novel model of recovery from sepsis in Drosophila melanogaster. Kaynar AM, et al. ICM. 2016

3. Biomarkers of sepsis. Faix JD. Crit Rev Clin Lab Sci. 2013

4. Grant funding w/ Dr. K Laudanski

\section{6}

Outcomes from critical care in pregnant and postpartum patients with COVID-19

C. Hermann ${ }^{1}$; E. Holndonner-Kirst ${ }^{1}$; B. Bódi, ${ }^{1}$; B. Mogyoródi, ${ }^{1}$; K. Tóth, ${ }^{1}$;

A. Kállai, ${ }^{1}$; E. Szegő, ; K. Kárpáthi, ; ' L. Takács, ${ }_{1}^{1}$; P. Golopencza, ; H. Gál, ${ }^{1}$; V.

Kesztyüs,

${ }^{1}$ Department of Anaesthesiology and Intensive Therapy, Semmelweis

University, Budapest, Hungary

Correspondence: $\mathrm{C}$. Hermann

Intensive Care Medicine Experimental 2021, 9(1): 001306

Introduction. Given the well described susceptibility of pregnant women to viral infections, several authors dealt with the morbidity and mortality of these patients during the SARS-CoV2 pandemic. Besides the conflicting results of papers investigating pregnant women with mild or moderate COVID-19 infection, relatively few information is available of critically ill patients 1,2 .

Objectives. Therefore, we analysed the data of pregnant and postpartum patients admitted to our tertiary intensive care unit with proven SARS-CoV2 positivity.

Methods. In our prospective, consecutive study, a wide range of clinical data were collected from medical databases. Continous variables are represented with medians and interquartile ranges (IQR), and non-parametric test were used for comparisons.

From 1st October 2020 to 30th April 2021, we admitted 88 COVID 19 patients under 50 years of age to our ICU. We analysed the data of 21 pregnant or postpartum patients and compared to their counterparts. Major outcomes were mortality and length of stay in the ICU.

Results. Pregnant and postpartum patients were significantly younger on admission, with median age of 35.2 years $(I Q R=31.2$ $37.7)$, compared to 43.4 years (IQR $=36.5-46.5)$ for the controls, respectively. 13 patients of the study group (61.9\%) were pregnant on admission, median gestational age was 29 weeks (IQR $=29-31$ ), 8 patients were admitted after caesarean section. Three of the study patients had hypertension, 7 had diabetes, 3 had hypothyroidism, and one patient had rheumatoid arthritis. Only one patient of the study group was current smoker.

The median APACHE II score for the pregnant or postpartum patients was 12 points (IQR $=8-16)$. On admission, 6 patients of the study group $(28.6 \%)$ required vasopressors, 4 patients $(19.0 \%)$ had positive PCT (>0.5 mg/L). Chest CT or X-ray on admission showed extensive $\left({ }^{3} 50 \%\right)$ involvement in 11 cases $(52.4 \%)$, and 13 patients $(61.9 \%)$ had consolidative lung areas in the study group. The median $\mathrm{PaO}_{2} / \mathrm{FiO}_{2}$ ratio for them was 229 (IQR $\left.=169-287\right)$ on admission, ten patients (47.6\%) were intubated on admission or within $12 \mathrm{~h}, 7$ $(33.3 \%)$ patients received non-invasive ventilation on the first day of ICU care.

$12(57.1 \%)$ vs. $46(69.7 \%)$ patients were ventilated invasively at some point during their intensive care unit stay in the study and control groups, respectively $(p=0.288)$.

The median length of ICU stay was similar in the two groups (7 days $(\mathrm{IQR}=3.5-13.5)$ for pregnant and postpartum patients and 7 days $(\mathrm{IQR}=4.0-11.0)$ for control, respectively, $\mathrm{p}=0.796)$.

Four patients $(19.0 \%)$ died in the study group, whereas 20 in the control group $(30.3 \%), p=0.315$.

Conclusion. We found no significant difference in terms of mortality, length of ICU stay and need of invasive ventilation between 
critically ill pregnant/postpartum patients and their counterparts. It must be considered that these patients may be overrepresented in HDUs and ICUs since preventive measures. Given the small sample size, further multicentre studies are required.

\section{Reference(s)}

1. 2Pierce-Williams R. A. M. et al. Clinical course of severe and critical coronavirus disease 2019 in hospitalized pregnancies: a United States cohort study. Am J Obstet Gynecol MFM 2, 100,134, https://doi.org/10.1016/j. ajogmf.2020.100134 (2020).

2. 1Collin J, Bystrom E, Carnahan A, Ahrne M. Pregnant and postpartum women with SARS-CoV-2 infection in intensive care in Sweden. Acta Obstet Gynecol Scand. 2020;99:819-822

\section{5}

Phenotypical characteristics of bacterial and Covid-19 associated sepsis

F. Perschinka ${ }^{1}$; T. Mayerhöfer ${ }^{1}$; GF. Lehner' ${ }^{1}$ SJ. Klein ${ }^{1}$; M. Joannidis ${ }^{1}$ 'Internal Medicine, Medical University Innsbruck, Innsbruck, Austria

Correspondence: F. Perschinka

Intensive Care Medicine Experimental 2021, 9(1): 001335

Introduction. In critically ill Covid-19 patients highly elevated cytokine levels were accused to be predictors for disease severity. Various investigations showed higher mortality in Covid-19 patients with high interleukin levels, proposing a "cytokine storm" which describes a systemic inflammatory response to infections and drugs that leads to excessive activation of immune cells and the generation of pro-inflammatory cytokines as major pathomechanism.

Objectives. Identify differences regarding inflammatory parameters between bacterial and Covid-19 associated sepsis in critically ill patients.

Methods. Eighteen patients treated with bacterial sepsis or septic shock at the medical ICU at the University Hospital Innsbruck, Austria, during September 2018 to October 2020, were selected. These patients were matched to a cohort of Covid-19 patients who were treated during the period from August 2020 to April 2021 at the same unit and included in the Tyrolean Covid-19 registry. Matching was performed in a 1:1 ratio by age, sex and invasive mechanical ventilation 7 days after ICU admission.

The two matched cohorts were compared regarding maximum IL-6, PCT and CRP within $48 \mathrm{~h}$ after ICU admission. Maximum (IL-6, PCT and (RP) levels were used for analysis.

Results. The maximum median IL-6 levels of 106.2 ng/l (IQR; 40.95140.68) in Covid-19 patients turned out to be far below the values observed in bacterial sepsis with a median of $4990 \mathrm{ng} / \mathrm{l}$ (IQR; 678.6$22,257.25)(p<0.001)$. Median CRP levels were clearly increased up to $12.44 \mathrm{mg} / \mathrm{dl}$ (IQR; 7.97-20.46) in Covid-19 but lower than those measured in the patients with bacterial sepsis presenting a median of $33.53 \mathrm{mg} / \mathrm{dl}$ (IQR; 24.94-49.31) ( $p<0.001)$. Additionally, PCT levels were hardly elevated with a median of $0.18 \mu \mathrm{g} / \mathrm{I}(\mathrm{IQR} ; 0.15-0.43$ ) in Covid-19 patients and significantly lower than those observed in bacterial sepsis $(26.77 \mu \mathrm{g} / \mathrm{l} ; \mathrm{IQR} ; 9.39-142.1 ; \mathrm{p}<0.001)$. Neither SAPS-III score (50 vs. $54 ; p=0.669$ ) nor mortality (4 vs $7 ; p=0.278$ ) showed significant differences between the groups.

Conclusion. During the first $48 \mathrm{~h}$ after ICU admission PCT levels were hardly elevated in Covid-19 patients. Concurrently, both CRP and especially IL-6 levels were moderately increased but remarkably lower compared to patients with bacterial sepsis. Overall, these data suggest that cytokine storm may play an inferior role in severe Covid-19 disease, if any at all.

\section{Reference(s)}

1. Klein SJ, Bellmann R, Dejaco H, Eschertzhuber S, Fries D, Furtwängler W, et al. Structured ICU resource management in a pandemic is associated with favorable outcome in critically ill COVID-19 patients. Wien Klin Wochenschr. 2020:132(21-22):653-63.

2. Hu B, Huang S, Yin L. The cytokine storm and COVID-19. J Med Virol. 2021;93(1):250-6.
3. Liu F, Li L, Xu M, Wu J, Luo D, Zhu Y, et al. Prognostic value of interleukin-6, C-reactive protein, and procalcitonin in patients with COVID-19. J Clin Virol. 2020:127:104,370

001342

Factors associated with mortality in patients with esophageal perforation in a third level ICU

I. Mataix-Ponce'; M. Mendoza-Prieto ${ }^{1}$; J. Navarro-Rodriguez ${ }^{1}$;

R. Posada-Ojeda'; S. Martin-Sastre ${ }^{1}$; A. Cuadra-Calahorra ${ }^{1}$; A.

Escoresca-Ortega,

${ }^{1}$ Critical Care Unit, Hospital Universitario Virgen del Rocío, Sevilla, Spain;

${ }^{2}$ Critical care, Virgen del Rocio Hospital, Sevilla, Spain

Correspondence: M. Mendoza-Prieto

Intensive Care Medicine Experimental 2021, 9(1): 001342

Introduction. Esophageal perforation is a potentially life-threating condition in which early diagnosis and immediate treatment decrease mortality, morbidity and long-term sequelae.

Objectives. To analyze epidemiological characteristics, complications and clinical outcomes of critical patients with diagnosis of esophageal perforation.

Methods. Retrospective observational study from January 2011 to December 2019 in a third level Hospital. All patients with diagnosis of esophageal perforation admitted to the Intensive Care Unit (ICU) were included. Demographic data, personal history, days of ICU stay, 30-day mortality and main complications were collected. We compared differencies between groups based on mortality. Qualitative variables were described as absolute number and frecuencies; quantitative variables as median and interquartile range (IR). Chi-squared test for qualitative variables and $U$ Mann Whitney test for quantitative ones were performed.

Results. A total of 26 patients were included, 22 (84.62\%) in the group of survivors. Demographic data, personal history and evolution of both groups are shown in Table 1. A higher mortality were observed in those patients who required amines, invasive respiratory support and those with a higher value of lactate at admission, without finding differences regarding mortality according to the treatment received. Median ICU stay was 8 (2.75 -27.75) days, with a mortality of $15.4 \%$

\begin{tabular}{|c|c|c|c|}
\hline & NON-DECEASED & DECEASED & $p$ value \\
\hline Male; n (\%) & $15(68)$ & $4(100)$ & 0.187 \\
\hline Age; median (IR) & $59(43.25-42)$ & $63.5(53.25-75.50)$ & 1 \\
\hline Hypertension; n(\%) & $13(59)$ & $2(50)$ & 0.821 \\
\hline Diabetes; n(\%) & $3(14)$ & 0 & 0.432 \\
\hline APACHE II; median (IR) & $10.5(6-14.25)$ & $23.5(15.5-28.5)$ & 0.104 \\
\hline \multicolumn{4}{|l|}{ Location of perforation } \\
\hline Proximal & $5(23)$ & 0 & 0.295 \\
\hline Medial & $4(18)$ & $2(50)$ & \\
\hline Distal & $13(59)$ & $2(50)$ & \\
\hline Etiology & & & 0.299 \\
\hline Spontaneous & $8(36)$ & $3(75)$ & \\
\hline latrogenic & $6(28)$ & 0 & \\
\hline Strange body & $8(36)$ & $1(25)$ & \\
\hline $\begin{array}{l}\text { Lactate value at admis- } \\
\text { sion; median (IR) }\end{array}$ & $1.6(1.5-1.8)$ & $12.8(5.3-19.25)$ & $0.008^{*}$ \\
\hline Amines; $\mathrm{n}(\%)$ & $7(32)$ & $4(100)$ & $0.011^{*}$ \\
\hline $\begin{array}{l}\text { Invasive respiratory } \\
\text { support; } \mathrm{n}(\%)\end{array}$ & $8(36)$ & $4(100)$ & $0.019 *$ \\
\hline Type of surgery; n(\%) & & & 0.310 \\
\hline
\end{tabular}




\begin{tabular}{llll}
\hline & NON-DECEASED & DECEASED & p value \\
\hline Primary closure & $5(23)$ & $2(50)$ & \\
Esophagectomy & $4(18)$ & $2(50)$ & \\
Endoscopic stent & $3(14)$ & 0 & \\
Focus drain & $1(5)$ & 0 & 0.208 \\
Mediastinitis; $n(\%)$ & $9(41)$ & $3(75)$ & \\
\hline
\end{tabular}

Table 1. Patients characteristics.

Conclusion. In our cohort, the type of treatment received did not influence global mortality in patients with esophageal perforation, just as described in the literature. The need for respiratory support, amines and higher lactate values at admission were associated with higher mortality.

\section{Reference(s)}

1. Aghajanzadeh M, Porkar NF, Ebrahimi H. Cervical esophageal perforation: a 10-year clinical experience in north of iran. Indian J Otolaryngol Head Neck Surg. 2015;67(Suppl 1):34-9.

2. Brinster CJ, Singhal S, Lee L, Marshall MB, Kaiser LR, Kucharczuk JC. Evolving options in the management of esophageal perforation. Ann Thorac Surg. 2004;77(4):1475-83

3. Puerta Vicente A, Priego Jiménez P, Cornejo López MÁ, García-Moreno Nisa F, Rodríguez Velasco G, Galindo Álvarez J, Lobo Martínez E. Management of Esophageal Perforation: 28-Year Experience in a Major Referral Center. Am Surg. 2018 May 1;84(5):684-689.

\section{2}

Aspergillus spp. isolation in critically ill patients with COVID-19 after one year of pandemic outbreak

JJ. Pineda-Capitán '; J. Rodriguez-Gomez'; E. Marfil'; R. Carmona-Flores'; J. Caston-Osorio ; ' L. Cristina'; J. Naranjo ; J. Muñoz-Trujillo'; R. Tejero²; JC. Pozo Laderas'; R. León ${ }^{1}$; C. De La Fuente Martos ${ }^{1}$

'Intensive Care Unit, Reina Sofia Universtiy Hospital-IMIBIC-University of Cordoba, Cordoba, Spain., Cordoba, Spain; ${ }^{2}$ Microbiology Unit, Reina Sofia Universtiy Hospital-Imibic-University of Cordoba, Cordoba, Spai, Córdoba, Córdoba, Spain; ${ }^{3}$ Infectious Disease, Reina Sofia Universtiy Hospital-Imibic-University of Cordoba, CORDOBA, SPAIN, CÓRDOBA, Córdoba, Spain

Correspondence: J. Rodriguez-Gomez

Intensive Care Medicine Experimental 2021, 9(1): 001362

Introduction. It's been reported an increased incidence of Aspergillus spp. isolation in critically ill patients with COVID-19.

Objectives. The aim of this study is to describe the main clinical and microbiological characteristics of critically ill patients with COVID-19 pneumonia and Aspergillus spp isolation during the first year of pandemic outbreak.

Methods. This is an observational and retrospective study carried out in one ICU. We evaluated all patients with confirmed COVID-19 pneumonia and Aspergillus spp isolation in respiratory samples during their ICU admission. Patients were recruited from March 2020 to March 2021. We described the main clinical and microbiological characteristics. Categorical variables were described as proportions, and continuous variables were described as median and p25-75.

Results. During the first year of pandemic outbreak there were admitted 267 critically ill patients with COVID-19 pneumonia in ICU; 23 (8.6\%) patients exhibit Aspergillus spp isolation in respiratory samples. The median age was $67(63-70)$ years and $14(60,9 \%)$. The more frequent comorbidities were hypertension, dyslipemia and obesity (56.5\%). Previous respiratory disease was present in $8(37 \%)$ patients and $11(47 \%)$ received chronic steroid therapy. At ICU admission APACHE II score and C-reactive protein were $4(9-18)$ and $171(69-212)$ $\mathrm{mg} / \mathrm{dL}$.

A. fumigatus was the most frequent Aspergillus spp isolated in 18 patients (78.3\%). A. terreus, A. niger and A. flavus were isolated in 5 (21.3\%), $4(17.4 \%)$ and $2(8,7 \%)$ patients. Of 50 positive respiratory samples, 29 (58\%) were obtained with bronchoscopy exploration and pseudomembranous plaques or ulcers were visible in $4(17,4 \%)$ patients. Bronchialveolar Galactomannan was solicited in $9(39.1 \%)$ patients, $6(26 \%)$ of them positive. 7 patients (30.4\%) experienced bacterial coinfection.

The median days from ICU admission to Aspergillus spp. isolation was 6 (2-13) days. All patients were treated for COVID-19 disease with corticosteroids $23(100 \%)$ and $4(17.3)$ of them with tociluzumab; 19 (82.6) remained under mechanical ventilation. The day of isolation, $20(86.9 \%)$ patients experienced respiratory deterioration, $15(65.2 \%)$ radiology deterioration, $11(47.8 \%)$ fever and worsening secretions $10(43,5 \%)$. According to CAPA criteria, probable invasive aspergillosis was diagnosed in 9 patients (40\%); all patients with invasive bronchi alveolar lavage were positive for Aspergillus spp. A total of 22 (95.6\%) patients received antifungal treatment, 1 was considered colonisation. The more frequent initially treatments were voriconazol and isavuconazol 17 (73.9) and 4 (17.4) patients and 5 (25.9) received combined therapy with caspofungin. ICU mortality was 11 (47,8\%).

Conclusion. In our study the incidence of Aspergillus spp isolation in critically ill patients with COVID-19 was $8 \%$. The most frequent isolation was A. fumigatus. Those patients experiece high mortality of $47 \%$.

\section{Reference(s)}

1. Pemán J, Ruiz-Gaitán A, García-Vidal C, Salavert M, Ramírez P, Puchades F, García-Hita M, Alastruey-Izquierdo A, Quindós G. Fungal co-infection in COVID-19 patients: Should we be concerned? Rev Iberoam Micol. 2020 Apr-Jun;37(2):41-46

2. Fekkar A, Lampros A, Mayaux J, Poignon C, Demeret S, Constantin JM, et al. Occurrence of invasive pulmonary fungal infections in patients with severe COVID-19 admitted to the ICU. Am J Respir Crit Care Med. 2021;203(3):307-17

3. Fekkar A, Lampros A, Mayaux J, Poignon C, Demeret S, Constantin JM, et al. Occurrence of invasive pulmonary fungal infections in patients with severe COVID-19 admitted to the ICU. Am J Respir Crit Care Med. 2021;203(3):307-17

4. Bartoletti M, Pascale R, Cricca M, Rinaldi M, Maccaro A, et al. Epidemiology of Invasive Pulmonary Aspergillosis Among Intubated Patients With COVID-19: A Prospective Study. Clin Infect Dis. 2020;(Xx):1-9

5. Koehler P, Bassetti M, Chakrabarti A, Chen SCA, Colombo AL, Hoenigl M, et al. Defining and managing COVID-19-associated pulmonary aspergilIosis: the $2020 \mathrm{ECMM} / \mathrm{ISHAM}$ consensus criteria for research and clinical guidance. Lancet Infect Dis. 2021;3099(20)

6. Lahmer T, Kriescher S, Herner A, Rothe K, Spinner CD, Schneider J, et al. Invasive pulmonary aspergillosis in critically ill patients with severe COVID-19 pneumonia: Results from the prospective AspCOVID-19 study. PLoS One [Internet]. 2021;16(3 March):1-16.

\section{8}

Predicting Values Of Coagulation Parameters Determining Mortality in Patients With COVID-19

N. Boyaci'; G. Koksal'; Y. Tezcan'; N. AKSOY'; A. Yalcin³; G. Aksu²; EK. Kaya²; O. Sen ${ }^{1}$

${ }^{1}$ Anesthesiology and Reanimation, University of Health Sciences,Haseki Training and Research Hospital, Istanbul, Turkey; ${ }^{2}$ Critical care, University of Health Sciences,Haseki Training and Research Hospital, Istanbul, Turkey; ${ }^{3}$ Pulmonary and critical care, University of Health Sciences, Haseki Training and Research Hospital, Istanbul, Turkey

Correspondence: N. Boyaci

Intensive Care Medicine Experimental 2021, 9(1): 001418

Introduction. Coronavirus disease 2019 (COVID-19, SARS Cov-2) is a viral infection that causes the death of millions of people throughout the world and a life-threatening disease that could lead to progressive respiratory failure and eventually progresses to an acute respiratory distress syndrome (ARDS) or ARDS-like illness in the lungs.Coagulation parameters recommended to be evaluated in COVID-19 patients are d-dimer, fibrinogen, fibrin degradation products (FDP), prothrombin time (PT), activated partial thromboplastin time (aPTT), and platelet count. In addition, monitoring ferritin, leukocyte, and lymphocyte 
counts are essential in predicting the course of the disease and mortality.

Objectives. In this study, we aimed to determine the predictive significance of initial coagulation parameters on the prognosis of COVID-19 in the $24 \mathrm{~h}$ of admission to the intensive care unit (ICU) and demographic data (age, gender), correlation with each other.

Methods. We conducted this retrospective clinical study with 255 patients with COVID-19 who were admitted to the adult intensive care unit (ICU) in Haseki Training and Research Hospital Sultangazi, Istanbul, between 03/11/2020-06/01/2021. Patients aged 18 years and older, who had positive PCR test, hypoxia with specific thorax computerized tomography (CT) involvement were included in to study. Ferritin, fibrinogen, D-dimer, PT, aPTT, INR, leukocyte, lymphocyte, thrombocyte measurements, acute physiology and chronic health evaluation II (APACHE II) and sequential organ failure assessment (SOFA) scores on the first day of ICU admission were collected for statistical analysis. In calculating the sample size of this study, the power for each variable was determined $80 \%$ at least, and type 1 error was $5 \%$. Whether the continuous measurements in the study were evenly distributed was checked with the Kolmogorov-Smirnov $(n>50)$ test. Since measurements were evenly distributed, nonparametric tests were used. Statistical significance (a) was $5 \%$ in calculations, and SPSS (IBM SPSS for Windows, ver.24) program was used for calculations. Results were considered significant $p<0,05$.

Results. High coagulation values are statistically significant in predicting mortality in our study $(p<0.05)$. Coagulation parameters are higher in male patients, and the tendency to thrombosis and

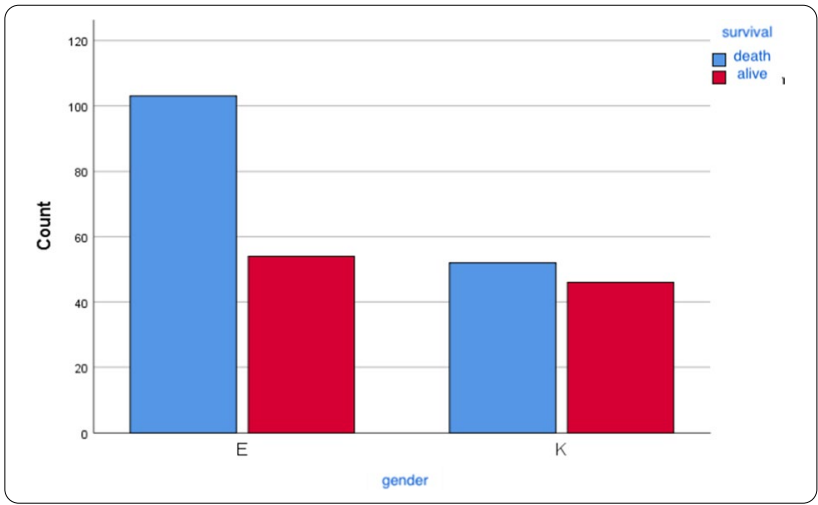

mortality was higher than in female patients (Table 1). Besides, daily SOFA score tracing, measurement of leukocyte count and lymphocyte ratio have significant correlations in evaluating the severity of the disease. Correlation coefficients and results have shown in Table 2.

\begin{tabular}{|c|c|c|c|c|c|c|c|c|}
\hline Table 2 & & AGE & Dimer & Ferritin & SOFA & Fib & CRP & WBC \\
\hline Dimer & $r$ &, 112 & & & & & & \\
\hline Ferritin & $r$ &,- 082 &, $187^{* *}$ & & & & & \\
\hline SOFA & $r$ &, 031 &, $193^{* *}$ &, $287^{* *}$ & & & & \\
\hline Fib & $r$ &,$- 131^{*}$ &,$- 147^{*}$ &, $165^{* *}$ & & & & \\
\hline CRP & $r$ &,- 006 &, 041 &, 086 & ,010 &, $144^{*}$ & & \\
\hline WBC & r &,- 090 &, $182^{* *}$ & $246^{* *}$ &, $475^{* *}$ &, $303^{* *}$ &,- 040 & \\
\hline LYMP & $r$ &,- 089 &,- 009 &,- 065 &,- 109 & ,037 &,$- 240^{* *}$ &,- 004 \\
\hline
\end{tabular}

${ }^{*} p<0,05 ;{ }^{* *} p<0,01 ; r$ : Spearman correlation coeficents

Conclusion. Trying to prevent thrombin formation from the moment of COVID-19 diagnosis can attenuate the excessive endothelial damage and thromboembolic events. Antiagreggan using alongside anticoagulant treatment in the early phase can decrease the severity of the disease.
Reference(s)

1. Levi M, Thachil J, Iba T, Levy J. Coagulation abnormalities and thrombosis in patients with COVID-19 infection. Lancet Haematol. 2020;S23523026(20): 30145-9.

2. Klok FA, Kruip MJHA, van der Meer NJM, Arbous MS, Gom- mers DAMPJ, Kant KM, Kaptein FHJ, van Paassen J, Stals MAM, Huisman MV, Endeman H (2020) Incidence of thrombotic complications in critically ill ICU patients with COVID-19. Thromb Res. On-line April10.

3. . Harenberg J, Favaloro E. COVID-19: progression of disease and intravascular coagulation-present status and future perspectives. Clin Chem Lab Med. 2020;58(7):1029-1036. https://doi.org/10.1515/cclm-2020-0502

\section{0}

Potential prognostic biomarkers in COVID19: role of mid-regional pro-adrenomedullin, methemoglobin and carboxyhemoglobin

CM. Oblitas'; F. Galeano-Valle'; J. López-Cano ${ }^{2}$; A. Monterrubio-Manrique ${ }^{2}$ M. García-Gámiz ; J. Ramírez-Navarro'; M. Sancho-González ${ }^{4}$; S.

Arenal-López ${ }^{4}$; L. ÁLvarez-Sala Walther'; P. Demelo-Rodríguez

${ }^{1}$ Internal Medicine, Gregorio Marañón Hospital, Madrid, Spain; ${ }^{2}$ Internal

Medicine, Complutense University of Madrid, Madrid, Spain; ${ }^{3}$ Clinical Biochemistry, Gregorio Marañón Hospital, Madrid, Spain; ${ }^{4}$ Intensive Care Medicine, Gregorio Marañón Hospital, Madrid, Spain

\section{Correspondence: C.M. Oblitas}

Intensive Care Medicine Experimental 2021, 9(1): 001420

Introduction. The current pandemic of coronavirus disease 2019 (COVID-19) caused by the severe acute respiratory coronavirus 2 (SARS(oV-2) is the major healthy issue worldwide in decades, with more than 192 countries affected and almost 3.5 millions of deaths.

Adrenomedullin is a biomarker of sepsis released in the setting of organ failure, but it has a very short plasma half-life. The middle-region proadrenomedullin (MR-proADM) which keeps a ratio 1:1 is usually measured instead. Other biomarkers that have been studied in sepsis include methemoglobin (MetHb) and carboxyhemoglobine ( $\mathrm{COHb})$.

Objectives. The main objective of our study is to determine whether plasma levels of MR-proADM, MetHb or $\mathrm{COHb}$ at ICU admission are associated with worse prognosis at 90 days in terms of mortality and a compound event (mortality, venous and arterial thrombosis).

Methods. A prospective single-center study including COVID-19 patients admitted in an ICU from a tertiary hospital was performed. MR-proADM, MetHb and $\mathrm{COHb}$ were measured in the first $72 \mathrm{~h}$ after admission.

Results. A total of 95 consecutive patients were included, with a mean age of 60.28 years and $67.4 \%$ were male. The $53.7 \%$ (51 patients) required mechanical ventilation and $6.3 \%$ ( 6 patients) needed venovenous extracorporeal membrane oxygenation. The mean length of hospital stay was 34.38 days (P25-P75, 17-43 days). The global mortality rate at 90 days was $25.3 \%$ (24 patients), being COVID-19 the main cause of death in $87.5 \%$ (21 patients). The compound event occurred in $32.6 \%$ (31 patients). MetHb and $\mathrm{COHb}$ were measured in $86 / 95$ patients, the mean values were 1.093\% (P25-P75, 0.875-1.325) and 1.579\% (P25-P75; 1.3-2.0), respectively. There were no significant differences between survivors and non-survivors for both MetHb (0.97 vs $1.13, p=0.057)$ and $\mathrm{COHb}(1.54$ vs $1.59, \mathrm{p}=0.85)$. On the other hand, the mean levels of MRproADM were significantly higher in both non-survivors (1.63 vs 1.18 , $\mathrm{p}=0.007$ ) and in those with compound event (1.46 vs $1.21, \mathrm{p}=0.036$ ). The best cut-off point was $1.0 \mathrm{nmol} / \mathrm{L}$ with an area under the receiver operating characteristic (AUROC) curve of 0.68 (Cl 95\%, 0.57-0.77) for mortality (Figure 1) with a negative predicted value (NPV) of $84.4 \%$, and an AUROC of 0.63 (Cl 95\%, 0.53-0.73) for compound event with a NPV of 76.6. The univariant analysis showed an OR 4.064 for mortality (Cl 95\% $1.52-10.85 ; p=0.005)$ and OR 2.69 for compound event (Cl 95\% $1.07-$ $6.74 ; p=0.035$ ); however, this association was not confirmed in multivariate analysis adjusted for age. 


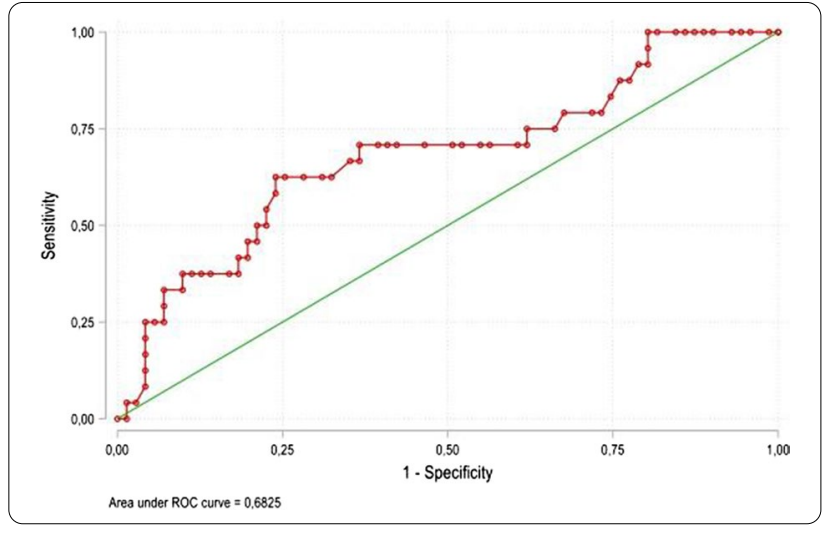

Conclusion. MR-proADM, but not MetHb or $\mathrm{COHb}$, is a useful biomarker for prognosis in terms of mortality and development of thrombotic complications at 90 days in COVID-19 patients admitted at ICU. The search for biomarkers is essential to better stratify our patients. More studies are needed to confirm these results.

\section{Reference(s)}

1. 1. Wiersinga WJ, Rhodes A, Cheng AC, Peacock SJ, Prescott HC. Pathophysiology, transmission, diagnosis, and treatment of Coronavirus disease 2019 (COVID-19): A review: A review. JAMA. 2020;324(8):782-93.

2. 2. Valenzuela-Sánchez F, Valenzuela-Méndez B, Rodríguez-Gutiérrez JF, Estella-García Á, González-García MÁ. New role of biomarkers: midregional pro-adrenomedullin, the biomarker of organ failure. Ann Transl Med. 2016:4:329.

3. 3. Hare GM, Tsui AK, Crawford JH, Patel RP. Is methemoglobin an inert bystander, biomarker or a mediator of oxidative stress-The example of anemia? Redox Biol. 2013;1:65-9.

4. 4. Yeo TW, Lampah DA, Kenangalem E, Tjitra E, Price RN, Anstey NM. Increased carboxyhemoglobin in adult falciparum malaria is associated with disease severity and mortality. J Infect Dis. 2013;208:813-7.

5. 5. Sozio E, Tascini C, Fabris M, D'Aurizio F, De Carlo C, Graziano E, et al. MRproADM as prognostic factor of outcome in COVID-19 patients. Sci Rep. $2021 ; 11: 5121$

6. None.

\section{4}

\section{Endothelial glycocalyx damage in patients with pneumonia} and septic shock and in patients with severe covid-19 infection on mechanical ventilation

D. Astapenko'; A. Ticha²; R. Hyspler²; A. Tomasova²; R. Cerna Parizkova'; S. Chua Huey ${ }^{1}$; V. Cerny ${ }^{3}$

${ }^{1}$ Dept. of Anesthesiology, Resuscitation and Intensive Care Medicine, Fakultní Nemocnice Hradec Králové, Hradec Králové, Czech Republic; ${ }^{2}$ Dept. of Clinical Biochemistry and Diagnostics, Fakultní nemocnice Hradec Králové, Hradec Králové, Czech Republic; ${ }^{3}$ Czech Republic, Masaryk Hospital, University J.E. Purkyne, ccc, Usti nad Labem, Czech Republic Correspondence: D. Astapenko

Intensive Care Medicine Experimental 2021, 9(1): 001424

Introduction. Endothelial glycocalyx (EG) plays a crucial role in the physiology of microcirculation. A novel coronavirus SARS-CoV-2 has been linked to severe endotheliopathy presenting as a dysfunction of microcirculation, microangiopathy, thrombosis, and EG damage. Comparison of the microcirculation dysfunction and EG damage in patients with severe COVID-19 and pneumonia with septic shock and mechanical ventilation of non-COVID origin has not been published.

Objectives. To compare the dysfunction of microcirculation and EG damage in adult patients with severe COVID-19 and severe pneumonia of non-COVID origin.
Methods. Adult patients with diagnoses of COVID-19 or pneumonia on mechanical ventilation were enrolled into the study in the second wave of COVID pandemia from September till December 2020. Laboratory data were followed for 3 consecutive days from admission. Blood and urine samples were collected for detecting markers of endothelial (P-selectine, thrombomodulin), EG function (syndecan-1), and urine albumin/creatinine ratio for glomerular integrity assessment. Sublingual microcirculation was assessed by Side-stream Dark Field (SDF) imaging (parameters: proportion of perfused vessels (PPV) and DeBacker score). The study was prospective, non-randomized, observational, and was approved by a local ethical committee.

Results. 13 non-COVID and 15 COVID-19 patients were enrolled in the study with an average age of 70 and 64 respectively. COVID patients had a significantly higher SOFA score on the day 2 and 3, higher PEEP on mechanical ventilation on admission and day 2 and pron position was used in each COVID patient. Blood lactate was higher on admission in non-COVID patients. Natremia was significantly higher on day 3 in COVID patients. The urine albumin/creatinine ratio was significantly higher on admission in non-COVID patients. Syndecan-1 was significantly higher all three days in COVID-19 patients. P-selectine and thrombomobulin had no difference. Parameters of sublingual microcirculation (PPV and DeBacker score) were significantly higher on day 2 in COVID patients $(p=0,04)$. In COVID patients therapeutic heparinisation by low molecular weight heparin was used.

Conclusion. In the 2 groups of patients in our study, the observed differences were due to the different pathophysiology of both types of pulmonary disease. In COVID patients the EG was significantly damaged confirming the hypothesis of endotheliopathy caused by the virus SARS-CoV-2. Therapeutic heparinisation led to improved parameters of sublingual microcirculation compared to non-COVID patients.

\section{Reference(s)}

1. Supported by MZ ČZ - RVO (UHHK, 00179906), PROGRES Q40/N8514002, and PROGRES Q40/06, all rights reserved.

\section{6}

Second wave of COVID-19 in a University hospital in Greece: Outcome and mortality risk factors among intubated ICUpatients with severe disease

V. Theodorou'; I. Retselas²; K. Nikolaou²; M. Nakou²; I. Lazana²; R.

Papadopoulou'²; A. Petala'; E. lagkoumpidou²; V. Papaioannou ${ }^{3}$

${ }^{1}$ Intensive Care, University Hospital of Alexandroupolis, Alexandroupoli, Greece; ${ }^{2}$ ntensive care, University Hospital of Alexandroupolis, Alexandroupoli, Greece; ${ }^{3} \mathrm{ICU}$, Democritus University of Thrace, THESSALONIKI, Greece

Correspondence: $\mathrm{V}$. Theodorou

Intensive Care Medicine Experimental 2021, 9(1): 001456

Introduction. The 28-day mortality and mortality risk factors for critically ill Covid- 19 mechanically ventilated adult patients still remain controversial.

Methods. 122 intubated severe Covid-19 patients between 1st September 2020 and 15th February 2021, were included retrospectively. The demographic and clinical data were compared between survivors and non-survivors. Cox regression analysis was used to explore risk factors for 28-day mortality. The primary outcome was 28-day mortality.

Results. The mean age was $65,71 \pm 10,52$, sex was $66 \%$ male and $88 \%$ had at least one co-morbidity (arterial hypertension, diabetes and obesity). Mean APACHE II score was $23.03( \pm 6.62)$ and SOFA score at admission $7.75( \pm 1.24)$. Severe ARDS (100\%) and distributive shock $(73 \%)$ were the main reasons for admission. All patients received dexamethasone, whereas $49(40 \%)$ received Interleukin-1 inhibitor and 24 (20\%) Interleukin-6 inhibitor.

The 28 -day mortality was $81.1 \%$. The leading cause of death was multiorgan failure (SOFA $14 \pm 4.07$ ).

Age higher than 60 years $(\mathrm{OR}=2.9 / p=0.031)$, APACHE II score higher than $23(\mathrm{OR}=3.7 / p=0.002)$ and $\mathrm{AKI}$ during ICU-hospitalization $(\mathrm{OR}=4.0 / p=0.001)$ were factors independently associated with 
28-days mortality. Mortality was not associated with either interleukin-1 or interleukin-6 inhibitor $(p=0.701)$.

Conclusion. The mortality of invasively ventilated COVID-19 patients still remains particularly high. High age, APACHE II score and AKI were found to be significantly associated with mortality.

\section{Reference(s)}

1. European guideline on managing adults in hospital with COVID-19 Lancet.com/respiratory Vol 9 May 2021

2. Aziz S. et al. Managing ICU surge during the COVID-19 crisis: Rapid Guidelines. Intensive Care Medicine (2020)

\section{7}

Investigation of Syndecan-1 as a marker of glycocalyx shedding in a human intravenous lipopolysaccharide model of sepsis R. McMullan'; E. Symington'; W. Sendama²; J. Simpson, ${ }^{2}$; D. McAuley ${ }^{3}$; JA. Silversides ${ }^{1}$; A. Rostron, ${ }^{4}$

'Wellcome wolfson institute for experimental medicine, Queen's University Belfast, Belfast, United Kingdom; ${ }^{2}$ Faculty of medical sciences, Newcastle University, Newcastle upon Tyne, United Kingdom; ${ }^{3}$ University, Queen's University Belfast, Belfast, United Kingdom; ${ }^{4}$ Integrated critical care unit, Sunderland Royal Hospital, Sunderland, United Kingdom Correspondence: R. McMullan

Intensive Care Medicine Experimental 2021, 9(1): 001457

Introduction. Endothelial damage is a hallmark feature of sepsis and is characterised by the disruption of the endothelial glycocalyx. The glycocalyx plays a crucial role in the regulation of endothelial permeability $[1,2]$. Syndecan-1 is a proteoglycan found on the surface of the glycocalyx [3].

Objectives. Our study aimed to produce a human model of glycocalyx degradation in order to aid future research into interventions acting on the endothelial glycocalyx.

We hypothesised that Syndecan-1 levels would increase, from baseline, in response to an intravenous lipopolysaccharide challenge in healthy volunteers.

Methods. Intravenous lipopolysaccharide ( $2 \mathrm{ng} / \mathrm{kg}$ ) was administered to healthy volunteers as a clinically relevant model of sepsis. Blood samples were taken at $0 \mathrm{~h}, 4 \mathrm{~h}$ and $24 \mathrm{~h}$. Samples were analysed using a validated Syndecan-1 ELISA technique.

GraphPad Prism version 8.4.0. was used for statistical analysis.

Results.

Serum Syndecan-1 levels in healthy volunteers injected with LPS

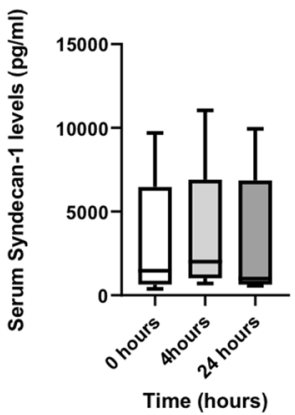

There was no significant change in mean (SD) serum syndecan-1 levels over $24 \mathrm{~h}(3313 \mathrm{pg} / \mathrm{ml}(3332)$ at $0 \mathrm{~h}, 3888 \mathrm{pg} / \mathrm{ml}$ (3864) at $4 \mathrm{~h}$ and $3389 \mathrm{pg} / \mathrm{ml}(3674)$ at $24 \mathrm{~h})$.

Conclusion. In our study syndecan-1 levels did not rise following administration of lipopolysaccharide. This model is currently not suitable to be used to investigate glycocalyx damage.

Future studies using this model could consider using a higher dose of intravenous lipopolysaccharide $(4 \mathrm{ng} / \mathrm{kg}$ ) and assessment of different time points.

\section{Reference(s)}

1. 1 Chelazzi C, Villa G, Mancinelli P, De Gaudio AR, Adembri C. Glycocalyx and sepsis-induced alterations in vascular permeability. Critical care. 2015 Dec;19(1):26.

2. 2 Schött U, Solomon C, Fries D, Bentzer P. The endothelial glycocalyx and its disruption, protection and regeneration: a narrative review. Scandinavian journal of trauma, resuscitation and emergency medicine. 2016 Dec 1;24(1):48.

3. 3 Alexopoulou AN, Multhaupt HA, Couchman JR. Syndecans in wound healing, inflammation and vascular biology. Int J Biochem Cell Biol 2007:39:505-28

4. 4 Chappell D, Bruegger D, Potzel J, Jacob M, Brettner F, Vogeser M, Conzen P, Becker BF, Rehm M. Hypervolemia increases release of atrial natriuretic peptide and shedding of the endothelial glycocalyx. Critical care. 2014 Oct 1;18(5):538

5. 5 Garrido AG, Figueiredo LF. Experimental models of sepsis and septic shock: an overview. Acta Cirurgica Brasileira. 2004 Apr;19(2):82-8.

6. 6 Rehm M, Bruegger D, Christ F, Conzen P, Thiel M, Jacob M, et al. Shedding of the endothelial glycocalyx in patients undergoing major vascular surgery with global and regional ischemia. Circulation 2007;116: 1896-906.

7. 7 Anand D, Ray S, Srivastava LM, Bhargava S. Evolution of serum hyaluronan and syndecan levels in prognosis of sepsis patients. Clin Biochem. 2016:49: 768-76.

8. Nil to add.

001460

Severe invasive Streptococcal Infections and clinical entities: a retrospective study

C. Carvalho ${ }^{1} ;$ H. Santos, ${ }^{1}$; J. Carvalho, ; R. Amaral

${ }^{1}$ Intensive Medicine, Centro Hospitalar De Trás-Os-Montes E Alto Douro, E.P.E., Vila Real, Portugal

Correspondence: $C$. Carvalho

Intensive Care Medicine Experimental 2021, 9(1): 001460

Introduction. Severe disease by invasive Streptococcal group was recognized not only by the occurrence of streptococcal toxic shock syndrome, but also by an increase in severity and incidence of other clinical entities including group A Streptococcus (GAS) pneumonia and soft tissue infection.

Objectives. The aim of the study was to elucidate the epidemiology of invasive streptococcus infection, in association with the clinical picture and outcome.

Methods. Retrospective observational study, involving all cases with severe streptococcal infection, between 2011-2021 at CHTMAD, using descriptive statistical analysis.

Results. 44 patients with severe Streptococcal infection patients were enrolled. Thirty $(72 \%)$ of these patients were male, the median age was 70 years, and 22 cases of infections were acquired in the community (50\%). Clinical manifestations were: soft tissue infection $(n=17)$, bacteremia $(n=10)$, urinary infection $(n=3)$, bone infection $(n=6)$ and pneumonia $(n=8)$. The main comorbidities of severe invasive Streptococcus infection were hypertension $(52 \%)$, immunosuppression (27\%), cancer (26\%) and kidney disease (19\%). At admission on ICU, the mean, Saps II, SOFA score and number of organ failures were 51 , 11 , and 3 , respectively. One-third of the patients were not treated with clindamycin. The ICU mortality was $33,3 \%$, and $21 \%$ in the subgroup with the use of adjunctive therapy, clindamycin.

Conclusion. Severe invasive Streptococcal infections are uncommon, although serious and often life threatening. The available evidence supports the need of identifying and removing any source of streptococcal infection, resuscitation and supportive care with aggressive fluid replacement and inotropes. First principle suggest that the earlier adjunctive therapy is started, the more effective and rapidly it will interrupt the inflammatory cascade. Future research is needed to elucidate which patients benefit most, and thus how early in the course of illness adjunctive therapy needs to be initiated for greatest benefit. 
Reference(s)

1. Steer AC, Lamagni T, Curtis N, Carapetis JR. Invasive group a streptococcal disease: epidemiology, pathogenesis and management. Drugs. 2012 Jun 18;72(9):1213-27. https://doi.org/10.2165/11634180-000000000-00000. PMID: 22686614; PMCID: PMC7100837

2. Steer, A. C., Lamagni, T., Curtis, N., \& Carapetis, J. R. (2012). Invasive Group A Streptococcal Disease. Drugs, 72(9), 1213-1227. https://doi.org/10. 2165/11634180-000000000-00000

3. Ispahani, P., Donald, F. E., \& Aveline, A. J. D. (1988). Streptococcus pyogenes bacteraemia: An old enemy subdued, but not defeated. Journal of Infection, 16(1), 37-46. https://doi.org/10.1016/s0163-4453(88)96073-2

4. Kristensen, B., \& Sch nheyder, H. C. (1995). A 13-year survey of bacteraemia due to -haemolytic streptococci in a Danish county. Journal of Medical Microbiology, 43(1), 63-67. https://doi.org/10.1099/00222 615-43-1-63

5. Streptococcus pyogenes Bacteraemia in Cambridge-A Review of 67 Episodes. (1988). QJM: An International Journal of Medicine. https://doi. org/10.1093/oxfordjournals.qjmed.a068228

e-Posters: Metabolism, endocrinology, liver failure and nutrition

\section{7}

Increased glucocorticoid receptor alpha expression and signaling in critically-ill COVID-19 patients

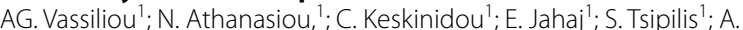
Zacharis'; E. Botoula ${ }^{2}$; A. Diamantopoulos²; D. Vassiliadi²; S. Tsagarakis ${ }^{2}$; A Kotanidou'; I. Dimopoulou

${ }^{1} 1$ st department of Critical Care Medicine \& Pulmonary Services, National and Kapodistrian University of Athens, Athens, Greece; ${ }^{2}$ Department of Endocrinology, Diabetes and Metabolism, Evaggelismos General Hospital, Athens, Greece

Correspondence: A.G. Vassiliou

Intensive Care Medicine Experimental 2021, 9(1): 000017

Introduction. Critical illness is characterized by increased serum cor tisol concentrations and bioavailability resulting from the activation of the hypothalamic-pituitary-adrenal axis, which constitutes an essential part of the stress response. The actions of glucocorticoids are mediated by a ubiquitous intracellular receptor protein, the glucocorticoid receptor (GCR). However, so far, data on COVID-19 and GCR-a expression are lacking

Objectives. To measure GCR-alpha expression and signaling in critically-ill COVID-19 patients.

Methods. Expression of GCR- $a$ and glucocorticoid-inducible leucine zipper (GILZ), and serum cortisol were measured on ICU admission in 26 COVID-19 patients and 33 non-COVID-19 patients, matched for age, sex, and disease severity. All patients were steroid-free.

Results. In COVID-19 patients, GCR- $a$ and GILZ mRNA expression were upregulated (4.7-fold, $p<0.01$ and 14-fold, $p<0.0001$, respectively), and cortisol was higher $(20.3 \mu \mathrm{g} / \mathrm{dl}$ vs. $14.3 \mu \mathrm{g} / \mathrm{dl}, \mathrm{p}<0.01)$, compared to the control group.

Conclusion. ICU COVID-19 patients showed upregulated GCR-a and GILZ expression, along with cortisol levels, compared to ICU nonCOVID-19 patients. Thus, infection with COVID-19 seems to be associated with increased stress response.

\section{7}

NUTRIC Score as a predictor of outcome in COVID-19 ARDS

Patients. A retrospective observational study

N. Kumar ; A. Kumar²; A. Kumar²; K. Singh²

${ }^{1}$ Trauma and Emergency, AlIMS Patna, Patna, India; ${ }^{2}$ Anaesthesiology,

AlIMS Patna, Patna, India

Correspondence: N. Kumar

Intensive Care Medicine Experimental 2021, 9(1): 000077
Introduction. The COVID-19 pandemic has been caused by the severe acute respiratory syndrome coronavirus 2 (SARS-CoV-2), as named by the International Committee on Taxonomy of Viruses.The nutritional assessment and the early nutritional care management of COVID19 patients must be integrated into the overall therapeutic strategy. Nutritional therapy plays one of the most important role in reducing the mortality in ICU especially in COVID-19 patients, so nutritional status of these patients must be evaluated before the administration of general treatment. Nutritional risk is defined as the risk of adverse effects on clinical outcomes which are dependent on nutritional factors.[1]The NRS-2002 is an simple, easy, efficient and highly sensitive tool that is accepted by health practitioners world-wide. [2] In general, patients with an NRS-2002 score $<3$ are not at nutritional risk, so nutritional support is not required during the first week of hospitalization. So periodic NRS-2002 screening of all COVID-19 patients is required once in every 3-5 days. A score of more than 3 score, required immediate nutritional intervention. The Nutrition Risk in the Critically ill (NUTRIC) score, another recommended screening tool, was developed first to be used specifically for ICU patients. [3] Till this date very little is known about the nutritional risks for critically ill COVID19 patients. Therefore, we conducted a retrospective observational single center study in COVID- 19 ICUs of All India Institute of medical Sciences Patna, India.

Objectives. Our main aims and objective was to assess the NUTRIC Score as a predictor of outcome in COVID-19 ARDS patients.

Methods. This retrospective observational study was conducted in COVID ICUs of All India Institute of medical Sciences Patna, India.A institutional ethical approval was taken from AlIMS Patna and informed consent was waived off. The study population was COVID19 ARDS patients who had been admitted to our ICU. Patients who were RT-PCR positive and fulfilling the criteria of ARDS were included in the study.[4] The Patients' demographic characteristics and clinica information were obtained from the hospital's medical records department. Each patient was evaluated according to the Acute Physiology and Chronic Health Evaluation II (APACHE II) [5] and sequential Organ Failure Assessment (SOFA) [6] scoring criteria and values of IL-6 within $72 \mathrm{~h}$ of their admission to the ICU.

Results. A total of 96 COVID-19 ARDS patients were admitted to ICU, out of which 80 COVID-19 ARDS patients met our inclusion criteria. We observed cut-off values of NUTRIC scores (>3.5) in COVID-19 ARDS patients have higher 28 days mortality risk (AUC 0.802) and higher need of mechanical ventilation (AUC 0.637). The NUTRIC cut-off Score of more than 3.5 was $95 \%$ specific and $62.2 \%$ sensitive in predicting 28-days mortality (Fig. 1). The NUTRIC cut-off score of more than 3.5 was $97.29 \%$ specific and $23.25 \%$ sensitive for detecting the need of mechanical ventilation.(Fig. 2).

Conclusion. The NUTRIC score greater than 3.5 at ICU admission has a higher 28 days ICU mortality and has higher need of mechanical ventilation.

\section{Reference(s)}

1. 6.Vincent JL, Moreno R, Takala J, Willatts S, De Mendonca A, Bruining H, et al. The SOFA (Sepsis-related organ failure assessment) score to describe organ dysfunction/failure. On behalf of the working group on sepsisrelated problems of the European society of intensive care medicine. Intensive Care Med 1996;22(7):707e10.

2. 5. Knaus WA, Draper EA, Wagner DP, Zimmerman JE. Apache II: a severity of disease classification system. Crit Care Med 1985;13(10):818e29.

3. 4.Force ADT, Ranieri VM, Rubenfeld GD, Thompson BT, Ferguson ND, Caldwell E et al. Acute respiratory distress syndrome: the Berlin Definition. JAMA. 2012;307(23):2526-33.

4. 3. Heyland DK, Dhaliwal $R$, Jiang $X$, Day AG. Identifying critically ill patients who benefit the most from nutrition therapy: the development and initial validation of a novel risk assessment tool. Crit Care 2011:15(6):R268.

5. 2.Li ZJ, Chen W. Essentials of nutritional support therapy in critically ill patients. Chinese Journal of Practical Surgery. 2018:38:289-92.

6. 1.Kondrup J, Allison SP, Elia M, Vellas B, Plauth M. ESPEN guidelines for nutrition screening 2002. Clin Nutr 2003:22(4):415e21.

7. Non-Funded. 


\section{7}

Nutritional situation of patients admitted for SARS-CoV-2 infection in our Unit of Intensive Care in a pandemic situation T. Amat Serna ${ }^{1}$; C. Aranda Martínez ${ }^{2}$; LB. Yaguez Mateos ${ }^{1}$; E. Pérez Mesa'; RF. R. ${ }^{3}$

${ }^{1}$ Medicina intensiva, Hospital of Jaen, Jaén, Spain; ${ }^{2}$ Medicina Intensiva, Hospital of Jaen, Jaén, Spain; ${ }^{3}$ Intensive care, Hopsital Neurotrauma-

tológico Universitario, Jaen, Spain

Correspondence: $C$. Aranda Martínez

Intensive Care Medicine Experimental 2021, 9(1): 000087

Introduction. To evaluate the nutritional status of patients admitted to the ICU with a diagnosis of COVID-19 infection during the pandemic, taking into account the recommendations of the SEMICYUC Metabolism and Nutrition Group.

Methods. Retrospective and prospective observational study to analyze the nutritional aspect of patients admitted to the Intensive Care Unit of the University Hospital of Jaén with COVID-19 Pneumonia, from March 2020 to November 2020, using the NUTRIC Score. A descriptive analysis was carried out using the SPSS.

Results. 71 patients were admitted to our ICU with SARS-CoV2 infection, 52 men with a median age of $64(42-80)$. Scores: APACHE II 14 (4-25), SOFA 6 (2-15) and NUTRIC 3 (1-7). At admission: 40\% of the patients required respiratory support with HFNO $(\mathrm{N}=29)$ and $45 \%$ of the patients required orotracheal intubation $(\mathrm{N}=38)$, of which 12 prone maneuvers.

During the first $48 \mathrm{~h}$ in our Unit, of the total number of patients, 51 patients received enteral nutrition (EN) and 19 hyperprotein shakes, on the other hand in 4 cases EN could not be maintained due to its non-tolerance despite optimizing treatment with oligomeric and prokinetic formulas and in 12 cases because the patient was in the PRONE position and with muscle relaxation. Of the total number of patients: 15 received parenteral nutrition, only 5 of them total parenteral nutrition (TPN) and the other 10 mixed nutrition because they did not meet EN requirements on the 6th day.

In the nutritional study, 18 patients presented high nutritional risk (25\%): 5 received hyperprotein shakes on admission, 12 NE in the first 48 h, 6 patients received parenteral nutrition during admission (4 mixed for not meeting nutritional requirements).

No statistically significant relationship has been found between the situation of high nutritional risk and a higher incidence of infection or mortality.

Conclusion. We have seen that the nutritional situation of the patient has been acted more quickly, taking into account their caloric needs and the recommendations of the guidelines. But the need for PN by prone position has still been frequent, it would be necessary to reevaluate these patients to identify possible improvements in order to apply them. This is an objective to improve in order to achieve the required nutritional support.

\section{3}

\section{Proning in critically ill COVID-19 patients with hypoxaemic} respiratory failure is associated with increased insulin requirements, regardless of steroid exposure

H. Griffiths' ${ }^{1}$; S. Lockhart ${ }^{1}$; M. Barne ${ }^{1}$; B. Petrisor ${ }^{1}$; A. Usman ${ }^{1}$; J.

Calvo-Latorre $^{2}$; L. Heales ${ }^{3}$; V. Bansiya ${ }^{2}$; R. Mahroof, ; A. Conway-Morris, ${ }^{4}$

'John Farman Intensive Care Unit, Addenbrooke's Hospital, Cambridge,

United Kingdom; ' ${ }^{2}$ Department of Diabetes and Endocrinology, Cam-

bridge University Hospitals NHS Foundation Trust, Cambridge, United Kingdom; ${ }^{3}$ Department of Anaesthetics \& Intensive Care Medicine, Cambridge University Hospitals NHS Foundation Trust, Cambridge, United Kingdom; ${ }^{4}$ University Division of Anaesthesia, Department of Medicine, University of Cambridge, Cambridge, United Kingdom

Correspondence: $\mathrm{H}$. Griffiths

Intensive Care Medicine Experimental 2021, 9(1): 000333

Introduction. We have previously demonstrated that severity of respiratory failure in COVID-19 pneumonitis is associated with increasing insulin requirements, a surrogate for systemic insulin resistance.
However, it is unclear if the increased insulin resistance observed is a direct result of hypoxia or due to other factors. To investigate this further, we hypothesised that in hypoxaemic respiratory failure, proning, and hence improvement in oxygenation, would be associated with a reduction in insulin requirements.

Methods. We performed a retrospective observational study of patients who underwent first episode prone positioning for acute hypoxaemic respiratory failure due to COVID-19 pneumonitis in our intensive care unit between March and July 2020. Hourly measurements of oxygenation, blood glucose and insulin dose among other co-variates were extracted from electronic medical records. As patients were typically proned for $16 \mathrm{~h}$, we compared data for this duration directly with the 16 -h period preceding proning via two-way repeated measurements ANOVA with time and Supine/Prone as covariates. For variables without consistent hourly measurements, we compared mean values for prone and supine position using paired t-tests.

Results. 26 patients met inclusion criteria and were included in our analysis. As expected, proning was associated with a significant improvement in oxygenation (Mean SpO2, Supine: $91.86 \%$, Prone: $93.7 \%, \mathrm{P}<0.001)$. Contrary to our hypothesis, prone positioning was associated with a small but statistically significant increase in insulin requirements. Importantly, there was no effect of proning on blood glucose (Mean Blood Glucose, Supine: $11.41 \mathrm{mmol} / \mathrm{L}$, Prone: $10.99 \mathrm{mmol} / \mathrm{L}, \mathrm{p}=0.77)$ suggesting that increased insulin requirements were a result of impaired insulin sensitivity, not differential intensity of glycaemic control. We reasoned that this could be attributed to differences in timing of corticosteroid administration. However, when steroid exposure was included in our model as a dichotomous variable the effect of proning on insulin requirements remained significant - excluding this as an explanation of this phenomenon.

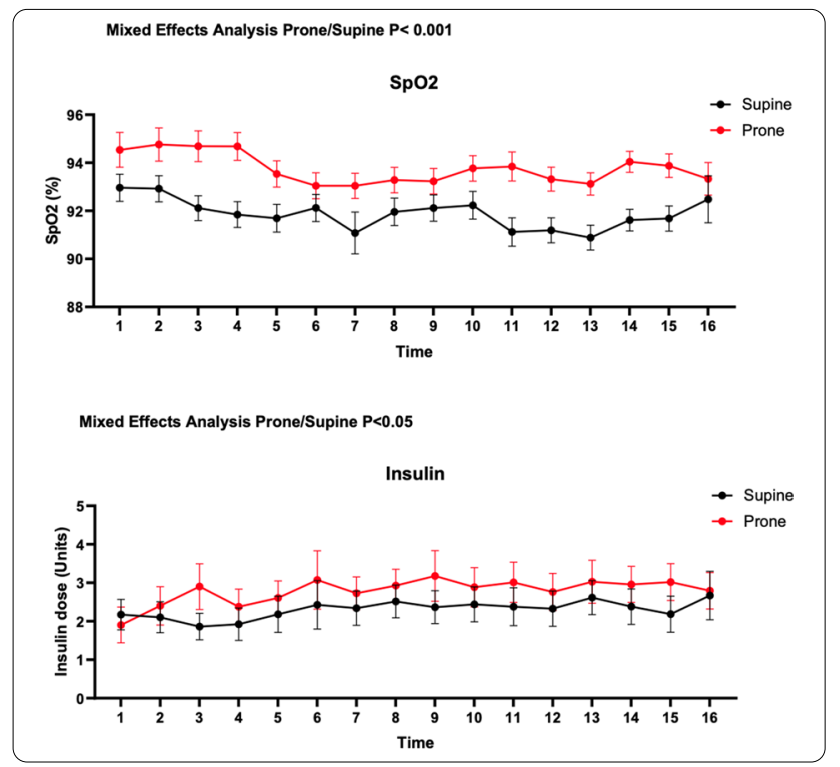

Conclusion. Our results provide preliminary evidence that relative hypoxia may be associated with an increase in insulin sensitivity, thus contradicting our original hypothesis. Interestingly, hypoxia enhances cellular insulin signalling in vitro. Our results provide a potential correlate of this in humans in vivo which may have implications for our understanding of the regulation of systemic metabolism in critical illness. Further work will characterise this association in a larger cohort incorporating comprehensive dietetic analysis.

\section{Reference(s)}

1. SL is supported by a National Institute of Health Research Academic Clinical Fellowship. 
2. ACM was supported by a Clinical Research Career Development Fellowship from the Wellcome Trust at the time of the work (WT 2055214/Z/16/Z) and is currently supported by an MRC Clinician Scientist Fellowship (MR/N006118/1).

\section{8}

\section{Detailed analysis of severe hyperlactatemia in ICU-survivors} shows a limited number of possible diagnoses

L. Wesdijk-Nijhof ${ }^{1}$; F. Smits ${ }^{1}$; P. Freire Jorge ${ }^{1}$; RA. Posma ${ }^{1}$; M. Nijsten ${ }^{1}$ ${ }^{1}$ Department of critical care, University Medical Center Groningen, Groningen, Netherlands

\section{Correspondence: M. Nijsten}

Intensive Care Medicine Experimental 2021, 9(1): 000348

Introduction. Lactate is the most powerful single biochemical predictor of outcome in a wide variety of critical disease states [1]. Although the overall prognosis of severe hyperlactatemia (SHL) is quite poor, immediate diagnosis of the underlying condition may be life-saving. Objectives. We analyzed if the main cause of SHL could be directly established in hospital survivors from information available at the time the blood gas analysis that showed SHL was performed.

Methods. All patients aged $\geq 18$ years in whom at least 2 lactates were measured and who were admitted to our ICU over a 14-year period were screened. SHL was defined as a maximum lactate $(\mathrm{ML}) \geq 12 \mathrm{mmol} / \mathrm{L}$ as measured by point-of-care blood gas analyzer. Hospital mortality was related with ML with univariate logistic regression. Hospital records of survivors with SHL were analyzed in detail for the underlying diagnosis by at least two independent investigators. We determined which diagnoses could immediately be made once SHL was detected.

Results. More than 33,000 adult patients $(62 \%$ mMales; mean $\pm S D$ age of $61 \pm 15$ years) with 870,000 lactate measurements were analyzed. Overall hospital mortality was $12.7 \%$. Patients with SLH of 12 and $20 \mathrm{mmol} / \mathrm{L}$ had predicted mortalities of $63 \%$ and $95 \%$ respectively according to univariate analysis. We identified 190 hospital survivors with an ML of $16.1 \pm 3.5$ (12.0 to 30.0$) \mathrm{mmol} / \mathrm{L}$. Most major causes explaining SHL (including cardiac arrest/cardiogenic shock 55\%; hemorrhagic shock 12\%; generalized tonic clonic seizures $10 \%$; acute liver failure $10 \%$ ) could immediately be determined with the information available at the time SHL was present. A major exception was metformin-associated lactic acidosis (MALA: 5\%).

Conclusion. This study underscores what is well-known, namely that ICU patients presenting with severe hyperlactatemia have a high hospital mortality. However analysis of survivors showed a limited number of possible diagnoses. In addition it showed that underlying causes were typically immediately evident in the majority of cases. As it is known that prompt treatment of MALA with hemodialysis is essential to improve outcome, MALA should be immediately considered if an obvious other cause for severe hyperlactatemia cannot be identified.

\section{Reference(s)}

1. None.

2. • [1] Alkozai et al. Systematic comparison of routine laboratory measurements with in-hospital mortality: ICU-Labome, a large cohort study of critically ill patients. Clin Chem Lab Med 2018.

3. - [2] Calello DP, et al. Extracorporeal Treatments in Poisoning Workgroup. Extracorporeal Treatment for Metformin Poisoning: Systematic Review and Recommendations From the Extracorporeal Treatments in Poisoning Workgroup. Crit Care Med 2015.

\section{3}

Prevalence of liver dysfunction in patients admitted for COVID-19 in intensive care

JE. Barrueco Francioni ${ }^{1}$; MD. Arias Verdú1 ; G. Seller Pérez ${ }^{1}$; C. Aragón

González; ; G. Quesada García; ME. Herrera Gutiérrez

${ }^{1}$ ICU, Hospital Regional Universitario de Málaga (Carlos de Haya), Málaga, Spain

Correspondence: J.E. Barrueco Francioni

Intensive Care Medicine Experimental 2021, 9(1): 000353
Introduction. COVID-19 is a novel disease, some investigators reports find liver dysfunction during COVID-19 but its significance is uncertain. Objectives. Evaluate occurrence of liver dysfunction in patient who requires admission to intensive care for COVID-19.

Methods. Retrospective observational study, including all patients admitted to our ICU with a diagnosis of COVID-19 in the first wave March-April 2020. We analyzed transaminases, bilirubin, INR and factor V Leiden. These variables were stratified as: (AST / ALT > 200); BT: $(<1.2-1.2$ at $6->6)$; INR: $(>2)$. The relationship of these variables with treatments and intra-ICU and hospital mortality was analyzed. The results are shown as percentages, mean (standard deviation) or median (interquartile range) and for the univariate analysis non-parametric tests were applied with a significance of 0.05 .

Results. 42 patients were analyzed, 21.4\% women, mean age 62.4 (14.6), SOFA at admission 7 (5-8) points, mortality in ICU $47.6 \%$ and hospital $50 \%$. No patient had a history of liver disease. $40.5 \%$ had BT $>1.2$ and $7.1 \%>6,19 \%$ had ALT $>200$ and $16.7 \%$ INR $>2$.

Regarding the medication, $95.2 \%$ received hydroxychloroquine; $85.7 \%$ azithromycin; $54.8 \%$ lopinavir + ritonavir; $59.5 \%$ corticosteroids; $50 \%$ tocilizumab, $4.7 \%$ Interferon and $2.4 \%$ remdesivir. We only found an increase in BT with the use of Lopinavir (1.11 \pm 0.6 vs $2.86 \pm 2.69, p$ $0.009)$ and interferon $(1.79 \pm 1.85$ vs $7.59 \pm 0.57, \mathrm{p}<0.001)$.

None of the parameters analyzed was related to mortality. ALT figures were $160 \pm 139$ in survivors vs $158 \pm 265$ in deaths; for BT $2.07 \pm 2.23$ vs $2.07 \pm 2.22$; for INR $1.73 \pm 1.27$ vs $1.56 \pm 0.58$ and for FV Leiden $110 \pm 23$ vs $105 \pm 27$, none of them were significant.

Conclusion. Although the percentage of cholestasis is high in our series, in part it could be due to a pharmacological effect. When assessing liver dysfunction by synthesis or cytolysis parameters, it seldom occurs in COVID-19 disease and does not seem to have an impact on its prognosis.

\section{8}

Preliminary experience with a new management protocol for diabetic ketoacidosis based on isotonic fluids and continuous subcutaneous glucose monitoring

C. Saito ' ; L. Gonzales-Fernandez²; J. Trastoy-Quintela²; E. Rodriguez-Ruiz ; A. Rodriguez-Nuñez ${ }^{2}$

${ }^{1}$ Medicina Intensiva, Complejo Hospitalario Santiago de Compostela, Santiago de Compostela, Spain; ${ }^{2}$ Uci pediátrica, Complejo Hospitalario Santiago de Compostela, Santiago de Compostela, Spain

Correspondence: C. Saito

Intensive Care Medicine Experimental 2021, 9(1): 000458

Introduction. Diabetic ketoacidosis (DKA) as diabetes debut is an acute critical disorder with a treatment that has been based on insulin infusion and unbalanced fluids. Such protocol often leads to multiple manipulations of the bags, changes of fluids composition and may be associated with quick drops of blood glucose levels. Based on the hypothesis that balanced isotonic fluids with continuous subcutaneous glucose monitoring and early introduction of intravenous glucose, would facilitate the patient management, we have designed a new management protocol of DKA.

Objectives. A protocol was designed through a process of discussion and consensus among PICU clinicians and endocrinologists from three Spanish hospitals (Oviedo, Vigo and Santiago), with the following objectives: a) To facilitate the initial fluid therapy with commercial balanced isotonic fluids avoiding the need of repetitive manipulation and addition of electrolytes, b) To prevent electrolyte alterations and their consequences during treatment, and c) Early introduction and continuous monitoring of subcutaneous glucose in order to avoid hypoglycemia.

Methods. Our PICU protocol includes 3 phases: I) Initial stabilization, II) Replacement of water deficit and initiation of insulin therapy and glucose administration, and III) Transition to oral feeding and initiation of subcutaneous insulin regimen.

Results. In first 8 months of application, we treated 7 children with ages between 11 months and 14 years. Their baseline blood glucose ranged between 346 and $606 \mathrm{mg} / \mathrm{dL}$ (median $521 \mathrm{mg} / \mathrm{dL}$ ). After the application of the protocol, only one brief episode of asymptomatic 
hypoglycemia $(60 \mathrm{mg} / \mathrm{dl})$ was detected, and only in one case the rate of glycemia decrease was greater than $100 \mathrm{mg} / \mathrm{dL} / \mathrm{hour}$ in the first $24 \mathrm{~h}$. Three patients showed mild hypokalemia at some time point with no clinical repercussions; they required intravenous supplementation. No other significant biochemical alterations were observed, nor were clinical manifestations or signs in possible relation to the applied protocol were detected. The measurements of the continuous subcutaneous glucose sensors were concordant with capillary or venous blood glucose values and had the added value of tendency monitoring. The attending pediatricians and nurses considered the new protocol as simpler and safer for patients than the classic regimen and reported that it requires fewer calculations and fluid manipulations.

Conclusion. A new treatment protocol for DKA based on balanced isotonic fluids, continuous monitoring of subcutaneous glucose and early introduction of glucose inputs, may improve patient care and facilitate achieving the therapeutic objectives without the need for multiple and potentially risky manipulations of fluids and electrolytes supplementation. In order to define the role, advantages and drawbacks of this new therapeutic plan, a randomized clinical trial should be done and seems to be warranted.

\section{4}

\section{Study of the incidence of dysphagia in ICU patients}

LDP. Armas-Navarro'; L. Mendoza-Segura'; BN. Santana-Lopez ${ }^{2}$; YG. Santana-Padilla33; JA. Alcaráz-Jimenez'; D. Simon-Bautista, ; L. Santana-Cabrera'

${ }^{1}$ Intensive Care Unit, Complejo Hospitalario Universitario Insular Materno Infantil de Gran Canaria, Las Palmas de Gran Canaria, Spain; ${ }^{2}$ Intensive care unit, University Hospital of Gran Canaria Dr. Negrin, Las Palmas de Gran Canaria, Spain; ${ }^{3}$ Surgery unit, Complejo Hospitalario Universitario Insular Materno Infantil de Gran Canaria, Las Palmas de Gran Canaria, Spain; ${ }^{4}$ Rehabilitation service, Complejo Hospitalario Universitario Insular Materno Infantil de Gran Canaria, Las Palmas de Gran Canaria, Spain

Correspondence: B.N. Santana-Lopez

Intensive Care Medicine Experimental 2021, 9(1): 000484

Introduction. Dysphagia is a problem in the ICU whose incidence is not clearly known, nor is there enough information on how to evaluate it.

Objectives. To know the differences between critically ill patients according to the presence or absence of dysphagia.

Methods. Prospective study carried out in an ICU, with extubated or tracheostomized patients who underwent a MECVV-type dysphagia test. Differences in demographic and prognostic characteristics were analyzed, depending on whether or not they had oropharyngeal dysphagia.

Results. During the study period, 77 dysphagia tests were carried out in critically ill patients. Of the total sample, $40.2 \%$ presented dysphagia during the test $(n=31)$.

In our sample, no significant differences were found in those patients who did not present dysphagia with those who did according to sex $(p=0.063)$, neurological diagnosis on admission $(p=0.143)$, or according to neurological history $(p=0.069)$, digestive $(p=0.351)$ or respiratory $(p=0.633)$. No differences were found according to the time of admission to the ICU $(p=0.610)$ or according to the nutritional status $(p=0.220)$.

However, there was a higher incidence of dysphagia in those patients in whom their stay in the ICU prior to the test was longer $(p=0.034)$.

Conclusion. In our sample of critically ill patients, we found no differences in demographic and prognostic characteristics, depending on whether or not they presented oropharyngeal dysphagia.

\section{Reference(s)}

1. 3. Perren A, Zürcher P, Schefold JC. Clinical Approaches to Assess Post-extubation Dysphagia (PED) in the Critically III. Dysphagia. 2019 Aug;34(4):475-486. https://doi.org/10.1007/s00455-019-09977-w

2. 2. Zuercher P, Moret CS, Dziewas R, Schefold JC. Dysphagia in the intensive care unit: epidemiology, mechanisms, and clinical management. Crit Care. 2019;23(1):103. https://doi.org/10.1186/s13054-019-2400-2
3. 1. Skoretz SA, Flowers HL, Martino R. The incidence of dysphagia following endotracheal intubation: a systematic review. Chest. 2010;137(3):66573. http://dx.doi.org/10.1378/chest.09-1823.

\section{2}

Analysis of the incidence of dysphagia in COVID positive patients in an ICU

LDP. Armas-Navarro'; L. Mendoza-Segura'; BN. Santana-Lopez²;

YG. Santana-Padilla ${ }^{3}$; JA. Alcaráz-Jimenez'; D. Simon-Bautista, ; L.

Santana-Cabrera

${ }^{1}$ Intensive Care Unit, Complejo Hospitalario Universitario Insular Materno Infantil de Gran Canaria, Las Palmas de Gran Canaria, Spain; ${ }^{2}$ Intensive Care Unit, University Hospital of Gran Canaria Dr. Negrin, Las Palmas de Gran Canaria, Spain; ${ }^{3}$ Surgery Unit, Complejo Hospitalario Universitario Insular Materno Infantil de Gran Canaria, Las Palmas de Gran Canaria, Spain; ${ }^{4}$ Rehabilitation service, Complejo Hospitalario Universitario Insular Materno Infantil de Gran Canaria, Las Palmas de Gran Canaria, Spain

Correspondence: B.N. Santana-Lopez

Intensive Care Medicine Experimental 2021, 9(1): 000492

Introduction. The incidence of dysphagia in COVID pathology in critically ill patients is a problem in the ICU of which its incidence is not known, nor is there enough information on how to evaluate it.

Objectives. To know the differences between critical patients with COVID pathology according to the presence or not of dysphagia.

Methods. Prospective study carried out in an ICU, with extubated or tracheostomized patients with COVID pathology who underwent a MECVV-type dysphagia test. Differences in demographic and prognostic characteristics were analyzed, depending on whether or not they had oropharyngeal dysphagia.

Results. During the study period, dysphagia testing was performed in 32 ICU patients with COVID infection. Of the total sample, $46.8 \%$ presented dysphagia during the test $(n=15)$. In our sample, no significant differences were found in those patients who did not present dysphagia with those who did according to age, nutritional status, or their medical history.

However, there was a higher incidence of dysphagia in those patients with longer admissions $(p=0.005)$, in whom their stay in the ICU prior to the test was longer $(p=0.006)$, in obese patients $(p=0.046)$ and also in patients with tracheostomy $(p=0.029)$.

Conclusion. In our sample of critical COVID patients, we did not find differences in demographic and prognostic characteristics, depending on whether or not they presented oropharyngeal dysphagia, except in the stay or the time in which the test could be performed, obese patients and those tracheostomized.

\section{Reference(s)}

1. 3. Perren A, Zürcher P, Schefold JC. Clinical Approaches to Assess Post-extubation Dysphagia (PED) in the Critically III. Dysphagia. 2019 Aug;34(4):475-486. https://doi.org/10.1007/s00455-019-09977-w

2. 2. Zuercher P, Moret CS, Dziewas R, Schefold JC. Dysphagia in the intensive care unit: epidemiology, mechanisms, and clinical management. Crit Care. 2019;23(1):103. http://dx.doi.org/10.1186/s13054-019-2400-2.

3. 1. Skoretz SA, Flowers HL, Martino R. The incidence of dysphagia following endotracheal intubation: a systematic review. Chest. 2010;137(3):66573. http://dx.doi.org/10.1378/chest.09-1823.

000533

Energy requirements of the critically ill patient with COVID-19 R. Marinho'; RP. Medeiros ${ }^{1}$; I. Magalhães ${ }^{2}$; R. Sousa ${ }^{3}$; M. Santos ${ }^{4}$; JP. Pinto ${ }^{5}$; A. Marinho

${ }^{1}$ Intensive Care Unit, Hospital Geral de Santo António, Porto, Portugal; ${ }^{2}$ Medical student, Abel Salazar Biomedical Sciences Institute-University of Porto, Porto, Portugal; ${ }^{3}$ Mgf, USF Terras de Souza, Paredes, Portugal; ${ }^{4}$ Clinical nutrition unit, Hospital Lusíadas Porto, Porto, Portugal; ${ }^{5} \mathrm{Clinical}$ nutrition unit, Centro Hospitalar do Médio Ave, Vila Nova de Famalicão, Portugal

Correspondence:Correspondence: R. Marinho

Intensive Care Medicine Experimental 2021, 9(1): 000533 
Introduction. Severe SARS-CoV-2 requires intensive care management with mechanical ventilation and other organ support. Patients with COVID-19 often require prolonged MV and ICU support, resulting in significant immobility, catabolic stress, and muscle wasting. These patients are at high risk of malnutrition during the period of critical care, as well as in the recovery phase of this illness, and may stay in hospital for a significant length of time. However, in the midst of a pandemic, the use of the indirect calorimetry to measure resting energy expenditure (REE) is restricted, so clinical nutrition societies have issued recommendations based on standardized formulas or bodyweight calculations. However, the predictions might correlate poorly with measured REE, as the clinical data is still scarce.

Objectives. The aims of this study were to assess the energy expenditure of critically ill patients with SARS-CoV-2 pneumonia requiring intubation and to compare our findings with the predictive equations and the nutrition that was administered and correlate to stress variables.

Methods. Unicentric retrospective observational study conducted in a large tertiary university hospital. Descriptive analysis of the results of 49 measurements, in 21 COVID19 patients hospitalized in an Intensive Care Unit between January and April of 2021. Data were obtained regarding resting energy expenditure (REE), energy expenditure per kilogram (EEKg) and respiratory exchange rate quotient (RER), by the method of indirect calorimetry. Stress variables such as hemodynamic support, sedation, ventilatory and prone methods were also evaluated.

Results. 19 patients were included in this study. Seventy-eight percent of the study subjects were male, with a mean age of $64.6 \pm 12.3$ years. Mean weight was $88.9 \pm 21.7 \mathrm{~kg}$ and $52.6 \%(\mathrm{n}=9)$ of patients are obese. The mean SOFA score at admission was $5.7 \pm 2.5$ and SAPS II score was $30.2 \pm 12.5$. The mortality rate was $52.6 \%$.

A total of 44 indirect calorimetry determinations were performed with a mean of resting energy expenditure was $1711.5 \pm 354.8 \mathrm{kcal} /$ day, $19.93 \pm 4.6 \mathrm{kcal} / \mathrm{Kg} /$ day when using the actual weight and $22.6 \pm 4.2 \mathrm{kcal} / \mathrm{Kg} / \mathrm{day}$ when using adjusted weight for obese patients. These correspond to $70.6 \%$ of energy requirements when compared to predicted using Harris-Benedict equation. During the first 21 days post-intubation there were no significant differences in the energy expenditure. With a difference of $-213 \pm 606 \mathrm{kcal} /$ day $(p=0.02)$ or $-1.95 \pm 7.2 \mathrm{kcal} / \mathrm{Kg} /$ day $(p=0.08)$.

The use of sedatives decreased the energy requirements and the use of vasopressor amines and prone position increased the energy requirements.

Conclusion. Although it was initially predicted that COVID in critically ill patients would lead to a hypercatabolic state, this was not the case in our sample. No significant difference in measured energy expenditure in the first 21 days was observed in this sample of COVID19 patients. In our sample the patients remained normocaloric as long as they remained in mechanical ventilation. Sedation decreased energy expenditure and prone position and use of vasopressor used increased it. However, it is important to note that we do not supply the required amount of Kcal in our sample.

\section{6}

\section{Loss of Muscle Mass and Nutritional Support in Critically III}

\section{Patients}

R. Marinho'; T. Mendoça ${ }^{2}$; R. Sousa ${ }^{3}$; V. Cesar' ; RP. Medeiros ${ }^{1}$; M. Santos ${ }^{4}$; JP. Pinto ${ }^{5}$ : A. Marinho ${ }^{1}$

'Intensive Care Unit, Hospital Geral de Santo António, Porto, Portugal; ${ }^{2}$ Internal medicine, Centro Hospitalar do Tâmega e Sousa, Penafiel, Portugal; ${ }^{3}$ Mgf, USF Terras de Souza, Paredes, Portugal; ${ }^{4}$ Clinical nutrition unit, APNEP, Porto, Portugal; ${ }^{5}$ Clinical nutrition unit, Centro Hospitalar do Médio Ave, Santo Tirso, Portugal

Correspondence: R. Marinho

Intensive Care Medicine Experimental 2021, 9(1): 000536

Introduction. The loss of muscle mass is an increasingly prevalent problem in critically ill patients, being strongly related to their prognosis and quality of life after leaving the Intensive Care Unit. Several studies have shown that the patient in critical condition undergoes metabolic changes, which translates into a marked hypermetabolism and hypercatabolism, with a negative nitrogen balance. Currently, it has been the target of several studies on how an adequate nutritional support can influence this process, in order to mitigate the loss of muscle mass.

Objectives. The aim of this study was to evaluate the loss of muscle mass in the first five days of intensite care stay, and to evaluate if there was a correlation between the loss of muscle mass and the nutritional support provided to patients, during this period of time.

Methods. A prospective observational study was carried out in a Intensive Care Unit between January and March 2020. On the day of admission and on the 5th day of stay, an ultrasound evaluation of the thickness of the biceps brachii and quadriceps femoris was performed. A Wilcoxon test for paired samples later used to verify the significance of the loss of muscle mass. The nutritional support during this time frame was also analyzed. The Spearman coefficient was used to assess whether there is a correlation between the two variables analyzed in this study. A value of $p<0.05$ was considered significant.

Results. 35 patients were evaluated. Average age of $64 \pm 15$ (64) years, being mostly male $(62.9 \%)$.The median SOFA score was $6 \pm 3(1-12)$ Median BMI was $26,62 \pm 5,16$. There as a loss of $1.0 \mathrm{~mm}(p=0.0001)$ in the thickness of the biceps brachii and $1.4 \mathrm{~mm}(p=0.0001)$ in the quadriceps femoris. Using the current recommendations of ASPEN, it was found that patients received nutritional support corresponding to $20.9 \%$ of their energy needs and $16.5 \%$ of their estimated protein needs. Statistically, there was no significant correlation between loss of muscle mass in the brachial biceps with protein intake $(\rho=-0.123$, $p=0.450)$ and energy intake $(\rho=-0.132, p=0.449)$. In the femoral quadriceps, there was also no significant correlation between loss of muscle mass and protein intake $(\rho=0.047, p=0.777)$ and energy intake $(\rho=0.046, p=0.793)$.

Conclusion. Muscle mass atrophy is an inevitable consequence in critically ill patients and is, in most cases, related to the worsening morbidity and mortality of these patients. Several studies point to ultrasound as a good tool to assess muscle thickness in critically ill patients, demonstrating that it offes reliable results.

In this sample, the nutritional support provided was considerably lower than that recommended by the international guidelines of ASPEN and ESPEN. Even taking into account the maximum $50-70 \%$ supply of nutritional needs in the first week of hospitalization referred to by multiple studies, the provided value was much lower. This disparity was even more accentuated regarding the protein intake.

Despite this apparent hyponutrition and generalized decrease in muscle mass in this sample, no statistically significant correlation was found between these two situations, which might be justified by the short evaluation time, the precocity of the same, or by overestimation the nutritional needs of these patients, in these critical conditions. In our study, the ultrasound documented that there is a loss of muscle mass in our patients in the first five days in an Intensite Care Unit.

000564

Statements about nutrition in patients with Sars-cov2's pneumonia admitted in the ICU

L. Hidalgo Manchado'; T. Rodríguez Fernández De Simón'; P. Fernandez Florido ; F. Villagran Ramirez²; J. Carrillo Acosta' ; F. Manzano Manzano ${ }^{1}$ Intensive Care Unit, University Hospital Complex of Granada, Granada, Spain; ${ }^{2}$ Intensive Care Unit, Hospital Virgen de las Nieves, Granada, Spain Correspondence: $\mathrm{L}$. Hidalgo

Intensive Care Medicine Experimental 2021, 9(1): 000564

Introduction. Sars Cov2 infection has become a worldwide pandemic causing multiple admissions in ICU with the need of mechanical ventilation, hence the need of artificial nutrition. The refeeding syndrome causes a high morbility and mortality in ICU patients being frequent in patients with artificial nutrition.

Objectives. To acknowledge the prognosis of the refeeding syndrome in patients with Sars-cov2's pneumonia.

Methods. We perform a retrospective, observational study that includes 71 patients admitted in the ICU between September and November 2020 with the dianognosis of Sars-cov2's pneumonia, with 
more than $24 \mathrm{~h}$ of mechanical ventilation and that received enteral or parenteral nutrition for more than 3 days.

We gather information about baseline features, SOFA score of the first day, mechanical ventilation duration and ICU mortality. We made a stadystical analysis with t-student, Chi-squared test and proportional hazards model.

We measure the levels of magnesium, phosphorus and vitamin $D$ in the beginning of artificial nutrition and $48 \mathrm{~h}$, a week, and two weeks later. Refeeding syndrome is defined as hypophosphatemia with a decrease of $30 \%$ of the baseline leves or a value of less than $1.86 \mathrm{mg} /$ dl.

Results. 71 patients were included with ages between $60 \pm 14$. $29.6 \%$ of the patients had diabetes and $21 \%$ dyslipidemia. Mortality in the ICU and in the hospital were $36,6 \%$ and $38 \%$, respectively. The medium duration of mechanical ventilation was $17 \pm 10$ days and the ICU stay was $18 \pm 10$ days. Refeeding syndrome was present in $21.6 \%$ of the patients, with a decrease of $18,4 \pm 13,4 \%$ in phosphatemia in the first week. The prevalence of magnesium deficiency was $12,8 \%$ and of hypovitaminosis D was $54.5 \%$. Enteral nutrition was used in $92,5 \%$ of the patients, with a duration of $13,8 \pm 13$ days, and parenteral nutrition was given to $12,7 \%$ of patients.

Patient's mortality was:

- With/witouth diabetes mellitus: $47,6 \%$ vs $25,7 \%(\mathrm{p}=0.09)$.

- With/witouth hypovitaminosis D: $40 \%$ vs $33,3 \%(\mathrm{p}=0,64)$.

- With/witouth refeeding syndrome: $45 \%$ vs $25 \%$ [RR 2,5 (0.62-9.9)] $(\mathrm{p}=0,18)$.

- With/witouth magnesium deficiency: $16,7 \%$ vs $34 \%$ [RR $0.38(0,4-$ 3.6)] $(\mathrm{p}=0.39)$.

The prognostic factors asocciated with an increased in mortality in the logistic model were the age (HR 1,03; IC95\% 0,98-1,09; $p=0,15$ ), the refeeding syndrome (HR 0,39; IC95\% 0.08-1,78; $p=0,22)$, dyslipidemia (HR 0,46; IC95\% 0,10-2,06; $p=0,31$ ) and diabetes mellitus (HR 4,35; IC95\% 1,09-17,35; $p=0,03$ ).

Conclusion. The refeeding syndrome is not associated with an increased in mortality in patients with Sars Cov2 pneumoniae in mechanical ventilation admitted in the ICU. However, the sample size was small. Diabetes mellitus was associated with a worse outcome.

\section{9}

Long-term kidney function after acute liver failure: a cohort analysis

P. Fidalgo, ${ }^{1}$; P. Povoa'; N. Germano, ${ }^{2}$; F. S. Cardoso ${ }^{2}$

${ }^{1}$ Polyvalent intensive care unit, São Francisco Xavier Hospital, CHLO, Lisbon, Portugal; ${ }^{2}$ Ucip 7, Hospital Curry Cabral, CHULC, Lisbon, Portugal Correspondence: $P$. Fidalgo

Intensive Care Medicine Experimental 2021, 9(1): 000579

Introduction. Acute liver failure (ALF) is a rare disease with high shortterm mortality and morbidity. Acute kidney injury (AKI) is a frequent complication of ALF and has been associated with worse short-term outcomes but little is known about its impact on long-term kidney function.

Objectives. Assess long-term kidney function after intensive care unit (ICU) admission due to ALF.

Methods. Retrospective cohort analysis of prospectively collected data from all consecutive adult patients ( $\geq 16$ years) admitted to a liver ICU at Curry Cabral Hospital, Lisbon, Portugal (100-120 liver transplants per year) between October 2013 and February 2020. Daily serum creatinine $(\mathrm{sCr}, \mathrm{mg} / \mathrm{dl})$ and renal replacement therapy (RRT) use were recorded for the first 7 days of ICU stay. We used the KDIGO criterion to define AKI and to calculate baseline $\mathrm{sCr}$, if not available. Long term kidney function was defined as 2 determinations of $\mathrm{s} C \mathrm{r}$, at least 3 months apart in steady state ${ }_{1}$, at least one year after index ICU admission. Estimated glomerular filtration rate (eGFR) was calculated using CKD-EPI formula ${ }^{2}$. Primary outcome was defined as eGFR at least one year after index ICU admission. Univariate comparisons used student t-test or Mann-Whitney test where appropriate.
Results. Among 104 patients with ALF included, mean (SD) age was 43.7 (18.0) years and $44(42.3 \%)$ were male. Non-paracetamol etiologies were found in $86(82.7 \%)$ patients.

On index ICU admission, mean (SD) SOFA score was 7.6 (4.8); 27 (26.0\%) patients had grade 3-4 hepatic encephalopathy (West Haven criteria), 21 (20.2\%) were mechanically ventilated, with mean (SD) PF ratio of 381.1 (126.3) $\mathrm{mmHg}, 19$ (18.3\%) were on vasopressors, and 14 (13.5\%) were on RRT. At this time point, median (IQR) serum INR, bilirubin, creatinine, and lactate were 2.5 (1.8-3.9), 9.1 (2.7-19.2) mg/dl, 0.86 (0.66-1.55) $\mathrm{mg} / \mathrm{dl}$, and $2.0(1.5-3.4) \mathrm{mmol} / \mathrm{l}$, respectively.

Overall, median (IOR) baseline eGFR was 79.3 (76.7-80.6) $\mathrm{ml} /$ $\mathrm{min} / 1.73 \mathrm{~m} 2$. During the first 7 days of ICU stay, $53(51.0 \%)$ patients developed AKI and $33(31.7 \%)$ required RRT, 23 (69.7\%) on a continuous RRT.

Overall, during a mean (SD) follow-up time of 2.4 (1.9) years, $34(32.7 \%)$ patients required liver transplant (LT) and 32 (30.8\%) died, 30 (93.8\%) of these during index hospital stay. Among 74 patients discharged alive from the hospital, 56 had available long-term kidney function, with a median (IQR) eGFR of $95.3(75.0-107.7) \mathrm{ml} / \mathrm{min} / 1.73 \mathrm{~m} 2$, for a mean (SD) follow-up time of 3.1 (1.6) years. Moreover, there was no significant difference between those patients who had on index ICU stay AKI or not [86.7 (72.7-103.3) vs $97.2(76.2-110.6) \mathrm{ml} / \mathrm{min} / 1.73 \mathrm{~m} 2$, $\mathrm{p}=0.34]$, those treated with RRT or not [102.2 (73.4-122.6) vs 95.0 (75.3-105.9) $\mathrm{ml} / \mathrm{min} / 1.73 \mathrm{~m} 2, \mathrm{p}=0.56]$, or those who underwent LT or not [93.2 (69.5-104.3) vs 97.1 (79.6-109.3) $\mathrm{ml} / \mathrm{min} / 1.73 \mathrm{~m} 2, \mathrm{p}=0.21$ ]

Conclusion. We present one of the first studies describing long-term kidney function after ICU admission due to ALF. In our cohort, among ALF survivors, long-term kidney function was largely preserved, irrespective of previous AKI, RRT use, or LT.

\section{Reference(s)}

1. 1. Kidney Disease: Improving Global Outcomes (KDIGO) Acute Kidney Injury Work Group. KDIGO Clinical Practice Guideline for Acute Kidney Injury. Kidney inter., Suppl. 2012; 2: 1-138.

2. 2.Kidney Disease: Improving Global Outcomes (KDIGO) CKD Work Group. KDIGO 2012 Clinical Practice Guideline for the Evaluation and Management of Chronic Kidney Disease. Kidney inter., Suppl. 2013; 3: 1-150.

\section{2}

Parenteral Nutrition: Epidemiology, patterns of administration and factors associated with mortality

JC. Lopez-Delgado'; L. Servia-Goixart'².T. Grau-Carmona ${ }^{3}$; E. Portugal-Rodriguez ; E. Mor-Marco ; C. Lorencio-Cardenas ; R. Gastaldo Simeón ${ }^{7}$; E. Navas-Moya ${ }^{8}$; JF. Martinez-Carmona ${ }^{9}$; JC. Yebenes-Reyes ${ }^{10}$ ${ }^{1}$ Intensive Care, Hospital Universitari de Bellvitge, L'Hospitalet de Llobregat (Barcelona), Spain; ${ }^{2}$ Intensive Care, Hospital Arnau de Vilanova, LLeida, Spain; ${ }^{3}$ Intensive care, Hospital 12 de Octubre, Madrid, Spain; ${ }^{4}$ Intensive Care, Hospital Clínico, Valladolid, Spain; Intensive Care, Hospital Germans Trias i Pujol, Badalona, Spain; ${ }^{\circ}$ Intensive care, Hospital Josep Trueta, Girona, Spain; Intensive Care Unit, Hospital de Manacor, Manacor, Spain; ${ }^{8}$ Intensive Care, Hospital Mútua de Terrassa, Terrassa, Spain; ${ }^{9}$ Intensive Care, Hospital Regional Universitario de Málaga, Málaga, Spain; ${ }^{10}$ Intensive Care, Hospital de Mataró, Mataró, Spain

Correspondence: J.C. Lopez-Delgado

Intensive Care Medicine Experimental 2021, 9(1): 000612

Introduction. The use of Parenteral Nutrition (PN) has evolved over the years due to technical improvements that make PN as safe as EN. Indeed, their use and when to administered PN in critical care has been controversial.

Objectives. To describe the use of PN in the ICU, as well as its different patterns of administration, and to evaluate nutritional variables associated with mortality.

Methods. National multicenter prospective observational study (37 hospitals) (NCT Registry: 03,634,943). Demographic data and comorbidities, reason for admission, nutritional assessment, caloric-protein dose administered (up to 14 days), laboratory variables, and complications, were collected. Statistical analysis was performed independently using univariant and multivariate analysis (SPSS 20.0). 
Results. 229 patients who received PN during their admission to the ICU were included. The mean age was $63.55 \pm 13.9$ years; $67.7 \%$ were men; BMI: $26.9 \pm 5.1 \mathrm{~kg} \cdot \mathrm{m}^{-2}$; APACHE II: $20.3 \pm 7.6 .48 \%$ and $46.7 \%$ were medical and surgical patients respectively. Only $23.6 \%(54)$ received early PN $(<48 \mathrm{~h})$. They received a mean caloric and protein dose of $19.1 \pm 6.7 \mathrm{kcal} / \mathrm{Kg} / \mathrm{d}$ and $0.99 \pm 0.4 \mathrm{~g} / \mathrm{Kg} / \mathrm{d}$ respectively.

112 (49\%) received total PN and 117 (51\%) received also enteral nutrition (mixed PN). Mortality in these subgroups was highly variable, with lower mortality for patients who received total PN $(24.11 \%$ vs $37.21 \%$ (NE-PN) vs $25.68 \%$ (PN-NE), as well as the patterns of administration in mixed PN (see Figure 1).

When analyzing factors associated with mortality, it was observed that a high NUTRIC Score (Hazard Ratio (HR): 1.334; 95\% Cl: 1.013-1.758; $\mathrm{P}=0.041$ ) was associated with higher mortality, while higher Prealbumin levels on day 7 they were associated with lower mortality (HR: 0.982; 95\% Cl: 0.971-0.994; $\mathrm{P}=0.002$ ).

Conclusion. There is great variability when PN is administered in the ICU, which may probably require a greater consensus and higher standardization among different hospitals. Nutritional risk, as well as laboratory variables, may be associated with mortality in these patients.

\section{9}

Effect of Ringer Lactate versus Plasmalyte on electrolytes

and metabolic acidosis in critically ill patients

M. Koopmans ${ }^{1}$; I. Stam ${ }^{1}$; RF. Crane'; B. Bogaard Van Den ${ }^{1}$

${ }^{1}$ Intensive Care, OLVG location East, Amsterdam, Netherlands

Correspondence: $\mathrm{M}$. Koopmans

Intensive Care Medicine Experimental 2021, 9(1): 000619

Introduction. Crystalloid intravenous fluids most notably differ in sodium and chloride content. Extensive use of intravenous fluids could lead to hypernatremia and hyperchloremic acidosis. We therefore set out to investigate the effect of Ringer Lactate (RL) and Plasmalyte (PL) on hypernatremia and hyperchloremic acidosis in critically ill patients. Methods. We performed a non-randomised before-after single-centre retrospective cohort study in a 20 bed mixed medical-surgical ICU of a teaching hospital (OLVG, Amsterdam, the Netherlands) comparing RL to $\mathrm{PL}$. The type of crystalloid fluid used was changed from $\mathrm{RL}$ to $\mathrm{PL}$ as of April 2019. We compared the year prior to the change (RL) to the year after introduction of PL. All adult ICU patients admitted between April 2018 and April 2020, with a length of stay $>24 \mathrm{~h}$ were included. The primary outcome was difference in incidence of hypernatremia defined as plasma sodium $>145 \mathrm{mmol} / \mathrm{L}$ during the first 7 days after admission.

Results. We included 1460 patients (mean age $65 \pm 13,64 \%$ male): 783 in RL group and 677 in PL group. Baseline characteristics were comparable for age, sex, BMI, admission category, APACHE II score, chloride and bicarbonate plasma levels. Sodium plasma level was slightly higher at baseline in Plasmalyte group (139 versus $138 \mathrm{mmol} / \mathrm{L}$, $p=0.03$ ). We found an increased incidence of hypernatremia in the PL group ( $27 \%$ vs $35 \%, p=0.001)$. There was no difference in total fluid balance at discharge or after 7 days between groups.

Conclusion. We found a higher incidence of hypernatremie in the PL group compared tot the RL group.

\section{2}

Persistent Inflammation, Immunosuppression and Chronic Catabolism Syndrome (PICS) in ICU Patients at the Fundación Santa Fe de Bogota University Hospital

V. Giraldo, ; A. Vergara ${ }^{2}$; FD. Camacho-Obando ${ }^{3}$; J. Carrizosa ${ }^{4}$

${ }^{1}$ Critical Care Medicine, Sede Quinta de Mutis, Universidad del Rosario, Bogotá, Colombia; ${ }^{2}$ Department of Surgery, Fundacion Santa Fe de Bogota University Hospital, Bogotá, Colombia; ${ }^{3}$ School of Medicine, Universidad de los Andes, Bogotá, Colombia; ${ }^{4}$ Critical Care Medicine, University Hospital Fundación Santa Fe de Bogotá, Bogota, Colombia Correspondence: J. Carrizosa

Intensive Care Medicine Experimental 2021, 9(1): 000652
Introduction. Patients admitted into an intensive care unit (ICU) are at risk of suffering a chronic critical illness (CCI) (1). 30 to $50 \%$ of patients with $\mathrm{CCl}$ develop a condition known as persistent inflammation, immunosuppression, and chronic catabolism syndrome (PICS) (2). PICS is associated with recurrent infections, poor wound healing, rehabilitation failure, and indolent death (3-6). Thus, identifying these patients is critical to address unique treatments.

Objectives. This study aims to describe PICS syndrome in patients admitted to the intensive care unit from 2019 to 2021 in a tertiary University Hospital in Bogotá, Colombia.

Methods. This was a retrospective longitudinal descriptive study. Mortality, age, admission diagnostic, lymphocytes count, albumin levels, weight change, and c-reactive protein levels data were collected in patients with a prolonged ICU stay (>14d).

Results. 63 patients with prolonged ICU stay were analyzed, 14,29\% of these patients died while hospitalized, $57,4 \%$ were 65 years old or older, the most common admission diagnostic was post-neurosurgical care $(36,51 \%), 42,8 \%$ of patients had albumin serum levels lower than $3.0 \mathrm{mg} / \mathrm{dL}$, a lower than 800 lymphocyte count was present in $46,03 \%$ of patients, $33,3 \%$ of patients had a $10 \%$ or more significant decrease in their weight from the moment of admission to discharge.

Conclusion. Our results are similar to those found in previous literature (3-6), suggesting that patients with a prolonged ICU stay are at risk of developing PICS as they present lymphopenia, weight loss, and low serum albumin levels.

\section{Reference(s)}

1. 1. Julie A. Stortz, MD1, Juan C. Mira, MD1, Steven L. Raymond, MD1, Tyler J. Loftus M, Tezcan Ozrazgat-Baslanti, PhD2, Zhongkai Wang, MS3, Gabriela L. Ghita, MPH3 C, Leeuwenburgh, PhD4, Mark S. Segal, MD, PhD2, Azra Bihorac, MD2 BAB, PhD3, Alicia M. Mohr, MD1, Philip A. Efron, MD1, Lyle L. Moldawer, PhD1 FA, Moore, MD1, and Scott C. Brakenridge M. Benchmarking clinical outcomes and the immunocatabolic phenotype of chronic critical illness after sepsis in surgical intensive care unit patients. J Trauma Acute Care Surg. 2018:123(24):342-9.

2. 2. Gentile LF, Cuenca AG, Efron PA, Ang D, Bihorac A, McKinley BA, et al. Persistent inflammation and immunosuppression: A common syndrome and new horizon for surgical intensive care. J Trauma Acute Care Surg. 2012;72(6):1491-501.

3. 3. Hiroyuki Horiguchi1 2, Tyler J. Loftus1, Russell B. Hawkins1 SLR, Julie A. Stortz1, McKenzie K. Hollen1, Brett P. Weiss1, Elizabeth S. Miller1 AB, Shawn D. Larson1, Alicia M. Mohr1, Scott C. Brakenridge1 HT, Ueno2 $\mathrm{H}$, Moore1 FA, et al. Innate Immunity in the Persistent Inflammation, Immunosuppression, and Catabolism Syndrome and Its Implications for Therapy. 2018;9(April):1-20.

4. 4. Mira JC, Gentile LF, Mathias BJ, Efron PA, Brakenridge SC, Mohr AM, et al. Sepsis Pathophysiology, Chronic Critical IIIness, and Persistent Inflammation- Immunosuppression and Catabolism Syndrome. 2016;(13):253-62.

5. 5. Kahn JM, Le T, Angus DC, Cox CE, Hough CL, White DB, et al. The epidemiology of chronic critical Illness in the United States. Crit Care Med. 2015:43(2):282-7.

6. 6. Iwashyna TJ, Hodgson CL, Pilcher D, Bailey M, van Lint A, Chavan S, et al. Timing of onset and burden of persistent critical illness in Australia and New Zealand: a retrospective, population-based, observational study. Lancet Respir Med [Internet]. 2016;4(7):566-73

\section{1}

Factors related to bloodstream infections in patients with central venous catheter and parenteral nutrition support

V. Nuñez-Ricardo, ; A. Vergara ${ }^{2}$; A. Cuatis, ${ }^{3}$; D. Merchan, ${ }^{3}$; H. Acevedo, ${ }^{4}$; A. Forero, ${ }^{5}$; JE. García, ; JC. León, ${ }^{5}$; J. Carrizosa ${ }^{6}$

${ }^{1}$ Metabolic support, Fundacion Santa Fe de Bogota University Hospital, Bogotá, Colombia; ${ }^{2}$ Department of surgery, Fundacion Santa Fe de Bogota University Hospital, Bogotá, Colombia; ${ }^{3}$ Critical care medicine, Universidad del Rosario, Bogotá, Colombia; ${ }^{4}$ Auditory department, Fundacion Santa Fe de Bogota University Hospital, Bogotá, Colombia; ${ }^{5}$ Nursing department, Fundacion Santa Fe de Bogota University Hospital, Bogotá, Colombia; ${ }^{6}$ Critical Care Medicine, University Hospital Fundación Santa Fe 
de Bogotá, Bogota, Colombia

Correspondence: J. Carrizosa

Intensive Care Medicine Experimental 2021, 9(1): 000701

Introduction. Infection of the bloodstream associated with the central venous catheter is one of the most frequent complications in inbound patients, especially in the ICU, and is considered even more remarkable in patients who receive parenteral nutrition, with an enormous impact on morbidity and mortality and costs of care; various associated factors have been identified.

Objectives. The objective was to evaluate the associated factors with bacteremia in patients with parenteral nutrition through a central catheter at the Fundación Santa Fe University Hospital, 2014-2020.

Methods. A cross-sectional study was carried out, based on the analysis of a retrospective and extensive database from the Fundación Santa Fe de Bogotá university hospital's metabolic support service.

Results. Results Out of 1653 patients, 76 were bacteremia cases with a calculated prevalence of $4.16 \%$, which is below the $5 \%$ national standard, although the rate of infection was 17.4 per 1000 catheter days. Age over 65 and having a baseline infectious process are associated factor with mortality.

Conclusion. A large cohort of patients with central venous catheter and parenteral nutrition was evaluated in which no high prevalence of bloodstream infection has seen. Those results suggest that parenteral nutrition could not be a risk factor for bacteriemia; however, evaluation in clinical trials are needed to confirm those results.

\section{6}

\section{Micronutrients in severe COVID-19 patients. Epidemiological} study of the first wave

L. Bielsa' ; T. Tomasa, ; V. Philibert ; V. Ruiz, ; E. Gonzalez, ; L. Raguer, ; L. Bordejé, $^{1}$

${ }^{1}$ Intensive Care Unit, Hospital Germans Trias i Pujol, Badalona, Spain Correspondence: T. Tomasa

Intensive Care Medicine Experimental 2021, 9(1): 000746

Introduction. Micronutrients are an integral part of the immune system, and the body needs optimal levels for effective immune function. It is well established that overt micronutrient deficiencies can adversely affect the immune system and predispose individuals to infections (1). Micronutrients could help in the fight against COVID-19 disease (2).

Objectives. Determine plasma levels of micronutrients (vitamins $A$, B6, C, D, E, and Zinc) upon at hospital admission in COVID-19 patients of the first wave.

Methods. Blood samples were drawn for the analyses of micronutrients in those patients admitted for severe COVID-19 within the first $24 \mathrm{~h}$ of hospital admission. The first wave means from March to May of 2020. At that time, critically ill patients were admitted to the hospital ward and the most severe ill patients to the different ICU units that were being created day by day.

Results. We included 195 patients for this observational study. We found $70 \%$ low levels at least of one micronutrient, and most had several deficits: $68.7 \%, 45.6 \%, 82 \%, 74.3 \%, 8.2 \%$, and $70.3 \%$, in $\mathrm{A}, \mathrm{B} 6, \mathrm{C}$, $D, E$, vitamins and Zinc, respectively. Vitamin A low levels ( $<0.3 \mathrm{mg} / \mathrm{L})$ were detected in 134 patients (68.7\%) with a mean value of 0.17 (0.06); Vitamin B6 low levels $(<3.6 \mathrm{ng} / \mathrm{mL}$ ) were detected in $89(45.6 \%)$ patients with a mean value of $2.2(0.9)$; Vitamin C low levels $(<0.4 \mathrm{mg} /$ $\mathrm{dL}$ ) were detected in 55 (82\%) patients (only measured in UCl patients) with a mean value of $0.14(0.05)$; Vitamin D low levels $(<20 \mathrm{ng} /$ $\mathrm{mL}$ ) were detected in $78(74.3 \%)$ patients with a mean value of 11.4 (4.3); Vitamin E low levels ( $<5 \mathrm{mg} / \mathrm{L})$ were only detected in $16(8.2 \%)$ patients with a mean value of 3.95 (0.87); and Zinc low levels $(<84 \mu \mathrm{g} /$ $\mathrm{dL}$ ) were detected in 137 (70.3\%) patients with a mean value of 63.5 (13.5). Characteristics of the sample: Mean age: 58.74 years; Gender: $63.1 \%$ male; Body Mass Index: $29.7 \mathrm{~kg} / \mathrm{m}^{2}$; Obese patients: $38.5 \%$; Dyslipidemia: 39\%; Arterial hypertension: 32.3\%; Mellitus diabetes: $16.4 \%$; Kidney disease: $16.9 \%$; Thromboembolic disease: $13.3 \%$. The different new created-ICU admitted 80 (41\%) patients: Invasive mechanical ventilation was supplied to 68 (85\%) patients. In-hospital mortality bay day 90 was $23.07 \%$.

Conclusion. 1 . We found $70 \%$ low levels at least of one micronutrient, and most had several deficits.

2. It would be interesting to conduct clinical trials with a combination of several micronutrients in the fight against covid-19.

\section{Reference(s)}

1. 2. Jovic TH, Ali SR, Ibrahim N, Jessop ZM, Tarassoli SP, Dobbs TD, Holford P, Thornton CA, Whitaker IS. Could Vitamins Help in the Fight Against COVID-19? Nutrients. 2020 Aug 23;12(9):2550. https://doi.org/10.3390/ nu12092550. PMID: 32842513; PMCID: PMC7551685.

2. 1. Gombart AF, Pierre A, Maggini S. A Review of Micronutrients and the Immune System-Working in Harmony to Reduce the Risk of Infection. Nutrients. 2020 Jan 16;12(1):236. https://doi.org/10.3390/nu12010236. PMID: 31963293; PMCID: PMC7019735

3. Does not apply.

\section{9}

Is there a place for indirect calorimetry in the later stages of critical illness?

S. Castro ${ }^{1}$; C. Monteiro ${ }^{1}$ E. Pinto, ${ }^{2}$; P. Pinto, ${ }^{1}$; AP. Alves ${ }^{3}$

${ }^{1}$ Serviço de medicina intensiva 1, Centro Hospitalar Universitário

do Algarve, Faro, Portugal; ${ }^{2}$ Universidade do algarve, Centro de Estudos e Desenvolvimento em Saúde, Faro, Portugal: ${ }^{3}$ Serviço de medicina intensiva 2, Centro Hospitalar Universitário do Algarve, Portimão, Portugal Correspondence: $\mathrm{S}$. Castro

Intensive Care Medicine Experimental 2021, 9(1): 000839

Introduction. The use of indirect calorimetry (IC) in monitoring the nutritional therapy of critically ill patients, as well as an increased protein intake without overfeeding, appears to be beneficial and are currently recommended by the latest European guidelines.

Objectives. We aimed to compare energy requirement (ER) assessed through IC with estimated ER using standard formulas and to compare estimated protein requirement $(1,3 \mathrm{gr} / \mathrm{Kg})$ with protein intake through artificial nutrition during the late acute and rehabilitation phases of critical illness.

Methods. We conducted an observational study comprising 21 IC tests in a non-random sample of adult critical patients, selected on account of their eligibility for IC assessment, as proposed by the manufacturer of the Q-NRG $+\mathrm{Cl}$ device that was used. ER was measured by IC and estimated using patients' adjusted weight (AW) as follows: 4 to 7 days in ICU, ER $=0.7 \times 25 \mathrm{Kcal} \times A W ; 8$ or more days in ICU, $\mathrm{ER}=25 \mathrm{Kcal} \times \mathrm{AW}$. Patients were classified according to length of ICU stay: 3-7 days: late acute stage; $\geq 8$ days: rehabilitation stage.

Results. When IC was used, 8 patients were in late acute stage (Group A), and 13 patients were in the rehabilitation stage (Group B). 4 tests for patients in Group B were done in canopy mode, after patient discharge from the ICU. We found statistically significant differences between ER estimated using standard formulas and ER assessed by IC in Group A $(p=0.036)$. In this group, standard formulas underestimated ER, on average, by $335 \pm 360.1 \mathrm{kcal}$. In group B, mean underestimation was $116 \pm 496.8 \mathrm{kcal}$, but differences between the two methods were not statistically significant $(p=0.701)$. Regarding protein, estimation resulted in significantly higher requirements than the amount that was able to be provided to patients, both in group A $(p=0.035)$ and in group $B(p=0.001)$. According to estimates, patients should have been provided, on average, with an additional $43,7 \pm 40,37 \mathrm{~g}$ (Group A) or 42,4 $\pm 22,26 \mathrm{~g}$ (Group B) of protein.

Conclusion. ER is significantly different according to the method used for estimation. When compared with IC, standard formulas, underestimated ER in late acute stage of illness during ICU stay. Although further studies are needed, strategies to increase protein intake must be developed. IC can be a useful tool for monitoring nutritional support in critical ill patients and it may even be feasible after ICU discharge. 
Reference(s)

1. P. Singer et al., "ESPEN guideline on clinical nutrition in the intensive care unit," Clin. Nutr., no. October, pp. 1-32, 2018.

\section{6}

Ischemic Colitis in the Critical Care Patient with SARS-COV-2

\section{Pneumonia}

C. Lorencio ${ }^{1}$; A. Horta Puig ${ }^{1}$; À. Castillo Niell ${ }^{1}$; A. Timoteo Delgado²; N

Ortega Torrecilla²; J. Gonzalez Londoño'; S. Cuenca Barrero

${ }^{1}$ Intensive Care Medicine, Hospital Universitari de Girona Dr Josep Trueta, Girona, Spain; ${ }^{2}$ Colon and Rectal Surgery Department, Hospital Universitari de Girona Dr Josep Trueta, Girona, Spain

Correspondence: J. Gonzalez Londoño

Intensive Care Medicine Experimental 2021, 9(1): 000916

Introduction. Gastrointestinal dysfunction (GID) is common in the ICU setting. Up to $60 \%$ of patients present some manifestation of DGI during their stay in the ICU. However, an increase in ischemic colitis (IC) has been observed in critically ill patients admitted to the ICU due to SARS-CoV2.

Objectives. The aim of the study is to present the characteristics of IC in a series of critical patients with SARS-CoV-2 pneumonia.

Methods. Retrospective analysis of a series of patients admitted to the ICU of a tertiary hospital with SARS-CoV2, that developed IC and required surgery.

Results. Between March/2020 and January/2021, 287 SARS-CoV2 patients requiring mechanical ventilation were admitted to the ICU of a tertiary hospital.

$8\left(2^{\prime} 78 \%\right)$ presented gangrenous IC (Brand \& Boley) requiring urgent surgery. $87.5 \%$ were men, the median age was 60.5 (IR 48.5-71) and the mean BMI was 32.5 (IR: 27-34). 5 patients showed at least one risk factor for IC. The median number of days from intubation to surgery was 13d (IR: 9.5-20.5). Fever (75\%) and abdominal distension (62.5\%) were the initiall symptoms. The location of the ischemia was the right colon in all cases. 6 patients required extended right hemicolectomy and 2, subtotal colectomies. In the histological study, 7 patients had transmural ischemia, and one patient was limited to the mucosa; predominantly right location was observed. There was no thrombosis or vasculitis in the specimens. Mortality was $37.5 \%$ (3/8), due to septic shock and refractory multi-organ failure.

Conclusion. The incidence of severe IC in critically ill patients due to COVID19 seems higher than that of the overall critically ill patients, without a clear relationship with thromboembolic phenomena in our series. The predominantly right location in all patients could represent an entity of its own secondary to virus infection. Fever and abdomina distension in these patients should lead to suspicion of this entity.

\section{8}

Variations in glycemic control in critical care patients with artificial nutrition

C. Lorencio'; J. Gonzalez Londoño'; JC. Lopez-Delgado²; L. Servià Goixart³ T. Grau-Carmona ${ }^{4}$; L. Bordejé, ${ }^{5}$; E. Mor-Marco ${ }^{5}$; P. Vera-Artazcoz ${ }^{6}$; L. Macaya Redín $^{7}$; E. Portugal-Rodriguez ${ }^{8}$; J. Marin-Corral ${ }^{9}$; JF. Martínez Carmona ${ }^{10}$; D. Monge-Donaire ${ }^{11}$; B. Llorente-Ruiz ${ }^{12}$; R. Iglesias-Rodriguez ${ }^{13}$; JJ. Trujillano ${ }^{14}$ ${ }^{1}$ Intensive Care Unit, Hospital Universitari de Girona Dr Josep Trueta, Girona, Spain; ${ }^{2}$ Intensive Care, Hospital Universitari de Bellvitge, L'Hospitalet de Llobregat (Barcelona), Spain: ${ }^{3}$ Intensive Care Unit, Hospital Universitari Arnau de Vilanova, Lleida, Spain; ${ }^{4}$ Intensive Care, Hospital 12 de Octubre, Madrid, Spain; ${ }^{5}$ Intensive Care Unit, Hospital Germans Trias i Pujol, Badalona, Spain; ${ }^{6}$ Intensive Care, Hospital Sant Pau, Barcelona, Spain; ${ }^{7}$ Intensive Care Unit, Royal Navarre Hospital, Pamplona, Spain; ${ }^{8}$ Intensive care unit, Hospital Clínico, Valladolid, Spain; ${ }^{9}$ Intensive care unit, IMIM, Barcelona, Spain; ${ }^{10}$ Intensive Care Unit, Hospital Carlos Haya, Málaga, Spain; ${ }^{11}$ Intensive Care, Hospital Virgen De La Concha, Zamora, Spain; ${ }^{12}$ Intensive Care Unit, Hospital Universitario Príncipe de Asturias, Alcala de Henares, Spain; ${ }^{13}$ Intensive Care Unit, Hospital General de Granollers, Granollers, Spain; ${ }^{14}$ Intensive care unit, University Hospital Arnau de Vilanova, Lleida, Spain

Correspondence: J. Gonzalez Londoño

Intensive Care Medicine Experimental 2021, 9(1): 000968
Introduction. Hyperglycemia is a common phenomenon in the critically ill patient and has been directly related to its morbidity and mortality. However, glycemic variability is postulated as a factor of poor prognosis of greater relevance than hyperglycemia per se.

Objectives. The aim of this study is to evaluate glycemic variability in patients who require artificial nutritional support.

Methods. All the data related to glycemic behavior in patients that were included in the ENPIC study were analyzed ([NCT: 03634943] multicenter registry on the nutritional practices of critically ill patients in 37 Spanish ICUs, from April 23 to June 15, 2019).

Results. A total of 629 patients were included: $67.6 \%$ were men, with a mean age of $61.8( \pm 15)$ years and a mean BMI of $27.7( \pm 5.2)$. The average APACHE II was 20 and the average time until nutritional therapy was started was $28 \mathrm{~h}$.

$63.4 \%$ of patients received enteral nutrition, $18.2 \%$ parenteral and $18.4 \%$ mixed.

$14.8 \%$ of patients maintained blood glucose $<120 \mathrm{mg} / \mathrm{dL}$ (Group A) $27.5 \%$ between 120 and $139 \mathrm{mg} / \mathrm{dL}$ (Group B), 35.8\% between 140 and $179 \mathrm{mg} / \mathrm{dL}$ (Group C) and 21.9\% > $180 \mathrm{mg} / \mathrm{dL}$ (Group D).

MORTALITY was higher in the groups with the worst glycemic control $(A=15.1 \%, B=19.7 \%, C=27.1 \%$ and $D=37.7 \%) \quad(p<0.05)$ and it was in these groups where greater glycemic variability was observed $(A=13.6 \mathrm{mg} / \mathrm{dL} \pm 6.9 \mathrm{mg} / \mathrm{dL}, B=16.8 \mathrm{mg} / \mathrm{dL} \pm 8 \mathrm{mg} /$ $\mathrm{dL}, \quad \mathrm{C}=24.65 \mathrm{mg} / \mathrm{dL} \pm 13.3 \mathrm{mg} / \mathrm{dL}, \quad \mathrm{D}=34.9 \mathrm{mg} / \mathrm{dL} \pm 15.9 \mathrm{mg} /$ $d L)(p<0.05)$ and higher insulin therapy requirement $(A=22.6 \%$, $B=57.2 \%, C=87.6 \%, D=97.8 \%)(p<0.05)$

The patients with worse glycemic control, had a higher incidence of at least one episode of glycemia greater than $180 \mathrm{mg} / \mathrm{dL}(\mathrm{A}=9.7 \%$ $B=46.2 \%, C=92.9 \%, D=100 \%)(p<0.05)$

Conclusion. Although the current guidelines recommend monitoring the patient's blood glucose below $150 \mathrm{mg} / \mathrm{dL}$, more than one-third of patients had higher blood glucose levels.

Hyperglycemia and increased glycemic variability were associated with higher mortality in our series. Patients with more hyperglycemia and more glycemic variability received more insulin.

\section{9}

\section{Hyperglycemia in critical care patients. Behavior} and complications

C. Lorencio ${ }^{1}$; J. Gonzalez Londoño²; JC. Lopez-Delgado 3 ; L. Servià Goixart ${ }^{4}$; T. Grau-Carmona5; JJ. Trujillano6; L. Bordejé, $;$; E. Mor-Marco; P. Vera-Artazcoz ; L. Macaya Redín?; E. Portugal-Rodriguez ${ }^{10}$; JF. Martínez Carmona $^{11}$; J. Marin-Corral ${ }^{12}$; D. Monge-Donaire ${ }^{13}$; B. Llorente-Ruiz ${ }^{14}$; R. Iglesias-Rodriguez ${ }^{15}$

IIntensive Care Unit, Hospital Universitari de Girona Dr Josep Trueta, Girona, Spain; ${ }^{2}$ Intensive Care Medicine, Hospital Universitari de Girona Dr Josep Trueta, Girona, Spain; ${ }^{3}$ Intensive Care, Hospital Universitari de Bellvitge, L'Hospitalet de Llobregat (Barcelona), Spain; ${ }^{4}$ Intensive Care Unit, Hospital Universitari Arnau de Vilanova, Lleida, Spain; ${ }^{5}$ Intensive Care, Hospital 12 de Octubre, Madrid, Spain; ${ }^{6}$ Intensive Care Unit, University Hospital Arnau de Vilanova, Lleida, Spain; ${ }^{7}$ Intensive Care Unit, Hospital Germans Trias i Pujol, Badalona, Spain; ${ }^{8}$ Intensive care, Hospital Sant Pau, Barcelona, Spain; ${ }^{9}$ Intensive care unit, Royal Navarre Hospital, Pamplona, Spain; ${ }^{10}$ Intensive Care Unit, Hospital Clínico, Valladolid, Spain; ${ }^{11}$ Intensive Care Unit, Hospital Carlos Haya, Málaga, Spain; ${ }^{12}$ Intensive Care Unit, Imim, Barcelona, Spain; ${ }^{13}$ Intensive Care, Hospital Virgen De La Concha, Zamora, Spain; ${ }^{14}$ Intensive Care Unit, Hospital Universitario Príncipe de Asturias, Alcala de Henares, Spain; ${ }^{15}$ Intensive Care Unit, Hospital General de Granollers, Granollers, Spain

Correspondence: J. Gonzalez Londoño

Intensive Care Medicine Experimental 2021, 9(1): 000969

Introduction. Hyperglycemia is a common phenomenon in the critically ill patient, which has been directly related to morbidity and mortality of these patients.

Objectives. The aim of this study is to evaluate the behavior of glycemia in patients who require artificial nutritional support.

Methods. All the data related to glycemic behavior in patients that were included in the ENPIC study were analyzed ([NCT: 03634943] 
multicenter registry on the nutritional practices of critically ill patients in 37 Spanish ICUs, from April 23 to June 15, 2019).

Results. A total of 629 patients were included: $67.6 \%$ were men, with a mean age of $61.8( \pm 15)$ years and a mean BMI of $27.7( \pm 5.2)$. The average APACHE II was 20 and the average time until nutritional therapy was started was $28 \mathrm{~h}$.

$63.4 \%$ of patients received enteral nutrition, $18.2 \%$ parenteral and $18.4 \%$ mixed.

$14.8 \%$ of patients maintained blood glucose $<120 \mathrm{mg} / \mathrm{dL}$ (Group A), $27.5 \%$ between 120 and $139 \mathrm{mg} / \mathrm{dL}$ (Group B), 35.8\% between 140 and $179 \mathrm{mg} / \mathrm{dL}$ (Group C) and 21.9\% > $180 \mathrm{mg} / \mathrm{dL}$ (Group D).

Mortality was higher in the groups with the worst glycemic control $(A=15.1 \%, B=19.7 \%, C=27.1 \%$ and $D=37.7 \%)(p<0.05)$.

Older patients had worse glycemic control $(A=51.8$ years, $B=59.4$, $C=64$ and $D=67.7)(p<0.05)$. Likewise, patients with a history of hypertension $(43.9 \%)$, or DM (25.9\%), presented worse glycemic control $(p<0.05)$. The mean BMI was higher in the worst glycemic control groups $(D=28.9, C=28, B=27.2, A=26.1)(p<0.05)$.

Conclusion. Although the current guidelines recommend controlling the critical patient's blood glucose below $150 \mathrm{mg} / \mathrm{dL}$, more than a third of patients had higher blood glucose levels. In our series, hyperglycemia was associated with higher mortality. Older patients, with higher BMI and the presence of comorbidities such as hypertension or DM, had worse glycemic control.

\section{4}

Comparison of The Effects of Stress Hormones (cortisol, insulin, tsh, $\mathrm{ft} 3, \mathrm{ft} 4$ ) and Metabolites (vanyl mandelic acid) Levels on Mortality in Patients Undergoing Invasive and Non-invasive Mechanical Ventilation in Adult Intensive Care Unit

N. AKSOY'; G. Koksal'; O. Sen ${ }^{1}$

${ }^{1}$ Anesthesiology and reanimation, University of Health Sciences,Haseki Training and Research Hospital, Istanbul, Turkey

Correspondence: N. AKSOY

Intensive Care Medicine Experimental 2021, 9(1): 001004

Introduction. Critical illness occurs as a result of severe surgery or severe medical illnesses. It is the failure of organ functions that can result in death if hemodynamic, drug and respiratory support is not provided (1). Mechanical ventilation is one of the most important supportive treatments used in intensive care unit. It can affect all organ systems that depend on the homeostatic balance between the lung and other organ systems. It is thought that combinations of endocrine measurements will provide a better result than a single hormone measurement in determining mortality. In our study, we aimed to investigate the effect of stress hormones (cortisol, blood glucose, insulin, TSH, fT3, fT4), catecholamine metabolite (VMA) and blood glucose levels on mortality of the patients undergoing invasive and non-invasive mechanical ventilator support therapy.

Objectives. To research the effect of metabolites (VMA) of stress hormones (cortisol, insulin, TSH, Ft3, Ft4) and blood glucose levels on mortality in patients undergoing invasive and non-invasive mechanical ventilator support treatment and the differences in measurements between the two groups.

Methods. From a total of 109 patients, 44 patients who is being received invasive mechanical ventilation support treatment and 65 patients who is being received non-invasive mechanical ventilation support treatment between September 2020 and February 2021 in Sultangazi Haseki Education and Research Hospital Adult Intensive Care Unit were included in the study. All patients aged 18-60 who were followed up with invasive and non-invasive mechanical ventilation were included in the study. Patients using steroids, ketoconazole, etomidate, thyroid hormones, insulin and patients suffer endocrinological disease, malnutrition, head trauma, malignancy, renal insufficiency required renal replacement therapy and postoperative patients were excluded from the study. Cortisol, insulin, TSH, fT3, fT4, vanyl mandelic acid in 24-h urine and blood sugar levels were measured at the same time (at 06:00) on the 1st, 3rd and 7th days of hospitalization. APACHE II scores of the patients were calculated.
Results. All data of 109 patients included in the study, 44 who received invasive mechanical ventilation support and 65 patients who received non-invasive mechanical ventilation support, were analysed and compared. The duration of mechanical ventilation was found higher in the invasive group. In the non-invasive and invasive groups, age was higher in the non-survival group. In the non-invasive group, insulin first day measurement was found to be higher in survivors. In the non-invasive mechanical ventilation group, the seventh day cortisol measurement was found to be higher than the cortisol measurement on the first day which was making the difference. In the invasive mechanical ventilation group, the TSH measurement on the seventh day was found to be higher than the TSH measurement on the third day which was making the difference. The APACHE II Score was higher in the non-surviving patient group.

Conclusion. In our study, the stress hormone response was compared and evaluated in patients who is being received invasive and noninvasive mechanical ventilation. Patients in both groups were hemodynamically stable. Stress response was found to be higher in the non-invasive mechanical ventilation group. Actually, the general condition of the patients in the non-invasive mechanical ventilation group is expected to be better. The depth of sedation applied here may have affected on these results. Being aware the change in hormone levels may affect the treatment decision and prognosis. Because the increase in cortisol level on the first day was found to be correlated with the increase of the APACHE II level. Again, the increase in insulin level on the first day was found to be correlated with the decrease in APACHE II level. Thyroid hormone replacement may be considered by following the clinical condition of the patients. Studies on the predictive effects of sedation levels, sedative medicals and cortisol-insulin-tyhroid levels in all patients undergoing invasive or non-invasive mechanical ventilation are required in larger patient groups.

\section{Reference(s)}

1. 4.De La Rosa, G., Vasquez, E. M., Quintero, A. M., Donado, J. H., Bedoya, M., Restrepo, A. H., Roncancio, G., Cadavid, C. A., Jaimes, F. A., \& Grupo de Investigacion en Cuidado Intensivo GICI-HPTU (2013). The potential impact of admission insulin levels on patient outcome in the intensive care unit. The journal of trauma and acute care surgery, 74(1), 270-275. https://doi.org/10.1097/TA.0b013e3182788042

2. 3.Wang, Y. F., Heng, J. F., Yan, J., \& Dong, L. (2018). Relationship between disease severity and thyroid function in Chinese patients with euthyroid sick syndrome. Medicine, 97(31), e11756. https://doi.org/10.1097/MD. 0000000000011756

3. 2.Sharshar, T., Bastuji-Garin, S., Polito, A., De Jonghe, B., Stevens, R. D., Maxime, V., Rodriguez, P., Cerf, C., Outin, H., Touraine, P., Laborde, K., \& Groupe de Réflexion et d'Etude des Neuromyopathies En Réanimation (2011). Hormonal status in protracted critical illness and in-hospital mortality. Critical care (London, England), 15(1), R47. https://doi.org/10.1186/ cc10010

4. 1. Van Den Berghe G: Novel insights into the neuroendocrinology of critical illness. Eur J Endocrinol, 143(1): 1-13, 2000.

\section{6}

Mortality in elderly versus very elderly septic patients: $A$ retrospective multi-center analysis

R. Rezar'; B. Mamandipoor ${ }^{2}$; V. Osmani '; C. Jung 33 ; B. Wernly ${ }^{4}$

${ }^{1}$ Cardiology and Intensive Care, Paracelsus Medical University Salzburg, Salzburg, Austria; ${ }^{2}$-health Research Group, Fondazione Bruno Kessler Research Institute, Trento, Italy; ${ }^{3}$ Dep. of Cardiology, Pulmonology and Angiology, University Hospital of Düsseldorf, Düsseldorf, Germany; ${ }^{4}$ Anesthesiology and Intensive Care, Paracelsus Medical University Salzburg, Salzburg, Austria

Correspondence: R. Rezar

Intensive Care Medicine Experimental 2021, 9(1): 001016

Introduction. Sepsis is a common disease entity with high morbidity and mortality. Also, the number of elderly people in the population is increasing, whereas this group in particular is more prone to sepsis with often severe courses due to their co-morbidities, limited fitness 
and certainly already compromised immune competence. All in all, the incidence of sepsis is known to increase, and the number of available intensive care beds in times of a pandemic is lower than it already was before. Also the therapeutic approach regarding septic patients has changed; in addition to general advances in modern medicine, treatment bundles have been propagated and led to a relatively standardized approach in septic patients. In many cases, patients can now be cured if they are treated correctly and in a timely manner. Nevertheless, it is often difficult to decide which of the oldest of the elderly will still benefit from intensive care. In this retrospective multi-center analysis we aimed to investigate differences regarding mortality in elderly versus very elderly patients.

Methods. A total of 9,385 patients with sepsis were included in this analysis. Patients were split into two groups, namely elderly (6579 years) and very elderly patients ( $>80$ years). In sum 6,184 patients could be assigned to the first group, and 3,201 individuals to the second group. The primary outcome was ICU mortality, the secondary outcomes were mechanical ventilation (MV) and vasopressor use. Multilevel logistic regression analysis was performed and fitted for three models (1: ICU as a random effect; 2: baseline characteristics; 3: management strategies). Furthermore, a stratified sensitivity analysis was performed.

Results. Very elderly patients evidenced lower body mass indices (BMl: $25 \pm 8 \mathrm{~kg} / \mathrm{m}^{2}$ vs. $28 \pm 10 \mathrm{~kg} / \mathrm{m}^{2} ; \mathrm{p}<0.001$ ), but higher serum creatinine concentrations $(1.5 \pm 1.3 \mathrm{mg} / \mathrm{dL}$ vs. $1.4 \pm 1.5 \mathrm{mg} / \mathrm{dL} ; \mathrm{p}=0.005)$, and higher baseline lactate concentrations $(2.0 \pm 1.9 \mathrm{mmol} / \mathrm{L}$ vs. $1.8 \pm 1.8 \mathrm{mmol} / \mathrm{L} ; \mathrm{p}=0.02)$. Also length of stay was shorter in the very elderly group $(50 \pm 67$ h. vs. $56 \pm 72$ h.; $p<0.001)$. Rates of vasopressor use were similar ( $34 \%$ vs. $33 \%$ ), whereas elderly patients received MV more often than very elderly patients $(23 \%$ vs. $18 \%, p<0.001)$. Regarding the primary endpoint mortality in very elderly patients was higher $(13 \%$ vs. $11 \%, p=0.91)$ and remained so after multivariable adjustment (aOR $1.3295 \% \mathrm{Cl} 1.09-1.59 ; \mathrm{p}=0.004$ ). This finding was also observed for the subgroup of patients with septic shock (mortality rates: $38 \%$ vs. $36 \%$; aOR $1.5095 \% \mathrm{Cl} 1.10-2.06 ; \mathrm{p}=0.01$ ).

Conclusion. In this multi-center analysis of 9,385 elderly and very elderly patients a higher mortality in individuals $>80$ years of age was shown. Despite the $2 \%$ difference in absolute numbers, the question is to what extent this is clinically relevant, which is why, from our point of view, ICU trials are a better way to determine who benefits from intensive care than chronological age alone.

\section{5}

"The bitter truth of sugar"-Euglycemic Diabetic Ketoacidosis due to Sodium-glucose Co-transporter-2 Inhibitors: A case series E. Pathrose ${ }^{1}$ : M. SHAH ${ }^{1}$

${ }^{1}$ Critical Care Medicine, Sir H. N. Reliance Foundation Hospital and Research Centre, Mumbai, India

Correspondence: E. Pathrose

Intensive Care Medicine Experimental 2021, 9(1): 001035

Introduction. Diabetic ketoacidosis (DKA) consists of a triad of blood sugar level greater than $250 \mathrm{mg} / \mathrm{dL}$., ketonemia of greater than $3 \mathrm{mmol} / \mathrm{L}$ and/or significant ketonuria and a blood $\mathrm{pH}$ less than 7.3 with an increased anion gap. Currently, the sodium-glucose co-transporter-2 inhibitors (SGLT-2i) are widely used in management of Type 2 diabetes. There have been several reports associating euglycemic diabetic ketoacidosis (EuDKA) and these SGLT-2i agents. We present three different patients who were on SGLT-2i and developed recurrent EuDKA post procedure or sepsis.

Methods. Clinical Case 1: A 66 years old gentleman, with Type 2 DM, was on Empaglifozin (10 mg OD), Metformin (500 mg BD), and Basal bolus therapy (Regular Insulin thrice a day and Degludec once a day). He presented with a history of loss of consciousness, vomiting and headache after traumatic head injury. Computed Tomography (CT) of the brain showed subarachnoid, subdural hemorrhage with minor basal temporal fracture without CSF leak. His plasma glucose was controlled with subcutaneous insulin. After 3 days, patient was shifted to the wards and was restarted on Empaglifozin. He was readmitted to the ICU within $24 \mathrm{~h}$ in view of altered sensorium and tachypnea.
Repeat CT brain was unremarkable as was the sepsis workup. Arterial blood gas (ABG) revealed a high anion gap metabolic acidosis (HAGMA) with normal lactate and blood glucose levels. Serum and urine ketones were positive and Euglycemic Diabetic Ketoacidosis (EuDKA) was established. He was treated with Intravenous (IV) insulin as per the DKA protocol and shifted to subcutaneous insulin once the AG and Ketones resolved after 24-48 h. As the patient was improving with closure of the gap and trace amounts of serum and urine ketones, the insulin dosing was being reduced. Astonishingly, there was a relapse of HAGMA with positive serum and urine ketones with euglycemia. He was restarted on the previous management till urine glucose was negative. This was approximately ten days after discontinuing Empaglifozin. In view of the EuDKA relapse and altered sensorium with negative cultures, lumbar puncture was done and the cerebrospinal fluid (CSF) analysis revealed findings of aseptic meningitis. He improved over the next 15 days.

Clinical Case 2: A 74 years old gentleman, with a history of Type 2 DM on OHA's (Empaglifozin $25 \mathrm{mg}$ + Linegliptin $5 \mathrm{mg}$ once daily) since last 5 years was diagnosed with Triple vessel coronary artery disease and was admitted for Coronary Artery Bypass Grafting (CABG) surgery. He was switched over to subcutaneous insulin $24 \mathrm{~h}$ prior to the surgery. Post-surgery, ABG showed HAGMA with normal lactates and glucose. Despite fluid resuscitation, subsequent $A B G$ showed no improvement. On reviewing the patient's home medications, it was found that that the patient was on SGLT-2i. Clinical suspicion of EuDKA led to further workup which turned positive. With our previous experience, we treated him with the above regimen till urine glucose was negative. This took us around 5 days.

Clinical Case 3: A 71 years old lady, with a history of Ischemic heart disease and Type 2 DM on SGLT-2i (Empaglifozin $12.5 \mathrm{mg}$ once daily), was admitted with abdominal distension, vomiting and constipation, noted since a couple of days. CT of the abdomen and pelvis showed a large bowel obstruction raising the suspicion of colonic cancer with bilateral hydronephrosis. Post-operative workup which included an ABG showed HAGMA with normal lactates and normal sugar levels. On the basis of the prior cases, EuDKA was suspected, proven and treated accordingly with the similar regimen till the urine glucose were negative. This also took 5 days.

Results. The treatment of recurrent EuDKA relies upon monitoring trends of urine glucose. IV insulin may need to be continued for a longer duration till urine glucose is negative. Also, we propose that these medications should be stopped at least $6-8$ days prior to any elective surgical procedure, contrary to the recommendation of 3 days. Conclusion. Euglycemic Diabetic Ketoacidosis is not only a life threatening emergency but also a diagnostic challenge, not only due to the absence of hyperglycemia but also due to a variety of triggers. Delay in diagnosis and management may lead to in-hospital morbidity and mortality.

\section{Reference(s)}

1. 1.Madaan T, Akhtar M, Najmi AK. Sodium glucose CoTransporter 2 (SGLT2) inhibitors: Current status and future perspective. European Journal of Pharmaceutical Sciences. 2016 Oct 10;93: 244-52. 2.Zinman B, Wanner C, Lachin JM, Fitchett D, Bluhmki E, Hantel S, Mattheus M, Devins T, Johansen OE, Woerle HJ, Broedl UC. Empagliflozin, cardiovascular outcomes, and mortality in type 2 diabetes. New England Journal of Medicine. 2015 Nov 26;373(22):2117-28. 3.Mahaffey KW, Jardine MJ, Bompoint S, Cannon CP, Neal B, Heerspink HJ, Charytan DM, Edwards R, Agarwal R, Bakris G, Bull S. Canagliflozin and cardiovascular and renal outcomes in type 2 diabetes mellitus and chronic kidney disease in primary and secondary cardiovascular prevention groups: results from the randomized CREDENCE trial. Circulation. 2019 Aug 27;140(9):739-50. 4.Norhammar A, Bodegård J, Nyström T, Thuresson M, Nathanson D, Eriksson JW. Dapagliflozin and cardiovascular mortality and disease outcomes in a population with type 2 diabetes similar to that of the DECLARE-TIMI 58 trial: a nationwide observational study. Diabetes, Obesity and Metabolism. 2019 May;21(5):1136-45. 5.McMurray JJ, DeMets DL, Inzucchi SE, Køber L, Kosiborod MN, Langkilde AM, Martinez FA, Bengtsson O, Ponikowski P, Sabatine MS, Sjöstrand M. A trial to evaluate the effect of the sodium-glucose co-transporter 2 inhibitor dapagliflozin 
on morbidity and mortality in patients with heart failure and reduced left ventricular ejection fraction (DAPA-HF). European journal of heart failure 2019 May;21 (5):665-75. 6.FDA Drug Safety Communication: FDA Warns that SGLT2 Inhibitors for Diabetes May Result in a Serious Condition of Too Much Acid in the Blood. [Last accessed on 2021-Apr-15]. http:// www.fda.gov/Drugs/DrugSafety/ucm446845.htm. [Ref list] 7.Gauthier PM, Szerlip HM. Metabolic acidosis in the intensive care unit. Critical care clinics. 2002 Apr 1;18(2):289-308. 8.Munro JF, Campbell IW, McCuish AC, Duncan LJ. Euglycaemic diabetic ketoacidosis. Br Med J. 1973 Jun 9;2(5866):578-80. 9.Peters AL, Buschur EO, Buse JB, Cohan P, Diner JC, Hirsch IB. Euglycemic diabetic ketoacidosis: a potential complication of treatment with sodium-glucose cotransporter 2 inhibition. Diabetes care. 2015 Sep 1;38(9):1687-93. 10. Hine J, Paterson H, Abrol E, RussellJones D, Herring R. SGLT inhibition and euglycaemic diabetic ketoacidosis. The Lancet Diabetes \& Endocrinology. 2015 Jul 1;3(7):503-4. 11. Roach P, Skierczynski P. Euglycemic diabetic ketoacidosis in a patient with type 2 diabetes after treatment with empagliflozin. Diabetes Care. 2016 Jan 1;39(1):e3-. 12.Barski L, Eshkoli T, Brandstaetter E, Jotkowitz A. Euglycemic diabetic ketoacidosis. European journal of internal medicine. 2019 May 1;63:9-14. 13.Bonora BM, Avogaro A, Fadini GP. Extraglycemic effects of SGLT2 inhibitors: a review of the evidence. Diabetes, metabolic syndrome and obesity: targets and therapy. 2020;13:161. 14.Diaz-Ramos A, Eilbert W Marquez D. Euglycemic diabetic ketoacidosis associated with sodiumglucose cotransporter-2 inhibitor use: a case report and review of the literature. International journal of emergency medicine. 2019 Dec;12(1):1-4. 15.Ferrannini E, Baldi S, Frascerra S, Astiarraga B, Barsotti E, Clerico A, Muscelli E. Renal handling of ketones in response to sodium-glucose cotransporter 2 inhibition in patients with type 2 diabetes. Diabetes care. 2017 Jun 1;40(6):771-6. 16.Saponaro C, Mühlemann M, Acosta-Montalvo A, Piron A, Gmyr V, Delalleau N, Moerman E, Thévenet J, Pasquetti G, Coddeville A, Cnop M. Interindividual heterogeneity of SGLT2 expression and function in human pancreatic islets. Diabetes. 2020 May 1;69(5):902-14. 17.Taylor SI, Blau JE, Rother KI. SGLT2 inhibitors may predispose to ketoacidosis. The Journal of Clinical Endocrinology \& Metabolism. 2015 Aug 1;100(8):2849-52. 18.FDA revises labels of SGLT2 inhibitors for diabetes to include warnings about too much acid in the blood and serious urinary tract infections.-FDA Drug Safety Communication.03/19/2020. https:// www.fda.gov/drugs/drug-safety-and-availability/fda-revises-labels-sglt2inhibitors-diabetes-include-warnings-about-too-much-acid-blood-andserious. 19.Zala A, Maple-Brown LJ, Shaw JE, Hare MJ. Current evidence and practical guidance for the use of sodium-glucose co-transporter-2 inhibitors in type 2 diabetes. Australian journal of general practice. 2021 Apr 1;50(4):225-30.

\section{2}

Is there a relationship between protein supply and mortality in patients with COVID 19?

I. Buonso'; S. Almeida, ${ }^{2}$; U. Flato, ${ }^{3}$; A. Wirgues, ${ }_{1}^{3}$; R. Ralio, ${ }^{3}$; M. Vilela, ${ }_{1}^{3}$; F. Costa, ${ }^{3}$; IS. Rocco, ${ }^{4}$; L. Almeida, ${ }^{3}$; A. Alves,

${ }^{1}$ Icu, Hospital Samaritano, São Paulo, Brazil; ${ }^{2}$ Icu, Hospital Samaritano Higienópolis, São Paulo, State of São Paulo, Brazil, Brazil; ${ }^{3} \mid \mathrm{cu}$, Hospital Samaritano Higienópolis, São Paulo, Brazil; ${ }^{4}{ }^{4} \mathrm{cu}$, Hospital Samaritano Higienopolis, São Paulo, Brazil; ${ }^{5}$ Icu, Hospital Samaritano Higienópolis, SAO PAULO, Brazil

Correspondence: I. Buonso

Intensive Care Medicine Experimental 2021, 9(1): 001042

Introduction. The nutritional therapy prescribed appropriately, taking into account the patient's critical phase shows that it is possible to improve the prognosis, decrease the ICU time, the use of mechanics ventilation, among other benefits. With the pandemic of COVID 19 and Brazil being one of the epicenters, since the UTIS ICU'S found itself with a large number of critically ill patients, dependent on mechanics ventilation and consequently needing enteral nutritional therapy. The main risk factors for COVID-19 were: immunosuppressed, elderly, disabled with comorbidity, taking advantage of the latter two groups most susceptible to malnutrition. The long stay in the ICU, in this group of patients, may worsen malnutrition already present or be its cause. Several publications have shown that the adequate protein supply can decrease catabolism and, as a consequence, these patients have better respiratory evolution. Due to the pandemic of COVID19 being something very recent, there are still few studies reaffirming a condition that has already been shown in other studies with patients of other diagnoses. Therefore, the aim of this study is to try to show whether there is a relationship between inadequate protein supply and fundamentally patient with COVID-19.

Objectives. To evaluate whether there is a relationship between increased mortality from COVID 19, relate the offer of protection during the period in which he received enteral nutritional therapy.

Methods. Retrospective, observational study, with analysis of 16 deaths by COVID-19, which occurred between the months of March and April/2020, of patients admitted to an ICU of a particular hospital in the city of São Paulo, Brazil. For inclusion criteria were adopted: adult patients who received enteral nutritional therapy for more than $72 \mathrm{~h}$. For calculations of nutritional offer, the ESPEN Guidelines for critical patients were used, considering the adequate offer or the minimum average value of $80 \%$. The data were analyzed using Excel planning and statistically defined.

Results. Of the 16 patients who died, $13(81 \%)$ received enteral nutritional therapy (TNE) and, of these, $9(69 \%)$ for a period longer than $72 \mathrm{~h}$, with the average age of those 71.6 years $(\sigma 7,12)$ and the average offer period for TNE was 13 days ( $\sigma 7.81)$. The average protein supply was $67.9 \%$ / day ( $\sigma 18.6)$ and only 2 patients (22.2\%) received less than $80 \%$ of the protein target used. There was a weak correlation ( $r$ 0.379) between the days of diet and protein supply. There was a medium correlation $(r 0.5250)$ between age and protein supply. There was a weak correlation between death and protein offer (r. 0.298).

Conclusion. Given the data, it is possible to conclude that an inadequate protein supply has contributed to the mortality of this group of patients, since only 2 patients $(22.2 \%)$ receive less than $80 \%$ of the goal achieved, this is the first time tested in scientific studies is essential for the recovery of patients in the ICU.

\section{Reference(s)}

1. Barazzoni R et al., ESPEN expert statements and practical guidance for nutritional management of individuals with SARS-CoV-2 infection, Clinical Nutrition, https://doi.org/10.1016/j.clnu.2020.03.022

\section{5}

Barriers to adequate nutritional supply in patients with COVID-19

I. Buonso'; S. Almeida, ; U. Flato, ; A. Wirgues, ; R. Ralio, ; M. Vilela, ; IS. Rocco, ${ }^{4}$; F. Costa, ; L. Almeida, ${ }^{3}$; A. Alves, ${ }^{3}$

${ }^{1} \mathrm{Icu}$, Hospital Samaritano, São Paulo, Brazil; ${ }^{2} \mathrm{cu}$, Hospital Samaritano Higienópolis, São Paulo, State of São Paulo, Brazil, Brazil; ${ }^{3} \mathrm{Icu}$, Hospital Samaritano Higienópolis, São Paulo, Brazil; ${ }^{4}{ }^{\mathrm{C} u}$, Hospital Samaritano Higienopolis, São Paulo, Brazil

Correspondence: I. Buonso

Intensive Care Medicine Experimental 2021, 9(1): 001045

Introduction. The nutritional therapy prescribed appropriately, taking into account the patient's critical phase shows that it is possible to improve the prognosis, decrease the ICU time, the use of mechanics ventilation, among other benefits. With the pandemic of COVID 19 and Brazil being one of the epicenters, since the UTIS found itself with a large number of critically ill patients, dependent on mechanics ventilation and consequently needing enteral nutritional therapy. The main risk factors for COVID-19 were: immunosuppressed, elderly, disabled with comorbidity, taking advantage of the latter two groups most susceptible to malnutrition. The long stay in the ICU, in this group of patients, may worsen malnutrition already present or be its cause. Several publications have shown that the SARS COV 2 virus has an impact not only on respiratory function, but also gastrointestinal, compromising nutrient absorption and impacting malnutrition.

Objectives. The purpose of this publication is to show the main challenges in the enteral nutritional supply of patients affected by COVID 1 9 admitted to the ICU.

Methods. Retrospective and observacional study, with 42 adults patients admitted to na ICU of a private hospital in São Paulo, Brazil, followed between march and april 2020, diagnosed with COVID 19 
and receaving enteral nutrition therapy for a period longer than $72 \mathrm{~h}$. Data are analyzed using Excell and statistically defined.

Results. In the total patients, 12 were female (29\%) and $30(71 \%)$ were male. The mean of age was 72 years. Only 15 patients (36\%) received at least $80 \%$ of the nutritional goals, based ond ESPEN Guidelines. The main causes of supply impedimente were: constipation (52\%), hemodinamic instability $(14,8 \%)$; gastroparesis $(14,8 \%)$; prone position $(11,1 \%)$; progression failure $(7,4 \%)$.

Conclusion. The data that show the main factor that causes the impossibility of a full nutritional offer are constipation, which are the early offer of neuromuscular block in patients, but are already receiving intestinal stimulants in conjunction with symbols. More therapeutic alternatives are needed to improve intestinal mobility, allowing the full nutritional offer that is extremely important in this group of patients.

\section{Reference(s)}

1. ROCCO, Barazzoni et al. Espen expert statements and practical guidance for nutritional management of individuals with sars-cov-2 infection, march 2020.

2. SINGER, Pierre et al. ESPEN guideline on clinical nutrition in the intensive care unit. Clinical Nutrition, set. 2018

\section{8}

Can adequate nutritional offer impact the results of strength and functionality of patients with COVID 19 in the ICU?

I. Buonso'; S. Almeida, ; U. Flato, ${ }^{3}$; A. Wirgues, ${ }^{3}$; R. Ralio, ${ }^{3}$ : M. Vilela ${ }^{3} ;$ IS. Rocco, ; F. Costa, ; L. Almeida, ${ }^{3}$; A. Alves,

${ }^{1}$ Icu, Hospital Samaritano, São Paulo, Brazil; ${ }^{2} \mid c u$, Hospital Samaritano Higienópolis, São Paulo, State of São Paulo, Brazil, Brazil; ${ }^{3} \mid c u$, Hospital Samaritano Higienópolis, São Paulo, Brazil; ${ }^{4}{ }^{1} \mathrm{Cu}$, Hospital Samaritano Higienopolis, São Paulo, Brazil; ${ }^{5}{ }^{1} c u$, Hospital Samaritano Higienópolis, Sao Paulo, Brazil

Correspondence: I. Buonso

Intensive Care Medicine Experimental 2021, 9(1): 001048

Introduction. The nutritional therapy prescribed appropriately, taking into account the patient's critical phase shows that it is possible to improve the prognosis, decrease the ICU time, the use of mechanics ventilation, among other benefits. With the pandemic of COVID 19 and Brazil being one of the epicenters, since the UTIS found itself with a large number of critically ill patients, dependent on mechanics ventilation and consequently needing enteral nutritional therapy. The main risk factors for COVID-19 were: immunosuppressed, elderly, disabled with comorbidity, taking advantage of the latter two groups most susceptible to malnutrition. The long stay in the ICU, in this group of patients, may worsen malnutrition already present or be its cause. Several publications have shown that the adequate protein supply can decrease catabolism and, its improve de results on force and mobility on ICUthat can be evaluated by dynamometry and PERME. Due to the pandemic of COVID19 being something very recent, there are still few studies reaffirming a condition that has already been shown in other studies with patients of other diagnoses.

Objectives. To compare whether the adequate nutritional offer can modify the results of dynamometry and PERME on admission and discharge from the ICU.

Methods. Observational, retrospective study with analysis of medical records of 10 patients admitted to the ICU of a private hospital in the city of São Paulo, Brazil. For the inclusion criteria, patients with more than $72 \mathrm{~h}$ of enteral nutritional therapy (ENT) were defined. To calculate the nutritional goal, the ESPEN GUIDELINES for critical patients were used and adequate protein supply was considered, average $>80 \%$ in the ENT period. The results of dynamometry (DIN) and PERME were collected from the physiotherapy assessment chart. Data were cross-checked with: DIN X PERME x protein supply and patients divided into 2 groups: G1 Adequated protein suply, subdivided into blocks: B1 PERME gained (PG) and positive DIN (DINP); B2 DINP and PERME maintenance (PERMM); B3 DIN without variation (DINWV) and PG; B4 negative DIN (DINN) and PG; B5 DIN not applicable (DINNA) and
PERME with loss (PWL). In group 2 there were non-adequated protein suply patients (average offer 44,\%) subdivided into blocks: B6 DINWV AND PG; B7 DINNN AND PG; B8 DINNA AND PERMM.

Results. $60 \%$ of the patients belonged to $\mathrm{G} 1$ (adequated protein suply), with $20 \%$ belong to $B 1,10 \%$ belong to $B 2,10 \%$ beolong $B 3$, $10 \%$ belong to $\mathrm{B} 4$ and $10 \%$ belong to $\mathrm{B} 5$. Of the non adequated protein suply group (40\%), $10 \%$ belong to $\mathrm{B} 6,20 \%$ belong to $\mathrm{B} 7$ and $10 \%$ belong $\mathrm{B} 8$.

Conclusion. In view of the results, it was possible to conclude that the adequate protein supply reflects on best results of mobility and muscle strength. And even the non adequated protein suply could influence, even with less impact, in both evaluations applied by the physiotherap.

\section{Reference(s)}

1. SINGER, Pierre et al. ESPEN guideline on clinical nutrition in the intensive care unit. Clinical Nutrition, set. 2018

2. Perme C, Nawa RK, Winkelman C, Masud F. A tool to assess mobility status in critically ill patients: the PERME Intensive Care Unit Mobility Score. Methodist Debakey Cardiovasc J. 2014 Jan-Mar;10(1):41-9.

\section{3}

Complication of the fluid therapy algorithm of diabetic

ketoacidosis: iatrogenic hyperchloremic acidosis

ZT. Sarikaya ${ }^{1}$; B. Gucyetmez ${ }^{1}$; E. Kilinc ${ }^{1}$; F. Tuzuner ${ }^{2}$; L. Telci ${ }^{3}$

${ }^{1}$ Anesthesiology and Reanimation, Acıbadem Mehmet Ali Aydınlar University, School of Medicine, Istanbul, Turkey; ${ }^{2}$ General Intensive Care Unit, Acibadem Hospital in Taksim, İstanbul, Turkey; ${ }^{3}$ General Intensive Care

Unit, Acıbadem International Hospital, Istanbul, Turkey

Correspondence: $\mathrm{B}$. Gucyetmez

Intensive Care Medicine Experimental 2021, 9(1): 001073

Introduction. Fluids with zero strong ion difference (SID) and chloride rich fluids such as $0.9 \% \mathrm{NaCl}, 0.45 \% \mathrm{NaCl}$ and $5 \%$ Dextrose $0.45 \% \mathrm{NaCl}$ are recommended by the fluid therapy algorithm of diabetic ketoacidosis (DKA) (1). The aim of the usage of these fluids in DKA is to reduce unmeasured anion acidosis by removing ß-hydroxybutyrate with $\mathrm{Na}$ from the kidneys (2). On the other hand, these fluids may cause hyperchloremic acidosis because of their low SID levels and high chloride contents $(3,4)$

Objectives. The aim of this study is to investigate the effect of the fluid therapy algorithm of DKA on metabolic acid-base status.

Methods. In the study, patients who were admitted to the intensive care unit (ICU) because of DKA in the last 15 years were retrospectively evaluated. Patients who were under 18 years old and who were administered fluid regimes apart from the algorithm were excluded. Demographic data, APACHE II and SOFA scores, blood gas samples at the ICU admission and 6th hour and outcomes were recorded. Strong ion gap $(\mathrm{SIG})$ and base-excess chloride $(\mathrm{BECl}=\mathrm{Na}-\mathrm{Cl}-32 \mathrm{mmol} / \mathrm{L})$ were calculated to detect unmeasured ions and chloride effect $(4,5)$. SPSS version 27 was used for statistical analysis.

Results. DKA was detected in the thirty-three $(0.2 \%)$ of 16,814 patients. Sixteen patients who were only administered fluids with zero SID and chloride rich fluids were included in the study. At the ICU admission, ketonuria, blood glucose, $\mathrm{pH}, \mathrm{PaCO}_{2}, \mathrm{Na}, \mathrm{Cl}, \mathrm{HCO}_{3}, \mathrm{SBE}$, SIG, SID and BECl were $3+(2+; 4+), 419 \pm 172,7.10 \pm 0.11,20.8 \pm 8.0$, $139 \pm 7.3,109 \pm 8.6,8.3 \pm 4.0,-21.3 \pm 5.8,17.6 \pm 6.7,33 \pm 6.7$ and $-3.1 \pm 7.1$ respectively. At the 6th hour, $\mathrm{pH}, \mathrm{PaCO}_{2}, \mathrm{Cl}, \mathrm{HCO}_{3}$, and SBE were significantly higher $(7.28 \pm 0.12,25.1 \pm 4.2,113 \pm 6.9,13.5 \pm 3.7$ and $-13.6 \pm 5.1 ; p<0.001, p=0.036, p=0.005, p=0.001$ and $p<0.001$ respectively) whereas blood glucose, $\mathrm{BECl}, \mathrm{SID}$ and SIG were significantly lower $(293 \pm 124,-7.1 \pm 7.1,30 \pm 6.1$ and $7.8 \pm 5.4 ; p=0.003$ $p=0.003, p=0.006$ and $p<0.001$ respectively) than their values at the ICU admission. Na values at the ICU admission and 6th hour were simi$\operatorname{lar}(139 \pm 7.3$ and $139 \pm 7.2 ; \mathrm{p}=0.890)$.

Conclusion. Although fluids with zero SID and chloride rich fluids reduce the increased SIG in the DKA, they cause iatrogenic hyperchloremic acidosis. Despite decreased SIG, the reason for the lack of expected increase in $\mathrm{HCO}_{3}$ and $\mathrm{SBE}$ and continued hypocapnia at the 6 th hour is the contribution of iatrogenic hyperchloremia to the 
acidosis. Hence, we think that the usage of fluids with zero SID and chloride rich fluids in DKA should be avoided and the fluid therapy algorithm of DKA should be reconsidered.

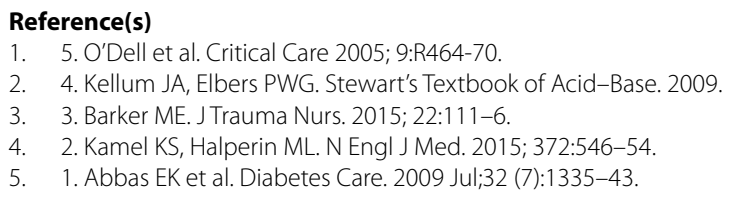

001133

Toxic epidermal necrolysis and hydroxychloroquine

W. Gadalla'; R. Kulkarni

${ }^{1}$ Intensive Care Unit, Kettering General Hospital, Kettering, United Kingdom

Correspondence: W. Gadalla

Intensive Care Medicine Experimental 2021, 9(1): 001133

Introduction. I am presenting a 71-year-old lady who developed Toxic Epidermal Necrolysis (TEN) after starting Hydroxychloroquine for rheumatoid arthritis. Our case shown rapid deterioration and more than $80 \%$ involvement of the skin.

Hydroxychloroquine is an 4-aminoquinoline with a low molecular weight $(333,9 \mathrm{Da}) 0.6,7,8,9 \mathrm{It}$ has been used for decades to treat rheumatologic conditions (with a dose of $6 \mathrm{mg} / \mathrm{kg}$ ) with a general good safety, even though in last years cases of skin reactions, also severe, have increasingly been reported. 10 .

Methods. A 71-year-old woman presented to the emergency department with diffuse erythematous rashes over her body 2 weeks after starting Hydroxychloroquine for treatment of rheumatoid arthritis. The lesion started as painful erythematous macules, progressed into blisters followed by diffuse exfoliation of the skin involving both upper and lower extremities, back, abdomen, head and neck. The symptoms developed within a few hours. Medical history was significant for Hypothyroidism, Chronic obstructive Pulmonary Disease and Rheumatoid arthritis. Her regular medications for $>5$ years before presentation included: Alendronate $70 \mathrm{mg}$ once/week, Prednisolone $5 \mathrm{mg}$ OD, Cocodamol PRN, Atorvastatin $10 \mathrm{mg}$ ON, Levothyroxine 50mcg OD and PRN salbutamol inhaler. Patient denied any known drug allergy. Her family also denied that she may be taking any herbal medications.

Physical examination at Emergency Department 7.4.2021 was remarkable for multiple areas of redness, blisters and extensive detachment of skin involving $80 \%$ body surface area. Oral ulcers were noted. No genital ulcers. Nikolsky sign was positive. Targeoid lesions on the thigh and extensive epidermolysis on the trunk.

The patient developed AKI secondary to dehydration and blood pressure dropped so she was admitted to intensive care unit on 14.4.2021. She required inotropic support and Continuous renal replacement therapy (CRRT) for 2 days. She was transferred to a burn unit at tertiary hospital and managed there for 5 more days before she passed.

Results. Laboratory investigations: white cell count of $15.4 \times 103$ / $\mathrm{cm}^{3}, \mathrm{~N} 14.2$ (Left shift neutrophilia with toxic granulation), $\mathrm{L} 0.7, \mathrm{Hb}$ of $130 \mathrm{~g} / \mathrm{L}$, and a platelet count $430 \times 103 / \mathrm{cm}^{3}$. Serum electrolytes, liver profile, prothrombin time, partial thromboplastic time and International Normalised Ratio were within normal limits. CRP on admission was 532. A punch skin biopsy was obtained. Blood, urine and wound cultures sent. Chest X-ray was unremarkable. COVID 19 swabs were negative. Creatinine $173 \mathrm{umol} / \mathrm{l}$ and urea of $13.4 \mathrm{mmol} / \mathrm{l}$ on admission which rapidly deteriorated to creatinine of 332 , urea 32 and $\mathrm{PH}$ of 7.17 $\mathrm{HCO}_{3}$ was 12 before she started on CRRT.

Skin biopsy: Oedema of the dermis and perivascular infiltrates of mononuclear cells. Necrotic epidermis. Increased histocytes cells within the dermis.

The patient was admitted to the ITU then transferred to burn unit Hydroxychloroquine was stopped immediately. The patient was managed with intravenous fluids, prophylactic antibiotics and proper wound care. Electrolytes were replaced as needed. She was monitored continuously for haemodynamic stability. She received CRRT for AKI and metabolic acidosis.
There was an episode of acute respiratory distress syndrome, for which she required ventilatory support for few days. Her AKI required her being on CRRT for 3 days. She was transferred from district general hospital to a burn unit at tertiary hospital.

Conclusion. Hydroxychloroquine as a causative agent of toxic epidermal necrolysis.

Toxic epidermal necrolysis (TEN) is a life-threatening adverse drug reaction that can occur secondary to use of a variety of drugs including Hydroxychloroquine.

\section{0}

Long-term survival of cirrhotic patients admitted to the ICU. A retrospective 10 years single-centre analysis

JL. García-Garmendia'; ; SL. Gallego-Lara ${ }^{1}$; M. Aguilar Cabello' ${ }^{1}$ C. Martinez Fernandez ${ }^{1}$; C. Colon Pallarés ${ }^{1}$; F. Maroto Monserrat ${ }^{1}$; M. Sanchez Santamaría

${ }^{1}$ Intensive Care Unit, Hospital San Juan de Dios del Aljarafe, Bormujos, Sevilla, Spain

Correspondence: J.L. García-Garmendia

Intensive Care Medicine Experimental 2021, 9(1): 001170

Introduction. Hepatic Cirrhosis is a comorbidity with high severity and mortality in the critically ill patient. Long-term survival has been reported as high as $77 \%$ ( 5 years). So, the appropriateness of admission of cirrhotic patients in the ICU is controversial.

Objectives. To describe the long-term survival of critically ill cirrhotic patients and to analyse factors associated with mortality.

Methods. Retrospective observational cohort single-centre study on critically ill patients with cirrhosis in a 200-bed hospital with an 8-bed ICU, from 2011 to 2020. Variables analysed were demographic, severity scores, diagnoses at admission, therapies in ICU and mortality (ICU, hospital, 6 months, 12 months and five years). Multivariate logistic regression analysis was made for mortality at different points. Significance level was $p<0,05$.

Results. There were 93 ICU admissions of 78 patients during the period study (3\% of total). Mean age was 61,1 (range 39-83), with $24 \%$ of female patients. Mean APACHE II score was 23,3 (range 3-40) and mean SOFA was 8,4 (range 0-19). CHID score was A (40\%), B (41\%) and C (19\%). Mean ICU stay was 7 days. Acute renal failure (ARF) was detected in $61 \%$, and renal replacement therapy (RRT) was needed in $15 \%$. Mechanical ventilation was used in $76 \%$ of patients. Fifty-six admissions were related with cirrhosis (with 26 gastrointestinal haemorrhage and 10 acute on chronic liver failure [ACLF] and others) and 37 were not related ( 8 pneumonia, 8 abdominal sepsis, 7 septic shock and others). Mortality at $6 \mathrm{~m}, 12 \mathrm{~m}$ and $5 \mathrm{y}$ were: $51,3 \%$, $55,7 \%$ and $67,5 \%$ for whole population; related vs. not related with cirrhosis were $55,6 \%$ vs $51,4 \%, 60,7 \%$ vs $60,6 \%$ and 65,5 vs $85,0 \%$; ARF vs. no-ARF were $72,2 \%$ vs $25,7 \%, 80,4$ vs. $30,3 \%$ and $84,4 \%$ vs $52,9 \%$. Gl haemorrhage mortality were $38,5 \%, 36 \%$ and $50 \%$ while ACLF mortality was $87,5 \%, 100 \%$ and $100 \%$ at $6 \mathrm{~m}, 12 \mathrm{~m}$ and $5 \mathrm{y}$. RRT mortality was $97,2 \%, 100 \%$ and $100 \%$ at $6 \mathrm{~m}, 12 \mathrm{~m}$ and $5 \mathrm{y}$. ARF, need of RRT, APACHE II and SOFA scores were variables independently related to hospital, $6 \mathrm{~m}, 12 \mathrm{~m}$ and a $5 y$ mortality, but only renal failure was included in all the models.

Conclusion. Long-term mortality in cirrhotic critically ill patients is high, but in ACLF and patients with renal failure is extremely high, while it was not in patients with GI haemorrhage.

\section{Reference(s)}

1. Crager S. Critically III Patients with End-Stage Liver Disease. Emerg Med Clin North Am. 2019 Aug;37(3):511-527. https://doi.org/10.1016/j.emc. 2019.03.008. Epub 2019 May 21. PMID: 31262418.

2. None. 
001212

What Is Your Gut Feeling About Probiotics in the Critically III? An ICU Stakeholder Survey

S. Zec'; J. Bakken²; B. Bauer ${ }^{3}$; S. Ahmad'; W. Freeman ${ }^{4}$; S. Khanna ${ }^{5}$; A. Shah ${ }^{6}$. K. Philbrick' L. Karnatovskaia

${ }^{1}$ Division of Pulmonary and Critical Care, Mayo Clinic, Rochester, United States of America; ${ }^{2}$ Section of Infectious Disease, St Luke's Hospital,

Duluth, United States of America; ${ }^{3}$ Division of General Internal Medicine, Mayo Clinic, Rochester, United States of America; ${ }^{4}$ Division of Neurosurgery and Critical Care, Mayo Clinic, Jacksonville, United States of America; ${ }^{5}$ Division of Gastroenterology, Mayo Clinic, Rochester, United States of America; ${ }^{6}$ Division of Infectious Diseases, Mayo Clinic, Rochester, United States of America; ${ }^{7}$ Division of Psychiatry, Mayo Clinic, Rochester, United States of America

Correspondence: S. Zec

Intensive Care Medicine Experimental 2021, 9(1): 001212

Introduction. Fermented foods, unlike supplements, facilitate digestion, provide nutrition, limit pathogen growth, and contain a wide diversity of microorganisms and signaling molecules that regulate metabolism. Their use in the critical care setting has been limited. Kefir, a fermented dairy product, has demonstrated anti-cancer, antimicrobial, immunomodulatory, anti-inflammatory, and psychobiotic effects in animal models and is one of the most common probiotic foods worldwide. It may also be relatively easy to administer in the intensive care setting, whether to prevent diarrheal illness or evaluate its role in minimizing delirium.

Objectives. To gauge stakeholder knowledge base and obtain feedback on the use of probiotics, particularly kefir, in the critically ill.

Methods. An anonymous web-based survey was administered to a multidisciplinary, interprofessional group of critical care providers at the Mayo Clinic campuses in Minnesota and Florida.

Results. 214 participants completed the survey (20\% response rate) $97(45 \%)$ bedside nurses, 97 (45\%) physicians, and 20 (9\%) critical care nurse practitioners/physician assistants. For the knowledgebased items, of the organ systems that could be affected by gut dysbiosis, the most endorsed systems were immune (79\%), hepatic $(65 \%)$, renal (58\%), and neural (57\%). Only $42 \%$ knew that over $70 \%$ of the human immune system is contained within the gastrointestinal tract. Over $90 \%$ of respondents considered that antimicrobials, physiologic stress, and proton-pump inhibitors have a role in alteration of the gut microbiome, with $62 \%$ agreeing that microbial diversity can be reduced within hours of critical illness onset. Regarding the role of probiotics, most (75\%) believed in its ability to prevent and treat diarrhea, followed by use in inflammatory or autoimmune conditions (58\%), urinary/vaginal infections $(54 \%)$, and as prophylaxis to viral infections (43\%). A quarter of responders expressed not knowing of the role of probiotics. Interestingly, 30\% endorsed that probiotics may improve mental wellbeing. $72 \%$ of respondents had heard of kefir and thought it to have more live active probiotic cultures compared to yogurt (58\%); a third were unsure. About half of responders did not know of the most effective way to deliver probiotics to the critically ill or whether kefir is safe in patients with lactose intolerance. Regarding overall feedback, perceived barriers to kefir administration included compromised gut integrity (53\%), immunosuppression (32\%), and poor prognosis (34\%); 33 participants saw no barriers. An overwhelming majority $(93 \%)$ welcomed conducting kefir research in critically ill populations.

Conclusion. Most providers are aware of the negative influence of gut dysbiosis in critically ill patients and recognize the need for additional research on finding an optimal probiotic source for this patient population.

\section{9}

Effect of nutrition practices and target calories on mortality in critically ill patients: A multi-center study across Turkish medical intensive care units

L. Talan, ; ND. Altintas'; B. Halacli, ; S. Temel, ${ }^{3}$; O. Cakin, ; M. Türkoğlu; ; B. Comert, ${ }_{1}^{6}$; G. Taskin, ; A. Zerman, ${ }^{8} ;$ T. Akbas, $^{9}$; E. Karakoc, ${ }^{10}$; K. Gonderen, ${ }^{11}$ iH. Akbudak, ${ }^{12}$; A. Topeli

'Department of Internal Medicine, Division of Intensive Care Medicine, Ankara University, Faculty of Medicine, Ankara, Turkey; ${ }^{2}$ Department of Internal Medicine, Division of Intensive Care Medicine, Hacettepe University, Faculty of Medicine, Ankara, Turkey; ${ }^{3}$ Department of Internal Medicine, Division of Intensive Care medicine, Erciyes University, School of Medicine, Kayseri, Turkey; ${ }^{4}$ Department of intensive care medicine, University of Health Sciences, Antalya Training and Research Hospital, Antalya, Turkey; ${ }^{5}$ Department of Internal Medicine, Division of Intensive Care Medicine, Gazi University, School of Medicine, Ankara, Turkey; ${ }^{6}$ Department of Internal Medicine, Division of intensive Care Medicine, Dokuz Eylül University, Faculty of Medicine, Izmir, Turkey; ${ }^{7}$ Department of intensive care medicine, University of Health Sciences, Gulhane Faculty of Medicine, Ankara, Turkey; ${ }^{8}$ Department of intensive care medicine, Kirsehir Ahi Evran University, Education and Research Hospital, Kırsehir, Turkey; ${ }^{9}$ Department of internal medicine, division of intensive care medicine, Duzce University, Faculty of Medicine, Bolu, Turkey; ${ }^{10}$ Department of internal medicine, division of intensive care medicine, Cukurova University, Faculty of Medicine, Adana, Turkey; ${ }^{11}$ Department of intensive care medicine, Kütahya University of Health Sciences, Faculty of Medicine, Kütahya, Turkey; ${ }^{12}$ Department of internal medicine, division of intensive care medicine, Pamukkale University, Faculty of Medicine, Denizli, Turkey Correspondence: N.D. Altintas

Intensive Care Medicine Experimental 2021, 9(1): 001459

Introduction. Nutritional planning is an important aspect of intensive care unit (ICU) management of critically ill patients. We aimed to evaluate the nutritional practices adopted in medical intensive care units of university and training research hospitals in our country, and the effect of attaining at least $70 \%$ of target calories on mortality in critically ill patients.

Methods. This study was planned as a multi-center, point prevalence study and medical ICU directors willing to participate were required to complete online questionnaires on a predetermined study date. Participants filled two separate online questionnaires: one on ICU characteristics and facilities, and one on patients included, and later they were questioned about the 28th day outcomes of patients. For statistical analysis, patients were grouped depending on whether they had received at least $70 \%$ of the target calories or not. The results are given as median (25th-75th percentile), percentages and odds ratios (OR) and $95 \%$ confidence interval $(\mathrm{Cl})$ limits.

Results. Medical ICUs from a total of 12 hospitals participated and 132 patients were included in the study. The median age was 71 years (57-83). The number of female patients was 63 (47.7\%). The median APACHE II score was $23(17-29)$ and median SOFA score was 6.5 (4-9.5). Eighty-seven (68\%) patients were fed enterally and in $79(60 \%)$ patients feeding was started within first $24 \mathrm{~h}$ of admission. Target calories were calculated using weight-based formulas. On the study day, the median caloric intake of the patients during the last $24 \mathrm{~h}$ was $1440 \mathrm{kcal}(1000-1700 \mathrm{kcal})$ and the median amount of protein intake

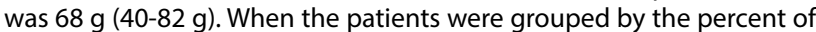
calories they received, it was observed that $90(68.2 \%)$ of the patients had received $\geq 70 \%$ of target calories. In this cohort of patients 28th day mortality was $43.9 \%$. Attainment of $<70 \%$ of target calories was found to be independently associated with mortality (OR: 3.7, 95\% Cl:1.1-12.3, p:0.035), when adjusted for sepsis, shock, admission with renal failure, postoperative admission, chronic renal failure, chronic obstructive pulmonary disease, SOFA score, need for vasopressors, serum albumin level, enteral feeding and parenteral feeding.

Conclusion. This multicenter study among medical ICUs in our country revealed that, nutrition therapy in medical ICUs led by intensivists were planned early after ICU admission. Being unable to attain target calories of at least $70 \%$ was found to be an independent risk factor for 28th day mortality. 
Reference(s)

1. Singer P, Blaser AR, Berger MM, Alhazzani W, Calder PC, Casaer MP, et al. ESPEN guideline on clinical nutrition in the intensive care unit. Clin Nutr. 2019;38(1):48-79.

2. None.

e-Posters: Neurointensive care

\section{7}

\section{Effects of passive leg raising test, fluid challenge} and norepinephrine on cerebral autoregulation in Covid-19 critically ill patients

E. Moncalvo ${ }^{1}$; A. Messina ${ }^{2}$; D. Battaglini, ${ }^{1}$; L. Ball ${ }^{3}$; I. Brunetti, ${ }^{1}$; M. Bassetti, ${ }^{4}$ DR. Giacobbe, ${ }^{5}$; A. Vena, ${ }^{5}$; N. Patroniti, ${ }^{6}$; M. Cecconi ${ }^{7}$; BF. Matta, ; L. Xiuyun ${ }^{9}$; M. Czosnyka ${ }^{10} ;$ P. Pelosi ${ }^{11}$; C. Robba ${ }^{12}$

${ }^{1}$ Anesthesia and Intensive Care, San Martino Policlinico Hospital, irccs for Oncology and Neurosci, IRCCS AOU San Martino, Genova, Italy ${ }^{2}$ Anesthesia and Intensive Care, Humanitas Research Hospital, Rozzano, Italy; ${ }^{3}$ Department of surgical Sciences and Integrated Diagnostic (disc), University of genoa, Genoa, it, Policlinico San martino Genova, Genoa, Italy; ${ }^{4}$ Infectious disease unit, san martino policlinico Hospital, irccs for Oncology and Neuroscience, Department of Health Sciences, University of Genoa, Genoa, Italy., Genova, Italy; ${ }^{5}$ Infectious Disease Unit, san Martino Policlinico Hospital, irccs for Oncology and Neuroscience, IRCCS AOU San Martino, Genova, Italy; ${ }^{6}$ Department of Surgical Sciences and Integrated Diagnostic (disc), University of Genoa, genoa, it, Anesthesia and Intensive Care, San Martino Policlinico Hospital, IRCCS for Oncology and Neurosci, Genoa, Italy; ${ }^{7}$ Anesthesia and intensive care, Humanitas Research Hospital, Milan, Italy; ${ }^{8}$ Neurosciences critical care unit, addenbrooke's hospital, cambridge university hospitals nhs fou, Department of Anesthesia,Addenbrooke's Hospital,Cambridge University Hospitals NHS, Cambridge, United Kingdom; ${ }^{9}$ Dipartimento di anestesiologia e medicina critica, Johns Hopkins University, Cambridge, United Kingdom; ${ }^{10}$ Department of Clinical Neurosciences, Brain Physics Laboratory, Division of Neurosurgery, University of Cambridge, Cambridge, United Kingdom; ${ }^{11}$ Departement of Anesthesia and Intensive Care, University of Genoa, Genova, Italy; ${ }^{12}$ Department of Anesthesia and Intensive Care, University of Genoa, Genova, United Kingdom

Correspondence: $\mathrm{E}$. Moncalvo

Intensive Care Medicine Experimental 2021, 9(1): 000027

Introduction. COVID-19 patients are at high risk for neurological complications consequent to several factors including persistent hypoxia and hypotension. So far, no data are available regarding the best strategy to adopt to maintain hemodynamic stability and the effect of different hemodynamic strategies and in particular of passive leg rising (PLR) test on cerebral hemodynamics is unclear.

Methods. Single center, observational prospective study conducted at San Martino Policlinico Hospital, Genova, Italy during the second wave of COVID-19 pandemic. Mechanically ventilated COVID-19 patients who had at least one episode of hypotension and received a PLR test were included.

We evaluated the effect of PLR test on static cerebral autoregulation (CA) measured through Transcranial Doppler (TCD), compared to other hemodynamic strategies normally applied for clinical reasons in case of hypotensive events [fluid bolus challenge (FC) and norepinephrine (NE)]. Secondary aims included the evaluation of the effect of PRL test, FC and NE on systemic hemodynamic variables (mean arterial pressure, MAP) cerebral oxygenation ( $\mathrm{rSO} 2$ ) and noninvasive intracranial pressure (nICP).

Results. Twenty-three patients were included and underwent PLR test. Among these, 22 patients received $F C$, and 14 received NE. A total of 59 hemodynamic maneuvers were studied. The median age was 62 years [Interquartile range, IQR $=57-68.5$ ], and $78.3 \%$ were males. Median CA index in the PLR test group was 58 [44-76,3], in the FC was 90.8 [74.2-100] and in the NE group was 100 [100-100]. CA was significantly higher in the norepinephrine and fluid challenge groups compared to the PLR test group (NE vs PLR test; $p<0.001$ and FC vs PLR test; $p<0.001)$, but no differences in CA were found between the FC and NE groups $(p=0.169)$. All the hemodynamic strategies resulted in a significant increase of mean arterial pressure (MAP), cerebral perfusion pressure (CPP) and rSO2. After PLR test, both nICPPI and nICPFVd were increased (12.9 [8.5-18] vs $15[10.5-19.7] \mathrm{mmHg}, \mathrm{p}=0.001$, and $18.6[17.7-19.6]$ vs $19.3[18.2-19.8] \mathrm{mmHg}, \mathrm{p}=0.009$, respectively). After FC and NE administration, nICPPI was not affected and nICPFVd was slightly increased in both cases (10.9 [6.7-15.7] vs 14.4 [5.6-21.1] $\mathrm{p}=0.004$, and 10 [6.9-21.3] vs 12.2 [5.5-22.6], $\mathrm{p}=0.022$, respectively). Conclusion. PLR test seems to have the worse effect on nICP and CA when compared with FC and NE administration. Regardless the hemodynamic strategy used, an increase of MAP resulted in an increase of rSO2. An individualized strategy aimed to assess both the hemodynamic and cerebral needs is warranted in patients at high risk of neurological complications.

Reference(s)

1. None.

2. Goyal, P.; Choi, J.J.; Pinheiro, L.C.; Schenck, E.J; Chen, R.; Jabri, A.; Satlin, M.J.; Campion, T.R.; Nahid, M.; Ringel, J.B.; et al. Clinical Characteristics of Covid-19 in New York City. N. Engl. J. Med. 2020, 382, 2372-2374, https:// doi.org/10.1056/NEJMc2010419.

3. Bassetti, M.; Vena, A.; Giacobbe, D.R.; The novel Chinese coronavirus (2019$\mathrm{nCoV}$ ) infections: Challenges for fighting the storm. Eur J. Clin. Invest. 2020, 50, e13209, https://doi.org/10.1111/eci.13209.

4. Grasselli, G.; Zangrillo, A.; Zanella, A.; Antonelli, M.; Cabrini, L.; Castelli, A.; Cereda, D.; Coluccello, A.; Foti, G.; Fumagalli, R.; et al. Baseline Characteristics and Outcomes of 1591 Patients Infected With SARS-CoV-2 Admitted to ICUs of the Lombardy Region, Italy. JAMA 2020, 323, 1574, https://doi. org/10.1001/jama.2020.5394.

5. Battaglini,D.; Brunetti, I.; Anania, P.; Fiaschi, P.; Zona, G.; Ball, L.; Giacobbe, D:R; Vena, A.; Bassetti,M.; Patroniti, N.; et al. Neurological Manifestations of Severe SARS-CoV-2 Infection: Potential Mechanisms and Implications of Individualized Mechanical Ventilation Settings. Front. Neurol. 2020, 11, 845, https://doi.org/10.3389/fneur 2020.00845 .

6. Battaglini,D.; Santori, G.; Chandraptham,K.; lannuzzi, F.; Bastianello M.; Tarantino, F; Ball, L.; Giacobbe, D.R; Vena, A.; Bassetti, M; et al.; Neurological Complications and Noninvasive Multimodal Neuromonitoring in Critically III Mechanically Ventilated COVID-19 Patients. Front. Neurol. 2020, 11, 602,114, https://doi.org/10.3389/fneur.2020.602114

7. Wu,Z.; McGoogan, J.M.; Characteristics of and Important Lessons From the Coronavirus Disease 2019 (COVID-19) Outbreak in China. JAMA 2020, 323, 1239, https://doi.org/10.1001/jama.2020.2648

8. Robba, C.: Battaglini, D.: Pelosi, P.; Rocco, R.M.P. Multiple organ dysfunction in SARS-CoV-2: MODS-CoV-2. Expert Rev. Respir. Med. 2020, 14, 865-868, https://doi.org/10.1080/17476348.2020.1778470

\section{8}

\section{A Rapid Systematic Review of The Effect of Fever Control} on Outcomes Following Traumatic Brain Injury

MM. Anwar ${ }^{1}$; S. Basser ${ }^{1}$; M. Beanlands ${ }^{1}$; A. Mclaughlan ${ }^{1}$; M. Murdoch ${ }^{1}$; A. Pollock'; JGC. Rodrigues'; H. Shamaz'; M. Song ${ }^{1}$; K. White ; T. Anderson²; J. Rhodes ${ }^{2}$

${ }^{1}$ Edinburgh Medical School, The University of Edinburgh, Edinburgh, United Kingdom; ${ }^{2}$ critical Care, Anaesthesia and Pain Medicine, University of Edinburgh and NHS Lothian, Edinburgh, United Kingdom

\section{Correspondence: M.M. Anwar}

Intensive Care Medicine Experimental 2021, 9(1): 000058

Introduction. Each year, 69 million people worldwide are estimated to sustain a Traumatic Brain Injury (TBI) [1]. TBI is a major cause of death and disability in those aged 1-40 in the UK [2]. Multiple studies suggest that fever is associated with poorer outcomes in TB patients, including increased mortality and morbidity [3]. Fever control remains standard in the treatment of TBI despite lack of evidence to support this. This rapid systematic review sought to summarise the current state of knowledge to determine whether fever is associated 
with poorer TBI outcomes and investigate if fever control is clinically required.

Methods. A literature search, using search terms relating to fever control, TBI and outcomes (including mortality and morbidity) was conducted on PubMed and Embase spanning the years 2011 to 2021. Eligible studies were randomised clinical trials, prospective observational and retrospective cohort studies that involved TBI patients $\geq 13$ years old and reported outcomes following interventions for fever control compared with the absence of such interventions. Eligible studies had to assess systemic temperature and define fever as $\geq 37.5^{\circ} \mathrm{C}$. A total of 307 studies were initially identified. In 2 rounds of screening, by pairs of authors working independently, these were reduced to only three studies fitting the inclusion/exclusion criteria [4-6]. The outcomes for hospital mortality and hospital length of stay (LoS) were extracted for statistical analysis on RevMan.

Results. When comparing interventions for fever control with no intervention, there was no statistically significant association with hospital mortality (Odds ratio $=0.8 ; 95 \% \mathrm{Cl} 0.46-1.41 ; \mathrm{I} 2=53 \% ; \mathrm{p}=0.45$ ) which could be a result of the heterogenous nature of that data. Conversely, there was a statistically significant association between shorter LoS in hospital and no fever control intervention (Mean difference $=3.63$, $95 \% \mathrm{Cl} 0.33-6.94 ; 12=0 \% ; p=0.03$ ). The LoS data was not heterogenous but heavily weighted towards one study with smaller standard errors.

Conclusion. There was no significant impact on hospital mortality when controlling fever. There was, however, statistical significance in hospital LoS, with the absence of fever control reducing its duration. This could be explained by confounding factors such as earlier mortality within the no fever control group. Although this review is not exhaustive it is unlikely to have missed a significant positive trial. In that context, it is interesting that the clinical use of fever control in $\mathrm{TBI}$ is so widespread in the absence of evidence to justify the resource implications.

\section{Reference(s)}

1. This study was not support through any grants

2. • [1] Dewan MC, Rattani A, Gupta S, Baticulon RE, Hung YC, Punchak M, Agrawal A, Adeleye AO, Shrime MG, Rubiano AM, Rosenfeld JV. Estimating the global incidence of traumatic brain injury. Journal of neurosurgery. 2018 Apr 27;130(4):1080-97

3. - [2] Lawrence T, Helmy A, Bouamra O, Woodford M, Lecky F, Hutchinson PJ. Traumatic brain injury in England and Wales: prospective audit of epidemiology, complications and standardised mortality. BMJ open. 2016 Nov 1;6(11)

4. • [3] Diringer MN, Reaven NL, Funk SE, Uman GC. Elevated body temperature independently contributes to increased length of stay in neurologic intensive care unit patients. Critical care medicine. $2004 \mathrm{Jul}$ 1;32(7):1489-95

5. - [4] Launey, Y., Nesseler, N., Le Cousin, A. et al. Effect of a fever control protocol-based strategy on ventilator-associated pneumonia in severely brain-injured patients. Crit Care, 2014; 18, 689.

6. • [5] Saxena MK, Taylor C, Billot L, Bompoint S, Gowardman J, Roberts $J A$, et al. The Effect of Paracetamol on Core Body Temperature in Acute Traumatic Brain Injury: A Randomised, Controlled Clinical Trial. PLoS ONE, 2015; 10(12): e0144740.

7. · [6] Weng WJ, Yang C, Huang XJ, et al. Effects of Brain Temperature on the Outcome of Patients with Traumatic Brain Injury: A Prospective Observational Study. J Neurotrauma, 2019; 36(7):1168-1174.

\section{1}

VCSF danger-associated molecular patterns after traumatic and non-traumatic acute brain injury

C. Santacruz Herrera

'Departament of Critical and Intensive Care Medicine, Fundacion Santa Fe de Bogota University Hospital, Bogotá, Colombia

Correspondence: C. Santacruz Herrera

Intensive Care Medicine Experimental 2021, 9(1): 000061
Introduction. In the early phase after acute brain injury (ABI), a series of proteins released in the ventricular cerebrospinal fluid (VCSF) may act together as danger-associated molecular patterns (DAMPs) to orchestrate the pathophysiological pathways associated with prognosis.

Objectives. To identify DAMPS and significant protein-protein interactions (PPI) in VCSF during episodes of elevated intra-cranial pressure (ICP), and their association with survival after ABI.

Methods. We collected vCSF samples for 5 consecutive days from ABI patients who required intraventricular catheterization for ICP monitoring. We studied 50 consecutive $A B I$ patients with spontaneous subarachnoid hemorrhage (sSAH, 46\%), traumatic brain injury (TBI, 30\%), intracranial hemorrhage $(\mathrm{ICH}, 12 \%)$, and other causes (12\%). vCSF proteins with significant intra- and inter-day differences in traumatic vs non-traumatic, high vs low ICP and surviving vs non-surviving $A B I-$ patients were compared using linear models. Significant proteins were selected for PPI using the STRING ${ }^{\circledR}$ database.

Results. Compared to ABI-patients with $\mathrm{ICP}<20 \mathrm{mmHg}$, patients with ICP $>30 \mathrm{mmHg}$ expressed the DAMP_ICP30 and theDAMP_death suggesting an increased risk for development of severe intra-cranial hypertension and death. DAMP_trauma was expressed exclusively in patients with a traumatic origin of ABI. PPI for these three DAMPS (DAMP_ICP30 PPI $<0.01 ;$ DAMP_death $\mathrm{PPI}<0.01$ and DAMP_trauma $\mathrm{PPI}=0.04)$ suggest that this particular set of proteins had more interactions among themselves than what would be expected for a random set of proteins and were biologically connected as a group for 5 consecutive days.

Conclusion. We describe signature vCSF DAMPs that were associated with high ICP and increased mortality after $A B I$.

\section{9}

Transcranial Doppler Ultrasound as a predictor of neurological outcome in Emergency Department patients with mild and moderate Traumatic Brain Injury

M. Shebl Zahra'; A. Sakrr ; AA. Elmorsy ${ }^{3} ;$ M. Megahed ${ }^{3}$; A. Elbeheiry ${ }^{4}$ ${ }^{1}$ Intensive Care Medicine, King's College Hospital, London, United Kingdom; ${ }^{2}$ Department of Emergency Medicine, Alexandria Faculty of Medicine, Alexandria, Egypt; ${ }^{3}$ Intensive Care Medicine, Alexandria Faculty of Medicine, Alexandria, Egypt; ${ }^{4}$ Department of Radiodiagnosis, Alexandria Faculty of Medicine, Alexandria, Egypt

Correspondence: M. Shebl Zahra

Intensive Care Medicine Experimental 2021, 9(1): 000079

Introduction. Traumatic brain injury (TBI) is a global health and socioeconomic problem (1). It is responsible of millions of ED visits, hospitalizations, and deaths with lifelong disability among survivors (2). Immediately after TBI, cerebral blood flow (CBF) is extremely low approaching the ischemic thresholds (3). Concurrently, velocities are strongly correlated to CBF. Identification of such disturbances can predict outcome in TBI when assessed early post-injury using Transcranial Doppler (TCD). TCD provides non-invasive real time measurements of CBF velocities as well as pulsatility index (PI) $(4,5)$.

Objectives. To assess the role of early use of Transcranial Doppler in predicting neurologic outcome after mild and moderate traumatic brain injury.

Methods. 120 TBI patients with Glasgow Coma Score (GCS) of 9-15 who were randomly selected, underwent TCD within $8 \mathrm{~h}$ post trauma. Middle cerebral artery (MCA) velocities and pulsatility index, as well as other clinical and neuroimaging data, were recorded. The primary end point was the development of secondary neurological deterioration (SND) at day 7 after injury. Secondary outcome measures were the Glasgow Outcome Scale extended (GOSE) at 1 months post-injury and in-hospital mortality.

Results. There was a significant relation between end diastolic velocity (EVD) as well as PI with SND at day 7 as well as GOSE at day 28 posttrauma. There was also significant correlation between PI and hospital mortality.

ROC curves for TCD measurements to predict In-hospital mortality. 


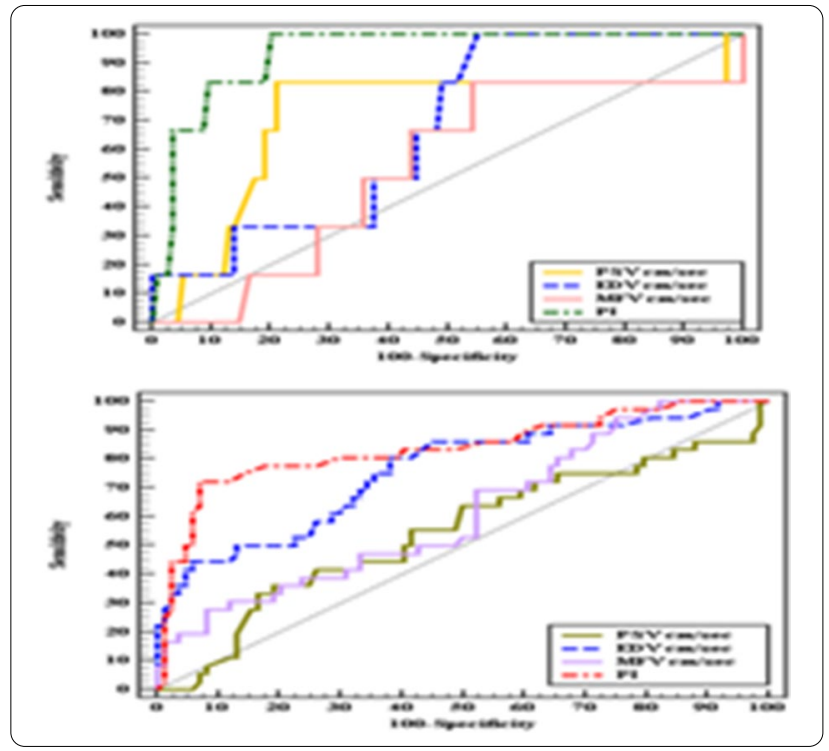

- Table $1 \mathrm{a}$. Characteristics of the patients: overall and according $\mathrm{DCl}$ or not

\begin{tabular}{|c|c|c|c|c|}
\hline & \multirow[b]{2}{*}{$\begin{array}{c}\text { Overall } \\
N=161\end{array}$} & \multicolumn{2}{|c|}{$\begin{array}{l}\text { Delayed Cerebral } \\
\text { Ischemia }\end{array}$} & \multirow[b]{2}{*}{$p$} \\
\hline & & $\begin{array}{c}\text { No } \\
N=117\end{array}$ & $\begin{array}{c}\text { Yes } \\
N=44\end{array}$ & \\
\hline Age (years) & & $59.4 \pm 12.6$ & & 0.006 \\
\hline Sex female & $110(68.3)$ & $78(66.7)$ & $32(72.7)$ & 0.461 \\
\hline Apache-ll at admission & $14.9 \pm 7.5$ & $14.6 \pm 7.9$ & $16.0 \pm 6.5$ & 0.288 \\
\hline SOFA at admission & $2(0-6)$ & $2(0.6)$ & $3(1 \cdot 7.2)$ & 0.185 \\
\hline Smoker & $61(38.1)$ & $39(33.6)$ & $22(50.0)$ & 0.057 \\
\hline Cocaine & $6(3.8)$ & $5(4.3)$ & $1(2.3)$ & 1 \\
\hline SDD & $58(37.9)$ & $39(34.5)$ & $19(47.5)$ & 0.146 \\
\hline Stroke & $53(32.9)$ & $19(16.2)$ & $34(77.3)$ & $<.001$ \\
\hline Deaths & $36(23.1)$ & $25(21.7)$ & $11(26.8)$ & 0.507 \\
\hline ICU-Deaths & 25 (15.6) & $17(14.5)$ & $8(18.6)$ & 0.529 \\
\hline Hospital-Deaths & $10(6.5)$ & $7(6.1)$ & $3(7.5)$ & 0.720 \\
\hline Arterial hypertension & $83(51.9)$ & $60(51.7)$ & $23(52.3)$ & 0.951 \\
\hline Diabetes mellitus & $24(15.0)$ & $19(16.4)$ & $5(11.4)$ & 0.428 \\
\hline Dyslipidemia & $44(27.5)$ & $32(27.6)$ & $12(27.3)$ & 0.968 \\
\hline Chronic obstructive disease & $1(0.6)$ & $1(0.9)$ & 0 & 1 \\
\hline Renal failurel & $5(3.1)$ & $4(3.5)$ & $1(2.3)$ & 1 \\
\hline Caucasian race & $144(94.7)$ & $104(95.4)$ & $40(93.0)$ & 0.688 \\
\hline Oriented & $75(46.6)$ & $63(53.9)$ & $12(27.3)$ & 0.004 \\
\hline Alert & $86(53.4)$ & $71(60.7)$ & $15(34.1)$ & 0.003 \\
\hline Confused & $27(16.8)$ & $17(14.5)$ & $10(22.7)$ & 0.215 \\
\hline Stuporous & $40(25.2)$ & $22(19.0)$ & $18(41.9)$ & 0.003 \\
\hline Bilateral mydriasis & $6(3.8)$ & $4(3.5)$ & $2(4.7)$ & 0.662 \\
\hline Anisochoric pupils & $14(8.8)$ & $6(5.2)$ & $8(18.6)$ & 0.022 \\
\hline Isochoric pupils & $142(89.3)$ & $107(922)$ & $35(81.4)$ & 0.079 \\
\hline One reactive pupil & $6(3.8)$ & $2(1.7)$ & $4(9.3)$ & 0.046 \\
\hline Both reactive pupils & $140(88.0)$ & $105(90.5)$ & $35(81.4)$ & 0.115 \\
\hline None-reactive pupils & $9(6.5)$ & $6(5.8)$ & $3(8.3)$ & 0.695 \\
\hline Right aneurysm & $37(23.4)$ & $24(21.1)$ & $13(29.6)$ & 0.258 \\
\hline Left aneurysm & $48(30.4)$ & $33(28.9)$ & $15(34.1)$ & 0.529 \\
\hline Bilateral aneurysm & $9(5.7)$ & $7(6.1)$ & $2(4.5)$ & 1 \\
\hline Aneurysm in the midline & $49(31.0)$ & $34(29.8)$ & $15(34.1)$ & 0.603 \\
\hline Anterior cerebral artery aneurysm & $14(8.9)$ & $9(7.9)$ & $5(11.6)$ & 0.532 \\
\hline Anterior communicating antery aneurysm & $49(30.6)$ & $37(31.9)$ & $12(27.3)$ & 0.571 \\
\hline Posterior communicating artery aneurysm & $24(15.2)$ & $14(12.3)$ & $10(22.7)$ & 0.101 \\
\hline Anterior cerebral artery aneurysm & $7(4.4)$ & $5(4.4)$ & $2(4.5)$ & 1 \\
\hline Middle Cerebral Artery aneurysm & $21(13.3)$ & $14(12.3)$ & $7(15.9)$ & 0.547 \\
\hline Posterior Cerebral Artery aneurysm & $8(5.1)$ & $5(4.4)$ & $3(6.8)$ & 0.686 \\
\hline Basilar Artery aneurysm & $14(8.9)$ & $12(10.5)$ & $2(4.5)$ & 0.352 \\
\hline Posterior inferior cerebellar antery anurysm & $9(5.7)$ & $8(7.0)$ & $1(2.3)$ & 0.446 \\
\hline
\end{tabular}

Data are frequencies (\%), means \pm SD and medians (IQR). SOD:

gelective digestive decontaminaton

ROC curves for TCD measurements to predict SND.

Conclusion. TCD, when done early after admission, is useful in identifying patients at risk for developing neurological deterioration after mild and moderate TBI. EDV and PI are good predictors of SND at one week as well as GOSE at one month. PI is a good predictor for in-hospital mortality.

\section{Reference(s)}

1. 5. Zaytoun T, Elsayed A, Beshay B, Elbeheiry A, Shebl M. Evaluation of cerebral autoregulation using transcranial doppler ultrasound in patients with moderate and severe traumatic brain injuries. Biolife 2017; 5(1):111-117.

2. 4. 13. Powers WJ, Rabinstein AA, Ackerson T, Adeoye OM, Bambakidis NC, Becker K, et al. 2018 guidelines for the early management of patients with acute ischemic stroke: a guideline for healthcare professionals from the American Heart Association/American Stroke Association. Stroke 2018; 49(3):e46-e99.

3. 3. 10. Bouzat P, Sala N, Payen J-F, Oddo M. Beyond intracranial pressure: optimization of cerebral blood flow, oxygen, and substrate delivery after traumatic brain injury. Ann Int Care 2013; 3(1):23.

4. 2. Peeters W, van den Brande R, Polinder S, Brazinova A, Steyerberg EW, Lingsma HF, et al. Epidemiology of traumatic brain injury in Europe. Acta neurochirurgica 2015; 157(10):1683-96.

5. 1. Centers for Disease Control Prevention. Report to congress on traumatic brain injury in the United States: epidemiology and rehabilitation. Atlanta, GA: CDC. 2016.

\section{1}

Decoding accelerometry for classification and prediction

of severe brain injury in the ICU

S. Bhattacharyay ${ }^{1}$; M. Wang ${ }^{2}$; P. Dziedzic ${ }^{3}$; E. Calvillo ${ }^{3}$; H. Kim³ ${ }^{3}$ E. Joshi ${ }^{2}$; J.

Rattray ${ }^{4}$; R. Etienne-Cummings ${ }^{4}$; P. Kudela ${ }^{5} ;$ R. Stevens ${ }^{6}$

${ }^{1}$ Department of Clinical Neurosciences, University of Cambridge,

Cambridge, United Kingdom; ${ }^{2}$ Department of Biomedical engineering, Johns Hopkins University, Baltimore, United States of America; ${ }^{3}$ Laboratory of computational intensive care medicine, Johns Hopkins University, Baltimore, United States of America; ${ }^{4}$ Department of Electrical and Computer Engineering, Johns Hopkins University, Baltimore, United States of America; ${ }^{5}$ Department of Neurosurgery, Johns Hopkins University, Baltimore, United States of America; ${ }^{6}$ Department of Anesthesiology and Critical Care Medicine, Johns Hopkins University School of Medicine, Baltimore, United States of America

Correspondence: S. Bhattacharyay

Intensive Care Medicine Experimental 2021, 9(1): 000181

Introduction. Levels of physical activity have been linked to important clinical outcomes in patients with severe brain injury (SBI) [1-5]; however, ICU management of SBI excludes any quantitative motion monitoring modalities.

Objectives. In this pilot study, we aim to determine whether motion features extracted from high-resolution, triaxial accelerometry are significantly associated with neurological scores of motor function and functional outcome in SBI patients.

Methods. The study was carried out in a cohort of patients admitted to the neurosciences critical care unit (NCCU) for SBI management. Six wireless sensors were placed on elbows, wrists, and ankles, sampling data at $10 \mathrm{~Hz}$. From accelerometry time series, we generated 6 types of features ( 2 time-, 3 frequency-, 1 wavelet-domain) from non-overlapping 5-s windows of filtered accelerometry data. Motion features across extremity sensors were binned in 19 observation windows (from $3 \mathrm{~min}$ to $24 \mathrm{~h}$ ) directly preceding nurse-assessed Glasgow Coma Scale (GCS) [6] evaluations $(n=653)$. Dimensionality reduction and feature importance ranking was performed using linear optimal lowrank (LOL) projection [7]. Embedded features were then used in logistic regression models to detect motor sub-scores of the GCS (GCSm) and predict Glasgow Outcome Scale - Extended (GOSE) [8] scores (at discharge and at 12 months) at each threshold of the scores.

Results. Sixty-nine patients with SBI underwent continuous motion sensing (median duration: $24 \mathrm{~h}$ ). Motor function detection models significantly discriminated (AUC: $0.70,95 \% \mathrm{Cl}: 0.53-0.85$ ) SBI patients capable of purposeful motion (GCSm $>4$ ) with 12 or more minutes of accelerometry. In retrospective case studies of patients who experienced a transition across the GCSm $>4$ threshold during the recording duration, we observe that the model can detect motor function transitions up to five hours before a clinical evaluation. Dynamic functional outcome prediction models significantly discriminate (AUC: $0.82,95 \% \mathrm{Cl}: 0.75-0.90$ ) patients of upper moderate disability or better (GOSE $>5$ ) at hospital discharge with 2 or more hours of recording. No 
association was detected between accelerometry-based features and GOSE scores at 12 months post-discharge.

Conclusion. In comatose patients with SBI, models trained on accelerometry-based features can discriminate purposeful from nonpurposeful motor activity, predict motor state transitions, and predict functional outcomes at hospital discharge. Further development and validation of this approach may enable individualized approaches to the care of patients with SBI.

\section{Reference(s)}

1. [8] Wilson JT, Pettigrew LE, Teasdale GM (1998) Structured interviews for the Glasgow Outcome Scale and the extended Glasgow Outcome Scale: guidelines for their use. J Neurotrauma. https://doi.org/10.1089/neu. 1998.15.573

2. [7] Vogelstein JT, Bridgeford E, Tang M, Zheng D, Burns R, Maggioni M (2017) Geometric Dimensionality Reduction for Subsequent Classification. arXiv preprint arXiv:1709.01233

3. [6] Teasdale G, Maas A, Lecky F, Manley G, Stocchetti N, Murray G (2014) The Glasgow Coma Scale at 40 years: standing the test of time. Lancet Neurol. https://doi.org/10.1016/S1474-4422(14)70120-6

4. [5] Schujmann DS, Lunardi AC, Fu C (2018) Progressive mobility program and technology to increase the level of physical activity and its benefits in respiratory, muscular system, and functionality of ICU patients: study protocol for a randomized controlled trial. Trials. https://doi.org/10.1186/ s13063-018-2641-4

5. [4] Parry SM, Puthucheary ZA (2015) The impact of extended bed rest on the musculoskeletal system in the critical care environment. Extrem Physiol Med. https://doi.org/10.1186/s13728-015-0036-7

6. [3] Drummond MJ, Timmerman KL, Markofski MM et al. (2013) Short-term bed rest increases TLR4 and IL-6 expression in skeletal muscle of older adults. Am J Physiol Regul Integr Comp Physiol. https://doi.org/10.1152/ ajpregu.00072.2013

7. [2] Reinkensmeyer DJ, Burdet E, Casadio M et al. (2016) Computational neurorehabilitation: modeling plasticity and learning to predict recovery. Journal of NeuroEngineering and Rehabilitation. https://doi.org/10.1186/ s12984-016-0148-3

8. [1] Bahouth MN, Power MC, Zink EK et al. (2018) Safety and Feasibility of a Neuroscience Critical Care Program to Mobilize Patients With Primary Intracerebral Hemorrhage. Arch Phys Med Rehabil. https://doi.org/10. 1016/j.apmr.2018.01.034

9. S.B. is currently funded by a Gates Cambridge fellowship.

000237

Phosphatase alkaline levels as predictor of poor outcomes in a cohort of subarachanoid hemorrhage pacients.

$A$ retrospective study

A. Diosdado'; E. Gouvêa-Bogossian²; FS. Taccone ${ }^{3}$; D. Daniela ${ }^{4}$; A. Minini ${ }^{5}$; M. Marco ${ }^{4}$; J. Creteur ${ }^{3}$

'Anaesthesiology and reanimation, Hospital del Mar, Barcelona, Spain ${ }^{2}$ Department of intensive care, Hospital Erasme, Bruxelles, Belgium;

${ }^{3}$ Soins intensif, ULB Erasme, Anderlecht, Belgium; ${ }^{4}$ Intensive care, Hospital Erasme, Bruxelles, Belgium; ${ }^{5}$ Intensive care unit, UZ Brussel, Jette, Belgium Correspondence: A. Diosdado

Intensive Care Medicine Experimental 2021, 9(1): 000237

Introduction. Increased levels of alkaline phosphatase (ALP) are often present in patients with stroke and Alzheimer disease and can be associated with poor outcomes (1) (2). The role of ALP in brain injury due to non-traumatic subarachnoid hemorrhage $(\mathrm{SAH})$ remains poorly investigated (3).

Objectives. To determinate if ALP levels during the hospital admission are related with worsen outcomes in pacients suffering for an SAH event.

Methods. All patients with non-traumatic SAH admitted to the intensive care unit (ICU) of Erasme Hospital (Brussels, Belgium) and who survived for at least $24 \mathrm{~h}$ were included. Patients with a history of liver cirrhosis, oncologic disease or with missing ALP levels were excluded.
Baseline information, clinical data, and radiologic data were collected, as well as serum ALP levels during the first 12 days after ICU admission. Mortality was assessed at hospital discharge and neurological outcome at 3 months, using the Glasgow Outcome Scale (GOS). Unfavorable Outcome (UO) was defined as GOS 1-3.

Results. 650 patients were included. Baseline and peak ALP were 64 [53-76] and 85 [65-121] IU/L, respectively. ALP levels remained unchanged from baseline during the first 5 days and therefore increased significantly from day 6 to 12. ALP values on admission were significantly higher in non-survivors $(n=185,28 \%)$ than survivors $(n=465,72 \%) ; 74$ [59-88] Vs 66 [53-77] IU/L, $p=0.038$, as in patients with UO $(n=270,42 \%)$ compared to others $(n=380,58 \%) ; 77$ [64-95] Vs 62 [51-80] IU/L, $p=0.017$. After mixed-model analysis, the time-course values of APL between patients with UO and favorable neurological outcome showed significant increased levels, $p=0.001$. No differences were seen when studying ALP variations over time between survivors and no-survivors, $p=0.16$. In the multivariable analysis, ALP levels on admission were not associated with mortality [OR 1.01 (0.99-1.03)] nor with UO [OR 0.99 (0.97-1.01)].

Conclusion. In this study, ALP levels were not independently associated with poor outcome after non-traumatic SAH.

\section{Reference(s)}

1. 3-Zhu, Yu, et al. "Serum Alkaline Phosphatase Level Is Associated with Angiographic Vasospasm, Delayed Cerebral Ischemia-Caused Clinical Deterioration, and Functional Outcome After Aneurysmal Subarachnoid Hemorrhage." Neurocritical Care, vol. 31, no. 3, 2019, pp. 466-475., https://doi.org/10.1007/s12028-019-00714-7.

2. 2-Zhong C, You S, Chen J, et al. Serum alkaline phosphatase, phosphate, and in-hospital mortality in acute ischemic stroke patients. J Stroke. Cerebrovasc Dis. 2018;27:257-66.

3. 1-Wannamethee SG, Sattar N, Papcosta O, Lennon L, Whincup PH (2013) Alkaline phosphatase, serum phosphate, and incident cardiovascular disease and total mortality in older men. Arterioscler Thromb Vasc Biol 33: $1070-1076$

\section{4}

Delayed cerebral ischemia associated risk factors in patients with subarachnoid hemorrhage in a neurotraumatic ICU

C. Sánchez Ramírez'; CF. Lübbe Vázquez'; C. Rodriguez Mata'; J J. Vicente Arranz'; J. López Pérez'; A. Padrón Mujica'; J. Garriga Segarra'; P. Saavedra-Santana ${ }^{2}$; S. Ruiz-Santana

'Intensive care medicine, University Hospital of Gran Canaria Dr. Negrin, Las Palmas de Gran Canaria, Spain; ${ }^{2}$ Mathematics and informatics deparment, University of Las Palmas:, Las Palmas de Gran Canaria, Spain

Correspondence: $C$. Sánchez Ramírez

Intensive Care Medicine Experimental 2021, 9(1): 000304

Introduction. Delayed cerebral ischemia (DCI) is a major contributor to the high morbidity and fatality case rates of aneurysmal subarachnoid hemorrhage (SAH). About $30 \%$ of the SAH patients develop DCI but it is difficult to predict which patients will develop it.

Objectives. To prospectively assess the DCl risk factors in patients with $\mathrm{SAH}$, admitted to a neurotraumatic ICU.

Methods. Prospectively collected data of patients admitted from October, 2013 to December 2020 to a 10-bed Neurotraumatic ICU. We analyzed: main diagnosis at admission; demographic data including sex and race; neurological data (clinical examination, pupils reactivity and size, and Glasgow Coma Score: GCS; localization and aneurysm size; presence of intracranial hematoma (ICH); presence and volume of intraventricular bleeding; days to develop vasoespam; development of DCl; Fisher scale, Modified Fisher scale (MFS), Hunt and Hess scale (HHS), Word Federation of Neurosurgeons (WFNS) scale; presence of vasoespasm in doppler or arteriography; delayed of admission in ICU; treatment of the aneurysm; complications, including infections; Glasgow Outcome Scale (GOS) at ICU discharge and 6 months after ICU discharge and several other risk factors. A univariate analysis of $\mathrm{DCl}$ was performed to obtain 
a predictive rule for the $\mathrm{DCl}$, a model for the prediction was obtained using the classification and regression trees (CART) procedure [1]. CART classifies data using a sequence of if-then rules. The basis of the decision tree algorithms is the binary recursive partitioning of the data. At each terminal node, the probability of DCl was estimated as the proportion of patients belonging to that node that developed the event. The tree was constructed according to the following algorithm: in the first stage, the tree grows until all cases are correctly classified, and in the second stage, we used the tenfold cross-validation method of successive pruning [1]. Finally, the tree that minimized the error measurement (deviance) was chosen. For this predictor the corresponding ROC curve was obtained and the AUC was estimated by means of a $95 \% \mathrm{Cl}$.

Results. One hundred sixty one SAH patients were collected, 44(27,3\%) of them developed DCl. Demographic data and types of admission are shown in Table 1a and $1 \mathrm{~b}$.

\begin{tabular}{|c|c|c|c|c|}
\hline & \multirow[b]{2}{*}{$\begin{array}{l}\text { Overall } \\
N=161\end{array}$} & \multicolumn{2}{|c|}{$\begin{array}{l}\text { Delayed Cerebral } \\
\text { Ischemia }\end{array}$} & \multirow[b]{2}{*}{$p$} \\
\hline & & $\begin{array}{c}\text { No } \\
N=117\end{array}$ & $\begin{array}{c}\text { Yes } \\
N=44\end{array}$ & \\
\hline Aneurysm clipping & & & & 0.149 \\
\hline Lumbar drainage & $2(1.2)$ & $1(0.8)$ & $1(2.3)$ & 0.473 \\
\hline Embolization of the aneurysm & $\$ 8(60.9)$ & $69(59.0)$ & $29(65.9)$ & 0.422 \\
\hline Embolization and surgery treatment & $2(1.2)$ & $1(0.8)$ & $1(2.3)$ & 0.473 \\
\hline Conservative treatment & $21(13.0)$ & $18(15.4)$ & $3(6.8)$ & 0.150 \\
\hline Decompressive craniectomy & $7(4.4)$ & $4(3.5)$ & $3(6.8)$ & 0.395 \\
\hline Intraoperative aneurysm rupture & $8(5.0)$ & $5(4.3)$ & $3(6.8)$ & 0.685 \\
\hline Died after treatment & $1(0.6)$ & 0 & $1(2.3)$ & 0.273 \\
\hline External ventricular device & $77(48.1)$ & $49(42.2)$ & $28(63.6)$ & 0.016 \\
\hline Cerebrospinal fluid fistula & $7(4.3)$ & $5(4.3)$ & $2(4.5)$ & 1 \\
\hline Hidrocephalus & $67(41.9)$ & $46(39.7)$ & $21(47.7)$ & 0.355 \\
\hline MV $>7$ days & 55 (34.2) & $35(29.9)$ & $20(45.5)$ & 0.064 \\
\hline $\mathrm{ICH}$ & $38(23.6)$ & $31(26.5)$ & $7(15.9)$ & 0.159 \\
\hline Frontal ICH & $26(16.2)$ & $21(18.1)$ & $5(11.4)$ & 0.302 \\
\hline Perisylvian ICH & $21(13.1)$ & $16(13.8)$ & $5(11.4)$ & 0.684 \\
\hline Temporal ICH & $19(11.9)$ & $17(14.7)$ & $2(4.5)$ & 0.078 \\
\hline Subdural hematoma & $13(8.1)$ & $10(8.6)$ & $3(6.8)$ & 1 \\
\hline Vasoespasm_doppler & $40(27.2)$ & $19(17.8)$ & $21(52.5)$ & $<.001$ \\
\hline Vasoespasm_Arteriography & $39(27.1)$ & $12(11.7)$ & $27(65.8)$ & $<001$ \\
\hline $\mathrm{ACl}$ & $15(9.3)$ & $12(10.3)$ & $3(6.8)$ & 0.762 \\
\hline Renal Replacement Therapy & $2(1.3)$ & $1(0.9)$ & $1(23)$ & 0.478 \\
\hline Rebleeding 72 nours & $7(4,3)$ & $4(3.4)$ & $3(6.8)$ & 0.393 \\
\hline Ventriculitis & $14(8.8)$ & $9(7.8)$ & $5(11.4)$ & 0.535 \\
\hline Fisher scale & $3.0(3.0 .4 .0)$ & $3.0(3.0-4.0)$ & $3.0(3.0-4.0)$ & 0.716 \\
\hline Fisher modified scale & $4.0(3.0-4.0)$ & $3.0(3.0-4.0)$ & $4.0(3.0-4.0)$ & 0.010 \\
\hline Hunt and Hess scale & $2.0(1.0-4.0)$ & $1.0(1.0-3.0)$ & $2.5(1.0-4.0)$ & 0.016 \\
\hline WFNS scale & $2.0(1.0 \cdot 4.0)$ & $2.0(1.0-4.0)$ & $2.0(1.0-4.5)$ & 0.029 \\
\hline APACHE-Vasoespasm & $15(8-18)$ & $13(8-18)$ & $17(9 \cdot 19)$ & 0.207 \\
\hline SOFA-Vasoespasm & $3.0(1.0 \cdot 6.5)$ & $3.0(0.2-5.8)$ & $4.0(1.0-8.0)$ & 0.340 \\
\hline Delayed admission after bleeding & $12(2-24)$ & $10(2-24)$ & $12(8-24)$ & 0.153 \\
\hline GCS on site & $15(12 \cdot 15)$ & $15(12-15)$ & $14(12-15)$ & 0.295 \\
\hline GCS in emergency room & $14(9-15)$ & $14(10-15)$ & $12(7-15)$ & 0.071 \\
\hline GCS at ICU admission & $13(4-15)$ & $14(6-15)$ & $9(4-15)$ & 0.094 \\
\hline
\end{tabular}

Anterior communicating (Aco) artery aneurysms were the most frequently found and most of them were located in the left side.The most frequent DCI patient complications were stroke $34(77,3 \%)$ and hydrocephalus 21 (47,7\%). Thirty six (23.1\%) SAH patients died. Eleven (26.8\%) $\mathrm{DCl}$ patients died. There was not statistical significant difference in mortality in DCl vs SAH patients. Classification tree showed: MFS $\leq 3$ and $\mathrm{HHS} \leq 3$ have a high probability of not having DCI $(86,6 \%)$. MFS $>3$ and $\mathrm{HHS}>3$ have probabilities of $50 \%$ of DCl. MFS $>3$ without intracraneal hematoma (ICH) have probabilities of $43,3 \%$.of DCI The AUC was 0.695 (0.609-0.780) (Figures 1-3).
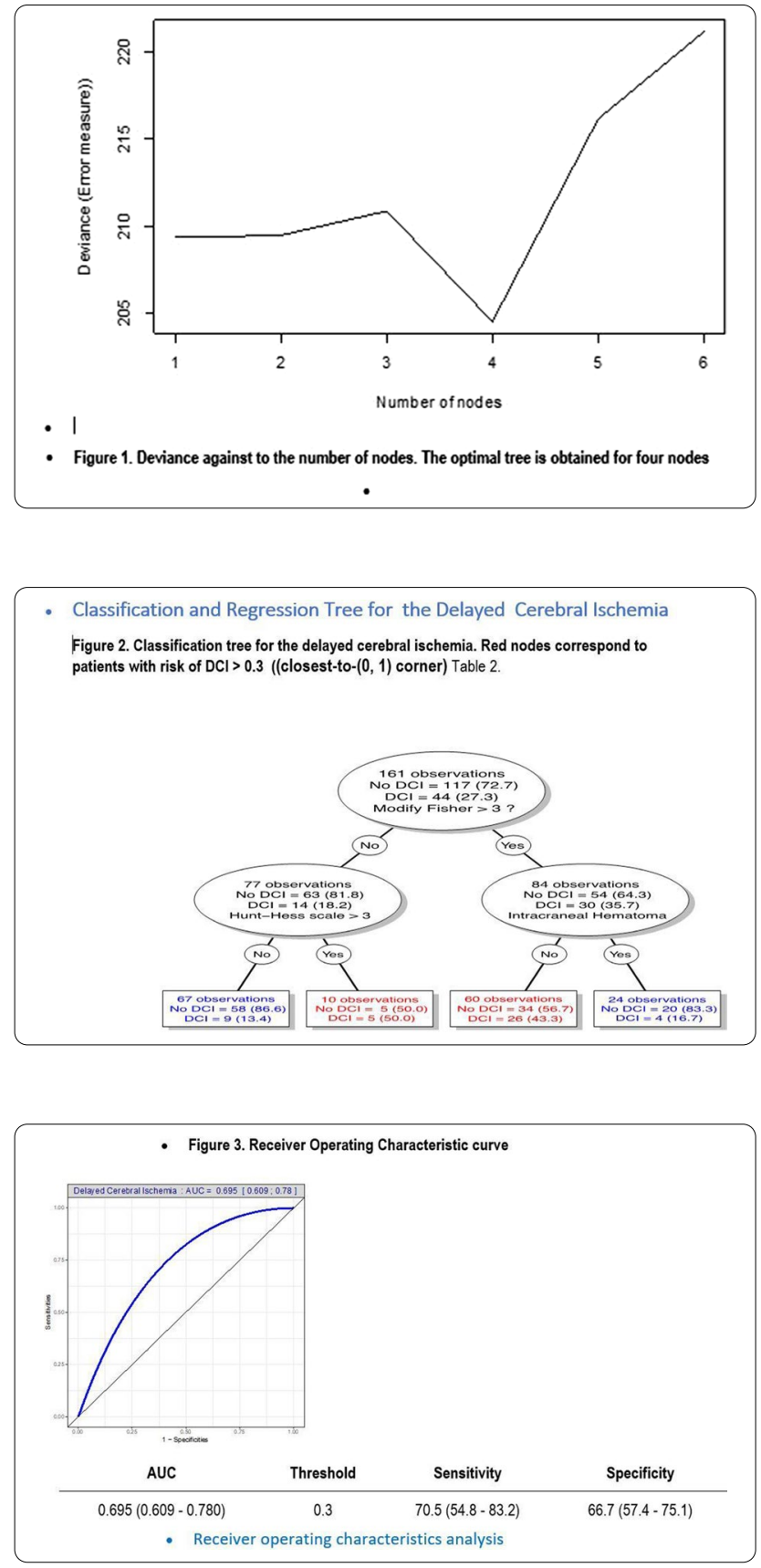
Conclusion. Our data show $27,3 \%$ of our studied patients had DCI. Classification tree showed: MFS $\leq 3$ and $\mathrm{HHS} \leq 3$ have a high probability of not having $\mathrm{DCl}(86,6 \%)$.MFS $>3$ and $\mathrm{HHS}>3$ have probabilities of $50 \%$ of DCl. MFS $>3$ without ICH have probabilities of $43,3 \%$ of DCI.The AUC was $0.695(0.609-0.780)$.Finally, mortality was not significantly greater in the studied versus total SAH patients.

\section{Reference(s)}

1. • [1] Breiman L, Freidman JH, Olshen RA, Stone CJ (1984) Classification and regression trees. Wadsworth, Belmon

\section{0}

Disability associated risk factors in patients with subarachnoid hemorrhage in a neurotraumatic ICU

C. Sánchez Ramírez'; CF. Lübbe Vázquez'; C. Rodriguez Mata'; JL. Vicente Arranz'; J. López Pérez'; A. Padrón Mujica' ; J. Garriga Segarra '; P. Saavedra-Santana ${ }^{2}$; S. Ruiz-Santana ${ }^{1}$

${ }^{1}$ Intensive Care Medicine, University Hospital of Gran Canaria Dr. Negrin, Las Palmas de Gran Canaria, Spain; ${ }^{2}$ Mathematics and Informatics Deparment, University of Las Palmas:, Las Palmas de Gran Canaria, Spain

Correspondence: $C$. Sánchez Ramírez

Intensive Care Medicine Experimental 2021, 9(1): 000310

Introduction. Aneurysmal subarachnoid hemorrhage $(\mathrm{SAH})$ is a worldwide health burden with serious outcomes such as permanent disability. It is important to learn the disability risk factors and identify those potentially preventable, to reduce them.

Objectives. To assess disability risk factors in patients admitted with subarachnoid hemorrhage (SAH), admitted in a neurotraumatic ICU.

Methods. Prospectively collected data of patients admitted from October, 2013 to December 2020 to a 10-bed Neurotraumatic ICU. We analyzed: main diagnosis at admission; demographic data, including sex and race; neurological data (clinical examination, pupils reactivity and size, and Glasgow Coma Score (GCS); location and aneurysm size; presence of intracranial hematoma $(\mathrm{ICH})$; presence and volume of intraventricular bleeding; days to develop vasoespam; development of delayed cerebral ischemia (DCl); Fisher scale, modified Fisher scale, Hunt and Hess scale, Word Federation of Neurosurgeons (WFNS) scale; presence of vasoespasm in doppler or arteriography; delayed of admission in ICU; treatment of the aneurysm; complications, including infections; Glasgow Outcome Scale (GOS) at ICU discharge and 6 months after ICU discharge and several other risk factors. Disability was defined as $\mathrm{GOS} \leq 3$. In order to identify those factors that maintain independent association with disability, a multivariate logistic regression analysis were performed. It was considered significant if $p \leq 0.05$. To determine the discriminatory capacity of the Score, an analysis of the receiver operating characteristics (ROC) was performed. The diagnostic capacity of the score was assessed using the area under the ROC curve, which was estimated using a 95\% confidence interval. For the chosen cut-off point, sensitivity, specificity, positive predictive value (PPV) and negative predictive value (NPV) were calculated.

Results. Among 187 SAH admitted patients, 71 (38.15\%) had GOS $\leq 3$. DCl was not significantly different between studied groups (Table 1).

\begin{tabular}{|c|c|c|c|c|}
\hline Table 1. Patients & $\begin{array}{c}\text { SAH } \\
N=187\end{array}$ & $\begin{array}{l}\mathrm{GOS}>3 \\
\mathrm{~N}=116\end{array}$ & $\begin{array}{l}\mathrm{GOS} \leq 3 \\
\mathrm{~N}=71\end{array}$ & $\mathbf{P}$ \\
\hline Age (years) & $56.5 \pm 14.4$ & $54.2 \pm 12.9$ & $60.3 \pm 15.8$ & 0.005 \\
\hline Sexfemale & $123(65.8)$ & $76(65.5)$ & $47(66.2)$ & 0.924 \\
\hline Apache-ll at admission & $14.8 \pm 7.7$ & $11.1 \pm 6.0$ & $20.8 \pm 6.2$ & $<0.001$ \\
\hline SOFA at admission & $3(0-6)$ & $1(0-3)$ & $6(3-9)$ & $<0.001$ \\
\hline Deaths & $41(22.8)$ & $3(27)$ & $38(55.1)$ & $<0.001$ \\
\hline ICU.Deans & $28(15.2)$ & 0 & $28(40.0)$ & $<0.001$ \\
\hline Hospital-Ceaths & $12(6.7)$ & $3(2 \pi)$ & $9(13.2)$ & 0.011 \\
\hline Arterial hypertension & $91(48.9)$ & $50(43.5)$ & $41(57.8)$ & 0.059 \\
\hline Number of platelets at ICU admission & $17(9.2)$ & $6(5.3)$ & $11(15.7)$ & 0.017 \\
\hline Urgent surgery at ICU admission & $23(12.4)$ & $9(7.9)$ & $14(19.7)$ & 0.018 \\
\hline Criented & $90(48.4)$ & $77(67.0\}$ & $13(18.3)$ & $<0.001$ \\
\hline Alert & $102(54.8)$ & $82(71.3)$ & $20(28.2)$ & $<0.001$ \\
\hline Contused & $30(16.1)$ & $14(122)$ & $16(22.5)$ & 0.062 \\
\hline Stuporous & $46(25.0)$ & $15(13.3)$ & $31(43.7)$ & $<0.001$ \\
\hline Bilateral mydrasis & $7(3.9)$ & $1(0.9)$ & $6(8.6)$ & 0.014 \\
\hline Anisochoric pupils & $18(9.9)$ & $5(4.5)$ & $13(18.6)$ & 0.002 \\
\hline Isochoric pupils & $158(87.3)$ & $105(94.6)$ & $53(75.7)$ & $<0.001$ \\
\hline One reacive pupil & $157(87.2)$ & $101(91.8)$ & $56(80.0)$ & 0.021 \\
\hline $\begin{array}{l}\text { Posterio inferior cerebellar arery } \\
\text { anurysm }\end{array}$ & $9(5.0)$ & $3(2.6)$ & $6(9.0)$ & 0.079 \\
\hline Aneurysm cipping & $35(19.0)$ & $15(13.2)$ & $20(28.6)$ & 0.010 \\
\hline Decompressive craniectiony & $10(5.4)$ & 0 & $10(14.1)$ & $<0.001$ \\
\hline External venticylar device & $85(47.2)$ & $31(27.9)$ & $54(78.3)$ & $<0.001$ \\
\hline Stroke & $55(30.1)$ & $22(19.3)$ & $33(47.8)$ & $<0.001$ \\
\hline Hydrocephaus & $74(40.7)$ & $25(22.1)$ & $49(71.0)$ & $<0.001$ \\
\hline$M N>7$ days & $61(33.3)$ & $13(11.4)$ & $48(69.6)$ & $<0.001$ \\
\hline $\mathrm{ICH}$ & $44(24.0)$ & $16(14.2)$ & $28(40.0)$ & $<0.001$ \\
\hline Fronta ICH & $28(15.5)$ & $6(5.4)$ & $22(31.4)$ & $<0.001$ \\
\hline Tempocal ICH & $24(13.3)$ & $9(8.1)$ & $15(21.4)$ & 0.010 \\
\hline Acste Cerebra Ischenia & $17(9.4)$ & $4(3.6)$ & $13(19.1)$ & $<0.001$ \\
\hline Delayer Cerebral Ischemia & $44(24.9)$ & $25(22.3)$ & $19(29.2)$ & 0.305 \\
\hline Fisher scale & $3(3.4)$ & $3(2.4)$ & $4(3.4)$ & $<0.001$ \\
\hline Fisher modifed scale & $4(3.4)$ & $3(2 \cdot 4)$ & $4(3-4)$ & $<0.001$ \\
\hline Hunt and Hess scale & $2(1.4)$ & $1(1-2)$ & $4(2 \cdot 5)$ & $<0.001$ \\
\hline WFNS scale & $2(1-4)$ & $1(1-2)$ & $4(2-5)$. & $<0.001$ \\
\hline APACHE-Vasoespasm & $145(8 \cdot 18)$ & $12(5 \cdot 17)$ & $18(17-23)$ & $<0.001$ \\
\hline SOFA-Vascespasm & $3(1 \cdot 6.2)$ & $2(0.3)$ & $7(5 \cdot 9)$ & $<0.001$ \\
\hline Delayed admission after bleeding & $12(2.2 \cdot 24.0)$ & $9(2-24)$ & $12(6-24)$ & 0.105 \\
\hline GCSon site & $15(12-15)$ & $15(14-15)$ & $13(7 \cdot 15)$ & $<0.001$ \\
\hline QCS in emergency rocm & $14(8.5-15)$ & $15(13-15)$ & $9(6-14)$ & $<0.001$ \\
\hline GCS at ICU admission & $13(4 \cdot 15)$ & $15(10.2 \cdot 15)$ & $6(3.9 .5)$ & $<0.001$ \\
\hline
\end{tabular}

Data are means $\pm \mathrm{CS}$, medians (RIQ) y frequencies (\%). MV: mecharical venolaton; $\mathrm{ICH}$ : intracrania heamtoma.

Twenty eight (14,97\%) died at ICU discharge and $12(6,41 \%)$ died in hospital. Decompresive craniectomy was performed in $10(5.4 \%) \mathrm{SAH}$ patients, all of them with $\mathrm{GOS} \leq 3$. The independent risk factors associated with mortality were: Apache II at ICU admission per unit OR $(95 \%$ Cl) 1.344 (1.192; 1.516); number of platelets $\times 103$ at ICU admission (NPLICU): OR: 27.010 (3.594; 202.990); hydrocephalus OR: 27.010 (3.594; 202.990) and mechanical ventilation (MV) > 7 days OR: 6.960 $(2.328 ; 20.808)$ (Table 2$)$.

Table 2. Multivariate logistic regression analysis $\mathrm{GOS} \leq 3$ at ICU discharge

\begin{tabular}{lccc}
\hline & P & BIC & Odd-Ratio $(95 \%$ IC $)$ \\
\hline Apache-Il at admission (per unit) & $<0.001$ & 154.4 & $1.344(1.192 ; 1.516)$ \\
Number of platelets at admission & $<0.001$ & 122.6 & $27.010(3.594 ; 202.990)$ \\
Hydrocephalus & 0.001 & 121.5 & $6.065(1.949 ; 18.872)$ \\
Mechanical ventilation $>7$ days & $<0.001$ & 124.1 & $6.960(2.328 ; 20.808)$ \\
\hline
\end{tabular}




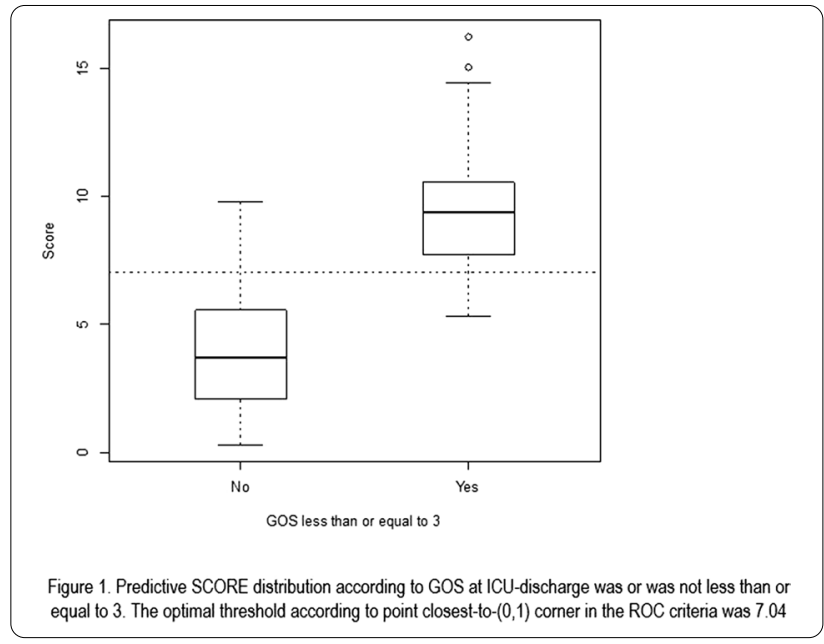

From the logistic model the next Score was deduced: 0,296 $\times$ APACHE $\mathrm{II}+3.296 \times \mathrm{NPLICU}+1.803 \times$ hydrocephalus $+1.940 \times \mathrm{MV}>$ de 7 days. The area under the curve (AUC) was 0.951 IC $(0.923 ; 0.979)$. Cut-off point:7.03;specificity: 0.87; sensitivity: 0.87; NPV: 0.91; PPV: 0.81 (Figures 1, 2)

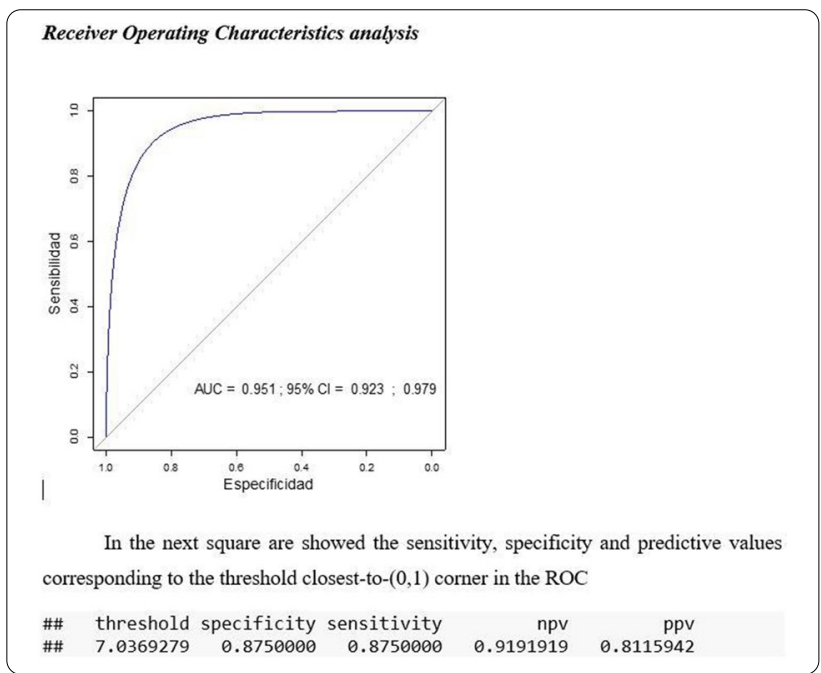

Conclusion. In SAH patients admitted to a neurotraumatic ICU, risk factors that were independently associated with disability were: Apache II at ICU admission per unit, number of platelets at ICU admission, hydrocephalus and MV $>7$ days. A predictive score was obtained.

000323

Risk factors associated with disability and mortality in patients in a neurotraumatic ICU with decompressive craniectomy, six months after discharge. A eigth year prospective study

C. Sánchez Ramírez'; CF. Lübbe Vázquez'; LDM. Díaz Suárez ; C. Rodriguez Mata ${ }^{1}$; JL. Vicente Arranz'; A. Padrón Mujica ${ }^{1}$; C. Vázquez Pineda ${ }^{1}$; P. Saavedra-Santana²; S. Ruiz-Santana

${ }^{1}$ Intensive Care Medicine, University Hospital of Gran Canaria Dr. Negrin, Las Palmas de Gran Canaria, Spain; ${ }^{2}$ Mathematics and Informatics Deparment, University of Las Palmas:, Las Palmas de Gran Canaria, Spain

Correspondence: C. Sánchez Ramírez

Intensive Care Medicine Experimental 2021, 9(1): 000323
Introduction. Second level therapeutic actions for controlling intracranial hypertension (ICH) proposed by the European Brain Itrauma Foundation include barbiturates, moderate hypothermia and decompressive craniectomy (DC), but outcomes are controversial. Our aim was to evaluate the factors associated with disability and mortality after ICU discharge in patients undergoing DC.

Objectives. To assess the factors associated with disability and mortality, after ICU discharge, in DC patients after 8 years.

Methods. Prospective study of patients admitted from January 1 , 2013 to January 2021 who required DC. DC was performed due ICH refractory to medical treatment. We analyzed: main admission diagnosis; demographic data; neurological data (clinical examination and Glasgow Coma Score: GCS); hypotension; type of craniectomy and DC complications; Rankin scale, and Glasgow outcome scale (GOS) at 30 , 60 days after ICU admission, at ICU discharge and 6 months after ICU discharge; preoperative serum lactate levels; hypo and hyperglycemia; application of mannitol or hypertonic saline solution before and after DC; leukocytes and platelets previous and after DC and other factors related to prognosis. Univariate analysis of disability (Rankin $>3$ ) and mortality in ICU and 6 months after ICU discharge were performed. Statistical significance was set at $p \leq 0.05$.

Results. Forty one DC patients were collected. Demographic data and types of admission are shown in Table 1.

\begin{tabular}{|c|c|c|c|c|}
\hline & \multirow[b]{2}{*}{$\begin{array}{l}\text { Global } \\
\mathrm{N}=41\end{array}$} & \multicolumn{2}{|c|}{ Rankin at ICU discharge } & \multirow[b]{2}{*}{$\mathrm{P}$} \\
\hline & & $\begin{array}{l}<3 \\
N=9\end{array}$ & $\begin{array}{r}23 \\
N=32 \\
\end{array}$ & \\
\hline Age (years) & $46.1(35.6-55.5)$ & $41.8(38.8-56.4)$ & $46.6(33.9-55.2)$ & 0.83 \\
\hline Sex female & $14(34.1)$ & $4(44.4)$ & $10(31.2)$ & 0.692 \\
\hline APACHE-II & $22.5(18.8-26)$ & $25.0(21.5-26.5)$ & $22(18-25)$ & 0.293 \\
\hline GCS at admission & $9.5(5.2-14)$ & $12(5-13)$ & $9(6-14)$ & 0.917 \\
\hline GCS at ICU admission & $3(3-7.5)$ & $3(3-10)$ & $3(3-7)$ & 0.693 \\
\hline ICU-Deaths & $7(17.5)$ & - & - & - \\
\hline Hospital-Deaths & $2(5.4)$ & - & - & . \\
\hline Discharge of the Hospital-Deaths & $3(8.6)$ & - & - & - \\
\hline Deaths & $12(33.3)$ & - & - & . \\
\hline Diabetes mellitus & $5(12.2)$ & 1 (11.1) & $4(12.5)$ & 1 \\
\hline Arterial hypertension & $7(17.1)$ & $3(33.3)$ & $4(12.5)$ & 0.165 \\
\hline Dislypemia & $8(19.5)$ & $2(22.2)$ & $6(18.8)$ & 1 \\
\hline Tumor & $4(10.0)$ & $1(12.5)$ & $3(9.4)$ & 1 \\
\hline SAH & $21(51.2)$ & $5(55.6)$ & $16(50.0)$ & 1 \\
\hline Stroke Malignant middle cerebral artery & $10(24.4)$ & $1(11.1)$ & $9(28.1)$ & 0.41 \\
\hline Acute subdural hematoma & $15(37.5)$ & $3(37.5)$ & $12(37.5)$ & 1 \\
\hline Obliteration of the cistems of the base & $16(40.0)$ & $1(12.5)$ & $15(46.9)$ & 0.11 \\
\hline Focal contusion with edema and expansivity & $16(40.0)$ & $5(62.5)$ & $11(34.4)$ & 0.229 \\
\hline Evacuated Injury & $8(20.0)$ & $2(25.0)$ & $6(18.8)$ & $0.65 c$ \\
\hline BTI & $15(36.6)$ & $5(55.6)$ & $10(31.2)$ & 0.24 \\
\hline OTl previous Hospital & $13(31.7)$ & $4(44.4)$ & $9(28.1)$ & 0.429 \\
\hline OTIEmergency & $12(29.3)$ & 1 (11.1) & $11(34.4)$ & $0.24 \mathrm{C}$ \\
\hline OTl on Surgery & $10(24.4)$ & $3(33.3)$ & $7(21.9)$ & 0.739 \\
\hline Transfusion prior to DC & $9(23.1)$ & $1(12.5)$ & $8(25.8)$ & 0.780 \\
\hline Pre-craniectomy seizures & $4(10.3)$ & & $4(12.9)$ & 0.563 \\
\hline Bilateral arreactive mydriasis prior to $D C$ & $3(7.7)$ & & $3(9.7)$ & 1 \\
\hline Both reactive pupils prior to DC & $27(69.2)$ & $6(85.7)$ & $21(65.6)$ & 0.403 \\
\hline One reactive pupil prior to DC & $4(10.5)$ & & $4(12.5)$ & 1 \\
\hline None-reactive pupils prior to DC & $5(12.8)$ & $1(14.3)$ & $4(12.5)$ & 1 \\
\hline Midline shitt on CT at admission & $5(0-8.8)$ & $1.5(0-8.5)$ & $5(0-8.8)$ & 0.596 \\
\hline Number of Platelets prior to $D C$ & $228(176-272)$ & $258(246-293)$ & $217(166-260)$ & 0.192 \\
\hline
\end{tabular}

Most DC were subarachnoid hemorrhages (SAH) 21(51.2\%). and brain trauma injury $15(36.6 \%)$.The most frequent complications were reoperation due to complications $16(39,02 \%$ of them) and hydrocephalus 15 (35,5\%).Six patients died at ICU discharge (14,6\%), 4 (66,6\%) of them were SAH. Rankin score at ICU discharge was 4,5 and GOS was 3. Rankin score at 6 months after ICU discharge was 5 and GOS was 3 . We did not find any significant risk factors associated with disability six months after ICU discharge (Table 1) Mortality 6 months after discharge was significantly associated with one arreactive pupil prior to DC and bilateral pupillary reactivity prior to DC, and the Midline shift on CT at admission (Table 2). 
Table 2. Univariate analysis of DC mortality 6 months after ICU discharge

\begin{tabular}{|c|c|c|c|}
\hline & $\begin{array}{l}\text { ALIVE } \\
\mathrm{N}=29\end{array}$ & $\begin{array}{l}\text { DEATH } \\
\mathrm{N}=12\end{array}$ & P-valor \\
\hline Age (years) & $46.6(31.5-56.4)$ & $45.5(37.7-54.3)$ & 0.892 \\
\hline Sex female & $8(27.6)$ & $6(50.0)$ & 0.278 \\
\hline APACHE-II & $22(16-25.2)$ & $23.5(22-26.5)$ & 0.156 \\
\hline Diabetes Mellitus & $3(10.3)$ & $2(16.7)$ & 0.620 \\
\hline Arterial hypertension & $6(20.7)$ & $1(8.3)$ & 0.651 \\
\hline Dislypemia & $6(20.7)$ & $2(16.7)$ & 1 \\
\hline Tumor & $3(10.7)$ & $1(8.3)$ & 1 \\
\hline SAH & $14(48.3)$ & $7(58.3)$ & 0.558 \\
\hline Stroke Malignant middle cerebral artery & $6(20.7)$ & $4(33.3)$ & 0.441 \\
\hline Acute subdural hematoma & $11(39.3)$ & $4(33.3)$ & 1 \\
\hline Obliteration of the cisterns of the base & $11(39.3)$ & $5(41.7)$ & 1 \\
\hline Hematoma no evacuado & $7(25.0)$ & $1(8.3)$ & 0.396 \\
\hline Focal contusion with edema and expansivity & $14(50.0)$ & $2(16.7)$ & 0.079 \\
\hline Evacuated Injury & $5(17.9)$ & $3(25.0)$ & 0.677 \\
\hline BTI & $11(37.9)$ & $4(33.3)$ & 1 \\
\hline Ninguna pupila reactiva previo CD & $3(11.1)$ & $2(16.7)$ & 0.634 \\
\hline OTI previous Hospital & $10(34.5)$ & $3(25.0)$ & 0.719 \\
\hline OTIEmergency & $7(24.1)$ & $5(41.7)$ & 0.285 \\
\hline OTI on Surgery & $8(27.6)$ & $2(16.7)$ & 0.783 \\
\hline Transfusion prior to DC & $6(21.4)$ & $3(27.3)$ & 0.403 \\
\hline Pre-craniectomy seizures & $1(3.6)$ & $3(27.3)$ & 0.060 \\
\hline Bilateral arreactive mydriasis prior to $\mathrm{CD}$ & $2(7.1)$ & $1(9.1)$ & 1 \\
\hline Both reactive pupis prior to DC & $23(85.2)$ & $4(33.3)$ & 0.002 \\
\hline One reactive pupil prior to DC & $0(0.0)$ & $4(33.3)$ & 0.007 \\
\hline None-reactive pupils prior to DC & $3(11.1)$ & $2(16.7)$ & 0.634 \\
\hline ICU-Deaths & - & $7(58.3)$ & - \\
\hline Hospital-Deaths & - & $2(16.7)$ & - \\
\hline Discharge of the Hospital-Deaths & - & $3(25.0)$ & . \\
\hline Midline shitt on CT at admission & $3(0-6.5)$ & $8.5(2.5-10)$ & 0.049 \\
\hline GCS on admission & $10(5-14.5)$ & $7(6-13)$ & 0.673 \\
\hline GCS at ICU admission & $3(3-9)$ & $3(3-5)$ & 0.482 \\
\hline Number of Platelets prior tod DC & $224(179-263)$ & $252(188-326)$ & 0.391 \\
\hline
\end{tabular}

Data are means and medians (IQR) y frequences (\%).SAH: subarachnoid hemorrhage,BTl: brain trauma injured; OTI. orotracheal Intubation; DC: decompressive craniectomy

Conclusion. DC patients showed low ICU mortality (14,6\%) Rankin and GOS reflected moderate/severe disability of these patients, at ICU and 6 months after ICU discharge. Mortality, 6 months after discharge, was significantly associated with one arreactive pupil prior to $\mathrm{DC}$, bilateral pupillary reactivity prior to $\mathrm{DC}$, and midline shift on $\mathrm{CT}$ at admission.

\section{4}

Effect of multimodal neuromonitoring on outcome of acute brain injury patients: a cohort study

S. Papoti ${ }^{1}$;. Karagewrgiou' ${ }^{1}$; E. Lazoudi ${ }^{1}$; N. Kapravelos ${ }^{1}$; C. Iasonidou ${ }^{1}$

${ }^{1}$ B icu, General Hospital of Thessaloniki "George Papanikolaou", Thessaloniki, Greece

Correspondence: S. Papoti

Intensive Care Medicine Experimental 2021, 9(1): 000324

Introduction. Patients with primary Acute Brain Injury $(\mathrm{ABI})$, including Intracranial hemorrhage (ICH), Traumatic Brain Injury (TBI) and Subarachnoid Hemorrhage (SAH) admitted in ICU need a close and specialized brain monitoring. There is evidence that a single neuromonitor cannot explain all pathophysiological mechanisms and that an individualized management approach should be based on multiple physiologic variables.

Objectives. The aim of our study is to evaluate whether variables collected from specialized brain catheters have an effect on survival and on preventing Secondary Brain Injuries (SBI) of $\mathrm{ABI}$ patients.

Methods. 95 patients with $\mathrm{ABI}$ (median age 54 years, 64.2\% males) under intracranial pressure (ICP) and partial brain tissue oxygen pressure $\left(\mathrm{PbtO}_{2}\right)$ monitoring were included in a four-year retrospective cohort study. 28-day survival and SBI development (brain edema, ischemia) were ascertained using data records from patients' files. Univariate and multivariate regression analysis were used to identify variables correlation with outcome.

Results. Out of 95 patients, 14 (14.7\%) had ICH, 42 (42.2\%) TBI and 39 (41.1\%) SAH. Their median GCS admission score was 8 while during discharge the GCS was 10 and had median length of stay in ICU 20 days (range 5-92). 71 patients (74.7\%) survived threw day 28 and $44(46.3 \%)$ of all did not develop SBI. A significant negative association was found between time of ICP $>20 \mathrm{mmHg} \mathrm{PbtO}_{2}<10 \mathrm{mmHg}$ and $\mathrm{SBI}$ development with 28-day survival. Furthermore, a positive correlation was observed between ICP $>20 \mathrm{mmHg}, \mathrm{PbtO}_{2}<15 \mathrm{mmHg}$ $\mathrm{PbtO}_{2}<10 \mathrm{mmHg}$ and $\mathrm{ABI}$ type with SBI. In multivariate analysis, the association of time of ICP $>20 \mathrm{mmHg}$ with day-28 survival and SBI development was confirmed, while time $\mathrm{PbtO}_{2}<15 \mathrm{mmHg}$ seemed to maintain its significance only for SBI presentation. What was interesting, is the negative association found between the delay of monitoring onset and the development of SBI.

Conclusion. Our data suggest that $\mathrm{PbtO}_{2}$ is an important physiological target of monitoring $\mathrm{ABI}$ patients affecting the survival and as a complement of ICP to help guide the management and prevent SBIs.

\section{Reference(s)}

1. 1. Lazaridis C, Robertson C. The role of multimodal invasive monitoring in acute traumatic brain injury. Neurosurg. Clinics of North America. 2016.

2. 1. Goyal K, Khandelwal A, Kedia S. Multimodal Neuromonitoring: Current Scenario in Neurocritical Care. J Neuroanaesth Crit Care. 2019;06(02):062-71

3. 1. Tasneem N, Samaniego EA, Pieper C, Leira EC, Adams HP, Hasan D, et al. Brain Multimodality Monitoring: A New Tool in Neurocritical Care of Comatose Patients. Crit Care Res Pract. 2017;2017:1-8.

\section{9}

Diagnostic criteria for delayed cerebral ischemia and outcome after non-traumatic subarachnoid hemorrhage

E. Bogossian 1; D. Daniela ; M. Marco ${ }^{1}$; N. Ndieugnou Djangang ${ }^{1}$; L. Peluso1; F. Annoni ${ }^{1}$; J. Creteur ${ }^{1}$; FS. Taccone

${ }^{1}$ Intensive Care, Hospital Erasme, Bruxelles, Belgium

Correspondence: $\mathrm{E}$. Bogossian

Intensive Care Medicine Experimental 2021, 9(1): 000349

Introduction. Delayed cerebral ischemia occurs in $20 \%-30 \%$ of patients with aneurysmal subarachnoid hemorrhage $(\mathrm{aSAH})$ and is one the most important preventable cause of poor neurological outcome [1]. The advances in imaging techniques and in multimodal monitoring can help diagnose potentially reversible $\mathrm{DCl}$ and improve outcome of aSAH.

Methods. This is a retrospective cohort study of patients admitted to the Intensive Care Unit (ICU) of Erasme Hospital (Brussels, Belgium) with aSAH from 2010-2020.We excluded patients who remained than $24 \mathrm{~h}$ in the ICU. We reported the prevalence and factors associated with the development of $\mathrm{DCl}$ using the following definitions: type 1: permanent neurological deficit and/or cerebral infarction on CT scan not explained by other causes and not related to procedures; type 2: new neurological deficit and cerebral vasospasm (diagnosed on transcranial doppler, angio-CT scan, angio-MRI and/or angiography); type 3: new neurological deficit or poor clinical exam associated with radiological evidence of vasospasm and neuromonitoring (brain oxygen pressure below $20 \mathrm{mmHg}$ or lateralization on EEG)/perfusion CT-scan compatible with ischemia. Early brain injury was defined as a Glasgow Coma Scale $<13$ on admission. Unfavorable neurological outcome at 3 months was defined as Glasgow Outcome Scale (GOS) of 1-3.

Results. Of 528 eligible patients, 208 (39\%) were diagnosed with DCl: $146(70 \%)$ with type 1 criteria, including 94 (44\%) patients with initial type $2 / 3$ DCI[FST1], and 62 (30\%) only with type $2 / 3$ criteria. Early brain injury was independently associated [FST2] with the development of $\mathrm{DCl}$. The DCl type 1, but not type 2 or 3, was associated with increased in-hospital mortality (HR 1.59 [95\% Cls 1.02-2.48] and UO (OR 9.60 [95\% Cls 5.31-17.37].

Conclusion. $\mathrm{DCl}$ remains an important determinant of poor outcome after $\mathrm{SAH}$. Patients diagnosed with $\mathrm{DCl}$ using imaging tests and multimodal monitoring have better prognosis that those with established neurological deficit or infarction on the CT scan. 
Reference(s)

1. [1]Francoeur, C.L.Mayer, S.A. Management of delayed cerebral ischemia after subarachnoid hemorrhage. Crit Care 2016;20(1):277.

\section{4}

Are current ventilatory settings still related to development of Acute Respiratory Distress Syndrome in patients with severe acute brain injury?

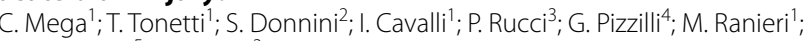
P. Malacarne ${ }^{5}$ L. Mascia $^{3}$

${ }^{1}$ Department of Medical and Surgical Sciences, Alma Mater Studiorum-Università di Bologna, Bologna, Italy; ${ }^{2}$ U.o anestesia e rianimazione, United Hospitals of Livorno Area Critica, Livorno, Italy; ${ }^{3}$ Department of Biomedical and Neuromotor Sciences, Alma Mater Studiorum-Università di Bologna, Bologna, Italy; ${ }^{4}$ U.o anestesia e rianimazione, Azienda Ospedaliero-Universitaria di Bologna_Policlinico S. Orsola-Malpighi, Bologna, Italy; ${ }^{5}$ U.o- anestesia e rianimazione-p.S., Azienda Ospedaliero Universitaria pisana, Pisa, Italy

Correspondence: C. Mega

Intensive Care Medicine Experimental 2021, 9(1): 000354

Introduction. Acute Respiratory Distress Syndrome (ARDS) occurs in $10-20 \%$ of patients with severe acute brain injury (ABI) and worsen clinical outcomes. Previous clinical studies showed that mechanical ventilation with large tidal volumes may contribute to the development of ARDS in patients that did not have ARDS at ICU admission. However, ventilatory management of $A B I$ still gives priority toward ventilatory settings that result in cerebral protection by tight $\mathrm{pCO} 2$ control and less attention is paid to use ventilatory setting aiming at minimize ventilator-induced lung injury (VILI).

Objectives. We performed a prospective observational study in patients with $A B I$ to verify if currently implemented ventilatory settings still represent a potential risk factor for the development of ARDS.

Methods. Patients with ABI (Glasgow Coma Score $(\mathrm{GCS}) \leq 8$ ) consecutively admitted to the ICU of Cisanello University Hospital, Pisa Italy were studied for the first week from admission. Patients' follow up included ICU and hospital mortality and LOS and at 28 and neurological outcome at 6 months. ARDS was defined according to the Berlin definition. Demographic and clinical data at admission and during ICU stay were collected. Logistic regression analysis was performed to assess independent predictors for ARDS development.

Results. During the study period (November 2015-March 2020), 203 consecutive patients (age $55 \pm 19$ years, $F: M=51: 152$ ) were screened; 26 patients met ARDS criteria already at day 0 and were separately analysed. Causes of ABI were: trauma in 148 (73\%), cerebrovascular in $29(14 \%)$, others in 26 pts (13\%). At ICU admission GCS was $7 \pm 1$, SAPS2 $44 \pm 10$ and SOFA $10 \pm 2$. Thirty-eight patients (21\%) developed ARDS on day 7 (range, 3-14). Chest CT-scan or x-ray were evaluated by radiologist and intensivist blinded to clinical data with inter-rater agreement of $0.88(95 \% \mathrm{Cl} 0.79-0.96)$. In the first $72 \mathrm{~h}$ of the study period, mean values of tidal volume (Vt/PBW), plateau pressure, respiratory rate (RR), PEEP, and $\mathrm{PaCO} 2$ were: $7.8 \mathrm{ml} / \mathrm{kg}$ (range 6.6-10.6), 18 $\mathrm{cmH} 2 \mathrm{O}$ (range 14-32), 16 breath/min (range 12-25), $6 \mathrm{cmH} 2 \mathrm{O}$ (range $5-12)$ and $36 \mathrm{mmHg}(28-40)$ respectively. A logistic regression model for ARDS development was built with demographics, severity scores, cause of $A B I$ and ventilatory parameters (either worst [first model] or mean values [second model] in the first $72 \mathrm{~h}$ ) as covariates. In the first model, maximum Vt/PBW (Figure 1) and respiratory rate were significantly associated with ARDS (OR 45.3 [95\% Cl 4.4-467.9] and $1.6[95 \%$ $\mathrm{Cl} 1.2-2.2]$, respectively) with sensitivity and specificity (0.92 and 0.99 , respectively; AUC 0.98$)$. In the second model, mean respiratory rate and plateau pressure were significantly associated with ARDS (OR 2.9 [95\% Cl 1.5-5.9] and 2.1 [95\% Cl 1.5-2.8], respectively) with sensitivity and specificity (0.97 and 0.99, respectively; AUC 0.99).

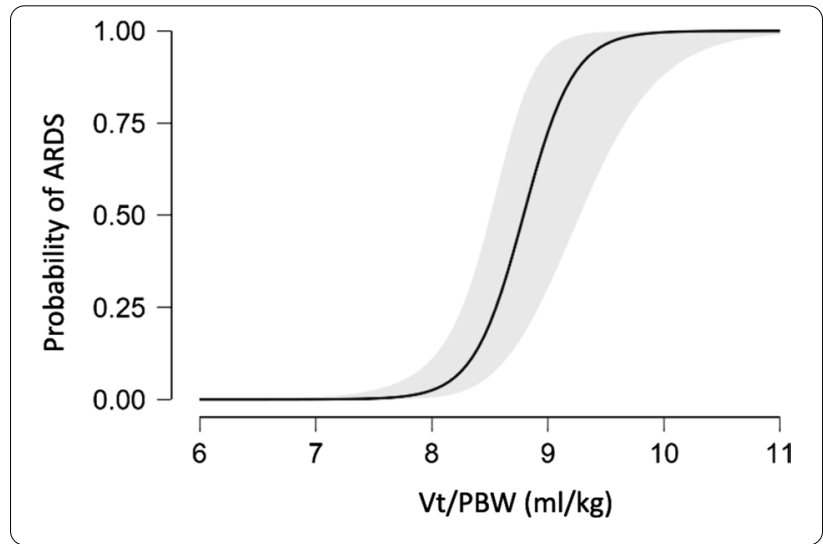

Figure 1. Probability of ARDS as a function of maximum Vt/PBW. Conclusion. Tidal volume, plateau pressure and respiratory rate were the strongest predictors of ARDS in ABI patients. These data confirm that in the current clinical management of $A B I$ the ventilatory strategy is strongly oriented towards a tight $\mathrm{PaCO}_{2}$ control overlooking potentially injurious ventilatory settings. Final follow up is under evaluation.

\section{Reference(s)}

1. -Mascia L, Zavala E, Bosma K, Pasero D, Decaroli D, Andrews P, Isnardi D, Davi A, Arguis MJ, Berardino M, Ducati A; Brain IT group. High tidal volume is associated with the development of acute lung injury after severe brain injury: an international observational study. Crit Care Med. 2007 Aug;35(8):1815-20. https://doi.org/10.1097/01.CCM.0000275269. 77467.DF. PMID: 17568331. -Robba C, Poole D, McNett M, Asehnoune K, Bösel J, Bruder N, Chieregato A, Cinotti R, Duranteau J, Einav S, Ercole A, Ferguson N, Guerin C, Siempos II, Kurtz P, Juffermans NP, Mancebo J, Mascia L, McCredie V, Nin N, Oddo M, Pelosi P, Rabinstein AA, Neto AS, Seder DB, Skrifvars MB, Suarez JI, Taccone FS, van der Jagt M, Citerio G, Stevens RD. Mechanical ventilation in patients with acute brain injury: recommendations of the European Society of Intensive Care Medicine consensus. Intensive Care Med. 2020 Dec;46(12):2397-2410. https://doi org/10.1007/s00134-020-06283-0. Epub 2020 Nov 11. PMID: 33175276 ; PMCID: PMC7655906

2. no funding provided.

\section{7}

Intra-arterial Nimodipine Infusion for Refractory Basilar Artery Vasospasm after Aneurysmatic Subarachnoid Hemorrhage: Case Report

V. Giraldo, ; ME. Eraso, ${ }^{2}$; J. Carrizosa ${ }^{3}$

${ }^{1}$ Critical Care Medicine, Sede Quinta de Mutis, Universidad del Rosario, Bogotá, Colombia; ${ }^{2}$ School of medicine, Universidad de los Andes, Bogotá, Colombia; ${ }^{3}$ Critical Care Medicine, University Hospital Fundación Santa Fe de Bogotá, Bogotá, Colombia

Correspondence: J. Carrizosa

Intensive Care Medicine Experimental 2021, 9(1): 000387

Introduction. Aneurysmatic subarachnoid haemorrhage (SAH) has an incidence of 2 to 16 cases per 100,000 people (1). Delayed cerebral ischemia (DCl) affects up to $30 \%$ of SAH patients, and most of them develop motor or cognitive deficits (2). Cerebral vasospasm (CVS) causes regional hypoperfusion and eventually $\mathrm{DCl}$, with the highest risk of vasospasm 7 to 10 days after bleeding (3). Vasospasm management includes hemodynamic augmentation with intravenous fluids and inotropic and vasopressor support (4). Oral Nimodipine therapy has proved to reduce vasospasm related to DCI incidence by $34 \%$ (5). Recent literature shows intra-arterial nimodipine infusion (IANI) as a safe and effective therapy for DCl after SAH (6). 
Objectives. This abstract aims to present a successfully treated patient with refractory basilar artery CVS using IANI.

Methods. Case description: A 52-year-old woman was admitted to ER with a three day-occipital headache, nausea, photophobia, phonophobia, and aphasia. A right-sided temporoparietal SAH due to a ruptured internal carotid artery aneurysm was noticed on her angiography CT. Augmented mean Flow velocities in transcranial doppler control with right-sided Lindegaard value of 4 after aneurysm clipping suggested cerebral vasospasm. The patient turned somnolent with a III CN palsy, and a bedside ultrasound identified low cardiac output. Hemodynamic augmentation with dobutamine and norepinephrine plus standard management at the neurocritical care unit. Symptoms persisted after the 7th post-clipping day with a severe right-sided carotid spasm shown in her pan-angiography. Intra-arterial nimodipine was administrated, with partial improvement and a brief consciousness recovery. Twelve hours later, she returned to an altered consciousness state. A new pan-angiography showed severe basilar artery vasospasm, treated again with intra-arterial nimodipine. Due to vasospasm persistence, an intravertebral microcatheter was placed, and a continuous $0.5 \mathrm{mg} /$ hour nimodipine infusion administrated.

Results. The patient recovered progressively with stable mental status, Lindegaard value of 1.2 bilaterally and Sviri ratio of 1.5 without any deficit at the clinical examination or brain ischemia on control CT scan after $72 \mathrm{~h}$ of continuous infusion of IANI.

Conclusion. Standard management post-SAH, individualized haemodynamic targets, and intravertebral IANI could be an option for managing refractory basilar artery vasospasm and preventing $\mathrm{DCl}$ as the basilar artery is difficult to access for continuous monitoring different from transcranial doppler.

Reference(s)

1. 1. Rouanet, C., \& Silva, G. S. (2019). Aneurysmal subarachnoid hemorrhage: current concepts and updates. Arquivos de Neuro-Psiquiatria, 77(11), 806-814

2. 2. Meirow, Y., Kanterman, J., \& Baniyash, M. (2015). Paving the road to tumor development and spreading: Myeloid-derived suppressor cells are ruling the fate. Frontiers in Immunology, 6(OCT)

3. 3. Budohoski, K. P., Guilfoyle, M., Helmy, A., Huuskonen, T., Czosnyka, M., Kirollos, R., Menon, D. K., Pickard, J. D., \& Kirkpatrick, P. J. (2014). The pathophysiology and treatment of delayed cerebral ischaemia following subarachnoid haemorrhage. Journal of Neurology, Neurosurgery and Psychiatry, 85(12), 1343-1353. https://doi.org/10.1136/jnnp-2014-307711

4. 4. Treggiari, M. M., \& Deem, S. (2009). Which H Is The Most Important In Triple-H Therapy For Cerebral Vasospasm? Current Opinion in Critical Care, 15(2), 83-86. https://doi.org/10.1097/MCC.0b013e32832922d1

5. 5. Goerge S. Allen, M. et all. (1983). Cerebral Arterial Spasm A Controlled Trial Of Nimodipine In Patients With Subarachnoid Hemorrhage. New England of Jorunal of Medicine, 308, 619-624. https://doi.org/10.1056/ NEJM198603273141302

6. 6. Helbok R, Zangerle A, Chemelli A, et al. Continuous intra-arterial nimodipine infusion in refractory symptomatic vasospasm after subarachnoid hemorrhage. Springerplus. 2016;5(1):1807. Published 2016 Oct 18. https://doi.org/10.1186/s40064-016-3495-4

\section{7}

\section{Changes in the diameter of an optical nerve sheath} with increasing intracranial pressure

N. Dovbish'; A. Gritsan

${ }^{1}$ Anaesthesiology and Intensive Care, Krasnoyarsk State Medical University, Krasnoyrsk Regional Clinical Hospital, Krasnoyarsk, Russia

Correspondence: N. Dovbish

Intensive Care Medicine Experimental 2021, 9(1): 000407

Introduction. High intracranial pressure (ICP) is a leading pathogenic factor in a lot of critical conditions associated with acute brain injury. Intracranial pressure (ICP) monitoring is limited by the lack of modern minimally invasive technologies. Ultrasound examination of the diameter of the optic nerve sheath is a relatively new application of ultrasound in emergency medical care.
Objectives. To assess the information content of ultrasound determination of the optic nerve sheath diameter in patients with intracerebral pathology (ischemic stroke (IS), hemorrhagic stroke in the form of intracerebral hemorrhage $(\mathrm{ICH})$, subarachnoid hemorrhage $(\mathrm{SAH})$ ) to determine the presence of increased intracranial pressure.

Methods. The study included 17 patients with acute cerebrovascular accidents who were treated at the Neuro Intensive Care Unit Krasnoyarsk regional hospital. The patients were: 10-men, 7-women; 12-with Al, 5-with ICH and 2-with SAH. All patients, upon admission to the clinic, underwent computed tomography scan of the brain. At the first stage (admission to the NICU), the level of consciousness was assessed using the Glasgow Coma Scale (GCS) and the ultrasound measurement of the optic nerve sheath diameter with a linear transducer using the SonoScape S8 ultrasound system. The optic nerve sheath diameter was measured at a depth of $3 \mathrm{~mm}$ posterior to the globe. At the II stage of the study (3-5 days from the moment of admission to the NICU), the level of consciousness was assessed according to the GCS; repeated ultrasound measurement of the optic nerve sheath diameter and CT scan of the brain was performed. Data are presented as median and 25 and 75 percentiles.

Results. At I stage the level of consciousness in patients by GCS was estimated at $14[13 ; 14]$ points, at II stage $-8[7 ; 11]$ points, $\mathrm{p} \leq 0.05$. Optical nerve sheath diameter at I stage $-0.62[0.6 ; 0.68] \mathrm{cm}$, at II stage $-0.67[0.65 ; 0.72] \mathrm{cm}, \mathrm{p} \leq 0.05$. At II stage according to CT scan of the brain all patients showed signs of increased ICP - were noted smoothing of the gyruses, sulceses were not observed, was a dislocation of the median structures and compression of the basal cisterns.

Conclusion. We noted a statistically significant increase in the diameter of the optic nerve sheath with an increase in ICP, which correlates both with a decrease in the level of consciousness in patients, assessed by GCS, and with computed tomographic signs of an increase in ICP.

\section{Reference(s)}

1. Wang LJ, Chen LM, Chen Y, et al. Ultrasonography assessments of optic nerve sheath diameter as a noninvasive and dynamic method of detecting changes in intracranial pressure. JAMA Ophthalmol 2018;136:250-6. https://doi.org/10.1001/jamaophthalmol.2017.6560

2. Dubourg J, Javouhey E, Geeraerts T, et al. Ultrasonography of optic nerve sheath diameter for detection of raised intracranial pressure: a systematic review and meta-analysis. Intensive Care Med 2011;37:1059-1068. https://doi.org/10.1007/s00134-011-2224-2

\section{8}

Aneurysmal subarachnoid hemorrhage: do serum sodium levels have impact on the outcome?

N. Jorge ; J. Fernandes ${ }^{1}$; E. Monteiro ${ }^{1}$; JA. Paiva

${ }^{1}$ Intensive Care Medicine Department, São João Universitary Hospital

Center, Porto, Portugal

Correspondence: N. Jorge

Intensive Care Medicine Experimental 2021, 9(1): 000448

Introduction. Dysnatremia is frequently observed after aneurysmal subarachnoid hemorrhage (aSAH) and has been identified as a possible contributor to adverse clinical outcome. However the effect of fluctuations in serum sodium levels is not well established in literature. Objectives. Assess the association of serum sodium levels with functional outcome after aSAH.

Methods. Retrospective analysis of aSAH patients admitted from 1st January 2018 to 31st December 2019 at a Neurocritical Care Unit in a University Hospital in Portugal. Serum sodium levels were measured at admission, 7, 10 and 14 days after aSAH. The functional outcome was defined according to the Glasgow Outcome Scale (GOS), considering GOS of 1, 2 or 3 as an unfavorable functional outcome. GOS was accessed on the 28th day and on the 3rd and 6th months of follow-up. Results. Sixty-five patients were analyzed, $66 \%$ females, with median age of 55 (47.5-66.5) years and previous Modified Rankin Score (mRS) of 0 or 1 . At admission, $25(39 \%)$ patients presented Hunt and Hess score of 4 or 5 and $58(89 \%)$ patients a Fisher score of 3 or 4 . ICU-mortality was $12 \%$. The mortality among those with registered medical follow-up was $9 \%$ (6 of 64) on the 28th day, 14\% (8 of 58) on the 3rd 
month and $14 \%$ ( 8 of 56 ) on the 6th month. Median serum sodium was $138(136-141) \mathrm{mmol} / \mathrm{L}$ at admission, 140 (137-145) $\mathrm{mmol} / \mathrm{L}$ on the 7th day, 139 (137-143) $\mathrm{mmol} / \mathrm{L}$ on the 10 th day and 140 (138-144) $\mathrm{mmol} / \mathrm{L}$ on the 14th day. Higher sodium serum values at admission $(p=0,002), 7$ th day $(p=0,009)$, 10th day $(p=0,001)$ and 14th day $(p=0,048)$ were associated with unfavorable functional outcome at 28th day of follow-up. This association was not observed on the 3rd and 6 th month. The analysis of serum sodium values as a categorical variable with a cut-off of $140 \mathrm{mmol} / \mathrm{L}$, showed that values equal to or above $140 \mathrm{mmol} / \mathrm{L}$ at admission $(p=0,005), 7$ th day $(p=0,006)$ and 10 th day $(p=0,001)$, were associated with worst functional outcomes at day 28 of follow-up.

Conclusion. Higher levels of serum sodium were associated with unfavorable functional outcome on the 28th day after aSAH, but not on the 3rd and 6th months.

\section{Reference(s)}

1. Beseoglu K, Etminan N, Steiger HJ, Hänggi D. The relation of early hypernatremia with clinical outcome in patients suffering from aneurysmal subarachnoid hemorrhage. Clin Neurol Neurosurg. 2014 Aug;123:164-8.

2. Mapa B, Taylor BE, Appelboom G, Bruce EM, Claassen J, Connolly ES Jr. Impact of Hyponatremia on Morbidity, Mortality, and Complications After Aneurysmal Subarachnoid Hemorrhage: A Systematic Review. World Neurosurg. 2016 Jan:85:305-14.

3. Qureshi Al, Suri MF, Sung GY, Straw RN, Yahia AM, Saad M, Guterman LR, Hopkins LN. Prognostic significance of hypernatremia and hyponatremia among patients with aneurysmal subarachnoid hemorrhage. Neurosurgery. 2002 Apr;50(4):749-55.

\section{5}

Biomarkers in the prognostic evaluation of ischemic stroke: Is there benefit in the measurements of TREM-1 and TREM-2 in the acute phase?

FN. Backes'; A. Souza, ${ }^{2}$ MM. Bianchin, ${ }^{3}$

${ }^{1}$ Intensive Care Medicine, Hospital de Clínicas de Porto Alegre, Porto Alegre, Brazil; ${ }^{2}$ Pharmacy, Hospital de Clínicas de Porto Alegre, Porto Alegre, Brazil; ${ }^{3}$ Neurology, Hospital de Clínicas de Porto Alegre, Porto Alegre, Brazil

Correspondence: F.N. Backes

Intensive Care Medicine Experimental 2021, 9(1): 000525

Introduction. Stroke is a complex process in which initial cerebral ischemia is followed by secondary injury from innate and adaptive immune responses. Cerebral ischemia causes the release of highly immunogenic components or damage-associated molecular patterns (DAMPs) from the brain into the systemic circulation. These DAMPs activate and recruit peripheral immune cells to injured brain regions. As a consequence, toxic or proinflammatory and protective or anti-inflammatory processes are activated after stroke. We report the results of a study focused on the evaluation of serum levels of triggering receptors expressed on myeloid cells (TREM proteins), a family of cell surface receptors that participate in a variety of cellular processes and are activated almost immediately after the onset of brain ischemia, to determine their prognostic value and association with validated stroke scales.

Methods. We investigated 50 patients with acute ischemic stroke who were admitted within $24 \mathrm{~h}$ of event onset at the intensive care unit or neurovascular emergency unit of the Hospital de Clínicas. All patients provided venous blood samples for the measurement of triggering receptor expressed on myeloid cells type 1 (TREM-1) and type 2 (TREM-2) within $24 \mathrm{~h}$ of the acute event and on the third and fifth days after the stroke. Neurological stroke severity and global disability were determined with the National Institutes of Health Stroke Scale (NIHSS) and modified Rankin Scale (mRS) at the same three times and at the time of hospital discharge. After four years the patients were reevaluated using the mRS. The patients were subdivided into two groups according to the NIHSS score (NIHSS $\leq 6$ and NIHSS $>6$ ) and the mRS score ( $m R S \leq 2$ and $m R S>2$ ), which were employed as neurological outcome measures.
Results. The mortality rate reached $28 \%$ after four years. TREM- 1 and TREM-2 levels were elevated in stroke patients compared to healthy controls with the same risk factors. The serum level of TREM- 1 within $24 \mathrm{~h}$ also presented the best correlation with the neurological outcomes at hospital discharge (NIHSS and TREM-1: $p=0.021 ; \mathrm{mRS}$ and TREM-1: $p=0.049$ ). Both neurologic scores showing favorable outcome (NIHSS $\leq 6$ and $m R S \leq 2$ ) at hospital discharge were correlated with the TREM-1 protein concentration within $24 \mathrm{~h}$, with low predictive value. The serum concentrations of TREM-1 protein within $24 \mathrm{~h}$ after stroke was significantly higher in patients with poor outcome (mRS $>2)$ at hospital discharge $(p=0.021)$.

Conclusion. Blood biomarkers may be useful in acute stroke by suggesting stroke severity, correlating with clinical findings, or providing prognostic value. In this study, TREM-1 was found to be the best prognostic biomarker.

Reference(s)

1. 4. Gervois P, Lambrichts I. The Emerging Role of Triggering Receptor Expressed on Myeloid Cells 2 as a Target for Immunomodulation in Ischemic Stroke. Front Immunol. 2019 Jul; 10: 1668-1675. https://doi.org/ 10.3389/fimmu.2019.01668

2. 3. Hu X, Li P, Guo Y, Wang H, Leak RK, Chen S, et al. Microglia/macrophage polarization dynamics reveal novel mechanism of injury expansion after focal cerebral ischemia. Stroke. 2012 Nov; 43(11): 3063-3070. https://doi. org/10.1161/STROKEAHA.112.659656.

3. 2. Shekhar S, Cunningham MW, Pabbidi MR, Wang S, Booz GW, Fan F. Targeting vascular inflammation in ischemic stroke: Recent developments on novel immunomodulatory approaches. Eur J Pharmacol. 2018 Aug; 833: 531-544. https://doi.org/10.1016/j.ejphar.2018.06.028

4. 1. Zhao SC, Ma LS, Chu ZH, Xu H, Wu WQ, Liu F. Regulation of microglial activation in stroke. Acta Pharmacol Sin. 2017 Apr; 38(4): 445-458. https://doi.org/10.1038/aps.2016.162.

5. This study was partially supported by the FIPE - HCPA.

\section{5}

IL-23 and IL-17 in Acute Ischemic Stroke: Correlation with Stroke Scales and Prognostic Value

FN. Backes ${ }^{1}$; A. Souza, ${ }^{2}$; MM. Bianchin, ${ }^{3}$

${ }^{1}$ Intensive Care Medicine, Hospital de Clínicas de Porto Alegre, Porto Alegre, Brazil; ${ }^{2}$ Pharmacy, Hospital de Clínicas de Porto Alegre, Porto Alegre, Brazil; ${ }^{3}$ Neurology, Hospital de Clínicas de Porto Alegre, Porto Alegre, Brazil

Correspondence: F.N. Backes

Intensive Care Medicine Experimental 2021, 9(1): 000545

Introduction. Ischemic stroke causes a broad spectrum of motor sensory and cognitive impairments. There is an urgent need for accurate outcome prediction after acute ischemic stroke for physicians, patients, and their families to aid in early and informed decision-making about therapies, palliative care, or rehabilitation. In clinical practice, the prognosis is based on well-validated stroke scales, but they have limitations, and blood biomarkers measurements may improve the predictive capability. Inflammation plays a crucial role in brain damage following stroke with different local and peripheral activation pathways involved in post-ischemic neurodegeneration and neuroprotection mechanisms. For this study, we selected two important interleukins involved in the post-ischemic inflammatory process: interleukin 23 (IL-23) and interleukin 17 (IL-17).

Methods. Fifty consecutive patients with acute ischemic stroke admitted to the neurovascular emergency unit or intensive care unit at Hospital de Clínicas de Porto Alegre within 24 h of stroke onset were enrolled. All patients provided venous blood samples for IL-23 and IL-17 measurements within $24 \mathrm{~h}$ of the acute event and on the third and fifth day after the stroke. Neurological stroke severity and global disability were determined with the National Institutes of Health Stroke Scale (NIHSS) within $24 \mathrm{~h}$ of the acute event, on the third and fifth day after the stroke, and at the time of hospital discharge. The modified Rankin Scale (mRS) was applied at the same times and after four years. For short-term and long-term neurological outcome 
analysis, the patients were subdivided into two groups: favorable (NIHSS $\leq 6$ and $m R S \leq 2$ ) and poor outcome (NIHSS $>6$ and $m R S>2$ ).

Results. Both neurological scores for a favorable outcome at hospital discharge that were related to IL-23 protein within $24 \mathrm{~h}$ and on the fifth day had a low predictive value. The other measurements did not show predictive capacity during the hospital observation time or after four years. There was a significant increase in median serum concentrations of IL-23 on the fifth day $(p<0.001)$ and in IL-17 median levels on the third day compared to the first $24 \mathrm{~h}$ after the acute injury $(p<0.001)$. However, there was no correlation between IL-23 and IL-17 levels with the dichotomization of neurological outcomes at hospital discharge and after four years.

Conclusion. Neither IL-23 nor IL-17 had sufficient predictive power to be of clinical use to predict outcome after stroke. Thus, the research for better biomarkers with clinical prognostic relevance and the potential to improve the bedside management of stroke patients needs to be continued.

\section{Reference(s)}

1. 5. Jiang C, Kong W, Wang Y, Ziai W, Yang Q, Zuo F, et al. Changes in the cellular immune system and circulating inflammatory markers of stroke patients. Oncotarget. 2017 Jan; 8(2): 3553-3567. https://doi.org/10. 18632/oncotarget.12201.

2. 4. Abbas A, Gregersen I, Holm S, Daissormont I, Bjerkeli V, Krohg-Sørensen $\mathrm{K}$, et al. Interleukin 23 levels are increased in carotid atherosclerosis: possible role for the interleukin 23/interleukin 17 axis. Stroke. 2015 Mar; 46(3): 793-799. https://doi.org/10.1161/STROKEAHA.114.006516.

3. 3. Zhao SC, Ma LS, Chu ZH, Xu H, Wu WQ, Liu F. Regulation of microglial activation in stroke. Acta Pharmacol Sin. 2017 Apr; 38(4): 445-458. https://doi.org/10.1038/aps.2016.162.

4. 2. Shichita T, Sakaguchi R, Suzuki M, Yoshimura A. Post-ischemic inflammation in the brain. Front Immunol. 2012 May; 3: 132-138. https://doi. org/10.3389/fimmu.2012.00132.

5. 1. Hu X, De Silva TM, Chen J, Faraci FM. Cerebral Vascular Disease and Neurovascular Injury in Ischemic Stroke. Circ Res. 2017 Feb; 120(3): 449-471. https://doi.org/10.1161/CIRCRESAHA.116.308427.

6. This study was partially supported by the FIPE - HCPA.

\section{4}

Rethinking hemoglobin threshold in aneurysmal subarachnoid hemorrhage

J. Fernandes ${ }^{1}$; N. Jorge ${ }^{1}$; E. Monteiro ${ }^{1}$; JA. Paiva

'Intensive Care Medicine Department, São João Universitary Hospital

Center, Porto, Portugal

Correspondence: J. Fernandes

Intensive Care Medicine Experimental 2021, 9(1): 000604

Introduction. Anemia is common in spontaneous subarachnoid hemorrhage (SAH). Despite the growing recognition that SAH patients may benefit from higher hemoglobin levels, the optimal hemoglobin concentration to trigger red blood cell transfusion is not clearly defined.

Objectives. To evaluate the impact of hemoglobin concentration on functional status after $\mathrm{SAH}$.

Methods. Retrospective analysis of all adult patients with aneurysmal SAH admitted from 1st January 2018 to 31st December 2019 at a Neurocritical Care Unit (NCCU) of an University Hospital. Functional outcome was evaluated by Glasgow Outcome Scale (GOS) on the 28th day and 3rd month after the acute neurological event, considering favorable GOS 4 or 5 and unfavorable GOS 1, 2 or 3.

Results. Sixty-five patients were included, 43 females (66.2\%), with a median age of 55 (IQR 47.5-66.5) years. Hunt and Hess score of 4 or 5 was observed in $25(38.5 \%)$ and Fisher score of 3 or 4 in $58(89.2 \%)$ NCCU mortality was $12,3 \%$. Among the patients with a registered medical follow-up, mortality was $9.4 \%$ (6 of 64 ) on the 28th day and $14.0 \%$ (8 of 57) on the 3rd month. Hemoglobin $<10 \mathrm{~g} / \mathrm{dL}$ was present in $5 \%$ of patients at presentation, in $39 \%$ at day 7 , in $34 \%$ at day 10 and in $59 \%$ at day 14 after the event. Lower hemoglobin levels at admission $(p=0,001)$, day $7(p<0,001)$, day $10(p<0,001)$ and day $14(p=0,001)$ were associated with unfavorable GOS on the 28 th day after the event. The threshold level that showed an association with unfavorable
GOS on the 28th day after the acute event was hemoglobin $<12 \mathrm{~g} /$ $\mathrm{dL}$ at admission $(\mathrm{p}<0,001)$ and $<10 \mathrm{~g} / \mathrm{dL}$ at day $7(\mathrm{p}=0,001)$, day 10 $(p<0,001)$ and day $14(p=0,004)$. Although there were an association between unfavorable GOS on the 3rd month after the acute event and lower hemoglobin levels at day $7(p=0,002)$ and day $10(p=0,04)$, no significant cut-off was identified. Hemoglobin level at day 21 did not impact 28 day or 3 month GOS. Regardless of the assessment day, hemoglobin level had no association with the development of vasospasm or delayed cerebral ischemia.

Conclusion. Functional outcome at 28 days after subarachnoid hemorrhage were significantly worse between patients who have hemoglobin concentration inferior to $10 \mathrm{~g} / \mathrm{dL}$ during the first 14 days of NCCU admission. Higher hemoglobin goals rather than a restrictive approach to transfusion may impact SAH outcomes.

\section{Reference(s)}

1. Vlaar AP, Oczkowski S, de Bruin S, Wijnberge M, Antonelli M, Aubron C, et al. Transfusion strategies in non-bleeding critically ill adults: a clinical practice guideline from the European Society of Intensive Care Medicine. Intensive Care Med. 2020 Apr:46(4):673-696. https://doi.org/10.1007/ s00134-019-05884-8.

2. English SW, Fergusson D, Chassé M, et al. Aneurysmal Subarachnoid Hemorrhage-Red Blood Cell Transfusion And Outcome (SAHaRA): a pilot randomised controlled trial protocol. BMJ Open. 2016:6:e012623. https:// doi.org/10.1136/bmjopen-2016-012623

3. Ayling OGS, Ibrahim GM, Alotaibi NM, Gooderham PA, Macdonald RL. Anemia After Aneurysmal Subarachnoid Hemorrhage Is Associated With Poor Outcome and Death. Stroke. 2018 Aug;49:1859-1865. https://doi. org/10.1161/STROKEAHA.117.020260.

\section{3}

Quality of Life of Patients Discharged from The Neurocritical Care Unit at A University Hospital in Bogotá, Colombia

J. Barrero'; JN. Carreño, ; J. Carrizosa

${ }^{1}$ Department of Critical and Intensive Care Medicine, Fundacion Santa Fe de Bogota University Hospital, Bogotá, Colombia

Correspondence: $\lrcorner$. Carrizosa

Intensive Care Medicine Experimental 2021, 9(1): 000643

Introduction. From the beginning of intensive care units, mortality has decreased, but the subsequent sequelae of the disease, impact on quality of life and economy, has been studied recently (1-4). In Colom$\mathrm{bia}$, there are few studies in neurocritical pathologies $(5,6)$. As seen in literature, neurocritical pathologies such as moderate or severe traumatic brain injury (TBI), subarachnoid haemorrhage $(\mathrm{SAH})$, ischemic stroke (ischemic stroke) or hemorrhagic stroke, subdural and epidural hematomas, can have an impact on quality of life $(7,8)$, in return for the usual activities (9)

Objectives. The main objective is to know patients' quality of life discharged from the intensive care unit due to a neurocritical pathology in 2017. Also, to know the time to return to work or usual daily activity. Methods. A descriptive cross-sectional study was carried out, patients discharged from the intensive care unit (ICU) with the diagnoses of moderate or severe traumatic brain injury (TBI), subarachnoid haemorrhage (SAH), ischemic or hemorrhagic stroke, subdural and epidural hematomas. Information from 60 patients was collected. An EQ-5D-5L survey was applied to them on quality of life, work activity before hospitalization in 2017 and current, re-entry to work or time to resume their usual activities.

Results. Sixty people answered the questionnaire, the respondents considered that the quality of life decreased by an average of $63.8 \%$, the quality-of-life modules most affected according to the EQ-5D-5L survey were: mobility and pain or discomfort. $38.3 \%$ never resumed their work activities, and 35\% never resumed their usual activities to the date of this survey.

Conclusion. Patients discharged from the ICU due to a neurocritical pathology have a quality-of-life compromise, and a third of the patients will never resume their usual or work activities three years after discharge. 
Reference(s)

1. 1. Oeyen SG, Vandijck DM, Benoit DD, Annemans L, Decruyenaere JM. Quality of life after intensive care: A systematic review of the literature. Crit Care Med. 2010;38(12):2386-400

2. 2. Williams TA, Leslie GD. Beyond the walls: A review of ICU clinics and their impact on patient outcomes after leaving hospital. Aust Crit Care. 2008;21(1):6-17.

3. 3. van Beusekom I, Bakhshi-Raiez F, de Keizer NF, Dongelmans DA, van der Schaaf M. Reported burden on informal caregivers of ICU survivors: a literature review. Crit Care [Internet]. 2015;20(1):16. Available from: http:// ccforum.com/content/20/1/16

4. 4. Kamdar BB, Suri R, Suchyta MR, Digrande KF, Sherwood KD, Colantuoni $E$, et al. Return to work after critical illness: A systematic review and metaanalysis. Thorax. 2020;75(1):17-27.

5. 5. Oliveros H, Martínez F, Lobelo R, Velásquez J, Gómez M, Granados M. Recursos utilizados y calidad de vida de los pacientes críticamente enfermos egresados de la unidad de cuidados intensivos. Acta Med Colomb. 2008:33(4):268-75.

6. 6. Cariello AN, Perrin PB, Rodríguez-Agudelo Y, Plaza SLO, Quijano-Martinez MC, Arango-Lasprilla JC. A multi-site study of traumatic brain injury in mexico and colombia: Longitudinal mediational and cross-lagged models of family dynamics, coping, and health-related quality of life. Int J Environ Res Public Health. 2020;17(17):1-16.

7. 7. Haacke C, Althaus A, Spottke A, Siebert U, Back T, Dodel R. Long-term outcome after stroke: Evaluating health-related quality of life using utility measurements. Stroke. 2006:37(1):193-8.

8. 8. Lu Q, Mårtensson J, Zhao Y, Johansson L. Living on the edge: Family caregivers' experiences of caring for post-stroke family members in China: A qualitative study. Int J Nurs Stud. 2019:94(22):1-8.

9. 9. Edwards JD, Kapoor A, Linkewich E, Swartz RH. Return to work after young stroke: A systematic review. Int J Stroke. 2018;13(3):243-56.

\section{8}

Outcomes related to transfusional trigger in patients with subarachnoid haemorrhage at 2600 MASL

CM. Palomino, ; JN. Carreño, ${ }^{2}$; J. Carrizosa

${ }^{1}$ Department of Critical and Intensive Care Medicine, Fundacion Santa Fe de Bogota University Hospital, Bogotá, Colombia; ${ }^{2}$ Department of Critical and Intensive Care Medicine, Fundacion Santa Fe de Bogota University Hospital, Bogotá, Colombia; ${ }^{3}$ Critical Care Medicine, University Hospital Fundación Santa Fe de Bogotá, Bogota, France

Correspondence: J. Carrizosa

Intensive Care Medicine Experimental 2021, 9(1): 000648

Introduction. Subarachnoid haemorrhage (SAH) is a devastating pathology of the central nervous system, with high morbidity and mortality. Clinical trials of pharmacological therapies may not be affected by the patient's geographic location, particularly by height, as therapy with blood products whose relationship with atmospheric pressure and oxygen transport generates areas of uncertainty in cities of higher altitude.

Objectives. To evaluate the relationship of favourable neurological functional outcome (modified Rankin $<3$ ) vs unfavourable (modified Rankin equal or higher than 4) with a cut-off point of $10 \mathrm{gr} / \mathrm{dl}$ haemoglobin before transfusion in patients diagnosed with subarachnoid haemorrhage, hospitalized in the neurocritical care unit of the Santa Fe de Bogotá Foundation at 2600 MASL in Bogotá, Colombia.

Methods. A retrospective case-control observational study based on a historical cohort of patients with SAH, from January 2010 to December 2018 in the Neurological Intensive Care Unit.

Results. No relationship was found between favourable functional outcome and transfusion with haemoglobin level with a cut-off point of $10 \mathrm{gr} / \mathrm{dL}$ OR 1,111 ( $\mathrm{p}=0.86595 \% \mathrm{Cl} 0.328-3.755)$

Conclusion. A favourable or unfavourable association between the haemoglobin cut-off point and the functional neurological outcome was not demonstrated for patients at 2600 MASL. It is not possible to recommend the transfusion threshold of $10 \mathrm{gr} / \mathrm{dL}$ for patients with SAH living in heights close to 2600 MASL based on these results. No deleterious blood transfusion effect was evident, no increase in episodes of spasm vessel or late cerebral ischemia.

\section{Reference(s)}

1. Kramer AH, Diringer MN, Suarez JI, Naidech AM, Macdonald LR, Le Roux PD. Red blood cell transfusion in patients with subarachnoid hemorrhage: a multidisciplinary North American survey. Crit Care [Internet]. 2011;15(1):1-11. Available from: http://dx.doi.org/10.1186/cc9977

2. Kramer AH, Zygun DA, Bleck TP, Dumont AS, Kassell NF, Nathan B. Relationship between hemoglobin concentrations and outcomes across subgroups of patients with aneurysmal subarachnoid hemorrhage. Neurocrit Care [Internet]. 2009;10. Available from: http://dx.doi.org/10.1007/ s12028-008-9137-0

3. Naidech AM, Shaibani A, Garg RK, Duran IM, Liebling SM, Bassin SL, et al. Prospective, randomized trial of higher goal hemoglobin after subarachnoid hemorrhage. Neurocrit Care. 2010/08/19. 2010;13(3):313-20.

4. Rosenberg NF, Koht A, Naidech AM. Anemia and transfusion after aneurysmal subarachnoid hemorrhage. J Neurosurg Anesth [Internet]. 2013;25(1):66-74. Available from: http://www.ncbi.nlm.nih.gov/pubmed/ 23047622

\section{7}

\section{Impact of Complications and Comorbidities on the outcome} after Aneurysmal Subarachnoid Haemorrhage

F. Sequeira, ; L. Linhares, ${ }_{1}^{\text {; }}$ A. Santos, ${ }^{1}$; S. Teixeira, '; P. Martins,

${ }^{1}$ Department of Intensive Care, Centro Hospitalar e Universitário de

Coimbra, Coimbra, Portugal

Correspondence: F. Sequeira

Intensive Care Medicine Experimental 2021, 9(1): 000657

Introduction. Aneurysmal subarachnoid haemorrhage (SAH) is a devastating disease associated with a mortality of more than $30 \%$. Aside from direct effects of SAH on the brain parenchyma and directly associated complications like rebleeding, delayed cerebral ischemia and hydrocephalus, there is evidence that further medical complications play a crucial role in the outcome after SAH. However, these complications might be preventable or at least controllable. A better outcome might be achievable with improved intensive care management.

Objectives. The aim of the study was to determine predictors of mortality among patients with subarachnoid hemorrhage hospitalized in an Intensive Care Unit.

Methods. Retrospective study of patients with subarachnoid hemorrhage admitted to a University Hospital's Intensive Care Unit during a 2-year period. Data were collected from the Intensive Care Unit computerized database.

Results. Nineteen patients with aneurismal subarachnoid haemorrhage were included. The important risk factors identified for the development of cerebral aneurysms: female sex $(79 \% ; n=15)$, hypertension ( $32 \% ; n=6)$, cigarette smoking $(10 \% ; n=2)$, high cholesterol levels (10\%; $n=2)$ and Ehlers-Danlos syndrome $(5 \% ; n=1) .79 \%$ of patients had the classic presentation with a sudden-onset, severe headache, which was an isolated finding in $58 \%$. The overall mortality was $42 \%$ (8 patients) and was significantly associated with septic shock (75\%), hydrocephalus (75\%) and hyponatraemia (37,5\%). There wasn't any relationship between the degree of consciousness on admission and the prognosis (the median of Glasgow Coma Scale was 6 in both patients who died and survived). Five patients operated (45\%), 1 embolized (9\%) and 1 (9\%) endovascularly treated had neurological sequelae. One endovascularly treated patient (12,5\%) and $4(50 \%)$ surgically treated died. The frequency of clinical vasospasm was $15 \%$.

Conclusion. Septic shock, hydrocephalus and hyponatraemia were important complications among critically ill patients with $\mathrm{SAH}$ and were associated increased mortality. We found no statistically significant differences in outcome between the embolized or endovascularly treated patients and those undergoing conventional surgery, but this is a small population. Improvement of strategies regarding prevention and treatment of complications might therefore be one the most important things to improve outcome after aneurysmal SAH.

\section{Reference(s)}

1. Abulhasan, Y. B. et al. Health Care-Associated Infections after Subarachnoid Hemorrhage. World Neurosurg 115, e393-e403 
2. Wartenberg, K. E. et al. Impact of medical complications on outcome after subarachnoid hemorrhage. Crit Care Med 34, 617-623

3. Solenski, N. J. et al. Medical complications of aneurysmal subarachnoid hemorrhage: a report of the multicenter, cooperative aneurysm study. Participants of the Multicenter Cooperative Aneurysm Study. Crit Care Med 23, 1007-1017

\section{7}

Risk factors related to mortality in ICU patients with ischemic stroke

A. Navarro-Cruz ; A. Ortega-Ordiales ${ }^{1}$; G. Giudetti ${ }^{1}$; P. Ruiz-Peña ${ }^{1}$; J. Luna-Castro ${ }^{1}$ MD. Arias Verdú ${ }^{1}$

${ }^{1}$ Intensive Care, Hospital Regional Universitario de Málaga (Carlos de Haya), Málaga, Spain

Correspondence: M.D. Arias Verdú

Intensive Care Medicine Experimental 2021, 9(1): 000687

\section{Introduction...}

Objectives. To describe the risk factors that are related to ICU mortality in patients admitted due to ischemic stroke.

Methods. We undertook a retrospective descriptive cohort study of 105 adult patients ( $56.2 \%$ male; mean age $66+12.5$ years) with ischemic stroke admitted to the ICU of a 3rd level Hospital during 2017-2019. Multivariate logistic regression analysis was performed to identify risk factors for ICU mortality, including age, NIHSS, fibrinolysis treatment and thrombectomy, decompressive craniectomy (DC), hemorrhagic transformation, and mechanical ventilation.

Results. Only $24 \%$ had a history of Atrial fibrilation, and less than $26 \%$ were taking oral anticoagulants. The NIHSS on admission was $16.23+5$, ASPECT: $8 \pm 1.54$ and Glasgow: $8 \pm 3.6$. The most frequently affected artery was the right ICA, followed by the right MCA, and the least affected one was the basilar. Thrombectomy was performed in $89 \%$ and fibrinolysis + thrombectomy in $27 \%$. The mean stay in the ICU was $6.18 \pm 7$ days and in hospital 29 days. $71.2 \%$ were intubated, a Decompresive Craniectomy (DC) was performed in 18\% and there was a hemorrhagic transformation in $22 \%$. Mortality in ICU was $23 \%$, and in hospital $36 \%$. The multivariate regression analysis (introducing two-by-two variables) showed that mechanical ventilation time was independently associated with mortality (OR $1.016,95 \% \mathrm{Cl} 1.003-$ 1.028; $\mathrm{P}=0.015$ ) adjusted for $\mathrm{NISHH}$ and fibrinolysis treatment plus thrombectomy. Other risk factors (CD, age, diabetes, HTA and AF) were not significantly associated with mortality when mechanical ventilation was included in the bi-variant model.

Conclusion. Mechanical ventilation on admission to the ICU was the main variable related to mortality in patients admitted due to ischemic stroke. For every hour of mechanical ventilation, the risk of mortality increases by $16 \%$.

\section{0}

Epidemiological characteristics of Intensive Care Unit patients with ischemic stroke

A. Navarro-Cruz; ; A. Ortega-Ordiales ${ }^{1}$; S. González-Soto ${ }^{1}$; R.

Barraso-González ; FJ. Gallardo-Puga ${ }^{1}$; MD. Arias Verdú ${ }^{\prime}$

${ }^{1}$ Intensive Care, Hospital Regional Universitario de Málaga (Carlos de Haya), Málaga, Spain

Correspondence: M.D. Arias Verdú

Intensive Care Medicine Experimental 2021, 9(1): 000690
Introduction. $x \ldots .$.

Objectives. To describe epidemiological characteristics of ischemic stroke patients admitted to the ICU.

Methods. We undertook a retrospective descriptive study of adult patients with ischemic stroke admitted to the ICU of a 3rd level hospital during 2017-2019. Description of results using mean $\pm d$, median, or \% and non-parametric tests.

Results. $\mathrm{N}=105$ patients. Men $56.19 \%$, mean age $66+12$. Only $24 \%$ had a history of Atrial fibrilation and less than $26 \%$ were taking oral anticoagulants. NIHSS at admission 16.2+5, ASPECT: $8 \pm 1$, and Glasgow: $8 \pm 3$.

The most affected artery was the right ICA, followed by the right MCA; the one affected the least was the basilar. Most frequent neurological complications: malignant infarction requiring decompressive craniectomy (DC) and hemorrhagic transformation.

Our patients come from 5 more hospitals in addition to ours (we are a reference centre), so the process times are at the recommended high limit. Thrombectomy was performed in $89 \%$, and fibrinolysis + thrombectomy in $27 \%$.

Average stay in ICU was $6 \pm 7$ days, and in hospital 29. Mortality in ICU: $23 \%$, and in hospital $36 \%$.

The only factor related to mortality by univariate analysis was mechanical ventilation on admission (X2 $=11.61, p<0.0001)$. Difficulty extubating patients in the Radiology Room was the first cause of request for admission to the ICU. We found no ss relationship with age, NIHSS, ASPECT, or neurological complications.

Conclusion. Ischemic stroke is a disabling disease with considerable mortality.

The most frequent neurological complications are malignant infarction requiring $\mathrm{DC}$ and hemorrhagic transformation.

The only factor related to mortality was mechanical ventilation.

The attention times are long because we are a referral centre and more than half of the patients come from other hospitals.

\section{5}

Low cerebral tissue oxygen pressure is correlated with impaired cardiac function after acute brain injury: A prospective study C. Santacruz Herrera?' D. De Backer ${ }^{2}$; J. Carrizosa ${ }^{3}$; T. Medina ${ }^{4}$; D. Reyes ${ }^{4} ;$ A. Quintairos $^{4}$; K. Puerto ${ }^{5}$; JN. Carreño, ${ }^{1}$

${ }^{1}$ Departament of Critical and Intensive Care Medicine, Fundacion Santa Fe de Bogota University Hospital, Bogotá, Colombia; ${ }^{2}$ Department of Intensive Care, CHIREC Hospitals, Université Libre de Bruxelles, Brussels, Belgium; ${ }^{3}$ Critical Care Medicine, University Hospital Fundación Santa Fe de Bogotá, Bogota, Colombia; ${ }^{4}$ Intensive Care Medicine, Fundacion Santa Fe De Bogota Hospital Universitario, Carrera 7b, Bogota, Colombia, Bogotá, Bogota, Colombia, Colombia; ${ }^{5}$ Intensive Care Medicine, Fundacion Santa Fe De Bogota Hospital Universitario, Carrera 7b, Bogota, Colombia, Bogot, Colombia

Correspondence: $C$. Santacruz Herrera

Intensive Care Medicine Experimental 2021, 9(1): 000695

Introduction. Current approaches to the management of severe acute brain injury $(A B I)$ are mostly based on the optimization of cerebral perfusion pressure through the manipulation of intracranial pressure (ICP) and mean arterial pressure. However, little information is available regarding the influence of cardiac output (CO) on cerebral $\mathrm{PtiO}_{2}$ following $\mathrm{ABI}$. 
Objectives. To evaluate the correlation between cerebral $\mathrm{PtiO}_{2}$ and cardiac output after severe $A B I$.

Methods. The study included 12 patients (mean age 61.9 years, $\mathrm{SD} \pm 10.3$ ) who had been admitted to the ICU (GCS 9.4, SD \pm 3.7 ) and required intraventricular catheterization for ICP, parenchymal $\mathrm{PtiO}_{2}$ and cerebral oxygen metabolism monitoring using a jugular ascending catheter. Of these, 50\% had experienced intracranial hemorrhage, $33 \%$ spontaneous sub-arachnoid hemorrhage, and $16 \%$ traumatic brain injury. All measurements were taken on day 1. Cardiac output was evaluated using a thermodilution catheter and was considered abnormal if $\mathrm{CO}<4.2 \mathrm{~L} / \mathrm{min}$. Differences between patients with high and low $\mathrm{CO}$ and correlations with $\mathrm{CO}$ values were evaluated using parametric tests, analysis of variance, and linear models.

Results. ABI patients with high ICP had lower $\mathrm{PtiO}_{2}$ (23.8 vs. $7.5 \mathrm{mmhg}$; $\mathrm{p}<0.01)$ and lower $\mathrm{CO}(5.5 \mathrm{vs}$. $2.9 \mathrm{~L} / \mathrm{min} ; \mathrm{p}<0.01)$ than patients with normal ICP. Impaired CO led to lower cerebral perfusion pressure (73.7 vs. $93.2 \mathrm{mmhg} ; \mathrm{p}=0.04$ ), lower cerebral $\mathrm{O} 2$ delivery (517.9 vs. $968.2 \mathrm{mlO}_{2} / \mathrm{min} / \mathrm{m}^{2} ; \mathrm{p}<0.01$ ), and lower $\mathrm{PtiO}_{2}(26.5$ vs. $10.5 \mathrm{mmhg}$; $\mathrm{p}<0.01 ; \mathrm{r}=+0.73 ; \mathrm{p}<0.01)$. A positive correlation with the lactateglucose index $(r=+0.6 ; p=0.04)$ suggested increased cerebral anaerobic metabolism during episodes of low CO.

Conclusion. A positive correlation was found between cerebral regional $\mathrm{PtiO}_{2}$ and $\mathrm{CO}$. Altered $\mathrm{CO}$ correlated with lower cerebral oxygen delivery and with anaerobic metabolism.

\section{9}

Amplitude Integrated EEG - derivation of normative values and abnormal patterns in children age 2 months to 16 years in a continuous bedside brain-monitor

L. MacDarby'; J. Mchugh,2.; G. Curley, ${ }^{3}$ : M. Healy,

${ }^{1}$ Critical Care, Children's Health Ireland at Crumlin, Dublin, Ireland; ${ }^{2}$ Neurophysiology department, Children's Health Ireland (CHI) at Crumlin, Dublin, Ireland; ${ }^{3}$ Anaesthesia and critical care, Beaumont Hospital, Dublin, Ireland Correspondence: L. MacDarby

Intensive Care Medicine Experimental 2021, 9(1): 000849

Introduction. Amplitude integrated electroencephalography (aEEG) is a bedside neuromonitoring tool, the utility of which, is increasingly being recognised in ICUs across Europe. Amplitude values for aEEG traces have been demonstrated to evolve with age from pre-terms to neonates but values have not been determined in children older than 3.5 months.

Objectives. We aim to determine normative values in children from the age of 2 months to 16 years, to allow accurate interpretation of aEEG recordings at the bedside, in real-time, in the paediatric intensive care unit.

We also present representative traces of abnormal aEEG features, as derived from raw EEG, to characterise background patterns seen in older children.

Methods. A retrospective observational cohort study design, was used to determine normative amplitude ranges and background characteristics.

Children from 2 months to 16 years, with normal brain function who had an EEG in Children's Health Ireland at Crumlin (CHI@C) between the years 2012-2020 were included. 350 normal electroencephalograms (EEGs) were gathered from the departmental EEG register in $\mathrm{CH}$ @ Crumlin. 5 min epochs of waking and sleeping EEG per patient were gathered, processed as per aEEG algorithm (Persyst software), and collated to produce an overall maximum and minimum amplitude and bandwidth value as reference values for each age group.

Supplementary aEEGs were derived from a sample of children in PICU with EEG evidence of seizure and/or encephalopathy.

Results. A two way MANOVA showed a statistically significant interaction between the effects of age and state on amplitude $(p<0.001)$.

Mean amplitude values vary with age, most notably increasing over the first two years of life before diminishing again in older children greater than 6 years of age.

A statistically significant increase in amplitude was seen during sleep in both the minimum and maximum amplitudes in children less than 6 years of age, but was no longer significant above 6 years as per Wilcoxon Rank Sum test.

Strong correlation exists between the upper and lower margin of aEEG trace in all ages, both awake and asleep.

Conclusion. aEEG traces change as a function of age and state but the differences are small in a cohort derived from this large and predominantly out-patient database. Interpretation of aEEG recordings in children over 3 months is facilitated by this dataset which is the first to compile normative aEEG values for an older pediatric cohort.

\section{1}

To assess stress response to Propofol infusion vs. Midazolam infusion in severe traumatic brain injury (TBI) patients measured by serum cortisol levels. A randomised control trial

A. Hazarika'; M. Kurni²; N. Kaloria ${ }^{2}$; D. Jonna ${ }^{2}$

${ }^{1}$ Anaesthesia and Intensive Care, Post Graduate Institute of Medical Education \& Research, Chandigarh, Chandigarh, India; ${ }^{2}$ Anaesthesia \& Intensive Care, Post Graduate Institute of Medical Education \& Research, Chandigarh, Chandigarh, India

Correspondence: A. Hazarika

Intensive Care Medicine Experimental 2021, 9(1): 000951

Introduction. Stress response following Traumatic brain injury (TBI) causes a lot of endogenous systemic alterations in the brain. The primary insult induces the hyperadrenergic response that may be a factor of many complications. Sedatives are useful in dealing with this response. In acute phase (first $24 \mathrm{~h}$ up to $48 \mathrm{~h}$ ) continuous infusion of opioid agents and sedatives is considered to protect the injured brain and reduce intracranial pressure (ICP)0.1 Several drugs are useful as sedatives but none of the sedatives are perfect. Propofol demonstrated favourable effects in reducing CBF, ICP, and CMRO2.2 Midazolam has been proven to reduce CMRO2, CBF and ICP.1 Both propofol and midazolam are being used to sedate TBI patient on mechanical ventilation. However, no study have shown the effect of propofol and midazolam sedation on the stress response during acute phase of TBI.So our study will try to assess the effect of these two drugs on stress response as measured by serum cortisol levels.

Objectives. To assess if intravenous propofol infusion provides better obtundation of stress response due to traumatic brain injury as 
compared to midazolam infusion when measured by change in serum cortisol levels. Changes in hemodynamic variables during $48 \mathrm{~h}$ of drug infusion, GOS after ICU discharge, complications and additional therapy for management.

Methods. This study was a single-institution double-blind prospective randomized controlled trial.The study was registered in the Clinical trial registry of India (www.ctri.nic.in) (CTRI/2019/11/022121) after institutional ethical clearance. Inclusion criteria: Adult patients of 18 years and above with GCS of 8 or less with isolated head injury on first CT finding requiring mechanical ventilation for $>48 \mathrm{~h}$ with normal coagulation profiles were included. Exclusion criteria: Refusal to give consent for the study, An elapsed time between trauma and ICU admission in excess of 24 h, GCS of 3 or less Patients undergoing operation/intervention/cardiac arrest within the study period,pregnant patients,patients whose baseline serum cortisol level less then $15 \mu \mathrm{g} / \mathrm{dl}$, known allergy or contraindication to infusion drugs, deranged liver function test (LFT)and renal function test (RFT), Randomization and allocation: Patients were randomized using computer-generated random number tables (www. randomisation.com) and allocated to one of the two groups.Patients in Group I were administered with 1\% Propofol infusion and Midazolam infusion was used in Group II. Study Protocol: For all patients, basic demographic data, baseline vital parameters including GCS, pupillary reactions, ONSD and investigations was recorded.The drug used along with its total dose were documented. All patients received fentanyl as a fixed analgesic. Two samples of serum cortisol level were taken: 1stWithin $24 \mathrm{~h}$ of ICU admission; 2nd-After $48 \mathrm{~h}$ of 1st sample.Sedation end point: To maintain RA-SS score of-3. Intracranial pressure was indirectly measured by optic nerve sheath diameter (ONSD). Neurological Outcome was measured at ICU discharge using GOS (Glasgow Outcome Scale).

Results. Of the 104 patients were recruited, 21 patients were excluded. Fig. 1 shows the CONSORT diagram.

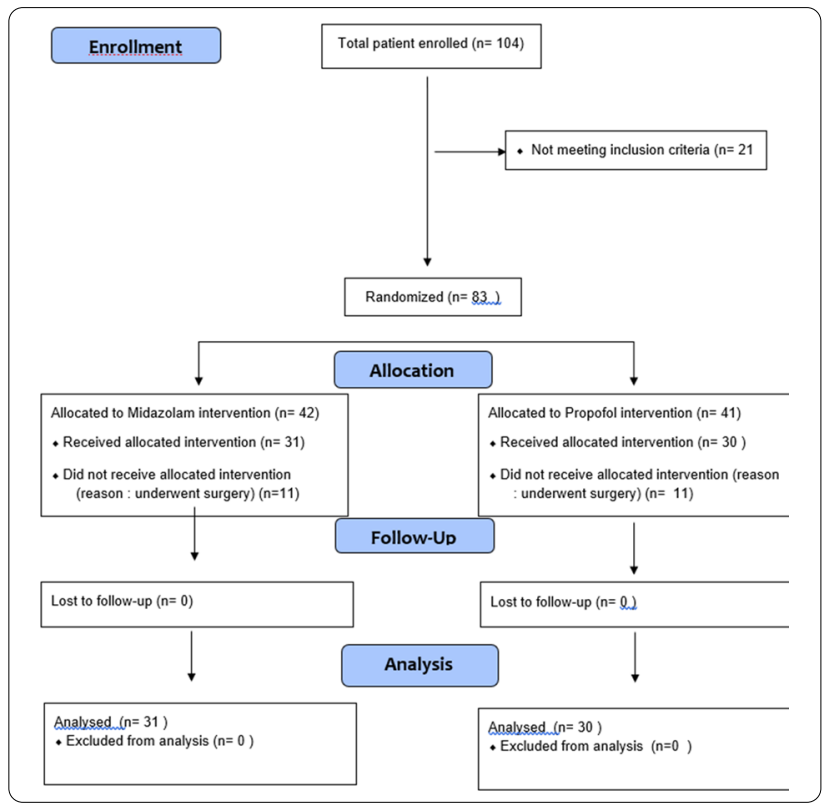

Table 2: Shows the initial and $48 \mathrm{~h}$ cortisol levels, hemodynamic variables and ONSD value between Propofol and Midazolam group.
Table 2: Shows the initial and 48 hrs cortisol levels, hemodynamic variables and ONSD value between Propofol and Midazolam group

\begin{tabular}{|c|c|c|c|}
\hline Particular & $\begin{array}{l}\text { Group I(n-31) } \\
\text { (Propofol) } \\
\text { (MEAN } \pm \text { S.D.) }\end{array}$ & $\begin{array}{l}\text { Group II(n-30) } \\
\text { (Midazolam) } \\
\text { (MEAN } 5 \text { S.D.) }\end{array}$ & $p$-value ${ }^{*}$ \\
\hline \multicolumn{4}{|l|}{ Serum Cortisol } \\
\hline Initial & $471.65 \pm 252.67$ & $494.61 \pm 147.21$ & 0.66 \\
\hline At 48 hours & $362.75 \pm 200.90$ & $380.72 \pm 112.23$ & 0.70 \\
\hline$\Delta$ Cortisol & $108.90 \pm 172.17$ & $113.89 \pm 178.57$ & 0.91 \\
\hline$p$-value ${ }^{*}$ & 0.03 & 0.01 & \\
\hline \multicolumn{4}{|l|}{ Heart rate } \\
\hline Initial & $87.40 \pm 15.34$ & $93.00 \pm 15.64$ & 0.16 \\
\hline At $48 \mathrm{hrs}$ & $79.65 \pm 10.79$ & $88.28 \pm 13.10$ & 0.006 \\
\hline$\Delta$ Heart rate & $7.75 \pm 15.28$ & $4.72 \pm 10.94$ & \\
\hline$p$-value ${ }^{\gamma}$ & 0.02 & 0.21 & \\
\hline \multicolumn{4}{|l|}{ Systolic blood pressure } \\
\hline Initial & $136.40 \pm 25.22$ & $129.56 \pm 21.18$ & 0.25 \\
\hline At $48 \mathrm{hrs}$ & $130.15 \pm 15.31$ & $128.17 \pm 17.53$ & 0.64 \\
\hline$\Delta$ Systolic blood pressure & $6.25 \pm 22.14$ & $1.39 \pm 20.92$ & 0.38 \\
\hline$p$-value ${ }^{\gamma}$ & 0.24 & 0.78 & \\
\hline \multicolumn{4}{|l|}{ Diastolic pressure } \\
\hline Initial & $86.80 \pm 19.70$ & $81.33 \pm 14.84$ & 0.22 \\
\hline At $48 \mathrm{hrs}$ & $81.25 \pm 11.27$ & $85.67 \pm 12.03$ & 0.14 \\
\hline$\Delta$ Diastolic pressure & $5.55 \pm 16.17$ & $-4.33 \pm 14.49$ & 0.01 \\
\hline$p$-value ${ }^{\gamma}$ & 0.17 & 0.21 & \\
\hline \multicolumn{4}{|l|}{ Mean arterial pressure } \\
\hline Initial & $103.35 \pm 20.55$ & $97.50 \pm 16.21$ & 0.22 \\
\hline At $48 \mathrm{hrs}$ & $97.50 \pm 11.66$ & $99.72 \pm 13.39$ & 0.49 \\
\hline$\Delta$ Mean arterial pressure & $5.85 \pm 15.96$ & $-2.22 \pm 15.77$ & 0.05 \\
\hline$p$-value ${ }^{\gamma}$ & 0.17 & 0.56 & \\
\hline \multicolumn{4}{|l|}{ ONSD (Right) } \\
\hline Initial & $5.52 \pm 0.97$ & $5.48 \pm 0.89$ & 0.86 \\
\hline At $48 \mathrm{hrs}$ & $4.39 \pm 1.02$ & $4.43 \pm .90$ & 0.87 \\
\hline$\triangle$ ONSD & $1.13 \pm 1.03$ & $1.05 \pm 1.13$ & 0.77 \\
\hline$p$-value & 0.00 & 0.00 & \\
\hline \multicolumn{4}{|l|}{ ONSD (Left) } \\
\hline Initial & $5.43 \pm 0.97$ & $5.34 \pm 0.73$ & \\
\hline At $48 \mathrm{hrs}$ & $4.44 \pm 0.95$ & $4.31 \pm 1.01$ & 0.60 \\
\hline$\triangle$ ONSD & $0.99 \pm 1.03$ & $1.03 \pm 0.98$ & 0.88 \\
\hline$p$-value ${ }^{\gamma}$ & 0.00 & 0.00 & \\
\hline
\end{tabular}

Table 3 Shows the Glasgow Outcome Score between the groups

Table 3: Shows the Glasgow Outcome Score between the groups
\begin{tabular}{|c|c|c|}
\hline GOS* $^{*}$ & $\begin{array}{c}\text { Group I(n-31) } \\
\text { (Propofol) }\end{array}$ & $\begin{array}{c}\text { Group II(n-30) } \\
\text { (Midazolam) }\end{array}$ \\
\hline 1 & 2 & 4 \\
\hline 2 & 0 & 1 \\
\hline 3 & 1 & 1 \\
\hline 4 & 3 & 3 \\
\hline 5 & 25 & 21 \\
\hline
\end{tabular}

Conclusion. Our study found that there is no significant difference between infusion propofol and midazolam in obtundation of stress response in TBI patients using serum cortisol as marker.There were no difference in the hemodynamic parameters, ONSD measurement and even on the outcome of the patients. The propofol infusion rate used in the study also had no complication/side effect on the study population. Quest for ideal sedative and marker to gauge its effect in Traumatic Brain Injury patients is still open. A large study will be required to effectively bring about a proper conclusion.

\section{Reference(s)}

1. 2.Adembri C, Venturi L, Pellegrini-Giampietro DE. Neuroprotective effects of propofol in acute cerebral injury. CNS Drug Rev. 2007;13:333-51.

2. 1.Choi HA, Jeon SB, Samuel S, Allison T, Lee K. Paroxysmal sympathetic hyperactivity after acute brain injury. Curr Neurol Neurosci Rep. 2013;13(8).

3. PGIMER, Chandigarh, India 


\section{8}

Exposure to non-lung protective mechanical ventilation is associated with outcome in critically ill patients with acute brain injury: a machine learning approach

N. Sengana'; H. Kim²; R. Stevens ${ }^{3}$

${ }^{1}$ Mechanical Engineering, Whiting School of engineering, Johns Hopkins University, Baltimore, United States of America; ${ }^{2}$ Department of Anesthesiology and Critical Care Medicine, Johns Hopkins University School of Medicine, Baltimore, United States of America; ${ }^{3}$ Anesthesiology and Critical Care Medicine, Johns Hopkins University, Baltimore, United States of America

Correspondence: R. Stevens

Intensive Care Medicine Experimental 2021, 9(1): 001108

Introduction. Patients admitted to the ICU with acute brain injury $(\mathrm{ABI})$ commonly receive mechanical ventilation (MV) which can be harmful due to physiological interactions on the brain and also ventilator-induced lung injury.

Objectives. Our aim is to explore the relationship between MV settings and the outcome of patients admitted to the ICU for management of acute brain injury. We hypothesize that MV settings are important determinants of clinical outcomes in this population.

Methods. The elCU database was searched for patients with traumatic brain injury (TBI) or stroke who were mechanically ventilated. A set of features was crafted based on current recommendations for lung-protective ventilation and included time "out-of-range" (OOR) for tidal volume per ideal body weight $\left(>8 \mathrm{ml} / \mathrm{kg}\right.$ ), plateau pressure $\left(>30 \mathrm{cmH}_{2} \mathrm{O}\right)$, PEEP $\left(<5 \mathrm{cmH}_{2} \mathrm{O}\right)$. Two models were created: (1) A clinical model which uses only the first value recorded for each feature; and (2) A combined model which includes predefined OOR MV variables. Outcomes were defined as "Unfavorable" for patients who died or whose discharge motor Glasgow Coma Score (mGCS) was $<5$, and "Favorable" for patients who were alive and had a $\mathrm{mGCS} \geq 5$ at discharge. Three different machine learning models (generalized linear model, XGBoost, and random forest) were trained using extracted features.

Results. Data were identified for a total of 839 TBI and 1,221 stroke ICU stays in which patients were mechanically ventilated. The best performing classifier was a random forest model. Clinical and combined models had an AUROC of $0.67 \pm 0.07$ and $0.79 \pm 0.13$ respectively for Stroke and an AUROC of $0.70 \pm 0.07$ and $0.81 \pm 0.07$ respectively for TBI. As shown in Figure 1, a number of OOR MV variables and respiratory physiologic variables contributed to the prediction of outcome. Duration of exposure to Out of Range (OOR) tidal volume, PEEP and plateau pressure were identified as top contributors with longer OOR durations predictive of unfavorable outcome.

Conclusion. These results suggest that exposure to non-lung protective MV may contribute to clinical and neurological outcomes in mechanically ventilated stroke and TBI patients.

\section{Reference(s)}

1. Robba C, Poole D, McNett M, Asehnoune K, Bösel J, Bruder N, Chieregato A, Cinotti R, Duranteau J, Einav S, Ercole A, Ferguson N, Guerin C, Siempos II. Kurtz P, Juffermans NP, Mancebo J, Mascia L, McCredie V, Nin N, Oddo M, Pelosi P, Rabinstein AA, Neto AS, Seder DB, Skrifvars MB, Suarez Jl, Taccone FS, van der Jagt M, Citerio G, Stevens RD. Mechanical ventilation in patients with acute brain injury: recommendations of the European Society of Intensive Care Medicine consensus. Intensive Care Med. 2020 Dec;46(12):2397-2410.

\section{8}

Neurological diagnostics in COVID-19 ICU Patients:

A Single-Centre Cohort Study

C. Bennewitz'; C. Teunissen²; J. Horn

${ }^{1}$ Intensive Care, Amsterdam UMC, Amsterdam, Netherlands; ${ }^{2}$ Neurobiochemistry, Amsterdam UMC, Amsterdam, Netherlands

Correspondence: J. Horn

Intensive Care Medicine Experimental 2021, 9(1): 001218
Introduction. COVID-19 primarily affects the respiratory system, however neurological complications have been reported too. It is unknown which diagnostic tests are most useful for brain injury identification in COVID-19 patients. We investigated the clinical practice of neurological diagnostics, including neurofilament light (NfL), in intensive care unit (ICU) patients from the first COVID-19 wave.

Methods. This retrospective study at the Amsterdam UMC, the Netherlands, included patients admitted to the ICU due to a COVID-19 infection between March and May 2020. Clinical data on patient characteristics, neurological diagnostics, and outcome was collected from the electronic files. Two groups were distinguished; patients with neuro-diagnostic testing (ND+) and patients without neuro-diagnostic testing (ND-). To compare NfL levels, the ND+ group was matched (age and gender) with ND- controls. NfL levels were assessed at ICU admission and day of neurological diagnostics or day 8 of ICU admission. Samples were available from the Amsterdam UMC COVID-19Biobank and analyzed using SimoaTM.

Results. Seventy-eight patients ( $24 \%$ female) were included and median age was 62 (IQR 55-70). ICU mortality was $45 \%$. In 27 patients neurological diagnostics were done; 39 brain CTs, 7 lumbar punctures, 5 brain MRIs and 4 EEGs. Main indication for neurological diagnostics was decreased consciousness (69\%). In 7 patients (26\%) brain abnormalities were detected: infarction, hemorrhage, white matter abnormalities, encephalopathy, focal epilepsy and neuro-inflammation.

Median NfL levels at ICU admission for ND + was $29.2 \mathrm{pg} / \mathrm{ml}$ (IQR 21.276.4), for matched-ND- $(n=23) 29.2 \mathrm{pg} / \mathrm{ml}$ (IQR 19.3-43.4). Median $\mathrm{NfL}$ levels at time of neurological diagnostics was $135.9 \mathrm{pg} / \mathrm{ml}$ (IQR 76.6-240.4), for matched-ND- $(\mathrm{n}=22) 92.5 \mathrm{pg} / \mathrm{ml}$ (IQR 63.5-136.0). No statistical differences were found.

Conclusion. Neurological diagnostics were performed in $35 \%$ of ICU COVID-19 patients, abnormalities were found in only $26 \%$. No differences in NfL levels were found between patients with or without neurological diagnostics.

\section{8}

Side of probe placement in Traumatic Brain Injury as an indicator of prolonged brain tissue hypoxia and poor outcome

S. Papoti ${ }^{1}$; I. Karagewrgiou'; ${ }^{1}$ E. Lazoudi'; N. Kapravelos ${ }^{1}$; C. Iasonidou

'B icu, General Hospital of Thessaloniki "George Papanikolaou", Thessa-

loniki, Greece

Correspondence: S. Papoti

Intensive Care Medicine Experimental 2021, 9(1): 001338

Introduction. Brain monitoring consists of several parameters that give information about its function. As already known, intracranial pressure (ICP) measurement reflects the global pressure inside the cranial cavity, while partial brain tissue oxygen $\left(\mathrm{PbtO}_{2}\right)$ concerns a regional measurement of brain tissue oxygen delivery, influenced by the underlying injury.

Objectives. To investigate an association between the side of probe placement in TBI patients and the time of $\mathrm{PbtO}_{2}$ below the lower threshold with the outcome during discharge from ICU.

Methods. A total of 44 patients were admitted in the ICU because of TBI with a median Glasgow Coma Scale (GCS) score of 9 (IQR 4.25). According to demographic characteristics of the patients, the majority of them were men $(84.1 \%)$ compared to women $(15.9 \%)$, with a median age of 36.5 years (IQR 28.5). The median ICU length-of-stay was 15.5 days (IQR 15.5) with a median GCS scale on discharge day of 14 (IQR 5.25). A special intraparenchemal catheter measuring ICP and $\mathrm{PbtO}_{2}$ on real time was inserted in all the patients of our study. In 31 patients $(70.5 \%)$ the catheter was placed in the injury side and in 13 (29.5\%) it was placed either in the opposite side or in diffused injured brain. The total time of $\mathrm{PbtO}_{2}$ below the threshold value of $10 \mathrm{mmHg}$ was measured and a favorable outcome of GCS 13-15 during discharge was examined.

Results. After statistical analysis of values measured, the median time of $\mathrm{PbtO}_{2}<10 \mathrm{mmHg}$ in underlying injured brain was $19 \mathrm{~h}$ (range 0-96) compared to $0 \mathrm{~h}$ (range $0-16)$ in normal appearing brain $(\mathrm{p}<0.05)$. A number of 30 patients $(68.2 \%)$ had a favorable outcome when discharged from ICU compared to $14(31.6 \%)$ who had a severe or 
moderate neurological outcome after the first resuscitation, needing a prolonged hospitalization and time of recovery. A significant $(p<0.05)$ negative association was found between time of $\mathrm{PbtO}_{2}<10 \mathrm{mmHg}$ and favorable outcome, $95 \% \mathrm{Cl}(0.95,0.99)$. In subgroup analysis, patients with catheter placement in injured brain had a significant $(p<0.05)$ lower total time of $\mathrm{PbtO}_{2}<10 \mathrm{mmHg}$ when the outcome was favorable compared to those with worse outcome (median time $45 \mathrm{~h}$ vs $16 \mathrm{~h}$ ). In the group of catheter placement in the non-injured side, an association between $\mathrm{PbtO}_{2}$ time below the threshold of $10 \mathrm{mmHg}$ and outcome could not be found.

Conclusion. Our data suggest that the side of probe placement in patients with traumatic brain injury can be an indicator of prolonged brain tissue hypoxia and is associated with early outcome during discharge from ICU.

\section{Reference(s)}

1. 1. Lucido L, Shibu P, Jovany C, Xiaoqi L, H. Julia, Shankar G et al. Position of probe determines prognostic information of brain tissue $\mathrm{PO} 2$ in severe traumatic brain injury. Neurosurgery. 2012;70(6):1492-1503.

2. 1. Oddo M, Levine JM, Mackenzie L, Frangos S, Feihl F, Kasner SE et al. Brain hypoxia is associated with short-term outcome after severe traumatic brain injury independently of intracranial hypertension and low cerebral perfusion pressure. Neurosurgery. 2011;69(5):1037-45.

\section{1}

Acute Bacterial Meningitis Risk Factors in Patients with Craniocerebral Gunshot Injuries

H. Sood ${ }^{1}$; A. Daneshmand, ${ }^{1}$; A. Cervantes, ${ }^{1}$; J. Yang, ${ }_{1}^{1}$; T. Brahmbatt, ${ }^{2}$; K. Yeh, ${ }^{3} ;$ C. Rupp, ${ }^{4} ;$ H. Dasenbrock, ${ }^{5}$; A. Nozari, ${ }^{4} ;$ K. Suchdev,

${ }^{1}$ Neurology, Boston University School of Medicine, Boston, United States of America; ${ }^{2}$ Surgery, Boston University School of Medicine, Boston, United States of America; ${ }^{3}$ Pharmacy, Boston University School of Medicine, Boston, United States of America; ${ }^{4}$ Anesthesiologist, Boston University School of Medicine, Boston, United States of America; ${ }^{5}$ Neurosurgery, Boston University School of Medicine, Boston, United States of America Correspondence: $\mathrm{H}$. Sood

Intensive Care Medicine Experimental 2021, 9(1): 001341

Introduction. Craniocerebral Gunshot Injuries (CGI) can predispose patients to acute bacterial meningitis (ABM) because of the possible spread of bacteria from the skin or contaminated spaces (sinuses, nasopharynx or middle ear) to CNS. Antibiotics are often given prophylactically, although the evidence to support their utility is limited.

Objectives. In our study, we sought to determine the incidence of meningitis, use of prophylactic antibiotics, and risk factors for patients who developed ABM after CGI.

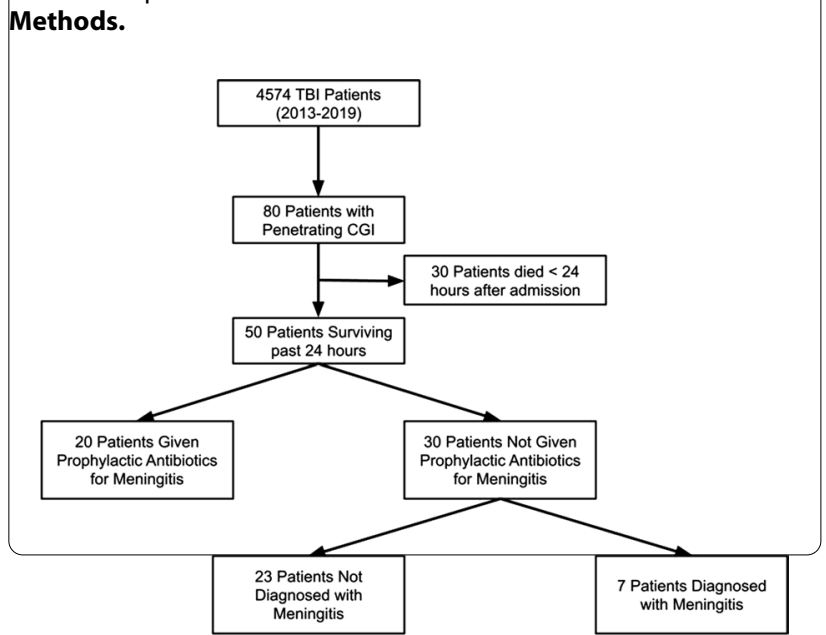

A retrospective chart review was performed of all patients who were admitted with a diagnosis of traumatic brain injury to an urban level 1 trauma center from January 1st, 2013 to December 31st, 2019 and patients with CGI were identified. All patients with CGI were screened for ABM within six weeks following admission, based on cerebrospinal fluid (CSF) analysis and neuroimaging. We also recorded demographic data, medical history, use of prophylactic antibiotics, incidence of skull fractures, CSF leaks, and neurosurgical procedures.

Results. Of 4,574 patients presenting with traumatic brain injury, 80 patients $(1.75 \%)$ had CGl. Of those, 50 patients $(62.50 \%)$ survived past the first 24 h. 20 out of these 50 patients were given prophylactic antibiotics for meningitis, ranging from 3-10 days (mean $=5.6$ days). Of the remaining 30 patients, 7 (23.33\%) were diagnosed with ABM based on neuroimaging in one patient, and CSF findings for the rest. Their age ranged from 17-40 years, and six of the seven patients were male. Skull fractures with CSF leak on presentation were present in 3 out of $7(42.86 \%)$ patients, and all 3 underwent a decompressive craniectomy for refractory intracranial pressure (ICP), within the first week of admission. Moreover, 5 out of the 7 patients had an external ventriculostomy drain (EVD) or ICP monitor placement. 1 out of 7 patients $(14.28 \%)$ died during the hospital stay whereas all others were discharged to rehab. Amongst the patients who received prophylactic antibiotics, none had a CSF leak and only 2 had an ICP monitor placed. Conclusion. We found that amongst 4574 TBI patients, the incidence of $A B M$ was $23.33 \%$ in $C G$ patients who did not receive antibiotics. Development of ABM was associated with the presence of CSF leaks or undergoing neurosurgical procedures. Patients with a CSF leak had a statistically significant increased odds ratio (OR) of developing ABM compared to patients without a CSF leak (OR 6.50,95\% Cl 1.35, 201.29). Similarly, patients undergoing neurosurgical procedures had a higher risk of developing ABM (OR 26.25, 95\% CI 2.94, 234.37). Our data suggest that these may be independent risk factors for developing meningitis and that this subset of patients with CGI may need antibiotic prophylaxis. Future prospective studies, especially in CGI patients with CSF leaks and $\mathrm{EVD/ICP}$ monitor placements, are needed to confirm our findings.

\section{Reference(s)}

1. Alvis-Miranda, H. R., M Rubiano, A., Agrawal, A., Rojas, A., Moscote-Salazar, L. R., Satyarthee, G. D., Calderon-Miranda, W. G., Hernandez, N. E., \& Zabaleta-Churio, N. (2016). Craniocerebral Gunshot Injuries; A Review of the Current Literature. Bulletin of emergency and trauma, 4(2), 65-74.

2. Button, K., loannidis, J., Mokrysz, C. et al. Power failure: why small sample size undermines the reliability of neuroscience. Nat Rev Neurosci 14, 365-376 (2013). https://doi.org/10.1038/nrn3475

3. Gönül, E., Baysefer, A., Kahraman, S., Ciklatekerlioğlu, O., Gezen, F., Yayla, O., \& Seber, N. (1997). Causes of infections and management results in penetrating craniocerebral injuries. Neurosurgical review, 20(3), 177-181. https://doi.org/10.1007/BF01105561

4. Jimenez, C. M., Polo, J., \& España, J. A. (2013). Risk factors for intracranial infection secondary to penetrating craniocerebral gunshot wounds in civilian practice. World neurosurgery, 79(5-6), 749-755. https://doi.org/ 10.1016/j.wneu.2012.06.025

5. Kim, T. W., Lee, J. K., Moon, K. S., Kwak, H. J., Joo, S. P., Kim, J. H., \& Kim, S. H. (2007). Penetrating gunshot injuries to the brain. The Journal of trauma, 62(6), 1446-1451.https://doi.org/10.1097/01.ta.0000222909.31666.db

001350

Chemical Vs. chemical and mechanical venothromoembolism prophylaxis in neurocritically ill patients

A. Alshaya ${ }^{1}$; F. Alanazi, ${ }^{1}$; R. Alzoman ${ }^{1}$; R. Faden, ${ }^{1}$; H. Alyahya ${ }^{1}$; O. Alshaya ${ }^{1}$

${ }^{1}$ Pharmacy Practice, King Saud bin Abdulaziz University for Health Sciences, Riyadh, Saudi Arabia

Correspondence: A. Alshaya

Intensive Care Medicine Experimental 2021, 9(1): 001350

Introduction. Patients admitted with neurocritical illness are presumed to be at high risk for venothromboembolism (VTE). The administration of chemical and/or mechanical VTE prophylaxis is a common practice in critically ill patients. Recent data did not show a significant 
difference in the incidence of VTE between chemical compared to chemical and mechanical VTE prophylaxis in critically ill patients with limited data in neurocritically ill population.

Objectives. To investigate the incidence of VTE between chemical alone compared to chemical and mechanical VTE prophylaxis in neurocritically ill patients.

Methods. Retrospective cohort study at a tertiary teaching hospital. Data were obtained from electronic medical records for all patients admitted with neurocritical illness from $1 / 1 / 2016$ to $1 / 12 / 2020$. Patients were excluded if they did not receive VTE prophylaxis during admission or were younger than 18 YO. The major outcome was symptomatic VTE based on clinical and radiological findings. Minor endpoints included severe or life-threatening bleeding based on GUSTO, intensive care unit (ICU) length of stay (LOS), and mortality at 28-days. Results. Two hundred and twelve patients were included in this study. Patients did not have any significant differences in their baseline characteristics. The incidence of VTE was not statistically significant between chemical only compared to chemical and mechanical VTE prophylaxis groups $(19 / 166(11.3 \%)$ vs $7 / 46(15.2 \%) ; P=0.49$. No difference between groups in their ICU LOS 6 [3-16.2] vs 6.5 [3-19]; $P=0.52$, nor their mortality $(18 / 166$ $(10.7 \%)$ vs $3 / 46(6.5 \%) ; P=0.38$, respectively. Less bleeding events were seen in the chemical prophylaxis group compared to the combined VTE prophylaxis group (19/166 (11.3\%) vs $12 / 46(26.1 \%) ; P=0.013$.

Conclusion. Our findings observed no difference between the administration of chemical prophylaxis alone compared to combined VTE prophylaxis in neurocritically ill patients. More data are needed to confirm this finding with more roboust methodology.

\section{Reference(s)}

1. Nyquist P, Bautista C, Jichici D, Burns J, Chhangani S, DeFilippis M, Goldenberg FD, Kim K, Liu-DeRyke X, Mack W, Meyer K. Prophylaxis of Venous Thrombosis in Neurocritical Care Patients: An Evidence-Based Guideline: A Statement for Healthcare Professionals from the Neurocritical Care Society. Neurocrit Care. 2016 Feb;24(1):47-60.

2. Cook D, Crowther M, Meade M, Rabbat C, Griffith L, Schiff D, Geerts W, Guyatt G. Deep venous thrombosis in medical-surgical critically ill patients: prevalence, incidence, and risk factors. Crit Care Med. 2005 Jul:33(7):1565-71.

3. Guyatt GH, AkI EA, Crowther M, Gutterman DD, Schuunemann HJ. Executive summary: Antithrombotic therapy and prevention of thrombosis, 9th ed. American college of chest physicians evidence-based clinical practice guidelines. Chest. 2012;141:75-47.

4. Arabi YM, Al-Hameed F, Burns KEA, et al. Adjunctive Intermittent Pneumatic Compression for Venous Thromboprophylaxis. N Engl J Med 2019;380:1305-15. https://doi.org/10.1056/NEJMoa1816150

\section{3}

\section{Sodium homeostasis in patients with traumatic brain injury} and its impact on mortality

N. Gaibino'; IC. Medeiros²; P. Mota ${ }^{3}$; SM. Fernandes ${ }^{1}$;. Moniz'; J. Ribeiro

${ }^{1}$ Serviço de medicina intensiva, Centro Hospitalar Universitário Lisboa Norte-Hospital de Santa Maria, Lisbon, Portugal: ${ }^{2}$ Fmul, FMUL, Lisbon, Portugal; ${ }^{3}$ Serviço de medicina intensiva, Hospital da Luz, Lisbon, Portugal Correspondence: N. Gaibino

Intensive Care Medicine Experimental 2021, 9(1): 001353

Introduction. Dysregulation of sodium homeostasis is associated with a higher risk of mortality in traumatic brain injury (TBI). Recently it has been reported that sodium shifts, even within its normal range, can have an impact on mortality, even though its prognostic value is still misunderstood. We hypothesize that sodium shifts during the first few days after moderate or severe TBI might be associated with mortality in this population.

Methods. Observational and retrospective study that included all patients with moderate to severe TBI admitted to a Neurocritical Intensive Care Unit (ICU) in a large urban, referral university hospital, between January 2017 and April 2019. We collected values of sodium obtained at morning evaluation in all patients at 24,48 and $72 \mathrm{~h}$ after ICU admission. Demographic data and survival was also documented.
Results. Eighty-six patients were identified with moderate (20\%) to severe $(80 \%) \mathrm{TBI}, 40 \%$ of which had also other trauma lesions. They were predominantly of male gender, with a mean age of $50 \pm 17$ years and $57 \%$ with at least one relevant comorbidity. At ICU admission they had a mean GCS of $8.2 \pm 3.1$ and a SAPS II score of 46.1. $\pm 20.0 .98 \%$ of patients were under invasive mechanical ventilation. Among the studied population $54 \%$ had intracranial pressure monitoring, 39\% were submitted to decompressive craniectomy and in $27 \%$ an external ventricular drain was placed. Pharmacological treatment was initiated in $9 \%$ of patients with thiopental and in $13 \%$ with mannitol.

Global ICU mortality at day 30 after TBI was $27 \%$. Sodium levels at admission were between $126-154 \mathrm{mmol} / \mathrm{L}$, with a mean of $139 \mathrm{mmol} / \mathrm{L}$. When we searched for sodium shifts, we found a higher sodium shift up to $72 \mathrm{~h}$ after admission $(8.2 \mathrm{mmol} / \mathrm{L})$ in patients who died when compared to sodium shift up to $72 \mathrm{~h}$ after admission $(3.1 \mathrm{mmol} / \mathrm{L})$ in patients who survived $(p=0.02)$. This difference was also seen for sodium shifts at $24(p=0.002)$ and $48 \mathrm{~h}(p=0.004)$ after admission. In a multivariate logistic regression, sodium shifts at $72 \mathrm{~h}$ were still associated with a mortality increase at 30 days after adjusting for SAPS II, administration of thiopental or mannitol to control elevated intracranial pressure, intracranial pressure monitoring or need for decompressive craniectomy.

Conclusion. In patients with moderate to severe TBI admitted to a neurocritical ICU, minimal sodium shift seems to be an independent mortality predictor. We believe that monitoring sodium shifts may affect decisions concerning the treatment of severe TBI patients, namely in face of elevated intracranial pressure.

\section{5}

Brain Connectivity Changes and Functional Recovery

after Aneurysmal Subarachnoid Hemorrhage

C. Weiner'; S. Nelson²; R. Stevens

'Department of Biomedical Engineering, Johns Hopkins University, Baltimore, United States of America; ${ }^{2}$ Departments of Neurosurgery and Neurology, Mount Sinai Health System, New York, United States of America; ${ }^{3}$ Anesthesiology and Critical Care Medicine, Johns Hopkins University, Baltimore, United States of America

Correspondence: R. Stevens

Intensive Care Medicine Experimental 2021, 9(1): 001355

Introduction. Aneurysmal subarachnoid hemorrhage (aSAH) is associated with high morbidity and mortality despite advances in management. Accurate methods to prognosticate in aSAH are lacking. Here, we used graph theory to analyze magnetic resonance diffusion tensor imaging (DTI) obtained from aSAH patients in the acute setting and evaluated its association with functional outcome and mortality at 6 months.

Objectives. The aim of this study was to predict 6-month aSAH outcome using a composite model that combines clinical and radiographic (graph theoretic) features.

Methods. We enrolled a prospective cohort of aSAH patients admitted to the ICU at a single institution. Clinical data abstracted included age, sex, days elapsed between SAH onset and MRI scan, admission World Federation of Neurosurgical Societies (WFNS) score, hydrocephalus presence, and presence of delayed cerebral ischemia. All patients underwent DTI as part of a multimodal MRI scan acquired during their acute hospitalization. Image volumes were preprocessed and used to generate a weighted undirected structural connectome of each patient's brain using 176 ROIs defined by the Johns Hopkins Eve atlas [1]. Regions of interest (ROls) were sorted into 4 groups: Default Mode Network (DMN), Executive Control Network (ECN), Salience Network (SAL), and Whole Brain. We calculated graph theoretic connectome features including Node Strength (NS), Betweenness Centrality (BC), Network Degree (ND), and Connectedness (C). Clinical and DTI graph features were used independently and in combination to train Random Forest (RF) and Logistic Regression classifiers to predict the dichotomized modified Rankin Score (mRS) at discharge and at six months after discharge (favorable outcome mRS 0-2, unfavorable outcome mRS 3-6). 
Results.

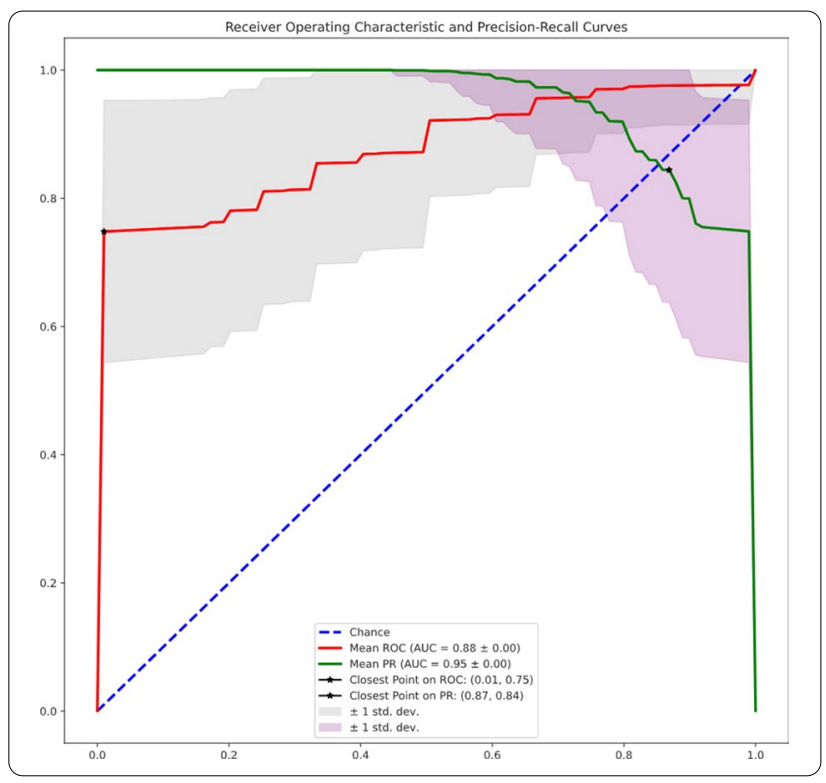

A total of 56 aSAH patients were enrolled in the cohort and underwent $\mathrm{DTI}$ a median (IQR) of $7(\mathrm{IQR}=8.5)$ days after admission. The best performing model (RF) combining clinical and DTI graph features had a mean (AUROC) of $0.880 \pm 0.001$ and an Area Under the Precision Recall Curve (AUPRC) of $0.950 \pm 0.001$ over the same 500 trials. This model was significantly more discriminative than the clinical model alone (AUROC of $0.810 \pm 0.011$, AUPRC of $0.910 \pm 0.001$ ). The highest ranked graph features for prediction were NS, BC, and ND.

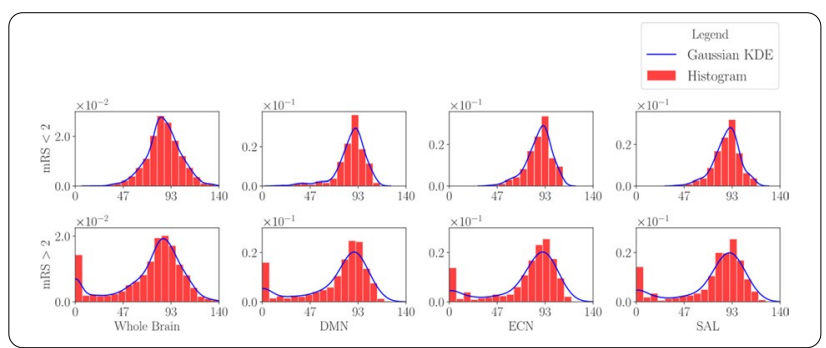

Conclusion. aSAH prognostic models performed best when informed by both radiographic metrics and clinical covariates. The highly predictive DTI graph features suggest a dynamic process of structural connectivity reorganization occurring in the early phase after aSAH. These results warrant prospective validation and integration with expanded feature domains such as neurophysiological time series.

\section{Reference(s)}

1. Atlas-based whole brain white matter analysis using large deformation diffeomorphic metric mapping: application to normal elderly and Alzheimer's disease participants. Oishi K, Faria A, Jiang H, Li X, Akhter K, Zhang J, Hsu JT, Miller MI, van Zijl PC, Albert M, Lyketsos CG, Woods R, Toga AW, Pike GB, Rosa-Neto P, Evans A, Mazziotta J, Mori S. Neuroimage. 2009 Jun;46(2):486-99.
001368

Cerebral autoregulation indexes in septic patients: Are they all the same?

J. Caldas, ${ }^{1}$; AA. Quispe-Cornejo ${ }^{2}$; IA. Crippa ${ }^{2}$; J. Creteur ${ }^{2}$; JL. Vincent ${ }^{2}$; R. Panerai ${ }^{3}$; FS. Taccone ${ }^{2}$

${ }^{1}$ Critical Care Unit, Escola Bahiana de Medicina e Saude Publica, Universidade de Salvador, UNIFACS, Salvador, Brazil; ${ }^{2}$ Department of Intensive Care, Erasme University Hospital, Brussels, Belgium; ${ }^{3}$ Department of Cardiovascular Sciences, University of Leicester \& NIHR Leicester Biomedical Research Centre, Leicester, United Kingdom

Correspondence: A.A. Quispe-Cornejo

Intensive Care Medicine Experimental 2021, 9(1): 001368

Introduction. Several studies have shown that sepsis is often associated with impaired cerebral autoregulation (CAR), which can predict the occurrence of brain dysfunction. However, different indexes are available, and may yield different results.

Objectives. To assess the agreement of two validated CAR indexes to categorize autoregulation in septic patients.

Methods. Prospectively collected data of septic patients (all with an arterial catheter) without intracranial disease, severe arrhythmias, treatment with extracorporeal membrane oxygenation or supra-aortic arteriopathy. Transcranial Doppler (DWL, Germany) was performed insonating the left middle cerebral artery (LMCA) with a 2-MHz probe. LMCA blood flow velocity (BFV) and arterial pressure (AP) signals were simultaneously recorded for at least 6 min; Pearson's correlation coefficient between AP and FV (Mean flow index, Mxa) was calculated using MATLAB (MathWorks, USA). Using the same recording, transfer function analysis of spontaneous fluctuations in AP and BFV was performed to estimate the ARI using custom FORTRAN software. Impaired CAR was defined as Mxa $>0.3$ and ARI $\leq 4$, respectively. The Cohen's kappa coefficient was used to measure the agreement between the two indexes.

Results. We studied 100 patients (median age 69 [60-78]) for a total of 132 recordings. Median Mxa and ARI were 0.32 [0.14-0.51] and 4.63 [2.99-6.21], respectively. The number of recordings showing impaired CAR for Mxa and ARI were $63(48 \%)$ and $49(37 \%)$, respectively. Mxa and ARI classified both impaired or intact CAR in 37 and 18 recordings, respectively. The Cohen's kappa coefficient was therefore 0.177 , suggesting poor agreement.

Conclusion. ARI and Mxa are not interchangeable to assess CAR in septic patients. The identification of different thresholds for impaired CAR with Mxa and ARI might lead to improved agreement.

001399

Endovascular embolization of an intracerebral aneurysm and a lost cerebral coil

S. Nissar'; 'S. NAWAZZ2; A. Sumayya2 ${ }^{2}$; A. Ayesha ${ }^{3}$; T. Abdulnasser ${ }^{3}$

${ }^{1}$ Sicu, Hamad Medical Corporation, Doha, Qatar; ${ }^{2}$ Medical Education, Hamad Medical Corporation, Doha, Qatar; ${ }^{3}$ Neurosurgery, Hamad Medi-

cal Corporation, Doha, Qatar

Correspondence: S. NAWAZ

Intensive Care Medicine Experimental 2021, 9(1): 001399

Introduction. Endovascular embolization of ruptured and unruptured cerebral aneurysm is common in clinical practice. The rare complication of endovascular embolization is coil misplacement. Most of the reported coil misplacement occurs due to premature release of coil into the cerebral circulation. We report a case of lost coil into the peripheral circulation due to failure to detach from the micro catheter at the right place.

Case: A young lady with no previous medical history, presented to the emergency department with worst headache of her life. She consciousness level deteriorated, and her GCS dropped to 5, requiring immediate intubation, sedation and ventilation. Computer tomography and angiography showed a giant supraclinoid aneurysm of left internal carotid artery. She was taken for endovascular coiling under General anesthesia and one coil was successfully inserted in the aneurysm. During the insertion of the second coil there was failure to get 
the coil detached from the microcatheter; and at withdrawal the coil got stuck at the level of carotid artery bifurcation. After several failed attempts to remove it, the coil was swept away into the general circulation and went undetected on fluoroscopy. Next day extremity x-ray showed coil in the left popliteal artery. Patient was taken for angiography; the misplaced coil was successfully removed, and the aneurysm was completely embolised. Her condition improved with supportive care and by day 14 she started to obey commands and was successfully extubated. She was transferred to the ward and discharged home after another week.

Methods. A retrospective case report of a rare complication and its outcome after endovascular embolization.

Results. Endovascular coiling has become a mainstay in the treatment of ruptured cerebral aneurysms due obvious benefits which include but are not limited to having a less invasive procedure and better clinical outcomes. 4 The main complications of endovascular embolization of cerebral aneurysms are rebleeding due to perforation (2.3 to 4.7\%), thromboembolism (2.5 to $14.5 \%$ ), parent artery occlusion (2 to $3 \%$ ), collapse of GDC $(8 \%)$ and protruding, migration or misplacement $(0.5$ to 3\%)0.5 These cerebral coil migration and misplacement can cause ischemic stroke or cause organ ischemia and infarctions.4 The aneurysm related risk factors for the coil protrusion, migration or misplacement are having a wide neck, large or very small aneurysms.6 The reported coil risk factors for migration and misplacement are insertion of small, flexible wires and tightly spiralled ones with diameter of around $1.5 \mathrm{~mm} .7$ Majority of cerebral coil misplacement or displacement occur in the cerebral circulation and one case of misplacement in the peroneal artery is reported in the literature. 6

In our patient the coil was difficult to detach from the micro catheter and during withdrawal the coil got stuck at the carotid artery bifurcation. Several attempt to remove the coil with snare led to losing it in the general circulation. Immediate fluoroscopy search failed to detect the coil in head, neck, thorax and abdomen. Fortunately, we were able to detect the lost coil in the left popliteal artery with $x$-ray without any embolic complications. In the follow-up angiography, the lost misplaced coil was successfully removed, and complete embolization of the aneurysm was achieved. Patient's follow-up angiography and outpatient clinic visits did not show any abnormality.

Conclusion. Concluding lines from our case are that coil can fail to be detached from the micro catheter and can get swept away in the general circulation with embolization in the peripheral circulation. Early removal of these misplaced coils is the key to avoid thromboembolic complications.

\section{Reference(s)}

1. 1. Guglielmi G. Guglielmi detachable coils. J Neurointerv Surg 2018; 10:e5

2. 2. Bekelis K, Gottlieb DJ, Su Y, et al. Comparison of clipping and coiling in elderly patients with unruptured cerebral aneurysms. J Neurosurg. 2017; 126(3):811-818

3. 3. Stidd DA, Johnson AK, Lopes DK. Manual Aspiration Technique to Retrieve a Prematurely Detached Coil during Cerebral Aneurysm Embolization. Neurointervention. 2014; 9(1):21-25

4. 4. Singh DP, Kwon SC, Huang L, Lee WJ. Retrieval of Distally Migrated Coils with Detachable Intracranial Stent during Coil Embolization of Cerebral Aneurysm. J Cerebrovasc Endovasc Neurosurg. 2016; 18(1):48-54

5. 5. Gatto LAM, Rocha LB, Koppe GL, Demartini Jr Z. Late coil migration after embolization of cerebral aneurysms-case series. Arq Bras Neurocir 2018; $37: 71-5$

6. 6. Nikoubashman O. Endovascular retrieval of a dislocated coil in the peroneal artery with a stent retriever. BJR 2015; $2: 1-2$

7. 7. Abe T, Hirohata M, Tanaka N, et al. Coil migration, malposition, stretching and retrieval. Interv Neuroradiol. 2000; 6 Suppl 1(Suppl 1):143-147
e-Posters: Nursing care and physiotherapy

\section{5}

Is targeted low tidal volume ventilation alone an effective method to achieve satisfactory driving pressure in mechanically ventilated patients with COVID-19?

A. Harriman"; J. Weblin ${ }^{2}$

${ }^{1}$ Therapy Services, Queen Elizabeth Hospital Birmingham, Birmingham, United Kingdom; ${ }^{2}$ Consultant Physiotherapist Critical Care, Queen Elizabeth Hospital Birmingham, Birmingham, United Kingdom

Correspondence: A. Harriman

Intensive Care Medicine Experimental 2021, 9(1): 000085

Introduction. Current evidence-based management for lung protective ventilation (LPV) in ARDS advocates the delivery of low tidal volumes (TV) and limited airway pressures $\left(<30 \mathrm{cmH}_{2} \mathrm{O}\right)(1)$. Recent focus has emphasised the importance of driving pressure (DP) in mechanical ventilation, with pressures above $15 \mathrm{cmH}_{2} \mathrm{O}$ being associated with increased mortality (2). Strategically targeted DP has not yet shown to be feasible in clinical practice, with low TV ventilation being the mainstay of LPV strategy.

Objectives. We aim to establish whether the current practice of targeted low TV ventilation of $6-8 \mathrm{ml} / \mathrm{kg}$ is an effective method alone in maintaining DP of less than $15 \mathrm{cmH}_{2} \mathrm{O}$ in mechanically ventilated patients with COVID-19.

Methods. Ventilation parameters including TV's, peak airway pressure, plateau pressure and DP was recorded once daily on a single COVID19 Intensive Care Unit at Queen Elizabeth Hospital Birmingham. 110 episodes were recorded from 34 patients over 5 days. TV's within 6-8 $\mathrm{ml} / \mathrm{kg}$ of ideal body weight and DP of $<15 \mathrm{cmH}_{2} \mathrm{O}$ was noted to be compliant.

Results. Target TV's were achieved in 78 (70.9\%) episodes, whilst target DP was achieved in $88(80 \%)$ episodes, with no significant difference in compliance between the two parameters $(P=0.558)$. ABG's at the time of data collection indicated respiratory acidosis of a $\mathrm{pH}<7.25$ on only 2 occasions. This may indicate overventilation, especially in the presence of elevated DP or non-compliant volumes; this was not investigated further at the time. When adherence to target TV's was achieved, DP was $<15 \mathrm{cmH}_{2} \mathrm{O}$ in $78.2 \%$ of cases. When DP $>15 \mathrm{cmH}_{2} \mathrm{O}$, peak airway pressure exceeded $30 \mathrm{cmH}_{2} \mathrm{O}$ in 14/22 cases, with plateau pressure $>30 \mathrm{cmH}_{2} \mathrm{O}$ on 7 on those occasions. DP was satisfactory in 11 cases in which TV's exceeded the limits of $6-8 \mathrm{ml} / \mathrm{kg}$.

Conclusion. Within the limitations of study size, compliance with targeted DP and TV target was found to be statistically similar. Adherence to patient specific TV targets alone may be an adequate method in maintaining satisfactory DP in the majority of ventilated patients. However, in the presence of significantly elevated airway pressures, the ability to achieve both TV and DP targets may not always be possible. In such incidences, personalised approach to LPV is warranted. Further research is necessary to establish whether low TV ventilation consistently results in satisfactory DP, and whether compliance with both parameters can be increased simultaneously.

\section{Reference(s)}

1. 2. Aoyama, H., Yamada, Y., \& Fan, E. (2018). The future of driving pressure: A primary goal for mechanical ventilation? 11 Medical and Health Sciences 1102 Cardiorespiratory Medicine and Haematology. Journal of Intensive Care, 6(1), 4-7.

2. Amato, M. B. P., Meade, M. O., Slutsky, A. S., Brochard, L., Costa, E. L. V, Schoenfeld, D. A., Stewart, T. E., Briel, M., Talmor, D., Mercat, A., Richard, J.-C. M., Carvalho, C. R. R., \& Brower, R. G. (2015). Driving Pressure and Survival in the Acute Respiratory Distress Syndrome. New England Journal of Medicine, 372(8), 747-755. 


\section{6}

Measurement of ulnar nerve stiffness with elastography in the prone position

E. Hernandez Gonzalez'; J. Izquierdo García ; M. Sanz Sanchezz; C. Román Moraleda'; E. Zamorano Zarate ${ }^{2}$; C. ÁLvarez Trimiño'; MD. Hungría Rodriguez'; A. Muñoz Rodriguez'; I. González Pizarro'; C. López López; H. Albertos Domínguez'; M. Briones Cantero'; JM. Rodríguez Gómez'; MC. Pérez Muñoz; N. García Blanco'; MZ. Pintos Díaz'; MP. Sanz Ayan4; M. Lopez Saez ${ }^{4}$; JC. Montejo-Gonzalez'; JI. Castillo Martín ${ }^{4}$

${ }^{1}$ Physiotherapy, University Hospital 12 de Octubre, Madrid, Spain; ${ }^{2}$ Physiotherapy, Fisioterapia Instituto Terapia y Movimiento, Madrid, Spain

${ }^{3}$ Nurse, University Hospital 12 de Octubre, Madrid, Spain; ${ }^{4}$ Rehabilitation, University Hospital 12 de Octubre, Madrid, Spain; ${ }^{5}$ Intensive care, University Hospital 12 de Octubre, Madrid, Spain

Correspondence: J. izquierdo garcia

Intensive Care Medicine Experimental 2021, 9(1): 000116

Introduction. Musculosketal sequels have been observed in patients with covid-19 admitted to a critical care unit requiring prone positioning. Shoulder stiffness and ulnar nerve compression neuropathy at the elbow is one of the most prevalent symptoms $\mathbf{1 , 2}$.

Ultrasond elastography measures in a non invasive manner tissue stiffness and mechanical changes in tissue properties. Mechanical shear waves used for elastography travel 1 to $10 \mathrm{~m} / \mathrm{second}(\mathrm{m} / \mathrm{s})$. This propagation speed depends on the stiffness of the tissue. The ultrasond machine translate $\mathrm{m} / \mathrm{s}$ into kilopascals $(\mathrm{kpa})$ which makes it posible to quantify the stiffness of the neural tissue3,4.

Objectives. Identify using elastography which prone position produces more stiffness and/or tension at the ulnar nerve level.

Methods. To carry out this study, 79 healthy volunteers, 27 men and 52 women, were evaluated.

$65 \%$ of the sample group is between $40-60$ years old.

The measurement is perfomed in the epitrochleo-olecranial canal of the elbow in the longitudinal plane of the ulnar nerve by placing the notch and edge of the probe in the axis between the olecranon and epitrochlea. The elastography probe is SHEAR WAVE.

Ulnar nerve measurements in 5 positions on each patient were performed. In each position, 3 measurements were taken along the path of the ulnar nerve in each positions. Measurements were taken in kpa. Then the average of measures 1, 2 and 3 were calculated.

Position 1: in a standing position.

Position 2: in a prone position with a $90^{\circ}$ shoulder abduction, a $90^{\circ}$ elbow flexion, and a homolateral rotation of the head.

Position 3: in a prone position with a $90^{\circ}$ shoulder abduction, a $90^{\circ}$ elbow flexion, and a counter lateral rotation of the head.

Position 4: in a prone position with arms along the body, and a homolateral rotation of the head.

Position 5: in a prone position with arms along the body and a counter rotation of the head.

Results. Position 1: 21,47 kpa.

Position 2: 42,21 kpa.

Position 3: 38,60 kpa.

Position 4: 31,49 kpa.

Position 5: 31,74 kpa.

Conclusion.

- According to the results obtained, positions 2 and 3 should be avoided, while positions 4 and 5 are recommend.

- Alternating forearm pronation and external and internal shoulder rotations are proposed as a postural treatment.

- Passive kinesitherapy on both limbs and the tensioning and gliding of the ulnar nerve are proposed as physioterapeutic treatments.

\section{Reference(s)}

1. Taljanovic, Mihra S et al. "Shear-Wave Elastography: Basic Physics and Musculoskeletal Applications." Radiographics: a review publication of the Radiological Society of North America, Inc vol. 37,3. 855-870. 2017, https://doi.org/10.1148/rg.2017160116
2. Sigrist, Rosa M S et al. "Ultrasound Elastography: Review of Techniques and Clinical Applications."Theranostics: vol. 7,5 1303-1329. 7 Mar. 2017, https://doi.org/10.7150/thno.18650

3. Schmid, Annina et al. "Entrapment neuropathies: a contemporary approach to pathophysiology, clinical assessment, and management", PAIN Reports: vol. 5-Issue 4-p e829. 2020, https://doi.org/10.1097/PR9. 0000000000000829

4. Munshi et al. "Prone position for acute respiratory distress syndrome: A systematic review and meta-análisis", Annals of the American Thoracic Society. 2017, https://doi.org/10.1513/AnnalsATS.201704-3430T. 5. No.

\section{1}

Multidisciplinary approach.Rehabilitation as a central team in the care of the ICU patients

D. Terzo ${ }^{1}$; E. Conoscenti ${ }^{2}$; G. Provenzale ${ }^{3}$; G. Martucci ${ }^{4}$; S. Blot ${ }^{5}$; FM. Bondi ${ }^{6}$. G. Enea ${ }^{1}$; G. Arena ${ }^{7}$; F. Tuzzolino ${ }^{8}$; A. Pietrosi ${ }^{9}$; A. Arcadipane ${ }^{10}$; A. Luca ${ }^{11}$ ${ }^{1}$ Rehabilitation Department, ISMETT, Palermo, Italy; ${ }^{2}$ Infection Control Department, ISMETT, Palermo, Italy; ${ }^{3}$ Senior Reporting System controller, ISMETT, Palermo, Italy; ${ }^{4}$ Anesthesia and intensive care unit, ISMETT, Palermo, Italy; ${ }^{5}$ Internal Medicine department, Ghent University Hospital, Gent, Belgium; ${ }^{6}$ Magnet program director, ISMETT, Palermo, Italy; ${ }^{7}$ Chief of nursing, ISMETT, Palermo, Italy; ${ }^{8}$ Statistician and Data Manager Department, IRCCS-ISMETT, Palermo, Italy; ${ }^{9}$ Director Management control and Decision Support Department, ISMETT, Palermo, Italy; ${ }^{10}$ Anesthesia and Intensive Care Unit, IRCCS-ISMETT, Palermo, Italy; ${ }^{11}$ General director, ISMETT, Palermo, Italy

Correspondence: D. Terzo

Intensive Care Medicine Experimental 2021, 9(1): 000141

Introduction. Early rehabilitation programs can reduce complications and patient length of stay and facilitate a faster turnover of ICU patients.

Objectives. Descriptive report of the activities of the Rehabilitation Service (RS) at IRCCS-ISMETT and the impact of early mobilization and fast-track programs on the average length of stay (ALOS).

Methods. The RS activity as described (2013-2019) considering the number of treatments and quality indicators.Moreover, for six of the most common DRG (Diagnosis Related Group) procedures, the hospital ALOS was compared to the Italian annual report of hospital discharges.

Results. ISMETT is a continuously growing center with a high complexity of care delivered to critical care patients.The hospital's capacity was 78 beds from 2013 to 2018, and 107 beds from 2019.The average age of the patients described is 58.1 (SD 19.1).Over $64.7 \%$ of inpatients were male.The complexity of care delivered was considerably high with an average DRG weight of 3.12 (SD 3.56, median 1.91, IQR 1.18-3.56) between 2013 and 2016 and an average increase of 3.63 (SD 3.94, median 2.40, IQR 1.18-4.65) between 2017 and 2019.The average number of occupied beds was $72.5 \%$ in $2013-2016$ and $78.2 \%$ from 2017 to 2019 . The RS team is committed to patient care and has a crucial role during all stages of the patient's hospitalization. Their commitment for the patients' clinical recovery stands out with 163,404 respiratory (RT) and physical (PT) treatments provided during 7-year span. A total of 73,263 RT and PT treatments were performed between 2017 and 2019 of which 62,247 on surgical patients, and 17,080 h (27.5\%) were spent in the ICU. From 2013, the RS team has had an important impact on surgical patient care with $94.3 \%$ of patients receiving at least one PT or RT treatment. From $2017,66 \%$ of surgical patients were treated in the first $24 \mathrm{~h}$ compared to previous years when only $45.8 \%$ (on average) were treated the same day of surgery.The combination of the fast-track protocol and the progressive mobility program supported by a "mobility" culture within the hospital, allowed the RS to provide 3,188 physical treatments with a percentage of 29.5 (28.6-30.4 $\mathrm{Cl} 95 \%$ ) of patients reaching a "sitting" position (2017 to 2019) versus the 2016 percentage of $32.4(30.8-34.0)(p=0.0015)$. A percentage of $9.1(8.6-9.7 \mathrm{Cl} 95 \%)$ of patients reaching a "standing" position (2017 to 2019) versus the 2016 percentage of 10.0 (9.0-11.1 Cl 95\%) $(p=0.1116)$ table 1 . The reduction of mobility level between $2017-2019$ vs 2016 is 
correlated to the RS implementation of research activities. In particular, in 2019 the time was eighteen times compared to 2018. Having said that and given the DRG weight increase over the years the reduction on mobility is justifiable. In 2017-2019, the ALOS at ISMETT was lower than the Italian national benchmark for six of the most common procedures 10.4 mean (SD 16.8, median 7, IQR 3-11).The Italian annual report of hospital discharge records shows an ALOS, for the same procedures, of 12.6 days (table 2 ).

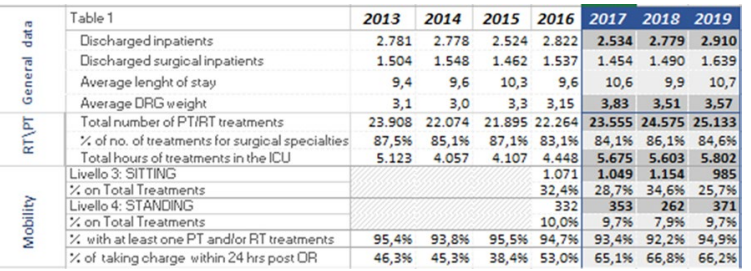

\begin{tabular}{|c|c|c|c|c|c|c|c|c|c|}
\hline \multirow[b]{3}{*}{ Procedures } & \multicolumn{6}{|c|}{ ISMETT } & \multirow{2}{*}{\multicolumn{3}{|c|}{$\begin{array}{c}\text { National Benchmarking } \\
\text { Lenght of stay }\end{array}$}} \\
\hline & \multicolumn{2}{|c|}{2017} & \multicolumn{2}{|c|}{2018} & \multicolumn{2}{|c|}{2019} & & & \\
\hline & n.cases & $\begin{array}{l}\text { lenght } \\
\text { of stay }\end{array}$ & n.ceses & $\begin{array}{l}\text { lenght } \\
\text { of stay }\end{array}$ & n.c cases & $\begin{array}{l}\text { lenght } \\
\text { of stay }\end{array}$ & 2017 & 2018 & 2019 \\
\hline Cardiac valve / cardiac catheterization & 233 & 12.3 & 242 & 12.4 & 243 & 11.8 & 14.2 & 14.1 & 13.6 \\
\hline Cardiac valve / without cardiac catheterization & 114 & 12.3 & 146 & 12.6 & 167 & 11.6 & 15.1 & 14.7 & 15.0 \\
\hline Pancreas, liver and shunt procedures with $\mathrm{CC}$ & 138 & 8.7 & 129 & 7.3 & 147 & 9.4 & 16.7 & 16.9 & 16.4 \\
\hline Major thoracic procedures & 86 & 7.7 & 90 & 8.1 & 113 & 8.2 & 9.8 & 9.5 & 9.4 \\
\hline Coronary artery bypass & 90 & 11.1 & 85 & 13.1 & 108 & 11.1 & 12.4 & 12.1 & 12.2 \\
\hline Pancreas, liver and shunt without $\subset \subset$ & 50 & 5.8 & 93 & 7.1 & 95 & 7.2 & 8.4 & 8.5 & 8.1 \\
\hline
\end{tabular}

Conclusion. The rehabilitation service within our critical care units constantly supports the patient care and recovery during the hospitalization. Speeding up the recovery times of the patient's functional abilities is feasible and can contribute to reduce the length of stay.

\section{Reference(s)}

1. Bertani et al. A comprehensive protocol for physiokinesis therapy and enhanced recovery after surgery in patients undergoing video assisted thoracoscopic surgery lobectomy. J Thorac Dis 2018;10(Suppl 4):S499-S511. https://doi.org/10.21037/jtd.2018.02.55

2. Pagano et al. ERAS Protocol for Perioperative Care of Patients Treated with Laparoscopic Nonanatomic Liver Resection for Hepatocellular Carcinoma: The ISMETT Experience. JOURNAL OF LAPAROENDOSCOPIC \& ADVANCED SURGICAL TECHNIQUES. https://doi.org/10.1089/lap.2020.0445

3. Mohamed D Hashem, Archana Nelliot and Dale M Needham. Early Mobilization and rehabilitation in the ICU: Moving Back to the Future. Respiratory Care 2016;61(7):971-979.

4. Hodgson et al. Early Mobilization of Patients in Intensive Care: Organization, Communication and safety Factors that Influence Translation into Clinical Practice. Critical Care (2018) 22:77

5. Hodgson CL, Tipping CJ. Physiotherapy management of intensive care unit-acquired weakness. 2016. Journal of Physiotherapy 63: 4-10.

6. Ann Parker, Thiti Sricharoenchai and Dale M. Needham. Early Rehabilitation in the Intensive Care Unit: Preventing Physical and Mental Health Impairments. Curr Phys Med Rehabil Reports. 2013 December; 1(4): 307-314.

7. Fuke R, et al. Early rehabilitation to prevent postintensive care syndrome in patients with critical illness: a systematic review and meta-analysis. BMJ Open 2018;8:e019998.

\section{8}

Persisting symptoms in patients following hospital admission with COVID-19: An observational cohort study

C. Pheasant, ; N. Dixon, ${ }^{2}$; P. Day, ; A. Roberts, ; N. Petruso-Osborn, ${ }_{1}^{5}$;. Lawley, ${ }^{4}$ E. Wilson, ${ }^{4}$; D. McWilliams ${ }^{6}$

${ }^{1}$ Associate Director of Allied Health Professionals, University Hospital Coventry \& Warwickshire, Coventry, United Kingdom; ${ }^{2}$ Major Trauma, University Hospital Coventry \& Warwickshire, Coventry, United Kingdom; ${ }^{3}$ Speech and Language therapy, University Hospital
Coventry \& Warwickshire, Coventry, United Kingdom; ${ }^{4}$ Physiotherapy, University Hospital Coventry \& Warwickshire, Coventry, United Kingdom; ${ }^{5}$ Occupational Therapy, University Hospital Coventry \& Warwickshire, Coventry, United Kingdom; ${ }^{6}$ Centre for Care Excellence, University Hospital Coventry \& Warwickshire, Coventry, United Kingdom Correspondence: D. McWilliams Intensive Care Medicine Experimental 2021, 9(1): 000218

Introduction. Severe acute respiratory syndrome coronavirus 2 (SARS CoV-2) is the novel coronavirus first detected in Wuhan, China, that causes coronavirus disease (COVID-19) (1). Due to the novel nature of the virus, little is known or reported regarding the actual longer term symptoms experienced by survivors following discharge from hospital. In England, the National Health Service has predicted that as many as $45 \%$ of people discharged from hospital would need some ongoing support from health and/or social care (2). Within our trust we recognised a gap in the lack of follow up services available for survivors of COVID-19, with an urgent need to understand what a follow up clinic might require from the multidisciplinary team.

Objectives. - To describe the prevalence of ongoing symptoms and associated burden on recovery for patients discharged from hospital following treatment for COVID-19.

- To identify differences in symptoms for patients with severe infection requiring admission to ICU in comparison to those who were hospitalized and received ward based care only.

Methods. This was a single centre, prospective cohort study conducted in patients admitted to a tertiary level, acute NHS hospital between 12 March 2020 and 14 May 2020. Patients were included in the analysis if they met the inclusion criteria of being adults $(\geq 18$ years of age), having a confirmed diagnosis of COVID-19 and being admitted to hospital for at least 4 days.

A post COVID-19 evaluation questionnaire was developed apriori by the multidisciplinary team (MDT) to evaluate aspects of recovery based on clinical experience and early reported outcomes from the literature. The final agreed domains included 29 questions to include an evaluation of any new or worsening 'physical', 'practical', 'emotional' and 'thinking' symptoms. Patients were contacted by telephone by a member of the MDT. Informed verbal consent was taken to proceed with the telephone consultation.

Primary outcome was the presence of new or worsening 'physical', 'practical', 'emotional' and 'thinking' symptoms. Group comparisons were performed using the Fisher's exact tests for categorical variables and Mann-Whitney $\mathrm{U}$ test for continuous variables. All analyses were performed using IBM SPSS 22 (IBM Corp.), with $\mathrm{P}<0.05$ deemed to be indicative of statistical significance throughout.

Results. A total of 228 eligible patients were identified, of whom 165 were contactable and included in the subsequent analysis. Inlcuded patients had a median (IQR) age of 54 (53-79), and spent a median (IQR) 10 (7-17) days in hospital. Physical symptoms were the most commonly reported, with $76 \%$ of patients reporting at least 1 ongoing physical symptom. The most common of these were fatigue $(47 \%)$ and breathlessness (38\%). Ongoing emotional symptoms were also reported by $44 \%$ of all patients.

Twenty nine patients required admission to an intensive care. These patients tended to be younger (57 vs 69 years, $p=0.006$ ), spent longer in hospital (median 19 vs 9 days, $\mathrm{p}<0.00001$ ), and were more likely to have ongoing physical domain symptoms ( $93 \%$ vs. $72 \%, P=0.048)$. No other significant differences were observed in reported symptoms between ICU and non-ICU admissions.

Table $1 \mathrm{~A}$ comparison of ongoing symptoms for patients admitted to ICU vs ward care only

\begin{tabular}{lllll}
\hline Domain & All Pateints & Ward Patients & ICU Patients & $\mathbf{p}$ \\
\hline Physical & $114 / 150(76 \%)$ & $87 / 121(72 \%)$ & $27 / 29(93 \%)$ & 0.048 \\
Practical & $53 / 147(36 \%)$ & $39 / 118(33 \%)$ & $14 / 29(48 \%)$ & 0.498 \\
Emotional & $64 / 146(44 \%)$ & $51 / 117(44 \%)$ & $13 / 29(45 \%)$ & 1.000 \\
Thinking & $44 / 148(30 \%)$ & $34 / 119(29 \%)$ & $10 / 29(34 \%)$ & 0.871 \\
\hline
\end{tabular}


Conclusion. COVID-19 survivors continue to experience physical and non-physical problems up to 12 weeks following hospital discharge. ICU survivors in particular were most likely to suffer ongoing physical problems. There is an urgent need for specialist MDT rehabilitation services to meet the individual needs of the Covid-19 survivors following hospital discharge.

\section{Reference(s)}

1. 1. World Health Organization. Novel coronavirus_China. 2020. Available at: http://www.who.int/csr/don/12-january2020-novel-coronavirus-8; 9china/en/.

2. 2. National Health Service. COVID-19 Hospital Discharge Service Requirements. Available at: https://www.england.nhs.uk/coronavirus/publi cation/covid-19-hospital-discharge-service-requirements/: NHS England, HM Government: Crown Copyright; 2020.

3. n/a.

\section{4}

RAND scores and outcomes in COVID-19 patients admitted to ICU, an observational study

S. Boyd'; I. Martin-Loeches ${ }^{2}$

${ }^{1}$ Anaesthesia and Intensive Care, St James hospital, Dublin, Ireland, Dublin, France; ${ }^{2}$ School of medicine, Trinity College Dublin, Dublin, Ireland

Correspondence: S. Boyd

Intensive Care Medicine Experimental 2021, 9(1): 000254

Introduction. Although many patients may have clinically good outcomes when discharged from ICU, they may not necessarily return to their baseline function.

The RAND 36-Item Health Survey(1) measures eight health concepts (described in Figure 1), as well as perceived change in health. This enables us to estimate patient outcomes after ICU discharge, although there is an element of subjectivity involved.

Methods. We performed a retrospective chart review on all 55 patients admitted to ICU with COVID-19, and recorded their overall outcomes.

We followed up all surviving patients, by phone, 3 months after ICU discharge. Of the 44 surviving patients, and only 39 were contactable. We used a questionnaire to calculate their RAND scores.

Results. The mean age of the patient cohort was 60.38 years, with a greater proportion of male patients to female patients (38:17). Cardiovascular disease, Diabetes and Obesity were the most common comorbidities, affecting over $20 \%$ of the patients.

The mean APACHE and SOFA scores on admission were 21.87 and 5.73 , respectively.

The mean mortality rate at 3 months post ICU discharge was $20.8 \%$. The mean ICU Length of Stay (LOS) was 18.37 days, and hospital LOS 36.04 days. 11.2 days was the mean duration of mechanical ventilation. Rand scores of the surviving patients can be seen in Figure 1.

Figure 1 (RAND scores).

\begin{tabular}{ll}
\hline $\mathbf{N}=\mathbf{3 9}$ & $\begin{array}{l}\text { Mean } \\
\text { [Standard } \\
\text { Deviation] }\end{array}$ \\
\hline Physical Functioning & $65.77[35.12]$ \\
Role Limitations due to Physical health & $51.28[43.28]$ \\
Role Limitations due to emotional problems & $76.05[35.88]$ \\
Energy/Fatigue & $48.33[35.88]$ \\
Emotional Well-being & $74.26[21.52]$ \\
Social Functioning & $73.28[27.05]$ \\
Pain & $66.82[26.09]$ \\
General Health & $56.67[19.58]$ \\
Health Change & $41.67[25.86]$
\end{tabular}

Conclusion. We can see how after discharge from ICU, most patients experienced a degree of limitation. Of note, most patients had perceived a change in their health.

It is important to be aware that, although a patient may be fit for discharge from ICU, there is still a long path to recovery ahead of them.

Reference(s)

1. 1. Ware, J.E., Jr., \& Sherbourne, C.D. "The MOS 36-Item Short-Form Health Survey (SF-36): I. Conceptual Framework and Item Selection.". Medical Care, 30:473-483, 1992.

2. We would like to acknowledge the hard work of all of the ICU staff throughout this pandemic.

000302

A support strategy for ICU staff that overcomes resistance to accepting psychological help

M. Maddock

${ }^{1}$ Intensive Care, North Bristol, NHS Trust, Bristol, France

Correspondence: M. Maddock

Intensive Care Medicine Experimental 2021, 9(1): 000302

Introduction. It is not unusual for staff in Intensive care Units (ICU) to be exposed to traumatic events. Even before the Covid-19 pandemic research had shown that those working in ICU were at higher risk of psychological and moral distress. A recent study suggested that $45 \%$ of ICU staff reported some signs of PTSD, severe depression, or severe anxiety disorder [1].

Several recommendations were made from this study which included "evidence-based staff support" and "rapid access to formal treatment". A report from Wuhan noted that despite offering mental health services in the form of online interventions and phone hotlines to frontline workers caring for Covid-19 patients "clinicians were often reluctant to participate in services identified as "mental health" interventions." [2].

This has also been our experience. After the death of an ICU colleague in 2020, 250 nurses were offered a 1:1 session with a clinical psychologist but only a 3 took up the offer. That is not to say that there was no need, rather, it seemed that there exists a reluctance to make use of such support at exceedingly difficult times and from others outside of the ICU team.

Objectives. To establish a method to overcome the barrier of resistance to accepting help in frontline staff, by developing advanced peer support processes amongst ICU nurses.

Methods. The development of a structured discussion format was created between the wellbeing lead of ICU and Consultant Clinical Psychologist in line with guidance from the Institute for Healthcare Improvements [3]. The discussion addressed individual's experiences during the Covid19 pandemic in ways that drew out the most difficult challenges, incidents of moral stress, team attitudes and support, adaptive-coping strategies, learning, and confidence for future working. Those conducting the discussions were supported by a two-day peer-to-peer training workshop and follow-on supervision. This discussion strategy were known as "check in chats".

Every nurse on ICU was offered a 1:1 "check in chat" with a member of the wellbeing team. The private, realtively informal chats (compared to a consultation with a counsellor) took place during working hours and on average took no more than 10 min to conduct.

During these check in chats and more specifically as individuals were reflecting on their experiences, peer supporters were able to assess if further input was needed and 'sow the seed' for more formal peer support.

An opportunity sample of these 1:1 discussions were recorded and and a content analysis was conducted by two independent raters to derive themes of individual experiences.

Results. Following on from the death of an ICU colleague Peer support was established and implemented on the unit in August 2020, only 2 nurses accessed this method of support in the following eight months. However, with the introduction of "check in chats" in March 2021, 8 nurses self-referred themselves for peer support in one 
month alone, 5 of which sought more specific mental health support after these sessions.

Conclusion. These results suggest that this strategy appears to have opened a doorway to accepting support. Peer Support needs to be integral and not a standalone support system. Further investigation is needed into the barriers that prevent healthcare professionals accessing mental health support.

\section{Reference(s)}

1. [1] Mental health of staff working in intensive care during COVID-19 N Greenberg, D Weston, C Hall, T Caulfield, V Williamson and K Fong Occup Med (Lond). 2021 Jan 13

2. 2] Epidemic psychiatry: The opportunities and challenges of COVID-19 Daniel Shalev* and Peter A. Shapiro Gen Hosp Psychiatry 2020 (MayJune) vol 64 pp 68-71

3. [3] Institute for Healthcare Improvement: IHI tool: Conversation and Action Guide to Support Staff Well-Being and Joy in Work During and After the Covid-19 Pandemic. ihi.org

\section{1}

\section{Crisis Resource Management to optimize patients' intensive care} admission: A qualitative study

JF. Jensen '; G. Bunkenborg'; J. Ramos²; M. ØRom²; K. Naver'; L. Shiv²; TU. Skram²

${ }^{1}$ Dept. of Anaesthesiology, Holbæk Hospital, Holbæk, Denmark; ${ }^{2}$ Dept of intensive care therapy, Nordsjællands Hospital—Hillerød, Dyrehavevej, Hillerød, Denmark, Hillerød, Denmark

Correspondence: J.F. Jensen

Intensive Care Medicine Experimental 2021, 9(1): 000401

Introduction. Improving patient safety is important as adverse events and errors cause serious harm and even death in the intensive care unit (ICU) (1). These events are related to problems with non-technical skills (i.e., communication, leadership) rather than lack of technical expertise. Crisis Resource Management (CRM) interventions in reallife ICU settings have been relatively under-investigated. A model for an optimized admittance at the ICU was developed to improved staffs' non-technical skills, increase patients' safety and quality of care. Inspired by principles of Crisis Resource Management (CRM) was the intervention tested in simulation-based multidisciplinary team training $(2,3)$. Studies in ICU settings have investigated principles of CRM in areas of advanced airway management, trauma, and critical care training in different acute scenarios such as septic shock, cardiac or respiratory problems $(4,5)$. Investigations of CRM training of the ICU admission procedure are lacking. The intervention was qualitatively evaluated.

Objectives. The ojective was to explore staffs' experiences of a quality improvement intervention in real life ICU admittance, after completing simulation-based multidisciplinary team training.

Methods. A qualitative approach using interview data with physicians $(n=5)$ and nurses $(n=15)$, who delivered the intervention with a maximum variation of ICU work experiences. Data were analyzed by thematic analysis and the consolidated criteria for reporting qualitative research (COREQ) was applied.

Results. The analysis reveal three themes: Core clinical activities and patient centeredness, Reflection on patient safety, and Transition into practice, which reflected the essential meaning of implementing the quality improvement intervention.

Conclusion. This study provides a contemporary understanding of the implementation process of a quality improvement intervention at ICU admittance. Implementation evolves through core clinical activities that facilitates reflection on patient safety and transition into clinical practice. It is relevant for clinical practice because it enables health care professionals to understand how this intervention can contribute to increase patients' safety and quality of care in the management of ICU admittance. Our study adds to the understanding of the implementation process by providing descriptions of staffs' experiences of a quality improvement intervention in real life ICU admittance staffs' experiences.
Reference(s)

1. 4. Flin R, Patey R, Glavin R, Maran N. Anaesthetists' non-technical skills. Br J Anaesth. Jul 2010;105(1):38-44

2. 5. Gundrosen S; Solligård E; Aadahl P. Team comptence among nurses in an intensive care unit: the feasibility of in situ simulation and assessing non-technical skills. Intensive Critical Care nurse. 2014;30(6):312-317.

3. 1. Haerkens $\mathrm{MH}$, Jenkins $\mathrm{DH}$, van der Hoeven JG. Crew resource management in the ICU: the need for culture change. Ann Intensive Care. Aug 22 2012;2(1):39

4. 2. Fletcher G, Flin R, McGeorge P, Glavin R, Maran N, Patey R. Anaesthetists' Non-Technical Skills (ANTS): evaluation of a behavioural marker system. Br J Anaesth. May 2003;90(5):580-588. 5. Flin R, Patey R, Glavin R, Maran N Anaesthetists' non-technical skills. Br J Anaesth. Jul 2010;105(1):38-44.

5. 3. Fletcher G, Flin R, McGeorge P, Glavin R, Maran N, Patey R. Anaesthetists' Non-Technical Skills (ANTS): evaluation of a behavioural marker system. Br J Anaesth. May 2003;90(5):580-588. 5. Flin R, Patey R, Glavin R, Maran N. Anaesthetists' non-technical skills. Br J Anaesth. Jul 2010;105(1):38-44.

6. TRYG Foundation

\section{5}

Inspiratory muscle training in adult UK intensive care units: A survey of current physiotherapy clinical practice

R. Davies ${ }^{1}$; K. Jerrard ${ }^{2}$

1Physiotherapy Department, King's College Hospital NHS Foundation Trust, London, United Kingdom; ${ }^{2}$ Physiotherapy department, Royal Victoria Hospital, Belfast Health and Social Care Trust, Belfast, United Kingdom Correspondence: $\mathrm{R}$. Davies

Intensive Care Medicine Experimental 2021, 9(1): 000415

Introduction. Inspiratory muscle training (IMT) is a safe and feasible treatment modality for critically ill patients presenting with respiratory muscle weakness(1). IMT has been shown to increase respiratory muscle strength, improve weaning outcomes and reduce intensive care unit (ICU) length of stay(2).

Objectives. To explore the clinical use of IMT by Physiotherapists working in adult UK ICUs.

Methods. An online survey was developed, including questions regarding IMT device selection, inclusion criteria, training regimens, outcome measures specific to IMT and staff training. The survey was open from November 2020 until January 2021 and disseminated via the Association of Chartered Physiotherapists in Respiratory Care and the UK Respiratory Physiotherapy Leaders group.

Results. Eligible responses were received from fourty-five ICUs. 11 ICUs (24\%) used IMT, 5 (11\%) were in the process of procurement and the majority $(n=29 ; 65 \%)$ did not use IMT.

Of the 11 ICUs using IMT and the 5 in the process of procurement, device selection varied. Mechanical threshold IMT devices were present in 10 ICUs (67\%). 5 ICUs (33\%) used tapered flow devices and 2 $(13 \%)$ utilised inspiratory trigger adjustment on the ventilator as a means of delivering IMT. Five of these respondents (33\%) stocked more than one type of device. One ICU did not specify the type of modality used.

Of the 11 ICUs using IMT, 5 (45\%) used IMT on ventilator dependent patients with an endotracheal tube, $9(82 \%)$ on ventilator dependent patients with a tracheostomy and $10(91 \%)$ on self-ventilating patients via a facemask. All respondents reported that IMT was a Physiotherapist led modality.

The most commonly reported clinical indications for IMT use were failure to wean $(n=8 ; 73 \%)$ and prolonged mechanical ventilation of $>7$ days $(n=5 ; 45 \%)$.

Eight ICUs (73\%) listed an IMT training regime. Five (36\%) cited the work of the same author(3), adopting a regime of a set threshold of $50 \%$ maximal inspiratory pressure (MIP), 5-6 breaths daily with incrementation of $1-2 \mathrm{CmH}_{2} \mathrm{O}$ every $1-2$ days.

The most commonly reported outcome measure, used to guide treatment parameters and determine effectiveness of IMT, was MIP ( $n=7$; 64\%).

Conclusion. The preliminary results indicate that IMT is not a common treatment modality used by Physiotherapists within adult UK ICUs. 
However, we acknowledge that only a small proportion of ICUs have been represented. No standardised approach for the use of IMT was identified by this survey. This highlights a need for increased education and the development of an evidence based national guideline, to enable a standardised approach to IMT delivery and promote its use within UK ICUs.

\section{Reference(s)}

1. (3) Bissett, B. et al. (2019) 'Inspiratory muscle training for intensive care patients: A multidisciplinary practical guide for clinicans'Australian Critical Care, 32, pp. 249-255

2. (2) Elkins, M and Dentice, R. (2015) Inspiratory muscle training facilitates weaning from mechanical ventilation among patients in the intensive care unit: A systematic review' Journal of Physiotherapy, 61(3), pp. 125-134.

3. (1) Vorona, S. et al. (2019) 'Inspiratory Muscle Rehabilitation in Critically II Adults. A Systematic Review and Meta-Analysis' Annals of the American Thoracic Society, 15(6), pp.735-744.

\section{5}

\section{Stakeholder feedback on reading ICU diary entries aloud in real} time to patients in the ICU

K. Johnson'; J. Temeyer'2; K. Philbrick³; L. Karnatovskaia

${ }^{1}$ Division of Pulmonary and Critical Care, Mayo Clinic, Rochester, United States of America; ${ }^{2}$ Department of Nursing, Mayo Clinic Hospital, Rochester, United States of America; ${ }^{3}$ Division of Psychiatry, Mayo Clinic, Rochester, United States of America

Correspondence: K. Johnson

Intensive Care Medicine Experimental 2021, 9(1): 000425

Introduction. The Intensive Care Unit (ICU) diary is a well-known intervention with mixed data of its effect on psychological outcomes of critical illness survivors. Entries are made by providers/family daily, and the patient reads these sometime after the ICU discharge. Indeed, memories of frightening/delusional (not factual) ICU experiences are the biggest potentially modifiable risk factor for psychological morbidity. Recent research on the formation of fear memories demonstrated that one can interfere with the initial process of fear conditioning during the time when initial memory consolidation occurs. Moreover, if mitigating information about a traumatic event is introduced during the time between memory formation and its subsequent recall, the emotional experience of the memory can be modified. Therefore, we explored whether the ICU diary intervention can be used in a novel way where entries are also read aloud to the patients right after they are written, providing patients with systematic real time orientation, and facilitating formation of factual memories.

Objectives. To obtain stakeholder feedback on the intervention.

Methods. Single center qualitative study. Patients, family members, nurses, physical and occupational therapists participating in the care of enrolled patients completed structured interviews regarding the intervention with subsequent qualitative analysis to identify common themes.

Results. The intervention (reading the entries aloud) required less than 5 min a day on average to complete. Thirty patients who had ICU diary entries read aloud, 3 family members (due to the no-visitor policy for most of the study duration), 31 nurses, 5 physical and 3 occupational therapists completed the interviews. Of the 15 patients who remembered the intervention, 14 responded favorably. Main themes were valuing the daily updates, being less confused about what was happening, feeling comforted at a time when most families could not visit, and appreciating when providers talked to them. All family members liked the intervention. All providers identified beneficial aspects of the intervention, emphasizing improved understanding by the patients of their hospital course, being reminded to talk to the patients and to stimulate their brain, establishing a meaningful connection with the patients, and enhancing the human element of patient care by interacting with them. Most common concerns included lack of time to complete the intervention, and the need for standardized implementation with suggestions to include this into the daily checklist. Interestingly, while 4 therapists expressed concern with the intervention potentially overwhelming sicker patients, none of the nursing or patient comments shared that concern.

Conclusion. Reading ICU diary entries aloud was viewed positively overall by patients, families, nursing staff, and therapists. Testing efficacy of this novel approach on psychological outcomes therefore appears warranted.

Reference(s)

1. Funding provided by the ZOLL Foundation.

\section{8}

Utilisation of proformas to improve prone positing during SARS-COV2: A closed-loop audit

L. Western ; A. Baldwin, 2.T. Ali,

${ }^{1}$ Anaesthetics and Critical Care, Buckinghamshire Healthcare NHS Trust, Aylesbury, United Kingdom; '2Anaesthetics and Critical Care, Buckinghamshire Healthcare NHS Trust, Oxford, United Kingdom

Correspondence: $L$. Western

Intensive Care Medicine Experimental 2021, 9(1): 000468

Introduction. Prone positing has been well established in intensive care for the treatment of ARDS [1]. SARS-COV2 presented a complex challenge, but with lung involvement bearing similarities to ARDS, proning was quickly established as a useful tool to aid management [2].

However, the procedure is not benign, particularly in mechanically ventilated patients. During the procedure, the patient's airway may be compromised, or lines disconnected. Furthermore, patients are vulnerable to unusual pressure areas and brachial plexopathies [3].

Objectives. A closed-loop local audit was conducted to assess adherence to new prone position related documentation and the standard of prone positioning in our unit for intubated patients during the early 2021 UK SARS-COV2 surge.

Methods. Local safety standard for invasive procedures (LocSSIP) proformas, for proning and supination, were adapted for use during SARS-COV2. A SSKINN (skin, surface, keep moving, incontinence, nutrition, and nerves) bundle form adapted to assess additional pressure areas for prone patients had been created. It was expected all forms would be completed. Documentation was retrospectively reviewed from inpatients notes and compared to nursing position charts, which record the patient's specific position every hour.

Prone position quality was assessed directly and compared to the official guidelines provided by the Intensive Care Society, UK [3].

Each loop lasted one week. The loops took place on the last weeks of January and February 2021. All patients proned in the period were included.

Results. The initial audit assessed five patients, each with multiple procedures. It found generally good utilisation of procedure LocSSIPs $(80-100 \%)$, however, they were infrequently sign and dated $(60 \%)$. SSKINN form use was not utilised appropriately (0\%). Prone positioning was poor (0\%); the most common error was over-abduction of the arm.

In response, LocSSIPs were edited to emphasise signing and assessment of pressure areas. Nursing staff were communicated to use the SSKINN bundle for all SARS-COV2 patients. Department emails promoted the importance of positioning.

The close loop assessed four patients. Sign and dating improved to $80 \%$. Prone adapted SSKINN bundle use was at $100 \%$. All patients prone position was appropriate (100\%). 
Conclusion. We demonstrate the utilisation of simple LocSSIPs and related documentation to improve the quality of prone positioning during the SARS-COV2 pandemic surge. We recommend other departments utilise appropriate LocSSIPs to mitigate risks of proning.

\section{Reference(s)}

1. [3] Prone Positioning in Adult Intensive Care Guideline, Intensive Care Society.

2. [2] Elharrar $X$, et al. Use of Prone Positioning in Nonintubated Patients With COVID-19 and Hypoxemic Acute Respiratory Failure. JAMA 2020;323:2336. https://doi.org/10.1001/jama.2020.8255.

3. [1] Guérin C, et al. Prone Positioning in Severe Acute Respiratory Distress Syndrome. N Engl J Med 2013;368:2159-68. https://doi.org/10.1056/ NEJMoa1214103.

\section{6}

\section{Support needed by relatives of ECMO patients: a qualitative} approach

M. Onrust ${ }^{1}$; F. Blokzijl ${ }^{2}$; I. van der Meulen ${ }^{1}$; W. Paans ${ }^{3}$; W. Dieperink ${ }^{1}$

${ }^{1}$ Department of Critical Care, University Medical Center Groningen, Groningen, Netherlands; ${ }^{2}$ Department of Cardiothoracic Surgery, University Medical Center Groningen, Groningen, Netherlands; ${ }^{3}$ Professorship Nursing Diagnosis, School of Nursing, Hanze University of Applied Sciences Groningen, Groningen, Netherlands

Correspondence: I. van der Meulen

Intensive Care Medicine Experimental 2021, 9(1): 000566

Introduction. Relatives of ECMO patients are at risk of developing long lasting psychological symptoms due to the impact of the critical illness and ICU stay. In addition, patients are being discharged from inpatient facilities at an earlier stage, giving relatives an increasingly important role. This also effects ECMO patients and their relatives. It is therefore important for healthcare professionals to know how they can support relatives adapt to the emotional challenges following an ICU admission.

Objectives. To identify meaningful and relevant forms of support and interventions for ECMO relatives that can help professionals in the ICU and throughout the care chain to support caregivers.

Methods. A qualitative, retrospective, descriptive study with an exploratory approach was conducted, using the method of 'storytelling'. Interviews were held with relatives of patients who received ECMO treatment in the University Medical Center Groningen during the period September 2018 to September 2019. Audio recordings of the conversations were transcribed verbatim. Data analysis was done by two researchers independently, with open coding first and axial coding second. Then selective coding divided the categories into groups. By incorporating the opinions of a team into the analysis, triangulation was applied.

Results. Eight relatives of ECMO patients participated in the study. The mean age of the participants was 48.6 (SD 9.8) and two of them were male. ECLS duration ranged from 4 to 39 days. Nine categories were found during data analysis related to professional support to ECMO relatives during and after ICU admission. The three overarching concepts were 'cultural dimensions', 'role perception' and 'health related aspects'.

Conclusion. Relatives of ECMO patients perceived 'taking part in the patient's disease process' as meaningful and relevant support by professionals during the ICU period. In addition, repeating the 'possibility of telephone contact at all times' was experienced as valuable support. With this, the relatives felt recognized in their role as representative of the patient. Known interventions used in the ICU to support relatives, were not mentioned by any of the relatives in the narratives, with the exception of counseling by Social Work. Transfer to a nursing ward, home or rehabilitation center caused feelings of helplessness and frustration when relatives did not feel heard and seen as a partner in dialogue of professionals. Relatives did not receive support from professionals for themselves and did not seem to be looking for it, despite the impact of the ICU admission on their own health.

\section{1}

Muscle function may predict physical function but not HRQoL in ICU survivors

H. Pool, ${ }^{1}$; A. Lupton-Smith ${ }^{1}$; S. Hanekom

${ }^{1}$ Physiotherapy, Stellenbosch University, Stellenbosch, South Africa

Correspondence: S. Hanekom

Intensive Care Medicine Experimental 2021, 9(1): 000581

Introduction. Changes in muscle function and muscle structure in critical ill population are widely reported(1). The relationship between these early measures of muscle function with physical function and health related quality of life (HRQoL) of survivors is not clear.

Objectives. The aim of this study was to explore the relationships between measures used to report on muscle function in ICU (Peripheral and respiratory) with measures of function and $\mathrm{HRQoL}$ in ICU survivors.

Methods. Prospective observational cohort study. Sonographic measurements of the diaphragm and rectus femoris, dynamometer of $M$ Quadriceps and M Deltoid; grip strength, maximum inspiratory pressure (MIP) and medical research council sum score (MRC-SS) were recorded at ICU and hospital discharge. Six minute walk distance (6MWD), Barthel Index (BI), and EQ-5D measures were completed at ICU-, hospital discharge and three months after discharge. All procedures standardised a priori and completed by a single assessor. Shapiro-Wilk to test for normality. Simple linear regression was used to explore Muscle function measures associated with functional capacity, functional ability and HRQoL of ICU Survivors. Due to the small sample size we report on single predictors using the adjusted R2, and unstandardised co-efficient. The f-statistic in ANOVA was used to report on significance of models. Significance was accepted at $P<0.05$. Results. 21 participants were included. The mean age of the sample was 68.29 (SD 14.38) years, and the mean SAPS 3 score was 57 (SD 15.53). Patients stayed in the unit 15.81 (SD 9.35) days and in the hospital for 20.95 (SD 11.48) days. MIP (R2 $0.458 p=0.005)$, grip strength (R2 $0.662 p=0.000)$ and MRC-SS (R2 $0.220 p=0.044$ ) at ICU discharge were identified as potential predictors for 6MWD and functional ability at hospital discharge. None of the muscle strength measures were associated with patient's perception of HRQoL or functional ability at three months after discharge from hospital (Table).

\begin{tabular}{|c|c|c|c|c|c|c|c|}
\hline & Predictor & $\begin{array}{l}\text { Adjusted } \\
\text { R2 }\end{array}$ & $\begin{array}{l}\text { Co- } \\
\text { efficient }\end{array}$ & $P=$ & $\begin{array}{l}\text { Adjusted } \\
\text { R2 }\end{array}$ & $\begin{array}{l}\text { Co-effi- } \\
\text { cient }\end{array}$ & $\mathrm{P}=$ \\
\hline & \multicolumn{3}{|c|}{ 6MWD Hospital DC } & \multicolumn{3}{|c|}{ BI Hospital D/C } & \\
\hline \multirow[t]{12}{*}{$\begin{array}{l}\text { ICU } \\
\qquad / C\end{array}$} & $\begin{array}{l}\text { RF Thickness } \\
(\mathrm{cm})\end{array}$ & -0.010 & 167.68 & 0.35 & -0.071 & 1.796 & 0.94 \\
\hline & DTF (mm) & 0.103 & -4.64 & 0.13 & 0.101 & -0.516 & 0.23 \\
\hline & MIP $(\mathrm{cm} \mathrm{H} 2 \mathrm{O})$ & 0.458 & 3.336 & $0.00^{* *}$ & 0.346 & 0.423 & $0.01 * *$ \\
\hline & MRC-SS & 0.220 & 7.827 & $0.04 * *$ & 0.639 & 1.733 & $0.00^{* *}$ \\
\hline & $\begin{array}{l}\text { Grip strength } \\
\quad(\mathrm{kg})\end{array}$ & 0.662 & 10.188 & $0.00^{* *}$ & 0.377 & 1.174 & $0.01^{* *}$ \\
\hline & \multicolumn{7}{|c|}{ EQ5D Hospital DC } \\
\hline & $\begin{array}{l}\text { RF thickness } \\
(\mathrm{cm})\end{array}$ & 0.064 & -10.305 & 0.18 & & & \\
\hline & DTF $(\mathrm{mm})$ & -0.026 & -0.105 & 0.44 & & & \\
\hline & $\mathrm{MIP}(\mathrm{cmH} 2 \mathrm{O})$ & 0.171 & 0.105 & $0.07^{*}$ & & & \\
\hline & MRC-SS & -0.019 & -0.148 & 0.41 & & & \\
\hline & $\begin{array}{l}\text { Grip strength } \\
\quad(\mathrm{kg})\end{array}$ & -0.070 & -0.21 & 0.89 & & & \\
\hline & BI 3 MONTHS & & & EQ5D 3 & MONTHS & & \\
\hline
\end{tabular}




\begin{tabular}{|c|c|c|c|c|c|c|c|}
\hline & Predictor & $\begin{array}{l}\text { Adjusted } \\
\text { R2 }\end{array}$ & $\begin{array}{l}\text { Co- } \\
\text { efficient }\end{array}$ & $\mathrm{P}=$ & $\begin{array}{l}\text { Adjusted } \\
\text { R2 }\end{array}$ & $\begin{array}{l}\text { Co-effi- } \\
\text { cient }\end{array}$ & $\mathrm{P}=$ \\
\hline \multirow[t]{5}{*}{$\begin{array}{r}\text { HOSP } \\
D / C\end{array}$} & $\begin{array}{l}\text { Dynam Quads } \\
\text { (N) }\end{array}$ & -0.055 & 0.120 & 0.21 & 0.03 & 0.093 & 0.26 \\
\hline & $\begin{array}{c}\text { Dynam Del- } \\
\text { toid (N) }\end{array}$ & 0.089 & 0.218 & 0.17 & 0.059 & 0.149 & 0.21 \\
\hline & $\mathrm{MIP}(\mathrm{cmH} 2 \mathrm{O})$ & -0.025 & 0.211 & 0.43 & -0.078 & 0.093 & 0.82 \\
\hline & MRC-SS & 0.213 & 1.347 & $0.06^{*}$ & -0.033 & 0.431 & 0.46 \\
\hline & $\begin{array}{l}\text { Grip strength } \\
\quad(\mathrm{kg})\end{array}$ & 0.116 & 0.757 & 0.23 & 0.087 & 0.653 & 0.16 \\
\hline
\end{tabular}

Conclusion. Muscle function at ICU discharge might be used to explain functional capacity and -ability of ICU survivors at hospital discharge. However, muscle function at ICU and hospital discharge does not explain patients' perceptions of HRQoL or functional ability at hospital discharge or at three months. This data will now be used to inform the design of a sufficiently powered study to develop a predictive model for functional ablity of ICU survivors.

\section{Reference(s) \\ 1. Puthucheary ZA, Rawal J, McPhail M, Connolly B, Ratnayake G, Chan P, et al. Acute skeletal muscle wasting in critical illness. JAMA. 2013;310(15):1591 \\ 2. NRF South Africa \\ 000590 \\ UK Emergency Medical Team (EMT) deployment to Armenia supporting the national COVID response \\ P. Mccready' ${ }^{1}$ R. Inglis, ${ }^{1}$ \\ ${ }^{1}$ Uk emt, UK EMT, Manchester, United Kingdom \\ Correspondence: $M$. Inglis \\ Intensive Care Medicine Experimental 2021, 9(1): 000590}

Introduction. The UK Emergency Medical Team (UK EMT) responded to a call from the Armenian Ministry of Health for support in the delivery of care to severe and critically ill patients with COVID-19. Two members of the team were stationed at the Surb Grigor Lusavorich (SGL) hospital for a 5-week period. SGL has a 50 -bed ICU which is dedicated exclusively to the care of patients with COVID-19.

Following an initial needs assessment, the UK EMT delivered a structured programme of training and supervision that covered both core ICU skills and COVID-specific content. The programme used interactive training methods including simulation, video case discussion and hands-on practical skills teaching.

Objectives. This study has two objectives:

1) To describe the process of developing and delivering a tailored COVID-19 training programme in the context of a global pandemic

2) To analyse the training programme to generate a list of lessons learned.

Methods. We used a case study approach to obtain an in-depth appreciation of this complex intervention. A case study is an 'empirical inquiry that investigates a contemporary phenomenon in depth and within its real-life context, especially when the boundaries between phenomenon and context are not clearly evident'1. Data was collected using semi-structured interviews and participant observation.

Results. Our key findings were:

In-service training during the COVID-19 pandemic needs to be flexible, adaptable and based on a local needs assessment.

Delivering face-to-face training to ICU staff in the context of COVID raises infection prevention and control challenges but they can be overcome with careful planning.

ICU simulation training can be delivered easily and cheaply using actors and an electronic tablet 'patient monitor' in the absence of a high fidelity manikin.

The participants reported feeling 'well supported' and 'cheered up' by the training team and appreciated the element of international solidarity.

Conclusion. Our study highlights important learning points for the design and delivery of COVID-training in the setting of a large, busy
ICU. It also has broader implications for the roll out of training in an emergency humanitarian context.

Reference(s)

1. Crowe S, Cresswell K, Robertson A, Huby G, Avery A, Sheikh A. The case study approach. BMC Med Res Methodol. Published online 2011:1. https://doi.org/10.1186/1471-2288-11-100

\section{6}

Facial pressure ulcers from prone positioning in COVID-19

patients

F. Binda ${ }^{1}$; F. Marelli'; A. Galazzi ; L. Villa'; E. Vinci'; I. Adamini' ${ }^{1}$ D.

Laquintana ${ }^{2}$

${ }^{1}$ Intensive Care Unit, Fondazione IRCCS Ca' Granda Ospedale Maggiore Policlinico, Milano, Italy; ${ }^{2}$ Direction of Healthcare Professions, Fondazione IRCCS Ca' Granda Ospedale Maggiore Policlinico, Milano, Italy

Correspondence: F. Binda

Intensive Care Medicine Experimental 2021, 9(1): 000726

Introduction. Patients with COVID-19 often required intensive care unit (ICU) admission due to the development of acute respiratory distress syndrome (ARDS). [1] Prone positioning can support the respiratory function in patients with severe ARDS although this therapy is not without risks like pressure ulcers (PU) in anatomical sites different from those typical of bedridden patients. [2].

Objectives. To report the prevalence of facial pressure ulcers associated with prone positioning in patients with COVID-19 admitted to the ICU hub of a tertiary-level hospital in Lombardy (Italy).

Methods. All patients with laboratory-confirmed SARS-CoV-2 infection and subsequently admitted to ICU of Foundation IRCCS $\mathrm{Ca}^{\prime}$ Granda Ospedale Maggiore Policlinico (Milan), between February 23 and June 30, 2020, were enrolled in this retrospective consecutive case-series study. The recorded data included demographic and clinical characteristics of patients. PU development and staging were evaluated using the National Pressure Ulcer Advisory Panel (NPUAP).

Results. During the study period, the prevalence of patients with facial PU related to PP was $30.2 \%$. A total of 28 pressure ulcers were recorded on the face. The most frequent NPUAP stage was stage II $(67.9 \%, 23 / 28)$, followed by stage I $(32.1 \%, 9 / 28)$. The stage III was not recorded on the face. The other results are summarized in the table.

\begin{tabular}{lccr}
\hline Demographic and clinical characteristics \\
\hline $\begin{array}{l}\text { Age (mean, SD) } \\
\text { Sex (male) }\end{array}$ & $51.7 \pm 11.0$ & BMI (mean, SD) & $28.9 \pm 4.6$ \\
& $\begin{array}{c}\text { Braden score (mean, } \\
\text { SD) }\end{array}$ & $9.2 \pm 1.7$ \\
$\begin{array}{l}\text { ICU lenght of stay } \\
\text { (days) }\end{array}$ & $\begin{array}{l}19.0(11.0-45.5) \\
\text { Mortality (\%) }\end{array}$ & $\begin{array}{l}\text { SOFA score (mean, } \\
\text { SD) }\end{array}$ & $6.7 \pm 3.1$ \\
& $29(46 \%)$ & $\begin{array}{c}\text { Number of PP cycle } \\
\text { (mean, SD) }\end{array}$ & $3.5 \pm 2.7$ \\
& &
\end{tabular}

Conclusion. PP in patients with COVID-19 was extensively used and related PU were a frequent event, especially on the face. As expected, the longer time spent in the PP and the greater number of PP sessions augmented the risk of pressure ulcers. Specifically nursing interventions, in particular preventive measures, should be implemented to reduce the prevalence of this phenomenon.

\section{Reference(s)}

1. 2) Moore Z, et al. J Wound Care. 2020 Jun 2;29(6):312-320.

2. 1) Qadri SK, et al. Pulm Ther. 2020 Dec;6(2):233-246. 
000750

Exploring Nursing Sensitive Outcomes in Pediatric Intensive Care Units: preliminary findings from a scoping review

M. Danielis ${ }^{1}$; A. Castellano ${ }^{1}$; E. Mattiussi

${ }^{1}$ Department of Medical Sciences, University of Udine, Udine, Italy

Correspondence: $M$. Danielis

Intensive Care Medicine Experimental 2021, 9(1): 000750

Introduction. There is increasing interest in the nursing contribution to critically ill patients' outcomes. Although some attempts have been made to conceptualize the nature of that contribution in pediatric setting, no published or ongoing reviews have emerged to date. In order to advance the quality of care for pediatric critically ill patients, existing evidence need to be better utilized.

Objectives. This review aims to describe what is the state of art of the research in the field of Nursing Sensitive Outcomes (NSO) in the Pediatric Intensive Care Unit (PICU), and to synthesize those outcomes that have been documented to date.

Methods. A scoping review was conducted, by following the framework proposed by Arksey and O'Malley. Medline (via PubMed) and Cumulative Index to Nursing and Allied Health (CINAHL) electronic databases were searched in April 2020. In addition, the reference list of included articles was screened. Two researchers screened publications based on the following inclusion criteria: (a) articles that reported NSO on pediatric critically ill patients admitted in PICU, (b) both primary and secondary studies without methodology limits, (c) publications written in English (d) without time frame limitation.

Results. Of the 2,293 records, 60 fully met the inclusion criteria, and were included. Publications were mainly authored between 2014 and $2020(n=42 ; 70.0 \%)$, and the majority $(n=30 ; 50.0 \%)$ was quasi-experimental in design. 156 nursing sensitive outcomes have emerged, categorized in 75 items, and grouped in four domains following Doran's classification (safety, clinical, functional and perceptual), and six subdomains (healthcare-associated infections [e.g., central line-associated bloodstream infection], critical incidents [e.g., pressure ulcers], general health [e.g., length of intermittent mandatory ventilation], goal assessment and monitoring [e.g., pain], psychosocial dimension [e.g., delirium], and physical dimension [e.g., oral mucositis]). The most commonly reported outcome was PICU length of stay in 18 studies, followed by mortality in 14 and ventilator-associated pneumonia in eight studies. Clinical domain was the most researched, with a total of 82 outcomes reported. No outcome attributable to the perceptual domain has emerged.

Conclusion. A large heterogeneity of outcomes suitable to monitoring nursing care in PICUs was found. The results also highlighted that some poorly developed areas needed improvement at the practice level. At organizational level, more robust administrative databases are needed to collect data at the bedside, and further research with an interventional approach will be helpful in producing more causal relationship between nursing activities and pediatric patients' outcomes.

\section{Reference(s)}

1. Munn, Z., Peters, M. D., Stern, C., Tufanaru, C., McArthur, A., \& Aromataris, E. (2018). Systematic review or scoping review? Guidance for authors when choosing between a systematic or scoping review approach. BMC medical research methodology, 18(1), 1-7.

2. Siow, E., Wypij, D., Berry, P., Hickey, P., \& Curley, M. A. (2013). The effect of continuity in nursing care on patient outcomes in the pediatric intensive care unit. JONA: The Journal of Nursing Administration, 43(7/8), 394-402.

\section{1}

Understanding the lived experiences of family members in the Intensive Care Unit: a systematic review and meta-synthesis E. Mattiussi ${ }^{1}$; C. Qualizza'; M. Danielis

'Department of Medical Sciences, University of Udine, Udine, Italy

Correspondence: $M$. Danielis

Intensive Care Medicine Experimental 2021, 9(1): 000751
Introduction. Intensive Care Unit (ICU) admission is a critical event that potentially affects the balance of the patient's whole family. The ICU experience has been reported to trigger negative effects on the lives of family members, who are at increased risk for psychological symptoms. These symptoms can affect their ability as caregivers in ICU, thus having an impact on patients' outcomes.

Objectives. The aim of this study was to investigate the lived experiences faced by family members during the ICU-stay of their loved one. Methods. A systematic review of qualitative studies with a metasynthesis was conducted. MEDLINE (PubMed) and the Cumulative Index to Nursing and Allied Health Literature (CINAHL) databases were searched between 2010-2020. The review methodology was guided by The Preferred Reporting Items for Systematic Reviews and Meta-Analyses (PRISMA) statement; and the Critical Appraisal Skills Programme (CASP) checklist was used to evaluate all included studies. Qualitative data synthesis was performed by using Sandelowski and Barroso's technique.

Results. Out of 1,812 original publications, 15 fulfilled the inclusion criteria, with a total of 216 family members interviewed. Ten categories have emerged, grouped into seven themes: (1) "The experience of the family members", (2) "The emotional experience of the family members", (3) "The environment", (4) "The health professional-family-patient relationship", (5) "The expectation of the family members", (6) "The support for the family members", and (7) "The need for the family members to be actively involved in care". The most represented category was negative feelings (66.6\%), followed by communication $(60.0 \%)$, while the least widespread category was environmental factors (13.3\%).

Conclusion. Findings suggest that family members have experienced the negative effects of the ICU-stay more keenly. Communication, closeness, support, hope and involvement in care were found to be key determinants in families' experiences. Although nursing care in the early stage of a critical illness is less oriented to the concerns of family members, more efforts should be made by the health care professionals to improve a family-centered care approach within a framework of humanization of care.

\section{Reference(s)}

1. La Calle, G. H., Oviés, Á. A., \& Tello, V. G. (2017). A plan for improving the humanisation of intensive care units. Intensive care medicine, 43(4) 547-549.

2. Davidson, J. E., Aslakson, R. A., Long, A. C., Puntillo, K. A., Kross, E. K., Hart, J, \& \& Curtis, J. R. (2017). Guidelines for family-centered care in the neonatal, pediatric, and adult ICU. Critical care medicine, 45(1), 103-128.

000762

Establishing consensus on the use of isotonic saline instillation during physiotherapy management of smoke inhalation in the intensive care unit-a repeated survey review 2020

A. Malik'; A. Hargreaves'; T. Lea

'Physiotherapy, Queen Elizabeth Hospital Birmingham, Birmingham, United Kingdom

Correspondence: A. Malik

Intensive Care Medicine Experimental 2021, 9(1): 000762

Introduction. Pulmonary injury from acute smoke inhalation is common in burns patients. Inhalation injury is a devastating complication in burns for up to one-third of patients and is a significant morbidity, increasing mortality by up to 20 times (Mercel et al., 2020; Dries and Endorf, 2013). Vigorous pulmonary toilet removes obstruction of the airway due to cast formation specific to the pathophysiology of inhalation injury (Toon et al., 2010). There is limited evidence for the routine use of saline within general Physiotherapy practice (Pathmanathan et al., 2014) and the use of saline for smoke inhalation injury is mainly based on clinical reasoning and physiology to remove carbonaceous soot that is adhered to the airway mucosa. The aim of this study is to gain an understanding of current practices with the use of isotonic saline when treating ventilated, smoke inhalation patients in the intensive care unit (ICU) setting. 
Objectives. The aim of this survey is to establish an understanding of current physiotherapy practice for the use of isotonic saline when treating ventilated, smoke inhalation patients in the intensive care unit.

Methods. Between June-September 2020, all lead ICU physiotherapists of burns centres across the UK were invited via email to participate in an online anonymous survey of a combination of 9 closed and open questions.

Results. A response rate of $69.2 \%(9 / 13)$ was obtained. $55.5 \%(5 / 9)$ centres reported using a protocol to guide management. $66.6 \%(6 / 9)$ centres reported use of saline however in varying volumes as demonstrated in Figure 1 below.

https://esicmlives2021.process.y-congress.com/ScientificProcess/ Data/40

Thematic analysis was completed and two key categories were identified; treatment modalities and frequency of review. Figure 2 below outlines treatment modalities.

https://esicmlives2021.process.y-congress.com/ScientificProcess/ Data/40/199/717/bc8477a8-1fed-4328-83cb-30376f9574a2/Uploa ds/Figure\%202\%20-\%20treatment\%20modalities.png

The study found that $55.5 \%(5 / 9)$ used a protocol to guide management. Furthermore, $66.6 \%$ (6/9) used isotonic saline prior to suctioning as standard practice in the chest physiotherapy management of smoke inhalation injuries. Of the centres using isotonic saline, $83.3 \%$ (5/6) used pulmonary lavage as part of physiotherapy intervention, this equates to $55.5 \%$ (5/9) of centres overall. $44.4 \%$ (4/9) completed a regular review; two centres reported this as four hourly. The result of this consensus survey suggests saline use is an accepted practice in smoke inhalation injury management. This was found to be based on expert clinical opinion, established practice and an understanding that saline aids removal of carbonaceous soot. However, physiotherapy treatment modalities and frequency varied. The main limitation to this study was that the survey was only conducted within the UK and is not representative of all UK burns centres due to return rate. Future research could be extended to international burns centres.

Conclusion. This study aimed to establish a consensus on the use of isotonic saline in physiotherapy management of smoke inhalation injuries in ventilated patients. A high percentage used saline instillation as a routine part of treatment and the majority of centres used a protocol to guide clinical practice. The use of saline in respiratory management remains a controversial treatment intervention and there remains insufficient evident to support routine use (Pathmanathan et al., 2014). Future research comparing respiratory management in smoke inhalation injury with and without saline instillation would be beneficial to further develop physiotherapy best practice.

\section{Reference(s)}

1. Toon, M.H., Maybauer, M.O., Greenwood, J.E., Maybauer, D.M., and Fraser, F.J. (2010) Management of acute smoke inhalation injury. Critical Care Resuscitation, 12, 53-61.

2. Pathmanathan, N., Beaumonth, N., and Gratrix, A. (2014) Respiratory physiotherapy in the critical care unit. Continuing Education in Anaesthesia Critical Care \& Pain [online], 15 (1), 20-25. Available from: https://doi. org/10.1093/bjaceaccp/mku005

3. Mlcak, R.P., Suman, O.E., and Herndon, D.N. (2007) Respiratory management of inhalation injury. Burns [online], 33(1), 2-13. Available from: https://doi.org/10.1016/..burns.2006.07.007

4. Mercel, A., Tsihlis, N.D., Maile, R., and Kibbe, M.R. (2020) Emerging therapies for smoke inhalation injury: a review. Journal of Translational Medicine [online], 18(1), 141. Available from: https://doi.org/10.1186/ s12967-020-02300-4
5. Dries, D.J., and Endorf, F.W. (2013) Inhalation injury: epidemiology, pathology, treatment strategies. Scandinavian Journal of Trauma, Resuscitation and Emergency Medicine [online], 21. Available from: https://doi.org/10. 1186/1757-7241-21-31

6. Chao, K-Y., Lin, Y-W., Chiang, C-E., and Tseng, C-W. (2019) Respiratory Management in Smoke Inhalation Injury. Journal of Burn Care \& Research. 40, 507-512.

7. We thank the physiotherapists who took part in our survey for their valuable contributions to our review and acknowledge the ongoing care they provide to smoke inhalation injury patients across the UK.

000808

Therapeutic Effectiveness of Diaphragmatic with Costal Breathing Exercises On C-19 PEFR Patients

K. T

${ }^{1}$ Physiotherapy Center, NIMHANS, Bangalore, India

Correspondence: $\mathrm{K}$. $\mathrm{T}$

Intensive Care Medicine Experimental 2021, 9(1): 000808

Introduction. COVID-19 symptoms can linger for weeks or even months after the initial infection. This phenomenon has been dubbed "long covid" by some. Because the coronavirus primarily affects the lungs, one of the long COVID-19 breathing symptoms after initial recovery can be ongoing breathlessness. The key symptoms of COVID19 virus are cough, fever, breathlessness, anxiety, delirium and agitation, but you may also have fatigue, muscle ache and headache.

Objectives. To find out the therapeutic effectiveness of diaphragmatic breathing with costal breathing exercises on C-19 patients.

Methods. DESIGN:

The study design was pre-test, post test experimental study design. PARTICIPANTS:

Sixty subjects aged 30 to 55 years with C-19 patients were selected under purposive sampling technique and assigned into two groups with 30 subjects each, one group remained as control group and other group received diaphragmatic breathing with costal breathing exercises.

INTERVENTIONS:

The patients were instructed to perform diaphragmatic breathing with coastal exercises, four times each, three times a day for four weeks. MAIN OUT COME MEASURES:

PEFR on Peak Flow Meter and dyspnea rating on Modified Borg Dyspnea scale were used for evaluation of both groups.

Results. Patients in the experimental group with diaphragmatic breathing and costal breathing exercises showed significantly better performance.

Conclusion. The author concluded that DBE with CBE improve the peak expiratory flow rate and reduce dyspnea level in C-19 Patients.

Reference(s)

1. 4. H.Denagardinar: "The Principles of Exercise Therapy" 4th edition[CBS publishers and Distributors, New Delhi, 1985]

2. 3. Tidys physiotherapy, Ann Thomson, Alison Skinner', John Piercy 12th edition [Varghese publishing house, Dadar Mumbai.

3. 2. Davidson's, Principles and practice of medicine, 2nd edition, Churchill Livingston 1998

4. 1. Barbara A, Physiotherapy for respiratory and cardiac problems, WB Saunders, 1986

5. My sincere thanks to Director of NIMHANS Bangalore given permission under go and investigate the effects of this study

6. This study observation/pilot study.Here by I declared that there is no financial implications on the part of this study 
000812

\section{Building patient safety: adverse events notification}

LCA. Leite ; C. Carvalho

${ }^{1}$ Intensive Care Department, University Hospital Center of Algarve, Faro, Faro, Portugal

\section{Correspondence: L.C.A. Leite}

Intensive Care Medicine Experimental 2021, 9(1): 000812

Introduction. Patient safety has been gradually recognized, within the scope of health quality, with clinical error and management emerging in its various areas and, specifically, the notification of incidents/adverse events. These events significantly impact the results in hospital health, and notification of them is considered one of the most efficient means of prevention.

Objectives. Improving the quality of health and safety of critically ill patients, through the introduction of a tool, adapted to the clinical context, of notification of Adverse events.

Methods. Running between December 2019 and March 2020 in a level III UCl, based on project work methodology, we introduced a closed adverse event notification system, within the intensive care department, evidence-based, combined with the opinions and suggestions of the medical and nursing staff. Previous to the operationalization of the system, a situation diagnosis questionnaire was drawn up, used an online form, sought to obtain a sociodemographic and professional characterization of the target population (Doctors and Nurses) and to carry out a reliable and concrete assessment of the team's knowledge, perception and experience in the use of notification systems. Inseparable from the situation diagnosis questionnaire, the free and informed consent was obtained through the same online form.

Results. Of the 69 professionals of the multidisciplinary team, $82.6 \%$ $(n=57)$ consented to participate, with the consent and filling out the questionnaire, accounting for $79 \%(n=42)$ of the total number of nurses and $94 \%(n=15))$ percentage of the Medical team, a sample that is considered significant and representative of the team. In view of the external constraints posed, only a sociodemographic and professional characterization of the Nurses will be carried out. there is a significant percentage of Specialist Nurses (39\%), mostly in Critical Care $(57 \%)$ with a high heterogeneity in the nursing team with regard to professional experience (average $=12$ years) and in intensive care (average $=9$ years).

Previous training in risk management and patient safety is clear for most professionals (67\%). In this context, greater knowledge (89\%) and use (54\%) of the adverse organization notification system of the Hospital Organization is highlighted in relation to the national system for notification of adverse events (39\% know but only $14 \%$ have already used it).

With regard to assessing the utility, ease of use and suitability of using the Hospital Organization's adverse event reporting system and the national system, a 10-item Likert scale was used. Thus, there is a reasonable assessment of the usefulness of the Hospital Organization's adverse event notification system (average of 6.12 , of $0-10$ ) that is combined with a deficient assessment in the most operational items, ease of use and suitability for the clinical context, 3.96 and 4.38 respectively. The low feedback rate of the notifications made $(10 \%)$ is noted. A tool proposal was built structured in 7 consecutive and sequential steps (Patient Identification, Incident Description, Incident Detection,
Severity Rating, Procedures Performed, Improvement Proposal, Notifier Identification), developed around 12 related axes (s) with the type (s) of adverse incident (s)/event (s) occurred. Fields of free completion were created in the various stages to supply the possibility of non-inclusion or inadequacy of the incident in the axes and / or in the options given or the possibility of adding relevant data to the notification that was not pre-contemplated. Likewise, the condition of anonymity was not considered mandatory, due to the possibility of obtaining more relevant information from the notifier, if he has identified himself.

Conclusion. It was possible and feasible to build a tool, in a closed system within the Intensive Care Department, integrated in the hospital's adverse event notification system.Thus, due to the declaration of the state of emergency and public calamity and the declaration, by the WHO, of Pandemic by SARS-COVID-19, it was not possible to follow the planning, previously outlined, in our clinical context, namely the collection and treatment of the notifications made, by the profound reorganization of the health organization, invalidating and delaying some of the planned activities. Likewise, when possible, they intend to move forward with delayed activities.

\section{Reference(s)}

1. Brunsveld-Reinders, A., Arbous, M., De Vos, R. \& De Jonge, E. (2016). Incident and error reporting systems in intensive care: a systematic review of the literature. International Journal for Quality in Health Care, 28(1), 2-13. https://doi.org/10.1093/intqhc/mzv100.

2. Heavner, J. \& Siner, J. (2015). Adverse Event Reporting and Quality Improvement in the Intensive care Unit. Clinics in Chest Medicine, 36(3), 461-467. https://doi.org/10.1016/j.ccm.2015.05.005.

3. Garrouste-Orgeas, M., Flaatten, H. \& Moreno, R. (2016). Understanding Medical Errors and Adverse Events in ICU Patients. Intensive Care Medicine, 42(1), 107-109. https://doi.org/10.1007/s00134-015-3968-x.

4. Ruivo, A., Ferrito, C \& Nunes, L. (2010). Metodologia de Projecto: Colectânea Descritiva de Etapas. Percursos, n. 15 (Janeiro-Março 2010). Acedido em 13 de Dezembro de 2019. Retirado de http://web.ess.ips.pt/ Percursos/pdfs/Revista_Percursos_15.pdf.

\section{4}

Achieving calm after the storm-recovering staff morale post Covid

M. Malaj ${ }^{1}$; C. Webb ${ }^{1} ;$ K. Billing ${ }^{1}$; D. Bryden ${ }^{1}$

${ }^{1}$ Critical care, Sheffield Teaching Hospitals, Sheffield, United Kingdom Correspondence: D. Bryden

Intensive Care Medicine Experimental 2021, 9(1): 000834

Introduction. Prior to the Covid pandemic one third of NHS staff reported being unwell from work related stress. [1]. Recent surveys suggest only $54 \%$ of ICU nursing staff working during the pandemic report good well-being [2]. Poor staff mental health is associated with increased risk to patients and workforce shortages. Multi-level interventions (training and a positive working environment complemented by specialist support if needed) have been shown to be most effective in improving staff wellbeing.

Objectives. We set out to research and promote positive staff experiences in Sheffield as a way to 
1. Support workforce recovery and mental health wellbeing

2. Encourage staff engagement as a marker of quality of patient care and staff recovery

Methods. Since March 2021 we have introduced Appreciative Inquiry (A) into critical care governance. Al is an approach focussing on strengths rather than deficits and is based on a 5D cycle [3]. (Figure 1).

As part of our discovery phase we have introduced a monthly 'Shout Out' to encourage staff to contribute positive written feedback about colleagues. Staff write positive comments on the working day and individuals they feel have helped them. Feedback is shared with the whole department to highlight the range and number of contributions. All nominations received are subsequently distributed to staff for use in revalidation portfolios.

Mortality and morbidity meetings have also been adjusted to allow for case presentations that meet the delivery and discovery aspects of $\mathrm{Al}$ by focussing on when good care delivery occurred in difficult circumstances eg facilitating organ donation during the pandemic. This enables staff to develop reflection within a positive supportive framework. Results. The initiative has received overwhelmingly positive feedback from all staff groups in the department. Full data is not yet available on the impact of changes as this is an iterative, cycle approach but quantitative and qualitative feedback will be presented for the 2021 meeting.

Conclusion. Simple primary level interventions promoting positive staff experiences and using appreciative inquiry techniques are costeffective outside of critical care. We believe developing a cycle of positivity within routine unit day to day functioning using an Al approach can provide a cheap, simple intervention to help support post pandemic staff recovery.

\section{Reference(s)}

1. 3. https://www.england.nhs.uk/south/wp-content/uploads/sites/6/2020/ 08/Appendix-7.2-Al-training.pdf

2. 2. Greenberg $\mathrm{N}$ et al. Occup Med (Lond) 2021 Apr 9;71(2):62-67. https:// doi.org/10.1093/occmed/kqaa220

3. 1. NHS Employers. https://www.nhsemployers.org/retention-and-staffexperience/health-and-wellbeing/taking-a-targeted-approach/taking-atargeted-approach/mental-health-in-the-workplace

\section{6}

Development and Implementation of a Critical Care 'Cheat Sheet' as a Visual Learning Tool

L. Russell ${ }^{1}$; L. Mcleish

${ }^{1}$ Intensive Therapy Unit, N H S Borders, Melrose, United Kingdom

Correspondence: $L$. Russell

Intensive Care Medicine Experimental 2021, 9(1): 000876

Introduction. Critical Care Nursing is a highly skilled role and advances in intensive care medicine demand a variety of specialist skills from the critical care nurse to safely apply in-depth knowledge to practice. Transition to the critical care environment for new and redeployed nurses is challenging due to the complex knowledge, skills and technical abilities that underpin safe and effective practice.

Objectives. We aimed to develop a visual learning tool to display complex information in a Manner that could be quickly consumed and easily understood. This aids comprehension, enhances quality of learning and retention of the material. We aimed to engage staff and encourage further information seeking. Displaying the 'Cheat Sheet' in prime position in the critical care area will allow staff to recognise when treatments are required and when deviations from normal values are evident.

\section{Methods}

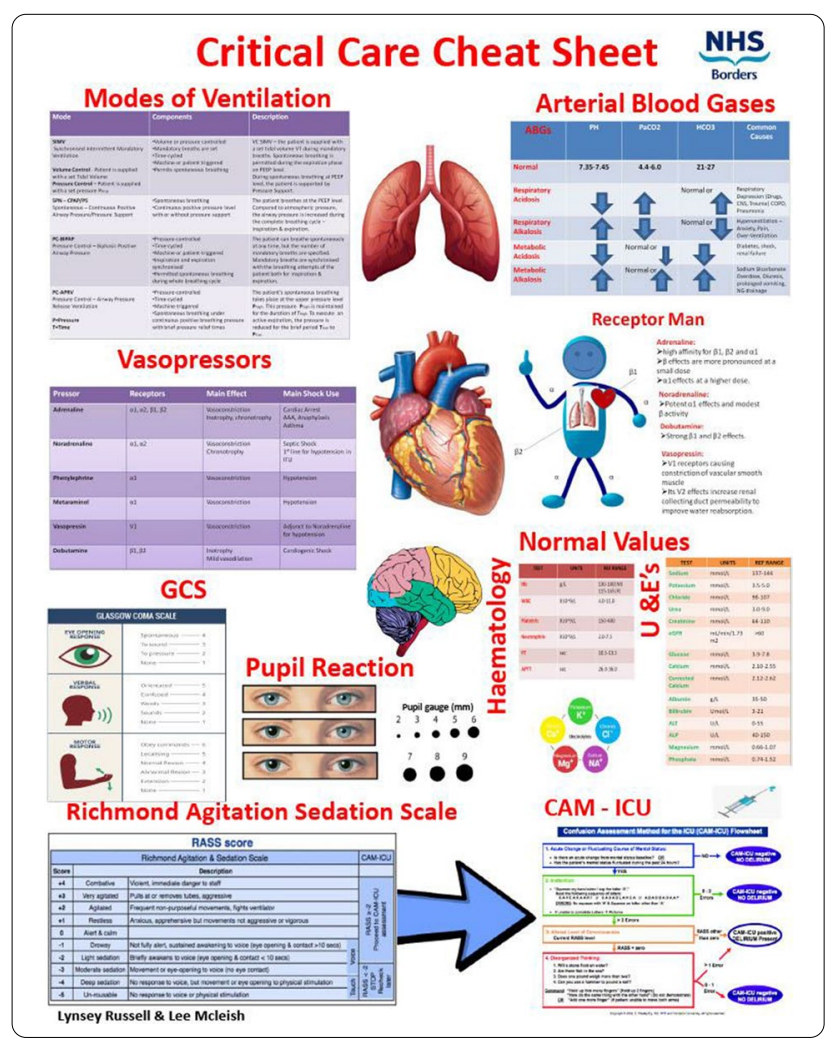

We conducted an informal survey of new and redeployed staff, asking which areas of critical care they perceived to be most daunting. We used the Plan, Do, Study Act (PDSA) cycle to produce multiple iterations of the resulting infographic. After discussion with nursing, medical and wider multi-disciplinary team colleagues, we produced the final version, focusing on Cardiorespiratory and neurological principles.

Results. Extensive qualitative feedback has been universally positive and we have had significant interest from other Critical Care Units throughout the country hoping to emulate our success.Staff reported that patients are receiving treatment quicker due to the presence of the 'Cheat Sheet'. Electrolyte replacement is initiated promptly and early stages of delirium are picked up and treated appropriately. It is now being shared widely throughout the UK.

Conclusion. The infographic 'Cheat Sheet' first and foremost enhances patient safety. To ensure equity of care delivery, it makes sense to strive for a critical care workforce that is trained to common standards, assuring quality of workforce across geographical boundaries. This leads to an enhanced patient journey and can reduce length of stay. Nurses utilising the 'Cheat Sheet' learn about the skills and knowledge needed to deliver safe high quality bedside care to the critically ill patient. Being able to involve and work closely with the multidisciplinary team to best meet the patient's needs is essential to providing person centred care.

\section{Reference(s)}

1. Deacon KS, Baldwin A, Donnelly KA, Freeman P, Himsworth AP, Kinoulty SM, Kynaston M, Platten J, Price AM, Rumsby N, Witton N (2017). The National Competency Framework for Registered Nurses in Adult Critical Care: An Overview. Journal of the Intensive Care Society. 10: 1-15 
000943

Exploring the impact of Intensive Care Unit admission on physical function in COVID-19 survivors: Clinical observations from a large London NHS Foundation Trust during the first wave of the COVID-19 pandemic

C. Bradley'; K. Jerrard ${ }^{2}$; C. Reilly, ${ }^{1}$

${ }^{1}$ Physiotherapy, King's College Hospital, London, United Kingdom; ${ }^{2}$ Physiotherapy, Royal Victoria Hospital, Belfast, United Kingdom

Correspondence: $C$. Bradley

Intensive Care Medicine Experimental 2021, 9(1): 000943

Introduction. Intensive Care Unit (ICU) survival is frequently accompanied by ICU acquired weakness, impaired mobility levels and reduced health related quality of life $(1,2)$. The COVID-19 pandemic caused a sudden and unprecedented surge in ICU admissions for severe acute respiratory failure. Whilst stretching ICU capacity and resources to its limits, little was known about the pathophysiology, treatment or physical impact on the patient of COVID-19.

Objectives. To explore the impact of ICU admission on physical function in COVID-19 survivors.

Methods. A retrospective clinical review of patients admitted to ICU with a primary diagnosis of COVID-19 between March-April 2020 was conducted at a large London NHS Foundation Trust. Electronic clinical notes were reviewed, and the following data extracted: age, ethnicity, sex, BMI, duration of sedation, duration of mechanical ventilation, ICU length of stay (LOS) and hospital LOS. Physical impairment was based on the Intensive Care Unit Mobility Score (ICUMS) and defined as significant $(\leq 3)$, moderate $(\leq 6)$, mild $(\geq 7)$ or none (score of 10$)$. Data were analysed using descriptive statistics and reported as absolute numbers, percentages (\%) and median (range).

Results. 178 patient clinical notes were identified, 134 clinical notes were excluded as 56 died, 2 were incidental findings of COVID-19, 58 had missing data due to paper notes or transfers in/out of the Trust and 18 were duplicate records. Forty-four patients were included in the final analysis; of which 26 (59\%) were male, $13(30 \%)$ white, age 53.5 (25-78) years, BMI 28.2 (19.9-59.3), duration of sedation 9 (0-39) days, duration of mechanical ventilation 15 (0-61) days, ICU LOS 19 (2-67) days and hospital LOS 38.5 (5-207) days.

$98 \%$ of patients had ongoing physical impairment and rehabilitation needs at step down from ICU: 19 (43\%) significant, 18 (41\%) moderate and $6(14 \%)$ mild physical impairment. $84 \%$ had either a severe or moderate level of impairment defined as being unable to mobilise away from the bedspace (ICUMS $\leq 6)$.

At hospital discharge $57 \%$ of patients had residual physical impairment and rehabilitation needs: 2 (5\%) significant, 1 (2\%) moderate, 22 (51\%) mild physical impairment.

Conclusion. These preliminary data suggest that ICU admission due to COVID-19 resulted in acute and long-term physical impairment and rehabilitation needs for individuals surviving COVID-19. Future research needs to explore if these data are representative of the national and global impact of COVID-19 and examine whether there are contributary factors to the degree of physical impairment experienced by ICU COVID-19 survivors. Moreover, these data highlight the importance of having core outcome measures within the ICU so that we can fully understand the acute and long-term needs of individuals surviving ICU admission, to inform rehabilitation provision and health care policy.

\section{Reference(s)}

1. 2. Physical Impairments Associated With Post-Intensive Care Syndrome: Systematic Review Based on the World Health Organization's International Classification of Functioning, Disability and Health Framework. Ohtake PJ, Lee AC, Scott JC, Hinman RS, Ali NA, Hinkson CR, Needham DM, Shutter L, Smith-Gabai H, Spires MC, Thiele A, Wiencek C, Smith JM. Phys Ther. 2018: 1;98(8):631-645
2. 1. Post-intensive care syndrome: Its pathophysiology, prevention, and future directions. Inoue S, Hatakeyama J, Kondo Y, Hifumi T, Sakuramoto H, Kawasaki T, Taito S, Nakamura K, Unoki T, Kawai Y, Kenmotsu Y, Saito M, Yamakawa K, Nishida O. Acute Med Surg. 2019: 25;6(3):233-246.

3. Nil.

001126

Do many hands make light work? Lessons learned about nursing team composition in Intensive Care Units during the first COVID-19 wave

D. Stalpers ${ }^{1}$; M. Veenstra ; D. Martini' ${ }^{2}$;. Lalleman²; L. Schoonhoven ${ }^{1}$ ${ }^{1}$ Julius Center for Health Sciences and primary Care; Nursing Science, University Medical Center Utrecht, Utrecht, Netherlands; ${ }^{2}$ Insitute of Nursing Studies, HU University of Applied Sciences Utrecht, Utrecht, Netherlands Correspondence: $M$. Veenstra

Intensive Care Medicine Experimental 2021, 9(1): 001126

Introduction. Teamwork is an essential part of most nurses' jobs. A combination of various characteristics, like team composition (skills mix) and staffing numbers determine team effectiveness. New techniques and growing complexity of clinical care ask for a variety of nursing qualities and changes in the current skill mix. In the Netherlands, hospital nurses with various educational backgrounds often fulfil the same tasks. Despite calls for change, developing more agile and flexible nursing teams is a lengthy and complex process. The COVID-19 pandemic underscores the need for an efficient skill mix. Due to the enormously increased work pressure on Intensive Care Units (ICUs), hospitals called on other healthcare professionals to support the ICU staff. During the first COVID-19 wave in April 2020, set teams were forced to work in newly composed teams with new dynamics. This type of teamwork can be described as cross-boundary teaming or teaming.

Objectives. The aim of this study was to provide insight from a workplace perspective into how teaming took place at ICUs in the Netherlands during the first COVID-19 wave. With these insights we aimed to describe lessons learned and to gain new knowledge on long-term team design in ICU nursing.

Methods. For this study 46 semi-structured interviews were conducted from June to September 2020. Supervisors, ICU nurses and healthcare personnel in a supporting role were interviewed in five different hospitals. An abductive approach was employed and data were coded and converted into themes via thematic analysis.

Results. Three main themes emerged from the interview data: commitment, teamwork and changing responsibilities. Within these themes the perspectives of the three groups of interviewees were shown. The acute crisis situation evoked strong feelings of solidarity and boundaries between healthcare professionals faded. This led to the opportunity to experiment with new ways of task and role division. At the same time, changes in responsibilities led to conflict and a strong need for task demarcation arose. All three steps of teaming were identified and illustrated from different interviewee perspectives. Interpersonal aspects and behaviors of successful teaming were also described.

Conclusion. Teaming took place at various ICUs during the first COVID-19 wave. Although the process of teaming was unplanned, positive results were seen. The lessons learned during the COVID-19 crisis can be useful for future change processes. Increasing staff shortages and growing complexity of nursing care underscore the need for change. Teaming could be a promising way of organizing team work in nursing. However, interviewed healthcare professionals addressed several challenges. Many hands do not necessarily make light work. To cope with current and future challenges, it is essential that nurses expertise and strengths are being used to the fullest.

\section{Reference(s)}

1. This work was supported by Dutch Ministry of Health, Welfare and Sports 
001134

Addressing skill shortages in Intensive Care Medicine; a qualitative and quantitative analysis of the introduction of an Advanced Critical Care Practitioner programme in a tertiary-level hospital

S. Sears-Aizenberg ${ }^{1}$; A. Wong ${ }^{2}$

${ }^{1}$ King's Critical Care, King's College Hospital, London, United Kingdom;

${ }^{2}$ King's Critical Care, King's College Hospital, London, United Kingdom

Correspondence: S. Sears-Aizenberg

Intensive Care Medicine Experimental 2021, 9(1): 001134

Introduction. The recent COVID19 pandemic has placed an unprecedented strain in healthcare systems across the world. In such challenging times, it is a timely reminder that the delivery of highquality care is dependent upon the availability of trained staff. However, such delivery of care has traditionally been the remit of doctors and nurses, supported by other allied healthcare professionals such as physiotherapists and dietitians. The Advanced Critical Care Practitioner (ACCP) is unique in that it is relatively novel outside of the USA and UK. Given the challenges of staffing (made more acute by COVID), we aimed to see if this dogma can be challenged.

Objectives. To assess the effectiveness and safety of ACCPs after having recieved Faculty of Intensive Care Medicine (FICM) accreditation in the delivery of intensive care services in a tertiary-level, teaching hospital in the UK.

Methods. A triangulation exercise, using qualitative and quantitative measures simultaneously, to assess the work output and response to the introduction of ACCPs. Quantitative data was captured over 4 weeks to identify the various clinical roles provided by the ACCPs on a shift-by-shift basis. These included the transfer of patients, insertion of vascular access, patient stabilisation etc. A multi-disciplinary questionnaire was designed to assess the experiences, attitudes and interactions of other colleagues who came into contact with the ACCP team.

Results. A total of 11 ACCPs were introduced into the critical care team. Analysis of the ACCP clinical duties highlighted that they are involved in the provision of critical care both within and outside the ICU (e.g. Emergency department).The majority of their work involved clinical review of sick patients as well as the establishment of central venous and arterial access for higher level of monitoring/ care.

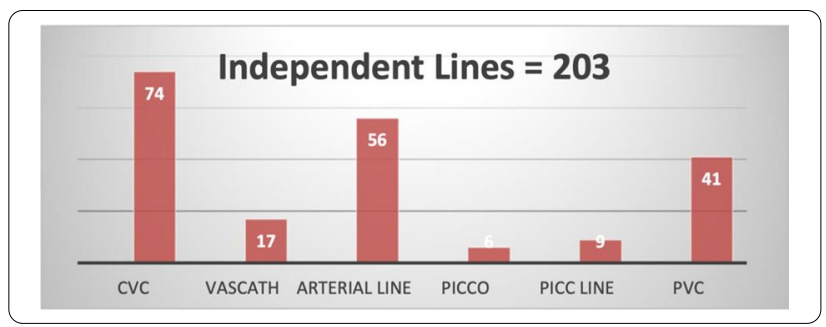

During this period, the majority of patient transfers (CT scans, transfer from ward to ICU) were performed without immediate, doctor supervision. $70 \%$ were independent and $30 \%$ were supervised.

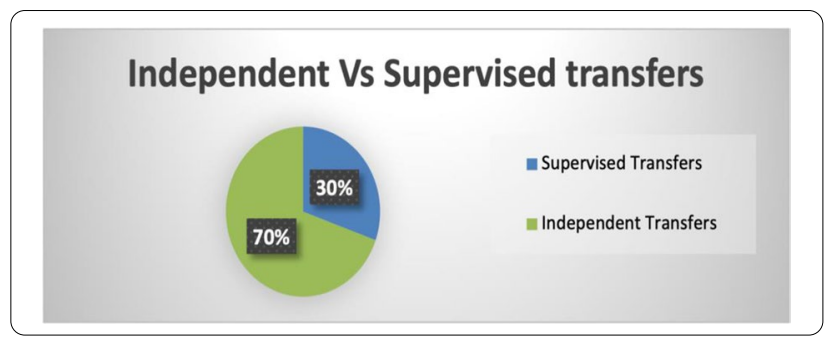

The survey highlighted the fact that the majority of established ICU staff interact with ACCPs on a regular basis with universally positive feedback.

Conclusion. The COVID pandemic is a timely reminder and an opportunity to address the shortage of trained specialist in ICM and evaluate the way we work and deliver care. Introducing ACCPs into our critical care workforce in 2019, we have demonstrated that they are both safe and effective in the provision of intensive care across the entire hospital setting and complement existing healthcare roles. The reduction in workload on doctor/nurses time on common task such as patient transfer and venous access means that resources can be more appropriately re-allocated.

\section{Reference(s)}

1. Advanced Critical Care Practitioner Membership |The Faculty of Intensive Care Medicine [Internet]. 2020 [cited 2020 Feb 23]. Available from: https://www.ficm.ac.uk/membership/advanced-critical-care-practition er-membership

2. Curriculum | The Faculty of Intensive Care Medicine [Internet]. [cited 2020 Nov 23]. Available from: https://www.ficm.ac.uk/accps/curriculum

\section{5}

VEMO: An Innovative Robotic Device for Early Mobilization in the Intensive Care Unit

M. Egger ; M. Steinböck'; V. Huge ${ }^{2} ;$ F. Müller $^{3}$

${ }^{1}$ Research Department, Schön Klinik Bad Aibling, Bad Aibling, Germany;

${ }^{2}$ Anesthesiology and Intensive Care, Schön Klinik Bad Aibling, Bad Aibling, Germany; ${ }^{3}$ Neurology, Schön Klinik Bad Aibling, Bad Aibling, Germany

Correspondence: $M$. Egger

Intensive Care Medicine Experimental 2021, 9(1): 001225

Introduction. Early mobilization in the Intensive Care Unit (ICU) has been shown to be safe and effective, but is also highly elaborate and time consuming, thereby limiting its frequent realization $(1,2)$. The new robotic device VEMO (Reactive Robotics, Munich, Germany), is the first robot especially developed for implementation in the ICU. It enables innovative early mobilization by verticalizing patients combined with step-like leg movements applied directly in the patient's bed Thus, no dangerous and resource intensive transfer onto a therapy device is needed and verticalization is highly simplified.

Objectives. Evaluating feasibility and safety of the VEMO in a neurological ICU and early rehabilitation setting.

Methods. The VEMO is currently assessed in a pilot project in early rehabilitation and the ICU at Schoen Clinic Bad Aibling, Germany. Therapies are conducted by a team of trained nurses and physiotherapists. Patients with neurological diseases and stable cardiovascular and neurologic conditions as well as patients undergoing weaning from mechanical ventilation are eligible for VEMO therapy.

Results. In the early rehabilitation setting, in 5 tracheotomized patients with severe disorders of consciousness (mean age $57.2 \pm 11.8$ years, 2 female) a series of 6 VEMO therapies per patient was administered. Patients responded well to the therapy, partly improved oxygen saturations or were more awake. Mean therapy duration was 17:35 \pm 04:39 min, mean verticalization angle was $49.0 \pm 8.6^{\circ}$, mean amount of steps was $535 \pm 150$. Therapy preparation took 10-20 min.Within the applications in ICU, VEMO therapy was accepted very well by ICU staff and patients and no adverse events occurred. VEMO therapy was conducted in patients during mechanical ventilation (tracheal cannula) as well as in spontaneously breathing patients within the weaning process. Central venous catheters, invasive arterial line devices as well as breathing hoses did not interfere with the VEMO therapy. In case of an emergency, treatment can be terminated within seconds. 


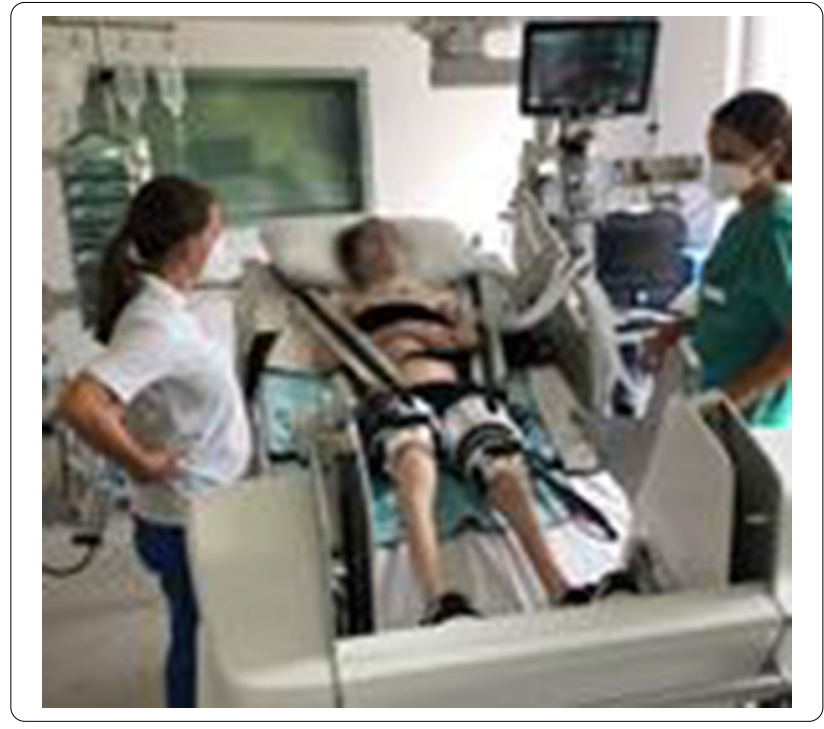

Figure 1: VEMO therapy with a ventilated patient after double lung transplantation.

Conclusion. The VEMO offers a safe early robotic-based mobilization in critically ill patients within the ICU. Verticalization can be realized with minimal time and staff resources, thus a high frequency of early mobilization seems achievable. Further evaluation comparing VEMO therapy to standard mobilization in the ICU needs to be performed in future trials.

\section{Reference(s)}

1. 1. Zhang L, Hu W, Cai Z, et al. Early mobilization of critically ill patients in the intensive care unit: A systematic review and meta-analysis. PloS one. 2019;14(10):e0223185.

2. 2. Parry SM, Knight LD, Connolly B, et al. Factors influencing physical activity and rehabilitation in survivors of critical illness: a systematic review of quantitative and qualitative studies. Intensive care med. 2017:43(4):531-542.

3. The study was partly funded by the German Federal Ministry of Education and Research, project MobIPaR (reference number: 16SV17731).

e-Posters: Paediatrics

\section{1}

Effects on Incidence of Hyperchloraemia Following Introduction of Plasma-Lyte 148 in PICU

S. McCallion'; L. Woodgate, ; L. Mckelvie'; C. Begg

${ }^{1}$ Paediatric Intensive Care Unit, Royal Hospital for Children, Glasgow,

Glasgow, United Kingdom

Correspondence: S. McCallion

Intensive Care Medicine Experimental 2021, 9(1): 000131

Introduction. The ideal type of maintenance fluid for paediatric patients lacks high level evidence. The shift from hypotonic to isotonic fluids has been adopted in many units following widespread concern regarding hyponatraemia.(1) However, with the use of $0.9 \%$ saline, comes an excess chloride load. Hyperchloraemia can result in metabolic acidosis and hyperchloraemia has been shown to increase risk of acute kidney injury $(2,3)$ and mortality $(4)$. Using balanced crystalloids has been shown to reduce the incidence of hyperchloraemia. (5) In our quaternary paediatric intensive care unit, our maintenance fluid practice changed in February 2017 with a new guideline recommending the use of Plasma-Lyte 148 over $0.9 \%$ saline for standard maintenance.
Objectives. Our primary aim was to assess if the practice change led to a reduction in episodes of hyperchloraemia. Our secondary aim was to review whether peak chloride levels were reduced.

Methods. Metavision electronic medical records were searched for all patients admitted to Paediatric Intensive Care Unit (PICU) over 3 time periods 1) between 01/12/2016-31/01/2017 (group 2016, pre-change), 2) 01/12/2017-31/01/2018 (group 2017) and 3) 01/12/2019-31/01/2020 (group 2019). Patients who had an episode of hyperchloraemia (Chloride $>107 \mathrm{mmol} / \mathrm{L}$ ) were included. Patients who had hyperchloraemia on admission, which normalised within $24 \mathrm{~h}$, or did not receive IV maintenance fluids in PICU were excluded. Data was gathered for patient demographics, length of stay, admission diagnosis, type of maintenance fluid prescribed and whether they were post-operative including post-operative from cardiac bypass surgery. Statistical analysis was completed using Microsoft Excel and R Studio.

Results. Following the introduction of Plasma-Lyte 148, the relative risk reduction of a patient having an episode of hyperchloraemia was 1.58 (95\% Cl 1.28-1.95). Incidence of hyperchloraemia over the year groups is shown in Table 1 . There was a statistically significant reduction in the incidence of hyperchloraemia after the introduction of Plasma-Lyte $148(x 2=17.6629 p=<0.005)$. Mean chloride levels in those with hyperchloraemia also reduced. In group 2016 mean chloride level $(95 \% \mathrm{Cl})$ was $114.94 \mathrm{mmol} / \mathrm{L}(113.67,116.21)$, in group 2017 it was $113.90 \mathrm{mmol} / \mathrm{L}(112.71,115.09)$ and in group 2020 it was $113.22 \mathrm{mmol} / \mathrm{L}(111.8,114.6)$. A linear regression analysis accounting for confounding variables demonstrated a statistically significant reduction in the peak chloride level in those patients with hyperchloraemia with a reduction in chloride levels in group 2017 and group 2019 by $-1.85 \mathrm{mmol} / \mathrm{L}(\mathrm{p}=<0.05)$ and $-1.89 \mathrm{mmol} / \mathrm{L}(\mathrm{p}=<0.05)$ respectively.

Table 1. Hyperchloraemia over year groups

\begin{tabular}{llll}
\hline $\begin{array}{l}\text { Year Group } \\
(\mathbf{n}=)\end{array}$ & $\begin{array}{l}\text { Group 2016 } \\
(\mathbf{n}=\mathbf{1 5 0 )}\end{array}$ & $\begin{array}{l}\text { Group 2017 } \\
(\mathbf{n}=\mathbf{1 6 1})\end{array}$ & $\begin{array}{l}\text { Group 2019 } \\
(\mathbf{n}=\mathbf{1 4 8})\end{array}$ \\
\hline $\begin{array}{c}\text { \% of hypechlo- } \\
\text { raemia }(\mathrm{n}=)\end{array}$ & $53.3 \%(\mathrm{n}=83)$ & $36.6 \%(\mathrm{n}=59)$ & $33.1 \%(\mathrm{n}=49)$ \\
$\begin{array}{c}\text { Mean Chloride } \\
\mathrm{mmol} / \mathrm{L}(95 \%\end{array}$ & $\begin{array}{c}114.94(113.67 \\
\text { to } 116.21)\end{array}$ & $\begin{array}{c}113.90(112.71 \\
\text { to } 115.09)\end{array}$ & $\begin{array}{c}113.22(111.9 \text { to } \\
114.6)\end{array}$ \\
$\mathrm{Cl})$ & & & \\
\hline
\end{tabular}

Conclusion. Introduction of Plasma-Lyte 148 as maintenance fluid reduced the incidence of hyperchloraemia in our PICU. Where hyperchloraemia occurs, the overall chloride value is also significantly lower.

\section{Reference(s)}

1. 1. Medicines and Healthcare products Regulatory Agency (MHRA). Intravenous $0.18 \%$ saline/4\% glucose solution ('hypotonic saline'): do not use in children except in specialist settings under expert supervision [Internet]. Medicines and Healthcare products Regulatory Agency (MHRA); 2012 Oct [cited 2021 Mar 20]. Available from: https://assets.publi shing.service.gov.uk/government/uploads/system/uploads/attachment_ data/file/852381/0.18_saline_or_4_glucose_IV_solution_safety_risks_ in_children.pdf

2. 2. Yunos NM, Bellomo R, Hegarty C, Story D, Ho L, Bailey M. Association Between a Chloride-Liberal vs Chloride-Restrictive Intravenous Fluid Administration Strategy and Kidney Injury in Critically III Adults. JAMA. 2012 Oct 17;308(15):1566

3. 3. Wilcox CS. Regulation of Renal Blood Flow by Plasma Chloride. J Clin Invest. 1983 Mar;71(3):726-35.

4. 4. Stenson EK, Cvijanovich NZ, Anas N, Allen GL, Thomas NJ, Bigham $M T$, et al. Hyperchloremia is associated with complicated course and mortality in pediatric patients with septic shock. Pediatr Crit Care Med J Soc Crit Care Med World Fed Pediatr Intensive Crit Care Soc. 2018 Feb;19(2):155-60.

5. 5. Bulfon AF, Alomani HL, Anton N, Comrie BT, Rochwerg B, Stef SA, et al. Intravenous Fluid Prescription Practices in Critically III Children: A Shift in Focus from Natremia to Chloremia? J Pediatr Intensive Care. 2019 Dec;8(4):218-25. 
000418

Mortality Trends in trauma patients in a Pediatric Intensive Care Unit

P. Kafaraki P. ; A. Spanaki ${ }^{1}$; E. Dardamani ${ }^{1}$; T. Tavladaki ${ }^{1}$; M. Miliaraki ${ }^{1}$; E. Vasilaki ${ }^{1}$; E. Geromarkaki' ${ }^{1}$ S. Ilia

${ }^{1}$ Paediatric Intensive Care Unit, University General Hospital of Heraklion, Heraklion, Greece

Correspondence: A. Spanaki

Intensive Care Medicine Experimental 2021, 9(1): 000418

Introduction. Injury is a leading cause of morbidity and mortality among children. Approximately $4 \%$ of injured children require hospital admission. The most critically injured will require admission to the pediatric intensive care unit (PICU), with high morbidity, while up to $17 \%$ of children discharged with a moderate to severe disability.

Objectives. The purpose of our study was to determine the epidemiology of death in our in a 7-bed PICU based in a University Hospital regarding trauma patients.

Methods. In this observational study, we retrospectively analyzed the characteristics of all the pediatric trauma patients, who died during their hospitalization in our Level III mixed PICU, in our University Hospital, during the last 5 years (1st January 2016-31th March 2021). The data collected included demographic profile, diagnosis admission, cause of death, length of stay and severity scores, such as PediatricRisk of Mortality (PRISM) III-24 and Pediatric Logistic Organ Dysfunction PELOD score. The study was conducted in our 7-bed pediatric intensive care unit (PICU) in a university hospital responsible for children not only from our island but from all the Southern islands of our country.

Results. From 758 PICU admissions during this 5 years period, we retrospectively identified 6 pediatric trauma patients, among total 90 children with trauma or accident, who died during their hospitalization in the PICU $(0.67 \%)$. The leading causes of death were traumatic head injury (only) (50\%), multiple injuries (33.3\%) and drowning (16.7\%). The median age was 7.5 years $(\min 0.5, \max 14)$ and it mainly concerned male patients (male:female $=4: 2$ ). The median length of stay of children who died in our PICU was 20 days. According to PRISM III-24, the median score of predicted mortality of these 6 patients who died was $68.45 \%$. According to PELOD score the median score of predicted mortality was $52.5 \%$.

Conclusion. Traumatic head injury was highly represented in our PICU but overall mortality was relatively low.

\section{Reference(s)}

1. - CDC Childhood Injury Report, 2008: Tracy et al.,2013-Slain et al. US children of minority race are less likely to be admitted to the pediatric intensive care unit after traumatic injury, a retrospective analysis of a single pediatric trauma center, Injury Epidemiology (2021) 8:14

\section{9}

Severe Multi-inflammatory syndrome in children temporally related to COVID-19 requiring intensive care unit admissions in a tertiary care centre in India-a diverse spectrum

A. CHATTOPADHYAY'; SK. Karnika ${ }^{2}$

${ }^{1}$ Pediatrics, Chacha Nehru Bal Chikitsalaya, New Delhi, France; ${ }^{2}$ Microbiology, Chacha Nehru Bal Chikitsalaya, New Delhi, India

Correspondence: A. CHATTOPADHYAY

Intensive Care Medicine Experimental 2021, 9(1): 000939

Introduction. The severe acute respiratory syndrome coronavirus-2 (SARS-CoV-2) pandemic has posed as a challenge to public health globally ever since it first emerged in Wuhan, China in December 2019. The virus was initially described to cause mild to asymptomatic infections in the pediatric age group except in children with underlying co - morbidities. However, from April 2020 onwards, several case series emerged about a mysterious hyperinflammatory syndrome akin to Kawasaki disease, Toxic Shock Syndrome and Macrophage Activation syndrome from UK [1-3], Italy [4], USA [5,6] France [7] and India [8]. The affected children had evidence of previous exposure to COVID 19 infection (3-6 weeks back), were premorbidly healthy, of school age to adolescents and presented with fever and predominant gastrointestinal symptoms. These reports prompted societies and public health agencies such as RCPCH (Royal College of Paediatrics and Child Health) [9], WHO[10] and $\mathrm{CDC}[11]$ to issue worldwide alerts and surveillance definitions in order to facilitate early detection and management of this postinfectious multi-inflammatory syndrome. These criteria focussed on the early detection of the inflammatory cascade leading to multi -organ failure. However, in tropical countries, in settings where certain rapid diagnostics may not be immediately available-these definitions may be inclusionary of several exanthematous infections with multi organ dysfunction often encountered in these regions.

While the epidemiology of this entity is still evolving, studies till date collectively report $68 \%$ of MIS patients require Intensive care unit admissions, $31 \%$ require ventilation, $4 \%$ need salvage therapy as ECMO and a mortality rate of $1.5 \%[2-5,12]$. To, the best of our knowledge, data from Asian countries on this aspect is still scarce8, [13-15]. We present here, our case series of 17 critically ill children with severe MISC, all of whom were seropositive for SARSCOV 2 Antibodies. Due to the public health emergency and severe shortage of beds, several ICUs were designated as COVID ICUs. As our ICU was a non-COVID facility, we referred all SARSCOV 2 RTPCR positive cases to COVID designated hospitals and our cohort comprises of severe seropositive MISC only We describe the clinical, laboratory data and echocardiographic findings and plot the variation in longitudinal trends over first $48 \mathrm{~h}$ among survivors and nonsurvivors.

Objectives. We aim to describe our experience in terms of clinical and laboratory attributes in children with Multi - inflammatory syndrome in children temporally related to COVID-19 (MISC).

Methods. Our PICU is a 12 -bedded unit of a tertiary care children's hospital in Eastern part of New Delhi, the capital of India. All children with Kawasaki like or Dengue like illness or suspected MIS-C, that required intensive care unit admission were evaluated based on the MIS-C diagnostic algorithm currently being followed in our PICU (Flowchart 1 ). We prospectively collected data of children (aged $\leq 12$ years), admitted to PICU between May 1, 2020 and January 31, 2021, fulfilling the case definition of MIS-C published by World Health Organization (WHO) or Centre for Disease Control (CDC) [10,11].

Results. Between 1st May, 2020 and 31st January, 2021, 365 patients were admitted to the PICU, out of which,34 patients were referred with clinical suspicion of MISC. Seventeen cases met criteria for MIS-C as per WHO/CDC Classification of which six patients expired (see Flowchart 2). Majority of MIS-C patients were males (64.7\%). All our patients were SARSCOV 2 antibody positive and SARSCOV2 RT PCR negative. The clinical and demographic variables are shown in Table 1. The median age of the children was 4 years (range between 1 year 6 months to 8 years). All patients were previously healthy with no previous co- morbidity. The median duration of symptoms were 7 days (IQR 6, 10). The most common symptom was high grade fever present in all 17 cases followed by rash and conjunctival redness present in 12 (70.6\%) cases each. Gastrointestinal symptoms such as diarrhoea and vomiting were present in $8(47 \%)$ caseswhereas abdominal pain was present in 7 (41.1\%) cases. Among those patients with shock at anytime during PICU stay $(n=13)$, majority -9 children (69.2\%) were Kawasaki Disease (KD) phenotype. These 9 children did not meet criteria for classical KD.

Lab markers (see Table 2):

Neutrophilia at admission and then at $48 \mathrm{~h}$ of PICU entry was seen in $(12 / 17,70.5 \%)$ and $(8 / 17,47.05 \%)$ respectively. Only $4(23.5 \%)$ patients had lymphopenia at admission. Neutrophil to lymphocyte ratio (NLR) was more than 5 in $(4 / 17,23.2 \%)$ children with MIS-C. Plotting the trends of laboratory investigations within the first $48 \mathrm{~h}$ of admission among survivors and nonsurvivors (Figure $1 \mathrm{a}-\mathrm{c}$ ) depicted, higher median LDH $[488.5(349.38,627.61)$ vs $6670(4316.4,17,656.81)] \mathrm{IU} / \mathrm{L}$, median creatinine $[0.65(0.41,0.89)$ vs $0.93(0.24,1.62)] \mathrm{mg} / \mathrm{dl}$, median AST $[95.8(14.2,177.5)$ vs $2476.16(232.5,5184.8)] \mathrm{IU} / \mathrm{L}$ in nonsurvivors. Children who presented with myocardial dysfunction had higher median Ferritin (ng/ml) [1506.24 (230.8, 3243) vs $348.5(176.06)]$, median CRP (mg/L) [217.6 $(128.1,307.1)$ vs $137.84(378,237.8)]$ and 
median LDH (IU) [1152.2 (441.5, 2745.1) vs 806.3(47, 1565.6)] (see Fig. 2). In terms of inflammatory markers (see Table 4), median C- reactive protein $(\mathrm{mg} / \mathrm{L})$ showed serial reduction in levels-at admission [median/IQR) was $210(132.6,246.9)$ ] whereas at Day 3, it dropped to $52.3(42,120)$. Procalcitonin $(\mathrm{ng} / \mathrm{ml})$ level (median/IQR) at admission to PICU was $6.35(0.77,50.02)$. Other investigations demonstrated, Ferritin at admission $(n=12)$, (Median, IQR) was $690(203,1324)$, LDH at admission and $48 \mathrm{~h}(\mathrm{n}=12)($ Median, IQR) was $505(229.5,1032)$ and $1391(525,15,000)$ respectively. Despite a mean D- dimer $(\mathrm{ng} / \mathrm{ml})$ $(n=7), 5093.85$ (1991.65) only 5 patients were prescribed Aspirin and none were given anticoagulants, due to high rates of DIC and active mucosal and gastrointestinal bleeding.

Organ dysfunction:

Myocardial dysfunction was most frequently encountered (12/17, $70.5 \%)$, followed by acute kidney injury (10/17, 58.8\%) and encephalopathy $(9 / 17,52.9 \%)$. Acute respiratory distress syndrome was seen in only $5(29.4 \%)$ cases. $6(35.3 \%)$ cases required noninvasive ventilation whereas invasive mechanical ventilation was required in $11(64.7 \%)$ cases due to respiratory failure. Tachyarrhythmias were seen in $8 / 17$ cases-most common being narrow complex tachycardia, diffuse ST segment changes (ST elevation/depression) followed by low voltage complexes in all leads. Median Troponin I $(\mathrm{ng} / \mathrm{ml}$ ) in patients with cardiac dysfunction $(n=8)$ was $0.11(0.03,0.35)$. Echocardiogram was performed in 8 patients with myocardial dysfunction, after stabilisation of acute state-mean ejection fraction was $62.25 \%(8.13)$ and only 1 patient had ejection fraction less than $50 \%$. Median Z scores $(n=6)$ of Left Anterior Descending artery, Right Coronary artery and Left Coronary artery were as-2.45 $(1,5.3), 2.69(0.74,7.82)$ and 2.32 $(1,4.4)$ respectively. Two patients were reported to have left anterior descending coronary artery aneurysms, and 2 children had right coronary artery aneurysm (z score 2.5 or greater).

Treatment:

13 patients were on vasopressors for shock. 10 (58.2\%) patients of MIS-C were administered Intravenous Immunoglobulin (Ivlg) whereas steroids (intravenous/oral) was given in 12 cases (70.5\%). Aspirin was prescribed in 5 children (29.4\%), while only 2 patients received IL-6 inhibitor (Tocilizumab) [received 2 doses] and both survived.

Conclusion. With our case series we wish to highlight the pattern of clinical and laboratory features in a cohort of MISC-seropositive for SARSCOV2 antibody. We suggest refining the spectra of phenotypes of MIS-C for tropical countries keeping other exanthematous infections that present with fulminant myocarditis and refractory shock in perspective.

\section{Reference(s)}

1. 1. Riphagen S, Gomez X, Gonzalez-Martinez C, Wilkinson N, Theocharis P. Hyperinflammatory shock in children during COVID-19 pandemic. The Lancet. 2020 May 23;395(10237):1607-8. 2. Whittaker E, Bamford A, Kenny J, Kaforou M, Jones CE, Shah P, et al. Clinical characteristics of 58 children with a pediatric inflammatory multisystem syndrome temporally associated with SARS-CoV-2. JAMA 2020 [Epub ahead of print]. 3. Ramcharan T, Nolan O, Lai CY, et al. Paediatric inflammatory multisystem syndrome: temporally associated with SARS-CoV-2 (PIMS-TS): cardiac features, management and short-term outcomes at a UK Tertiary Paediatric Hospital [published online ahead of print, 2020 Jun 12]. Pediatr Cardiol. 2020;1-11. https://doi.org/10.1007/s00246-020-02391-2. 4. Verdoni L, Mazza A, Gervasoni A, Martelli L, Ruggeri M, Ciuffreda M, Bonanomi E, D'Antiga L. An outbreak of severe Kawasaki-like disease at the Italian epicentre of the SARS-CoV-2 epidemic: an observational cohort study. The Lancet. 2020 Jun 6;395(10239):1771-8. 5. Feldstein LR, Rose EB, Horwitz SM, et al. Multisystem Inflammatory Syndrome in U.S. Children and Adolescents. N Engl J Med. 2020;383(4):334-346. https://doi.org/10.1056/NEJMoa2021680. 6. Dufort EM, Koumans EH, Chow EJ, et al. Multisystem inflammatory syndrome in children in New York State. N Engl J Med 2020;383(4):347-58. 7. European Centre for Disease Prevention and Control. Pediatric inflammatory multisystem syndrome and SARS-CoV-2 infection in children - 15 May 2020. ECDC: Stockholm; 2020. 8. Balasubramanian S, Nagendran TM, Ramachandran B, Ramanan AV. Hyper-inflammatory syndrome in a child with COVID-19 treated successfully with intravenous immunoglobulin and tocilizumab. Indian Pediatr. 2020; S097475591600180. 9. Royal
College of Pediatrics and Child Health. Guidance-Pediatric multisystem inflammatory syndrome temporally associated with COVID-19, 2020. Available from: https://www.rcpch.ac.uk/resources/guidance-pediatricmultisystem-inflammatory-syndrometemporally- associated-covid-19. Accessed March 24, 2020. 10. Multisystem inflammatory syndrome in children and adolescents with COVID-19. 15 May 2020 Scientific brief: World Health Organisation. Available from: https://www.who.int/publi cations-detail/multisystem-inflammatory-syndrome-in-children-and adolescents- with-covid-19. Accessed March 22, 2021. 11. Centers for Disease Control and Prevention. Emergency preparedness and response: Health alert network. [May 14, 2020]. Available from: https://emergency. cdc.gov/han/2020/han00432.asp. Accessed March 21, 2021. 12. Radia T, Williams N, Agrawal P, Harman K, Weale J, Cook J, Gupta A. Multi-system inflammatory syndrome in children \& adolescents (MIS-C): A systematic review of clinical features and presentation. Paediatric respiratory reviews. 2020 Aug 11. 13. She J, Liu L, Liu W. COVID-19 epidemic: Disease characteristics in children. J Med Virol. 2020;92(7):747-754. 14. Kim YJ et al. (2020) Defining association between COVID-19 and the multisystem inflammatory syndrome in children through the pandemic. Journal of Korean Medical Science 35, e204. 15. Rauf A et al. (2020) Multisystem inflammatory syndrome with features of atypical Kawasaki disease during COVID-19 pandemic. Indian Journal of Pediatrics 87, 745-747.

\section{8}

\section{Mortality Trends in a Paediatric Intensive Care Unit}

E. Dardamani ${ }^{1}$; A. Spanaki ${ }^{1}$;. Tavladaki ${ }^{1}$; M. Miliaraki ${ }^{1}$; E. Vasilaki ${ }^{1}$; E.

Geromarkaki'; S. Ilia ${ }^{1}$

${ }^{1}$ Paediatric Intensive Care Unit, University General Hospital of Heraklion, Heraklion, Greece

Correspondence: E. Dardamani

Intensive Care Medicine Experimental 2021, 9(1): 000958

Introduction. Studies evaluating mortality rates in Paediatric Intensive Care Units (PICUs) across South-East European countries are lacking. The purpose of our study was to determine the epidemiology of mortality in a 7-bed PICU based in a University Hospital.

Methods. We retrospectively collected data from paediatric patients died during their stay in Level III mixed PICU, University Hospital from January 2016 to March 2021. The data collected included demographic profile, diagnosis at admission, cause of death, length of stay and severity scores, Pediatric Risk of Mortality (PRISM) and Pediatric Logistic Organ Dysfunction (PELOD) score.

Results. Out of 758 admitted children during this 5-year period, 213 $(28,1 \%)$ were day-care clinic patients and were excluded. From the remaining 545 patients, crude mortality was $5,32 \%(n=29)$, males were $328(60,2 \%)$, with no statistical significance concerning age or sex. The median age was $5(1,5-11,5)$ years and median length of PICU stay was $4(2-10)$ days. Mortality rate was found higher in infants $(6,03 \%)$ and adolescents $(7,89 \%)$ without reaching statistical significance. Mortality was not associated with the length of stay of children. The leading admission diagnoses of non-survived children were sepsis $(31 \%)$, trauma $(17 \%)$, neurological disorders $(17 \%)$ and malignancies (14\%). Mortality was significantly associated with co-morbidities, severity of critical illness, and the need for mechanical ventilation (all $\mathrm{p}<0,001)$. PELOD predicted mortality (\%) was 87 (23-99) for the nonsurvived children vs $0,1(0-6)$ for the survived children and PRISM predicted mortality $(\%) 83,7(32,5-94,5)$ vs $0,05(0-7,6)$ respectively.

Conclusion. Mortality rate in critically ill paediatric population remains low. Risk factors for mortality were co-morbidities, the need for mechanical ventilation and clinical severity. Sepsis and trauma are major factors for paediatric mortality, yet preventable.

Paediatric clinical severity scores are useful tools for the prediction of in-hospital deaths of children and can help clinicians to identify highrisk patients with poor prognosis. 
Reference(s)

1. Burns JP, Sellers DE, Meyer EC, Lewis-Newby M, Truog RD. Epidemiology of Death in the Pediatric Intensive Care Unit at Five U.S. Teaching Hospitals. Crit Care Med. 2014 Sep;42(9):2101-8.

2. Abdelatif RG, Mohammed MM, Mahmoud RA, Bakheet MAM, Gima M Nakagawa S. Characterization and Outcome of Two Pediatric Intensive Care Units with Different Resources. Critical Care Research and Practice. 2020 Mar 17;2020:e5171790

\section{0}

Magnetic Resonance Imaging in children with Status Epilepticus E. Choulaki ${ }^{1}$; M. Raissaki ${ }^{2}$; G. Briassoulis ${ }^{3}$; M. Miliaraki ${ }^{4}$; P. Briassoulis ${ }^{4}$; S. Ilia $^{3}$ ${ }^{1}$ Postgraduate Program "Emergency and Intensive Care of Children,

Adolescents and Young Adults", University of Crete, School of Medicine, Heraklion, Greece; ${ }^{2}$ Radiology Department, University Hospital, University of Crete, School of Medicine, Heraklion, Greece; ${ }^{3}$ Postgraduate Program "Emergency and Intensive Care of Children, Adolescents and Young Adults", University of Crete, School of Medicine, PICU, Heraklion, Greece; ${ }^{4}$ Pediatric Intensive Care Unit, University Hospital, University of Crete, School of Medicine, Heraklion, Greece

Correspondence: S. Ilia

Intensive Care Medicine Experimental 2021, 9(1): 000980

Introduction. Refractory (RSE) and super-refractory status epilepticus (SRSE) are challenging neurologic emergencies in childhood, associated with high morbidity and mortality. 1,2 The role of magnetic resonance imaging (MRI) in investigating brain pathology associated with RSE and SRSE is of particular importance.

Objectives. The aim of this study was to record the MRI findings in children hospitalized in PICU with SE, with emphasis on possible correlations between qualitative or quantitative $M R I$ characteristics and clinical-laboratory data, SE types and etiological factors, prognostic indicators and outcome.

Methods. PICU patients with different types of SE were enrolled during 2009-2020. MRI quantitative parameters like diffusion values in $A D C$ maps and the signal intensity in regions of interest (ROI) in FLAIR sequences were measured. The absolute $(\delta R O I)$ and percentage $(K)$ difference in signal intensity between the qualitatively abnormal and the contralateral normal cortex were calculated.

Results. The study included 5 children (12.5\%) diagnosed with SE, 19 with RSE (47.5\%) and 15 with SRSE (37.5\%). Out of 40 patients, 18 had normal MRI (45\%), 14 (35\%) abnormal with peri-ictal abnormalities and $8(20 \%)$ abnormal without peri-ictal abnormalities. Mean age differed significantly between seizure type (SE 0.83 vs. RSE 3 vs. SRSE 8 years; $p=0.023)$. FIRES was the most common final diagnosis $(28.6 \%)$ in children with abnormal MRI with peri-ictal abnormalities followed by NORSE $(21.4 \%)$ and autoimmune disease $(21.4 \%)$, while genetic abnormalities (75\%) were found in children with abnormal MRI without peri-ictal abnormalities. Regarding MRIs, detection of hyperintensity in FLAIR, compared to T2-w sequence was more common in the group of patients with peri-ictal abnormalities. PICU outcome was associated with FLAIR hyperintensity of the basal ganglia $(p=0.012)$, the hippocampus $(p=0.025)$, the brainstem $(p=0.026)$, the corpus callosum $(p=0.026)$, or the parietal $(p=0.024)$, frontal $(p=0.003)$ and occipital cortex $(p=0.003)$. Regarding the quantitative measurements, there was a correlation $(p=0.031)$ between signal intensity values in FLAIR at the right hippocampus (transverse plane) and outcome. The highest intensity values were associated with unfavorable outcome ( $361 \pm 9.9$ in the mortality group vs. $327 \pm 23.3$ in survivors). The mean value of $\mathrm{k}$ in normal MRI was $5 \%$, while in abnormal peri-ictal MRI the mean value of $\mathrm{K}$ was $16 \%$ higher than the optically normal cortex.

Conclusion. Peri-ictal abnormalities are frequently seen in MRIs of children with SE, especially those with SRSE diagnosed with FIRES, NORSE or autoimmune disease. Signal intensity values in FLAIR at the right hippocampus are associated with outcome.

\section{Reference(s)}

1. Dubey, D., Kalita, J. \& Misra, U. K. Status epilepticus: Refractory and superrefractory. Neurol. India 65, S12-S17 (2017).
2. Vasquez, A., Farias-Moeller, R. \& Tatum, W. Pediatric refractory and superrefractory status epilepticus. Seizure 68, 62-71 (2019).

000981

Metabolic and nutritional characteristics, diagnostic predictors and outcome of Long-Stay Patients in a Pediatric Intensive Care Unit

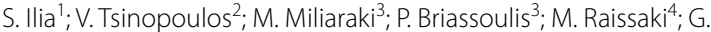

Briassoulis $^{1}$

1Postgraduate Program "Emergency and Intensive Care of Children, Adolescents and Young Adults", University of Crete, School of Medicine, PICU, Heraklion, Greece; ${ }^{2}$ Postgraduate Program "Emergency and Intensive Care of Children, Adolescents and Young Adults", University of Crete, School of Medicine, Heraklion, Greece; ${ }^{3}$ Pediatric Intensive Care Unit, University Hospital, University of Crete, School of Medicine, Heraklion, Greece; ${ }^{4}$ Radiology Department, University Hospital, University of Crete, School of Medicine, Heraklion, Greece

Correspondence: $\mathrm{S}$. Ilia

Intensive Care Medicine Experimental 2021, 9(1): 000981

Introduction. A special group of "Long-Stay" ICU patients (LSPs) has been recognized in recent years. Moreover, little is known about the degree of hypercatabolism and muscle mass loss of LSPs. Recent studies have highlighted the urea: creatinine ratio $(\mathrm{Ur} / \mathrm{Cr}$ ) as a reliable indicator of the catabolic state of patients with severe illness or trauma. To date, the nutritional/metabolic status and caloric/protein needs of pediatric LSPs and their possible prognostic importance have not been studied.

Objectives. The primary aim of this study is to identify early risk factors for LSPs, and to assess whether the PICU length of stay (LOS) differs significantly among different diagnoses and age categories. A secondary aim is to compare the longitudinal caloric and protein coverage of LSPs with the age-recommended energy protein needs of PICU patients, in line with recent guidelines.

Methods. This is a retrospective study of LSPS (LOS of more than 14 days) who were hospitalized in PICU during the period of 20092020. A number of short-stay patients (SSPs) (LOS of less than 14 days) was used as a control group. Patients' clinical, laboratory characteristics, disease severity scoring systems (PRISM, PeLOD, TISS), as well as their energy and protein intake during hospitalization were recorded.

Results. A total of 249 patients were included in the study, of whom 134 were LSPs and 115 SSPs. LSPs were malnourished with a mean weight $z$-score of $-0.45(p=0.001)$ and had greater severity of illness based on PRISM, PELOD and TISS $(p<0.001)$, more comorbidities $(p=0.013)$, and more re-admissions compared to SSPs $(p=0.004)$ LSPs also had a higher risk for ARDS, sepsis $(p=0.065)$ or multiple organ dysfunction syndrome $(p=0.011)$, and higher mortality rates than SSPs (13.4\% vs. 3.5\%, $p=0.004)$. LSPs were given more calories and proteins than SSP $(p=0.035)$, but neither group reached the recommended by ESPNIC or ESPEN daily caloric (Schofield equation) or protein coverage recommendations. The energy balance was less negative $(-10.9 \pm 22$ vs. $-16.3 \pm 21 \mathrm{kcal} / \mathrm{kg} / \mathrm{day}, \mathrm{p}=0.165)$, and the administered calories higher in LSPs compared to SSPs $(36 \pm 25$ vs. $26.7 \pm 24 \mathrm{kcal} / \mathrm{kg} /$ day, $\mathrm{p}=0.045)$. The proteins administered in LSPs were only marginally higher compared to SSPs $(1.45 \pm 1.2$ vs. $1.39 \pm 0.9 \mathrm{gr} / \mathrm{kg} / \mathrm{day}, \mathrm{p}=0.793)$. An increasing trend $(26 \%)$ per week was observed in the ur/cr ratio in LSPs (7th day, $60.2 \pm 30$ vs. $51.9 \pm 37$, $p=0.45)$. Malnutrition $(p=0.005)$, multi-organ failure $(p=0.034)$, and mechanical ventilation $(p=0.006)$ were associated with the probability of long-term hospitalization. PRISM and PELOD scores $(p=0.001)$, mechanical ventilation duration $(p=0.001)$ and PICU LOS $(p=0.02)$ were all strong predictors of mortality.

Conclusion. LSPs are malnourished with higher morbidity and mortality compared to SSPs. Since hyper-catabolism due to severe disease cannot be avoided despite aggressive intestinal feeding regimens, new protocols are required to support the increased metabolic needs of LSPs. 
001356

Does maternal admission to HDU affect neonatal admission to NICU?

S. Amer'; J. Gardner'² N. Logan, 2; K. Litchfield ${ }^{3}$

${ }^{1}$ Anaesthetics, Queen Elizabeth University Hospital, Glasgow, United Kingdom; ${ }^{2}$ Anaesthetics, Queen Elizabeth University Hospital, Govan Road, Glasgow, UK, Glasgow, UK, United Kingdom; ${ }^{3}$ Department of anaesthesia, critical care, and pain medicine, Royal Infirmary of Edinburgh, University of Edinburgh, Edinburgh, United Kingdom

Correspondence: S. Amer

Intensive Care Medicine Experimental 2021, 9(1): 001356

Introduction. We hypothesised that babies born during Obstetric HDU (oHDU) admission, (when the mother is unwell enough to be in oHDU), would be more likely to need Neonatal ICU (NICU) than those delivered before admission, (when the mother was well or not unwell enough to be in oHDU).

Methods. Admissions to our oHDUs are recorded in the Scottish Intensive Care Society Audit Group Wardwatcher (WW) database. Requirement for NICU was recorded within WW for Princess Royal Maternity from 11th May 2016; for Queen Elizabeth University Hospital from 2nd March 2017. Information governance and ethical approval gained. Data was exported from WW 10th January 2020. Admissions within two calendar days of delivery were included. Analysis of the 977 maternal admissions was performed using $\mathrm{R}$ and Excel. Results.

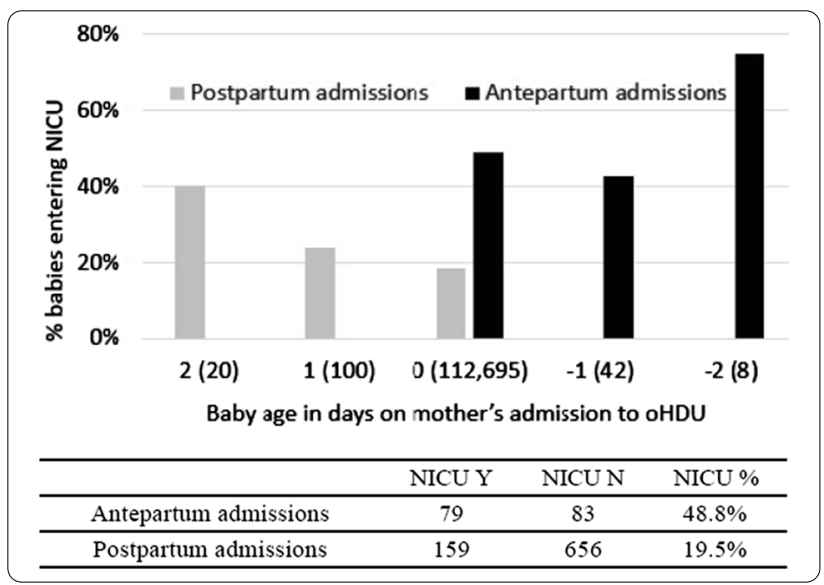

Conclusion. There is large difference between NICU requirements between babies born to mothers already unwell enough for oHDU compared to those that are not. Chi-squared $p<0.001$. Considering maternal admission as a predictor gives the low sensitivity of $33 \%$ but a good specificity $88 \%$, positive predictive value $49 \%$ and a negative predictive value $80 \%$. We recommend the presence of neonatal support for deliveries to patients receiving oHDU level care. Whilst realising that numerically more babies needing NICU are born to mothers entering HDU after their birth.

\section{Reference(s)}

1. 1. The best start: five -year plan for maternity and neonatal care, A five-year forward plan for the improvement of maternity and neonatal services in Scotland. ISBN: 9781786527127 Published: 20 Jan 2017.

2. 2. Knight M, Nair M, Tuffnell D, Shakespeare J, Kenyon S, Kurinczuk JJ (Eds.) on behalf of MBRRACE-UK. Saving Lives, Improving Mothers'Care-Lessons learned to inform maternity care from the UK and Ireland Confidential Enquiries into Maternal Deaths and Morbidity 2013-15. Oxford: National Perinatal Epidemiology Unit, University of Oxford 2017
e-Posters: Perioperative clinical studies

000074

MAIN MONITORING PERFORMANCES OF CARBON EXCHANGE

AFFECTING THE POST-OPERATING COURSE COGNITIVE DYSFUNCTION Dubivska SS Hryhorov Yu.B. Kharkov National Medical University Kharkov, Ukraine

S. Dubivska

${ }^{1}$ Anestasiology, Kharkiv National Medical University, Kharkiv, Ukraine Correspondence: S. Dubivska

Intensive Care Medicine Experimental 2021, 9(1): 000074

Introduction. The purpose of this study is to determine the content of the major carbohydrate metabolism in patients with acute surgical pathology, depending on the age after administration to general anesthesia patients.

Objectives. The aim of this study is to determine the content of the main indicators of carbohydrate metabolism in patients with acute surgical pathology depending on age after the application of general anesthesia to patients

Methods. The study of 130 patients was conducted in surgical departments of different profiles at the Kharkov City Clinical Hospital for Emergency and Emergency Medical Services. prof. A.I. Meshchaninov. Surgery was performed under conditions of general multicomponent anesthesia with artificial ventilation using propofol and fentanyl.

The patients were divided into three groups, respectively: 1 group $(\mathrm{n}=46)$-young patients (18-43 years); mean age $30.1 \pm 1.0$ years, 24 people, 22 women. Group $2(n=43)$ were middle-aged patients (44-59 years); mean age $49.3 \pm 5.1$ years, 18 males, 25 females. Group $3(n=41)$-elderly patients $(60-80$ years); mean age $74.4 \pm 6.1$ years, 22 people, 19 women. According to the purpose and objectives of the study, the blood biochemical markers of carbohydrate metabolism-glucose, pyruvate and lactate contentwere determined in patients' blood.

Results. The absence of changes in the content of this indicator in the blood of patients of the young age group indicate that humoral mechanisms of regulation of the content of this monosaccharide, which is necessary for the energy supply of the processes of vital activity of the organism, are functioning at a sufficient level to ensure glucose homeostasis. Hyperglycemia in patients of this age group before surgery and in the first week after anesthesia and surgery may be caused by an increase in the blood stress hormones: adrenaline, cortisol. Regarding the monitoring of carbohydrate metabolism-blood glucose in elderly patients, they have hyperglycemia in all periods of the study. A month later, it practically returns to the values before surgery.

Conclusion. Studies of anaerobic glycolysis metabolites such as pyruvate and lactate in the serum of patients of different age groups revealed certain changes in these indicators, the severity of which was dependent on age and duration after anesthesia and surgery.

\section{4}

Prediction and Detection of Delirium During Perioperative Care

V. Kalogianni ${ }^{1}$; M. Vizcaychipi ${ }^{2}$

${ }^{1}$ Medicine, Imperial College London, Hammersmith, United Kingdom;

${ }^{2}$ Intensive care unit, Chelsea and Westminster Hospital, London, United Kingdom

Correspondence: $\mathrm{V}$. Kalogianni

Intensive Care Medicine Experimental 2021, 9(1): 000094

Introduction. Delirium is common, affecting up to $50 \%$ of orthopaedic surgical patients. It leads to increased mortality, morbidity and cost to the hospitals. A tool currently validated in critical care patients that predicts risk of developing delirium is the 'E-PREDELIRIC' score. It is an enhanced version of the PREDELIRIC delirium prediction score for critical care patients, where E stands for "early". It involves nine parameters: age, history of cognitive impairment, history of alcohol abuse, blood urea nitrogen (BUN) at time of 
admission to critical care, admission category, urgent admission, mean arterial pressure (MAP) at the time of critical care admission, use of corticosteroids, and respiratory failure. These parameters are routinely collected in the emergency department and other areas of urgent admission, so it is worth investigating the validity of this tool in other settings, for instance orthopaedic surgical patients.

Objectives. To assess pre-operative delirium risk in emergency orthopaedic trauma surgical patients.

To measure rates of delirium in these patients.

To undertake initial validation of the E-PREDELIRIC delirium prediction tool in orthopaedic surgical patients.

Methods. Patient data from two surgical categories were collected prospectively over a 4-week period in February 2021 from a London Trust consisting of two teaching hospitals. Eligible patients ( $\geq 18$ years, orthopaedic surgery in the Emergency Orthopaedic \& Trauma or NCEPOD surgical list, operation date from 1st-28th February) were identified, and their patient notes were utilised to retrospectively calculate their EPREDELIRIC score. Their notes were then reviewed to assess for development of delirium postoperatively, either with a definitive diagnosis or with descriptors in the entries (confusion, agitation, aggression, fluctuation in cognition). The rates of development of delirium were compared against the EPREDELIRIC score to assess the sensitivity and specificity of the score.

Results. From 71 patients identified (31 males-40 females, mean age $=72$ years), $17(23.9 \%)$ developed delirium post-operatively. The average E-PREDELIRIC score calculated was $35.8 \%(\mathrm{~min}=5 \%$, $\max =75 \%)$

\begin{tabular}{lllllll}
\hline EPREDELIRIC\% & Sensitivity & Specificity & PPV & NPV & FP & FN \\
\hline$>30$ & 1 & 0.56 & 0.42 & 1 & 24 & 0 \\
$>\mathbf{4 0}$ & $\mathbf{0 . 8 2}$ & $\mathbf{0 . 8 3}$ & $\mathbf{0 . 6 1}$ & $\mathbf{0 . 9 4}$ & $\mathbf{1 0}$ & $\mathbf{3}$ \\
$>50$ & 0.71 & 0.93 & 0.75 & 0.91 & 4 & 5 \\
$>60$ & 0.53 & 0.97 & 0.82 & 0.87 & 2 & 8 \\
\hline
\end{tabular}

$\mathrm{PPV}=$ Positive Predictive Value, NPV $=$ Negative Predictive Value, $\mathrm{FP}=$ False Positives, $\mathrm{FN}=$ False Negatives.

From analysis of the results, it seems that for the specific cohort, the cut-off precentage (\%) score that could be used in practice to identify high risk patients is $40 \%$. This is because, though $30 \%$ seems to identify all patients at risk, it is not specific enough to discriminate between high risk patients. Other cut-off values, like 50 and $60 \%$, have a high rate of $\mathrm{FN}$ patients that would be missed in the risk stratification.

$40 \%$ from the table is an appropriate compromise between sensitivity and specificity to clinically identify high risk patients and monitor them appropriately.

Conclusion. The study was limited by the small sample size and the dependence on subjective interpretation of the patients' status (both by clinician dictating notes and by reviewer) as sometimes there was no definitive diagnosis of 'delirium' recorded in the notes. However, initial analysis shows that there is evidence to support research into the use of the EPREDELIRIC score into surgical patients to risk stratify delirium development postoperatively. Further validation is required to identify what the EPREDELIRIC cut-off score should be on a larger cohort and further investigate its use in other surgical cohorts, as the score might be dependent on the admission category. In any case, this is not to say that clinical judgment should not be advised in risk stratification where appropriate, especially when risk factors are in place for a patient that are not included in the EPREDELIRIC equation.

\section{Reference(s)}

1. Inouye SK, Westendorp RG, Saczynski JS. Delirium in elderly people. The Lancet. 2014; 383 (9920): 911-922. Available from: https://doi.org/10. 1016/S0140-6736(13)60688-1 Available from: https://www.sciencedirect. com/science/article/pii/S0140673613606881.

2. lamaroon A, Wongviriyawong T, Sura-arunsumrit P, Wiwatnodom N, Rewuri N, Chaiwat O. Incidence of and risk factors for postoperative delirium in older adult patients undergoing noncardiac surgery: a prospective study. BMC Geriatrics. 2020; 20 (1): 40. Available from: https:// doi.org/10.1186/s12877-020-1449-8 Available from: https://doi.org/10. 1186/s12877-020-1449-8.

3. Wassenaar A, van den Boogaard M, van Achterberg T, Slooter AJC, Kuiper MA, Hoogendoorn ME, et al. Multinational development and validation of an early prediction model for delirium in ICU patients. Intensive care medicine. 2015; 41 (6): 1048-1056. Available from: https://doi.org/ 10.1007/s00134-015-3777-2 Available from: https://doi.org/10.1007/ s00134-015-3777-2

4. Rengel KF, Pandharipande PP, Hughes CG. Postoperative delirium. La Presse Médicale. 2018; 47 (4, Part 2): e53-e64. Available from: https://doi. org/10.1016/j.lpm.2018.03.012 Available from: https://www.sciencedirect. com/science/article/pii/S075549821830157X.

5. With many thanks to Ms. Sundhiya Mandalia for her input on the statistical analysis of this research and her continuous support.

\section{2}

Intravenous lidocaine as an alternative analgesia method for the patients undergoing liver resection

AV. Biliaiev ${ }^{1}$; A. Mazur ${ }^{2}$; A. Lysenko ${ }^{2}$; O. Khomenko ${ }^{2}$; R. Zatsarynnyy ${ }^{2}$

${ }^{1}$ Pediatric Anaesthesiology and Intensive Care, Shupyk National Healthcare University of Ukraine, Kyiv, Ukraine; ${ }^{2}$ Anaesthesiology and Intensive Care, A. A. Shalimov National Institute of Surgery and Transplantology, Kyiv, Ukraine

Correspondence: $\mathrm{O}$. Khomenko

Intensive Care Medicine Experimental 2021, 9(1): 000202

Introduction. Liver resection is widely performed for the treatment of hepatic neoplasms, both benign and malignant as well as for living donation (1). Irrespective of the approach, the procedure can be associated with significant postoperative pain (2). Effective pain control can facilitate early mobilization and reduce postoperative complications (3). One of the reasons for the ERAS society's recommendation against epidural analgesia in liver surgery is the presence of postoperative coagulopathy (4). Alternative pain control technics required for this type of the patients.

Objectives. To evaluate effectiveness of using intravenous lidocaine infusion in perioperative period for the optimal pain management after open liver resection procedures.

Methods. We conducted a single center pilot study to which 39 patients with resection of $40-70 \%$ of liver parenchyma were included. All patients received inhalation anesthesia with sevoflurane and intraoperative analgesia with phentanile $0.005 \%$ during the surgery. The first group (20 patients) received continuous epidural infusion of lidocaine $1.5 \%$. Second group (9 patients) - received intravenous lidocaine $1.5 \%$ 2-4 mg per $\mathrm{kg}$ intraoperatively. Third-control group included 10 patients. For analysis of the analgesia we used intraoperative fentanyl consumption, VAS pain score during the first (PODO) and second (POD1) days after the surgery. We also analyzed the time (in min) when first dose of analgetics was administer to the patient after the surgery. One-Way ANOVA test, Including Tukey HSD was used to evaluate the difference. The result accepted as significant at $p<0.05$.

Results. Patients in first group received mean $1225 \pm 601.6 \mathrm{mkg}$ of phentanile, in second and third groups $1771.11 \pm 735.5$ and $2090.0 \pm 636.7 \mathrm{mkg}$ respectively ( $f$-ratio value is 6.60184 , the $p$-value is 0.00361 ). According to the Tukey's HSD procedure there is no difference between phentanile consumption in first and second groups ( $p=0.11777)$ but the difference is significant between epidural and control groups $(p=0.00733)$. Patients in control group received significantly higher dose of phentanile intraoperatively. During the PODO patients of the 1-st group had mean $4.5 \pm 2$ points by VAS score, in 2-nd group $-5 \pm 2.3$ and in 3-rd group $7.6 \pm 1$ points (the $f$-ratio value is 8.79657 , the $p$-value is 0.000903 ). Again, there was no significant difference between first and second groups $(p=0.82944)$, but in this case patients of control group had a pain score significantly higher than patients of both study groups ( $p=0.00321$ with the first group and $p=0.01447$ with the second one). There was no significant difference in VAS score at POD1 between the groups (mean VAS scores $3.8 \pm 2$, $4.6 \pm 1.3,5,6 \pm 2$, the $f$-ratio value is 2.4977 , the $p$-value is 0.099232 ). There was no statistically significant difference between groups in time of administration of the first dose of analgetics after the surgery: 
$375.3 \pm 649.7 \mathrm{~min}$ in first group, $283 \pm 478.6 \mathrm{~min}$ in second group and $237 \pm 359.3$ in third group (the $f$-ratio value is 1.54711 , the $p$-value is 0.227476).

Conclusion. Liver resection is painful procedure, which requires complex approach for the perioperative pain management. According to this pilot study, intravenous $1.5 \%$ lidocaine administration in dose 2-4 mg per kg can reduce intraoperative phentanile consumption and improve postoperative coagulopathy as an alternative to the epidural analgesia. Future multicenter randomized control studies required to confirm the result.

\section{Reference(s)}

1. 4. Vandana Agarwal, Jigeeshu V. Divatia. Enhanced recovery after surgery in liver resection: current concepts and controversies. Korean J Anesthesiol. 2019;72(2):119-129. Published online March 6, 2019. https://doi.org/ $10.4097 /$ kja.d.19.00010

2. 3. Yip VS, Dunne DFJ, Samuels S, et al. Adherence to early mobilisation: key for successful enhanced recovery after liver resection. Eur I Surg Oncol 2016;42:1561-7.

3. 2. Aragon RJ, Solomon NL. Techniques of hepatic resection. J Gastrointest Oncol. 2012;3(1):28-40. https://doi.org/10.3978/j.issn.2078-6891.2012.006

4. 1. Dieu A, Huynen P, Lavand'homme P, et alPain management after open liver resection: Procedure-Specific Postoperative Pain Management (PROSPECT) recommendationsRegional Anesthesia \& Pain Medicine Published Online First: 12 January 2021. https://doi.org/10.1136/ rapm-2020-101933

\section{2}

Usefulness of bioelectrical impedance analysis as a guidance for fluid management in critically ill patients after operation: ECW ratio as the marker of adequate fluid status

JC. Yoon'; EY. Kim²

${ }^{1}$ Department of Surgery, The Catholic University of Korea Seoul St. Mary's Hospital, Seoul, Republic of Korea; ${ }^{2}$ Division of Trauma and Surgical Critical Care, Department of Surgery, The Catholic University of Korea Seoul

St. Mary's Hospital, Seoul, Republic of Korea

Correspondence: E.Y. Kim

Intensive Care Medicine Experimental 2021, 9(1): 000212

Introduction. We determined the relationship between changes in bioelectrical impedance analysis (BIA) parameters and response of critically ill patients to fluid therapy during early postoperative period. Associations between BIA values indicating volume status of postoperative patient and clinical outcomes were also evaluated.

Methods. From May 2019 to April 2020, patients who were admitted to the surgical intensive care unit (SICU) of our institution at more than $48 \mathrm{~h}$ after surgery were enrolled. Volume status was measured with a portable BIA device every morning for five days from SICU admission. Overhydration was defined as the case where extracellular water (ECW) ratio $>0.390$ measured by BIA. Participants were daily classified into an overhydration or a normohydration group. The relationship between daily hydration status and postoperative outcome was evaluated.

Results. Of 190 participants, most of them showed overhydration status during the first $48 \mathrm{~h}$ after surgery. SOFA score at SICU admission and the overhydration status on day 3 were significant predictors of postoperative morbidities (SOFA score, OR: 1.163; overhydration status at day 3, OR: 1.182) and in-hospital mortality (SOFA score, OR: 3.151; overhydration status at day 3, OR: 2.040). Cut-off values of overhydration status by ECW ratio at day 3 for predicting postoperative morbidities and in-hospital mortality were $>0.3985$ and $>0.4145$, respectively.

Conclusion. BIA would be a useful and convenient tool to assess the volume status of patients requiring intensive fluid resuscitation in early postoperative period. Overhydration status by ECW ratio on postoperative day 3 needs careful monitoring and appropriate interventions to improve clinical outcomes.
000239

The Impact of Visceral Fat on Oxygenation After Open Abdominal Aortic Aneurysm Repair? A Retrospective Observational Study in Japan

M. Nishizaki ${ }^{1}$ Y. Okamoto ${ }^{2}$

${ }^{1}$ University of Glasgow, Glasgow, United Kingdom; ${ }^{2}$ Department of Anesthesiology, Otakanomori Hospital, Kashiwa, Japan

Correspondence: $M$. Nishizaki

Intensive Care Medicine Experimental 2021, 9(1): 000239

Introduction. Obesity has negative effects on respiratory function, such as obesity hypoventilation syndrome, but it is still not known how obesity can inhibit respiratory function. Therefore, we focused on visceral fat and investigated oxygenation after open abdominal aortic aneurysm repair.

This study was presented at the 46th annual meeting of the Japanese society of intensive care medicine in Japanese.

Objectives. The objective of this study is to explore the relationship between visceral fat and oxygenation after open abdominal aortic aneurysm repair.

Methods. This study was a retrospective observational study in Japan and included adult patients who received open abdominal aortic aneurysm repair in a Japanese local hospital over the course between November 2014 and June 2018. The investigated clinical outcome was $\mathrm{PaO}_{2} / \mathrm{FiO}_{2}$ ratio $(\mathrm{P} / \mathrm{F})$ after six hours from the surgery. Besides, explanatory variables were visceral fat area (VFA), subcutaneous fat area (SFA), age, emergency, smoking habit, the operation time. VFA and SFA were calculated using computer tomography (CT). We analysed data using multiple linear regression (MLR).

Results. Of 78 patients, 16 and 62 were female $(21 \%)$ and male (79\%) respectively. Also, 30 patients received emergency operation (38\%), and patients who needed mechanical ventilation after the surgery stood at $38(49 \%)$. The average $\mathrm{P} / \mathrm{F}$ was $350.08 \pm 113.15$. As a result of MLR with backward stepwise, VAT and emergency were extracted. The MLR model produced $R=0.413$ and $R^{2}=0.170$, and the studied patients' $\mathrm{P} / \mathrm{F}$ is equal to -0.599 (VFA)-71.546(emergency). In addition, the standardised partial regression coefficients of VFA and emergency were -0.278 and $-0.310(p<0.01)$.

Conclusion. For $\mathrm{P} / \mathrm{F}$ after open abdominal aortic aneurysm repair, emergency and VFA are likely to be harmful. Also, not SFA but VFA can harm the oxygenation. When obesity is evaluated before the surgery, VFA can be a far more effective way to predict oxygenation after the surgery than BMI, SFA and Body weight.

\section{Reference(s)}

1. Choe EK, Kang HY, Lee Y,et al.. The longitudinal association between changes in lung function and changes in abdominal visceral obesity in Korean non-smokers. PLoS One. 2018;13(2)

2. The authors do not receive any funding related to this study and have any potential conflicts of interest.

\section{7}

\section{EUROSCORE II as mortality predictor in infective endocarditis} surgery

VA. Hortigüela Martín; R. Hernandez Estefanía; AM. Ioan; E. Rosas

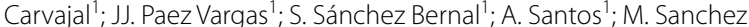
Casado $^{3}$; G. Aldamiz Echevarria Del Castillo²; M. Perez Marquez ; C. Pérez Calvo

${ }^{1}$ Intensive Care Medicine, Hospital Universitario Fundación Jiménez Díaz, Madrid, Spain; ${ }^{2}$ Cardiac Surgery, Hospital Universitario Fundación Jiménez Díaz, Madrid, Spain; ${ }^{3}$ Intensive Care Medicine, Hospital Universitario Virgen de la Salud, Toledo, Spain; ${ }^{4}$ Intensive Care Medicine, Hospital Universitario Rey Juan Carlos, Móstoles, Spain

Correspondence: A.M. loan

Intensive Care Medicine Experimental 2021, 9(1): 000267

Introduction. The estimated annual incidence of infective endocarditis is approximately 30 cases per million inhabitants in general population. It is most common in prosthetic valves, patients 
with prior episodes and elderly patients. Early diagnosis and surgical management play a decisive role in the outcomes, as well as a correct selection of the patient based on diagnostic scales such as EUROSCORE II.

Objectives. To evaluate EUROSCORE II as mortality predictor in patients who had undergone infective endocarditis surgery.

Methods. Patients operated by the same surgical team from two hospitals ("Fundación Jiménez Díaz" and "Rey Juan Carlos" Hospital in Madrid, Spain) have been evaluated, between 2010 and 2019. Discrimination capacity using ROC curve, standardized mortality ratio (SMR), observed to expected mortality ratio using the Hosmer-Lemeshow $(\mathrm{H}-\mathrm{L})$ goodness-of-fit test, have been assessed. All $p<0,05$ values were considered significant.

Results. 103 patients were included with a mean age of $67.7 \pm 11.8$ y.o., $67 \%$ of them man. $40,78 \%$ (42) of the patients underwent emergency surgery. $14,56 \%$ (15) of the patients died.

EUROSCORE II predicted mortality was $31,03 \%$, real mortality was $14,6 \%$ (15 patients). ROC curve: 0,847 (Cl95\%: 0,759-0,934; $p<0,001)$. SMR: 0,47 (CI95\%: 0,32-0,87). H-L test: 3,962; $p=0,861$.

Conclusion. EUROSCORE II, as mortality predictor in patients who underwent infective endocarditis surgery from our sample, presents both an adequate calibration and discrimination capacity. However, it overestimates mortality. This could be explained by the progressive optimization of the indication and surgical technique in time.

\section{2}

Patient experiences of intensive care post-liver transplantation D. Patel ${ }^{1}$; P. Featherstone,

'Intensive Care, Addenbrooke's Hospital, Cambridge, United Kingdom Correspondence: D. Patel

Intensive Care Medicine Experimental 2021, 9(1): 000312

Introduction. Liver transplantation (LT) is the only effective treatment for end-stage liver disease. Advances in peri-operative care have led to an expansion in the annual number of liver transplants undertaken. At Addenbrooke's Hospital, Cambridge, all patients undergoing LT are routinely admitted to the intensive care unit (ICU) post-operatively for resuscitation, stabilisation, and extubation. However, ICU admission is increasingly recognised to be associated with physical and psychological sequelae that continue beyond hospitalisation, including post-traumatic stress disorder (PTSD). Although PTSD has not been well-characterised in the LT population, it has a prevalence of up to $25 \%$ in general ICU patients. Addressing PTSD in LT patients is of value as it is associated with non-compliance with medical therapy (avoidance behaviour)(1). Planned admission to the ICU also represents a 'scheduled trauma' and therefore offers the opportunity to provide patients with pre-operative information which might mitigate the subsequent development of psychological sequelae.

Objectives. To understand patient experiences of ICU post LT in order to improve the quality and content of information given to patients prior to LT.

Methods. A focus group of patients who had undergone LT at Addenbrookes Hospital was convened and asked about their experience of ICU post-LT. Data was examined by thematic analysis.

Results. 10 patients ( 7 males, 3 females) who had undergone LT between 2004 and 2020 participated in the focus group. 6 patients received no information regarding ICU admission prior to LT. The remaining 4 knew they would be admitted to ICU, but did not know what this entailed. 4 patients continued to experience symptoms beyond LT that negatively impacted their lives. The dominant themes expressed included: 1) delirium with vivid nightmares and hallucinations; 2) endotracheal intubation which led to difficulty communicating, a feeling of vulnerability, and discomfort; 3 ) intermittent memory of ICU with difficulty in constructing a timeline of events; 4) psychological sequelae of ICU and LT. Prior to LT, patients suggested the importance of reassurance of ICU admission and the potential sequelae, further information about ICU for relatives, and patient diaries while on ICU to help complete their memories.

Conclusion. PTSD post ICU admission is seen in the LT population. This may be improved through more detailed information regarding
ICU admission prior to LT, fast-track extubation protocols which minimise duration of intubation, and improved psychological support following discharge.

\section{Reference(s)}

1. 1: Supelana C et al. PTSD in solid organ transplant recipients: Current understanding and future implications. Pediatr Transplant. 2016 Feb; 20(1): 23-33.

\section{6}

Persistent Ventilation Following Surgery-Admissions to an Intensive Care Unit Over 2 Years

D. Cottam ${ }^{1}$; N. Shahzad'; D. Lesch, ; S. Green, ; B. Creagh-Brown ${ }^{1}$

${ }^{1}$ Intensive Care and Perioperative Medicine, Royal Surrey County Hospital, Guildford, United Kingdom

Correspondence: D. Cottam

Intensive Care Medicine Experimental 2021, 9(1): 000416

Introduction. Following the end of surgery under general anaesthesia it is usual to awaken the patient and remove their artificial airway. There are some circumstances where invasive mechanical ventilation via an artificial airway is continued postoperatively. There is a paucity of data to guide decision-making about the risks and benefits of persistent postoperative ventilation, and little is described in the literature about its epidemiology.

Methods. The Royal Surrey County Hospital is a tertiary centre for oesophago-gastric, hepato-pancreatico-biliary, gynae-oncology and maxillofacial/ear-nose-throat surgery. There is no cardiac, thoracic, vascular or transplant surgery. We reviewed the demographics, clinical indications and outcomes of patients requiring persistent postoperative ventilation in our intensive care unit. WardWatcher (Critical care audit Ltd) software was interrogated, and data cross referenced with ICCA (Philips). Data was collated in Excel (MS) and analysed with GraphPad (Prism). Analyses were retrospective and did not include paper notes (anaesthetic record and operation note).

Results. Between 01/01/18 and 31/12/19 there were 1718 patients admitted from recovery or theatres to our intensive care unit following a procedure. Of these, 227 had persistent ventilation (13.2\%). We identified 131 patients for review after excluding those with surgery involving the airway (77), repeat admissions (9), patients who didn't have surgery (endoscopy/interventional radiology, 13) and those ventilated pre-operatively (3). These patients utilised 1345 bed-days over 2 years.

Although the majority of cases were emergency admissions, 33\% $(43 / 131)$ followed elective surgery, predominantly oesophageal or HPB surgery. Duration of ventilation was short, ICU stay was not prolonged and ICU mortality was low; however, hospital length of stay was prolonged and overall, there was considerable in-hospital mortality. Admission arterial blood gas analysis showed that $80 \%$ met oxygenation criteria for ARDS, while the median $\mathrm{pH}$ was slightly low with a mildly elevated $\mathrm{PaCO} 2$ and lactate. Average initial vasopressor requirements were moderate. Additional reasons for persistent ventilation commonly included recent bleeding/transfusion as well as concerns about adequacy of self-ventilation. The duration of surgery was not well documented, so we have not reported it. The commonest time of arrival in the ICU was the early evening, between 20:00 and 21:00.

Table 1: Patients with Persistent Postoperative Ventilation

\begin{tabular}{llll}
\hline & All $(\mathbf{n}=\mathbf{1 3 1})$ & $\begin{array}{l}\text { Emergen- } \\
\text { cies }(\mathbf{n}=\mathbf{8 8})\end{array}$ & $\begin{array}{l}\text { Elective } \\
(\mathbf{n}=\mathbf{4 3})\end{array}$ \\
\hline $\begin{array}{l}\text { Age, Median (IQR) } \\
\text { Days of Ventilation, } \\
\text { Median (IQR) }\end{array}$ & $69(57-79)$ & $72(61-82)$ & $62(57-71)$ \\
ICU LOS, Median (IQR) & $5(4-2)$ & $2(1-2)$ & $2(2-2)$ \\
\hline
\end{tabular}




\begin{tabular}{llll}
\hline & All $(\mathbf{n}=\mathbf{1 3 1})$ & $\begin{array}{l}\text { Emergen- } \\
\text { cies }(\mathbf{n}=\mathbf{8 8})\end{array}$ & $\begin{array}{l}\text { Elective } \\
(\mathbf{n}=\mathbf{4 3})\end{array}$ \\
\hline $\begin{array}{l}\text { Hospital LOS, Median } \\
\text { (IQR) }\end{array}$ & $19(9-30)$ & $21(12-32)$ & $15(6-27)$ \\
$\begin{array}{l}\text { Died on ICU, } \mathbf{n}(\%) \\
\text { Died in Hospital, } \mathbf{n}(\%)\end{array}$ & $9(6 \%)$ & $7(8 \%)$ & $1(2 \%)$ \\
\hline
\end{tabular}

Table 2: Physiological Derangement/Organ support

\begin{tabular}{lll}
\hline Oxygenation & Mild ARDS (PFR $\leq 39.9 \mathrm{kPa}), \mathrm{n}(\%)$ & $74(56 \%)$ \\
& Moderate ARDS (PFR $\leq 26.6 \mathrm{kPa}), \mathrm{n}(\%)$ & $25(19 \%)$ \\
& Severe ARDS (PFR $\leq 13.3 \mathrm{kPa}), \mathrm{n}(\%)$ & $6(5 \%)$ \\
\multirow{5}{*}{ Ventilation } & $\mathrm{PaCO}_{2}(\mathrm{kPa}), \mathrm{mead}(\mathrm{s} . \mathrm{d})$. & $5.89(0.9)$ \\
& $\mathrm{pH}$, median (IQR) & $7.3(7.25-7.36)$ \\
Circulation & $\begin{array}{c}\text { Noradrenaline (mcg/kg/min), median } \\
\text { (IQR) }\end{array}$ & $0.13(0.06-0.26)$ \\
\hline
\end{tabular}

Conclusion. Persistent ventilation following surgery is common on our ICU, typically following emergency laparotomy. There are limitations to this retrospective service evaluation, but this can provide a foundation for further work to better characterise this patient group and their outcomes.

\section{6}

\section{0-day clinical outcomes and Quality of Recovery} following endo-CABG: A prospective cohort study

D. Lenskens '; J. Claessens, ${ }^{2}$; JP. Ory, ${ }_{1}$; J. Dewandre, ${ }_{1}$; I. Callebaut ${ }^{1}$; A.

Yilmaz, ${ }^{2}$ B. Stessel, ${ }^{1}$

'Department of Anesthesisology and Intensive Care, Jessa Ziekenhuis, Hasselt, Belgium; ${ }^{2}$ Department of Cardio-thoracic Surgery, Jessa Ziekenhuis, Hasselt, Belgium

Correspondence: D. Lenskens

Intensive Care Medicine Experimental 2021, 9(1): 000766

Introduction. To bypass the disadvantages and potential complications associated with cardiac surgery via open sternotomy, a new minimally invasive endoscopic coronary artery bypass grafting (EndoCABG) technique to treat patients with single- and multi-vessel coronary artery disease has been developed. Quality of recovery (QoR) is a complex phenomenon covering many dimensions and has rarely been assessed in a broader perspective. Quality of Life (QoL) questionnaires fulfil the requirements for assessing late QoR (1 month to 1 year). The aim of this study is to assess QoR and 30-day clinical outcomes after Endo-CABG.

Methods. In this prospective, observational, cohort study, all adult patients scheduled for an elective endo-CABG in the Jessa hospital, Hasselt, Belgium were asked to participate. Both clinical outcomes and QoR were evaluated at baseline, 14 days, 30 days and 90 days after surgery. QoR was measured with both the 36 -item Short Form survey (SF36) and EuroQOL-5D (EQ-5D) questionnaire. Statistical analyses were performed with the Friedman rank sum test, followed by the Wilcoxon signed rank test adjusted with Bonferroni correction. A p-value $<0.05$ was considered statistically significant.

Results. From November 25th, 2019 until October 31st, 2020, 86 patients undergoing Endo-CABG were included. Clinical outcomes are shown in Table 1. QoR is presented in Table 2. Thirty days after Endo-CABG, QoL, measured with the EQ-5D VAS is returned to baseline level $(70(20)$ vs $70(20), p=1.00)$. Moreover, 90 days after surgery, QoL is significantly improved compared to baseline (70 (20) vs 80 (20), $\mathrm{p}<0.01$ ). This improvement at 90 days is also observed in the general health and physical functioning domains of the SF-36.

Conclusion. Endo-CABG seems to be associated not only with good 30-day clinical outcomes but also with a fast QoR with return to baseline levels of the QoL already 30 days after surgery and an improvement of the QoL 90 days after surgery.
Table 1. Outcomes

\begin{tabular}{|l|c}
\hline & $\begin{array}{c}\text { Patients undergoing Endo-CABG } \\
\mathrm{N}=86\end{array}$ \\
\hline CPB time (min) & $90.72 \pm 30.13$ \\
\hline Clamping time (min) & $53.06 \pm 18.61$ \\
\hline Ventilation time (min) & $7.37 \pm 6.75$ \\
\hline Early Revision & $6(6.97 \%)$ \\
\hline ICU LOS (hours) & $49.65 \pm 28.17$ \\
\hline Hospital LOS (days) & $5.42 \pm 2.26$ \\
\hline 30 day mortality & $1(1.16 \%)$ \\
\hline
\end{tabular}

Data are presented as mean \pm SD or as frequencies $(\%)$

Table 2. Results from SF 36 and EQ5-D questionnaires in patients undergoing EndoCABG ( $n=86)$.

\begin{tabular}{|c|c|c|c|c|c|}
\hline & Baseline & 14 days & 30 days & 90 days & $p$-value \\
\hline \multicolumn{6}{|l|}{ SF-36 } \\
\hline $\begin{array}{l}\text { Physical } \\
\text { functioning }\end{array}$ & $82.75(43.75)$ & $70.0(40)$ & $85(25)$ & $90(20)$ & $<0.001$ \\
\hline Role physical & 75 (93.75) & $0(50)$ & $50(100)$ & $100(75)$ & 0.06 \\
\hline Pain & $100(31.87)$ & $80(42.5)$ & $90(28.75)$ & $100(20)$ & $<0.01$ \\
\hline General health & $75(20)$ & $70(25)$ & 75 (15) & $80(20)$ & $<0.01$ \\
\hline PCS & 57.15 (14.15) & $49.43(12.46)$ & $54.72(10.65)$ & $61.64(9.31)$ & 0.53 \\
\hline Role emotional & $100(33.33)$ & $100(0)$ & $100(0)$ & $100(0)$ & 0.01 \\
\hline $\begin{array}{l}\text { Emotional } \\
\text { wellbeing }\end{array}$ & $80(32)$ & $88(20)$ & $92(24)$ & $92(16)$ & 0.53 \\
\hline Energy fatigue & $80(40)$ & $70(30)$ & $80(25)$ & $90(25)$ & 0.13 \\
\hline $\begin{array}{l}\text { Social } \\
\text { functioning }\end{array}$ & $100(46.87)$ & $50(100)$ & $75(75)$ & $75(100)$ & $<0.01$ \\
\hline \multicolumn{6}{|l|}{$E Q-5 D$} \\
\hline Index & $0.82(0.30)$ & $0.76(0.21)$ & $0.84(0.17)$ & $0.87(0.21)$ & $<0.001$ \\
\hline VAS & $70(20)$ & $65(24.25)$ & $70(20)$ & $80(20)$ & $<0.001$ \\
\hline
\end{tabular}

000829

Histidine-Rich Glycoprotein as a Novel Biomarker to Predict Postoperative Complications

M. Oiwa ${ }^{1}$; K. Kuroda ${ }^{1}$; N. Kawanoue ${ }^{1}$; H. Morimatsu

${ }^{1}$ Anesthesiology and Resuscitology, Okayama University Graduate School of Medicine, Dentistry and Pharmaceutical Sciences, Okayama, Japan

Correspondence: M. Oiwa

Intensive Care Medicine Experimental 2021, 9(1): 000829

Introduction. Histidine-Rich Glycoprotein (HRG) is a glycoprotein of approximately $75 \mathrm{kDa}$ and is present in plasma at $60-150 \mu \mathrm{g} / \mathrm{ml} 1) 2$ ). In our previous study, we reported that decrease in HRG may be useful biomarker for diagnosing sepsis, assessing patient severity and predicting patient outcome 3 ). This study also included postoperative patients and suggested that plasma HRG levels in postoperative patients were lower than normal. In addition, HRG has been reported to decrease in the acute inflammation phase and to be inversely correlated with CRP 4). However, it is not known whether plasma HRG levels can predict postoperative complications.

Objectives. The purpose of this study was to investigate the usefulness of HRG as a biomarker for predicting postoperative complications by examining the differences in plasma HRG levels in the presence and absence of postoperative complications, and comparing the ability of HRG and other biomarkers (CRP, WBC, procalcitonin, and presepsin) to predict postoperative complications.

Methods. The study was single center, prospective and observational investigation, and approved by the Ethics Committee of Okayama University. One hundred and fifty adult patients, excluding pregnant women, who were admitted to the intensive care unit after surgery at Okayama University Hospital were included in the study. Plasma HRG levels and other biomarker levels were measured on the day after surgery. Plasma HRG levels were measured by ELISA. The patients were classified into two groups according to the presence or absence of complications within 7 days after surgery. We defined postoperative complications as grade II or higher in the Clavien-Dindo classification. 
Data are presented as median [interquartile range], and $p$ value of less than 0.05 was considered statistically significant.

Results. Plasma HRG levels in the complication group $(n=60 ; 21.5 \mu \mathrm{g} /$ $\mathrm{mL}$ [18.12-25.74 $\mu \mathrm{g} / \mathrm{mL}]$ ) were significantly lower than those in noncomplication group $(\mathrm{n}=90 ; 25.46 \mu \mathrm{g} / \mathrm{mL}$ [21.05-31.63 $\mu \mathrm{g} / \mathrm{mL}])$ $(p<0.001)$. HRG level was significantly associated with postoperative complications ( $\mathrm{HR}, 0.92 ; 95 \% \mathrm{Cl}, 0.88-0.96 ; \mathrm{p}<0.001)$, and when adjusted according to age, ASA-PS, operative time, intraoperative infusion balance, and Surgery Apgar Score, this level remained an independent prediction factor (adjusted HR, 0.95; 95\% Cl, 0.91-0.99; $p=0.04$ ). ROC curve analysis for predicting postoperative complications showed that the area under the curve for HRG was 0.69, and at a cutoff value of $24.21 \mu \mathrm{g} / \mathrm{mL}$, the sensitivity and specificity of HRG level associated with postoperative complications were $73 \%$ and $57 \%$, respectively. Although HRG was not superior to presepsin, it was better at predicting postoperative complications than CRP and WBC.

Conclusion. HRG may be useful as a biomarker for predicting not only sepsis but also postoperative complications.

\section{Reference(s)}

1. 1) Poon IK, et al.: Histidine-rich glycoprotein: the Swiss Army knife of mammalian plasma. Blood 117: 2093-2101, 2011

2. 2) Corrigan JJ, et al.: Histidine-rich glycoprotein levels in children: the effect of age. Thromb Res 59: 681-686, 1990

3. 3) Kuroda K, et al.: Decrease in Histidine-Rich Glycoprotein as a Novel Biomarker to Predict Sepsis Among Systemic Inflammatory Response Syndrome. Crit Care Med 46: 570-576, 2018

4. 4) Saigo K, et al.: Histidine-Rich Glycoprotein as a Negative Acute Phase Reactant. Am J Hematol 34: 149-150, 1990

\section{2}

Fluid responsiveness assessment by passive leg raising and mini-fluid challenge in major surgery, a prospective study G. lacopetti ${ }^{1}$ L. Foti ${ }^{1}$ : M. Suppressa'; G. Villa ${ }^{1}$ : A. Messina ${ }^{2}$; Z. Ricci ${ }^{3} \cdot S$. Romagnoli

${ }^{1}$ Health Sciences Department, Section of Anesthesiology, Intensive Care and Pain Medicine, Careggi University Hospital, Firenze, Italy; ${ }^{2}$ Anesthesia and Intensive Care, Humanitas Research Hospital, Rozzano, Italy; ${ }^{3}$ Health Sciences Department, Section of Anesthesiology, Intensive Care and Pain Medicine, University Hospital Meyer, Firenze, Italy

Correspondence: $\mathrm{G}$. lacopetti

Intensive Care Medicine Experimental 2021, 9(1): 000872

Introduction. Guidelines recommend 2-h fasting for clear liquids to ensure euvolemia before surgery [1]. Few methods are available to assess fluid responsiveness in awake spontaneously breathing patients: Passive Leg Raising (PLR) and mini-fluid challenge $(\mathrm{mFC})$ are among them. Fluid responders (FRs) are those who increase Stroke Volume $(\Delta S V)>10 \%$ after PLR or mFC. The Starling ${ }^{\mathrm{TM}} \mathrm{SV}$ monitor (Cheetah Medical-Baxter) identifies FRs thanks to bioreactance technology [2].

Objectives. To evaluate fluid responsiveness preoperatively by PLR, and intraoperatively by $\mathrm{mFC}$.

Methods. Preoperative PLR test was performed to patients undergoing major surgery before the anesthesia induction. FRs received $250 \mathrm{ml}$ of balanced crystalloid solution and fluid responsiveness was re-tested at the end of the bolus and again until a non-FR state was achieved. Baseline intraoperative fluid therapy was set at $5 \mathrm{~mL} / \mathrm{kg} / \mathrm{h}$ until the end of surgery. Hourly, mFCs with $100 \mathrm{ml}$ of crystalloids balanced solutions were performed and FRs received the $250 \mathrm{ml}$-fluid bolus. Blood [Hb]-concentration was measured before surgery and hourly.

Results. Twenty-two patients were enrolled. Fifty \% of the patients were FRs. Average fasting time was $12(3.16) \mathrm{h}$ in FRs and 13.8(4.35)h in nonFRs. After the first crystalloid bolus, 9 (82\%) FRs became non-FRs. Intraoperatively, $47 \mathrm{mFCs}$ were performed in the first $3 \mathrm{~h}$ and 9 FRs were found (19\%). In all FRs, after the bolus, the mFC test was re-performed and no patient resulted to be FR. The $[\mathrm{Hb}]$ variation before and after $60 \mathrm{~min}$ from surgery start was $0.71(0.66) \mathrm{g} / \mathrm{dL}$ for FRs and $0.32(0.56) \mathrm{g} / \mathrm{dL}$ for non-FRs.

Conclusion. Preoperative fasting does not correlate with PLR test. After having tested for fluid responsiveness and corrected volemia, $[\mathrm{Hb}]$-concentration can be used as a sensitive indicator of fluid expansion-restriction in non-bleeding patients. PLR and mFC tests, are easy, reliable and may be efficient tools to guide volume optimization during major surgery.

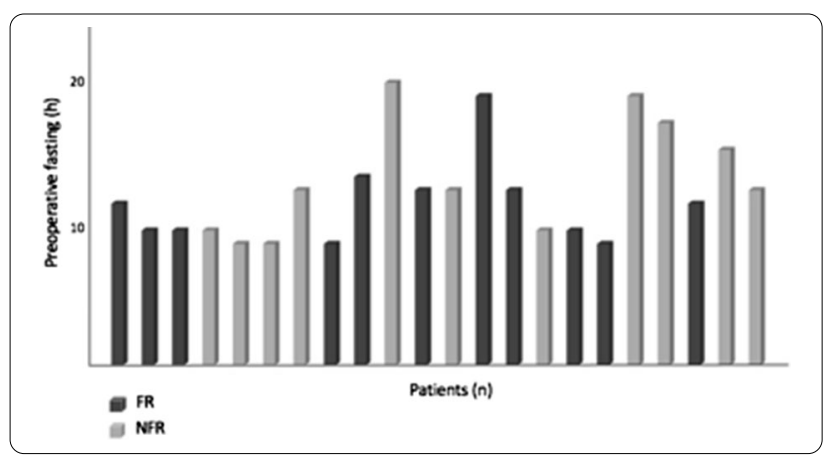

Reference(s)

1. 1. Scott MJ, Baldini G, Fearon KC et al. Enhanced Recovery After Surgery (ERAS) for gastrointestinal surgery, part 1: pathophysiological considerations. Acta Anaesthesiol Scand. 2015 Nov;59(10):1212-31

2. 2. Raval NY, Squara P, Cleman M, Yalamanchili K, Winklmaier M, Burkhoff D. Multicenter evaluation of noninvasive cardiac output measurement by bioreactance technique. J Clin Monit Comput. 2008 Apr;22(2):113-9.

000882

Comparing the carbon dioxide gap and central venous oxygen saturations as predictors of fluid responsiveness in patients post major abdominal surgery

V. Jambunathan ${ }^{\text {1 }}$ H. Gardiner'; M. Ardissino ${ }^{3}$; J. Handy ${ }^{4}$

${ }^{1}$ Anaesthesia and Critical Care Medicine, Sheffield Teaching Hospitals NHS Foundation Trust, Wakefield, United Kingdom; ${ }^{2}$ Anaesthesia, Imperial College Healthcare NHS Trust, London, United Kingdom; ${ }^{3}$ Medicine, Imperial College Healthcare NHS Trust, London, United Kingdom; ${ }^{4}$ Anaesthesia and Critical Care, The Royal Marsden Hospital, London, United Kingdom Correspondence: $\mathrm{V}$. Jambunathan

Intensive Care Medicine Experimental 2021, 9(1): 000882

Introduction. Patients undergoing major abdominal surgery often require haemodynamic support as a result of the fluid shifts associated with the surgical stress response and require fluid balance optimization [1]. There are multiple invasive and non-invasive techniques to measure cardiac output (CO) and assess fluid balance. Central venous oxygen saturation $(\mathrm{ScvO} 2)$ is widely used to guide fluid resuscitation. The central venous-arterial carbon dioxide difference has a growing body of evidence supporting its use as a surrogate for $\mathrm{CO}$ in sepsis as it reflects tissue blood flow relative to metabolic demand [2,3]. It has been shown to identify patients with ScvO2 $>70 \%$ who still require fluid resuscitation [4].

Objectives. To establish whether a high $\mathrm{CO}_{2}$ gap $(>1 \mathrm{kPa})$ predicts fluid responsiveness in patients post major abdominal surgery in comparison to $\mathrm{ScvO}_{2}$.

Methods. It was a retrospective cross-sectional study. Data was collected from patients admitted to post op surgical HDU following elective major Upper Gl surgery (Ivor-Lewis, gastrectomy, Whipple's), hepato-biliary surgery (hepatectomy, pancreatectomy), pelvic exenterations, major Colorectal and retroperitoneal sarcoma operations between 05/10/2020 and 31/10/2020. Paired arterial and central venous blood gases were taken to guide fluid therapy and the outcome was assessed clinically (improvement in blood pressure, urine output, reduction of vasopressor requirement).

Results. Data was collected on $\mathbf{4 0}$ occasions. 


\begin{tabular}{lllll}
\hline Mean Age & $\begin{array}{l}\text { Mean } \\
\text { Weight }\end{array}$ & $\begin{array}{l}\text { Mean } \\
\text { Height }\end{array}$ & $\begin{array}{l}\text { Mean } \\
\text { ScvO2 }\end{array}$ & $\begin{array}{l}\text { Mean CO2 } \\
\text { gap }\end{array}$ \\
\hline $66 \mathrm{yrs}$ & $72.1 \mathrm{~kg}$ & $167.8 \mathrm{~cm}$ & 63.11 & 1.232 \\
\hline
\end{tabular}

We then assessed the optimal threshold point using two approaches: the Youden $[=\max ($ sensitivities + specificities)] approach and the top left point $[=\min ((1$-sensitivities $) \wedge 2+(1-$ specificities $) \wedge 2)]$ approach. We assessed the predictive value of $\mathrm{CO}_{2}$ gap (Figure 1) and $\mathrm{ScvO} 2$ (Fig. 2) by plotting the ROC curve and returning metrics on the optimal threshold value for prediction of the outcome.

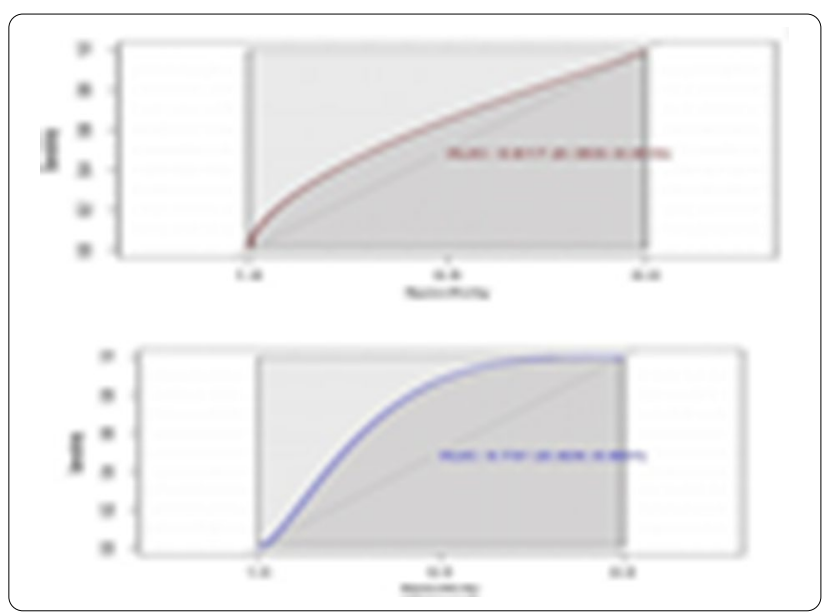

The ROC curve of the $\mathrm{CO}_{2}$ Gap was visually and quantitatively better than the ROC curve and parameters for the $\mathrm{ScvO}_{2}$. This provides evidence that within the data collected, paired $\mathrm{CO}_{2}$ could be a more informative blood gas parameter to aid the assessment of fluid status in this patient population.

The optimal value for predicting fluid responsiveness was of a $\mathrm{CO}_{2}$ gap of $1.05 \mathrm{kPa}$.

Conclusion. A central venous-arterial $\mathrm{CO} 2$ gap of $>1.05 \mathrm{kPa}$ appears to be a better to guide fluid resuscitation than $\mathrm{ScvO}_{2}$ following major abdominal surgery in this patient population. This makes it a useful adjunct or alternative to the multiple cardiac output monitors available, particularly in resource poor environments.

\section{Reference(s)}

1. Nill.

2. 4. Vallée et al. Intensive Care Med. 2008

3. 3. Vallet et al. Intensive Care Med. 2013

4. 2. Mallat et al. Eur J Anaesthesiol. 2014

5. 1. Strunden et al. Ann Intensive care 2011

\section{3}

Hemoynamic changes in the early period following liver transplantation

I. Saez de la Fuente ${ }^{1}$; J. Saez De La Fuente ${ }^{2}$; J. Gutiérrez, ; M. Gonzalez-Fernandez ; M. Sánchez-Bayton Griffith³; A. Marcos-Morales4; R. Munoz-Calahorro ${ }^{5}$ J JA. Sanchez-Izquierdo ; JC. Montejo-Gonzalez ${ }^{6}$ ${ }^{1}$ Critical Care, University Hospital 12 de Octubre, Madrid, Spain;

${ }^{2}$ Pharmacy, Hospital Ramón y Cajal, Madrid, Spain; ${ }^{3}$ Medicina intensiva, University Hospital 12 de Octubre, Madrid, Spain; ${ }^{4}$ Intensive Care, Hospital Doce de Octubre, Madrid, France; ${ }^{5}$ Intensive Care Medicine, Hospital

12 De Octubre, Madrid, Spain; ${ }^{6}$ Intensive Care, Hospital 12 De Octubre,
Madrid, Spain

Correspondence: I. Saez de la Fuente

Intensive Care Medicine Experimental 2021, 9(1): 000923

Introduction. Following successful orthotopic liver trans-plantation (OLT), many patients still manifest a "hyperdynamic" cardiovascular state as evidenced by a decrease in systemic vascular resis- tance (SVR), an increase in cardiac output (CO), and a normal or low normal systemic blood pressure (SBP).

Objectives. Study the hemodynamics in the inmediate post-trasplant period and compare patients regarding their Child-Pugh status.

Methods. Prospective observational study including all patients admitted to the ICU after liver transplantation over a 3-years study period. Hemodynamic variables were recorded at the ICU admission and in different study periods until the first $72 \mathrm{~h}$. Patients were classified according to their Child Pugh status.

Data statistical analysis was done using SPSS software. Quantitative variables are expressed as mean with standard deviation (SD) and qualitative variables as absolute and relative frequencies. The statistical significance of the proportion comparison was addressed by the chi-square test, Mann Whitney U test or Student's t-Test with a statistical significance $p<0.05$.

Results. During the study period, 150 patients were admitted to the ICU after a liver transplantation (80\% male) with a median age of 55,6 (IQR 51-63). The main causes of the liver transplant were alcoholism (58\%), hepatocellular carcinoma (46\%) and hepatitis C virus infection (40\%). Median MELD score was 13 (IQR 9-18) and Child Pugh score was 8 (IQR 6-9); Median ICU stay was 5 days (IQR 4-8,25), with an overall ICU mortality of $6,7 \%$.

An hyperdinamic state was registered at ICU admission, with a median cardiac index (CI) of $4,5 \mathrm{I} / \mathrm{min} / \mathrm{m}^{2}$ (IQR 3,65-5,5), a median systemic vascular resistance index (SVRI) of 1378 dynes $\times \mathrm{sec} / \mathrm{cm}^{5} / \mathrm{m}^{2}$ (IQR 1045-1733) and a median pulmonary resistance index (PVRI) of 160 dynes $\times \mathrm{sec} / \mathrm{cm}^{5} / \mathrm{m}^{2}$ (IQR 96,2-241,5). During the study period no differences were recorded between $\mathrm{Cl}$ values while SVRI [1532,8 at $24 \mathrm{~h}$ (IRQ 1175,51-1857,14); $\mathrm{p}=0,026]$ and PVRI [233 at $48 \mathrm{~h}$ (IQR 119,6473,3); $p=0,039]$.

According to Child Pugh status, no statically significant differences were recorded.

Conclusion. Hyperdynamic circulation persists in the inmediate posttrasplant period independently the Child Pugh status.

\section{Reference(s)}

1. Glauser F.L.. Systemic hemodynamic and cardiac function changes in patients undergoing orthotopic liver transplantation. Chest. 1990;98:1210-15

2. Della Rocca G, Costa M.G, Coccia C, Pompei L, Pietropaoli P. Preload and haemodynamic assessment during liver transplantation: a comparison betwwen the pulmonary artery catheter and transpulmonary indicator dilution techniques. Eur J Anaesthesiol. 2002 Dec;19(12):868-75.

3. Hori T, Ogura Y, Kamei H, Kurata N, Kainuma M, et al.. Systemic hemodynamics in advanced cirrhosis: Concerns during perioperative period of liver transplantation. World Journal of Hepatology. 2016; 8(25):1047-1060

4. Navasa M, Feu F, García-Pagán J.C, Jiménez W, Llach J, Rimola A, Bosch J, Rodés J. Hemodynamic and Humoral Changes After Liver Transplantation in Patients with Cirrhosis. Hepatology. 1993; 17(3):355-60.

5. Al-Hamoudi W, Alqahtani S, Tandon P, Ma M, Lee S. S.. Hemodynamics in the immediate post- transplantation period in alcoholic and viral cirrhosis.. World Journal of Gastroenterology. 2010; 16(5):608-612. 
001111

Significant Intraoperative hypotension and Serious Cardiac complication in Hypertensive Patients Underwent Surgery for Fracture Neck of Femur \& Admitted to the General SICU

S. Kongsayreepong

${ }^{1}$ \{street_address\}, Bangkok, Thailand

Correspondence: S. Kongsayreepong

Intensive Care Medicine Experimental 2021, 9(1): 001111

Introduction. Patients who underwent surgery for fracture neck of femur were extreme age with multiple comorbidities and high risk for postoperative complications. Hypotension and serious cardiac complication were found commonly intraoperative resulting in early higher mortality and morbidity especially in high risk patient. Still less information about predictors of these intraoperative hypotension and serious cardiac complications were reported.

Objectives. To study the incidence, predictors \& outcome (30 days mortality) of significant intraoperative hypotension $(\mathrm{IOH}$ : $\mathrm{SBP}<90 \mathrm{mmHg}>10 \mathrm{~min})$ ) and serious cardiac complications in high risk patients underwent surgery for fracture neck femur \& admitted to the general SICU.

Methods. This prospective observational study was done in 135 consecutive high risk hypertensive patients underwent only surgery for fracture neck of femur and admitted to the general SICU during Sep 2014-Oct 2020. Study data: patient's demographic data, co-morbidities; preoperative fluid \& antihypertensive agent, ASA physical status, detail of anesthesia \& surgery, intraoperative I/O, inotrope/vasopressor, significant cardiac events (bradycardia, significant hypotension and duration, cardiac arrhythmias, PMI, cardiac arrest), early (within $72 \mathrm{~h}$ ) postoperative organ dysfunctions (stroke, delirium, PMI, AKI, liver dysfunction).

Results. Among the studied patients (age $71 \pm 11 \mathrm{yrs}$ ), $65 \%$ were ASAIII, $18 \%$ were poor control HTN \&17\% had CAD, 38\% had CAD, $29 \%$ didn't have any premedication, 3.0, $15.0 \& 39.4$ percent received ARB/ ACEI, beta-blocker \& vasodilator respectively. $61 \%$ receive spinal. $61 \%$ had $\mathrm{IOH}$ that $53 \%$ had early organ dysfunction which $2 / 3$ were new AKI \& PMI ( $1 / 3$ serious cardiac arrhythmias most were significant bradycardia in patients who receive beta blocker). Data from multivariate analysis showed that ASA IV [OR $5.36(0.95,30.25)]$, poor control hypertension [OR $4.26(1.41,12.91)]$, preoperative vasodilator only [OR 6.20 $(1.63,23.56)]$, beta-blocker only [OR $16.17(3.45,75.88)]$, vasodilator plus and beta-blocker [OR $25.12(4.57,138.15]$, spinal anesthesia [OR $6.65(2.11,20.92]$ and ZNN \& cemented bipolar hemiarthroplasty [OR $3.85(1.07,13.87]$ were significant predictors of $\mathrm{IOH}$.

Conclusion. Care should be considered whether to continue or withhold preoperative antihypertensive agents in high risk hypertensive agent underwent surgery for fracture neck of femur.

\section{Reference(s)}

1. Leslie K, et al. Neuraxial block, death and serious cardiovascular morbidity in the POISE trial. Br J Anaesth 2013:111:382-90.
001300

Harmful alcohol use is associated with intra- and postoperative complications in cardiac surgery patients. A retrospective study N. Eliisa ; M. Hynninen ${ }^{1}$; R. Suojaranta ${ }^{2}$; S. Dahlbacka ${ }^{3}$; J. Hastbacka, ${ }^{4}$ ${ }^{1}$ Intensive Care Units, Department of Anaesthesiology, Intensive Care and Pain Medicine, University of Helsinki and Helsinki University Hospital, Helsinki, Finland; 'Department of Cardiology, Heart and Lung Center, University of Helsinki and Helsinki University Hospital, Helsinki, Finland; ${ }^{3}$ Department of Cardiac Surgery, Heart and Lung Center, HUS, Helsinki, Finland; ${ }^{4}$ Department of Anesthesiology and Intensive care, HUS, Helsinki, Finland

Correspondence: E. Nissilä

Intensive Care Medicine Experimental 2021, 9(1): 001300

Introduction. Alcohol metabolism contributes to tissue damage by multiple pathophysiological mechanisms and impairs cardiovascular function (1-3). Preoperative harmful alcohol use has been associated with increased risk for postoperative complications (4).

Objectives. To assess the association of preoperative harmful alcohol with perioperative complications in cardiac surgery patients.

Methods. Cardiac surgical patients (excluding transplant- and Left Ventricular Assist Device (LVAD) patient) receiving postoperative care in the intensive care unit of Helsinki University Hospital during 2017 were studied. Preoperatively, data on alcohol use has been routinely collected by using the AUDIT-C (Alcohol use disorder identification test-consumption) questionnaire. Hazardous alcohol use was defined as $\geq 5$ (of 12) points for females and $\geq 6$ six points for males. We obtained retrospectively the following data from the Finnish Intensive Care Consortium (FICC) database, medical records and hospital laboratory records: Duration of the operation, cumulative doses of vasoactive medication used, postoperative antibiotic treatment and its indications, postoperative arrhythmias, perioperative amount of blood products, ICU re-admissions and length of stay (ICU LOS).

Results. During this one-year period AUDIT-C scores were available for $758(82.5 \%$ of all) cardiac surgery patients. The prevalence of hazardous alcohol use was $14.1 \%$. Patients with hazardous alcohol use were younger [median age 59 (IQR 52-67) vs 69 (IQR 63-74) years $\left(p<0.001^{*}\right)$, respectively] and more often male $93.5 \%$ vs $71.9 \%$ $\left(p<0.001^{*}\right)$, than other patients. We found that harmful alcohol use is associated with higher cumulative dose of intraoperative vasoactive medication, platelets and fresh frozen plasma (Table 1). Postoperatively, hazardous alcohol use was associated with increased incidence of severe infections (sepsis, mediastinitis, endocarditis, pneumonia, sternal osteitis) $2.8 \%$ vs $7.5 \%\left(p=0.013^{*}\right)$ and re-admissions to ICU $2.8 \%$ vs $6.5 \%\left(p=0.043^{*}\right)$. We found no difference in the duration of operation, intraoperative blood loss, ICU LOS, incidence of arrhythmias or mortality between patients with non-hazardous and hazardous alcohol use.

\begin{tabular}{llll}
\hline $\begin{array}{l}\text { Intraoperative } \\
\text { cumulative dose } \\
\text { Median [IQR] }\end{array}$ & $\begin{array}{l}\text { Non-Harmful } \\
\text { alcohol use, } \\
\mathbf{N}=\mathbf{7 5 8}\end{array}$ & $\begin{array}{l}\text { Harmful alcohol } \\
\text { use, } \\
\mathbf{N}=107\end{array}$ & \\
\hline $\begin{array}{l}\text { Fresh frozen } \\
\quad \text { plasma }\end{array}$ & $500[500-750]$ & $1000[800-2200]$ & $\mathrm{p}=0.018^{*}$ \\
$\begin{array}{l}\text { Platelets } \\
\text { Noradrenalin (mg) }\end{array}$ & $\begin{array}{c}1.056[400-600] \\
1,968]\end{array}$ & $600[400-800]$ & $\mathrm{p}=0.031^{*}$ \\
Milrinone $(\mathrm{mg})$ & $2.3[1.5-3.2]$ & $1.356[0.752-2.42]$ & $\mathrm{p}=0.034^{*}$ \\
& & $2.8[2.3-3.7]$ & $\mathrm{p}=0.025^{*}$
\end{tabular}

Conclusion. Cardiac surgery patients with a history of hazardous alcohol use are younger and predominantly male. Compared with other cardiac surgery patients, patients with hazardous alcohol use receive more coagulation enhancing products and vasoactive medication and have more often severe infections and ICU re-admissions.

Reference(s)

1. 1. Piano MR. Alcohol's Effects on the Cardiovascular System. Alcohol Res 2017;38(2):219-241. 2. Rehm J, Roerecke M. Cardiovascular effects 
of alcohol consumption. Trends Cardiovasc Med 2017 November 01;27(8):534-538. 3. Molina PE, Gardner JD, Souza-Smith FM, Whitaker AM. Alcohol abuse: critical pathophysiological processes and contribution to disease burden. Physiology (Bethesda) 2014 May 01;29(3):203215. 4. Eliasen M, Gronkjaer M, Skov-Ettrup LS, Mikkelsen SS, Becker U, Tolstrup JS, et al. Preoperative alcohol consumption and postoperative complications: a systematic review and meta-analysis. Ann Surg 2013 December 01;258(6):930-942.

2. We thank Mika Erikson and Olli Kiiski, for their invaluable help with data acquisition

\section{0}

High incidence of perioperative hypovolemia in elderly patients undergoing major surgery

A. Bouwhuis ${ }^{1}$; C. Reuijl, ${ }^{1}$; C. Van Den Brom, ${ }^{2}$; S. Loer ${ }^{1}$; C. Bulte ${ }^{1}$

${ }^{1}$ Department of Anesthesiology, Amsterdam UMC, location VU University Medical Center, Amsterdam, Netherlands; ${ }^{2}$ Department of Intensive Care, Amsterdam UMC, location Amsterdam Medical Center, Amsterdam, Netherlands

Correspondence: A. Bouwhuis

Intensive Care Medicine Experimental 2021, 9(1): 001320

Introduction. Elderly patients have a higher risk of perioperative complications such as postoperative delirium and acute kidney injury $(\mathrm{AKI})$, both linked to tissue hypoperfusion. Fluid management plays a key role in the optimization of tissue perfusion. Physiological changes of aging and comorbid conditions decrease the elderly's tolerance towards a reduced circulatory volume, which might increase postoperative complications in elderly. The incidence of perioperative hypovolemia in the elderly is, however, unknown.

Objectives. The aim of this study was to determine the incidence of perioperative hypovolemia in the elderly population undergoing major surgery, and its relation to postoperative outcome.

Methods. This study was an open, prospective observational trial performed at a tertiary Dutch hospital between May 2018 and January 2021. Patients of 70 years or older and scheduled for major elective surgery, defined as an expected postoperative stay of at least two nights, were included after obtaining informed consent. Hypovolemia was defined by a $>10 \%$ increase in stroke volume upon a passive leg raising (PLR) maneuver, as measured by the Nexfin; a noninvasive cardiac output measuring device. Measurements were performed at four consecutive time points: preoperatively and 2, 24 and 48 h postoperatively. For secondary analyses, clinical outcome including major complications (Clavien-Dindo grade $\geq 3$ ) were collected from electronic patient files.

Results. One hundred forty-three patients (median age 75 [IQR $72-78])$ were included, of which the majority $(99 / 143,69 \%)$ had a history of cardiovascular disease. The most common performed procedure was open gastrointestinal surgery $(63 / 143,44 \%)$. Hypovolemia incidence was highest preoperatively: 74\% (101/136) (baseline mean stroke volume: $67 \pm 17 \mathrm{ml}$ and $77 \pm 18 \mathrm{ml}$ after PLR) followed by $67 \%$, $62 \%$ and $67 \%$ at the consecutive postoperative time points. Neither patient characteristics nor procedural details were associated with preoperative hypovolemia. Intraoperative vasopressor use was high $(93 \%)$ irrespective of volume status. Postoperative AKI occurred in $5.2 \%(5 / 95)$ of preoperative hypovolemic patients compared to $2.9 \%$ $(1 / 34)$ in normovolemic patients $(p=0.588)$. Postoperative delirium developed in $3.1 \%(3 / 95)$ of patients with preoperative hypovolemia versus $5.9 \%(2 / 34)$ with initial normovolemia $(p=0.472)$. Twenty-four patients developed a major postoperative complication; of which $15 \%$ (15/101) were preoperatively hypovolemic and 21\% (7/34) normovolemic $(p=0.523)$.

Conclusion. Perioperative hypovolemia incidence is high among elderly patients undergoing major elective surgery, in particular preoperatively. This study did not show an association with postoperative outcome, but as complications are frequent, perioperative volume status in elderly should have the attention of anesthesiologists and intensivists.

\section{Reference(s) \\ 1. None applicable.}

\section{6}

Time to critical care admission of urgent/emergent surgical patients admitted through the Emergency Room - impact of Covid-19 pandemic

J. Carvalho, ${ }^{1}$; C. Carvalho, ${ }^{1}$; R. Amaral ${ }^{1}$; H. Santos ${ }^{1}$; A. Santos, ${ }^{1}$; F. Esteves, ${ }^{1}$ ${ }^{1}$ Intensive Medicine, Centro Hospitalar De Trás-Os-Montes E Alto Douro, E.P.E., Vila Real, Portugal

Correspondence: J. Carvalho

Intensive Care Medicine Experimental 2021, 9(1): 001346

Introduction. Surgical patients often present in the emergency room (ER) with life threatening situations that require urgent surgical treatment and critical care (CC) admission in the post-operative period. 1 Considering this, the time to surgical treatment and CC admission is crucial. 2 In Portugal, the first case of Covid-19 was diagnosed in 2 nd of March of 2020. Covid-19 pandemic affected hospital organization and patients circuit flows and some studies even described a delayed time to diagnosis and time to surgical intervention.3 We intend to study if the Covid-19 pandemic affected the time to CC admission, being this dependent not only on the time to surgery but also on the availability of intensive care unit (ICU) beds, in all patients admitted through the ER that underwent urgent/emergent surgery.

Methods. Retrospective study of patients admitted in the ER that required urgent/emergent surgical treatment and post-operative ICU admission, between March and December of 2019 and the homologous period of 2020. Data were collected through the review of the electronic patient file: sex, age, date and hour of ER and ICU admission, length of stay in hospital and in ICU, duration of invasive mechanical ventilation (IMV), severity scores at $24 \mathrm{~h}$ and mortality rate.

Results. Between March and December of 2019, 30 patients fulfilled the inclusion criteria: twenty-two (73.3\%) were males, the mean age was 67.2 ( \pm 15.4$)$ years, $6(20 \%)$ were victims of trauma and $24(80 \%)$ were submitted to IMV. Within the same period of 2020, 24 patients were included in our study: $16(67 \%)$ were males, the mean age was $56( \pm 16.9)$ years, $7(29 \%)$ were victims of trauma and $19(79 \%)$ were under IMV.

Comparing the elapsed time between ER and ICU admission, we did not find any statistically significant difference-median of $487 \mathrm{~min}$ (258 to 923) in 2019 vs 481 (IQR 302 to 1283) in 2020. ICU length of stay was 4 days ( 1 to 6 ) in 2019 vs 5 ( 3 to 9) in 2020, hospital length of stay was 16 days (9 to 32) in 2019 vs 14 (10 to 28) in 2020 and duration of IMV was 3 days ( 1 to 5 ) in 2019 vs 3 ( 1 to 8 ) in 2020. ICU mortality rate was $16.7 \%(n=5)$ in 2019 vs $4 \%(n=1)$ in 2020 and hospital mortality rate $26.7 \%(n=8)$ in 2019 vs $12 \%(n=3)$ in 2020 . Regarding severity scores, SAPS II was $47( \pm 16)$ in 2019 vs $44( \pm 16)$ in 2020 and APACHE II $19( \pm 8)$ in 2019 vs $17( \pm 9)$ in 2020 . Concerning all the studied variables, no statistically significant difference was found.

Conclusion. In our hospital Covid-19 pandemic did not seem to change the time to admission in critical care, patient circuit flow or outcome of patients requiring urgent/emergent surgical treatment admitted through the ER. This could be explained since our institution always had the capacity to have an available operating room and critical care bed for this type of patients. Being a retrospective study clinical records may not be timely accorded to the reality and the low sampling size may be another factor that influenced the results. 
Reference(s)

1. 3. Reichert M, Sartelli M, Weigand MA, Doppstadt C, Hecker M, ReinischLiese A, Bender F, Askevold I, Padberg W, Coccolini F, Catena F, Hecker A; WSES COVID-19 emergency surgery survey collaboration group. Impact of the SARS-CoV-2 pandemic on emergency surgery services-a multinational survey among WSES members. World J Emerg Surg. 2020 Dec 9;15(1):64. https://doi.org/10.1186/s13017-020-00341-0. PMID: 33298131; PMCID: PMC7724441

2. 2. Mclsaac Dl, Abdulla K, Yang H, Sundaresan S, Doering P, Vaswan SG, Thavorn K, Forster AJ. Association of delay of urgent or emergency surgery with mortality and use of health care resources: a propensity score-matched observational cohort study. CMAJ. 2017 Jul 10;189(27):E905-E912. https://doi.org/10.1503/cmaj.160576. PMID: 28694308; PMCID: PMC5505757.

3. 1. Onwochei DN, Fabes J, Walker D, Kumar G, Moonesinghe SR. Critical care after major surgery: a systematic review of risk factors for unplanned admission. Anaesthesia. 2020 Jan;75 Suppl 1:e62-e74. https://doi.org/10. 1111/anae.14793. PMID: 31903563.

\section{2}

Effect of different oxygen saturation targets on surgical site infection in patients admitted to surgical intensive care unit after abdominal surgery

L. Wang ${ }^{1}$; K. Yan ${ }^{1}$; W. Qin ${ }^{1}$

${ }^{1}$ Department of Critical Care Medicine, West China Hospital, Sichuan

University, Chengdu, China

\section{Correspondence: $L$. Wang}

Intensive Care Medicine Experimental 2021, 9(1): 001402

Introduction. Perioperative high fraction of inspired oxygen $\left(\mathrm{FiO}_{2}\right)$ was recommended in surgical patients to avoid surgical site infection (SSI) [1, 2]. It is now increasingly accepted that hyperoxemia have the potential to harm critically ill patients [3-5]. Generally, critically ill patients develop hyperoxemia as a result of liberal oxygen supplementation practices. In other acute medical conditions, oxygen should be administered if the pulse oximetry $\left(\mathrm{SpO}_{2}\right)$ is less than $92 \%$ and titrated to a target $\mathrm{SpO}_{2}$ range of $92 \%-96 \%$ [6]. There is still a paucity of studies describing the relationship between higher $\mathrm{SpO}_{2}$ targets with the decreased SSI occurrence in patients admitted to the surgical intensive care unit (SICU) after abdominal surgery.

Objectives. To explore whether a higher target $\mathrm{SpO}_{2}$ is associated with decreased SSI occurrence in patients admitted to the SICU after abdominal surgery.

Methods. We retrospectively extracted bedside continuous $\mathrm{SpO}_{2}$ monitoring data in the first $24 \mathrm{~h}$ for patients admitted to the SICU after abdominal surgery. For each eligible patient, continuous $\mathrm{SpO}_{2}$ monitoring data were processed at 30-s intervals. According to whether SSI developed, patients were assigned to the No-SSI or SSI group. The primary outcome was the percentage time and cumulative percentage of time spent at different $\mathrm{SpO}_{2}$ levels.

Results. A total of 79 patients were enrolled in this study; 14 patients developed SSI and were allocated to the SSI group and 65 patients without SSI were allocated to the No-SSI group. The mean (standard deviation) percentage of time for an $\mathrm{SpO}_{2}$ of $99 \%$ was $19.4 \%(18.3)$ in the No-SSI group and $20.7 \%(17.7)$ in the SSI group $(p=0.798)$. The mean (standard deviation) percentage of time spent with an $\mathrm{SpO}_{2}$ of $100 \%$ was $64.3 \%$ (31.7) in the No-SSI group and $55.4 \%(30.3)$ in the SSI group $(p=0.341)$. The median (interquartile range) cumulative percentage of time spent with an $\mathrm{SpO}_{2}>96 \%$ was $99.8 \%$ (98.5-100.0) in the No-SSI group and $99.3 \%(97.2-99.9)$ in SSI group $(p=0.136)$. A median $93.7 \%$ and $88.4 \%$ of time was at an $\mathrm{SpO}_{2} \geq 99 \%$ in the No-SSI and SSI groups, respectively $(p=0.119)$.

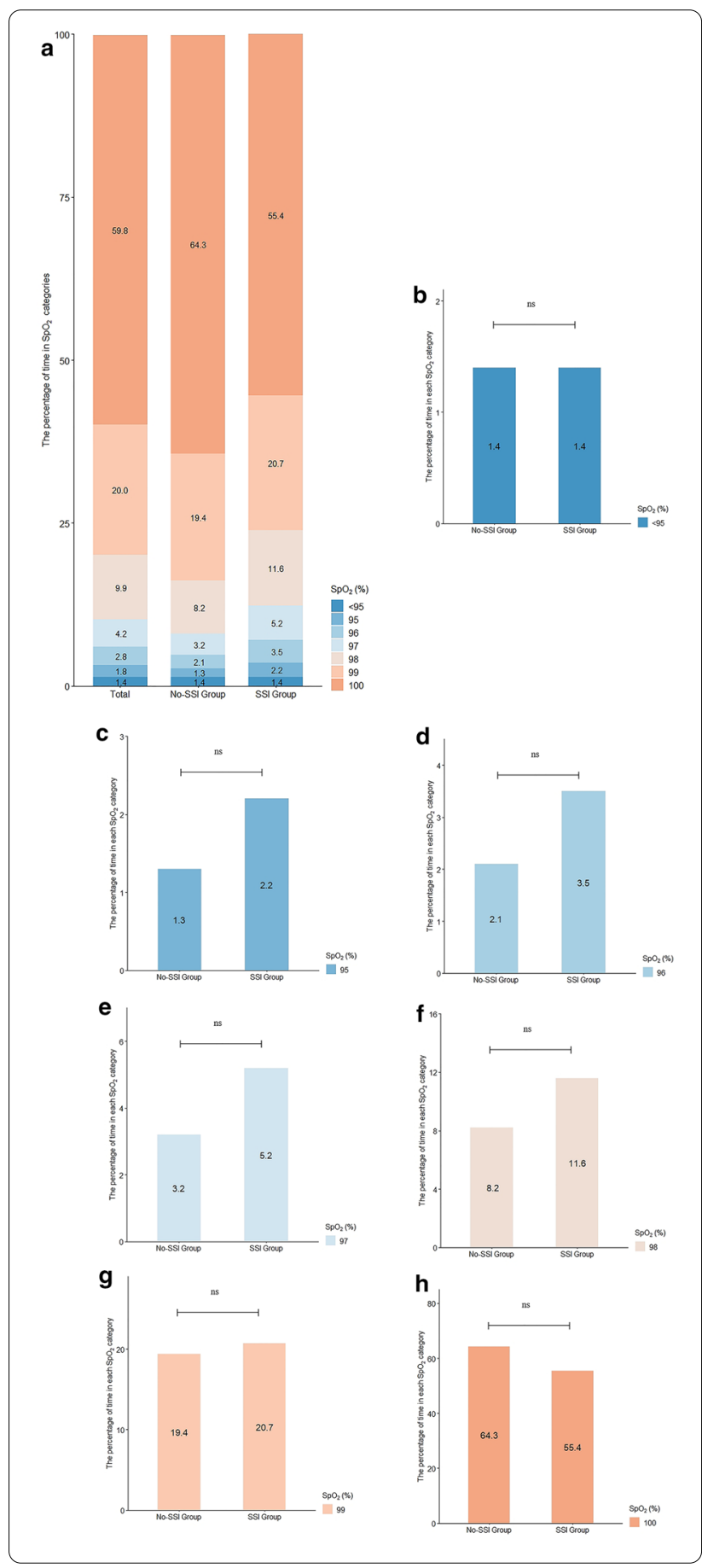

Conclusion. The percentage and cumulative percentage of time spent at higher $\mathrm{SpO}_{2}$ levels were not related to $\mathrm{SSI}$ reduction in patients admitted to the SICU after abdominal surgery.

\section{Reference(s)}

1. [1]. Berrios-Torres Sl, Umscheid CA, Bratzler DW, Leas B, Stone EC, Kelz RR, et al. Centers for Disease Control and Prevention Guideline for the Prevention of Surgical Site Infection, 2017. JAMA Surg. 2017;152(8):784-91. 
2. [2]. WHO Guidelines Approved by the Guidelines Review Committee. Global Guidelines for the Prevention of Surgical Site Infection. Geneva: World Health Organization (c) World Health Organization 2018; 2018.

3. [3]. Helmerhorst HJ, Schultz MJ, van der Voort PH, de Jonge E, van Westerloo DJ. Bench-to-bedside review: the effects of hyperoxia during critical illness. Crit Care. 2015;19(1):284.

4. [4]. Hedenstierna G, Meyhoff CS. Oxygen toxicity in major emergency surgery-anything new? Intensive Care Med. 2019;45(12):1802-5.

5. [5]. Martin DS, Grocott MP. Oxygen therapy in critical illness: precise control of arterial oxygenation and permissive hypoxemia. Crit Care Med. 2013:41(2):423-32.

6. [6]. Siemieniuk RAC, Chu DK, Kim LH, Guell-Rous MR, Alhazzani W, Soccal PM, et al. Oxygen therapy for acutely ill medical patients: a clinical practice guideline. BMJ. 2018;363:k4169.

7. The National Natural Science Foundation of China $(81801892,81901998)$

8. Sichuan Health Commission Research Project (19PJ237).

e-Posters: Poisoning/Toxicology/Pharmacology

\section{9}

Coagulation disturbances during tigecycline treatment in ICU patients

A. Tsimogianni ; C. Sklavou ${ }^{1}$; V. Panousi ; G. Katsagani ${ }^{2}$; G. Krimpeni ${ }^{1}$; S. Georgiou

${ }^{1}$ Intensive Care Unit, Saint Savvas Hospital, Athens, Greece; ${ }^{2}$ Intensive Care Unit, Saint Savvas, Athens, Greece

Correspondence: A. Tsimogianni

Intensive Care Medicine Experimental 2021, 9(1): 000089

Introduction. Tigecycline is a well tolerated, broad spectrum antibiotic with mainly gastro-intestinal side effects but with few reports about its effect on coagulation factors.

Objectives. To assess tigecycline effect on coagulation function in ICU patients.

Methods. From August 2020 to February 2021 all cases receiving tigecycline admitted to the ICU of Saint Savvas Hospital were identified. Inclusion criteria were: age above 18, treatment with tigecycline and absence of preexisting clotting abnormalities. INR, APTT, Fibrinogen, PLT and Creatinine were evaluated before and post treatment. Demographic data, SOFA and APACHE II scores and the bacterial strains isolated were recorded. Their short-term prognosis was also assessed.

Results.: Overall, 14 cases on tigecycline treatment were recorded aged from 19 to 83,8 of them males. Acinetobacter baumanii was isolated in 3 of the cases, Klebsiella pneumoniae in one, cocci in 3 cases and mixed infections in 6 samples. The Sofa score on admission was between 4 and 10, (mean 7.86) and APACHE II SCORE was between 9-26 ( mean 16.2). The INR and APTT were significantly prolonged during tigecycline treatment from 1.08 (SE 0.04) to 1.52 (SE 0.08) and from 33.13 (SE 1.41) to 48.49 (SE 3.61) respectively $(P<0.05)$ and INR improved post treatment, $P<0.005$. Fibrinogen decreased significantly from 606.71 (SE 65.28) to 222.64 (SE24.99) during treatment and improved post treatment, $p<0.05$. These changes occurred after 4-16 (mean 10.6) days of treatment. PLT and creatinine did not show any significant difference. 11 out of 14 patients survived and were discharged from the ICU to the ward (78.6\% survival), one remains in ICU and 2 patients died.

Conclusion. Although many parameters may affect clotting and PLT in ICU patients, tigecycline seems to have an independent significant but transient effect on coagulation. Clinicians should be aware of this effect and closely monitor INR, Fibrinogen and APTT in these patients, especially after the first week of treatment.
000272

Gradient-boosting machine learning model to predict average creatinine clearance of the next two days

CY. Huang ${ }^{1}$; M. Schetz ; J. Gunst ; M. Casaer ${ }^{1}$; G. Van den Berghe ${ }^{1}$; F. Güiza ${ }^{1}$; G. Meyfroidt ${ }^{1}$

${ }^{1}$ Laboratory of Intensive Care Medicine, Academic Department of Cellular and Molecular Medicine, KU Leuven, Leuven, Belgium

Correspondence: C.Y. Huang

Intensive Care Medicine Experimental 2021, 9(1): 000272

Introduction. Glomerular filtration rate (GFR) is frequently used to determine drug dosage, especially for hydrophilic drugs such as betalactam anti-microbials. GFR is often estimated in clinical practice by calculating creatinine clearance $(\mathrm{CrCl})$. Predicting $\mathrm{CrCl}$ could allow for a more pro-active drug dosing, avoiding sub-optimal drug plasma levels in acute kidney injury as well as hyper-clearance patients.

Objectives. The aim of this study was to develop and internally validate a prediction model for the average $\mathrm{CrCl}$ of the next two days, based on the routinely collected clinical data in the intensive care unit (ICU). The average $\mathrm{CrCl}$ of the next two days was chosen as predictive endpoint to ensure a more stable indication of the future renal function.

Methods. This study was based on the large EPaNIC multicenter randomized controlled trial database [1], which was split into a development and validation cohort. Here, we present the results of the development cohort only, consisting of 1054 adult ICU patients with available $\mathrm{CrCl}$ on the present day and the next two days. Clinical data were retrieved from the EPaNIC research database and from the clinical patient data management system database. $\mathrm{CrCl}$ was calculated by daily $24 \mathrm{~h}$ urine output (UO), urinary creatinine (UCr), and serum creatinine (SCr). The models were developed with a gradient boosting machine-learning algorithm. Model performance (Mean \pm SD) was internally validated with tenfold cross validation for coefficient of determination (R2), mean absolute error (MAE), and root mean square error (RMSE).

Results. Of the 1054 patients, equivalent to 9848 patient-days, who had $\mathrm{CrCl}$ on the present day and the next two days, 333 patients (31.59\%), equivalent to 1693 patient-days (17.19\%), had average $\mathrm{CrCl}$ of the next two days above $130 \mathrm{ml} / \mathrm{min} / 1.73 \mathrm{~m} 2$, a widely used cutoff for hyper-clearance definition. Fig. 1 depicts the internally validated model performance, where RMSE of $24.05 \pm 2.07$, MAE of $15.59 \pm 0.90$, and $\mathrm{R} 2$ of $0.80 \pm 0.03$ were reported. The five most predictive variables were mean $\mathrm{CrCl}, \mathrm{CrCl}$ of the previous day, age, $\mathrm{SCr}$ of the previous day, and UO of the previous day.

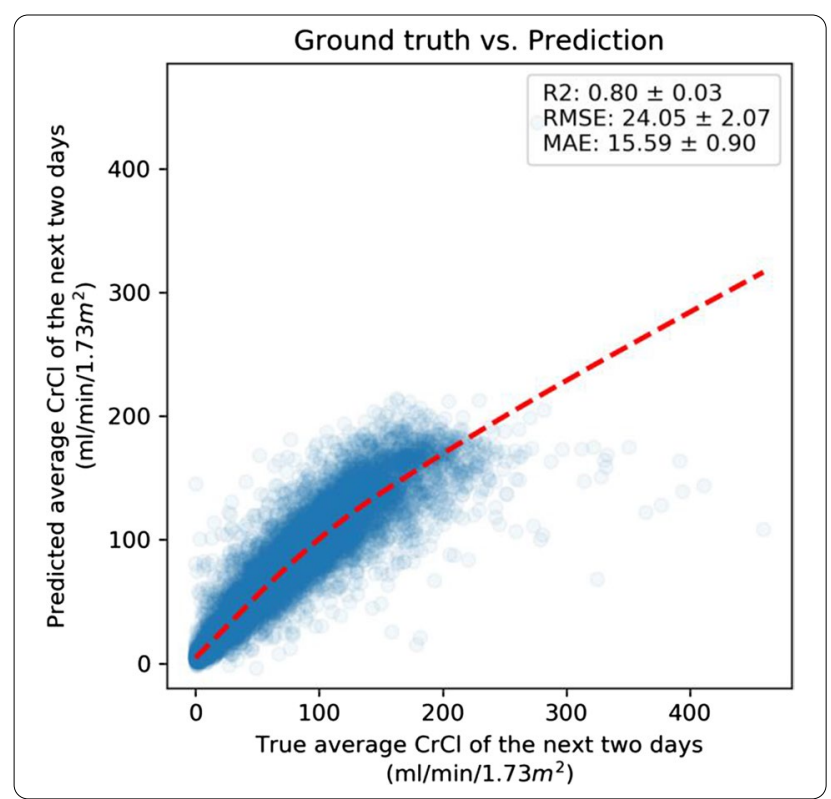


Conclusion. The average $\mathrm{CrCl}$ of the next two days can be accurately predicted on a daily basis during ICU stay, based on the clinical data routinely collected in the ICU. These predictions can be further incorporated into a pharmacokinetic model to support a more optimized dose regimen. The robustness of developed model will be examined in the external validation cohort.

\section{5}

ELISA for polymyxin B therapeutic drug monitoring in critically ill patients

Y. Surovoy'; M. Burkin²; I. Galvidis²; S. Tsarenko ${ }^{3}$

${ }^{1}$ Moscow State University, Moscow, Russia; ${ }^{2}$ Laboratory of Bioconjugates,

I. I. Mechnikov Research Institute for Vaccines and Sera, Moscow, Russia;

${ }^{3}$ Intensive Care, Moscow City Clinical Hospital № 52, Moscow, Russia

Correspondence: $Y$. Surovoy

Intensive Care Medicine Experimental 2021, 9(1): 000565

Introduction. Polymyxin B (PMB) is one of the most important antimicrobial agents in intensive care units with high prevalence of multidrug-resistant bacteria, often it is the only agent active against hospital-acquired microorganisms. Unfortunately PMB is characterized by a narrow therapeutic window and high toxicity. An easy method to measure PMB serum concentration and to study its pharmacokinetics in critically ill patients could increase therapy efficacy and safety.

Objectives. The main objective of this study was development of a PMB therapeutic drug monitoring method based on enzyme-linked immunoanalysis (ELISA) which is both precise and handy and could be used to study PMB pharmacokinetics in critically ill patients.

Methods. Specific rabbit anti-PMB antibodies were used to develop several formats of ELISA for PMB quantitative determination in human serum. This method was applied for preliminary pharmacokinetic study in 3 patients with sepsis, caused by A. baumannii and K. pneumoniae. These patients received $100 \mathrm{mg}$ or $125 \mathrm{mg}$ PMB twice daily. Blood samples were collected on the third day of therapy in 7 time-points between drug infusions.

Results. High sensitivity of assay allowed to determine PMB serum concentration with working range $1-100 \mathrm{ng} / \mathrm{ml}$ after simple $100-$ fold dilution. This obviated labor-intensive sample processing and reduced possible matrix effects.Comparison of PMB serum concentration data obtained by ELISAs and LC-MS/MS revealed good correlation $\left(r^{2}=0,82-0,99\right)$. Direct ELISA formats with coated antibodies and conjugated antigen decreased analysis run-time on $1 \mathrm{~h}(90 \mathrm{~min})$. This method was applied for preliminary pharmacokinetic study in 3 intensive care patients with sepsis. PMB exposition values in terms of AUC $_{0-24}$ were 107,165 and $72 \mathrm{mg}^{*} \mathrm{~h} / \mathrm{ml}$ and indicated high risk of toxicity in two patients (AUC $>100 \mathrm{mg} * \mathrm{~h} / \mathrm{ml}$ ). These preliminary data are suggestive of high individual PMB pharmacokinetics variability and highlight a point for PMB therapeutic drug monitoring application in critically ill patients.

Conclusion. The developed method for PMB serum concentration measurement is easy, precise and relatively fast $(90 \mathrm{~min})$ and might aid in clinical decision-making. This method will be used in further PMB pharmacokinetic studies in subgroups of critically ill patients with the most severe forms of disease.

\section{6}

\section{Addiction of patients in intensive care unit at the time} of the COVID19

H. Maamouri'; M. Fatnassi²; A. Ben Jazia'² N. Brahmi ${ }^{3}$

${ }^{1}$ Medical ICU, Hôpital Abderrahmen Mami de pneumo-phtisiologie,

Ariana, Tunisia; ${ }^{2}$ Intensive Care, Centre d'assistance médicale urgenteCAMU, Tunis, Tunisia; ${ }^{3}$ Intensive Care Unit, Centre d'assistance médicale
urgente-CAMU, Tunis, Tunisia

Correspondence: $\mathrm{H}$. Maamouri

Intensive Care Medicine Experimental 2021, 9(1): 000646

Introduction. The COVID 19 pandemic disproportionately disrupts the daily lives of people. Isolation, boredom and idleness promote addictive and worsen addiction in people suffering from psychoactive substance use disorder (PSD). The objective of our study was to discribe the socioeconomic profile, clinical charcteristics, complications and evolution of addicted patients hospitalized in intensive care uni at the time of the covid.

Methods. We conducted a prospective study during three months in our toxicological ICU from April to July 2020. We included all the patients admitted for any type of addiction and confirmed by toxic research.

Results. During the study 45 patients were included. They were male in $93 \%$, mostly single (82\%) with a mean age of $31.5 \pm 15.5$ [14-73] The level of study was primary school $(65 \%)$, middle $(8.8 \%)$, secondary school (17\%) and higher education ( $8.8 \%)$. Patients lived mainly in northeast $(53 \%)$. Twelve patients $(32 \%)$ lived with their siblings. The average number of people per household was $4.05 \pm 2.06$ [1-9]. The questioning of patients found an addiction to tobacco (55\%), buprenorphine $(6.6 \%)$,cannabis $(11 \%)$, trihexyphenidyle $(8.8 \%)$ and opiate poisoning in one case.The reason for hospitalization was mainly an overdose of PSD (98\%) and an endocarditis associated with respiratory distress in one case. Poisoning was secondary to ingestion of alcohol frelate $(53 \%)$ as well as overdoses with cannabis $(11 \%)$, buprenorphine (6.6\%), Datura strammonium (4.4\%) and extasy (4.4\%). Complications related to addiction were toxicoma withdrawal syndrome $(26.6 \%)$, hepatisis C and HIV $(4.4 \%)$, neuropathy retrobulbar optic (6.6\%), toxic leucoencephalopathy (4.4\%), bilateral blindess ( $2 \%)$, amputation of the upper limb (2\%), infectious endocarditis with septic localization (2\%), pneumithorax (2\%). Mechanical ventilation was required in $21 \%$ of cases and hemodialysis in $27 \%$ of cases. The mean lenght of stay in intensive care was $7.4 \pm 15.9$ days [1-94] with a mortality of $13 \%$.

Conclusion. The lack of social, sporting activities, isolation and confinement relating to the global health crisis due to COVID 19 has led the most fragile and poor people to the supply of PSD that lead to death or sequelae disabling.

\section{3}

Baclofen poisoning admitted in intensive care unit

H. Maamouri ${ }^{1}$; M. Fatnassi ${ }^{2}$; A. Ben Jazia ${ }^{2}$; G. Yosra ${ }^{3}$; N. Brahmi ${ }^{3}$

${ }^{1}$ Medical Icu, Hôpital Abderrahmen Mami de pneumo-phtisiologie,

Ariana, Tunisia; ${ }^{2}$ Intensive care, Centre d'assistance médicale urgente-

CAMU, Tunis, Tunisia; ${ }^{3}$ Intensive care unit, Centre d'assistance médicale

urgente-CAMU, Tunis, Tunisia

Correspondence: $\mathrm{H}$. Maamouri

Intensive Care Medicine Experimental 2021, 9(1): 000653

Introduction. Baclofen also named "Lioreseal» is a muscle relaxant used in spastic contractures during multiple sclerosis or tetanus. There was a renewed interest in baclofen in 2008 since the marketing of $\mathrm{Dr}$ Ameisen's book on its efects on alcohol withdrawal. In Tunisia Baclofen poisoing is not common but is unusual. The aim of this study was to describe the epidemiological characteristics of baclofen poisoning.

Methods. We coducted a retrospective study, descriptive, monocentric study that included patients admitted to Intensive care unit of the Mahmoud Yaacoub Center of Emergency Medical Assistance from January 2010 to Septemeber 2020 admitted for acute voluntary baclofen poisoning.

Results. During the period of the study, thirty two patients were included. The mean age was $28 \pm 12$ years [13-54]. There was a female predominance with a sex ratio of 0,33 . Fifteen patients $(47 \%)$ were treated with baclofen, three of whom were alcoholics. All the intoxications were related to a suicide attempt. The mean IGSII score was 28 [12-62]. An Agited coma was noted in seventeen patients (53\%) and two patients had a simple seizure that resolved on benzodiazepine. Four patients had hypothermia with a mean of $36.5^{\circ} \mathrm{C}$ of which one 
patient had Osborn J wave on electrocardiogram. Twelve patients initially presented with sinus bradycardia requiring atropine in seven of these patients. Seven patients presented with filing responsive shock $(22 \%)$, with need of vasoactive drugs in five patients (15\%). Twenty five patients $(78 \%)$ required mechanical ventilation. There was a significant correlation between supposedly ingested dose and coma, bradycardia, hypotension and hypothermia. The mean lenght of stay was 4 days [1-12] with a mean duration of mechanical ventilation of 2.5 days $[1-5]$. There were no cases of death.

Conclusion. Baclofen poisoning is rare but remain serious if management is not optimized. There is a correlation between the supposedly ingested dose and clinical symptoms.

\section{2}

\section{AMINOPHYLLINE IN THE ICU—an old drug with many tricks!}

\section{S. Gowda S'; IM. Dr'ㄹ. DD. Dr ${ }^{3}$}

1 Junior Consultant, Critical Care Medicine, Bangalore Baptist Hospital, Bengaluru, India; ${ }^{2}$ Head of the Department, Critical Care Medicine, Bangalore Baptist Hospital, Bengaluru, India; ${ }^{3}$ Consultant Intensivist, Bangalore Baptist Hospital, Bengaluru, India

Correspondence: S. Gowda S

Intensive Care Medicine Experimental 2021, 9(1): 000662

Introduction. Aminophylline, the ethylene diamine salt of theophylline is a 50 year old established bronchodilator. It has fallen out of popularity with the advent of newer drugs with lesser side effects, due to its drug interactions, the lack of use, absence of basic discussion in teaching centers and the misconceptions due to limited knowledge in its use. Also its low cost and enhanced marketing of newer agents is also a contributory factor.

Aminophylline, in addition to its effect on airway has been demonstrated to have other beneficial effects like renoprotection, diuresis, anti-inflammatory action and used in weaning difficulties. But the data to support this is scant, especially in critically ill adult patients.

Objectives. We therefore conducted a prospective, observational, single arm study in a tertiary care hospital to assess the efficacy of aminophylline as a renoprotective and anti-inflammatory agent in critically ill adult patients.

Methods. All patients admitted to the adult ICU who were prescribed aminophylline for severe bronchospasm over a $24 \mathrm{~h}$ period were included in the study. The use and dosing of aminophylline infusion was at the discretion of the treating intensivist (no loading dose was given to any patient and maximum infusion dose did not exceed $100 \mathrm{mcg} / \mathrm{kg} / \mathrm{hour}$ in any patient) Patients with h/o xanthine allergy, ongoing arrhythmias, liver disease, seizure disorder, chronic kidney disease/ acute kidney injury on haemodialysis, patients who received diuretics, newer antibiotics or steroids during the $24 \mathrm{~h}$ aminophylline infusion were excluded from the study.

Data was collected at baseline prior to the administration of aminophylline, and then again $24 \mathrm{~h}$ after the initiation of therapy.

Collected data consisted of demographics, diagnosis, medications and vital signs. In addition, data was obtained to assess the antiinflammatory and renoprotective effects of aminophylline.

RENAL FUNCTION: S.creatinine at baseline and $24 \mathrm{~h}$ post aminophylline infusion along with $24 \mathrm{~h}$ urine output.

ANTI-INFLAMMATORY: CRP, total leukocyte count at baseline and $24 \mathrm{~h}$ post infusion.

Results. A total of 53 patients were included in the study. Wilcoxon signed rank test was performed to compare baseline values with post aminophylline infusion values. There was significant improvement in terms of renoprotective effects, with increase in urine output $(p=0.0001)$ and decrease in serum creatinine levels $(p=0.016)$. CRP levels were significantly lower $(p=0.006)$ compared to preinfusion values, but the change in total white cell counts was not significant $(p=0.235)$. Side effects of aminophylline were detected in 12 out of 53 patients, with tachycardia being the most common side effect.

Conclusion. Aminophylline demonstrates renoprotective, diuretic and anti-inflammatory effects in critically ill adult patients. Intensivists should definitely consider reinstating aminophylline to their ICU drug formulary and it should not be shelved as an archaic agent.

\section{Reference(s)}

1. 5. Tamburro R, Thomas N, Ceneviva G, Dettorre M, Brummel G, Lucking S. A Prospective Assessment of the Effect of Aminophylline Therapy on Urine Output and Inflammation in Critically III Children. Frontiers in Pediatrics. 2014;2

2. 4. Axelrod D, Sutherland S, Anglemyer A, Grimm P, Roth S. A DoubleBlinded, Randomized, Placebo-Controlled Clinical Trial of Aminophylline to Prevent Acute Kidney Injury in Children Following Congenital Heart Surgery With Cardiopulmonary Bypass*. Pediatric Critical Care Medicine. 2016:17(2):135-143.

3. 3. RuDusky B. Aminophylline: Exploring Cardiovascular Benefits Versus Medical Malcontent. Angiology. 2005:56(3):295-304.

4. 2. Park K, Trout L, Xu C, Wang M, Tamburro R, Halstead E. No Requirement for Targeted Theophylline Levels for Diuretic Effect of Aminophylline in Critically III Children. Pediatric Critical Care Medicine. 2018;19(8):e425-e432

5. 1. Parker M, Willatts S. A pilot study to investigate the effects of an infusion of aminophylline on renal function following major abdominal surgery. Anaesthesia. 2001;56(7):670-689

6. NIL.

\section{2}

Interest of the protocolized weaning test of mechanical ventilation during toxic coma

S. Amri' ; A. Ben Jazia '; R. Fadhel' ; M. Fatnassi ${ }^{1}$; H. Maamouri ; N. Brahmi ${ }^{1}$ ${ }^{1}$ Intensive Care, Centre d'assistance médicale urgente-CAMU, Tunis,

Tunisia

Correspondence: A. Ben Jazia

Intensive Care Medicine Experimental 2021, 9(1): 000682.

Introduction. The protocolized mechanical ventilation withdrawal test is a recommended step in the weaning and extubation process for ventilated patients in intensive care unit (ICU). It makes it possible to reveal latent causes of failure and thus prevent the consequent excess mortality. Patients admitted to ICU for toxic reasons requiring mechanical ventilation represent a special population due to a lower incidence of co-morbidities and often short ventilation time $(<72 \mathrm{~h})$.

Objectives. Our objective was to assess the value of protocolizing the procedure in this population.

Methods. This was a prospective, monocentric and evaluative study, conducted during the year 2020, from June to December in the Toxicological ICU Department. Patients admitted ICU and ventilated for toxic reasons who met the criteria for extubation before $72 \mathrm{~h}$ of ventilation were randomised into two groups: one group received a protocolized withdrawal (switching to VS-AIDE ZEEP for $30 \mathrm{~min}$ ) and one group received a non-protocolized withdrawal based on basic clinical parameters only (neurological status, blood pressure, pulse and pulsed $\mathrm{O} 2$ saturation). The main judging criterion was the success of extubation and discharge from intensive care without sequelae.

Results. Fourty three patients were enrolled during the study period. Mean age was $31( \pm 10)$ and sex ratio was 0.8 . Mean severity scores were $24( \pm 3)$ [16-28] et $9( \pm 2)[4-14]$ for IGS II and Apache II, respectively. Patients are followed by psychiatry in $53.5 \%$ of cases, diabetic and hypertensive in $5(9.3 \%)$. Chloralose was the most common toxicant (34. 9\%) followed by carbamazepine (11.2\%). The majority (92\%) of patients were sedated with Propofol. Neurological distress was the main indication of ventilation $(100 \%)$, respiratory distress was associated in 4 cases (10\%). The two groups were homogeneous in terms of age, sex, and medical history $(p>0.05)$. The two withdrawal protocols were similar in this population of patients ventilated for toxic reasons for a period not exceeding $72 \mathrm{~h}(\mathrm{P}=1)$.

Conclusion. This study suggests that there is no problem with ventilatory weaning in patients ventilated for toxic reasons for a short period not exceeding $72 \mathrm{~h}$. 
001201

The effect of intermediate tinzaparin thromboprophylaxis on Anti-Factor Xa levels in critically ill patients with COVID-19 early in the pandemic

E. London" ; DO. Hamilton ${ }^{2}$; A. Waite, ${ }^{2}$; I. Welters, ${ }^{2}$; N. Robin, ${ }^{1}$; S. Ridler ${ }^{1}$

${ }^{1}$ Critical Care, Countess of Chester Hospital NHS Foundation Trust, Chester, United Kingdom; ${ }^{2}$ Critical Care, Royal Liverpool and Broadgreen University Hospitals NHS Trust, Liverpool, United Kingdom

Correspondence: D.O. Hamilton

Intensive Care Medicine Experimental 2021, 9(1): 001201

Introduction. There is a high prevalence of venous thromboembolism (VTE) in critically ill patients with COVID-19 [1]. This has led a number of European guidelines to suggest using an 'intermediate' dose for thromboprophylaxis based on expert opinion [1]. Monitoring anti-Factor Xa (anti-FXa) levels has previously been suggested for patients on the Intensive Care Unit (ICU) as sub-therapeutic levels can be common in critical illness [2].

In this study we retrospectively examined the anti-FXa levels achieved by 'intermediate' dose tinzaparin administration in patients on ICU with COVID-19 early in the pandemic.

Methods. All patients admitted to ICU with COVID-19 who had an anti-FXa level sent whilst on thromboprophylaxis were included and data were collected from health records. The study period was between March 4th and April 24th 2020. Given the observation of a high number of thrombotic events early in the pandemic, a twicedaily dosing regimen for subcutaneous tinzaparin administration was implemented, based on weight and renal function, rather than once per day typically used for patients without COVID-19. Peak anti-FXa levels were taken four hours after the third dose on admission or after dose changes. A level of $0.2-0.4 \mathrm{iU} / \mathrm{ml}$ was accepted.

Results. Twelve patients were included in the study time period. Six patients were male, mean age was $60.8 \pm 9.1$ and mean APACHE II score was $15.4 \pm 3.9$. Seven patients were ventilated and two received renal replacement therapy. ICU mortality was 33.3\%.

Mean tinzaparin dose was $9083 \pm 2382$ units and first anti-FXa levels were 0.12 (IQR 0.10-0.24). Only four (33.3\%) patients had anti-FXa levels in range on first measurement. Five patients later achieved adequate anti-FXa levels after dose increments and the mean dose to achieve this was $10,300 \pm 2387$ units. Figure 1 below shows antiFXa levels by daily tinzaparin dosing throughout admission. Seven patients were later anticoagulated for confirmed or presumed VTE. There was no correlation between D-dimer levels and anti-FXa levels. There were no significant bleeding events during the study period.

Conclusion. These results suggest that even intermediate dosing of tinzaparin may be inadequate to achieve target anti-FXa levels thereby leaving patients susceptible to developing VTE. This phenomenon may be due to systemic inflammation or specific COVID-19-induced activation of the clotting system.

However, whether titrating doses to achieve target levels reduces VTE rates or improves clinical outcomes is yet to be established and the optimal dose for thromboprophylaxis, particularly in patients now commonly receiving immunomodulation, requires further study.

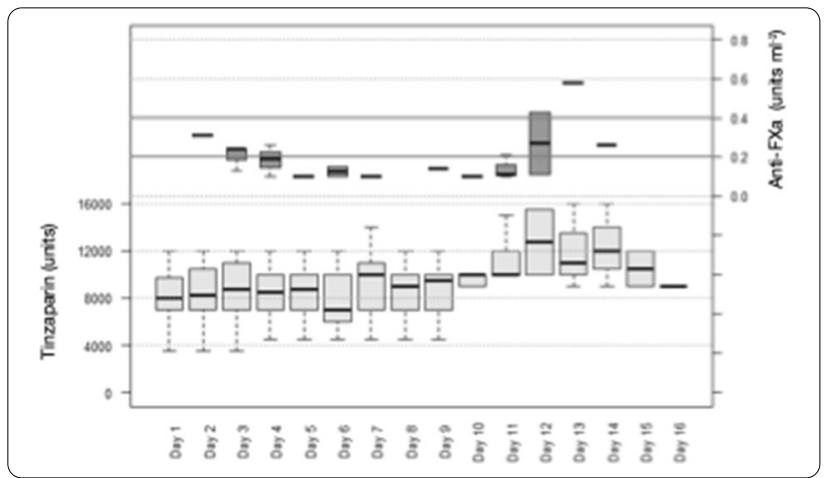

Figure 1
Reference(s)

1. 1. Waite AAC, Hamilton DO, Pizzi R et al. Hypercoagulopathy in Severe COVID-19: Implications for Acute Care. Thrombosis Haemostasis 2020;120:1654-1667

2. 2. Robinson S, Zincuk A, Strøm T, et al. Enoxaparin, effective dosage for intensive care patients: double-blinded, randomised clinical trial. Critical Care 2010; 14(2): R41.

3. Nil

001229

Diagnosis and management of anaphylaxis in toxicological intensive care unit

R. Fadhel ${ }^{1}$; A. Ben Jazia ${ }^{1}$; S. Amri'; A. Smiri' ${ }^{2}$ H. Maamouri ${ }^{1}$; N. Brahmi ${ }^{1}$

${ }^{1}$ Intensive Care, Centre d'assistance médicale urgente-CAMU, Tunis,

Tunisia; ${ }^{2}$ Intensive Care, CAMU, Tunis, Tunisia

Correspondence: A. Ben Jazia

Intensive Care Medicine Experimental 2021, 9(1): 001229

Introduction. Anaphylaxis is a potentially life-threatening allergic reaction.The identification of potential triggers and risk factors may improve the outcome of this entity. However, few data are available on patients who have previously experienced anaphylaxis and were admitted to intensive care unit (ICU).

Objectives. The objective of this study is to describe the epidemiological, clinical features, management and outcome of patients with anaphylaxis in ICU.

Methods. Prospective, observational study including patients over the age of 14 who were admitted to toxicological ICU between 2007 and 2019 for anaphylaxis. Collection of epidemiological, clinical and therapeutic parameters.

Results. Inclusion of 134 patients with a mean age of $42 \pm 17$ years and sex ratio $=1.3$. History of alleric reaction and atopic disease were noted in 27 patients (20\%). The different allergenic components and triggers were n (\%): drugs 75 (56), hymenoptera sting 28 (24.6), recent food 31(23). Clinical signs n (\%): cutaneous manifestations 98 (86), cardiovascular signs 40 (35), respiratory signs 46 (40.4), digestive signs 18 (15) and neurological signs 15 (13.2). An anaphylactic shock was recorded in 44 patients (38.6\%). Adrenaline was used in 49 patients (43\%). Fluid resuscitation was given to 52 patients (45.6). Corticosteroids and antihistamines were prescribed respectively in $89.5 \%$ and 82.5 of patients. Three patients were intubated.Two patients had cardiac arrest and one death was recorded.

Conclusion. Our findings reflect the need for standardized anaphylaxis criteria among physicians treating anaphylaxis as well as the implementation of anaphylaxis guidelines regarding diagnosis and treatment.

\section{3}

Paracetamol Induced Acute Liver Failure in a Tertiary ICU and National Liver Transplant Centre: Prognostic Factors and Outcomes

J. Kennedy ${ }^{1}$; Y. Besharatian²; A. Haren²; S. Nair, ${ }^{1}$

${ }^{1}$ Intensive Care Unit, St Vincent's University Hospital, Dublin, Ireland:

2Intensive Care Unit, St Vincent's University Hospital, Dublin, Ireland

Correspondence: A. Haren

Intensive Care Medicine Experimental 2021, 9(1): 001303

Introduction. Acute liver failure (ALF) in Europe is most frequently drug-induced in nature. Due to its accessibility, paracetamol is the most commonly overdosed medication in the USA and accounts for $57 \%$ of ALFs in the UK. Treatment includes supportive care, N-acetylcysteine (NAC) and orthopic liver transplantion (OLT) based on fulfilment of the King's College Criteria.

Objectives. We aimed to examine the associations of paracetamol induced ALF in ICU patients with predictive values, in order to improve future management.

Methods. Our cohort included 91 patients admitted to ICU from 2010-2020. Relevant data was retrospectively analysed, including laboratory values, psychiatric comorbidities, dose ingested and SOFA scores pertaining ICU paracetamol toxicity admissions. We compared 
these with outcomes including ventilation and dialysis requirement, OLT and death. Figures reflect parameters recorded at the time of ICU admission. Data was collected and analysed using Microsoft Excel.

Results. The average age amongst our cohort was 37. Mean ingested dose was $34 \mathrm{~g} .17 \%$ of overdoses were accidental. $79 \%$ of patients suffered from psychiatric comorbidities. $62 \%$ of patients survived $(25 \%$ post OLT and $37 \%$ with NAC/supportive care alone). Whilst $80.2 \%$ of patients required ventilation and $71.4 \%$ required CRRT. SOFA score and fulfilment of the King's College Criteria served as a reliable prognostic indicator, as demonstrated in Table 1.

\begin{tabular}{lcll}
\hline Table 1 & Supportive Only & OLT & RIP \\
\hline LACTATE & 3.6 & 7 & 9.5 \\
$\mathrm{pH}$ & 7.34 & 7.3 & 7.2 \\
INR & 4.07 & 6.8 & 7.7 \\
CREATININE & 149.13 & 206 & 237 \\
SOFA & 7.85 & 10.42 & 12.6 \\
\hline
\end{tabular}

Conclusion. Paracetamol induced ALF remains a significant burden on the health service. The King's College Criteria components and SOFA scores accurately predicted negative outcomes in patients with paracetamol induced ALF, with SOFA scores $62 \%$ higher in those who died versus those who received supportive care only. This highlights importance of early clinical examination, biochemical investigations and severity score calculations to improve timely diagnosis and appropriate management of paracetamol induced ALF.

\section{Reference(s)}

1. Kuffner E, Heard K, O'Malley G, Management of Acetaminophen Toxicity in the Intensive Care Unit, Journal of Intensive Care Medicine 14 (4), 157-165

2. Wendon J, Cordoba J, Dhawan A et al., EASL Clinical Practice Guidelines on the management of Acute (fulminant) Liver Failure, Journal of Hepatology, 66 (5), 1047-1081

e-Posters: Sedation, analgesia and delirium

\section{5}

Does sedation for interhospital transfer present an unacceptable risk of accidental awareness under anaesthesia?

N. Plummer'; K. James²; L. Hodierne'; G. Gibbon, '; M. Barley²

${ }^{1}$ Critical Care, Queen's Medical Centre Nottingham, Nottingham, United Kingdom; ${ }^{2}$ Anaesthesia, Queen's Medical Centre Nottingham, Nottingham, United Kingdom

Correspondence: N. PlumSmer

Intensive Care Medicine Experimental 2021, 9(1): 000055

Introduction. Interhospital transfer of the critically ill adult frequently involves sedation to facilitate ventilation, with neuromuscular blockade (NMB) often employed to assist this. Although NAP5[1] highlighted the increased risk of accident awareness (AAGA) with fixed-rate propofol infusions, and AAGBI guidelines for transfer[2] and use of total intravenous anaesthesia [3] recommend using depth of anaesthesia (DOA) monitors and target controlled infusions (TCl) to ensure appropriate sedation for transfer, their use in transfer persists.

Methods. We retrospectively reviewed all interhospital transfers of mechanically ventilated patients in the our local Critical Care Network from Oct 17 to Feb 19 (most recent complete dataset) to review regional practice. Where NMB was not explicitly documented, two authors assessed independently the likelihood of its use based on ventilatory mode and clinical indicators.

Results. 225 transfers were reviewed. NMB was documented or highly suspected in $70 \%$. Fixed-rate propofol infusions were used in $83 \%$ of these, and as a single agent in $43 \%$. Neither TCI nor DOA monitoring were employed on any transfer. Mean propofol dose where NMB was employed was $17.4 \pm 9.3 \mathrm{ml} / \mathrm{h}$, and was unadjusted in brain-injured $(22 \%)$ or cardiac $(14 \%)$ patients, or when used alongside another anaesthetic agent. There was a weak association of reduced dosing with increasing patient age $(p=0.021)$, and no association between propofol dose and vasopressor requirements $(p=0.770)$. Increasing dose was associated with a reduced heart rate $(p=0.035)$ but unassociated with changes in MAP $(p=0.059)$.

Plotting physiological variables against propofol dose highlights a cohort of undersedated, hypotensive patients; and another with abnormally high heart rates and mean arterial pressure suggesting physiological features of awareness.

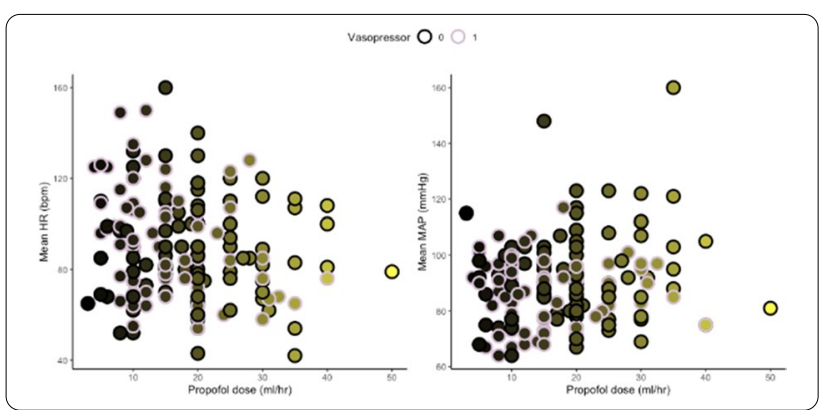

Conclusion. Routine use of NMBs and fixed-rate propofol infusions during interhospital transfer is common. Doses used equate to a Cet of $1.4 \mathrm{mcg} / \mathrm{ml}$ [4], which is insufficient to prevent waking in the average adult. That similarly low infusion rates were utilised irrespective of patient factors or co-infusions suggest transferring doctors may not take these into account during the transfer of critically-ill, paralysed patients, nor recognise patients at risk of awareness. It's also apparent that recommended standards of care and monitoring for TIVA are not applied when it is used outside of the operating theatre.

\section{Reference(s)}

1. 1. Pandit, et al. 5th National Audit Project (NAP5) on accidental awareness during general anaesthesia. BJA 2014;113:549-559

2. 2. Nathanson, et al. Guidelines for the safe transfer of the brain injured patient: trauma and stroke, 2019. Anaesthesia 2020;75:234-246

3. 3. Nimmo, et al. Guidelines for the safe practice of total intravenous anaesthesia (TIVA). Anaesthesia 2018;74:211-224

4. 4. Eleveld, et al. Pharmacokinetic-pharmacodynamic model for propofol for broad application in anaesthesia and sedastion. BJA 2018;120:942-959

\section{4}

Does rocking motion calm delirious patients in ICU? A multicenter randomized clinical trial (RockingICU)

M. Collet'; MN. Gitte' ${ }^{2}$; B. Benita ${ }^{3}$; L. Eva ${ }^{4}$; F. Susanne ${ }^{5}$; L. Anne ${ }^{3}$;. Linette ${ }^{6}$; L. Theis $^{7} ;$ I. Egerod ${ }^{8}$

${ }^{1}$ \{street_address\}, Copenhagen, Denmark; ${ }^{2}$ Intensive Care, Rigshospitalet, Copenhagen, Denmark; Intensive Care, Rigshospitalet, København, Denmark: ${ }^{4}$ Intensive Care, Odense University Hospital, Odense, Denmark; ${ }^{5}$ Intensive Care, Sydvestjysk Sygehus, Esbjerg, Denmark; ${ }^{6}$ Intensive Care, Skejby Sygehus, Aarhus, Denmark; ${ }^{7}$ Public Health, University of Copenhagen, Copenhagen, Denmark; ${ }^{8}$ Department of Neuroanaesthesiolgoy, Rigshospitalet, København, Denmark

Correspondence: M. Collet

Intensive Care Medicine Experimental 2021, 9(1): 000084

Introduction. Rocking chair therapy has been explored in patients with dementia to promote the feeling of relaxation (1). To our knowledge, no study has evaluated the effect of rocking chair therapy in critical ill patients admitted to an Intensive Care Unit (ICU) with delirium.

Objectives. We hypothesized that delirious patients receiving rocking chair therapy would have fewer days with delirium and coma and improve 1 level closer to 0 in the Richmond Agitation and Sedation Scale (RASS) before and after rocking chair therapy. The aim is to investigate the effect of a therapeutic rocking chair with or without rocking motion on the duration of delirium and intensity of agitation in critically ill patients admitted to the ICU. 
Methods. The RockingICU trial is an investigator-initiated, pragmatic, multicentre, parallel-grouped, centrally randomised, stratified, dataanalyst-blinded trial with adequate allocation sequence generation and allocation concealment.

We are including patients for 1:1 web-based randomisation, stratified by site in patients 18 years or older with a positive delirium score identified by a validated delirium screening tool. We are excluding patients mainly due to mobilisation restriction, body weight exceeding $130 \mathrm{~kg}$, inability to provide consent, and presence of multi resistant bacteria or viral droplet infections.

The trial period is the time spent in the ICU from randomisation to a maximum of two-weeks. The intervention is a rocking chair therapy and patients allocated to the intervention group will receives daily minimum 20 min of rocking therapy. The integrated music option will be turned on, unless the patient declines music. The program used is "Refresh", which is recommended for power napping and to dampen arousal. If the patients are comfortable in the rocking chair or have fallen asleep, the program can be repeated for an additional 20 min. Patients allocated to the control group will be transferred to the rocking chair, but the rocking motion and music will not be activated.

The primary outcome measure is number of days alive without coma and delirium in the two-week follow-up period. The secondary outcome measures are: number of days alive and without life support within two-weeks follow-up, length of stay in the ICU, daily RASS, the delta RASS before and after the intervention, number of patients that received benzodiazepines, antipsychotics or continues infusion of sedatives when in the rocking chair (both intervention and control), number of times the interventions was discontinued, number of patients that received other treatment against delirium and the nurses 'estimate of the patients experiences of comfort while in the rocking chair. The general plan is to perform analysis on the Intention-to-treat (ITT) population as well as per-protocol population (PPP). The primary conclusion of the trial will be based on the ITT analysis. The PPP is defined as randomised participants except those having one or major protocol violations, such as eligible patients not receiving the allocated intervention $>2$ days, ineligible patients receiving the allocated intervention $>2$ days (as delirium negative) or ineligible patients withdrawn from the intervention. A power calculation with a risk reduction of $20 \%$, a power of $80 \%$ with an alpha cut-off on $5 \%$ and further $20 \%$ inclusion gives 76 patients in intervention and control group reaching a total of $n=152$ inclusion in the trial.

The trial is registered at clincialtrials.gov (NCT04401514) and at The Capital region of Denmark ethical committees $(\mathrm{H}-180556774)$ and the Danish Data Protection Agency (P-2020-114). We are including patients after the achievement of consent for participation according to national regulations.

Results. Progress: By March 24th, 202157 patients have been included. Seven of eight sites have been initiated and the eight will start including patients by the beginning of April.

Conclusion. Expected outcomes: The RockingICU trial will provide important new knowledge and raise research questions regarding nonpharmacological interventions to alleviate delirium in ICU patients.

\section{Reference(s)}

1. Snyder M, Tseng YH, Brandt C, et al. A glider swing intervention for people with dementia. Geriatr Nurs (Minneap). 2001;22(2):86-90.

\section{5}

Sedoanalgesia protocol and delirium in patients admitted with COVID-19-associated acute respiratory distress syndrome (ARDS): initial experience in a medical Intensive Care Unit (ICU) in a secondary-level university Hospital

JL. Martinez Melgar'; E. Moreno Lopez²; I. Gallego Barbachano'; A. Ortega Montes ${ }^{1}$; E. Sanmartin Mantiñan'; A. De La Campa Gestido'; J. Casal Lorenzo'; J. Cenoz Osinaga'; T. Sanchez De Dios ${ }^{1}$; P. Posada Gonzalez ${ }^{1}$ Intensive Care Unit, Complexo Hospitalario Universitario de Pontevedra, Pontevedra, Spain; ${ }^{2}$ Anesthesia and Perioperative Care, Complexo Hospitalario Universitario de Ferrol, Ferrol, Spain

Correspondence: E. Moreno Lopez

Intensive Care Medicine Experimental 2021, 9(1): 000285
Introduction. In December 2019, a novel coronavirus (SARS-CoV-2) causing an acute respiratory distress syndrome (ARDS) emerged in China, and spread throughout the world, becoming a pandemic.

Objectives. To describe our experience with a sedoanalgesia protocol and delirium in COVID-19-associated acute respiratory distress syndrome patients admitted to a medical ICU in a secondary-level university Hospital.

Methods. Retrospective and descriptive study carried out in MarchApril 2020 in patients admitted with COVID-19-associated ARDS Parameters analyzed: gender, age range, comorbidities, severity scoring systems, deep sedoanalgesia strategie (DS) with bispectral index monitoring (BIS) in patients with moderate or severe ARDS, requiring prone positioning or neuromuscular blockade (NMB), and dynamic sedoanalgesia strategie (DyS): superficial level of sedation using Richmond Agitation Sedation Scale (RASS) (objective: - 2 and +1 ). Deep sedoanalgesia strategie: initially midazolam (MDZ) + fentanyl (FNT) + Propofol (PF) (if MDZ dosage $>0.25 \mathrm{mg} / \mathrm{kg} / \mathrm{h}$ or tolerance) + ketamine (KET) (if PF dosage $>4 \mathrm{mg} / \mathrm{kg} / \mathrm{h}$ ) and/or continuous NMB. Dynamic sedoanalgesia strategie: initially PF+FNT or $\mathrm{PF}+$ remifentanil (RF) (if renal failure or $\mathrm{BMI}>30 \mathrm{~kg} / \mathrm{m}^{2}$ ) or PF + KET (if hemodynamic instability). Management of delirium/agitation (D\&A): dexmedetomidine (DEX) + PF or DEX + RF or alpha-2 adrenergic receptor agonists (a-2 A): DEX o clonidine (CL) or neuroleptics (Nr).

Results. 22 patients were included (19 male/ 3 female), mean age: $67.5 \pm 6.1$ years old $(<60: 4,60-70: 13(59 \%),>70: 7)$. APACHE II: $26 \pm 7$, SOFA: $7.5 \pm 1.9$. Comorbidities: COPD:4, obesity(BMI $>30 \mathrm{~kg} /$ $\mathrm{m}^{2}$ ): 5 (22.7\%), hypertension: 13(59\%), diabetes: 4. DS strategie required using MDZ+FNT: 22 patients, MDZ+FNT+PF: 18 and $\mathrm{MDZ}+\mathrm{FNT}+\mathrm{PF}+\mathrm{KET}$ : 15. NMB was needed in 14 patients (cisatracurium: 10/ rocuronium: 4). In DyS strategie PF+FNT was used in 11 patients, PF + KET in 15 and PF + RF in 15. In terms of management of D\&A: DEX+PF: 7, DEX + RF: 11, a-2 A: 11 (DEX: 7 and CL: 4) and Nr: 11 (typical: 7 and atypical: 4 ).

Conclusion. Deep sedation strategie using MDZ + FNT was used in $100 \%$ and $\mathrm{MDZ}+\mathrm{FNT}+\mathrm{PF}$ in $82 \%$, needing neuromuscular blockade $64 \%$. Dynamic sedation strategie with $\mathrm{PF}+\mathrm{RF}$ was used in $68 \%$, as well as PF + KET. For management of delirium and agitation, the medication used was DEX + RF: $50 \%$ and $a-2$ A: $50 \%$

\section{3}

The impact of late onset delirium on critical care length of stay L. Dearden ; A. Parker, ; J. Hanison, ${ }^{1}$; A. Wilson,

${ }^{1}$ Adult Critical Care, Manchester Royal Infirmary, Manchester, United Kingdom; ${ }^{2}$ Critical Care, Manchester Royal Infirmary, Manchester, United Kingdom

\section{Correspondence: L. Dearden}

Intensive Care Medicine Experimental 2021, 9(1): 000393

Introduction. Delirium is a common problem in critical care and has been reported in nearly one third of patients during their critical care admission(1). Delirium has been observed to increase length of stay(1) and cost of stay(2), is an independent risk factor for mortality(1) and increases the risk long term cognitive impairment(3). Less is known about the impact of the time of onset of delirium and the impact this has on ICU stay.

Objectives. To assess the impact of late onset versus early onset delirium on length of stay in a large general adult Intensive Care Unit (ICU). Methods. We reviewed electronic patient records of all patients admitted to our general adult critical care unit between 1st January 2017 and 31st December 2020. Our case mix is a combined medical and surgical unit.

Data was extracted from the IntelliSpace critical care information system (Philips NC, Netherlands) which is used for all clinical documentation in our ICU. We extracted data for the CAM-ICU delirium screen which is used routinely in our unit as well as length of stay data.

We categorised patients with a positive delirium screen according to whether delirium was identified during the first 3 days (early onset) or after day 3 (late onset), to distinguish those whose presentation 
included delirium from those who developed delirium whilst in the ICU.

Results. We reviewed 8179 patient episodes. 1179 patients (14.4\%) had a positive CAM-ICU assessment on at least one occasion. Of these, $681 / 1179$ patients $(57.8 \%)$ were identified with early onset delirium and $498 / 1179(42.2 \%)$ were identified with late onset delirium. ICU length of stay for patients with late onset delirium was significantly increased compared to those with early onset delirium $(p<0.001)$. Length of stay was $24.5( \pm 22.0)$ days in this group. This was more than twice the length of the mean ICU stay for all patients with potential to develop late onset delirium (i.e. length of stay greater than 3 days).

\begin{tabular}{lllll}
\hline & $\begin{array}{l}\text { All patients } \\
(\mathbf{n}=\mathbf{8 1 7 9})\end{array}$ & $\begin{array}{l}\text { All patients } \\
\text { with } \\
\text { length of } \\
\text { stay }>\mathbf{3} \text { days } \\
(\mathbf{n}=\mathbf{3 6 7 9 )}\end{array}$ & $\begin{array}{l}\text { Early Onset } \\
\text { Delirium } \\
(\mathbf{n}=\mathbf{6 8 1})\end{array}$ & $\begin{array}{l}\text { Late Onset } \\
\text { Delirium } \\
(\mathbf{n}=\mathbf{4 9 8})\end{array}$ \\
\hline $\begin{array}{l}\text { Mean } \\
\text { length of } \\
\text { stay (days) }\end{array}$ & & $10.9(12.7)$ & $8.1(10.8)$ & $24.5(22.0)$ \\
(SD) & & & & \\
\hline
\end{tabular}

Table 1. ICU mean length of stay (all patients vs patients with positive CAM-ICU).

Conclusion. Late onset delirium occurring more than 3 days after admission to critical care is associated with a three-fold increase in length of stay compared to early onset delirium and appears to have a proportionally greater impact on length stay than early onset delirium. One limitation of this study is that patients who arrive intubated are more difficult to assess for delirium. They may have a more significant illness than those who arrive un-intubated and we have not stratified patients by illness severity on admission.

Further research into the prognostic implications and management of delirium onset at different time points during critical illness provides an interesting avenue for further research.

\section{Reference(s)}

1. Nil.

2. 1.Salluh Jl, Wang H, Schneider EB, Nagarajan N, Yenokyan G, Damluji A, et al. Outcome of delirium in critically ill patients: systematic review and meta-analysis. BMJ. 2015;350:h2538

3. 2.Vasilevskis E, Chandrasekhar R, Holtze C, Graves J, Speroff T, Girard TD et al. The Cost of ICU Delirium and Coma in the Intensive Care Unit Patient. Med Care. 2018;56(10):890-897.

4. 3.Pandharipande PP, Girard TD, Jackson JC, Morandi A, Thompson JL, Pun $B T$, et al. Long-term cognitive impairment after critical illness. N Engl J Med. 2013;369(14):1306-16.

\section{3}

\section{Early experience with Erector Spinae Block performed}

by Intensivists

M. Varela' ; C. Nunes ${ }^{1}$; L. Flores ${ }^{1}$; T. Capelas ${ }^{1}$; A. Krystopchuk' ; D. Nuñez ${ }^{1}$, J. Moreno ${ }^{1}$

${ }^{1}$ Intensive Care Medicine 1, University Hospital Center of Algarve, Faro, Faro, Portugal

\section{Correspondence: M. Varela}

Intensive Care Medicine Experimental 2021, 9(1): 000483

Introduction. Erector spinae block (ESPB) is a loco-regional block that was initially described in 2016 and since then has been used by anesthesiologists for pain management in rib fractures, abdominal, pelvic and limb pain. It may serve as a replacement for epidural blocks, due to its simplicity and better safety profile. We recently started using this technique for pain management in our Intensive Care Unit (ICU) and Intermediate Care Unit (IntCU).

Objectives. We aimed to describe our early experience with performing ESPB in our ICU and IntCU by intensivists.
Methods. We collected data on every patient to which we applied the ESPB in our units, since its introduction on the 10th of November 2020, until the 10th of May 2021, namely: patient characteristics, reason for the ESPB, perceived pain, analgesic and sedative drugs and their dosages, before the ESPB and $1 \mathrm{~h}, 6 \mathrm{~h}$ and $24 \mathrm{~h}$ after the block. We also recorded technical details (patient position, placement of ESPB), drug dosages given for the ESPB and complications associated with this technique.

Data was analyzed using Microsoft Office 365 Excel v16.0.13901.20148.

Results. ESPB was performed in 9 patients ( 3 in the ICU and 6 in the IntCU) for the following indications: rib fracture (4), lower limb fracture (2), acute pancreatitis (1), major abdominal surgery (2). The 2 patients who had abdominal surgery were septic at the time of ESBP and one had an INR over 2.

Bilateral blocks were performed in those patients with acute pancreatitis and major abdominal surgery. All but one catheter were placed with ultrasound guidance. There were no complications related to its insertion.

The most common prescription was an initial Ropivacaine bolus dose of $20 \mathrm{cc}$ of $100 \mathrm{mg}$, followed by $15 \mathrm{cc}$ of $30 \mathrm{mg}$, in each catheter, as suggested by our department's protocol. No toxicity was noted. In the 2 patients on intravenous fentanyl, complete withdrawal was possible $1 \mathrm{~h}$ after the ESPB.

Of the 2 patients who had major abdominal surgery, successful extubation was possible $2 \mathrm{~h}$ after the ESPB was performed, one of which had failed extubation the day before in relation to severe and uncontrolled abdominal pain.

The ESPB catheter remained in place for an average of 5,07 days and a maximum number of 10 days. One catheter had to be replaced after being inadvertently exteriorized.

Conclusion. ESPB can be easily learned by intensivists and safely applied in the ICU and IntCU for the management of pain, especially in septic patients in whom an epidural block is contra-indicated.

Further studies are needed in critically ill patients to determine whether ESPB can decrease opioid dose, increase ventilator-free days and lower length of stay.

Reference(s)

1. None.

2. Forero M, Adhikary SD, Lopez H, Tsui C, Chin KJ. The erector spinae plane block: a novel analgesic technique in thoracic neuropathic pain. Reg Anesth Pain Med. 2016;41(5):621-627

3. Singh N, Nagaraja P, Ragavendran S, Asai O, Bhavya G, Manjunath N et al. Comparison of continuous thoracic epidural analgesia with bilateral erector spinae plane block for perioperative pain management in cardiac surgery. Annals of Cardiac Anaesthesia. 2018;21(3):323

4. Mantuani D, Luftig P, Herring A, Mian M, Nagdev A. Successful emergency pain control for acute pancreatitis with ultrasound guided erector spinae plane blocks. Am J Emerg Med. 2020;38(6):1298.e5-1298.e7

5. Kot P, Rodriguez P, Granell M, Cano B, Rovira L, Morales I et al. The erector spinae plane block: a narrative review. Korean Journal of Anesthesiology. 2020;72(3):209-220

6. Josh Luftig P, Mantuani D, Herring A, Dixon B, Clattenburg E, Nagdev A. The authors reply to the optimal dose and volume of local anesthetic for erector spinae plane blockade for posterior rib fractures. The American Journal of Emergency Medicine. 2018;36(6):1103-1104

\section{5}

Sedation with Dexmedetomidine in neuromuscular patients in our Intensive Care Unit

O. Moreno Romero'; M. Muñoz Garach'; J. Tejero Aranguren'; ME. Poyatos Aguilera

${ }^{1}$ Intensive Care Unit, Hospital Universitario Clínico San Cecilio, Granada, Spain

Correspondence: $\mathrm{O}$. Moreno Romero

Intensive Care Medicine Experimental 2021, 9(1): 000505 
Introduction. The use of standard sedation drugs in neuromuscular patients elevates the risk for CNS deterioration and thus, complications. The use of non depressant drugs such as dexmedetomidine has changed the onset for invasive procedures in these particularly patients in the last decade.

Objectives. To evaluate the complications, and thus the safeness, of dexmedetomidine for the mild to moderate sedation of neuromuscular patients enroled in our Home Mechanical Ventilation Unit (HMVU) ruled by intensive care physicians who underwent invasive procedures.

Methods. We performed a 24-month prospective study (January 2019-January 2021) of all neuromuscular patients who underwent an invasive procedure and where sedated with dexmedetomidine, in a continuous infusion from $0.4-0.6 \mathrm{mcg} / \mathrm{kg} / \mathrm{h}$. Variables registered: sex age, type of neuromuscular disease (muscular dystrophy, amyotrophic lateral sclerosis ALS, dyaphragmatic paralysis, other congenital neuromyopathies), type of procedure (percutaneous gastrostomy, fiberoptic bronchoscopy, emergent digestive endoscopy), complications (mild, moderate, severe and death).

Results. In 24 month we registered 110 sedations in our neuromuscular patients. 65 male (69\%). Mean age of 49 y (19-72). ALS $53(48,2 \%)$, muscular dystrophy (Steinert, Duchenne, Becker..) 35 $(31,8 \%)$, other congenital neuro-myopathies (Charcot-Marie-Tooth, Bethlem myopathy, Pompe's disease...) 15 (13,6\%), dyaphragmatic paralysis $7(6,4 \%)$. Procedures: percutaneous gastrostomy $72(65,6 \%)$, fiberoptic bronchoscopy $28(25,4 \%)$, emergent digestive endoscopy 10 (9\%). Complications: 80 (73\%) mild (bradicardia, hypotension); 16 (15\%) moderate (1st dregree AV block); 14 (12\%) severe ( 7 with severe hypotension that needed phenylephrine, 4 presented 2nd AV block and 3 with 3d AV block); no deaths were registered. Only in 5 (4.5\%) patients (3 with 3rd degree block and 2 with severe hypotension), dexmedetomidine needed to be suspended, on the rest, the pause and start after 5 min with an infusion rate of $50 \%$ less dosage solved the problem and the patient ended the procedure safely.

Conclusion. The use of dexmedetomidine in continous infusion from 0.4 to $0.6 \mathrm{mcg} / \mathrm{kg} / \mathrm{h}$ in patients with neuromuscular disease for mild to moderate sedation appears to be a safe sedation drug in these particular patients. Only $12 \%$ had severe complications and in $4.5 \%$ the treatment needed to be suspended. No deaths were registered during sedations.

\section{3}

\section{Did we care about sedation and analgesia during COVID} pandemia?

A. Vallejo de la Cueva'; I. Perez Francisco ${ }^{2}$; A. Quintano Rodero'; M. Martin Martínez ; N. Aretxabala Kortajarena'; H. Barrasa Gonzalez'; J. Argaluza Escudero $^{2}$; A. Iglesias Rodriguez ${ }^{1}$; M. Martin Rogado ; A. Muniozguren Puertas

${ }^{1}$ Intensive Care Unit, Araba University Hospital, Gasteiz, Spain; ${ }^{2}$ Bioaraba, Instituto de Investigación Sanitaria Bioaraba, Gasteiz, Spain

Correspondence: A. Quintano Rodero

Intensive Care Medicine Experimental 2021, 9(1): 000563

Introduction. Due to SARS-CoV-2 pandemia, ICU capacity at University Hospital of Araba expanded 216\% during March 2020 (1). The excess of assistencial work and the complexity of these patients may had decreased our quality of assistance related to sedation and analgesia (2) in these patients.

Objectives. To compare the use of analgesics, sedative and antipsychotic drugs during the firts month of Pandemia and an hystoric cohort after the implementation of a new analgesia-based sedation and delirium protocol and monitoring throw validated ICU scales.

Methods. Observational study in a 1st Level Hospital with a target area of 400,000 patients, with an ICU of 31 High Dependency Beds and 4 Intermediate Dependency Beds expanded to 67 High Dependency Beds during the first Pandemic month. Two periods will be compared: PRE-COVID period (1/2 to 30/3/15) and COVID period (4/3 al 24/3/21). Inclussion criteria: $>18$ years, ICU admission $>48 \mathrm{~h}$, need of iv infussion of analgesic and sedative drugs.

Results. $\mathrm{N}=146$ PRE COVID vs COVID: 91 vs 55 . Age $62,75 \pm 16,52$ vs $62,57 \pm 12,43$, APACHE II $19,55 \pm 8,59$ vs $15,41 \pm 4,24(p, 001)$. Male $\%$ 65,9 vs 41,8

\begin{tabular}{llll}
\hline Type of Admission \% & PRE-COVID & COVID & P \\
\hline Neurocritical & 24,2 & & \\
Post-surgery & 25,3 & & \\
Sepsis & 18,7 & 3,5 & \\
ARDS & 15,4 & 96,4 & \\
Cardiac & 8,8 & & \\
Polytrauma & 5,5 & &, 001 \\
Use of Scales \% & & & $<, 001$ \\
RASS & 64,8 & 89,1 & $<, 001$ \\
BPS/ESCID & 20,9 & 94,5 & \\
CAM-ICU & 5,5 & 65,5 & \\
Use of Drugs \% & & & $<, 004$ \\
Sedatives & 69 & 74,5 & \\
Opioids & 90,1 & 86,4 &, 005 \\
Muscle relaxants & 4,4 & 67,3 & $<, 001$ \\
Dosage & & & \\
Propofol (mg/kg/h) & $0,74(0-3,57)$ & $1,65(0-2,5)$ & \\
Midazolam (mg/kg/h) & $0,08(0,1-0,07)$ & $0,19(0-0,25)$ & \\
Morphine & $0,03(0-15)$ & & $1(0-2)$ \\
Fentanyl (mg/kg/h) & $0,9(0,07-42)$ & $6(0-6)$ & \\
Remifentanyl (kg/h) & $6(0-12)$ & $49,1 \%$ & \\
NSAIDS \% & 30,8 & 54,5 & \\
Antipsychotics \% & 7,7 & & \\
\hline
\end{tabular}

In those COVID patients who needed muscle relaxants, $77,8 \%$ were monitored with BIS and $69,4 \%$ with TOFF. $70,6 \%$ of the COVID patients were oversedated at some point during the admission.

Conclusion. Despite the excess of assistencial work, levels of sedation, analgesia and delirium were more frequently measured through validated scales. This may suggest that the protocol has been consolidated in our unit.

COVID patients with ARDS required more days intubated and ventilated despite lower levels of severity.

As a result of increased monitoring CAM-ICU, we used more frequently antipsychotic drugs in COVID period.

We should improve analgesia in our patients in order to avoid oversedation.

Reference(s)

1. Barr J, Fraser GL, Puntillo K, et al. Clinical practice guidelines for the management of pain, agitation, and delirium in adult patients in the intensive care unit. Crit Care Med. 2013;41(1):263-306.

2. Barrasa H, Rello J, Tejada S, et al. SARS-CoV-2 in Spanish Intensive Care Units: Early experience with 15-day survival in Vitoria. Anaesth Crit Care Pain Med. 2020; S2352-5568(20)30,064-3 
000613

Analgesia and sedation in mechanically ventilated patients with COVID-19

A. Wieczorek'; K. Fidler ${ }^{1}$; J. Janc

${ }^{1}$ Intensive Care Unit, 4. Military Clinical Hospital SP ZOZ, Wrocław, Poland

Correspondence: A. Wieczorek

Intensive Care Medicine Experimental 2021, 9(1): 000613

Introduction. Practices of PAIDS in ARDS patients have been challenged during the COVID-19 pandemic. There are no guidelines which recommend management of analgesia and sedation for patients who require deep sedation, with extended durations of mechanical ventilation and are mostly overweighed, have high respiratory drive and desire to minimize nurse entry into COVID-19 patients rooms.

Objectives. Aim of the study was to analyze management of analgesia and sedation in COVID-19 ARDS patients.

Methods. Retrospective analysis of medical records patients hospitalized in our ICU during 2nd wave of COVID-19.

Results. 48 patients with moderate and severe ARDS who required intubation and mechanical ventilation were hospitalized (17 female and 31 man), the average age was 63 . Excluded from analysis were patients on ECMO, NIV, who died $<48 \mathrm{~h}$ after admission. All patients required deep sedation (RASS $-4 ;-5)$ and mean time of deep sedation was 9 days; $30(62 \%)$ of them were prone positioning, $20(20 \%)$ were paralized. According to local protocol was in analgesia infusion of remifentanyl used which was switched (changed) within 3-5 days to fentanyl. Propofol was first choice of sedative drug but according to ventilator dyssynchrony $95 \%$ patients required multimodal sedation with infusion of midazolam and ketamine. In $80 \%$ patients doses of drugs were in maximum doses per body weight. Observed side effects were hypotention (100\%), bradycardia (50\%), hypertriglyceridemia (14\%), gut hypomotility (76\%). 12 patients developed delirium, 5 patients were discharged.

Conclusion. We observed prolonged respiratory failure with exaggerated use of sedative-analgesic infusions, cumulative drugs side effects. Promotion of interruption or light sedation, avoidance of deliriogenic pharmacotherapies has been impossible to apply in COVID-19 mild and severe ARDS.

\section{Reference(s)}

1. Chanques G. et al. Analgesia and sedation in patients with ARDS. IntensiveCareMed. Intensive Care Med. 2020 Dec:46(12):2342-2356.

2. 3. Payen et al. Sedation for critically ill patients with COVID-19: Which specificities? One size does not fit all. Anaesth Crit Care Pain Med 39(2020)341-343

3. 1. Devlin, John W. et al. Clinical Practice Guidelines for the Prevention and Management of Pain, Agitation/Sedation, Delirium, Immobility, and Sleep Disruption in Adult Patients in the ICU. Critical Care Medicine: September 2018-Volume 46-Issue 9-p e825-e873

4. No grant acknowledgment

000704

Incidence and risk factors for delirium in critically ill patients admitted to an ICU in a middle-income country

H. Tirape-Castro ${ }^{1}$; PP. Arias-Sanchez ; M. Pacheco-Sanmartin, ${ }^{1}$; C. Ochoa-Duran, ${ }^{1}$; M. Pesantez-Jara ${ }_{1}^{1}$; O. Bustamante-Celleri, ${ }^{1} ; \mathrm{H}$. Aguirre-Bermeo ${ }^{1}$

${ }^{1}$ Intensive Care Unit, Hospital Vicente Corral Moscoso, Cuenca, Ecuador

Correspondence: $\mathrm{H}$. Aguirre-Bermeo

Intensive Care Medicine Experimental 2021, 9(1): 000704

Introduction. Delirium frequently occurs in critically ill patients. Its prompt diagnosis and the identification of risk factors could modify the patient clinical outcomes.

Methods. A prospective cohort study of critically ill patients admitted to our ICU in a middle-income country (Ecuador). We excluded patients under 18 years old, pregnant women, patients with alcohol misuse (drink more than 14 units $(112 \mathrm{~g}$ ) of alcohol a week or history of a withdrawal syndrome), and patients with psychotropic treatments. Delirium was diagnosed using the Confusion Assessment Method for the ICU (CAM-ICU) score. All patients were treated according to our ICU sedation and analgesia protocol, which establishes the use of light sedation according to the Richmond agitation-sedation scale (RASS -2) and daily awakenings.

Variables: 1. Sedation and analgesia were defined as: the use of at least one of the following drugs: Fentanyl, Remifentanil, Propofol, Dexmedetomidine, Midazolam. 2. Overnight interruptions (from 8 p.m. to 8 a.m.) were divided into two groups: a. 15 or fewer interruptions and b. 16 or more interruptions; the variable was determined considering the usual care of 2 to 3 interruptions for drug administration and the hourly vital signs measurements. 3. Alcohol drinking history: use of alcohol without meeting alcohol misuse definition.

Results. We collected 101 patients. Twenty-Seven (26.7\%) of them developed delirium. Twenty-two were males (81\%), and five were females (19\%), $p=0.01$.

The data is presented in the table.

\begin{tabular}{llll}
\hline Variable & $\begin{array}{l}\text { Delirium } \\
\text { group } \\
\text { (n=27) }\end{array}$ & $\begin{array}{l}\text { Non-Delirium } \\
\text { group (n= 74) }\end{array}$ & $\mathbf{p =}$ \\
\hline Age, years & $44 \pm 22$ & $50 \pm 19$ & 0.14 \\
SAPS III & $52 \pm 15$ & $54 \pm 14$ & 0.67 \\
Alcohol drinking history & $7(26)$ & $7(10)$ & 0.03 \\
Invasive Mechanical Ventilation & $17(63)$ & $37(50)$ & 0.25 \\
$\geq 16$ average overnight inter- & $17(63)$ & $33(45)$ & 0.10 \\
$\quad$ ruptions & $10(37)$ & $41(55)$ & 0.10 \\
$\leq 15$ average overnight inter- & & & 0.16 \\
$\quad$ ruptions & $17(63)$ & $35(45)$ & 0.05 \\
Sedation and analgesia & $8 \pm 9$ & $5 \pm 5$ & 0.59 \\
ICU length of stay, days & $3(11)$ & $11(14)$ & 0.32 \\
In ICU mortality & $6(22)$ & $13(32)$ & \\
Mortality 90-day after ICU admis- & & & \\
$\quad$ sion & & &
\end{tabular}

Data are presented in mean \pm standard deviation and number (percentage) as correspond.

Conclusion. In our sample, delirium incidence was similar to the described in critically ill patients from high-income countries. Alcoho drinking history and prolonged ICU length of stay showed a statistical association with delirium incidence. An ICU sedation and analgesia protocol may influence the incidence of delirium.

000814

Subsyndromal delirium in Intensive Care Unit, a multicenter prospective study

C. Paulino ${ }^{1}$; V. Costa, ${ }^{2}$; C. Conceição, ${ }^{3}$; R. Ivo ${ }^{3}$; P. Povoa ${ }^{2}$

${ }^{1}$ Polyvalente Intensive Care Unit, Hospital de São Francisco Xavier/ Hospital da Luz, Lisboa, Portugal; ${ }^{2}$ Polyvalent Intensive Care Unit, São Francisco Xavier Hospital, CHLO, Lisbon, Portugal; ${ }^{3}$ Intensive Care Unit, Hospital de São Francisco Xavier, Lisbon, Portugal

Correspondence: $C$. Paulino

Intensive Care Medicine Experimental 2021, 9(1): 000814

Introduction. Subsyndromal delirium (SSD) is a condition characterized by a less severe acute form of acute brain disfunction. To date, SSD has been reported as an intermediate stage between delirium and normal mental state.

Objectives. We aimed to investigate the prevalence of SSD, the association between SSD and clinical outcomes and understanding if SSD is an independent entity or precedes delirium.

Methods. Prospective, observational, multicenter clinical study, involving five Intensive Care Units. A daily evaluation of delirium and SSD was done with Intensive Care Delirium Screening Checklist (ICDSC) and Confusion Assessment Method-ICU (CAM-ICU). Scales 
were applied daily until ICU discharge/ death or up to 14 days of ICU stay. SSD was considered present if CAM-ICU assessment was negative but the patient exhibited at least 1 CAM-ICU feature, or if the ICDSC score was 1-3 points.

Results. Between 1 August 2018, and 30 March 2020, 137 patients were included. The median age was 71 years (IQR 59 to 78), 79 females (57.7\%), with a median Apache II of 22 (IQR 14 to27). 67 (52,3\%) were under invasive mechanical ventilation at some point during first 14 days of ICU stay with a median duration of ventilation of 1 day (IQR 0 to 5) and maximum of 14 days. Median length of ICU stay of 6 days (IQR 0 to 11 ) and hospital stay of 24 days (IQR 14-40); ICU and hospital mortality were $7.3 \%$ and $20.4 \%$ respectively.

Sedative infusion was common, propofol (46.1\%), morphine (27.3\%), remifentanil (21.9\%) and midazolam (15.6\%) were the most used drugs. Propofol, remifentanil, midazolam and dexmedetomidine were associated with delirium and SSD risk $(p<0,001)$ (when CAM-ICU scale was used).

A total of 1179 mental status assessments where performed during the study, 613 with CAM-ICU and 566 with ICDSC. CAM-ICU was positive for delirium in 105 episodes $(11,7 \%)$ and for SSD in $169(18.8 \%)$ which allowed identify of 46 delirious patients (35.9\%) and $43(33.6 \%)$ with SSD. ICDSC detected 143 (15.9\%) episodes of delirium and 105 (11.7\%) episodes of SSD, with a total of 58 delirious patients $(45.3 \%)$ and 28 (21.9\%) with SSD.

Apache II, mechanical ventilation and length of ICU stay were associated with a higher risk of delirium and SSD $(p<0.001)$ with both scales. Conclusion. SSD has a high prevalence with both scales, between $21.9 \%$ and $33.6 \%$, but lower than delirium. Both neurologic disfunctions are in association with Apache II, mechanical ventilation and length of ICU stay. Routine monitoring of SSD enables detection of less severe forms of neurologic disfunction and could aid the development of strategies to reduce SSD, delirium and associated sequelae.

\section{Reference(s)}

1. Corona A, Colombo R, Catena E. Early Identification of Subsyndromal Delirium in the Critically III: Don't Let the Delirium Rise! Crit Care Med. 2016 Mar;44(3):644-5. https://doi.org/10.1097/CCM.00000000000001544. PubMed 26901554

2. Yamada C, Iwawaki Y, Harada K, Fukui M, Morimoto M, Yamanaka R. Frequency and risk factors for subsyndromal delirium in an intensive care unit. Intensive Crit Care Nurs. 2018 Aug; 47:15-22. https://doi.org/10. 1016/j.iccn.2018.02.010. Epub 2018 Mar 30. PubMed 29606481

3. Serafim RB, Soares M, Bozza FA, Lapa E Silva JR, Dal-Pizzol F, Paulino MC, Povoa P, Salluh JIF. Outcomes of subsyndromal delirium in ICU: a systematic review and meta-analysis. Crit Care. 2017 Jul 12;21(1):179. https://doi. org/10.1186/ s13054-017-1765-3. Review. PubMed 28697802

4. Brummel NE, Boehm LM, Girard TD, Pandharipande PP, Jackson JC, Hughes CG, Patel MB, Han JH, Vasilevskis EE, Thompson JL, Chandrasekhar R, Bernard GR, Dittus RS, Ely EW. Subsyndromal Delirium and Institutionalization Among Patients With Critical IIIness. Am J Crit Care. 2017 Nov;26(6):447-455. https://doi.org/10.4037/ajcc2017263. PubMed 29092867

5. Boettger S, Nuñez DG, Meyer R, Richter A, Schubert M, Jenewein J. Subsyndromal delirium in the intensive care setting: Phenomenological characteristics and discrimination of subsyndromal delirium versus no and full-syndromal delirium. Palliat Support Care. 2018 Feb;16(1):3-13. https://doi.org/10.1017/S1478951517000104. Epub 2017 Mar 6. PubMed 28262089

6. Orion Corporation- Orion Pharma.

\section{2}

Association of natural light exposure and delirium according to the presence or absence of windows in the intensive care unit HJ. Lee'; HY. Lee'; SM. Lee'; L. Jinwoo²

'Internal Medicine, Seoul National University Hospital, Seoul, Republic of Korea; ${ }^{2}$ Department of Pulmonary and Critical Care, Seoul National University Hospital and Seoul National University College of Medicine, Seoul, Republic of Korea

Correspondence: H.J. Lee
Intensive Care Medicine Experimental 2021, 9(1): 000822

Introduction. Patients in the intensive care unit (ICU) have increased risks of delirium, which is associated with worse outcomes. As pharmacologic treatments for delirium are ineffective, prevention is important. Nonpharmacologic preventive strategies include exposure to natural light and restoring circadian rhythm. We investigated the effect of exposure to natural light via windows on delirium in the ICU.

Methods. This retrospective cohort study assessed all patients admitted to the medical ICU (MICU) of a university-affiliated hospital between January and June 2020 for eligibility. The ICU included 12 isolation rooms, six with and six without windows. Patients with ICU stays of $>48 \mathrm{~h}$ were included and were divided into groups based on their admission to a single room with (Window group) or without windows (Windowless group). The primary outcome was the cumulative incidence of delirium. The secondary outcomes were the numbers of delirium- and mechanical ventilation-free days, ICU and hospital length of stay, and in-ICU and 28-day mortalities.

Results. Among 150 patients, 83 patients were classified as Window group (55.3\%) and 67 patients as Windowless group (44.7\%). The cumulative incidence of delirium was significantly lower in the Window group compared to the Windowless group (21.7\% vs. $43.3 \%$; relative risk [RR] 1.996, 95\% confidence interval [95\% Cl], 1.220 3.265). Other secondary outcomes did not differ between groups. Admission to a room without a window was independently associated with an increased risk of delirium (adjusted odds ratio, 3.168, $95 \% \mathrm{Cl}, 1.428-7.030$ ). In Cox proportional regression analysis, admission to a room with windows was also associated with a decreased risk of the time to first delirium event (adjusted hazard ratio $=0.462$ $95 \% \mathrm{Cl} 0.251-0.853$ ).

Table 1. Main outcomes.

\begin{tabular}{llll}
\hline & $\begin{array}{l}\text { Windowless } \\
(\mathbf{n = 6 7 )}\end{array}$ & Window $(\mathbf{n}=\mathbf{8 3})$ & P-value \\
\hline Primary outcome & & & \\
\hline $\begin{array}{l}\text { Delirium incidence } \\
\text { Secondary outcomes }\end{array}$ & $29(43.3)$ & $18(21.7)$ & 0.005 \\
$\begin{array}{l}\text { Delirium-free days } \\
\text { Agitation }\end{array}$ & $1.4 \pm 2.5$ & $2.2 \pm 3.5$ & 0.105 \\
$\begin{array}{l}\text { Ventilation-free } \\
\quad \text { days }\end{array}$ & $3.8 \pm 5.3$ & $6(7.2)$ & 0.486 \\
ICU LOS, days & $10.5 \pm 11.8$ & $3.34 \pm 5.0$ & 0.615 \\
Hospital LOS, days & $47.6 \pm 36.0$ & $10.6 \pm 13.1$ & 0.955 \\
ICU mortality & $15(22.4)$ & $50.2 \pm 37.9$ & 0.670 \\
28-day mortality & $16(23.9)$ & $15(18.1)$ & 0.511 \\
\hline
\end{tabular}

Data are presented as mean \pm standard or numbers (percentage) for continuous and categorical variables, respectively. ICU, intensive care unit; LOS, length of stay. 


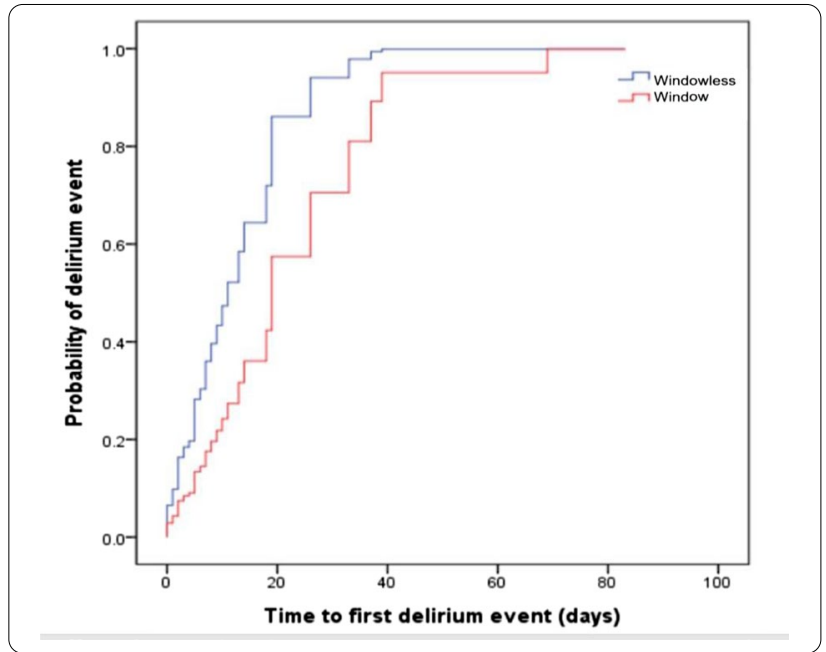

Figure 1. Cox proportional hazard model of the time to first delirium event during the intensive care unit (ICU) stay according to Windowless (room without windows) or Window (room with windows) groups Conclusion. Exposure to natural light through windows was associated with a decreased risk of delirium as compared to admission to a single windowless room. Natural light exposure was also associated with a decreased risk of delirium and time to the first delirium event.

\section{Reference(s)}

1. Zaal IJ, Spruyt CF, Peelen LM, van Eijk MM, Wientjes R, Schneider MM, et al. Intensive care unit environment may affect the course of delirium. Intensive Care Med 2013;39:481-8

2. Balas MC, Burke WJ, Gannon D, Cohen MZ, Colburn L, Bevil C, et al. Implementing the awakening and breathing coordination, delirium monitoring/management, and early exercise/mobility bundle into everyday care: opportunities, challenges, and lessons learned for implementing the ICU Pain, Agitation, and Delirium Guidelines. Crit Care Med 2013;41:S116-27.

3. Lin SM, Liu CY, Wang CH, Lin HC, Huang CD, Huang PY, et al. The impact of delirium on the survival of mechanically ventilated patients. Crit Care Med 2004;32:2254-9.

4. Ouimet S, Kavanagh BP, Gottfried SB, Skrobik Y. Incidence, risk factors and consequences of ICU delirium. Intensive Care Med 2007;33:66-73.

5. Ely EW, Shintani A, Truman B, Speroff T, Gordon SM, Harrell FE, Jr., et al. Delirium as a predictor of mortality in mechanically ventilated patients in the intensive care unit. Jama 2004;291:1753-62.

6. Pandharipande P, Cotton BA, Shintani A, Thompson J, Pun BT, Morris JA, Jr., et al. Prevalence and risk factors for development of delirium in surgical and trauma intensive care unit patients. J Trauma 2008;65:34-41.

7. Micek ST, Anand NJ, Laible BR, Shannon WD, Kollef MH. Delirium as detected by the CAM-ICU predicts restraint use among mechanically ventilated medical patients. Crit Care Med 2005;33:1260-5.

\section{6}

Efficacy of melatonin in prevention of delirium in critically ill adults: A Randomised Controlled Trial

A. Bandyopadhyay ${ }^{1}$; LN. Yaddanapudi ${ }^{2}$; V. saini $^{3}$; N. Sahni ${ }^{1}$; S. Grover ${ }^{4}$ ${ }^{1}$ Anaesthesia and Intensive Care, Post Graduate Institute of Medical Education \& Research, Chandigarh, Chandigarh, India; ${ }^{2}$ Anaesthesia and intensive care, Post Graduate Institute of Medical Education \& Research, Chandigarh, India; ${ }^{3}$ Anaesthesia and critical care, post graduate institute of medical education and research, Chandigarh, India; ${ }^{4}$ Psychiatry, Post Graduate Institute of Medical Education \& Research, Chandigarh, Chandigarh, India

Correspondence: A. Bandyopadhyay

Intensive Care Medicine Experimental 2021, 9(1): 000936
Introduction. Disruption of circadian rhythm and consequent sleep disturbance predispose to the development of intensive care unit (ICU) acquired delirium. We aimed to determine whether prophylactic melatonin decreases the prevalence of delirium in critically ill adults.

Methods. In this open label randomized controlled trial critically ill adults received either usual standard care alone (Group C) or or in combination with enteral melatonin $3 \mathrm{mg}$ once day at 9 PM (Group M). The primary outcome was the prevalence of delirium at $24 \mathrm{~h}$ of ICU stay. Secondary outcomes were prevalence of delirium at days 3 and 7, ICU mortality, length of ICU stay, duration of mechanical ventilation and Glasgow outcome scores at discharge. Multivariable logistic regression was used to develop models to predict delirium in ICU.

Results. 108 patients were included in the final analysis with 54 patients in each group. At $24 \mathrm{~h}$ of ICU stay, there was no difference in prevalence of delirium between groups $M$ and C (29.6 vs. $46.2 \%$;RR $0.6,95 \%$ C.I. $0.38-1.05, p=0.11$ ). None of thesecondary outcomes showed a statstically significant difference between the two groups. SOFA score on day 1 was an independent risk factor for developing new onset delirium in ICU with adjusted odds ratio of $2.60(95 \% \mathrm{Cl}$ : 1.24-5.45; $p=0.01$ )

Conclusion. Enteral melatonin $3 \mathrm{mg}$ is not more effective in decreasing the prevalence of delirium compared to standard care in critically ill adults.

\section{1}

There is no such a thing as a Difficult to Sedate COVID19 Patient R. Knafelj'; M. Noc

${ }^{1}$ Univesity Medical Center Ljubljana, MICU, Ljubljana, Slovenia

Correspondence: R. Knafelj

Intensive Care Medicine Experimental 2021, 9(1): 000991

Introduction. Sedation and mechanical ventilation is cornerstone supportive therapy in ARDS management. Patients with COVID-19 are typically ventilated for more then 10 days during which time increased doses of benzodiazepines together with propofol, opioids and NMBAs are used. Over time drug clearance is decreased and prolonged awakening, delayed weaning and delirium is commonly seen. Also, in some countries shortage of fentanyl, midazolam and propofol has been reported. With sevoflurane no active metabolites, tachyphylaxis, tolerence or dependance has been described. It has predictable and favourable pharmacokinetics and dynamics with anti-inflammatory effects in patients with ARDS. No data is available on using sevoflurane for sedation in COVID-19 patients requiring invasive mechanical ventilation.

Objectives. To assess feasibility of volatile sedation with sevoflurane in COVID-19 patients requiring mechanical ventilation.

Methods. 25 consecutive patients with COVID-19 referred to tertiary center for advanced ventilatory support and/or rescue therapies were switched from midazolam and/or midazolam + propofol based sedation to single agent sedation using sevofluran (Anaconda, Sedana Medical Sweden). Sedation depth was assessed by Richmond Agitation Sedation Scale (RASS) targeting -4 and Bispectral Index (BIS, Medtronic, US) targeting BIS 40-50. End expiratory sevoflurane concentration vas measured using Vamos (Dräger, Germany). All patients received continuous analgesia (fentanyl) and NMBA.

Results. In cohort of 25 invasively ventilated patients $69 \%$ were male with BMI $29 \pm 5$ and APACHE $17 \pm 5.18$ (72\%) patients received iNO $(20 \mathrm{ppm})$. Anaconda was placed in inspiratory limb in $3(12 \%)$ patients. *dose changes for fentanyl, propofol, midazolam and rocuronium only, boluses included.

\begin{tabular}{llll}
\hline & Day 1 & Day 3 & P \\
\hline Midazolam only & $12(48 \%)$ & 0 & \\
Midazolam (mg/h) & $42.5 \pm 18.3$ & 0 & \\
Fentanyl (microg/h) & $155 \pm 65$ & $160 \pm 32$ & NS \\
Propofol only & 0 & 0 & \\
\hline
\end{tabular}




\begin{tabular}{llll}
\hline & Day 1 & Day 3 & P \\
\hline Rocuronium (mg/h) & $48 \pm 7$ & $26 \pm 4$ & $<0.005$ \\
Propofol (mg/h) & $267 \pm 47$ & 0 & $<0.005$ \\
Infusion pump manipulations* (N) & $18 \pm 7$ & $8 \pm 4$ & $<0.005$ \\
Sevoflurane (end expiratory vol\%) & NA & $1,6267 \pm 0.2$ & \\
BIS & 40 & 41 & NS \\
Vt $(\mathrm{mL} / \mathrm{kg} \mathrm{IBM})$ & $6.7 \pm 0.8$ & $6.2 \pm 0.5$ & NS \\
DP $\left(\mathrm{cmH}_{2} \mathrm{O}\right)$ & $14 \pm 6$ & $11 \pm 4$ & NS \\
\hline
\end{tabular}

Conclusion. Sevoflurane via Anaconda provided adequate dept of sedation in all COVID-19 patients. Dose needed for adequate dept of sedation was higher than reported in other patient populations. Less infusion pump manipulation was needed during sevoflurane only sedation. Further research is warranted.

\footnotetext{
Reference(s)

1. Jerath A, Ferguson ND, Cuthbertson. Inhalational volatile-based sedation for COVID-19 pneumonia and ARDS. Intensive Care Med https://doi.org/ 10.1007/s00134-020-06154-8

2. None.
}

\section{0}

Whole blood thiamine (WBT) and delirium occurrence in the Intensive Care Unit (ICU)

M. Mumin ${ }^{1}$; C. McKenzie ${ }^{2}$; V. Page ; D. Strain ${ }^{4}$; B. Blackwood ${ }^{5}$; PA. Hopkins ${ }^{6}$; D. Hadfield ${ }^{6}$; E. Cunningham ${ }^{7}$; M. Ostermann ${ }^{8}$; A. Slooter ${ }^{9}$; DF. Mc Auley ${ }^{5}$; PE. Spronk ${ }^{10}$

'Pharmacy, King's College Hospital, London, United Kingdom; ${ }^{2}$ Pharmacy, Kings College Hospital, London, France; ${ }^{3}$ Intensive Care, Watford General Hospital, Watford, United Kingdom; ${ }^{4}$ Medicine, University of Exeter, Exeter, United Kingdom; ${ }^{5}$ Centre for Experimental Medicine, Queen's University Belfast, Belfast, United Kingdom; ${ }^{6}$ Critical care, King's College Hospital, London, United Kingdom; ${ }^{7}$ Centre for Explerimental Medicine, Queen's University Belfast, Belfast, United Kingdom; ${ }^{8}$ Intensive care, Guys \& St Thomas Nhs Foundation Trust, London, United Kingdom; ${ }^{9}$ Intensive care, University Medical Center Utrecht, Utrecht, Netherlands; ${ }^{10}$ Intensive care, Gelre ziekenhuizen Apeldoorn, Apeldoorn, Netherlands

Correspondence: $C$. McKenzie

Intensive Care Medicine Experimental 2021, 9(1): 001000

Introduction. Thiamine di-phosphate (TDP) is an essential cofactor in glucose metabolism, glutamate transformation and in cholinesterase activity, all reported in delirium occurrence [1]. We proposed that a deficiency in Whole Blood Thiamine (WBT) could increase risk of delirium occurrence in patients admitted to the Intensive Care Unit (ICU).

Objectives. To establish whether there is a relationship between deficiency in WBT and ICU delirium occurrence in a cohort of ICU admissions from Gelre Hospital, Netherlands.

Methods. An anonymised patient dataset was approved and obtained from Gelre ICU. This was a secondary analysis of a previous study on WBT in ICU patients (2). Delirium was assessed twice a day, using confusion assessment method-intensive care unit (CAM-ICU). A day in delirium was defined as 1 or more positive CAM-ICU scores in $24 \mathrm{~h}$. The pathology range for WBT deficiency was $\leq 100 \mathrm{nmol} /$ litre. An initial analysis was carried out to explore whether normal levels of WBT at $\mathrm{t}-0 \mathrm{~h}, \mathrm{t} 24 \mathrm{hrs}$ or $\mathrm{t} 48 \mathrm{hrs}$ resulted in a lower incidence of delirium during ICU admission. The analysis is reported as odds ratio (OR) and $95 \%$ confidence interval $(\mathrm{Cl})$.

Results. The original ICU patient cohort was admitted between 2009 to 2010 . There were 57 patients and WBT was reported at $\mathrm{t}-0, \mathrm{t}-24$ and $\mathrm{t}-48$. Analysis reported a comarable rate of delirium in those with normal WBT (> $100 \mathrm{nmol} /$ litre) compared to those that were WBT deficient ( $<100 \mathrm{nmol} /$ litre) at t $-0, \mathrm{t}-24$ and $\mathrm{t}-48$, (OR: 0.68 [95\% confidence interval (Cl): $0.23-1.97]$ ), (OR: 0.63 [95\% Cl: $0.20-2.00]$ ) and (OR: 0.70 [95\% Cl: $0.21-2.31]$ ) respectively. Regression analysis was performed with age and sepsis as confounding variables, no significant differences were observed.

Table 1. Relationship between WBT on ICU admission, t-24 h, t-48 $h$ and delirium occurrence in ICU.

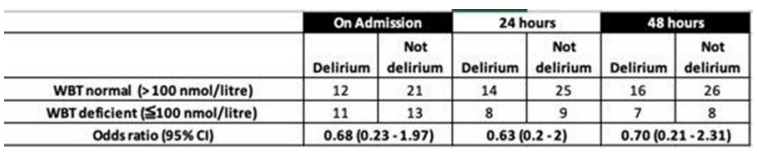

Conclusion. In this small dateset, no relationship could be detected between WBT and delirium occurrence on ICU admission, and 24 and $48 \mathrm{~h}$ post admission. The lack of significance with regards to confounding variable of sepsis and age could be attributed to small patient numbers. Further analysis with a larger dataset is needed to investigate the research question.

\section{Reference(s)}

1. (1) Kealy J, Murray C, Griffin EW, Lopez-Rodriguez AB, Healy D, Tortorelli LS, et al. Acute Inflammation Alters Brain Energy Metabolism in Mice and Humans: Role in Suppressed Spontaneous Activity, Impaired Cognition, and Delirium. The Journal of neuroscience: the official journal of the Society for Neuroscience 2020 July;40(29):5681-5696. (2).van Snippenburg W, Reijnders MGJ, Hofhuis JGM, de Vos R, Kamphuis S, Spronk PE. Thiamine Levels During Intensive Insulin Therapy in Critically III Patients. Journal of intensive care medicine 2017;32(9):559-564.

\section{3}

Introducing inhaled anaesthetics to a small intensive care unit during the COVID-19 pandemic

J. Coules ${ }^{1}$; D. Holmquist ${ }^{2}$

${ }^{1}$ Princess Alexandra Hospital, Harlow, United Kingdom: ${ }^{2}$ Critical care,

Princess Alexandra Hospital, Harlow, United Kingdom

Correspondence: J. Coules

Intensive Care Medicine Experimental 2021, 9(1): 001003

Introduction. Volatile anaesthetics are regularly used for induction and maintenance of general anaesthesia in the operating room but have thus far had limited use in intensive care units (ICU). These anaesthetic agents have ideal pharmacokinetic properties when compared to intravenous (IV) sedatives, with rapid onset and offset, organ-independent breakdown, and minimal adverse effects (1). The recent introduction of anaesthetic delivery systems into existing ventilator circuits allows volatile anaesthetics to sedate mechanically ventilated patients (2). During the coronavirus (COVID-19) pandemic, increasing patient numbers requiring mechanical ventilation and shortages of IV sedatives further encouraged the use of inhaled sedation (3). However, little is known about the impact of inhaled sedation on the outcomes of critically ill patients.

Objectives. The objective of this project was to assess the effect of inhaled sedation in comparison to IV sedation in mechanically ventilated patients in ICU.

Methods. A retrospective cohort study and descriptive analysis was conducted of all patients who were mechanically ventilated for $120 \mathrm{~h}$ or more on the ICU of Princess Alexandra Hospital, Harlow between May and December 2020. Patients were divided into three groups based on the sedation they received: inhaled, IV, or both. The median values of each outcome were compared between groups. Length of ventilation, time to extubation, mortality, incidence of adverse effects (delirium, hypercapnia, excessive secretions with/without emergency bronchoscopy) and average cost of sedatives per hour were assessed. Patients who received both forms of sedation were assessed for any changes in adverse effects or cost when the sedative type was altered. Results. 34 patients were included in the study: 24 received IV sedation, 2 received inhaled sedation, and 8 received both. No differences were noted in length of ventilation or time to extubation. There was a 
similar incidence of adverse effects in all groups, and in patients who received both. The use of inhaled sedatives had a lower average cost per hour, as shown in Figure 1: the median value of IV sedation was $£ 7.15$ whilst inhaled sedation was $£ 4.71$. All patients who received both had a lower average cost when on inhaled sedation, shown in Fig. 2. 15 patients were COVID-19 positive on admission (11 on IV sedation, 4 on both sedative types).

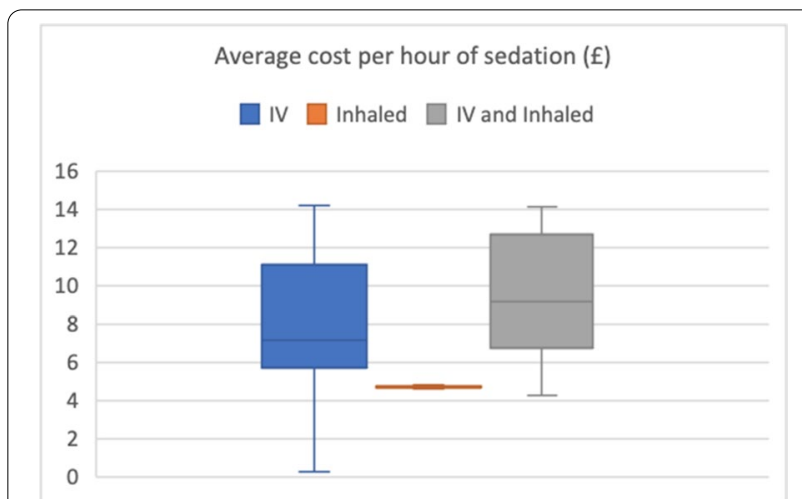

Figure 1: A box plot comparing the average cost per hour of sedation, showing median values, interquartile range, and minimum and maximum values for each group, with cost in pounds (GBP) on the $y$-axis.

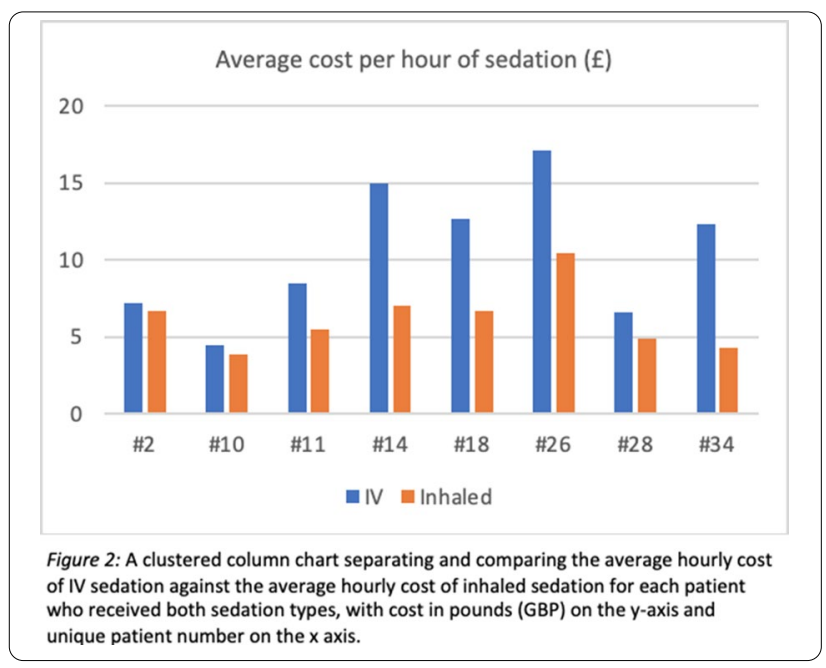

Conclusion. The small, variable group sizes and heterogeneous patient population limits the ability to draw conclusions about the effect of inhaled sedation. In spite of this, the above findings indicate that the use of inhaled sedation is a safe method of sedation in ICU, provides similar outcomes to IV sedation, and may confer a costsaving benefit. This is of particular relevance in anticipation of future waves of COVID-19 patients. Larger-scale prospective studies are needed to further assess these findings and identify which patients will most benefit from inhaled sedation on ICU.

\section{Reference(s)}

1. 3. Nieuwenhuijs-Moeke GJ, Jainandunsing JS, Struys MMRF. Sevoflurane, a sigh of relief in COVID-19? Br J Anaesth. 2020 Aug;125(2):118-121.

2. 2. Farrell R, Oomen G, Carey P. A technical review of the history, development and performance of the anaesthetic conserving device "Ana-

ConDa" for delivering volatile anaesthetic in intensive and post-operative critical care. J Clin Monit Comput. 2018 Aug;32(4):595-604.
3. 1. Kim HY, Lee JE, Kim HY, Kim J. Volatile sedation in the intensive care unit: A systematic review and meta-analysis. Medicine (Baltimore). 2017 Dec;96(49):e8976.

001047

Sedation with dexmedetomidine in ICU patients receiving noninvasive ventilation: a meta-analysis with trial sequential analysis

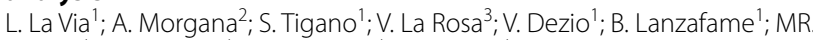
Valenti ${ }^{4}$ : P. Murabito ${ }^{4}$; F. Sanfilippo ${ }^{4}$; M. Astuto ${ }^{4}$

${ }^{1}$ School of Anaesthesia and Intensive Care, University Hospital "G. Rodolico", Catania, Italy; ${ }^{2}$ School of Anaesthesia and Intensive Care, Magna Græcia University, Catanzaro, Italy; ${ }^{3}$ Department of Anesthesiology and Intensive Care, Humanitas Istituto Clinico Catanese, Catania, Italy; ${ }^{4}$ Department of Anesthesiology and Intensive Care, University Hospital "G. Rodolico", Catania, Italy

Correspondence: $L$. La Via

Intensive Care Medicine Experimental 2021, 9(1): 001047

Introduction. Several studies have been published on the efficacy of Dexmedetomidine as sedation strategy in critically ill patients not tolerating noninvasive ventilation (NIV). We conducted a meta-analysis with Trial Sequential Analyses (TSAs) to evaluate the beneficial effects of dexmedetomidine as compared to other sedation strategies.

Methods. In this preliminary analysis we screened MEDLINE and EMBASE for randomized clinical trials (RCTs), up to May 5th, 2021. We calculated Relative Risk (RR) for dichotomous outcomes and standardized mean difference (MD) for continuous outcomes, with $95 \%$ confidence interval $(\mathrm{CI})$ using a random-effect model. For the TSA, the information size was computed assuming an alpha risk of $5 \%$ with a power of $80 \%$. Our primary outcome was the reduction of intubation rate. Analyses were performed with separation in subgroups according to the sedation strategy in the control group (placebo, midazolam, haloperidol, various).

Results. Twelve RCTs were included in our analysis. Dexmedetomidine, compared with other sedation strategies or placebo, reduced the risk of intubation ( $\mathrm{RR} 0.54 ; 95 \% \mathrm{Cl}, 0.41-0.71$ ). This effect was evident across subgroups and significant when dexmedetomidine was compared to midazolam ( $3 \mathrm{RCTs}, \mathrm{p}=0.003$ ) or haloperidol ( $2 \mathrm{RCTs}$, $\mathrm{p}=0.008$ ). Dexmedetomidine also reduced the incidence of delirium (RR, 0.34; 95\% $\mathrm{Cl}, 0.22-0.54$ ) and the Intensive Care Unit length of stay (ICU-LOS: MD, -2.4 days; $95 \% \mathrm{Cl},-3.51$ to -1.29 days). However, dexmedetomidine strategy was associated with increased risk of bradycardia (RR, 2.80; $95 \% \mathrm{Cl}, 1.92-4.07)$ and hypotension (RR, $1.98 ; 95 \% \mathrm{Cl}$, 1.32-2.98).The five TSAs conducted on these outcomes demonstrated that all results are robust (Figure 1), with Z-line crossing the alphaspending boundary. Mortality was not different between groups but TSA showed that more studies are needed.

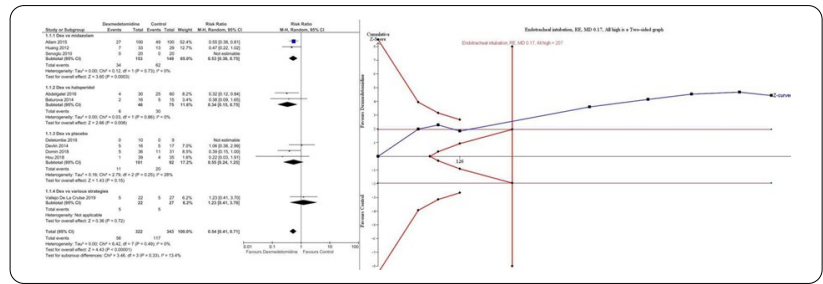

Figure 1. Forest plot analysis and Trial Sequential Analysis on tracheal intubation in ICU patients requiring NIV randomized to dexmedetomidine or other sedation strategies with subgroup analysis. Analysis performed with random effect model and Mantel-Haenszel $(\mathrm{M}-\mathrm{H}) . \mathrm{Cl}$ : confidence interval, RRR: relative risk reduction.

Conclusion. In critically ill patients receiving NIV, dexmedetomidine is associated with decreased risk of intubation, particularly if the alternatives are midazolam or haloperidol. A strategy of sedation with dexmedetomidine reduces also the incidence of delirium and shortens the ICU-LOS, while increasing the risks of bradycardia and hypotension. 
More studies are needed to evaluate the efficacy of dexmedetomidine in reducing mortality in this patient population.

\section{2}

Efficacy of halopeRIdol to decrease the burden of Delirium In adult Critically ill patiEnts (EuRIDICE): a Randomized Clinical Trial L. Smit, ; A. Slooter ${ }^{2}$; J. Devlin ${ }^{3}$; Z. Trogrlic ${ }^{1}$; N. Hunfeld ${ }^{1}$; RJ. Osse, ${ }^{4}$; H. Ponssen, ; A. Brouwers ${ }^{6}$; J. Schoonderbeek, ; K. Simons ${ }^{8}$; MVD. Boogaard, ${ }^{9}$; J. Lens, ${ }^{10}$; D. Boer, ${ }^{11}$; D. Gommers ${ }^{12}$; W. Rietdijk ${ }^{13}$; M. van der Jagt ${ }^{1}$

${ }^{1}$ Intensive Care, Erasmus MC, Rotterdam, Netherlands; ${ }^{2}$ ntensive Care, University Medical Center Utrecht, Utrecht, Netherlands; ${ }^{3}$ Bouve College of Health Sciences, Northeastern University, Boston, United States of America; ${ }^{4}$ Psychiatry, Erasmus MC, Rotterdam, Netherlands; ${ }^{5}$ Intensive Care, Albert Schweitzer Hospital, Dordrecht, Netherlands; ${ }^{6}$ Intensive care, Franciscus Gasthuis, Rotterdam, Netherlands; Intensive care, Ikazia Hospital, Rotterdam, Netherlands; ${ }^{8}$ Intensive care, Jeroen Bosch Hospital, $s^{\prime}$ Hertogenbosch, Netherlands; ${ }^{9}$ Intensive care medicine, Radboud University Medical Center, Nijmegen, Netherlands; ${ }^{10}$ Intensive care, IJsselland Hospital, Rotterdam, Netherlands; ${ }^{11}$ Intensive care, Maasstad Hospital, Rotterdam, Netherlands; ${ }^{12}$ Department of intensive care, Erasmus MC, Rotterdam, Netherlands; ${ }^{13}$ Pharmacy, Erasmus University Medical Center, Rotterdam, Netherlands

Correspondence: M. van der Jagt

Intensive Care Medicine Experimental 2021, 9(1): 001182

Introduction. The efficacy of haloperidol for treatment of delirium and associated adverse outcomes in critically ill adults has not been extensively studied.

Objectives. To determine whether haloperidol when compared to placebo reduces delirium and delirium-related adverse outcomes in critically ill adults.

Methods. Design: Randomized double-blind, placebo-controlled trial conducted between February 2018 and January 2020.

Setting: Eight Dutch intensive care units (ICU).

Participants: Adult ICU patients with delirium, assessed with the Intensive Care Delirium Screening Checklist or Confusion Assessment Method for the ICU, not having an acute neurologic injury or dementia.

Intervention: Intravenous haloperidol (or placebo) $2.5 \mathrm{mg}$ every $8 \mathrm{~h}$, and titrated to a maximum of $5 \mathrm{mg}$ every $8 \mathrm{~h}$ if delirium persisted, for up to 14 days.

Main outcomes and measures: The primary outcome was ICU deliriumand coma-free days (DCFD) up to 14 days after randomization. Secondary outcomes included efficacy and safety endpoints associated with haloperidol and delirium.

Results. The trial was prematurely terminated by the Data Safety Monitoring Board after 132 enrolled patients ( 65 haloperidol; 67 placebo), because of futility to find differences in the primary DCFD outcome. Mean age was 64 years (SD 15), mean APACHE IV score 73.1 (SD 33.9), and 90/132 (68\%) were male. There was no difference in DCFDs (RR $1.16,95 \% \mathrm{Cl} 0.76-1.77, \mathrm{p}=0.502)$. Haloperidol reduced overall benzodiazepine use (adjusted OR $0.41,95 \% \mathrm{Cl} 0.18$ to $0.89, \mathrm{p}=0.028$ ) and the risk of agitated-related events (falling out of bed: adjusted OR 0.32 $95 \% \mathrm{Cl} 0.11$ to $0.84, \mathrm{p}=0.026$ ), but increased opioid dosages (adjusted difference $146.0 \%, 95 \% \mathrm{Cl} 9.0 \%$ to $447.4 \%, \mathrm{p}=0.031)$. No patients were withdrawn because of safety concerns related to haloperidol, of which the incidence did not differ between groups.

Conclusion. Our findings do not support the routine use of haloperidol to reduce delirium in critically ill adults. However, differences in some clinically relevant secondary outcomes, such as agitation-related events, may indicate effects of haloperidol on specific delirium symptoms, requiring further research.

\section{Reference(s)}

1. Smit, L et al. Efficacy of haloperidol to decrease the burden of delirium in adult critically ill patientes (EURIDICE): study protocol for a prospective randomised multi-centre double-blind placebo-controlled clinical trial in the Netherlands. BMJ Open 2020;10:e036735.

2. ZonMW NL62689.078.17
001266

Delirium in COVID-19 patients admitted to the Intensive Care Unit: A one-year retrospective observational study on risk factors C. Díaz-Rodríguez'; D. Pérez-Torres ${ }^{1}$; I. Canas-Pérez'; MM. García-García'; AM. Prieto-Lamo ${ }^{1}$; C. Cuenca-Rubio'; R. Herrán-Monge ${ }^{1}$; JÁ.

Berezo-García ; 'LM. Tamayo-Lomas ${ }^{1}$; PM. Enríquez-Giraudo ${ }^{1}$

${ }^{1}$ Servicio de medicina intensiva, Hospital Universitario Río Hortega, Val-

ladolid, Spain

Correspondence: $C$. Díaz-Rodríguez

Intensive Care Medicine Experimental 2021, 9(1): 001266

Introduction. Delirium is an acute confusional state characterized by an alteration of consciousness with reduced ability to focus, sustain or shift attention. It is a multifactorial disorder, with an enormous impact upon patients admitted to the Intensive Care Unit (ICU). These patients experience prolonged hospitalizations, functional and cognitive decline and higher mortality (1).

Primary prevention is essential: there are numerous non-pharmacologic interventions with an impact in modifiable risk factors, with proven efficacy to reduce the incidence of delirium (2). However, implementation of these interventions during the COVID-19 pandemic was scarce.

Objectives. To assess the risk factors to develop delirium in COVID-19 patients admitted to the ICU.

Methods. We conducted a retrospective observational single-centre study in the ICU of a University Hospital in Spain, from March 2020 to March 2021. All the patients who were admitted to the ICU with acute respiratory distress syndrome and PCR-confirmed COVID-19 were included. Demographics, clinical and epidemiological data were recorded. Results are expressed as mean \pm standard deviation, median (interquartile range) or frequency (\%). Association between each risk factor and delirium was assessed with Chi-square or MannWhitney $\mathrm{U}$ tests, as appropriate. All the statistically significant and clinically relevant associations were included in a multivariate logistic regression model.

Results. We included 235 patients, with a prevalence of delirium of $37 \%$. Baseline characteristics: $73 \%$ male, aged $62 \pm 12$, body mass index $29(26-33) \mathrm{Kg} / \mathrm{m}^{2}$, APACHE-II 16(13-20) points. ICU-acquired weakness $39 \%$. ICU length of stay (LOS) $19(10-33)$ days, hospital LOS 27(19-43) days. Mechanical ventilation (MV) 91\%, 18(10-33) days; prone position $70 \%$, vv-ECMO $13 \%$. Days of neuromuscular blockade (NMB) 7(4-10), days of sedation 18(10-31), days under benzodiazepine (BZD) treatment 14(7-25).

For each potential risk factor, we compared the group of patients who did not develop delirium vs the group of patients with delirium: APACHE-II $16(13-20)$ vs $16(13-20), p=0.92$; ICU-acquired weakness $32 \%$ vs $50 \%, \mathrm{p}<0.01$; ICU LOS $16(7-26)$ vs $26(15-48)$ days, $\mathrm{p}<0.01$; hospital LOS 25(16-34) vs 37(22-63) days, $p<0.01$; days of MV $14(5-$ $25)$ vs $21(12-40), p<0.01$; days of NMB $5(2-11)$ vs $6(4-11), p=0.20$; days of sedation $12(5-24)$ vs $21(12-38), p<0.01$; days under BZD treatment $9(4-19)$ vs $16(7-38), \mathrm{p}<0.01$.

A multivariate logistic regression model including all the statistically significant and clinically relevant associations from bivariate analysis showed a sensitivity of $39 \%$, a specificity of $92 \%$, a positive predictive value of $72 \%$, a negative predictive value of $73 \%$, and an area under ROC curve of 0.74 to predict delirium, $p<0.01$ (Table).

\begin{tabular}{lcllll}
\hline & Coefficient & $\begin{array}{l}\text { Standard } \\
\text { error }\end{array}$ & OR & 95\% Cl & p \\
\hline Hospital LOS, per day & 0.046 & 0.02 & 1.047 & $1.01-1.08$ & 0.003 \\
$\begin{array}{l}\text { Days of MV, per day } \\
\text { Days of sedation, per day }\end{array}$ & -0.034 & 0.03 & 0.966 & $0.91-1.03$ & 0.296 \\
$\begin{array}{l}\text { Days under BZD treat- } \\
\text { ment, per day }\end{array}$ & 0.062 & 0.03 & 0.965 & $0.90-1.03$ & 0.311 \\
$\begin{array}{l}\text { ICU-acquired weakness } \\
\text { cons }\end{array}$ & 0.254 & 0.64 & 1.290 & $0.49-3.41$ & 0.609 \\
\hline
\end{tabular}


Conclusion. In our series, bivariate analysis showed longer courses of sedation, benzodiazepine treatment and mechanical ventilation, with more frequency of ICU-acquired weakness and more prolonged ICU and hospital LOS in patients who developed delirium, while we did not find any differences as per disease severity and days under neuromuscular blockade.

In our multivariate analysis, prolonged treatment with benzodiazepines and prolonged hospitalization were significantly associated with the development of delirium.

\section{Reference(s)}

1. 1. Girard TD, Jackson JC, Pandharipande PP, et al. Delirium as a predictor of long-term cognitive impairment in survivors of critical illness. Crit Care Med 2010; 38:1513.

2. 2. Hshieh TT, Yue J, Oh E, et al. Effectiveness of multicomponent nonpharmacological delirium interventions: a meta-analysis. JAMA Intern Med 2015; 175:512

\section{4}

\section{Role of AMT Score for Assessment of Acute Delirium \& Early} Diagnosis of Dementia

K. Ahmad'; Y. Kumar, ${ }^{2}$; A. Mohamed ${ }^{2}$; S. Kadri'; N. Fischer ${ }^{2}$

${ }^{1}$ Acute Medicine \& Intensive care, King's Mill Hospital, Sutton-in-Ashfield Nottinghamshire, United Kingdom; ${ }^{2}$ Acute Medicine, King's Mill Hospital Sutton-in-Ashfield Nottinghamshire, United Kingdom

Correspondence: K. Ahmad

Intensive Care Medicine Experimental 2021, 9(1): 001294

Introduction. Acute delirium is a common presentation in acute medicine \& intensive care. AMT (Abbreviated Mental Test) sore is a simple clinical tool for assessment of acute delirium \& screening of dementia. AMT score can be calculated in Nerve Centre, an online tool for clinical data collection. AMT Score ranges from $0-10$. If AMT Score $<7$, there is high likelihood for dementia \& patients needs to refer to RRLP (Rapid Response Psychiatry Liaison Team) for further assessment. An AMT Score $>8$, there is less likelihood for possibility of dementia.

Objectives. To check the frequency of acute delirium in vulnerable age group of $65 \&$ above. To find out role of AMT (Abbreviated Mental Test) Score for assessment of acute delirium \& early diagnosis of dementia. If AMT score is $<7$, there is high likelihood for dementia as compared to if AMT score of 8 or above where dementia is unlikely. Total AMT score is of 10 with rang of $0-10$.

Methods. An audit loop was completed by checking role of AMT score on Nerve Centre (one of online clinical tool) for assessment of acute delirium \& early diagnosis of dementia. A prospective analysis was done in all vulnerable age group of $65 \&$ above. The data was collected within $72 \mathrm{~h}$ of admission into acute medical unit.

Results. In first audit group, among total sample size of 394 mean age was found 79.79 (Age range of 65-100). Acute delirium was found in $28 \%$ cases as compared to normal individuals of $72 \%$ in all vulnerable age group of 65 \& above. Mean AMT score was found 7.37 (AMT Range of $0-10$ ). In $38 \%$ cases AMT score was found $0-7$ (with likely dementia) as compared to $62 \%$ with AMT score of 8-10 (unlikely dementia). Overall AMT score was checked in $60 \%$ of cases among this age group of $65 \&$ above.

In the second Audit group (Re-audit) from sample size of 152, the mean age was found with 80.5 (Age Range of 65-101). Acute delirium was diagnosed in $23 \%$ cases. Average AMT Score was found 8.1 (AMT Range of $0-10$ ). In $27 \%$ cases AMT score was found 0-7 (with likelihood of dementia) as compared to in $73 \%$ cases having AMT score 8-10 (unlikely for dementia). In all cases with AMT score of $<7$, RRLP (Rapid Response Psychiatry Liaison Team) referral was done (100\%). AMT Score was completed overall in $63 \%$ cases as compared to $60 \%$ completed in first audit report. These results remained quite comparable with UK National Audit of 2018-2019 for checking AMT Score for assessment of acute delirium \& dementia in general hospitals in age of 65 \& above, where AMT assessment was completed in $58 \%$ cases as compared to our audit results of $60 \% \& 63 \%$ respectively.

It was found quite significant correlation between acute delirium \& likelihood of dementia in both of our audit reports ( $28 \%$ delirium cases vs $38 \%$ likelihood of dementia with AMT Score of $0-7$ in first audit, \& $23 \%$ cases with acute delirium were having likelihood of dementia in $27 \%$ with AMT score of $0-7$ in 2 nd audit).

\begin{tabular}{clllllll}
\hline & $\begin{array}{l}\text { Sam- } \\
\text { ple } \\
\text { Size } \\
\text { (n) }\end{array}$ & $\begin{array}{l}\text { Mean } \\
\text { Age }\end{array}$ & $\begin{array}{l}\text { Mean } \\
\text { AMT } \\
\text { Score }\end{array}$ & $\begin{array}{l}\text { Delirium } \\
\text { by SQiD } \\
\text { Question }\end{array}$ & $\begin{array}{l}\text { Dementia } \\
\text { Likely } \\
\text { (AMTs } \\
\text { 0-7) }\end{array}$ & $\begin{array}{l}\text { Dementia } \\
\text { Unlikely } \\
\text { (AMTs } \\
\mathbf{8 - 1 0 )}\end{array}$ & $\begin{array}{l}\text { Overall } \\
\text { AMTs } \\
\text { Checked }\end{array}$ \\
\hline Audit 1 & 394 & 79.79 & 7.37 & $28 \%$ & $38 \%$ & $62 \%$ & $60 \%$ \\
& & & & $(110 / 394)$ & $(150 / 394)$ & $(244 / 394)$ & $(236 / 394)$ \\
$\begin{array}{c}\text { Audit 2 } \\
\text { (Re- } \\
\text { Audit) }\end{array}$ & 152 & 80.5 & 8.1 & $23 \%$ & $27 \%$ & $73 \%$ & $63 \%$ \\
\hline
\end{tabular}

Conclusion. AMT Score is a simple $\&$ reliable clinical tool to detect acute delirium \& helps in early diagnosis of dementia. Acute delirium has quite significant correlation for early diagnosis of dementia in vulnerable age group of $65 \&$ above.

001423

AHDID Study: Does after-hours discharge of ICU patients treated for delirium affect their outcome?

MY. Singh ${ }^{1}$; L. Salim, ; N. Talwarr ; K. Karpe,

${ }^{1}$ Intensive Care, Canberra Hospital, Garran, Australia; ${ }^{2}$ Medical student, The Australian National University, Canberra, Australia; ${ }^{3}$ Intensive care, Monash Health-Casey Hospital, Berwick, Australia; ${ }^{4}$ Renal medicine, Canberra Hospital, Garran, Australia

Correspondence: M.Y. Singh

Intensive Care Medicine Experimental 2021, 9(1): 001423

Introduction. While delirium has been acknowledged as a common syndrome in ICU patients, it remains widely unrecognised and underdiagnosed. It is a matter of concern and is independent predictor of mortality. So is after-hours discharge though some recent large scale prospective study has refuted this claim. We investigated the outcome of patients treated for delirium in ICU and whether their time of discharge influenced their hospital outcome.

Objectives. The primary outcome considered in this study was postICU all-cause hospital mortality. The secondary outcomes were ICU readmissions, and ICU and hospital lengths of stay.

Methods. This was a single-centre retrospective cohort study conducted at Canberra Hospital, the largest and only tertiary universityaffiliated public hospital in the region. The ICU at Canberra Hospital is a Level-III 31-bed unit including dedicated cardiothoracic and paediatric bays, which admits over 2400 patients each year, with only up to $30 \%$ being elective post-operative patients. After-hour ICU discharge was defined as those occurring between 18:00-07:59 $\mathrm{h}$ and thus, discharge during work-hours occurred between 08:00 $\mathrm{h}$ and 17:59 h. Due to the retorspective design of the study, we identified patients treated for delirium from the pharmacotherapy they received (dexmedetomidine, haloperidol, olanzapine, quetiapine and risperidone). For this reason, patients who were admitted for drug overdoses were also excluded as they are often administered with anti-psychotics during their ICU stay for agitation due to drug withdrawal and suffer delirium. The continuous variables in the data set were expressed in mean and standard deviation or median and interquartile range, while categorial variables were presented as actual numbers and percentages. The epidemiological model was developed using post-ICU all-cause hospital mortality as the outcome variable and being treated for delirium in the ICU as the exposure variable. The covariates thought to influence mortality as described above were also included in the analysis. Both univariate and multivariate logistic regression analyses were done using Stata version 14 (StataCorp LP, College Station, TX, USA). A P-value of less than 0.05 was taken to indicate statistical significance.

Results. 1410 patients were included in the study. In our study cohort, $233(16.5 \%)$ were treated for delirium and $346(24.5 \%)$ were discharged after-hours. Overall, patients treated for delirium had higher mortality $(\mathrm{OR} 2.35,95 \% \mathrm{Cl} 1.10-5.04 ; \mathrm{P}<0.05)$ and after-hour discharge was not (OR 1.41, $95 \mathrm{Cl} 0.66-3.01 ; \mathrm{P}>0.05)$. There was a large 
variation in hourly mortality rate as per the time of ICU discharge for patients treated for delirium compared to those who were not. However, among patients treated for delirium, there was no statistical difference in all-cause hospital mortality between those discharged after-hours and work-hours. Only 2/54(3.7\%) patients treated for delirium discharged after-hours died in the hospital compared to $8 / 179(4.46 \%)$ discharged in work-hours (OR $0.82,95 \% \mathrm{Cl} 0.17-3.99$; $\mathrm{P}>0.05$ ). Patients treated for delirium had longer ICU (median, 5 days vs. 2 days) and hospital stay (median, 17 vs. 9 days). Overal, the readmission rates were $5.6 \%$ and we found no statistically difference in readmission rates for patients treated for delirium $(6.9 \%)$ to those not treated (5.4\%). On multivariate analysis, delirium was not associated with statistically significant risk factor for increased mortality. Other factors like age, APACHE II score, cirrhosis, acute renal failure in ICU, treatment with olanzapine emerged as an independent risk predictor for all-cause hospital mortality. A linear regression analysis was also performed on the available patient characteristics and their total hospital lengths of stay (LOS). Most significantly, neither having treatment for delirium in the ICU $(R 2=0.0008, P=0.2858)$ nor being discharged from the ICU after-hours $(R 2=0.0002, P=0.5694)$ could statistically significantly predict a longer hospital LOS.

Conclusion. The low event rate in our study, failed to demonstrate that after-hours discharge of patients treated for delirium had worse outcome. This study forms the basis for larger future research on delirium and its pharmacotherapy in the ICU to better inform clinical practice and outcomes.

\section{Reference(s)}

1. Singh MY, Nayyar V, Clark PT, Kim C. Does after-hours discharge of ICU patients influence outcome? Crit Care Resusc. 2010;12:156-61.

2. Santamaria JD, Duke GJ, Pilcher D V., Cooper DJ, Moran J, Bellomo R. Timing of discharge from the intensive care unit and subsequent mortality a prospective, multicenter study. Am J Respir Crit Care Med. 2015;191:1033-9.

3. Cavallazzi R, Saad M, Marik PE. Delirium in the ICU: An overview. Ann Intensive Care. 2012;2:1-11

\section{0}

Evaluation of ICU staff exposure to anaesthetic gas during inhaled anaesthesia with AnaConDa device in the intensive care unit

RS. Contreras'; G. Marín Vivo; ${ }^{1}$ MJ. Sala Latorre ${ }^{2}$; MJ. Jimenez Alcala ${ }^{2}$; P. Torrella Llauger²; C. Palmada Ibars²; E. Gallart Vive ${ }^{2}$; P. Giron Espot²; R.

Ferrer Roca

${ }^{1}$ Critical Care Department, Vall d'Hebron University Hospital, Vall d'Hebron Research Institute, Barcelona, Spain; ${ }^{2}$ Intensive Care Department, Vall d'Hebron University Hospital, Barcelona, Spain

Correspondence: P. Torrella Llauger

Intensive Care Medicine Experimental 2021, 9(1): 001430

Introduction. Recently, inhaled anaesthesia is gaining interest outside the operating room setting for its applications in the critical care patient. Some devices have been developed for this purpose, as the AnaConDa ${ }^{\circledR}$ device. However, safety concerns arose regarding staff exposure. We conducted this study in order to test the safety of this device.

Objectives. To identify the staff exposure levels of anaesthetic gas in the ICU using an AnaConDa.

Methods. Measurements were obtained simulating a $12 \mathrm{~h}$ nurse shift in an ICU box at the Vall d'Hebron University Hospital. Basic prevention measures were implemented, including the use of a passive absorption system, general box ventilation, and specific staff training.

Passive gas detectors were used, and 4 measurement points were identified: 1) near the patient airway, 2) at the nurse station, 3) at the gas analyser exit port, 4) on the nurse in charge of the patient.

Results. The resulting measurements were compared with reference values established in Spain by the competent authority. The maximum permitted dose is set at $50 \mathrm{ppm}$ in an $8 \mathrm{~h}$ shift. Our measured values were $0,80 \mathrm{ppm}$ near the patient airway, $0,13 \mathrm{ppm}$ at the nurse station, 0,93 in the gas analyser and 0,4 on the nurse.
Conclusion. Our measurements reveal exposure levels to anaesthetic gas way below the permitted level. That demonstrates the efficacy of the preventive measures and establishes inhaled anesthesia with an AnaConDa device as a safe option in the ICU.

e-Posters: Systemic diseases

\section{7}

Duration of symptoms at presentation and mortality in severe COVID-19: a retrospective analysis

S. Cavinato ; M. Lana, ; J. Blake, ${ }^{1}$;. Eswarappa, ; R. Kumar, ${ }^{1}$

${ }^{1}$ Intensive Care, Kingston Hospital, LONDON, United Kingdom

Correspondence: S. Cavinato

Intensive Care Medicine Experimental 2021, 9(1): 000417

Introduction. Coronavirus 2019 (COVID-19) is a multisystem disease that typically presents with initial symptoms of cough, fever, myalgia, headache and diarrhoea.(1) The clinical course of severe COVID-19 has been described,(2) with dyspnoea present after approximately one week. The symptom dynamics show a high variability,(3) and it is unclear whether the duration of symptoms prior to hospital presentation is associated with mortality in severe COVID-19.

Objectives. We aimed to assess if there is an association between the duration of symptoms prior to hospital presentation and mortality in patients with COVID-19 who were admitted to the high dependency unit (HDU) or intensive care (ICU).

Methods. A retrospective analysis was performed of patients admitted to HDU or ICU with COVID-19 in a district general hospital in London between 17th March 2020 and 31st January 2021. The medical charts were reviewed, and patients were divided into two groups: those who presented within 7 days of initial symptoms $(\leq 7 \mathrm{~d})$ and those who presented with symptoms longer than 7 days $(>7 \mathrm{~d})$. The mortality rate, ventilator-free survival and characteristics of the two groups were then compared. Patients who did not have the onset of symptoms recorded or did not have a mortality outcome by 28th February 2021 were excluded from the analysis.

Results. A total of 301 patients were admitted to ICU or HDU with COVID-19 during the time period. After exclusion, 280 were included in the analysis, of which 136 patients had symptoms $\leq 7 \mathrm{~d}$ at presentation and 144 had symptoms $>7 \mathrm{~d}$. The mean duration of symptoms at presentation was 8.2 days (SD 4.5), and median was 7 days. Mortality was significantly higher in the $\leq 7 \mathrm{~d}$ group ( $44 \%$ vs $29 \%, p=0.009$ ). Survival without mechanical ventilation was significantly higher in the $>7 \mathrm{~d}$ group ( $53 \%$ vs $38 \%, p=0.01$ ). The mean age in the $\leq 7 \mathrm{~d}$ group was 58.2 years (SD 16.6) vs 59.1 (SD 13.5) in the $>7 d$ group, and mean BMI was 28.3 (SD 8.1) in $\leq 7 \mathrm{~d}$ group vs 29.0 (SD 6.8) in $>7 \mathrm{~d}$ group. Mean length of stay for survivors was 15.4 days (SD 19.0) in the $\leq 7 \mathrm{~d}$ group and 15.1 days (SD 21.1) in the $>7 \mathrm{~d}$ group. There was no significant statistical difference between the two groups in rates of T2DM, hypertension, asthma, COPD, ischaemic heart disease or heart failure.

Conclusion. In this single-centre retrospective analysis of patients admitted to ICU or HDU with COVID-19, patients who presented to hospital within 7 days of symptom onset had a significantly higher mortality than those who presented after 7 days. Survival without mechanical ventilation was significantly higher in those who presented after 7 days.

\section{Reference(s)}

1. 3. Mizrahi, B., Shilo, S., Rossman, H. et al. Longitudinal symptom dynamics of COVID-19 infection. Nat Commun 11, 6208 (2020). https://doi.org/10. 1038/s41467-020-20053-y

2. 2. Zhou F, Yu T, Du R, et al. Clinical course and risk factors for mortality of adult inpatients with COVID-19 in Wuhan, China: a retrospective cohort study. Lancet 2020;395:1054-62.

3. 1. Centers for Disease Control and Prevention. Interim clinical guidance for management of patients with confirmed coronavirus disease (COVID19). 2020 (https://www.cdc.gov/coronavirus/2019-ncov/hcp/clinicalguidance-management -patients.html). 


\section{6}

Pressure injury associated risk factors in adult intensive care unit patients

CI. Lemos' ; S. Chaves ${ }^{1}$; S. Vieira' ; C. Gouveia²; E. Henriques ${ }^{3}$; S. Ferreira, C. Lume ${ }^{1}$; M. Mota ${ }^{1}$; S. Correia ${ }^{1}$; C. Graça ${ }^{1}$; T. Camacho'; M. Jardim'; N. Fernandes ${ }^{1}$; G. Silva'; ${ }^{1}$ M. Câmara ${ }^{1}$; C. Pereira'; JJ. Nóbrega

${ }^{1}$ Serviço de Medicina Intensiva, Hospital Dr. Nélio Mendonça, Funchal,

Portugal; ${ }^{2}$ Serviço de medicina interna, Hospital de São Francisco Xavier, Lisboa, Portugal; ${ }^{3}$ Gabinete de estatística do centro dra. maria isabel mendonça, Hospital Dr. Nélio Mendonça, Funchal, Portugal

Correspondence: C.I. Lemos

Intensive Care Medicine Experimental 2021, 9(1): 000466

Introduction. Pressure injuries are localized lesions due to pressure that cause disability, compromise quality of life and extend the length of hospital stay. Patients of intensive care units (ICU) are extremely prone to developing pressure injuries due to immobility, hemodynamic instability, poor tissue perfusion and high exposure to medical devices.

Objectives. The aim of this study was to access which variables were associated with higher mortality.

Methods. This is a retrospective observational study in an intensive care unit, with patients admitted over a two-year period $(n=123)$. The authors proceeded to collect data regarding age, gender, Braden Scale, lesion location and stage, body mass index (BMI), mechanical ventilation, sedation, vasopressor use, renal function replacement therapy (RFRT), Acute Physiology and Chronic Health Evaluation II (APACHE II), Simplified Acute Physiology Score II (SAPS II), Sequential Organ Failure Assessment (SOFA), Mortality Probability Models II (MPM II) and length of stay in the Intensive Care Unit.

Results. The average age was 68 years, scored an average of 9 on the Braden scale (very high risk), mean BMI 27 (overweight) and the average length of stay in the ICU was 11 days. The majority of patients were male $(92,7 \%)$, with RFRT $(56,9 \%)$, vasopressor support $(76,4 \%)$ and a substantial percentage of patients was sedated and under mechanical ventilation $(80,5 \%)$. The sacral region and heels were the most affected anatomical sites accounting for $55,3 \%$ and $26,8 \%$ of all pressure injuries, respectively. Most lesions were stage II and 72,4\% were associated with a high Braden scale score. Prevalence was 10,8\% and mortality was $65,9 \%$. The categorical variables were tested by the Chi-square test and the continuous variables were tested by the non-parametric Mann-Whitney test and Student's t-test. The variables that are independently related to mortality are mechanical ventilation $(p<0,0001)$, sedation $(p<0,0001), R F R T(p<0.0001)$, vasopressor use $(p<0,0001), B M I(p<0,0001)$, SAPS II $(p<0,0001)$ and length of stay in the ICU $(p<0,0001)$.

Conclusion. Pressure injuries are a common burden and a substantial complication for healthcare systems so their prevention deserves increased clinical awareness.

\section{Reference(s)}

1. 1. 2. Sala JJ, Mayampurath A, Solmos S et al. (2020) Predictors of pressure injury development in critically ill adults: a retrospective cohort study. Intensive Crit Care Nurse.

2. 1. Sala JJ, Mayampurath A, Solmos S et al. (2020) Predictors of pressure injury development in critically ill adults: a retrospective cohort study. Intensive Crit Care Nurse.

\section{0}

Mortality risk factors and stratifications on critically ill hospitalized patients with covid 19 pneumonia in hispanic community of Laredo

RJ. Deliz; S. neppala ${ }^{2}$; M. Sierra-Hoffman ${ }^{3}$

${ }^{1}$ Medicine, Laredo Medical Center, Laredo, United States of America; ${ }^{2}$ Internal Medicine, University of Incarnate Word, laredo, United States of America; ${ }^{3}$ Medicine, Texas A\&M, Victoria, United States of America Correspondence: R.J. Deliz

Intensive Care Medicine Experimental 2021, 9(1): 000790
Introduction. Obesity, diabetes mellitus type 2 and hypertension has been associated with adverse outcomes in COVID 19 pneumonia. The city of Laredo has $97 \%$ hispanic population with a prevalence of $35.7 \%$ obesity. Hispanics in USA had 3.5 times of increased death and 2.3 times of hospitalization rate compared to caucasians. The variables causing death and hospitalizations need to be investigated.

Objectives. Identify variables causing death, complications, hospitalization related to obesity and diabetes mellitus type 2 comparing ICU to non ICU patients.

Methods. Variables reported as mean with standard deviation with percentages. Fisher's exact test was used to compare variables between groups among multiple proportions. Relative risk (RR) and 95\% confidence interval $(\mathrm{Cl})$ were calculated along with p-values. Clinical outcomes were compared between groups using multivariable logistic regression adjusting for potential counfounding covariables. Body mass index (BMI) subclassification were divided for diabetes mellitus type 2 with non diabetic patients.

Results. 950 hispanic patients were included in the study (male-502, female-448) admitted as inpatients in Laredo Medical Center hospital. $224(23.6 \%)$ were transfered to ICU with $91.1 \%$ invasive mechanical ventilation and $8.9 \%$ with high flow oxygen therapy. 726 were distributed to telemetry units requiring non invasive mechanical ventilation (27\%), CPAP $5 \%$, and non high flow oxygen therapy $67 \%$. From the whole population $2 \%$ were underweight, $16 \%$ had normal BMI, $29 \%$ were overweight, $26 \%$ with obesity class $1,15 \%$ obesity class 2 , and $12 \%$ obesity class 3 . The mean age was 57.74 years old range 21-98 years old. The lenght of stay was 11.08 days. Population of study: hypertension $56.1 \%$, diabetes mellitus type $252.5 \%$, hyperlipidemia $32.94 \%$, and congestive heart failure $13.78 \% .19 .05 \%$ of patients died during the hospitalization. An increase of mortality was found in obesity class 2 (RR4.14, 95\% $\mathrm{Cl}=2.2-7.7$, $\mathrm{p}$ value $<0.0001$ ) and obesity class 3 (RR $6.0,95 \% \mathrm{Cl}=1.3-4.6$, p value 0.0001$)$ compared with those with normal BMI. ARDS, atrial fibrillation, acute kidney injurie were more common in obese population (table available but not included due to limitations in space). There was a higher mortality in diabetic pateiths belonging to class 2 and 3 obesity compared to nondiabetics in obesity class 2 (RR:6.42 vs 3.74) and class 3 (RR:8.14 vs. 6.55) respectively. Obese patients were most likely to be intubated on mechanical ventilation: class 1 RR 2.61, class 2 RR 4.14, class 3 RR 6.0 .

Conclusion. This study demonstrates a correlation with covid -19 deaths associated with subclases of obesity with and, without diabetes mellitus type 2 indicating a higher mortality with the grade of obesity. Diabetes mellitus mortality was higher in combination with higher degress of obesity. The risk of intubation and mechanical ventilation correlated with higher levels of obesity.

\section{Reference(s)}

1. Apicella,M, Campopiano MC, Mantuano, M et al. COVID 19 in people with diabetes: understanding the reasons for worse outcomes. The Lancet Diabetes \& Endocrinology, https://doi.org/10.1016/S2213-8587(20)30238$2(2020)$

2. 2. De Santi F, Zoppini G, Locatelli F, et al. Type 2 diabetes is associated with an increased prevalence of respiratory symptoms as compared to the general population. BMC Pulm Med. 2017;17 (1): 55-60.

3. 1. Huang, Y, Lu Y, Huang, YM, et al. Obesity in patients with COVID 19: a systematic review and meta-analysis. Metabolism. 2020;113:154378. https://doi.org/10.1016/j.metabol.2020.154378.

\section{8}

Renal tubular acidosis in pregnant COVID-19 patients

S. Humbel ${ }^{1}$; S. Unseld ${ }^{1}$; PD. Wendel Garcia' ${ }^{1}$;. David ${ }^{1}$; R. Andermatt ${ }^{1}$ ${ }^{1}$ Institute of Intensive Care Medicine, University Hospital Zurich, Zurich, Switzerland

Correspondence: $\mathrm{R}$. Andermatt

Intensive Care Medicine Experimental 2021, 9(1): 001208

Introduction. Metabolic acidosis is a common problem in critically ill patients and often a consequence of acute kidney injury or lactic acidosis. With normal renal function and normal anion gap (AG), the 
diagnostic approach involves a urine analysis and the differential diagnosis are more complex. Renal tubular acidosis (RTA) is a rare disorder $(10$ in 100,000) in which, despite a well-preserved glomerular filtration rate (GFR), metabolic acidosis develops because of either a defect in the secretion of $\mathrm{H}+$ (Type I) or the inability to reabsorb bicarbonate (Type II) [1]. RTA has not been reported in COVID-19 patients, but single cases have been associated with pregnancy [2].

Objectives. We have observed a high percentage of normal AG (hyperchloremic) metabolic acidosis in pregnant critically ill COVID19 patients and systematically analyzed the prevalence, etiology and clinical course.

Methods. Retrospective analysis from patient data monitoring system and chart review.

Results. From February 2020 to April 2021321 COVID-19 patients were admitted to the intensive care units (ICU) of the University Hospital Zurich. Ninety-five (29.5\%) were women and 8 were either pregnant or just had C-section before admission to the ICU (age 24 to 42 years, $32 \pm 2.7$ gestational week). Seven women had symptomatic COVID-19 and were included in the study. Despite a normal renal function (eGFR $137 \pm 17 \mathrm{ml} / \mathrm{min} / \mathrm{m}^{2} \mathrm{BSA}$ ), 6 out of these 7 patients (85.7\%) either presented themselves on admission or during ICU-stay with an AG negative metabolic acidosis $(\mathrm{pH} 7.27 \pm 0.1$, min $\mathrm{HCO} 3-$ $15.6 \pm 3.1 \mathrm{mmol} / \mathrm{l}, \mathrm{pCO}_{2} 3.8 \pm 1.0$ ). Despite the generally rare incidence of RTA, we found that 3 of these 6 acidotic women fulfilled diagnostic criteria for RTA. Sodium bicarbonate was given over $2.3 \pm 0.5$ days in $43 \%$ of them. All women recovered from RTA within less than 7 days.

Conclusion. Here we report for the first time that metabolic acidosis might be very common (85\%) in critically ill pregnant COVID-19 patients and further half of the women had evidence of an extremely rare tubulopathy. We want to point out that the diagnosis of RTA was not made in all of these patients during their ICU stay, but only afterwards, which underlines the relevance of our findings for raising awareness among intensivists treating (pregnant) COVID-19 patients. It remains to be demonstrated if this observation is an indirect epiphenomenon or a putative direct viral effect on the tubular epithelium.

\section{Reference(s)}

1. 1. Palmer, B.F., E. Kelepouris, and D.J. Clegg, Renal Tubular Acidosis and Management Strategies: A Narrative Review. Adv Ther, 2021. 38(2): p. 949-968.

2. 2. Seeger, H., et al., Complicated pregnancies in inherited distal renal tubular acidosis: importance of acid-base balance. J Nephrol, 2017. 30(3): p. $455-460$

\section{6}

\section{Adult with a Paediatric Diagnosis after Covid-19 Infection}

P. Szawarski ${ }^{1}$; R. Whittaker, ${ }^{2}$; M. Riyat ${ }^{3}$; A. Mandal, ${ }^{4}$

${ }^{1}$ Intensive Care and Anaesthesia, Frimley Health NHS Foundation Trust, Slough, United Kingdom; ${ }^{2}$ Critcal care and thetares, Frimley Health NHS Foundation Trust-Wexham Park Hospital, Slough, United Kingdom; ${ }^{3}$ Intensive care and anaesthesia, Frimley Health NHS Foundation Trust, Slough, United Kingdom; ${ }^{4}$ Department of cardiology, Frimley Health NHS Foundation Trust, Slough, United Kingdom

Correspondence: P. Szawarski

Intensive Care Medicine Experimental 2021, 9(1): 001226

Introduction. The impact of Covid-19 infection on myocardium in adults is complex. In young patients whilst thrombosis, strongly linked to Covid-19 infection remains a possibility, viral myocarditis and immune mediated myocardial dysfunction such as described in context of Paediatric Multisystem inflammatory syndrome temporarily associated with Covid-19 (PIMS-TS (or MIS-C) are two important differential diagnoses. Failure to recognise this unusual condition may preclude successful treatment.

Methods. We would like to present a case of successful management of an adult with PIMS TS.

Results. 20 year old Caucasian male has been admitted with a two day history of fever, dizziness, abdominal discomfort and maculopapular rash. He had a history of asymptomatic Covid-19 infection six weeks prior to the admission. He was initially treated for sepsis. He had hypotension that was refractory to fluid administration and was admitted to the intensive care unit for vasopressors. There he continued to deteriorate with evidence of pulmonary oedema, increasing vasopressor requirements, eventually requiring intubation. His echo showed normal left ventricle cavity size with significantly reduced LVEF of $33 \%$ by Simpson's biplane. Peak global longitudinal strain (GLS) was $5.5 \%$. There was global hypokinesia predominantly affecting the left ventricle. His NT-pro BNP peaked at $>35,000 \mathrm{ng} / \mathrm{L}$ and troponin T peaked at $904 \mathrm{ng} / \mathrm{L}$. The diagnosis was tentatively revised to PIMS-TS. Treatment with IVIG and methylprednisolone was commenced resulting in improvement in clinical condition and resolution of echocardiographic myocardial dysfunction and return of NT-proBNP and troponin T to normal values. At follow up MRI showed patchy fibrosis.

Conclusion. PIMS TS should be a differential diagnosis in adults presenting with acute heart failure in context of Covid-19 pandemic. The diagnosis although described primarily in children should be considered in older patients as well, as failure to do so may preclude successful therapy.

\section{3}

The Effect of Corticosteroid Treatment on Secondary Bacterial Infection and Mortality Rates of COVID-19 Patients in ICU

E. Uysal'; IÖ. Turan, ${ }^{1} ;$ H. Ankaralı, ${ }^{2} ;$ A. Baştuğ ${ }^{3}$

${ }^{1}$ Intensive Care Unit, Ankara Sehir Hastanesi, Ankara, Turkey: ${ }^{2}$ Biostatistics and medical informatics, Istanbul Medeniyet University, İstanbul, Turkey;

${ }^{3}$ Clinic of infectious diseases and clinical microbiology, Ankara Şehir

Hastanesi, Ankara, Turkey

Correspondence:Correspondence: $E$. Uysal

Intensive Care Medicine Experimental 2021, 9(1): 001413

Introduction. There are recommendations and studies for the corticosteroid treatment of cytokine storm and ARDS in COVID-19 disease. Our aim is to evaluate the rates of secondary infections and mortality in COVID-19 patients receiving various doses of corticosteroid treatment in the ICU.

Methods. In the COVID Intensive Care Unit, 622 patients were retrospectively analyzed and recorded in terms of age, gender, duration of mechanical ventilation, length of stay (LOS) in intensive care, CRP, procalcitonin, $\mathrm{LDH}$, IL-6, lymphocyte, D-dimer, ferritin values and corticosteroid doses as well as blood, urine and deep tracheal aspiration growths. The patients were examined in 6 groups as those who never took corticosteroids, those who took methylprednisolone in doses of $250 \mathrm{mg}$, >250 mg, $80 \mathrm{mg}, 40 \mathrm{mg}$ and $6 \mathrm{mg}$ dexamethasone. Only one patient received hydrocortisone. So we excluded this patient.

In addition, the patients were classified according to the "cytokine storm score" (CSS) by calculating their laboratory values. Patients with scores $\geq 2$ were accepted as patients with cytokine storm. The data was evaluated to determine if there has been significant relationships between corticosteroid doses and the rates of secondary infection and mortality.

Results. Most of the patients received dexamethasone treatment of $6 \mathrm{mg}$ (190 patients, 30\%), whereas $66(10 \%)$ patients were not given any corticosteroids. Among the patients receiving methylprednisolone, 120 of them received $>250 \mathrm{mg}(19 \%), 90(14 \%)$ received $250 \mathrm{mg}$, $101(16 \%)$ received $80 \mathrm{mg}$, and $54(8.7 \%)$ received $40 \mathrm{mg}$. There was no significant difference between the patients who did not use corticosteroids and those who received $40 \mathrm{mg}$ methylprednisolone according to ICU LOS, but the LOS of both groups were shorter than the other corticosteroid groups $(p<0.001)$.

There were 37 patients in the CSS $<2$ group and 584 in the CSS $\geq 2$ group. Mortality rates were found to be significantly lower in patients who received methylprednisolone in doses of $>250 \mathrm{mg}$ in the CSS $>2$ group when compared with the other doses $(p=0.046)$. There was no significant difference between all the groups according to the frequency of secondary infection.

Conclusion. It has been observed that the use of different doses of corticosteroids in intensive care patients with COVID-19 does not increase the rate of secondary infection. In patients with cytokine storm the mortality rate due to COVID 19 may be lower with methylprednisolone in doses of $>250 \mathrm{mg}$. 
e-Posters: Transfusion and haemostasis disorders

\section{6}

Anaemia amongst intensive care survivors and association with days alive and at home: An observational study

S. van der Laan ${ }^{1}$; T. Billah, ${ }^{2}$; C. Chi, ${ }^{2}$; C. Lai, ${ }^{2}$, E. Litton, ${ }^{1}$

${ }^{1}$ Intensive Care Unit, Fiona Stanley Hospital, Murdoch, Australia; ${ }^{2}$ Medical student, Curtin University, Bentley, Australia

Correspondence: $\mathrm{S}$. van der Laan

Intensive Care Medicine Experimental 2021, 9(1): 000056

Introduction. Anaemia is highly prevalent at the time of intensive care discharge and is persistent for a high proportion of ICU survivors [1]. Whether anaemia is a driver of impaired recovery after critical illness is uncertain.

Objectives. The aim of this study was to test the hypothesis that, in adult intensive care survivors, anaemia at the time of intensive care discharge independently predicts decreased days alive and at home to Day 90 (DAAH90). Secondary outcomes of interest were hospital length of stay following index ICU admission, ICU and hospital readmission and all-cause mortality to day 90 .

Methods. This retrospective cohort study was conducted in the 30-bed mixed medical and surgical ICU of a tertiary centre in Perth, Western Australia. All patients $>16$ years of age, discharged alive from their index ICU admission, and without documented treatment limitations were included in the study. The primary outcome was DAAH90, Secondary outcomes included the components of DAAH90 (Day 90 all-cause mortality and total hospital length of stay amongst survivors), and other markers of morbidity including ICU readmission during the index hospitalisation and hospital readmission. The ICU clinical information system and the state-wide hospital administration system were used to extract the relevant data.

Results. Between 2017 and 2019, there were 6358 ICU admissions eligible for inclusion in the study. There were 2886 (45.4\%) patients discharged from ICU with a haemoglobin of $<100 \mathrm{~g} / \mathrm{L}$. Amongst these patients, the haemoglobin at hospital discharge was $<100 \mathrm{~g} / \mathrm{L}$ in $1948(69.7 \%)$. The DAAH90 for ICU survivors with a discharge haemoglobin $<100 \mathrm{~g} / \mathrm{L}$ was 80 versus 85 for those with a haemoglobin of $>100 \mathrm{~g} / \mathrm{L}$, median difference 5 [95\% confidence interval (Cl) 4.4-5.5], $\mathrm{P}<0.0001$. The association followed a severity-response relationship with more severe anaemia predicting lower DAAH90. A haemoglobin of $<100 \mathrm{~g} / \mathrm{L}$ at ICU discharge was independently associated with prolonged hospitalisation amongst ICU survivors, increased risk of ICU readmission, increased risk of hospital readmission, but not all-cause mortality to Day 90.

Conclusion. Anaemia is highly prevalent amongst ICU survivors and frequently persistent at hospital discharge. A haemoglobin of $<100 \mathrm{~g} / \mathrm{L}$ at ICU discharge was an independent risk factor for fewer days alive and at home to Day 90, and increased risk of ICU and hospital readmission.

\section{Reference(s)}

1. 1. Walsh TS, Lee RJ, Maciver CR, et al. Anemia during and at discharge from intensive care: the impact of restrictive blood transfusion practice. Intensive Care Med 2006; 32: 100-9.

\section{3}

Cold vs Room temperature: Which provides better platelet hemostasis for short term storage of whole blood?

E. Kusudo ${ }^{1}$; Y. Murata ${ }^{1}$; S. Kawamoto ${ }^{1}$; K. Fukuda ${ }^{1}$

${ }^{1}$ Department of Anesthesia, Kyoto University Hospital, Kyoto, Japan Correspondence: E. Kusudo

Intensive Care Medicine Experimental 2021, 9(1): 000073

Introduction. Acute normovolemic hemodilution (ANH) could be an alternative transfusion method to allogenic blood transfusion, which is valuable and at risk for donor-derived infections. In cardiovascular surgery with cardiopulmonary bypass where platelet function is severely compromised, transfusion of blood with preserved platelet function is desirable to reduce blood loss. It has been reported that the hemostatic capacity of platelets stored at cold temperature for several days is higher than that of platelets stored at room temperature [1]. However, there have been no reports of evaluating platelet function under cold storage in $\mathrm{ANH}$, where whole blood was conventionally stored at room temperature for several hours.

Objectives. The aim of this study is to determine whether changing the storage temperature to 4 degrees Celsius from 22 degrees Celsius increases platelet hemostasis when we store whole blood for $6 \mathrm{~h}$.

Methods. The protocol was approved by the ethical committee. Blood was collected from 6 healthy adults with documented consent and mixed with the anticoagulant CPDA (citrate-phosphate-dextroseadenine) currently used in ANH. The sample blood was stored at 4 degrees Celsius (group C) or 22 degrees Celsius (group R) for $6 \mathrm{~h}$. The platelet count was then adjusted to the same number of platelets to measure adenosine diphosphate (ADP)-induced platelet aggregability by light transmission aggregometry, and the expression of CD62P (P-selectin), a marker of platelet quality degradation using flow cytometry. We also performed thromboelastography (TEG), blood count and blood gas analysis.

Results. ADP-induced maximum platelet aggregation rate was significantly higher in group $C$ than group R. There was no significant difference between the two groups in P-selectin expression, TEG and blood count. Group $\mathrm{C}$ had significant higher $\mathrm{pH}$ and lower lactate levels compared to group $\mathrm{R}$ (Table).

\begin{tabular}{|c|c|c|c|c|}
\hline Parameter & Before storage & Group C & Group $R$ & Pvalue \\
\hline \multicolumn{5}{|l|}{ Maximum plateletentager agregation rate } \\
\hline ADP $2 \mu \mathrm{M}(\%)$ & $23.08 \pm 21.43$ & $24.83 \pm 14.63$ & $8.17 \pm 3.49$ & $0.001 *$ \\
\hline ADP $4 \mu M(\%)$ & $45.67 \pm 21.05$ & $47.67 \pm 14.77$ & $22.17 \pm 5.43$ & $0.004 \cdots$ \\
\hline ADP $6 \mu M(\%)$ & $51.92 \pm 16.75$ & $51.67 \pm 15.38$ & $33.33 \pm 14.70$ & $0.025 *$ \\
\hline P-selectin expression (MFI) & $16.69 \pm 5.17$ & $70.43 \pm 33.67$ & $59.27 \pm 19.27$ & 0.463 \\
\hline \multicolumn{5}{|l|}{ TEG } \\
\hline CRT-MA $(\mathrm{mm})$ & $61.33 \pm 1.96$ & $59.77 \pm 3.57$ & $61.35 \pm 3.43$ & 0.184 \\
\hline (CRT-MA)-(CFF-MA) (mm) & $46.10 \pm 3.75$ & $43.27 \pm 2.05$ & $43.87 \pm 2.28$ & $\begin{array}{l}0.620 \\
0.620\end{array}$ \\
\hline Platelet count $\left(\times 10^{9} \mathrm{~L}\right)$ & $183.5 \pm 29.02$ & $195.0 \pm 33.89$ & $182.7 \pm 40.16$ & 0.082 \\
\hline Hemoglobin $(g / d \mathrm{~L})$ & $13.07 \pm 0.75$ & $13.65 \pm 1.49$ & $13.60 \pm 1.78$ & 0.758 \\
\hline Mean corpuscular volume (\%) & $89.22 \pm 2.71$ & $88.78 \pm 2.79$ & $89.18 \pm 2.72$ & 0.153 \\
\hline & $6.968 \pm 0.042$ & $6.958 \pm 0.026$ & $6.938 \pm 0.036$ & $0.025 *$ \\
\hline Lactate (mg/dL) & $13.27 \pm 4.51$ & $16.70 \pm 3.95$ & $24.73 \pm 3.30$ & 0.010 \\
\hline \multicolumn{5}{|c|}{ 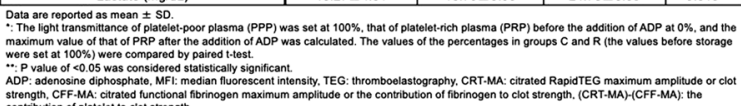 } \\
\hline
\end{tabular}

Conclusion. Six-hour cold storage of whole blood can preserve ADPinduced platelet aggregability, and might be adequate for platelet hemostasis in cardiovascular surgery with cardiopulmonary bypass.

Reference(s)

1. Grant-in-Aid for Scientific Research (KAKENHI): 19 K18293

2. [1] G.A. Becker, M. Tuccelli, T. Kunicki, M.K. Chalos, R.H. Aster. Studies of platelet concentrates stored at $22 \mathrm{C}$ and 4 C. Transfusion 1973;13(2):61-68

000205

Coagulopathy following intra-operative major haemorrhage protocol activation

R. Gillespie'; R. Noori' ${ }^{1}$; S. Williams

${ }^{1}$ Critical Care/Anaesthesia, Aberdeen Royal Infirmary, Aberdeen, United Kingdom

Correspondence: R. Gillespie

Intensive Care Medicine Experimental 2021, 9(1): 000205

Introduction. Blood product administration during intra-operative major haemorrhage is guided by a major haemorrhage protocol (MHP). Standard 'shock packs' of blood products are provided empirically, with subsequent products administered under clinician judgement supported with point-of-care tests (blood gas, viscoelastic assays) and standard lab tests (blood count, coagulation screen, fibrinogen).

Objectives. Are patients coaguloathic on admission to critical care following intra-operative major haemorrhage? What blood products did 
these patients receive? Was a viscoelastic assay (ROTEM) done during intra-operative major haemorrhage?

Methods. In this retrospective audit, 69 MHP activations occurred at Aberdeen Royal Infirmary between January 2019-March 2020. Of which, 19 were intra-operative and resulted in critical care admission. Three cases were excluded due to inadequate documentation or no blood products administered. Data was extracted from electronic patient records and point-of-care testing equipment databases.

Results. Of the 16 patients, 6 were coagulopathic (38\%) on admission to ICU, with criteria derived from the UK Association of Anaesthetists' guidance on 'use of blood components' 2016: INR $>1.5$ (2 patients), Fibrinogen $<1.0 \mathrm{~g} / \mathrm{L}$ ( 1 patient), Platelets $<50 \times 10^{\wedge} 6(0$ patients). In addition, patients with APTT $>40 \mathrm{~s}$ ( 3 patients) were classified as coagulopathic. The heterogenous cohort comprised of 9 female patients and a breadth of procedures including nephrectomy, Whipples, aortic aneurysm repair, macroadenoma debulking among others.

Coagulopathic patients and non-coagulopathic patients had similar average estimated blood loss (EBL) at 3600 and $3800 \mathrm{mls}$, respectively. The range was wide at $1000-12000 \mathrm{mls}$. Coagulopathic patients had an average transfusion ratio of Red Cells:Fresh Frozen Plasma:Platelets of 13:4:1 and received $3600 \mathrm{mls}$ of crystalloid whereas non-coagulopathic patients had an average ratio of 8:6:1 and $2700 \mathrm{mls}$ of crystalloid.

Table 1 Mean blood product administration and transfusion ratios for coagulopathic vs non-coagulopathic patients

\begin{tabular}{|c|c|c|c|c|}
\hline Ratio & Coagulopathic & & $\begin{array}{l}\text { Non- } \\
\text { Coagulo- } \\
\text { pathic }\end{array}$ & Ratio \\
\hline & 6 & $\begin{array}{l}\text { Number of patients } \\
\quad(\text { total }=16)\end{array}$ & 10 & \\
\hline 13 & 7.5 & Packed Red Cells (units) & 6.8 & 8 \\
\hline 4 & 2.3 & $\begin{array}{l}\text { Fresh Frozen Plasma } \\
\text { (units) }\end{array}$ & 4.1 & 6 \\
\hline \multirow[t]{5}{*}{1} & 0.5 & Platelets (units) & 1.3 & 1 \\
\hline & 0.3 & Cryoprecipitate (pools) & 0.5 & \\
\hline & 3600 & Crystalloid (mls) & 2700 & \\
\hline & 500 & Colloid (mls) & 700 & \\
\hline & 3 & ROTEM used? (frequency) & 4 & \\
\hline
\end{tabular}

Seven patients (44\%) had viscoelastic assays (ROTEM) tested during intra-operative major haemorrhage. Those who had ROTEM tests (vs those without) had greater average EBL (4800 vs $2800 \mathrm{mls}$ ) and more balanced transfusion ratios (7:4:1 vs 12:6:1).

Conclusion. Evidence from military and trauma medicine supports 'haemostatic resuscitation' in major haemorrhage aiming for blood transfusion ratios that approach physiological ratios, rather than 'volume resuscitation' with crystalloid. Evidence to inform blood product administration for intra-operative major haemorrhage is sparse.

Over a third of patients with intra-operative major haemorrhage were coagulopathic on admission to critical care post-operatively. Coagulopathic patients had higher transfusion ratios and received more crystalloid.

\section{Reference(s)}

1. Dhara S, Moore EE, Yaffe MB, Moore HB, Barrett CD. Modern Management of Bleeding, Clotting, and Coagulopathy in Trauma Patients: What Is the Role of Viscoelastic Assays? Curr Trauma Rep. 2020 Mar;6(1):69-81. https://doi.org/10.1007/s40719-020-00183-w. Epub 2020 Jan 23. PMID: 32864298; PMCID: PMC7453728.

2. Association of Anaesthetists. The use of blood products and their alternatives. 2016 July.Available at: https://anaesthetists.org/Home/Resourcespublications/Guidelines/Blood-components-and-their-alternatives

3. Holcomb JB, Jenkins D, Rhee P, Johannigman J, Mahoney P, Mehta S, Cox ED, Gehrke MJ, Beilman GJ, Schreiber M, Flaherty SF, Grathwohl KW,
Spinella PC, Perkins JG, Beekley AC, McMullin NR, Park MS, Gonzalez EA, Wade CE, Dubick MA, Schwab CW, Moore FA, Champion HR, Hoyt DB, Hess JR. Damage control resuscitation: directly addressing the early coagulopathy of trauma. J Trauma. 2007 Feb;62(2):307-10. https://doi. org/10.1097/TA.0b013e3180324124. PMID: 17297317

\section{8}

Analysis of Pulmonary Embolism Severity Index (PESI)

\section{Contributing Factors in COVID-19 Patients}

M. Cindryani'; N. Juwita²; T. Gde Agung Senapathi ${ }^{2} ;$ M. Wiryana ${ }^{2}$

${ }^{1}$ Anestesi dan Terapi Intensif, Universitas Indonesia, Jakarta, Indonesia; ${ }^{2}$ Anestesiologi dan terapi intensif, Udayana University, Denpasar, Indonesia

Correspondence: M. Cindryan

Intensive Care Medicine Experimental 2021, 9(1): 000268

Introduction. Pulmonary embolism has been greatly predicted as one of mortality cause in COVID-19. Due to incidence of thrombotic complications in ICU patients with COVID-19 is remarkably high (31\%)1, ISTH recommended to apply thrombosis prophylaxis in all COVID-19 patients admitted to ICU. A meta-analysis by Liao in 2020 had shown incidence and mortality rate of COVID-19 patients developing pulmonary embolism (PE) was 15.3\% (95\%: 9.8-21.9) and 45.1\% (95\%: 22.0-69.4) even with high statistical heterogeneity among studies reporting PE incidence $(I 2: 92.0 \%, p<0.001) 2$. Unfortunately, there is no established scoring system for assessing risk of pulmonary embolism in COVID-19.

Objectives. To observe any associations between contributing factors of pulmonary embolism in COVID-19 patients and Pulmonary Embolism Severity Index (PESI) score.3

Methods. We collected data from 20 admitted ICU patients from December 2020-January 2021 based on their characteristics and several related factors that might be contributed to pulmonary embolism. We also counted their Pulmonary Embolism Severity Index (PESI) score and we did bivariate analysis of those factors.

Results. There are numerical variables such as D-dimer, INR, Respiratory Rate (RR), P/F Ratio, Procalcitonin, CRP, Ferritin, temperature, platelet, and categorical variables such as antiviral type, comorbidities, smoking history, and ventilation type. Only ventilation type in which divided into high flow type and mechanical ventilation type who are shown significant in their associations with PESI score.

\begin{tabular}{|c|c|c|c|c|c|}
\hline \multicolumn{6}{|c|}{ Independent Samples Test } \\
\hline & & \multicolumn{4}{|c|}{ t-test for Equality of Means } \\
\hline & & $\begin{array}{l}\text { Sig. } \\
\text { (2-tailed) }\end{array}$ & $\begin{array}{l}\text { Mean dif- } \\
\text { ference }\end{array}$ & $\begin{array}{l}\text { Std. error } \\
\text { difference }\end{array}$ & $\begin{array}{l}95 \% \text { confi- } \\
\text { dence inter- } \\
\text { val of the } \\
\text { difference }\end{array}$ \\
\hline & & & & & Lower \\
\hline \multirow[t]{2}{*}{ PESI } & $\begin{array}{l}\text { Equal variances } \\
\text { assumed }\end{array}$ & 0.001 & 40.212 & 10.299 & 18.575 \\
\hline & $\begin{array}{l}\text { Equal variances not } \\
\text { assumed }\end{array}$ & 0.003 & 40.212 & 10.916 & 16.367 \\
\hline
\end{tabular}

\begin{tabular}{lrlllll}
\hline Coefficients & \multicolumn{2}{l}{} \\
\hline Model & \multicolumn{2}{l}{$\begin{array}{l}\text { Unstandardized Coef- } \\
\text { ficients }\end{array}$} & $\begin{array}{l}\text { Standardized } \\
\text { Coefficients }\end{array}$ & t & Sig \\
\cline { 2 - 7 } & B & Std. Error & Beta & & \\
\hline $1 \quad$ (Constant) & 146.391 & 20.938 & & 6.992 & 0.000 \\
ventilation & -38.318 & 10.930 & -0.645 & -3.506 & 0.003 \\
comorbidities & -16.482 & 13.176 & -0.223 & -1.251 & 0.229 \\
soking & 4.342 & 11.217 & 0.072 & 0.387 & 0.704 \\
\hline
\end{tabular}

There is also statistically significant relationship between PESI scoring and mortality risk between ICU COVID-19 patients. 


\begin{tabular}{llll}
\hline Step & $\begin{array}{l}\boldsymbol{- 2} \text { Log likeli- } \\
\text { hood }\end{array}$ & $\begin{array}{l}\text { Cox \& Snell R } \\
\text { Square }\end{array}$ & $\begin{array}{l}\text { Nagelkerke } \mathbf{R} \\
\text { Square }\end{array}$ \\
\hline 1 & $17.363 \mathrm{a}$ & 0.124 & 0.196 \\
\hline
\end{tabular}

In mechanically ventilated patients, there is a higher probability of PESI scoring which leads to a higher risk of PE. Subjects with a higher PESI score of 17.36 points would have a 1.2 times higher risk of mortality.

Conclusion. There is a statistically significant relationship between the type of ventilation and the PESI score probably suggested by positive pressure ventilation and subsequent increased intra-thoracic pressure. There is also statistically significant relationship between PESI scoring and mortality risk between ICU COVID-19 patients. In mechanically ventilated patients, there is a higher probability of PESI scoring which leads to a higher risk of PE and mortality.

\section{Reference(s)}

1. 1. Klok FA, Kruip MJHA, van der Meer NJM, et al. Incidence of thrombotic complications in critically ill ICU patients with COVID-19. J Thromb Res. 2020;191:145-147. https://doi.org/10.1016/j.thromres.2020.04.013.

2. 2. Liao S., Shao S., Chen Y., Chen YC, Hung M. Incidence and mortality of pulmonary embolism in COVID-19: a systematic review and meta-analysis. Critical Care (2020) 24:464 https://doi.org/10.1186/ s13054-020-03175-z.

3. 3. Aujesky, D.2005. Available at: https://www.mdcalc.com/pulmonaryembolism-severity-index-pesi.

4. No disclosure

\section{4}

"Ash Without A Fire": Discordance between D-Dimer Levels and Systemic Fibrinolytic Activity in Critically III COVID-19

\section{Patients}

G. Garcia Rosenbaum¹; R. Dujardin²; JS. Raval, ${ }^{3} ;$ MA. Rollins-Raval, ${ }^{3} ;$ N. Juffermans ${ }^{2} ;$ ND. Nielsen ${ }^{4}$

${ }^{1}$ Department of Internal Medicine, University of New Mexico School of Medicine, Albuquerque, United States of America; ${ }^{2}$ Department of Intensive Care, Amsterdam UMC, locatie AMC, Amsterdam, Netherlands; ${ }^{3}$ Department of Pathology, University of New Mexico School of Medicine, Albuquerque, United States of America; ${ }^{4}$ Departments of Internal Medicine and Pathology, The University of New Mexico, Albuquerque, United States of America

Correspondence: N.D. Nielsen

Intensive Care Medicine Experimental 2021, 9(1): 000494

Introduction. The deposition of microthrombi into various organs, particularly the lung and kidneys, has been posited as a major pathophysiological mechanism in severe COVID-19. However, very little is known of the regulation of the thrombolytic processes responsible for the clearance of these thrombi. Markedly elevated D-dimer levels are a consistently observed phenomenon in COVID-19, even in the absence of macrothrombi (i.e.: DVT, PE), leading to the supposition that very large numbers of microthrombi must be present. In order to better understand the processes leading to these highly elevated D-dimer levels and related microthrombosis-mediated organ injury, we initiated a prospective study of thrombotic and fibrinolytic activity in patients with COVID-19 admitted to our ICU.

Methods. In this prospective cohort study of critically ill COVID-19 adult patients, the following coagulation parameters were measured within $48 \mathrm{~h}$ of ICU admission: activated partial thromboplastin time (aPTT), prothrombin time (PT), D-dimer, plasminogen activity levels, plasminogen activator inhibitor 1 (PAI-1), Factor XIII (FXIII), and maximum lysis percentage (ML\%) on ROTEM. We also analyzed the cohort of patients with and without venous thromboembolism (VTE) and patients who died or were discharged alive from ICU. Additionally, we performed correlations using Spearman's rho between: a) Admission SOFA score and PAI-1, plasminogen and FXIII; and b) Admission D-dimer and PAI-1, plasminogen and FXIII.
Results. Results from 91 patients showed a mean age of 61 (IQR: 51-69), 63.7\% male, mean BMI 31.6 (26.9-34.0), and mean SOFA score of 6 (4-10). PT, aPTT, and platelet count were 14.2 (12.7-16.5 secs), $31.0(27.0-35.5 \mathrm{secs})$ and $142(127-16510 \wedge 9 / \mathrm{L})$ respectively. D-dimer levels were markedly elevated, with a mean value of $2300 \mathrm{ng} / \mathrm{mL}$ (IQR: $1300-6800 \mathrm{ng} / \mathrm{mL}$; normal<500). Mean Plasminogen activity was $107 \%$ (normal: $71-144 \%$ ), mean PAl-1 activity was $15.7 \%$ (normal: 2-25\%), mean FXIII activity was $94.5 \%$ (normal 69-143\%). Mean EXTEM ML was 5\% (IQR: 3-7\%; normal < 15\%) and FIBTEM ML 2\% (0-4\%).

VTE was diagnosed in 25 patients $(27 \%)$, but patients with and without VTE showed no significant differences in D-dimer, plasminogen, PAI-1 or FXIII levels.

Spearman's rho showed a significant correlation between admission SOFA and PAI-1 levels $(p=0.01)$ and a negative linear correlation between admission D-dimer and plasminogen $(p=0.01)$.

Overall, even in the setting of very high D-dimer levels, plasminogen, PAI-1 and FXIII were all within normal range and clot lysis activity was low normal on ROTEM testing.

Conclusion. The primary findings of our study were that despite very high D-dimer levels being present, systemic fibrinolytic activity was normal or depressed-D-dimer levels were on average 4 times normal range, while functional clot lysis activity assessed by maximum lysis (ML) on ROTEM assays ranged from low-normal to nil and enzymatic fibrinolytic activity as measured by plasminogen activity was within the normal range. Further, PAI-1 levels (which if elevated, could explain suppressed plasminogen activity) were also normal, as were Factor XIII levels (which if elevated, would lead to "clot resistance" to fibrinolysis). Notably, these laboratory findings were seen both in patients found to have VTE and those without.

This presents a striking paradox-how can evidence of massive clot dissolution be present without evidence of similarly massive fibrinolytic activity? A proposed theory, bolstered by our study, is that in the setting of COVID-19, massive fibrinolysis is taking place within organ tissue beds and not systemically. This "intra-organ" fibrinolysis is mediated not by systemic fibrinolytic mechanisms but by local fibrinolytic pathways, mediated by the likes of urokinase plasminogen activator and streptokinase.

This theory requires more research, as the implications for clinical management of the microthromboses of COVID-19 are significantnamely, systemic therapies to augment or accelerate fibrinolysis are likely to be ineffective, and specific organ-targeted therapies may be required instead.

\section{Reference(s)}

1. Medcalf RL, et al. Fibrinolysis and COVID-19: A plasmin paradox. J Thromb Haemost 2020;18:2118-2122.

2. Ranucci M, et al. COVID-19 Associated Coagulopathy: Biomarkers of Thrombin Generation and Fibrinolysis Leading the Outcome. J Clin Med 2020;9:3487.

3. Ibanez C., et al. High D dimers and low global fibrinolysis exist in COVID19 patients: what is going on in there? J. Thrombosis Thrombolysis 2021;51:308-312.

4. Creel-Bulos C, et al. Fibrinolysis Shutdown and Thrombosis in a COVID-19 ICU. Shock 2021;55(3):316-320.

\section{7}

Thromboembolic phenomena in patients with covid-19 admitted to the ICU of Virgen de las Nieves Hospital

I. de Dios Chacón ${ }^{1}$; D. García Huertas²; V. Martínez de Pinillos Sánchez ${ }^{1}$

${ }^{1}$ Icu, University Hospital Complex of Granada, Granada, Spain; ${ }^{2}$ Intensive care unit, University Hospital Complex of Granada, Granada, Spain

Correspondence: I. de Dios Chacón

Intensive Care Medicine Experimental 2021, 9(1): 000527

Introduction. Recent studies have shown an increased prevalence of thromboembolic disease in critically ill patients with the novel SARSCoV-2 disease (COVID-19).

Objectives. To determine the incidence of thromboembolic phenomena, whether venous thrombosis (proximal and/or distal) as well as 
pulmonary embolism, in patients with SARS-CoV-2 pneumonia admitted to our intesive care unit during pandemic months.

Methods. We retrospectively analyzed COVID-19 patients that were admitted to our intensive care unit (ICU) between March 14, 2020 and March 30, 2021. Demographics parameters, laboratory and radiologic evaluations were analyzed.

Results. 315 patients were enrolled: 218 men (69\%) and 97 women (31\%), with a mean age of $60.43 \pm 13$ years. Doppler ultrasound was performed in 110 patients (35\%) and CT angiography in $55(17 \%)$ patients due to suspicion of thrombotic phenomena, based on clinical examination and $\mathrm{D}$-dimer values (median $\mathrm{D}$-dimer in these patients of $8.9 \mathrm{mg} / \mathrm{l}) .65$ thrombotic phenomena were found in 63 patiens (20\%). Venous thrombosis was identified in 48 patients $(15.2 \%)$ by venous doppler. Of these, 20 were proximal (41.6\%) and 29 distal (58.4\%). The mean D-dimer value was $14.8 \mathrm{mg} / \mathrm{l}$ (median 7.09). There was a higher incidence of venous thrombosis in men, 38 cases $(79.2 \%)$, while in women there were 10 cases $(20.8 \%)(p<0.05)$.

Pulmonary embolism was found in 17 patients (5.4\%). This group has a mean D-dimer value of $28.13 \mathrm{mg} / \mathrm{l}$ (median 16.01). The incidence of pulmonary embolism in men was higher: 13 cases $(76.5 \%)$ vs 4 cases $(23.5 \%)$ in women $(p<0.05)$.

The patients with thrombotic phenomena were older (61.87 years versus 60.16$)$ but without statistical significance $(p=0.47)$. Regarding mortality, it was $34.92 \%(n=20)$ in patients who presented with thrombotic phenomena, compared to $27.38 \%(n=69)$ who did not, without statistical significant differences $(p=0.49)$.

Conclusion. Thrombotic phenomena were frecuently detected as a complication in patients with SARS-CoV-2 pneumonia who were admitted to our unit, mainly venous thrombosis. Differences related to sex were found, being higher the incidence in men than in women. Although mortality was higher in patients with thrombotic events we did not found statistical differences between both groups.

\section{1}

\section{Retrospective evaluation of economy in blood products} transfusion for cardiac surgery patients thanks to a point-of-care viscoelastic test

A. Roussiaux ${ }^{1}$; JY. Bigeon ${ }^{1}$; C. Halchini' ; P. Burtin ${ }^{1}$; N. Ducrocq ${ }^{1}$; E. Lorne ${ }^{1}$ ${ }^{1}$ Anesthésie et réanimation, Clinique du Millénaire, Montpellier, France Correspondence: A. Roussiaux

Intensive Care Medicine Experimental 2021, 9(1): 000801

Introduction. Viscoelastic tests are relevant for postoperative bleeding management of cardiac surgery patients requiring cardiopulmonary bypass (CPB) (1) but a systematic review and meta-analysis did not demonstrate a reduction in the proportion of patients receiving any blood product (2).

Objectives. We aim to assess the real transfusion savings achieved through the implementation of a point-of-care (POC) viscoelastic tool in our practice.

Methods. We performed a retrospective analysis on two groups of patients. The first one pooled the 140 surgical patients who underwent cardiac surgery with CPB between September and December 2018 while the transfusion attitude was guided by clinical practice and guidances. The second group of 131 patients consisted of those operated between September and December 2019, after more than 6 months of practice with the sonorheometric haemostasis evaluation device Quantra ${ }^{\circledR}$ (Stago Biocare, France).

The data collected were blood loss volumes as well as any blood products transfused during the first $48 \mathrm{~h}$ in the intensive care unit (ICU).

Results. The demographics, the surgical case mix performed and the overall blood loss volumes $(753 \mathrm{ml}$ vs $770 \mathrm{ml}$ ) were comparable in both populations during the ICU stay. The comparison of transfusion attitudes shows that, although not using Quantra ${ }^{\circledR}$ systematically for each patient operated on, we used significantly more fibrinogen concentrate but significantly less fresh frozen plasma (in fact none in the Quantra ${ }^{\circledR}$ group). Although not being statistically significant, the number of patients receiving platelets was reduced by $68 \%$ (16 vs 5 patients; odd ratio $3,25[1,16 ; 9,14]$ ) and by $85 \%$ for packed red blood cells (120 vs 18 patients; odd ratio $37,67[18,96 ; 74,85])$ after the implementation of the device in our department. Regarding transfusion-related costs only in the low-bleeding risk sub-category of patients undergoing coronary artery bypass graft(s) witch accounted for $40 \%$ of the whole population, the $88 \%$ reduction in the average transfusion cost per patient is very significant $(p<0.0001)$. This important shift might be explained by the team's evolution towards practices that now aim to treat each of the biological anomalies leading to insufficient haemostasis in a specific way and no longer globally.

Conclusion. The availability of Quantra ${ }^{\circledR}$ in our department rapidly led to significant positive changes in transfusion practices, with a marked reduction in the number of patients receiving blood products. This evolution was not associated with increased blood-loss volumes. Although not used in every single patient case, such a tool has allowed for the cardiac surgery team to improve transfusion management, towards the "essential only", resulting in a significant reduction in the global transfusion costs.

\section{Reference(s)}

1. (2) C. Lodewyks et al. Point-of-care viscoelastic hemostatic testing in cardiac surgery patients: a systematic review and meta-analysis. J Can Anesth 2018;65:1333-1347

2. (1) Agarwal S, Abdelmotieleb M. Viscoelastic testing in cardiac surgery. Transfusion. 2020;60 (Suppl 6):S52-S60

\section{6}

Incidence and predictors of venous thromboembolic complications in ICU-admitted COVID-19 patients

M. Bychinin ${ }^{1}$;I. Mandel ${ }^{1}$; T. Klypa'; P. Avdonin'² S. Andreichenko ${ }^{1}$

${ }^{1}$ Intencive Care Unit, Federal Research and Clinical Centre, FMBA of Russia, Moscow, Russia; ${ }^{2}$ Laboratory Medicine, Koltzov Institute of Developmental Biology of Russian Academy of Sciences, Moscow, Russia

Correspondence: $M$. Bychinin

Intensive Care Medicine Experimental 2021, 9(1): 001006

Introduction. COVID-19 may predispose to venous thromboembolic (VTE) complications due to excessive inflammation, hypoxia, immobilisation and diffuse intravascular coagulation. While initial observations showed a VTE incidence of 15-25\% [1], more recent studies found that VTE risk may be as high as $85 \%$ despite all patients receiving pharmacological thromboprophylaxis [2]. The diagnosis of VTE can be challenging in critically ill patients with COVID-19. Thereby, the use of the predictors with the ability to differentiate between patients with high and low risk for VTE is likely to improve diagnostic efficiency and early commencement of anticoagulant therapy.

Objectives. The aim of the study was to assess the incidence and identify the predictors of VTE complications in COVID-19 patients admitted to intensive care unit (ICU).

Methods. Two hundred ICU-admitted patients presenting with severe or critical COVID-19 were included in this retrospective study. Patients were treated with a dose of nadroparin 5700 IU subcutaneous twice a day.

Results. The VTE complications were found in 67 (33,5\%) out of 200 patients. Four $(6 \%)$ patients had pulmonary embolism, $94 \%$ deep venous thrombosis and other thromboses, including catheter-associated and superficial vein thrombosis. Patients displaying VTE complications, unlike those without such complications, had more extensive lung pathology (CT scan), and more frequently required the use of vasopressors $(79,1 \%$ vs $59,4 \%, p=0,005)$ as well as mechanical ventilation $(89,6 \%$ vs $60,2 \%, p=0,0001)$. The survival rate was lower in patients with VTE complications (23 (34,3\%) vs $76(57,1 \%), p=0,003)$. The levels of von Willebrand factor antigen (vWF: Ag), interleukin 6 (IL6), antithrombin III (AT III), and protein C were significantly different in patients with VTE complications, compared with non-VTE patients. According to ROC analysis, vWF:Ag above $455 \%$ (AUC-0,852 $(0,69$; $1,00), p=0,008)$ was highly predictive of the risk of VTE on days 1 and 7 (Figure 1 and Fig. 2). AT III below $72 \%$ (AUC-0,77 $(0,55 ; 0,99)$, $\mathrm{p}=0,04)$ and IL6 above $256 \mathrm{pg} / \mathrm{ml}(\mathrm{AUC}-0,85(0,65 ; 1,00), \mathrm{p}=0,053)$ were prognostic factors of VTE on day 1 (Figure 1). Protein $C$ levels below $81,5 \%$ at admission (AUC-0,79 $(0,59-0,99), p=0,042$ ) was predictive of VTE complications by day 7 (Fig. 2). 
Conclusion. The rate of VTE complications was 33,5\% in ICU-admitted patients. The vWF:Ag, AT III, IL-6, and protein C can be used to predict VTE complications in patients with COVID-19.

\section{Reference(s)}

1. 1. Cui S, Chen S, et al. Prevalence of venous thromboembolism in patients with severe novel coronavirus pneumonia. J Thromb Haemost. 2020;18(6):1421-1424

2. 2. Ren $B$, Yan F, Deng Z, et al. Extremely High Incidence of Lower Extremity Deep Venous Thrombosis in 48 Patients With Severe COVID-19 in Wuhan. Circulation. 2020;142(2):181-183.

3. Laboratory research was carried out within the framework of the theme of the state program for fundamental scientific research No. 0088-2021-0008

\section{4}

Restrictive versus liberal red-cell transfusion strategy in ICU patients: a Meta-analysis with Trial Sequential Analysis L. La Via'; A. Morgana ${ }^{2}$; S. Tigano ${ }^{1}$; F. Perna ${ }^{3}$; E. Tringali ${ }^{3}$; F. Merola ${ }^{1}$; S. Messina 2 ; P. Murabito ${ }^{3}$; F. Sanfilippo ${ }^{3}$; M. Astuto ${ }^{3}$

${ }^{1}$ School of Anaesthesia and Intensive Care, University Hospital "G. Rodolico", Catania, Italy; ${ }^{2}$ School of Anaesthesia and Intensive Care, Magna Græcia University, Catanzaro, Italy; ${ }^{3}$ Department of Anesthesiology and Intensive Care, University Hospital "G. Rodolico", Catania, Italy Correspondence: L. La Via

Intensive Care Medicine Experimental 2021, 9(1): 001074

Introduction. Transfusion of red blood cell is very frequently administered in patients admitted to intensive care unit (ICU). Current recommendations suggest that a restrictive transfusion strategy (if hemoglobin $[\mathrm{Hb}$ ] level is below $7 \mathrm{~g} / \mathrm{dL}$ ) is as effective as a liberal strategy (with variable cut-offs for transfusions ranging between 9 and $10 \mathrm{~g} / \mathrm{dL}$ ). We conducted a meta-analysis with Trial Sequential Analysis (TSA) to evaluate the effects of a restrictive transfusion strategy compared to a liberal transfusion strategy in critically ill adults.

Methods. In this preliminary analysis we screened MEDLINE and EMBASE for randomized clinical trials (RCTs), up to May 7th, 2021. We calculated relative risk (RR) for the primary outcome of all-cause mortality at longest follow-up, using a random-effect model with 95\% confidence interval $(\mathrm{Cl})$. Meta-analysis was performed with separation in subgroups according to the clinical setting of patients (general ICU, septic shock, surgical ICU, cardiac surgery ICU, traumatic brain injury). For the TSA, the information size was computed assuming an alpha risk of $5 \%$ with a power of $80 \%$. The RR reduction was set at $20 \%$, estimating a mortality in the liberal transfusion group of $45 \%$, according to published evidence.

Results. Fourteen RCTs were included in our analysis (10.496 patients in total). All-cause mortality was not statistically different when the restrictive transfusion strategy was compared with the liberal transfusion strategy (RR 1.02, 95\% confidence interval $[\mathrm{Cl}] 0.91,1.15, \mathrm{p}=0.09$ ). None of the subgroup presented significant differences in mortality between the two strategies. The TSA conducted on the outcome showed that the sample size required (11.635 patients) was not yet reached, but the Z-line crossed the futility line, demonstrating the robustness of these finding which may not need further investigation (Figure 1). However, these data are pooled from clinically heterogeneous populations and research on specific subgroups may be valuable.

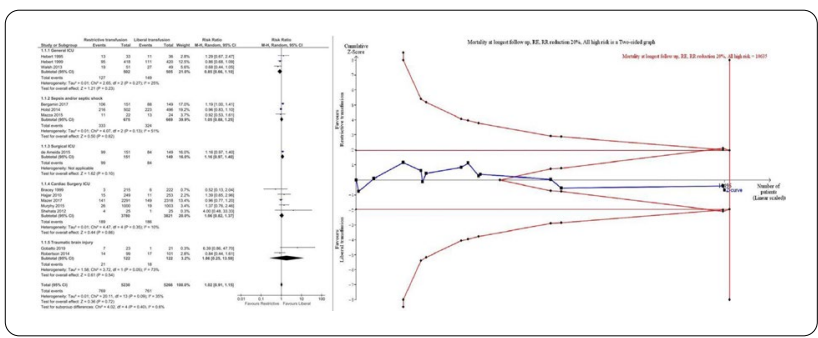

Figure 1. Forest plot analysis and Trial Sequential Analysis on mortality at longest follow-up in ICU patients randomized to restrictive transfusion or liberal transfusion of red blood cell with subgroup analysis. Analysis performed with random effect model and Mantel-Haenszel $(\mathrm{M}-\mathrm{H})$. Cl: confidence interval, RRR: relative risk reduction, RE: Random effect.

Conclusion. There is currently no evidence that a restrictive red-cell transfusion strategy reduces overall mortality as compared to a liberal transfusion strategy in critically ill patients. Future research should be focused on specific populations of ICU patients.

\section{Reference(s)}

1. Holst LB, Haase N, Wetterslev J, Wernerman J, Guttormsen AB, Karlsson S, et al.; TRISS Trial Group; Scandinavian Critical Care Trials Group. Lower versus higher hemoglobin threshold for transfusion in septic shock. N Engl J Med. 2014 Oct; 371(15): 1381-91.

001193

Prolonged clotting time does not increase the risk of bleeding after central venous catheter placement in severely thrombocytopenic critically ill patients

FEHP van Baarle 1 . APJ Vlaar'. BJ. Biemond ${ }^{2}$

${ }^{1}$ Intensive Care, Amsterdam UMC, locatie AMC, Amsterdam, Netherlands; ${ }^{2}$ Haematology, Amsterdam UMC, locatie AMC, Amsterdam, Netherlands Correspondence: F.E.H.P. van Baarle

Intensive Care Medicine Experimental 2021, 9(1): 001193

Introduction. ICU patients often undergo central venous catheter (CVC) placement for vasoactive medication and/or haemodialysis. 1 CVC placement is frequent in the setting of thrombocytopenia and/or coagulopathy.2 Studies on bleeding after CVC placement have mostly included patients with normal or limitedly disturbed coagulation parameters. There is conflicting evidence on the predictive value of traditional coagulation parameters (platelet count (PC), International Normalised Ratio (INR) and activated partial prothrombin time (APTT)) on bleeding after CVC placement and whether prophylactic correction with transfusion is needed. 3

Objectives. To find out whether increased INR and/or APTT during severe thrombocytopenia $(P C \leq 50 \times 109 / L)$ increases the bleeding risk after CVC placement over isolated thrombocytopenia. Secondly, to describe transfusion practice and the influence of platelet transfusion on periprocedural bleeding risk.

Methods. A single centre retrospective cohort study in an academic mixed ICU in the Netherlands, between February 2016 and February 2020. Patients were eligible if they underwent CVC placement during severe thrombocytopenia $(P C \leq 50 \times 109 / L)$. Coagulopathy was defined as INR $>1.5$ and/or APTT $>45 \mathrm{~s}$. The primary outcome was any bleeding at the CVC insertion site $<24 \mathrm{~h}$ after CVC placement. Multiple imputation and inverse probability of treatment weighting were used to account for missing data and the non-randomised nature of the study.4-5.

Results. 284 CVC placements in 180 patients with severe thrombocytopenia were included, 111 (40\%) during coagulopathy, 167 (60\%) with normal INR and APTT. There were 34 episodes of bleeding at the CVC insertion site $(12.1 \%)$, of which $24(8.5 \%)$ were grade 1,8 $(2.9 \%)$ were grade 2 and $2(0.7 \%)$ were grade 3 . In the coagulopathy group there were $14(12.6 \%)$ bleeding episodes, in the normal coagulation group there were 20 (11.8\%). Distribution of bleeding grades was similar in both groups. After correction for confounders, there was no effect of coagulopathy on bleeding: odds ratio $(95 \%$ Cl) 0.86 (0.22-3.32).

Prior to CVC placement $113(40.5 \%)$ patients received platelet transfusion, and $12(4.3 \%)$ patients received plasma transfusion. Platelet transfusions were mostly given due to the platelet count being below threshold levels (37\%), or prophylactically for CVC placement $(41 \%)$, and equally distributed between coagulopathy and normal coagulation groups. Plasma transfusions were mostly given for bleeding elsewhere (42\%) and prophylactically for CVC placement $(25 \%)$ and slightly more frequent in the coagulopathy group (7 vs. 5 patients). Bleeding at the CVC insertion site occurred in 18 
patients that received platelet transfusion (15.9\%) and 17 patients that did not (10.2\%). After correction for confounders, there was no effect of platelet transfusion on bleeding: odds ratio $(95 \% \mathrm{Cl}) 1.95$ (0.30-12.7).

Conclusion. Prolonged INR and APTT do not increase bleeding risk in severely thrombocytopenic ICU patients undergoing CVC placement. Prophylactic transfusion in patients with combined coagulopathy and severe thrombocytopenia does not reduce risk of bleeding.

\section{Reference(s)}

1. 5. Austin PC, Stuart EA. Moving towards best practice when using inverse probability of treatment weighting (IPTW) using the propensity score to estimate causal treatment effects in observational studies. Statist Med 2015; 34: 3661-3679

2. 4. Van Buuren S, Groothuis-Oudshoorn K: mice: Multivariate Imputation by Chained Equations in R. J Stat Soft 2011; 33

3. 3. Van de Weerdt EK, Biemond BJ, Baake B, et al.: Central venous catheter placement in coagulopathic patients: risk factors and incidence of bleeding complications. Transfusion 2017; 57: 2512-2525

4. 2. Levi M, Sivapalaratnam S: Hemostatic abnormalities in critically ill patients. Intern Emerg Med 2015; 10: 287-296

5. 1. Taylor RW, Palagiri AV: Central venous catheterization. Crit Care Med 2007; 35: 1390-1396

6. This study is funded by ZonMW [Zorgonderzoek Medische Wetenschappen] (part of the NWO [Nederlandse Organisatie voor Wetenschappelijk Onderzoek; the Dutch Organization for Scientific Research], Den Haag, The Netherlands), project number 843002625 . The sponsors of this work were not involved in the study design, the collection, analysis, and interpretation of data, the writing of the report, or the decision to submit the manuscript for publication.

001198

A Machine Learning Model for Pulmonary Embolism Prediction Using a Large Multi-Center Database

L. Tewala ${ }^{1}$ H. Kim²; R. Stevens ${ }^{2}$

${ }^{1}$ Department of Biophysics, Johns Hopkins University, Baltimore, United

States of America; ${ }^{2}$ Anesthesiology and Critical Care Medicine, Johns

Hopkins University, Baltimore, United States of America

Correspondence: R. Stevens

Intensive Care Medicine Experimental 2021, 9(1): 001198

Introduction. Pulmonary embolism (PE) is a frequent and lifethreatening complication in critically ill patients. While risk scores have been proposed, there is an unmet need for accurate methods that predict the likelihood for PE.

Objectives. The aim was to build a data-driven computational model to predict PE in patients admitted to the ICU.

Methods. From a multisite clinical database of 208 US institutions we identified 1315 patients, who were admitted to the ICU and then developed PE. We used time-dependent data from the $6 \mathrm{~h}$ before the patient was diagnosed with PE. We trained three different machine learning (ML) algorithms, gradient boosting (XGboost), random forest (RF), and generalized linear models (GLM) on two sets of data. The demographics dataset included 71 features, while the laboratory dataset included 215 features, some of which are time-dependent.

Results. ML models using the laboratory dataset discriminated well (mean \pm SD values for XGboost: AUROC $0.86 \pm 0.01$, sensitivity: $0.80 \pm 0.03$, specificity: $0.75 \pm 0.02$ ) and performance was even better (AUROC: $0.82 \pm 0.01$, sensitivity: $0.83 \pm 0.03$, specificity: $0.67 \pm 0.03$ ) with the demographic dataset. Highly ranked features strongly associated with PE included admission to the ICU from the emergency department or the operating room, higher anion gap, higher prothrombin time (PT), and higher partial thromboplastin time (PTT).

Conclusion. Results demonstrate the potential value of ML models using physiological and clinical variables to help predict the onset of $\mathrm{PE}$ in the ICU. Following external validation and testing against the established risk scores, this study aims to produce a model that could accurately predict PE in real-time in the ICU.
001273

The Role of ROTEM analysis in ICU patients with severe COVID-19 pneumonia

E. Lazoudi ${ }^{1}$; P. Ioannidis ${ }^{1}$; T. Schizodimos ${ }^{1}$; I. Karageorgiou ${ }^{1}$; A. Kosmas ${ }^{1}$; E. Siomos $^{1}$; N. Kapravelos'; C. lasonidou ${ }^{1}$

'B icu, GENERAL HOSPITAL "GEORGE PAPANIKOLAOU", Thessaloniki, Greece, Greece

Correspondence: $\mathrm{E}$. Lazoudi

Intensive Care Medicine Experimental 2021, 9(1): 001273

Introduction. COVID-19 associated coagulopathy (CAC), characterized by hypercoagulability and increased risk of thrombotic complications, is of important consideration in the management of pts with COVID-19. Traditional coagulation tests have shown several limitations in their ability to reliably detect coagulation disorders. ROTEM technology provides a rapid and dynamic assessment of haemostasis. ROTEM also measures hypercoagulability in various clinical scenarios which is not detected by routine coagulation tests.

Objectives. The aim of this study was to investigate consequences of severe COVID-19 infection and inflammatory state on global haemostasis using standard coagulation parameters and Thromboelastometry.

Methods. This retrospective evaluation comprised ICU pts with severe COVID-19 pneumonia. Blood samples were obtained on admission. ROTEM analysis (EXTEM, INTEM, FIBTEM tests) standard laboratory coagulation tests (PT, INR, aPTT, platelets count, fibrinogen concentration), D-dimers, interleukin-6 (IL-6), procalcitonin were evaluated. We compared ROTEM profile with the standard coagulation tests. We examined the relationship of hypercoagulability with the inflammatory state. The coagulation profile and the association with the outcome was evaluated. Using ROTEM analysis hypercogulability was defined as MCF in EXTEM $>72$ and MCF in FIBTEM $>25$.

Results. 24 patients were included in our study. 58.3\% (14/24) of patients were male. Mean age was $62.5 \pm 10.16$ years. Mortality in ICU was $50 \%$. Mean length of stay (LOS) was $23.3 \pm 10.9$ days. Blood tests showed high levels of inflammatory biomarkers (mean \pm SD) i.e., IL-6 $(86.55 \pm 95.78 \mathrm{pg} / \mathrm{ml})$, PCT $(0.38 \pm 0.44)$, Ferritine $(1233.65 \pm 1234.3 \mathrm{ng} / \mathrm{ml})$, Fibrinogen $(5.1 \pm 1.12 \mathrm{~g} / \mathrm{L})$ and D-dimers $(2.57 \pm 3.76 \mathrm{mg} / \mathrm{ml})$. ROTEM analysis showed normal CT values in EXTEM and INTEM, high MCF in EXTEM $(73.46 \pm 7.18)$ and high MCF in FIBTEM $(38.5 \pm 12.56) .45 .8 \%$ of the pts had increased MCF in EXTEM and and $79.2 \%$ in FIBTEM. Mortality rate was increased in pts with abnormal MCF in EXTEM (54.5\% vs 46.1\%), but without statistically significant difference. We observed no difference in LOS in ICU between these groups.

Conclusion. Our study showed an hyperinflammatory responce on admission in ICU in pts with severe COVID-19 pneumonia. The inflammation state was associated with severe hypercoagulability rather than a consumption coagulopathy. Probably, standard coagulation tests fail to detect the severity of prothrombotic phenotypes. Using ROTEM we are able to detect hypercoagulability. Further randomized studies are needed to establish whether these pts could benefit of an early anticoagulant therapy, in terms of improving outcomes.

\section{Reference(s)}

1. Eug. Yuriditsky et al. Thromboelastography Profiles of Crtically III Patients with Coronavirus Disease 2019. Critical Care Med. 2020

2. J. Hartmann et al. The Role of TEG Analysis in Patients with COVID-19-Associated Coagulopathy: A Systematic Review. Diagnostics 2012 1, 11, 172

001326

Tailored thromboprophylaxis strategy for Covid-19-associated coagulopathy during the second and third waves of the pandemic: report from a portuguese center

M. Pereira, ${ }^{1}$; J. Moreira, ${ }^{1}$; M. Remelhe ${ }^{1}$; M. Pinto, ${ }^{1}$ E. Neutel,

${ }^{1}$ Department of Anesthesia Intensive Care and Emergency, University Hospital Centre of Porto (CHUPorto), Porto, Portugal

Correspondence:Correspondence: $M$. Remelhe

Intensive Care Medicine Experimental 2021, 9(1): 001326 
Introduction. Since 2019, the severe acute respiratory syndrome by coronavirus-2 (SARS-CoV-2) has caused a worldwide pandemic of coronavirus disease (COVID-19). Accumulated evidence and clinical experience have revealed that patients with COVID-19 have been frequently found to suffer from a coagulopathy disorder, now considered a crucial factor of the pathophysiology of the disease, leading to thrombotic complications.

Objectives. The aim of the present study was to assess the incidence and clinical patterns of thrombotic events in patients admitted to a COVID-19 ICU after the 1st wave of the pandemic.

Methods. This study included patients aged $18+$ years old,who were admitted to a COVID-19 ICU in a tertiary hospital,between October 2020 and February 2021.Thrombotic events were diagnosed during routine clinical care. The Hospital's Immunohemotherapy department anticoagulation guidelines were followed, which included an enhanced thromboprophylaxis dose.Investigators searched clinical notes, laboratory and radiology reports to create a database. Statistical analyses were conducted using SPSS v25.This study was approved by the Hospital Ethics department, which waived the need for informed consent.

Results. Among the 74 hospitalized COVID-19 patients in our ICU, the median age was 69 (min-max, 31-89) years.Thromboembolic events occurred in $20(27 \%)$ patients (they could have more than one event), in a total of 28 events: $15(54 \%)$ pulmonary embolism; $9(32 \%)$ arterial thromboembolisms (7 limb ischemia, 2 ischemic strokes); $2(7 \%)$ deep vein thrombosis; 2 (7\%) line clothings. 4 patients had events on prophylactic anticoagulation dose $(20 \%), 11$ on intermediate $(55 \%)$ and 5 on therapeutic (35\%). No life threatening haemorrhagic events were observed. We registered 2 small intracranial haemorrhages and 6 mucosal bleed (total of $11 \%$ haemorrhagic events). Smoking habits (OR 8.7, p 0.015) was the only significant comorbidity associated with the events, even after adjustment. Bloods at hospital admission showed that higher $\mathrm{D}$-dimer $(\mathrm{p}=0.007, \mathrm{OR} 1.0)$ and leucocyte counts $(p=0.023, O R 1.18)$ were associated with thrombotic events. After adjustment, only D-dimer remained relevant. Patients with events had significantly longer hospital (median 30 days, $p=0.031$ ) and ICU length of stay (median 11 days, $p=0.012$ ). All-cause mortality at 30 days was $39 \%$ and was not significantly higher in those with thrombotic events $(p=0.327$, OR 1.7).Adjusted factors associated with mortality at 30 days were increased age and $C$ reactive protein at admission. A logistic regression with the different anticoagulation schemes did not show any difference in mortality outcome $(p=0.621$, OR 1.20).

Conclusion. While thrombosis is observed in other acute infections, the thrombotic risk appears to be higher in COVID-19. This coagulopathy may have several contributing factors, cytokine storm, hypoxic injury, endothelial dysfunction, hypercoagulability, and/or increased platelet activity.Reports from the first wave of the pandemic showed that many European centres used enhanced strategies of thromboprophylaxis with beneficial outcomes. Since then, dexamethasone has been widely used as it is the only drug clearly proven to reduce mortality, and its effect on the physiopathology of the coagulopathy has yet to be fully studied.In our ICU, $27 \%$ of the patients had thrombotic events even on dexamethasone and thromboprophylaxis.Despite its several limitations, our study shows that even with immunosuppression, thrombotic events are frequent, and heparin resistance still plays a strong role, since events still occur in face of anticoagulation.

\section{3}

Evaluation of coagulation function by ROTEM analysis in critically ill patients with severe COVID-19 pneumonia

P. Ioannidis ${ }^{1}$; E. Lazoudi ${ }^{1}$; I. Karageorgiou' ${ }^{1}$; M. Sileli ${ }^{1}$; E. Siomos ${ }^{1}$; A.

Kosmas $^{1}$; N. Kapravelos ${ }^{1}$; C. Iasonidou ${ }^{1}$

'B' icu, General Hospital of Thessaloniki "George Papanikolaou", ThessaIoniki, Greece

Correspondence: E. Lazoudi

Intensive Care Medicine Experimental 2021, 9(1): 001343
Introduction. Critically ill patients with COVID-19 pneumonia suffer both high thrombotic and bleeding risk. Until now, the effect of COVID-19 infection on haemostatic functions remain unspecified. Conventional coagulation tests only detect limited parts of the coagulation system. ROTEM is point-of-care tool that provides detailed information on clotting kinetics from clot formation through degradation.

Objectives. The aim of this retrospective study was to investigate consequences of severe COVID-19 infection on global haemostasis using standard coagulation parameters and whole blood ROTEM over time. Methods. We performed a retrospective study on critically ill pts admitted to our ICU with severe COVID-19 pneumonia and we evaluated coagulation function using ROTEM analysis on admission day (T0), on day 5 (T5) and on day 10 (T10). At the same time, standard laboratory coagulation tests (PT, INR, aPTT, platelets count, fibrinogen concentration) and inflammatory biomarkers i.e., D-dimers, interleukin-6 (IL-6), procalcitonin were evaluated. APACHE II and SOFA score were calculated. We evaluated the coagulation parameters over time and the association with the inflammatory state and the outcome. Using ROTEM analysis hypercogulability (abnormal value) was defined as MCF in EXTEM $>72$ and MCF in FIBTEM $>25$.

Results. 24 patients were included in our study. 58.3\% (14/24) of patients were male. Mean age was $62.5 \pm 10.16$ years. Mortality in ICU was $50 \%$. Mean long of stay (LOS) was $23.3 \pm 10.9$ days. Laboratory results of biomarkers at T0 showed high levels (mean $\pm \mathrm{SD}$ ) of IL-6 $(86.55 \pm 95.78 \mathrm{pg} / \mathrm{ml})$, PCT $(0.38 \pm 0.44)$, Ferritine $(1233.65 \pm 1234.3 \mathrm{ng} /$ $\mathrm{ml})$, Fibrinogen $(5.1 \pm 1.12 \mathrm{~g} / \mathrm{L})$ and $\mathrm{D}$-dimers $(2.57 \pm 3.76 \mathrm{mg} / \mathrm{ml})$ that reduced in the follow-up period. ROTEM analysis at T0 showed normal $C T$ values in EXTEM and INTEM but high MCF in EXTEM (73.46 \pm 7.18$)$ and MCF in FIBTEM (38.5 \pm 12.56$) .45 .8 \%$ of the pts had increased MCF in EXTEM and and $79.2 \%$ in FIBTEM. These values were decreased at T10 as only $38.1 \%$ and $66.7 \%$ of the pts had increased MCF in EXTEM and FIBTEM, respectively. Mortality rate was increased in pts with persistent abnormal MCF in EXTEM (50\% vs $61.5 \%$, p: 0.67$)$ and abnormal MCF in FIBTEM (42.9\% vs $85.7 \%$, p:0.16) at T10. We observed no difference in LOS in ICU between these groups.

Conclusion. Our study showed increased inflammatory biomarkers (i.e., IL-6, PCT) on admission in ICU in pts with severe COVID-19 pneumonia. The inflammatory state was associated with a state of severe hypercoagulability as confirmed using ROTEM analysis. This hypercoagulable state persists in first five days but it decreases after 10 days without returning to normal values in most pts. Further studies in pts with COVID-19 pneumonia are needed to elucidate the optimal use of ROTEM in this population and probably the benefits of appropiate anticoagulation therapy.

\section{Reference(s)}

1. eug. Yuriditsky. Thromboeladtography profiles of critically ill patients with Coronavirus disease 2019. Critical Care MED 2020

2. J. Hartmann. the role of TEG Analysis in patients with COVID-19 associated coagulopathy: a systematic review Diagnostic 2021, 11, 172

\section{2}

Coagulation abnormalities and severity of lung injury in ICU patients with COVID-19. Is there a correlation?

T. Schizodimos ${ }^{1}$; P. Ioannidis ${ }^{1}$; I. Karageorgiou' ${ }^{1}$; N. Kapravelos ${ }^{1}$; C. lasonidou ${ }^{1}$

'B icu, General Hospital "G. Papanikolaou", Thessaloniki, Greece

Correspondence: $T$. Schizodimos

Intensive Care Medicine Experimental 2021, 9(1): 001462

Introduction. Patients with COVID-19 and especially those admitted to the intensive care unit (ICU) are at high risk of developing thromboembolic events due to coagulation abnormalities. Early detection and treatment of coagulation abnormalities are of paramount importance for the clinical outcomes of patients with severe COVID-19. The use of conventional coagulation tests and rotational thromboelastometry (ROTEM) may have a major role in this purpose.

Objectives. To investigate the correlation between various measured coagulation abnormalities and the severity of lung injury in ICU patients with COVID-19. 
Methods. An observational (preliminary) study was performed in a Greek ICU including 31 patients with COVID-19. We divided them in two groups based on lung injury severity score (LISS) at day 1 . The first group included patients with LISS $<3$ and the second group patients with LISS $\geq 3$. Conventional coagulation tests and ROTEM were performed at days 1, 5, 10 and 15 . The main coagulation parameters measured and evaluated were D-dimer, fibrinogen, INR and platelet plasma levels and ROTEM- EXTEM and FIBTEM maximum clot firmness (MCF).

Results. Included 31 patients had a mean age of $63.5( \pm 8.8)$ years, Charlson Comorbidity Index $(\mathrm{CCl}) 2.2( \pm 0.9)$ and APACHE II score 16.2 ( \pm 3.1$) .15$ patients (48.4\%) had LISS $<3$ and 16 patients $(51.6 \%)$ had LISS $\geq 3$. D-dimer and fibrinogen plasma levels were increased in both groups. Fibrinogen plasma levels were higher in group LISS $\geq 3$ and significantly different between the two groups at days $1(p=0.03)$ and $5(p=0.021)$. INR remained relatively constant in all patients over time, while platelet count decreased. FIBTEM and EXTEM MCF were increased in all patients from day 1 to day 15 , with clearly higher values in group LISS $\geq 3$. Of all the coagulation parameters, the most statistically significant positive correlation was between platelets and EXTEM MCF, especially in group LISS $\geq 3$.

Conclusion. COVID-19 patients are characterized by a hypercoagulability state with reduced fibrinolysis. Our study shows a trend of positive correlation between the magnitude of coagulation abnormalities and the severity of lung injury. Certainly, there remains a need for more, high quality trials in ICU patients with COVID-19.

\section{Reference(s)}

1. 2. Van Veenendaal N, Scheeren TWL, Meijer K, et al. Rotational thromboelastometry to assess hypercoagulability in COVID-19 patients. Thromb Res. 2020;196:379-381.

2. 1. Kruse JM, Magomedov A, Kurreck A, et al. Thromboembolic complications in critically ill COVID-19 patients are associated with impaired fibrinolysis. Crit Care. 2020;24(1):676

3. None.

\section{e-Posters: Trauma}

\section{0}

Predictors of poor outcome in case of multiple trauma with severe thoracic trauma during the early posttraumatic period

M. Stupnytskyi ; O. Biletskyi, ;. V. Zhukov, ${ }^{3}$;. Gorbatch,

${ }^{1}$ Intensive Care Department in the Cardiovascular Surgery Clinic, Military Medical Clinical Center of the Western Region, L'viv, Ukraine; ${ }^{2}$ Anesthesiology and Intensive Care Department for patients with combined trauma Prof. O.I. Meshchaninov Kharkiv City Clinical Emergency Hospital, Kharkiv, Ukraine; ${ }^{3}$ Department of biological chemistry, Kharkiv National Medical University, Kharkiv, Ukraine

Correspondence: M. Stupnytskyi

Intensive Care Medicine Experimental 2021, 9(1): 000010

Introduction. Patients with blunt multiple trauma with severe thoracic trauma (BMTSTT) can change their clinical stability very rapidly hence intense monitoring, especially during early posttraumatic period, is mandatory [1]. Pathophysiology of polytrauma is complex and consists from certain stages of systemic reactions with different predominant mechanisms so different time periods of the early posttraumatic period can have own specific markers for outcome prediction $[2,3]$.

Objectives. The goal of this study was to determine simple and easy to perform predictive criteria for multiple trauma patients with severe thoracic trauma during early posttraumatic period.

Methods. This prospective observational cohort study was conducted on 73 male patients 20-68 y.o. with BMTSTT (ISS = 18-57; AIS tho$\mathrm{rax}=3-4$; survival/non-survival $=42 / 31$ ) in department of anesthesiology and intensive care for patients with combined trauma of the Prof O.I. Meshchaninov Kharkiv City Clinical Emergency Hospital. Examinations were performed on the 1st-2nd (11-34 h.), 3d-4th (48-75 h.) and 5th-6th (97-122 h.) days after trauma. ROC-analyses were performed in each of the time periods for 39 clinical and laboratory variables and cut-off values were calculated according to Youden's index.

Results. There were no statistical differences between survivors and non-survivors in terms to age, admission time, type of chest injuries, injured body regions, etiology of polytrauma and the number of patients with concomitant alcohol exposure. The highest odds ratios (OR) for negative outcome prediction were estimated for TRISS $<0.834$ OR 15.54 (4.68-51.59) $\mathrm{p}<0.0001, \mathrm{RTS}<7.004$ OR 12.57 (4.1-38.5) $p<0.0001$ and AIShead $>4$ OR $9.84(1.12-86.63) p=0.0372$. For the 1 st-2nd day after trauma most relevant predictive signs were total protein (TP) concentration $<49.36 \mathrm{~g} / \mathrm{L}$ OR 18.09 (5.37-60.92) $\mathrm{p}<0.0001$ Creatinine $>143.1 \mu \mathrm{mol} / \mathrm{L}$ OR $13.5(3.94-46.23) \mathrm{p}<0.0001$, oxygen content $<147.2 \mathrm{ml} / \mathrm{L}$ OR $10.27(2.69-39.06) \mathrm{p}=0.0001$. For the $3 \mathrm{~d}-4$ th day after trauma most significant predictive markers were TP $<53.83 \mathrm{~g} / \mathrm{L}$ OR $47(2.678-824.7) \mathrm{p}<0.0001$, band neutrophils $>13.5 \%$ OR 44.73 $(5.23-382.6) p<0.0001$ and $W B C>11.68 \mathrm{G} / \mathrm{L}$ OR 14.87 (3.74-59.05) $\mathrm{p}<0.0001$. For the 5th-6th day after trauma $-\mathrm{TP}<53.49 \mathrm{~g} / \mathrm{L}$ OR 36 (4.38-295.7) $\mathrm{p}<0.0001$, monocytes $<3.921 \%$ OR $18.5 \quad(4.89-69.96)$ $\mathrm{p}<0.0001$ and $\mathrm{RBC}<3.283 \mathrm{~T} / \mathrm{L}$ OR $18.18(3.46-95.51) \mathrm{p}=0.0001$.

Conclusion. These findings suggest that in general the same clinical and laboratory signs can't be used for outcome prediction during several days of the early posttraumatic period in case of BMSTT as each of the investigated time periods is characterized by own specific predictive markers. Predictive effectiveness of laboratory markers is different depending on time period. These simple criteria can be helpful for trauma team for monitoring clinical course of polytraumatized patients via recognition those at high risk of negative outcome to improve quality of patient care through early intensive focused care.

\section{Reference(s)}

1. [1] Fallouh H, Dattani-Patel R, Rathinam S. Blunt thoracic trauma. Surgery 2017; 35: 262-268.

2. [2] Stahel PF, Flierl MA, Moore EE. 'Metabolic staging' after major trauma a guide for clinical decision making? Scand J Trauma Resusc Emerg Med 2010: 18: 18-20.

3. [3] Aller M, Arias J, Alonso-poza A, et al. A Review of metabolic staging in severely injured patients. Scand J Trauma Resusc Emerg Med 2010; 18: 27.

\section{0}

Treatment of Polytraumatized Patients in a Level 1 Trauma Center between 1995 and 2019. 25-Year Experience-what has changed? V. Weihs' ${ }^{1}$; S. Frenzel' ; M. Dedeyan ${ }^{1}$; F. Hruska ${ }^{1}$; K. Staats' ${ }^{1}$; S. Hajdu'; L. Negrin':S. Aldrian

'Department of Orthopedics and Trauma Surgery, Medical University of Vienna, Wien, Austria

Correspondence: $\mathrm{V}$. Weihs

Intensive Care Medicine Experimental 2021, 9(1): 000060

Introduction. To analyze changes of the injury patterns, the mortality rates and the clinical characteristics of polytraumatized patients over the past 25 years.

Methods. Data analyses focused on the changes of the clinical characteristics, injury patterns and mortality rates of 1251 consecutive polytraumatized patients treated between January 1992 and December 2019 in a single level 1 trauma center.

Results. A significant increase of the rates of geriatric polytraumatized patients with a significant increase of the average age by 1.74 years per year could be seen over time. Contrarily, the injury severity showed a significant decrease over time especially in the body regions abdomen and extremities with a significant decrease of mean the injury severity score (ISS). Significantly higher rates of traumatic brain injury (TBI) could be found in the geriatric group of polytraumatized patients, although no changes in the overall rates of TBI could be detected. Characteristic changes in the injury patterns with a significant increase in low energy traumas could be detected. Regarding the late-phase mortality the relative risk increased by $2.2 \%$ per patient's year and the relative risk of in-hospital mortality increased by $1.9 \%$ per patient's year, whereas the overall mortality rate remained constant over time. Severe TBI, age beyond 65 years of age and ISS scores 
between 25 and 50 points remained as independent prognostic factors on the late-phase survival of polytraumatized patients.

Conclusion. Significant characteristic changes in the demographic variables and clinical characteristics of polytraumatized patients could be seen over the past 25 years. Although the injury severity decreased over time, no change in the mortality rates could be seen. With a constant increase in the mean age of polytraumatized patients, these findings might reflect the higher rates of frail polytraumatized patients.

\section{4 \\ Has lockdown had any effect on Major Trauma patients presentation? \\ B. Muñoz Molina'; G. Mcneill'; D. Kerslake² \\ ${ }^{1} \mathrm{ICU}$, Hospital de La Princesa, Madrid, Spain; ${ }^{2} \mathrm{ICu}$, Royal Infirmary of Edin- \\ burgh, Edinburgh, United Kingdom \\ Correspondence: B. Muñoz Molina \\ Intensive Care Medicine Experimental 2021, 9(1): 000144}

Introduction. SARS-CoV-2 pandemic has changed the paradigm of what we call normality, more obviously on healthcare level.

Objectives. We wanted to assess if lockdown in Scotland, started on 16th March 2020, has had any impact on demographic and presentation of Major Trauma patients. We hypothesised the type and range of presentation might have changed due to the Scottish government lockdown restrictions.

Methods. Retrospective, observational, descriptive study, on patients diagnosed with Major Trauma admitted to the ICU of a Major Trauma Centre, between March 2019 and December 2020, comparing between two cohorts, before and after lockdown.

We collected demographic, clinical and therapeutical variables including: age, gender, previous medical conditions and treatment, social and psychiatric background, presenting mechanism and injuries, complications and mortality.

We used $x^{2}$ test and Fisher's exact test, considering statistical significance when $\mathrm{p}<0.05$.

Results. A total of 145 patients were included in the analysis, 79 (54.5\%) before vs $66(45.5 \%)$ after lockdown.

112 were men (77.2\%), 62 vs 50.33 were women (22.8\%), 17 vs 16 . $\mathrm{p}=0.086$.

We divided the sample in 3 age groups, $<30,30-65$ and $>65$ years old. We saw a decreased on cases in $<30$, total $32(22.1 \%), 23$ vs 9 , and in $>65$, total $25(17.2 \%), 15$ vs $10, p=0$.

Within young population, this could be related to school and university closures or more unemployment. In elderly, more stay at home or more restrictive criteria to ICU admissions.

Analysing substance misuse and psychiatric background, there was no trend to a raise on cases after lockdown:

Alcohol: 63 (43.4\%), 31 vs 32.

. Drugs: 31 (21.4\%), 19 vs 12.

- Mental disorder: 66 (45.5\%), 32 vs 34.

Regarding the mechanism of trauma, most were RTC, 48 (33.1\%), with a decrease after lockdown, 30 vs 18 patients. The second cause were accidental falls from height, $29(20 \%), 17$ vs 12 . Accidents due to bikes increased, 2 vs 5, most amongst group 30-65 years old ( $p=0.32$ ), and apparently more related to drugs misuse $(p=0.015)$. In falls secondary to suicide attempts, incidence was similar, 8 vs 7 ; related to drugs misuse $(p=0.039)$ but not to other mental background.

Global mortality was 15 patients $(10.34 \%), 9$ vs 6 deaths.

Most of the patients had GCS $>12,103(71 \%), p=0$, and ISS $<50,124$ $(85.5 \%), p=0$. There was an increase in cases with $\mathrm{GCS}<8$ (8 vs 17) or ISS $>50$ (5 vs 11 ) after lockdown, statistically related with mortality, $\mathrm{p}=0.012$ and $\mathrm{p}=0.028$ respectively.

Mean stay in ICU was 5.96 vs 8.78 days, SD 6 vs 13.05 respectively.

Conclusion. We found differences in age, probably due to a higher rate of elderly patients staying at home, and a middle-age group that most possibly were active workers.

We didn't see an increase in cases related with mental health after lockdown.

There was a trend to a lower number of RTC after lockdown, and an increase in accidents due to the use of bicycles. This may relate to the documented reduction in car travel during lockdown period, as well as the documented raise in bicycle use.

Even though mortality seems less after lockdown, the morbidity and the mean stay in ICU appear to be higher.

\section{1}

SHOCK INDEX ASSOCIATED WITH AGE AND GLASGOW COMA SCORE (SIXA/G) AS PREDICTOR OF MORTALITY IN THE INITIAL CARE OF THE POLYTRAUMA PATIENT

P. Juárez Sanjuan ${ }^{1}$;V. Juárez-Sanjuann²; C. Rodríguez-Mata, ${ }^{3}$; J.

García-Biosque Rodríguez; ; J. Garriga Segarra ${ }^{3}$; M. Artiles-Armas ${ }^{4}$; D.

Ortiz-López, ; S. Castillo-Acosta²; L. Cano-Contreras, ${ }^{2}$; D. Guerrero, ; j. Freixinet-Gilart,

${ }^{1}$ Emergency Department Critical Care, University Hospital of Gran Canaria Dr. Negrin, Las Palmas de Gran Canaria, Spain; ${ }^{T}$ Thoracic Surgery, University Hospital of Gran Canaria Dr. Negrin, Las Palmas de Gran Canaria, Spain: ${ }^{3}$ Intensive Care Medicine, University Hospital of Gran Canaria Dr. Negrin, Las Palmas de Gran Canaria, Spain; ${ }^{4}$ General Surgery, University Hospital of Gran Canaria Dr. Negrin, Las Palmas de Gran Canaria, Spain

Correspondence: P. Juárez Sanjuan

Intensive Care Medicine Experimental 2021, 9(1): 000201

Introduction. Polytrauma is one of the leading causes of death in the population under 45. Prognostic severity scores provide rapid information on polytrauma.The Injury Severity Score (ISS) is based on anatomical injuries and its calculation is complex. The Revised Trauma Score (RTS) and Glasgow-Age-Systolic Blood Pressure (GAP) scores require scoring systems that can delay diagnosis. The shock index (SI) is the division between the heart rate (HR) and the systolic blood pressure (SBP) is a simple and fast tool in order to identify shock.

Objectives. To study whether the addition of age and the Glasgow Coma Score (GCS) increase the predictive capacity of mortality of the $\mathrm{SI}$ in the initial care of the polytrauma patient and compare it with GAP, RTS and ISS.

Methods. Observational study of patients admitted to the Critical Care Unit of the Emergeny Department of a Third-Level Hospital with a diagnosis of trauma between 2015 and 2020. 500 patients were included, those who died before their arrival at the hospital or received negative chronotropic treatment were excluded. $S$ (FC/SBP), SI associated with GCS (SI/G), age (SIXA) and both (SIXA/G) were calculated. The area under the curve (AUROC) of each of them was calculated for in-hospital mortality (MI) and the first $24 \mathrm{~h}$ mortality (M24). The AUROC of the SIA/G was also compared with those of the GAP, RTS and ISS scores.

Results. 433 patients were analyzed with a median age of 45 (range $31-60 ; 80.1 \%$ males). The most frequent mechanism of injury was falls $(42.3 \%)$, followed by traffic accident $(26.8 \%)$ and blunt trauma was the predominant type $(88.9 \%)$. The most frequent injury was head trauma (TBI) $(43.6 \%)$ followed by rib fractures $(31.4 \%)$. In the first $24 \mathrm{~h} 30$ patients (6.9\%) died, increasing to $47(10.9 \%)$ throughout admission. All types of shock index (SI, SIxA, SI/G and SIXA/G) were significantly related to mortality, however, the SIA/G presented the highest AUROC for $\mathrm{MI}(0.879 ; \mathrm{Cl} 0.83-0.93)$ and for $\mathrm{M} 24(0.875 ; \mathrm{Cl}$ $0.82-0.93)$.The SIA/G value of 3.3 points showed a sensitivity of $82 \%$ and a specificity of $80 \%$ for $\mathrm{Ml}$ and $86 \%$ and $78 \%$ for M24. Of the group of patients with SIA/G $\approx 3.3,32.1 \%$ died on admission compared to $2.5 \%$ of those with SIA/G $<3.3(\mathrm{p}<0.001$, OR $18.3[95 \% \mathrm{CI}$ $8.2-41]$ ). The survival curves of Kaplan-Meier showed that patients with $\mathrm{SIA} / \mathrm{G}$ value $\approx 3.3$ presented a risk of dying almost 9 times higher $(\mathrm{HR}=8.75 ;[\mathrm{Cl} 4.02-19])$. The AUROC of the SIA/G was higher than that of the rest of the scores for the MI (AUROC SIA/G 0.88, AUROC GAP 0.86, AUROC RTS 0.80, AUROC ISS 0.86).

Conclusion. SIA/G is the best score predicting mortality in polytraumatized patients, being an easy tool to calculate at bedside. A higher SIA/G ( $\gtrsim 3.3$ ) indicates a higher MI and M24, so the application upon arrival of the patient to the Emergency Department can facilitate the diagnosis and treatment in the "golden hour" of polytrauma. 
Reference(s)

1. - McNab A, Burns B, Bhullar I, Chesire D, Kerwin A. An analysis of shock index as a correlate for outcomes in trauma by age group. Surgery 2013 154(2): 384-7

2. - Kimura A, Tanaka N. Reverse shock index multiplied by Glasgow Coma Scale score ( $\mathrm{rSIG}$ ) is a simple measure with high discriminant ability for mortality risk in trauma patients: an analysis of the Japan Trauma Data Bank. Crit Care. 2018 Apr 11;22(1):87. Asian blunt trauma Victims. World J Surg. 2012;36:81-8.

3. - Zarzaur BL, Croce MA, Fischer PE, Magnotti LJ, Fabian TC. New vitals after injury: shock index for the young and age $x$ shock index for the old. J surg res. 2008;147(2):229-36. Remove [x]

4. - King RW, Plewa MC, Buderer NM, Knotts FB. Shock index as a marker for sig- nificant injury in trauma patients. Acad Emerg Med. 1996 Nov;3(11):1041-5

5. - Cannon CM, Braxton CC, Kling-Smith M, Mahnken JD, Carlton E, Moncure M. Utility of the shock index in predicting mortality in traumatically injured patients. J Trauma. 2009;67:1426-30

6. - Baker SP, O'Neill B, Haddon W, Long WB. The injury severity score: a method for describing patients with multiple injuries and evaluating emergency care. J Trauma. 1974;14:187-96

7. - Kondo Y, Abe T, Kohshi K, Tokuda Y, Cook EF, Kukita I. Revised trauma scor- ing system to predict in-hospital mortality in the emergency department: Glagow Coma Scale, Age, and Systolic Blood Pressure score. Crit Care. 2011;15:R191

8. - Parks JK,Elliott AC, Gentilello LM, Shafi S. Systemic hypotension is a late marker of shock after trauma: a validation study of Advanced Trauma Life Support principles in a large national sample. Am. J. Surg. 2006;192: 727-31

9. - The authors declare that there is no conflict of interest

10. The author(s) received no financial support for the research, authorship, and/or publication of this article.

\section{1}

Characteristics and outcomes of abdominal compartment syndrome in patients with trauma: a descriptive study from a nationwide cohort in Japan

A. Komori ${ }^{1}$; H. Iriyama ${ }^{1}$; T. Kainoh ${ }^{1}$; K. Inaba ${ }^{1}$; K. Narahashi ${ }^{1}$; M. Aoki ${ }^{2}$; T. Abe $^{1}$

${ }^{1}$ Department of Emergency and Critical Care Medicine, Tsukuba Memorial Hospital, Tsukuba, Japan; ${ }^{2}$ Advanced Medical Emergency Department and Critical Care Center, Japan Red Cross Maebashi Hospital, Maebashi, Japan

Correspondence: A. Komor

Intensive Care Medicine Experimental 2021, 9(1): 000391

Introduction. Abdominal compartment syndrome (ACS) is a rare complication associated with poor outcomes [1]. Little research using a nationwide database has been done on clinical characteristics and outcomes of ACS among trauma patients.

Objectives. We aimed to describe the characteristics, patterns, and outcomes of trauma patients with ACS based on data collected from a nationwide trauma registry in Japan.

Methods. This retrospective cohort study using data from the Japan Trauma Data Bank (JTDB) was conducted to describe the baseline characteristics, treatments, concomitant complications, and outcomes of all trauma patients with ACS from 2004 to 2017. ACS was diagnosed by the physician in charge.

Results. Among 294,274 trauma patients registered in the JTDB from 2004 to 2017, a total of 295 (0.1\%) patients developed ACS following trauma. The median age was 58 [interquartile range (IQR), 35-71] years, and $211(71.5 \%)$ of the patients were male. The median body temperature on arrival at emergency departments was $35.8^{\circ} \mathrm{C}$ (IQR, 35.0-36.4). The abdomen was the most frequent injury site classified over 3 by the abbreviated injury scale $(57.3 \%)$, followed by the lower extremities including pelvis (46.4\%) and the thorax (46.4\%). The median injury severity score was 29 (IQR, 20-43). The most frequent concomitant complications were disseminated intravascular coagulopathy (34.6\%), followed by thrombocytopenia (26.1\%), and sepsis (17.3\%). The treatments and interventions administered at emergency departments included blood transfusion within $24 \mathrm{~h}(66.1 \%)$, oral intubation or ventilator use (54.2\%), central venous line use (41.7\%), and emergency trans-arterial embolization $(41.7 \%)$. The most common primary major surgery was celiotomy (48.5\%). Of those, $18.0 \%$ underwent the operation within $1 \mathrm{~h}$, and $58.3 \%$ received surgery within $3 \mathrm{~h}$ of arriving at emergency departments. A total of $95.2 \%$ patients were admitted to intensive care units (ICU). The overall in-hospital mortality was $58.5 \%$, and of those $3.4 \%$ died at emergency department.

Conclusion. ACS was one of the most severe complications related to trauma. Almost all patients were admitted to ICU and over half of the trauma patients died if they developed ACS.

\section{4}

Temperature trajectories and hospital mortality among patients with severe trauma: a retrospective cohort study in Japan H. Iriyama ${ }^{1}$; A. Komori ${ }^{1}$; T. Kainoh ${ }^{1}$; K. Inaba ${ }^{1}$; K. Narahashi ${ }^{1}$; M. Aoki ${ }^{2}$;T. $\mathrm{Abe}^{1}$

'Department of Emergency and Critical Care Medicine, Tsukuba Memorial Hospital, 1187-299 Kaname, Tsukuba, Ibaraki, Japan, Tsukuba, Japan; ${ }^{2}$ Advanced Medical Emergency Department and Critical Care Center, Japan Red Cross Maebashi Hospital, Maebashi, Japan

\section{Correspondence: $\mathrm{H}$. Iriyama}

Intensive Care Medicine Experimental 2021, 9(1): 000554

Introduction. Hypothermia is a component of the lethal triad of trauma. Previous studies have focused on hypothermia at hospital admission. However, little is known about body temperature after admission among patients with trauma.

Objectives. We aimed to evaluate the association between body temperature trajectories and hospital mortality among patients with severe trauma.

Methods. This is a retrospective cohort study using a nationwide trauma registry named the Japan Trauma Data Bank (JTDB), compiled from 2004 to 2017. The patient inclusion criteria were as follows: blunt or penetrating trauma, age $\geq 16$ years old, Injury Severity Scores (ISSs) $\geq 16$, ICU admission, information on body temperature at the emergency department, and information on the presence of hypothermia after admission. Hypothermia at the emergency department (ED) was defined as $<36.0^{\circ} \mathrm{C}$. We defined the following four body temperature trajectory groups: normothermia (absence of hypothermia at the ED and during hospitalization), late-onset hypothermia (absence of hypothermia at the ED and presence of hypothermia during hospitalization), initial hypothermia (presence of hypothermia at the ED and absence of hypothermia during hospitalization), and persistent hypothermia (presence of hypothermia at the ED and during hospitalization). We conducted logistic regression analysis to evaluate body temperature trajectories and in-hospital mortality. We adjusted for variables that were selected based on prior studies and univariate analysis, such as age, gender, mechanism of injury, vital signs at the ED (systolic blood pressure, Glasgow Coma Scale, and respiratory rate), ISS, number of comorbidities, emergency procedures (intubation, ventilation, vasopressor, intra-aortic balloon occlusion, central venous catheter placement, transfusion, and transcatheter arterial embolization), and surgeries.

Results. Of those who were registered in the JTDB database, 55,054 patients were included in this study. Among them, 39,256 (71.3\%) were male, and the median age was 61 years (interquartile range (IQR), 40-74). The median ISS was 24 (IQR: 17-29). A total of 50,496 (91.7\%) patients were in the normothermia group, $94(0.2 \%)$ were in late-onset hypothermia, 4,209 (7.7\%) were in initial hypothermia, and $255(0.5 \%)$ were in persistent hypothermia. Hospital mortalities numbered 5,304 (10.8\%) in normothermia, 50 (56.8\%) in late-onset hypothermia, 901 $(22.1 \%)$ in initial hypothermia, and 62 (24.9\%) in persistent hypothermia, respectively.

In logistic regression analysis, late-onset hypothermia (odds ratio (OR) 2.69; 95\% confidence interval (Cl) 1.42-5.08), initial hypothermia (OR 1.24; 95\% Cl 1.16-1.33), and persistent hypothermia (OR 1.70; 95\% $\mathrm{Cl} 1.22-2.36)$ were independently associated with hospital mortality compared to normothermia. 
Conclusion. Temperature trajectories were associated with in-hospital mortality in patients with severe trauma. It is imperative to maintain body temperature not only at initial admission but also afterwards in patients with trauma.

\section{5 \\ Implementation of the French lyophilized plasma in a trauma center for severe trauma patients. A retrospective study \\ T. LEBOUVIER'; Y. Launey, ; F. Delamaire'; P. Seguin, \\ 135, CHU Rennes-Pontchaillou Hospital, Rennes, France \\ Correspondence: T. LEBOUVIER \\ Intensive Care Medicine Experimental 2021, 9(1): 000745}

Introduction. The blood products transfusion is an essential part of damage control resuscitation for severe trauma patients with hemorrhagic shock. The French and European guidelines on management for major bleeding and coagulopathy following trauma recommended early transfusion of packed red blood cells (pRBC) and fresh frozen plasma (FFP) with a ratio of 1:1 to 1:2 $(1,2)$. Freeze-dried plasma, called the French lyophilized plasma (FLYP) may be a solution to overcome the logistical constraints of transporting and thawing FFP. To that extent, the administration of FLYP further reduce the time to first plasma transfusion and may be essential to reduce coagulopathy. Few data and contradictory results are reported (3-5).

Objectives. In this context, the aim of our study was to evaluate the implementation of FLYP as first line of plasma transfusion in trauma patients who required massive transfusion on a Trauma Center.

Methods. This retrospective single-center study was performed in a University Hospital Trauma Center from January 2015 to June 2020. Since June 2017, FLYP was available in the Trauma Center for patients with hemorrhagic shock requiring massive transfusion, but not available in the prehospital setting. Two consecutive periods were compared: From January 1, 2015 to May, 312017 before FLYP availability (period 1-FFP group) and from June, 12017 to June, 30 2020, after FLYP availability (period 2-FLYP group). The main objective was to compare, in patients who received massive transfusion, the time to first plasma transfusion and the ability to obtain a plasma:pRBC ratio superior to 1:2 between this two periods. Secondary Objectives were to evaluate the impact of FLYP infusion on the number of RBC transfused before first plasma transfusion. According to the French law, the present study was approved by the local ethical committee of our hospital.

In the FFP group, transfusion was started with 2 pRBCs stored in the trauma Center. Fresh frozen plasma and others pRBCs requirements was provided by the French Blood Establishment. In the FLYP group, the transfusion protocol was modified to include FLYP instead of FFP and transfusion was activated when trauma patients had an Assessment of Blood Consumption score $(A B C) \geq 2$ (6).

Results. Between January 1, 2015 and June 30, 2020, 204 patients were admitted in the trauma center, 62 received massive transfusion and 40 were analyzed (FFP group, $n=12$ and FLYP group, $n=28$ ). Patients in the FLYP group were significantly older and tends to be more severe head injury. The times required to reach hospital and those spent in the trauma center did not differ between the two groups as well as the time between the trauma and an effective hemostasis. All the patients received tranexamic acid an fibrinogen in the prehospital and hospital setting until effective hemostasis. The time until the first transfusion of plasma was significantly reduced in the FLYP group with a median of $35(20-45)$ min vs. $59(36-88) \mathrm{min}$ in the FFP group $(\mathrm{p}<0.01)$. The number of pRBC transfused before the first plasma was significantly lower in the FLYP group 2 (IQR 1-3) vs. 4 (IQR $3-4)$ in the FFP group $(p<0.01)$. The plasma: $R B C$ ratio $\geq 1: 2$ was significantly more frequently observed after FLYP implementation. Prehospital transfusion of pRBC did not differ between the 2 groups as cristalloids infusion. The number of pRBC, FFP and platelets transfused during the first $24 \mathrm{~h}$ were similar in both groups. At the end of the trauma center management, there was no difference between the 2 groups according to the number of patients who had a prothrombin time $\geq 50 \%$ or a fibrinogen level $>1.5 \mathrm{~g} / \mathrm{l}$. No side effects were observed in either the FFP or FLYP group.

Mortality in the trauma center, at $24 \mathrm{~h}$ and at day 30 did not differ between the 2 groups.

Conclusion. Our study suggests that the French lyophilized plasma allowed an early transfusion of plasma during massive transfusion of severe trauma patients. It significantly reduces time for in hospital plasma delivery and allowed to obtain a plasma:RBC ratio $\geq 1: 2$ in the trauma center according to the guidelines but did not modify the quantity of crystalloids infused nor the coagulation test at the end of the trauma center management.

\section{Reference(s)}

1. 1. Duranteau J, Asehnoune K, Pierre S, Ozier Y, Leone M, Lefrant J-Y. Recommandations sur la réanimation du choc hémorragique. Anesth Réanimation. 2015 Feb; 1 (1):62-74.

2. 2. Spahn DR, Bouillon B, Cerny V, Duranteau J, Filipescu D, Hunt BJ, et al. The European guideline on management of major bleeding and coagulopathy following trauma: fifth edition. Crit Care. 2019 Dec;23(1):98.

3. 3. Vitalis V, Carfantan C, Montcriol A, Peyrefitte S, Luft A, Pouget T, et al. Early transfusion on battlefield before admission to role 2: A preliminary observational study during "Barkhane" operation in Sahel. Injury. 2018 May;49(5):903-10

4. 4. Nguyen C, Bordes J, Cungi P-J, Esnault P, Cardinale M, Mathais Q et al. Use of French lyophilized plasma transfusion in severe trauma patients is associated with an early plasma transfusion and early transfusion ratio improvement. J Trauma Acute Care Surg. 2018 May;84(5):780-5.

5. 5. Garrigue D, Godier A, Glacet A, Labreuche J, Kipnis E, Paris C, et al. French lyophilized plasma versus fresh frozen plasma for the initial management of trauma-induced coagulopathy: a randomized open-label trial. J Thromb Haemost. 2018 Mar;16(3):481-9.

6. 6. Nunez TC, Voskresensky IV, Dossett LA, Shinall R, Dutton WD, Cotton BA. Early Prediction of Massive Transfusion in Trauma: Simple as ABC (Assessment of Blood Consumption)? J Trauma Inj Infect Crit Care. 2009 Feb;66(2):346-52.

\section{6}

Relation of a hypocoagulable ROTEM profile to other coagulation tests in trauma patients

R. Dujardin'; DJ. Kleinveld ${ }^{2}$; K. Baksaas-Aasen ${ }^{3}$; C. Gaarder ${ }^{3}$; K. Brohi'; R.

Davenportt; N. Curry ${ }^{5}$; S. Stanworth ${ }^{5}$; P. Johansson ${ }^{6}$; J. Stensballe ; M.

Maegele ${ }^{8}$; NP. Juffermans ${ }^{2}$

${ }^{1}$ Department of Intensive Care Medicine, Amsterdam UMC, locatie AMC, Amsterdam, Netherlands; ${ }^{2}$ Laboratory of Experimental Intensive Care and Anesthesiology, Amsterdam UMC, locatie AMC, Amsterdam, Netherlands; ${ }^{3}$ Department of Traumatology, University Hospital Oslo, Oslo, Norway; ${ }^{4}$ Center for Trauma Sciences, Queen Mary University of London, London, United Kingdom; ${ }^{5}$ Nhs Blood and Transplant, John Radcliffe Hospital, Oxford, United Kingdom; ${ }^{6}$ Transfusion medicine, Copenhagen University Hospital, Rigshospitalet, København, Denmark; ${ }^{7}$ Department of Anesthesiology, Copenhagen University Hospital, Rigshospitalet, København, Denmark; ${ }^{8}$ Department of Traumatology and Orthopedic Surgery, Krankenhaus Merheim, Köln, Germany

Correspondence: R. Dujardin

Intensive Care Medicine Experimental 2021, 9(1): 000846

Introduction. Coagulation disturbances following trauma contribute to mortality [1]. Rotational thromboelastometry (ROTEM) is increasingly used to diagnose coagulopathy and guide treatment in trauma patients. However, the relation of a hypocoagulable ROTEM profile to other coagulation tests is unknown.

Objectives. To investigate how a hypocoagulable ROTEM profile reflects in vivo haemostasis in trauma patients.

Methods. This study is conducted as part of the prospective, multicentre, Activation of Coagulation and Inflammation in Trauma (ACIT) trial. All trauma patients $\geq 18$ years with full trauma team activation between January 2008 and April 2015 were included. Patients were excluded if they received $>2 \mathrm{~L}$ i.v. fluids before hospital admission, 
arrived $>2 \mathrm{~h}$ after injury or suffered from burns covering $>5 \%$ of the body.

For the analysis, two phases of hypocoagulability were distinguished. The initiation phase was defined as a prolonged EXTEM clotting time (CT) of $\geq 80 \mathrm{~s}$ (s). The propagation phase (representing clot strength) was defined as an EXTEM clotting amplitude at $5 \mathrm{~min}$ (CA5) of $<40 \mathrm{~mm}$ $(\mathrm{mm})$. Coagulation tests were compared between hypocoagulable and non-hypocoagulable groups.

Results. ROTEM data of 1968 trauma patients were available, of whom 304 (15.4\%) had a prolonged EXTEM CT and 511 (26.0\%) had a decreased EXTEM CA 5.

Patients with an EXTEM CT $\geq 80 \mathrm{~s}$ had a prolonged prothrombin time (PT, 12.4 vs $11.4 \mathrm{~s}, \mathrm{p}<0.01)$ and a prolonged activated partial thromboplastin time (aPTT, 26.0 vs $23.0 \mathrm{~s}, \mathrm{p}<0.01$ ), lower levels of factor II (77.4 vs $88.3 \mathrm{IU}, \mathrm{p}<0.01)$, of factor $\mathrm{V}(60.3$ vs $80.3 \mathrm{IU}, \mathrm{p}<0.01)$, of factor VII (80.1 vs $87.0 \mathrm{IU}, p<0.01)$ and of factor $X(78.1$ vs $87.0 \mathrm{IU}, p<0.01)$ when compared to patients with a normal EXTEM CT. Furthermore, a prolonged EXTEM CT was associated with an increase in $12 \mathrm{~h}(8.6 \%$ vs. $1.5 \%, \mathrm{p}<0.01)$ and 28 day mortality $(25.1 \%$ vs $6.6 \%, \mathrm{p}<0.01)$.

Patients with an EXTEM CA5 $<40 \mathrm{~mm}$ had decreased platelet counts (188 vs $237 \times 109 / L, p<0.01$ ), lower fibrinogen ( 1.9 vs. $2.6 \mathrm{~g} / \mathrm{L}, \mathrm{p}<0.01)$ and lower factor XIII levels (91.0 vs $108.4 \mathrm{IU}, \mathrm{p}<0.01$ ) when compared to patients with a normal EXTEM CA5. Also, a prolonged EXTEM CA5 was associated with an increase in $12 \mathrm{~h}(7.6 \%$ vs $0.8 \%, \mathrm{p}<0.01)$ and 28 day mortality $(20.7 \%$ vs $5.3 \%, p<0.01)$.

Conclusion. In trauma patients, an EXTEM CT $\geq 80 \mathrm{~s}$ reflected a modest prolongation of PT and APTT and a modest decrease in coagulation factor levels, most notably of factor V. An EXTEM CA5 $<40 \mathrm{~mm}$ reflected low fibrinogen. Although platelet count and factor XIII were also lower, these tests largely remained within normal ranges. Both phases of hypocoagulability as detected by ROTEM are relevant given their association with increased mortality. These exploratory results provide insight in the meaning of a deranged ROTEM profile.

\section{Reference(s)}

1. [1] Brohi K, Cohen MJ, Davenport RA. Acute coagulopathy of trauma: mechanism, identification and effect. Curr Opin Crit Care. 2007:13(6):680-5.

\section{0}

\section{Epidemiology of burn injuries in Scotland}

C. McGovern ${ }^{1}$; K. Puxty²; M. Shaw ${ }^{2}$;T. Quasim²

${ }^{1}$ Intensive Care Unit, NHS Greater Glasgow and Clyde, Glasgow, United

Kingdom; ${ }^{2}$ Intensive care unit, Glasgow Royal Infirmary, Glasgow, United Kingdom

Correspondence: C. McGovern

Intensive Care Medicine Experimental 2021, 9(1): 001100

Introduction. The incidence of burn injuries across Scotland has been falling due to educational, legislative and other measures (Sarhadi et al.). However, the burden of what are often life-changing injuries cannot be underestimated and there remains a need to accurately describe the epidemiology of these injuries with a view to identifying and acting upon modifiable risk factors.

Objectives. To describe the characteristics of burn injuries requiring hospital admission across Scotland, identify common features associated with such injuries and describe the differences between adult and paediatric ( $<16$ years old) burn injury patterns.

Methods. All patients admitted to hospital under the care of a specialist burn team between 2012 and 2015 were identified using the COBIS (Care of Burns in Scotland) national network database and linked to acute hospital administrative data (SMR01). Differences between adult and paediatric variables were assessed using Pearson's Chi squared, Wilcoxon rank sum or Fisher's exact test.

Results. A total of 1977 individuals were admitted to Scottish hospitals with a primary diagnosis of a burn injury. Male gender accounted for more injuries (63\%) with the majority of burns (90\%) involving less than 10\% TBSA (total body surface area). Patients from more deprived geographical areas, as measured by the SIMD (Scottish Index of Multiple Deprivation), were more likely to suffer a burn injury with individuals from the lowest two quintiles accounting for $57 \%$ of all injuries.

There were significant differences between adult and paediatric burn injury patterns. Although males consistently accounted for more injuries in both groups, this was more pronounced among adult patients $(66 \%$ vs $59 \%, p=0.004)$. Flame burns were also a more common feature among adults (29\%), being relatively rare in children $(6.0 \%)$ $(p<0.001)$, with injuries from hot liquids being the most common mechanism of injury among children (62\%).

The ethnic background among adult patients were similar to that seen among the general Scottish population (census data), with $2 \%$ of patients being of Asian of Afro-Caribbean descent. However, children from ethnic minority backgrounds seemed to make up a much higher proportion of injuries in this cohort $(9.2 \%)(p<0.001)$.

Conclusion. Significant differences exist between the pattern of burn injuries and demographics of burn injuries between adults and children. Although patients from areas of socioeconomic deprivation seem to be at higher risk of sustaining such injuries, this is more pronounced among children. Additionally, children from an ethnic minority background are at much higher risk of sustaining a burn injury, a fact that has been echoed in various similar studies from other developed nations (Tan et al.). Such socioeconomic and ethnic differences should help inform public health interventions to improve household safety for children in deprived areas and among families of ethnic minorities.

\section{Reference(s)}

1. Tan KT, Prowse PM, Falder S. Ethnic differences in burn mechanism and severity in a UK paediatric population. Burns. 2012;38(4):551-555. Ethnicity, Identity, Language and Religion | Scotland's Census.

3. Sarhadi NS, Reid WH, Murray GD, Williamson J. Flame burn admissions and fire fatalities in Scotland with particular reference to the Strathclyde (Glasgow) region, and their prevention. Burns. 2001;27(7):731-738.

4. Access to data was partially funded by a grant from the NIAA (National Institute of Academic Anaesthesia) and Association of Anaesthetists.

\section{9}

Relationship between the administration of noradrenaline and adverse outcomes in burn patients

A. Salvador-Rodríguez, ; D. Vasco; D. Manzano, ; J. Martins ${ }^{3}$; S. Ortiz, ; P. Fernandez Andújar ${ }^{4} ;$ O. Peñuelas ${ }^{5}$; JÁ. Lorente,

${ }^{1}$ Getafe University Hospital, Universidad Europea de Madrid (Campus Villaviciosa), Villaviciosa de Odón, Spain; ${ }^{2}$ Intensive Care Unit, Getafe University Hospital, Getafe, Spain; ${ }^{3}$ Nephrology Service, Getafe University Hospital, Getafe, Spain; ${ }^{4}$ Facultad de medicina, Universidad Europea de Madrid (Campus Villaviciosa), Villaviciosa de Odón, Spain; ${ }^{5}$ Getafe University Hospital, CIBERES, Madrid, Spain; ${ }^{6}$ Getafe University Hospital. universidad europea de madrid, CIBERES, Madrid, Spain

Correspondence: $O$. Peñuelas

Intensive Care Medicine Experimental 2021, 9(1): 001209

Introduction. Here is controversy as to whether the administration of noradrenaline (NA) to burn patients during the resuscitation phase is harmful. This information is important to better design resuscitation strategies associated with better outcomes.

Objectives. To demonstrate that the administration of NA to burn patients during the resuscitation phase (day 0 to day 3 after injury) is associated with adverse outcomes after resuscitation.

Methods. We conducted an observational, cohort, retrospective analysis of a database of burn patients admitted to the intensive care burn unit (ICBU) of Hospital Universitario de Getafe, from 1991 to 2017. Inclusion criteria were age 16 or older, and admission with any body surface area (BSA) burned or inhalation injury. Exclusion criteria were admission for reasons other than acute burns, admission $>24 \mathrm{~h}$ after injury, and ICBU length of stay $<3$ days. Categorical and quantitative variables were compared with the Chi square and the Student's $t$ test, respectively. Univariable and multivariable analyses were performed to prove the association between the use of NA during the 
resuscitation phase as the independent variable and the primary outcome variable (ICBU mortality) and other secondary outcome variables. The strength of the association was measured by the relative risk (RR), odds ratio (OR), adjusted OR, and their $95 \%$ confidence interval (CI). A p value $<0.05$ was considered statistically significant. Values are $\mathrm{n}(\%)$, or means \pm SEM. The study was carried out after approval by the Ethics Committee.

Results. We studied 960 patients (age $46.8 \pm 19.4$ years, $27.8 \%$ female total BSA burned $27.6 \pm 19.9 \%$, inhalation injury $33.0 \%$, on mechanical ventilation $38.0 \%$, receiving NA during the resuscitation phase $15.5 \%$, mortality $12.2 \%)$. The administration of NA was significantly associated with a higher ICBU mortality (34.9 vs. $8.0 \%$, RR $4.354(95 \%$ $\mathrm{Cl}$ : 3.162-5.997), a higher incidence of acute renal failure (72.5 vs. $46.5 \%)$ and requirement of renal replacement therapy (13.4 vs. $3 \%)$, higher serum creatinine concentration on days 4 to 7 after admission, and a higher SOFA score on day $7(4.20 \pm 0.28$ vs. $1.38 \pm 0.08)$ and day 14 ( $3.04 \pm 0.29$ vs. $0.87 \pm 0.07)$ ( $p<0.001$ for all comparisons). In multivariable logistic regression analysis, adjusting for variables known to be related to mortality (age, BSA burned, inhalation injury, female sex, SAPS II), there was a significant adjusted association between the administration of NA and the primary outcome variable (ICBU mortality) (adjusted OR 3.887, 95\% Cl 2.321-6.509, $\mathrm{p}<0.001$ ).

Conclusion. The administration of NA to burn patients during the resuscitation phase is associated with poor outcomes. Although we have not proven a causal relationship, the evidence provided supports the concept that the use of NA in burn patients early after injury could be, independently of other factors, deleterious.

\section{7}

Barotrauma in mechanically ventilated patients with COVID-19 ARDS: Incidence, outcome and potential risk factors

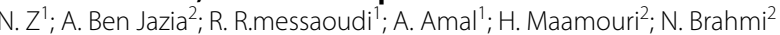
${ }^{1}$ Intensive Care, CAMU, Tunis, Tunisia; ${ }^{2}$ Intensive care, Centre d'assistance médicale urgente-CAMU, Tunis, Tunisia

Correspondence: A. Ben Jazia

Intensive Care Medicine Experimental 2021, 9(1): 001367

Introduction. Barotrauma, defined by the presence of air outside the pleural space is one of the complications of acute respiratory distress syndrome (ARDS) related to COVID-19.

It could be pneumomediastinum (PMD), subcutaneous emphysema (SE), or pneumothorax (PNX).

Objectives. This study aims to determine the incidence of barotrauma in coronavirus disease 2019 (COVID-19) acute respiratory distress syndrome (ARDS) and its outcome.

It also aims to identify risk factors associated with the development of barotrauma in COVID-19 patients.

Methods. It is a retrospective study including 104 COVID-19 patients admitted in our ICU in Tunisia from September 2020 to May 2021.

All patients received the same basic medications.and. Patients requiring mechanical ventilation had protective ventilation and comparable peak airway pressure targets.

Results. Barotrauma occurred in a total of 8 patients $(7.69 \%)$ with a median age of 65.37 years and an average male and female prevalance of $87.5 \%$ and $12.5 \%$, respectively.

All of them were under mechanical ventilation.

Three patients developped PNX (37.5\%), three patients developped both PMD and SE (37.5\%) and two patients (25\%) developped PNX, PMD and SE at the same time.

Median respirator parameters were $10 \mathrm{~cm} \mathrm{H}_{2} \mathrm{O}$ of positive end-expiratory pressure (PEEP), and $6 \mathrm{ml} / \mathrm{kg}$ of tidal volume (VT) with a plateau pressure (Pplateau) target $<30 \mathrm{~cm} \mathrm{H}_{2} \mathrm{O}$.

The mortality in patients with barotrauma was $100 \%$.

Conclusion. Barotrauma is a possible complication in COVID-19 patients with ARDS especially those requiring mechanical ventilation even with the application of protective ventilation.

It may result from lung and airways injury caused by COVID-19.

Its occurrence increases mortality.
e-Posters: Ethics

000231

Eligibility and enrollment of pregnant or breastfeeding individuals in critical care trials

S. Miljanic ${ }^{1}$; S. Jorgensen, ${ }^{1}$; S. Lapinsky, ${ }^{2}$; F. Leung, ${ }^{3}$; N. Tabbara, ${ }^{1}$; . Malhamé $^{4}$; L. Burry ${ }^{2}$

'Department of Pharmacy, Mount Sinai Hospital, Toronto, Canada;

${ }^{2}$ Critical care, Mount Sinai Hospital, Toronto, Canada; ${ }^{3}$ Leslie dan faculty

of Pharmacy, University of Toronto, Toronto, Canada; ${ }^{4}$ Department

of Medicine, Division of General Internal Medicine, McGill University

Health Centre, Montréal, Canada

Correspondence: S. Miljanic

Intensive Care Medicine Experimental 2021, 9(1): 000231

Introduction. The assumption that pregnant and breastfeeding individuals are vulnerable in clinical research and need to be excluded from interventional trials has been challenged. It is unclear whether efforts to increase their inclusion in clinical trials have been successful in critical care research.

Objectives. To characterize the representation of pregnant and breastfeeding individuals in critical care randomized controlled trials (RCTs).

Methods. Critical care-related RCTs published between 2017 and 2019 in five high-impact general and three critical care specialty journals were screened. We excluded trials specific to oncology, pediatrics, geriatrics, male or maternal or obstetrical health and publications of follow-up or subgroup analyses. Key variables of interest included enrolment period, geographic region, sponsor, pregnancy and breastfeeding inclusion/exclusion, criteria for exclusion, and interventions. Descriptive statistics were calculated.

Results. Two authors independently screened 2186 manuscripts of which 147 met inclusion criteria. Only two (1.4\%) included pregnant individuals, 88 (60\%) explicitly stated exclusion, and 57 (39\%) did not specify. Thirty-two (22\%) studies explicitly excluded breastfeeding individuals, all of which had also stated pregnancy exclusion; the remaining 115 (78\%) studies did not specify. Of the studies that explicitly excluded pregnant individuals, the majority were pharmacological interventions $(34 / 88,39 \%)$, most commonly inotropes, vasopressors, or fluids, followed by non-surgical procedures $(21 / 88,24 \%)$. Few studies $(10 / 88,11 \%)$ provided justification for exclusion of pregnant individuals. The most commonly provided justifications were that pregnant individuals were a vulnerable population unable to provide consent, or that the safety of the interventions was unknown in these individuals. Two RCTs of vitamins $C$ and $D$ explicitly excluded pregnancy stating unknown safety, which is inconsistent with currently available data. The two studies that included pregnant individuals were conducted in the USA and evaluated the use of anti-influenza immune plasma for the treatment of severe influenza. Both studies enrolled very few pregnant individuals (1/140 [0.7\%] and 2/98 [2\%]).

Conclusion. Only $1.4 \%$ of critical care RCTs explicitly included pregnant individuals. Pregnant individuals comprised $\leq 2 \%$ of the study population when they were included. Reporting of breastfeeding inclusion criteria was absent in most RCTs. Justification for exclusion was rarely provided. Further research to identify and address barriers to equitable inclusion of pregnant and breastfeeding individuals in critical care RCTs is needed.

\section{Reference(s)}

1. Beigel JH, Aga E, Elie-Turenne MC, et al. Anti-influenza immune plasma for the treatment of patients with severe influenza A: a randomised, doubleblind, phase 3 trial. Lancet Respir Med. 2019;7(11):941-950. https://doi. org/10.1016/S2213-2600(19)30199-7

2. van der Zande ISE, van der Graaf R, Oudijk MA, van Delden JJM. Vulnerability of pregnant women in clinical research. J Med Ethics. 2017;43(10):657-663. https://doi.org/10.1136/medethics-2016-103955

3. Beigel $\mathrm{JH}$, Tebas $\mathrm{P}$, Elie-Turenne MC, et al. Immune plasma for the treatment of severe influenza: an open-label, multicentre, phase 2 randomised study [published correction appears in Lancet Respir Med. 2017 
Jul;5(7):e26]. Lancet Respir Med. 2017;5(6):500-511. https://doi.org/10. 1016/S2213-2600(17)30174-1

4. The authors received no financial support for the research, or authorship of this abstract.

\section{4}

\section{IS IT POSSIBLE TO DONATE WITH RT-PCR POSITIVE} FOR SARS-CoV-2

M. Mateos Llosa'; C. Manso Murcia'²; AM. GÓMEZ DEL PULGAR VILLANUEVA'; MD. Victoria Rodenas ${ }^{3}$; AB. Pérez Pérez'; P. Rivera Sánchez'; M. Granados Madero ${ }^{3}$; N. López Hernández'; J. Moya Sánchez; M. Royo-Villanova Reparaz'; R. Jara Rubio'; M. Carrillo Cobarro'; A. López Domínguez'

${ }^{1}$ Intensive Care Unit, Hospital clinico universitario Virgen de la Arrixaca, Murcia, Spain; ${ }^{2}$ Intensive Care Unit, Hospital Clínico Universitario Virgen de la Arrixaca, El Palmar, Spain; ${ }^{3}$ Servicio de Medicina Intensiva, Hospital Clínico Universitario Virgen de la Arrixaca, El Palmar, Spain

Correspondence: M. Mateos Llosa

Intensive Care Medicine Experimental 2021, 9(1): 000334

Introduction. The COVID-19 pandemic is conditioning important changes in the field of organ donation. Although no case of donorderived COVID-19 infection has been described, there is a theoretical possibility of infection transmission.

Methods. We present a 21-year-old organ donor with a history of cured COVID-19 (more than 14 days from the onset of symptoms and more than $72 \mathrm{~h}$ asymptomatic), but with PCR for SARS-CoV-2 positive in which it was decided to continue with the donation process. The donor was admitted due to severe head trauma, presenting positive RT-PCR in nasopharyngeal exudate, as well as positive IgG serology for SARS-CoV-2, which is why it was decided not to isolate. He evolved to brain death persisting positive PCR result.

Results. For the study of SARS-CoV-2 infection a real-time RT-PCR was performed that detects 3 different genes: $E$ (envelope), RdRp (RNA polymerase-RNA-dependent) and gene $\mathrm{N}$ (nucleocapsid). As an urgent technique, a real-time RT-PCR detecting two targets (S and ORF1ab) was used. The serological study was carried out using a chemiluminescence microparticle immunoassay technique. The microbiological results performed on the donor are shown in Table 1. Heart transplantation was performed without notable incidences, with a favorable evolution and discharge from hospital of the patient, who did not present symptoms compatible with COVID-19. Moreover, RT-PCR for SARS-CoV-2 and serological controls were negative during admission. Conclusion. We consider that the option of donation could be considered even with a positive RT-PCR for SARS-CoV-2, if certain circumstances exist in the donor: i) asymptomatic or oligosymptomatic COVID-19; ii) long-term asymptomatic period; iii) Elevated RT-PCR CT, particularly if the positivity is for certain genetic targets; iv) positivity for IgG antibodies. With these requirements, the benefit of transplantation in terms of survival and quality of life would prevail in decision-making.

Table 1. COVID-19 in the donor

\begin{tabular}{|c|c|c|c|c|c|c|}
\hline \multirow[t]{2}{*}{ Date } & \multicolumn{5}{|c|}{ RT-PCR } & \multirow{2}{*}{$\begin{array}{l}\text { IgG SARS- } \\
\text { CoV-2 }\end{array}$} \\
\hline & $E(C t)$ & $\operatorname{RdRp}(\mathrm{Ct})$ & $N(C t)$ & $S(C t)$ & $\begin{array}{c}\text { ORF1ab } \\
\text { (Ct) }\end{array}$ & \\
\hline Day 0 & $+(35.7)$ & $+(37.6)$ & $+(35.2)$ & & & \\
\hline Day +37 & - & - & $+(34.7)$ & & & + \\
\hline Day +44 & & & & $+(32.2)$ & $+(33.1)$ & \\
\hline $\begin{array}{l}\text { Day +44 } \\
\text { diferido* }\end{array}$ & - & - & - & & & \\
\hline \multicolumn{7}{|c|}{ Ct: Cycle threshold; } \\
\hline
\end{tabular}

000350

Comparison of patient profile, health status and advance directive completion in ICU patients during two waves of the COVID-19 pandemic: a retrospective chart review study

W. Van Oosterwyck ; G. Joni ${ }^{2}$; E. De Waele ${ }^{1}$

${ }^{1}$ Intensive care, UZ Brussel, Jette, Belgium; ${ }^{2}$ Department family medicine \& chronic care, Vrije Universiteit Brussel, Jette, Belgium

Correspondence: W. Van Oosterwyck

Intensive Care Medicine Experimental 2021, 9(1): 000350

Introduction. In Europe, the COVID-19 pandemic presented itself in different waves, flooding the health care system with severely ill patients. Medical knowledge increased over time and treatment was optimised. Nevertheless there is limited data on the use of Advanced Care Directives (ACD) in COVID-19 patients, especially concerning admission to the Intensive Care Unit (ICU). [1,2] ACDs lower the chance of futile therapies and subsequently prolongation of suffering and could be crucial in managing restricted resources. $[3,4]$.

The bundle of different measures and established guidelines since the first wave may have an impact on the patient profile of ICU admissions, outcome and the level of limitations in ACDs. [5,6].

Objectives. We aimed to describe whether there were differences during COVID-19 wave 1 and 2 among tertiary ICU patient demographics, pre-existing health status, prevalence of ACDs (including the degree of limitation) and in-hospital mortality.

Methods. We conducted a retrospective chart review of patients admitted to the ICU in a tertiary centre in Brussels, Belgium during both COVID-19 waves. We extracted data from patients with a positive PCR-test for COVID-19 in combination with respiratory distress. The data included demographic characteristics, clinical frailty score and comorbidities using the Charlson Comorbidity Index (CCI), ACDs (date and degree) and in-hospital mortality. The study was approved by the Ethics Committee University Hospital Brussels. We present descriptive statistics, frequencies and Fisher's exact or t-test statistics to examine differences between the two waves.

Results. We extracted data from 176 patients admitted to ICU between March 14th and May 12th 2020 during the first wave and September 4th 2020 and January 1st 2021 for the second. During wave 1 , the mean age was 64.4 , with $39.5 \%$ of patients being female. Other than gender ratio ( 1.53 vs 4, p0.0073), demographics were similar during wave 2 . Frailty ( 2.91 vs $2.91, \mathrm{p} 0.97), \mathrm{CCl}(3.78$ vs $3.84, \mathrm{p} 0.86)$ and ten-year survival chance ( $53 \%$ vs $56 \%$, p 0.77 ) were not statistically different. During the second wave mortality was higher but not significant ( $38 \%$ vs $43 \%$, p 0.54). Completion of ACDs before or on admission was significantly higher during wave 1 (74\% vs 49\%, p0.0011). [Table 1] The degree of limitation of therapy during the second wave was eventually equal although they were drafted later in the admission.

Table 1. ACD codes.

\begin{tabular}{ll}
\hline ACD & Limitation of therapy \\
\hline Code 0 & Full therapy \\
Code 1 & No CRP \\
Code 2 & No intubation, renal replacement therapy \\
Code 3 & Comfort care \\
\hline
\end{tabular}

Conclusion. ICU patients had similar demographics and comorbidities during two waves of COVID-19. Mortality was slightly higher but not significantly between the waves. During wave 2 significantly more ACDs were filled out at a later stage of the ICU stay. However, no significant differences were found in degree of limitations.

Reference(s)

1. 1. Gupta A, Bahl B, Rabadi S, Mebane A, Levey R, Vasudevan V. Value of Advance Care Directives for Patients With Serious Illness in the Era of COVID Pandemic: A Review of Challenges and Solutions. Am J Hosp Palliat Med 2021;38(2):191-8. 
2. 2. Clarfield AM, Dwolatzky T, Brill S, et al. Israel Ad Hoc COVID-19 Committee: Guidelines for Care of Older Persons During a Pandemic. J Am Geriatr Soc 2020;68(7):1370-5.

3. 3. Farrell TW, Francis $L$, Brown $T$, et al. Rationing Limited Healthcare Resources in the COVID-19 Era and Beyond: Ethical Considerations Regarding Older Adults. J Am Geriatr Soc 2020;68(6):1143-9.

4. 4. Detering $K M$, Hancock $A D$, Reade MC, Silvester W. The impact of advance care planning on end of life care in elderly patients: Randomised controlled trial. BMJ 2010;340(7751):847.

5. 5. Radovanovic D, Pini S, Franceschi E, et al. Characteristics and outcomes in hospitalized COVID-19 patients during the first 28 days of the spring and autumn pandemic waves in Milan: An observational prospective study. Respir Med 2021;178(January).

6. 6. Adams C. Goals of Care in a Pandemic: Our Experience and Recommendations. J Pain Symptom Manage 2020;60(July):15-7.

7. No grants were received. No conflict of interest to declare.

\section{1}

Heart transplantation, a new program of donation after the circulatory determination death

\section{Mateos Llosa'; C. Manso Murcia²; AM. GÓMEZ DEL PULGAR}

VILLANUEVA ${ }^{1} ; A B$. Pérez Pérez ${ }^{1}$; MD. Victoria Rodenas²; P. Rivera Sánchez ${ }^{1}$;

M. Granados Madero²; A. López Domínguez ; J. Moya Sánchez ${ }^{1}$; M. Carrillo

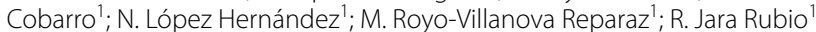
${ }^{1}$ Intensive Care Unit, Hospital clinico universitario Virgen de la Arrixaca, Murcia, Spain; ${ }^{2}$ Intensive Care Unit, Hospital Clínico Universitario Virgen de la Arrixaca, Murcia, Spain

Correspondence: M. Mateos Llosa

Intensive Care Medicine Experimental 2021, 9(1): 000351

Introduction. Heart transplantation is currently the most effective treatment to improve the prognosis of patients with end-stage heart failure. In recent years, controlled donation after circulatory determination of death (CDCD) with regional normothermic thoracoabdominal perfusion (TA-NRP) has been developed in Spain.

We present the initial experiences in cardiac transplantation from CDCD donors with TA-NRP at the Hospital Virgen de la Arrixaca in Murcia.

Methods. A total of 3 heart transplantations have been performed at our center using TA-NRP.

The donors were patients under 45 years of age admitted to the ICU in whom the medical team and legal representatives of the patients had made the decision for withdrawal of life support therapy (WLST).

WLST was performed in the operating room in all cases. Donor followup was performed using a Swan-Ganz catheter and continuous transesophageal echocardiography (TEE). Brain activity was monitored using the bispectral index.

After limitation, the 3 vessels of the aortic arch were clamped to exclude cerebral circulation. Simultaneously, the patient was intubated and mechanical ventilation was initiated.

In all cases the heart reverted to sinus rhythm within $1 \mathrm{~min}$. According to TEE and mean arterial pressure monitoring, dobutamine infusion was started in one of the donors; noradrenaline was not necessary in any of the cases.

The left ventricular ejection fraction and the cardiac index at the time of validation was optimal.

Results. The retrieved hearts were transplanted directly into recipients located in the adjacent operating room. All recipients experienced an excellent outcome, being easily weaned off from cardiopulmonary bypass. None of them required mechanical support after transplantation. All recipients had a short ICU stay and were discharged home with an excellent evolution and optimal cardiac index.

Conclusion. Transplantation from CDCD donors represents an important source of grafts in countries with an adequate legal framework.

TA-NRP may become a way to make CDCD donor heart transplantation feasible, reducing the costs of ex situ machine devices by making this procedure economically affordable.
000430

How doctors actually (do not) involve families in decisions to continue or discontinue life-sustaining treatment in neonatal, pediatric and adult intensive care: A qualitative study

A. Akkermans'; J. Lamerichs, ${ }^{2}$; M. Schultz ${ }^{3}$;TGV. Cherpanath, ; JBM.

Van Woensel ${ }^{4}$; M. Van Heerde ${ }^{4}$; AH. Van Kaam5. M. Van De Loo ${ }^{6}$; AM. Stiggelbout ${ }^{7}$; EMA. Smets ${ }^{1}$; MA. De Vos ${ }^{8}$

${ }^{1}$ Department of Medical Psychology, Amsterdam UMC, locatie AMC, Amsterdam, Netherlands; ${ }^{2}$ Department of Language, Literature and Communication, Vrije Universiteit Amsterdam, Amsterdam, Netherlands; ${ }^{3}$ Department of Intensive Care Medicine, Amsterdam UMC, locatie AMC, Amsterdam, Netherlands; ${ }^{4}$ Department of Pediatric Intensive Care, Emma Children's Hospital, Amsterdam UMC, locatie AMC, Amsterdam, Netherlands; ${ }^{5}$ Department of Neonatology, Amsterdam UMC, Amsterdam, Netherlands; ${ }^{6}$ Department of Neonatology, Amsterdam UMC, locatie AMC, Amsterdam, Netherlands; ${ }^{7}$ Department of Biomedical Data Science, Leiden University Medical Center (LUMC), Leiden, Netherlands; ${ }^{8}$ Department of Pediatrics, Amsterdam UMC, locatie AMC, Amsterdam, Netherlands

Correspondence: A. Akkermans

Intensive Care Medicine Experimental 2021, 9(1): 000430

Introduction. In intensive care units, decisions about the continuation or discontinuation of life-sustaining treatment often concern decisions in the 'gray zone'. These decisions often involve family members as surrogate decision-makers. Families vary in their preferences to what extent they wish to be involved.

Intensive care doctors have to find the right balance between sharing crucial decisions with families of patients on the one hand and not overburdening them on the other hand. This requires a tailored approach instead of a model based approach.

Objectives. To explore how doctors involve families in the decisionmaking process regarding life-sustaining treatment on the neonatal, pediatric and adult intensive care.

Methods. The explorative analysis of 101 audio-recorded conversations focused on doctors' communicative behaviors to (not) involve families of patients in the decision-making process, using inductive thematic analysis. 104 family members (61\% female, 39\% male) and 71 doctors ( $60 \%$ female, $40 \%$ male) of 36 patients ( $53 \%$ female, $47 \%$ male) from the neonatal, pediatric and adult intensive care of a large university medical center participated.

Results. Our results show that in a majority of conversations, doctors displayed a variety of communicative behaviors to involve families in the decision-making process. We identified eight relevant and distinct communicative behaviors. Doctors' sequential communicative behaviors either reflected similar approaches-a shared approach or a physician-driven approach - or reflected vacillating between both approaches. Doctors more often displayed a physician-driven or a vacillating approach than a shared approach, especially in the adult intensive care. Doctors did not verify whether their chosen approach matched the families' decision-making preferences. Families showed very little active input due to doctors' communicative behaviors.

Conclusion. Even though tailoring doctors' communication to families' preferences is advocated, it does not seem to be integrated into actual practice in the neonatal, pediatric and adult intensive care. To allow for true tailoring, doctors' awareness regarding the impact of their communicative behaviors is key. For this purpose, two simple questions can be helpful: (1) 'Which communicative behaviors do/ did I use in this situation?' and (2) 'Why do/did I use these behaviors?' Two important factors to consider in answering the latter question are: 'how gray is the decision?' and 'does my approach fit this family's communication preferences and needs?'.

Educational initiatives should focus especially on improving doctors' skills in tactfully exploring families' decision-making preferences and in mutually sharing knowledge, values and treatment preferences. Peer-to-peer coaching and recurring mirror interviews with families can create more awareness.

Despite cultural differences regarding medical decision-making, countries experience similar dilemmas of whether, in how far, and how to 
involve families of patients in the decision-making process. Our exploration can thus be regarded internationally relevant.

\section{Reference(s)}

1. De Vos MA, Van der Heide A, Maurice-Stam H, et al. The process of endof-life decision-making in pediatrics: a national survey in the Netherlands. Pediatrics. 2011; 127(4): e1004-e1012. https://doi.org/10.1542/peds. 2010-2591

2. Kon AA, Davidson JE, Morrison W, et al. Shared Decision-Making in Intensive Care Units. Executive Summary of the American College of Critical Care Medicine and American Thoracic Society Policy Statement. Am J Respir Crit Care Med. 2016; 193(12): 1334-1336. https://doi.org/10.1164/ rccm.201602-0269ED

3. Joffe H. Thematic analysis. In: Harper D and Thompson A, ed., Qualitative research methods in mental health and psychotherapy: a guide for students and practitioners.

4. Guest G, MacQueen K and Namey E. Applied Thematic Analysis. London: SAGE, 2012

5. De Vos MA, Seeber AA, Gever SKM, et al. Parents who wish no further treatment for their child. J Med Ethics. 2015; 41 (2): 195-200. https://doi. org/10.1136/medethics-2013-101395

6. Scheunemann LP, Ernecoff NC, Buddadhumaruk P, et al. Clinician-Family Communication About Patients'Values and Preferences in Intensive Care Units. JAMA Intern Med. 2019; 179(5): 676-684. https://doi.org/10.1001/ jamainternmed.2019.0027

7. Wilkinson D. Death or Disability?: The 'Carmentis Machine' and decisionmaking for critically ill children. Oxford: Oxford University Press, 2013.

8. Curtis JR and Tonelli MR. Shared Decision-Making in the ICU. Value, Challenges, and Limitations. Am J Respir Crit Care Med. 2011; 183(7): 840-841. https://doi.org/10.1164/rccm.201011-1836ED

9. Norman G. What Few Appreciate:The Gray Zone of Medical Decisions. https://www.linkedin.com/pulse/20140324195914-10808081-what few-appreciate-the-gray-zone-of-medical-decisions (2014, accessed 21 August 2020)

10. Davidson JE, Aslakson RA, Long AC et al. Guidelines for Family-Centered Care in the Neonatal, Pediatric, and Adult ICU. Crit Care Med. 2017; 45(1): 103-128. https://doi.org/10.1097/CCM.0000000000002169

11. This study is part of the research project'FamICom', which was supported by by ZonMw [project number 844001316]. ZonMw is the Dutch organization for healthcare research and innovation.

\section{1}

End of life care and withdrawal in ECMO: A National survey

\section{exploring preferences and practices within the UK}

R. Lewis'; A. Vuylsteke ${ }^{2}$; C. Harvey ${ }^{3}$; M. Garcia ${ }^{4}$; I. Scott ${ }^{5}$; R. Beale ${ }^{6}$; S. Ledot, L. Camporota ${ }^{8}$

${ }^{1} \mathrm{ICU}$, Ion, London, United Kingdom: ${ }^{2}$ Department of anaesthesia and intensive care, Royal Papworth Hospital NHS Foundation Trust, Cambridge, United Kingdom; ${ }^{3}$ Department of anaesthesia and intensive care, Glenfield Hospital, Glenfield, United Kingdom; ${ }^{4} \mathrm{Icu}$, Wythenshawe Hospital, Wythenshawe, United Kingdom; ${ }^{5} \mathrm{Icu}$, Aberdeen Royal Infirmary, Aberdeen, United Kingdom; ${ }^{6}$ Pulmonary, adult critical care and sleep, Guy's and St Thomas' NHS Foundation Trust, London, United Kingdom; ${ }^{7}$ Adult intensive care unit, Royal Brompton, London, United Kingdom;

${ }^{8}$ Critical care, St Thomas' Hospital, London, United Kingdom

Correspondence: R. Lewis

Intensive Care Medicine Experimental 2021, 9(1): 000491

Introduction. Discontinuation of ECMO when no longer deemed beneficial is challenging. The limited consensus for shared decisionmaking can lead to moral distress in healthcare professionals, conflict and complex grief in families. Within the UK there is currently limited guidance to shared and equitable decision-making. This survey aims to explore preferences and practices of end of life care (EOLC) and withdrawal of ECMO across the UK Network.

Methods. The Survey questionnaire was developed using an iterative process: from internal pilot to further retesting to refine and finalise question stems, ensuring face validity and clarity. The final survey created consisted of three sections and 19 questions, including 12 statements. The questionnaire included free text option to ensure capture of comments or examples. The survey was presented at the UK ECMO network annual meeting in October 2020 and then distributed to representatives of the multidisciplinary teams in UK ECMO centres.

Results. There was a total of 36 responses. $83 \%$ of respondents reported not having a framework approach to EOLC or withdrawal from ECMO, despite $91.7 \%$ having been involved with a difficult withdrawal process. Interestingly, $61 \%$ of respondent found it hard to identify or define "futility". Although $94.4 \%$ would not have a pre-defined timeframe to institute withdrawal, $64 \%$ were in favour of discussing the concept of "ECMO trial" at the onset of the ECMO run and that this should be a multidisciplinary process $(94.4 \%) .8 / 12$ statements met strong majority agreement. Strongest agreement was for early palliative care involvement (83.4\%), staff psychological (94.4\%) and bereavement support (100\%).

Conclusion. EOLC and withdrawal decisions in ECMO is challenging and distressing. The responses clearly indicate need for frameworks to support the shared decision making. It has also highlighted key priority areas to be explored as part of ongoing work aiming to produce meaningful guidance in ECMO.

\section{0}

Patient rejection for Intensive Care Unit admission in the time of COVID-19 pandemia

O. Moreno Romero'; M. Muñoz Garach

${ }^{1}$ Intensive Care Unit, Hospital Universitario Clínico San Cecilio, Granada,

Spain

Correspondence: $\mathrm{O}$. Moreno Romero

Intensive Care Medicine Experimental 2021, 9(1): 000510

Introduction. Intensive care physicians face rejection to ICU admission in their every day job. During COVID-19 pandemia, specially due to lack of space and younger patients that needed intensive care support and ventilation therapy, the rejection has become even more difficult and stressing worldwide.

Objectives. To analyse the rejection for ICU admission of patients before and after the COVID 19 pandemia was initiated in our hospital. Methods. We performed a 24-month retrospective study (March 2019-March 2021) of all patients rejected for ICU admission divided in two periods, before COVID pandemia (March 2019-March 2020), and after COVID pandemia was stablished (March 2020-March 2021). We compared the results and patients features in these two periods. Variables registered: sex, age, APACHE II, functional disability, in-hospital death rate.

Results. In 24 months we rejected 1905 patients for ICU admission: 857 in the first period and 1048 in the second. Before pandemia: men $53 \%$, mean age $77 y$ (34-97), APACHE II 18 \pm 8.2 , moderate-severe dissability $71 \%$, in hospital death rate $46 \%$. After pandemia began: men $65 \%$, mean age 69y (41-85), APACHE II 19 \pm 8.7 , moderate-severe dissability $57 \%$, in hospital death rate $56 \%$.

Conclusion. The rejection for ICU admission after COVID 19 pandemia began in our hospital, reduce the mean age (from 77 to $69 \mathrm{y}$ ) and moderate-severe dissability rate (from 71 to $57 \%$ ) of patients rejected and also elevated the in-hospital death rate from 46 to $56 \%$ although APACHE II keeped alike (18 vs 19).

\section{5}

Abdominal and thoracic organs donation from controlled donors after circulatory death: a single Italian centre experience

P. Fassini ${ }^{1}$; C. Mietto, ${ }^{1}$; V. Salice,

${ }^{1}$ Rianimazione, Ospedale di Legnano, Legnano, Italy

Correspondence: P. Fassini

Intensive Care Medicine Experimental 2021, 9(1): 000515

Introduction. Controlled donation after circulatory death (CDCD) has emerged as a valuable resource for reducing the gap between recipients and available organs. In Italy, protocols for CDCD have improved donor supply even though a very conservative law about determination of death1. Long warm ischemia time causes a huge ischemic organ suffering. Normothermic Regional Perfusion (NRP) allows 
abdominal organs donation, but thoracic organs preservation and procurement still remain a challenge.

Methods. In 2017 we implemented a cDCD protocol. Withdrawal of life sustaining therapy (WLST) is decided before any proposal of donation. After checking patient's will and eligibility, WLST could start, providing comfort for patient and relatives, light sedation, extubation and prevention of stridor and dyspnea. After 20 min 'no touch period' we arrange NRP and orotracheal intubation, following the protocol previously published by Valenza et al2. All organs retrieved underwent a machine perfusion reconditioning.

Results. From 2017 to 2021 we performed 9 CDCD. Mean age was $55 \pm 6$ years, baseline ALT and AST were $131 \pm 214$ and $105 \pm 65 \mathrm{U} / \mathrm{l}$ respectively and creatinine was $1.08 \pm 0.54 \mathrm{mg} / \mathrm{dl}$. Baseline lactate was 2,4 \pm 3.1 . Mean total WIT and NRP duration were $50 \pm 10 \mathrm{~min}$ and $226 \pm 30 \mathrm{~min}$, respectively. All nine livers were successfully transplanted. Transaminases increased during NRP in almost all cases, with values greater than $1000 \mathrm{U} / \mathrm{l}$ in 5 cases. Figure 2 shows transaminases trends during NRP. Lactate decreased in all cases (Fig. 3). Two donors were classified as unsuitable for kidney donation due to acute kidney injury and no matching recipient was found in one case. Moreover, three lungs were successfully transplanted following ex vivo lung perfusion (EVLP); follow up at 2 years is good. No primary organ disfunction occurred. Overall follow-up at 3 months showed good function of the transplanted organs.

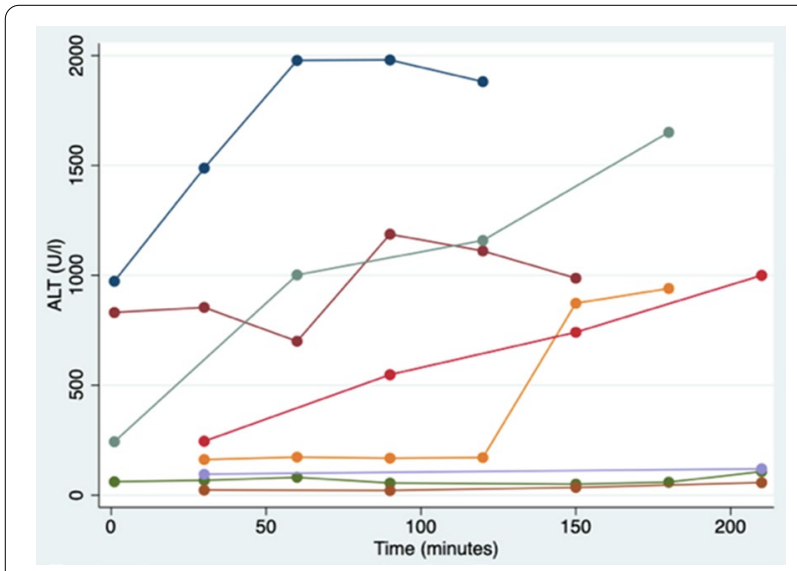

Fig. 2 Transaminases trends

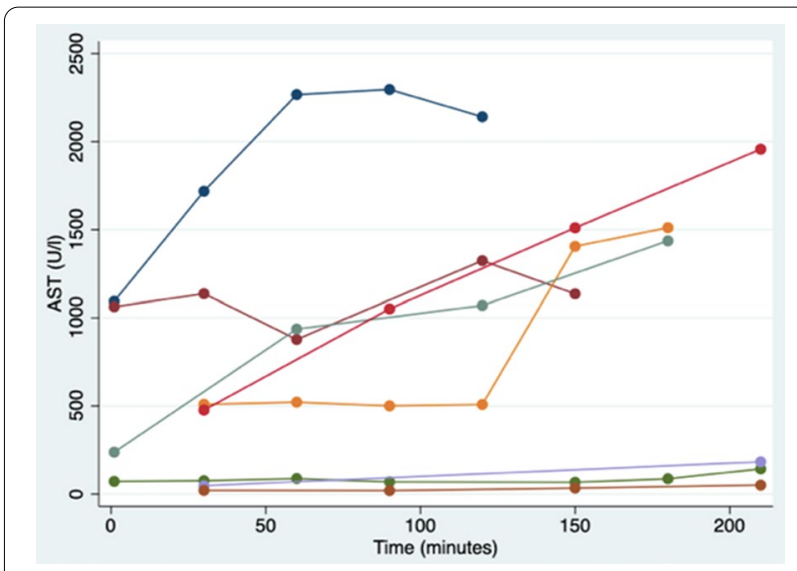

Fig. 3 Lactate trend.
Conclusion. CDCD protocol allows solid organ donation after cardiac death, preserving the dead donor rule and the Italian law. NRP allows ischemia reversal and lactate reduction. Transamineses don't predict liver good function. Lactate seems to correlate with good recovery. Extubation doesn't preclude lung donation. Combined lung and abdominal organ procurement is still a challenge, however thanks to team synergy we succed in.

Reference(s)

1. 1. "Why can't I give you my organs after my heart has stopped beating?" Giannini A et al., Minerva Anestesiol 2016 Mar; 82 (3): 359-68

2. 2. Successful Transplantationof lungs from an uDCD Preserved in situ by Alveolar recruitment Maneuvers and Assessed by EVLP Valenza F et al., Am J Transplant 2016 Apr; 16(4): 1312-8

3. None.

\section{0}

SPECT to verify cerebral circulatory arrest during donation in controlled asystole with normothermic regional perfusion: an ethical issue

C. Manso Murcia'; M. Mateos Llosa ; AB. Pérez Pérez; AM. GÓMEZ DEL PULGAR VILLANUEVA ${ }^{1}$; MD. Victoria Rodenas ${ }^{1}$; N. López Hernández ${ }^{1}$; P.

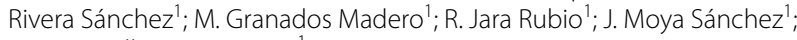
M. Royo-Villanova Reparaz ${ }^{1}$

${ }^{1}$ Intensive Care Unit, Hospital clinico universitario Virgen de la Arrixaca, Murcia, Spain

Correspondence: $C$. Manso Murcia

Intensive Care Medicine Experimental 2021, 9(1): 000670

Introduction. Controlled asystole donation has been proposed as a necessary alternative within a constantly growing transplantation system. The donation process implies strict compliance with ethical principles. The maintenance of the cerebral circulatory arrest during the maneuvers to maintain the perfusion of the rest of the organs should be a maximum in every procedure, and different solutions have been proposed to avoid the flow in the central nervous system.

Objectives. The aim of the study is to verify that during the donation after circulatory death there is an absence of cerebral blood flow and that this condition is irreversible. In this way, we want to demonstrate that there is no recovery of cerebral flow from the thoracoabdominal collateral circulation through the vertebral artery.

Methods. A experimental, prospective, one-center study was performed in Hospital Virgen de la Arrixaca (Murcia, Spain). Portable gammagraphy (Sentinella ${ }^{\circledR}$ ) is performed to donors after circulatory death ( 5 min delayed for legal reasons) with normothermic regional perfusion (NRP). In abdominal NRP, a intraaortic balloon occludes completely the descending aorta to prevent recovery of brain flow. In case of thoracoabdominal NRP, cardiac surgeon clamps the supraaortics vessels and connects the aortic arch vessels to a collecting bag. A Nuclear physician administrates technetium 99 in return cannula of extracorporeal circulation system at the beginning of NRP. The absence of perfusion in the cerebral hemispheres and brainstem confirms the abscense of resuscitation.

Results. Between January and February 2021, 3 donors have been studied. 2 cases were abdominal NRP and 1 of them thoracoabdominal NRP. Average time until cardiac arrest was $12.7 \mathrm{~min}$. None of them showed cerebral perfusion using the portable gamma-camera. In addition, there were no modifications with Bispectral Index, remaining at value 0 .

Conclusion. Absence of cerebral reperfusion using a portable gamma-camera has shown that cessation of brain circulation is permanent after starting the extracorporeal circulation, using the current protocols. This preliminary results shows that this type of donation is ethically and legally valid. 
Reference(s)

1. F.F. K, I.V. P. 99mTc-hexamethylpropyleneamine oxime (HMPAO) —its diagnostic potentials for assessing the cerebral blood supply (a review) [Internet]. Vol. 34, Meditsinskaia radiologiia. 1989 [cited 2021 Apr 8]. p. 29-35. Available from: https://pubmed.ncbi.n/m.nih.gov/2685504/

2. Eynon CA, Murphy PG, Smith M, Danbury C, Manara A. Heart trans-plantation after declaration of death by cardiorespiratory criteria. J Heart Lung Transplant 2009; 29: 232-3

3. Farsides B. Respecting wishes and avoiding conflict: understand-ing the ethical basis for organ donation and retrieval. Br JAnaesth 2012; 108 (Suppl. 1): i73-i79

4. Bell MDD. Non-heart beating organ donation: old procurementstrategy—new ethical problems. J Med Ethics 2003; 29: 176-81

5. Manara AR, Murphy PG, O'Callaghan G. Donation after circulatory death Br J Anaesth. 2012 Jan;108 Suppl 1:i108-21. https://doi.org/10.1093/bja/ aer357. PMID: 22194426

\section{1}

Impact of restricting ICU visits during the health crisis: a qualitative study with caregivers

V. Deltour ${ }^{1}$; P. Gerard ${ }^{2}$; JP. Quenot ${ }^{3} ;$ A. Laurent ${ }^{4} ;$ AL. Poujol $^{5}$

${ }^{1}$ Psychologie, University of Burgundy, Dijon, France; ${ }^{2}$ Psychologie, University of Burgundy, Dijon, France: ${ }^{3}$ Medical intensive care unit, Chu Dijon, Dijon, France; ${ }^{4}$ Psy-DREPI, University of Burgundy, Dijon, France; ${ }^{5}$ Psychopathologies, Ecole de Psychologues Praticiens, Paris, France

Correspondence: $V$. Deltour

Intensive Care Medicine Experimental 2021, 9(1): 000691

Introduction. In the emergency of the crisis, the principles of collegiality and presence of the families in the intensive care unit have been undermined (1). This lack of consultation and connection between caregivers and families is contrary to the recommendations of French resuscitation societies.

Objectives. Based on the Psy-COVID study, we will present our first results concerning the experience of caregivers of the absence of families in intensive care units.Based on the Psy-COVID study, we will present our first results concerning the experience of caregivers of the absence of families in intensive care units.

Methods. Our population consists of 85 caregivers (31 physicians, 11 interns, 30 nurses and 13 orderlies) from 19 French intensive care units. Telephone interviews with these professionals were conducted 3 months after the peak of the first crisis. The interviews were analyzed according to the phenomenological approach of the IPA (2).

Results. The preliminary results of the qualitative analysis underline that the absence of families in the ICU leads to a loss of meaning in practice for the caregivers. On the contrary, some caregivers may experience the restriction as a relief in connection with a decrease in workload. We observe that this ambivalence towards the restriction is a source of guilt. To cope with this restriction, caregivers have been inventive in finding new ways to compensate for this absence (videoconferencing, letters, telephone...).

Conclusion. The restriction of visits had an impact on the caregivers but also allowed the development of new ways of communicating with the families.

\section{Reference(s)}

1. Smith, J. A. (2009). Interpretative phenomenological analysis: Theory, method and research. SAGE.

2. Azoulay, E., Cariou, A., Bruneel, F., Demoule, A., Kouatchet, A., Reuter, D., Souppart, V., Combes, A., Klouche, K., Argaud, L., Barbier, F., Jourdain, M., Reignier, J., Papazian, L., Guidet, B., Géri, G., Resche-Rigon, M., Guisset, O., Labbé, V., ... Kentish-Barnes, N. (2020). Symptoms of Anxiety, Depression, and Peritraumatic Dissociation in Critical Care Clinicians Managing Patients with COVID-19. A Cross-Sectional Study. American Journal of Respiratory and Critical Care Medicine, 202(10), 1388-1398. https://doi. org/10.1164/rccm.202006-25680C

3. Grant by Fondation de France.
001236

Changes in withholding and withdrawal life-sustainig treatments trhough time in one spanish intensive care unit

S. MANRIQUE ${ }^{1}$; S. Canelles ${ }^{1}$; X. Daniel ${ }^{1}$; P. Angel ${ }^{1}$; C. Ferre ${ }^{1}$; M. Bodí ${ }^{1}$

${ }^{1}$ MEDICINA INTENSIVA, Hospital Universitari de Tarragona Joan XXIII, Tar-

ragona, Spain

Correspondence: S. MANRIQUE

Intensive Care Medicine Experimental 2021, 9(1): 001236

Introduction. Research in Medicine has allowed treatment of patients with growth of complexity. It is not only important to treat but also to alleviate when treatment is futile o there is a suffering for the patient. In this context, the practice of life-sustaining treatment (LST) limitation is increasing in all Intensive Care Units (ICU). However, there are not unified criteria and protocols because of the diversity in each country.

Objectives. Evaluate patients admitted in our ICU with a withholding or withdrawal LST applied, comparing the type and their progress over 6 years.

Methods. Retrospective study of cohorts carried out in a 30-bed polyvalent ICU. Inclusion criteria: Patients admitted to ICU between 2014 and 2019. All data were acquired from our Clinical Information System (Centricity Critical Care from General Electric) database through Extract, Transform and Load processes. Demographics, severity scores, length of stay and mortality were registered. Those who had one LST cessation applied type of LST, reason why, time from income until the decision of withholding or withdrawing and time to death were also recorded. Patients with or without LST cessation were compared. Statistical analysis was performed with SPSS v.25.0. P values $<0.05$ were considered significant.

Results. There were 5467 patients admitted in ICU, 64.3\% male, median age 63 years old (50-73), with median APACHE 20 (15-26) and length of stay 3.9 days (2-8.1). Medical admission was in $69.8 \%$ and global mortality of $15.8 \%$. Of all patients, $819(15 \%)$ had at least one LST registered. $64.2 \%$ were male, with median age 72 years old (3-79), 76.6\% medical admission and median APACHE 27 (22-32). In 38.7\%, LST limiting decision was recorded on the first $24 \mathrm{~h}$ of ICU admission. Withholding treatment was established in $76.2 \%$, whereas withdrawing was in $22.2 \%$. In withholding group, renal replacement therapy (RRT) and vasoactive drugs (VD) were the most frequent $(67.5 \%$ and $67.7 \%$ ), and invasive mechanical ventilation (IMV) and vasoactive drugs for withdrawing group $(91.1 \%$ vs. $70.1 \%)$. There were no differences between hypoventilation and terminal extubation (58\% vs. $42 \%)$. Futility (64\%) and comorbidity (53.4\%) were the most common reasons to recess LST. Median time after the establishment and death in ICU was 1.08 days (1-6), 2 days (1-3) in the withholding group and 1 day $(0-1)$ in the withdrawing group. In-hospital mortality rate was $71 \%(63.7 \%$ in ICU, $98 \%$ for withdrawing and $46.5 \%$ for withholding). Patients with any LST limitation are older, with a higher APACHE score, length of stay and mortality compared to those with not, statistically significant. Through the period 2014-2019, LST cessation rate remained unchanged (about 13\%). There is a trend in favor of increasing withdrawing LST ( $25 \%$ in 2014 vs. $34 \%$ in 2019), though the most frequent is withholding (average of $67.2 \%$ ).

Conclusion. LST cessation rate remains unchanged (13\%). Patients with any LST limitation are older, severely ill, with higher ICU length of stay and mortality. RRT, VD and IMV were the most frequent LST. Futility and comorbidity were the most common reasons to establish LST recess. Although there is no statistical significance between withholding and withdrawing therapies in the period studied, the latest one seems to be increasing in time.

\section{Reference(s)}

1. SM Lobo. Decision-Making on Withholding or Withdrawing Life Support in the ICU. A worldwide perspective. 152\#2 CHEST AUGUST 2017.

2. Azoulay E. End-of-life practices in 282 intensive care units: data from the SAPS 3 database. Intensive Care Med (2009) 35:623-630.

3. NM Mark. Global variability in withholding and withdrawal of life-sustaining treatment in the intensive care unit: a systematic review. Intensive Care Med. https://doi.org/10.1007/s00134-015-3810-5.

4. CL Sprung. Changes in End-of-Life Practices in European Intensive Care Units From 1999 to 2016. JAMA Published online October 2, 2019. 
5. Blazquez.V. Factors related to limitation of life support within $48 \mathrm{~h}$ of intensive care unit admission: A multicenter study. Med Intensiva. 2018

6. CL Sprung. End-of-Life Practices in European Intensive Care Units. The Ethics study. JAMA, August 13, 2003-Vol 290, No. 6.

\section{8}

Characteristics and evaluation of cadaveric organ donor potential and comparison of organ donation practices among intensive care units of tertiary care hospital of Sri Lanka

W. Chinthaka'; A. Galliyadda ${ }^{2}$

${ }^{1}$ Anesthesia and Critical Care, National Hospital Kandy, Kandy, Sri Lanka;

2Emergency Medicine, National Hospital-Kandy, Kandy, Sri Lanka

Correspondence: $W$. Chinthaka

Intensive Care Medicine Experimental 2021, 9(1): 001428

Introduction. Organ donation and transplantation plays a key role in the management of the advance end organ failure. The organ demand is steadily increasing and with the advancement of transplant medicine; island wide transplant services also improving which contributes to the increase organ demand. Currently in Sri Lanka major source of organ is from live donors and it is limited. It was therefore necessary to determine whether significant numbers of potential cadaveric organ donors in Sri Lanka do not become actual donors and, if so, to evaluate the reasons for this.

Objectives. To describe the characteristics and to evaluate the cadaveric organ donor potential and to compare organ donation practices among intensive care units of National Hospital, Kandy.

Methods. Hospital based cross sectional analytical study of ICU death victims of year 2019 in intensive care units of National Hospital, Kandy. Determined the sample size necessary with 95\% confidence, and a margin of error of $5 \%$. Assumed a population proportion of 0.5 .571 ICU deaths were recorded during the study period and 370 (64.7\%) were studied using random sampling.

Outcome measures: The proportion of eligible kidney, liver, lung and/or heart donors, the proportion of brain stem tests carried out in potential donors, the proportion of organ donation consideration among brain death victims, the proportion of approaches to next of kin with regard to organ donation among brain death victims, the proportion of consents for organ retrieval among brain death, the proportion of organ donations among brain death victims.

Results. From 370 deaths $22.1 \%(n=82)$ were identified as potential brain death victims. Among them $97 \%(n=80)$ were identified as potential organ donors. Actual donation rate was $2.4 \%(n=2) .80$ lung donors, 55 liver donors, 62 kidney donors and 71 heart donors were missed.55.1\% of deaths $(n=189)$ were not informed to the coroner and in $48 \%(n=178)$ organ donation request were not made. Only $8.5 \%(n=7)$ brain death tests were done among the potential brain death patients, 3 were diagnosed and only 2 patients were referred to transplant team. Intention of organ donation was documented only in 1 patient $(0.2 \%)$

Conclusion. The donor rate could have been increased by overcoming reluctance of ICU consultants in confirming brain deaths in potential candidates and making timely referral to transplant team. Lack of documentation of donor intension in records can be a major obstacle in making such referrals and in the absence of referrals the reluctance to confirm brain death become an inevitable cycle.
Reference(s)

1. Barber K, Falvey S, Hamilton C, Collett D, Rudge C. Potential for organ donation in the United Kingdom: audit of intensive care records. BMJ. 2006 May 13;332(7550):1124-7.

2. Madsen M, Bøgh L. Estimating the Organ Donor Potential in Denmark: A Prospective Analysis of Deaths in Intensive Care Units in Northern Denmark. Transplantation Proceedings. 2005 Oct 1;37(8):3258-9.

3. Flodén A, Kelvered M, Frid I, Backman L. Causes Why Organ Donation Was Not Carried Out Despite the Deceased Being Positive to Donation. Transplantation Proceedings. 2006 Oct 1;38(8):2619-21.

4. Vadysinghe A.N, Dassanayaka P.B, Edussuirya D.H \& Rukshana M.J.F. Tissue Donation and Transplantation Program in Sri Lanka A Medico Legal Review. Sri Lanka Journal of Forensic Medicine, Science \& Law-May 2018Vol.9 No.1

5. Dilshan Priyankara, Makarim Mohamed, Krishakeesen Kamalanthan, Edirisooriya Maddumage Manoj.Public Knowledge on Brain Death and Organ Donation in a Selected Sri Lankan population. Sri Lankan Journal of Anaesthesiology: 27(2):157-159(2019)

6. None.

e-Posters: Data Science

000138

Machine Learning-based Algorithm for predicting the need for postoperative ventilation after elective surgery

D. Andonov'; B. Ulm¹; M. Blobner'; A. Podtschaske ; M. Graessner²; B. Jungwirth²; S. Kagerbauer,

${ }^{1}$ Department of Anaesthesiology and Intensive Care, Klinikum rechts der Isar der Technischen Universität München, München, Germany; ${ }^{2}$ Department of Anaesthesiology and Intensive Care, University Hospital Of Ulm, Ulm, Germany Correspondence: D. Andonov Intensive Care Medicine Experimental 2021, 9(1): 000138

Introduction. Mechanical ventilation results in the increased risk of pulmonary complications.[1] Since the capacity of ICUs is limited especially in the current pandemic situation, it is essential to identify the patients prone to this risk.[2] Machine learning techniques become more and more popular and can be applied for medical purposes.[3] Here, we present the results of our trained ML model which predicts the patients' need for postoperative ventilation after elective surgery.

Objectives. The aim of this project is to create a model based on machine-based learning algorithms to calculate the risk for postoperative need for ventilation after elective surgery.

Methods. After ethical approval (ethics committee number 253/19, ClinicalTrials.gov ID NCT04092933), we analyzed the data of 60,398 non-ICU-patients who underwent elective non-cardiac surgery. Namely, the analysis was based on the pre-operative data which was accumulated in our hospital information and patient data management system (PDMS) from January 2014 to March 2020. We trained an Extreme Gradient Boosting (XGBoost) model with a 80/20 split for training and validation set. The 10,921 features used contained procedural data (28), surgical codes (>9000), laboratory parameter (988), data from preoperative assessment (181) and medication (201). The area under receiver operating curves (AUROC) for postoperative ventilation during hospital stay was found to be 91.4. Finally, we also identified the importance of variables by information gain (IG).

Results. The model predicts the need of post-operative ventilation after elective surgery based on 1033 of 10,921 features with an AUROC 
of 91.4 (Cl: 89.1-93.6) in the test dataset. Median duration of ventilation was $145 \mathrm{~h}$ (IQR 41.0-415.0 h). The 5 most important features were found to be the number of ordered red blood cell concentrates, the number of ordered fresh frozen plasma, haematocrit, body-massindex (BMI) and thyroid-stimulating hormone (TSH). Thus, comparing the obtained AUROC-values, the developed model demonstrates better performance than some previously reported results. [4]

Conclusion. Our ML-based model can help to identify patients at risk for postoperative mechanical ventilation early and accordingly allocate the capacity of the ICU facilities and adjust OR schedules. The study is to be continued in order to estimate the feasibility of applying this method in real clinical settings as well as developing a tool to support triage decisions for optimizing the patient care.

\section{Reference(s)}

1. 1. Clark, P. A. \& Lettieri, C. J. Clinical model for predicting prolonged mechanical ventilation. J. Crit. Care 28, 880.e1-880.e7 (2013).

2. 2. Thevathasan, T. et al. The Impact of Postoperative Intensive Care Unit Admission on Postoperative Hospital Length of Stay and Costs: A Prespecified Propensity-Matched Cohort Study. Anesth. Analg. 129, 753-761 (2019).

3. 3. Ngiam, K. Y. \& Khor, I. W. Big data and machine learning algorithms for health-care delivery. Lancet Oncol. 20, e262-e273 (2019).

4. 4. Bihorac, A., Ozrazgat-Baslanti, T. \& Ebadi, A. MySurgeryRisk: Development and Validation of a Machine-learning Risk Algorithm for Major Complications and Death After Surgery. Ann. Surg. 269, 652-662 (2019).

\section{0}

Effects of combining two machine learning models on mortality prediction in critically ill patients

YC. Yeh ${ }^{1}$; TJ. Lee, ${ }^{2}$; YS. Yu, ${ }^{3}$; F. Lai, ${ }^{3}$; DS. Liu, ${ }^{4}$

'Department of Anesthesiology, National Taiwan University Hospital, Taipei, Taiwan; ${ }^{2}$ Department of Internal Medicine, National Taiwan University Hospital, Taipei, Taiwan; ${ }^{3}$ Graduate Institute of Biomedical Electronics and Bioinformatics, National Taiwan University, Taipei, Taiwan; ${ }^{4}$ Department of Computer Science and Information Engineering, National Taiwan University, Taipei, Taiwan

Correspondence: $Y . C$. Yeh

Intensive Care Medicine Experimental 2021, 9(1): 000300

Introduction. Current machine learning algorithms are unable to train a perfect mortality prediction model with both high sensitivity and high precision from imbalanced dataset (1). In clinical practice, diagnosing a low-incidence/high-risk diseases often use a two-test strategy to maximize both sensitivity and precision (2).

Objectives. This study aimed to investigate whether combining one high-sensitivity machine learning model and one high-precision machine learning model could improve both the sensitivity and precision of mortality prediction in critically ill patients.

Methods. This study was approved by the hospital research ethics committee (REC number 202004016RINB) and registered on the ClinicalTrials.gov protocol registration system (ID NCT04541849). Data of critically ill adult patients were extracted from a standardized database in a medical centre and randomly allocated to the training, validation, and testing datasets. Seven machine learning algorithms were used for model training with the training dataset. Two machine learning models with high sensitivity and high precision, respectively, were selected for mortality prediction.

Results. A total of 4,228 patients were randomly allocated to training, validation, and testing datasets. Of the 843 patients in the testing dataset, 366 patients were classified into the high-risk group with a 30 -day mortality of $29.8 \%$ (95\% confidence interval [Cl], 25.1\%-34.5\%) using the high-sensitivity machine learning model. Subsequently, the high-precision machine learning model subclassified patients in the high-risk group into two subgroups with significantly different mortality $(22.4 \%$ [95\% Cl, $17.9 \%-27 \%$ ] vs. $82.2 \%$ [95\% Cl, $71.1 \%-93.4 \%]$, $\mathrm{p}<0.001)$. In Figure 1

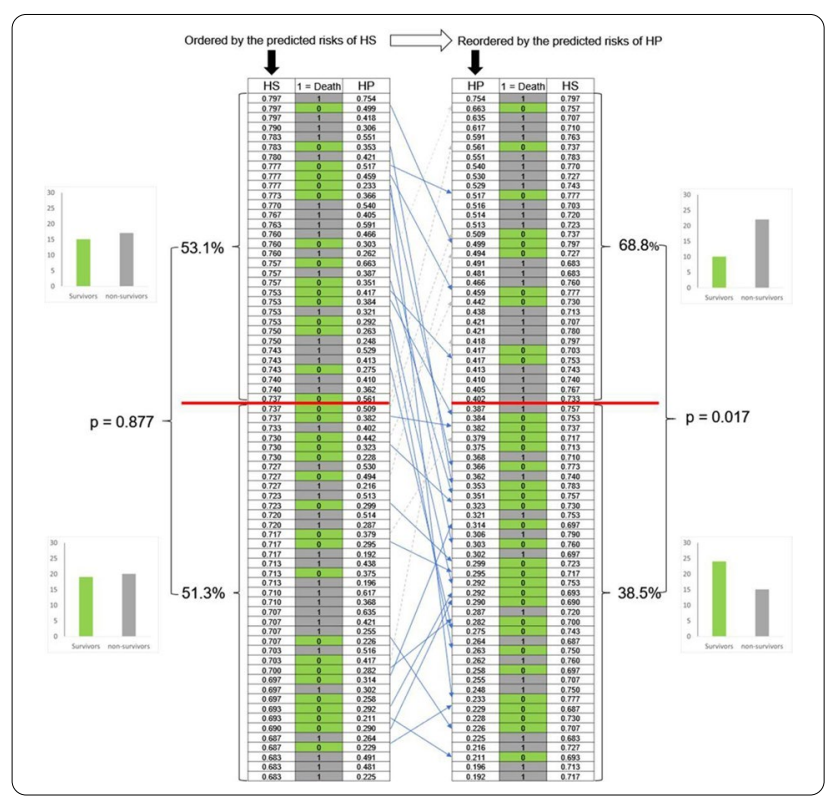

the patients with predicted risk of the high-sensitivity $\mathrm{ML}$ model (ranged within 0.681 to 0.8 ) were subclassified by the high-precision $\mathrm{ML}$ model into two subgroups with different mortality $(38.5 \%[95 \% \mathrm{Cl}$, $23.3 \%-53.7 \%]$ vs. $68.8 \%[95 \% \mathrm{Cl}, 52.7 \%-84.8 \%], \mathrm{p}=0.017)$ with a cutoff predicted risk of 0.4 .

Conclusion. Our results suggest that combining one high-sensitivity machine learning model with one high-precision machine learning model has the potential to improve mortality prediction and enable better clinical interpretation in critically ill patients.

\author{
Reference(s) \\ 1. 2. Iba et al. J Thromb Haemost 2019; 17: 1265-8. \\ 2. 1. Hill et al. British Journal of Anaesthesia 2019; 123: 877-86 \\ 3. National Taiwan University Hospital (NTUH 109-A150) \\ 4. Ministry of Science and Technology, Taiwan (109-2634-F-002-029)
}

000329

Systematic review and comparison of ICU datasets - A decision guide for clinicians and data scientists

CM. Sauer ${ }^{1}$; TA. Dam ${ }^{1}$; L. Celi, ; A. Girbes ${ }^{1}$; P. Elbers ${ }^{1}$

${ }^{1}$ Intensive Care Medicine, Amsterdam UMC, locatie VUmc, Amsterdam, Netherlands; ${ }^{2}$ Laboratory for Computational Physiology, Massachusetts Institute of Technology, Cambridge, United States of America

Correspondence: C.M. Sauer

Intensive Care Medicine Experimental 2021, 9(1): 000329

Introduction. The publication of large, rich, single patient level datasets has significantly propelled clinical research possibilities in the intensive care unit (ICU) and beyond. After publication of MIMIC-III, additional datasets have been released, thus warranting a comparison of their data completeness and richness to allow scientists to choose the most appropriate dataset(s) for their clinical problem.

Methods. A systematic search of published and pre-print articles was performed to identify all publicly available, adult, critical care, patient level databases. Subsequently, databases were compared using a priori defined categories, such as demographics, patient characteristics and data richness. Databases were queried using SQL and (where necessary) data analysis was performed using $R$.

Results. Out of $300+$ publications a total of 4 ICU databases were identified (MIMIC-IV, elCU-CRD, AmsterdamUMCDB, HiRiD). Number of unique patient admissions varied significantly between datasets, 
with elCU-CRD being the largest ( $>130 \mathrm{~K}$ ) and AmsterdamUMCDB the smallest ( $>23 \mathrm{k}$ ). ICU mortality and intensity of treatment also varied, with 28-day mortality rates and frequency of ventilation being lowest in eICU-CRD. Frequency of lab values tended to be highest in MIMICIV, while frequency of vital signs was highest in AmsterdamUMCDB. Conclusion. Several high-quality ICU databases are currently available. The research question, and thus required sample size, presence of covariates and frequency of measurements, should inform which database to use. Due to the underlying differences between the datasets, we suggest using at least two databases to ensure generalizability of findings.

\section{Reference(s)}

1. Johnson AEW, Pollard TJ, Shen L, et al. (2016) MIMIC-III, a freely accessible critical care database. Sci Data 3:1-9. https://doi.org/10.1038/sdata.2016. 35

2. Pollard TJ, Johnson AEW, Raffa JD, et al. (2018) The elCU Collaborative Research Database, a freely available multi-center database for critical care research. Sci Data 5:1-13. https://doi.org/10.1038/sdata.2018.178

3. Thoral PJ, Peppink JM, Driessen RH, et al. (2021) Sharing ICU Patient Data Responsibly Under the Society of Critical Care Medicine/European Society of Intensive Care Medicine Joint Data Science Collaboration: The Amsterdam University Medical Centers Database (AmsterdamUMCdb) Example.

4. Faltys, M., Zimmermann, M., Lyu, X., et al. (2021). HiRID, a high time-resolution ICU dataset (version 1.1.1). PhysioNet. https://doi.org/10.13026/ nkwc-js72.

\section{4}

Machine learning to understand unmet needs for multiclass outcome prediction in the ICU after traumatic brain injury

S. Bhattacharyay ${ }^{1}$; E. Steyerberg'ㄹ. A. Maas ${ }^{3}$; D. Menon ${ }^{1}$; A. Ercole

${ }^{1}$ Division of Anaesthesia, University of Cambridge, Cambridge, United Kingdom; ${ }^{2}$ Department of Biomedical Data Sciences, Leiden University Medical Center (LUMC), Leiden, Netherlands; ${ }^{3}$ Department of Neurosurgery, Antwerp University Hospital, Edegem, Belgium

Correspondence: $\mathrm{S}$. Bhattacharyay

Intensive Care Medicine Experimental 2021, 9(1): 000384

Introduction. Well validated models exist only for dichotomous outcome prognosis after traumatic brain injury (TBI). Dichotomisation, however, fails to capture the broad continuum of outcomes which may be crucial for shared decision-making and treatment stratification in the ICU. On the other hand, multiclass TBI outcome prediction performance is poor, especially for intermediate outcomes, but it is unclear whether this is due to inflexible modelling strategies (i.e., model complexity and outcome encoding) or inadequate disease characterisation by existing predictors.

Objectives. We assess multiclass prediction of the Glasgow Outcome Scale-Extended (GOSE) [1] at 6 months from standard TBI predictors as a function of endpoint encoding (multinomial vs ordinal) and model complexity (logistic regression vs deep learning).

Methods. We use data from a prospective cohort of 3,573 patients from the CENTER-TBI [2] study, extracted using Opal [3]. We use the IMPACT [4] extended predictor set (10 covariates collected within $24 \mathrm{~h}$ of ED/ICU admission) including age, clinical severity scores, secondary insult indicators, CT characteristics, and lab values. Missing values are multiply imputed $(m=100)$, and we train 4 multiclass prediction model types: multinomial logistic regression (MLR), ordinal logistic regression (OLR), neural network with a multinomial output layer (DeepM), and neural network with an ordinal output layer (DeepO). Model performance and calibration is assessed with repeated crossvalidation (20 repeats, 5 folds). We also calculate predictor significance (Shapley values) [5].

Results. As shown in Fig 1, discrimination of GOSE does not vary significantly with model type based on $95 \% \mathrm{Cls}$. Even the most flexible models yield AUCs with only modest discrimination (AUC between 0.61 and 0.66 ) for intermediate outcomes. MLR, OLR, and DeepO predictions are well-calibrated to true GOSE distributions, but DeepM learns to negatively bias predictions of GOSE 8 to increase sensitivity to intermediate outcomes. Classification performance is consistently poor (mean accuracy $\leq 46 \%$ ) due to excessive categorisation into GOSE 1 or GOSE 8 effectively reducing a multiclass problem to a dichotomous one. Based on paired Wilcoxon tests for predictor significance $(a=0.05)$ age, motor Glasgow Coma Score, Marshall CT classification, and pupil reactivity are the most significant while the lab values (glucose and $\mathrm{Hb}$ ) and presence of an epidural haematoma are the least.

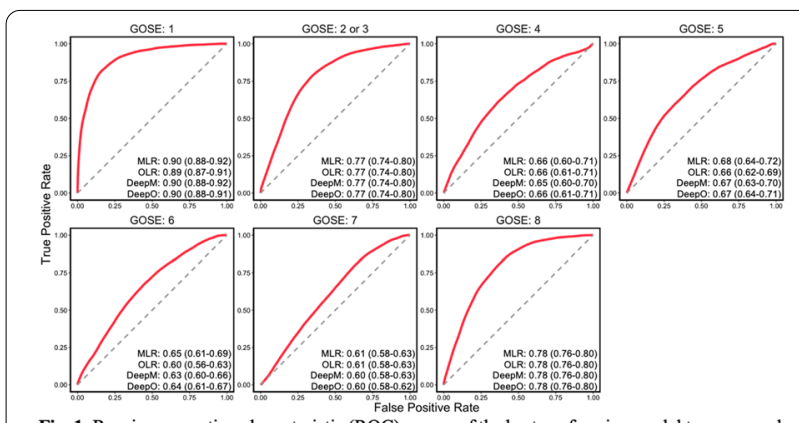

Fig. 1: Receiver operating characteristic (ROC) curves of the best-performing model type per each GOSE score at 6 months post-injury on the validation sets. The values in each box represent the mean area under the curve (with $95 \%$ confidence interval in parentheses) per model type.

Conclusion. Since the poor performance of GOSE prediction models is independent of complexity and outcome encoding, it follows that features known to predict dichotomous outcomes are insufficient for multiclass prediction. This result suggests that either admission characterisation is incomplete for intermediate outcomes or these outcomes are better explained by events during ICU stay. This motivates the search for a better classification of TBI that is related to a more nuanced understanding of outcome.

\section{Reference(s)}

1. [2] Maas AIR, Menon DK, Steyerberg EW et al. (2014) Collaborative European NeuroTrauma Effectiveness Research in Traumatic Brain Injury (CENTER-TBI): A Prospective Longitudinal Observational Study. Neurosurgery. https://doi.org/10.1227/NEU.0000000000000575

2. [1] McMillan T, Wilson L, Ponsford J, Levin H, Teasdale G, Bond M (2016) The Glasgow Outcome Scale - 40 years of application and refinement. Nature Reviews Neurology. https://doi.org/10.1038/nrneurol.2016.89

3. [5] Lundberg SM, Lee S (2017) A Unified Approach to Interpreting Model Predictions. NIPS'17: Proceedings of the 31st International Conference on Neural Information Processing Systems 30:4765-4774. https://doi.org/10 $5555 / 3295222.3295230$

4. [4] Steyerberg EW, Mushkudiani N, Perel P et al. (2008) Predicting Outcome after Traumatic Brain Injury: Development and International Validation of Prognostic Scores Based on Admission Characteristics. PLOS Medicine. https://doi.org/10.1371/journal.pmed.0050165

5. [3] Doiron D, Marcon Y, Fortier I, Burton P, Ferretti V (2017) Software Application Profile: Opal and Mica: open-source software solutions for epidemiological data management, harmonization and dissemination. Int J Epidemiol. https://doi.org/10.1093/ije/dyx180

6. S.B. is a recipient of the Gates Cambridge Scholarship. S.B. would like to thank Abhishek Dixit (University of Cambridge) for helping him extract the data.

\section{3}

An Al-based cardiovascular monitoring tool for sepsis identification in ICU

M. Mollura'; LW. Lehman ${ }^{2}$; R. Barbieri ${ }^{3}$

${ }^{1}$ DEIB, Politecnico di Milano, Milano, Italy; ${ }^{2}$ Institute for Medical Engineering \& Science (IMES), Massachusetts Institute of Technology, Cambridge, United States of America; ${ }^{3}$ Dipartimento di elettronica, informazione e bioingegneria, Politecnico di Milano, Milan, Italy

Correspondence: M. Mollura

Intensive Care Medicine Experimental 2021, 9(1): 000433 
Introduction. Sepsis is one of the major causes of mortality in ICU with an occurrence up to $40 \%$ worldwide [1]. It is considered a serious public health issue with 1 in 3 hospitalizations that end in death with sepsis [2]. Sepsis showed a strong influence on cardiovascular functioning in terms of both myocardial and cardiac autonomic dysfunction [3]. The major effects of sepsis on the cardiovascular system can be summarized as follows: a systolic and diastolic cardiac dysfunction, an increased heart rate despite an overall reduction in autonomic modulation of heart activity and an impairment in the baroreflex sensitivity.

Objectives. Our study is aimed at exploring the ability of ECG and arterial blood pressure (ABP) waveforms, recorded in the first hour of ICU stay, in recognizing patients with sepsis with an Al-based physiological and cardiovascular monitoring tool.

Methods. We extracted the first hour of ECG and ABP waveforms of patients admitted in the ICU, from the publicly available MIMIC III database on PhysioNet [4,5]. The final population includes 142 patients, $50 \%$ of whom with sepsis.

The ECG and ABP signals were processed in order to extract the R-peak occurrences from the ECG and the systolic, diastolic and onset occurrences and values from the pressure signal. 68 features were extracted from the heart rate and blood pressure variability domain through mathematical modelling of the closed loop cardiovascular system, which allowed also for the extraction of baroreflex gain [6]. Finally, 7 confoundings comprising the undergoing sedative and vasoactive agent treatment and mechanical ventilation as well as age, gender, diabetes and hypertension were included.

A logistic regression model was then trained on a $80 \%$ training set and tested on the remaining $20 \%$ of data.

Results. Best results on the test set show an $A U R O C=0.91$ and AUPRC $=0.90$, thus highlighting the ability of continuously recorded vital signs in recognizing septic patients.

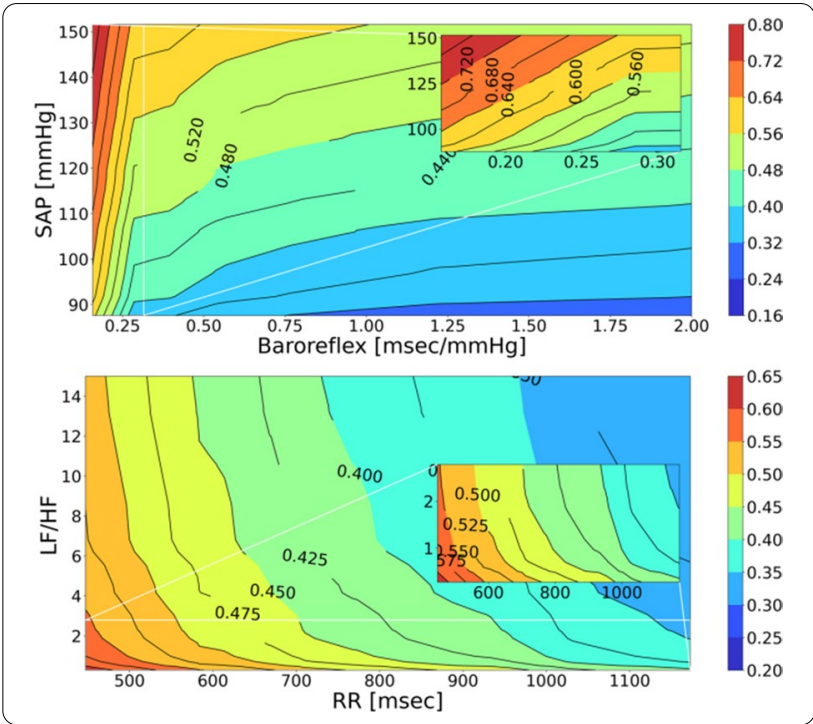

The figure depicts the results of the explainability analysis on model decision rules. The model's strategy results to be consistent with previously summarized clinical considerations about sepsis. Indeed, a lower (impaired) baroreflex gain despite the high systolic pressure values is associated with a higher probability of being septic. Similarly, the second plot shows that an increased heart rate (low RR-interval) is associated with higher sepsis probability and this probability increases when a reduced sympatho-vagal balance (LF/HF) is observed, corresponding to a reduced sympathetic activity or to an overall reduction of autonomic activity.

Conclusion. Our results show that continuously recorded patients' vital signs may help in automatic early identification of sepsis in the early hours of ICU stay. The proposed Al-based physiological monitoring also allows for the extraction of indices able to describe the state of the cardiovascular control system and providing key insights on cardiovascular functioning.

\section{Reference(s)}

1. [1]: Sakr, Y., Jaschinski, U., Wittebole, X., Szakmany, T., Lipman, J., Ñamendys-Silva, S. A., Martin-Loeches, I., Leone, M., Lupu, M.-N., \& Vincent, J.-L. (2018). Sepsis in Intensive Care Unit Patients: Worldwide Data From the Intensive Care over Nations Audit. Open Forum Infectious Diseases, 5(12). https://doi.org/10.1093/ofid/ofy31

2. [2]: Fay K, Sapiano MRP, Gokhale R, et al. Assessment of Health Care Exposures and Outcomes in Adult Patients With Sepsis and Septic Shock. JAMA Netw Open. 2020; 3(7): e206004. https://doi.org/10.1001/jaman etworkopen.2020.6004

3. [4]: Johnson, A. E. W., Pollard, T. J., Shen, L., Lehman, L. H., Feng, M., Ghassemi, M., Moody, B., Szolovits, P., Anthony Celi, L., \& Mark, R. G. (2016). MIMIC-III, a freely accessible critical care database. Scientific Data, 3(1). https://doi.org/10.1038/sdata.2016.35

4. [5]: Goldberger, A. L., Amaral, L. A., Glass, L., Hausdorff, J. M., Ivanov, P. C., Mark, R. G., Mietus, J. E., Moody, G. B., Peng, C. K., \& Stanley, H. E. (2000). PhysioBank, PhysioToolkit, and PhysioNet: components of a new research resource for complex physiologic signals. Circulation, 101(23), E215-E220. https://doi.org/10.1161/01.cir.101.23.e215

5. [6]: Chen, Z., Purdon, P.. Pierce, E., Harrell, G., Brown, E., \& Barbieri, R. (2009). Assessment of Baroreflex Control of Heart Rate During General Anesthesia Using a Point Process Method. Proceedings of the ... IEEE International Conference on Acoustics, Speech, and Signal Processing. ICASSP (Conference), 2009, 333-336. https://doi.org/10.1109/ICASSP.2009.49595

6. [3]: Wang H. (2019). Cardiac Autonomic Nervous System and Sepsis-Induced Cardiac Dysfunction. In: Fu X., Liu L. (eds.) Severe Trauma and Sepsis. Springer, Singapore. https://doi.org/10.1007/ 978-981-13-3353-8_6

\section{0}

Decoding digital health signatures for prediction of delirium in the intensive care unit

K. Gong, ; R. Lu' ; T. Bergamaschi'; A. Sanyall'; J. Guo ; H. Kim² ${ }^{2}$ R. Stevens ${ }^{2}$

${ }^{1}$ Biomedical Engineering, Johns Hopkins University, Baltimore, United States of America; ${ }^{2}$ Anesthesiology and Critical Care Medicine, Johns Hopkins University, Baltimore, United States of America

Correspondence: R. Stevens

Intensive Care Medicine Experimental 2021, 9(1): 000450

Introduction. ICU delirium is frequent, associated with unfavorable outcomes, increased costs, and may be preventable. There is an unmet need for accurate methods to predict risk of delirium. Here, we trained machine learning models to predict ICU delirium using physiological and clinical features in large clinical database.

Objectives. The aim of this study was to leverage machine learning applied to early physiological and clinical data to predict delirium onset using physiological and clinical features in large clinical database.

Methods. The first model (A) was designed to predict delirium onset at any time during the ICU stay. Data from the first $24 \mathrm{~h}$ following ICU admission were extracted from the multi-center elCU database. A second "dynamic" model (B) was built to predict onset of delirium in the next hour to twelve hours. Features extracted from data included demographics, medical history, labs, medications, nurse charting, comorbidities, treatments, and physiological time series. Similar features were extracted from the MIMIC-III database for external validation. The top features were analyzed using logistic regression (LR), random forest (RF), or Shapley values, and then a statistically pruned feature space for each model was obtained. These pruned feature spaces included about 150 variables, with slight variations depending on the model, lead time, and data window used. For both models, three algorithms were evaluated: LR, RF, and gradient boosting [CatBoost]). Model performance was evaluated using nested crossvalidation and area under the receiver operating characteristic curve 
(AUROC), precision-recall, and calibration metrics, and compared to the established PRE-DELIRIC model.

Results. 27,939 patient stays that had delirium scoring were identified. For model A, the mean AUROC (95\% confidence interval) was 0.785 $(0.767,0.802)$, obtained using the Catboost algorithm, and significantly higher than PRE-DELIRIC with a mean AUROC of $0.732(0.704,0.760)$ $(p=0.003)$. This model validated successfully in the MIMIC-III population (AUROC of 0.799). Model B had a mean AUROC of 0.890 (0.879, 0.900 ) when predicting delirium $1 \mathrm{~h}$ in advance. Performance was sustained in the MIMIC-III dataset, with an AUROC of 0.888. Among the top features in both models were GCS, RASS, APACHE scores, and age. Physiological time series features did not increase either model's performance.

Conclusion. Machine learning applied to features from the first $24 \mathrm{~h}$ after admission predicted ICU delirium with clinically relevant accuracy and higher than the reference PRE-DELIRIC score. Results suggest that high-resolution data contain information on delirium risk overlooked in current prediction systems. A dynamic model applied to features from hours before the onset of delirium had even greater predictive performance. These results warrant prospective validation and integration with expanded feature domains such as neurophysiological time series and neuroimaging.

\section{Reference(s)}

1. Salluh, J. I. F., Wang, H., Schneider, E. B., Nagaraja, N., Yenokyan, G., Damluji, A., ... Stevens, R. D. (2015). Outcome of delirium in critically ill patients: Systematic review and meta-analysis. BMJ (Online), 350, 1-10. [h2538]. https://doi.org/10.1136/bmj.h2538 Oh, E. S., Fong, T. G., Hshieh, T. T., \& Inouye, S. K. (2017). Delirium in Older Persons: Advances in Diagnosis and Treatment. JAMA, 318(12), 1161-1174. https://doi.org/10.1001/jama. 2017.12067

\section{8}

\section{A Machine Learning Model to Predict Responsiveness} in Non-Neurological ICU Patients

H. Xu'; J. Desman '; M. Igboko'; Q. Huang ${ }^{1}$; Z. Wang ${ }^{1}$; A. Kodibagkar'; R. Winslow'; J. Greenstein ${ }^{1}$; R. Stevens ${ }^{2}$

'Biomedical Engineering, Johns Hopkins University, Baltimore, United States of America; ${ }^{2}$ Anesthesiology and Critical Care Medicine, Johns Hopkins University, Baltimore, United States of America

Correspondence: $\mathrm{H}$. Xu

Intensive Care Medicine Experimental 2021, 9(1): 000518

Introduction. The acute loss of responsiveness is a frequent medical emergency that requires rapid and precise intervention to maximize the chances of survival, awakening, and neurological recovery. It is commonly associated with a primary neurological disorder, such as stroke or traumatic brain injury; however, decreased responsiveness is also frequently seen in critically ill patients without a primary neurological disorder.

Objectives. To predict the level of responsiveness at discharge in patients admitted to the ICU without a primary neurological diagnosis. We hypothesized that accurate prediction can be achieved by training machine learning classifiers with high-resolution data acquired in the first $24 \mathrm{~h}$ following ICU admission.

Methods. We leveraged the elCU database $(200,859$ ICU stays) as a train-and-test set and the MIMIC-IV database $(69,619$ ICU stays) for external validation. We included adult patients who did not have a primary neurological diagnosis at ICU admission and whose ICU length of stay was at least 2 days and no more than 7 days. The level of responsiveness was evaluated using the motor subscore of the Glasgow Coma Scale (mGCS). Binary classification models were trained on the full population and subgroups classified by the responsiveness levels at ICU admission: responsive admissions (mGCS =6; RA Group), and unresponsive admissions ( $\mathrm{mGCS}<6$; UA Group). Feature selection removed features with coverage $<85 \%$ and imputed the remaining with medians. The final feature space contained physiological signals, lab values, medications, and demographics extracted from the first $24 \mathrm{~h}$ of ICU stay. After performance comparisons, we chose gradient boosted decision trees (GB) to predict the level of responsiveness at ICU discharge.

Results. The preprocessed dataset consisted of 37,568 ICU stays (RA Group: 28,905; UA Group: 8,663) for elCU and 20,127 ICU stays (RA Group: 12,559; UA Group: 7,568) for MIMIC-IV. After fivefold cross validation, we evaluated the models with median AUROC, and GB trained on the full population had the best performance (Fig. 1). Other model performance metrics are provided in Table 1. Predictive features which were consistently highly ranked included physiological signals such as respiration rate, systemic blood pressure and heart rate, and lab features, including blood urea nitrogen and red blood cell count.

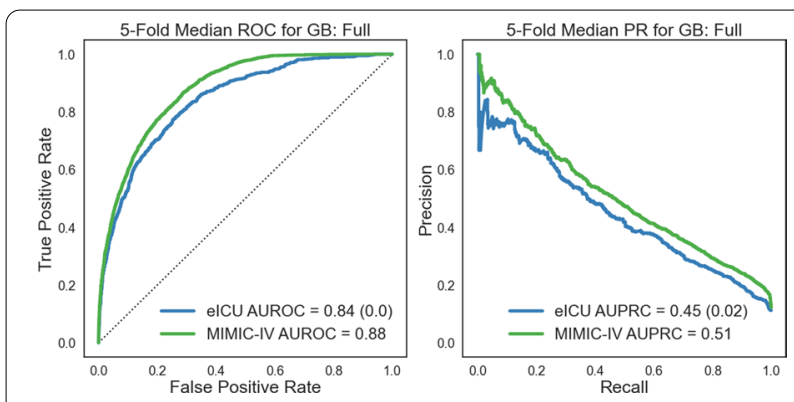

Figure 1.5-Fold Median Performance Curves for Full Population.

\begin{tabular}{|c|c|c|c|c|c|c|}
\hline \multirow{2}{*}{$\begin{array}{l}\text { Performance } \\
\text { Metrics }\end{array}$} & \multicolumn{2}{|c|}{ RA Group } & \multicolumn{2}{|c|}{ UA Group } & \multicolumn{2}{|c|}{ Full Population } \\
\hline & elCU & MIMIC-IV & eICU & MIMIC-IV & elCU & MIMIC-IV \\
\hline Sensitivity & 0.77 & 0.85 & 0.69 & 0.76 & 0.77 & 0.78 \\
\hline Specificity & 0.71 & 0.82 & 0.72 & 0.75 & 0.75 & 0.79 \\
\hline $\begin{array}{l}\text { Positve Predic- } \\
\text { tive Value }\end{array}$ & 0.15 & 0.22 & 0.45 & 0.41 & 0.27 & 0.31 \\
\hline $\begin{array}{l}\text { Negative } \\
\text { Predictive } \\
\text { Value }\end{array}$ & 0.98 & 0.99 & 0.88 & 0.93 & 0.97 & 0.97 \\
\hline Brier Score & 0.05 & 0.18 & 0.15 & 0.12 & 0.07 & 0.07 \\
\hline
\end{tabular}

Table 1.5-fold Median Performance Metrics for Population Groups.

Conclusion. In patients admitted to the ICU with a non-neurological diagnosis, a machine learning model using data collected in the first $24 \mathrm{~h}$ of ICU admission can accurately predict responsiveness at discharge. These predictions could inform strategies to prevent neurological deterioration or enhance neurological function during critical illness.

\section{Reference(s)}

1. Goldberger, A., Amaral, L., Glass, L., Hausdorff, J., Ivanov, P. C., Mark, R. \& Stanley, H. E. (2000). PhysioBank, PhysioToolkit, and PhysioNet: Components of a new research resource for complex physiologic signals. Circulation [Online]. 101 (23), pp. e215-e220.

2. Johnson, A., Bulgarelli, L., Pollard, T., Horng, S., Celi, L. A., \& Mark, R. (2020). MIMIC-IV (version 0.4). PhysioNet. https://doi.org/10.13026/a3wn-hq05.

3. The elCU Collaborative Research Database, a freely available multi-center database for critical care research. Pollard TJ, Johnson AEW, Raffa JD, Celi LA, Mark RG and Badawi O. Scientific Data (2018). http://dx.doi.org/10. 1038/sdata.2018.178.

4. Pollard, T., Johnson, A., Raffa, J., Celi, L. A., Badawi, O., \& Mark, R. (2019). elCU Collaborative Research Database (version 2.0). PhysioNet. https:// doi.org/10.13026/C2WM1R 
000621

Rapid Viewing and Annotation of Large High-Density Time Series Datasets

G. Welter ${ }^{1}$; A. Wertz ${ }^{1}$; V. Leonard ${ }^{1}$; S. La Vattiata ${ }^{1}$;T. Pellathy, 2 ; S. Helman²; M Hravnak $^{2}$; G. Clermont ${ }^{3}$; M. Pinsky ${ }^{3}$; A. Dubrawski ${ }^{1}$

${ }^{1}$ Auton Lab, School of Computer Science, Carnegie Mellon University, Pittsburgh, United States of America; ${ }^{2}$ School of Nursing, University of Pittsburgh, Pittsburgh, United States of America; ${ }^{3}$ School of Medicine, University of Pittsburgh, Pittsburgh, United States of America

Correspondence: $G$. Welter

Intensive Care Medicine Experimental 2021, 9(1): 000621

Introduction. Large multi-domain, multi-resolution timeseries datasets, prevalent in applications from Artificial Intelligence (AI) to decision support in intensive care, are particularly challenging to distribute for review, analysis, and annotation by experts due to their size, bandwidth limitations, and the need for specialized software to process them. Yet these datasets are foundational to Al-augmented decision support in data-rich clinical environments. The ability to interactively reveal useful information and prepare it for training Al models is required to reap benefits of using such data in practice.

Objectives. We present an open-source, versatile, scalable, portable web-based interactive tool for Al researchers and clinicians to interactively analyze large multivariate time series datasets.

Methods. We built an open-source web-based tool for viewing, analyzing, and annotating large, multi-domain time series datasets [1], and applied it to electronic health record and telemetry data. The too handles arbitrarily large amounts of data and performs efficiently over standard internet connections. It optimizes usage of computer memory and user query response vs. amount of data storage. Multi-resolution representations are computed upon loading the data, enabling browsing it at interactive speeds. Files are stored in interoperable HDF5 [2]-based AUData [3] or CCDEF [4] formats. Efficiencies of design and data management enable the tool to run on common computer hardware. A clinically intuitive web-based graphical user interface is portable and can be used on a local computer or by remote clients on any operating system with a web browser and network connection.

Results. The tool serves three main functions: review, analysis, and annotation. For review, it presents time series graphically using a cus tomizable user interface allowing for rapidly panning and zooming to any timescale desired. For analysis, the tool has a well-documented application programming interface through which analysts can manage projects and files, run analyses, browse results, and create pattern sets and annotation assignments. An extendable library of analytic functions includes configurable exceedance detection and signal quality assessment functions. The tool is currently used to support team-based clinical data annotation for training Al models in applications including bedside artifactual alert adjudication, forecasting cardio-respiratory insufficiency, and phenotyping response to stress and treatment in intensive care medicine.

Conclusion. The Auton Universal Viewer (AUViewer) [5] is an opensource tool designed to accelerate reviewing, analyzing, and annotating large multi-domain multi-resolution time series data, thus making data accessible, interoperable, and reusable [6]. We encourage its broad use across healthcare to facilitate clinical annotation, and boost productivity of analysts, researchers, and developers building Al-driven clinical decision support systems.

\section{Reference(s)}

1. Wilkinson MD et al. The FAIR Guiding Principles for scientific data management and stewardship. Sci Data [Internet]. 2016 Mar 15

2. Software available at https://github.com/autonlab/auviewer

3. CCDef. Available from: https://ccdef.org

4. AUData. Available from: https://github.com/autonlab/audata

5. HDF5. Available from: https://www.hdfgroup.org/solutions/hdf5/

6elter $\mathrm{G}$ et al. Auton Universal Viewer, arXiv, 2021

7. This work has been supported by DARPA (FA8750-17-2-0130), clinical context has been supported by NIH (1R01-GM117622-03, 2R01-NR013912-07)

\section{8}

Escalation in the elderly for COVID-19: Predicting outcomes for those at risk

N. Plummer ; J. West, 2 ; T. Card, ${ }^{2}$; C. Crooks, ${ }^{2}$

${ }^{1}$ Critical Care, Queen's Medical Centre Nottingham, Nottingham, United Kingdom; ${ }^{2}$ School of medicine, University of Nottingham, Nottingham, United Kingdom

\section{Correspondence: N. Plummer}

Intensive Care Medicine Experimental 2021, 9(1): 000838

Introduction. Although COVID-19 disproportionately impacts older adults [1] there is limited data on outcomes in this cohort to guide appropriatate decision making regarding escalation of care.

Objectives. We wished to better understand risk factors for poor outcomes to inform decision making regarding escalation to critical care in the elderly suffering from SARS-CoV-2 infection.

Methods. We conducted a retrospective cohort study of all patients admitted to Nottingham University Hospitals aged 65 or over when SARS-CoV-2 was diagnosed. Data were extracted from all available electronic records, linked, and anonymised. Focusing on patients felt suitable for escalation, we developed a logistic regression mode to identify risk-factors for mortality at 28 days. Initial models of mortality dependent purely on admission NEWS2 [2] and ISCARIC-4C[3]) were used, with all other covariates available to the model selection algorithm.

Results. Of 3,768 patients over $65,1,351$ were felt to be suitable for escalation. In hospital mortality was $16 \%$. Admission NEWS2 $(A \cup C=0.706)$ and ISCARIC-4C $(A \cup C=0.672)$ are comparable in predicting 28 day mortality $(\mathrm{p}=0.215)$. When including BMI (and age and comorbidities with NEWS2, as already included in ISCARIC-4C) in the model, both models improved, with NEWS2 (AUC $=0.767$ ) outperforming ISCARIC-4C (AUC $=0.717, p=0.005)$.

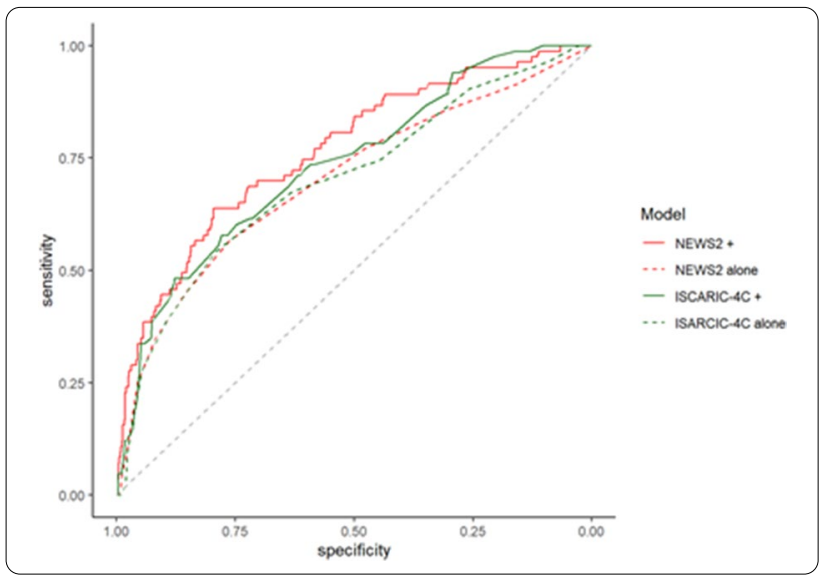

Conclusion. NEWS2 has a stronger discriminative power than ISARIC4C in predicting 28-day mortality from COVID-19 the elderly. Age, comorbidities, and low BMI are all strongly associated with mortality, potentially reflecting physiological reserve. We suggest adequate reserve may therefore be the primary driver of decision making in the elderly, and express caution in applying COVID-19 specific scoring systems derived using younger patients in this cohort.

Reference(s)

1. 1. Daoust J-F (2020) Elderly people and responses to COVID-19 in 27 Countries. PLoS ONE 15(7): e0235590. https://doi.org/10.1371/journal. pone.023559

2. 2. Carr, E., Bendayan, R., Bean, D. et al. Evaluation and improvement of the National Early Warning Score (NEWS2) for COVID-19: a multihospital study. BMC Med 19, 23 (2021). https://doi.org/10.1186/ s12916-020-01893-3

3. 3. Knight S R, Ho A, Pius R, Buchan I, Carson G, Drake T M et al. Risk stratification of patients admitted to hospital with covid-19 using the ISARIC 
WHO Clinical Characterisation Protocol: development and validation of the 4C Mortality Score BMJ 2020; 370:m3339 https://doi.org/10.1136/ bmj.m3339

\section{5}

Using Electronic Patient Records to Automate and Improve

Trainee Logbooks

G. Singh'; R. Osullivan²; A. Parker, 2; A. Wilson,2 ; J. Hanison,3, A. Martin,

${ }^{1}$ Critical Care, Manchester University NHS Foundation Trust, Manches-

ter, United Kingdom; ${ }^{2}$ Adult Critical Care, Manchester Royal Infirmary,

Manchester, United Kingdom; ${ }^{3}$ Critical Care, Manchester Royal Infirmary,

Manchester, United Kingdom

Correspondence: $\mathrm{G}$. Singh

Intensive Care Medicine Experimental 2021, 9(1): 001065

Introduction. UK trainees in Intensive Care Medicine (ICM) are required to keep a logbook of cases they manage and this is part of the Annual Review of Competency Progression throughout their training.[1] Typically, log books are manually recorded which may be time consuming and lacks any practical way for trainers to validate the contents. The literature demonstrates that logbooks can be difficult to maintain with recall deteriorating over time.[2].

Electronic patient records (EPR) offer a theoretical ability to automate this process. Our unit sets out to establish an electronic logbook for trainees using routinely collected information in order to reduce the time required from trainees as well as improve accuracy and validity of the data.

Methods. Data was extracted from our IntelliSpace critical care information system (Philips NC, Netherlands) using structured query language (SQL). We recorded document author, time stamp, type of form being completed and a patient episode identifier. Details of procedures were obtained from structured data fields within forms. ICNARC data about primary and secondary admission diagnoses were obtained from a separate database maintained by our data team. $\mathrm{R}$ markdown was used to construct a logbook for each trainee with a single change in clinician identification code updating all other data. [3] This is then published as a PDF document. We included frequency tables for each clinical document type, intubations (including time and date performed) and central line placement (including line type and line site). A record of cases is produced based on the presence of a significant clinical review (e.g. daily review). A list of primary and secondary ICNARC diagnoses is provided but no other patient identifiers. We undertook an iterative approach to improvements over 18 months based on informal feedback from trainees and Supervisors after each rotation. At this point we surveyed all ICM trainees in the Northwest region (England) to gain an understanding as to the attitudes of trainees towards logbooks.

Results. 18 ICM trainees responded to the survey (Table 1). All trainees reported currently using a logbook app or excel spreadsheet to maintain their logbook. 13/18 (73\%) reported significant difficulty in keeping their logbook up to date. The majority of trainees reported dedicating at least one hour per week to maintaining their logbook though there was considerable variation. 12/18 (67\%) reported that they would prefer an automated logbook and 14/18 (78\%) reported that they would like to be able to compare their own data with their peers.

Conclusion. Current standard practice is for trainees to self-record their logbook but most trainees would prefer an automated electronic logbook. As more units move towards EPR's, automatically generated logbook should become the standard of practice as they are efficient, verifiable and reduce workload for trainees. We describe one such method which has been successfully implemented in our unit.

Reference(s)

1. 1. Intensive Care Medicine Curriculum for a CCT In.; FICM 2019. Accessed May 16, 2021. www.ficm.ac.uk

2. 2. Schüttpelz-Brauns K, Narciss E, Schneyinck C, et al. Twelve tips for successfully implementing logbooks in clinical training. Med Teach. 2016;38(6):564-569. https://doi.org/10.3109/0142159X.2015.1132830

3. 3. JJ Allaire and Yihui Xie and Jonathan McPherson and Javier Luraschi and Kevin Ushey and Aron Atkins and Hadley Wickham and Joe Cheng and Winston Chang and Richard lannone (2020). rmarkdown: Dynamic Documents for R. R package version 2.3. URL https://rmarkdown.rstudio. com.

\section{8}

A study of factors associated with ICU patient's expenditure in a Japanese university hospital: a retrospective, single-center study

S. ikumi ${ }^{1}$;. T. Shiga ${ }^{2}$;Y. Iwasaki ${ }^{3}$; Y. Kaiho ${ }^{4}$; K. Saito ${ }^{2}$; M. Yamauchi ${ }^{1}$

${ }^{1}$ Anesthesiology and Perioperative Medicine, Tohoku University Graduate School of Medicine, Sendai, Japan; ${ }^{2}$ Intensive Care of Medicine, Tohoku University Hospital, Sendai, Japan; ${ }^{3}$ Intensive Care Unit, Jichi University Saitama Medical Center, Saitama, Japan; ${ }^{4}$ Anesthesiology Department, Tohoku University Hospital, Sendai, Japan

Correspondence: S. ikumi

Intensive Care Medicine Experimental 2021, 9(1): 001118

Introduction. Nursing Care Needs (NCN) is a scoring system unique to Japan, in which scores are assigned based on monitoring, procedures, and patient conditions and is reflected in the number of medical fees in the ICU. However, the correlation between the level of NCN and the amount of expenditure is not evident.

Objectives. The objectives of this study were: 1) to evaluate the correlation between NCN and the expenditure, 2) to evaluate the correlation between SOFA, APACHEIl, and the expenditure, although it has been reported that the expenditure is higher in patients with higher physiological severity, and 3) to examine the characteristics of patients with higher expenditure.

Methods. The present study was conducted in the 30-bed ICU of "Tohoku University Hospital" between April 1, 2017, and March 31, 2018. The data sources were the diagnosis procedure combination/ per-diem payment system (DPC/PDPS), patient-based administrative data, including clinical information and claims. For each patient, income, expenditure, and deficit were calculated. The debt was categorized into the top 10\% deficit (Large deficit group; LDG) and the remaining (Non-Large deficit group; NLDG), and the patient background was analyzed. The departmental cost accounting was calculated using a top-down approach. Using a bottom-up approach, we also calculated the income as a department. Parameters were evaluated with Pearson's Chi-squared test or Wilcoxon signed-rank test. Spearman's correlation coefficients were calculated for the amount of expenditure per patient, and NCN, SOFA, and APACHEII, $p<0.05$, were considered significant. All analyses were conducted the statistical software package $\mathrm{R}$ version 4.0.2.

Results. 2077 patients underwent this analysis. The median [IQR] of NCN was 14 [11-19], SOFA was 2 [1-4], APACHEIl was 12 [9-15]. The surgical patients were fewer in LDG (LDG $48.5 \%$ vs. NLDG $80.1 \%$; $p<0.001$ ). Length of stays in ICU was longer in LDG (LDG 10.59 days 
vs. NLDG 3.09 days; $p<0.001)$. Mortality was significantly higher in LDG (LDG 9.6\% vs. NLDG $2.1 \% ; p<0.001)$. NCN and total expenditure of hospitalization in ICU did not correlate $(\rho=0.0168, p=0.44)$. In contrast, SOFA and APACHEII both correlated with the expenditure (SOFA: $\rho=0.402, p<0.001$, APACHEll: $\rho=0.372, p<0.001$ ). According to the cost accounting result, the ICU department's total expenditure was $\$ 10,935,569$ (2.85\% of the hospital expenditure). As for the breakdown of ICU department, labor was $\$ 6,940,937$ (63.4\% of the ICU expenditure), medicine was $\$ 1,297,733(11.8 \%)$, medical consumption article was $\$ 1,245,532(11.3 \%)$, commission was $\$ 713,455(6.5 \%)$, and depreciation was $\$ 737,912(6.74 \%)$. The income as a department was $\$ 7,688,7144$, and the department had a negative balance.

Conclusion. The amount of expenditure for ICU patients did not correlate with NCN but was related to SOFA and APACHE II. LDG was characterized by many non-operative patients, extended ICU stays, and high mortality.

\section{Reference(s)}

1. JSPS KAKENHI Grant Numbers 19K18311

2. Institute for Health Economics and Policy

\section{1}

\section{Developing Interpretable Mortality Prediction Models}

\section{for Intensive Care}

L. Cowan'; L. Moss' $;$ K. Puxty²; M. Shaw ${ }^{2}$

${ }^{1}$ School of Medicine, Dentistry and Nursing, University of Glasgow Glasgow, United Kingdom; ${ }^{2}$ Academic Unit of Anaesthesia, Critical Care and peri-operative medicine, University of Glasgow, Glasgow, United Kingdom

Correspondence: L. Cowan

Intensive Care Medicine Experimental 2021, 9(1): 001171

Introduction. Mortality prediction in the intensive care unit (ICU) using scores such as APACHE II is integral to assessing patient illness severity and evaluating unit performance [1]. Recently, techniques from the field of machine learning have been applied to ICU patient data to develop models with high levels of predictive accuracy $[2,3]$. However, these models tend to be 'black boxes' (i.e., it is difficult to understand how they make predictions). This lack of interpretability could potentially undermine the clinical acceptance of such approaches [4].

Objectives. To use machine learning techniques to develop interpretable models for the prediction of in-hospital mortality for ICU patients and to assess their performance when compared to the existing APACHE II model.

Methods. Data for 5372 patients admitted to the ICU at Glasgow Royal Infirmary from 2014 to 2019 were extracted from electronic record systems and de-identified. The dataset was cleaned and divided into training data (67\%) and testing data (33\%). Machine learning algorithms were applied to patient data to produce an intrinsically interpretable decision tree (DT) model, in addition to three 'black box' models-a random forest (RF), an artificial neural network (ANN), and a gradient boosting machine (GBM), for which variable importance was determined post-hoc. The hyperparameters of each model were tuned via grid-search resampling of the training data using 5 -fold cross-validation. The final optimised model was trained on the complete training set. In the testing data, discrimination of each model was determined using the area under the receiving operator characteristic curve (AUROC) and calibration by the Brier score.

Results. No significant differences were found between the AUROCs of the RF, ANN, GBM and APACHE Il; all models had higher AUROCs than the DT. The RF and GBM had lower Brier scores than APACHE Il; calibration of the DT was not significantly different to that of APACHE II. All models had lower Brier scores than the ANN. In post-hoc interpretation analyses, the most important variable for all black box models to make their predictions was the motor component of the Glasgow coma scale.

Conclusion. Results demonstrate the trade-off between interpretability and accuracy; while the simple DT model is fully interpretable, its predictions are less accurate than the more complex models. The APACHE II model, being fully interpretable and having comparable accuracy to the 'black box' models, remains the optimal solution.

\section{Reference(s)}

1. [1] Knaus WA, Draper EA, Wagner DP, Zimmerman JE. APACHE II: A severity of disease classification system. Crit Care Med. 1985;13(10):818-29.

2. [2] Holmgren G, Andersson P, Jakobsson A, Frigyesi A. Artificial neural networks improve and simplify intensive care mortality prognostication: A national cohort study of 217,289 first-time intensive care unit admissions. J Intensive Care. 2019;7(1):1-8

3. [3] Pirracchio R, Petersen ML, Carone M, Rigon MR, Chevret S, van der Laan MJ. Mortality prediction in intensive care units with the Super ICU Learner Algorithm (SICULA): A population-based study. Lancet Respir Med. 2015:3(1):42-52

4. [4] Elshawi R, Al-Mallah MH, Sakr S. On the interpretability of machine learning-based models for predicting hypertension. BMC Med Inform Decis Mak. 2019;19(1):146.

\section{0}

Mortality Prediction Models for Patients With COVID-19 Based on Their First Day of Admission to the Intensive Care Unit: A Retrospective Study in a Public Hospital in Barcelona

R. Rigo-Bonin ${ }^{1}$; VD. Gumucio Sanguino ${ }^{2}$; S. Ventura-Pedret ${ }^{1}$; AL. Corral ${ }^{2}$; SM. Pons ${ }^{2}$; JL. Pérez Fernández ${ }^{2}$; MP. Fuset ${ }^{2}$; J. Sabater Riera ${ }^{2}$

${ }^{1}$ Clinical Laboratory, Bellvitge Hospital, Hospitalet de Llobregat, Spain; ${ }^{2}$ Intensive Care Medicine, Bellvitge Hospital, Hospitalet de Llobregat,

Spain

Correspondence: V.D. Gumucio Sanguino

Intensive Care Medicine Experimental 2021, 9(1): 001260

Introduction. During the strain of the Coronavirus disease 2019 (COVID-19) pandemia the intensive care units (ICU) in many hospitals were forced to implement triage policies for critically ill COVID-19 patients because of the limited number of beds available. Therewise, it seems essential to identify clinical and analytical factors that may allow adequate prognostication and management of critically ill patients with COVID-19.

Objectives. To propose mortality prediction models based on binary logistic regression (BLR) and artificial neural network (ANN) analyses of data collected in the first $24 \mathrm{~h}$ of intensive care unit (ICU) admission for patients with COVID-19 infection.

Methods. A single-centre, retrospective observational study, in the ICU of a tertiary hospital in Barcelona. Two hundred and forty ICU adult patients with COVID-19 infection admitted between March 15 2020 , and June 30,2020. We collect variables from the first day of ICU admission that can prognosticate a fatal outcome in ICU patients with COVID-19, as well as binary logistic regression (BLR) and artificial neural network (ANN) models to predict the mortality of these patients.

Results. We constructed BLR and ANN mortality prediction models on a random training set of 176 admissions and used the remaining 64 as a validation set. BLR was assessed using the Wald Forward Stepwise method, and the ANN model was constructed using multilayer perceptron architecture. Both models had a good fit (the Hosmer-Lemeshow $C$ statistics were not significant; $p$-values $>0.05$; and the $95 \%$ confidence intervals of the standardized mortality ratio included 1). The ANN model had significantly higher performance than the BLR model for prediction of non-survivor patients (area under the receiver operating characteristic curve value of 0.919 vs $0.770 ; p<0.01$ ). Variables such as age, cancer, previous treatment with mask or nasal conventional oxygen therapy, plasma creatinine, plasma lactate and plasma lactate dehydrogenase were present in both models, and they were important predictors of death in COVID-19 patients based on the first $24 \mathrm{~h}$ of ICU admission.

Conclusion. Our study provides valuable information on which clinical and analytical variables obtained in the first day of ICU admission could predict a fatal outcome for ICU patients with COVID-19. 


\section{5}

Using machine learning to support early prediction of feeding intolerance in critically ill patients

O. Raphaeli' ; C. Hajaj ; I. Bendavid²; A. Goldstein ${ }^{1}$; E. Chen ${ }^{1}$; P. Singer ${ }^{2}$ ${ }^{1}$ Industrial Engineering and Management, Ariel University, Ariel, Israel;

${ }^{2}$ Intensive care unit, Rabin Medical Center, Petakh Tikwa, Israel

Correspondence: $\bigcirc$. Raphaeli

Intensive Care Medicine Experimental 2021, 9(1): 001285

Introduction. Enteral feeding is the default strategy for medical nutritional therapy in the ICU, however not always possible to achieve. Feeding intolerance in enterally fed patients is a common problem, associated with adverse outcomes in critically ill patients. Yet, it is not clear whether enteral feeding intolerance can be predicted in advance and which physiological biomarkers and interventions, can characterize patients that are likely to be intolerant to enteral feeding.

Objectives. The aim of the study is to apply machine learning models to predict enteral feeding intolerance (EFI) in first week using patients characteristics in $72 \mathrm{~h}$ of ICU admission.

Methods. We included adult ICU patients (2012-2018) admitted at the ICU, for more than $48 \mathrm{~h}$, in Beilinson hospital, Israel. EFI in first week is defined according to accepted definitions including the occurrence of Gl symptoms, "large" gastric volumes and "inadequate" delivery of enteral nutrition (Reintam Blaser et al., 2020). Admission conditions, medications and lab results along $72 \mathrm{~h}$ from admission were analyzed by machine learning $(\mathrm{ML})$ classification algorithms. Prediction performance was assessed by the area under the curve (AUROC) of ten-fold cross-validation and validation sets. The study was approved by the ethics committee of Beilinson.

Results. The dataset comprised of 2,026 enterally fed patients. The median (IQR) age was 62 (48-72) years, BMI 26.5 (23-31). Main admission conditions: surgical (47\%), trauma (27\%) and medical (25\%). The prevalence of $\mathrm{Fl}$ in the first week in our cohort was $66 \%$. Five $\mathrm{ML}$ algorithms were trained and tested (Python package software). The best performing algorithm was Extra Trees Classifier that achieved AUC of $88 \%$. The results show that the best input variables to predict $\mathrm{EFI}$ in first week of admission include: Sepsis, BMI > 30, metabolic acidosis, need for vasopressors and high $\mathrm{PaCO}_{2}$, Phosphate and Potassium.

Conclusion. Specific features are associated with intolerance to enteral feeding and machine learning model can recognize them. This may allow the physician to switch to parenteral nutrition earlier and not increase risks of worse outcome.

\section{Reference(s)}

1. Reintam Blaser A., Deane AM, Preiser JC, Arabi YM, Jakob SM. Enteral Feeding Intolerance: Updates in Definitions and Pathophysiology. Nutr Clin Pract. 2021 Feb;36(1):40-49

2. Singer $\mathrm{P}$, Weinberger $\mathrm{H}$, Tadmor $\mathrm{B}$. Which nutritional regimen for the comorbid complex intensive care unit patient? World Rev Nutr Diet 2013;105:169-178

3. Singer P, Blaser AR, Berger MM, Alhazzani W, Calder PC, Casaer MP, Hiesmayr M, Mayer K, Montejo JC, Pichard C, Preiser JC, van Zanten ARH, Oczkowski S, Szczeklik W, Bischoff SC. ESPEN guideline on clinical nutrition in the intensive care unit. Clin Nutr. 2019 Feb;38(1):48-79

4. Bloch E, Rotem T, Cohen J, Singer P, Aperstein Y. Machine Learning Models for Analysis of Vital Signs Dynamics: A Case for Sepsis Onset Prediction. Journal of Healthcare Engineering. 2019 (1): 1-12

5. This study is supported by Fresenius-Kabi.

\section{2}

Overmortality and disproportionate resource use during Covid-19 pandemic in the ICU

NM. Messerich ${ }^{1}$; GR. Kleger ${ }^{1}$

${ }^{1}$ Medical ICU, Kantonsspital St.Gallen, St. Gallen, Switzerland

Correspondence:: N.M. Messerich

Intensive Care Medicine Experimental 2021, 9(1): 001442

Introduction. On March 11th, WHO declared SARS-CoV-2 infection as a pandemic. Switzerland was affected by a first wave (March to
Jul) and a second wave (Oct-Dec) in 2020. A remarkable number of patients developed severe ARDS requiring intensive care unit (ICU) care and mostly invasive mechanical ventilation. ICU resources were severely strained due to high mortality, prolonged ICU length of stay (LOS), lack of ICU beds, need of specialized equipment, and specially trained ICU staff.

To assess the quality and performance of an ICU longitudinally, we regularly track two parameters: Standardized $28 \mathrm{~d}$ mortality rate (SMR) as an outcome parameter and standardized resource use (SRU) as a surrogate marker for effectiveness.

Objectives. Analysis of the outcome and effectiveness during the first and second waves of the Covid-19 pandemic in 2020 compared with previous years.

Methods. We analyzed prospectively obtained data of our quality assessment data set of our 12-bed medical ICU. SMR was calculated longitudinally (2012 to 2020) based on a recalibrated original SAPS II model. SRU was calculated longitudinally using the method of Rothen et al., but using SAPS II. Both models were calibrated specifically to our ICU using pooled patient data from 2007 to 2013, generalized additive models (GAM), and a bootstrap sampling procedure.

Results. In 2020 we cared for 1'170 patients, including 137 with severe SARS-CoV-2 infection. 87 (64\%) of these patients had been ventilated invasively and $22(16 \%)$ had vV-ECMO. $38(28 \%)$ patients died in the hospital and median ICU-LOS was 9 (IQR 3 to 18) days. This resulted in an SMR of 1.05 and SRU of 1.03, which is substantially higher than in previous years when we observed a steadily decreasing SMR and SRU (Fig. 1 and 2).

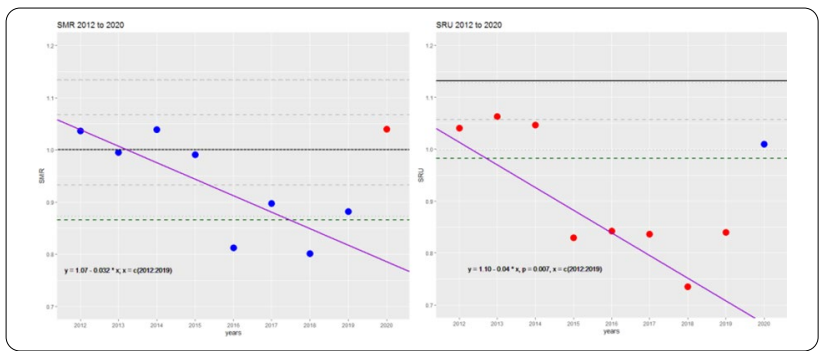

Conclusion. During the first Covid-19 pandemic year, the SMR in our ICU increased considerably compared with previous years, while resource use also increased. This reflects the high proportion of patients with Covid-19 who were hospitalized in the ICU during 2020. Mortality and resource consumption were disproportionally high in the first year of the Covid-19 pandemic.

Reference(s)

1. Rothen HU. Intensive Care Med 2007; 33: 1329-36

e-Posters: Translational Biology

000926

Cardioprotection with thiometallate sulfide donors: a role for antioxidant selenoproteins

J. Holt ${ }^{1}$; M. Singer ${ }^{1}$; A. Dyson

${ }^{1}$ Bloomsbury Institute of Intensive Care Medicine, University College London, London, United Kingdom

Correspondence: A. Dyson

Intensive Care Medicine Experimental 2021, 9(1): 000926

Introduction. Ammonium tetrathiomolybdate (ATTM), a sulfidedonor, modulates metabolism and protects against ischaemia/reperfusion (I/R) injury in rats [1]. Evidence of cross-talk between sulfur and selenium biology has also been described [2].

Objectives. To assess the impact of ATTM on selenoprotein (glutathione peroxidase; GPx) and non-selenoprotein (catalase) antioxidant enzyme activity in heart tissue from pigs subjected to myocardial I/R injury. 
Methods. Tissues were obtained from terminally anaesthetised female Large White pigs $(69 \pm 5 \mathrm{~kg})$ at $24 \mathrm{~h}$-post I/R. The ischaemic insult and therapy comprised left anterior descending coronary artery occlusion for 1-h, and ATTM (or equivalent saline) as an IV bolus and 1-h infusion (onset 12-min before reperfusion), respectively. Biopsy samples were obtained from the ischaemic penumbra and remote [not at risk] left ventricle (LV). GPx and catalase enzyme activities (both detoxify hydrogen peroxide) were assessed biochemically by colorimetry using tissue homogenate and a spectrophotometric plate reader.

Results. ATTM treatment increased GPx activity in both regions (penumbra and remote LV). By contrast, catalase activity, elevated in the penumbra in controls, was unaffected by ATTM-treatment.

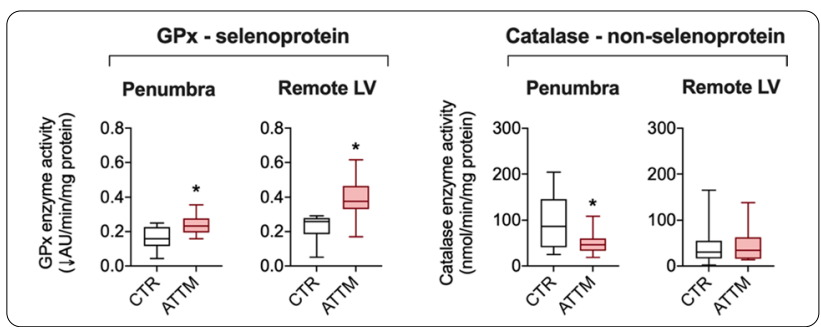

Figure: Glutathione peroxidase (GPx) and catalase enzyme activity. $A U$, arbitrary units; $L V$, left ventricle. Data: median, IQR and range. * $p<0.05$ vs control (CTR); unpaired T-test (Prism 9). Sample size: control, $n=9$; ATTM-treated, $\mathrm{n}=11$.
Conclusion. ATTM-treatment differentially affected the activity of two antioxidant enzymes. A consistent increase in GPx activity indicates a systemic effect from IV administration of ATTM rather than a direct response to I/R. Catalase activity, elevated in the ischaemic penumbra in controls, was unaffected by ATTM treatment, possible due less oxidative stress/damage and/or compensation by selenoprotein activity in treated animals. The elevation in selenoprotein activity with ATTM could represent an additional molecular mode of action that affords cardioprotection in large mammals.

\section{Reference(s)}

1. 2. Greasley A, et al. 2019. Oxid Med Cell Longev. 6494306.

2. 1. Dyson A, et al. 2017. PLoSMED. 14:e1002310.

3. University College London

\section{About this supplement}

These abstracts have been published as part of Intensive Care Medicine Experimental Volume 9 Supplement 1, 2021: ESICM LIVES 2021. The full contents of the supplement are available online at https://icm-experimental.sprin geropen.com/articles/supplements/volume-9-supplement-1. Please note that this is part 2 of 2.

\section{Publisher's Note}

Springer Nature remains neutral with regard to jurisdictional claims in published maps and institutional affiliations. 